\title{
Chemistrll and the

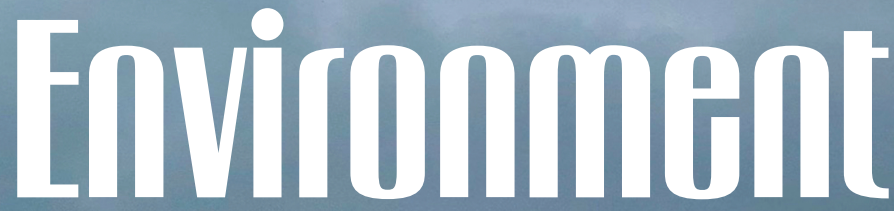 A chemistry perspective for discussion of environmental issues
}

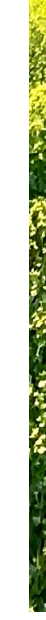




\title{
CHEMISTRY AND
} THE

\section{ENVIRONMENT}

\author{
A Chemistry Perspective for \\ discussion of Environmental Issues
}

\section{DEVIN R LATIMER}

OpenStax/University of Winnipeg Library

Houston, USA/Winnipeg, Canada 


\section{(a) (1)}

Chemistry and the Environment by OSCRiceUniversity/DevinRLatimer is licensed under a Creative Commons Attribution 4.0 International License, except where otherwise noted.

Ebook ISBN 978-0-9868123-4-7

Print ISBN 978-0-9868123-5-4

Chemistry and the Environment by Devin R Latimer (c) August 21, 2020) was adapted from the OpenStax book Chemistry Ze by Paul Flowers, Klaus Theopold, Richard Langley and William R. Robinson at OSCRiceUniversity (c) Jul 19, 2019 OpenStax). Textbook content produced by OpenStax (c) Jul 19, 2019) licensed under a Creative Commons Attribution License 4.0 license, except for the following changes and additions which are (c) August 19, 2020 by Devin R. Latimer, and are licensed under a Creative Commons Attribution License 4.0 license.

Sections Added: Introductory Focus, Focus on Air Pollution, Focus on The Ozone Layer, Focus on Climate Change, Focus on Energy, Focus on Nuclear Energy, Focus on Alternative Energy, Focus on Water Quality, Focus on Acid Precipitation, Focus on Polymers.

Various sections have had paragraphs added and discussions edited.

Sections Removed: 1.5 Measurement Uncertainty, Accuracy, and Precision, 3.2 Determining Empirical and Molecular Formulas, 4.5 Quantitative Chemical Analysis, 5.2 Calorimetry, 5.3 Enthalpy, 10.3 Phase Transitions, 10.4 Phase Diagrams, 10.6 Lattice Structures in Crystalline Solids, 11.4 Colligative Properties, 13.2 Equilibrium Constants, 13.3 Shifting Equilibria, 13.4 Equilibrium Calculations, 14.3 Relative Strengths of Acids and Bases, 14.4 Hydrolysis of Salts, 14.5 Polyprotic Acids, 14.6 Buffers, 14.7 Acid-Base Titrations, 17.2 Review of Redox Chemistry, 17.3 Electrode and Cell Potentials, 17.4 Potential, Free Energy and Equilibrium, 17.6 Corrosion, 17.7 Electrolysis, 20.3 Aldehydes, Ketones, Carboxylic Acids and Esters, 20.4 Amides and Amines, 21.5 Uses of Radioisotopes, Appendix E: Water Properties, Appendix F: Composition of Commercial Acids and Bases, Appendix G: Standard 
Thermodynamic Properties for Selected Substances, Appendix $\mathrm{H}$ : Ionization Constants of Weak Acids, Appendix I: Ionization Constants of Weak Bases, Appendix J: Solubility Products, Appendix K: Formation Constants for Complex lons, Appendix L: Standard Electrode (Half-Cell) Potentials

Chapters Removed: 8. Advanced Theories of Chemical Bonding, 9. Gases, 12. Kinetics, 15. Equilibria of Other Reactions Classes, 16. Thermodynamics, 18. Representative Metals, Metalloids and Nonmetals, 19. Transition Metals and Coordination Chemistry

Partial sections have also been removed and various errata corrected in the editing of this book.

Under this license, any user of this textbook or the textbook contents herein must provide proper attribution as follows:

The OpenStax name, OpenStax logo, OpenStax book covers, OpenStax CNX name, and OpenStax CNX logo are not subject to the creative commons license and may not be reproduced without the prior and express written consent of Rice University. For questions regarding this license, please contact support@openstax.org.

- If you use this textbook as a bibliographic reference, then you should cite it as follows: Chemistry and the Environment by Devin R. Latimer (@ August 21, 2020) adapted from OpenStax, Chemistry 2e. OpenStax CNX. Jul 19, 2019 http://cnx.org/contents/ 7fccc9cf-9b71-44f6-800b-f9457fd64335@7.4.

Cover photo by Steve Schlachter

Cover design by Keri Latimer 


\section{Contents}

Preface xi

Introductory Focus 1

\section{Part I: Essential Ideas}

1. Introduction $\quad 7$

2. Chemistry in Context 9

3. Phases and Classification of Matter 23

4. Physical and Chemical Properties 48

5. Measurements 59

6. Mathematical Treatment of 76

Measurement Results

\section{Part II: Atoms, Molecules and Ions}

7. Introduction 99

8. Early Ideas in Atomic Theory 101

9. Evolution of Atomic Theory 113

10. Atomic Structure and Symbolism 128

11. Chemical Formulas 152

12. The Periodic Table 170 
13. Molecular and Ionic Compounds

183

14. Chemical Nomenclature

201

Part III: Composition of Substances and Solutions

15. Introduction 223

16. Formula Mass and the Mole Concept 225

17. Molarity 256

18. Other Units for Solution Concentrations 276

\section{Part IV: Stoichiometry of Chemical Reactons}

19. Introduction

20. Writing and Balancing

293

Chemical Equations

21. Classifying Chemical Reactions

22. Reaction Stoichiometry

334

23. Reaction Yields

349

Focus on Air 


\section{Part V: Thermochemistry}

24. Introduction 373

25. Energy Basics 375

Part VI: Electronic Structure and Periodic Properties of Elements

26. Introduction 393

27. Electromagnetic Energy 395

28. The Bohr Model 416

29. Development of Quantum Theory 431

30. Electronic Structure of Atoms 461 (Electron Configurations)

\section{Part VII: Chemical Bonding and Molecular Geometry}

31. Introduction

32. Ionic Bonding

493

33. Covalent Bonding 505

34. Lewis Symbols and Structures

522

35. Formal Charges and Resonance 558

36. Strengths of Ionic and Covalent Bonds 577

37. Molecular Structure and Polarity 
Focus on The Ozone Layer

Focus on Climate Change 655

Focus on Energy 673

\section{Part VIII: Liquids and Solids}

38. Introduction 689

39. Intermolecular Forces 691

40. Properties of Liquids $\quad 722$

41. The Solid State of Matter 737

Part IX: Solutions and Colloids

42. Introduction 757

43. The Dissolution Process 759

$\begin{array}{ll}\text { 44. Electrolytes } & 773\end{array}$

$\begin{array}{ll}\text { 45. Solubility } & 782\end{array}$

46. Colloids 803

Focus on Water Quality $\quad 821$ 


\section{Part X: Fundamental Equilibrium Concepts}

47. Introduction 835

48. Chemical Equilibria 837

Part XI: Acid-Base Equilibria

49. Introduction 847

50. Bronsted-Lowry Acids and Bases 849

51. $\mathrm{pH}$ and $\mathrm{pOH} \quad 863$

Focus on Acid Precipitation 876

Part XII: Electrochemistry

52. Introduction 883

53. Galvanic Cells 885

54. Batteries and Fuel Cells 896

Focus on Alternative Energy 909

\section{Part XIII: Organic Chemistry}

55. Introduction 919

56. Hydrocarbons 922

57. Alcohols and Ethers 966 
Focus on Polymers

981

\section{Part XIV: Nuclear Chemistry}

58. Introduction

991

59. Nuclear Structure and Stability

993

60. Nuclear Equations

1010

61. Radioactive Decay

1020

62. Transmutation and Nuclear Energy

1051

63. Biological Effects of Radiation

1082

Focus on Nuclear Energy

1099

Appendix

1109

The Periodic Table

Essential Mathematics

Units and Conversion Factors

1121

Fundamental Physical Constants

1124

Half-Lives for Several Radioactive Isotopes

1126 


\section{PREfACE}

Welcome to Chemistry and the Environment, a book adapted for a chemistry-focused environmental issues course from Chemistry $2 e$, an OpenStax resource. This textbook was written to increase student access to highquality learning materials, maintaining highest standards of academic rigor at little to no cost.

\section{About OpenStax}

OpenStax is a nonprofit based at Rice University, and it's our mission to improve student access to education. Our first openly licensed college textbook was published in 2012, and our library has since scaled to over 25 books for college and $\mathrm{AP}^{\circledR}$ courses used by hundreds of thousands of students. OpenStax Tutor, our low-cost personalized learning tool, is being used in college courses throughout the country. Through our partnerships with philanthropic foundations and our alliance with other educational resource organizations, OpenStax is breaking down the most common barriers to learning and empowering students and instructors to succeed. 
About OpenStax resources

Customization

Chemistry and the Environment and Chemistry $2 e$ are licensed under a Creative Commons Attribution 4.0 International (CC BY) license, which means that you can distribute, remix, and build upon the content, as long as you provide attribution to the authors, OpenStax and its content contributors.

Because our books are openly licensed, you are free to use the entire book or pick and choose the sections that are most relevant to the needs of your course. Feel free to remix the content by assigning your students certain chapters and sections in your syllabus, in the order that you prefer. You can even provide a direct link in your syllabus to the sections in the web view of your book.

Instructors also have the option of creating a customized version of their OpenStax book. The custom version can be made available to students in low-cost print or digital form through their campus bookstore. Visit the Instructor Resources section of your book page on OpenStax.org for more information.

Note: One of the equation editors used in this text displays chemical reactions with no arrow between reactants and products (ie. If there is a space between molecules but no "+" sign between those molecules, then that is where the reaction arrow belongs). For example, $\mathrm{C}$ $+\mathrm{O}_{2} \mathrm{CO}_{2}$ indicates the reaction $\mathrm{C}+\mathrm{O}_{2} \rightarrow \mathrm{CO}_{2}$

Errata

All OpenStax textbooks undergo a rigorous review 
process. However, like any professional-grade textbook, errors sometimes occur. Since our books are web based, we can make updates periodically when deemed pedagogically necessary. If you have a correction to suggest, submit it through email to d.latimer@uwinnipeg.ca and the link on the book page on OpenStax.org. Subject matter experts review all errata suggestions. OpenStax is committed to remaining transparent about all updates, so you will also find a list of past errata changes on your book page on OpenStax.org.

\section{Format}

Chemistry and the Environment is designed to accompany a one-semester course in chemistry-based discussions of important environmental issues such as air pollution, the ozone layer, climate change and water quality. Chemical principles are introduced, followed by environmental 'focus' sections to base discussions on the scientific principles and societal intricacies of the individual topics. Instructors can also use the focus sections as a resource for presentation slides.

Chemistry $2 e$ is designed to meet the scope and sequence requirements of the two-semester general chemistry course. The textbook provides an important opportunity for students to learn the core concepts of chemistry and understand how those concepts apply to their lives and the world around them. The book also includes a number of innovative features, including interactive exercises and real-world applications, designed to enhance student learning. The second edition has been revised to incorporate clearer, more current, and 
more dynamic explanations, while maintaining the same organization as the first edition. Substantial improvements have been made in the figures, illustrations, and example exercises that support the text narrative.

Coverage and scope

The Chemistry $2 e$ textbook adheres to the scope and sequence of most general chemistry courses nationwide. We strive to make chemistry, as a discipline, interesting and accessible to students. With this objective in mind, the content of this textbook has been developed and arranged to provide a logical progression from fundamental to more advanced concepts of chemical science. Topics are introduced within the context of familiar experiences whenever possible, treated with an appropriate rigor to satisfy the intellect of the learner, and reinforced in subsequent discussions of related content. The organization and pedagogical features were developed and vetted with feedback from chemistry educators dedicated to the project.

Changes to the second edition

OpenStax only undertakes second editions when significant modifications to the text are necessary. In the case of Chemistry $2 e$, user feedback indicated that we needed to focus on a few key areas, which we have done in the following ways:

Content revisions for clarity and accuracy. The revision plan varied by chapter based on need. About five chapters were extensively rewritten and another twelve chapters 
were substantially revised to improve the readability and clarity of the narrative.

Example and end-of-chapter exercises. The example and end-of-chapter exercises in several chapters were subjected to a rigorous accuracy check and revised to correct any errors, and additional exercises were added to several chapters to more fully support chapter content.

Art and illustrations. Under the guidance of the authors and expert scientific illustrators, especially those wellversed in creating accessible art, the OpenStax team made changes to much of the art in the first edition of Chemistry. The revisions included correcting errors, redesigning illustrations to improve understanding, and recoloring for overall consistency.

Accessibility improvements. As with all OpenStax books, the first edition of Chemistry was created with a focus on accessibility. We have emphasized and improved that approach in the second edition. To accommodate users of specific assistive technologies, all alternative text was reviewed and revised for comprehensiveness and clarity. Many illustrations were revised to improve the color contrast, which is important for some visually impaired students. Overall, the OpenStax platform has been continually upgraded to improve accessibility.

\section{Pedagogical foundation and features}

Throughout Chemistry $2 e$, you will find features that draw the students into scientific inquiry by taking selected topics a step further. Students and educators alike will appreciate discussions in these feature boxes.

- Chemistry in Everyday Life ties chemistry 
concepts to everyday issues and real-world applications of science that students encounter in their lives. Topics include cell phones, solar thermal energy power plants, plastics recycling, and measuring blood pressure.

- How Sciences Interconnect feature boxes discuss chemistry in context of its interconnectedness with other scientific disciplines. Topics include neurotransmitters, greenhouse gases and climate change, and proteins and enzymes.

- Portrait of a Chemist presents a short bio and an introduction to the work of prominent figures from history and present day so that students can see the "faces" of contributors in this field as well as science in action.

Comprehensive art program

Our art program is designed to enhance students' understanding of concepts through clear, effective illustrations, diagrams, and photographs.

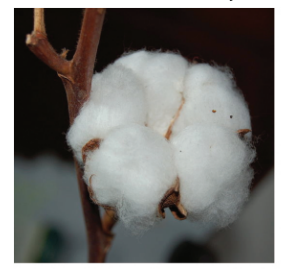

(a)

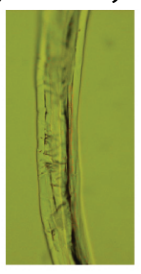

(b)

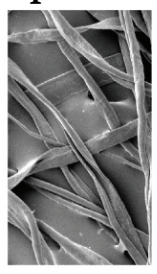

(c)

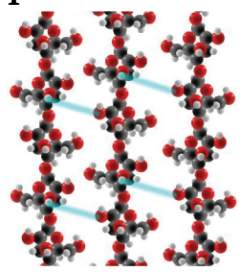

(d)

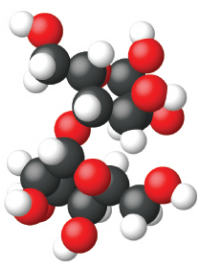

(e) 


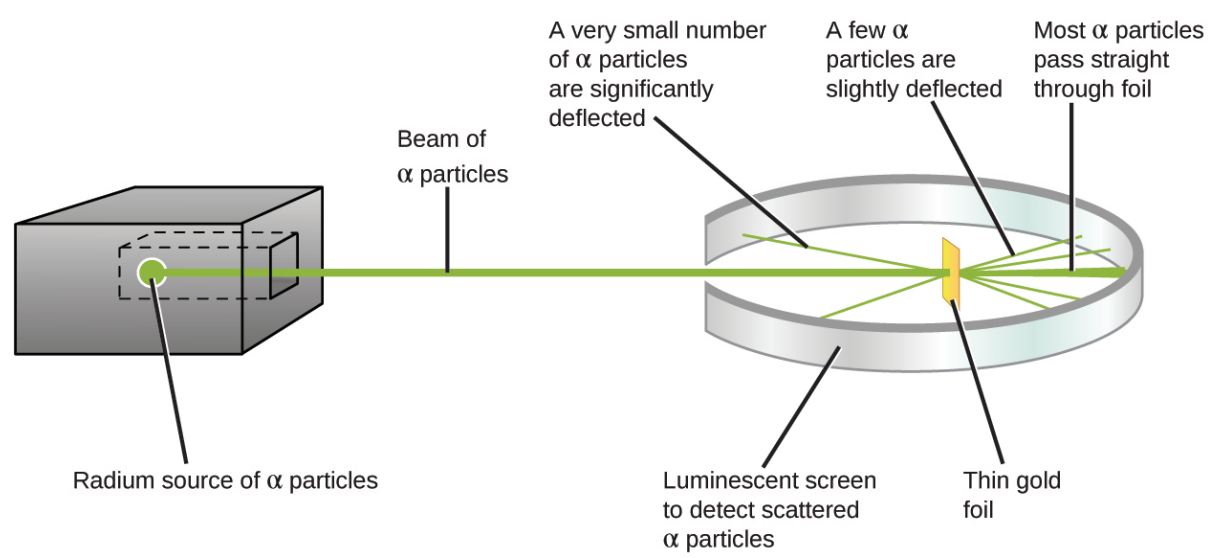

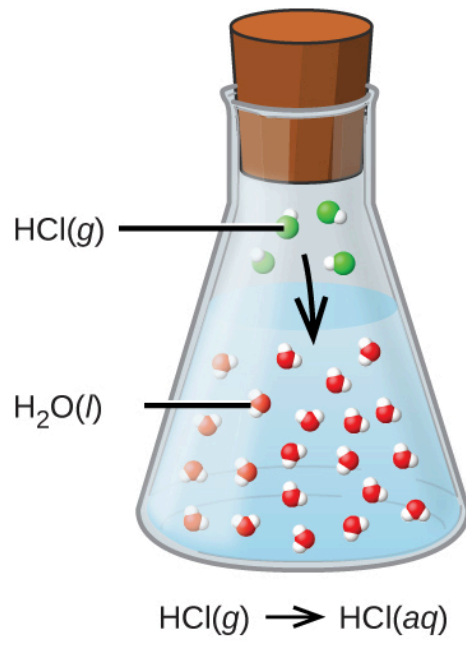

(a)
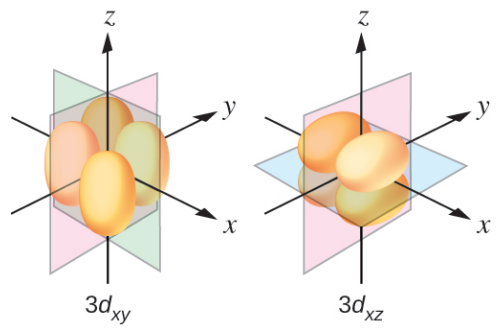

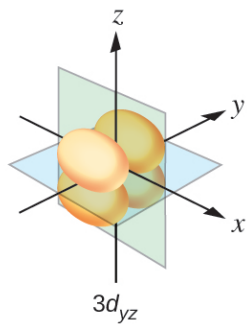

$3 d_{y z}$

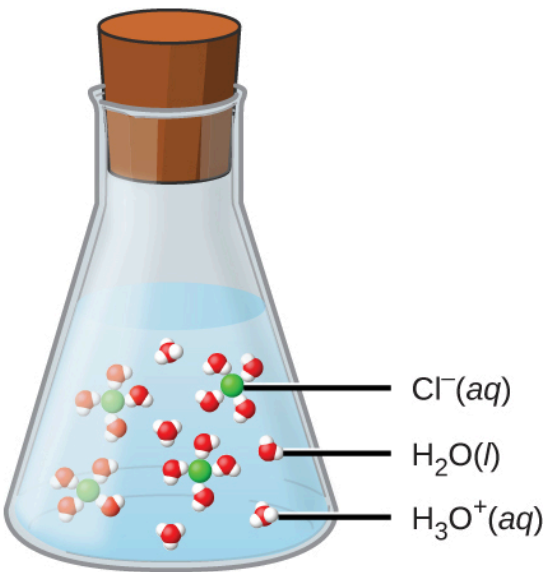

$$
\mathrm{HCl}(a q)+\mathrm{H}_{2} \mathrm{O}(l) \rightarrow \mathrm{H}_{3} \mathrm{O}^{+}(a q)+\mathrm{Cl}^{-}(a q)
$$

(b)

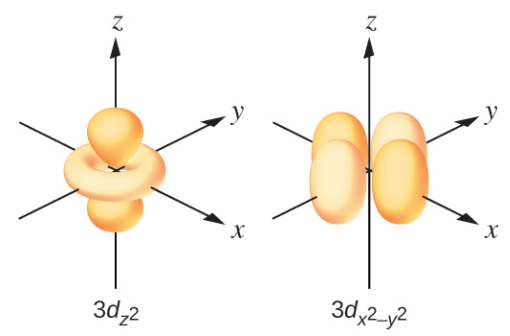


$\mathrm{H}-\stackrel{\mathrm{I}}{\mathrm{I}}-\mathrm{H}$
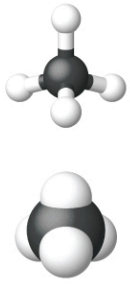

methane

$\mathrm{CH}_{4}$
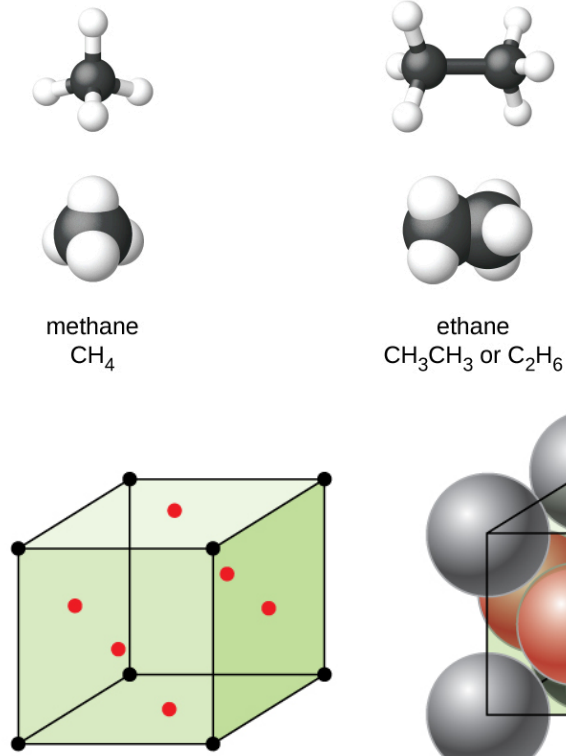

ethane
$\mathrm{CH}_{3} \mathrm{CH}_{3}$ or $\mathrm{C}_{2} \mathrm{H}_{6}$
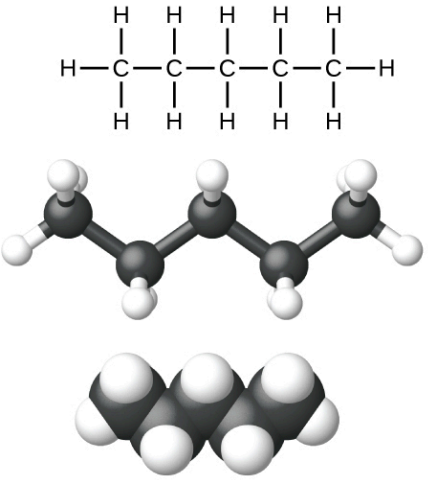

pentane $\mathrm{CH}_{3} \mathrm{CH}_{2} \mathrm{CH}_{2} \mathrm{CH}_{2} \mathrm{CH}_{3}$ or $\mathrm{C}_{5} \mathrm{H}_{12}$
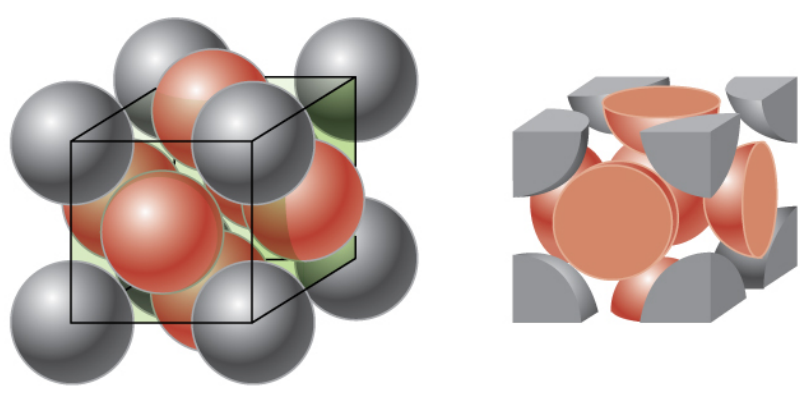

Face-centered cubic structure

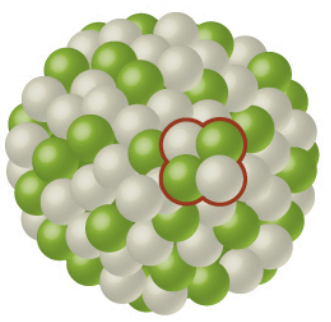

Parent nucleus uranium-238

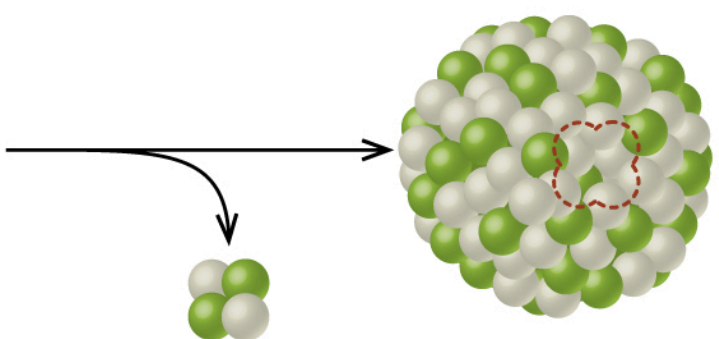

$\alpha$ particle
Daughter nucleus thorium-234

Interactives that engage

Chemistry $2 e$ incorporates links to relevant interactive exercises and animations that help bring topics to life through our Link to Learning feature. Examples include:

- PhET simulations

- IUPAC data and interactives 
- TED Talks

Assessments that reinforce key concepts

In-chapter Examples walk students through problems by posing a question, stepping out a solution, and then asking students to practice the skill with a "Check Your Learning" component. The book also includes assessments at the end of each chapter so students can apply what they've learned through practice problems.

Additional resources

Student and instructor resources

We've compiled additional resources for both students and instructors, including Getting Started Guides, an instructor solutions manual, and PowerPoint slides. Instructor resources require a verified instructor account, which you can apply for when you log in or create your account on OpenStax.org. Take advantage of these resources to supplement your OpenStax book.

\section{Community Hubs}

OpenStax partners with the Institute for the Study of Knowledge Management in Education (ISKME) to offer Community Hubs on OER Commons - a platform for instructors to share community-created resources that support OpenStax books, free of charge. Through our Community Hubs, instructors can upload their own materials or download resources to use in their own courses, including additional ancillaries, teaching 
material, multimedia, and relevant course content. We encourage instructors to join the hubs for the subjects most relevant to your teaching and research as an opportunity both to enrich your courses and to engage with other faculty.

To reach the Community Hubs, visit www.oercommons.org/hubs/OpenStax.

Technology partners

As allies in making high-quality learning materials accessible, our technology partners offer optional lowcost tools that are integrated with OpenStax books. To access the technology options for your text, visit your book page on OpenStax.org.

About the authors

Chemistry and the Environment

\section{Devin R. Latimer, University of Winnipeg}

Devin Ronald Latimer earned a B.Sc.(Honours) from the University of Winnipeg (Canada) in 1987 and a M.Sc. in Inorganic Chemistry from the University of Sydney (Australia) in 1992. After one year studying nuclear magnetic resonance spectroscopy at Dalhousie University (Canada), he joined the faculty of the University of Winnipeg where he has been teaching courses in organic, environmental and introductory chemistry for over 25 years. 
Chemistry $2 e$, Senior contributing authors

Paul Flowers, University of North Carolina at Pembroke

Dr. Paul Flowers earned a BS in Chemistry from St. Andrews Presbyterian College in 1983 and a $\mathrm{PhD}$ in Analytical Chemistry from the University of Tennessee in 1988. After a one-year postdoctoral appointment at Los Alamos National Laboratory, he joined the University of North Carolina at Pembroke in the fall of 1989. Dr. Flowers teaches courses in general and analytical chemistry, and conducts experimental research involving the development of new devices and methods for microscale chemical analysis.

\section{Klaus Theopold, University of Delaware}

Dr. Klaus Theopold (born in Berlin, Germany) received his Vordiplom from the Universität Hamburg in 1977. He then decided to pursue his graduate studies in the United States, where he received his $\mathrm{PhD}$ in inorganic chemistry from UC Berkeley in 1982. After a year of postdoctoral research at MIT, he joined the faculty at Cornell University. In 1990, he moved to the University of Delaware, where he is a Professor in the Department of Chemistry and Biochemistry and serves as an Associate Director of the University's Center for Catalytic Science and Technology. Dr. Theopold regularly teaches graduate courses in inorganic and organometallic chemistry as well as general chemistry.

\section{Richard Langley, Stephen F. Austin State University} Dr. Richard Langley earned BS degrees in Chemistry and Mineralogy from Miami University of Ohio in the early 1970s and went on to receive his $\mathrm{PhD}$ in Chemistry from 
the University of Nebraska in 1977. After a postdoctoral fellowship at the Arizona State University Center for Solid State Studies, Dr. Langley taught in the University of Wisconsin system and participated in research at Argonne National Laboratory. Moving to Stephen F. Austin State University in 1982, Dr. Langley today serves as Professor of Chemistry. His areas of specialization are solid state chemistry, synthetic inorganic chemistry, fluorine chemistry, and chemical education.

\section{William R. Robinson, PhD}

Contributing authors

Mark Blaser, Shasta College

Simon Bott, University of Houston

Donald Carpenetti, Craven Community College Andrew Eklund, Alfred University

Emad El-Giar, University of Louisiana at Monroe

Don Frantz, Wilfrid Laurier University

Paul Hooker, Westminster College

Jennifer Look, Mercer University

George Kaminski, Worcester Polytechnic Institute

Carol Martinez, Central New Mexico Community College

Troy Milliken, Jackson State University

Vicki Moravec, Trine University

Jason Powell, Ferrum College

Thomas Sorensen, University of Wisconsin-Milwaukee Allison Soult, University of Kentucky

\section{Reviewers}

Casey Akin, College Station Independent School District Lara AL-Hariri, University of Massachusetts-Amherst 
Sahar Atwa, University of Louisiana at Monroe

Todd Austell, University of North Carolina-Chapel Hill

Bobby Bailey, University of Maryland-University College

Robert Baker, Trinity College

Jeffrey Bartz, Kalamazoo College

Greg Baxley, Cuesta College

Ashley Beasley Green, National Institute of Standards and

Technology

Patricia Bianconi, University of Massachusetts

Lisa Blank, Lyme Central School District

Daniel Branan, Colorado Community College System

Dorian Canelas, Duke University

Emmanuel Chang, York College

Carolyn Collins, College of Southern Nevada

Colleen Craig, University of Washington

Yasmine Daniels, Montgomery College-Germantown

Patricia Dockham, Grand Rapids Community College

Erick Fuoco, Richard J. Daley College

Andrea Geyer, University of Saint Francis

Daniel Goebbert, University of Alabama

John Goodwin, Coastal Carolina University

Stephanie Gould, Austin College

Patrick Holt, Bellarmine University

George A. Kaminski, Worcester Polytechnic Institute

Kevin Kolack, Queensborough Community College

Amy Kovach, Roberts Wesleyan College

Judit Kovacs Beagle, University of Dayton

Krzysztof Kuczera, University of Kansas

Marcus Lay, University of Georgia

Pamela Lord, University of Saint Francis

Oleg Maksimov, Excelsior College

John Matson, Virginia Tech 
Katrina Miranda, University of Arizona

Douglas Mulford, Emory University

Mark Ott, Jackson College

Adrienne Oxley, Columbia College

Richard Pennington, Georgia Gwinnett College

Rodney Powell, Coastal Carolina Community College

Jeanita Pritchett, Montgomery College-Rockville

Aheda Saber, University of Illinois at Chicago

Raymond Sadeghi, University of Texas at San Antonio

Nirmala Shankar, Rutgers University

Jonathan Smith, Temple University

Bryan Spiegelberg, Rider University

Ron Sternfels, Roane State Community College

Cynthia Strong, Cornell College

Kris Varazo, Francis Marion University

Victor Vilchiz, Virginia State University

Alex Waterson, Vanderbilt University

JuchaoYan, Eastern New Mexico University

Mustafa Yatin, Salem State University

Kazushige Yokoyama, State University of New York at

Geneseo

Curtis Zaleski, Shippensburg University

Wei Zhang, University of Colorado-Boulder 


\section{Introductory Focus}

Drastic environmental change has often come during times of revolution - be it scientific, societal or ideological. This text offers a chemical background to support discussions of environmental issues of our times. In this, you will be introduced to the basics of chemistry and then we will use that information to focus on discussions involved in the following topics: Air Pollution, The Ozone Layer, Climate Change, Energy (Fossil Fuels, Nuclear Energy, Alternative Energy), Water Quality, Acid Precipitation and Polymers.

The figure below represents the trend in world population over the past 5000 years. Noteworthy are the bumps and dips in population over that time, but perhaps most striking is the sharp increase in population leading up to today at the far right end of the graph. Population continues to grow at the rate of approximately $1 \%$ worldwide. It is an interesting exercise to refer to a site such as worldometers.info to see the world population as of today and then calculate $1 \%(0.01)$ of that number. Divide by 365 to get the daily population growth and you will see that human population is growing by approximately a quarter million people every day.

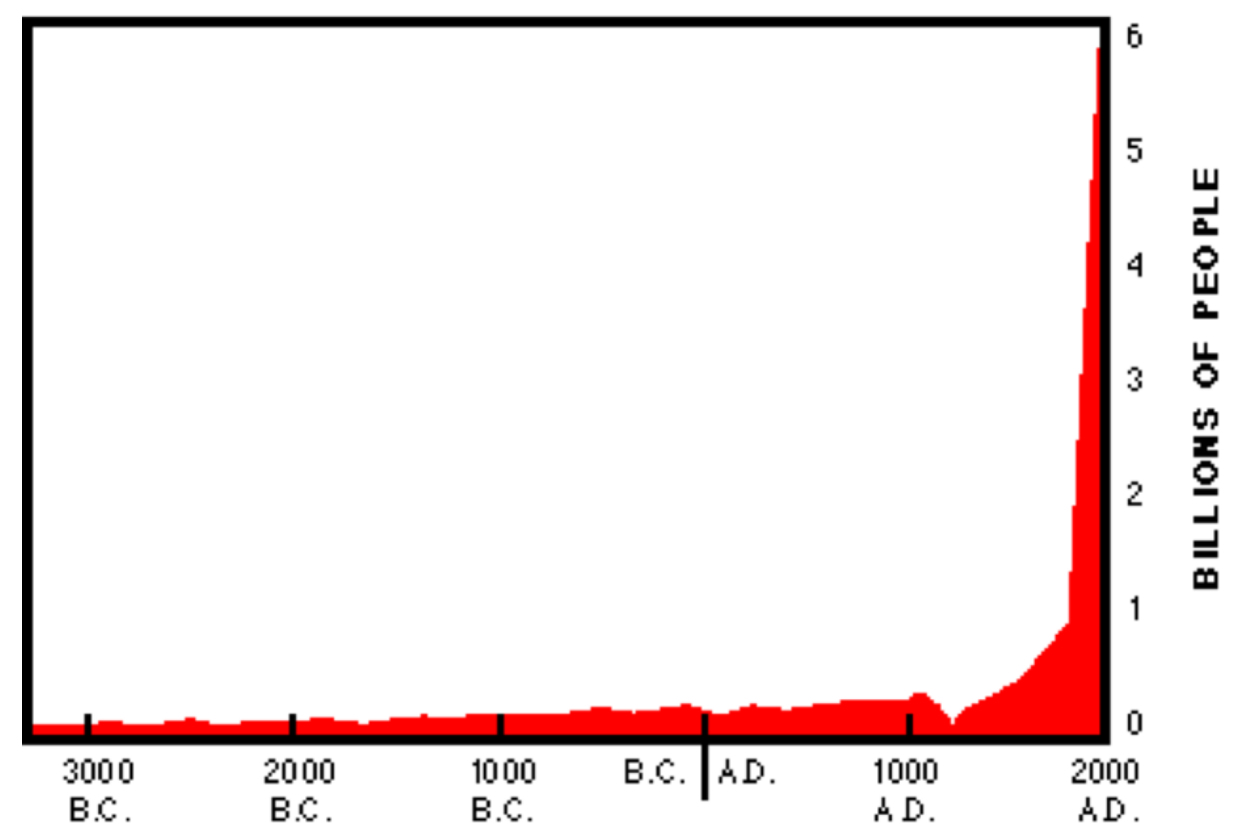


Likewise, our use of energy shows a large upsurge in the $20^{\text {th }}$ century that continues today with energy use growing at the rate of approximately $2 \%$.

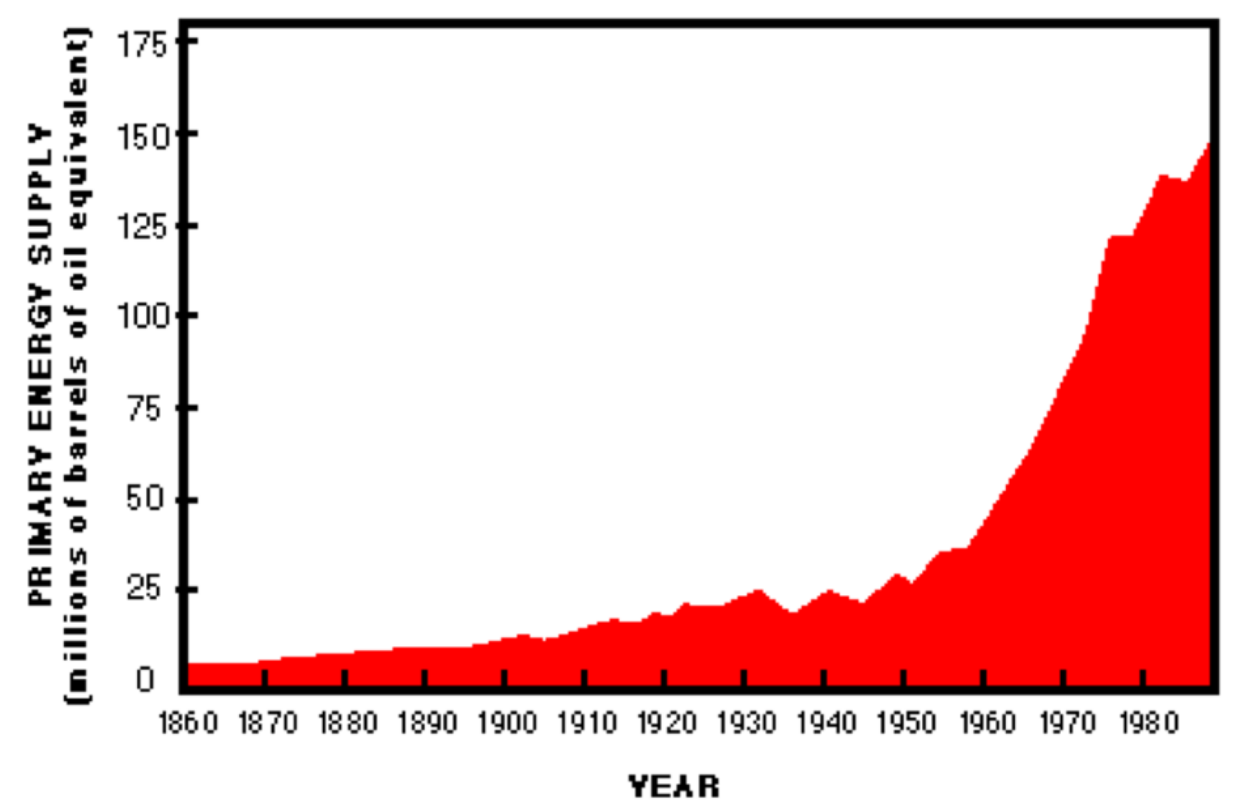

It is in this context that we begin the work-book style discussions ('Focus' sections) in this text. A staggering number of human beings using a staggering amount of energy and materials on a finite and complicated planet is having drastic environmental consequences. We deal with these consequences every day - some quite successfully, some less successfully. Studying the scientific principles involved in tandem with the debates involved with these issues is crucial in deciding on the best paths to move forward. 

PART I

\section{ESSENTIAL IDEAS}





\section{1. \\ INTRODUCTION}

\section{Learning Objectives}

- Chemistry in Context

- Phases and Classification of Matter

- Physical and Chemical Properties

- Measurements

- Mathematical Treatment of Measurement Results

Chemical substances and processes are essential for our existence, providing sustenance, keeping us clean and healthy, fabricating electronic devices, enabling transportation, and much more. (credit "left": modification of work by "vxla"/Flickr; credit "left middle": modification of work by "the Italian voice"/Flickr; credit "right middle": modification of work by Jason Trim; credit "right": modification of work by "gosheshe"/Flickr) 


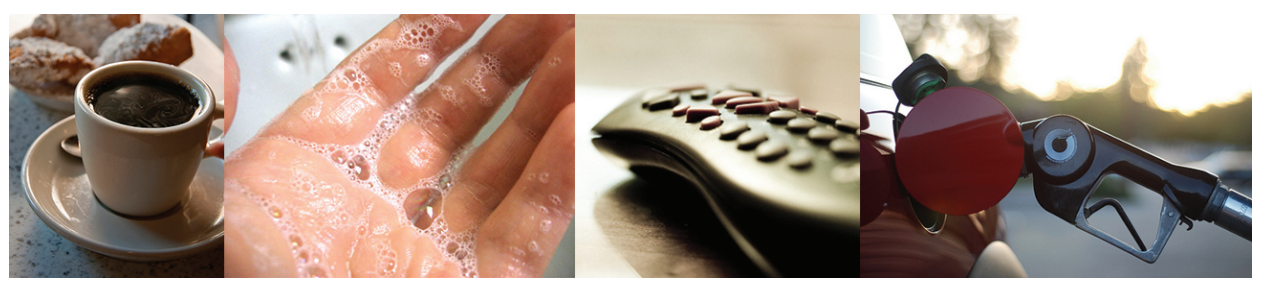

Your alarm goes off and, after hitting "snooze" once or twice, you pry yourself out of bed. You make a cup of coffee to help you get going, and then you shower, get dressed, eat breakfast, and check your phone for messages. On your way to school, you stop to fill your car's gas tank, almost making you late for the first day of chemistry class. As you find a seat in the classroom, you read the question projected on the screen: "Welcome to class! Why should we study chemistry?"

Do you have an answer? You may be studying chemistry because it fulfills an academic requirement, but if you consider your daily activities, you might find chemistry interesting for other reasons. Most everything you do and encounter during your day involves chemistry. Making coffee, cooking eggs, and toasting bread involve chemistry. The products you use-like soap and shampoo, the fabrics you wear, the electronics that keep you connected to your world, the gasoline that propels your car-all of these and more involve chemical substances and processes. Whether you are aware or not, chemistry is part of your everyday world. In this course, you will learn many of the essential principles underlying the chemistry of modern-day life. 
2.

\section{CHEMISTRY IN CONTEXT}

\section{Learning Objectives}

By the end of this module, you will be able to:

- Outline the historical development of chemistry

- $\quad$ Provide examples of the importance of chemistry in everyday life

- Describe the scientific method

- Differentiate among hypotheses, theories, and laws

- Provide examples illustrating macroscopic, microscopic, and symbolic domains

Throughout human history, people have tried to convert matter into more useful forms. Our Stone Age ancestors chipped pieces of flint into useful tools and carved wood into statues and toys. These endeavors involved changing the shape of a substance without changing the substance 
itself. But as our knowledge increased, humans began to change the composition of the substances as well-clay was converted into pottery, hides were cured to make garments, copper ores were transformed into copper tools and weapons, and grain was made into bread.

Humans began to practice chemistry when they learned to control fire and use it to cook, make pottery, and smelt metals. Subsequently, they began to separate and use specific components of matter. A variety of drugs such as aloe, myrrh, and opium were isolated from plants. Dyes, such as indigo and Tyrian purple, were extracted from plant and animal matter. Metals were combined to form alloys-for example, copper and tin were mixed together to make bronze-and more elaborate smelting techniques produced iron. Alkalis were extracted from ashes, and soaps were prepared by combining these alkalis with fats. Alcohol was produced by fermentation and purified by distillation.

Attempts to understand the behavior of matter extend back for more than 2500 years. As early as the sixth century BC, Greek philosophers discussed a system in which water was the basis of all things. You may have heard of the Greek postulate that matter consists of four elements: earth, air, fire, and water. Subsequently, an amalgamation of chemical technologies and philosophical speculations was spread from Egypt, China, and the eastern Mediterranean by alchemists, who endeavored to transform "base metals" such as lead into "noble metals" like gold, and to create elixirs to cure disease and extend life ((Figure)).

This portrayal shows an alchemist's workshop circa 1580. Although alchemy made some useful contributions to 
how to manipulate matter, it was not scientific by modern standards. (credit: Chemical Heritage Foundation)

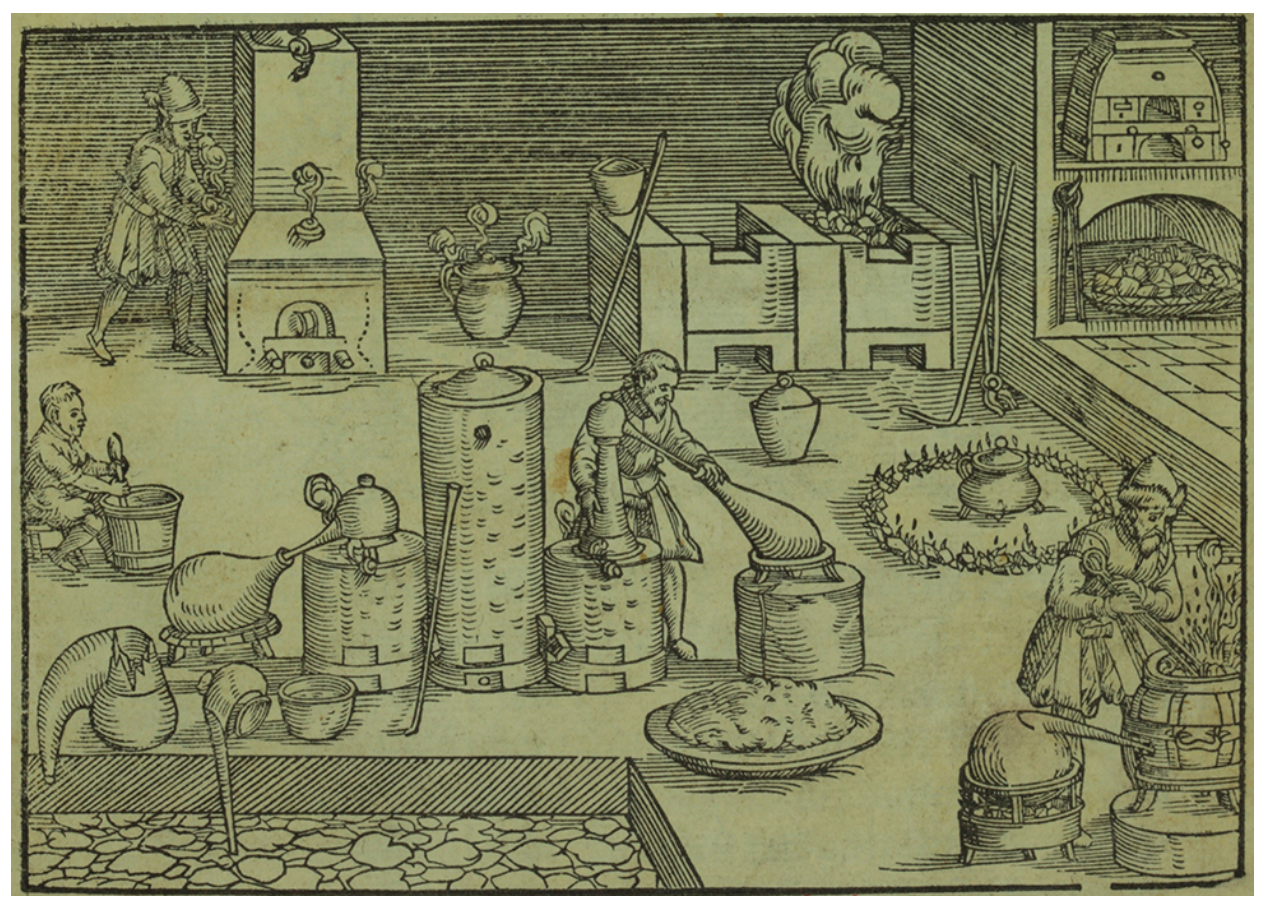

From alchemy came the historical progressions that led to modern chemistry: the isolation of drugs from natural sources, metallurgy, and the dye industry. Today, chemistry continues to deepen our understanding and improve our ability to harness and control the behavior of matter.

Chemistry: The Central Science

Chemistry is sometimes referred to as "the central science" due to its interconnectedness with a vast array of other STEM disciplines (STEM stands for areas of study in the science, technology, engineering, and math fields). Chemistry and the language of chemists play vital roles in biology, medicine, materials science, forensics, 
environmental science, and many other fields ((Figure)). The basic principles of physics are essential for understanding many aspects of chemistry, and there is extensive overlap between many subdisciplines within the two fields, such as chemical physics and nuclear chemistry. Mathematics, computer science, and information theory provide important tools that help us calculate, interpret, describe, and generally make sense of the chemical world. Biology and chemistry converge in biochemistry, which is crucial to understanding the many complex factors and processes that keep living organisms (such as us) alive. Chemical engineering, materials science, and nanotechnology combine chemical principles and empirical findings to produce useful substances, ranging from gasoline to fabrics to electronics. Agriculture, food science, veterinary science, and brewing and wine making help provide sustenance in the form of food and drink to the world's population. Medicine, pharmacology, biotechnology, and botany identify and produce substances that help keep us healthy. Environmental science, geology, oceanography, and atmospheric science incorporate many chemical ideas to help us better understand and protect our physical world. Chemical ideas are used to help understand the universe in astronomy and cosmology.

Knowledge of chemistry is central to understanding a wide range of scientific disciplines. This diagram shows just some of the interrelationships between chemistry and other fields. 


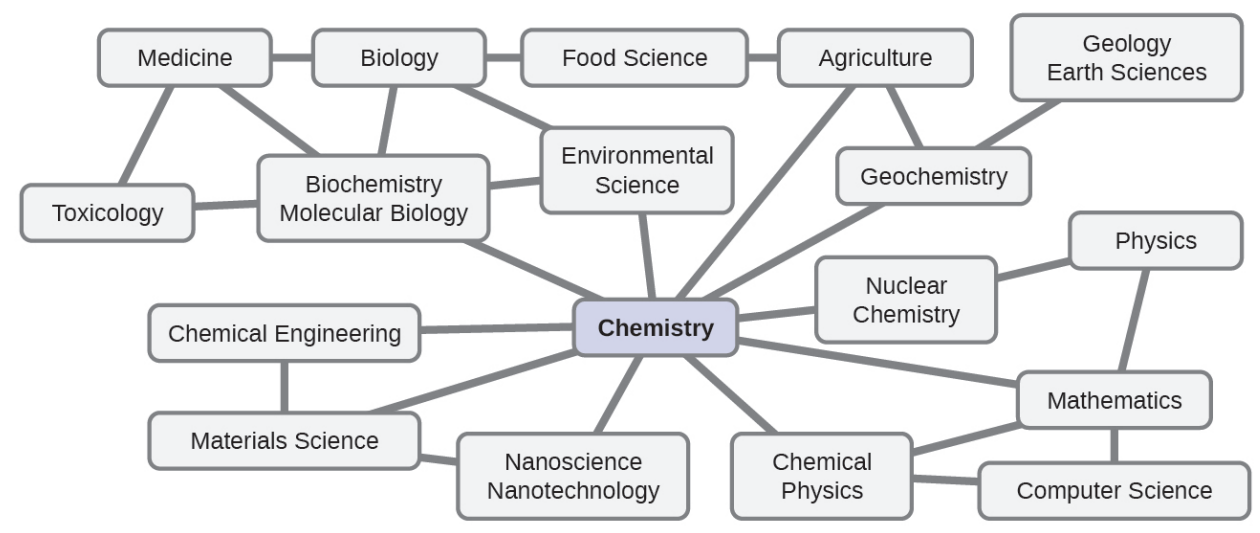

What are some changes in matter that are essential to daily life? Digesting and assimilating food, synthesizing polymers that are used to make clothing, containers, cookware, and credit cards, and refining crude oil into gasoline and other products are just a few examples. As you proceed through this course, you will discover many different examples of changes in the composition and structure of matter, how to classify these changes and how they occurred, their causes, the changes in energy that accompany them, and the principles and laws involved. As you learn about these things, you will be learning chemistry, the study of the composition, properties, and interactions of matter. The practice of chemistry is not limited to chemistry books or laboratories: It happens whenever someone is involved in changes in matter or in conditions that may lead to such changes.

The Scientific Method

Chemistry is a science based on observation and experimentation. Doing chemistry involves attempting to answer questions and explain observations in terms of the laws and theories of chemistry, using procedures that are 
accepted by the scientific community. There is no single route to answering a question or explaining an observation, but there is an aspect common to every approach: Each uses knowledge based on experiments that can be reproduced to verify the results. Some routes involve a hypothesis, a tentative explanation of observations that acts as a guide for gathering and checking information. A hypothesis is tested by experimentation, calculation, and/or comparison with the experiments of others and then refined as needed.

Some hypotheses are attempts to explain the behavior that is summarized in laws. The laws of science summarize a vast number of experimental observations, and describe or predict some facet of the natural world. If such a hypothesis turns out to be capable of explaining a large body of experimental data, it can reach the status of a theory. Scientific theories are well-substantiated, comprehensive, testable explanations of particular aspects of nature. Theories are accepted because they provide satisfactory explanations, but they can be modified if new data become available. The path of discovery that leads from question and observation to law or hypothesis to theory, combined with experimental verification of the hypothesis and any necessary modification of the theory, is called the scientific method ((Figure)).

The scientific method follows a process similar to the one shown in this diagram. All the key components are shown, in roughly the right order. Scientific progress is seldom neat and clean: It requires open inquiry and the reworking of questions and ideas in response to findings. 


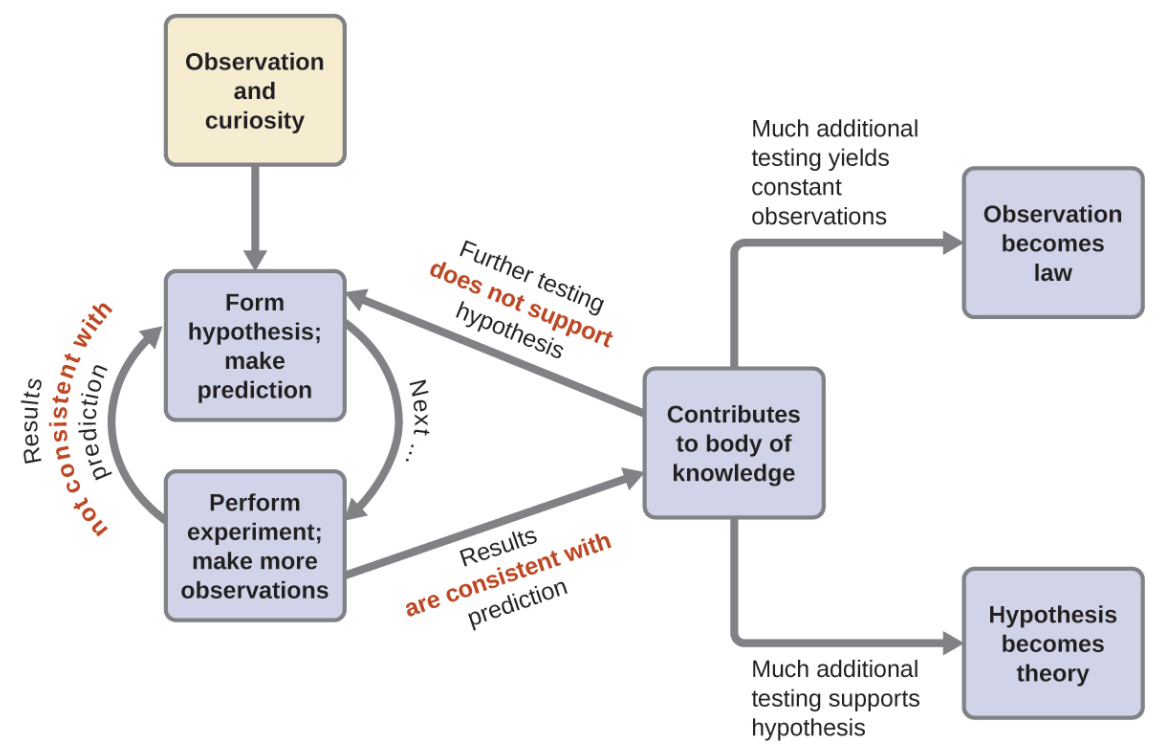

The Domains of Chemistry

Chemists study and describe the behavior of matter and energy in three different domains: macroscopic, microscopic, and symbolic. These domains provide different ways of considering and describing chemical behavior.

Macro is a Greek word that means "large." The macroscopic domain is familiar to us: It is the realm of everyday things that are large enough to be sensed directly by human sight or touch. In daily life, this includes the food you eat and the breeze you feel on your face. The macroscopic domain includes everyday and laboratory chemistry, where we observe and measure physical and chemical properties such as density, solubility, and flammability.

Micro comes from Greek and means "small." The microscopic domain of chemistry is often visited in the imagination. Some aspects of the microscopic domain are visible through standard optical microscopes, for 
example, many biological cells. More sophisticated instruments are capable of imaging even smaller entities such as molecules and atoms (see (Figure) (b)).

However, most of the subjects in the microscopic domain of chemistry are too small to be seen even with the most advanced microscopes and may only be pictured in the mind. Other components of the microscopic domain include ions and electrons, protons and neutrons, and chemical bonds, each of which is far too small to see.

The symbolic domain contains the specialized language used to represent components of the macroscopic and microscopic domains. Chemical symbols (such as those used in the periodic table), chemical formulas, and chemical equations are part of the symbolic domain, as are graphs, drawings, and calculations. These symbols play an important role in chemistry because they help interpret the behavior of the macroscopic domain in terms of the components of the microscopic domain. One of the challenges for students learning chemistry is recognizing that the same symbols can represent different things in the macroscopic and microscopic domains, and one of the features that makes chemistry fascinating is the use of a domain that must be imagined to explain behavior in a domain that can be observed.

A helpful way to understand the three domains is via the essential and ubiquitous substance of water. That water is a liquid at moderate temperatures, will freeze to form a solid at lower temperatures, and boil to form a gas at higher temperatures ((Figure)) are macroscopic observations. But some properties of water fall into the microscopic domain-what cannot be observed with the naked eye. The description of water as comprising two 
hydrogen atoms and one oxygen atom, and the explanation of freezing and boiling in terms of attractions between these molecules, is within the microscopic arena. The formula $\mathrm{H}_{2} \mathrm{O}$, which can describe water at either the macroscopic or microscopic levels, is an example of the symbolic domain. The abbreviations $(g)$ for gas, $(s)$ for solid, and $(l)$ for liquid are also symbolic.

(a) Moisture in the air, icebergs, and the ocean represent water in the macroscopic domain. (b) At the molecular level (microscopic domain), gas molecules are far apart and disorganized, solid water molecules are close together and organized, and liquid molecules are close together and disorganized. (c) The formula $\mathrm{H}_{2} \mathrm{O}$ symbolizes water, and $(g),(s)$, and $(l)$ symbolize its phases. Note that clouds actually comprise either very small liquid water droplets or solid water crystals; gaseous water in our atmosphere is not visible to the naked eye, although it may be sensed as humidity. (credit a: modification of work by "Gorkaazk"/Wikimedia Commons)

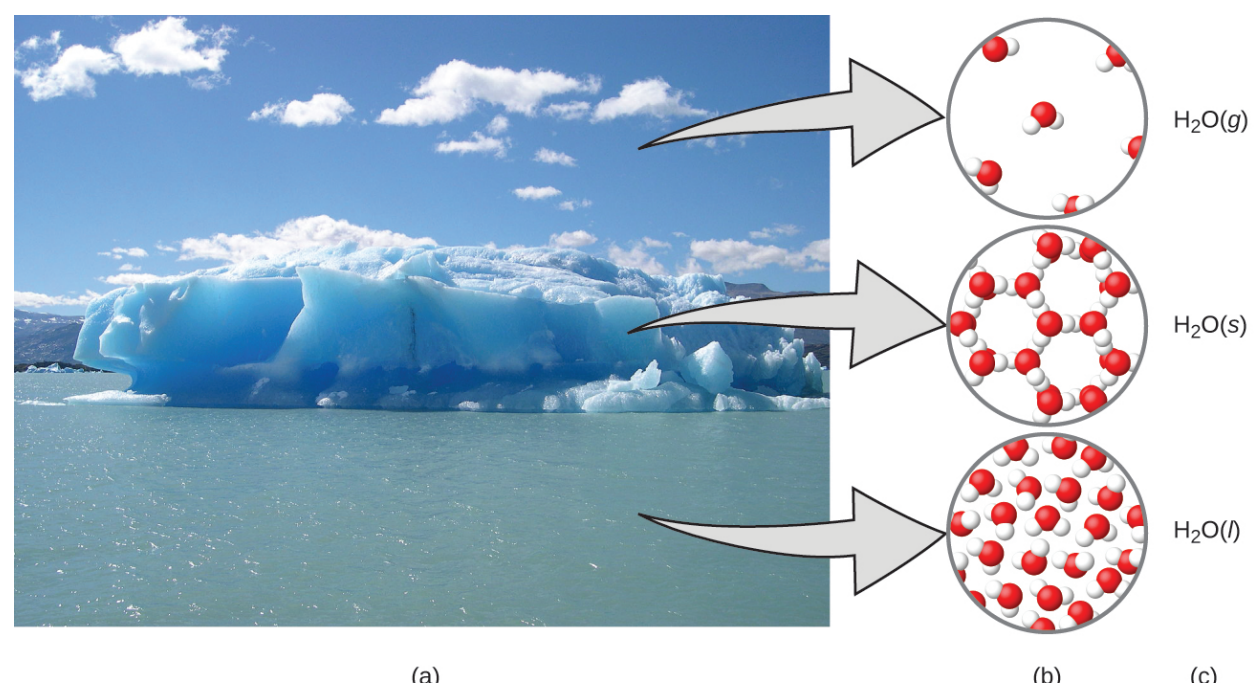


Key Concepts and Summary

Chemistry deals with the composition, structure, and properties of matter, and the ways by which various forms of matter may be interconverted. Thus, it occupies a central place in the study and practice of science and technology. Chemists use the scientific method to perform experiments, pose hypotheses, and formulate laws and develop theories, so that they can better understand the behavior of the natural world. To do so, they operate in the macroscopic, microscopic, and symbolic domains. Chemists measure, analyze, purify, and synthesize a wide variety of substances that are important to our lives.

Chemistry End of Chapter Exercises

Explain how you could experimentally determine whether the outside temperature is higher or lower than $0{ }^{\circ} \mathrm{C}(32$ ${ }^{\circ} \mathrm{F}$ ) without using a thermometer.

Place a glass of water outside. It will freeze if the temperature is below $0{ }^{\circ} \mathrm{C}$.

Identify each of the following statements as being most similar to a hypothesis, a law, or a theory. Explain your reasoning.

(a) Falling barometric pressure precedes the onset of bad weather.

(b) All life on earth has evolved from a common, primitive organism through the process of natural selection.

(c) My truck's gas mileage has dropped significantly, probably because it's due for a tune-up. 
Identify each of the following statements as being most similar to a hypothesis, a law, or a theory. Explain your reasoning.

(a) The pressure of a sample of gas is directly proportional to the temperature of the gas.

(b) Matter consists of tiny particles that can combine in specific ratios to form substances with specific properties.

(c) At a higher temperature, solids (such as salt or sugar) will dissolve better in water.

(a) law (states a consistently observed phenomenon, can be used for prediction); (b) theory (a widely accepted explanation of the behavior of matter); (c) hypothesis (a tentative explanation, can be investigated by experimentation)

Identify each of the underlined items as a part of either the macroscopic domain, the microscopic domain, or the symbolic domain of chemistry. For any in the symbolic domain, indicate whether they are symbols for a macroscopic or a microscopic feature.

(a) The mass of a lead pipe is $14 \mathrm{lb}$.

(b) The mass of a certain chlorine atom is $35 \mathrm{amu}$.

(c) A bottle with a label that reads $\underline{\mathrm{Al}}$ contains aluminum metal.

(d) $\underline{\mathrm{Al}}$ is the symbol for an aluminum atom.

Identify each of the underlined items as a part of either the macroscopic domain, the microscopic domain, or the symbolic domain of chemistry. For those in the symbolic domain, indicate whether they are symbols for a macroscopic or a microscopic feature. 
(a) A certain molecule contains one $\underline{\mathrm{H}}$ atom and one $\mathrm{Cl}$ atom.

(b) Copper wire has a density of about $8 \mathrm{~g} / \mathrm{cm}^{3}$.

(c) The bottle contains 15 grams of Ni powder.

(d) A sulfur molecule is composed of eight sulfur atoms.

(a) symbolic, microscopic; (b) macroscopic; (c) symbolic, macroscopic; (d) microscopic

According to one theory, the pressure of a gas increases as its volume decreases because the molecules in the gas have to move a shorter distance to hit the walls of the container. Does this theory follow a macroscopic or microscopic description of chemical behavior? Explain your answer.

The amount of heat required to melt $2 \mathrm{lbs}$ of ice is twice the amount of heat required to melt $1 \mathrm{lb}$ of ice. Is this observation a macroscopic or microscopic description of chemical behavior? Explain your answer.

Macroscopic. The heat required is determined from macroscopic properties.

Glossary

chemistry

study of the composition, properties, and interactions of matter 


\section{hypothesis}

tentative explanation of observations that acts as a guide for gathering and checking information

law

statement that summarizes a vast number of experimental observations, and describes or predicts some aspect of the natural world macroscopic domain

realm of everyday things that are large enough to sense directly by human sight and touch

\section{microscopic domain}

realm of things that are much too small to be sensed directly

\section{scientific method}

path of discovery that leads from question and observation to law or hypothesis to theory, combined with experimental verification of the hypothesis and any necessary modification of the theory

\section{symbolic domain}

specialized language used to represent components of the macroscopic and microscopic domains, such as chemical symbols, chemical formulas, chemical equations, graphs, drawings, and calculations

\section{theory}

well-substantiated, comprehensive, testable explanation of a particular aspect of 
22

Devin R Latimer

nature 
3.

\section{PHASES AND \\ CLASSIFICATION OF \\ MATTER}

\section{Learning Objectives}

By the end of this section, you will be able to:

- Describe the basic properties of each physical state of matter: solid, liquid, and gas

- Distinguish between mass and weight

- Apply the law of conservation of matter

- Classify matter as an element, compound, homogeneous mixture, or heterogeneous mixture with regard to its physical state and composition

- Define and give examples of atoms and molecules 
Matter is defined as anything that occupies space and has mass, and it is all around us. Solids and liquids are more obviously matter: We can see that they take up space, and their weight tells us that they have mass. Gases are also matter; if gases did not take up space, a balloon would not inflate (increase its volume) when filled with gas.

Solids, liquids, and gases are the three states of matter commonly found on earth ((Figure)). A solid is rigid and possesses a definite shape. A liquid flows and takes the shape of its container, except that it forms a flat or slightly curved upper surface when acted upon by gravity. (In zero gravity, liquids assume a spherical shape.) Both liquid and solid samples have volumes that are very nearly independent of pressure. A gas takes both the shape and volume of its container.

The three most common states or phases of matter are solid, liquid, and gas.

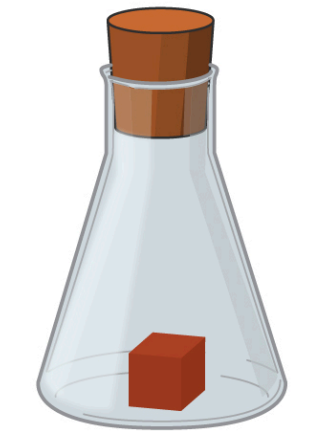

Solid

Has fixed shape and volume

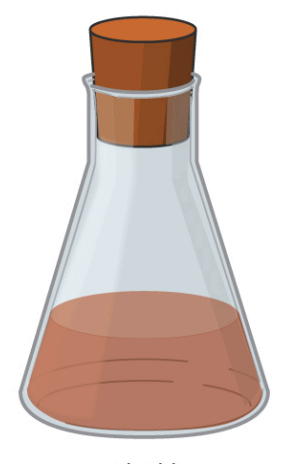

Liquid

Takes shape of container Forms horizontal surface Has fixed volume

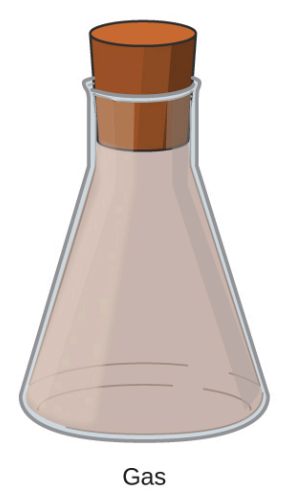

Expands to fill container

A fourth state of matter, plasma, occurs naturally in the interiors of stars. A plasma is a gaseous state of matter that contains appreciable numbers of electrically charged particles ((Figure)). The presence of these charged 
particles imparts unique properties to plasmas that justify their classification as a state of matter distinct from gases. In addition to stars, plasmas are found in some other high-temperature environments (both natural and manmade), such as lightning strikes, certain television screens, and specialized analytical instruments used to detect trace amounts of metals.

A plasma torch can be used to cut metal. (credit: "Hypertherm"/Wikimedia Commons)

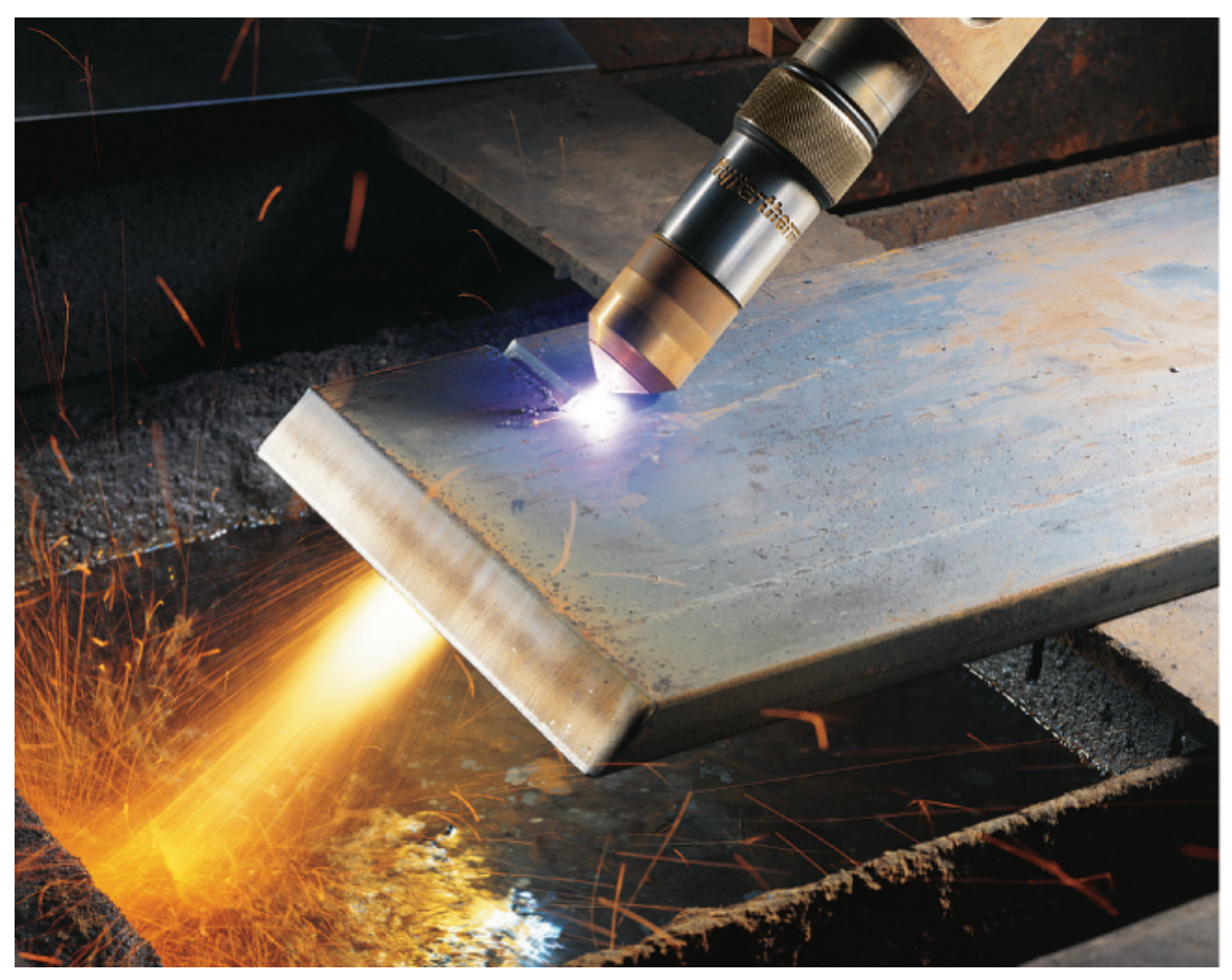

In a tiny cell in a plasma television, the plasma emits ultraviolet light, which in turn causes the display at that location to appear a specific color. The composite of these tiny dots of color makes up the image that you see. Watch this video to learn more about plasma and the places you encounter it. 
Some samples of matter appear to have properties of solids, liquids, and/or gases at the same time. This can occur when the sample is composed of many small pieces. For example, we can pour sand as if it were a liquid because it is composed of many small grains of solid sand. Matter can also have properties of more than one state when it is a mixture, such as with clouds. Clouds appear to behave somewhat like gases, but they are actually mixtures of air (gas) and tiny particles of water (liquid or solid).

The mass of an object is a measure of the amount of matter in it. One way to measure an object's mass is to measure the force it takes to accelerate the object. It takes much more force to accelerate a car than a bicycle because the car has much more mass. A more common way to determine the mass of an object is to use a balance to compare its mass with a standard mass.

Although weight is related to mass, it is not the same thing. Weight refers to the force that gravity exerts on an object. This force is directly proportional to the mass of the object. The weight of an object changes as the force of gravity changes, but its mass does not. An astronaut's mass does not change just because she goes to the moon. But her weight on the moon is only one-sixth her earthbound weight because the moon's gravity is only onesixth that of the earth's. She may feel "weightless" during her trip when she experiences negligible external forces (gravitational or any other), although she is, of course, never "massless."

The law of conservation of matter summarizes many scientific observations about matter: It states that there is no detectable change in the total quantity of matter present 
when matter converts from one type to another (a chemical change) or changes among solid, liquid, or gaseous states (a physical change). Brewing beer and the operation of batteries provide examples of the conservation of matter ((Figure)). During the brewing of beer, the ingredients (water, yeast, grains, malt, hops, and sugar) are converted into beer (water, alcohol, carbonation, and flavoring substances) with no actual loss of substance. This is most clearly seen during the bottling process, when glucose turns into ethanol and carbon dioxide, and the total mass of the substances does not change. This can also be seen in a lead-acid car battery: The original substances (lead, lead oxide, and sulfuric acid), which are capable of producing electricity, are changed into other substances (lead sulfate and water) that do not produce electricity, with no change in the actual amount of matter.

(a) The mass of beer precursor materials is the same as the mass of beer produced: Sugar has become alcohol and carbon dioxide. (b) The mass of the lead, lead oxide, and sulfuric acid consumed by the production of electricity is exactly equal to the mass of lead sulfate and water that is formed.

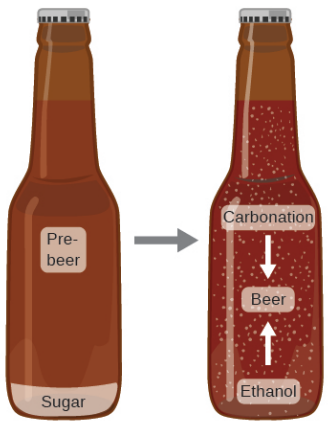

(a)

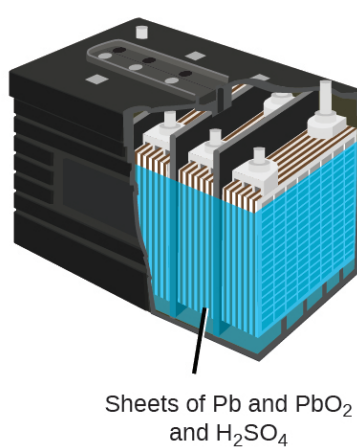

and $\mathrm{H}_{2} \mathrm{SO}_{4}$

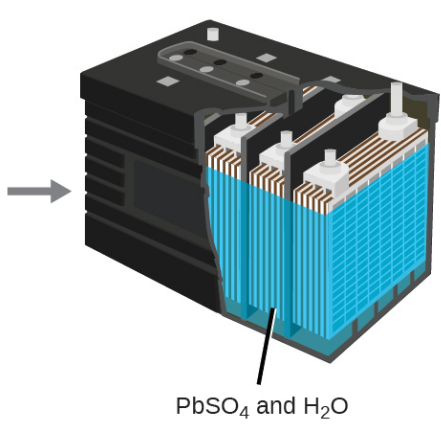

(b) 
Although this conservation law holds true for all conversions of matter, convincing examples are few and far between because, outside of the controlled conditions in a laboratory, we seldom collect all of the material that is produced during a particular conversion. For example, when you eat, digest, and assimilate food, all of the matter in the original food is preserved. But because some of the matter is incorporated into your body, and much is excreted as various types of waste, it is challenging to verify by measurement.

\section{Classifying Matter}

Matter can be classified into several categories. Two broad categories are mixtures and pure substances. A pure substance has a constant composition. All specimens of a pure substance have exactly the same makeup and properties. Any sample of sucrose (table sugar) consists of $42.1 \%$ carbon, $6.5 \%$ hydrogen, and $51.4 \%$ oxygen by mass. Any sample of sucrose also has the same physical properties, such as melting point, color, and sweetness, regardless of the source from which it is isolated.

Pure substances may be divided into two classes: elements and compounds. Pure substances that cannot be broken down into simpler substances by chemical changes are called elements. Iron, silver, gold, aluminum, sulfur, oxygen, and copper are familiar examples of the more than 100 known elements, of which about 90 occur naturally on the earth, and two dozen or so have been created in laboratories.

Pure substances that can be broken down by chemical changes are called compounds. This breakdown may 
produce either elements or other compounds, or both. Mercury(II) oxide, an orange, crystalline solid, can be broken down by heat into the elements mercury and oxygen ((Figure)). When heated in the absence of air, the compound sucrose is broken down into the element carbon and the compound water. (The initial stage of this process, when the sugar is turning brown, is known as caramelization-this is what imparts the characteristic sweet and nutty flavor to caramel apples, caramelized onions, and caramel). Silver(I) chloride is a white solid that can be broken down into its elements, silver and chlorine, by absorption of light. This property is the basis for the use of this compound in photographic films and photochromic eyeglasses (those with lenses that darken when exposed to light).

(a) The compound mercury(II) oxide, (b) when heated, (c) decomposes into silvery droplets of liquid mercury and invisible oxygen gas. (credit: modification of work by Paul Flowers)

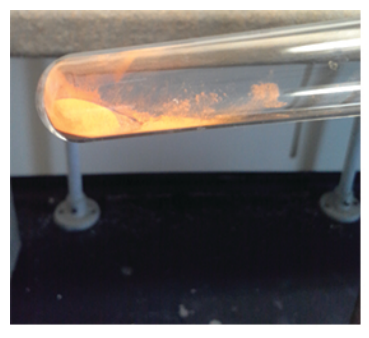

(a)

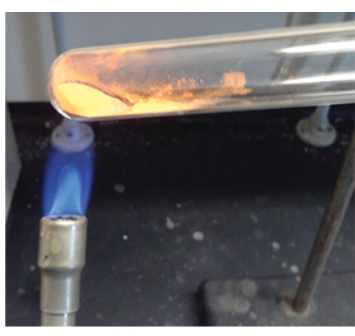

(b)

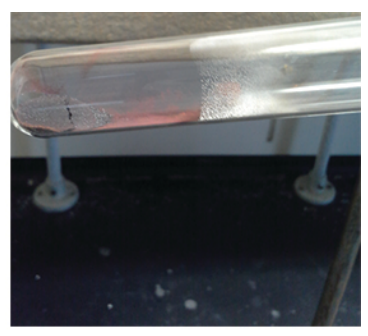

(c)

Many compounds break down when heated. This site shows the breakdown of mercury oxide, $\mathrm{HgO}$. You can also view an example of the photochemical decomposition of silver chloride $(\mathrm{AgCl})$, the basis of early photography.

The properties of combined elements are different from 
those in the free, or uncombined, state. For example, white crystalline sugar (sucrose) is a compound resulting from the chemical combination of the element carbon, which is a black solid in one of its uncombined forms, and the two elements hydrogen and oxygen, which are colorless gases when uncombined. Free sodium, an element that is a soft, shiny, metallic solid, and free chlorine, an element that is a yellow-green gas, combine to form sodium chloride (table salt), a compound that is a white, crystalline solid.

A mixture is composed of two or more types of matter that can be present in varying amounts and can be separated by physical changes, such as evaporation (you will learn more about this later). A mixture with a composition that varies from point to point is called a heterogeneous mixture. Italian dressing is an example of a heterogeneous mixture ((Figure)). Its composition can vary because it may be prepared from varying amounts of oil, vinegar, and herbs. It is not the same from point to point throughout the mixture-one drop may be mostly vinegar, whereas a different drop may be mostly oil or herbs because the oil and vinegar separate and the herbs settle. Other examples of heterogeneous mixtures are chocolate chip cookies (we can see the separate bits of chocolate, nuts, and cookie dough) and granite (we can see the quartz, mica, feldspar, and more).

A homogeneous mixture, also called a solution, exhibits a uniform composition and appears visually the same throughout. An example of a solution is a sports drink, consisting of water, sugar, coloring, flavoring, and electrolytes mixed together uniformly ((Figure)). Each drop of a sports drink tastes the same because each drop 
contains the same amounts of water, sugar, and other components. Note that the composition of a sports drink can vary-it could be made with somewhat more or less sugar, flavoring, or other components, and still be a sports drink. Other examples of homogeneous mixtures include air, maple syrup, gasoline, and a solution of salt in water.

(a) Oil and vinegar salad dressing is a heterogeneous mixture because its composition is not uniform throughout. (b) A commercial sports drink is a homogeneous mixture because its composition is uniform throughout. (credit a "left": modification of work by John Mayer; credit a "right": modification of work by Umberto Salvagnin; credit b "left: modification of work by Jeff Bedford)

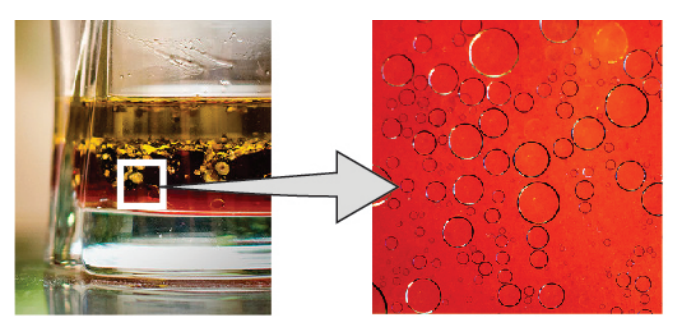

(a)

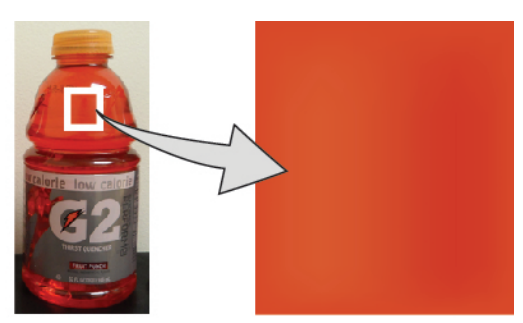

(b)

Although there are just over 100 elements, tens of millions of chemical compounds result from different combinations of these elements. Each compound has a specific composition and possesses definite chemical and physical properties that distinguish it from all other compounds. And, of course, there are innumerable ways to combine elements and compounds to form different mixtures. A summary of how to distinguish between the various major classifications of matter is shown in ((Figure)).

Depending on its properties, a given substance can be 
classified as a homogeneous mixture, a heterogeneous mixture, a compound, or an element.

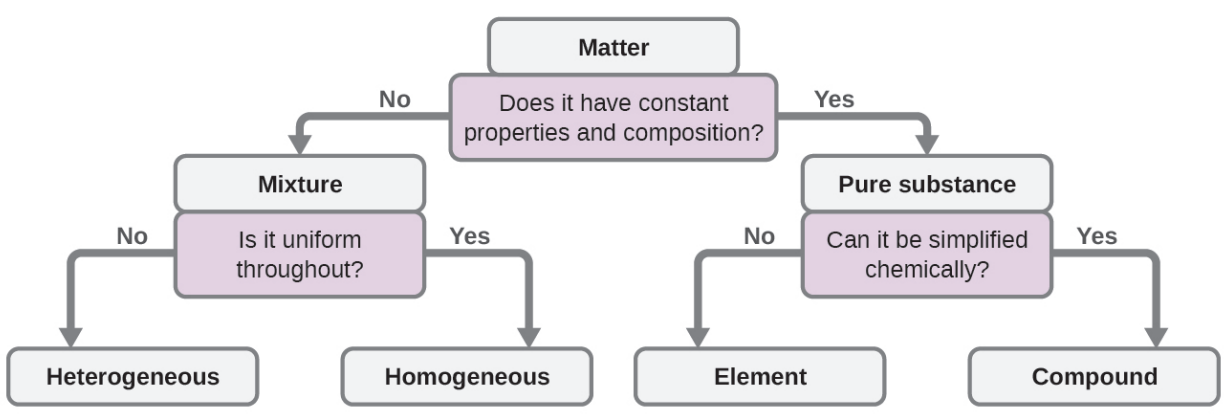

Eleven elements make up about 99\% of the earth's crust and atmosphere ((Figure)). Oxygen constitutes nearly one-half and silicon about one-quarter of the total quantity of these elements. A majority of elements on earth are found in chemical combinations with other elements; about one-quarter of the elements are also found in the free state.

\section{Elemental Composition of Earth}

$\begin{array}{llllll}\text { Element } & \text { Symbol } & \begin{array}{l}\text { Percent } \\ \text { Mass }\end{array} & \text { Element } & \text { Symbol } & \begin{array}{l}\text { Percent } \\ \text { Mass }\end{array} \\ \text { oxygen } & \mathrm{O} & 49.20 & \text { chlorine } & \mathrm{Cl} & 0.19 \\ \text { silicon } & \mathrm{Si} & 25.67 & \text { phosphorus } & \mathrm{P} & 0.11 \\ \text { aluminum } & \mathrm{Al} & 7.50 & \text { manganese } & \mathrm{Mn} & 0.09 \\ \text { iron } & \mathrm{Fe} & 4.71 & \text { carbon } & \mathrm{C} & 0.08 \\ \text { calcium } & \mathrm{Ca} & 3.39 & \text { sulfur } & \mathrm{S} & 0.06 \\ \text { sodium } & \mathrm{Na} & 2.63 & \text { barium } & \mathrm{Ba} & 0.04 \\ \text { potassium } & \mathrm{K} & 2.40 & \text { nitrogen } & \mathrm{N} & 0.03 \\ \text { magnesium } & \mathrm{Mg} & 1.93 & \text { fluorine } & \mathrm{F} & 0.03 \\ \text { hydrogen } & \mathrm{H} & 0.87 & \text { strontium } & \mathrm{Sr} & 0.02 \\ \text { titanium } & \mathrm{Ti} & 0.58 & \text { all others } & - & 0.47\end{array}$


Atoms and Molecules

An atom is the smallest particle of an element that has the properties of that element and can enter into a chemical combination. Consider the element gold, for example. Imagine cutting a gold nugget in half, then cutting one of the halves in half, and repeating this process until a piece of gold remained that was so small that it could not be cut in half (regardless of how tiny your knife may be). This minimally sized piece of gold is an atom (from the Greek atomos, meaning “indivisible”) ((Figure)). This atom would no longer be gold if it were divided any further.

(a) This photograph shows a gold nugget. (b) A scanningtunneling microscope (STM) can generate views of the surfaces of solids, such as this image of a gold crystal.

Each sphere represents one gold atom. (credit a: modification of work by United States Geological Survey; credit b: modification of work by “Erwinrossen”/Wikimedia Commons)

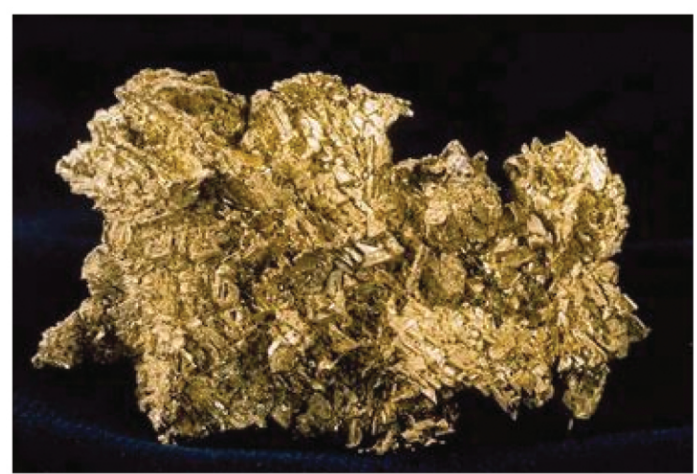

(a)

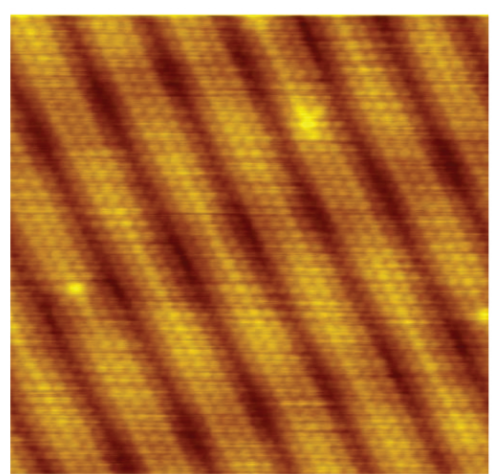

(b)

The first suggestion that matter is composed of atoms is attributed to the Greek philosophers Leucippus and 
Democritus, who developed their ideas in the 5th century BCE. However, it was not until the early nineteenth century that John Dalton (1766-1844), a British schoolteacher with a keen interest in science, supported this hypothesis with quantitative measurements. Since that time, repeated experiments have confirmed many aspects of this hypothesis, and it has become one of the central theories of chemistry. Other aspects of Dalton's atomic theory are still used but with minor revisions (details of Dalton's theory are provided in the chapter on atoms and molecules).

An atom is so small that its size is difficult to imagine. One of the smallest things we can see with our unaided eye is a single thread of a spider web: These strands are about $1 / 10,000$ of a centimeter $(0.0001 \mathrm{~cm})$ in diameter. Although the cross-section of one strand is almost impossible to see without a microscope, it is huge on an atomic scale. A single carbon atom in the web has a diameter of about 0.000000015 centimeter, and it would take about 7000 carbon atoms to span the diameter of the strand. To put this in perspective, if a carbon atom were the size of a dime, the cross-section of one strand would be larger than a football field, which would require about 150 million carbon atom "dimes" to cover it. ((Figure)) shows increasingly close microscopic and atomic-level views of ordinary cotton.

These images provide an increasingly closer view: (a) a cotton boll, (b) a single cotton fiber viewed under an optical microscope (magnified 40 times), (c) an image of a cotton fiber obtained with an electron microscope (much higher magnification than with the optical microscope); and ( $\mathrm{d}$ and e) atomic-level models of the fiber (spheres of 
different colors represent atoms of different elements).

(credit c: modification of work by

"Featheredtar"/Wikimedia Commons)

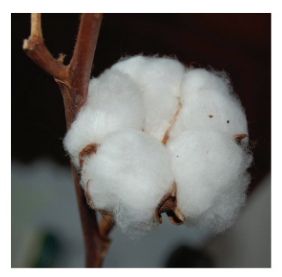

(a)

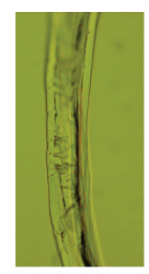

(b)

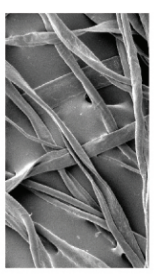

(c)

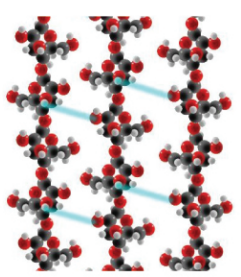

(d)

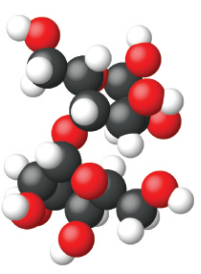

(e)

An atom is so light that its mass is also difficult to imagine. A billion lead atoms (1,000,000,000 atoms) weigh about $310^{-13}$ grams, a mass that is far too light to be weighed on even the world's most sensitive balances. It would require over 300,000,000,000,000 lead atoms (300 trillion, or $310^{14}$ ) to be weighed, and they would weigh only 0.0000001 gram.

It is rare to find collections of individual atoms. Only a few elements, such as the gases helium, neon, and argon, consist of a collection of individual atoms that move about independently of one another. Other elements, such as the gases hydrogen, nitrogen, oxygen, and chlorine, are composed of units that consist of pairs of atoms ((Figure)). One form of the element phosphorus consists of units composed of four phosphorus atoms. The element sulfur exists in various forms, one of which consists of units composed of eight sulfur atoms. These units are called molecules. A molecule consists of two or more atoms joined by strong forces called chemical bonds. The atoms in a molecule move around as a unit, much like the cans of soda in a six-pack or a bunch of keys joined together on a single key ring. A molecule may consist of two or more identical atoms, as in the molecules found in the elements 
hydrogen, oxygen, and sulfur, or it may consist of two or more different atoms, as in the molecules found in water. Each water molecule is a unit that contains two hydrogen atoms and one oxygen atom. Each glucose molecule is a unit that contains 6 carbon atoms, 12 hydrogen atoms, and 6 oxygen atoms. Like atoms, molecules are incredibly small and light. If an ordinary glass of water were enlarged to the size of the earth, the water molecules inside it would be about the size of golf balls.

The elements hydrogen, oxygen, phosphorus, and sulfur form molecules consisting of two or more atoms of the same element. The compounds water, carbon dioxide, and glucose consist of combinations of atoms of different elements.
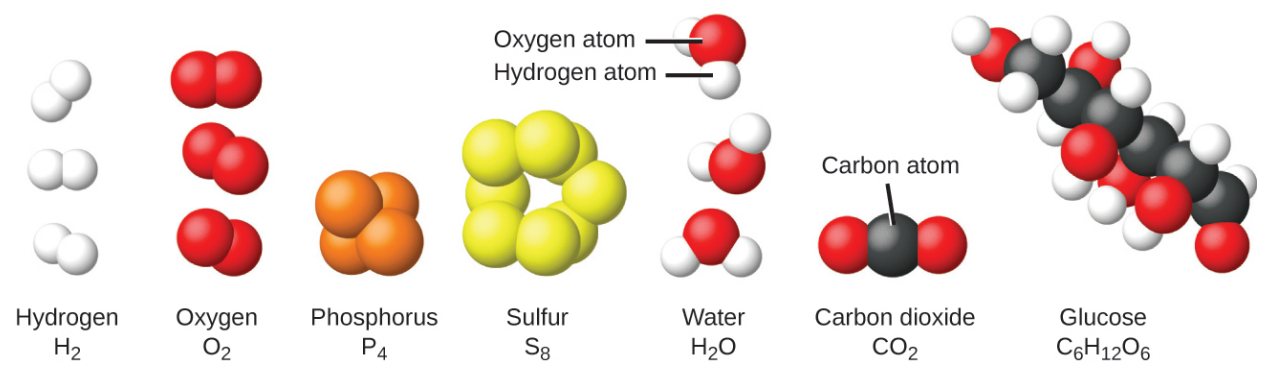

Decomposition of Water / Production of Hydrogen

Water consists of the elements hydrogen and oxygen combined in a 2 to 1 ratio. Water can be broken down into hydrogen and oxygen gases by the addition of energy. One way to do this is with a battery or power supply, as shown in ((Figure)).

The decomposition of water is shown at the macroscopic, microscopic, and symbolic levels. The battery provides an electric current (microscopic) that decomposes water. At the macroscopic level, the liquid separates into the gases hydrogen (on the left) and oxygen (on the right). 
Symbolically, this change is presented by showing how liquid $\mathrm{H}_{2} \mathrm{O}$ separates into $\mathrm{H}_{2}$ and $\mathrm{O}_{2}$ gases.

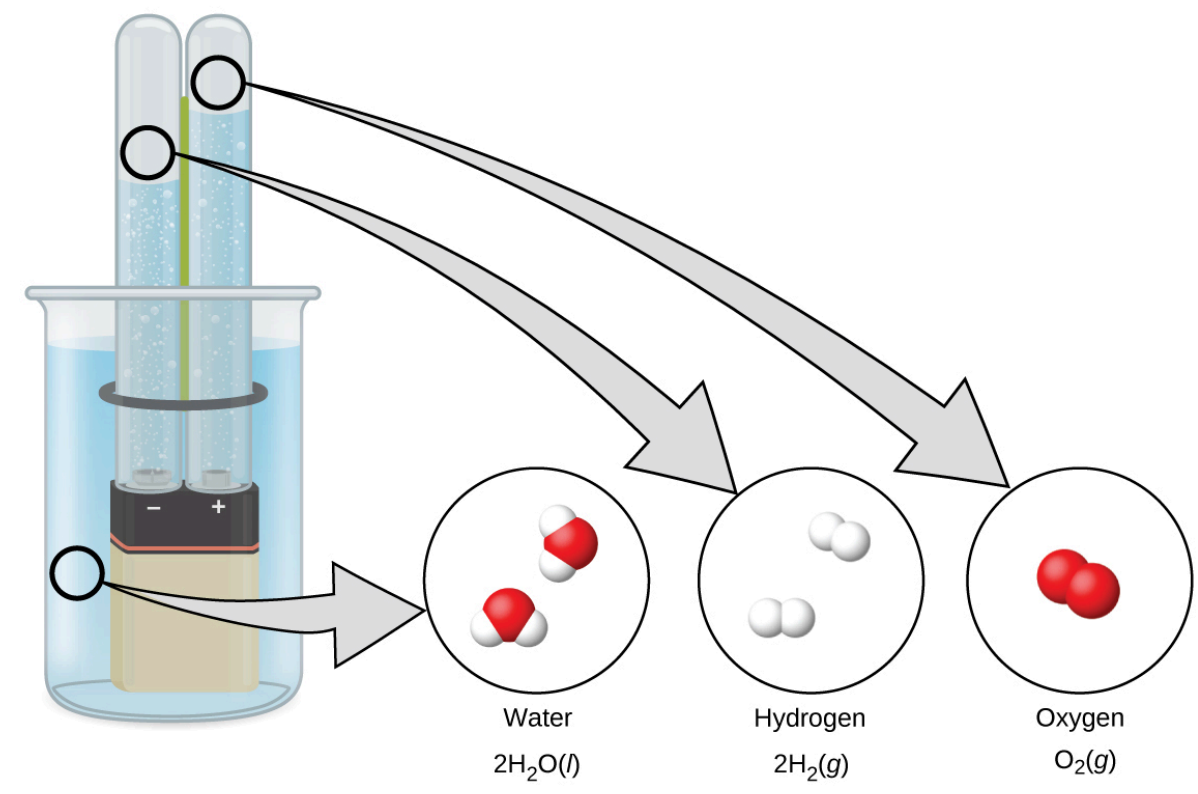

The breakdown of water involves a rearrangement of the atoms in water molecules into different molecules, each composed of two hydrogen atoms and two oxygen atoms, respectively. Two water molecules form one oxygen molecule and two hydrogen molecules. The representation for what occurs, $2 \mathrm{H}_{2} \mathrm{O}(l) 2 \mathrm{H}_{2}(g)+\mathrm{O}_{2}(g)$, will be explored in more depth in later chapters.

The two gases produced have distinctly different properties. Oxygen is not flammable but is required for combustion of a fuel, and hydrogen is highly flammable and a potent energy source. How might this knowledge be applied in our world? One application involves research into more fuel-efficient transportation. Fuel-cell vehicles (FCV) run on hydrogen instead of gasoline ((Figure)). They are more efficient than vehicles with internal combustion 
engines, are nonpolluting, and reduce greenhouse gas emissions, making us less dependent on fossil fuels. FCVs are not yet economically viable, however, and current hydrogen production depends on natural gas. If we can develop a process to economically decompose water, or produce hydrogen in another environmentally sound way, FCVs may be the way of the future.

A fuel cell generates electrical energy from hydrogen and oxygen via an electrochemical process and produces only water as the waste product.

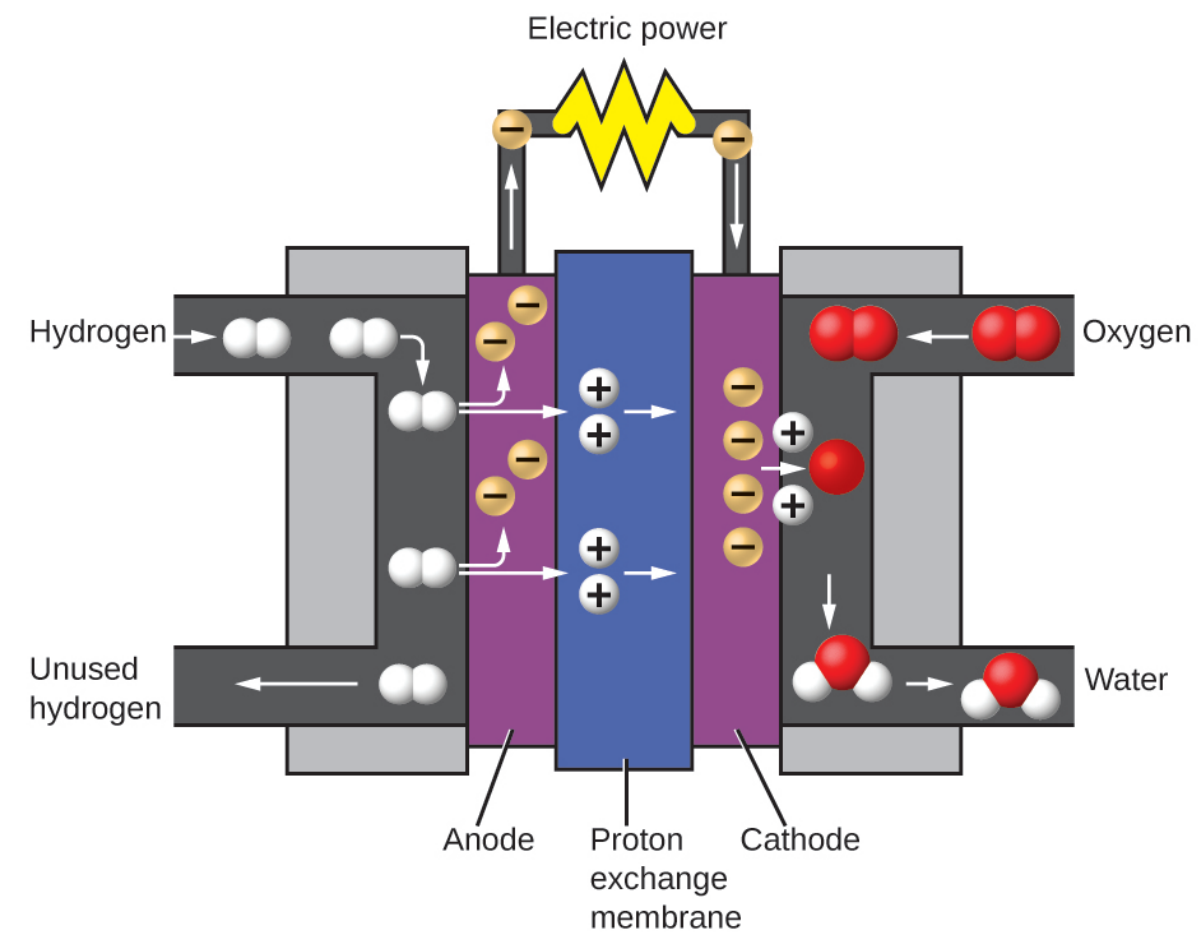

Chemistry of Cell Phones

Imagine how different your life would be without cell phones ((Figure)) and other smart devices. Cell phones are made from numerous chemical substances, which are extracted, refined, purified, and assembled using an extensive and in-depth understanding of chemical 
principles. About $30 \%$ of the elements that are found in nature are found within a typical smart phone. The case/ body/frame consists of a combination of sturdy, durable polymers composed primarily of carbon, hydrogen, oxygen, and nitrogen [acrylonitrile butadiene styrene (ABS) and polycarbonate thermoplastics], and light, strong, structural metals, such as aluminum, magnesium, and iron. The display screen is made from a specially toughened glass (silica glass strengthened by the addition of aluminum, sodium, and potassium) and coated with a material to make it conductive (such as indium tin oxide). The circuit board uses a semiconductor material (usually silicon); commonly used metals like copper, tin, silver, and gold; and more unfamiliar elements such as yttrium, praseodymium, and gadolinium. The battery relies upon lithium ions and a variety of other materials, including iron, cobalt, copper, polyethylene oxide, and polyacrylonitrile.

Almost one-third of naturally occurring elements are used to make a cell phone. (credit: modification of work by John Taylor)

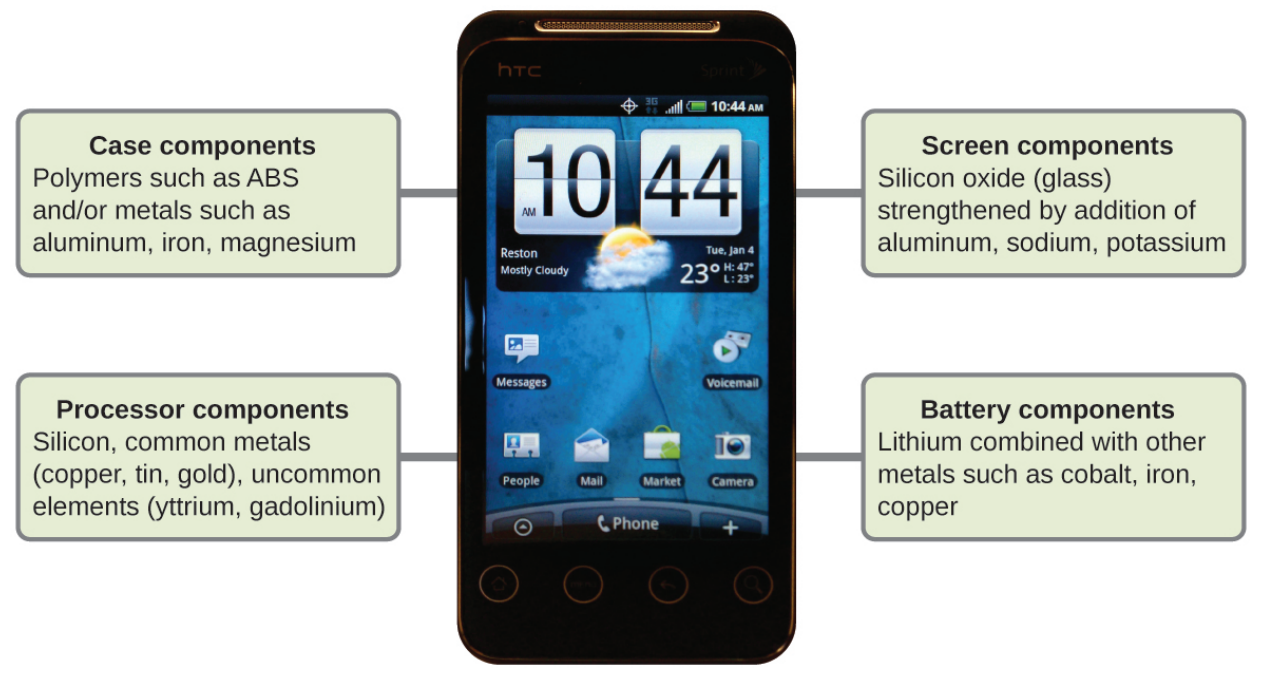


Key Concepts and Summary

Matter is anything that occupies space and has mass. The basic building block of matter is the atom, the smallest unit of an element that can enter into combinations with atoms of the same or other elements. In many substances, atoms are combined into molecules. On earth, matter commonly exists in three states: solids, of fixed shape and volume; liquids, of variable shape but fixed volume; and gases, of variable shape and volume. Under hightemperature conditions, matter also can exist as a plasma. Most matter is a mixture: It is composed of two or more types of matter that can be present in varying amounts and can be separated by physical means. Heterogeneous mixtures vary in composition from point to point; homogeneous mixtures have the same composition from point to point. Pure substances consist of only one type of matter. A pure substance can be an element, which consists of only one type of atom and cannot be broken down by a chemical change, or a compound, which consists of two or more types of atoms.

\section{Chemistry End of Chapter Exercises}

Why is an object's mass, rather than its weight, used to indicate the amount of matter it contains?

What properties distinguish solids from liquids? Liquids from gases? Solids from gases?

Liquids can change their shape (flow); solids can't. Gases can undergo large volume changes as pressure changes; 
liquids do not. Gases flow and change volume; solids do not.

How does a heterogeneous mixture differ from a homogeneous mixture? How are they similar?

How does a homogeneous mixture differ from a pure substance? How are they similar?

The mixture can have a variety of compositions; a pure substance has a definite composition. Both have the same composition from point to point.

How does an element differ from a compound? How are they similar?

How do molecules of elements and molecules of compounds differ? In what ways are they similar?

Molecules of elements contain only one type of atom; molecules of compounds contain two or more types of atoms. They are similar in that both are comprised of two or more atoms chemically bonded together.

How does an atom differ from a molecule? In what ways are they similar?

Many of the items you purchase are mixtures of pure compounds. Select three of these commercial products and prepare a list of the ingredients that are pure compounds.

Answers will vary. Sample answer: Gatorade contains water, sugar, dextrose, citric acid, salt, sodium chloride, 
monopotassium phosphate, and sucrose acetate isobutyrate.

Classify each of the following as an element, a compound, or a mixture:
(a) copper
(b) water
(c) nitrogen
(d) sulfur
(e) air
(f) sucrose
(g) a substance composed of molecules each of which contains two iodine atoms

(h) gasoline

Classify each of the following as an element, a compound, or a mixture:
(a) iron
(b) oxygen
(c) mercury oxide
(d) pancake syrup
(e) carbon dioxide
(f) a substance composed of molecules each of which contains one hydrogen atom and one chlorine atom
(g) baking soda
(h) baking powder

(a) element; (b) element; (c) compound; (d) mixture; (e) compound; (f) compound; (g) compound; (h) mixture

A sulfur atom and a sulfur molecule are not identical. What is the difference? 
How are the molecules in oxygen gas, the molecules in hydrogen gas, and water molecules similar? How do they differ?

In each case, a molecule consists of two or more combined atoms. They differ in that the types of atoms change from one substance to the next.

Why are astronauts in space said to be "weightless," but not "massless"?

Prepare a list of the principal chemicals consumed and produced during the operation of an automobile.

Gasoline (a mixture of compounds), oxygen, and to a lesser extent, nitrogen are consumed. Carbon dioxide and water are the principal products. Carbon monoxide and nitrogen oxides are produced in lesser amounts.

Matter is everywhere around us. Make a list by name of fifteen different kinds of matter that you encounter every day. Your list should include (and label at least one example of each) the following: a solid, a liquid, a gas, an element, a compound, a homogenous mixture, a heterogeneous mixture, and a pure substance.

When elemental iron corrodes it combines with oxygen in the air to ultimately form red brown iron(III) oxide called rust. (a) If a shiny iron nail with an initial mass of $23.2 \mathrm{~g}$ is weighed after being coated in a layer of rust, would you expect the mass to have increased, decreased, or remained the same? Explain. (b) If the mass of the iron 
nail increases to $24.1 \mathrm{~g}$, what mass of oxygen combined with the iron?

(a) Increased as it would have combined with oxygen in the air thus increasing the amount of matter and therefore the mass. (b) $0.9 \mathrm{~g}$

As stated in the text, convincing examples that demonstrate the law of conservation of matter outside of the laboratory are few and far between. Indicate whether the mass would increase, decrease, or stay the same for the following scenarios where chemical reactions take place:

(a) Exactly one pound of bread dough is placed in a baking tin. The dough is cooked in an oven at $350{ }^{\circ} \mathrm{F}$ releasing a wonderful aroma of freshly baked bread during the cooking process. Is the mass of the baked loaf less than, greater than, or the same as the one pound of original dough? Explain.

(b) When magnesium burns in air a white flaky ash of magnesium oxide is produced. Is the mass of magnesium oxide less than, greater than, or the same as the original piece of magnesium? Explain.

(c) Antoine Lavoisier, the French scientist credited with first stating the law of conservation of matter, heated a mixture of tin and air in a sealed flask to produce tin oxide. Did the mass of the sealed flask and contents decrease, increase, or remain the same after the heating?

Yeast converts glucose to ethanol and carbon dioxide during anaerobic fermentation as depicted in the simple chemical equation here: 
glucose ethanol + carbon dioxide

(a) If $200.0 \mathrm{~g}$ of glucose is fully converted, what will be the total mass of ethanol and carbon dioxide produced?

(b) If the fermentation is carried out in an open container, would you expect the mass of the container and contents after fermentation to be less than, greater than, or the same as the mass of the container and contents before fermentation? Explain.

(c) If $97.7 \mathrm{~g}$ of carbon dioxide is produced, what mass of ethanol is produced?

(a) $200.0 \mathrm{~g}$; (b) The mass of the container and contents would decrease as carbon dioxide is a gaseous product and would leave the container. (c) $102.3 \mathrm{~g}$

Glossary

atom

smallest particle of an element that can

enter into a chemical combination

compound

pure substance that can be decomposed into two or more elements

element

substance that is composed of a single type of atom; a substance that cannot be decomposed by a chemical change

gas

state in which matter has neither definite 


\section{volume nor shape \\ heterogeneous mixture}

combination of substances with a

composition that varies from point to point

homogeneous mixture

(also, solution) combination of substances

with a composition that is uniform

throughout

liquid

state of matter that has a definite volume but indefinite shape

law of conservation of matter

when matter converts from one type to another or changes form, there is no detectable change in the total amount of matter present

mass

fundamental property indicating amount of matter

\section{matter}

anything that occupies space and has mass mixture

matter that can be separated into its components by physical means

\section{molecule}

bonded collection of two or more atoms of the same or different elements

plasma

gaseous state of matter containing a large number of electrically charged atoms and/or molecules 


\section{pure substance}

homogeneous substance that has a constant composition

solid

state of matter that is rigid, has a definite shape, and has a fairly constant volume weight

force that gravity exerts on an object 
4.

\section{PHYSICAL AND CHEMICAL PROPERTIES}

Learning Objectives

By the end of this section, you will be able to:

- Identify properties of and changes in matter as physical or chemical

- Identify properties of matter as extensive or intensive

The characteristics that distinguish one substance from another are called properties. A physical property is a characteristic of matter that is not associated with a change in its chemical composition. Familiar examples of physical properties include density, color, hardness, melting and boiling points, and electrical conductivity. Some physical properties, such as density and color, may be observed without changing the physical state of the 
matter. Other physical properties, such as the melting temperature of iron or the freezing temperature of water, can only be observed as matter undergoes a physical change. A physical change is a change in the state or properties of matter without any accompanying change in the chemical identities of the substances contained in the matter. Physical changes are observed when wax melts, when sugar dissolves in coffee, and when steam condenses into liquid water ((Figure)). Other examples of physical changes include magnetizing and demagnetizing metals (as is done with common antitheft security tags) and grinding solids into powders (which can sometimes yield noticeable changes in color). In each of these examples, there is a change in the physical state, form, or properties of the substance, but no change in its chemical composition.

(a) Wax undergoes a physical change when solid wax is heated and forms liquid wax. (b) Steam condensing inside a cooking pot is a physical change, as water vapor is changed into liquid water. (credit a: modification of work by "95jb14"/Wikimedia Commons; credit b: modification of work by "mjneuby”/Flickr)

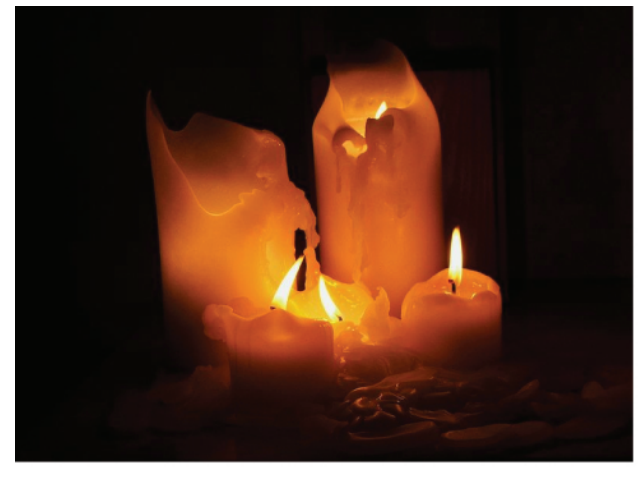

(a)

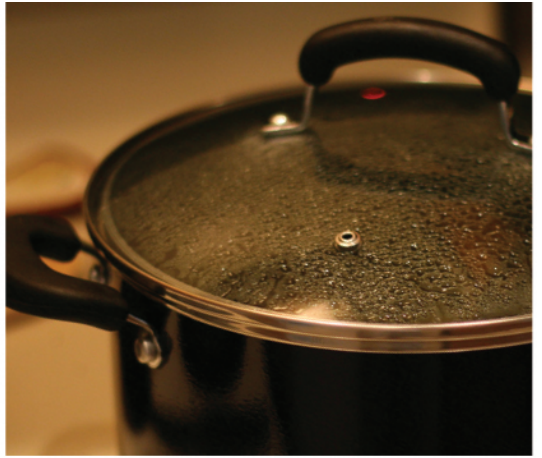

(b) 
The change of one type of matter into another type (or the inability to change) is a chemical property. Examples of chemical properties include flammability, toxicity, acidity, and many other types of reactivity. Iron, for example, combines with oxygen in the presence of water to form rust; chromium does not oxidize ((Figure)). Nitroglycerin is very dangerous because it explodes easily; neon poses almost no hazard because it is very unreactive.

(a) One of the chemical properties of iron is that it rusts;

(b) one of the chemical properties of chromium is that it does not. (credit a: modification of work by Tony Hisgett; credit b: modification of work by "Atoma"/Wikimedia Commons)

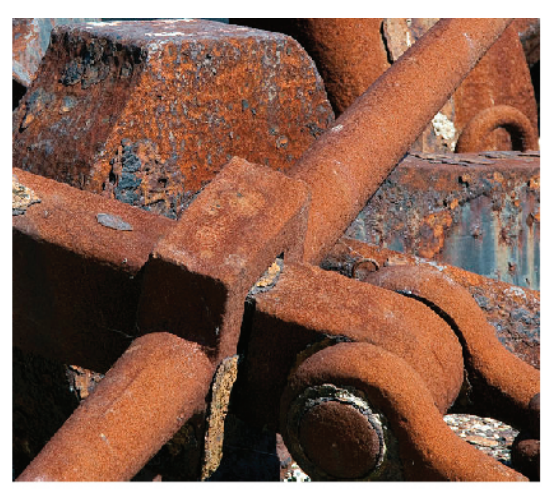

(a)

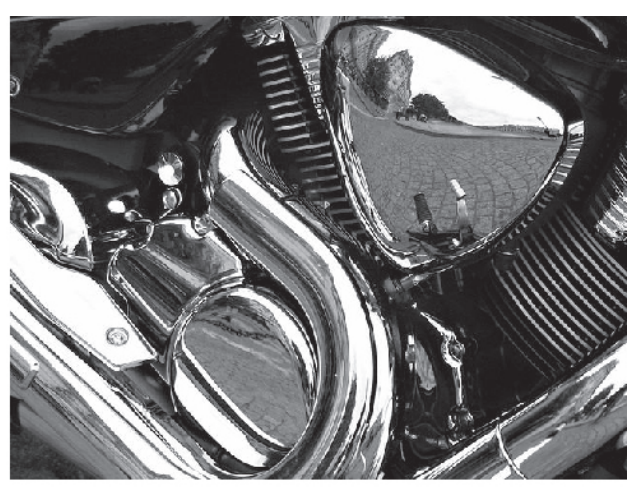

(b)

A chemical change always produces one or more types of matter that differ from the matter present before the change. The formation of rust is a chemical change because rust is a different kind of matter than the iron, oxygen, and water present before the rust formed. The explosion of nitroglycerin is a chemical change because the gases produced are very different kinds of matter from the original substance. Other examples of chemical changes include reactions that are performed in a lab 
(such as copper reacting with nitric acid), all forms of combustion (burning), and food being cooked, digested, or rotting ((Figure)).

(a) Copper and nitric acid undergo a chemical change to form copper nitrate and brown, gaseous nitrogen dioxide. (b) During the combustion of a match, cellulose in the match and oxygen from the air undergo a chemical change to form carbon dioxide and water vapor. (c) Cooking red meat causes a number of chemical changes, including the oxidation of iron in myoglobin that results in the familiar red-to-brown color change. (d) A banana turning brown is a chemical change as new, darker (and less tasty) substances form. (credit b: modification of work by Jeff Turner; credit c: modification of work by Gloria Cabada-Leman; credit d: modification of work by Roberto Verzo) 


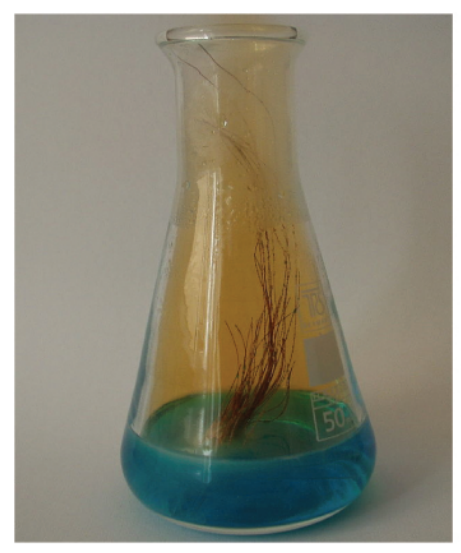

(a)

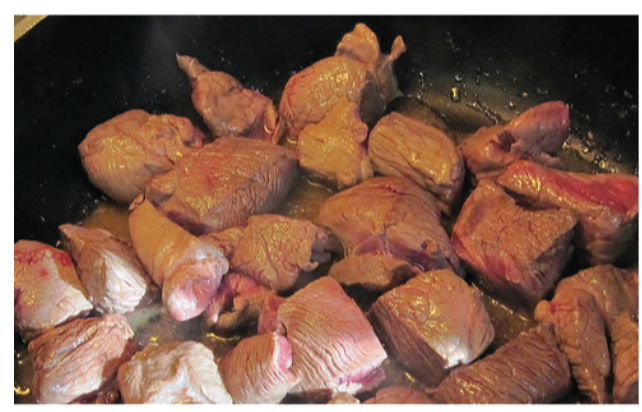

(c)

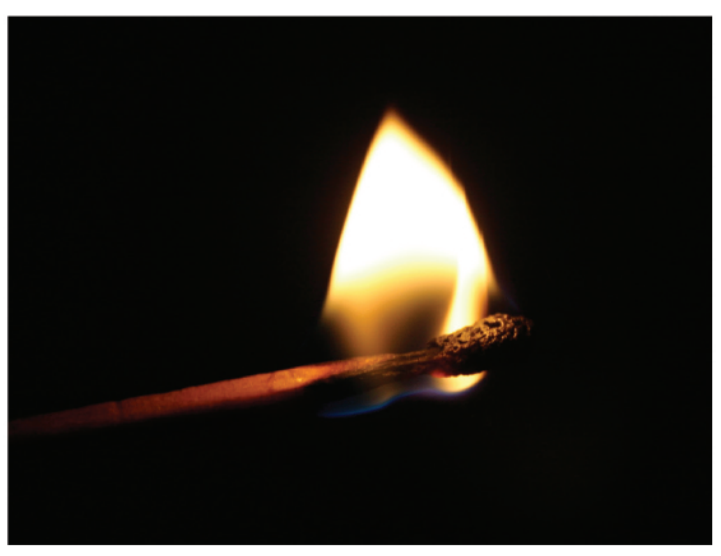

(b)

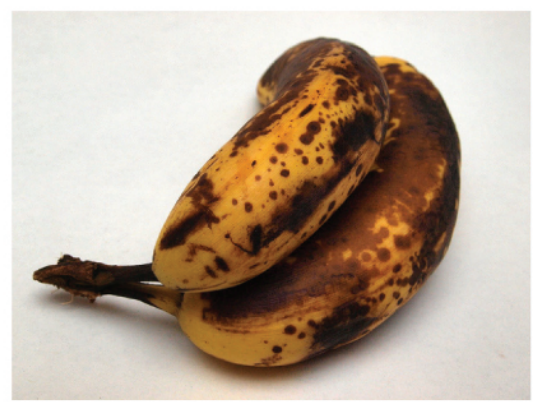

(d)

Properties of matter fall into one of two categories. If the property depends on the amount of matter present, it is an extensive property. The mass and volume of a substance are examples of extensive properties; for instance, a gallon of milk has a larger mass than a cup of milk. The value of an extensive property is directly proportional to the amount of matter in question. If the property of a sample of matter does not depend on the amount of matter present, it is an intensive property. Temperature is an example of an intensive property. If the gallon and cup of milk are each at $20{ }^{\circ} \mathrm{C}$ (room temperature), when they are combined, the temperature remains at $20{ }^{\circ} \mathrm{C}$. As another example, consider the distinct but related properties of heat and temperature. A drop of hot cooking oil spattered on your arm causes brief, minor discomfort, 
whereas a pot of hot oil yields severe burns. Both the drop and the pot of oil are at the same temperature (an intensive property), but the pot clearly contains much more heat (extensive property).

Hazard Diamond

You may have seen the symbol shown in (Figure) on containers of chemicals in a laboratory or workplace. Sometimes called a "fire diamond" or "hazard diamond," this chemical hazard diamond provides valuable information that briefly summarizes the various dangers of which to be aware when working with a particular substance.

The National Fire Protection Agency (NFPA) hazard diamond summarizes the major hazards of a chemical substance.

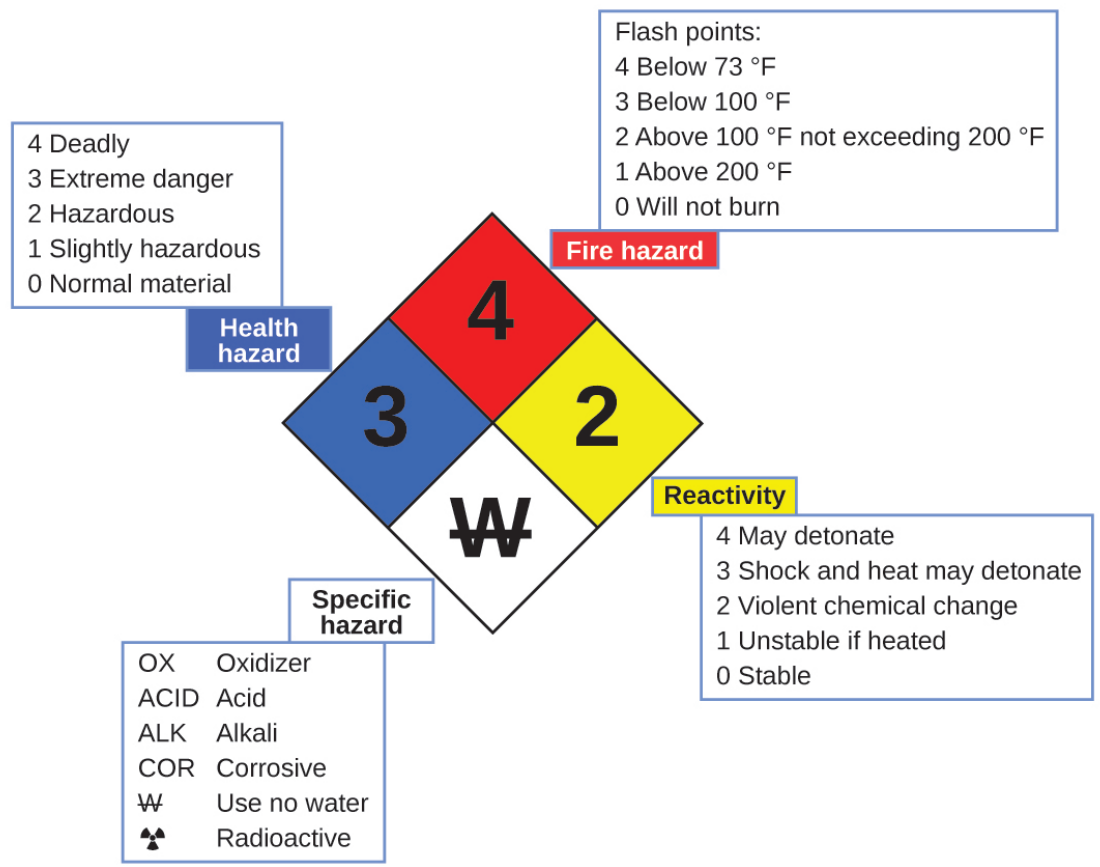

The National Fire Protection Agency (NFPA) 704 Hazard 
Identification System was developed by NFPA to provide safety information about certain substances. The system details flammability, reactivity, health, and other hazards. Within the overall diamond symbol, the top (red) diamond specifies the level of fire hazard (temperature range for flash point). The blue (left) diamond indicates the level of health hazard. The yellow (right) diamond describes reactivity hazards, such as how readily the substance will undergo detonation or a violent chemical change. The white (bottom) diamond points out special hazards, such as if it is an oxidizer (which allows the substance to burn in the absence of air/oxygen), undergoes an unusual or dangerous reaction with water, is corrosive, acidic, alkaline, a biological hazard, radioactive, and so on. Each hazard is rated on a scale from 0 to 4 , with 0 being no hazard and 4 being extremely hazardous.

While many elements differ dramatically in their chemical and physical properties, some elements have similar properties. For example, many elements conduct heat and electricity well, whereas others are poor conductors. These properties can be used to sort the elements into three classes: metals (elements that conduct well), nonmetals (elements that conduct poorly), and metalloids (elements that have intermediate conductivities).

The periodic table is a table of elements that places elements with similar properties close together ((Figure)). You will learn more about the periodic table as you continue your study of chemistry.

The periodic table shows how elements may be grouped according to certain similar properties. Note the background color denotes whether an element is a metal, 
metalloid, or nonmetal, whereas the element symbol color indicates whether it is a solid, liquid, or gas.
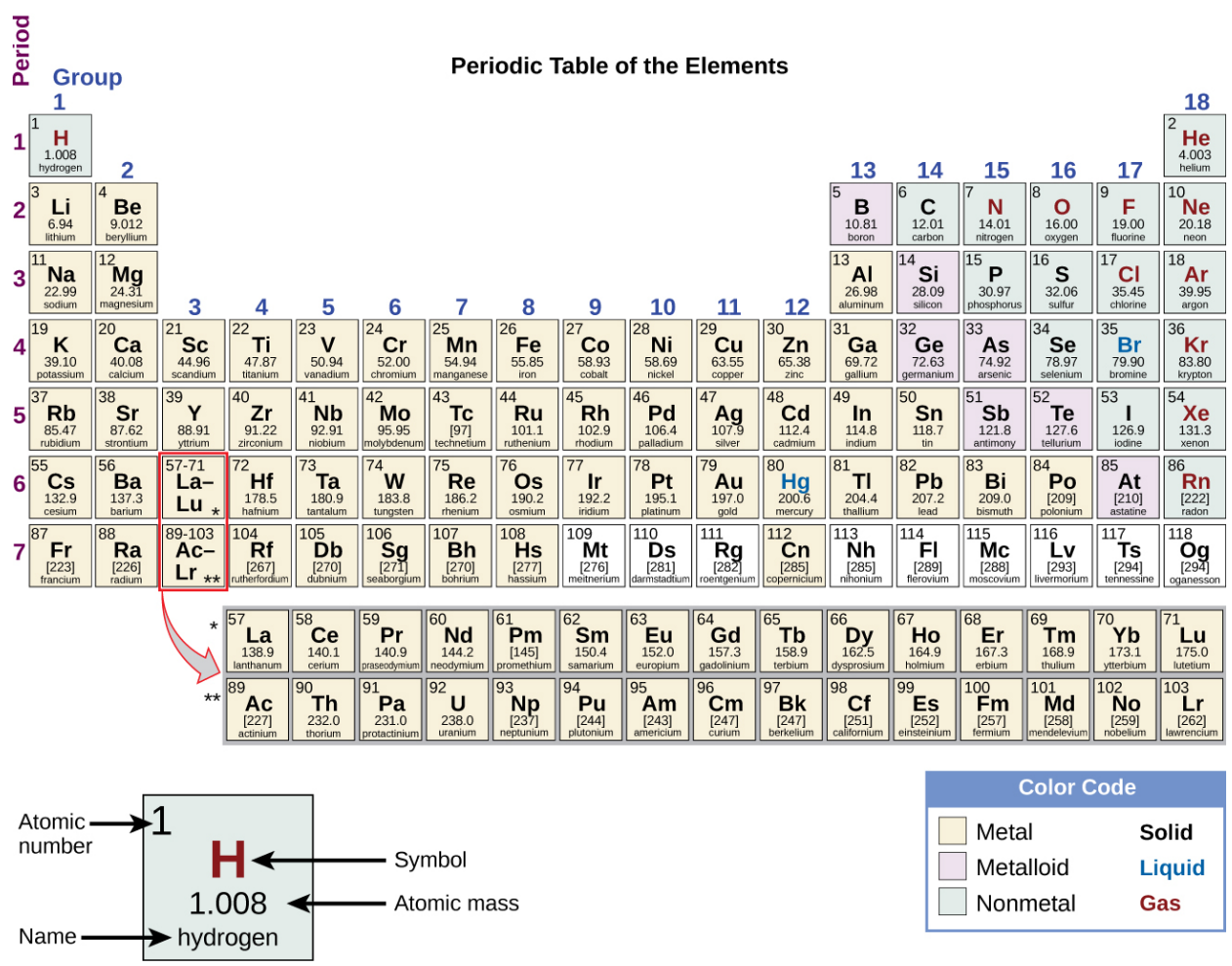

\begin{tabular}{|l|l|}
\multicolumn{2}{|c|}{ Color Code } \\
$\square$ Metal & Solid \\
$\square$ Metalloid & Liquid \\
$\square$ Nonmetal & Gas \\
\hline
\end{tabular}

Key Concepts and Summary

All substances have distinct physical and chemical properties, and may undergo physical or chemical changes. Physical properties, such as hardness and boiling point, and physical changes, such as melting or freezing, do not involve a change in the composition of matter. Chemical properties, such flammability and acidity, and chemical changes, such as rusting, involve production of matter that differs from that present beforehand.

Measurable properties fall into one of two categories. Extensive properties depend on the amount of matter present, for example, the mass of gold. Intensive properties do not depend on the amount of matter 
present, for example, the density of gold. Heat is an example of an extensive property, and temperature is an example of an intensive property.

Chemistry End of Chapter Exercises

Classify the six underlined properties in the following paragraph as chemical or physical:

Fluorine is a pale yellow gas that reacts with most substances. The free element melts at $-220^{\circ} \mathrm{C}$ and boils at $-188^{\circ} \mathrm{C}$. Finely divided metals burn in fluorine with a bright flame. Nineteen grams of fluorine will react with 1.0 gram of hydrogen.

Classify each of the following changes as physical or chemical:

(a) condensation of steam

(b) burning of gasoline

(c) souring of milk

(d) dissolving of sugar in water

(e) melting of gold

(a) physical; (b) chemical; (c) chemical; (d) physical; (e) physical

Classify each of the following changes as physical or chemical:

(a) coal burning

(b) ice melting

(c) mixing chocolate syrup with milk

(d) explosion of a firecracker

(e) magnetizing of a screwdriver 
The volume of a sample of oxygen gas changed from $10 \mathrm{~mL}$ to $11 \mathrm{~mL}$ as the temperature changed. Is this a chemical or physical change?

physical

A 2.0-liter volume of hydrogen gas combined with 1.0 liter of oxygen gas to produce 2.0 liters of water vapor. Does oxygen undergo a chemical or physical change?

Explain the difference between extensive properties and intensive properties.

The value of an extensive property depends upon the amount of matter being considered, whereas the value of an intensive property is the same regardless of the amount of matter being considered.

Identify the following properties as either extensive or intensive.

(a) volume

(b) temperature

(c) humidity

(d) heat

(e) boiling point

The density (d) of a substance is an intensive property that is defined as the ratio of its mass $(\mathrm{m})$ to its volume (V). density $=\frac{\text { mass }}{\text { volume }} \quad \mathrm{d}=\frac{\mathrm{m}}{\mathrm{V}}$

Considering that mass and volume are both extensive properties, explain why their ratio, density, is intensive. 
Being extensive properties, both mass and volume are directly proportional to the amount of substance under study. Dividing one extensive property by another will in effect "cancel" this dependence on amount, yielding a ratio that is independent of amount (an intensive property).

Glossary

chemical change

change producing a different kind of matter from the original kind of matter

chemical property

behavior that is related to the change of one kind of matter into another kind of matter

extensive property

property of a substance that depends on the amount of the substance

intensive property

property of a substance that is independent of the amount of the substance physical change

change in the state or properties of matter that does not involve a change in its chemical composition

physical property

characteristic of matter that is not associated with any change in its chemical composition 


\section{5.}

\section{MEASUREMENTS}

\section{Learning Objectives}

By the end of this section, you will be able to:

- Explain the process of measurement

- Identify the three basic parts of a quantity

- Describe the properties and units of length, mass, volume, density, temperature, and time

- Perform basic unit calculations and conversions in the metric and other unit systems

Measurements provide much of the information that informs the hypotheses, theories, and laws describing the behavior of matter and energy in both the macroscopic and microscopic domains of chemistry. Every measurement provides three kinds of information: the size or magnitude of the measurement (a number); a standard of comparison for the measurement (a unit); and 
an indication of the uncertainty of the measurement. While the number and unit are explicitly represented when a quantity is written, the uncertainty is an aspect of the measurement result that is more implicitly represented and will be discussed later.

The number in the measurement can be represented in different ways, including decimal form and scientific notation. (Scientific notation is also known as exponential notation; a review of this topic can be found in Appendix B.) For example, the maximum takeoff weight of a Boeing 777-200ER airliner is 298,000 kilograms, which can also be written as $2.9810^{5} \mathrm{~kg}$. The mass of the average mosquito is about 0.0000025 kilograms, which can be written as 2.5 $10^{-6} \mathrm{~kg}$.

Units, such as liters, pounds, and centimeters, are standards of comparison for measurements. A 2-liter bottle of a soft drink contains a volume of beverage that is twice that of the accepted volume of 1 liter. The meat used to prepare a 0.25 -pound hamburger weighs one-fourth as much as the accepted weight of 1 pound. Without units, a number can be meaningless, confusing, or possibly life threatening. Suppose a doctor prescribes phenobarbital to control a patient's seizures and states a dosage of "100" without specifying units. Not only will this be confusing to the medical professional giving the dose, but the consequences can be dire: $100 \mathrm{mg}$ given three times per day can be effective as an anticonvulsant, but a single dose of $100 \mathrm{~g}$ is more than 10 times the lethal amount.

The measurement units for seven fundamental properties ("base units") are listed in (Figure). The standards for these units are fixed by international agreement, and they are called the International System 
of Units or SI Units (from the French, Le Système International d'Unités). SI units have been used by the United States National Institute of Standards and Technology (NIST) since 1964. Units for other properties may be derived from these seven base units.

\section{Base Units of the SI System}

Property Measured Name of Unit Symbol of Unit

$\begin{array}{lll}\text { length } & \text { meter } & \mathrm{m} \\ \text { mass } & \text { kilogram } & \mathrm{kg} \\ \text { time } & \text { second } & \mathrm{s} \\ \text { temperature } & \text { kelvin } & \mathrm{K} \\ \text { electric current } & \text { ampere } & \mathrm{A} \\ \text { amount of substance } & \text { mole } & \mathrm{mol} \\ \text { luminous intensity } & \text { candela } & \mathrm{cd}\end{array}$

Everyday measurement units are often defined as fractions or multiples of other units. Milk is commonly packaged in containers of 1 gallon (4 quarts), 1 quart (0.25 gallon), and one pint (0.5 quart). This same approach is used with SI units, but these fractions or multiples are always powers of 10. Fractional or multiple SI units are named using a prefix and the name of the base unit. For example, a length of 1000 meters is also called a kilometer because the prefix kilo means "one thousand," which in scientific notation is $10^{3}$ (1 kilometer $=1000 \mathrm{~m}=10^{3} \mathrm{~m}$ ). The prefixes used and the powers to which 10 are raised are listed in (Figure). 
Common Unit Prefixes

Prefix Symbol Factor Example

\begin{tabular}{|c|c|c|c|}
\hline femto & $\mathrm{f}$ & $10^{-15}$ & $\begin{array}{l}1 \text { femtosecond }(\mathrm{fs})=110^{-15} \mathrm{~s} \\
(0.000000000000001 \mathrm{~s})\end{array}$ \\
\hline pico & $\mathrm{p}$ & $10^{-12}$ & $\begin{array}{l}1 \text { picometer }(\mathrm{pm})=110^{-12} \mathrm{~m} \\
(0.000000000001 \mathrm{~m})\end{array}$ \\
\hline nano & $\mathrm{n}$ & $10^{-9}$ & $\begin{array}{l}4 \text { nanograms (ng) }=410^{-9} \mathrm{~g}(0.000000004 \\
\text { g) }\end{array}$ \\
\hline micro & $\mu$ & $10^{-6}$ & 1 microliter $(\mu \mathrm{L})=110^{-6} \mathrm{~L}(0.000001 \mathrm{~L})$ \\
\hline milli & $\mathrm{m}$ & $10^{-3}$ & $\begin{array}{l}2 \text { millimoles }(\mathrm{mmol})=210^{-3} \mathrm{~mol}(0.002 \\
\mathrm{mol})\end{array}$ \\
\hline centi & c & $10^{-2}$ & 7 centimeters $(\mathrm{cm})=710^{-2} \mathrm{~m}(0.07 \mathrm{~m})$ \\
\hline deci & $d$ & $10^{-1}$ & 1 deciliter $(\mathrm{dL})=110^{-1} \mathrm{~L}(0.1 \mathrm{~L})$ \\
\hline kilo & $\mathrm{k}$ & $10^{3}$ & 1 kilometer $(\mathrm{km})=110^{3} \mathrm{~m}(1000 \mathrm{~m})$ \\
\hline mega & M & $10^{6}$ & $\begin{array}{l}3 \text { megahertz }(\mathrm{MHz})=310^{6} \mathrm{~Hz}(3,000,000 \\
\mathrm{Hz})\end{array}$ \\
\hline giga & G & $10^{9}$ & $\begin{array}{l}8 \text { gigayears }(\mathrm{Gyr})=8 \quad 10^{9} \mathrm{yr}(8,000,000,000 \\
\mathrm{yr})\end{array}$ \\
\hline tera & $\mathrm{T}$ & $10^{12}$ & $\begin{array}{l}5 \text { terawatts }(\mathrm{TW})=510^{12} \mathrm{~W} \\
(5,000,000,000,000 \mathrm{~W})\end{array}$ \\
\hline
\end{tabular}

Need a refresher or more practice with scientific notation? Visit this site to go over the basics of scientific notation.

SI Base Units

The initial units of the metric system, which eventually evolved into the SI system, were established in France during the French Revolution. The original standards for the meter and the kilogram were adopted there in 1799 and eventually by other countries. This section introduces four of the SI base units commonly used in chemistry. Other SI units will be introduced in subsequent chapters. 
Length

The standard unit of length in both the SI and original metric systems is the meter (m). A meter was originally specified as $1 / 10,000,000$ of the distance from the North Pole to the equator. It is now defined as the distance light in a vacuum travels in $1 / 299,792,458$ of a second. A meter is about 3 inches longer than a yard ((Figure)); one meter is about 39.37 inches or 1.094 yards. Longer distances are often reported in kilometers $\left(1 \mathrm{~km}=1000 \mathrm{~m}=10^{3} \mathrm{~m}\right)$, whereas shorter distances can be reported in centimeters $\left(1 \mathrm{~cm}=0.01 \mathrm{~m}=10^{-2} \mathrm{~m}\right)$ or millimeters $(1 \mathrm{~mm}=0.001 \mathrm{~m}=$ $\left.10^{-3} \mathrm{~m}\right)$.

The relative lengths of $1 \mathrm{~m}, 1 \mathrm{yd}, 1 \mathrm{~cm}$, and $1 \mathrm{in}$. are shown (not actual size), as well as comparisons of $2.54 \mathrm{~cm}$ and 1 in., and of $1 \mathrm{~m}$ and $1.094 \mathrm{yd}$.

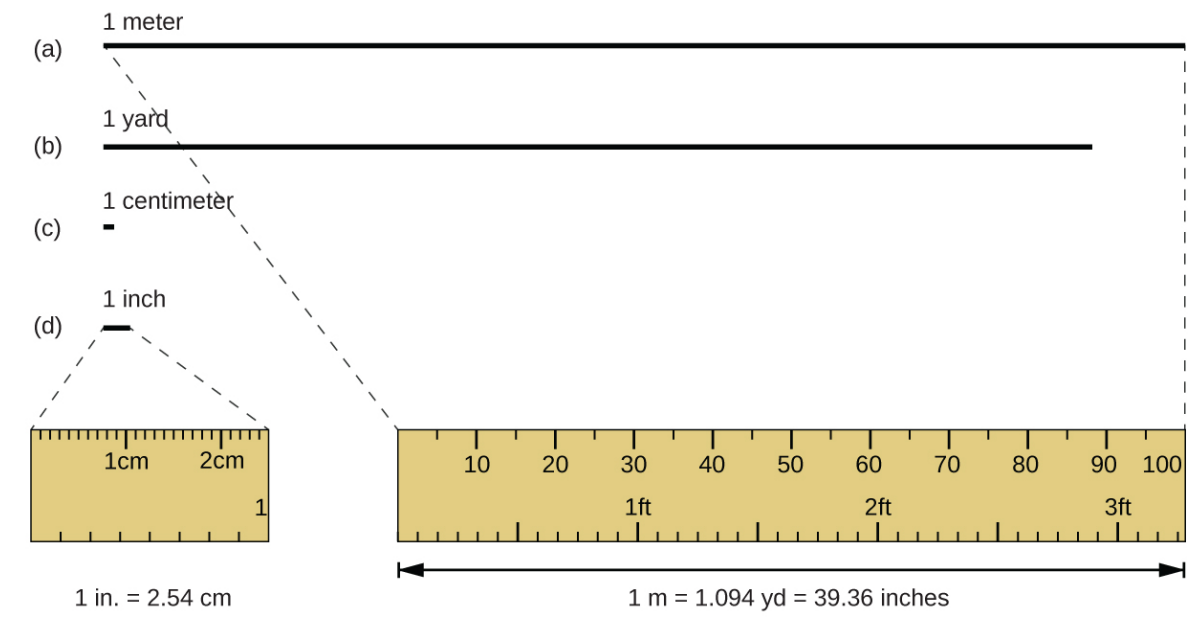

Mass

The standard unit of mass in the SI system is the kilogram (kg). The kilogram was previously defined by the International Union of Pure and Applied Chemistry 
(IUPAC) as the mass of a specific reference object. This object was originally one liter of pure water, and more recently it was a metal cylinder made from a platinumiridium alloy with a height and diameter of $39 \mathrm{~mm}$ ((Figure)). In May 2019, this definition was changed to one that is based instead on precisely measured values of several fundamental physical constants. ${ }^{1}$. One kilogram is about 2.2 pounds. The gram (g) is exactly equal to $1 / 1000$ of the mass of the kilogram $\left(10^{-3} \mathrm{~kg}\right)$.

This replica prototype kilogram as previously defined is housed at the National Institute of Standards and Technology (NIST) in Maryland. (credit: National Institutes of Standards and Technology)

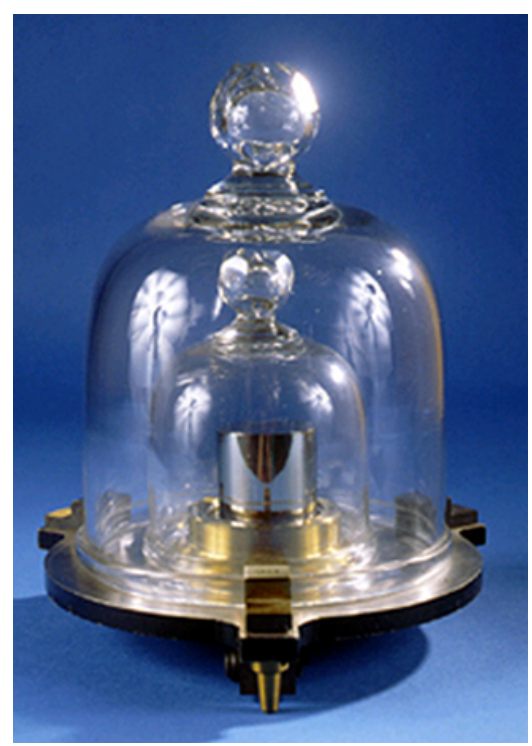

\section{Temperature}

Temperature is an intensive property. The SI unit of temperature is the kelvin (K). The IUPAC convention is to use kelvin (all lowercase) for the word, $\mathrm{K}$ (uppercase) for the unit symbol, and neither the word "degree" nor the degree symbol $\left({ }^{\circ}\right)$. The degree Celsius $\left({ }^{\circ} \mathrm{C}\right)$ is also allowed 
in the SI system, with both the word "degree" and the degree symbol used for Celsius measurements. Celsius degrees are the same magnitude as those of kelvin, but the two scales place their zeros in different places. Water freezes at $273.15 \mathrm{~K}\left(0{ }^{\circ} \mathrm{C}\right)$ and boils at $373.15 \mathrm{~K}\left(100{ }^{\circ} \mathrm{C}\right)$ by definition, and normal human body temperature is approximately $310 \mathrm{~K}\left(37^{\circ} \mathrm{C}\right)$. The conversion between these two units and the Fahrenheit scale will be discussed later in this chapter.

\section{Time}

The SI base unit of time is the second (s). Small and large time intervals can be expressed with the appropriate prefixes; for example, 3 microseconds $=0.000003 \mathrm{~s}=3$ $10^{-6}$ and 5 megaseconds $=5,000,000 \mathrm{~s}=510^{6} \mathrm{~s}$. Alternatively, hours, days, and years can be used.

Derived SI Units

We can derive many units from the seven SI base units. For example, we can use the base unit of length to define a unit of volume, and the base units of mass and length to define a unit of density.

\section{Volume}

Volume is the measure of the amount of space occupied by an object. The standard SI unit of volume is defined by the base unit of length ((Figure)). The standard volume is a cubic meter $\left(\mathrm{m}^{3}\right)$, a cube with an edge length of exactly one meter. To dispense a cubic meter of water, we could build a cubic box with edge lengths of exactly one meter. 
This box would hold a cubic meter of water or any other substance.

A more commonly used unit of volume is derived from the decimeter $(0.1 \mathrm{~m}$, or $10 \mathrm{~cm})$. A cube with edge lengths of exactly one decimeter contains a volume of one cubic decimeter $\left(\mathrm{dm}^{3}\right)$. A liter $(\mathrm{L})$ is the more common name for the cubic decimeter. One liter is about 1.06 quarts.

A cubic centimeter $\left(\mathrm{cm}^{3}\right)$ is the volume of a cube with an edge length of exactly one centimeter. The abbreviation cc (for cubic centimeter) is often used by health professionals. A cubic centimeter is equivalent to a milliliter $(\mathrm{mL})$ and is $1 / 1000$ of a liter.

(a) The relative volumes are shown for cubes of $1 \mathrm{~m}^{3}, 1$ $\mathrm{dm}^{3}(1 \mathrm{~L})$, and $1 \mathrm{~cm}^{3}$ (1 $\mathrm{mL}$ ) (not to scale). (b) The diameter of a dime is compared relative to the edge length of a $1-\mathrm{cm}^{3}(1-\mathrm{mL})$ cube.

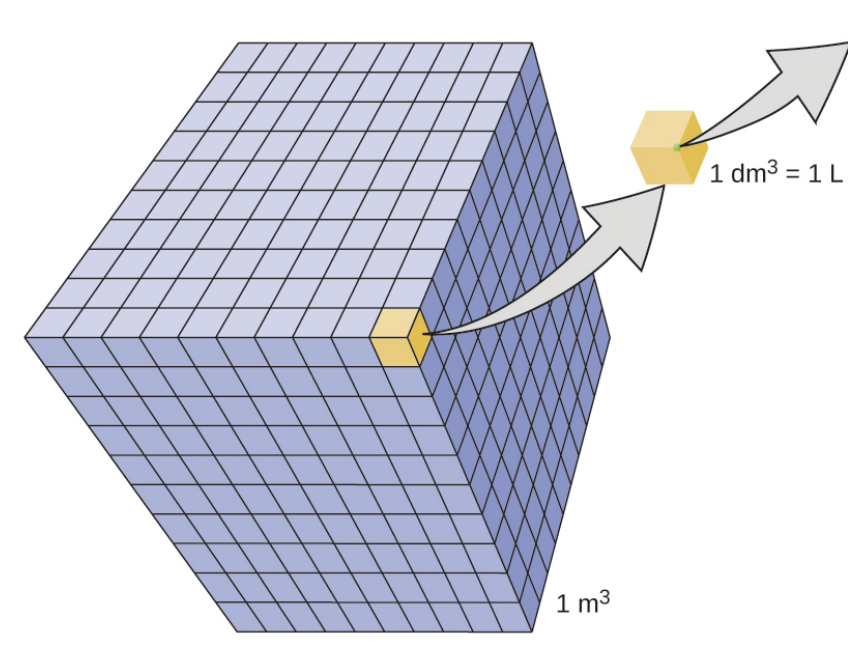

(a)

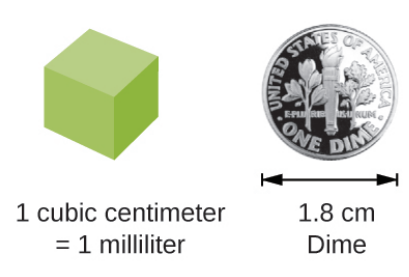

(b)

Density

We use the mass and volume of a substance to determine 
its density. Thus, the units of density are defined by the base units of mass and length.

The density of a substance is the ratio of the mass of a sample of the substance to its volume. The SI unit for density is the kilogram per cubic meter $\left(\mathrm{kg} / \mathrm{m}^{3}\right)$. For many situations, however, this as an inconvenient unit, and we often use grams per cubic centimeter $\left(\mathrm{g} / \mathrm{cm}^{3}\right)$ for the densities of solids and liquids, and grams per liter $(\mathrm{g} / \mathrm{L})$ for gases. Although there are exceptions, most liquids and solids have densities that range from about $0.7 \mathrm{~g} / \mathrm{cm}^{3}$ (the density of gasoline) to $19 \mathrm{~g} / \mathrm{cm}^{3}$ (the density of gold). The density of air is about $1.2 \mathrm{~g} / \mathrm{L}$. (Figure) shows the densities of some common substances.

\section{Densities of Common Substances}

Solids

ice $\left(\right.$ at $\left.0{ }^{\circ} \mathrm{C}\right) 0.92 \mathrm{~g} /$

$\mathrm{cm}^{3}$

oak (wood) $0.60-0.90$

$\mathrm{g} / \mathrm{cm}^{3}$

iron $7.9 \mathrm{~g} / \mathrm{cm}^{3}$

copper $9.0 \mathrm{~g} / \mathrm{cm}^{3}$

lead $11.3 \mathrm{~g} / \mathrm{cm}^{3}$

silver $10.5 \mathrm{~g} / \mathrm{cm}^{3}$

gold $19.3 \mathrm{~g} / \mathrm{cm}^{3}$
Liquids

water $1.0 \mathrm{~g} / \mathrm{cm}^{3} \quad$ dry air $1.20 \mathrm{~g} / \mathrm{L}$

ethanol $0.79 \mathrm{~g} / \mathrm{cm}^{3} \quad$ oxygen $1.31 \mathrm{~g} / \mathrm{L}$

acetone $0.79 \mathrm{~g} / \mathrm{cm}^{3} \quad$ nitrogen $1.14 \mathrm{~g} / \mathrm{L}$

glycerin $1.26 \mathrm{~g} / \mathrm{cm}^{3}$

olive oil $0.92 \mathrm{~g} / \mathrm{cm}^{3} \quad$ helium $0.16 \mathrm{~g} / \mathrm{L}$

gasoline $0.70-0.77$ $\mathrm{g} / \mathrm{cm}^{3}$

mercury $13.6 \mathrm{~g} / \mathrm{cm}^{3}$ radon $9.1 \mathrm{~g} / \mathrm{L}$

While there are many ways to determine the density of an object, perhaps the most straightforward method involves separately finding the mass and volume of the object, and 
then dividing the mass of the sample by its volume. In the following example, the mass is found directly by weighing, but the volume is found indirectly through length measurements.

density $=\frac{\text { mass }}{\text { volume }}$

Calculation of Density Gold-in bricks, bars, and coins-has been a form of currency for centuries. In order to swindle people into paying for a brick of gold without actually investing in a brick of gold, people have considered filling the centers of hollow gold bricks with lead to fool buyers into thinking that the entire brick is gold. It does not work: Lead is a dense substance, but its density is not as great as that of gold, $19.3 \mathrm{~g} / \mathrm{cm}^{3}$. What is the density of lead if a cube of lead has an edge length of $2.00 \mathrm{~cm}$ and a mass of $90.7 \mathrm{~g}$ ?

Solution The density of a substance can be calculated by dividing its mass by its volume. The volume of a cube is calculated by cubing the edge length.

volume of lead cube $=2.00 \mathrm{~cm} 2.00 \mathrm{~cm} 2.00 \mathrm{~cm}=8.00 \mathrm{~cm}^{3}$

density $=\frac{\text { mass }}{\text { volume }}=\frac{90.7 \mathrm{~g}}{8.00 \mathrm{~cm}^{3}}=\frac{11.3 \mathrm{~g}}{1.00 \mathrm{~cm}^{3}}=11.3 \mathrm{~g} / \mathrm{cm}^{3}$

(We will discuss the reason for rounding to the first decimal place in the next section.)

Check Your Learning (a) To three decimal places, what is the volume of a cube $\left(\mathrm{cm}^{3}\right)$ with an edge length of $0.843 \mathrm{~cm}$ ?

(b) If the cube in part (a) is copper and has a mass of $5.34 \mathrm{~g}$, what is the density of copper to two decimal places?

Answer:

(a) $0.599 \mathrm{~cm}^{3}$; (b) $8.91 \mathrm{~g} / \mathrm{cm}^{3}$

To learn more about the relationship between mass, volume, and density, use this interactive simulator to 
explore the density of different materials, like wood, ice, brick, and aluminum.

\section{Using Displacement of Water to Determine Density This} PhET simulation illustrates another way to determine density, using displacement of water. Determine the density of the red and yellow blocks.

Solution When you open the density simulation and select Same Mass, you can choose from several 5.00-kg colored blocks that you can drop into a tank containing 100.00 L water. The yellow block floats (it is less dense than water), and the water level rises to $105.00 \mathrm{~L}$. While floating, the yellow block displaces $5.00 \mathrm{~L}$ water, an amount equal to the weight of the block. The red block sinks (it is more dense than water, which has density $=1.00 \mathrm{~kg} / \mathrm{L}$ ), and the water level rises to $101.25 \mathrm{~L}$.

The red block therefore displaces $1.25 \mathrm{~L}$ water, an amount equal to the volume of the block. The density of the red block is:

$$
\text { density }=\frac{\text { mass }}{\text { volume }}=\frac{5.00 \mathrm{~kg}}{1.25 \mathrm{~L}}=4.00 \mathrm{~kg} / \mathrm{L}
$$

Note that since the yellow block is not completely submerged, you cannot determine its density from this information. But if you hold the yellow block on the bottom of the tank, the water level rises to $110.00 \mathrm{~L}$, which means that it now displaces $10.00 \mathrm{~L}$ water, and its density can be found:

$$
\text { density }=\frac{\text { mass }}{\text { volume }}=\frac{5.00 \mathrm{~kg}}{10.00 \mathrm{~L}}=0.500 \mathrm{~kg} / \mathrm{L}
$$

Check Your Learning Remove all of the blocks from the water and add the green block to the tank of water, placing it approximately in the middle of the tank. Determine the density of the green block.

Answer:

$2.00 \mathrm{~kg} / \mathrm{L}$ 
Key Concepts and Summary

Measurements provide quantitative information that is critical in studying and practicing chemistry. Each measurement has an amount, a unit for comparison, and an uncertainty. Measurements can be represented in either decimal or scientific notation. Scientists primarily use SI (International System) units such as meters, seconds, and kilograms, as well as derived units, such as liters (for volume) and $\mathrm{g} / \mathrm{cm}^{3}$ (for density). In many cases, it is convenient to use prefixes that yield fractional and multiple units, such as microseconds $\left(10^{-6}\right.$ seconds) and megahertz ( $10^{6}$ hertz), respectively.

Key Equations

- density $=\frac{\text { mass }}{\text { volume }}$

Chemistry End of Chapter Exercises

Is one liter about an ounce, a pint, a quart, or a gallon?

Is a meter about an inch, a foot, a yard, or a mile?

about a yard

Indicate the SI base units or derived units that are appropriate for the following measurements:

(a) the length of a marathon race (26 miles 385 yards)

(b) the mass of an automobile

(c) the volume of a swimming pool

(d) the speed of an airplane

(e) the density of gold 
(f) the area of a football field

(g) the maximum temperature at the South Pole on April 1, 1913

Indicate the SI base units or derived units that are appropriate for the following measurements:

(a) the mass of the moon

(b) the distance from Dallas to Oklahoma City

(c) the speed of sound

(d) the density of air

(e) the temperature at which alcohol boils

(f) the area of the state of Delaware

(g) the volume of a flu shot or a measles vaccination

(a) kilograms; (b) meters; (c) kilometers/second; (d) kilograms/cubic meter; (e) kelvin; (f) square meters; (g) cubic meters

Give the name and symbol of the prefixes used with SI units to indicate multiplication by the following exact quantities.

(a) $10^{3}$

(b) $10^{-2}$

(c) 0.1

(d) $10^{-3}$

(e) $1,000,000$

(f) 0.000001

Give the name of the prefix and the quantity indicated by the following symbols that are used with SI base units.

(a) c

(b) $\mathrm{d}$

(c) $\mathrm{G}$ 
(d) $\mathrm{k}$

(e) $\mathrm{m}$

(f) $n$

(g) $\mathrm{p}$

(h) $\mathrm{T}$

(a) centi-, $10^{-2}$; (b) deci-, $10^{-1}$; (c) Giga-, $10^{9}$; (d) kilo-, $10^{3}$; (e) milli-, $10^{-3}$; (f) nano-, $10^{-9}$; (g) pico-, $10^{-12}$; (h) tera-, $10^{12}$

A large piece of jewelry has a mass of $132.6 \mathrm{~g}$. A graduated cylinder initially contains $48.6 \mathrm{~mL}$ water. When the jewelry is submerged in the graduated cylinder, the total volume increases to $61.2 \mathrm{~mL}$.

(a) Determine the density of this piece of jewelry.

(b) Assuming that the jewelry is made from only one substance, what substance is it likely to be? Explain.

Visit this PhET density simulation and select the Same Volume Blocks.

(a) What are the mass, volume, and density of the yellow block?

(b) What are the mass, volume and density of the red block?

(c) List the block colors in order from smallest to largest mass.

(d) List the block colors in order from lowest to highest density.

(e) How are mass and density related for blocks of the same volume?

(a) $8.00 \mathrm{~kg}, 5.00 \mathrm{~L}, 1.60 \mathrm{~kg} / \mathrm{L}$; (b) $2.00 \mathrm{~kg}, 5.00 \mathrm{~L}, 0.400 \mathrm{~kg} /$ L; (c) red < green < blue < yellow; (d) If the volumes are 
the same, then the density is directly proportional to the mass.

Visit this PhET density simulation and select Custom Blocks and then My Block.

(a) Enter mass and volume values for the block such that the mass in $\mathrm{kg}$ is less than the volume in L. What does the block do? Why? Is this always the case when mass < volume?

(b) Enter mass and volume values for the block such that the mass in $\mathrm{kg}$ is more than the volume in L. What does the block do? Why? Is this always the case when mass > volume?

(c) How would (a) and (b) be different if the liquid in the tank were ethanol instead of water?

(d) How would (a) and (b) be different if the liquid in the tank were mercury instead of water?

Visit this PhET density simulation and select Mystery Blocks.

(a) Pick one of the Mystery Blocks and determine its mass, volume, density, and its likely identity.

(b) Pick a different Mystery Block and determine its mass, volume, density, and its likely identity.

(c) Order the Mystery Blocks from least dense to most dense. Explain.

(a) (b) Answer is one of the following. A/yellow: mass $=65.14 \mathrm{~kg}$, volume $=3.38 \mathrm{~L}$, density $=19.3 \mathrm{~kg} / \mathrm{L}$, likely identity $=$ gold. $\mathrm{B} /$ blue: mass $=0.64 \mathrm{~kg}$, volume $=1.00 \mathrm{~L}$, density $=0.64 \mathrm{~kg} / \mathrm{L}$, likely identity $=$ apple. $\mathrm{C} /$ green: mass $=4.08 \mathrm{~kg}$, volume $=5.83 \mathrm{~L}$, density $=0.700 \mathrm{~kg} / \mathrm{L}$, likely 
identity = gasoline. $\mathrm{D} / \mathrm{red}$ : mass $=3.10 \mathrm{~kg}$, volume $=3.38$ $\mathrm{L}$, density $=0.920 \mathrm{~kg} / \mathrm{L}$, likely identity $=$ ice; and E/purple: mass $=3.53 \mathrm{~kg}$, volume $=1.00 \mathrm{~L}$, density $=3.53 \mathrm{~kg} / \mathrm{L}$, likely identity = diamond. (c) B/blue/apple $(0.64 \mathrm{~kg} / \mathrm{L})<\mathrm{C} /$ green/ gasoline $(0.700 \mathrm{~kg} / \mathrm{L})<\mathrm{D} / \mathrm{red} /$ ice $(0.920 \mathrm{~kg} / \mathrm{L})<\mathrm{E} /$ purple/ diamond $(3.53 \mathrm{~kg} / \mathrm{L})<$ A/yellow/gold $(19.3 \mathrm{~kg} / \mathrm{L})$

Footnotes

- 1For details see https://www.nist.gov/pml/ weights-and-measures/si-units-mass

Glossary

\section{Celsius $\left({ }^{\circ} \mathrm{C}\right)$}

unit of temperature; water freezes at $0^{\circ} \mathrm{C}$ and boils at $100^{\circ} \mathrm{C}$ on this scale

cubic centimeter $\left(\mathrm{cm}^{3}\right.$ or $\left.\mathrm{cc}\right)$

volume of a cube with an edge length of exactly $1 \mathrm{~cm}$

cubic meter $\left(\mathrm{m}^{3}\right)$

SI unit of volume

density

ratio of mass to volume for a substance or object

kelvin (K)

$\mathrm{SI}$ unit of temperature; $273.15 \mathrm{~K}=0{ }^{\circ} \mathrm{C}$

\section{kilogram (kg)}

standard SI unit of mass; $1 \mathrm{~kg}=$ approximately 2.2 pounds 
length

measure of one dimension of an object

liter (L)

(also, cubic decimeter) unit of volume; $1 \mathrm{~L}=$ $1,000 \mathrm{~cm}^{3}$

meter (m)

standard metric and SI unit of length; $1 \mathrm{~m}=$ approximately 1.094 yards milliliter $(\mathrm{mL})$

$1 / 1,000$ of a liter; equal to $1 \mathrm{~cm}^{3}$ second (s)

Sl unit of time SI units (International System of Units)

standards fixed by international agreement in the International System of Units (Le Système International d'Unités) unit standard of comparison for measurements volume amount of space occupied by an object 
6.

\section{MATHEMATICAL} TREATMENT OF

\section{MEASUREMENT RESULTS}

\section{Learning Objectives}

By the end of this section, you will be able to:

- Explain the dimensional analysis (factor label) approach to mathematical calculations involving quantities

- Use dimensional analysis to carry out unit conversions for a given property and computations involving two or more properties

It is often the case that a quantity of interest may not be easy (or even possible) to measure directly but instead must be calculated from other directly measured properties and appropriate mathematical relationships. 
For example, consider measuring the average speed of an athlete running sprints. This is typically accomplished by measuring the time required for the athlete to run from the starting line to the finish line, and the distance between these two lines, and then computing speed from the equation that relates these three properties:

speed $=\frac{\text { distance }}{\text { time }}$

An Olympic-quality sprinter can run $100 \mathrm{~m}$ in approximately $10 \mathrm{~s}$, corresponding to an average speed of $\frac{100 \mathrm{~m}}{10 \mathrm{~s}}=10 \mathrm{~m} / \mathrm{s}$

Note that this simple arithmetic involves dividing the numbers of each measured quantity to yield the number of the computed quantity $(100 / 10=10)$ and likewise dividing the units of each measured quantity to yield the unit of the computed quantity $(\mathrm{m} / \mathrm{s}=\mathrm{m} / \mathrm{s})$. Now, consider using this same relation to predict the time required for a person running at this speed to travel a distance of $25 \mathrm{~m}$. The same relation among the three properties is used, but in this case, the two quantities provided are a speed $(10 \mathrm{~m} / \mathrm{s})$ and a distance $(25 \mathrm{~m})$. To yield the sought property, time, the equation must be rearranged appropriately:

time $=\frac{\text { distance }}{\text { speed }}$

The time can then be computed as:

$\frac{25 \mathrm{~m}}{10 \mathrm{~m} / \mathrm{s}}=2.5 \mathrm{~s}$

Again, arithmetic on the numbers $(25 / 10=2.5)$ was accompanied by the same arithmetic on the units $(\mathrm{m} / \mathrm{m} /$ $\mathrm{s}=\mathrm{s}$ ) to yield the number and unit of the result, $2.5 \mathrm{~s}$. Note that, just as for numbers, when a unit is divided by 
an identical unit (in this case, $\mathrm{m} / \mathrm{m}$ ), the result is " 1 "-or, as commonly phrased, the units "cancel."

These calculations are examples of a versatile mathematical approach known as dimensional analysis (or the factor-label method). Dimensional analysis is based on this premise: the units of quantities must be subjected to the same mathematical operations as their associated numbers. This method can be applied to computations ranging from simple unit conversions to more complex, multi-step calculations involving several different quantities.

Conversion Factors and Dimensional Analysis

A ratio of two equivalent quantities expressed with different measurement units can be used as a unit conversion factor. For example, the lengths of $2.54 \mathrm{~cm}$ and 1 in. are equivalent (by definition), and so a unit conversion factor may be derived from the ratio, $\frac{2.54 \mathrm{~cm}}{1 \mathrm{in} .}(2.54 \mathrm{~cm}=1$ in. $)$ or $2.54 \frac{\mathrm{cm}}{\mathrm{in}}$

Several other commonly used conversion factors are given in (Figure).

\section{Common Conversion Factors}

Length

$1 \mathrm{~m}=1.0936 \mathrm{yd}$

1 in. $=2.54 \mathrm{~cm}$

(exact)

$1 \mathrm{~km}=0.62137 \mathrm{~m}$

$1 \mathrm{mi}=1609.3 \mathrm{~m}$

\section{Volume}

$1 \mathrm{~L}=1.0567 \mathrm{qt}$

$1 \mathrm{qt}=0.94635 \mathrm{~L} \quad 1 \mathrm{lb}=453.59 \mathrm{~g}$

$1 \mathrm{ft}^{3}=28.317 \mathrm{~L} \quad \underset{\mathrm{g}}{1 \text { (avoirdupois) } \mathrm{oz}=28.349}$

1 tbsp $=14.787$

$\mathrm{mL}$
Mass

$1 \mathrm{~kg}=2.2046 \mathrm{lb}$

1 (troy) oz $=31.103 \mathrm{~g}$ 
When a quantity (such as distance in inches) is multiplied by an appropriate unit conversion factor, the quantity is converted to an equivalent value with different units (such as distance in centimeters). For example, a basketball player's vertical jump of 34 inches can be converted to centimeters by:

$34 \overline{\operatorname{in}} \frac{2.54 \mathrm{~cm}}{1 \overline{\text { )in. }}}=86 \mathrm{~cm}$

Since this simple arithmetic involves quantities, the premise of dimensional analysis requires that we multiply both numbers and units. The numbers of these two quantities are multiplied to yield the number of the product quantity, 86 , whereas the units are multiplied to yield $\frac{\text { in. } \mathrm{cm}}{\text { in. }}$. Just as for numbers, a ratio of identical units is also numerically equal to one, $\frac{i n}{\mathrm{in}}$. $=1$, and the unit product thus simplifies to $\mathrm{cm}$. (When identical units divide to yield a factor of 1, they are said to "cancel.") Dimensional analysis may be used to confirm the proper application of unit conversion factors as demonstrated in the following example.

Using a Unit Conversion Factor The mass of a competition frisbee is $125 \mathrm{~g}$. Convert its mass to ounces using the unit conversion factor derived from the relationship $1 \mathrm{oz}=28.349 \mathrm{~g}$ ((Figure)).

Solution Given the conversion factor, the mass in ounces may be derived using an equation similar to the one used for converting length from inches to centimeters.

$x \mathrm{oz}=125 \mathrm{~g}$ unit conversion factor

The unit conversion factor may be represented as:

$\frac{1 \mathrm{oz}}{28.349 \mathrm{~g}}$ and $\frac{28.349 \mathrm{~g}}{1 \mathrm{oz}}$ 
The correct unit conversion factor is the ratio that cancels the units of grams and leaves ounces.

$$
\begin{aligned}
x \mathrm{oz} & =125 \overline{) \mathrm{g}} \frac{1 \mathrm{oz}}{28.349 \overline{\mathrm{g}}} \\
& =\left(\frac{125}{28.349}\right) \mathrm{oz} \\
& =4.41 \mathrm{oz} \text { (three significant figures) }
\end{aligned}
$$

Check Your Learning Convert a volume of 9.345 qt to liters.

Answer:

$8.844 \mathrm{~L}$

Beyond simple unit conversions, the factor-label method can be used to solve more complex problems involving computations. Regardless of the details, the basic approach is the same-all the factors involved in the calculation must be appropriately oriented to ensure that their labels (units) will appropriately cancel and/or combine to yield the desired unit in the result. As your study of chemistry continues, you will encounter many opportunities to apply this approach.

Computing Quantities from Measurement Results and Known Mathematical Relations What is the density of common antifreeze in units of $\mathrm{g} / \mathrm{mL}$ ? A 4.00-qt sample of the antifreeze weighs $9.26 \mathrm{lb}$.

Solution Since density $=\frac{\text { mass }}{\text { volume' }}$ we need to divide the mass in grams by the volume in milliliters. In general: the number of units of $B=$ the number of units of $A$ unit conversion factor. The necessary conversion factors are given in (Figure): $1 \mathrm{lb}=453.59 \mathrm{~g} ; 1 \mathrm{~L}=1.0567 \mathrm{qt} ; 1 \mathrm{~L}=1,000 \mathrm{~mL}$. Mass may be converted from pounds to grams as follows:

$$
9.26 \overline{\mathrm{lb}} \frac{453.59 \mathrm{~g}}{1 \overline{\mathrm{lb}}}=4.2010^{3} \mathrm{~g}
$$


Volume may be converted from quarts to millimeters via two steps:

1. Convert quarts to liters.

$$
4 . 0 0 \longdiv { ) \mathrm { qt } } \frac { 1 \mathrm { L } } { 1 . 0 5 6 7 \overline { \mathrm { qt } } } = 3 . 7 8 \mathrm { L }
$$

2. Convert liters to milliliters.

$$
3.78 \overline{\mathrm{L}} \frac{1000 \mathrm{~mL}}{1 \overline{\mathrm{L}}}=3.7810^{3} \mathrm{~mL}
$$

Then,

$$
\text { density }=\frac{4.2010^{3} \mathrm{~g}}{3.7810^{3} \mathrm{~mL}}=1.11 \mathrm{~g} / \mathrm{mL}
$$

Alternatively, the calculation could be set up in a way that uses three unit conversion factors sequentially as follows:

$$
\frac{9.26 \overline{\mathrm{lb}}}{4.00 \overline{\mathrm{qt}}} \frac{453.59 \mathrm{~g}}{1 \overline{\mathrm{lb}}} \frac{1.0567 \overline{\mathrm{qt}}}{1 \overline{\mathrm{L}}} \frac{1 \overline{\mathrm{L}}}{1000 \mathrm{~mL}}=1.11 \mathrm{~g} / \mathrm{mL}
$$

Check Your Learning What is the volume in liters of $1.000 \mathrm{oz}$, given that $1 \mathrm{~L}=1.0567 \mathrm{qt}$ and $1 \mathrm{qt}=32 \mathrm{oz}$ (exactly)?

Answer:

$2.956-10^{-2} \mathrm{~L}$

Computing Quantities from Measurement Results and Known Mathematical Relations While being driven from Philadelphia to Atlanta, a distance of about 1250 km, a 2014 Lamborghini Aventador Roadster uses $213 \mathrm{~L}$ gasoline.

(a) What (average) fuel economy, in miles per gallon, did the Roadster get during this trip?

(b) If gasoline costs ?3.80 per gallon, what was the fuel cost for this trip?

Solution (a) First convert distance from kilometers to miles:

$1250 \overline{\mathrm{km}} \frac{0.62137 \mathrm{mi}}{1 \overline{\mathrm{km}}}=777 \mathrm{mi}$

and then convert volume from liters to gallons: 


$$
213 \overline{\mathrm{LL}} \frac{1.0567 \overline{\mathrm{qt}}}{1 \overline{\mathrm{LL}}} \frac{1 \mathrm{gal}}{4 \overline{\mathrm{qt}}}=56.3 \mathrm{gal}
$$

Finally,

$$
\text { (average) mileage }=\frac{777 \mathrm{mi}}{56.3 \mathrm{gal}}=13.8 \mathrm{miles} / \text { gallon }=13.8 \mathrm{mpg}
$$

Alternatively, the calculation could be set up in a way that uses all the conversion factors sequentially, as follows:

$$
\frac{1250 \overline{\mathrm{km}}}{213 \overline{\mathrm{L}}} \frac{0.62137 \mathrm{mi}}{1 \overline{\mathrm{km}}} \frac{1 \overline{\mathrm{L}}}{1.0567 \overline{\mathrm{qt}}} \frac{4 \overline{\mathrm{qt}}}{1 \mathrm{gal}}=13.8 \mathrm{mpg}
$$

(b) Using the previously calculated volume in gallons, we find:

$$
56.3 \overline{\text { gal }} \frac{3.80}{1 \overline{\text { gal }}}=214
$$

Check Your Learning A Toyota Prius Hybrid uses $59.7 \mathrm{~L}$ gasoline to drive from San Francisco to Seattle, a distance of $1300 \mathrm{~km}$ (two significant digits).

(a) What (average) fuel economy, in miles per gallon, did the Prius get during this trip?

(b) If gasoline costs ?3.90 per gallon, what was the fuel cost for this trip?

Answer:

(a) $51 \mathrm{mpg}$; (b) ?62

Conversion of Temperature Units

We use the word temperature to refer to the hotness or coldness of a substance. One way we measure a change in temperature is to use the fact that most substances expand when their temperature increases and contract when their temperature decreases. The mercury or alcohol in a common glass thermometer changes its volume as the temperature changes, and the position of the trapped 
liquid along a printed scale may be used as a measure of temperature.

Temperature scales are defined relative to selected reference temperatures: Two of the most commonly used are the freezing and boiling temperatures of water at a specified atmospheric pressure. On the Celsius scale, 0 ${ }^{\circ} \mathrm{C}$ is defined as the freezing temperature of water and $100{ }^{\circ} \mathrm{C}$ as the boiling temperature of water. The space between the two temperatures is divided into 100 equal intervals, which we call degrees. On the Fahrenheit scale, the freezing point of water is defined as $32{ }^{\circ} \mathrm{F}$ and the boiling temperature as $212^{\circ} \mathrm{F}$. The space between these two points on a Fahrenheit thermometer is divided into 180 equal parts (degrees).

Defining the Celsius and Fahrenheit temperature scales as described in the previous paragraph results in a slightly more complex relationship between temperature values on these two scales than for different units of measure for other properties. Most measurement units for a given property are directly proportional to one another $(\mathrm{y}=\mathrm{mx})$. Using familiar length units as one example: length in feet $=\left(\frac{1 \mathrm{ft}}{12 \mathrm{in} .}\right)$ length in inches

where $\mathrm{y}=$ length in feet, $\mathrm{x}=$ length in inches, and the proportionality constant, $\mathrm{m}$, is the conversion factor. The Celsius and Fahrenheit temperature scales, however, do not share a common zero point, and so the relationship between these two scales is a linear one rather than a proportional one $(\mathrm{y}=\mathrm{mx}+\mathrm{b})$. Consequently, converting a temperature from one of these scales into the other requires more than simple multiplication by a conversion 
factor, $\mathrm{m}$, it also must take into account differences in the scales' zero points (b).

The linear equation relating Celsius and Fahrenheit temperatures is easily derived from the two temperatures used to define each scale. Representing the Celsius temperature as $x$ and the Fahrenheit temperature as $y$, the slope, $m$, is computed to be:

$m=\frac{y}{x}=\frac{212 \mathrm{~F}-32 \mathrm{~F}}{100 \mathrm{C}-0 \mathrm{C}}=\frac{180 \mathrm{~F}}{100 \mathrm{C}}=\frac{9 \mathrm{~F}}{5 \mathrm{C}}$

The $y$-intercept of the equation, $b$, is then calculated using either of the equivalent temperature pairs, $\left(100{ }^{\circ} \mathrm{C}, 212\right.$ $\left.{ }^{\circ} \mathrm{F}\right)$ or $\left(0{ }^{\circ} \mathrm{C}, 32{ }^{\circ} \mathrm{F}\right)$, as:

$b=y-m x=32 \mathrm{~F}-\frac{9 \mathrm{~F}}{5 \mathrm{C}} 0 \mathrm{C}=32 \mathrm{~F}$

The equation relating the temperature $(T)$ scales is then:

$T_{\mathrm{F}}=\left(\frac{9 \mathrm{~F}}{5 \mathrm{C}} T_{\mathrm{C}}\right)+32 \mathrm{C}$

An abbreviated form of this equation that omits the measurement units is:

$T_{\mathrm{F}}=\left(\frac{9}{5} T_{\mathrm{C}}\right)+32$

Rearrangement of this equation yields the form useful for converting from Fahrenheit to Celsius:

$T_{\mathrm{C}}=\frac{5}{9}\left(T_{\mathrm{F}}-32\right)$

As mentioned earlier in this chapter, the SI unit of temperature is the kelvin (K). Unlike the Celsius and Fahrenheit scales, the kelvin scale is an absolute temperature scale in which 0 (zero) $\mathrm{K}$ corresponds to the lowest temperature that can theoretically be achieved. Since the kelvin temperature scale is absolute, a degree symbol is not included in the unit abbreviation, K. The 
early 19th-century discovery of the relationship between a gas's volume and temperature suggested that the volume of a gas would be zero at $-273.15^{\circ} \mathrm{C}$. In 1848, British physicist William Thompson, who later adopted the title of Lord Kelvin, proposed an absolute temperature scale based on this concept (further treatment of this topic is provided in this text's chapter on gases).

The freezing temperature of water on this scale is 273.15 K and its boiling temperature is $373.15 \mathrm{~K}$. Notice the numerical difference in these two reference temperatures is 100 , the same as for the Celsius scale, and so the linear relation between these two temperature scales will exhibit a slope of $1 \frac{\mathrm{K}}{\mathrm{C}}$. Following the same approach, the equations for converting between the kelvin and Celsius temperature scales are derived to be:

$T_{\mathrm{K}}=T_{\mathrm{C}}+273.15$

$T_{\mathrm{C}}=T_{\mathrm{K}}-273.15$

The 273.15 in these equations has been determined experimentally, so it is not exact. (Figure) shows the relationship among the three temperature scales. The Fahrenheit, Celsius, and kelvin temperature scales are compared. 


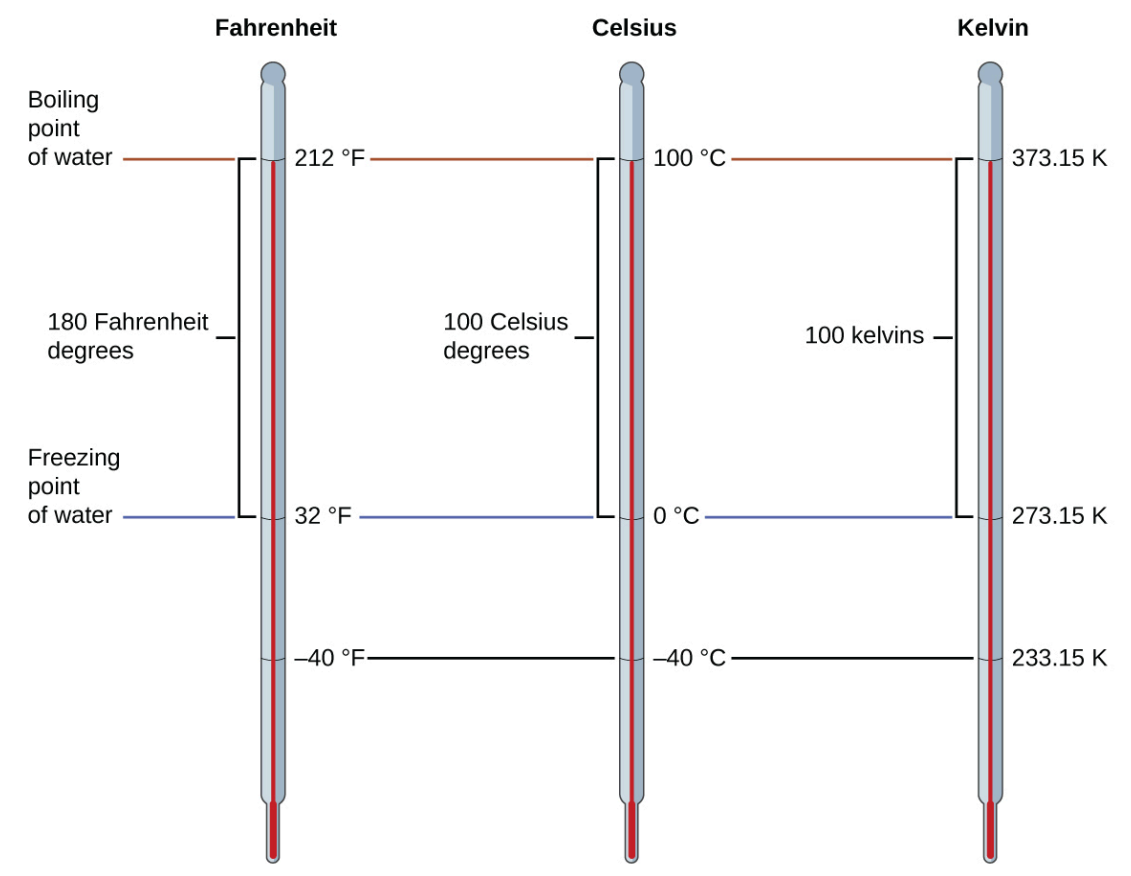

Although the kelvin (absolute) temperature scale is the official SI temperature scale, Celsius is commonly used in many scientific contexts and is the scale of choice for nonscience contexts in almost all areas of the world. Very few countries (the U.S. and its territories, the Bahamas, Belize, Cayman Islands, and Palau) still use Fahrenheit for weather, medicine, and cooking.

Conversion from Celsius Normal body temperature has been commonly accepted as $37.0^{\circ} \mathrm{C}$ (although it varies depending on time of day and method of measurement, as well as among individuals). What is this temperature on the kelvin scale and on the Fahrenheit scale?

Solution

$$
\begin{aligned}
& \mathrm{K}=\mathrm{C}+273.15=37.0+273.2=310.2 \mathrm{~K} \\
& \mathrm{~F}=\frac{9}{5} \mathrm{C}+32.0=\left(\frac{9}{5} 37.0\right)+32.0=66.6+32.0=98.6 \mathrm{~F}
\end{aligned}
$$

Check Your Learning Convert $80.92{ }^{\circ} \mathrm{C}$ to $\mathrm{K}$ and ${ }^{\circ} \mathrm{F}$. 
Answer:

$354.07 \mathrm{~K}, 177.7^{\circ} \mathrm{F}$

Conversion from Fahrenheit Baking a ready-made pizza calls for an oven temperature of $450^{\circ} \mathrm{F}$. If you are in Europe, and your oven thermometer uses the Celsius scale, what is the setting? What is the kelvin temperature?

Solution

$\mathrm{C}=\frac{5}{9}(\mathrm{~F}-32)=\frac{5}{9}(450-32)=\frac{5}{9} 418=232 \mathrm{C}$ set oven to $230 \mathrm{C} \quad$ (two significant figures) $\mathrm{K}=\mathrm{C}+273.15=230+273=503 \mathrm{~K} 5.010^{2} \mathrm{~K} \quad$ (two significant figures)

Check Your Learning Convert $50{ }^{\circ} \mathrm{F}$ to ${ }^{\circ} \mathrm{C}$ and $\mathrm{K}$.

Answer:

$10{ }^{\circ} \mathrm{C}, 280 \mathrm{~K}$

Key Concepts and Summary

Measurements are made using a variety of units. It is often useful or necessary to convert a measured quantity from one unit into another. These conversions are accomplished using unit conversion factors, which are derived by simple applications of a mathematical approach called the factor-label method or dimensional analysis. This strategy is also employed to calculate sought quantities using measured quantities and appropriate mathematical relations.

Key Equations

- $T_{\mathrm{C}}=\frac{5}{9}\left(T_{\mathrm{F}}-32\right)$

- $T_{\mathrm{F}}=\left(\frac{9}{5} T_{\mathrm{C}}\right)+32$

- $T_{\mathrm{K}}=\mathrm{C}+273.15$ 
88

$$
\text { - } T_{\mathrm{C}}=\mathrm{K}-273.15
$$

Chemistry End of Chapter Exercises

Write conversion factors (as ratios) for the number of:

(a) yards in 1 meter

(b) liters in 1 liquid quart

(c) pounds in 1 kilogram

(a) $\frac{1.0936 \mathrm{yd}}{1 \mathrm{~m}}$; (b) $\frac{0.94635 \mathrm{~L}}{1 \mathrm{qt}}$; (c) $\frac{2.2046 \mathrm{lb}}{1 \mathrm{~kg}}$

Write conversion factors (as ratios) for the number of:

(a) kilometers in 1 mile

(b) liters in 1 cubic foot

(c) grams in 1 ounce

The label on a soft drink bottle gives the volume in two units: $2.0 \mathrm{~L}$ and $67.6 \mathrm{fl} \mathrm{oz}$. Use this information to derive a conversion factor between the English and metric units. How many significant figures can you justify in your conversion factor?

$\frac{2.0 \mathrm{~L}}{67.6 \mathrm{fl} \mathrm{oz}}=\frac{0.030 \mathrm{~L}}{1 \mathrm{fl} \mathrm{oz}}$

Only two significant figures are justified.

The label on a box of cereal gives the mass of cereal in two units: 978 grams and $34.5 \mathrm{oz}$. Use this information to find a conversion factor between the English and metric units. How many significant figures can you justify in your conversion factor?

Soccer is played with a round ball having a circumference between 27 and $28 \mathrm{in}$. and a weight between 14 and $16 \mathrm{oz}$. 
What are these specifications in units of centimeters and grams?

$68-71 \mathrm{~cm} ; 400-450 \mathrm{~g}$

A woman's basketball has a circumference between 28.5 and 29.0 inches and a maximum weight of 20 ounces (two significant figures). What are these specifications in units of centimeters and grams?

How many milliliters of a soft drink are contained in a 12.0-oz can?

$355 \mathrm{~mL}$

A barrel of oil is exactly 42 gal. How many liters of oil are in a barrel?

The diameter of a red blood cell is about $310^{-4} \mathrm{in}$. What is its diameter in centimeters?

$810^{-4} \mathrm{~cm}$

The distance between the centers of the two oxygen atoms in an oxygen molecule is $1.2110^{-8} \mathrm{~cm}$. What is this distance in inches?

Is a 197-lb weight lifter light enough to compete in a class limited to those weighing $90 \mathrm{~kg}$ or less?

yes; weight $=89.4 \mathrm{~kg}$

A very good 197-lb weight lifter lifted $192 \mathrm{~kg}$ in a move 
called the clean and jerk. What was the mass of the weight lifted in pounds?

Many medical laboratory tests are run using $5.0 \mu \mathrm{L}$ blood serum. What is this volume in milliliters?

$5.010^{-3} \mathrm{~mL}$

If an aspirin tablet contains $325 \mathrm{mg}$ aspirin, how many grams of aspirin does it contain?

Use scientific (exponential) notation to express the following quantities in terms of the SI base units in (Figure):
(a) $0.13 \mathrm{~g}$
(b) $232 \mathrm{Gg}$
(c) $5.23 \mathrm{pm}$
(d) $86.3 \mathrm{mg}$
(e) $37.6 \mathrm{~cm}$
(f) $54 \mu \mathrm{m}$
(g) $1 \mathrm{Ts}$
(h) $27 \mathrm{ps}$
(i) $0.15 \mathrm{mK}$

(a) $1.310^{-4} \mathrm{~kg}$; (b) $2.3210^{8} \mathrm{~kg}$; (c) $5.2310^{-12} \mathrm{~m}$; (d) 8.63 $10^{-5} \mathrm{~kg}$; (e) $3.7610^{-1} \mathrm{~m}$; (f) $5.410^{-5} \mathrm{~m}$; (g) $110^{12} \mathrm{~s}$; (h) $2.710^{-11} \mathrm{~s}$; (i) $1.510^{-4} \mathrm{~K}$

Complete the following conversions between SI units.
(a) $612 \mathrm{~g}=$ $\mathrm{mg}$
(b) $8.160 \mathrm{~m}=$ $\mathrm{cm}$
(c) $3779 \mu \mathrm{g}=$ g
(d) $781 \mathrm{~mL}=$ $\mathrm{L}$ 
(e) $4.18 \mathrm{~kg}=$

(f) $27.8 \mathrm{~m}=$ g

(g) $0.13 \mathrm{~mL}=$

(h) $1738 \mathrm{~km}=$ $\mathrm{m}$

(i) $1.9 \mathrm{Gg}=$ g

Gasoline is sold by the liter in many countries. How many liters are required to fill a 12.0-gal gas tank?

$45.4 \mathrm{~L}$

Milk is sold by the liter in many countries. What is the volume of exactly $1 / 2$ gal of milk in liters?

A long ton is defined as exactly $2240 \mathrm{lb}$. What is this mass in kilograms?

$1.016010^{3} \mathrm{~kg}$

Make the conversion indicated in each of the following:

(a) the men's world record long jump, $29 \mathrm{ft} 4 \frac{1}{4} \mathrm{in}$., to meters

(b) the greatest depth of the ocean, about $6.5 \mathrm{mi}$, to kilometers

(c) the area of the state of Oregon, $96,981 \mathrm{mi}^{2}$, to square kilometers

(d) the volume of 1 gill (exactly $4 \mathrm{oz}$ ) to milliliters

(e) the estimated volume of the oceans, 330,000,000 $\mathrm{mi}^{3}$, to cubic kilometers.

(f) the mass of a 3525-lb car to kilograms

(g) the mass of a 2.3-oz egg to grams

Make the conversion indicated in each of the following: 
(a) the length of a soccer field, $120 \mathrm{~m}$ (three significant figures), to feet

(b) the height of Mt. Kilimanjaro, at $19,565 \mathrm{ft}$, the highest mountain in Africa, to kilometers

(c) the area of an 8.5- $\times 11$-inch sheet of paper in $\mathrm{cm}^{2}$

(d) the displacement volume of an automobile engine, 161 in. $^{3}$, to liters

(e) the estimated mass of the atmosphere, $5.6 \times 10^{15}$ tons, to kilograms

(f) the mass of a bushel of rye, $32.0 \mathrm{lb}$, to kilograms

(g) the mass of a 5.00-grain aspirin tablet to milligrams $(1$ grain $=0.00229 \mathrm{oz})$

(a) $394 \mathrm{ft}$; (b) $5.9634 \mathrm{~km}$; (c) $6.010^{2}$; (d) $2.64 \mathrm{~L}$; (e) 5.1 $10^{18} \mathrm{~kg}$; (f) $14.5 \mathrm{~kg}$; (g) $324 \mathrm{mg}$

Many chemistry conferences have held a 50-Trillion Angstrom Run (two significant figures). How long is this run in kilometers and in miles? $\left(1 \AA=110^{-10} \mathrm{~m}\right)$

A chemist's 50-Trillion Angstrom Run (see (Figure)) would be an archeologist's 10,900 cubit run. How long is one cubit in meters and in feet? $\left(1 \AA=110^{-8} \mathrm{~cm}\right)$

$0.46 \mathrm{~m} ; 1.5 \mathrm{ft} / \mathrm{cubit}$

The gas tank of a certain luxury automobile holds 22.3 gallons according to the owner's manual. If the density of gasoline is $0.8206 \mathrm{~g} / \mathrm{mL}$, determine the mass in kilograms and pounds of the fuel in a full tank.

As an instructor is preparing for an experiment, he requires $225 \mathrm{~g}$ phosphoric acid. The only container readily 
available is a $150-\mathrm{mL}$ Erlenmeyer flask. Is it large enough to contain the acid, whose density is $1.83 \mathrm{~g} / \mathrm{mL}$ ?

Yes, the acid's volume is $123 \mathrm{~mL}$.

To prepare for a laboratory period, a student lab assistant needs $125 \mathrm{~g}$ of a compound. A bottle containing $1 / 4 \mathrm{lb}$ is available. Did the student have enough of the compound?

A chemistry student is $159 \mathrm{~cm}$ tall and weighs $45.8 \mathrm{~kg}$. What is her height in inches and weight in pounds?

62.6 in (about $5 \mathrm{ft} 3 \mathrm{in}$.) and $101 \mathrm{lb}$

In a recent Grand Prix, the winner completed the race with an average speed of $229.8 \mathrm{~km} / \mathrm{h}$. What was his speed in miles per hour, meters per second, and feet per second?

Solve these problems about lumber dimensions.

(a) To describe to a European how houses are constructed in the US, the dimensions of "two-by-four" lumber must be converted into metric units. The thickness width length dimensions are $1.50 \mathrm{in} .3 .50 \mathrm{in} .8 .00 \mathrm{ft}$ in the US. What are the dimensions in $\mathrm{cm} \mathrm{cm} \mathrm{m}$ ?

(b) This lumber can be used as vertical studs, which are typically placed 16.0 in. apart. What is that distance in centimeters?

(a) $3.81 \mathrm{~cm} 8.89 \mathrm{~cm} 2.44 \mathrm{~m}$; (b) $40.6 \mathrm{~cm}$

The mercury content of a stream was believed to be above the minimum considered safe -1 part per billion (ppb) by weight. An analysis indicated that the concentration was 
0.68 parts per billion. What quantity of mercury in grams was present in $15.0 \mathrm{~L}$ of the water, the density of which is $0.998 \mathrm{~g} / \mathrm{ml}$ ? $\left(1 \mathrm{ppb} \mathrm{Hg}=\frac{1 \mathrm{ng} \mathrm{Hg}}{1 \mathrm{~g} \text { water }}\right)$

Calculate the density of aluminum if $27.6 \mathrm{~cm}^{3}$ has a mass of $74.6 \mathrm{~g}$.

$2.70 \mathrm{~g} / \mathrm{cm}^{3}$

Osmium is one of the densest elements known. What is its density if $2.72 \mathrm{~g}$ has a volume of $0.121 \mathrm{~cm}^{3}$ ?

Calculate these masses.

(a) What is the mass of $6.00 \mathrm{~cm}^{3}$ of mercury, density = $13.5939 \mathrm{~g} / \mathrm{cm}^{3}$ ?

(b) What is the mass of $25.0 \mathrm{~mL}$ octane, density $=0.702$ $\mathrm{g} / \mathrm{cm}^{3}$ ?

(a) $81.6 \mathrm{~g}$; (b) $17.6 \mathrm{~g}$

Calculate these masses.

(a) What is the mass of $4.00 \mathrm{~cm}^{3}$ of sodium, density = $0.97 \mathrm{~g} / \mathrm{cm}^{3}$ ?

(b) What is the mass of $125 \mathrm{~mL}$ gaseous chlorine, density $=3.16 \mathrm{~g} / \mathrm{L}$ ?

Calculate these volumes.

(a) What is the volume of $25 \mathrm{~g}$ iodine, density $=4.93 \mathrm{~g} /$ $\mathrm{cm}^{3}$ ?

(b) What is the volume of $3.28 \mathrm{~g}$ gaseous hydrogen, density $=0.089 \mathrm{~g} / \mathrm{L}$ ?

(a) $5.1 \mathrm{~mL}$; (b) $37 \mathrm{~L}$ 
Calculate these volumes.

(a) What is the volume of 11.3 g graphite, density $=2.25$ $\mathrm{g} / \mathrm{cm}^{3}$ ?

(b) What is the volume of $39.657 \mathrm{~g}$ bromine, density $=$ $2.928 \mathrm{~g} / \mathrm{cm}^{3}$ ?

Convert the boiling temperature of gold, $2966{ }^{\circ} \mathrm{C}$, into degrees Fahrenheit and kelvin.

$5371^{\circ} \mathrm{F}, 3239 \mathrm{~K}$

Convert the temperature of scalding water, $54{ }^{\circ} \mathrm{C}$, into degrees Fahrenheit and kelvin.

Convert the temperature of the coldest area in a freezer, $-10^{\circ} \mathrm{F}$, to degrees Celsius and kelvin.

$-23^{\circ} \mathrm{C}, 250 \mathrm{~K}$

Convert the temperature of dry ice, $-77^{\circ} \mathrm{C}$, into degrees Fahrenheit and kelvin.

Convert the boiling temperature of liquid ammonia, -28.1 ${ }^{\circ} \mathrm{F}$, into degrees Celsius and kelvin.

$-33.4^{\circ} \mathrm{C}, 239.8 \mathrm{~K}$

The label on a pressurized can of spray disinfectant warns against heating the can above $130{ }^{\circ} \mathrm{F}$. What are the corresponding temperatures on the Celsius and kelvin temperature scales?

The weather in Europe was unusually warm during the summer of 1995 . The TV news reported temperatures as 
high as $45{ }^{\circ} \mathrm{C}$. What was the temperature on the Fahrenheit scale?

\section{$113^{\circ} \mathrm{F}$}

Glossary

dimensional analysis

(also, factor-label method) versatile mathematical approach that can be applied to computations ranging from simple unit conversions to more complex, multi-step calculations involving several different quantities

\section{Fahrenheit}

unit of temperature; water freezes at $32^{\circ} \mathrm{F}$ and boils at $212^{\circ} \mathrm{F}$ on this scale

\section{temperature}

intensive property representing the hotness or coldness of matter unit conversion factor

ratio of equivalent quantities expressed with different units; used to convert from one unit to a different unit 


\section{PART II \\ ATOMS, MOLECULES, \\ AND IONS}



7.

\section{INTRODUCTION}

\section{Learning Objectives}

- Early Ideas in Atomic Theory

- Evolution of Atomic Theory

- Atomic Structure and Symbolism

- Chemical Formulas

- $\quad$ The Periodic Table

- Molecular and Ionic Compounds

- Chemical Nomenclature

Analysis of molecules in an exhaled breath can provide valuable information, leading to early diagnosis of diseases or detection of environmental exposure to harmful substances. (credit: modification of work by Paul Flowers) 


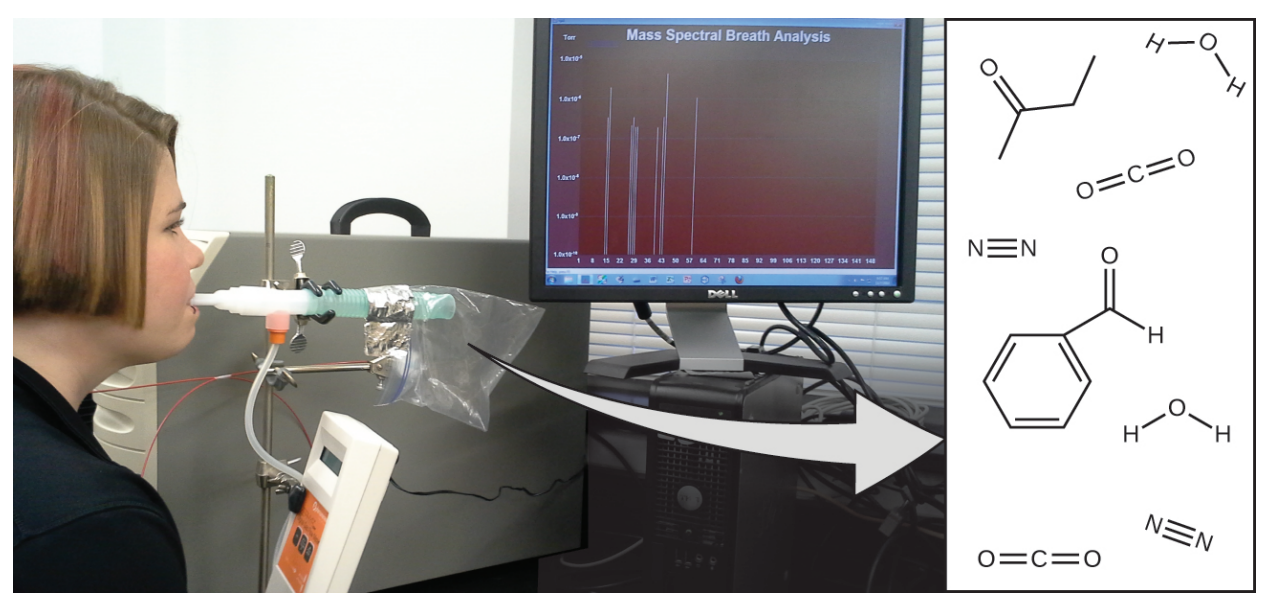

Your overall health and susceptibility to disease depends upon the complex interaction between your genetic makeup and environmental exposure, with the outcome difficult to predict. Early detection of biomarkers, substances that indicate an organism's disease or physiological state, could allow diagnosis and treatment before a condition becomes serious or irreversible. Recent studies have shown that your exhaled breath can contain molecules that may be biomarkers for recent exposure to environmental contaminants or for pathological conditions ranging from asthma to lung cancer. Scientists are working to develop biomarker "fingerprints" that could be used to diagnose a specific disease based on the amounts and identities of certain molecules in a patient's exhaled breath. An essential concept underlying this goal is that of a molecule's identity, which is determined by the numbers and types of atoms it contains, and how they are bonded together. This chapter will describe some of the fundamental chemical principles related to the composition of matter, including those central to the concept of molecular identity. 
8.

\section{EARLY IDEAS IN ATOMIC \\ THEORY}

\section{Learning Objectives}

By the end of this section, you will be able to:

- State the postulates of Dalton's atomic theory

- Use postulates of Dalton's atomic theory to explain the laws of definite and multiple proportions

The earliest recorded discussion of the basic structure of matter comes from ancient Greek philosophers, the scientists of their day. In the fifth century BC, Leucippus and Democritus argued that all matter was composed of small, finite particles that they called atomos, a term derived from the Greek word for "indivisible." They thought of atoms as moving particles that differed in shape and size, and which could join together. Later, 
Aristotle and others came to the conclusion that matter consisted of various combinations of the four "elements"-fire, earth, air, and water-and could be infinitely divided. Interestingly, these philosophers thought about atoms and "elements" as philosophical concepts, but apparently never considered performing experiments to test their ideas.

The Aristotelian view of the composition of matter held sway for over two thousand years, until English schoolteacher John Dalton helped to revolutionize chemistry with his hypothesis that the behavior of matter could be explained using an atomic theory. First published in 1807, many of Dalton's hypotheses about the microscopic features of matter are still valid in modern atomic theory. Here are the postulates of Dalton's atomic theory.

1. Matter is composed of exceedingly small particles called atoms. An atom is the smallest unit of an element that can participate in a chemical change.

2. An element consists of only one type of atom, which has a mass that is characteristic of the element and is the same for all atoms of that element ((Figure)). A macroscopic sample of an element contains an incredibly large number of atoms, all of which have identical chemical properties.

A pre-1982 copper penny (left) contains approximately $3 \quad 10^{22}$ copper atoms (several dozen are represented as brown spheres at the right), each of which has the same chemical 
properties. (credit: modification of work by “slgckgc"/Flickr)

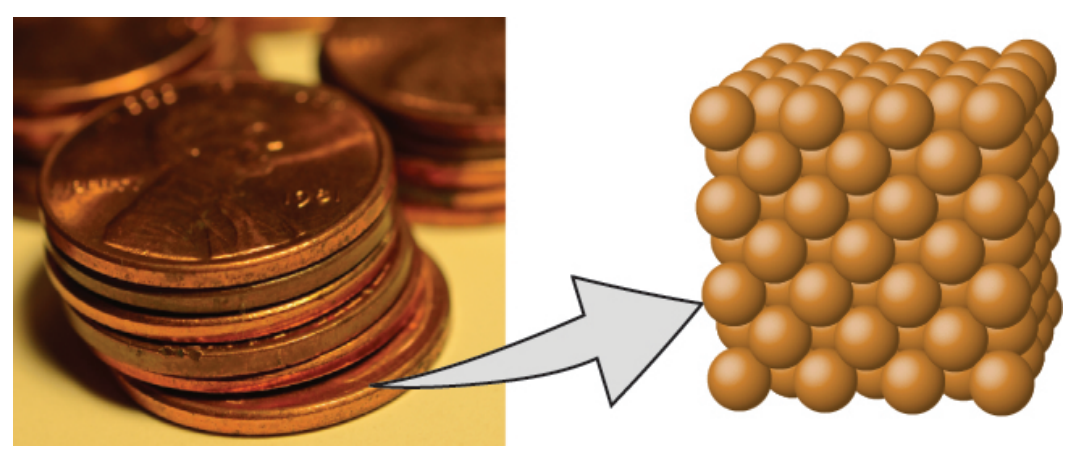

3. Atoms of one element differ in properties from atoms of all other elements.

4. A compound consists of atoms of two or more elements combined in a small, whole-number ratio. In a given compound, the numbers of atoms of each of its elements are always present in the same ratio ((Figure)).

Copper(II) oxide, a powdery, black compound, results from the combination of two types of atoms-copper (brown spheres) and oxygen (red spheres)-in a 1:1 ratio. (credit: modification of work by "Chemicalinterest"/Wikimedia Commons)

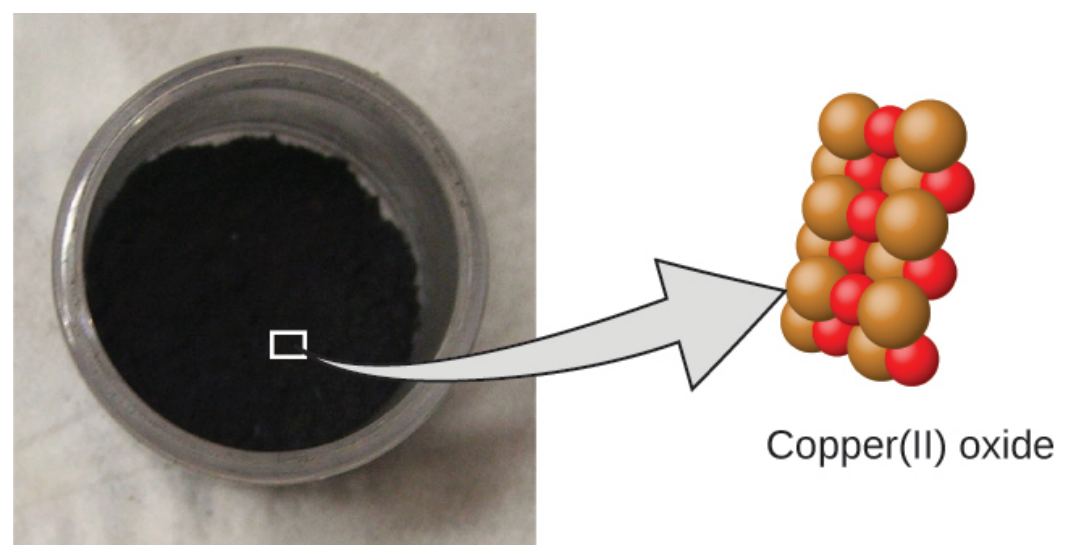


5. Atoms are neither created nor destroyed during a chemical change, but are instead rearranged to yield substances that are different from those present before the change ((Figure)).

When the elements copper (a shiny, red-brown solid, shown here as brown spheres) and oxygen (a clear and colorless gas, shown here as red spheres) react, their atoms rearrange to form a compound containing copper and oxygen (a powdery, black solid). (credit copper: modification of work by http://images-ofelements.com/copper.php)
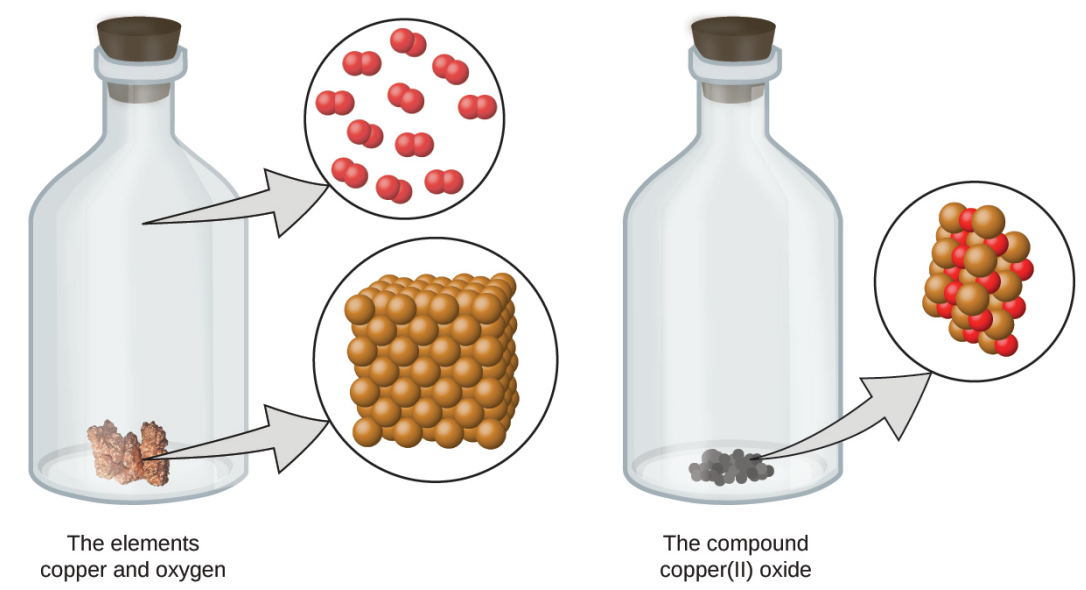

Dalton's atomic theory provides a microscopic explanation of the many macroscopic properties of matter that you've learned about. For example, if an element such as copper consists of only one kind of atom, then it cannot be broken down into simpler substances, that is, into substances composed of fewer types of atoms. And if atoms are neither created nor destroyed during a chemical change, then the total mass of matter present when matter changes from one type to another will remain constant (the law of conservation of matter). 
Testing Dalton's Atomic Theory In the following drawing, the green spheres represent atoms of a certain element. The purple spheres represent atoms of another element. If the spheres touch, they are part of a single unit of a compound. Does the following chemical change represented by these symbols violate any of the ideas of Dalton's atomic theory? If so, which one?

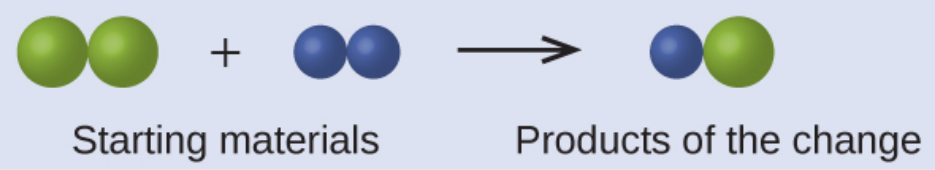

Solution The starting materials consist of two green spheres and two purple spheres. The products consist of only one green sphere and one purple sphere. This violates Dalton's postulate that atoms are neither created nor destroyed during a chemical change, but are merely redistributed. (In this case, atoms appear to have been destroyed.)

Check Your Learning In the following drawing, the green spheres represent atoms of a certain element. The purple spheres represent atoms of another element. If the spheres touch, they are part of a single unit of a compound. Does the following chemical change represented by these symbols violate any of the ideas of Dalton's atomic theory? If so, which one?

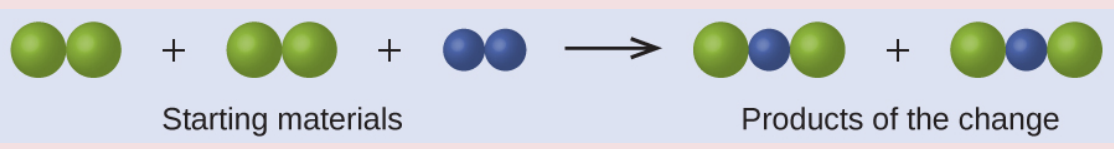

\section{Answer:}

The starting materials consist of four green spheres and two purple spheres. The products consist of four green spheres and two purple spheres. This does not violate any of Dalton's postulates: Atoms are neither created nor destroyed, but are redistributed in small, whole-number ratios. 
Dalton knew of the experiments of French chemist Joseph Proust, who demonstrated that all samples of a pure compound contain the same elements in the same proportion by mass. This statement is known as the law of definite proportions or the law of constant composition. The suggestion that the numbers of atoms of the elements in a given compound always exist in the same ratio is consistent with these observations. For example, when different samples of isooctane (a component of gasoline and one of the standards used in the octane rating system) are analyzed, they are found to have a carbon-tohydrogen mass ratio of 5.33:1, as shown in (Figure).

\section{Constant Composition of Isooctane}

Sample Carbon Hydrogen Mass Ratio
A
$14.82 \mathrm{~g} \quad 2.78 \mathrm{~g}$
$\frac{14.82 \mathrm{~g} \text { carbon }}{2.78 \mathrm{~g} \text { hydrogen }}=\frac{5.33 \mathrm{~g} \text { carbon }}{1.00 \mathrm{~g} \text { hydrogen }}$
B
$22.33 \mathrm{~g} \quad 4.19 \mathrm{~g}$
$\frac{22.33 \mathrm{~g} \text { carbon }}{4.19 \mathrm{~g} \text { hydrogen }}=\frac{5.33 \mathrm{~g} \text { carbon }}{1.00 \mathrm{~g} \text { hydrogen }}$
C
$19.40 \mathrm{~g} \quad 3.64 \mathrm{~g}$
$\frac{19.40 \mathrm{~g} \text { carbon }}{3.63 \mathrm{~g} \text { hydrogen }}=\frac{5.33 \mathrm{~g} \text { carbon }}{1.00 \mathrm{~g} \text { hydrogen }}$

It is worth noting that although all samples of a particular compound have the same mass ratio, the converse is not true in general. That is, samples that have the same mass ratio are not necessarily the same substance. For example, there are many compounds other than isooctane that also have a carbon-to-hydrogen mass ratio of 5.33:1.00.

Dalton also used data from Proust, as well as results from his own experiments, to formulate another interesting law. The law of multiple proportions states that when two elements react to form more than one compound, a fixed mass of one element will react with masses 
of the other element in a ratio of small, whole numbers. For example, copper and chlorine can form a green, crystalline solid with a mass ratio of $0.558 \mathrm{~g}$ chlorine to $1 \mathrm{~g}$ copper, as well as a brown crystalline solid with a mass ratio of 1.116 $\mathrm{g}$ chlorine to $1 \mathrm{~g}$ copper. These ratios by themselves may not seem particularly interesting or informative; however, if we take a ratio of these ratios, we obtain a useful and possibly surprising result: a small, whole-number ratio.

$\frac{\frac{1.116 \mathrm{~g} \mathrm{Cl}}{1 \mathrm{~g} \mathrm{Cu}}}{\frac{0.558 \mathrm{~g} \mathrm{Cl}}{1 \mathrm{~g} \mathrm{Cu}}}=\frac{2}{1}$

This 2-to-1 ratio means that the brown compound has twice the amount of chlorine per amount of copper as the green compound.

This can be explained by atomic theory if the copperto-chlorine ratio in the brown compound is 1 copper atom to 2 chlorine atoms, and the ratio in the green compound is 1 copper atom to 1 chlorine atom. The ratio of chlorine atoms (and thus the ratio of their masses) is therefore 2 to 1 ((Figure)).

Compared to the copper chlorine compound in (a), where copper is represented by brown spheres and chlorine by green spheres, the copper chlorine compound in (b) has twice as many chlorine atoms per copper atom. (credit a: modification of work by "Benjah-bmm27"/Wikimedia Commons; credit b: modification of work by "Walkerma"/Wikimedia Commons) 


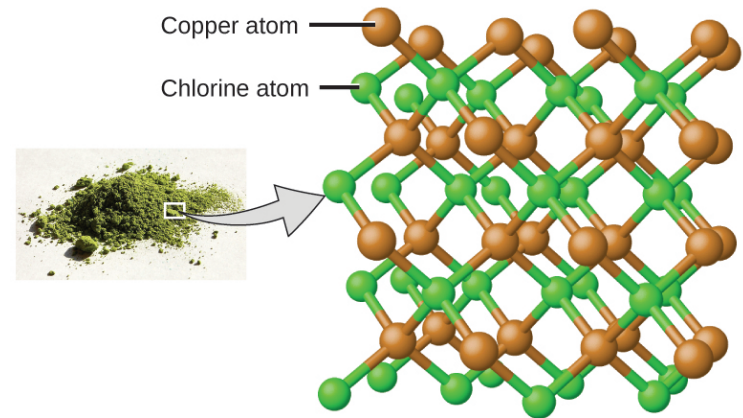

(a)

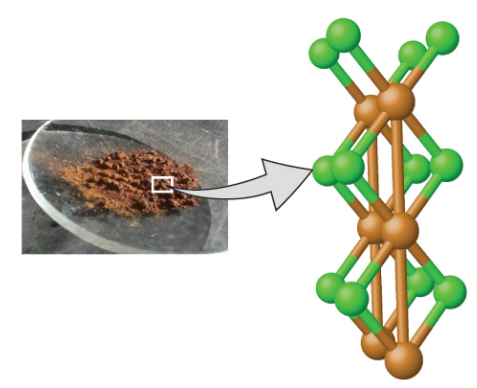

(b)

Laws of Definite and Multiple Proportions A sample of compound A (a clear, colorless gas) is analyzed and found to contain $4.27 \mathrm{~g}$ carbon and $5.69 \mathrm{~g}$ oxygen. A sample of compound B (also a clear, colorless gas) is analyzed and found to contain $5.19 \mathrm{~g}$ carbon and $13.84 \mathrm{~g}$ oxygen. Are these data an example of the law of definite proportions, the law of multiple proportions, or neither? What do these data tell you about substances $A$ and $B$ ?

Solution In compound A, the mass ratio of carbon to oxygen is:

$\frac{1.33 \mathrm{~g} \mathrm{O}}{1 \mathrm{~g} \mathrm{C}}$

In compound $\mathrm{B}$, the mass ratio of carbon to oxygen is:

\section{$\frac{2.67 \mathrm{~g} \mathrm{O}}{1 \mathrm{~g} \mathrm{C}}$}

The ratio of these ratios is:

$$
\frac{\frac{1.33 \mathrm{~g} \mathrm{O}}{1 \mathrm{~g} \mathrm{C}}}{\frac{2.67 \mathrm{~g} \mathrm{O}}{1 \mathrm{~g} \mathrm{C}}}=\frac{1}{2}
$$

This supports the law of multiple proportions. This means that $A$ and $B$ are different compounds, with $A$ having one-half as much oxygen per amount of carbon (or twice as much carbon per amount of oxygen) as B. A possible pair of compounds that would fit this relationship would be $A=C O$ and $\mathrm{B}=\mathrm{CO}_{2}$.

Check Your Learning A sample of compound X (a clear, 
colorless, combustible liquid with a noticeable odor) is analyzed and found to contain $14.13 \mathrm{~g}$ carbon and $2.96 \mathrm{~g}$ hydrogen. A sample of compound $Y$ (a clear, colorless, combustible liquid with a noticeable odor that is slightly different from X's odor) is analyzed and found to contain 19.91 $\mathrm{g}$ carbon and $3.34 \mathrm{~g}$ hydrogen. Are these data an example of the law of definite proportions, the law of multiple proportions, or neither? What do these data tell you about substances $\mathrm{X}$ and $\mathrm{Y}$ ?

Answer:

In compound $X$, the mass ratio of carbon to hydrogen is $\frac{14.13 \mathrm{~g} \mathrm{C}}{2.96 \mathrm{~g} \mathrm{H}}$. In compound $\mathrm{Y}$, the mass ratio of carbon to oxygen is $\frac{19.91 \mathrm{~g} \mathrm{C}}{3.34 \mathrm{~g} \mathrm{H}}$. The ratio of these ratios is $\frac{\frac{14.13 \mathrm{~g} \mathrm{C}}{2.96 \mathrm{~g} \mathrm{H}}}{\frac{19.91 \mathrm{~g} \mathrm{C}}{3.34 \mathrm{~g} \mathrm{H}}}=\frac{4.77 \mathrm{~g} \mathrm{C} / \mathrm{g} \mathrm{H}}{5.96 \mathrm{~g} \mathrm{C} / \mathrm{g} \mathrm{H}}=0.800=\frac{4}{5}$. This small, whole-number ratio supports the law of multiple proportions. This means that $X$ and $Y$ are different compounds.

Key Concepts and Summary

The ancient Greeks proposed that matter consists of extremely small particles called atoms. Dalton postulated that each element has a characteristic type of atom that differs in properties from atoms of all other elements, and that atoms of different elements can combine in fixed, small, whole-number ratios to form compounds. Samples of a particular compound all have the same elemental proportions by mass. When two elements form different compounds, a given mass of one element will combine with masses of the other element in a small, wholenumber ratio. During any chemical change, atoms are neither created nor destroyed. 
Chemistry End of Chapter Exercises

In the following drawing, the green spheres represent atoms of a certain element. The purple spheres represent atoms of another element. If the spheres of different elements touch, they are part of a single unit of a compound. The following chemical change represented by these spheres may violate one of the ideas of Dalton's atomic theory. Which one?

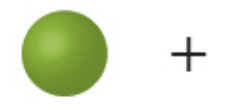

Starting materials

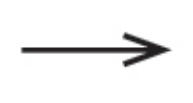

Products of the change

The starting materials consist of one green sphere and two purple spheres. The products consist of two green spheres and two purple spheres. This violates Dalton's postulate that that atoms are not created during a chemical change, but are merely redistributed.

Which postulate of Dalton's theory is consistent with the following observation concerning the weights of reactants and products? When 100 grams of solid calcium carbonate is heated, 44 grams of carbon dioxide and 56 grams of calcium oxide are produced.

Identify the postulate of Dalton's theory that is violated by the following observations: $59.95 \%$ of one sample of titanium dioxide is titanium; $60.10 \%$ of a different sample of titanium dioxide is titanium.

This statement violates Dalton's fourth postulate: In a given compound, the numbers of atoms of each type (and thus also the percentage) always have the same ratio. 
Samples of compound $\mathrm{X}, \mathrm{Y}$, and $\mathrm{Z}$ are analyzed, with results shown here.

$\begin{array}{llcl}\text { Compound } & \text { Description } & \begin{array}{l}\text { Mass of } \\ \text { Carbon }\end{array} & \begin{array}{l}\text { Mass of } \\ \text { Hydrogen }\end{array} \\ \mathrm{X} & \begin{array}{l}\text { clear, colorless, liquid with } \\ \text { strong odor }\end{array} & 1.776 \mathrm{~g} & 0.148 \mathrm{~g} \\ \mathrm{Y} & \begin{array}{l}\text { clear, colorless, liquid with } \\ \text { strong odor }\end{array} & 1.974 \mathrm{~g} & 0.329 \mathrm{~g} \\ \mathrm{Z} & \begin{array}{l}\text { clear, colorless, liquid with } \\ \text { strong odor }\end{array} & 7.812 \mathrm{~g} & 0.651 \mathrm{~g}\end{array}$

Do these data provide example(s) of the law of definite proportions, the law of multiple proportions, neither, or both? What do these data tell you about compounds X, Y, and $\mathrm{Z}$ ?

Glossary

\section{Dalton's atomic theory}

set of postulates that established the fundamental properties of atoms

\section{law of constant composition}

(also, law of definite proportions) all samples of a pure compound contain the same elements in the same proportions by mass

\section{law of definite proportions}

(also, law of constant composition) all samples of a pure compound contain the same elements in the same proportions by 
mass

\section{law of multiple proportions}

when two elements react to form more than one compound, a fixed mass of one element will react with masses of the other element in a ratio of small whole numbers 


\section{9.}

\section{EVOLUTION OF ATOMIC THEORY}

Learning Objectives

By the end of this section, you will be able to:

- $\quad$ Outline milestones in the development of modern atomic theory

- Summarize and interpret the results of the experiments of Thomson, Millikan, and Rutherford

- Describe the three subatomic particles that compose atoms

- Define isotopes and give examples for several elements

If matter is composed of atoms, what are atoms composed of? Are they the smallest particles, or is there something smaller? In the late 1800s, a number of scientists 
interested in questions like these investigated the electrical discharges that could be produced in lowpressure gases, with the most significant discovery made by English physicist J. J. Thomson using a cathode ray tube. This apparatus consisted of a sealed glass tube from which almost all the air had been removed; the tube contained two metal electrodes. When high voltage was applied across the electrodes, a visible beam called a cathode ray appeared between them. This beam was deflected toward the positive charge and away from the negative charge, and was produced in the same way with identical properties when different metals were used for the electrodes. In similar experiments, the ray was simultaneously deflected by an applied magnetic field, and measurements of the extent of deflection and the magnetic field strength allowed Thomson to calculate the charge-to-mass ratio of the cathode ray particles. The results of these measurements indicated that these particles were much lighter than atoms ((Figure)).

(a) J. J. Thomson produced a visible beam in a cathode ray tube. (b) This is an early cathode ray tube, invented in 1897 by Ferdinand Braun. (c) In the cathode ray, the beam (shown in yellow) comes from the cathode and is accelerated past the anode toward a fluorescent scale at the end of the tube. Simultaneous deflections by applied electric and magnetic fields permitted Thomson to calculate the mass-to-charge ratio of the particles composing the cathode ray. (credit a: modification of work by Nobel Foundation; credit b: modification of work by Eugen Nesper; credit c: modification of work by “Kurzon”/Wikimedia Commons) 


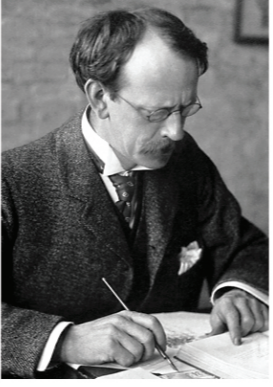

(a)

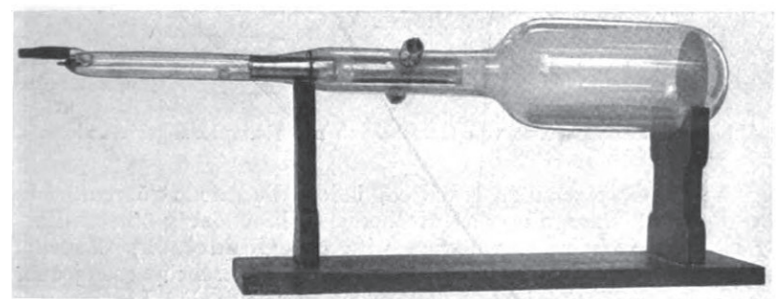

(b)

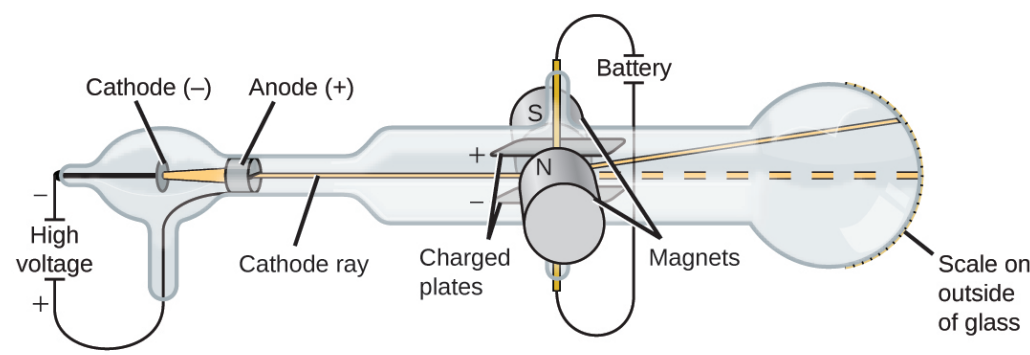

(c)

Based on his observations, here is what Thomson proposed and why: The particles are attracted by positive $\left.{ }^{+}\right)$charges and repelled by negative $(-)$charges, so they must be negatively charged (like charges repel and unlike charges attract); they are less massive than atoms and indistinguishable, regardless of the source material, so they must be fundamental, subatomic constituents of all atoms. Although controversial at the time, Thomson's idea was gradually accepted, and his cathode ray particle is what we now call an electron, a negatively charged, subatomic particle with a mass more than one thousandtimes less that of an atom. The term "electron" was coined in 1891 by Irish physicist George Stoney, from "electric ion."

Click here to hear Thomson describe his discovery in his own voice. 
In 1909, more information about the electron was uncovered by American physicist Robert A. Millikan via his “oil drop" experiments. Millikan created microscopic oil droplets, which could be electrically charged by friction as they formed or by using X-rays. These droplets initially fell due to gravity, but their downward progress could be slowed or even reversed by an electric field lower in the apparatus. By adjusting the electric field strength and making careful measurements and appropriate calculations, Millikan was able to determine the charge on individual drops ((Figure)).

Millikan's experiment measured the charge of individual oil drops. The tabulated data are examples of a few possible values.

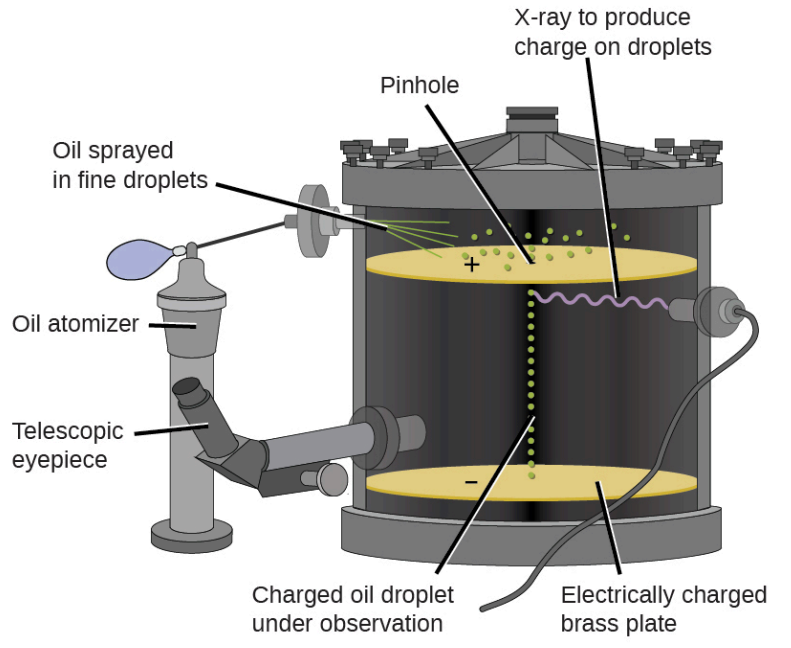

\begin{tabular}{|c|c|}
\hline Oil drop & $\begin{array}{c}\text { Charge in } \\
\text { coulombs (C) }\end{array}$ \\
\hline A & $4.8 \times 10^{-19} \mathrm{C}$ \\
\hline B & $3.2 \times 10^{-19} \mathrm{C}$ \\
\hline C & $6.4 \times 10^{-19} \mathrm{C}$ \\
\hline D & $1.6 \times 10^{-19} \mathrm{C}$ \\
\hline E & $4.8 \times 10^{-19} \mathrm{C}$ \\
\hline
\end{tabular}

Looking at the charge data that Millikan gathered, you may have recognized that the charge of an oil droplet is always a multiple of a specific charge, $1.610^{-19} \mathrm{C}$. Millikan concluded that this value must therefore be a fundamental charge-the charge of a single electron-with his measured charges due to an excess of 
one electron (1 times $1.610^{-19} \mathrm{C}$ ), two electrons (2 times $1.610^{-19} \mathrm{C}$ ), three electrons (3 times $1.610^{-19} \mathrm{C}$ ), and so on, on a given oil droplet. Since the charge of an electron was now known due to Millikan's research, and the charge-to-mass ratio was already known due to Thomson's research $\left(1.75910^{11} \mathrm{C} / \mathrm{kg}\right)$, it only required a simple calculation to determine the mass of the electron as well.

Mass of electron $=1.60210^{-19} \mathrm{C} \frac{1 \mathrm{~kg}}{1.75910^{11} \mathrm{C}}=9.10710^{-31} \mathrm{~kg}$

Scientists had now established that the atom was not indivisible as Dalton had believed, and due to the work of Thomson, Millikan, and others, the charge and mass of the negative, subatomic particles-the electrons-were known. However, the positively charged part of an atom was not yet well understood. In 1904, Thomson proposed the "plum pudding" model of atoms, which described a positively charged mass with an equal amount of negative charge in the form of electrons embedded in it, since all atoms are electrically neutral. A competing model had been proposed in 1903 by Hantaro Nagaoka, who postulated a Saturn-like atom, consisting of a positively charged sphere surrounded by a halo of electrons ((Figure)).

(a) Thomson suggested that atoms resembled plum pudding, an English dessert consisting of moist cake with embedded raisins ("plums"). (b) Nagaoka proposed that atoms resembled the planet Saturn, with a ring of electrons surrounding a positive "planet." (credit a: modification of work by "Man vyi"/Wikimedia Commons; credit b: modification of work by "NASA"/Wikimedia Commons) 


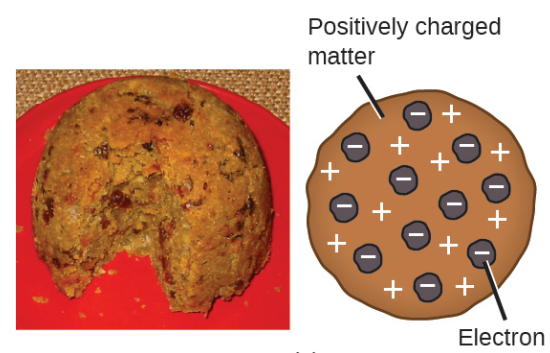

(a)

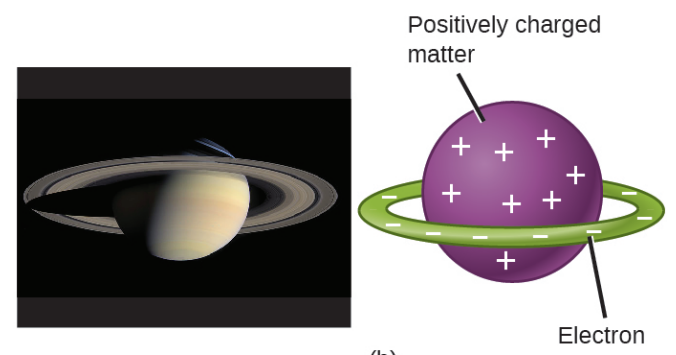

(b)

The next major development in understanding the atom came from Ernest Rutherford, a physicist from New Zealand who largely spent his scientific career in Canada and England. He performed a series of experiments using a beam of high-speed, positively charged alpha particles ( $\alpha$ particles) that were produced by the radioactive decay of radium; $\alpha$ particles consist of two protons and two neutrons (you will learn more about radioactive decay in the chapter on nuclear chemistry). Rutherford and his colleagues Hans Geiger (later famous for the Geiger counter) and Ernest Marsden aimed a beam of $\alpha$ particles, the source of which was embedded in a lead block to absorb most of the radiation, at a very thin piece of gold foil and examined the resultant scattering of the $\alpha$ particles using a luminescent screen that glowed briefly where hit by an $\alpha$ particle.

What did they discover? Most particles passed right through the foil without being deflected at all. However, some were diverted slightly, and a very small number were deflected almost straight back toward the source ((Figure)). Rutherford described finding these results: "It was quite the most incredible event that has ever happened to me in my life. It was almost as incredible as if you fired a 15-inch shell at a piece of tissue paper and it came back and hit you."1 
Geiger and Rutherford fired $\alpha$ particles at a piece of gold foil and detected where those particles went, as shown in this schematic diagram of their experiment. Most of the particles passed straight through the foil, but a few were deflected slightly and a very small number were significantly deflected.

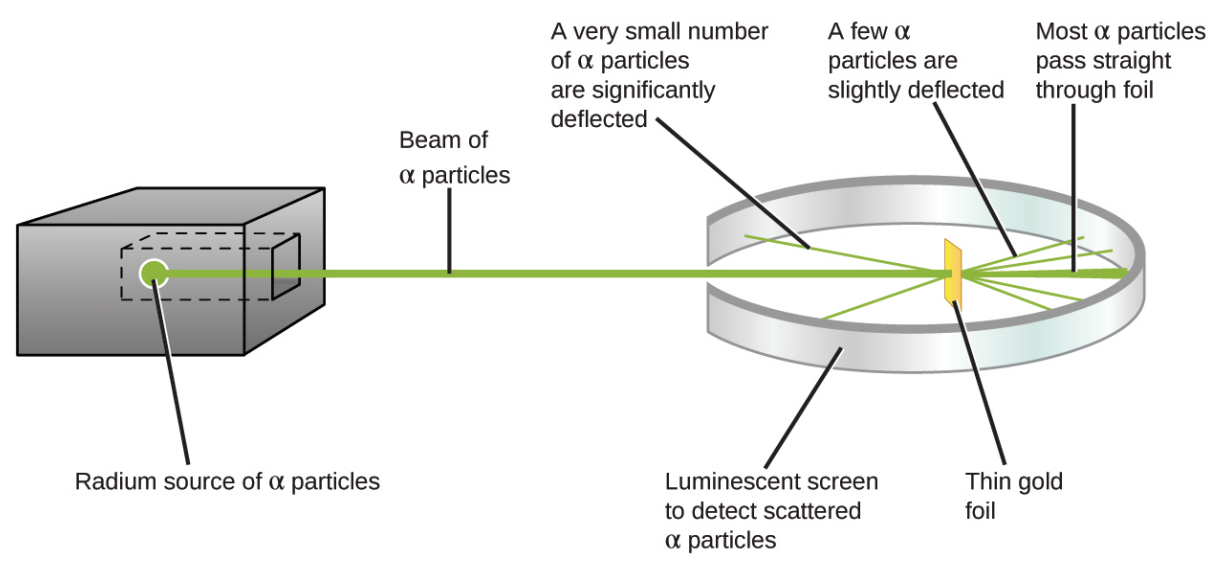

Here is what Rutherford deduced: Because most of the fast-moving $\alpha$ particles passed through the gold atoms undeflected, they must have traveled through essentially empty space inside the atom. Alpha particles are positively charged, so deflections arose when they encountered another positive charge (like charges repel each other). Since like charges repel one another, the few positively charged $\alpha$ particles that changed paths abruptly must have hit, or closely approached, another body that also had a highly concentrated, positive charge. Since the deflections occurred a small fraction of the time, this charge only occupied a small amount of the space in the gold foil. Analyzing a series of such experiments in detail, Rutherford drew two conclusions:

1. The volume occupied by an atom must consist of 
a large amount of empty space.

2. A small, relatively heavy, positively charged body, the nucleus, must be at the center of each atom.

View this simulation of the Rutherford gold foil experiment. Adjust the slit width to produce a narrower or broader beam of $\alpha$ particles to see how that affects the scattering pattern.

This analysis led Rutherford to propose a model in which an atom consists of a very small, positively charged nucleus, in which most of the mass of the atom is concentrated, surrounded by the negatively charged electrons, so that the atom is electrically neutral ((Figure)). After many more experiments, Rutherford also discovered that the nuclei of other elements contain the hydrogen nucleus as a "building block," and he named this more fundamental particle the proton, the positively charged, subatomic particle found in the nucleus. With one addition, which you will learn next, this nuclear model of the atom, proposed over a century ago, is still used today.

The $\alpha$ particles are deflected only when they collide with or pass close to the much heavier, positively charged gold nucleus. Because the nucleus is very small compared to the size of an atom, very few $\alpha$ particles are deflected. Most pass through the relatively large region occupied by electrons, which are too light to deflect the rapidly moving particles. 


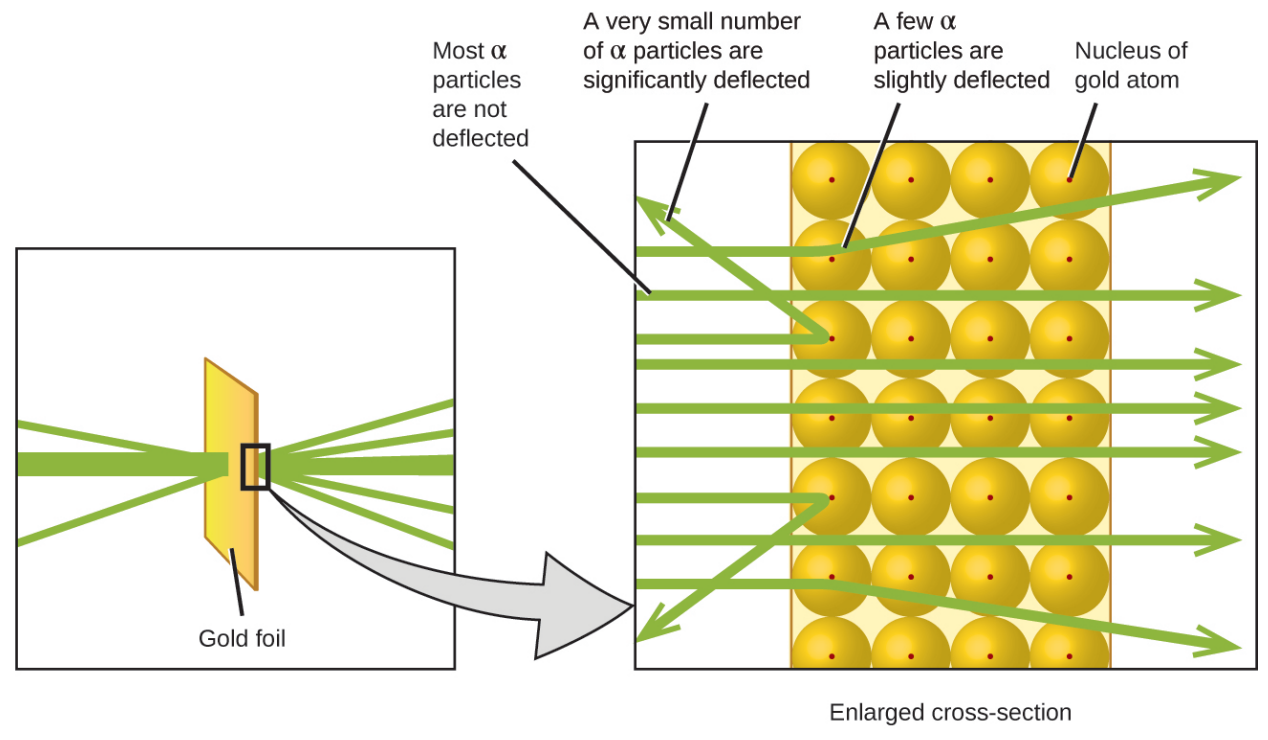

The Rutherford Scattering simulation allows you to investigate the differences between a "plum pudding" atom and a Rutherford atom by firing $\alpha$ particles at each type of atom.

Another important finding was the discovery of isotopes. During the early 1900s, scientists identified several substances that appeared to be new elements, isolating them from radioactive ores. For example, a "new element" produced by the radioactive decay of thorium was initially given the name mesothorium. However, a more detailed analysis showed that mesothorium was chemically identical to radium (another decay product), despite having a different atomic mass. This result, along with similar findings for other elements, led the English chemist Frederick Soddy to realize that an element could have types of atoms with different masses that were chemically indistinguishable. These different types are called isotopes-atoms of the same element that differ in 
mass. Soddy was awarded the Nobel Prize in Chemistry in 1921 for this discovery.

One puzzle remained: The nucleus was known to contain almost all of the mass of an atom, with the number of protons only providing half, or less, of that mass. Different proposals were made to explain what constituted the remaining mass, including the existence of neutral particles in the nucleus. As you might expect, detecting uncharged particles is very challenging, and it was not until 1932 that James Chadwick found evidence of neutrons, uncharged, subatomic particles with a mass approximately the same as that of protons. The existence of the neutron also explained isotopes: They differ in mass because they have different numbers of neutrons, but they are chemically identical because they have the same number of protons. This will be explained in more detail later in this chapter.

Key Concepts and Summary

Although no one has actually seen the inside of an atom, experiments have demonstrated much about atomic structure. Thomson's cathode ray tube showed that atoms contain small, negatively charged particles called electrons. Millikan discovered that there is a fundamental electric charge-the charge of an electron. Rutherford's gold foil experiment showed that atoms have a small, dense, positively charged nucleus; the positively charged particles within the nucleus are called protons. Chadwick discovered that the nucleus also contains neutral particles called neutrons. Soddy demonstrated that atoms of the same element can differ in mass; these are called isotopes. 
Chemistry End of Chapter Exercises

The existence of isotopes violates one of the original ideas of Dalton's atomic theory. Which one?

Dalton originally thought that all atoms of a particular element had identical properties, including mass. Thus, the concept of isotopes, in which an element has different masses, was a violation of the original idea. To account for the existence of isotopes, the second postulate of his atomic theory was modified to state that atoms of the same element must have identical chemical properties.

How are electrons and protons similar? How are they different?

How are protons and neutrons similar? How are they different?

Both are subatomic particles that reside in an atom's nucleus. Both have approximately the same mass. Protons are positively charged, whereas neutrons are uncharged.

Predict and test the behavior of $\alpha$ particles fired at a "plum pudding" model atom.

(a) Predict the paths taken by $\alpha$ particles that are fired at atoms with a Thomson's plum pudding model structure. Explain why you expect the $\alpha$ particles to take these paths.

(b) If $\alpha$ particles of higher energy than those in (a) are fired at plum pudding atoms, predict how their paths will differ from the lower-energy $\alpha$ particle paths. Explain your reasoning.

(c) Now test your predictions from (a) and (b). Open 
the Rutherford Scattering simulation and select the "Plum Pudding Atom" tab. Set "Alpha Particles Energy" to "min," and select "show traces." Click on the gun to start firing $\alpha$ particles. Does this match your prediction from (a)? If not, explain why the actual path would be that shown in the simulation. Hit the pause button, or "Reset All." Set "Alpha Particles Energy" to "max," and start firing $\alpha$ particles. Does this match your prediction from (b)? If not, explain the effect of increased energy on the actual paths as shown in the simulation.

Predict and test the behavior of $\alpha$ particles fired at a Rutherford atom model.

(a) Predict the paths taken by $\alpha$ particles that are fired at atoms with a Rutherford atom model structure. Explain why you expect the $\alpha$ particles to take these paths.

(b) If $\alpha$ particles of higher energy than those in (a) are fired at Rutherford atoms, predict how their paths will differ from the lower-energy $\alpha$ particle paths. Explain your reasoning.

(c) Predict how the paths taken by the $\alpha$ particles will differ if they are fired at Rutherford atoms of elements other than gold. What factor do you expect to cause this difference in paths, and why?

(d) Now test your predictions from (a), (b), and (c). Open the Rutherford Scattering simulation and select the "Rutherford Atom" tab. Due to the scale of the simulation, it is best to start with a small nucleus, so select " 20 " for both protons and neutrons, "min" for energy, show traces, and then start firing $\alpha$ particles. Does this match your prediction from (a)? If not, explain why the actual path would be that shown in the simulation. Pause or 
reset, set energy to "max," and start firing a particles. Does this match your prediction from (b)? If not, explain the effect of increased energy on the actual path as shown in the simulation. Pause or reset, select " 40 " for both protons and neutrons, "min" for energy, show traces, and fire away. Does this match your prediction from (c)? If not, explain why the actual path would be that shown in the simulation. Repeat this with larger numbers of protons and neutrons. What generalization can you make regarding the type of atom and effect on the path of $\alpha$ particles? Be clear and specific.

(a) The Rutherford atom has a small, positively charged nucleus, so most $\alpha$ particles will pass through empty space far from the nucleus and be undeflected. Those $\alpha$ particles that pass near the nucleus will be deflected from their paths due to positive-positive repulsion. The more directly toward the nucleus the $\alpha$ particles are headed, the larger the deflection angle will be. (b) Higher-energy $\alpha$ particles that pass near the nucleus will still undergo deflection, but the faster they travel, the less the expected angle of deflection. (c) If the nucleus is smaller, the positive charge is smaller and the expected deflections are smaller-both in terms of how closely the $\alpha$ particles pass by the nucleus undeflected and the angle of deflection. If the nucleus is larger, the positive charge is larger and the expected deflections are larger-more $\alpha$ particles will be deflected, and the deflection angles will be larger. (d) The paths followed by the $\alpha$ particles match the predictions from (a), (b), and (c). 


\section{Footnotes}

- 1Ernest Rutherford, "The Development of the Theory of Atomic Structure," ed. J. A. Ratcliffe, in Background to Modern Science, eds. Joseph Needham and Walter Pagel, (Cambridge, UK: Cambridge University Press, 1938), 61-74. Accessed September 22, 2014, https://ia600508.us.archive.org/3/items/ backgroundtomode $032734 \mathrm{mbp} /$ backgroundtomode032734mbp.pdf.

Glossary

alpha particle (a particle)

positively charged particle consisting of two protons and two neutrons

electron

negatively charged, subatomic particle of relatively low mass located outside the nucleus

\section{isotopes}

atoms that contain the same number of protons but different numbers of neutrons neutron

uncharged, subatomic particle located in the nucleus

\section{nucleus}

massive, positively charged center of an atom made up of protons and neutrons 
proton

positively charged, subatomic particle located in the nucleus 


\section{0.}

\section{ATOMIC STRUCTURE AND SYMBOLISM}

Learning Objectives

By the end of this section, you will be able to:

- Write and interpret symbols that depict the atomic number, mass number, and charge of an atom or ion

- $\quad$ Define the atomic mass unit and average atomic mass

- $\quad$ Calculate average atomic mass and isotopic abundance

The development of modern atomic theory revealed much about the inner structure of atoms. It was learned that an atom contains a very small nucleus composed of positively charged protons and uncharged neutrons, surrounded by a much larger volume of space containing negatively 
charged electrons. The nucleus contains the majority of an atom's mass because protons and neutrons are much heavier than electrons, whereas electrons occupy almost all of an atom's volume. The diameter of an atom is on the order of $10^{-10} \mathrm{~m}$, whereas the diameter of the nucleus is roughly $10^{-15} \mathrm{~m}$-about 100,000 times smaller. For a perspective about their relative sizes, consider this: If the nucleus were the size of a blueberry, the atom would be about the size of a football stadium ((Figure)).

If an atom could be expanded to the size of a football stadium, the nucleus would be the size of a single blueberry. (credit middle: modification of work by "babyknight"/Wikimedia Commons; credit right: modification of work by Paxson Woelber)
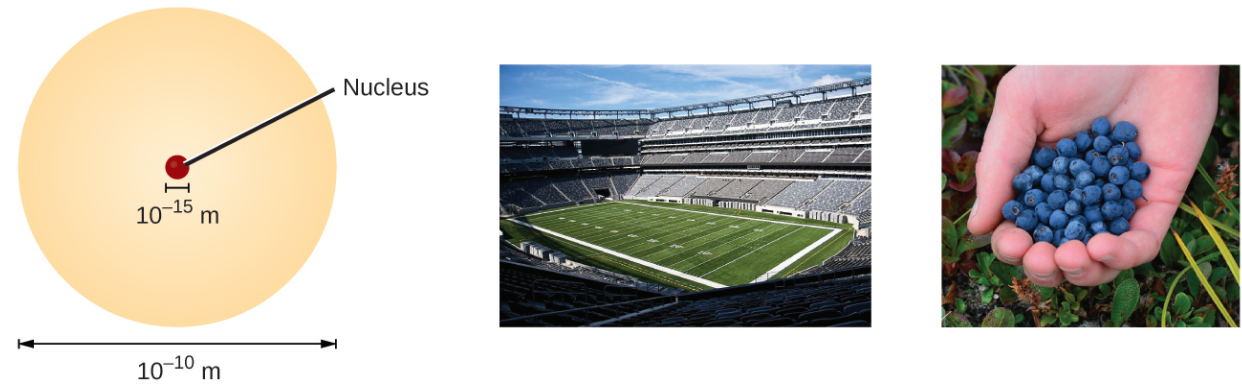

Atoms - and the protons, neutrons, and electrons that compose them-are extremely small. For example, a carbon atom weighs less than $210^{-23} \mathrm{~g}$, and an electron has a charge of less than $2 \quad 10^{-19} \mathrm{C}$ (coulomb). When describing the properties of tiny objects such as atoms, we use appropriately small units of measure, such as the atomic mass unit (amu) and the fundamental unit of charge (e). The amu was originally defined based on hydrogen, the lightest element, then later in terms of oxygen. Since 1961, it has been defined with regard to 
the most abundant isotope of carbon, atoms of which are assigned masses of exactly $12 \mathrm{amu}$. (This isotope is known as "carbon-12" as will be discussed later in this module.) Thus, one amu is exactly $\frac{1}{12}$ of the mass of one carbon-12 atom: $1 \mathrm{amu}=1.660510^{-24} \mathrm{~g}$. (The Dalton (Da) and the unified atomic mass unit $(\mathrm{u})$ are alternative units that are equivalent to the amu.) The fundamental unit of charge (also called the elementary charge) equals the magnitude of the charge of an electron (e) with $\mathrm{e}=1.60210^{-19} \mathrm{C}$.

A proton has a mass of $1.0073 \mathrm{amu}$ and a charge of 1+. A neutron is a slightly heavier particle with a mass $1.0087 \mathrm{amu}$ and a charge of zero; as its name suggests, it is neutral. The electron has a charge of $1-$ and is a much lighter particle with a mass of about $0.00055 \mathrm{amu}$ (it would take about 1800 electrons to equal the mass of one proton). The properties of these fundamental particles are summarized in (Figure). (An observant student might notice that the sum of an atom's subatomic particles does not equal the atom's actual mass: The total mass of six protons, six neutrons, and six electrons is $12.0993 \mathrm{amu}$, slightly larger than 12.00 amu. This "missing" mass is known as the mass defect, and you will learn about it in the chapter on nuclear chemistry.)

Properties of Subatomic Particles

$\begin{array}{llllll}\text { Name } & \text { Location } & \begin{array}{l}\text { Charge } \\ (\mathbf{C})\end{array} & \begin{array}{l}\text { Unit } \\ \text { Charge }\end{array} & \begin{array}{l}\text { Mass } \\ (\mathbf{a m u})\end{array} & \text { Mass (g) } \\ \text { electron } & \begin{array}{l}\text { outside } \\ \text { nucleus }\end{array} & \begin{array}{l}-1.602 \\ 10^{-19}\end{array} & 1- & 0.00055 & 0.00091 \\ & & 1.602 & 1+ & & 1.00727 \\ \text { proton } & \text { nucleus } & 10^{-19} & 1.67262 & 10^{-24} \\ & & & & & 1.67493 \\ \text { neutron } & \text { nucleus } & 0 & 0 & 1.00866 & 10^{-24}\end{array}$


The number of protons in the nucleus of an atom is its atomic number ( $\mathrm{Z})$. This is the defining trait of an element: Its value determines the identity of the atom. For example, any atom that contains six protons is the element carbon and has the atomic number 6 , regardless of how many neutrons or electrons it may have. A neutral atom must contain the same number of positive and negative charges, so the number of protons equals the number of electrons. Therefore, the atomic number also indicates the number of electrons in an atom. The total number of protons and neutrons in an atom is called its mass number (A). The number of neutrons is therefore the difference between the mass number and the atomic number: $A-Z=$ number of neutrons.

atomic number $(\mathrm{Z})=$ number of protons

mass number $(\mathrm{A})=$ number of protons + number of neutrons $\mathrm{A}-\mathrm{Z}=$ number of neutrons

Atoms are electrically neutral if they contain the same number of positively charged protons and negatively charged electrons. When the numbers of these subatomic particles are not equal, the atom is electrically charged and is called an ion. The charge of an atom is defined as follows:

Atomic charge $=$ number of protons - number of electrons

As will be discussed in more detail later in this chapter, atoms (and molecules) typically acquire charge by gaining or losing electrons. An atom that gains one or more electrons will exhibit a negative charge and is called an anion. Positively charged atoms called cations are formed when an atom loses one or more electrons. For example, a neutral sodium atom $(Z=11)$ has 11 electrons. If this 
atom loses one electron, it will become a cation with a $1+$ charge $(11-10=1+)$. A neutral oxygen atom $(Z=8)$ has eight electrons, and if it gains two electrons it will become an anion with a $2-$ charge $(8-10=2-)$.

Composition of an Atom lodine is an essential trace element in our diet; it is needed to produce thyroid hormone. Insufficient iodine in the diet can lead to the development of a goiter, an enlargement of the thyroid gland ((Figure)).

(a) Insufficient iodine in the diet can cause an enlargement of the thyroid gland called a goiter. (b) The addition of small amounts of iodine to salt, which prevents the formation of goiters, has helped eliminate this concern in the US where salt consumption is high. (credit a: modification of work by "Almazi"/Wikimedia Commons; credit b: modification of work by Mike Mozart)

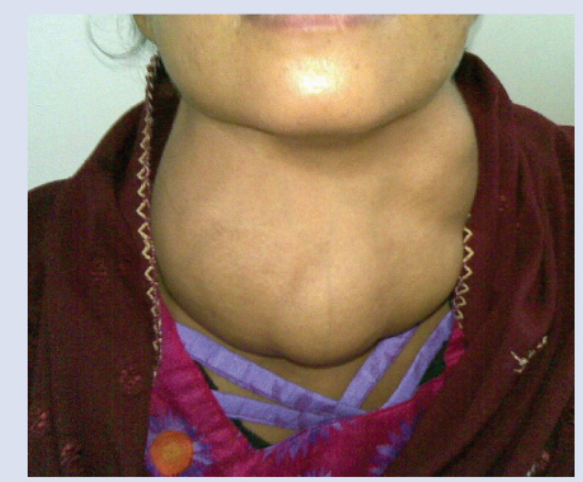

(a)

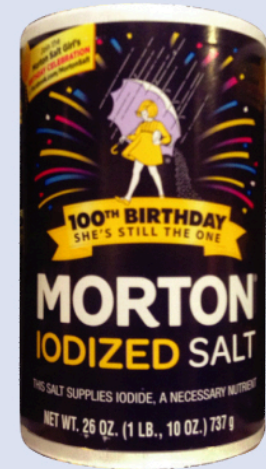

(b)

The addition of small amounts of iodine to table salt (iodized salt) has essentially eliminated this health concern in the United States, but as much as $40 \%$ of the world's population is still at risk of iodine deficiency. The iodine atoms are added as anions, and each has a 1- charge and a mass number of 127 . Determine the numbers of protons, neutrons, and electrons in one of these iodine anions.

SolutionThe atomic number of iodine (53) tells us that a 
neutral iodine atom contains 53 protons in its nucleus and 53 electrons outside its nucleus. Because the sum of the numbers of protons and neutrons equals the mass number, 127 , the number of neutrons is $74(127-53=74)$. Since the iodine is added as a 1- anion, the number of electrons is 54 [53-(1-) = 54].

Check Your LearningAn ion of platinum has a mass number of 195 and contains 74 electrons. How many protons and neutrons does it contain, and what is its charge?

Answer:

78 protons; 117 neutrons; charge is 4+

Chemical Symbols

A chemical symbol is an abbreviation that we use to indicate an element or an atom of an element. For example, the symbol for mercury is $\mathrm{Hg}$ ((Figure)). We use the same symbol to indicate one atom of mercury (microscopic domain) or to label a container of many atoms of the element mercury (macroscopic domain).

The symbol Hg represents the element mercury regardless of the amount; it could represent one atom of mercury or a large amount of mercury. 


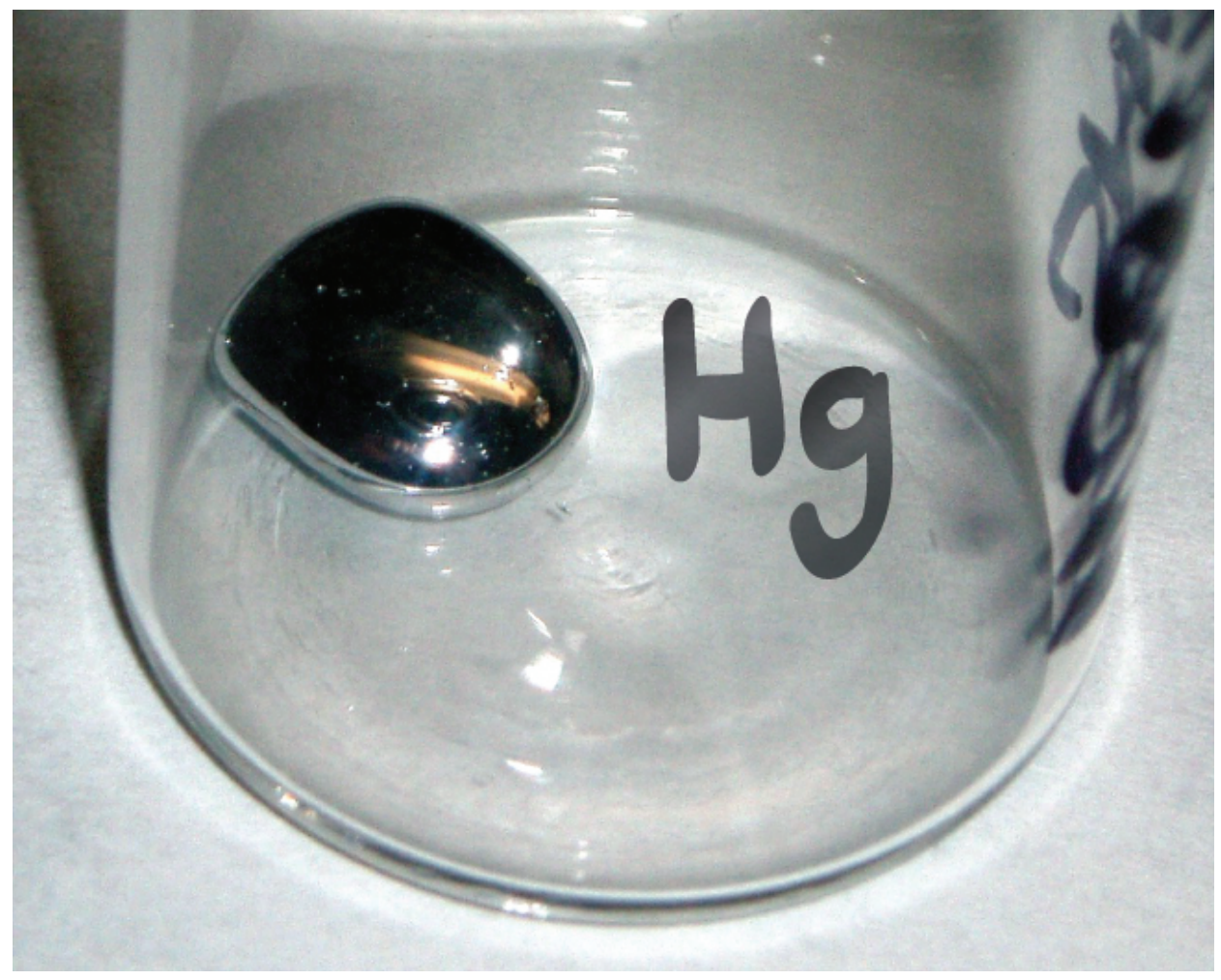

The symbols for several common elements and their atoms are listed in (Figure). Some symbols are derived from the common name of the element; others are abbreviations of the name in another language. Most symbols have one or two letters, but three-letter symbols have been used to describe some elements that have atomic numbers greater than 112 . To avoid confusion with other notations, only the first letter of a symbol is capitalized. For example, Co is the symbol for the element cobalt, but $\mathrm{CO}$ is the notation for the compound carbon monoxide, which contains atoms of the elements carbon $(\mathrm{C})$ and oxygen $(\mathrm{O})$. All known elements and their symbols are in the periodic table in (Figure) (also found in Appendix A). 
Some Common Elements and Their Symbols

$\begin{array}{llll}\text { Element } & \text { Symbol } & \text { Element } & \text { Symbol } \\ \text { aluminum } & \mathrm{Al} & \text { iron } & \mathrm{Fe} \text { (from ferrum) } \\ \text { bromine } & \mathrm{Br} & \text { lead } & \mathrm{Pb} \text { (from plumbum) } \\ \text { calcium } & \mathrm{Ca} & \text { magnesium } & \mathrm{Mg} \\ \text { carbon } & \mathrm{C} & \text { mercury } & \mathrm{Hg} \text { (from hydrargyrum) } \\ \text { chlorine } & \mathrm{Cl} & \text { nitrogen } & \mathrm{N} \\ \text { chromium } & \mathrm{Cr} & \text { oxygen } & \mathrm{O} \\ \text { cobalt } & \mathrm{Co} & \text { potassium } & \mathrm{K} \text { (from kalium) } \\ \text { copper } & \mathrm{Cu} \text { (from cuprum) } & \text { silicon } & \mathrm{Si} \\ \text { fluorine } & \mathrm{F} & \text { silver } & \mathrm{Ag} \text { (from argentum) } \\ \text { gold } & \mathrm{Au} \text { (from aurum) } & \text { sodium } & \mathrm{Na} \text { (from natrium) } \\ \text { helium } & \mathrm{He} & \text { sulfur } & \mathrm{S} \\ \text { hydrogen } & \mathrm{H} & \text { tin } & \mathrm{Sn} \text { (from stannum) } \\ \text { iodine } & \mathrm{I} & \text { zinc } & \mathrm{Zn}\end{array}$

Traditionally, the discoverer (or discoverers) of a new element names the element. However, until the name is recognized by the International Union of Pure and Applied Chemistry (IUPAC), the recommended name of the new element is based on the Latin word(s) for its atomic number. For example, element 106 was called unnilhexium (Unh), element 107 was called unnilseptium (Uns), and element 108 was called unniloctium (Uno) for several years. These elements are now named after scientists (or occasionally locations); for example, element 106 is now known as seaborgium (Sg) in honor of Glenn Seaborg, a Nobel Prize winner who was active in the discovery of several heavy elements. 
Visit this site to learn more about IUPAC, the International Union of Pure and Applied Chemistry, and explore its periodic table.

\section{Isotopes}

The symbol for a specific isotope of any element is written by placing the mass number as a superscript to the left of the element symbol ((Figure)). The atomic number is sometimes written as a subscript preceding the symbol, but since this number defines the element's identity, as does its symbol, it is often omitted. For example, magnesium exists as a mixture of three isotopes, each with an atomic number of 12 and with mass numbers of 24,25 , and 26, respectively. These isotopes can be identified as ${ }^{24} \mathrm{Mg},{ }^{25} \mathrm{Mg}$, and ${ }^{26} \mathrm{Mg}$. These isotope symbols are read as "element, mass number" and can be symbolized consistent with this reading. For instance, ${ }^{24} \mathrm{Mg}$ is read as "magnesium 24," and can be written as "magnesium-24" or "Mg-24." ${ }^{25} \mathrm{Mg}$ is read as "magnesium 25 ," and can be written as "magnesium-25" or "Mg-25." All magnesium atoms have 12 protons in their nucleus. They differ only because a ${ }^{24} \mathrm{Mg}$ atom has 12 neutrons in its nucleus, a ${ }^{25} \mathrm{Mg}$ atom has 13 neutrons, and a ${ }^{26} \mathrm{Mg}$ has 14 neutrons.

The symbol for an atom indicates the element via its usual two-letter symbol, the mass number as a left superscript, the atomic number as a left subscript (sometimes omitted), and the charge as a right superscript. 


\section{Mass number $-4 \quad 2+-$ Charge $\mathrm{He}$}

Atomic number -2

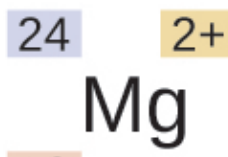

12 (often omitted)

Information about the naturally occurring isotopes of elements with atomic numbers 1 through 10 is given in (Figure). Note that in addition to standard names and symbols, the isotopes of hydrogen are often referred to using common names and accompanying symbols. Hydrogen-2, symbolized ${ }^{2} \mathrm{H}$, is also called deuterium and sometimes symbolized D. Hydrogen-3, symbolized ${ }^{3} \mathrm{H}$, is also called tritium and sometimes symbolized $\mathrm{T}$. 
Nuclear Compositions of Atoms of the Very Light Elements

\begin{tabular}{|c|c|c|c|c|c|c|}
\hline Element & Symbol & $\begin{array}{l}\text { Atomic } \\
\text { Number }\end{array}$ & $\begin{array}{l}\text { Number } \\
\text { of } \\
\text { Protons }\end{array}$ & $\begin{array}{l}\text { Number } \\
\text { of } \\
\text { Neutrons }\end{array}$ & $\begin{array}{l}\text { Mass } \\
\text { (amu) }\end{array}$ & $\begin{array}{l}\text { \% Natural } \\
\text { Abundance }\end{array}$ \\
\hline \multirow{3}{*}{ hydrogen } & $\begin{array}{l}{ }_{1}^{1} \mathrm{H} \\
\text { (protium) }\end{array}$ & 1 & 1 & 0 & 1.0078 & 99.989 \\
\hline & $\begin{array}{l}{ }_{1}^{2} \mathrm{H} \\
\text { (deuterium) }\end{array}$ & 1 & 1 & 1 & 2.0141 & 0.0115 \\
\hline & $\begin{array}{l}{ }_{1}^{3} \mathrm{H} \\
\text { (tritium) }\end{array}$ & 1 & 1 & 2 & 3.01605 & - (trace) \\
\hline \multirow{2}{*}{ helium } & ${ }_{2}^{3} \mathrm{He}$ & 2 & 2 & 1 & 3.01603 & 0.00013 \\
\hline & ${ }_{2}^{4} \mathrm{He}$ & 2 & 2 & 2 & 4.0026 & 100 \\
\hline \multirow{2}{*}{ lithium } & ${ }_{3}^{6} \mathrm{Li}$ & 3 & 3 & 3 & 6.0151 & 7.59 \\
\hline & ${ }_{3}^{7} \mathrm{Li}$ & 3 & 3 & 4 & 7.0160 & 92.41 \\
\hline beryllium & ${ }_{4}^{9} \mathrm{Be}$ & 4 & 4 & 5 & 9.0122 & 100 \\
\hline \multirow{2}{*}{ boron } & ${ }_{5}^{10} \mathrm{~B}$ & 5 & 5 & 5 & 10.0129 & 19.9 \\
\hline & ${ }_{5}^{11} \mathrm{~B}$ & 5 & 5 & 6 & 11.0093 & 80.1 \\
\hline \multirow{3}{*}{ carbon } & ${ }_{6}^{12} \mathrm{C}$ & 6 & 6 & 6 & 12.0000 & 98.89 \\
\hline & ${ }_{6}^{13} \mathrm{C}$ & 6 & 6 & 7 & 13.0034 & 1.11 \\
\hline & ${ }_{6}^{14} \mathrm{C}$ & 6 & 6 & 8 & 14.0032 & - (trace) \\
\hline \multirow{2}{*}{ nitrogen } & ${ }_{7}^{14} \mathrm{~N}$ & 7 & 7 & 7 & 14.0031 & 99.63 \\
\hline & ${ }_{7}^{15} \mathrm{~N}$ & 7 & 7 & 8 & 15.0001 & 0.37 \\
\hline \multirow{3}{*}{ oxygen } & ${ }_{8}^{16} \mathrm{O}$ & 8 & 8 & 8 & 15.9949 & 99.757 \\
\hline & ${ }_{8}^{17} \mathrm{O}$ & 8 & 8 & 9 & 16.9991 & 0.038 \\
\hline & ${ }_{8}^{18} \mathrm{O}$ & 8 & 8 & 10 & 17.9992 & 0.205 \\
\hline
\end{tabular}


Nuclear Compositions of Atoms of the Very Light Elements

\begin{tabular}{|c|c|c|c|c|c|c|}
\hline Element & Symbol & $\begin{array}{l}\text { Atomic } \\
\text { Number }\end{array}$ & $\begin{array}{l}\text { Number } \\
\text { of } \\
\text { Protons }\end{array}$ & $\begin{array}{l}\text { Number } \\
\text { of } \\
\text { Neutrons }\end{array}$ & $\begin{array}{l}\text { Mass } \\
\text { (amu) }\end{array}$ & $\begin{array}{l}\text { \% Natural } \\
\text { Abundance }\end{array}$ \\
\hline \multirow[t]{2}{*}{ fluorine } & ${ }_{9}^{19} \mathrm{~F}$ & 9 & 9 & 10 & 18.9984 & 100 \\
\hline & ${ }_{10}^{20} \mathrm{Ne}$ & 10 & 10 & 10 & 19.9924 & 90.48 \\
\hline \multirow[t]{2}{*}{ neon } & ${ }_{10}^{21} \mathrm{Ne}$ & 10 & 10 & 11 & 20.9938 & 0.27 \\
\hline & ${ }_{10}^{22} \mathrm{Ne}$ & 10 & 10 & 12 & 21.9914 & 9.25 \\
\hline
\end{tabular}

Use this Build an Atom simulator to build atoms of the first 10 elements, see which isotopes exist, check nuclear stability, and gain experience with isotope symbols.

Atomic Mass

Because each proton and each neutron contribute approximately one amu to the mass of an atom, and each electron contributes far less, the atomic mass of a single atom is approximately equal to its mass number (a whole number). However, the average masses of atoms of most elements are not whole numbers because most elements exist naturally as mixtures of two or more isotopes.

The mass of an element shown in a periodic table or listed in a table of atomic masses is a weighted, average mass of all the isotopes present in a naturally occurring sample of that element. This is equal to the sum of each individual isotope's mass multiplied by its fractional abundance.

average mass $=\sum_{i}(\text { fractional abundance isotopic mass })_{i}$

For example, the element boron is composed of two 
isotopes: About $19.9 \%$ of all boron atoms are ${ }^{10} \mathrm{~B}$ with a mass of $10.0129 \mathrm{amu}$, and the remaining $80.1 \%$ are ${ }^{11} \mathrm{~B}$ with a mass of $11.0093 \mathrm{amu}$. The average atomic mass for boron is calculated to be:

boron average mass $=(0.19910 .0129 \mathrm{amu})+(0.80111 .0093 \mathrm{amu})$

$=1.99 \mathrm{amu}+8.82 \mathrm{amu}$

$=10.81 \mathrm{amu}$

It is important to understand that no single boron atom weighs exactly $10.8 \mathrm{amu} ; 10.8 \mathrm{amu}$ is the average mass of all boron atoms, and individual boron atoms weigh either approximately $10 \mathrm{amu}$ or $11 \mathrm{amu}$.

Calculation of Average Atomic Mass A meteorite found in central Indiana contains traces of the noble gas neon picked up from the solar wind during the meteorite's trip through the solar system. Analysis of a sample of the gas showed that it consisted of $91.84 \%{ }^{20} \mathrm{Ne}$ (mass $19.9924 \mathrm{amu}$ ), $0.47 \%{ }^{21} \mathrm{Ne}$ (mass $20.9940 \mathrm{amu}$ ), and $7.69 \%{ }^{22} \mathrm{Ne}$ (mass $21.9914 \mathrm{amu}$ ). What is the average mass of the neon in the solar wind?

\section{Solution}

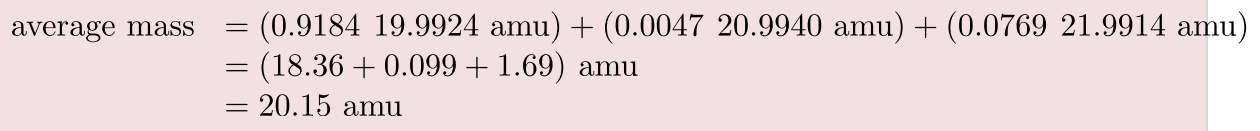

The average mass of a neon atom in the solar wind is 20.15 amu. (The average mass of a terrestrial neon atom is 20.1796 amu. This result demonstrates that we may find slight differences in the natural abundance of isotopes, depending on their origin.)

Check Your Learning A sample of magnesium is found to contain $78.70 \%$ of ${ }^{24} \mathrm{Mg}$ atoms (mass $23.98 \mathrm{amu}$ ), $10.13 \%$ of ${ }^{25} \mathrm{Mg}$ atoms (mass $24.99 \mathrm{amu}$ ), and $11.17 \%$ of ${ }^{26} \mathrm{Mg}$ atoms (mass $25.98 \mathrm{amu}$ ). Calculate the average mass of a Mg atom.

Answer: 
$24.31 \mathrm{amu}$

We can also do variations of this type of calculation, as shown in the next example.

Calculation of Percent Abundance Naturally occurring chlorine consists of ${ }^{35} \mathrm{Cl}$ (mass $34.96885 \mathrm{amu}$ ) and ${ }^{37} \mathrm{Cl}$ (mass $36.96590 \mathrm{amu}$ ), with an average mass of $35.453 \mathrm{amu}$. What is the percent composition of $\mathrm{Cl}$ in terms of these two isotopes?

Solution The average mass of chlorine is the fraction that is ${ }^{35} \mathrm{Cl}$ times the mass of ${ }^{35} \mathrm{Cl}$ plus the fraction that is ${ }^{37} \mathrm{Cl}$ times the mass of ${ }^{37} \mathrm{Cl}$.

average mass $=\left(\right.$ fraction of ${ }^{35} \mathrm{Cl}$ mass of $\left.{ }^{35} \mathrm{Cl}\right)+\left(\right.$ fraction of ${ }^{37} \mathrm{Cl}$ mass of $\left.{ }^{37} \mathrm{Cl}\right)$

If we let $x$ represent the fraction that is ${ }^{35} \mathrm{Cl}$, then the fraction that is ${ }^{37} \mathrm{Cl}$ is represented by $1.00-x$.

(The fraction that is ${ }^{35} \mathrm{Cl}+$ the fraction that is ${ }^{37} \mathrm{Cl}$ must add up to 1 , so the fraction of ${ }^{37} \mathrm{Cl}$ must equal 1.00 - the fraction of ${ }^{35} \mathrm{Cl}$.)

Substituting this into the average mass equation, we have:

$$
\begin{aligned}
35.453 \mathrm{amu} & =(x 34.96885 \mathrm{amu})+[(1.00-x) 36.96590 \mathrm{amu}] \\
35.453 & =34.96885 x+36.96590-36.96590 x \\
1.99705 x & =1.513 \\
x & =\frac{1.513}{1.99705}=0.7576
\end{aligned}
$$

So solving yields: $x=0.7576$, which means that $1.00-0.7576$ $=0.2424$. Therefore, chlorine consists of $75.76 \%{ }^{35} \mathrm{Cl}$ and $24.24 \%{ }^{37} \mathrm{Cl}$.

Check Your Learning Naturally occurring copper consists of ${ }^{63} \mathrm{Cu}$ (mass $62.9296 \mathrm{amu}$ ) and ${ }^{65} \mathrm{Cu}$ (mass $64.9278 \mathrm{amu}$ ), with an average mass of $63.546 \mathrm{amu}$. What is the percent composition of $\mathrm{Cu}$ in terms of these two isotopes?

Answer:

69.15\% Cu-63 and 30.85\% Cu-65 
Visit this site to make mixtures of the main isotopes of the first 18 elements, gain experience with average atomic mass, and check naturally occurring isotope ratios using the Isotopes and Atomic Mass simulation.

The occurrence and natural abundances of isotopes can be experimentally determined using an instrument called a mass spectrometer. Mass spectrometry (MS) is widely used in chemistry, forensics, medicine, environmental science, and many other fields to analyze and help identify the substances in a sample of material. In a typical mass spectrometer ((Figure)), the sample is vaporized and exposed to a high-energy electron beam that causes the sample's atoms (or molecules) to become electrically charged, typically by losing one or more electrons. These cations then pass through a (variable) electric or magnetic field that deflects each cation's path to an extent that depends on both its mass and charge (similar to how the path of a large steel ball bearing rolling past a magnet is deflected to a lesser extent that that of a small steel BB). The ions are detected, and a plot of the relative number of ions generated versus their mass-to-charge ratios (a mass spectrum) is made. The height of each vertical feature or peak in a mass spectrum is proportional to the fraction of cations with the specified mass-to-charge ratio. Since its initial use during the development of modern atomic theory, MS has evolved to become a powerful tool for chemical analysis in a wide range of applications.

Analysis of zirconium in a mass spectrometer produces a mass spectrum with peaks showing the different isotopes of Zr. 

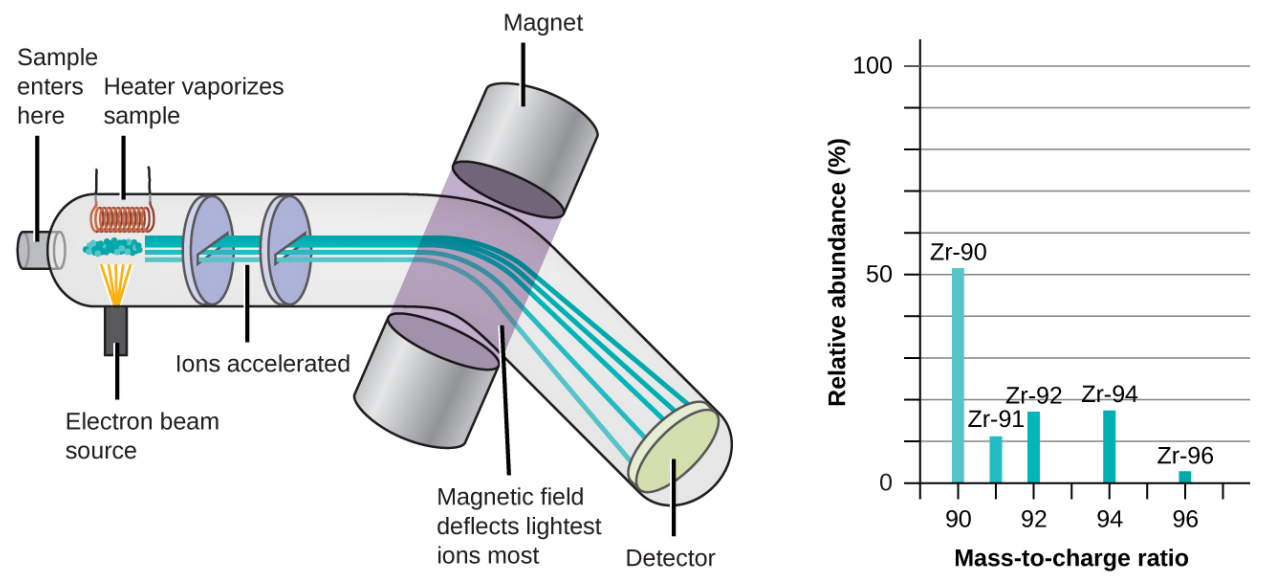

See an animation that explains mass spectrometry. Watch this video from the Royal Society for Chemistry for a brief description of the rudiments of mass spectrometry.

Key Concepts and Summary

An atom consists of a small, positively charged nucleus surrounded by electrons. The nucleus contains protons and neutrons; its diameter is about 100,000 times smaller than that of the atom. The mass of one atom is usually expressed in atomic mass units (amu), which is referred to as the atomic mass. An amu is defined as exactly $\frac{1}{12}$ of the mass of a carbon-12 atom and is equal to $1.660510^{-24} \mathrm{~g}$.

Protons are relatively heavy particles with a charge of $1+$ and a mass of $1.0073 \mathrm{amu}$. Neutrons are relatively heavy particles with no charge and a mass of $1.0087 \mathrm{amu}$. Electrons are light particles with a charge of 1- and a mass of $0.00055 \mathrm{amu}$. The number of protons in the nucleus is called the atomic number $(\mathrm{Z})$ and is the property that defines an atom's elemental identity. The sum of the numbers of protons and neutrons in the nucleus is called the mass number and, expressed in amu, is approximately 
equal to the mass of the atom. An atom is neutral when it contains equal numbers of electrons and protons.

Isotopes of an element are atoms with the same atomic number but different mass numbers; isotopes of an element, therefore, differ from each other only in the number of neutrons within the nucleus. When a naturally occurring element is composed of several isotopes, the atomic mass of the element represents the average of the masses of the isotopes involved. A chemical symbol identifies the atoms in a substance using symbols, which are one-, two-, or three-letter abbreviations for the atoms.

Key Equations

- average mass $=\sum_{i}(\text { fractional abundance isotopic mass })_{i}$

Chemistry End of Chapter Exercises

In what way are isotopes of a given element always different? In what way(s) are they always the same?

Write the symbol for each of the following ions:

(a) the ion with a $1+$ charge, atomic number 55, and mass number 133

(b) the ion with 54 electrons, 53 protons, and 74 neutrons

(c) the ion with atomic number 15 , mass number 31, and a 3- charge

(d) the ion with 24 electrons, 30 neutrons, and a 3+ charge

(a) ${ }^{133} \mathrm{Cs}^{+}$; (b) ${ }^{127} \mathrm{I}^{-}$; (c) ${ }^{31} \mathrm{P}^{3-}$; (d) ${ }^{57} \mathrm{Co}^{3+}$ 
Write the symbol for each of the following ions:

(a) the ion with a 3+ charge, 28 electrons, and a mass number of 71

(b) the ion with 36 electrons, 35 protons, and 45 neutrons

(c) the ion with 86 electrons, 142 neutrons, and a 4+ charge

(d) the ion with a 2+ charge, atomic number 38, and mass number 87

Open the Build an Atom simulation and click on the Atom icon.

(a) Pick any one of the first 10 elements that you would like to build and state its symbol.

(b) Drag protons, neutrons, and electrons onto the atom template to make an atom of your element.

State the numbers of protons, neutrons, and electrons in your atom, as well as the net charge and mass number.

(c) Click on "Net Charge" and "Mass Number," check your answers to (b), and correct, if needed.

(d) Predict whether your atom will be stable or unstable. State your reasoning.

(e) Check the "Stable/Unstable" box. Was your answer to (d) correct? If not, first predict what you can do to make a stable atom of your element, and then do it and see if it works. Explain your reasoning.

(a) Carbon-12, ${ }^{12} \mathrm{C}$; (b) This atom contains six protons and six neutrons. There are six electrons in a neutral ${ }^{12} \mathrm{C}$ atom. The net charge of such a neutral atom is zero, and the mass number is 12. (c) The preceding answers are correct. (d) The atom will be stable since C-12 is a stable 
isotope of carbon. (e) The preceding answer is correct. Other answers for this exercise are possible if a different element of isotope is chosen.

Open the Build an Atom simulation.

(a) Drag protons, neutrons, and electrons onto the atom template to make a neutral atom of Oxygen-16 and give the isotope symbol for this atom.

(b) Now add two more electrons to make an ion and give the symbol for the ion you have created.

Open the Build an Atom simulation.

(a) Drag protons, neutrons, and electrons onto the atom template to make a neutral atom of Lithium- 6 and give the isotope symbol for this atom.

(b) Now remove one electron to make an ion and give the symbol for the ion you have created.

(a) Lithium-6 contains three protons, three neutrons, and three electrons. The isotope symbol is ${ }^{6} \mathrm{Li}$ or ${ }_{3}^{6} \mathrm{Li}$. (b) ${ }^{6} \mathrm{Li}^{+}$ or ${ }_{3}^{6} \mathrm{Li}^{+}$

Determine the number of protons, neutrons, and electrons in the following isotopes that are used in medical diagnoses:

(a) atomic number 9, mass number 18, charge of 1-

(b) atomic number 43, mass number 99, charge of 7+

(c) atomic number 53, atomic mass number 131, charge of $1-$

(d) atomic number 81, atomic mass number 201, charge of $1+$

(e) Name the elements in parts (a), (b), (c), and (d). 
The following are properties of isotopes of two elements that are essential in our diet. Determine the number of protons, neutrons and electrons in each and name them.

(a) atomic number 26 , mass number 58 , charge of $2+$

(b) atomic number 53, mass number 127 , charge of $1-$

(a) Iron, 26 protons, 24 electrons, and 32 neutrons; (b) iodine, 53 protons, 54 electrons, and 74 neutrons

Give the number of protons, electrons, and neutrons in neutral atoms of each of the following isotopes:
(a) ${ }_{5}^{10} \mathrm{~B}$
(b) ${ }_{80}^{199} \mathrm{Hg}$
(c) ${ }_{29}^{63} \mathrm{Cu}$
(d) ${ }_{6}^{13} \mathrm{C}$
(e) ${ }_{34}^{77} \mathrm{Se}$

Give the number of protons, electrons, and neutrons in neutral atoms of each of the following isotopes:
(a) ${ }_{3}^{7} \mathrm{Li}$
(b) ${ }_{52}^{125} \mathrm{Te}$
(c) ${ }_{47}^{109} \mathrm{Ag}$
(d) ${ }_{7}^{15} \mathrm{~N}$
(e) ${ }_{15}^{31} \mathrm{P}$

(a) 3 protons, 3 electrons, 4 neutrons; (b) 52 protons, 52 electrons, 73 neutrons; (c) 47 protons, 47 electrons, 62 neutrons; (d) 7 protons, 7 electrons, 8 neutrons; (e) 15 protons, 15 electrons, 16 neutrons

Click on the site and select the "Mix Isotopes" tab, hide the "Percent Composition" and "Average Atomic Mass" boxes, and then select the element boron. 
(a) Write the symbols of the isotopes of boron that are shown as naturally occurring in significant amounts.

(b) Predict the relative amounts (percentages) of these boron isotopes found in nature. Explain the reasoning behind your choice.

(c) Add isotopes to the black box to make a mixture that matches your prediction in (b). You may drag isotopes from their bins or click on "More" and then move the sliders to the appropriate amounts.

(d) Reveal the "Percent Composition" and "Average Atomic Mass" boxes. How well does your mixture match with your prediction? If necessary, adjust the isotope amounts to match your prediction.

(e) Select "Nature's" mix of isotopes and compare it to your prediction. How well does your prediction compare with the naturally occurring mixture? Explain. If necessary, adjust your amounts to make them match "Nature's" amounts as closely as possible.

Repeat (Figure) using an element that has three naturally occurring isotopes.

Let us use neon as an example. Since there are three isotopes, there is no way to be sure to accurately predict the abundances to make the total of 20.18 amu average atomic mass. Let us guess that the abundances are 9\% $\mathrm{Ne}-22,91 \% \mathrm{Ne}-20$, and only a trace of $\mathrm{Ne}-21$. The average mass would be $20.18 \mathrm{amu}$. Checking the nature's mix of isotopes shows that the abundances are 90.48\% $\mathrm{Ne}-20$, 9.25\% Ne-22, and $0.27 \% \mathrm{Ne}-21$, so our guessed amounts have to be slightly adjusted. 
An element has the following natural abundances and isotopic masses: 90.92\% abundance with $19.99 \mathrm{amu}$, 0.26\% abundance with $20.99 \mathrm{amu}$, and $8.82 \%$ abundance with $21.99 \mathrm{amu}$. Calculate the average atomic mass of this element.

Average atomic masses listed by IUPAC are based on a study of experimental results. Bromine has two isotopes, ${ }^{79} \mathrm{Br}$ and ${ }^{81} \mathrm{Br}$, whose masses (78.9183 and $80.9163 \mathrm{amu}$, respectively) and abundances (50.69\% and 49.31\%, respectively) were determined in earlier experiments. Calculate the average atomic mass of bromine based on these experiments.

$79.90 \mathrm{amu}$

Variations in average atomic mass may be observed for elements obtained from different sources. Lithium provides an example of this. The isotopic composition of lithium from naturally occurring minerals is $7.5 \%{ }^{6} \mathrm{Li}$ and 92.5\% ${ }^{7} \mathrm{Li}$, which have masses of $6.01512 \mathrm{amu}$ and 7.01600 amu, respectively. A commercial source of lithium, recycled from a military source, was $3.75 \%{ }^{6} \mathrm{Li}$ (and the rest $\left.{ }^{7} \mathrm{Li}\right)$. Calculate the average atomic mass values for each of these two sources.

The average atomic masses of some elements may vary, depending upon the sources of their ores. Naturally occurring boron consists of two isotopes with accurately known masses $\left({ }^{10} \mathrm{~B}, 10.0129 \mathrm{amu}\right.$ and $\left.{ }^{11} \mathrm{~B}, 11.0931 \mathrm{amu}\right)$. The actual atomic mass of boron can vary from 10.807 to 10.819 , depending on whether the mineral source is from Turkey or the United States. Calculate the percent 
abundances leading to the two values of the average atomic masses of boron from these two countries.

Turkey source: $26.49 \%$ (of 10.0129 amu isotope); US source: $25.37 \%$ (of 10.0129 amu isotope)

The ${ }^{18} \mathrm{O}:{ }^{16} \mathrm{O}$ abundance ratio in some meteorites is greater than that used to calculate the average atomic mass of oxygen on earth. Is the average mass of an oxygen atom in these meteorites greater than, less than, or equal to that of a terrestrial oxygen atom?

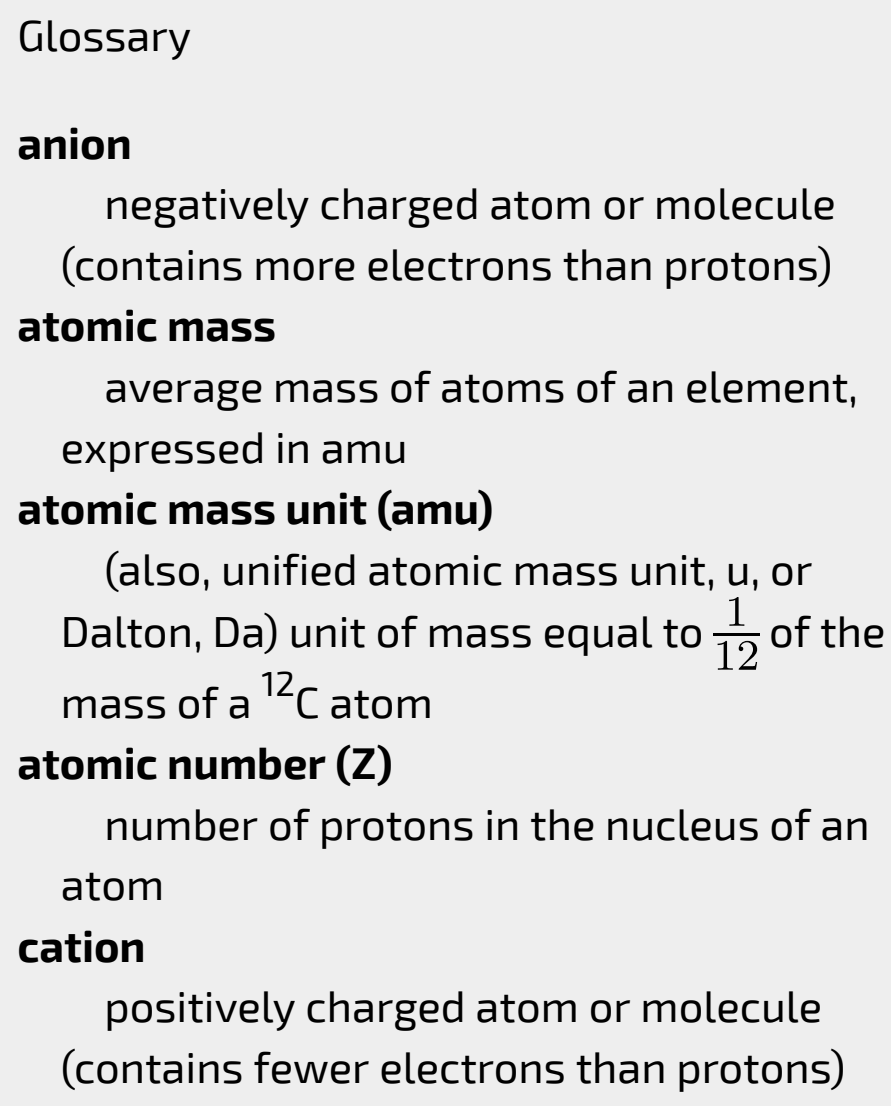


chemical symbol

one-, two-, or three-letter abbreviation used to represent an element or its atoms

\section{Dalton (Da)}

alternative unit equivalent to the atomic mass unit

\section{fundamental unit of charge}

(also called the elementary charge) equals the magnitude of the charge of an electron (e) with $\mathrm{e}=1.60210^{-19} \mathrm{C}$

ion

electrically charged atom or molecule (contains unequal numbers of protons and electrons)

mass number $(A)$

sum of the numbers of neutrons and protons in the nucleus of an atom unified atomic mass unit (u)

alternative unit equivalent to the atomic mass unit 


\section{CHEMICAL FORMULAS}

Learning Objectives

By the end of this section, you will be able to:

- Symbolize the composition of molecules using molecular formulas and empirical formulas

- Represent the bonding arrangement of atoms within molecules using structural formulas

A molecular formula is a representation of a molecule that uses chemical symbols to indicate the types of atoms followed by subscripts to show the number of atoms of each type in the molecule. (A subscript is used only when more than one atom of a given type is present.) Molecular formulas are also used as abbreviations for the names of compounds.

The structural formula for a compound gives the same information as its molecular formula (the types and numbers of atoms in the molecule) but also shows how 
the atoms are connected in the molecule. The structural formula for methane contains symbols for one $\mathrm{C}$ atom and four $\mathrm{H}$ atoms, indicating the number of atoms in the molecule ((Figure)). The lines represent bonds that hold the atoms together. (A chemical bond is an attraction between atoms or ions that holds them together in a molecule or a crystal.) We will discuss chemical bonds and see how to predict the arrangement of atoms in a molecule later. For now, simply know that the lines are an indication of how the atoms are connected in a molecule. A ball-and-stick model shows the geometric arrangement of the atoms with atomic sizes not to scale, and a spacefilling model shows the relative sizes of the atoms.

A methane molecule can be represented as (a) a molecular formula, (b) a structural formula, (c) a balland-stick model, and (d) a space-filling model. Carbon and hydrogen atoms are represented by black and white spheres, respectively.

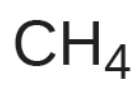

(a)

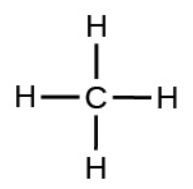

(b)

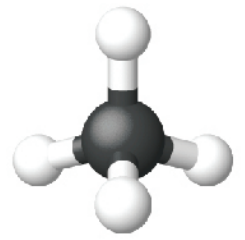

(c)

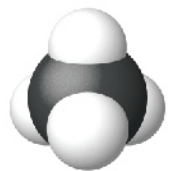

(d)

Although many elements consist of discrete, individual atoms, some exist as molecules made up of two or more atoms of the element chemically bonded together. For example, most samples of the elements hydrogen, oxygen, and nitrogen are composed of molecules that contain two atoms each (called diatomic molecules) and thus have the molecular formulas $\mathrm{H}_{2}, \mathrm{O}_{2}$, and $\mathrm{N}_{2}$, respectively. Other 
elements commonly found as diatomic molecules are fluorine $\left(\mathrm{F}_{2}\right)$, chlorine $\left(\mathrm{Cl}_{2}\right)$, bromine $\left(\mathrm{Br}_{2}\right)$, and iodine $\left(\mathrm{I}_{2}\right)$. The most common form of the element sulfur is composed of molecules that consist of eight atoms of sulfur; its molecular formula is $\mathrm{S}_{8}$ ((Figure)).

A molecule of sulfur is composed of eight sulfur atoms and is therefore written as $\mathrm{S}_{8}$. It can be represented as (a) a structural formula, (b) a ball-and-stick model, and (c) a space-filling model. Sulfur atoms are represented by yellow spheres.

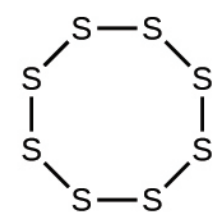

(a)

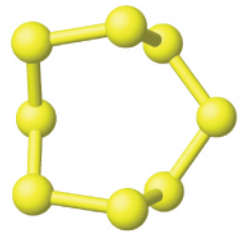

(b)

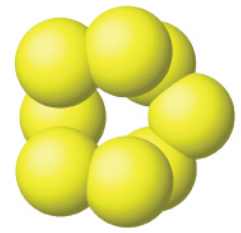

(c)

It is important to note that a subscript following a symbol and a number in front of a symbol do not represent the same thing; for example, $\mathrm{H}_{2}$ and $2 \mathrm{H}$ represent distinctly different species. $\mathrm{H}_{2}$ is a molecular formula; it represents a diatomic molecule of hydrogen, consisting of two atoms of the element that are chemically bonded together. The expression $2 \mathrm{H}$, on the other hand, indicates two separate hydrogen atoms that are not combined as a unit. The expression $2 \mathrm{H}_{2}$ represents two molecules of diatomic hydrogen ((Figure)).

The symbols $\mathrm{H}, 2 \mathrm{H}, \mathrm{H}_{2}$, and $2 \mathrm{H}_{2}$ represent very different entities. 


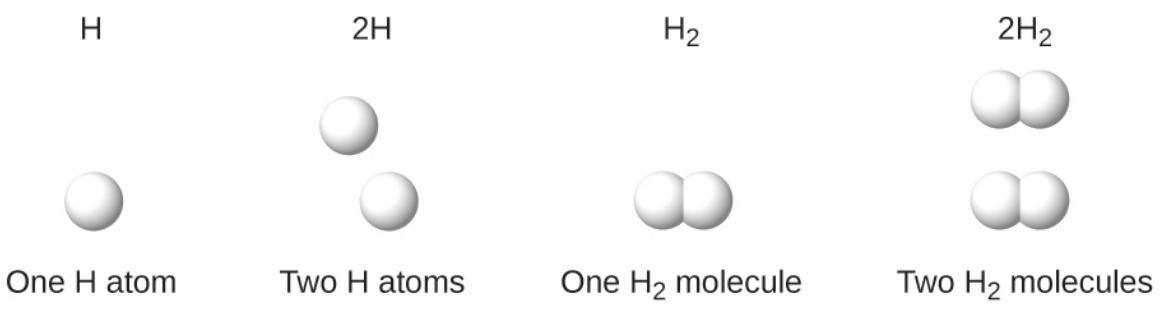

Compounds are formed when two or more elements chemically combine, resulting in the formation of bonds. For example, hydrogen and oxygen can react to form water, and sodium and chlorine can react to form table salt. We sometimes describe the composition of these compounds with an empirical formula, which indicates the types of atoms present and the simplest whole-number ratio of the number of atoms (or ions) in the compound. For example, titanium dioxide (used as pigment in white paint and in the thick, white, blocking type of sunscreen) has an empirical formula of $\mathrm{TiO}_{2}$. This identifies the elements titanium (Ti) and oxygen (O) as the constituents of titanium dioxide, and indicates the presence of twice as many atoms of the element oxygen as atoms of the element titanium ((Figure)).

(a) The white compound titanium dioxide provides effective protection from the sun. (b) A crystal of titanium dioxide, $\mathrm{TiO}_{2}$, contains titanium and oxygen in a ratio of 1 to 2 . The titanium atoms are gray and the oxygen atoms are red. (credit a: modification of work by “osseous”/Flickr) 


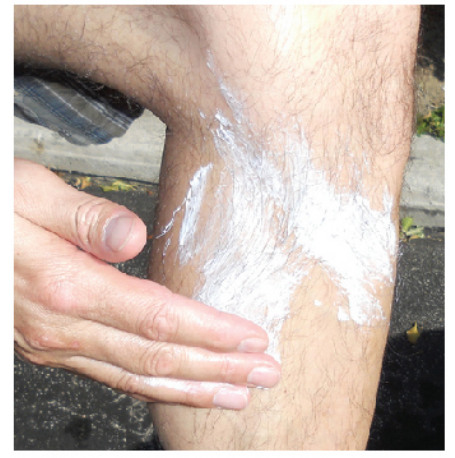

(a)

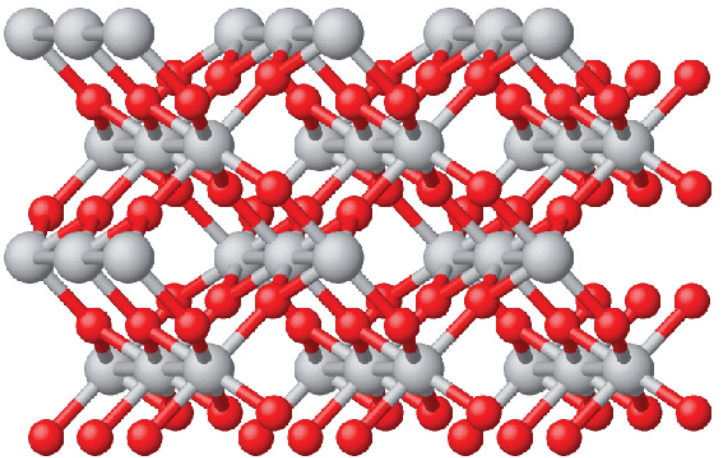

(b)

As discussed previously, we can describe a compound with a molecular formula, in which the subscripts indicate the actual numbers of atoms of each element in a molecule of the compound. In many cases, the molecular formula of a substance is derived from experimental determination of both its empirical formula and its molecular mass (the sum of atomic masses for all atoms composing the molecule). For example, it can be determined experimentally that benzene contains two elements, carbon $(\mathrm{C})$ and hydrogen $(\mathrm{H})$, and that for every carbon atom in benzene, there is one hydrogen atom. Thus, the empirical formula is $\mathrm{CH}$. An experimental determination of the molecular mass reveals that a molecule of benzene contains six carbon atoms and six hydrogen atoms, so the molecular formula for benzene is $\mathrm{C}_{6} \mathrm{H}_{6}$ ((Figure)).

Benzene, $\mathrm{C}_{6} \mathrm{H}_{6}$, is produced during oil refining and has many industrial uses. A benzene molecule can be represented as (a) a structural formula, (b) a ball-andstick model, and (c) a space-filling model. (d) Benzene is a clear liquid. (credit d: modification of work by Sahar Atwa) 


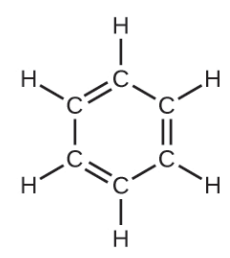

(a)

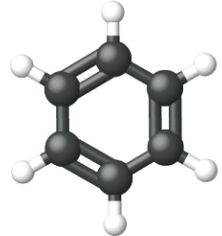

(b)

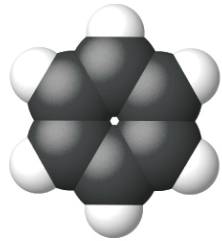

(c)

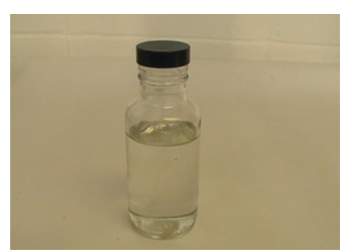

(d)

If we know a compound's formula, we can easily determine the empirical formula. (This is somewhat of an academic exercise; the reverse chronology is generally followed in actual practice.) For example, the molecular formula for acetic acid, the component that gives vinegar its sharp taste, is $\mathrm{C}_{2} \mathrm{H}_{4} \mathrm{O}_{2}$. This formula indicates that a molecule of acetic acid ((Figure)) contains two carbon atoms, four hydrogen atoms, and two oxygen atoms. The ratio of atoms is $2: 4: 2$. Dividing by the lowest common denominator (2) gives the simplest, whole-number ratio of atoms, $1: 2: 1$, so the empirical formula is $\mathrm{CH}_{2} \mathrm{O}$. Note that a molecular formula is always a whole-number multiple of an empirical formula.

(a) Vinegar contains acetic acid, $\mathrm{C}_{2} \mathrm{H}_{4} \mathrm{O}_{2}$, which has an empirical formula of $\mathrm{CH}_{2} \mathrm{O}$. It can be represented as (b) a structural formula and (c) as a ball-and-stick model. (credit a: modification of work by "HomeSpot HQ"/Flickr)

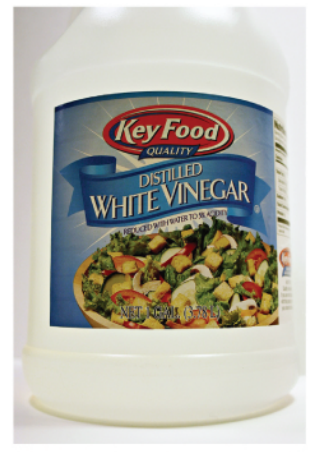

(a)

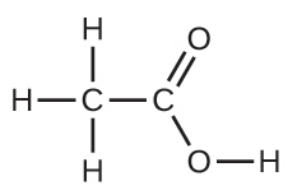

(b)

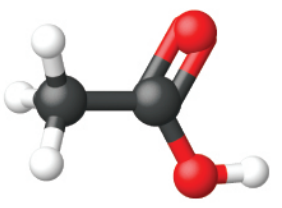

(c) 
Empirical and Molecular Formulas Molecules of glucose (blood sugar) contain 6 carbon atoms, 12 hydrogen atoms, and 6 oxygen atoms. What are the molecular and empirical formulas of glucose?

Solution The molecular formula is $\mathrm{C}_{6} \mathrm{H}_{12} \mathrm{O}_{6}$ because one molecule actually contains $6 \mathrm{C}, 12 \mathrm{H}$, and 60 atoms. The simplest whole-number ratio of $\mathrm{C}$ to $\mathrm{H}$ to $\mathrm{O}$ atoms in glucose is $1: 2: 1$, so the empirical formula is $\mathrm{CH}_{2} \mathrm{O}$.

Check Your Learning A molecule of metaldehyde (a pesticide used for snails and slugs) contains 8 carbon atoms, 16 hydrogen atoms, and 4 oxygen atoms. What are the molecular and empirical formulas of metaldehyde?

Answer:

Molecular formula, $\mathrm{C}_{8} \mathrm{H}_{16} \mathrm{O}_{4}$; empirical formula, $\mathrm{C}_{2} \mathrm{H}_{4} \mathrm{O}$

You can explore molecule building using an online simulation.

Lee Cronin

What is it that chemists do? According to Lee Cronin ((Figure)), chemists make very complicated molecules by "chopping up" small molecules and "reverse engineering" them. He wonders if we could "make a really cool universal chemistry set" by what he calls "app-ing" chemistry. Could we "app" chemistry?

In a 2012 TED talk, Lee describes one fascinating possibility: combining a collection of chemical "inks" with a 3D printer capable of fabricating a reaction apparatus (tiny test tubes, beakers, and the like) to fashion a "universal toolkit of chemistry." This toolkit could be used to create custom-tailored drugs to fight a new superbug or to "print" medicine personally configured to your genetic 
makeup, environment, and health situation. Says Cronin, "What Apple did for music, I'd like to do for the discovery and distribution of prescription drugs." "View his full talk at the TED website.

Chemist Lee Cronin has been named one of the UK's 10 most inspirational scientists. The youngest chair at the University of Glasgow, Lee runs a large research group, collaborates with many scientists worldwide, has published over 250 papers in top scientific journals, and has given more than 150 invited talks. His research focuses on complex chemical systems and their potential to transform technology, but also branches into nanoscience, solar fuels, synthetic biology, and even artificial life and evolution. (credit: image courtesy of Lee Cronin) 


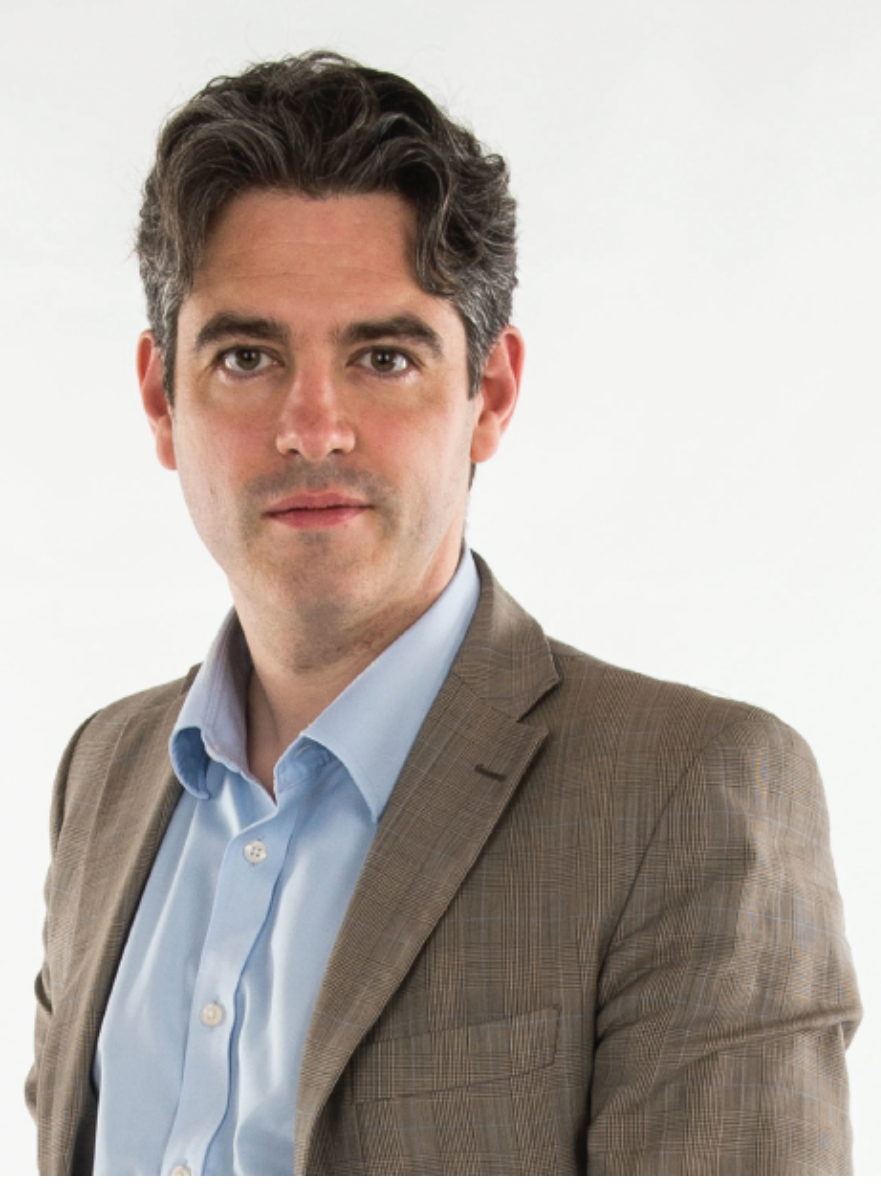

It is important to be aware that it may be possible for the same atoms to be arranged in different ways: Compounds with the same molecular formula may have different atom-to-atom bonding and therefore different structures. For example, could there be another compound with the same formula as acetic acid, $\mathrm{C}_{2} \mathrm{H}_{4} \mathrm{O}_{2}$ ? And if so, what would be the structure of its molecules?

If you predict that another compound with the formula $\mathrm{C}_{2} \mathrm{H}_{4} \mathrm{O}_{2}$ could exist, then you demonstrated good chemical insight and are correct. Two $\mathrm{C}$ atoms, four $\mathrm{H}$ atoms, and two $\mathrm{O}$ atoms can also be arranged to form a methyl formate, which is used in manufacturing, as an insecticide, and for quick-drying finishes. Methyl formate 
molecules have one of the oxygen atoms between the two carbon atoms, differing from the arrangement in acetic acid molecules. Acetic acid and methyl formate are examples of isomers-compounds with the same chemical formula but different molecular structures ((Figure)). Note that this small difference in the arrangement of the atoms has a major effect on their respective chemical properties. You would certainly not want to use a solution of methyl formate as a substitute for a solution of acetic acid (vinegar) when you make salad dressing.

Molecules of (a) acetic acid and methyl formate (b) are structural isomers; they have the same formula $\left(\mathrm{C}_{2} \mathrm{H}_{4} \mathrm{O}_{2}\right)$ but different structures (and therefore different chemical properties).

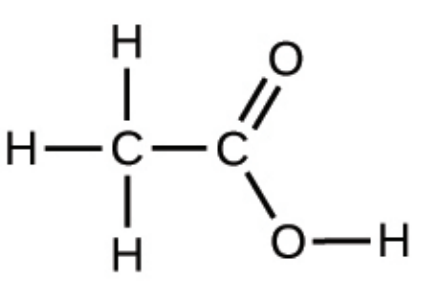

Acetic acid

$\mathrm{C}_{2} \mathrm{H}_{4} \mathrm{O}_{2}$

(a)<smiles>COC=O</smiles>

Methyl formate

$\mathrm{C}_{2} \mathrm{H}_{4} \mathrm{O}_{2}$

(b)

Many types of isomers exist ((Figure)). Acetic acid and methyl formate are structural isomers, compounds in which the molecules differ in how the atoms are connected to each other. There are also various types of spatial isomers, in which the relative orientations of the atoms in space can be different. For example, the compound carvone (found in caraway seeds, spearmint, 
and mandarin orange peels) consists of two isomers that are mirror images of each other. $S$-(+)-carvone smells like caraway, and $R-(-)$-carvone smells like spearmint.

Molecules of carvone are spatial isomers; they only differ in the relative orientations of the atoms in space. (credit bottom left: modification of work by

"Miansari66"/Wikimedia Commons; credit bottom right: modification of work by Forest \& Kim Starr)
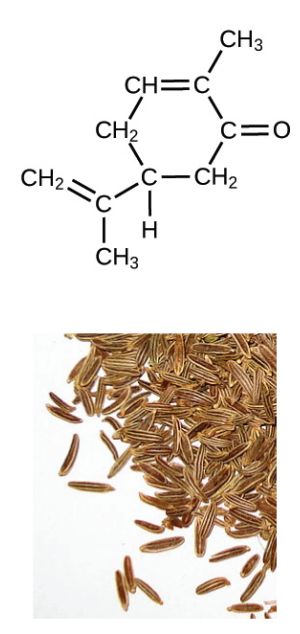

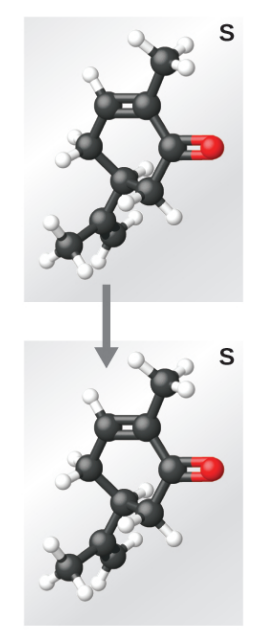

(+)-Carvone $\mathrm{C}_{10} \mathrm{H}_{14} \mathrm{O}$

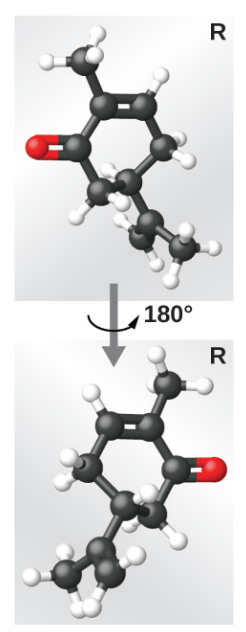

(-)-Carvone
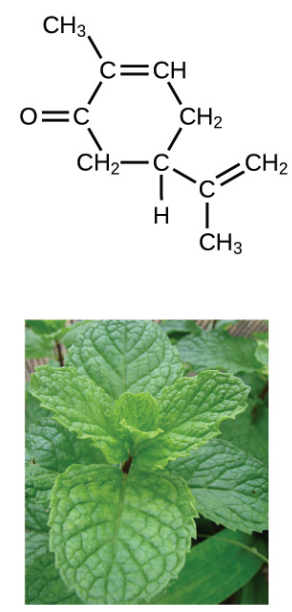

Select this link to view an explanation of isomers, spatial isomers, and why they have different smells (select the video titled "Mirror Molecule: Carvone”).

Key Concepts and Summary

A molecular formula uses chemical symbols and subscripts to indicate the exact numbers of different atoms in a molecule or compound. An empirical formula gives the simplest, whole-number ratio of atoms in a compound. A structural formula indicates the bonding arrangement of the atoms in the molecule. Ball-and-stick 
and space-filling models show the geometric arrangement of atoms in a molecule. Isomers are compounds with the same molecular formula but different arrangements of atoms.

Chemistry End of Chapter Exercises

Explain why the symbol for an atom of the element oxygen and the formula for a molecule of oxygen differ.

The symbol for the element oxygen, $\mathrm{O}$, represents both the element and one atom of oxygen. A molecule of oxygen, $\mathrm{O}_{2}$, contains two oxygen atoms; the subscript 2 in the formula must be used to distinguish the diatomic molecule from two single oxygen atoms.

Explain why the symbol for the element sulfur and the formula for a molecule of sulfur differ.

Write the molecular and empirical formulas of the following compounds:

(a)

$$
\mathrm{O}=\mathrm{C}=\mathrm{O}
$$

(b)

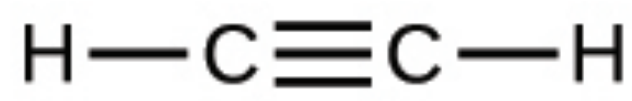

(c) 


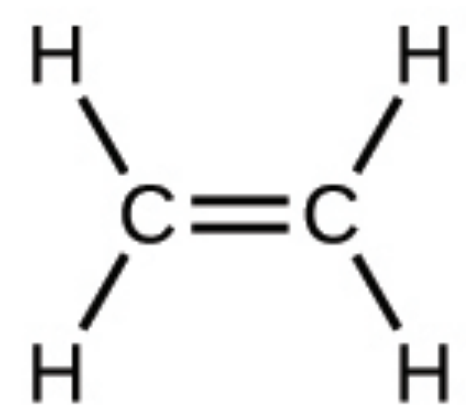

(d)

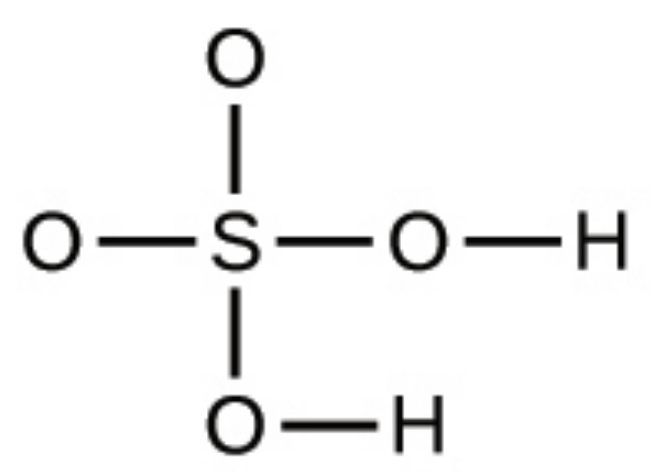

(a) molecular $\mathrm{CO}_{2}$, empirical $\mathrm{CO}_{2}$; (b) molecular $\mathrm{C}_{2} \mathrm{H}_{2}$, empirical $\mathrm{CH}$; (c) molecular $\mathrm{C}_{2} \mathrm{H}_{4}$, empirical $\mathrm{CH}_{2}$; (d) molecular $\mathrm{H}_{2} \mathrm{SO}_{4}$, empirical $\mathrm{H}_{2} \mathrm{SO}_{4}$

Write the molecular and empirical formulas of the following compounds:

(a)

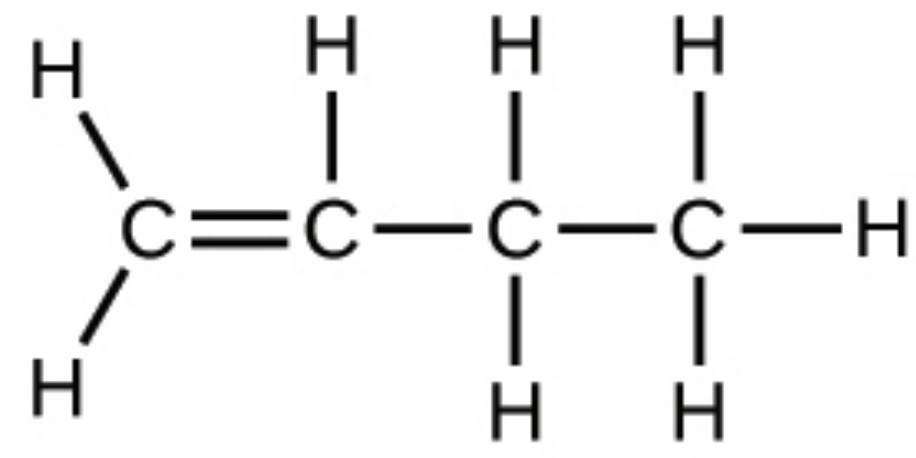

(b) 


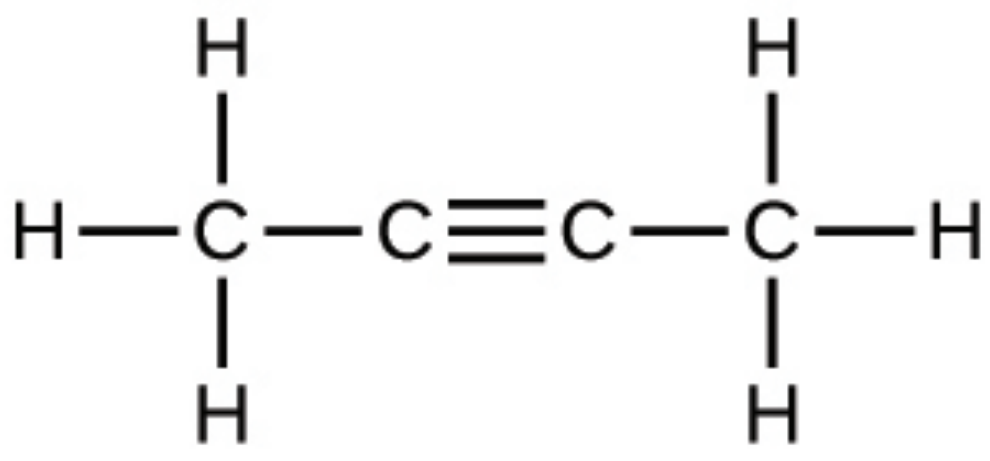

(C)

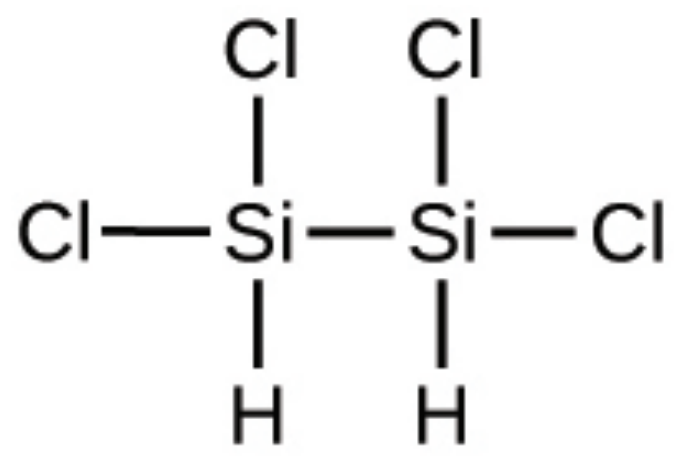

(d)

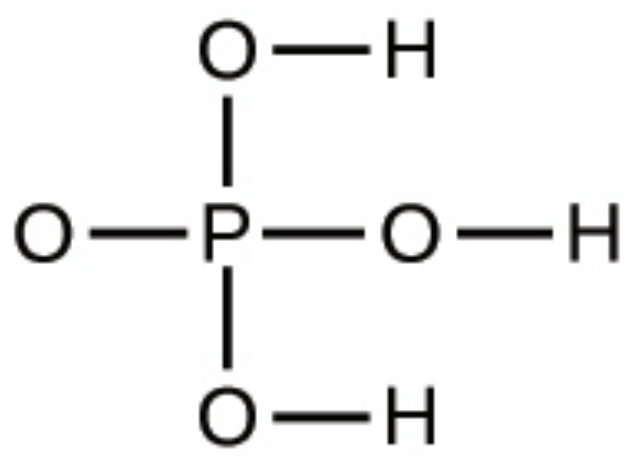

Determine the empirical formulas for the following compounds:

(a) caffeine, $\mathrm{C}_{8} \mathrm{H}_{10} \mathrm{~N}_{4} \mathrm{O}_{2}$

(b) fructose, $\mathrm{C}_{12} \mathrm{H}_{22} \mathrm{O}_{11}$

(c) hydrogen peroxide, $\mathrm{H}_{2} \mathrm{O}_{2}$

(d) glucose, $\mathrm{C}_{6} \mathrm{H}_{12} \mathrm{O}_{6}$ 
(e) ascorbic acid (vitamin C), $\mathrm{C}_{6} \mathrm{H}_{8} \mathrm{O}_{6}$

(a) $\mathrm{C}_{4} \mathrm{H}_{5} \mathrm{~N}_{2} \mathrm{O}$; (b) $\mathrm{C}_{12} \mathrm{H}_{22} \mathrm{O}_{11}$; (c) $\mathrm{HO}$; (d) $\mathrm{CH}_{2} \mathrm{O}$; (e) $\mathrm{C}_{3} \mathrm{H}_{4} \mathrm{O}_{3}$

Determine the empirical formulas for the following compounds:

(a) acetic acid, $\mathrm{C}_{2} \mathrm{H}_{4} \mathrm{O}_{2}$

(b) citric acid, $\mathrm{C}_{6} \mathrm{H}_{8} \mathrm{O}_{7}$

(c) hydrazine, $\mathrm{N}_{2} \mathrm{H}_{4}$

(d) nicotine, $\mathrm{C}_{10} \mathrm{H}_{14} \mathrm{~N}_{2}$

(e) butane, $\mathrm{C}_{4} \mathrm{H}_{10}$

Write the empirical formulas for the following compounds:

(a)

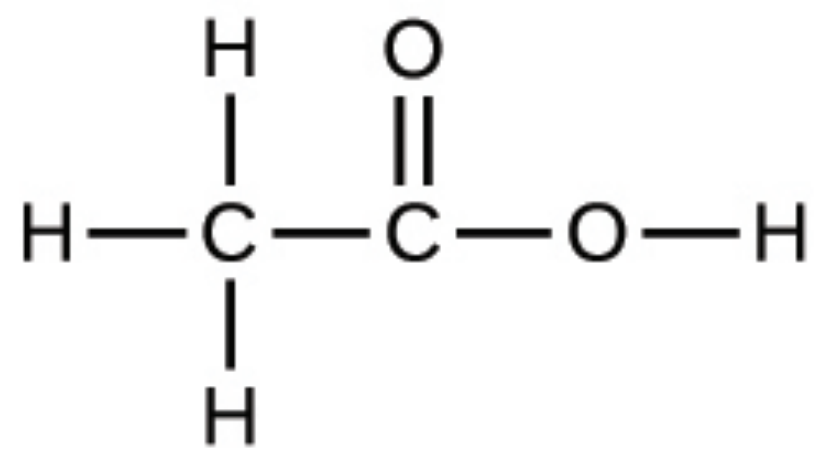

(b)<smiles>CC(C)=O</smiles>

(a) $\mathrm{CH}_{2} \mathrm{O}$; (b) $\mathrm{C}_{2} \mathrm{H}_{4} \mathrm{O}$

Open the Build a Molecule simulation and select the "Larger Molecules" tab. Select an appropriate atom's "Kit" 
to build a molecule with two carbon and six hydrogen atoms. Drag atoms into the space above the "Kit" to make a molecule. A name will appear when you have made an actual molecule that exists (even if it is not the one you want). You can use the scissors tool to separate atoms if you would like to change the connections. Click on "3D" to see the molecule, and look at both the space-filling and ball-and-stick possibilities.

(a) Draw the structural formula of this molecule and state its name.

(b) Can you arrange these atoms in any way to make a different compound?

Use the Build a Molecule simulation to repeat (Figure), but build a molecule with two carbons, six hydrogens, and one oxygen.

(a) Draw the structural formula of this molecule and state its name.

(b) Can you arrange these atoms to make a different molecule? If so, draw its structural formula and state its name.

(c) How are the molecules drawn in (a) and (b) the same? How do they differ? What are they called (the type of relationship between these molecules, not their names).?

(a) ethanol 


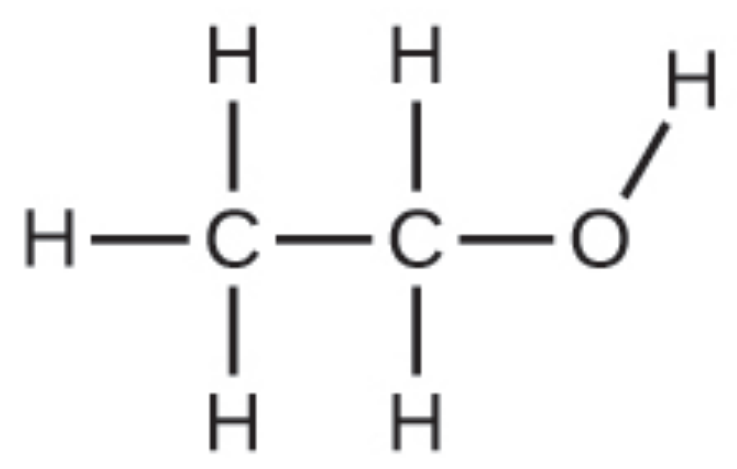

(b) methoxymethane, more commonly known as dimethyl ether

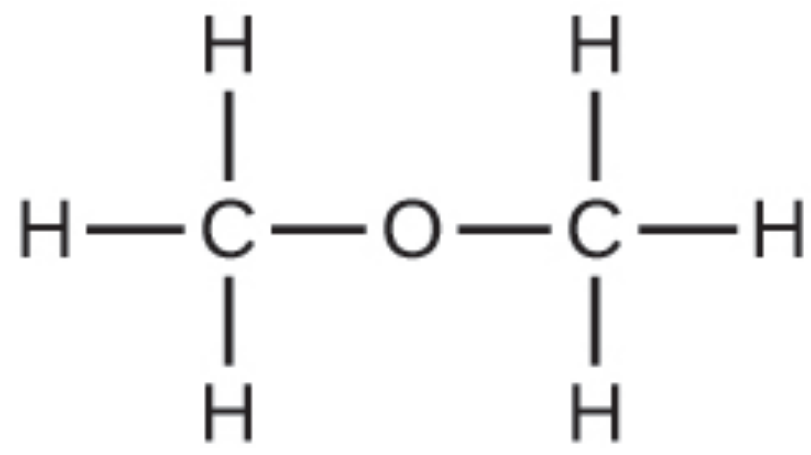

(c) These molecules have the same chemical composition (types and number of atoms) but different chemical structures. They are structural isomers.

Use the Build a Molecule simulation to repeat (Figure), but build a molecule with three carbons, seven hydrogens, and one chlorine.

(a) Draw the structural formula of this molecule and state its name.

(b) Can you arrange these atoms to make a different molecule? If so, draw its structural formula and state its name.

(c) How are the molecules drawn in (a) and (b) the same? How do they differ? What are they called (the type of relationship between these molecules, not their names)? 
Footnotes

- 1 Lee Cronin, “Print Your Own Medicine," Talk presented at TED Global 2012, Edinburgh, Scotland, June 2012.

Glossary

empirical formula

formula showing the composition of a compound given as the simplest wholenumber ratio of atoms

isomers

compounds with the same chemical formula but different structures

molecular formula

formula indicating the composition of a molecule of a compound and giving the actual number of atoms of each element in a molecule of the compound.

spatial isomers

compounds in which the relative orientations of the atoms in space differ structural formula

shows the atoms in a molecule and how they are connected structural isomer one of two substances that have the same molecular formula but different physical and chemical properties because their atoms are bonded differently 
12.

\section{THE PERIODIC TABLE}

Learning Objectives

By the end of this section, you will be able to:

- $\quad$ State the periodic law and explain the organization of elements in the periodic table

- $\quad$ Predict the general properties of elements based on their location within the periodic table

- Identify metals, nonmetals, and metalloids by their properties and/or location on the periodic table

As early chemists worked to purify ores and discovered more elements, they realized that various elements could be grouped together by their similar chemical behaviors. One such grouping includes lithium (Li), sodium $(\mathrm{Na})$, and potassium $(\mathrm{K})$ : These elements all are shiny, conduct heat and electricity well, and have similar chemical properties. A second grouping includes calcium (Ca), strontium (Sr), 
and barium (Ba), which also are shiny, good conductors of heat and electricity, and have chemical properties in common. However, the specific properties of these two groupings are notably different from each other. For example: $\mathrm{Li}, \mathrm{Na}$, and $\mathrm{K}$ are much more reactive than are $\mathrm{Ca}, \mathrm{Sr}$, and $\mathrm{Ba}$; $\mathrm{Li}, \mathrm{Na}$, and $\mathrm{K}$ form compounds with oxygen in a ratio of two of their atoms to one oxygen atom, whereas $\mathrm{Ca}, \mathrm{Sr}$, and $\mathrm{Ba}$ form compounds with one of their atoms to one oxygen atom. Fluorine $(\mathrm{F})$, chlorine $(\mathrm{Cl})$, bromine ( $\mathrm{Br})$, and iodine (I) also exhibit similar properties to each other, but these properties are drastically different from those of any of the elements above.

Dimitri Mendeleev in Russia (1869) and Lothar Meyer in Germany (1870) independently recognized that there was a periodic relationship among the properties of the elements known at that time. Both published tables with the elements arranged according to increasing atomic mass. But Mendeleev went one step further than Meyer: He used his table to predict the existence of elements that would have the properties similar to aluminum and silicon, but were yet unknown. The discoveries of gallium (1875) and germanium (1886) provided great support for Mendeleev's work. Although Mendeleev and Meyer had a long dispute over priority, Mendeleev's contributions to the development of the periodic table are now more widely recognized ((Figure)).

(a) Dimitri Mendeleev is widely credited with creating (b) the first periodic table of the elements. (credit a: modification of work by Serge Lachinov; credit b: modification of work by "Den fjättrade ankan"/Wikimedia Commons) 


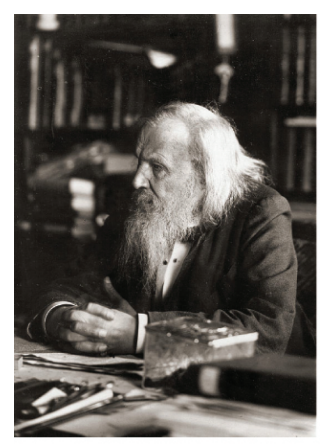

(a)

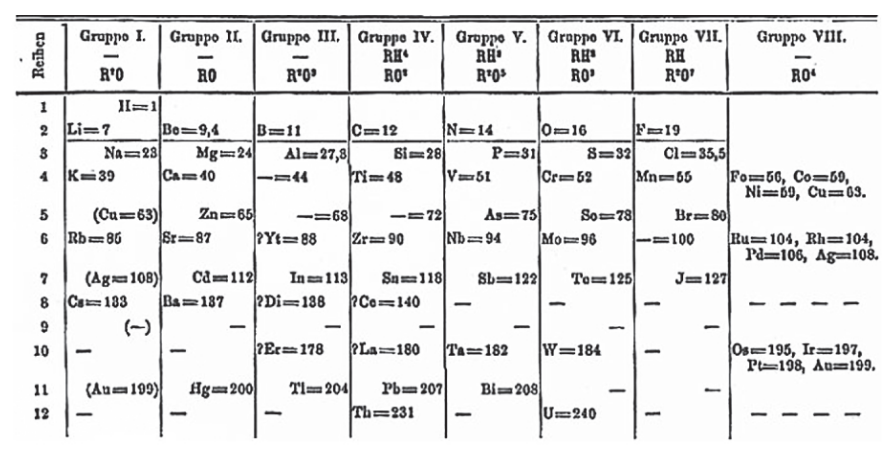

(b)

By the twentieth century, it became apparent that the periodic relationship involved atomic numbers rather than atomic masses. The modern statement of this relationship, the periodic law, is as follows: the properties of the elements are periodic functions of their atomic numbers. A modern periodic table arranges the elements in increasing order of their atomic numbers and groups atoms with similar properties in the same vertical column ((Figure)). Each box represents an element and contains its atomic number, symbol, average atomic mass, and (sometimes) name. The elements are arranged in seven horizontal rows, called periods or series, and 18 vertical columns, called groups. Groups are labeled at the top of each column. In the United States, the labels traditionally were numerals with capital letters. However, IUPAC recommends that the numbers 1 through 18 be used, and these labels are more common. For the table to fit on a single page, parts of two of the rows, a total of 14 columns, are usually written below the main body of the table. Elements in the periodic table are organized according to their properties. 
흫
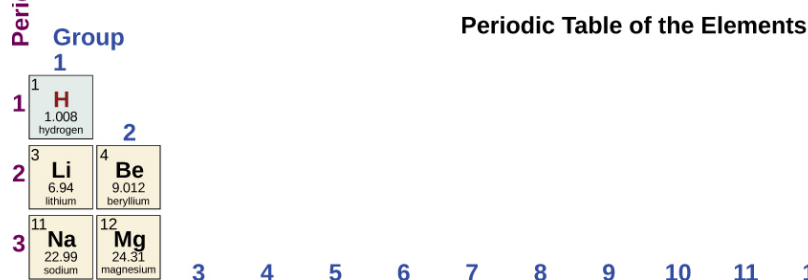

Periodic Table of the Elements
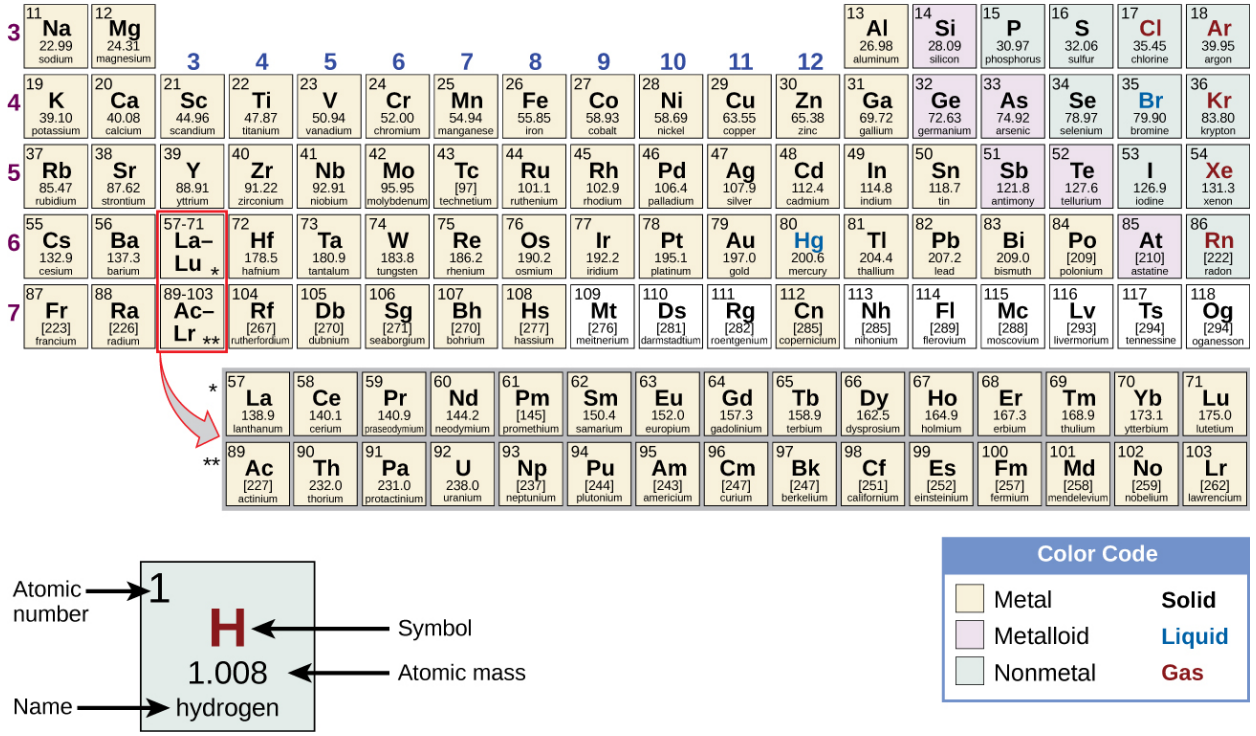

\begin{tabular}{|ll|}
\hline \multicolumn{3}{|c|}{ Color Code } \\
\hline$\square$ Metal & Solid \\
$\square$ Metalloid & Liquid \\
$\square$ Nonmetal & Gas \\
\hline
\end{tabular}

Many elements differ dramatically in their chemical and physical properties, but some elements are similar in their behaviors. For example, many elements appear shiny, are malleable (able to be deformed without breaking) and ductile (can be drawn into wires), and conduct heat and electricity well. Other elements are not shiny, malleable, or ductile, and are poor conductors of heat and electricity. We can sort the elements into large classes with common properties: metals (elements that are shiny, malleable, good conductors of heat and electricity-shaded yellow); nonmetals (elements that appear dull, poor conductors of heat and electricity-shaded green); and metalloids (elements that conduct heat and electricity moderately well, and possess some properties of metals and some properties of nonmetals-shaded purple).

The elements can also be classified into the main-group elements (or representative elements) in the columns 
labeled 1,2, and 13-18; the transition metals in the columns labeled $3-12^{1}$; and inner transition metals in the two rows at the bottom of the table (the top-row elements are called lanthanides and the bottom-row elements are actinides; (Figure)). The elements can be subdivided further by more specific properties, such as the composition of the compounds they form. For example, the elements in group 1 (the first column) form compounds that consist of one atom of the element and one atom of hydrogen. These elements (except hydrogen) are known as alkali metals, and they all have similar chemical properties. The elements in group 2 (the second column) form compounds consisting of one atom of the element and two atoms of hydrogen: These are called alkaline earth metals, with similar properties among members of that group. Other groups with specific names are the pnictogens (group 15), chalcogens (group 16), halogens (group 17), and the noble gases (group 18, also known as inert gases). The groups can also be referred to by the first element of the group: For example, the chalcogens can be called the oxygen group or oxygen family. Hydrogen is a unique, nonmetallic element with properties similar to both group 1 and group 17 elements. For that reason, hydrogen may be shown at the top of both groups, or by itself.

The periodic table organizes elements with similar properties into groups. 


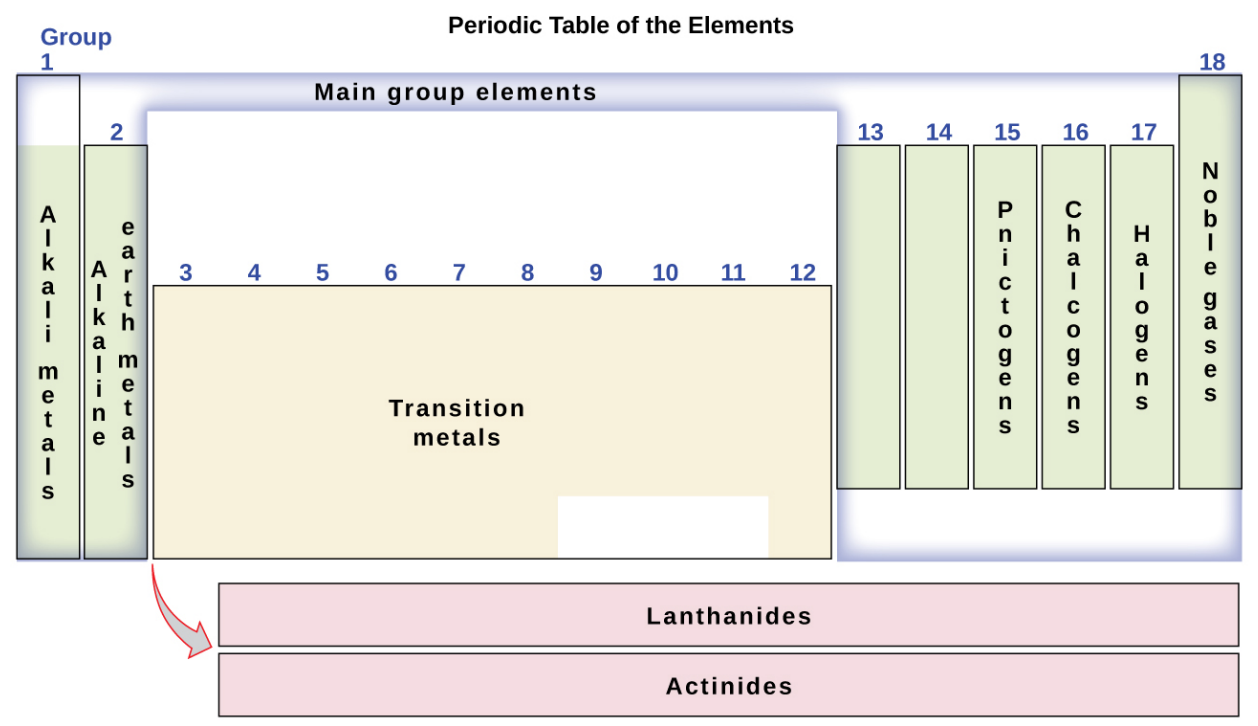

Click on this link for an interactive periodic table, which you can use to explore the properties of the elements (includes podcasts and videos of each element). You may also want to try this one that shows photos of all the elements.

Naming Groups of Elements Atoms of each of the following elements are essential for life. Give the group name for the following elements:

(a) chlorine

(b) calcium

(c) sodium

(d) sulfur

Solution The family names are as follows:
(a) halogen
(b) alkaline earth metal
(c) alkali metal
(d) chalcogen 
Check Your Learning Give the group name for each of the following elements:

(a) krypton

(b) selenium

(c) barium

(d) lithium

Answer:

(a) noble gas; (b) chalcogen; (c) alkaline earth metal; (d) alkali metal

In studying the periodic table, you might have noticed something about the atomic masses of some of the elements. Element 43 (technetium), element 61 (promethium), and most of the elements with atomic number 84 (polonium) and higher have their atomic mass given in square brackets. This is done for elements that consist entirely of unstable, radioactive isotopes (you will learn more about radioactivity in the nuclear chemistry chapter). An average atomic weight cannot be determined for these elements because their radioisotopes may vary significantly in relative abundance, depending on the source, or may not even exist in nature. The number in square brackets is the atomic mass number (an approximate atomic mass) of the most stable isotope of that element.

Key Concepts and Summary

The discovery of the periodic recurrence of similar properties among the elements led to the formulation of the periodic table, in which the elements are arranged 
in order of increasing atomic number in rows known as periods and columns known as groups. Elements in the same group of the periodic table have similar chemical properties. Elements can be classified as metals, metalloids, and nonmetals, or as a main-group elements, transition metals, and inner transition metals. Groups are numbered 1-18 from left to right. The elements in group 1 are known as the alkali metals; those in group 2 are the alkaline earth metals; those in 15 are the pnictogens; those in 16 are the chalcogens; those in 17 are the halogens; and those in 18 are the noble gases.

\section{Chemistry End of Chapter Exercises}

Using the periodic table, classify each of the following elements as a metal or a nonmetal, and then further classify each as a main-group (representative) element, transition metal, or inner transition metal:
(a) uranium
(b) bromine
(c) strontium
(d) neon
(e) gold
(f) americium
(g) rhodium
(h) sulfur
(i) carbon
(j) potassium

(a) metal, inner transition metal; (b) nonmetal, representative element; (c) metal, representative element; (d) nonmetal, representative element; (e) metal, 
transition metal; (f) metal, inner transition metal; (g) metal, transition metal; (h) nonmetal, representative element; (i) nonmetal, representative element; (j) metal, representative element

Using the periodic table, classify each of the following elements as a metal or a nonmetal, and then further classify each as a main-group (representative) element, transition metal, or inner transition metal:
(a) cobalt
(b) europium
(c) iodine
(d) indium
(e) lithium
(f) oxygen
(g) cadmium
(h) terbium
(i) rhenium

Using the periodic table, identify the lightest member of each of the following groups:

(a) noble gases

(b) alkaline earth metals

(c) alkali metals

(d) chalcogens

(a) He; (b) Be; (c) Li; (d) O

Using the periodic table, identify the heaviest member of each of the following groups:
(a) alkali metals
(b) chalcogens
(c) noble gases 
(d) alkaline earth metals

Use the periodic table to give the name and symbol for each of the following elements:

(a) the noble gas in the same period as germanium

(b) the alkaline earth metal in the same period as selenium

(c) the halogen in the same period as lithium

(d) the chalcogen in the same period as cadmium

(a) krypton, Kr; (b) calcium, Ca; (c) fluorine, F; (d) tellurium, Te

Use the periodic table to give the name and symbol for each of the following elements:

(a) the halogen in the same period as the alkali metal with 11 protons

(b) the alkaline earth metal in the same period with the neutral noble gas with 18 electrons

(c) the noble gas in the same row as an isotope with 30 neutrons and 25 protons

(d) the noble gas in the same period as gold

Write a symbol for each of the following neutral isotopes. Include the atomic number and mass number for each.

(a) the alkali metal with 11 protons and a mass number of 23

(b) the noble gas element with 75 neutrons in its nucleus and 54 electrons in the neutral atom

(c) the isotope with 33 protons and 40 neutrons in its nucleus

(d) the alkaline earth metal with 88 electrons and 138 neutrons 

(a) ${ }_{11}^{23} \mathrm{Na}$
(b) ${ }_{54}^{129} \mathrm{Xe}$;
(c) ${ }_{33}^{73} \mathrm{As}$;
(d) ${ }_{88}^{226} \mathrm{Ra}$

Write a symbol for each of the following neutral isotopes. Include the atomic number and mass number for each.

(a) the chalcogen with a mass number of 125

(b) the halogen whose longest-lived isotope is radioactive

(c) the noble gas, used in lighting, with 10 electrons and 10 neutrons

(d) the lightest alkali metal with three neutrons

Footnotes

- 1 Per the IUPAC definition, group 12 elements are not transition metals, though they are often referred to as such. Additional details on this group's elements are provided in a chapter on transition metals and coordination chemistry.

Glossary

actinide

inner transition metal in the bottom of the bottom two rows of the periodic table alkali metal element in group 1

alkaline earth metal element in group 2 chalcogen element in group 16 
group

vertical column of the periodic table halogen

element in group 17

hydrate

compound containing one or more water molecules bound within its crystals inert gas

(also, noble gas) element in group 18 inner transition metal

(also, lanthanide or actinide) element in the bottom two rows; if in the first row, also called lanthanide, or if in the second row, also called actinide

lanthanide

inner transition metal in the top of the bottom two rows of the periodic table main-group element

(also, representative element) element in groups 1, 2, and 13-18

metal

element that is shiny, malleable, good conductor of heat and electricity metalloid element that conducts heat and electricity moderately well, and possesses some properties of metals and some properties of nonmetals

\section{noble gas}

(also, inert gas) element in group 18 


\section{nonmetal}

element that appears dull, poor conductor of heat and electricity

period

(also, series) horizontal row of the periodic table

periodic law

properties of the elements are periodic function of their atomic numbers.

periodic table

table of the elements that places elements with similar chemical properties close together

pnictogen

element in group 15

representative element

(also, main-group element) element in

columns 1,2 , and $12-18$

series

(also, period) horizontal row of the period table

transition metal

element in groups 3-12 (more strictly

defined, 3-11; see chapter on transition

metals and coordination chemistry) 


\section{3. \\ MOLECULAR AND IONIC COMPOUNDS}

Learning Objectives

By the end of this section, you will be able to:

- Define ionic and molecular (covalent) compounds

- $\quad$ Predict the type of compound formed from elements based on their location within the periodic table

- Determine formulas for simple ionic compounds

In ordinary chemical reactions, the nucleus of each atom (and thus the identity of the element) remains unchanged. Electrons, however, can be added to atoms by transfer from other atoms, lost by transfer to other atoms, or shared with other atoms. The transfer and sharing of electrons among atoms govern the chemistry of the 
elements. During the formation of some compounds, atoms gain or lose electrons, and form electrically charged particles called ions ((Figure)).

(a) A sodium atom $(\mathrm{Na})$ has equal numbers of protons and electrons (11) and is uncharged. (b) A sodium cation $\left(\mathrm{Na}^{+}\right)$has lost an electron, so it has one more proton (11) than electrons (10), giving it an overall positive charge, signified by a superscripted plus sign.

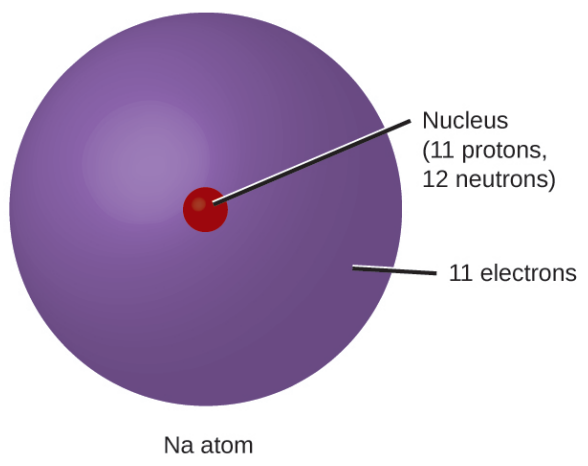

(a)

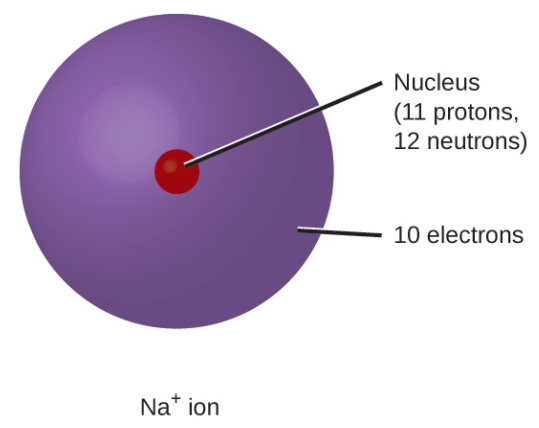

(b)

You can use the periodic table to predict whether an atom will form an anion or a cation, and you can often predict the charge of the resulting ion. Atoms of many maingroup metals lose enough electrons to leave them with the same number of electrons as an atom of the preceding noble gas. To illustrate, an atom of an alkali metal (group 1) loses one electron and forms a cation with a 1+ charge; an alkaline earth metal (group 2) loses two electrons and forms a cation with a $2+$ charge, and so on. For example, a neutral calcium atom, with 20 protons and 20 electrons, readily loses two electrons. This results in a cation with 20 protons, 18 electrons, and a $2+$ charge. It has the same number of electrons as atoms of the preceding noble gas, argon, and is symbolized $\mathrm{Ca}^{2+}$. The name of a metal ion 
is the same as the name of the metal atom from which it forms, so $\mathrm{Ca}^{2+}$ is called a calcium ion.

When atoms of nonmetal elements form ions, they generally gain enough electrons to give them the same number of electrons as an atom of the next noble gas in the periodic table. Atoms of group 17 gain one electron and form anions with a 1- charge; atoms of group 16 gain two electrons and form ions with a 2- charge, and so on. For example, the neutral bromine atom, with 35 protons and 35 electrons, can gain one electron to provide it with 36 electrons. This results in an anion with 35 protons, 36 electrons, and a 1 - charge. It has the same number of electrons as atoms of the next noble gas, krypton, and is symbolized $\mathrm{Br}^{-}$. (A discussion of the theory supporting the favored status of noble gas electron numbers reflected in these predictive rules for ion formation is provided in a later chapter of this text.)

Note the usefulness of the periodic table in predicting likely ion formation and charge ((Figure)). Moving from the far left to the right on the periodic table, main-group elements tend to form cations with a charge equal to the group number. That is, group 1 elements form $1+$ ions; group 2 elements form 2+ ions, and so on. Moving from the far right to the left on the periodic table, elements often form anions with a negative charge equal to the number of groups moved left from the noble gases. For example, group 17 elements (one group left of the noble gases) form 1- ions; group 16 elements (two groups left) form 2- ions, and so on. This trend can be used as a guide in many cases, but its predictive value decreases when moving toward the center of the periodic table. In fact, transition metals and some other metals often exhibit 
variable charges that are not predictable by their location in the table. For example, copper can form ions with a $1+$ or $2+$ charge, and iron can form ions with a $2+$ or $3+$ charge.

Some elements exhibit a regular pattern of ionic charge when they form ions.

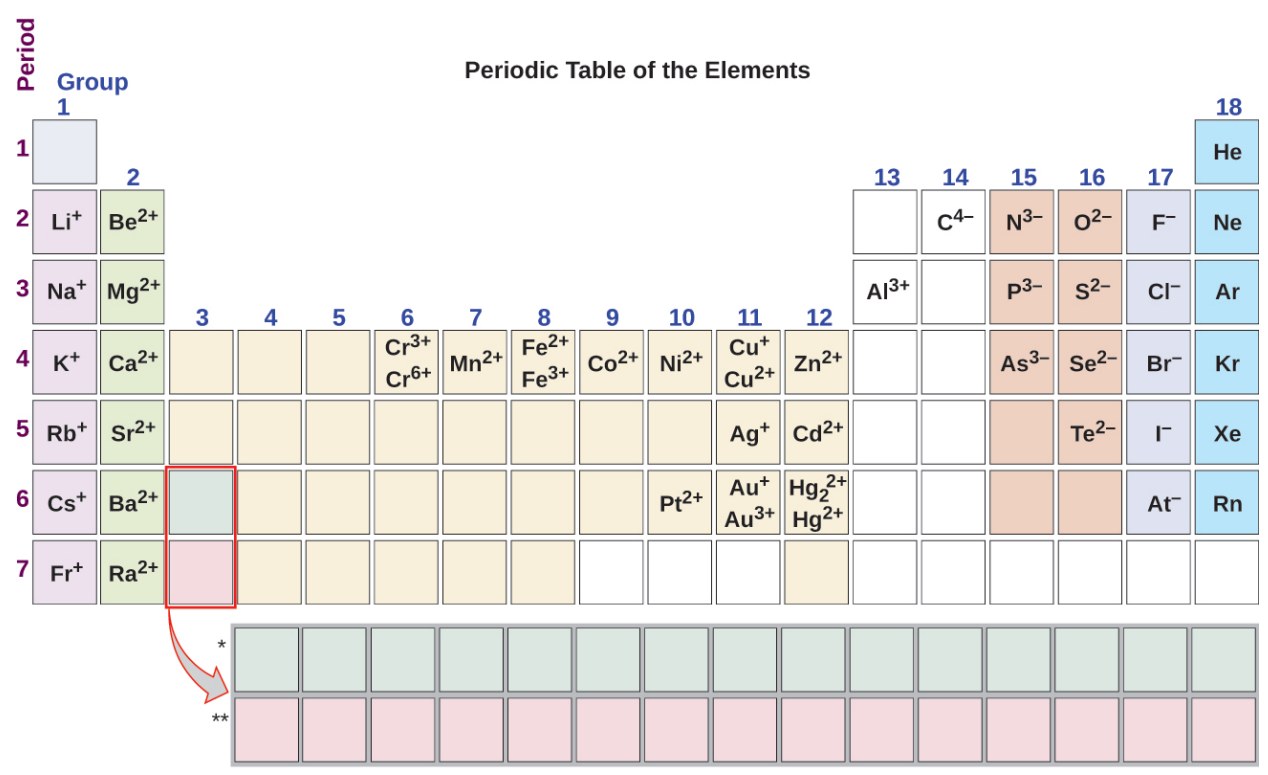

Composition of lons An ion found in some compounds used as antiperspirants contains 13 protons and 10 electrons. What is its symbol?

Solution Because the number of protons remains unchanged when an atom forms an ion, the atomic number of the element must be 13. Knowing this lets us use the periodic table to identify the element as Al (aluminum). The Al atom has lost three electrons and thus has three more positive charges (13) than it has electrons (10). This is the aluminum cation, $\mathrm{Al}^{3+}$.

Check Your Learning Give the symbol and name for the ion with 34 protons and 36 electrons.

Answer: 
$\mathrm{Se}^{2-}$, the selenide ion

Formation of lons Magnesium and nitrogen react to form an ionic compound. Predict which forms an anion, which forms a cation, and the charges of each ion. Write the symbol for each ion and name them.

Solution Magnesium's position in the periodic table (group 2 ) tells us that it is a metal. Metals form positive ions (cations). A magnesium atom must lose two electrons to have the same number electrons as an atom of the previous noble gas, neon. Thus, a magnesium atom will form a cation with two fewer electrons than protons and a charge of $2+$. The symbol for the ion is $\mathrm{Mg}^{2+}$, and it is called a magnesium ion.

Nitrogen's position in the periodic table (group 15) reveals that it is a nonmetal. Nonmetals form negative ions (anions). A nitrogen atom must gain three electrons to have the same number of electrons as an atom of the following noble gas, neon. Thus, a nitrogen atom will form an anion with three more electrons than protons and a charge of 3-. The symbol for the ion is $\mathrm{N}^{3-}$, and it is called a nitride ion.

Check Your Learning Aluminum and carbon react to form an ionic compound. Predict which forms an anion, which forms a cation, and the charges of each ion. Write the symbol for each ion and name them.

Answer:

Al will form a cation with a charge of $3+: \mathrm{Al}^{3+}$, an aluminum ion. Carbon will form an anion with a charge of $4-: \mathrm{C}^{4-}, \mathrm{a}$ carbide ion.

The ions that we have discussed so far are called monatomic ions, that is, they are ions formed from only one atom. We also find many polyatomic ions. These ions, 
which act as discrete units, are electrically charged molecules (a group of bonded atoms with an overall charge). Some of the more important polyatomic ions are listed in (Figure). Oxyanions are polyatomic ions that contain one or more oxygen atoms. At this point in your study of chemistry, you should memorize the names, formulas, and charges of the most common polyatomic ions. Because you will use them repeatedly, they will soon become familiar. 
Common Polyatomic Ions

\begin{tabular}{|c|c|c|c|}
\hline Name & Formula & Related Acid & Formula \\
\hline ammonium & $\mathrm{NH}_{4}{ }^{+}$ & & \\
\hline hydronium & $\mathrm{H}_{3} \mathrm{O}^{+}$ & & \\
\hline peroxide & $\mathrm{O}_{2}{ }^{2-}$ & & \\
\hline hydroxide & $\mathrm{OH}^{-}$ & & \\
\hline acetate & $\mathrm{CH}_{3} \mathrm{COO}^{-}$ & acetic acid & $\mathrm{CH}_{3} \mathrm{COOH}$ \\
\hline cyanide & $\mathrm{CN}^{-}$ & hydrocyanic acid & $\mathrm{HCN}$ \\
\hline azide & $\mathrm{N}_{3}^{-}$ & hydrazoic acid & $\mathrm{HN}_{3}$ \\
\hline carbonate & $\mathrm{CO}_{3}{ }^{2-}$ & carbonic acid & $\mathrm{H}_{2} \mathrm{CO}_{3}$ \\
\hline bicarbonate & $\mathrm{HCO}_{3}^{-}$ & & \\
\hline nitrate & $\mathrm{NO}_{3}-$ & nitric acid & $\mathrm{HNO}_{3}$ \\
\hline nitrite & $\mathrm{NO}_{2}{ }^{-}$ & nitrous acid & $\mathrm{HNO}_{2}$ \\
\hline sulfate & $\mathrm{SO}_{4}{ }^{2-}$ & sulfiric acid & $\mathrm{H}_{2} \mathrm{SO}_{4}$ \\
\hline hydrogen sulfate & $\mathrm{HSO}_{4}^{-}$ & & \\
\hline sulfite & $\mathrm{SO}_{3}{ }^{2-}$ & sulfurous acid & $\mathrm{H}_{2} \mathrm{SO}_{3}$ \\
\hline hydrogen sulfite & $\mathrm{HSO}_{3}-$ & & \\
\hline phosphate & $\mathrm{PO}_{4} 3-$ & phosphoric acid & $\mathrm{H}_{3} \mathrm{PO}_{4}$ \\
\hline hydrogen phosphate & $\mathrm{HPO}_{4}{ }^{2-}$ & & \\
\hline $\begin{array}{l}\text { dihydrogen } \\
\text { phosphate }\end{array}$ & $\mathrm{H}_{2} \mathrm{PO}_{4}^{-}$ & & \\
\hline perchlorate & $\mathrm{ClO}_{4}^{-}$ & perchloric acid & $\mathrm{HClO}_{4}$ \\
\hline chlorate & $\mathrm{ClO}_{3}{ }^{-}$ & chloric acid & $\mathrm{HClO}_{3}$ \\
\hline chlorite & $\mathrm{ClO}_{2}{ }^{-}$ & chlorous acid & $\mathrm{HClO}_{2}$ \\
\hline
\end{tabular}


Common Polyatomic Ions

$\begin{array}{llll}\text { Name } & \text { Formula } & \begin{array}{l}\text { Related Acid } \\ \text { hypochlorous }\end{array} & \text { Formula } \\ \text { hypochlorite } & \mathrm{ClO}^{-} & \mathrm{HClO} \\ \text { chromate } & \mathrm{CrO}_{4}^{2-} & \text { chromic acid } & \mathrm{H}_{2} \mathrm{Cr}_{2} \mathrm{O}_{4} \\ \text { dichromate } & \mathrm{Cr}_{2} \mathrm{O}_{7}{ }^{2-} & \text { dichromic acid } & \mathrm{H}_{2} \mathrm{Cr}_{2} \mathrm{O}_{7} \\ \text { permanganate } & \mathrm{MnO}_{4}- & \begin{array}{l}\text { permanganic } \\ \text { acid }\end{array} & \mathrm{HMnO}_{4}\end{array}$

Note that there is a system for naming some polyatomic ions; -ate and -ite are suffixes designating polyatomic ions containing more or fewer oxygen atoms. Per- (short for "hyper") and hypo- (meaning "under") are prefixes meaning more oxygen atoms than -ate and fewer oxygen atoms than -ite, respectively. For example, perchlorate is $\mathrm{ClO}_{4}{ }^{-}$, chlorate is $\mathrm{ClO}_{3}{ }^{-}$, chlorite is $\mathrm{ClO}_{2}{ }^{-}$and hypochlorite is $\mathrm{ClO}^{-}$. Unfortunately, the number of oxygen atoms corresponding to a given suffix or prefix is not consistent; for example, nitrate is $\mathrm{NO}_{3}{ }^{-}$while sulfate is $\mathrm{SO}_{4}{ }^{2-}$. This will be covered in more detail in the next module on nomenclature.

The nature of the attractive forces that hold atoms or ions together within a compound is the basis for classifying chemical bonding. When electrons are transferred and ions form, ionic bonds result. Ionic bonds are electrostatic forces of attraction, that is, the attractive forces experienced between objects of opposite electrical charge (in this case, cations and anions). When electrons are "shared" and molecules form, covalent bonds result. Covalent bonds are the attractive forces between the positively charged nuclei of the bonded atoms and one 
or more pairs of electrons that are located between the atoms. Compounds are classified as ionic or molecular (covalent) on the basis of the bonds present in them.

Ionic Compounds

When an element composed of atoms that readily lose electrons (a metal) reacts with an element composed of atoms that readily gain electrons (a nonmetal), a transfer of electrons usually occurs, producing ions. The compound formed by this transfer is stabilized by the electrostatic attractions (ionic bonds) between the ions of opposite charge present in the compound. For example, when each sodium atom in a sample of sodium metal (group 1) gives up one electron to form a sodium cation, $\mathrm{Na}^{+}$, and each chlorine atom in a sample of chlorine gas (group 17) accepts one electron to form a chloride anion, $\mathrm{Cl}^{-}$, the resulting compound, $\mathrm{NaCl}$, is composed of sodium ions and chloride ions in the ratio of one $\mathrm{Na}^{+}$ion for each $\mathrm{Cl}^{-}$ion. Similarly, each calcium atom (group 2) can give up two electrons and transfer one to each of two chlorine atoms to form $\mathrm{CaCl}_{2}$, which is composed of $\mathrm{Ca}^{2+}$ and $\mathrm{Cl}^{-}$ ions in the ratio of one $\mathrm{Ca}^{2+}$ ion to two $\mathrm{Cl}^{-}$ions.

A compound that contains ions and is held together by ionic bonds is called an ionic compound. The periodic table can help us recognize many of the compounds that are ionic: When a metal is combined with one or more nonmetals, the compound is usually ionic. This guideline works well for predicting ionic compound formation for most of the compounds typically encountered in an introductory chemistry course. However, it is not always true (for example, aluminum chloride, $\mathrm{AlCl}_{3}$, is not ionic). 
You can often recognize ionic compounds because of their properties. Ionic compounds are solids that typically melt at high temperatures and boil at even higher temperatures. For example, sodium chloride melts at 801 ${ }^{\circ} \mathrm{C}$ and boils at $1413^{\circ} \mathrm{C}$. (As a comparison, the molecular compound water melts at $0{ }^{\circ} \mathrm{C}$ and boils at $100{ }^{\circ} \mathrm{C}$.) In solid form, an ionic compound is not electrically conductive because its ions are unable to flow ("electricity" is the flow of charged particles). When molten, however, it can conduct electricity because its ions are able to move freely through the liquid ((Figure)).

Sodium chloride melts at $801{ }^{\circ} \mathrm{C}$ and conducts electricity when molten. (credit: modification of work by Mark Blaser and Matt Evans)

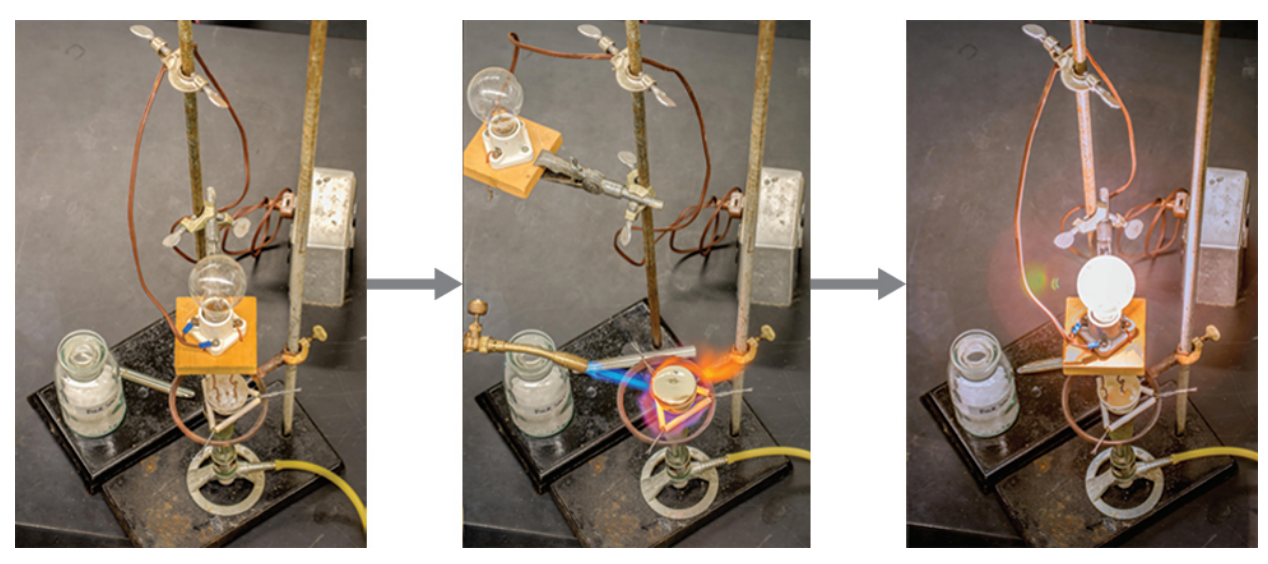

Watch this video to see a mixture of salts melt and conduct electricity.

In every ionic compound, the total number of positive charges of the cations equals the total number of negative charges of the anions. Thus, ionic compounds are electrically neutral overall, even though they contain positive and negative ions. We can use this observation 
to help us write the formula of an ionic compound. The formula of an ionic compound must have a ratio of ions such that the numbers of positive and negative charges are equal.

Predicting the Formula of an Ionic Compound The gemstone sapphire ((Figure)) is mostly a compound of aluminum and oxygen that contains aluminum cations, $\mathrm{Al}^{3+}$, and oxygen anions, $\mathrm{O}^{2-}$. What is the formula of this compound?

Although pure aluminum oxide is colorless, trace amounts of iron and titanium give blue sapphire its characteristic color. (credit: modification of work by Stanislav Doronenko)

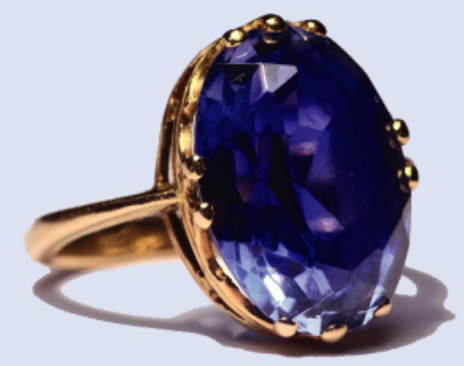

Solution Because the ionic compound must be electrically neutral, it must have the same number of positive and negative charges. Two aluminum ions, each with a charge of $3+$, would give us six positive charges, and three oxide ions, each with a charge of $2-$, would give us six negative charges. The formula would be $\mathrm{Al}_{2} \mathrm{O}_{3}$.

Check Your Learning Predict the formula of the ionic compound formed between the sodium cation, $\mathrm{Na}^{+}$, and the sulfide anion, $\mathrm{S}^{2-}$.

Answer:

$\mathrm{Na}_{2} \mathrm{~S}$

Many ionic compounds contain polyatomic ions ((Figure)) as the cation, the anion, or both. As with simple ionic 
compounds, these compounds must also be electrically neutral, so their formulas can be predicted by treating the polyatomic ions as discrete units. We use parentheses in a formula to indicate a group of atoms that behave as a unit. For example, the formula for calcium phosphate, one of the minerals in our bones, is $\mathrm{Ca}_{3}\left(\mathrm{PO}_{4}\right)_{2}$. This formula indicates that there are three calcium ions $\left(\mathrm{Ca}^{2+}\right)$ for every two phosphate $\left(\mathrm{PO}_{4}{ }^{3-}\right)$ groups. The $\mathrm{PO}_{4}{ }^{3-}$ groups are discrete units, each consisting of one phosphorus atom and four oxygen atoms, and having an overall charge of $3-$. The compound is electrically neutral, and its formula shows a total count of three $\mathrm{Ca}$, two $\mathrm{P}$, and eight $\mathrm{O}$ atoms.

Predicting the Formula of a Compound with a Polyatomic Anion Baking powder contains calcium dihydrogen phosphate, an ionic compound composed of the ions $\mathrm{Ca}^{2+}$ and $\mathrm{H}_{2} \mathrm{PO}_{4}{ }^{-}$. What is the formula of this compound?

Solution The positive and negative charges must balance, and this ionic compound must be electrically neutral. Thus, we must have two negative charges to balance the $2+$ charge of the calcium ion. This requires a ratio of one $\mathrm{Ca}^{2+}$ ion to two $\mathrm{H}_{2} \mathrm{PO}_{4}{ }^{-}$ions. We designate this by enclosing the formula for the dihydrogen phosphate ion in parentheses and adding a subscript 2 . The formula is $\mathrm{Ca}\left(\mathrm{H}_{2} \mathrm{PO}_{4}\right)_{2}$.

Check Your Learning Predict the formula of the ionic compound formed between the lithium ion and the peroxide ion, $\mathrm{O}_{2}{ }^{2-}$ (Hint: Use the periodic table to predict the sign and the charge on the lithium ion.)

Answer:

$\mathrm{Li}_{2} \mathrm{O}_{2}$

Because an ionic compound is not made up of single, 
discrete molecules, it may not be properly symbolized using a molecular formula. Instead, ionic compounds must be symbolized by a formula indicating the relative numbers of its constituent ions. For compounds containing only monatomic ions (such as $\mathrm{NaCl}$ ) and for many compounds containing polyatomic ions (such as $\mathrm{CaSO}_{4}$ ), these formulas are just the empirical formulas introduced earlier in this chapter. However, the formulas for some ionic compounds containing polyatomic ions are not empirical formulas. For example, the ionic compound sodium oxalate is comprised of $\mathrm{Na}^{+}$and $\mathrm{C}_{2} \mathrm{O}_{4}{ }^{2-}$ ions combined in a 2:1 ratio, and its formula is written as $\mathrm{Na}_{2} \mathrm{C}_{2} \mathrm{O}_{4}$. The subscripts in this formula are not the smallest-possible whole numbers, as each can be divided by 2 to yield the empirical formula, $\mathrm{NaCO}_{2}$. This is not the accepted formula for sodium oxalate, however, as it does not accurately represent the compound's polyatomic anion, $\mathrm{C}_{2} \mathrm{O}_{4}{ }^{2-}$.

\section{Molecular Compounds}

Many compounds do not contain ions but instead consist solely of discrete, neutral molecules. These molecular compounds (covalent compounds) result when atoms share, rather than transfer (gain or lose), electrons. Covalent bonding is an important and extensive concept in chemistry, and it will be treated in considerable detail in a later chapter of this text. We can often identify molecular compounds on the basis of their physical properties. Under normal conditions, molecular compounds often exist as gases, low-boiling liquids, and 
low-melting solids, although many important exceptions exist.

Whereas ionic compounds are usually formed when a metal and a nonmetal combine, covalent compounds are usually formed by a combination of nonmetals. Thus, the periodic table can help us recognize many of the compounds that are covalent. While we can use the positions of a compound's elements in the periodic table to predict whether it is ionic or covalent at this point in our study of chemistry, you should be aware that this is a very simplistic approach that does not account for a number of interesting exceptions. Shades of gray exist between ionic and molecular compounds, and you'll learn more about those later.

Predicting the Type of Bonding in Compounds Predict whether the following compounds are ionic or molecular:

(a) $\mathrm{Kl}$, the compound used as a source of iodine in table salt

(b) $\mathrm{H}_{2} \mathrm{O}_{2}$, the bleach and disinfectant hydrogen peroxide

(c) $\mathrm{CHCl}_{3}$, the anesthetic chloroform

(d) $\mathrm{Li}_{2} \mathrm{CO}_{3}$, a source of lithium in antidepressants

Solution (a) Potassium (group 1) is a metal, and iodine (group 17 ) is a nonmetal; $\mathrm{Kl}$ is predicted to be ionic.

(b) Hydrogen (group 1) is a nonmetal, and oxygen (group 16) is a nonmetal; $\mathrm{H}_{2} \mathrm{O}_{2}$ is predicted to be molecular.

(c) Carbon (group 14) is a nonmetal, hydrogen (group 1) is a nonmetal, and chlorine (group 17) is a nonmetal; $\mathrm{CHCl}_{3}$ is predicted to be molecular.

(d) Lithium (group 1) is a metal, and carbonate is a polyatomic ion; $\mathrm{Li}_{2} \mathrm{CO}_{3}$ is predicted to be ionic. 
Check Your Learning Using the periodic table, predict whether the following compounds are ionic or covalent:

(a) $\mathrm{SO}_{2}$

(b) $\mathrm{CaF}_{2}$

(c) $\mathrm{N}_{2} \mathrm{H}_{4}$

(d) $\mathrm{Al}_{2}\left(\mathrm{SO}_{4}\right)_{3}$

Answer:

(a) molecular; (b) ionic; (c) molecular; (d) ionic

Key Concepts and Summary

Metals (particularly those in groups 1 and 2) tend to lose the number of electrons that would leave them with the same number of electrons as in the preceding noble gas in the periodic table. By this means, a positively charged ion is formed. Similarly, nonmetals (especially those in groups 16 and 17, and, to a lesser extent, those in Group 15) can gain the number of electrons needed to provide atoms with the same number of electrons as in the next noble gas in the periodic table. Thus, nonmetals tend to form negative ions. Positively charged ions are called cations, and negatively charged ions are called anions. Ions can be either monatomic (containing only one atom) or polyatomic (containing more than one atom).

Compounds that contain ions are called ionic compounds. Ionic compounds generally form from metals and nonmetals. Compounds that do not contain ions, but instead consist of atoms bonded tightly together in molecules (uncharged groups of atoms that behave as a single unit), are called covalent compounds. Covalent compounds usually form from two nonmetals. 
Chemistry End of Chapter Exercises

Using the periodic table, predict whether the following chlorides are ionic or covalent: $\mathrm{KCl}, \mathrm{NCl}_{3}, \mathrm{ICl}, \mathrm{MgCl}_{2}, \mathrm{PCl}_{5}$, and $\mathrm{CCl}_{4}$.

Ionic: $\mathrm{KCl}, \mathrm{MgCl}_{2}$; Covalent: $\mathrm{NCl}_{3}, \mathrm{ICl}, \mathrm{PCl}_{5}, \mathrm{CCl}_{4}$

Using the periodic table, predict whether the following chlorides are ionic or covalent: $\mathrm{SiCl}_{4}, \mathrm{PCl}_{3}, \mathrm{CaCl}_{2}, \mathrm{CsCl}$, $\mathrm{CuCl}_{2}$, and $\mathrm{CrCl}_{3}$.

For each of the following compounds, state whether it is ionic or covalent. If it is ionic, write the symbols for the ions involved:

(a) $\mathrm{NF}_{3}$

(b) $\mathrm{BaO}$

(c) $\left(\mathrm{NH}_{4}\right)_{2} \mathrm{CO}_{3}$

(d) $\mathrm{Sr}\left(\mathrm{H}_{2} \mathrm{PO}_{4}\right)_{2}$

(e) $\mathrm{IBr}$

(f) $\mathrm{Na}_{2} \mathrm{O}$

(a) covalent; (b) ionic, $\mathrm{Ba}^{2+}, \mathrm{O}^{2-}$; (c) ionic, $\mathrm{NH}_{4}^{+}$, $\mathrm{CO}_{3}{ }^{2-}$; (d) ionic, $\mathrm{Sr}^{2+}, \mathrm{H}_{2} \mathrm{PO}_{4}^{-}$; (e) covalent; (f) ionic, $\mathrm{Na}^{+}, \mathrm{O}^{2-}$

For each of the following compounds, state whether it is ionic or covalent, and if it is ionic, write the symbols for the ions involved:

(a) $\mathrm{KClO}_{4}$

(b) $\mathrm{Mg}\left(\mathrm{C}_{2} \mathrm{H}_{3} \mathrm{O}_{2}\right)_{2}$

(c) $\mathrm{H}_{2} \mathrm{~S}$

(d) $\mathrm{Ag}_{2} \mathrm{~S}$ 
(e) $\mathrm{N}_{2} \mathrm{Cl}_{4}$

(f) $\mathrm{Co}\left(\mathrm{NO}_{3}\right)_{2}$

For each of the following pairs of ions, write the formula of the compound they will form:
(a) $\mathrm{Ca}^{2+}, \mathrm{S}^{2-}$
(b) $\mathrm{NH}_{4}^{+}, \mathrm{SO}_{4}^{2-}$
(c) $\mathrm{Al}^{3+}, \mathrm{Br}^{-}$
(d) $\mathrm{Na}^{+}, \mathrm{HPO}_{4}{ }^{2-}$
(e) $\mathrm{Mg}^{2+}, \mathrm{PO}_{4}{ }^{3-}$

(a) $\mathrm{CaS}$; (b) $\left(\mathrm{NH}_{4}\right)_{2} \mathrm{SO}_{4}$; (c) $\mathrm{AlBr}_{3}$; (d) $\mathrm{Na}_{2} \mathrm{HPO}_{4}$; (e) $\mathrm{Mg}_{3}$ $\left(\mathrm{PO}_{4}\right)_{2}$

For each of the following pairs of ions, write the formula of the compound they will form:
(a) $\mathrm{K}^{+}, \mathrm{O}^{2-}$
(b) $\mathrm{NH}_{4}+, \mathrm{PO}_{4}{ }^{3-}$
(c) $\mathrm{Al}^{3+}, \mathrm{O}^{2-}$
(d) $\mathrm{Na}^{+}, \mathrm{CO}_{3}{ }^{2-}$
(e) $\mathrm{Ba}^{2+}, \mathrm{PO}_{4}{ }^{3-}$

Glossary

covalent bond

attractive force between the nuclei of a molecule's atoms and pairs of electrons between the atoms 


\section{covalent compound}

(also, molecular compound) composed of molecules formed by atoms of two or more different elements

ionic bond

electrostatic forces of attraction between the oppositely charged ions of an ionic compound

ionic compound

compound composed of cations and anions combined in ratios, yielding an electrically neutral substance

molecular compound (also, covalent compound) composed of molecules formed by atoms of two or more different elements monatomic ion ion composed of a single atom oxyanion

polyatomic anion composed of a central atom bonded to oxygen atoms polyatomic ion ion composed of more than one atom 


\section{4.}

\section{CHEMICAL}

\section{NOMENCLATURE}

Learning Objectives

By the end of this module, you will be able to:

- Derive names for common types of inorganic compounds using a systematic approach

Nomenclature, a collection of rules for naming things, is important in science and in many other situations. This module describes an approach that is used to name simple ionic and molecular compounds, such as $\mathrm{NaCl}, \mathrm{CaCO}_{3}$, and $\mathrm{N}_{2} \mathrm{O}_{4}$. The simplest of these are binary compounds, those containing only two elements, but we will also consider how to name ionic compounds containing polyatomic ions, and one specific, very important class of compounds known as acids (subsequent chapters in this text will focus on these compounds in great detail). 
We will limit our attention here to inorganic compounds, compounds that are composed principally of elements other than carbon, and will follow the nomenclature guidelines proposed by IUPAC. The rules for organic compounds, in which carbon is the principle element, will be treated in a later chapter on organic chemistry.

Ionic Compounds

To name an inorganic compound, we need to consider the answers to several questions. First, is the compound ionic or molecular? If the compound is ionic, does the metal form ions of only one type (fixed charge) or more than one type (variable charge)? Are the ions monatomic or polyatomic? If the compound is molecular, does it contain hydrogen? If so, does it also contain oxygen? From the answers we derive, we place the compound in an appropriate category and then name it accordingly.

Compounds Containing Only Monatomic lons

The name of a binary compound containing monatomic ions consists of the name of the cation (the name of the metal) followed by the name of the anion (the name of the nonmetallic element with its ending replaced by the suffix -ide). Some examples are given in (Figure). 


\section{Names of Some Ionic Compounds}

$\mathrm{NaCl}$, sodium chloride $\quad \mathrm{Na}_{2} \mathrm{O}$, sodium oxide

$\mathrm{KBr}$, potassium bromide $\mathrm{CdS}$, cadmium sulfide

$\mathrm{CaI}_{2}$, calcium iodide $\quad \mathrm{Mg}_{3} \mathrm{~N}_{2}$, magnesium nitride

$\mathrm{CsF}$, cesium fluoride $\quad \mathrm{Ca}_{3} \mathrm{P}_{2}$, calcium phosphide

LiCl, lithium chloride $\quad \mathrm{Al}_{4} \mathrm{C}_{3}$, aluminum carbide

Compounds Containing Polyatomic Ions

Compounds containing polyatomic ions are named similarly to those containing only monatomic ions, i.e. by naming first the cation and then the anion. Examples are shown in (Figure).

\section{Names of Some Polyatomic Ionic Compounds}

$\mathrm{KC}_{2} \mathrm{H}_{3} \mathrm{O}_{2}$, potassium acetate $\quad \mathrm{NH}_{4} \mathrm{Cl}$, ammonium chloride

$\mathrm{NaHCO}_{3}$, sodium bicarbonate $\mathrm{CaSO}_{4}$, calcium sulfate

$\mathrm{Al}_{2}\left(\mathrm{CO}_{3}\right)_{3}$, aluminum carbonate $\mathrm{Mg}_{3}\left(\mathrm{PO}_{4}\right)_{2}$, magnesium phosphate

Ionic Compounds in Your Cabinets

Every day you encounter and use a large number of ionic compounds. Some of these compounds, where they are found, and what they are used for are listed in (Figure). Look at the label or ingredients list on the various products that you use during the next few days, and see if you run into any of those in this table, or find other ionic compounds that you could now name or write as a formula. 
Everyday Ionic Compounds

Ionic Compound

$\mathrm{NaCl}$, sodium chloride

KI, potassium iodide

$\mathrm{NaF}$, sodium fluoride

$\mathrm{NaHCO}_{3}$, sodium bicarbonate

$\mathrm{Na}_{2} \mathrm{CO}_{3}$, sodium carbonate

$\mathrm{NaOCl}$, sodium hypochlorite

$\mathrm{CaCO}_{3}$ calcium carbonate

$\mathrm{Mg}(\mathrm{OH})_{2}$, magnesium hydroxide

$\mathrm{Al}(\mathrm{OH})_{3}$, aluminum hydroxide

$\mathrm{NaOH}$, sodium hydroxide

$\mathrm{K}_{3} \mathrm{PO}_{4}$, potassium phosphate

$\mathrm{MgSO}_{4}$, magnesium sulfate

$\mathrm{Na}_{2} \mathrm{HPO}_{4}$, sodium hydrogen phosphate

$\mathrm{Na}_{2} \mathrm{SO}_{3}$, sodium sulfite
Use

ordinary table salt

added to "iodized" salt for thyroid health

ingredient in toothpaste

baking soda; used in cooking (and as antacid)

washing soda; used in cleaning agents

active ingredient in household bleach

ingredient in antacids

ingredient in antacids

ingredient in antacids

lye; used as drain cleaner

food additive (many purposes)

added to purified water

anti-caking agent; used in powdered products

preservative

\section{Compounds Containing a Metal Ion with a Variable}

\section{Charge}

Most of the transition metals and some main group metals can form two or more cations with different charges. Compounds of these metals with nonmetals are named with the same method as compounds in the first category, except the charge of the metal ion is specified by a Roman numeral in parentheses after the name of the metal. The 
charge of the metal ion is determined from the formula of the compound and the charge of the anion. For example, consider binary ionic compounds of iron and chlorine. Iron typically exhibits a charge of either $2+$ or $3+$ (see (Figure)), and the two corresponding compound formulas are $\mathrm{FeCl}_{2}$ and $\mathrm{FeCl}_{3}$. The simplest name, "iron chloride," will, in this case, be ambiguous, as it does not distinguish between these two compounds. In cases like this, the charge of the metal ion is included as a Roman numeral in parentheses immediately following the metal name. These two compounds are then unambiguously named iron(II) chloride and iron(III) chloride, respectively. Other examples are provided in (Figure).

$\begin{array}{ll}\text { Some Ionic Compounds with Variably Cha } \\ \text { Compound } & \text { Name } \\ \mathrm{FeCl}_{2} & \text { iron(II) chloride } \\ \mathrm{FeCl}_{3} & \text { iron(III) chloride } \\ \mathrm{Hg}_{2} \mathrm{O} & \text { mercury(I) oxide } \\ \mathrm{HgO} & \text { mercury(II) oxide } \\ \mathrm{SnF}_{2} & \text { tin(II) fluoride } \\ \mathrm{SnF}_{4} & \text { tin(IV) fluoride }\end{array}$

Out-of-date nomenclature used the suffixes -ic and -ous to designate metals with higher and lower charges, respectively: Iron(III) chloride, $\mathrm{FeCl}_{3}$, was previously called ferric chloride, and iron(II) chloride, $\mathrm{FeCl}_{2}$, was known as ferrous chloride. Though this naming convention has been largely abandoned by the scientific community, it remains in use by some segments of industry. For example, you may see the words stannous 
fluoride on a tube of toothpaste. This represents the formula $\mathrm{SnF}_{2}$, which is more properly named tin(II) fluoride. The other fluoride of tin is $\mathrm{SnF}_{4}$, which was previously called stannic fluoride but is now named tin(IV) fluoride.

Ionic Hydrates

Ionic compounds that contain water molecules as integral components of their crystals are called hydrates. The name for an ionic hydrate is derived by adding a term to the name for the anhydrous (meaning "not hydrated") compound that indicates the number of water molecules associated with each formula unit of the compound. The added word begins with a Greek prefix denoting the number of water molecules (see (Figure)) and ends with "hydrate." For example, the anhydrous compound copper(II) sulfate also exists as a hydrate containing five water molecules and named copper(II) sulfate pentahydrate. Washing soda is the common name for a hydrate of sodium carbonate containing 10 water molecules; the systematic name is sodium carbonate decahydrate.

Formulas for ionic hydrates are written by appending a vertically centered dot, a coefficient representing the number of water molecules, and the formula for water. The two examples mentioned in the previous paragraph are represented by the formulas copper(II) sulfate pentahydrate $\mathrm{CuSO}_{4} \bullet 5 \mathrm{H}_{2} \mathrm{O}$ sodium carbonate decahydrate $\mathrm{Na}_{2} \mathrm{CO}_{3} \bullet 10 \mathrm{H}_{2} \mathrm{O}$ 


\section{Nomenclature Prefixes}

$\begin{array}{llll}\text { Number } & \text { Prefix } & \text { Number } & \text { Prefix } \\ 1 \text { (sometimes omitted) } & \text { mono- } & 6 & \text { hexa- } \\ 2 & \text { di- } & 7 & \text { hepta- } \\ 3 & \text { tri- } & 8 & \text { octa- } \\ 4 & \text { tetra- } & 9 & \text { nona- } \\ 5 & \text { penta- } & 10 & \text { deca- }\end{array}$

Naming lonic Compounds Name the following ionic compounds

(a) $\mathrm{Fe}_{2} \mathrm{~S}_{3}$

(b) CuSe

(c) GaN

(d) $\mathrm{MgSO}_{4} \cdot 7 \mathrm{H}_{2} \mathrm{O}$

(e) $\mathrm{Ti}_{2}\left(\mathrm{SO}_{4}\right)_{3}$

Solution The anions in these compounds have a fixed negative charge $\left(\mathrm{S}^{2-}, \mathrm{Se}^{2-}, \mathrm{N}^{3-}, \mathrm{Cl}^{-}\right.$, and $\mathrm{SO}_{4}{ }^{2-}$, and the compounds must be neutral. Because the total number of positive charges in each compound must equal the total number of negative charges, the positive ions must be $\mathrm{Fe}^{3+}$, $\mathrm{Cu}^{2+}, \mathrm{Ca}^{3+}, \mathrm{Cr}^{3+}$, and $\mathrm{Ti}^{3+}$. These charges are used in the names of the metal ions:
(a) iron(III) sulfide
(b) copper(II) selenide
(c) gallium(III) nitride
(d) magnesium sulfate heptahydrate
(e) titanium(III) sulfate 
Check Your Learning Write the formulas of the following ionic compounds:

(a) chromium(III) phosphide

(b) mercury(II) sulfide

(c) manganese(II) phosphate

(d) copper(I) oxide

(e) iron(III) chloride dihydrate

Answer:

(a) $\mathrm{CrP}$; (b) $\mathrm{HgS}$; (c) $\mathrm{Mn}_{3}\left(\mathrm{PO}_{4}\right)_{2}$; (d) $\mathrm{Cu}_{2} \mathrm{O}$; (e) $\mathrm{FeCl}_{3} \cdot 2 \mathrm{H}_{2} \mathrm{O}$

Erin Brokovich and Chromium Contamination

In the early 1990s, legal file clerk Erin Brockovich ((Figure)) discovered a high rate of serious illnesses in the small town of Hinckley, California. Her investigation eventually linked the illnesses to groundwater contaminated by $\mathrm{Cr}(\mathrm{VI})$ used by Pacific Gas \& Electric (PG\&E) to fight corrosion in a nearby natural gas pipeline. As dramatized in the film Erin Brokovich (for which Julia Roberts won an Oscar), Erin and lawyer Edward Masry sued PG\&E for contaminating the water near Hinckley in 1993. The settlement they won in 1996-?333 million-was the largest amount ever awarded for a directaction lawsuit in the US at that time.

(a) Erin Brockovich found that $\mathrm{Cr}(\mathrm{VI})$, used by PG\&E, had contaminated the Hinckley, California, water supply. (b) $\mathrm{The} \mathrm{Cr}(\mathrm{VI})$ ion is often present in water as the polyatomic ions chromate, $\mathrm{CrO}_{4}{ }^{2-}$ (left), and dichromate, $\mathrm{Cr}_{2} \mathrm{O}_{7}{ }^{2-}$ (right). 


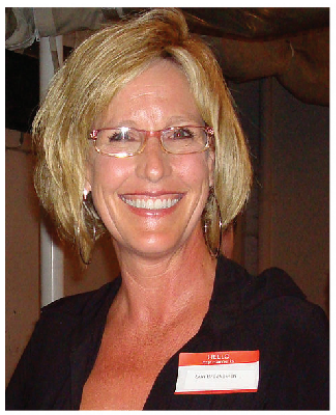

(a)

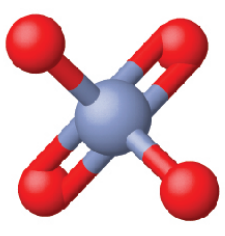

(b)

Chromium compounds are widely used in industry, such as for chrome plating, in dye-making, as preservatives, and to prevent corrosion in cooling tower water, as occurred near Hinckley. In the environment, chromium exists primarily in either the $\mathrm{Cr}(\mathrm{III})$ or $\mathrm{Cr}(\mathrm{VI})$ forms. $\mathrm{Cr}$ (III), an ingredient of many vitamin and nutritional supplements, forms compounds that are not very soluble in water, and it has low toxicity. But $\mathrm{Cr}(\mathrm{VI})$ is much more toxic and forms compounds that are reasonably soluble in water. Exposure to small amounts of $\mathrm{Cr}(\mathrm{VI})$ can lead to damage of the respiratory, gastrointestinal, and immune systems, as well as the kidneys, liver, blood, and skin.

Despite cleanup efforts, $\mathrm{Cr}(\mathrm{VI})$ groundwater contamination remains a problem in Hinckley and other locations across the globe. A 2010 study by the Environmental Working Group found that of 35 US cities tested, 31 had higher levels of $\mathrm{Cr}(\mathrm{VI})$ in their tap water than the public health goal of 0.02 parts per billion set by the California Environmental Protection Agency.

Molecular (Covalent) Compounds

The bonding characteristics of inorganic molecular compounds are different from ionic compounds, and they 
are named using a different system as well. The charges of cations and anions dictate their ratios in ionic compounds, so specifying the names of the ions provides sufficient information to determine chemical formulas. However, because covalent bonding allows for significant variation in the combination ratios of the atoms in a molecule, the names for molecular compounds must explicitly identify these ratios.

\section{Compounds Composed of Two Elements}

When two nonmetallic elements form a molecular compound, several combination ratios are often possible. For example, carbon and oxygen can form the compounds $\mathrm{CO}$ and $\mathrm{CO}_{2}$. Since these are different substances with different properties, they cannot both have the same name (they cannot both be called carbon oxide). To deal with this situation, we use a naming method that is somewhat similar to that used for ionic compounds, but with added prefixes to specify the numbers of atoms of each element. The name of the more metallic element (the one farther to the left and/or bottom of the periodic table) is first, followed by the name of the more nonmetallic element (the one farther to the right and/or top) with its ending changed to the suffix -ide. The numbers of atoms of each element are designated by the Greek prefixes shown in (Figure).

When only one atom of the first element is present, the prefix mono- is usually deleted from that part. Thus, $\mathrm{CO}$ is named carbon monoxide, and $\mathrm{CO}_{2}$ is called carbon dioxide. When two vowels are adjacent, the $a$ in the Greek 
prefix is usually dropped. Some other examples are shown in (Figure).

$\begin{array}{llll}\begin{array}{l}\text { Names of Some Molecular Compounds Composed of Two } \\ \text { Elements }\end{array} \\ \text { Compound } & \text { Name } & \text { Compound } & \text { Name } \\ \mathrm{SO}_{2} & \text { sulfur dioxide } & \mathrm{BCl}_{3} & \begin{array}{l}\text { boron trichloride } \\ \mathrm{SO}_{3}\end{array} \\ \text { sulfur trioxide } & \mathrm{SF}_{6} & \begin{array}{l}\text { sulfur hexafluoride } \\ \text { phosphorus } \\ \text { pentafluoride }\end{array} \\ \mathrm{NO}_{2} & \text { nitrogen dioxide } & \mathrm{PF}_{5} & \begin{array}{l}\text { tetraphosphorus } \\ \text { decaoxide }\end{array} \\ \mathrm{N}_{2} \mathrm{O}_{4} & \begin{array}{l}\text { dinitrogen } \\ \text { tetroxide }\end{array} & \mathrm{P}_{4} \mathrm{O}_{10} & \text { iodine heptafluoride } \\ \mathrm{N}_{2} \mathrm{O}_{5} & \begin{array}{l}\text { dinitrogen } \\ \text { pentoxide }\end{array} & \mathrm{IF}_{7} & \end{array}$

There are a few common names that you will encounter as you continue your study of chemistry. For example, although $\mathrm{NO}$ is often called nitric oxide, its proper name is nitrogen monoxide. Similarly, $\mathrm{N}_{2} \mathrm{O}$ is known as nitrous oxide even though our rules would specify the name dinitrogen monoxide. (And $\mathrm{H}_{2} \mathrm{O}$ is usually called water, not dihydrogen monoxide.) You should commit to memory the common names of compounds as you encounter them.

Naming Covalent Compounds Name the following covalent compounds:
(a) $\mathrm{SF}_{6}$
(b) $\mathrm{N}_{2} \mathrm{O}_{3}$
(c) $\mathrm{Cl}_{2} \mathrm{O}_{7}$
(d) $\mathrm{P}_{4} \mathrm{O}_{6}$ 
Solution Because these compounds consist solely of nonmetals, we use prefixes to designate the number of atoms of each element:

(a) sulfur hexafluoride

(b) dinitrogen trioxide

(c) dichlorine heptoxide

(d) tetraphosphorus hexoxide

Check Your Learning Write the formulas for the following compounds:

(a) phosphorus pentachloride

(b) dinitrogen monoxide

(c) iodine heptafluoride

(d) carbon tetrachloride

Answer:

(a) $\mathrm{PCl}_{5}$; (b) $\mathrm{N}_{2} \mathrm{O}$; (c) $\mathrm{IF}_{7}$; (d) $\mathrm{CCl}_{4}$

The following website provides practice with naming chemical compounds and writing chemical formulas. You can choose binary, polyatomic, and variable charge ionic compounds, as well as molecular compounds.

Binary Acids

Some compounds containing hydrogen are members of an important class of substances known as acids. The chemistry of these compounds is explored in more detail in later chapters of this text, but for now, it will suffice to note that many acids release hydrogen ions, $\mathrm{H}^{+}$, when dissolved in water. To denote this distinct chemical property, a mixture of water with an acid is given a name 
derived from the compound's name. If the compound is a binary acid (comprised of hydrogen and one other nonmetallic element):

1. The word "hydrogen" is changed to the prefix hydro-

2. The other nonmetallic element name is modified by adding the suffix $-i c$

3. The word "acid" is added as a second word

For example, when the gas $\mathrm{HCl}$ (hydrogen chloride) is dissolved in water, the solution is called hydrochloric acid. Several other examples of this nomenclature are shown in (Figure).

\author{
Names of Some Simple Acids \\ Name of Gas \\ $\mathrm{HF}(g)$, hydrogen fluoride $\quad \mathrm{HF}(a q)$, hydrofluoric acid \\ $\mathrm{HCl}(g)$, hydrogen chloride $\mathrm{HCl}(a q)$, hydrochloric acid \\ $\operatorname{HBr}(g)$, hydrogen bromide $\operatorname{HBr}(a q)$, hydrobromic acid \\ HI $(g)$, hydrogen iodide $\quad \mathrm{HI}(a q)$, hydroiodic acid \\ $\mathrm{H}_{2} \mathrm{~S}(g)$, hydrogen sulfide $\quad \mathrm{H}_{2} \mathrm{~S}(a q)$, hydrosulfuric acid
}

\section{Oxyacids}

Many compounds containing three or more elements (such as organic compounds or coordination compounds) are subject to specialized nomenclature rules that you will learn later. However, we will briefly discuss the important compounds known as oxyacids, compounds that contain hydrogen, oxygen, and at least one other element, and are 
bonded in such a way as to impart acidic properties to the compound (you will learn the details of this in a later chapter). Typical oxyacids consist of hydrogen combined with a polyatomic, oxygen-containing ion. To name oxyacids:

1. Omit "hydrogen"

2. Start with the root name of the anion

3. Replace -ate with -ic, or -ite with -ous

4. Add "acid"

For example, consider $\mathrm{H}_{2} \mathrm{CO}_{3}$ (which you might be tempted to call "hydrogen carbonate"). To name this correctly, "hydrogen" is omitted; the -ate of carbonate is replace with $-i c$; and acid is added-so its name is carbonic acid. Other examples are given in (Figure). There are some exceptions to the general naming method (e.g., $\mathrm{H}_{2} \mathrm{SO}_{4}$ is called sulfuric acid, not sulfic acid, and $\mathrm{H}_{2} \mathrm{SO}_{3}$ is sulfurous, not sulfous, acid).

\section{Names of Common Oxyacids}

$\begin{array}{lll}\text { Formula } & \text { Anion Name } & \text { Acid Name } \\ \mathrm{HC}_{2} \mathrm{H}_{3} \mathrm{O}_{2} & \text { acetate } & \text { acetic acid } \\ \mathrm{HNO}_{3} & \text { nitrate } & \text { nitric acid } \\ \mathrm{HNO}_{2} & \text { nitrite } & \text { nitrous acid } \\ \mathrm{HClO}_{4} & \text { perchlorate } & \text { perchloric acid } \\ \mathrm{H}_{2} \mathrm{CO}_{3} & \text { carbonate } & \text { carbonic acid } \\ \mathrm{H}_{2} \mathrm{SO}_{4} & \text { sulfate } & \text { sulfuric acid } \\ \mathrm{H}_{2} \mathrm{SO}_{3} & \text { sulfite } & \text { sulfurous acid } \\ \mathrm{H}_{3} \mathrm{PO}_{4} & \text { phosphate } & \text { phosphoric acid }\end{array}$


Key Concepts and Summary

Chemists use nomenclature rules to clearly name compounds. Ionic and molecular compounds are named using somewhat-different methods. Binary ionic compounds typically consist of a metal and a nonmetal. The name of the metal is written first, followed by the name of the nonmetal with its ending changed to -ide. For example, $\mathrm{K}_{2} \mathrm{O}$ is called potassium oxide. If the metal can form ions with different charges, a Roman numeral in parentheses follows the name of the metal to specify its charge. Thus, $\mathrm{FeCl}_{2}$ is iron(II) chloride and $\mathrm{FeCl}_{3}$ is iron(III) chloride. Some compounds contain polyatomic ions; the names of common polyatomic ions should be memorized. Molecular compounds can form compounds with different ratios of their elements, so prefixes are used to specify the numbers of atoms of each element in a molecule of the compound. Examples include $\mathrm{SF}_{6}$, sulfur hexafluoride, and $\mathrm{N}_{2} \mathrm{O}_{4}$, dinitrogen tetroxide. Acids are an important class of compounds containing hydrogen and having special nomenclature rules. Binary acids are named using the prefix hydro-, changing the -ide suffix to $-i c$, and adding "acid;" $\mathrm{HCl}$ is hydrochloric acid. Oxyacids are named by changing the ending of the anion (-ate to -ic and -ite to -ous), and adding “acid;" $\mathrm{H}_{2} \mathrm{CO}_{3}$ is carbonic acid.

Chemistry End of Chapter Exercises

Name the following compounds:

(a) $\mathrm{CsCl}$

(b) $\mathrm{BaO}$ 
(c) $\mathrm{K}_{2} \mathrm{~S}$

(d) $\mathrm{BeCl}_{2}$

(e) $\mathrm{HBr}$

(f) $\mathrm{AlF}_{3}$

(a) cesium chloride; (b) barium oxide; (c) potassium sulfide; (d) beryllium chloride; (e) hydrogen bromide; (f) aluminum fluoride

Name the following compounds:
(a) $\mathrm{NaF}$
(b) $\mathrm{Rb}_{2} \mathrm{O}$
(c) $\mathrm{BCl}_{3}$
(d) $\mathrm{H}_{2} \mathrm{Se}$
(e) $\mathrm{P}_{4} \mathrm{O}_{6}$
(f) $\mathrm{ICl}_{3}$

Write the formulas of the following compounds:
(a) rubidium bromide
(b) magnesium selenide
(c) sodium oxide
(d) calcium chloride
(e) hydrogen fluoride
(f) gallium phosphide
(g) aluminum bromide
(h) ammonium sulfate

(a) $\mathrm{RbBr}$; (b) $\mathrm{MgSe}$; (c) $\mathrm{Na}_{2} \mathrm{O}$; (d) $\mathrm{CaCl}_{2}$; (e) $\mathrm{HF}$; (f) $\mathrm{GaP}$; (g) $\mathrm{AlBr}_{3} ;(\mathrm{h})\left(\mathrm{NH}_{4}\right)_{2} \mathrm{SO}_{4}$

Write the formulas of the following compounds:

(a) lithium carbonate

(b) sodium perchlorate 

(c) barium hydroxide
(d) ammonium carbonate
(e) sulfuric acid
(f) calcium acetate
(g) magnesium phosphate
(h) sodium sulfite

Write the formulas of the following compounds:

(a) chlorine dioxide

(b) dinitrogen tetraoxide

(c) potassium phosphide

(d) silver(I) sulfide

(e) aluminum fluoride trihydrate

(f) silicon dioxide

(a) $\mathrm{ClO}_{2}$; (b) $\mathrm{N}_{2} \mathrm{O}_{4}$; (c) $\mathrm{K}_{3} \mathrm{P}$; (d) $\mathrm{Ag}_{2} \mathrm{~S}$; (e) $\mathrm{AIF}_{3} \cdot 3 \mathrm{H}_{2} \mathrm{O}$; (f) $\mathrm{SiO}_{2}$

Write the formulas of the following compounds:
(a) barium chloride
(b) magnesium nitride
(c) sulfur dioxide
(d) nitrogen trichloride
(e) dinitrogen trioxide
(f) tin(IV) chloride

Each of the following compounds contains a metal that can exhibit more than one ionic charge. Name these compounds:
(a) $\mathrm{Cr}_{2} \mathrm{O}_{3}$
(b) $\mathrm{FeCl}_{2}$
(c) $\mathrm{CrO}_{3}$
(d) $\mathrm{TiCl}_{4}$ 
(e) $\mathrm{CoCl}_{2} \cdot 6 \mathrm{H}_{2} \mathrm{O}$

(f) $\mathrm{MoS}_{2}$

(a) chromium(III) oxide; (b) iron(II) chloride; (c) chromium(VI) oxide; (d) titanium(IV) chloride; (e) cobalt(II) chloride hexahydrate; (f) molybdenum(IV) sulfide

Each of the following compounds contains a metal that can exhibit more than one ionic charge. Name these compounds:

(a) $\mathrm{NiCO}_{3}$

(b) $\mathrm{MoO}_{3}$

(c) $\mathrm{Co}\left(\mathrm{NO}_{3}\right)_{2}$

(d) $\mathrm{V}_{2} \mathrm{O}_{5}$

(e) $\mathrm{MnO}_{2}$

(f) $\mathrm{Fe}_{2} \mathrm{O}_{3}$

The following ionic compounds are found in common household products. Write the formulas for each compound:

(a) potassium phosphate

(b) copper(II) sulfate

(c) calcium chloride

(d) titanium(IV) oxide

(e) ammonium nitrate

(f) sodium bisulfate (the common name for sodium hydrogen sulfate)

(a) $\mathrm{K}_{3} \mathrm{PO}_{4}$; (b) $\mathrm{CuSO}_{4}$; (c) $\mathrm{CaCl}_{2}$; (d) $\mathrm{TiO}_{2}$; (e) $\mathrm{NH}_{4} \mathrm{NO}_{3}$; (f) $\mathrm{NaHSO}_{4}$ 
The following ionic compounds are found in common household products. Name each of the compounds:
(a) $\mathrm{Ca}\left(\mathrm{H}_{2} \mathrm{PO}_{4}\right)_{2}$
(b) $\mathrm{FeSO}_{4}$
(c) $\mathrm{CaCO}_{3}$
(d) $\mathrm{MgO}$
(e) $\mathrm{NaNO}_{2}$
(f) $\mathrm{KI}$

What are the IUPAC names of the following compounds?

(a) manganese dioxide

(b) mercurous chloride $\left(\mathrm{Hg}_{2} \mathrm{Cl}_{2}\right)$

(c) ferric nitrate $\left[\mathrm{Fe}\left(\mathrm{NO}_{3}\right)_{3}\right]$

(d) titanium tetrachloride

(e) cupric bromide $\left(\mathrm{CuBr}_{2}\right)$

(a) manganese(IV) oxide; (b) mercury(I) chloride; (c) iron(III) nitrate; (d) titanium(IV) chloride; (e) copper(II) bromide

Glossary

binary acid

compound that contains hydrogen and one other element, bonded in a way that imparts acidic properties to the compound (ability to release $\mathrm{H}^{+}$ions when dissolved in water)

binary compound

compound containing two different 
elements.

nomenclature

system of rules for naming objects of interest

oxyacid

compound that contains hydrogen, oxygen, and one other element, bonded in a way that imparts acidic properties to the compound (ability to release $\mathrm{H}^{+}$ions when dissolved in water) 


\section{PART III \\ COMPOSITION OF \\ SUBSTANCES AND \\ SOLUTIONS}





\section{5. \\ INTRODUCTION}

The water in a swimming pool is a complex mixture of substances whose relative amounts must be carefully maintained to ensure the health and comfort of people using the pool. (credit: modification of work by Vic Brincat)

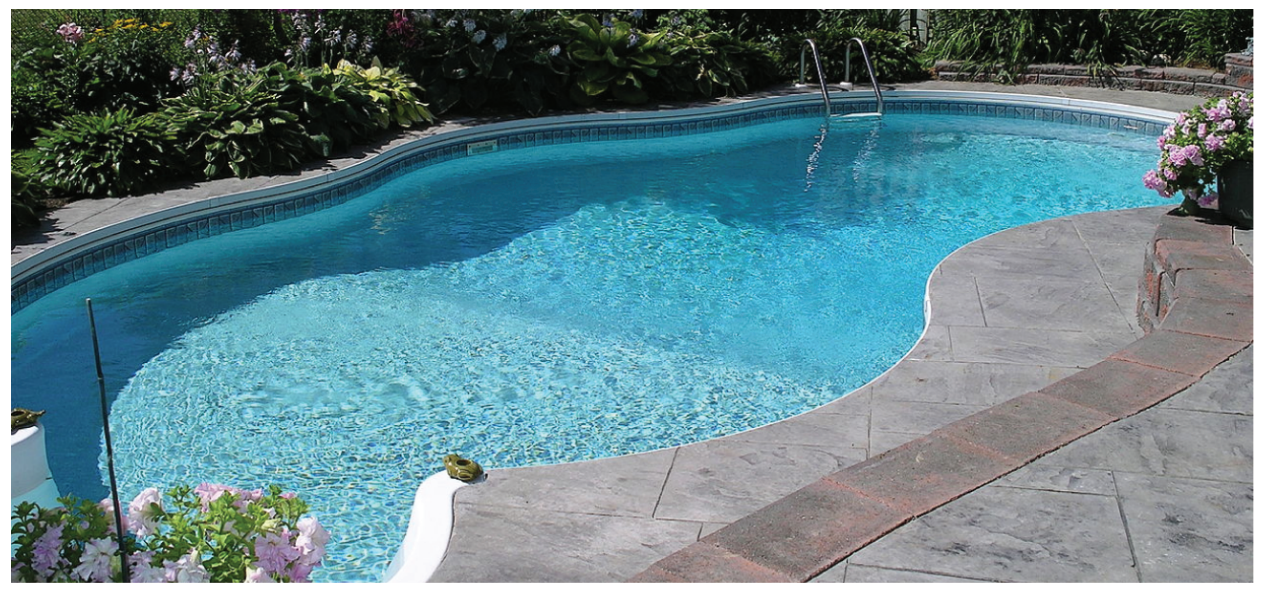

Swimming pools have long been a popular means of recreation, exercise, and physical therapy. Since it is impractical to refill large pools with fresh water on a frequent basis, pool water is regularly treated with 
chemicals to prevent the growth of harmful bacteria and algae. Proper pool maintenance requires regular additions of various chemical compounds in carefully measured amounts. For example, the relative amount of calcium ion, $\mathrm{Ca}^{2+}$, in the water should be maintained within certain limits to prevent eye irritation and avoid damage to the pool bed and plumbing. To maintain proper calcium levels, calcium cations are added to the water in the form of an ionic compound that also contains anions; thus, it is necessary to know both the relative amount of $\mathrm{Ca}^{2+}$ in the compound and the volume of water in the pool in order to achieve the proper calcium level. Quantitative aspects of the composition of substances (such as the calciumcontaining compound) and mixtures (such as the pool water) are the subject of this chapter. 


\section{6.}

\section{FORMULA MASS AND THE MOLE CONCEPT}

Learning Objectives

By the end of this section, you will be able to:

- Calculate formula masses for covalent and ionic compounds

- $\quad$ Define the amount unit mole and the related quantity Avogadro's number Explain the relation between mass, moles, and numbers of atoms or molecules, and perform calculations deriving these quantities from one another

Many argue that modern chemical science began when scientists started exploring the quantitative as well as the qualitative aspects of chemistry. For example, Dalton's atomic theory was an attempt to explain the results of measurements that allowed him to calculate the relative 
masses of elements combined in various compounds. Understanding the relationship between the masses of atoms and the chemical formulas of compounds allows us to quantitatively describe the composition of substances.

Formula Mass

An earlier chapter of this text described the development of the atomic mass unit, the concept of average atomic masses, and the use of chemical formulas to represent the elemental makeup of substances. These ideas can be extended to calculate the formula mass of a substance by summing the average atomic masses of all the atoms represented in the substance's formula.

\section{Formula Mass for Covalent Substances}

For covalent substances, the formula represents the numbers and types of atoms composing a single molecule of the substance; therefore, the formula mass may be correctly referred to as a molecular mass. Consider chloroform $\left(\mathrm{CHCl}_{3}\right)$, a covalent compound once used as a surgical anesthetic and now primarily used in the production of tetrafluoroethylene, the building block for the "anti-stick" polymer, Teflon. The molecular formula of chloroform indicates that a single molecule contains one carbon atom, one hydrogen atom, and three chlorine atoms. The average molecular mass of a chloroform molecule is therefore equal to the sum of the average atomic masses of these atoms. (Figure) outlines the calculations used to derive the molecular mass of chloroform, which is $119.37 \mathrm{amu}$.

The average mass of a chloroform molecule, $\mathrm{CHCl}_{3}$, is 
$119.37 \mathrm{amu}$, which is the sum of the average atomic masses of each of its constituent atoms. The model shows the molecular structure of chloroform.

\begin{tabular}{|c|c|c|c|c|c|}
\hline Element & Quantity & $\begin{array}{c}\text { Average atomic } \\
\text { mass (amu) }\end{array}$ & $\begin{array}{c}\text { Subtotal } \\
(\mathbf{a m u})\end{array}$ \\
\hline $\mathrm{C}$ & 1 & $\times$ & 12.01 & $=$ & 12.01 \\
\hline $\mathrm{H}$ & 1 & $\times$ & 1.008 & $=$ & 1.008 \\
\hline $\mathrm{Cl}$ & 3 & $\times$ & 35.45 & $=$ & 106.35 \\
\hline \multicolumn{3}{|c|}{ Molecular mass } & 119.37 \\
\hline
\end{tabular}

Likewise, the molecular mass of an aspirin molecule, $\mathrm{C}_{9} \mathrm{H}_{8} \mathrm{O}_{4}$, is the sum of the atomic masses of nine carbon atoms, eight hydrogen atoms, and four oxygen atoms, which amounts to $180.15 \mathrm{amu}$ ((Figure)).

The average mass of an aspirin molecule is $180.15 \mathrm{amu}$. The model shows the molecular structure of aspirin, $\mathrm{C}_{9} \mathrm{H}_{8} \mathrm{O}_{4}$.

\begin{tabular}{|c|c|c|c|c|c|}
\hline Element & Quantity & $\begin{array}{c}\text { Average atomic } \\
\text { mass (amu) }\end{array}$ & $\begin{array}{c}\text { Subtotal } \\
(\mathbf{a m u})\end{array}$ \\
\hline $\mathrm{C}$ & 9 & $\times$ & 12.01 & $=$ & 108.09 \\
\hline $\mathrm{H}$ & 8 & $\times$ & 1.008 & $=$ & 8.064 \\
\hline $\mathrm{O}$ & 4 & $\times$ & 16.00 & $=$ & 64.00 \\
\hline \multicolumn{3}{|c|}{ Molecular mass } & 180.15 \\
\hline
\end{tabular}

Computing Molecular Mass for a Covalent Compound Ibuprofen, $\mathrm{C}_{13} \mathrm{H}_{18} \mathrm{O}_{2}$, is a covalent compound and the active ingredient in several popular nonprescription pain medications, such as Advil and Motrin. What is the molecular mass (amu) for this compound?

Solution Molecules of this compound are composed of 13 carbon atoms, 18 hydrogen atoms, and 2 oxygen atoms. 
Following the approach described above, the average molecular mass for this compound is therefore:

\begin{tabular}{|c|c|c|c|c|c|}
\hline Element & Quantity & $\begin{array}{c}\text { Average atomic } \\
\text { mass (amu) }\end{array}$ & $\begin{array}{c}\text { Subtotal } \\
\text { (amu) }\end{array}$ \\
\hline $\mathrm{C}$ & 13 & $\times$ & 12.01 & $=$ & 156.13 \\
\hline $\mathrm{H}$ & 18 & $\times$ & 1.008 & $=$ & 18.144 \\
\hline $\mathrm{O}$ & 2 & $\times$ & 16.00 & $=$ & 32.00 \\
\hline \multicolumn{5}{|c|}{ Molecular mass } & 206.27 \\
\hline
\end{tabular}

Check Your Learning Acetaminophen, $\mathrm{C}_{8} \mathrm{H}_{9} \mathrm{NO}_{2}$, is a covalent compound and the active ingredient in several popular nonprescription pain medications, such as Tylenol. What is the molecular mass (amu) for this compound?

Answer:

$151.16 \mathrm{amu}$

Formula Mass for lonic Compounds

Ionic compounds are composed of discrete cations and anions combined in ratios to yield electrically neutral bulk matter. The formula mass for an ionic compound is calculated in the same way as the formula mass for covalent compounds: by summing the average atomic masses of all the atoms in the compound's formula. Keep in mind, however, that the formula for an ionic compound does not represent the composition of a discrete molecule, so it may not correctly be referred to as the "molecular mass."

As an example, consider sodium chloride, $\mathrm{NaCl}$, the chemical name for common table salt. Sodium chloride is an ionic compound composed of sodium cations, $\mathrm{Na}^{+}$, and chloride anions, $\mathrm{Cl}^{-}$, combined in a 1:1 ratio. The formula mass for this compound is computed as 58.44 amu (see (Figure)). 
Table salt, $\mathrm{NaCl}$, contains an array of sodium and chloride ions combined in a $1: 1$ ratio. Its formula mass is 58.44 amu.

\begin{tabular}{|c|c|c|c|c|c|}
\hline Element & Quantity & & $\begin{array}{c}\text { Average atomic } \\
\text { mass (amu) }\end{array}$ & Subtotal \\
\hline $\mathrm{Na}$ & 1 & $\times$ & 22.99 & $=$ & 22.99 \\
\hline $\mathrm{Cl}$ & 1 & $\times$ & 35.45 & $=$ & 35.45 \\
\hline \multicolumn{5}{r}{ Formula mass } & 58.44 \\
\hline
\end{tabular}

Note that the average masses of neutral sodium and chlorine atoms were used in this computation, rather than the masses for sodium cations and chlorine anions. This approach is perfectly acceptable when computing the formula mass of an ionic compound. Even though a sodium cation has a slightly smaller mass than a sodium atom (since it is missing an electron), this difference will be offset by the fact that a chloride anion is slightly more massive than a chloride atom (due to the extra electron). Moreover, the mass of an electron is negligibly small with respect to the mass of a typical atom. Even when calculating the mass of an isolated ion, the missing or additional electrons can generally be ignored, since their contribution to the overall mass is negligible, reflected only in the nonsignificant digits that will be lost when the computed mass is properly rounded. The few exceptions to this guideline are very light ions derived from elements with precisely known atomic masses.

Computing Formula Mass for an lonic Compound Aluminum sulfate, $\mathrm{Al}_{2}\left(\mathrm{SO}_{4}\right)_{3}$, is an ionic compound that is used in the manufacture of paper and in various water purification processes. What is the formula mass (amu) of this compound? 
Solution The formula for this compound indicates it contains $\mathrm{Al}^{3+}$ and $\mathrm{SO}_{4}{ }^{2-}$ ions combined in a 2:3 ratio. For purposes of computing a formula mass, it is helpful to rewrite the formula in the simpler format, $\mathrm{Al}_{2} \mathrm{~S}_{3} \mathrm{O}_{12}$. Following the approach outlined above, the formula mass for this compound is calculated as follows:

\begin{tabular}{|c|c|c|c|c|c|}
\hline Element & Quantity & $\begin{array}{c}\text { Average atomic } \\
\text { mass (amu) }\end{array}$ & $\begin{array}{c}\text { Subtotal } \\
(\mathbf{a m u})\end{array}$ \\
\hline $\mathrm{Al}$ & 2 & $\times$ & 26.98 & $=$ & 53.96 \\
\hline $\mathrm{S}$ & 3 & $\times$ & 32.06 & $=$ & 96.18 \\
\hline $\mathrm{O}$ & 12 & $\times$ & 16.00 & $=$ & 192.00 \\
\hline & \multicolumn{3}{c}{ Molecular mass } & 342.14 \\
\hline
\end{tabular}

Check Your Learning Calcium phosphate, $\mathrm{Ca}_{3}\left(\mathrm{PO}_{4}\right)_{2}$, is an ionic compound and a common anti-caking agent added to food products. What is the formula mass (amu) of calcium phosphate?

Answer:

310.18 amu

The Mole

The identity of a substance is defined not only by the types of atoms or ions it contains, but by the quantity of each type of atom or ion. For example, water, $\mathrm{H}_{2} \mathrm{O}$, and hydrogen peroxide, $\mathrm{H}_{2} \mathrm{O}_{2}$, are alike in that their respective molecules are composed of hydrogen and oxygen atoms. However, because a hydrogen peroxide molecule contains two oxygen atoms, as opposed to the water molecule, which has only one, the two substances exhibit very different properties. Today, sophisticated instruments allow the direct measurement of these defining microscopic traits; however, the same traits were originally derived from the measurement of macroscopic 
properties (the masses and volumes of bulk quantities of matter) using relatively simple tools (balances and volumetric glassware). This experimental approach required the introduction of a new unit for amount of substances, the mole, which remains indispensable in modern chemical science.

The mole is an amount unit similar to familiar units like pair, dozen, gross, etc. It provides a specific measure of the number of atoms or molecules in a sample of matter. One Latin connotation for the word "mole" is "large mass" or "bulk," which is consistent with its use as the name for this unit. The mole provides a link between an easily measured macroscopic property, bulk mass, and an extremely important fundamental property, number of atoms, molecules, and so forth. A mole of substance is that amount in which there are $6.02214076 \times 10^{23}$ discrete entities (atoms or molecules). This large number is a fundamental constant known as Avogadro's number $\left(N_{\mathrm{A}}\right)$ or the Avogadro constant in honor of Italian scientist Amedeo Avogadro. or the Avogadro constant in honor of Italian scientist Amedeo Avogadro. This constant is properly reported with an explicit unit of "per mole," a conveniently rounded version being $6.022 \times 10^{23} / \mathrm{mol}$.

Consistent with its definition as an amount unit, 1 mole of any element contains the same number of atoms as 1 mole of any other element. The masses of 1 mole of different elements, however, are different, since the masses of the individual atoms are drastically different. The molar mass of an element (or compound) is the mass in grams of 1 mole of that substance, a property expressed in units of grams per mole ( $\mathrm{g} / \mathrm{mol}$ ) (see (Figure)).

Each sample contains $6.022 \times 10^{23}$ atoms $-1.00 \mathrm{~mol}$ of 
atoms. From left to right (top row): $65.4 \mathrm{~g}$ zinc, $12.0 \mathrm{~g}$ carbon, $24.3 \mathrm{~g}$ magnesium, and $63.5 \mathrm{~g}$ copper. From left to right (bottom row): $32.1 \mathrm{~g}$ sulfur, $28.1 \mathrm{~g}$ silicon, $207 \mathrm{~g}$ lead, and $118.7 \mathrm{~g}$ tin. (credit: modification of work by Mark Ott)

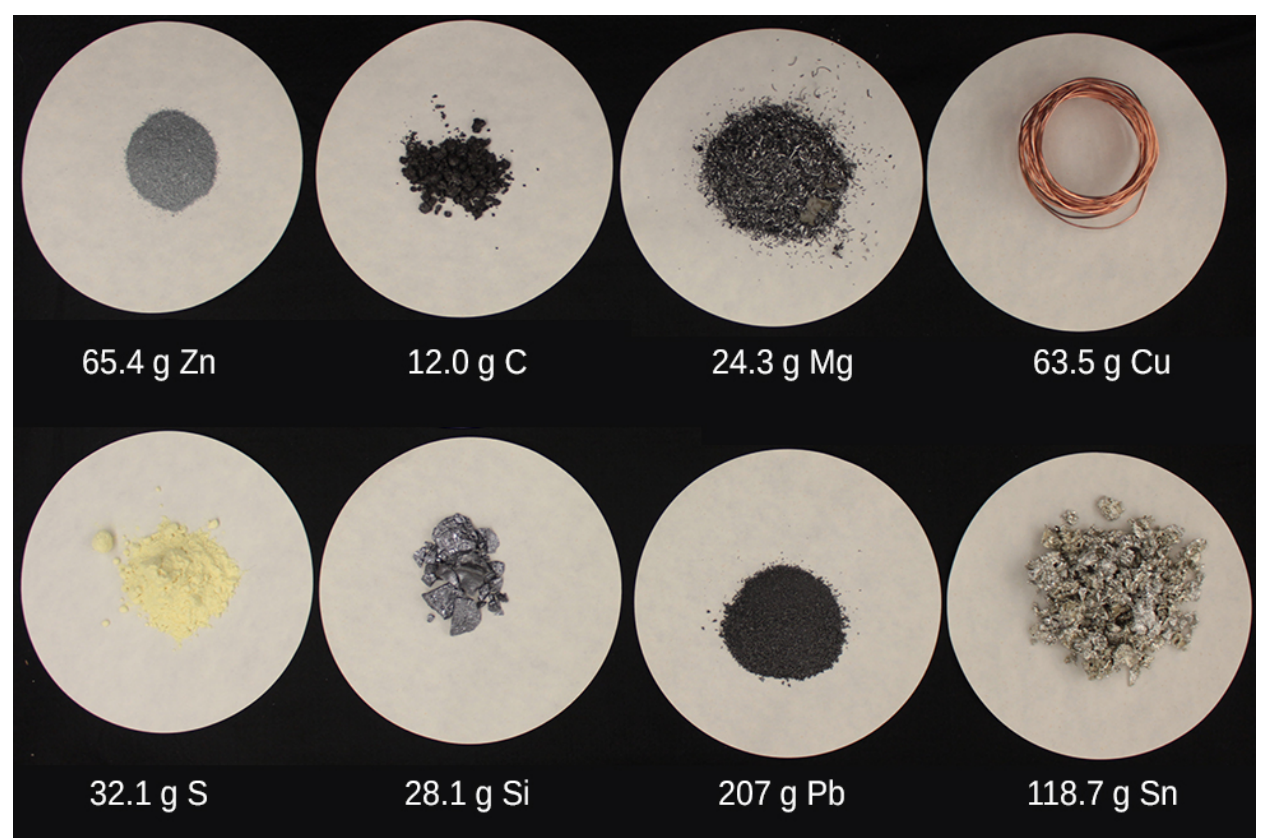

The molar mass of any substance is numerically equivalent to its atomic or formula weight in amu. Per the amu definition, a single ${ }^{12} \mathrm{C}$ atom weighs $12 \mathrm{amu}$ (its atomic mass is $12 \mathrm{amu}$ ). A mole of ${ }^{12} \mathrm{C}$ weighs $12 \mathrm{~g}$ (its molar mass is $12 \mathrm{~g} / \mathrm{mol}$ ). This relationship holds for all elements, since their atomic masses are measured relative to that of the amu-reference substance, ${ }^{12} \mathrm{C}$. Extending this principle, the molar mass of a compound in grams is likewise numerically equivalent to its formula mass in amu ((Figure)).

Each sample contains $6.02 \times 10^{23}$ molecules or formula units $-1.00 \mathrm{~mol}$ of the compound or element. Clock-wise from the upper left: $130.2 \mathrm{~g}$ of $\mathrm{C}_{8} \mathrm{H}_{17} \mathrm{OH}$ (1-octanol, 
formula mass $130.2 \mathrm{amu}$ ), $454.4 \mathrm{~g}$ of $\mathrm{HgI}_{2}$ (mercury(II) iodide, formula mass $454.4 \mathrm{amu}$ ), $32.0 \mathrm{~g}$ of $\mathrm{CH}_{3} \mathrm{OH}$ (methanol, formula mass $32.0 \mathrm{amu}$ ) and $256.5 \mathrm{~g}$ of $\mathrm{S}_{8}$ (sulfur, formula mass $256.5 \mathrm{amu}$ ). (credit: Sahar Atwa)

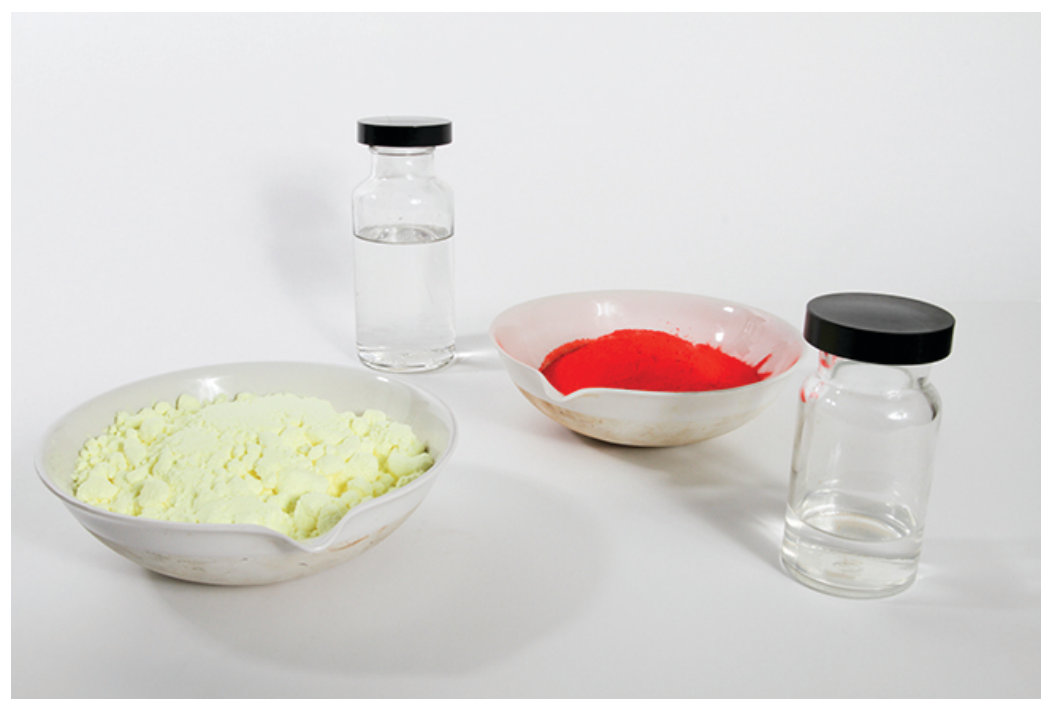

$\begin{array}{lllll}\text { Element } & \begin{array}{l}\text { Average Atomic Mass } \\ (\mathbf{a m u})\end{array} & \begin{array}{l}\text { Molar Mass (g/ } \\ \text { mol) }\end{array} & \begin{array}{l}\text { Atoms/ } \\ \text { Mole }\end{array} \\ \mathrm{C} & 12.01 & 12.01 & 6.022 & 10^{23} \\ \mathrm{H} & 1.008 & 1.008 & 6.022 & 10^{23} \\ \mathrm{O} & 16.00 & 16.00 & 6.022 & 10^{23} \\ \mathrm{Na} & 22.99 & 22.99 & 6.022 & 10^{23} \\ \mathrm{Cl} & 35.45 & 35.45 & 6.022 & 10^{23}\end{array}$

While atomic mass and molar mass are numerically equivalent, keep in mind that they are vastly different in terms of scale, as represented by the vast difference in the magnitudes of their respective units (amu versus g). To appreciate the enormity of the mole, consider a small drop of water weighing about $0.03 \mathrm{~g}$ (see (Figure)). Although this represents just a tiny fraction of 1 mole 
of water ( $18 \mathrm{~g})$, it contains more water molecules than can be clearly imagined. If the molecules were distributed equally among the roughly seven billion people on earth, each person would receive more than 100 billion molecules.

The number of molecules in a single droplet of water is roughly 100 billion times greater than the number of people on earth. (credit: "tanakawho"/Wikimedia commons)

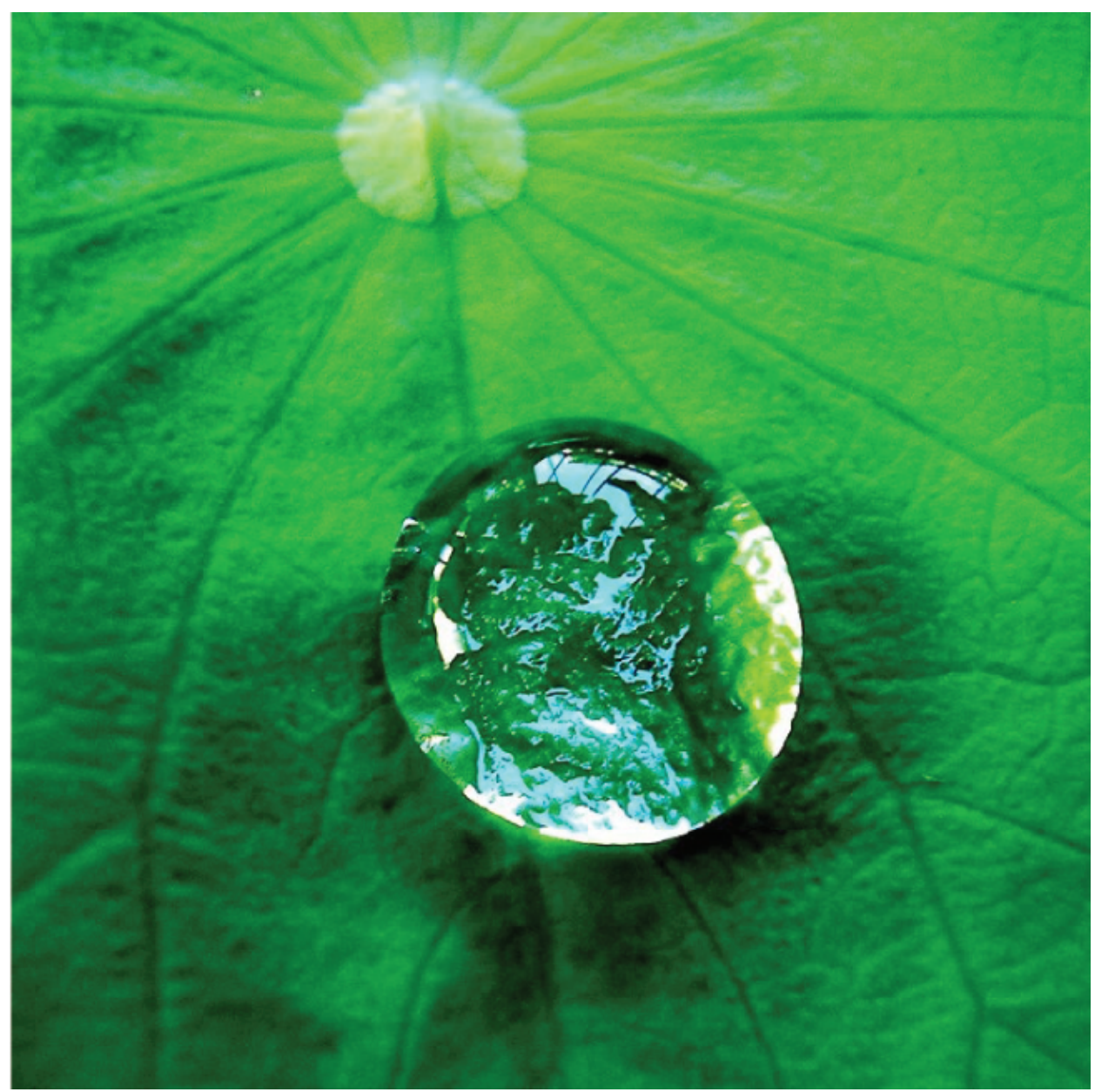

The mole is used in chemistry to represent $6.022 \times 10^{23}$ of something, but it can be difficult to conceptualize such a large number. Watch this video and then complete the 
"Think" questions that follow. Explore more about the mole by reviewing the information under "Dig Deeper."

The relationships between formula mass, the mole, and Avogadro's number can be applied to compute various quantities that describe the composition of substances and compounds, as demonstrated in the next several example problems.

Deriving Moles from Grams for an Element According to nutritional guidelines from the US Department of Agriculture, the estimated average requirement for dietary potassium is $4.7 \mathrm{~g}$. What is the estimated average requirement of potassium in moles?

Solution The mass of $\mathrm{K}$ is provided, and the corresponding amount of $\mathrm{K}$ in moles is requested. Referring to the periodic table, the atomic mass of $\mathrm{K}$ is $39.10 \mathrm{amu}$, and so its molar mass is $39.10 \mathrm{~g} / \mathrm{mol}$. The given mass of $\mathrm{K}(4.7 \mathrm{~g})$ is a bit more than one-tenth the molar mass (39.10 g), so a reasonable "ballpark" estimate of the number of moles would be slightly greater than $0.1 \mathrm{~mol}$.

The molar amount of a substance may be calculated by dividing its mass ( $\mathrm{g}$ ) by its molar mass ( $\mathrm{g} / \mathrm{mol}$ ):

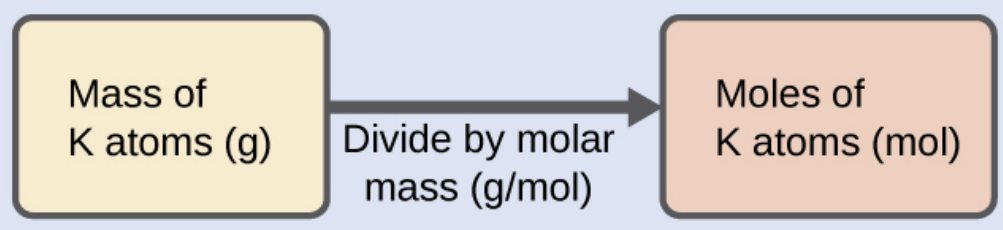

The factor-label method supports this mathematical approach since the unit " $g$ " cancels and the answer has units of "mol:"
$4.7 \overline{) \mathrm{g}} \mathrm{K}$
$\left(\frac{\mathrm{mol} \mathrm{K}}{39.10 \overline{) g}}\right)$
$0.12 \mathrm{~mol} \mathrm{~K}$ 
The calculated magnitude $(0.12 \mathrm{~mol} \mathrm{~K})$ is consistent with our ballpark expectation, since it is a bit greater than $0.1 \mathrm{~mol}$.

Check Your Learning Beryllium is a light metal used to fabricate transparent $\mathrm{X}$-ray windows for medical imaging instruments. How many moles of Be are in a thin-foil window weighing $3.24 \mathrm{~g}$ ?

Answer:

$0.360 \mathrm{~mol}$

Deriving Grams from Moles for an Element $A$ liter of air contains $9.210^{-4} \mathrm{~mol}$ argon. What is the mass of Ar in a liter of air?

Solution The molar amount of Ar is provided and must be used to derive the corresponding mass in grams. Since the amount of Ar is less than 1 mole, the mass will be less than the mass of 1 mole of $A r$, approximately $40 \mathrm{~g}$. The molar amount in question is approximately one-one thousandth $\left(\sim 10^{-3}\right)$ of a mole, and so the corresponding mass should be roughly oneone thousandth of the molar mass $(\sim 0.04 \mathrm{~g})$ :

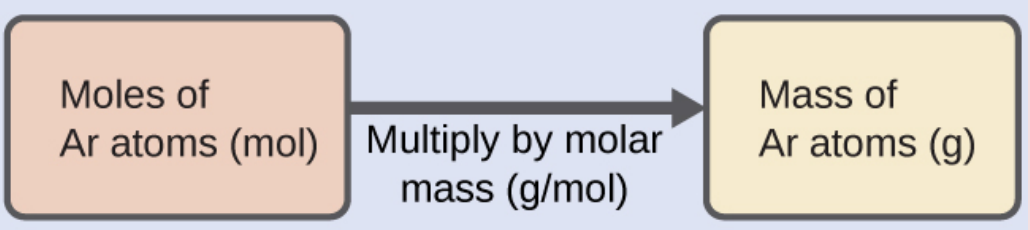

In this case, logic dictates (and the factor-label method supports) multiplying the provided amount ( $\mathrm{mol}$ ) by the molar mass (g/mol):

$$
9.210^{-4} \overline{) \mathrm{mol}} \mathrm{Ar}\left(\frac{39.95 \mathrm{~g}}{\overline{\overline{\mathrm{mol} \mathrm{Ar}}}}\right)=0.037 \mathrm{~g} \mathrm{Ar}
$$

The result is in agreement with our expectations, around $0.04 \mathrm{~g}$ Ar.

Check Your Learning What is the mass of $2.561 \mathrm{~mol}$ of gold? Answer: 
$504.4 \mathrm{~g}$

Deriving Number of Atoms from Mass for an Element Copper is commonly used to fabricate electrical wire ((Figure)). How many copper atoms are in $5.00 \mathrm{~g}$ of copper wire?

Copper wire is composed of many, many atoms of $\mathrm{Cu}$. (credit: Emilian Robert Vicol)

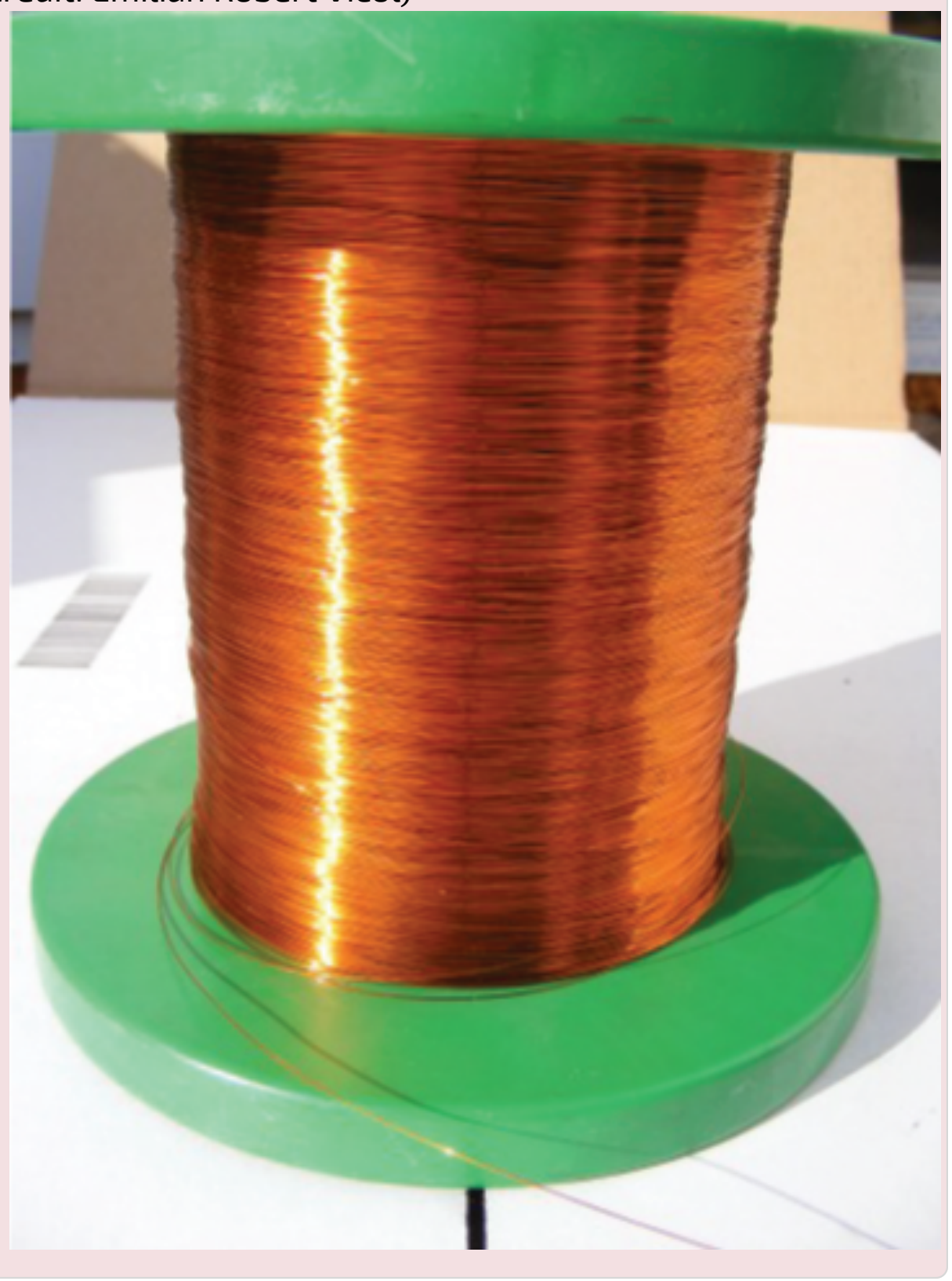


Solution The number of $\mathrm{Cu}$ atoms in the wire may be conveniently derived from its mass by a two-step computation: first calculating the molar amount of $\mathrm{Cu}$, and then using Avogadro's number $\left(N_{A}\right)$ to convert this molar amount to number of $\mathrm{Cu}$ atoms:

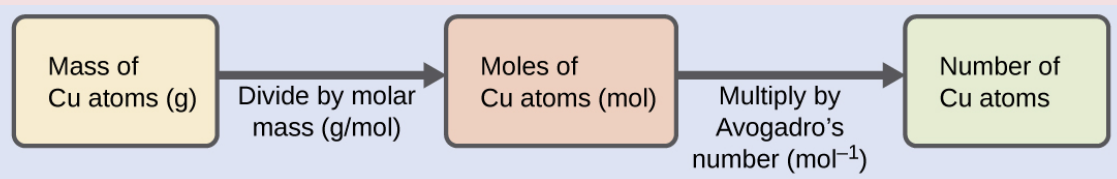

Considering that the provided sample mass $(5.00 \mathrm{~g})$ is a little less than one-tenth the mass of 1 mole of Cu ( 64 g), a reasonable estimate for the number of atoms in the sample would be on the order of one-tenth $N_{A}$, or approximately $10^{22}$ Cu atoms. Carrying out the two-step computation yields:

$5.00 \overline{\mathrm{g}} \mathrm{Cu}\left(\frac{\overline{\frac{\mathrm{mol}}{6 u}}}{63.55 \overline{) \mathrm{g}}}\right)\left(\frac{6.022}{\overline{10^{23}} \text { atoms }}\right)=4.7410^{22}$ atoms of copper

The factor-label method yields the desired cancellation of units, and the computed result is on the order of $10^{22}$ as expected.

Check Your Learning A prospector panning for gold in a river collects $15.00 \mathrm{~g}$ of pure gold. How many Au atoms are in this quantity of gold?

Answer:

$4.58610^{22}$ Au atoms

Deriving Moles from Grams for a Compound Our bodies synthesize protein from amino acids. One of these amino acids is glycine, which has the molecular formula $\mathrm{C}_{2} \mathrm{H}_{5} \mathrm{O}_{2} \mathrm{~N}$. How many moles of glycine molecules are contained in $28.35 \mathrm{~g}$ of glycine?

Solution Derive the number of moles of a compound from its mass following the same procedure used for an element in (Figure): 


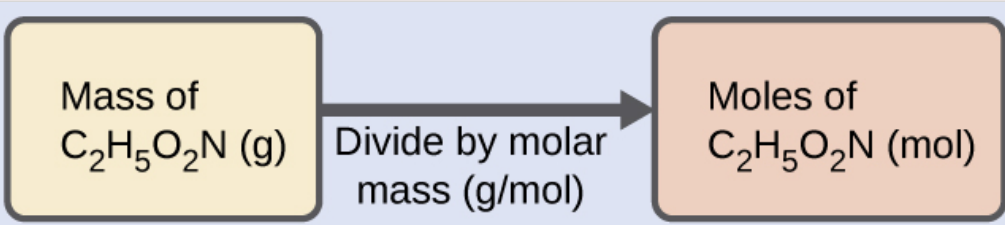

The molar mass of glycine is required for this calculation, and it is computed in the same fashion as its molecular mass. One mole of glycine, $\mathrm{C}_{2} \mathrm{H}_{5} \mathrm{O}_{2} \mathrm{~N}$, contains 2 moles of carbon, 5 moles of hydrogen, 2 moles of oxygen, and 1 mole of nitrogen:

\begin{tabular}{|c|c|c|c|c|c|}
\hline Element & $\begin{array}{c}\text { Quantity } \\
\text { (mol element/ } \\
\text { mol compound) }\end{array}$ & $\begin{array}{c}\text { Molar mass } \\
\text { (g/mol element) }\end{array}$ & $\begin{array}{c}\text { Subtotal } \\
\text { (g/mol compound) }\end{array}$ \\
\hline C & 2 & $\times$ & 12.01 & $=$ & 24.02 \\
\hline H & 5 & $\times$ & 1.008 & $=$ & 5.040 \\
\hline O & 2 & $\times$ & 16.00 & $=$ & 32.00 \\
\hline N & 1 & $\times$ & 14.007 & $=$ & 14.007 \\
\hline \multicolumn{7}{|c|}{ Molecular mass (g/mol compound) } & 75.07 \\
\hline
\end{tabular}

The provided mass of glycine ( $28 \mathrm{~g})$ is a bit more than onethird the molar mass $(\sim 75 \mathrm{~g} / \mathrm{mol})$, so the computed result is expected to be a bit greater than one-third of a mole $(\sim 0.33$ mol). Dividing the compound's mass by its molar mass yields:

$28.35 \overline{) g}$ glycine $\left(\frac{\text { mol glycine }}{75.07 \overline{) g}}\right)=0.378 \mathrm{~mol}$ glycine

This result is consistent with the rough estimate.

Check Your Learning How many moles of sucrose, $\mathrm{C}_{12} \mathrm{H}_{22} \mathrm{O}_{11}$, are in a 25 -g sample of sucrose?

Answer:

$0.073 \mathrm{~mol}$

Deriving Grams from Moles for a Compound Vitamin C is a covalent compound with the molecular formula $\mathrm{C}_{6} \mathrm{H}_{8} \mathrm{O}_{6}$. The recommended daily dietary allowance of vitamin $\mathrm{C}$ for children aged 4-8 years is $1.4210^{-4} \mathrm{~mol}$. What is the mass of this allowance in grams? 
Solution As for elements, the mass of a compound can be derived from its molar amount as shown:

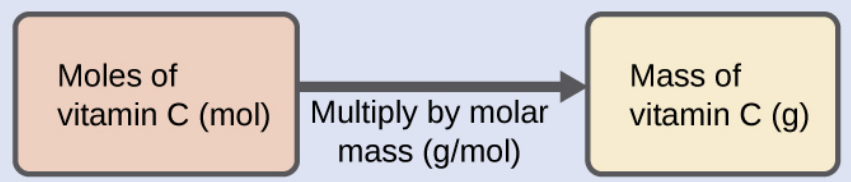

The molar mass for this compound is computed to be $176.124 \mathrm{~g} / \mathrm{mol}$. The given number of moles is a very small fraction of a mole ( $10^{-4}$ or one-ten thousandth); therefore, the corresponding mass is expected to be about one-ten thousandth of the molar mass ( $0.02 \mathrm{~g}$ ). Performing the calculation yields:

$1.4210^{-4} \overline{\mathrm{mol}}$ vitamin $\mathrm{C}\left(\frac{176.124 \mathrm{~g}}{\overline{\overline{\text { mol vitamin C }}}}\right)=0.0250 \mathrm{~g}$ vitamin $\mathrm{C}$

This is consistent with the anticipated result.

Check Your Learning What is the mass of $0.443 \mathrm{~mol}$ of hydrazine, $\mathrm{N}_{2} \mathrm{H}_{4}$ ?

Answer:

$14.2 \mathrm{~g}$

Deriving the Number of Atoms and Molecules from the Mass of a Compound A packet of an artificial sweetener contains $40.0 \mathrm{mg}$ of saccharin $\left(\mathrm{C}_{7} \mathrm{H}_{5} \mathrm{NO}_{3} \mathrm{~S}\right)$, which has the structural formula:<smiles>O=C1NS(=O)(=O)c2ccccc21</smiles>

Given that saccharin has a molar mass of $183.18 \mathrm{~g} / \mathrm{mol}$, how many saccharin molecules are in a 40.0-mg (0.0400-g) 
sample of saccharin? How many carbon atoms are in the same sample?

Solution The number of molecules in a given mass of compound is computed by first deriving the number of moles, as demonstrated in (Figure), and then multiplying by Avogadro's number:

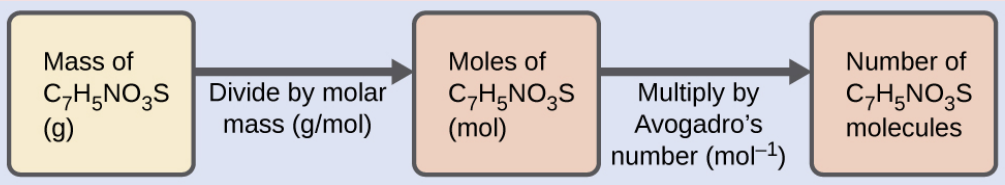

Using the provided mass and molar mass for saccharin yields:

$0.0400) \bar{g} \mathrm{C}_{7} \mathrm{H}_{5} \mathrm{NO}_{3} \mathrm{~S}\left(\frac{\overline{\mathrm{mol} \mathrm{C}_{7} \mathrm{H}_{5} \mathrm{NO}_{3} \mathrm{~S}}}{183.18 \overline{) \mathrm{g} \mathrm{C}} \mathrm{H}_{7} \mathrm{NO}_{3} \mathrm{~S}}\right)\left(\frac{6.02210^{23} \mathrm{C}_{7} \mathrm{H}_{5} \mathrm{NO}_{3} \mathrm{~S} \text { molecules }}{1) \mathrm{mol}_{7} \mathrm{H}_{5} \mathrm{NO}_{3} \mathrm{~S}}\right)$

$=1.3110^{20} \mathrm{C}_{7} \mathrm{H}_{5} \mathrm{NO}_{3} \mathrm{~S}$ molecules

The compound's formula shows that each molecule contains seven carbon atoms, and so the number of $C$ atoms in the provided sample is:

$1.3110^{20} \mathrm{C}_{7} \mathrm{H}_{5} \mathrm{NO}_{3} \mathrm{~S}$ molecules $\left(\frac{7 \mathrm{C} \text { atoms }}{1 \mathrm{C}_{7} \mathrm{H}_{5} \mathrm{NO}_{3} \mathrm{~S} \text { molecule }}\right)=9.1710^{20} \mathrm{C}$ atoms

Check Your Learning How many $\mathrm{C}_{4} \mathrm{H}_{10}$ molecules are contained in $9.213 \mathrm{~g}$ of this compound? How many hydrogen atoms?

Answer:

$9.54510^{22}$ molecules $\mathrm{C}_{4} \mathrm{H}_{10} ; 9.54510^{23}$ atoms $\mathrm{H}$

Counting Neurotransmitter Molecules in the Brain

The brain is the control center of the central nervous system ((Figure)). It sends and receives signals to and from muscles and other internal organs to monitor and control their functions; it processes stimuli detected by sensory organs to guide interactions with the external world; and it houses the complex physiological processes that give 
rise to our intellect and emotions. The broad field of neuroscience spans all aspects of the structure and function of the central nervous system, including research on the anatomy and physiology of the brain. Great progress has been made in brain research over the past few decades, and the BRAIN Initiative, a federal initiative announced in 2013, aims to accelerate and capitalize on these advances through the concerted efforts of various industrial, academic, and government agencies (more details available at www.whitehouse.gov/share/braininitiative).

(a) A typical human brain weighs about $1.5 \mathrm{~kg}$ and occupies a volume of roughly $1.1 \mathrm{~L}$. (b) Information is transmitted in brain tissue and throughout the central nervous system by specialized cells called neurons (micrograph shows cells at $1600 \times$ magnification).

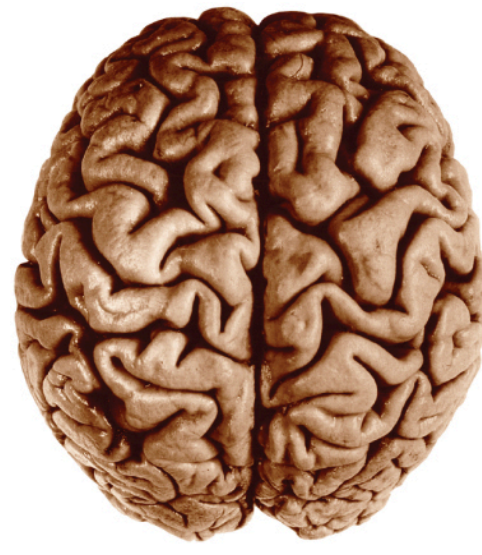

(a)

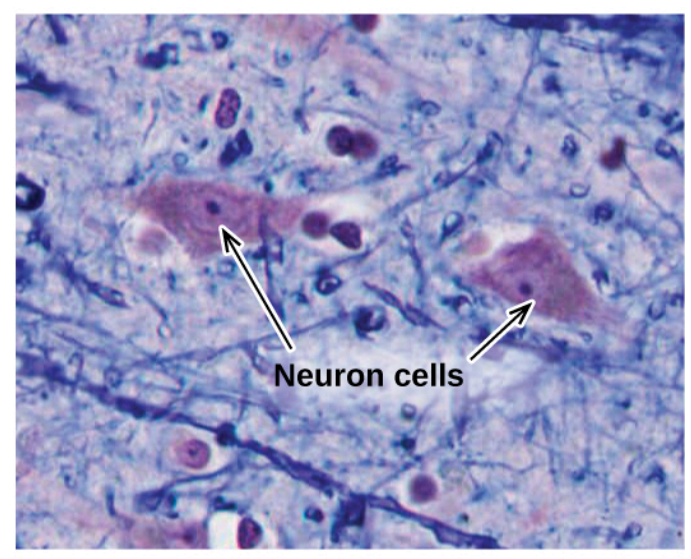

(b)

Specialized cells called neurons transmit information between different parts of the central nervous system by way of electrical and chemical signals. Chemical signaling occurs at the interface between different neurons when one of the cells releases molecules (called 
neurotransmitters) that diffuse across the small gap between the cells (called the synapse) and bind to the surface of the other cell. These neurotransmitter molecules are stored in small intracellular structures called vesicles that fuse to the cell wall and then break open to release their contents when the neuron is appropriately stimulated. This process is called exocytosis (see (Figure)). One neurotransmitter that has been very extensively studied is dopamine, $\mathrm{C}_{8} \mathrm{H}_{11} \mathrm{NO}_{2}$. Dopamine is involved in various neurological processes that impact a wide variety of human behaviors. Dysfunctions in the dopamine systems of the brain underlie serious neurological diseases such as Parkinson's and schizophrenia.

(a) Chemical signals are transmitted from neurons to other cells by the release of neurotransmitter molecules into the small gaps (synapses) between the cells. (b)

Dopamine, $\mathrm{C}_{8} \mathrm{H}_{11} \mathrm{NO}_{2}$, is a neurotransmitter involved in a number of neurological processes.

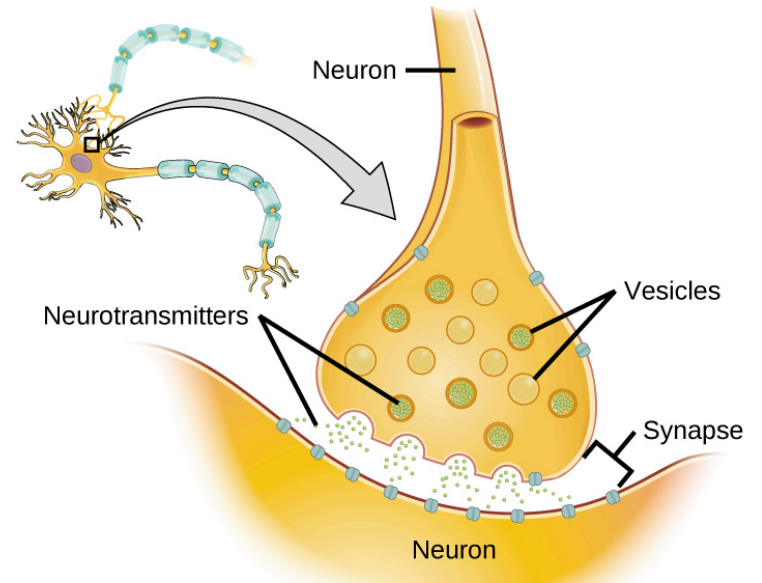

(a)

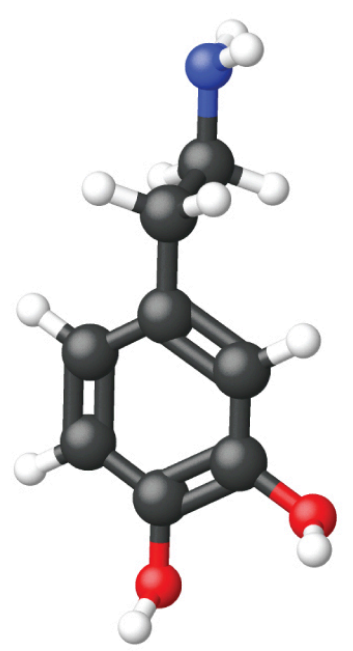

(b)

One important aspect of the complex processes related 
to dopamine signaling is the number of neurotransmitter molecules released during exocytosis. Since this number is a central factor in determining neurological response (and subsequent human thought and action), it is important to know how this number changes with certain controlled stimulations, such as the administration of drugs. It is also important to understand the mechanism responsible for any changes in the number of neurotransmitter molecules released-for example, some dysfunction in exocytosis, a change in the number of vesicles in the neuron, or a change in the number of neurotransmitter molecules in each vesicle.

Significant progress has been made recently in directly measuring the number of dopamine molecules stored in individual vesicles and the amount actually released when the vesicle undergoes exocytosis. Using miniaturized probes that can selectively detect dopamine molecules in very small amounts, scientists have determined that the vesicles of a certain type of mouse brain neuron contain an average of 30,000 dopamine molecules per vesicle (about $510^{-20} \mathrm{~mol}$ or $50 \mathrm{zmol}$ ). Analysis of these neurons from mice subjected to various drug therapies shows significant changes in the average number of dopamine molecules contained in individual vesicles, increasing or decreasing by up to three-fold, depending on the specific drug used. These studies also indicate that not all of the dopamine in a given vesicle is released during exocytosis, suggesting that it may be possible to regulate the fraction released using pharmaceutical therapies. ${ }^{1}$ 
Key Concepts and Summary

The formula mass of a substance is the sum of the average atomic masses of each atom represented in the chemical formula and is expressed in atomic mass units. The formula mass of a covalent compound is also called the molecular mass. A convenient amount unit for expressing very large numbers of atoms or molecules is the mole. Experimental measurements have determined the number of entities composing 1 mole of substance to be 6.022 $10^{23}$, a quantity called Avogadro's number. The mass in grams of 1 mole of substance is its molar mass. Due to the use of the same reference substance in defining the atomic mass unit and the mole, the formula mass (amu) and molar mass $(\mathrm{g} / \mathrm{mol})$ for any substance are numerically equivalent (for example, one $\mathrm{H}_{2} \mathrm{O}$ molecule weighs approximately 18 amu and 1 mole of $\mathrm{H}_{2} \mathrm{O}$ molecules weighs approximately $18 \mathrm{~g}$ ).

Chemistry End of Chapter Exercises

What is the total mass (amu) of carbon in each of the following molecules?

(a) $\mathrm{CH}_{4}$

(b) $\mathrm{CHCl}_{3}$

(c) $\mathrm{C}_{12} \mathrm{H}_{10} \mathrm{O}_{6}$

(d) $\mathrm{CH}_{3} \mathrm{CH}_{2} \mathrm{CH}_{2} \mathrm{CH}_{2} \mathrm{CH}_{3}$

(a) $12.01 \mathrm{amu}$; (b) $12.01 \mathrm{amu}$; (c) $144.12 \mathrm{amu}$; (d) 60.05 amu

What is the total mass of hydrogen in each of the molecules? 

(a) $\mathrm{CH}_{4}$
(b) $\mathrm{CHCl}_{3}$
(c) $\mathrm{C}_{12} \mathrm{H}_{10} \mathrm{O}_{6}$
(d) $\mathrm{CH}_{3} \mathrm{CH}_{2} \mathrm{CH}_{2} \mathrm{CH}_{2} \mathrm{CH}_{3}$

Calculate the molecular or formula mass of each of the following:
(a) $\mathrm{P}_{4}$
(b) $\mathrm{H}_{2} \mathrm{O}$
(c) $\mathrm{Ca}\left(\mathrm{NO}_{3}\right)_{2}$
(d) $\mathrm{CH}_{3} \mathrm{CO}_{2} \mathrm{H}$ (acetic acid)
(e) $\mathrm{C}_{12} \mathrm{H}_{22} \mathrm{O}_{11}$ (sucrose, cane sugar)

(a) $123.896 \mathrm{amu}$; (b) $18.015 \mathrm{amu}$; (c) $164.086 \mathrm{amu}$; (d) $60.052 \mathrm{amu}$; (e) $342.297 \mathrm{amu}$

Determine the molecular mass of the following compounds:

(a)

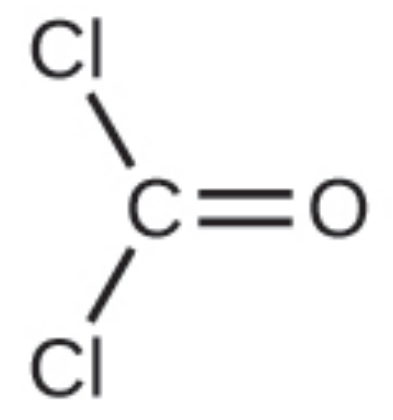

(b)

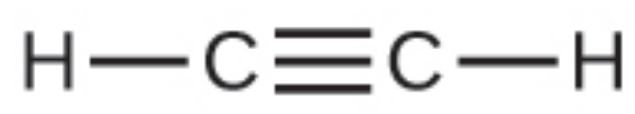

(c) 


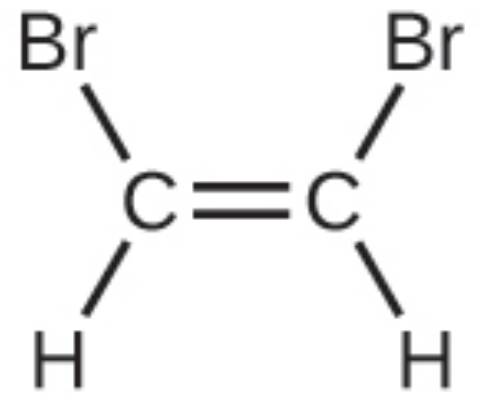

(d)

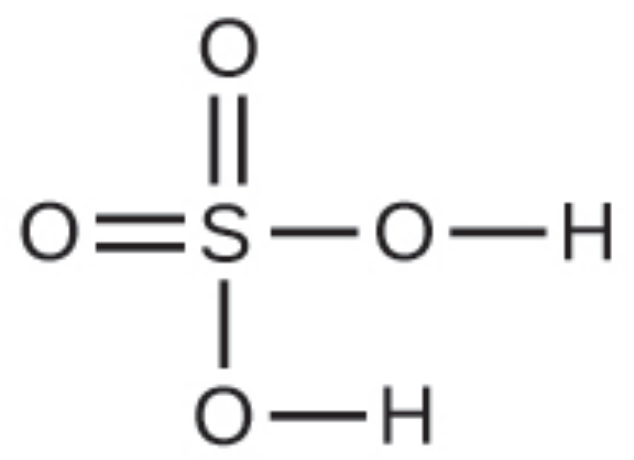

Determine the molecular mass of the following compounds:

(a)

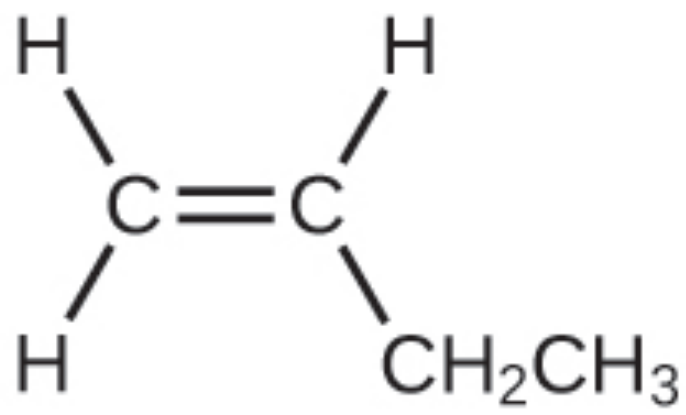

(b)

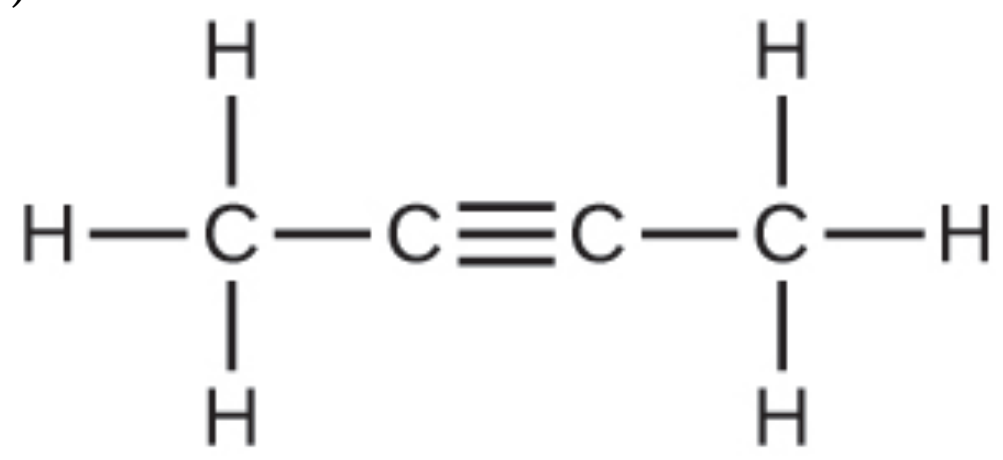


(c)

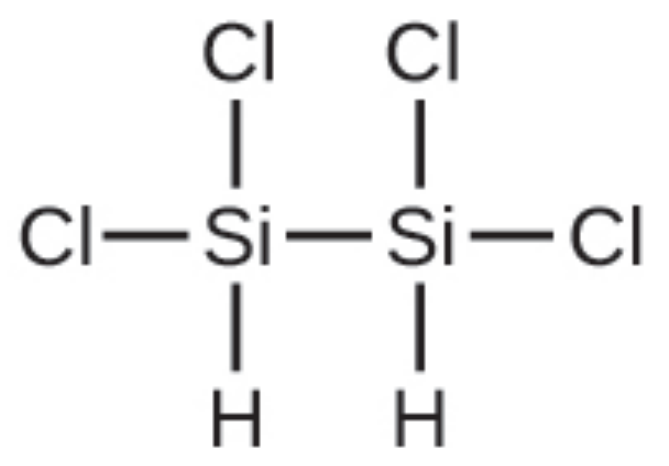

(d)

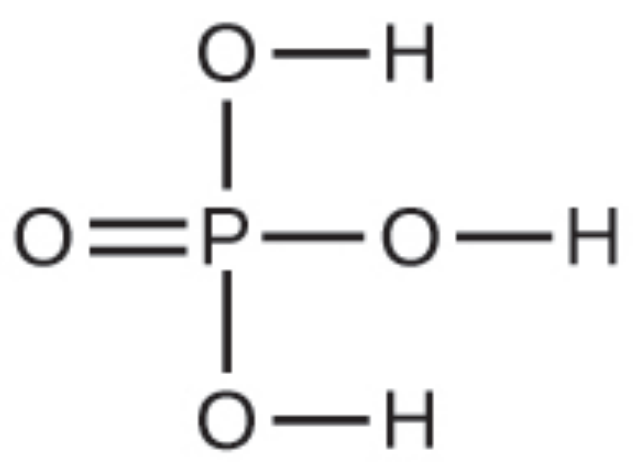

(a) $56.107 \mathrm{amu}$; (b) $54.091 \mathrm{amu}$; (c) $199.9976 \mathrm{amu}$; (d) $97.9950 \mathrm{amu}$

Which molecule has a molecular mass of $28.05 \mathrm{amu}$ ?

(a)

$$
\mathrm{H}-\mathrm{C} \equiv \mathrm{C}-\mathrm{H}
$$

(b)

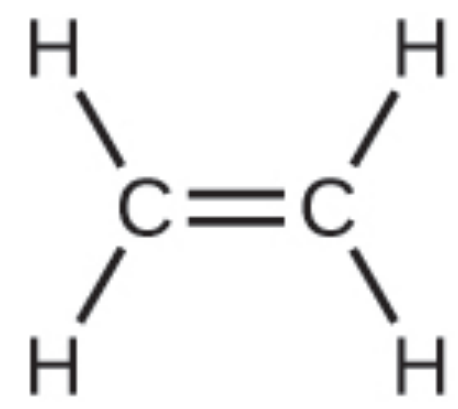

(c) 


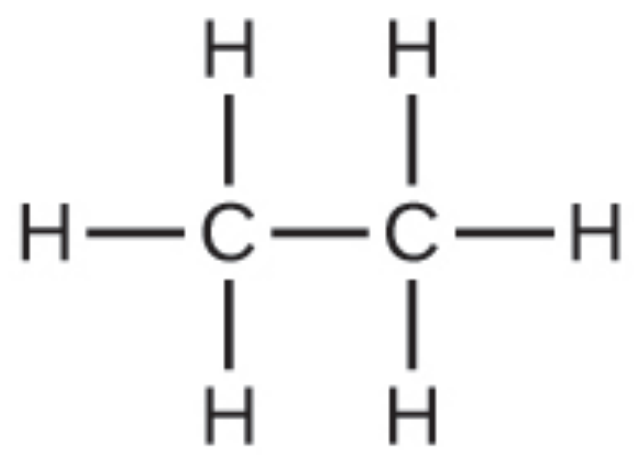

Write a sentence that describes how to determine the number of moles of a compound in a known mass of the compound using its molecular formula.

Use the molecular formula to find the molar mass; to obtain the number of moles, divide the mass of compound by the molar mass of the compound expressed in grams.

Compare 1 mole of $\mathrm{H}_{2}, 1$ mole of $\mathrm{O}_{2}$, and 1 mole of $\mathrm{F}_{2}$.

(a) Which has the largest number of molecules? Explain why.

(b) Which has the greatest mass? Explain why.

Which contains the greatest mass of oxygen: $0.75 \mathrm{~mol}$ of ethanol $\left(\mathrm{C}_{2} \mathrm{H}_{5} \mathrm{OH}\right), 0.60 \mathrm{~mol}$ of formic acid $\left(\mathrm{HCO}_{2} \mathrm{H}\right)$, or 1.0 mol of water $\left(\mathrm{H}_{2} \mathrm{O}\right)$ ? Explain why.

Formic acid. Its formula has twice as many oxygen atoms as the other two compounds (one each). Therefore, 0.60 mol of formic acid would be equivalent to $1.20 \mathrm{~mol}$ of a compound containing a single oxygen atom.

Which contains the greatest number of moles of oxygen atoms: $1 \mathrm{~mol}$ of ethanol $\left(\mathrm{C}_{2} \mathrm{H}_{5} \mathrm{OH}\right), 1 \mathrm{~mol}$ of formic acid $\left(\mathrm{HCO}_{2} \mathrm{H}\right)$, or $1 \mathrm{~mol}$ of water $\left(\mathrm{H}_{2} \mathrm{O}\right)$ ? Explain why. 
How are the molecular mass and the molar mass of a compound similar and how are they different?

The two masses have the same numerical value, but the units are different: The molecular mass is the mass of 1 molecule while the molar mass is the mass of $6.02210^{23}$ molecules.

Calculate the molar mass of each of the following compounds:
(a) hydrogen fluoride, HF
(b) ammonia, $\mathrm{NH}_{3}$
(c) nitric acid, $\mathrm{HNO}_{3}$
(d) silver sulfate, $\mathrm{Ag}_{2} \mathrm{SO}_{4}$
(e) boric acid, $\mathrm{B}(\mathrm{OH})_{3}$

Calculate the molar mass of each of the following:

(a) $\mathrm{S}_{8}$

(b) $\mathrm{C}_{5} \mathrm{H}_{12}$

(c) $\mathrm{Sc}_{2}\left(\mathrm{SO}_{4}\right)_{3}$

(d) $\mathrm{CH}_{3} \mathrm{COCH}_{3}$ (acetone)

(e) $\mathrm{C}_{6} \mathrm{H}_{12} \mathrm{O}_{6}$ (glucose)

(a) $256.528 \mathrm{~g} / \mathrm{mol}$; (b) $72.150 \mathrm{~g} \mathrm{~mol}^{-1}$; (c) $378.103 \mathrm{~g} \mathrm{~mol}^{-1}$; (d) $58.080 \mathrm{~g} \mathrm{~mol}^{-1}$; (e) $180.158 \mathrm{~g} \mathrm{~mol}^{-1}$

Calculate the empirical or molecular formula mass and the molar mass of each of the following minerals:

(a) limestone, $\mathrm{CaCO}_{3}$

(b) halite, $\mathrm{NaCl}$

(c) beryl, $\mathrm{Be}_{3} \mathrm{Al}_{2} \mathrm{Si}_{6} \mathrm{O}_{18}$

(d) malachite, $\mathrm{Cu}_{2}(\mathrm{OH})_{2} \mathrm{CO}_{3}$

(e) turquoise, $\mathrm{CuAl}_{6}\left(\mathrm{PO}_{4}\right)_{4}(\mathrm{OH})_{8}\left(\mathrm{H}_{2} \mathrm{O}\right)_{4}$ 
Calculate the molar mass of each of the following:

(a) the anesthetic halothane, $\mathrm{C}_{2} \mathrm{HBrClF}_{3}$

(b) the herbicide paraquat, $\mathrm{C}_{12} \mathrm{H}_{14} \mathrm{~N}_{2} \mathrm{Cl}_{2}$

(c) caffeine, $\mathrm{C}_{8} \mathrm{H}_{10} \mathrm{~N}_{4} \mathrm{O}_{2}$

(d) urea, $\mathrm{CO}\left(\mathrm{NH}_{2}\right)_{2}$

(e) a typical soap, $\mathrm{C}_{17} \mathrm{H}_{35} \mathrm{CO}_{2} \mathrm{Na}$

(a) $197.382 \mathrm{~g} \mathrm{~mol}^{-1}$; (b) $257.163 \mathrm{~g} \mathrm{~mol}^{-1}$; (c) $194.193 \mathrm{~g}$ $\mathrm{mol}^{-1}$; (d) $60.056 \mathrm{~g} \mathrm{~mol}^{-1}$; (e) $306.464 \mathrm{~g} \mathrm{~mol}^{-1}$

Determine the number of moles of compound and the number of moles of each type of atom in each of the following:

(a) $25.0 \mathrm{~g}$ of propylene, $\mathrm{C}_{3} \mathrm{H}_{6}$

(b) $3.0610^{-3} \mathrm{~g}$ of the amino acid glycine, $\mathrm{C}_{2} \mathrm{H}_{5} \mathrm{NO}_{2}$

(c) $25 \mathrm{lb}$ of the herbicide Treflan, $\mathrm{C}_{13} \mathrm{H}_{16} \mathrm{~N}_{2} \mathrm{O}_{4} \mathrm{~F}(1 \mathrm{lb}=$ $454 \mathrm{~g})$

(d) $0.125 \mathrm{~kg}$ of the insecticide Paris Green, $\mathrm{Cu}_{4}\left(\mathrm{AsO}_{3}\right)_{2}\left(\mathrm{CH}_{3} \mathrm{CO}_{2}\right)_{2}$

(e) $325 \mathrm{mg}$ of aspirin, $\mathrm{C}_{6} \mathrm{H}_{4}\left(\mathrm{CO}_{2} \mathrm{H}\right)\left(\mathrm{CO}_{2} \mathrm{CH}_{3}\right)$

Determine the mass of each of the following:

(a) $0.0146 \mathrm{~mol} \mathrm{KOH}$

(b) 10.2 mol ethane, $\mathrm{C}_{2} \mathrm{H}_{6}$

(c) $1.610^{-3} \mathrm{~mol} \mathrm{Na}_{2} \mathrm{SO}_{4}$

(d) $6.85410^{3}$ mol glucose, $\mathrm{C}_{6} \mathrm{H}_{12} \mathrm{O}_{6}$

(e) $2.86 \mathrm{~mol} \mathrm{Co}\left(\mathrm{NH}_{3}\right)_{6} \mathrm{Cl}_{3}$

(a) $0.819 \mathrm{~g}$; (b) $307 \mathrm{~g}$; (c) $0.23 \mathrm{~g}$; (d) $1.23510^{6} \mathrm{~g}$ (1235 kg);

(e) $765 \mathrm{~g}$

Determine the number of moles of the compound and 
determine the number of moles of each type of atom in each of the following:

(a) $2.12 \mathrm{~g}$ of potassium bromide, $\mathrm{KBr}$

(b) $0.1488 \mathrm{~g}$ of phosphoric acid, $\mathrm{H}_{3} \mathrm{PO}_{4}$

(c) $23 \mathrm{~kg}$ of calcium carbonate, $\mathrm{CaCO}_{3}$

(d) $78.452 \mathrm{~g}$ of aluminum sulfate, $\mathrm{Al}_{2}\left(\mathrm{SO}_{4}\right)_{3}$

(e) $0.1250 \mathrm{mg}$ of caffeine, $\mathrm{C}_{8} \mathrm{H}_{10} \mathrm{~N}_{4} \mathrm{O}_{2}$

Determine the mass of each of the following:

(a) $2.345 \mathrm{~mol} \mathrm{LiCl}$

(b) $0.0872 \mathrm{~mol}$ acetylene, $\mathrm{C}_{2} \mathrm{H}_{2}$

(c) $3.310^{-2} \mathrm{~mol} \mathrm{Na}_{2} \mathrm{CO}_{3}$

(d) $1.2310^{3}$ mol fructose, $\mathrm{C}_{6} \mathrm{H}_{12} \mathrm{O}_{6}$

(e) $0.5758 \mathrm{~mol} \mathrm{FeSO}_{4}\left(\mathrm{H}_{2} \mathrm{O}\right)_{7}$

(a) $99.41 \mathrm{~g}$; (b) $2.27 \mathrm{~g}$; (c) $3.5 \mathrm{~g}$; (d) $222 \mathrm{~kg}$; (e) $160.1 \mathrm{~g}$

The approximate minimum daily dietary requirement of the amino acid leucine, $\mathrm{C}_{6} \mathrm{H}_{13} \mathrm{NO}_{2}$, is $1.1 \mathrm{~g}$. What is this requirement in moles?

Determine the mass in grams of each of the following:

(a) $0.600 \mathrm{~mol}$ of oxygen atoms

(b) 0.600 mol of oxygen molecules, $\mathrm{O}_{2}$

(c) $0.600 \mathrm{~mol}$ of ozone molecules, $\mathrm{O}_{3}$

(a) $9.60 \mathrm{~g}$; (b) $19.2 \mathrm{~g}$; (c) $28.8 \mathrm{~g}$

A 55-kg woman has $7.510^{-3} \mathrm{~mol}$ of hemoglobin (molar mass $=64,456 \mathrm{~g} / \mathrm{mol}$ ) in her blood. How many hemoglobin molecules is this? What is this quantity in grams?

Determine the number of atoms and the mass of 
zirconium, silicon, and oxygen found in $0.3384 \mathrm{~mol}$ of zircon, $\mathrm{ZrSiO}_{4}$, a semiprecious stone.

zirconium: $2.03810^{23}$ atoms; $30.87 \mathrm{~g}$; silicon: $2.03810^{23}$ atoms; $9.504 \mathrm{~g}$; oxygen: $8.15110^{23}$ atoms; $21.66 \mathrm{~g}$

Determine which of the following contains the greatest mass of hydrogen: $1 \mathrm{~mol}$ of $\mathrm{CH}_{4}, 0.6 \mathrm{~mol}$ of $\mathrm{C}_{6} \mathrm{H}_{6}$, or 0.4 mol of $\mathrm{C}_{3} \mathrm{H}_{8}$.

Determine which of the following contains the greatest mass of aluminum: $122 \mathrm{~g}$ of $\mathrm{AlPO}_{4}, 266 \mathrm{~g}$ of $\mathrm{Al}_{2} \mathrm{C}_{6}$, or 225 $\mathrm{g}$ of $\mathrm{Al}_{2} \mathrm{~S}_{3}$.

$\mathrm{AlPO}_{4}: 1.000 \mathrm{~mol}$, or $26.98 \mathrm{~g} \mathrm{Al} ; \mathrm{Al}_{2} \mathrm{Cl}_{6}: 1.994 \mathrm{~mol}$, or $53.74 \mathrm{~g} \mathrm{Al} ; \mathrm{Al}_{2} \mathrm{~S}_{3}: 3.00 \mathrm{~mol}$, or $80.94 \mathrm{~g} \mathrm{Al}$; The Al2S3 sample thus contains the greatest mass of Al.

Diamond is one form of elemental carbon. An engagement ring contains a diamond weighing 1.25 carats $(1$ carat $=$ $200 \mathrm{mg})$. How many atoms are present in the diamond?

The Cullinan diamond was the largest natural diamond ever found (January 25, 1905). It weighed 3104 carats (1 carat $=200 \mathrm{mg})$. How many carbon atoms were present in the stone?

\section{$3.11310^{25} \mathrm{C}$ atoms}

One 55-gram serving of a particular cereal supplies 270 mg of sodium, $11 \%$ of the recommended daily allowance. How many moles and atoms of sodium are in the recommended daily allowance? 
A certain nut crunch cereal contains 11.0 grams of sugar (sucrose, $\mathrm{C}_{12} \mathrm{H}_{22} \mathrm{O}_{11}$ ) per serving size of 60.0 grams. How many servings of this cereal must be eaten to consume 0.0278 moles of sugar?

0.865 servings, or about 1 serving.

A tube of toothpaste contains $0.76 \mathrm{~g}$ of sodium monofluorophosphate $\left(\mathrm{Na}_{2} \mathrm{PO}_{3} \mathrm{~F}\right)$ in $100 \mathrm{~mL}$.

(a) What mass of fluorine atoms in mg was present?

(b) How many fluorine atoms were present?

Which of the following represents the least number of molecules?

(a) $20.0 \mathrm{~g}$ of $\mathrm{H}_{2} \mathrm{O}(18.02 \mathrm{~g} / \mathrm{mol})$

(b) $77.0 \mathrm{~g}$ of $\mathrm{CH}_{4}(16.06 \mathrm{~g} / \mathrm{mol})$

(c) $68.0 \mathrm{~g}$ of $\mathrm{CaH}_{2}(42.09 \mathrm{~g} / \mathrm{mol})$

(d) $100.0 \mathrm{~g}$ of $\mathrm{N}_{2} \mathrm{O}(44.02 \mathrm{~g} / \mathrm{mol})$

(e) $84.0 \mathrm{~g}$ of $\mathrm{HF}(20.01 \mathrm{~g} / \mathrm{mol})$

$20.0 \mathrm{~g} \mathrm{H}_{2} \mathrm{O}$ represents the least number of molecules since it has the least number of moles.

Footnotes

- 10miatek, Donna M., Amanda J. Bressler, AnnSofie Cans, Anne M. Andrews, Michael L. Heien, and Andrew G. Ewing. “The Real Catecholamine Content of Secretory Vesicles in the CNS Revealed by Electrochemical Cytometry." Scientific Report 3 (2013): 1447, accessed January 14, 2015, doi:10.1038/srep01447. 
Glossary

\section{Avogadro's number $\left(N_{A}\right)$}

experimentally determined value of the number of entities comprising 1 mole of substance, equal to $6.02210^{23} \mathrm{~mol}^{-1}$

\section{formula mass}

sum of the average masses for all atoms represented in a chemical formula; for covalent compounds, this is also the molecular mass

molar mass

mass in grams of 1 mole of a substance mole

amount of substance containing the same number of atoms, molecules, ions, or other entities as the number of atoms in exactly 12 grams of ${ }^{12} \mathrm{C}$ 
17.

\section{MOLARITY}

\section{Learning Objectives}

By the end of this section, you will be able to:

- Describe the fundamental properties of solutions

- Calculate solution concentrations using molarity

- Perform dilution calculations using the dilution equation

Preceding sections of this chapter focused on the composition of substances: samples of matter that contain only one type of element or compound. However, mixtures-samples of matter containing two or more substances physically combined-are more commonly encountered in nature than are pure substances. Similar to a pure substance, the relative composition of a mixture plays an important role in determining its properties. The relative amount of oxygen in a planet's atmosphere determines its ability to sustain aerobic life. The relative 
amounts of iron, carbon, nickel, and other elements in steel (a mixture known as an "alloy") determine its physical strength and resistance to corrosion. The relative amount of the active ingredient in a medicine determines its effectiveness in achieving the desired pharmacological effect. The relative amount of sugar in a beverage determines its sweetness (see (Figure)). This section will describe one of the most common ways in which the relative compositions of mixtures may be quantified.

Sugar is one of many components in the complex mixture known as coffee. The amount of sugar in a given amount of coffee is an important determinant of the beverage's sweetness. (credit: Jane Whitney)

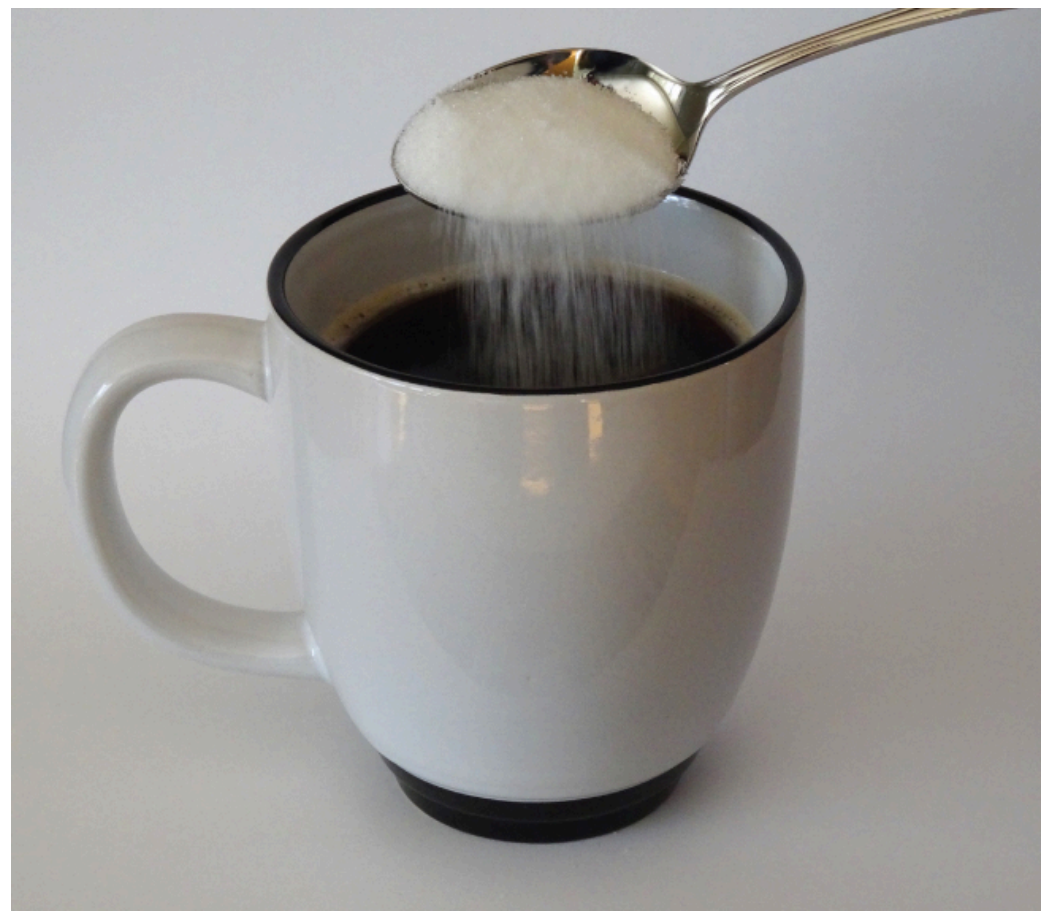

Solutions

Solutions have previously been defined as homogeneous mixtures, meaning that the composition of the mixture 
(and therefore its properties) is uniform throughout its entire volume. Solutions occur frequently in nature and have also been implemented in many forms of manmade technology. A more thorough treatment of solution properties is provided in the chapter on solutions and colloids, but provided here is an introduction to some of the basic properties of solutions.

The relative amount of a given solution component is known as its concentration. Often, though not always, a solution contains one component with a concentration that is significantly greater than that of all other components. This component is called the solvent and may be viewed as the medium in which the other components are dispersed, or dissolved. Solutions in which water is the solvent are, of course, very common on our planet. A solution in which water is the solvent is called an aqueous solution.

A solute is a component of a solution that is typically present at a much lower concentration than the solvent. Solute concentrations are often described with qualitative terms such as dilute (of relatively low concentration) and concentrated (of relatively high concentration).

Concentrations may be quantitatively assessed using a wide variety of measurement units, each convenient for particular applications. Molarity $(M)$ is a useful concentration unit for many applications in chemistry. Molarity is defined as the number of moles of solute in exactly 1 liter $(1 \mathrm{~L})$ of the solution:

$$
M=\frac{\text { mol solute }}{\mathrm{L} \text { solution }}
$$

\section{Calculating Molar Concentrations A 355-mL soft drink}


sample contains 0.133 mol of sucrose (table sugar). What is the molar concentration of sucrose in the beverage?

Solution Since the molar amount of solute and the volume of solution are both given, the molarity can be calculated using the definition of molarity. Per this definition, the solution volume must be converted from $\mathrm{mL}$ to $\mathrm{L}$ :

$$
M=\frac{\text { mol solute }}{\mathrm{L} \text { solution }}=\frac{0.133 \mathrm{~mol}}{355 \mathrm{~mL} \frac{1 \mathrm{~L}}{1000 \mathrm{~mL}}}=0.375 \mathrm{M}
$$

Check Your Learning A teaspoon of table sugar contains about $0.01 \mathrm{~mol}$ sucrose. What is the molarity of sucrose if a teaspoon of sugar has been dissolved in a cup of tea with a volume of $200 \mathrm{~mL}$ ?

Answer:

$0.05 \mathrm{M}$

Deriving Moles and Volumes from Molar Concentrations How much sugar $(\mathrm{mol})$ is contained in a modest $\operatorname{sip}(\sim 10 \mathrm{~mL})$ of the soft drink from (Figure)?

Solution Rearrange the definition of molarity to isolate the quantity sought, moles of sugar, then substitute the value for molarity derived in (Figure), $0.375 \mathrm{M}$ :

$$
\begin{gathered}
M=\frac{\text { mol solute }}{\mathrm{L} \text { solution }} \\
\text { mol solute }=M \text { L solution }
\end{gathered}
$$

mol solute $=0.375 \frac{\text { mol sugar }}{\mathrm{L}}\left(10 \mathrm{~mL} \frac{1 \mathrm{~L}}{1000 \mathrm{~mL}}\right)=0.004 \mathrm{~mol}$ sugar

Check Your Learning What volume $(\mathrm{mL})$ of the sweetened tea described in (Figure) contains the same amount of sugar ( $\mathrm{mol}$ ) as $10 \mathrm{~mL}$ of the soft drink in this example?

Answer:

$80 \mathrm{~mL}$ 
Calculating Molar Concentrations from the Mass of Solute Distilled white vinegar ((Figure)) is a solution of acetic acid, $\mathrm{CH}_{3} \mathrm{CO}_{2} \mathrm{H}$, in water. A 0.500-L vinegar solution contains $25.2 \mathrm{~g}$ of acetic acid. What is the concentration of the acetic acid solution in units of molarity?

Distilled white vinegar is a solution of acetic acid in water.

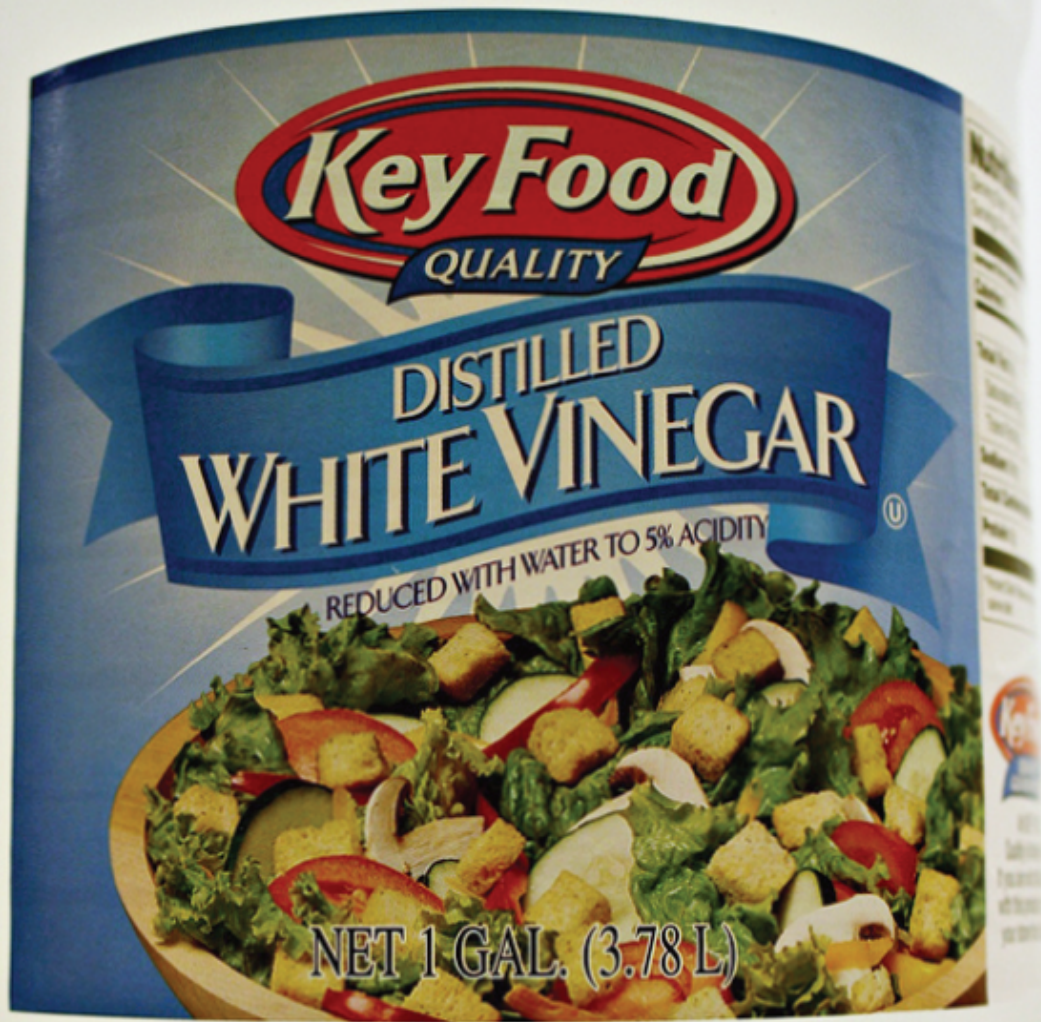

Solution As in previous examples, the definition of molarity is the primary equation used to calculate the quantity sought. Since the mass of solute is provided instead of its molar amount, use the solute's molar mass to obtain the amount of solute in moles:

$$
M=\frac{\text { mol solute }}{\mathrm{L} \text { solution }}=\frac{25.2 g \mathrm{CH}_{3} \mathrm{CO}_{2} \mathrm{H} \frac{1 \mathrm{~mol} \mathrm{CH}_{3} \mathrm{CO}_{2} \mathrm{H}}{60.052 \mathrm{~g} \mathrm{CH}_{3} \mathrm{CO}_{2} \mathrm{H}}}{0.500 \mathrm{~L} \text { solution }}=0.839 \mathrm{M}
$$




$$
\begin{aligned}
& M=\frac{\text { mol solute }}{\mathrm{L} \text { solution }}=0.839 M \\
& M=\frac{0.839 \text { mol solute }}{1.00 \mathrm{~L} \text { solution }}
\end{aligned}
$$

Check Your Learning Calculate the molarity of $6.52 \mathrm{~g}$ of $\mathrm{CoCl}_{2}$ $(128.9 \mathrm{~g} / \mathrm{mol})$ dissolved in an aqueous solution with a total volume of $75.0 \mathrm{~mL}$.

Answer:

$0.674 M$

Determining the Mass of Solute in a Given Volume of Solution How many grams of $\mathrm{NaCl}$ are contained in $0.250 \mathrm{~L}$ of a 5.30-M solution?

Solution The volume and molarity of the solution are specified, so the amount ( $\mathrm{mol}$ ) of solute is easily computed as demonstrated in (Figure):

$$
\begin{gathered}
M=\frac{\text { mol solute }}{\mathrm{L} \text { solution }} \\
\text { mol solute }=M \text { L solution }
\end{gathered}
$$

mol solute $=5.30 \frac{\mathrm{mol} \mathrm{NaCl}}{\mathrm{L}} 0.250 \mathrm{~L}=1.325 \mathrm{~mol} \mathrm{NaCl}$

Finally, this molar amount is used to derive the mass of $\mathrm{NaCl}$ :

$1.325 \mathrm{~mol} \mathrm{NaCl} \frac{58.44 \mathrm{~g} \mathrm{NaCl}}{\mathrm{mol} \mathrm{NaCl}}=77.4 \mathrm{~g} \mathrm{NaCl}$

Check Your Learning How many grams of $\mathrm{CaCl}_{2}$ (110.98 g/ $\mathrm{mol}$ ) are contained in $250.0 \mathrm{~mL}$ of a $0.200-M$ solution of calcium chloride?

Answer:

$5.55 \mathrm{~g} \mathrm{CaCl} 2$

When performing calculations stepwise, as in (Figure), it is important to refrain from rounding any intermediate calculation results, which can lead to rounding errors in the final result. In (Figure), the molar amount of $\mathrm{NaCl}$ 
computed in the first step, $1.325 \mathrm{~mol}$, would be properly rounded to $1.32 \mathrm{~mol}$ if it were to be reported; however, although the last digit (5) is not significant, it must be retained as a guard digit in the intermediate calculation. If the guard digit had not been retained, the final calculation for the mass of $\mathrm{NaCl}$ would have been $77.1 \mathrm{~g}$, a difference of $0.3 \mathrm{~g}$.

In addition to retaining a guard digit for intermediate calculations, rounding errors may also be avoided by performing computations in a single step (see (Figure)). This eliminates intermediate steps so that only the final result is rounded.

Determining the Volume of Solution Containing a Given Mass of Solute In (Figure), the concentration of acetic acid in white vinegar was determined to be $0.839 \mathrm{M}$. What volume of vinegar contains $75.6 \mathrm{~g}$ of acetic acid?

Solution First, use the molar mass to calculate moles of acetic acid from the given mass:

$$
\text { g solute } \frac{\text { mol solute }}{\text { g solute }}=\text { mol solute }
$$

Then, use the molarity of the solution to calculate the volume of solution containing this molar amount of solute:

mol solute $\frac{\mathrm{L} \text { solution }}{\text { mol solute }}=\mathrm{L}$ solution

Combining these two steps into one yields:

g solute $\frac{\text { mol solute }}{\text { g solute }} \frac{\mathrm{L} \text { solution }}{\text { mol solute }}=\mathrm{L}$ solution

$75.6 \mathrm{~g} \mathrm{CH}_{3} \mathrm{CO}_{2} \mathrm{H}\left(\frac{\mathrm{mol} \mathrm{CH}_{3} \mathrm{CO}_{2} \mathrm{H}}{60.05 \mathrm{~g}}\right)\left(\frac{\mathrm{L} \text { solution }}{0.839 \mathrm{~mol} \mathrm{CH}_{3} \mathrm{CO}_{2} \mathrm{H}}\right)=1.50 \mathrm{~L}$ solution

Check Your Learning What volume of a 1.50-M KBr solution contains $66.0 \mathrm{~g} \mathrm{KBr}$ ?

Answer:

$0.370 \mathrm{~L}$ 
Dilution of Solutions

Dilution is the process whereby the concentration of a solution is lessened by the addition of solvent. For example, a glass of iced tea becomes increasingly diluted as the ice melts. The water from the melting ice increases the volume of the solvent (water) and the overall volume of the solution (iced tea), thereby reducing the relative concentrations of the solutes that give the beverage its taste ((Figure)).

Both solutions contain the same mass of copper nitrate. The solution on the right is more dilute because the copper nitrate is dissolved in more solvent. (credit: Mark Ott)

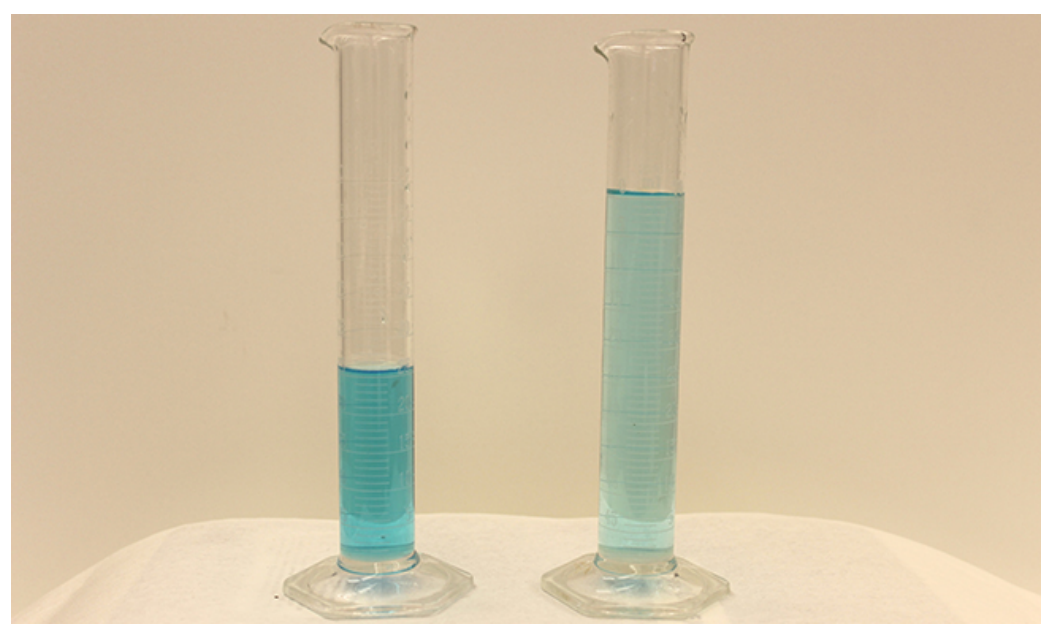

Dilution is also a common means of preparing solutions of a desired concentration. By adding solvent to a measured portion of a more concentrated stock solution, a solution of lesser concentration may be prepared. For example, commercial pesticides are typically sold as solutions in which the active ingredients are far more concentrated than is appropriate for their application. Before they can 
be used on crops, the pesticides must be diluted. This is also a very common practice for the preparation of a number of common laboratory reagents.

A simple mathematical relationship can be used to relate the volumes and concentrations of a solution before and after the dilution process. According to the definition of molarity, the molar amount of solute in a solution $(n)$ is equal to the product of the solution's molarity $(M)$ and its volume in liters $(L)$ :

$n=M L$

Expressions like these may be written for a solution before and after it is diluted:

$n_{1}=M_{1} L_{1}$

$n_{2}=M_{2} L_{2}$

where the subscripts "1" and "2" refer to the solution before and after the dilution, respectively. Since the dilution process does not change the amount of solute in the solution, $n_{1}=n_{2}$. Thus, these two equations may be set equal to one another:

$M_{1} L_{1}=M_{2} L_{2}$

This relation is commonly referred to as the dilution equation. Although this equation uses molarity as the unit of concentration and liters as the unit of volume, other units of concentration and volume may be used as long as the units properly cancel per the factor-label method. Reflecting this versatility, the dilution equation is often written in the more general form:

$C_{1} V_{1}=C_{2} V_{2}$

where $C$ and $V$ are concentration and volume, respectively. 
Use the simulation to explore the relations between solute amount, solution volume, and concentration and to confirm the dilution equation.

Determining the Concentration of a Diluted Solution If 0.850 $\mathrm{L}$ of a 5.00-M solution of copper nitrate, $\mathrm{Cu}\left(\mathrm{NO}_{3}\right)_{2}$, is diluted to a volume of $1.80 \mathrm{~L}$ by the addition of water, what is the molarity of the diluted solution?

Solution The stock concentration, $C_{1}$, and volume, $V_{1}$, are provided as well as the volume of the diluted solution, $V_{2}$. Rearrange the dilution equation to isolate the unknown property, the concentration of the diluted solution, $C_{2}$ :

$$
\begin{gathered}
C_{1} V_{1}=C_{2} V_{2} \\
C_{2}=\frac{C_{1} V_{1}}{V_{2}}
\end{gathered}
$$

Since the stock solution is being diluted by more than twofold (volume is increased from $0.85 \mathrm{~L}$ to $1.80 \mathrm{~L}$ ), the diluted solution's concentration is expected to be less than one-half 5 $M$. This ballpark estimate will be compared to the calculated result to check for any gross errors in computation (for example, such as an improper substitution of the given quantities). Substituting the given values for the terms on the right side of this equation yields:

$$
C_{2}=\frac{0.850 \mathrm{~L} 5.00 \frac{\mathrm{mol}}{\mathrm{L}}}{1.80 \mathrm{~L}}=2.36 \mathrm{M}
$$

This result compares well to our ballpark estimate (it's a bit less than one-half the stock concentration, $5 \mathrm{M}$ ).

Check Your Learning What is the concentration of the solution that results from diluting $25.0 \mathrm{~mL}$ of a $2.04-M$ solution of $\mathrm{CH}_{3} \mathrm{OH}$ to $500.0 \mathrm{~mL}$ ?

Answer:

$0.102 \mathrm{M} \mathrm{CH}_{3} \mathrm{OH}$ 
Volume of a Diluted Solution What volume of $0.12 \mathrm{M} \mathrm{HBr}$ can be prepared from $11 \mathrm{~mL}(0.011 \mathrm{~L})$ of $0.45 \mathrm{M} \mathrm{HBr}$ ?

Solution Provided are the volume and concentration of a stock solution, $V_{1}$ and $C_{1}$, and the concentration of the resultant diluted solution, $C_{2}$. Find the volume of the diluted solution, $V_{2}$ by rearranging the dilution equation to isolate $V_{2}$ :

$$
\begin{gathered}
C_{1} V_{1}=C_{2} V_{2} \\
V_{2}=\frac{C_{1} V_{1}}{C_{2}}
\end{gathered}
$$

Since the diluted concentration $(0.12 \mathrm{M})$ is slightly more than one-fourth the original concentration $(0.45 \mathrm{M})$, the volume of the diluted solution is expected to be roughly four times the original volume, or around $44 \mathrm{~mL}$. Substituting the given values and solving for the unknown volume yields:

$$
\begin{gathered}
V_{2}=\frac{(0.45 M)(0.011 \mathrm{~L})}{(0.12 M)} \\
V_{2}=0.041 \mathrm{~L}
\end{gathered}
$$

The volume of the $0.12-M$ solution is $0.041 \mathrm{~L}(41 \mathrm{~mL})$. The result is reasonable and compares well with the rough estimate.

Check Your Learning A laboratory experiment calls for 0.125 $\mathrm{MHNO}_{3}$. What volume of $0.125 \mathrm{M} \mathrm{HNO}_{3}$ can be prepared from $0.250 \mathrm{~L}$ of $1.88 \mathrm{MHNO}_{3}$ ?

Answer:

$3.76 \mathrm{~L}$

Volume of a Concentrated Solution Needed for Dilution What volume of $1.59 \mathrm{M} \mathrm{KOH}$ is required to prepare $5.00 \mathrm{~L}$ of $0.100 \mathrm{M}$ $\mathrm{KOH}$ ?

Solution Given are the concentration of a stock solution, $C_{1}$, and the volume and concentration of the resultant diluted 
solution, $V_{2}$ and $C_{2}$. Find the volume of the stock solution, $V_{1}$ by rearranging the dilution equation to isolate $V_{1}$ :

$$
\begin{gathered}
C_{1} V_{1}=C_{2} V_{2} \\
V_{1}=\frac{C_{2} V_{2}}{C_{1}}
\end{gathered}
$$

Since the concentration of the diluted solution $0.100 \mathrm{M}$ is roughly one-sixteenth that of the stock solution $(1.59 \mathrm{M})$, the volume of the stock solution is expected to be about onesixteenth that of the diluted solution, or around 0.3 liters. Substituting the given values and solving for the unknown volume yields:

$$
\begin{gathered}
V_{1}=\frac{(0.100 M)(5.00 \mathrm{~L})}{1.59 M} \\
V_{1}=0.314 \mathrm{~L}
\end{gathered}
$$

Thus, $0.314 \mathrm{~L}$ of the $1.59-M$ solution is needed to prepare the desired solution. This result is consistent with the rough estimate.

Check Your Learning What volume of a $0.575-M$ solution of glucose, $\mathrm{C}_{6} \mathrm{H}_{12} \mathrm{O}_{6}$, can be prepared from $50.00 \mathrm{~mL}$ of a $3.00-M$ glucose solution?

Answer:

$0.261 \mathrm{~L}$

Key Concepts and Summary

Solutions are homogeneous mixtures. Many solutions contain one component, called the solvent, in which other components, called solutes, are dissolved. An aqueous solution is one for which the solvent is water. The concentration of a solution is a measure of the relative amount of solute in a given amount of solution. Concentrations may be measured using various units, with one very useful unit being molarity, defined as the 
number of moles of solute per liter of solution. The solute concentration of a solution may be decreased by adding solvent, a process referred to as dilution. The dilution equation is a simple relation between concentrations and volumes of a solution before and after dilution.

Chemistry End of Chapter Exercises

Explain what changes and what stays the same when 1.00 $\mathrm{L}$ of a solution of $\mathrm{NaCl}$ is diluted to $1.80 \mathrm{~L}$.

What information is needed to calculate the molarity of a sulfuric acid solution?

We need to know the number of moles of sulfuric acid dissolved in the solution and the volume of the solution.

A 200-mL sample and a 400-mL sample of a solution of salt have the same molarity. In what ways are the two samples identical? In what ways are these two samples different?

Determine the molarity for each of the following solutions:

(a) $0.444 \mathrm{~mol}$ of $\mathrm{CoCl}_{2}$ in $0.654 \mathrm{~L}$ of solution

(b) $98.0 \mathrm{~g}$ of phosphoric acid, $\mathrm{H}_{3} \mathrm{PO}_{4}$, in $1.00 \mathrm{~L}$ of solution

(c) $0.2074 \mathrm{~g}$ of calcium hydroxide, $\mathrm{Ca}(\mathrm{OH})_{2}$, in $40.00 \mathrm{~mL}$ of solution

(d) $10.5 \mathrm{~kg}$ of $\mathrm{Na}_{2} \mathrm{SO}_{4} \cdot 10 \mathrm{H}_{2} \mathrm{O}$ in $18.60 \mathrm{~L}$ of solution

(e) $7.010^{-3} \mathrm{~mol}$ of $\mathrm{I}_{2}$ in $100.0 \mathrm{~mL}$ of solution

(f) $1.810^{4} \mathrm{mg}$ of $\mathrm{HCl}$ in $0.075 \mathrm{~L}$ of solution 
(a) $0.679 M$; (b) $1.00 M$; (c) $0.06998 M$; (d) $1.75 M$; (e) 0.070 $M$; (f) $6.6 M$

Determine the molarity of each of the following solutions:

(a) $1.457 \mathrm{~mol} \mathrm{KCl}$ in $1.500 \mathrm{~L}$ of solution

(b) $0.515 \mathrm{~g}$ of $\mathrm{H}_{2} \mathrm{SO}_{4}$ in $1.00 \mathrm{~L}$ of solution

(c) $20.54 \mathrm{~g}$ of $\mathrm{Al}\left(\mathrm{NO}_{3}\right)_{3}$ in $1575 \mathrm{~mL}$ of solution

(d) $2.76 \mathrm{~kg}$ of $\mathrm{CuSO}_{4} \cdot 5 \mathrm{H}_{2} \mathrm{O}$ in $1.45 \mathrm{~L}$ of solution

(e) $0.005653 \mathrm{~mol}$ of $\mathrm{Br}_{2}$ in $10.00 \mathrm{~mL}$ of solution

(f) $0.000889 \mathrm{~g}$ of glycine, $\mathrm{C}_{2} \mathrm{H}_{5} \mathrm{NO}_{2}$, in $1.05 \mathrm{~mL}$ of solution

Consider this question: What is the mass of the solute in $0.500 \mathrm{~L}$ of $0.30 \mathrm{M}$ glucose, $\mathrm{C}_{6} \mathrm{H}_{12} \mathrm{O}_{6}$, used for intravenous injection?

(a) Outline the steps necessary to answer the question.

(b) Answer the question.

(a) determine the number of moles of glucose in $0.500 \mathrm{~L}$ of solution; determine the molar mass of glucose; determine the mass of glucose from the number of moles and its molar mass; (b) $27 \mathrm{~g}$

Consider this question: What is the mass of solute in 200.0 $\mathrm{L}$ of a $1.556-M$ solution of $\mathrm{KBr}$ ?

(a) Outline the steps necessary to answer the question.

(b) Answer the question.

Calculate the number of moles and the mass of the solute in each of the following solutions:

(a) $2.00 \mathrm{~L}$ of $18.5 \mathrm{M} \mathrm{H}_{2} \mathrm{SO}_{4}$, concentrated sulfuric acid

(b) $100.0 \mathrm{~mL}$ of $3.810^{-5} \mathrm{M} \mathrm{NaCN}$, the minimum lethal concentration of sodium cyanide in blood serum 
(c) $5.50 \mathrm{~L}$ of $13.3 \mathrm{M} \mathrm{H}_{2} \mathrm{CO}$, the formaldehyde used to "fix" tissue samples

(d) $325 \mathrm{~mL}$ of $1.8 \quad 10^{-6} M \mathrm{FeSO}_{4}$, the minimum concentration of iron sulfate detectable by taste in drinking water

(a) $37.0 \mathrm{~mol} \mathrm{H}_{2} \mathrm{SO}_{4}, 3.6310^{3} \mathrm{~g} \mathrm{H}_{2} \mathrm{SO}_{4}$; (b) $3.810^{-6} \mathrm{~mol}$ $\mathrm{NaCN}, 1.910^{-4} \mathrm{~g} \mathrm{NaCN}$; (c) $73.2 \mathrm{~mol} \mathrm{H}_{2} \mathrm{CO}, 2.20 \mathrm{~kg} \mathrm{H} \mathrm{CO}$; (d) $5.910^{-7} \mathrm{~mol} \mathrm{FeSO}_{4}, 8.910^{-5} \mathrm{~g} \mathrm{FeSO}_{4}$

Calculate the number of moles and the mass of the solute in each of the following solutions:

(a) $325 \mathrm{~mL}$ of $8.2310^{-5} \mathrm{M} \mathrm{KI}$, a source of iodine in the diet

(b) $75.0 \mathrm{~mL}$ of $2.210^{-5} \mathrm{M} \mathrm{H}_{2} \mathrm{SO}_{4}$, a sample of acid rain

(c) $0.2500 \mathrm{~L}$ of $0.1135 \mathrm{M} \mathrm{K}_{2} \mathrm{CrO}_{4}$, an analytical reagent used in iron assays

(d) $10.5 \mathrm{~L}$ of $3.716 \mathrm{M}\left(\mathrm{NH}_{4}\right)_{2} \mathrm{SO}_{4}$, a liquid fertilizer

Consider this question: What is the molarity of $\mathrm{KMnO}_{4}$ in a solution of $0.0908 \mathrm{~g}$ of $\mathrm{KMnO}_{4}$ in $0.500 \mathrm{~L}$ of solution?

(a) Outline the steps necessary to answer the question.

(b) Answer the question.

(a) Determine the molar mass of $\mathrm{KMnO}_{4}$; determine the number of moles of $\mathrm{KMnO}_{4}$ in the solution; from the number of moles and the volume of solution, determine the molarity; (b) $1.1510^{-3} M$

Consider this question: What is the molarity of $\mathrm{HCl}$ if $35.23 \mathrm{~mL}$ of a solution of $\mathrm{HCl}$ contain $0.3366 \mathrm{~g}$ of $\mathrm{HCl}$ ?

(a) Outline the steps necessary to answer the question.

(b) Answer the question. 
Calculate the molarity of each of the following solutions:

(a) $0.195 \mathrm{~g}$ of cholesterol, $\mathrm{C}_{27} \mathrm{H}_{46} \mathrm{O}$, in $0.100 \mathrm{~L}$ of serum, the average concentration of cholesterol in human serum

(b) $4.25 \mathrm{~g}$ of $\mathrm{NH}_{3}$ in $0.500 \mathrm{~L}$ of solution, the concentration of $\mathrm{NH}_{3}$ in household ammonia

(c) $1.49 \mathrm{~kg}$ of isopropyl alcohol, $\mathrm{C}_{3} \mathrm{H}_{7} \mathrm{OH}$, in $2.50 \mathrm{~L}$ of solution, the concentration of isopropyl alcohol in rubbing alcohol

(d) $0.029 \mathrm{~g}$ of $\mathrm{I}_{2}$ in $0.100 \mathrm{~L}$ of solution, the solubility of $\mathrm{I}_{2}$ in water at $20^{\circ} \mathrm{C}$

(a) $5.0410^{-3} \mathrm{M}$; (b) $0.499 \mathrm{M}$; (c) $9.92 \mathrm{M}$; (d) $1.110^{-3} \mathrm{M}$

Calculate the molarity of each of the following solutions:

(a) $293 \mathrm{~g} \mathrm{HCl}$ in $666 \mathrm{~mL}$ of solution, a concentrated $\mathrm{HCl}$ solution

(b) $2.026 \mathrm{~g} \mathrm{FeCl}_{3}$ in $0.1250 \mathrm{~L}$ of a solution used as an unknown in general chemistry laboratories

(c) $0.001 \mathrm{mg} \mathrm{Cd}^{2+}$ in $0.100 \mathrm{~L}$, the maximum permissible concentration of cadmium in drinking water

(d) $0.0079 \mathrm{~g} \mathrm{C}_{7} \mathrm{H}_{5} \mathrm{SNO}_{3}$ in one ounce $(29.6 \mathrm{~mL})$, the concentration of saccharin in a diet soft drink.

There is about $1.0 \mathrm{~g}$ of calcium, as $\mathrm{Ca}^{2+}$, in $1.0 \mathrm{~L}$ of milk. What is the molarity of $\mathrm{Ca}^{2+}$ in milk?

$0.025 M$

What volume of a $1.00-M \mathrm{Fe}\left(\mathrm{NO}_{3}\right)_{3}$ solution can be diluted to prepare $1.00 \mathrm{~L}$ of a solution with a concentration of $0.250 M$ ?

If $0.1718 \mathrm{~L}$ of a $0.3556-M \mathrm{C}_{3} \mathrm{H}_{7} \mathrm{OH}$ solution is diluted to 
a concentration of $0.1222 M$, what is the volume of the resulting solution?

$0.5000 \mathrm{~L}$

If $4.12 \mathrm{~L}$ of a $0.850 \mathrm{M}-\mathrm{H}_{3} \mathrm{PO}_{4}$ solution is be diluted to a volume of $10.00 \mathrm{~L}$, what is the concentration of the resulting solution?

What volume of a $0.33-M \mathrm{C}_{12} \mathrm{H}_{22} \mathrm{O}_{11}$ solution can be diluted to prepare $25 \mathrm{~mL}$ of a solution with a concentration of $0.025 \mathrm{M}$ ?

\section{$1.9 \mathrm{~mL}$}

What is the concentration of the $\mathrm{NaCl}$ solution that results when $0.150 \mathrm{~L}$ of a $0.556-M$ solution is allowed to evaporate until the volume is reduced to $0.105 \mathrm{~L}$ ?

What is the molarity of the diluted solution when each of the following solutions is diluted to the given final volume?

(a) $1.00 \mathrm{~L}$ of a $0.250-M$ solution of $\mathrm{Fe}\left(\mathrm{NO}_{3}\right)_{3}$ is diluted to a final volume of $2.00 \mathrm{~L}$

(b) $0.5000 \mathrm{~L}$ of a $0.1222-M$ solution of $\mathrm{C}_{3} \mathrm{H}_{7} \mathrm{OH}$ is diluted to a final volume of $1.250 \mathrm{~L}$

(c) $2.35 \mathrm{~L}$ of a $0.350-M$ solution of $\mathrm{H}_{3} \mathrm{PO}_{4}$ is diluted to a final volume of $4.00 \mathrm{~L}$

(d) $22.50 \mathrm{~mL}$ of a $0.025-M$ solution of $\mathrm{C}_{12} \mathrm{H}_{22} \mathrm{O}_{11}$ is diluted to $100.0 \mathrm{~mL}$

(a) $0.125 \mathrm{M}$; (b) $0.04888 \mathrm{M}$; (c) $0.206 \mathrm{M}$; (d) $0.0056 \mathrm{M}$ 
What is the final concentration of the solution produced when $225.5 \mathrm{~mL}$ of a $0.09988-M$ solution of $\mathrm{Na}_{2} \mathrm{CO}_{3}$ is allowed to evaporate until the solution volume is reduced to $45.00 \mathrm{~mL}$ ?

A 2.00-L bottle of a solution of concentrated $\mathrm{HCl}$ was purchased for the general chemistry laboratory. The solution contained $868.8 \mathrm{~g}$ of $\mathrm{HCl}$. What is the molarity of the solution?

\section{$11.9 M$}

An experiment in a general chemistry laboratory calls for a 2.00-M solution of $\mathrm{HCl}$. How many $\mathrm{mL}$ of $11.9 \mathrm{M} \mathrm{HCl}$ would be required to make $250 \mathrm{~mL}$ of $2.00 \mathrm{M} \mathrm{HCl}$ ?

What volume of a $0.20-M \mathrm{~K}_{2} \mathrm{SO}_{4}$ solution contains $57 \mathrm{~g}$ of $\mathrm{K}_{2} \mathrm{SO}_{4}$ ?

\section{$1.6 \mathrm{~L}$}

The US Environmental Protection Agency (EPA) places limits on the quantities of toxic substances that may be discharged into the sewer system. Limits have been established for a variety of substances, including hexavalent chromium, which is limited to $0.50 \mathrm{mg} / \mathrm{L}$. If an industry is discharging hexavalent chromium as potassium dichromate $\left(\mathrm{K}_{2} \mathrm{Cr}_{2} \mathrm{O}_{7}\right)$, what is the maximum permissible molarity of that substance?

Key Equations

$$
\text { - } M=\frac{\text { mol solute }}{\text { L solution }}
$$


- $C_{1} V_{1}=C_{2} V_{2}$

Glossary

aqueous solution

solution for which water is the solvent

concentrated

qualitative term for a solution containing solute at a relatively high concentration

\section{concentration}

quantitative measure of the relative amounts of solute and solvent present in a solution

\section{dilute}

qualitative term for a solution containing solute at a relatively low concentration

\section{dilution}

process of adding solvent to a solution in order to lower the concentration of solutes

\section{dissolved}

describes the process by which solute components are dispersed in a solvent molarity (M) unit of concentration, defined as the number of moles of solute dissolved in 1 liter of solution

\section{solute}

solution component present in a concentration less than that of the solvent solvent solution component present in a 
concentration that is higher relative to other components 


\section{8.}

\section{OTHER UNITS FOR SOLUTION CONCENTRATIONS}

\section{Learning Objectives}

By the end of this section, you will be able to:

- $\quad$ Define the concentration units of mass percentage, volume percentage, mass-volume percentage, parts-per-million (ppm), and parts-perbillion ( $\mathrm{ppb})$

- Perform computations relating a solution's concentration and its components' volumes and/or masses using these units

The previous section introduced molarity, a very useful measurement unit for evaluating the concentration of solutions. However, molarity is only one measure of 
concentration. This section will describe some other units of concentration that are commonly used in various applications, either for convenience or by convention.

\section{Mass Percentage}

Earlier in this chapter, percent composition was introduced as a measure of the relative amount of a given element in a compound. Percentages are also commonly used to express the composition of mixtures, including solutions. The mass percentage of a solution component is defined as the ratio of the component's mass to the solution's mass, expressed as a percentage:

mass percentage $=\frac{\text { mass of component }}{\text { mass of solution }} 100$

Mass percentage is also referred to by similar names such as percent mass, percent weight, weight/weight percent, and other variations on this theme. The most common symbol for mass percentage is simply the percent sign, \%, although more detailed symbols are often used including \%mass, \%weight, and (w/w)\%. Use of these more detailed symbols can prevent confusion of mass percentages with other types of percentages, such as volume percentages (to be discussed later in this section).

Mass percentages are popular concentration units for consumer products. The label of a typical liquid bleach bottle ((Figure)) cites the concentration of its active ingredient, sodium hypochlorite $(\mathrm{NaOCl})$, as being $7.4 \%$. A 100.0-g sample of bleach would therefore contain $7.4 \mathrm{~g}$ of $\mathrm{NaOCl}$.

Liquid bleach is an aqueous solution of sodium hypochlorite $(\mathrm{NaOCl})$. This brand has a concentration of 
7.4\% $\mathrm{NaOCl}$ by mass.

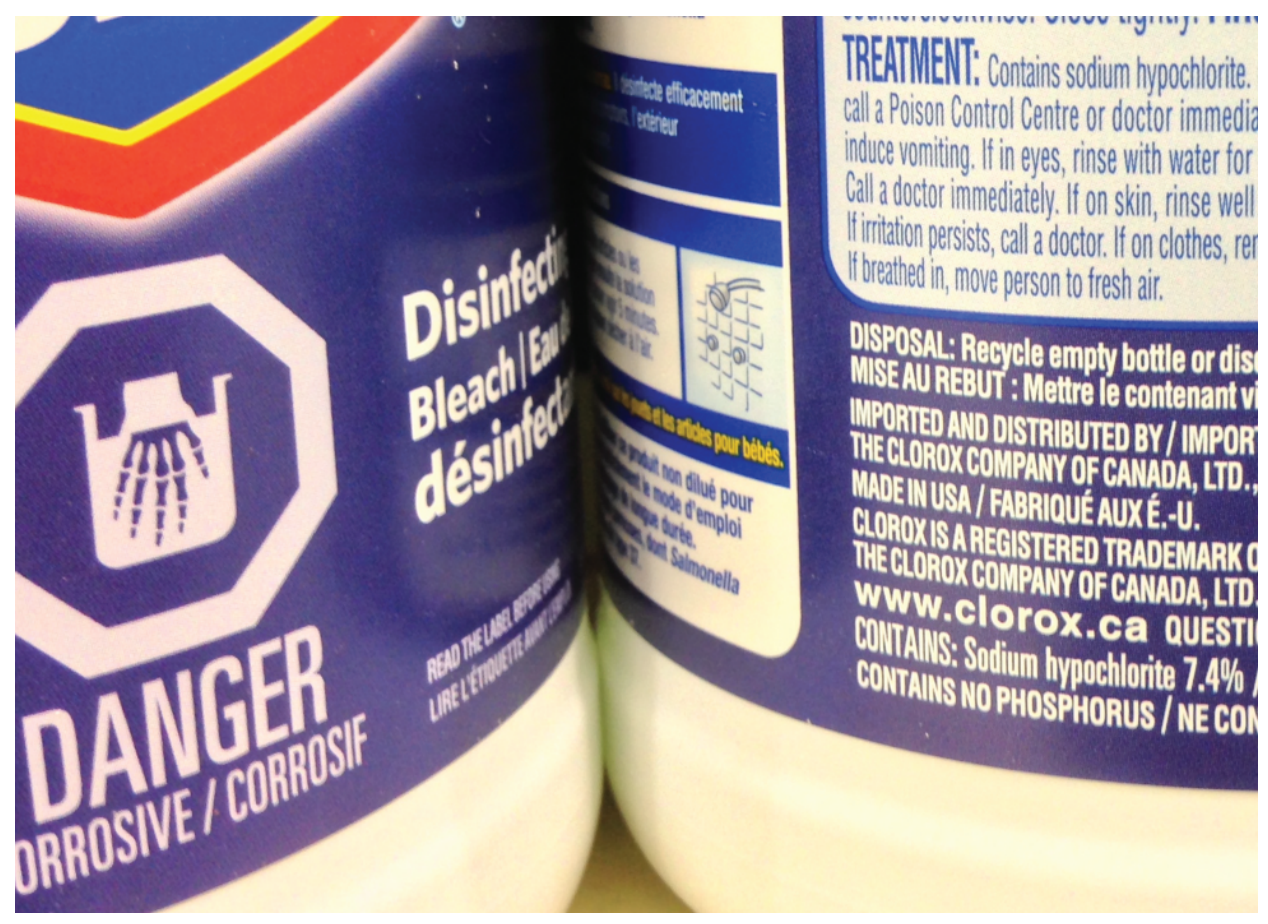

Calculation of Percent by Mass A 5.0-g sample of spinal fluid contains $3.75 \mathrm{mg}(0.00375 \mathrm{~g})$ of glucose. What is the percent by mass of glucose in spinal fluid?

Solution The spinal fluid sample contains roughly $4 \mathrm{mg}$ of glucose in $5000 \mathrm{mg}$ of fluid, so the mass fraction of glucose should be a bit less than one part in 1000, or about $0.1 \%$. Substituting the given masses into the equation defining mass percentage yields:

The computed mass percentage agrees with our rough estimate (it's a bit less than $0.1 \%$ ).

Note that while any mass unit may be used to compute a mass percentage ( $\mathrm{mg}, \mathrm{g}, \mathrm{kg}, \mathrm{oz}$, and so on), the same unit must be used for both the solute and the solution so that the mass units cancel, yielding a dimensionless ratio. In this case, the solute mass unit in the numerator was converted from $\mathrm{mg}$ to $\mathrm{g}$ 
to match the units in the denominator. Alternatively, the spinal fluid mass unit in the denominator could have been converted from $g$ to $\mathrm{mg}$ instead. As long as identical mass units are used for both solute and solution, the computed mass percentage will be correct.

Check Your Learning A bottle of a tile cleanser contains $135 \mathrm{~g}$ of $\mathrm{HCl}$ and $775 \mathrm{~g}$ of water. What is the percent by mass of $\mathrm{HCl}$ in this cleanser?

Answer:

$14.8 \%$

\section{Calculations using Mass Percentage "Concentrated"} hydrochloric acid is an aqueous solution of $37.2 \% \mathrm{HCl}$ that is commonly used as a laboratory reagent. The density of this solution is $1.19 \mathrm{~g} / \mathrm{mL}$. What mass of $\mathrm{HCl}$ is contained in $0.500 \mathrm{~L}$ of this solution?

Solution The $\mathrm{HCl}$ concentration is near $40 \%$, so a $100-\mathrm{g}$ portion of this solution would contain about $40 \mathrm{~g}$ of $\mathrm{HCl}$. Since the solution density isn't greatly different from that of water (1 $\mathrm{g} / \mathrm{mL})$, a reasonable estimate of the $\mathrm{HCl}$ mass in $500 \mathrm{~g}(0.5 \mathrm{~L})$ of the solution is about five times greater than that in a $100 \mathrm{~g}$ portion, or $540=200 \mathrm{~g}$. In order to derive the mass of solute in a solution from its mass percentage, the mass of the solution must be known. Using the solution density given, convert the solution's volume to mass, and then use the given mass percentage to calculate the solute mass. This mathematical approach is outlined in this flowchart:

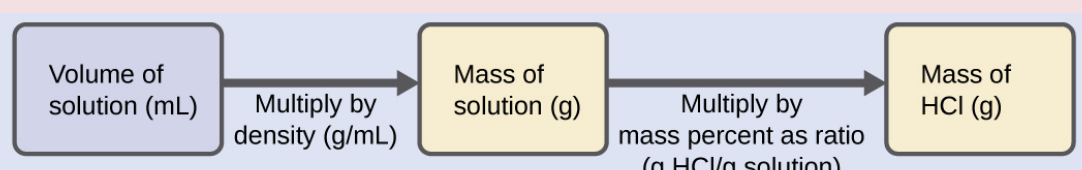
(g HCl/g solution)

For proper unit cancellation, the $0.500-\mathrm{L}$ volume is 
converted into $500 \mathrm{~mL}$, and the mass percentage is expressed as a ratio, $37.2 \mathrm{~g} \mathrm{HCl} / \mathrm{g}$ solution:

$500 \mathrm{~mL}$ solution $\left(\frac{1.19 \mathrm{~g} \text { solution }}{\mathrm{mL} \text { solution }}\right)\left(\frac{37.2 \mathrm{~g} \mathrm{HCl}}{100 \mathrm{~g} \text { solution }}\right)=221 \mathrm{~g} \mathrm{HCl}$ This mass of $\mathrm{HCl}$ is consistent with our rough estimate of approximately $200 \mathrm{~g}$.

Check Your Learning What volume of concentrated $\mathrm{HCl}$ solution contains $125 \mathrm{~g}$ of $\mathrm{HCl}$ ?

Answer:

$282 \mathrm{~mL}$

\section{Volume Percentage}

Liquid volumes over a wide range of magnitudes are conveniently measured using common and relatively inexpensive laboratory equipment. The concentration of a solution formed by dissolving a liquid solute in a liquid solvent is therefore often expressed as a volume percentage, \%vol or $(\mathrm{v} / \mathrm{v}) \%$ :

volume percentage $=\frac{\text { volume solute }}{\text { volume solution }} 100$

\section{Calculations using Volume Percentage Rubbing alcohol} (isopropanol) is usually sold as a $70 \%$ vol aqueous solution. If the density of isopropyl alcohol is $0.785 \mathrm{~g} / \mathrm{mL}$, how many grams of isopropyl alcohol are present in a $355 \mathrm{~mL}$ bottle of rubbing alcohol?

Solution Per the definition of volume percentage, the isopropanol volume is $70 \%$ of the total solution volume. Multiplying the isopropanol volume by its density yields the requested mass:

(355 mL solution) $\left(\frac{70 \mathrm{~mL} \text { isopropyl alcohol }}{100 \mathrm{~mL} \text { solution }}\right)\left(\frac{0.785 \mathrm{~g} \text { isopropyl alcohol }}{1 \mathrm{~mL} \text { isopropyl alcohol }}\right)=195 \mathrm{~g}$ isopropyl alchol Check Your Learning Wine is approximately $12 \%$ ethanol 
$\left(\mathrm{CH}_{3} \mathrm{CH}_{2} \mathrm{OH}\right)$ by volume. Ethanol has a molar mass of $46.06 \mathrm{~g} /$ mol and a density $0.789 \mathrm{~g} / \mathrm{mL}$. How many moles of ethanol are present in a $750-\mathrm{mL}$ bottle of wine?

Answer:

1.5 mol ethanol

Mass-Volume Percentage

"Mixed" percentage units, derived from the mass of solute and the volume of solution, are popular for certain biochemical and medical applications. A mass-volume percent is a ratio of a solute's mass to the solution's volume expressed as a percentage. The specific units used for solute mass and solution volume may vary, depending on the solution. For example, physiological saline solution, used to prepare intravenous fluids, has a concentration of $0.9 \%$ mass/volume $(\mathrm{m} / \mathrm{v})$, indicating that the composition is $0.9 \mathrm{~g}$ of solute per $100 \mathrm{~mL}$ of solution. The concentration of glucose in blood (commonly referred to as "blood sugar") is also typically expressed in terms of a mass-volume ratio. Though not expressed explicitly as a percentage, its concentration is usually given in milligrams of glucose per deciliter $(100 \mathrm{~mL})$ of blood ((Figure)).

"Mixed" mass-volume units are commonly encountered in medical settings. (a) The $\mathrm{NaCl}$ concentration of physiological saline is $0.9 \%(\mathrm{~m} / \mathrm{v})$. (b) This device measures glucose levels in a sample of blood. The normal range for glucose concentration in blood (fasting) is around $70-100 \mathrm{mg} / \mathrm{dL}$. (credit a: modification of work by "The National Guard"/Flickr; credit b: modification of work by Biswarup Ganguly) 
Two pictures are shown labeled a and b. Picture a depicts a clear, colorless solution in a plastic bag being held in a person's hand. Picture $b$ shows a person's hand holding a detection meter with a digital readout screen while another hand holds someone's finger up to the end of the meter. The meter is pressed to the drop of blood that is at the end of the person's finger.

Parts per Million and Parts per Billion

Very low solute concentrations are often expressed using appropriately small units such as parts per million (ppm) or parts per billion (ppb). Like percentage ("part per hundred") units, ppm and ppb may be defined in terms of masses, volumes, or mixed mass-volume units. There are also ppm and ppb units defined with respect to numbers of atoms and molecules.

The mass-based definitions of ppm and ppb are given here:

$\mathrm{ppm}=\frac{\text { mass solute }}{\text { mass solution }} 10^{6} \mathrm{ppm}$

$\mathrm{ppb}=\frac{\text { mass solute }}{\text { mass solution }} 10^{9} \mathrm{ppb}$

Both ppm and ppb are convenient units for reporting the concentrations of pollutants and other trace contaminants in water. Concentrations of these contaminants are typically very low in treated and natural waters, and their levels cannot exceed relatively low concentration thresholds without causing adverse effects on health and wildlife. For example, the EPA has identified the maximum safe level of fluoride ion in tap water to be $4 \mathrm{ppm}$. Inline water filters are designed to reduce the 
concentration of fluoride and several other trace-level contaminants in tap water ((Figure)).

(a) In some areas, trace-level concentrations of contaminants can render unfiltered tap water unsafe for drinking and cooking. (b) Inline water filters reduce the concentration of solutes in tap water. (credit a: modification of work by Jenn Durfey; credit b: modification of work by "vastateparkstaff"/Wikimedia commons)

Two pictures are shown labeled $\mathrm{a}$ and $\mathrm{b}$. Picture $\mathrm{a}$ is $\mathrm{a}$ close-up shot of water coming out of a faucet. Picture $\mathrm{b}$ shows a machine with the words, "Filtered Water Dispenser." This machine appears to be inside a refrigerator.

Calculation of Parts per Million and Parts per Billion Concentrations According to the EPA, when the concentration of lead in tap water reaches $15 \mathrm{ppb}$, certain remedial actions must be taken. What is this concentration in ppm? At this concentration, what mass of lead ( $\mu$ g) would be contained in a typical glass of water $(300 \mathrm{~mL})$ ?

Solution The definitions of the ppm and ppb units may be used to convert the given concentration from $\mathrm{ppb}$ to $\mathrm{ppm}$. Comparing these two unit definitions shows that ppm is 1000 times greater than ppb $\left(1 \mathrm{ppm}=10^{3} \mathrm{ppb}\right)$. Thus:

$$
15 \mathrm{ppb} \frac{1 \mathrm{ppm}}{10^{3} \mathrm{ppb}}=0.015 \mathrm{ppm}
$$

The definition of the ppb unit may be used to calculate the requested mass if the mass of the solution is provided. Since the volume of solution ( $300 \mathrm{~mL}$ ) is given, its density must be used to derive the corresponding mass. Assume the density of tap water to be roughly the same as that of pure water $(\sim 1.00$ 
$\mathrm{g} / \mathrm{mL}$ ), since the concentrations of any dissolved substances should not be very large. Rearranging the equation defining the ppb unit and substituting the given quantities yields:

$$
\begin{gathered}
\mathrm{ppb}=\frac{\text { mass solute }}{\text { mass solution }} 10^{9} \mathrm{ppb} \\
\text { mass solute }=\frac{\text { ppb mass solution }}{10^{9} \mathrm{ppb}}
\end{gathered}
$$

mass solute $=\frac{15 \mathrm{ppb} 300 \mathrm{~mL} \frac{1.00 \mathrm{~g}}{\mathrm{~mL}}}{10^{9} \mathrm{ppb}}=4.510^{-6} \mathrm{~g}$

Finally, convert this mass to the requested unit of micrograms:

$$
4.510^{-6} \mathrm{~g} \frac{1 \mathrm{~g}}{10^{-6} \mathrm{~g}}=4.5 \mathrm{~g}
$$

Check Your Learning A 50.0-g sample of industrial wastewater was determined to contain $0.48 \mathrm{mg}$ of mercury. Express the mercury concentration of the wastewater in ppm and ppb units.

Answer:

$9.6 \mathrm{ppm}, 9600 \mathrm{ppb}$

\section{Section Summary}

In addition to molarity, a number of other solution concentration units are used in various applications. Percentage concentrations based on the solution components' masses, volumes, or both are useful for expressing relatively high concentrations, whereas lower concentrations are conveniently expressed using ppm or ppb units. These units are popular in environmental, medical, and other fields where mole-based units such as molarity are not as commonly used.

Consider this question: What mass of a concentrated 
solution of nitric acid (68.0\% $\mathrm{HNO}_{3}$ by mass) is needed to prepare $400.0 \mathrm{~g}$ of a $10.0 \%$ solution of $\mathrm{HNO}_{3}$ by mass?

(a) Outline the steps necessary to answer the question.

(b) Answer the question.

(a) The dilution equation can be used, appropriately modified to accommodate mass-based concentration units: . This equation can be rearranged to isolate mass 1 and the given quantities substituted into this equation. (b) $58.8 \mathrm{~g}$

What mass of a $4.00 \% \mathrm{NaOH}$ solution by mass contains $15.0 \mathrm{~g}$ of $\mathrm{NaOH}$ ?

What mass of solid $\mathrm{NaOH}(97.0 \% \mathrm{NaOH}$ by mass) is required to prepare $1.00 \mathrm{~L}$ of a $10.0 \%$ solution of $\mathrm{NaOH}$ by mass? The density of the $10.0 \%$ solution is $1.109 \mathrm{~g} / \mathrm{mL}$.

\section{$114 \mathrm{~g}$}

What mass of $\mathrm{HCl}$ is contained in $45.0 \mathrm{~mL}$ of an aqueous $\mathrm{HCl}$ solution that has a density of $1.19 \mathrm{~g} \mathrm{~cm}^{-3}$ and contains $37.21 \% \mathrm{HCl}$ by mass?

The hardness of water (hardness count) is usually expressed in parts per million (by mass) of $\mathrm{CaCO}_{3}$, which is equivalent to milligrams of $\mathrm{CaCO}_{3}$ per liter of water. What is the molar concentration of $\mathrm{Ca}^{2+}$ ions in a water sample with a hardness count of $175 \mathrm{mg} \mathrm{CaCO} / \mathrm{L}$ ?

\section{$1.7510^{-3} M$}

The level of mercury in a stream was suspected to be 
above the minimum considered safe (1 part per billion by weight). An analysis indicated that the concentration was 0.68 parts per billion. Assume a density of $1.0 \mathrm{~g} / \mathrm{mL}$ and calculate the molarity of mercury in the stream.

In Canada and the United Kingdom, devices that measure blood glucose levels provide a reading in millimoles per liter. If a measurement of $5.3 \mathrm{~m} M$ is observed, what is the concentration of glucose $\left(\mathrm{C}_{6} \mathrm{H}_{12} \mathrm{O}_{6}\right)$ in $\mathrm{mg} / \mathrm{dL}$ ?

$95 \mathrm{mg} / \mathrm{dL}$

A throat spray is $1.40 \%$ by mass phenol, $\mathrm{C}_{6} \mathrm{H}_{5} \mathrm{OH}$, in water. If the solution has a density of $0.9956 \mathrm{~g} / \mathrm{mL}$, calculate the molarity of the solution.

Copper(I) iodide (CuI) is often added to table salt as a dietary source of iodine. How many moles of $\mathrm{CuI}$ are contained in $1.00 \mathrm{lb}$ (454 g) of table salt containing 0.0100\% CuI by mass?

\section{$2.3810^{-4} \mathrm{~mol}$}

A cough syrup contains $5.0 \%$ ethyl alcohol, $\mathrm{C}_{2} \mathrm{H}_{5} \mathrm{OH}$, by mass. If the density of the solution is $0.9928 \mathrm{~g} / \mathrm{mL}$, determine the molarity of the alcohol in the cough syrup.

D5W is a solution used as an intravenous fluid. It is a 5.0\% by mass solution of dextrose $\left(\mathrm{C}_{6} \mathrm{H}_{12} \mathrm{O}_{6}\right)$ in water. If the density of $\mathrm{D} 5 \mathrm{~W}$ is $1.029 \mathrm{~g} / \mathrm{mL}$, calculate the molarity of dextrose in the solution.

$0.29 \mathrm{~mol}$ 
Find the molarity of a $40.0 \%$ by mass aqueous solution of sulfuric acid, $\mathrm{H}_{2} \mathrm{SO}_{4}$, for which the density is $1.3057 \mathrm{~g} / \mathrm{mL}$.

Key Equations

- Percent by mass $=\frac{\text { mass of solute }}{\text { mass of solution }} 100$

- $\mathrm{ppm}=\frac{\text { mass solute }}{\text { mass solution }} 10^{6} \mathrm{ppm}$

- $\mathrm{ppb}=\frac{\text { mass solute }}{\text { mass solution }} 10^{9} \mathrm{ppb}$

Glossary

mass percentage

ratio of solute-to-solution mass expressed

as a percentage

mass-volume percent

ratio of solute mass to solution volume, expressed as a percentage

parts per billion (ppb)

ratio of solute-to-solution mass multiplied by $10^{9}$

parts per million (ppm)

ratio of solute-to-solution mass multiplied by $10^{6}$

\section{volume percentage}

ratio of solute-to-solution volume

expressed as a percentage 



\section{PART IV}

\section{STOICHIOMETRY OF CHEMICAL REACTIONS}



19.

\section{INTRODUCTION}

\section{Learning Objectives}

- Writing and Balancing Chemical Equations

- Classifying Chemical Reactions

- Reaction Stoichiometry

- Reaction Yields

Many modern rocket fuels are solid mixtures of substances combined in carefully measured amounts and ignited to yield a thrust-generating chemical reaction. (credit: modification of work by NASA) 


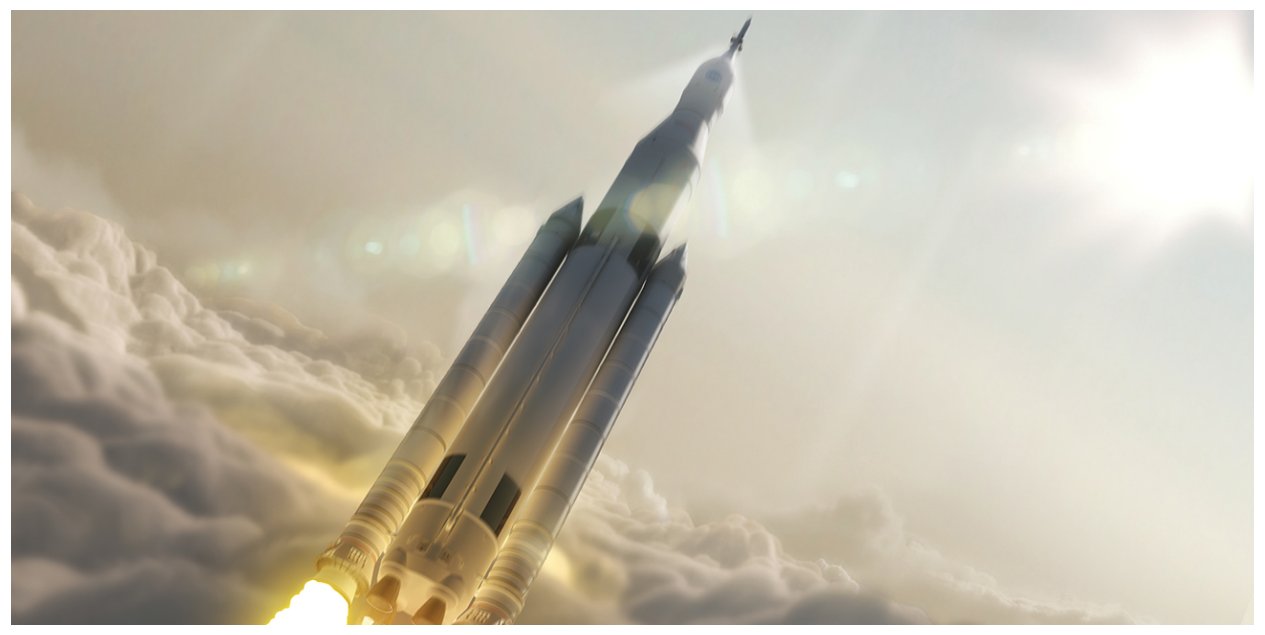

Solid-fuel rockets are a central feature in the world's space exploration programs, including the new Space Launch System being developed by the National Aeronautics and Space Administration (NASA) to replace the retired Space Shuttle fleet ((Figure)). The engines of these rockets rely on carefully prepared solid mixtures of chemicals combined in precisely measured amounts. Igniting the mixture initiates a vigorous chemical reaction that rapidly generates large amounts of gaseous products. These gases are ejected from the rocket engine through its nozzle, providing the thrust needed to propel heavy payloads into space. Both the nature of this chemical reaction and the relationships between the amounts of the substances being consumed and produced by the reaction are critically important considerations that determine the success of the technology. This chapter will describe how to symbolize chemical reactions using chemical equations, how to classify some common chemical reactions by identifying patterns of reactivity, and how to determine the quantitative relations between the amounts of substances involved in chemical reactions - that is, the reaction stoichiometry. 
20.

\section{WRITING AND BALANCING CHEMICAL EQUATIONS}

Note: One of the equation editors used in this text displays chemical reactions with no arrow between reactants and products (ie. If there is a space between molecules but no "+" sign between those molecules, then that is where the reaction arrow belongs). For example, $\mathrm{C}$ $+\mathrm{O}_{2} \mathrm{CO}_{2}$ indicates the reaction $\mathrm{C}+\mathrm{O}_{2} \rightarrow \mathrm{CO}_{2}$

\section{Learning Objectives}

By the end of this section, you will be able to:

- Derive chemical equations from narrative descriptions of chemical reactions.

- Write and balance chemical equations in molecular, total ionic, and net ionic formats.

An earlier chapter of this text introduced the use of 
element symbols to represent individual atoms. When atoms gain or lose electrons to yield ions, or combine with other atoms to form molecules, their symbols are modified or combined to generate chemical formulas that appropriately represent these species. Extending this symbolism to represent both the identities and the relative quantities of substances undergoing a chemical (or physical) change involves writing and balancing a chemical equation. Consider as an example the reaction between one methane molecule $\left(\mathrm{CH}_{4}\right)$ and two diatomic oxygen molecules $\left(\mathrm{O}_{2}\right)$ to produce one carbon dioxide molecule $\left(\mathrm{CO}_{2}\right)$ and two water molecules $\left(\mathrm{H}_{2} \mathrm{O}\right)$. The chemical equation representing this process is provided in the upper half of (Figure), with space-filling molecular models shown in the lower half of the figure.

The reaction between methane and oxygen to yield carbon dioxide and water (shown at bottom) may be represented by a chemical equation using formulas (top).

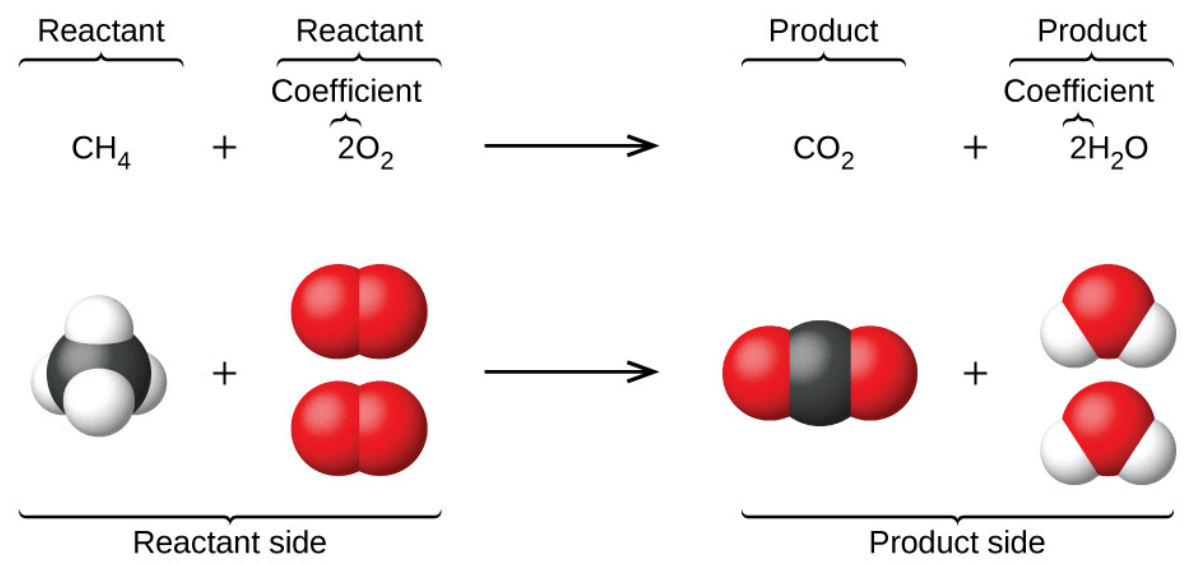

This example illustrates the fundamental aspects of any chemical equation:

1. The substances undergoing reaction are called 
reactants, and their formulas are placed on the left side of the equation.

2. The substances generated by the reaction are called products, and their formulas are placed on the right side of the equation.

3. Plus signs $(+)$ separate individual reactant and product formulas, and an arrow () separates the reactant and product (left and right) sides of the equation.

4. The relative numbers of reactant and product species are represented by coefficients (numbers placed immediately to the left of each formula). A coefficient of 1 is typically omitted.

It is common practice to use the smallest possible wholenumber coefficients in a chemical equation, as is done in this example. Realize, however, that these coefficients represent the relative numbers of reactants and products, and, therefore, they may be correctly interpreted as ratios. Methane and oxygen react to yield carbon dioxide and water in a $1: 2: 1: 2$ ratio. This ratio is satisfied if the numbers of these molecules are, respectively, 1-2-1-2, or 2-4-2-4, or 3-6-3-6, and so on ((Figure)). Likewise, these coefficients may be interpreted with regard to any amount (number) unit, and so this equation may be correctly read in many ways, including:

- One methane molecule and two oxygen molecules react to yield one carbon dioxide molecule and two water molecules.

- One dozen methane molecules and two dozen 
oxygen molecules react to yield one dozen carbon dioxide molecules and two dozen water molecules.

- One mole of methane molecules and 2 moles of oxygen molecules react to yield 1 mole of carbon dioxide molecules and 2 moles of water molecules.

Regardless of the absolute numbers of molecules involved, the ratios between numbers of molecules of each species that react (the reactants) and molecules of each species that form (the products) are the same and are given by the chemical reaction equation.

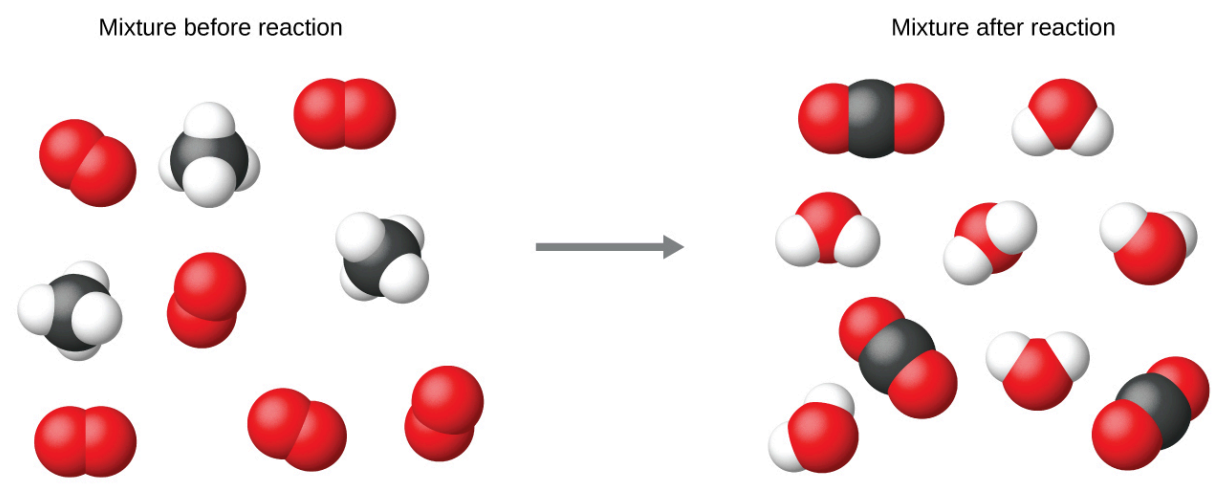

Balancing Equations

The chemical equation described in section 4.1 is balanced, meaning that equal numbers of atoms for each element involved in the reaction are represented on the reactant and product sides. This is a requirement the equation must satisfy to be consistent with the law of conservation of matter. It may be confirmed by simply summing the numbers of atoms on either side of the arrow and comparing these sums to ensure they are equal. Note 
that the number of atoms for a given element is calculated by multiplying the coefficient of any formula containing that element by the element's subscript in the formula. If an element appears in more than one formula on a given side of the equation, the number of atoms represented in each must be computed and then added together. For example, both product species in the example reaction, $\mathrm{CO}_{2}$ and $\mathrm{H}_{2} \mathrm{O}$, contain the element oxygen, and so the number of oxygen atoms on the product side of the equation is

$\left(1 \mathrm{CO}_{2}\right.$ molecule $\left.\frac{2 \mathrm{O} \text { atoms }}{\mathrm{CO}_{2} \text { molecule }}\right)+\left(2 \mathrm{H}_{2} \mathrm{O}\right.$ molecules $\left.\frac{1 \mathrm{O} \text { atom }}{\mathrm{H}_{2} \mathrm{O} \text { molecule }}\right)=4 \mathrm{O}$ atoms

The equation for the reaction between methane and oxygen to yield carbon dioxide and water is confirmed to be balanced per this approach, as shown here:

\section{$\mathrm{CH}_{4}+2 \mathrm{O}_{2} \mathrm{CO}_{2}+2 \mathrm{H}_{2} \mathrm{O}$}

$\begin{array}{llll}\text { Element } & \text { Reactants } & \text { Products } & \text { Balanced? } \\ \text { C } & 11=1 & 11=1 & 1=1 \text {, yes } \\ \text { H } & 41=4 & 22=4 & 4=4 \text {, yes } \\ \text { O } & 22=4 & (12)+\left(\begin{array}{ll}2 & 1\end{array}\right)=4 & 4=4 \text {, yes }\end{array}$

A balanced chemical equation often may be derived from a qualitative description of some chemical reaction by a fairly simple approach known as balancing by inspection. Consider as an example the decomposition of water to yield molecular hydrogen and oxygen. This process is represented qualitatively by an unbalanced chemical equation:

$\mathrm{H}_{2} \mathrm{O} \mathrm{H}_{2}+\mathrm{O}_{2} \quad$ (unbalanced) 
Comparing the number of $\mathrm{H}$ and $\mathrm{O}$ atoms on either side of this equation confirms its imbalance:

\section{Element Reactants Products Balanced?}

$\begin{array}{llll}\mathrm{H} & 12=2 & 12=2 & 2=2 \text {, yes } \\ \mathrm{O} & 11=1 & 12=2 & 1 \neq 2, \text { no }\end{array}$

The numbers of $\mathrm{H}$ atoms on the reactant and product sides of the equation are equal, but the numbers of $\mathrm{O}$ atoms are not. To achieve balance, the coefficients of the equation may be changed as needed. Keep in mind, of course, that the formula subscripts define, in part, the identity of the substance, and so these cannot be changed without altering the qualitative meaning of the equation. For example, changing the reactant formula from $\mathrm{H}_{2} \mathrm{O}$ to $\mathrm{H}_{2} \mathrm{O}_{2}$ would yield balance in the number of atoms, but doing so also changes the reactant's identity (it's now hydrogen peroxide and not water). The $\mathrm{O}$ atom balance may be achieved by changing the coefficient for $\mathrm{H}_{2} \mathrm{O}$ to 2 .

$\mathbf{2} \mathrm{H}_{2} \mathrm{O} \mathrm{H}_{2}+\mathrm{O}_{2} \quad$ (unbalanced)

$\begin{array}{llll}\text { Element } & \text { Reactants } & \text { Products } & \text { Balanced? } \\ \mathrm{H} & 22=4 & 12=2 & 4 \neq 2 \text {, no } \\ \mathrm{O} & 21=2 & 12=2 & 2=2 \text {, yes }\end{array}$

The $\mathrm{H}$ atom balance was upset by this change, but it is easily reestablished by changing the coefficient for the $\mathrm{H}_{2}$ product to 2 .

$2 \mathrm{H}_{2} \mathrm{O} 2 \mathrm{H}_{2}+\mathrm{O}_{2} \quad$ (balanced) 


\section{Element Reactants Products Balanced?}

$\begin{array}{lllll}\mathrm{H} & 2 & 2=4 & 22=4 & 4=4 \text {, yes } \\ \mathrm{O} & 2 & 1=2 & 12=2 & 2=2 \text {, yes }\end{array}$

These coefficients yield equal numbers of both $\mathrm{H}$ and $\mathrm{O}$ atoms on the reactant and product sides, and the balanced equation is, therefore:

$2 \mathrm{H}_{2} \mathrm{O} 2 \mathrm{H}_{2}+\mathrm{O}_{2}$

Balancing Chemical Equations Write a balanced equation for the reaction of molecular nitrogen $\left(\mathrm{N}_{2}\right)$ and oxygen $\left(\mathrm{O}_{2}\right)$ to form dinitrogen pentoxide.

Solution First, write the unbalanced equation.

$$
\mathrm{N}_{2}+\mathrm{O}_{2} \mathrm{~N}_{2} \mathrm{O}_{5} \quad \text { (unbalanced) }
$$

Next, count the number of each type of atom present in the unbalanced equation.

$\begin{array}{llll}\text { Element } & \text { Reactants } & \text { Products } & \text { Balanced? } \\ \mathrm{N} & 12=2 & 12=2 & 2=2 \text {, yes } \\ \mathrm{O} & 12=2 & 15=5 & 2 \neq 5 \text {, no }\end{array}$

Though nitrogen is balanced, changes in coefficients are needed to balance the number of oxygen atoms. To balance the number of oxygen atoms, a reasonable first attempt would be to change the coefficients for the $\mathrm{O}_{2}$ and $\mathrm{N}_{2} \mathrm{O}_{5}$ to integers that will yield 100 atoms (the least common multiple for the 0 atom subscripts in these two formulas).

$$
\mathrm{N}_{2}+\mathbf{5} \mathrm{O}_{2} \mathbf{2} \mathrm{N}_{2} \mathrm{O}_{5} \quad \text { (unbalanced) }
$$




$\begin{array}{llll}\text { Element } & \text { Reactants } & \text { Products } & \text { Balanced? } \\ \mathrm{N} & 12=2 & \mathbf{2} 2=4 & 2 \neq 4 \text {, no } \\ \mathrm{O} & \mathbf{5 2}=10 & \mathbf{2} 5=10 & 10=10 \text {, yes }\end{array}$

The $\mathrm{N}$ atom balance has been upset by this change; it is restored by changing the coefficient for the reactant $\mathrm{N}_{2}$ to 2 .

$$
2 \mathrm{~N}_{2}+5 \mathrm{O}_{2} 2 \mathrm{~N}_{2} \mathrm{O}_{5}
$$

$\begin{array}{llll}\text { Element } & \text { Reactants } & \text { Products } & \text { Balanced? } \\ \mathrm{N} & 22=4 & 22=4 & 4=4 \text {, yes } \\ \mathrm{O} & 52=10 & 25=10 & 10=10 \text {, yes }\end{array}$

The numbers of $\mathrm{N}$ and $\mathrm{O}$ atoms on either side of the equation are now equal, and so the equation is balanced.

Check Your Learning Write a balanced equation for the decomposition of ammonium nitrate to form molecular nitrogen, molecular oxygen, and water. (Hint: Balance oxygen last, since it is present in more than one molecule on the right side of the equation.)

Answer:

$2 \mathrm{NH}_{4} \mathrm{NO}_{3} 2 \mathrm{~N}_{2}+\mathrm{O}_{2}+4 \mathrm{H}_{2} \mathrm{O}$

It is sometimes convenient to use fractions instead of integers as intermediate coefficients in the process of balancing a chemical equation. When balance is achieved, all the equation's coefficients may then be multiplied by a whole number to convert the fractional coefficients to integers without upsetting the atom balance. For example, consider the reaction of ethane $\left(\mathrm{C}_{2} \mathrm{H}_{6}\right)$ with oxygen to yield $\mathrm{H}_{2} \mathrm{O}$ and $\mathrm{CO}_{2}$, represented by the unbalanced equation:
$\mathrm{C}_{2} \mathrm{H}_{6}+\mathrm{O}_{2} \mathrm{H}_{2} \mathrm{O}+\mathrm{CO}_{2}$
(unbalanced) 
Following the usual inspection approach, one might first balance $\mathrm{C}$ and $\mathrm{H}$ atoms by changing the coefficients for the two product species, as shown:
$\mathrm{C}_{2} \mathrm{H}_{6}+\mathrm{O}_{2} 3 \mathrm{H}_{2} \mathrm{O}+2 \mathrm{CO}_{2}$
(unbalanced)

This results in seven $\mathrm{O}$ atoms on the product side of the equation, an odd number-no integer coefficient can be used with the $\mathrm{O}_{2}$ reactant to yield an odd number, so a fractional coefficient, $\frac{7}{2}$, is used instead to yield a provisional balanced equation:

$\mathrm{C}_{2} \mathrm{H}_{6}+\frac{7}{2} \mathrm{O}_{2} 3 \mathrm{H}_{2} \mathrm{O}+2 \mathrm{CO}_{2}$

A conventional balanced equation with integer-only coefficients is derived by multiplying each coefficient by 2 : $2 \mathrm{C}_{2} \mathrm{H}_{6}+7 \mathrm{O}_{2} 6 \mathrm{H}_{2} \mathrm{O}+4 \mathrm{CO}_{2}$

Finally with regard to balanced equations, recall that convention dictates use of the smallest whole-number coefficients. Although the equation for the reaction between molecular nitrogen and molecular hydrogen to produce ammonia is, indeed, balanced, $3 \mathrm{~N}_{2}+9 \mathrm{H}_{2} 6 \mathrm{NH}_{3}$

the coefficients are not the smallest possible integers representing the relative numbers of reactant and product molecules. Dividing each coefficient by the greatest common factor, 3 , gives the preferred equation:

$\mathrm{N}_{2}+3 \mathrm{H}_{2} 2 \mathrm{NH}_{3}$

Use this interactive tutorial for additional practice balancing equations. 
Additional Information in Chemical Equations

The physical states of reactants and products in chemical equations very often are indicated with a parenthetical abbreviation following the formulas. Common abbreviations include $s$ for solids, $l$ for liquids, $g$ for gases, and $a q$ for substances dissolved in water (aqueous solutions, as introduced in the preceding chapter). These notations are illustrated in the example equation here:

$2 \mathrm{Na}(s)+2 \mathrm{H}_{2} \mathrm{O}(l) 2 \mathrm{NaOH}(a q)+\mathrm{H}_{2}(g)$

This equation represents the reaction that takes place when sodium metal is placed in water. The solid sodium reacts with liquid water to produce molecular hydrogen gas and the ionic compound sodium hydroxide (a solid in pure form, but readily dissolved in water).

Special conditions necessary for a reaction are sometimes designated by writing a word or symbol above or below the equation's arrow. For example, a reaction carried out by heating may be indicated by the uppercase Greek letter delta $(\Delta)$ over the arrow.

$\mathrm{CaCO}_{3}(s) \quad \mathrm{CaO}(s)+\mathrm{CO}_{2}(g)$

Other examples of these special conditions will be encountered in more depth in later chapters.

\section{Equations for lonic Reactions}

Given the abundance of water on earth, it stands to reason that a great many chemical reactions take place in aqueous media. When ions are involved in these reactions, the chemical equations may be written with various levels of detail appropriate to their intended use. To illustrate 
this, consider a reaction between ionic compounds taking place in an aqueous solution. When aqueous solutions of $\mathrm{CaCl}_{2}$ and $\mathrm{AgNO}_{3}$ are mixed, a reaction takes place producing aqueous $\mathrm{Ca}\left(\mathrm{NO}_{3}\right)_{2}$ and solid $\mathrm{AgCl}$ :

$\mathrm{CaCl}_{2}(a q)+2 \mathrm{AgNO}_{3}(a q) \mathrm{Ca}\left(\mathrm{NO}_{3}\right)_{2}(a q)+2 \mathrm{AgCl}(s)$

This balanced equation, derived in the usual fashion, is called a molecular equation because it doesn't explicitly represent the ionic species that are present in solution. When ionic compounds dissolve in water, they may dissociate into their constituent ions, which are subsequently dispersed homogenously throughout the resulting solution (a thorough discussion of this important process is provided in the chapter on solutions). Ionic compounds dissolved in water are, therefore, more realistically represented as dissociated ions, in this case:
$\mathrm{CaCl}_{2}(a q) \mathrm{Ca}^{2+}(a q)+2 \mathrm{Cl}(a q)$
$2 \mathrm{AgNO}_{3}(a q) 2 \mathrm{Ag}^{+}(a q)+2 \mathrm{NO}_{3}(a q)$
$\mathrm{Ca}\left(\mathrm{NO}_{3}\right)_{2}(a q) \mathrm{Ca}^{2+}(a q)+2 \mathrm{NO}_{3}(a q)$

Unlike these three ionic compounds, $\mathrm{AgCl}$ does not dissolve in water to a significant extent, as signified by its physical state notation, $s$.

Explicitly representing all dissolved ions results in a complete ionic equation. In this particular case, the formulas for the dissolved ionic compounds are replaced by formulas for their dissociated ions:

$\underset{2 \mathrm{AgCl}(s)}{\mathrm{Ca}^{2+}(a q)+2 \mathrm{Cl}(a q)+2 \mathrm{Ag}^{+}(a q)+2 \mathrm{NO}_{3}(a q) \mathrm{Ca}^{2+}(a q)+2 \mathrm{NO}_{3}(a q)+}$

Examining this equation shows that two chemical species are present in identical form on both sides of the arrow, 
$\mathrm{Ca}^{2+}(a q)$ and $\mathrm{NO}_{3}(a q)$. These spectator ions-ions whose presence is required to maintain charge neutrality-are neither chemically nor physically changed by the process, and so they may be eliminated from the equation to yield a more succinct representation called a net ionic equation:

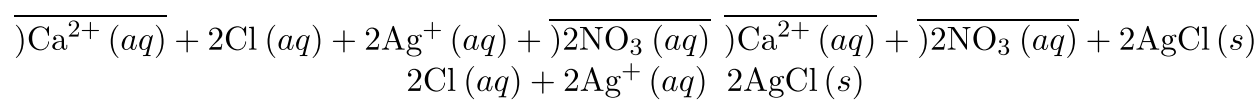

Following the convention of using the smallest possible integers as coefficients, this equation is then written:

$\mathrm{Cl}(a q)+\mathrm{Ag}^{+}(a q) \quad \mathrm{AgCl}(s)$

This net ionic equation indicates that solid silver chloride may be produced from dissolved chloride and silver(I) ions, regardless of the source of these ions. These molecular and complete ionic equations provide additional information, namely, the ionic compounds used as sources of $\mathrm{Cl}^{-}$and $\mathrm{Ag}^{+}$.

Molecular and Ionic Equations When carbon dioxide is dissolved in an aqueous solution of sodium hydroxide, the mixture reacts to yield aqueous sodium carbonate and liquid water. Write balanced molecular, complete ionic, and net ionic equations for this process.

Solution Begin by identifying formulas for the reactants and products and arranging them properly in chemical equation form:

$$
\mathrm{CO}_{2}(a q)+\mathrm{NaOH}(a q) \quad \mathrm{Na}_{2} \mathrm{CO}_{3}(a q)+\mathrm{H}_{2} \mathrm{O}(l)
$$

Balance is achieved easily in this case by changing the coefficient for $\mathrm{NaOH}$ to 2, resulting in the molecular equation for this reaction: 
$\mathrm{CO}_{2}(a q)+2 \mathrm{NaOH}(a q) \quad \mathrm{Na}_{2} \mathrm{CO}_{3}(a q)+\mathrm{H}_{2} \mathrm{O}(l)$

The two dissolved ionic compounds, $\mathrm{NaOH}$ and $\mathrm{Na}_{2} \mathrm{CO}_{3}$, can be represented as dissociated ions to yield the complete ionic equation:

$\mathrm{CO}_{2}(a q)+2 \mathrm{Na}^{+}(a q)+2 \mathrm{OH}(a q) 2 \mathrm{Na}^{+}(a q)+\mathrm{CO}_{3}{ }^{2-}(a q)+\mathrm{H}_{2} \mathrm{O}(l)$

Finally, identify the spectator ion(s), in this case $\mathrm{Na}^{+}(a q)$, and remove it from each side of the equation to generate the net ionic equation:

$$
\begin{aligned}
& \mathrm{CO}_{2}(a q)+\overline{2 \mathrm{Na}^{+}(a q)}+2 \mathrm{OH}(a q) \overline{2^{\mathrm{Na}^{+}(a q)}}+\mathrm{CO}_{3}{ }^{2-}(a q)+\mathrm{H}_{2} \mathrm{O}(l) \\
& \mathrm{CO}_{2}(a q)+2 \mathrm{OH}(a q) \mathrm{CO}_{3}{ }^{2-}(a q)+\mathrm{H}_{2} \mathrm{O}(l)
\end{aligned}
$$

Check Your Learning Diatomic chlorine and sodium hydroxide (lye) are commodity chemicals produced in large quantities, along with diatomic hydrogen, via the electrolysis of brine, according to the following unbalanced equation:

$$
\mathrm{NaCl}(a q)+\mathrm{H}_{2} \mathrm{O}(l) \stackrel{\text { electricity }}{\rightarrow} \mathrm{NaOH}(a q)+\mathrm{H}_{2}(g)+\mathrm{Cl}_{2}(g)
$$

Write balanced molecular, complete ionic, and net ionic equations for this process.

Answer:

$$
2 \mathrm{NaCl}(a q)+2 \mathrm{H}_{2} \mathrm{O}(l) 2 \mathrm{NaOH}(a q)+\mathrm{H}_{2}(g)+\mathrm{Cl}_{2}(g) \quad \text { (molecular) }
$$

Key Concepts and Summary

Chemical equations are symbolic representations of chemical and physical changes. Formulas for the substances undergoing the change (reactants) and substances generated by the change (products) are separated by an arrow and preceded by integer coefficients indicating their relative numbers. Balanced equations are 
those whose coefficients result in equal numbers of atoms for each element in the reactants and products. Chemical reactions in aqueous solution that involve ionic reactants or products may be represented more realistically by complete ionic equations and, more succinctly, by net ionic equations.

Chemistry End of Chapter Exercises

What does it mean to say an equation is balanced? Why is it important for an equation to be balanced?

An equation is balanced when the same number of each element is represented on the reactant and product sides. Equations must be balanced to accurately reflect the law of conservation of matter.

Consider molecular, complete ionic, and net ionic equations.

(a) What is the difference between these types of equations?

(b) In what circumstance would the complete and net ionic equations for a reaction be identical?

Balance the following equations:

(a) $\mathrm{PCl}_{5}(s)+\mathrm{H}_{2} \mathrm{O}(l) \mathrm{POCl}_{3}(l)+\mathrm{HCl}(a q)$

$\mathrm{Cu}(s)+\mathrm{HNO}_{3}(a q) \mathrm{Cu}\left(\mathrm{NO}_{3}\right)_{2}(a q)+\mathrm{H}_{2} \mathrm{O}(l)+\mathrm{NO}(g)$

(c) $\mathrm{H}_{2}(g)+\mathrm{I}_{2}(s) \mathrm{HI}(s)$

(d) $\mathrm{Fe}(s)+\mathrm{O}_{2}(g) \mathrm{Fe}_{2} \mathrm{O}_{3}(s)$

(e) $\mathrm{Na}(s)+\mathrm{H}_{2} \mathrm{O}(l) \mathrm{NaOH}(a q)+\mathrm{H}_{2}(g)$

$\left(\mathrm{NH}_{4}\right)_{2} \mathrm{Cr}_{2} \mathrm{O}_{7}(s) \mathrm{Cr}_{2} \mathrm{O}_{3}(s)+\mathrm{N}_{2}(g)+\mathrm{H}_{2} \mathrm{O}(g)$ 
(g) $\mathrm{P}_{4}(s)+\mathrm{Cl}_{2}(g) \mathrm{PCl}_{3}(l)$

(h) $\mathrm{PtCl}_{4}(s) \mathrm{Pt}(s)+\mathrm{Cl}_{2}(g)$

(a) $\mathrm{PCl}_{5}(s)+\mathrm{H}_{2} \mathrm{O}(l) \mathrm{POCl}_{3}(l)+2 \mathrm{HCl}(a q)$; (b) $3 \mathrm{Cu}(s)+8 \mathrm{HNO}_{3}(a q) 3 \mathrm{Cu}\left(\mathrm{NO}_{3}\right)_{2}(a q)+4 \mathrm{H}_{2} \mathrm{O}(l)+2 \mathrm{NO}(g)$;

$$
\mathrm{H}_{2}(g)+\mathrm{I}_{2}(s) 2 \mathrm{HI}(s) \text {; }
$$

$4 \mathrm{Fe}(s)+3 \mathrm{O}_{2}(g) 2 \mathrm{Fe}_{2} \mathrm{O}_{3}(s)$;

$2 \mathrm{Na}(s)+2 \mathrm{H}_{2} \mathrm{O}(l) 2 \mathrm{NaOH}(a q)+\mathrm{H}_{2}(g)$;

$\left(\mathrm{NH}_{4}\right)_{2} \mathrm{Cr}_{2} \mathrm{O}_{7}(s) \mathrm{Cr}_{2} \mathrm{O}_{3}(s)+\mathrm{N}_{2}(g)+4 \mathrm{H}_{2} \mathrm{O}(g)$;

(g)

$$
\mathrm{P}_{4}(s)+6 \mathrm{Cl}_{2}(g) 4 \mathrm{PCl}_{3}(l) \text {; }
$$

$\mathrm{PtCl}_{4}(s) \mathrm{Pt}(s)+2 \mathrm{Cl}_{2}(g)$

Balance the following equations:

(a)

$\mathrm{Ag}(s)+\mathrm{H}_{2} \mathrm{~S}(g)+\mathrm{O}_{2}(g) \mathrm{Ag}_{2} \mathrm{~S}(s)+\mathrm{H}_{2} \mathrm{O}(l)$

(b) $\mathrm{P}_{4}(s)+\mathrm{O}_{2}(g) \mathrm{P}_{4} \mathrm{O}_{10}(s)$

(c) $\mathrm{Pb}(s)+\mathrm{H}_{2} \mathrm{O}(l)+\mathrm{O}_{2}(g) \mathrm{Pb}(\mathrm{OH})_{2}(s)$

(d) $\mathrm{Fe}(s)+\mathrm{H}_{2} \mathrm{O}(l) \mathrm{Fe}_{3} \mathrm{O}_{4}(s)+\mathrm{H}_{2}(g)$

(e) $\mathrm{Sc}_{2} \mathrm{O}_{3}(s)+\mathrm{SO}_{3}(l) \mathrm{Sc}_{2}\left(\mathrm{SO}_{4}\right)_{3}(s)$

(f)

$\mathrm{Ca}_{3}\left(\mathrm{PO}_{4}\right)_{2}(a q)+\mathrm{H}_{3} \mathrm{PO}_{4}(a q) \mathrm{Ca}\left(\mathrm{H}_{2} \mathrm{PO}_{4}\right)_{2}(a q)$

(g) $\mathrm{Al}(s)+\mathrm{H}_{2} \mathrm{SO}_{4}(a q) \quad \mathrm{Al}_{2}\left(\mathrm{SO}_{4}\right)_{3}(s)+\mathrm{H}_{2}(g)$

(h) $\mathrm{TiCl}_{4}(s)+\mathrm{H}_{2} \mathrm{O}(g) \quad \mathrm{TiO}_{2}(s)+\mathrm{HCl}(g)$

Write a balanced molecular equation describing each of the following chemical reactions.

(a) Solid calcium carbonate is heated and decomposes to solid calcium oxide and carbon dioxide gas.

(b) Gaseous butane, $\mathrm{C}_{4} \mathrm{H}_{10}$, reacts with diatomic oxygen gas to yield gaseous carbon dioxide and water vapor.

(c) Aqueous solutions of magnesium chloride and 
sodium hydroxide react to produce solid magnesium hydroxide and aqueous sodium chloride.

(d) Water vapor reacts with sodium metal to produce solid sodium hydroxide and hydrogen gas.

$$
\mathrm{CaCO}_{3}(s) \mathrm{CaO}(s)+\mathrm{CO}_{2}(g)
$$

$2 \mathrm{C}_{4} \mathrm{H}_{10}(g)+13 \mathrm{O}_{2}(g) 8 \mathrm{CO}_{2}(g)+10 \mathrm{H}_{2} \mathrm{O}(g)$; (c) $\mathrm{MgCl}_{2}(a q)+2 \mathrm{NaOH}(a q) \mathrm{Mg}(\mathrm{OH})_{2}(s)+2 \mathrm{NaCl}(a q)$; (d) $2 \mathrm{H}_{2} \mathrm{O}(g)+2 \mathrm{Na}(s) 2 \mathrm{NaOH}(s)+\mathrm{H}_{2}(g)$

Write a balanced equation describing each of the following chemical reactions.

(a) Solid potassium chlorate, $\mathrm{KClO}_{3}$, decomposes to form solid potassium chloride and diatomic oxygen gas.

(b) Solid aluminum metal reacts with solid diatomic iodine to form solid $\mathrm{Al}_{2} \mathrm{I}_{6}$.

(c) When solid sodium chloride is added to aqueous sulfuric acid, hydrogen chloride gas and aqueous sodium sulfate are produced.

(d) Aqueous solutions of phosphoric acid and potassium hydroxide react to produce aqueous potassium dihydrogen phosphate and liquid water.

Colorful fireworks often involve the decomposition of barium nitrate and potassium chlorate and the reaction of the metals magnesium, aluminum, and iron with oxygen.

(a) Write the formulas of barium nitrate and potassium chlorate.

(b) The decomposition of solid potassium chlorate leads to the formation of solid potassium chloride and diatomic oxygen gas. Write an equation for the reaction.

(c) The decomposition of solid barium nitrate leads to 
the formation of solid barium oxide, diatomic nitrogen gas, and diatomic oxygen gas. Write an equation for the reaction.

(d) Write separate equations for the reactions of the solid metals magnesium, aluminum, and iron with diatomic oxygen gas to yield the corresponding metal oxides. (Assume the iron oxide contains $\mathrm{Fe}^{3+}$ ions.)

(a)

$$
\mathrm{Ba}\left(\mathrm{NO}_{3}\right)_{2}, \quad \mathrm{KClO}_{3} ;
$$

$2 \mathrm{KClO}_{3}(s) 2 \mathrm{KCl}(s)+3 \mathrm{O}_{2}(g)$;

$2 \mathrm{Ba}\left(\mathrm{NO}_{3}\right)_{2}(s) 2 \mathrm{BaO}(s)+2 \mathrm{~N}_{2}(g)+5 \mathrm{O}_{2}(g)$; $2 \mathrm{Mg}(s)+\mathrm{O}_{2}(g) 2 \mathrm{MgO}(s)$;

$4 \mathrm{Al}(s)+3 \mathrm{O}_{2}(g) 2 \mathrm{Al}_{2} \mathrm{O}_{3}(s)$;

$4 \mathrm{Fe}(s)+3 \mathrm{O}_{2}(g) 2 \mathrm{Fe}_{2} \mathrm{O}_{3}(s)$

Fill in the blank with a single chemical formula for a covalent compound that will balance the equation:

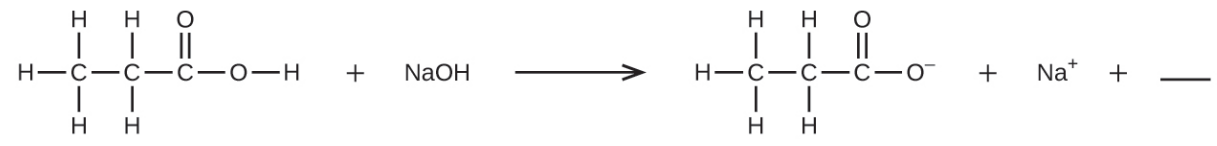

Aqueous hydrogen fluoride (hydrofluoric acid) is used to etch glass and to analyze minerals for their silicon content. Hydrogen fluoride will also react with sand (silicon dioxide).

(a) Write an equation for the reaction of solid silicon dioxide with hydrofluoric acid to yield gaseous silicon tetrafluoride and liquid water.

(b) The mineral fluorite (calcium fluoride) occurs extensively in Illinois. Solid calcium fluoride can also be prepared by the reaction of aqueous solutions of calcium chloride and sodium fluoride, yielding aqueous sodium 
chloride as the other product. Write complete and net ionic equations for this reaction.

(a) $4 \mathrm{HF}(a q)+\mathrm{SiO}_{2}(s) \mathrm{SiF}_{4}(g)+2 \mathrm{H}_{2} \mathrm{O}(l)$; (b) complete ionic equation: $2 \mathrm{Na}^{+}(a q)+2 \mathrm{~F}(a q)+\mathrm{Ca}^{2+}(a q)+2 \mathrm{Cl}(a q) \quad \mathrm{CaF}_{2}(s)+2 \mathrm{Na}^{+}(a q)+2 \mathrm{Cl}(a q)$, net ionic equation: $2 \mathrm{~F}(a q)+\mathrm{Ca}^{2+}(a q) \mathrm{CaF}_{2}(s)$

A novel process for obtaining magnesium from sea water involves several reactions. Write a balanced chemical equation for each step of the process.

(a) The first step is the decomposition of solid calcium carbonate from seashells to form solid calcium oxide and gaseous carbon dioxide.

(b) The second step is the formation of solid calcium hydroxide as the only product from the reaction of the solid calcium oxide with liquid water.

(c) Solid calcium hydroxide is then added to the seawater, reacting with dissolved magnesium chloride to yield solid magnesium hydroxide and aqueous calcium chloride.

(d) The solid magnesium hydroxide is added to a hydrochloric acid solution, producing dissolved magnesium chloride and liquid water.

(e) Finally, the magnesium chloride is melted and electrolyzed to yield liquid magnesium metal and diatomic chlorine gas.

From the balanced molecular equations, write the complete ionic and net ionic equations for the following:

$\mathrm{K}_{2} \mathrm{C}_{2} \mathrm{O}_{4}(a q)+\mathrm{Ba}(\mathrm{OH})_{2}(a q) 2 \mathrm{KOH}(a q)+\mathrm{BaC}_{2} \mathrm{O}_{4}(s)$ 
(b)

$\mathrm{Pb}\left(\mathrm{NO}_{3}\right)_{2}(a q)+\mathrm{H}_{2} \mathrm{SO}_{4}(a q) \mathrm{PbSO}_{4}(s)+2 \mathrm{HNO}_{3}(a q)$ (c)

$\mathrm{CaCO}_{3}(s)+\mathrm{H}_{2} \mathrm{SO}_{4}(a q) \mathrm{CaSO}_{4}(s)+\mathrm{CO}_{2}(g)+\mathrm{H}_{2} \mathrm{O}(l)$

$2 \mathrm{~K}^{+}(a q)+\mathrm{C}_{2} \mathrm{O}_{4}{ }^{2-}(a q)+\mathrm{Ba}^{2+}(a q)+2 \mathrm{OH}(a q) 2 \mathrm{~K}^{+}(a q)+2 \mathrm{OH}(a q)+\mathrm{BaC}_{2} \mathrm{O}_{4}(s) \quad$ (complete) $\mathrm{Ba}^{2+}(a q)+\mathrm{C}_{2} \mathrm{O}_{4}{ }^{2-}(a q) \mathrm{BaC}_{2} \mathrm{O}_{4}(s) \quad$ (net)

(b)

$\mathrm{Pb}^{2+}(a q)+2 \mathrm{NO}_{3}(a q)+2 \mathrm{H}^{+}(a q)+\mathrm{SO}_{4}{ }^{2-}(a q) \mathrm{PbSO}_{4}(s)+2 \mathrm{H}^{+}(a q)+2 \mathrm{NO}_{3}(a q) \quad$ (complete) $\mathrm{Pb}^{2+}(a q)+\mathrm{SO}_{4}^{2-}(a q) \mathrm{PbSO}_{4}(s) \quad$ (net)

(c)

$\mathrm{CaCO}_{3}(s)+2 \mathrm{H}^{+}(a q)+\mathrm{SO}_{4}^{2-}(a q) \mathrm{CaSO}_{4}(s)+\mathrm{CO}_{2}(g)+\mathrm{H}_{2} \mathrm{O}(l) \quad$ (complete)

$\mathrm{CaCO}_{3}(s)+2 \mathrm{H}^{+}(a q)+\mathrm{SO}_{4}^{2-}(a q) \mathrm{CaSO}_{4}(s)+\mathrm{CO}_{2}(g)+\mathrm{H}_{2} \mathrm{O}(l)$ (net)

Glossary

balanced equation

chemical equation with equal numbers of

atoms for each element in the reactant and

product

chemical equation

symbolic representation of a chemical reaction

coefficient

number placed in front of symbols or formulas in a chemical equation to indicate their relative amount 


\section{complete ionic equation}

chemical equation in which all dissolved ionic reactants and products, including spectator ions, are explicitly represented by formulas for their dissociated ions molecular equation chemical equation in which all reactants and products are represented as neutral substances

net ionic equation

chemical equation in which only those dissolved ionic reactants and products that undergo a chemical or physical change are represented (excludes spectator ions)

\section{product}

substance formed by a chemical or physical change; shown on the right side of the arrow in a chemical equation

reactant

substance undergoing a chemical or physical change; shown on the left side of the arrow in a chemical equation

\section{spectator ion}

ion that does not undergo a chemical or physical change during a reaction, but its presence is required to maintain charge neutrality 
21.

\section{CLASSIFYING CHEMICAL REACTIONS}

Learning Objectives

By the end of this section, you will be able to:

- Define and recognize two common types of chemical reactions (acid-base, and oxidationreduction)

- Identify common acids and bases

Acid-Base Reactions

An acid-base reaction is one in which a hydrogen ion, $\mathrm{H}^{+}$, is transferred from one chemical species to another. Such reactions are of central importance to numerous natural and technological processes, ranging from the chemical transformations that take place within cells and the lakes 
and oceans, to the industrial-scale production of fertilizers, pharmaceuticals, and other substances essential to society. The subject of acid-base chemistry, therefore, is worthy of thorough discussion, and a full chapter is devoted to this topic later in the text.

For purposes of this brief introduction, we will consider only the more common types of acid-base reactions that take place in aqueous solutions. In this context, an acid is a substance that will dissolve in water to yield hydronium ions, $\mathrm{H}_{3} \mathrm{O}^{+}$. As an example, consider the equation shown here:

$$
\mathrm{HCl}(a q)+\mathrm{H}_{2} \mathrm{O}(a q) \mathrm{Cl}(a q)+\mathrm{H}_{3} \mathrm{O}^{+}(a q)
$$

The process represented by this equation confirms that hydrogen chloride is an acid. When dissolved in water, $\mathrm{H}_{3} \mathrm{O}^{+}$ions are produced by a chemical reaction in which $\mathrm{H}^{+}$ ions are transferred from $\mathrm{HCl}$ molecules to $\mathrm{H}_{2} \mathrm{O}$ molecules ((Figure)).

When hydrogen chloride gas dissolves in water, (a) it reacts as an acid, transferring protons to water molecules to yield (b) hydronium ions (and solvated chloride ions).

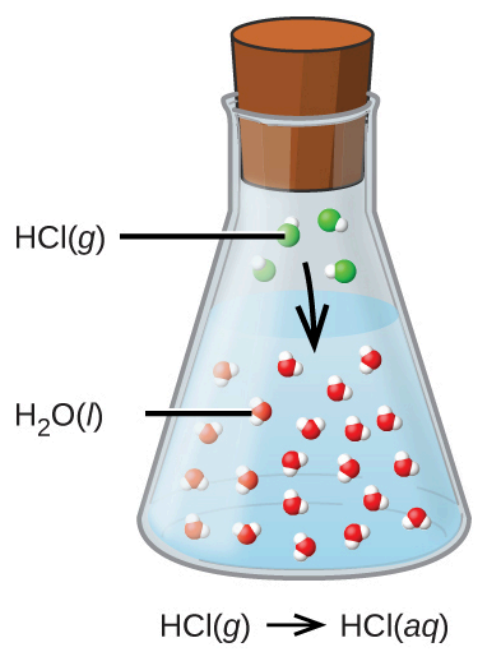

(a)

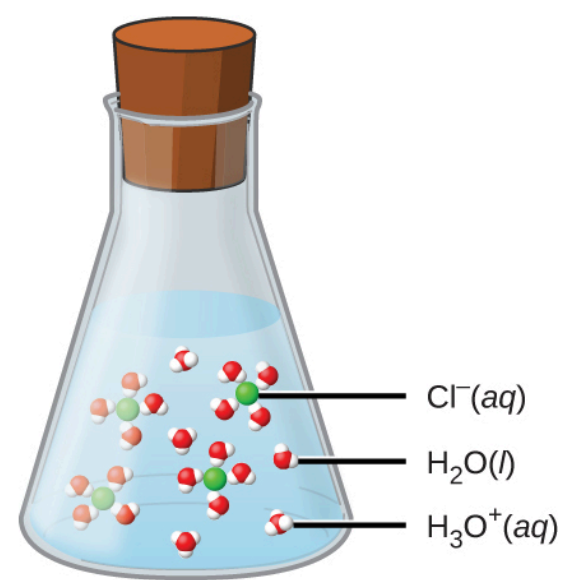

$\mathrm{HCl}(a q)+\mathrm{H}_{2} \mathrm{O}(l) \rightarrow \mathrm{H}_{3} \mathrm{O}^{+}(a q)+\mathrm{Cl}^{-}(a q)$

(b) 
The nature of $\mathrm{HCl}$ is such that its reaction with water as just described is essentially 100\% efficient: Virtually every $\mathrm{HCl}$ molecule that dissolves in water will undergo this reaction. Acids that completely react in this fashion are called strong acids, and $\mathrm{HCl}$ is one among just a handful of common acid compounds that are classified as strong ((Figure)). A far greater number of compounds behave as weak acids and only partially react with water, leaving a large majority of dissolved molecules in their original form and generating a relatively small amount of hydronium ions. Weak acids are commonly encountered in nature, being the substances partly responsible for the tangy taste of citrus fruits, the stinging sensation of insect bites, and the unpleasant smells associated with body odor. A familiar example of a weak acid is acetic acid, the main ingredient in food vinegars:

$\mathrm{CH}_{3} \mathrm{CO}_{2} \mathrm{H}(a q)+\mathrm{H}_{2} \mathrm{O}(l) \mathrm{CH}_{3} \mathrm{CO}_{2}(a q)+\mathrm{H}_{3} \mathrm{O}^{+}(a q)$

When dissolved in water under typical conditions, only about $1 \%$ of acetic acid molecules are present in the ionized form, $\mathrm{CH}_{3} \mathrm{CO}_{2}{ }^{-}$((Figure)). (The use of a doublearrow in the equation above denotes the partial reaction aspect of this process, a concept addressed fully in the chapters on chemical equilibrium.)

(a) Fruits such as oranges, lemons, and grapefruit contain the weak acid citric acid. (b) Vinegars contain the weak acid acetic acid. (credit a: modification of work by Scott Bauer; credit b: modification of work by BrückeOsteuropa/Wikimedia Commons) 


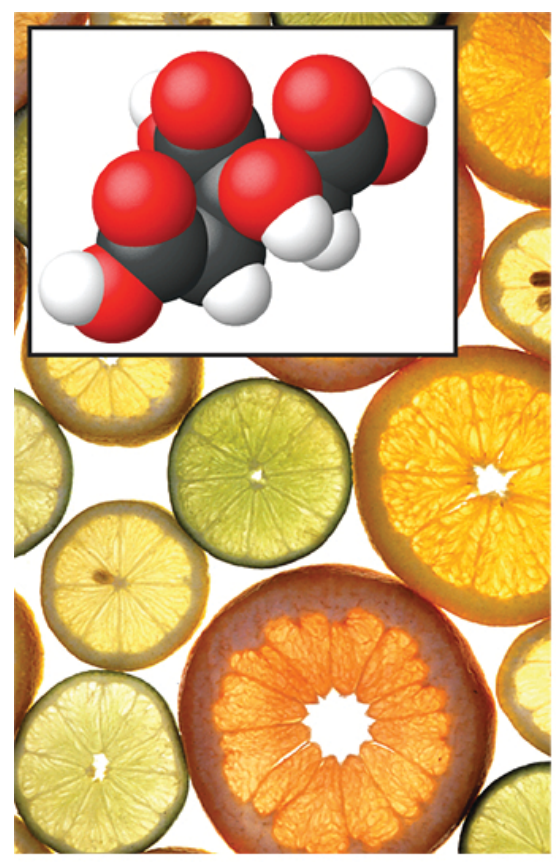

(a)

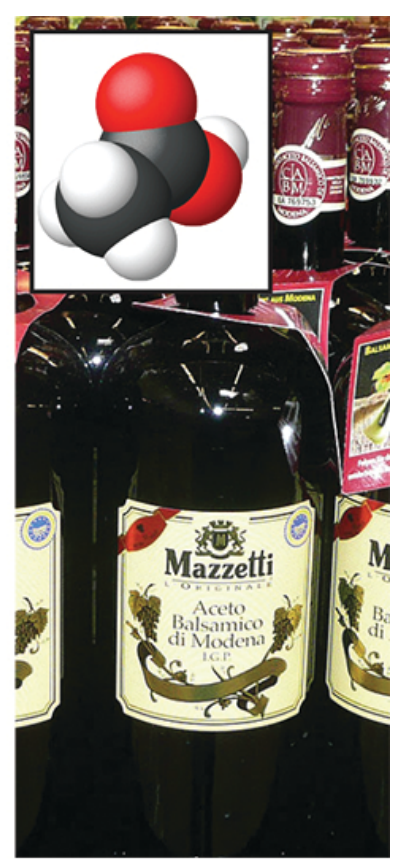

(b)

Common Strong Acids

Compound Formula Name in Aqueous Solution

$\mathrm{HBr}$

hydrobromic acid

$\mathrm{HCl}$

hydrochloric acid

$\mathrm{HI}$

hydroiodic acid

$\mathrm{HNO}_{3}$

nitric acid

$\mathrm{HClO}_{4}$

perchloric acid

$\mathrm{H}_{2} \mathrm{SO}_{4}$

sulfuric acid

A base is a substance that will dissolve in water to yield hydroxide ions, $\mathrm{OH}^{-}$. The most common bases are ionic compounds composed of alkali or alkaline earth metal cations (groups 1 and 2) combined with the hydroxide ion-for example, $\mathrm{NaOH}$ and $\mathrm{Ca}(\mathrm{OH})_{2}$. Unlike the acid compounds discussed previously, these compounds do not react chemically with water; instead they dissolve and 
dissociate, releasing hydroxide ions directly into the solution. For example, $\mathrm{KOH}$ and $\mathrm{Ba}(\mathrm{OH})_{2}$ dissolve in water and dissociate completely to produce cations $\left(\mathrm{K}^{+}\right.$and $\mathrm{Ba}^{2+}$, respectively) and hydroxide ions, $\mathrm{OH}^{-}$. These bases, along with other hydroxides that completely dissociate in water, are considered strong bases.

Consider as an example the dissolution of lye (sodium hydroxide) in water:

$\mathrm{NaOH}(s) \mathrm{Na}^{+}(a q)+\mathrm{OH}(a q)$

This equation confirms that sodium hydroxide is a base. When dissolved in water, $\mathrm{NaOH}$ dissociates to yield $\mathrm{Na}^{+}$ and $\mathrm{OH}^{-}$ions. This is also true for any other ionic compound containing hydroxide ions. Since the dissociation process is essentially complete when ionic compounds dissolve in water under typical conditions, $\mathrm{NaOH}$ and other ionic hydroxides are all classified as strong bases.

Unlike ionic hydroxides, some compounds produce hydroxide ions when dissolved by chemically reacting with water molecules. In all cases, these compounds react only partially and so are classified as weak bases. These types of compounds are also abundant in nature and important commodities in various technologies. For example, global production of the weak base ammonia is typically well over 100 metric tons annually, being widely used as an agricultural fertilizer, a raw material for chemical synthesis of other compounds, and an active ingredient in household cleaners ((Figure)). When dissolved in water, ammonia reacts partially to yield hydroxide ions, as shown here:

$\mathrm{NH}_{3}(a q)+\mathrm{H}_{2} \mathrm{O}(l) \mathrm{NH}_{4}+(a q)+\mathrm{OH}(a q)$ 
This is, by definition, an acid-base reaction, in this case involving the transfer of $\mathrm{H}^{+}$ions from water molecules to ammonia molecules. Under typical conditions, only about $1 \%$ of the dissolved ammonia is present as $\mathrm{NH}_{4}{ }^{+}$ions. Ammonia is a weak base used in a variety of applications. (a) Pure ammonia is commonly applied as an agricultural fertilizer. (b) Dilute solutions of ammonia are effective household cleansers. (credit a: modification of work by National Resources Conservation Service; credit b: modification of work by pat00139)

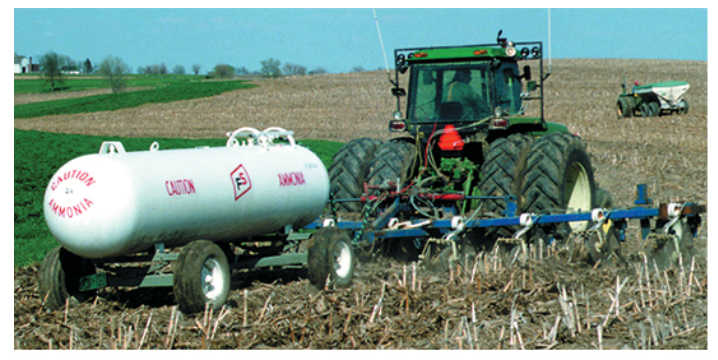

(a)

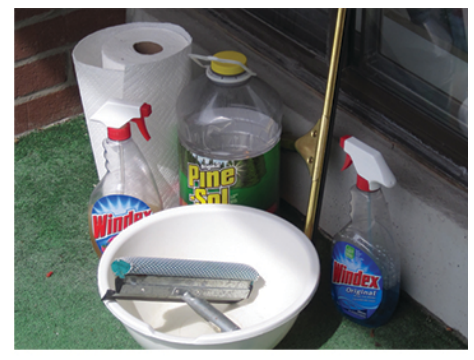

(b)

A neutralization reaction is a specific type of acid-base reaction in which the reactants are an acid and a base (but not water), and the products are often a salt and water acid + base salt + water

To illustrate a neutralization reaction, consider what happens when a typical antacid such as milk of magnesia (an aqueous suspension of solid $\mathrm{Mg}(\mathrm{OH})_{2}$ ) is ingested to ease symptoms associated with excess stomach acid $(\mathrm{HCl})$ : $\mathrm{Mg}(\mathrm{OH})_{2}(s)+2 \mathrm{HCl}(a q) \mathrm{MgCl}_{2}(a q)+2 \mathrm{H}_{2} \mathrm{O}(l)$.

Note that in addition to water, this reaction produces a salt, magnesium chloride. 
Writing Equations for Acid-Base Reactions Write balanced chemical equations for the acid-base reactions described here:

(a) the weak acid hydrogen hypochlorite reacts with water

(b) a solution of barium hydroxide is neutralized with a solution of nitric acid

Solution (a) The two reactants are provided, $\mathrm{HOCl}$ and $\mathrm{H}_{2} \mathrm{O}$. Since the substance is reported to be an acid, its reaction with water will involve the transfer of $\mathrm{H}^{+}$from $\mathrm{HOCl}$ to $\mathrm{H}_{2} \mathrm{O}$ to generate hydronium ions, $\mathrm{H}_{3} \mathrm{O}^{+}$and hypochlorite ions, $\mathrm{OCl}^{-}$.

$$
\mathrm{HOCl}(a q)+\mathrm{H}_{2} \mathrm{O}(l) \mathrm{OCl}(a q)+\mathrm{H}_{3} \mathrm{O}^{+}(a q)
$$

A double-arrow is appropriate in this equation because it indicates the $\mathrm{HOCl}$ is a weak acid that has not reacted completely.

(b) The two reactants are provided, $\mathrm{Ba}(\mathrm{OH})_{2}$ and $\mathrm{HNO}_{3}$. Since this is a neutralization reaction, the two products will be water and a salt composed of the cation of the ionic hydroxide $\left(\mathrm{Ba}^{2+}\right)$ and the anion generated when the acid transfers its hydrogen ion $\left(\mathrm{NO}_{3}\right)$.

$$
\mathrm{Ba}(\mathrm{OH})_{2}(a q)+2 \mathrm{HNO}_{3}(a q) \quad \mathrm{Ba}\left(\mathrm{NO}_{3}\right)_{2}(a q)+2 \mathrm{H}_{2} \mathrm{O}(l)
$$

Check Your Learning Write the net ionic equation representing the neutralization of any strong acid with an ionic hydroxide. (Hint: Consider the ions produced when a strong acid is dissolved in water.)

Answer:

$$
\mathrm{H}_{3} \mathrm{O}^{+}(a q)+\mathrm{OH}(a q) 2 \mathrm{H}_{2} \mathrm{O}(l)
$$

\section{Stomach Antacids}

Our stomachs contain a solution of roughly $0.03 \mathrm{M} \mathrm{HCl}$, which helps us digest the food we eat. The burning sensation associated with heartburn is a result of the acid 
of the stomach leaking through the muscular valve at the top of the stomach into the lower reaches of the esophagus. The lining of the esophagus is not protected from the corrosive effects of stomach acid the way the lining of the stomach is, and the results can be very painful. When we have heartburn, it feels better if we reduce the excess acid in the esophagus by taking an antacid. As you may have guessed, antacids are bases. One of the most common antacids is calcium carbonate, $\mathrm{CaCO}_{3}$. The reaction,

$\mathrm{CaCO}_{3}(s)+2 \mathrm{HCl}(a q) \mathrm{CaCl}_{2}(a q)+\mathrm{H}_{2} \mathrm{O}(l)+\mathrm{CO}_{2}(g)$

not only neutralizes stomach acid, it also produces $\mathrm{CO}_{2}(g)$, which may result in a satisfying belch.

Milk of Magnesia is a suspension of the sparingly soluble base magnesium hydroxide, $\mathrm{Mg}(\mathrm{OH})_{2}$. It works according to the reaction:

$\mathrm{Mg}(\mathrm{OH})_{2}(s) \mathrm{Mg}^{2+}(a q)+2 \mathrm{OH}(a q)$

The hydroxide ions generated in this equilibrium then go on to react with the hydronium ions from the stomach acid, so that:

$\mathrm{H}_{3} \mathrm{O}^{+}+\mathrm{OH} 2 \mathrm{H}_{2} \mathrm{O}(l)$

This reaction does not produce carbon dioxide, but magnesium-containing antacids can have a laxative effect. Several antacids have aluminum hydroxide, $\mathrm{Al}(\mathrm{OH})_{3}$, as an active ingredient. The aluminum hydroxide tends to cause constipation, and some antacids use aluminum hydroxide in concert with magnesium hydroxide to balance the side effects of the two substances. 
Culinary Aspects of Chemistry

Examples of acid-base chemistry are abundant in the culinary world. One example is the use of baking soda, or sodium bicarbonate in baking. $\mathrm{NaHCO}_{3}$ is a base. When it reacts with an acid such as lemon juice, buttermilk, or sour cream in a batter, bubbles of carbon dioxide gas are formed from decomposition of the resulting carbonic acid, and the batter "rises." Baking powder is a combination of sodium bicarbonate, and one or more acid salts that react when the two chemicals come in contact with water in the batter.

Many people like to put lemon juice or vinegar, both of which are acids, on cooked fish ((Figure)). It turns out that fish have volatile amines (bases) in their systems, which are neutralized by the acids to yield involatile ammonium salts. This reduces the odor of the fish, and also adds a "sour" taste that we seem to enjoy.

A neutralization reaction takes place between citric acid in lemons or acetic acid in vinegar, and the bases in the flesh of fish. 


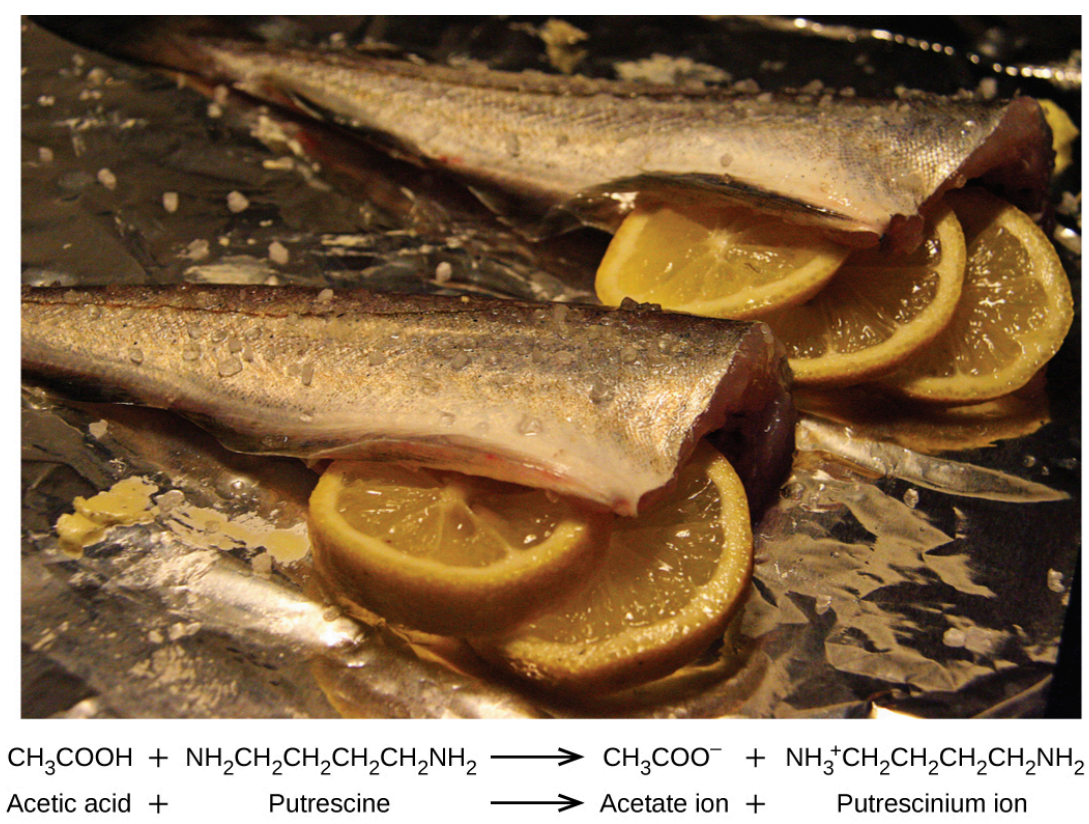

Pickling is a method used to preserve vegetables using a naturally produced acidic environment. The vegetable, such as a cucumber, is placed in a sealed jar submerged in a brine solution. The brine solution favors the growth of beneficial bacteria and suppresses the growth of harmful bacteria. The beneficial bacteria feed on starches in the cucumber and produce lactic acid as a waste product in a process called fermentation. The lactic acid eventually increases the acidity of the brine to a level that kills any harmful bacteria, which require a basic environment. Without the harmful bacteria consuming the cucumbers they are able to last much longer than if they were unprotected. A byproduct of the pickling process changes the flavor of the vegetables with the acid making them taste sour.

Explore the microscopic view of strong and weak acids and bases. 
Oxidation-Reduction Reactions

Earth's atmosphere contains about 20\% molecular oxygen, $\mathrm{O}_{2}$, a chemically reactive gas that plays an essential role in the metabolism of aerobic organisms and in many environmental processes that shape the world. The term oxidation was originally used to describe chemical reactions involving $\mathrm{O}_{2}$, but its meaning has evolved to refer to a broad and important reaction class known as oxidation-reduction (redox) reactions. A few examples of such reactions will be used to develop a clear picture of this classification.

Some redox reactions involve the transfer of electrons between reactant species to yield ionic products, such as the reaction between sodium and chlorine to yield sodium chloride:

$2 \mathrm{Na}(s)+\mathrm{Cl}_{2}(g) 2 \mathrm{NaCl}(s)$

It is helpful to view the process with regard to each individual reactant, that is, to represent the fate of each reactant in the form of an equation called a half-reaction: $2 \mathrm{Na}(s) 2 \mathrm{Na}^{+}(s)+2 \mathrm{e}^{-}$ $\mathrm{Cl}_{2}(g)+2 \mathrm{e}^{-} 2 \mathrm{Cl}(s)$

These equations show that $\mathrm{Na}$ atoms lose electrons while $\mathrm{Cl}$ atoms (in the $\mathrm{Cl}_{2}$ molecule) gain electrons, the " $s$ " subscripts for the resulting ions signifying they are present in the form of a solid ionic compound. For redox reactions of this sort, the loss and gain of electrons define the complementary processes that occur:

oxidation $=$ loss of electrons reduction $=$ gain of electrons 
In this reaction, then, sodium is oxidized and chlorine undergoes reduction. Viewed from a more active perspective, sodium functions as a reducing agent (reductant), since it provides electrons to (or reduces) chlorine. Likewise, chlorine functions as an oxidizing agent (oxidant), as it effectively removes electrons from (oxidizes) sodium.

reducing agent $=$ species that is oxidized oxidizing agent $=$ species that is reduced

Some redox processes, however, do not involve the transfer of electrons. Consider, for example, a reaction similar to the one yielding $\mathrm{NaCl}$ :

$\mathrm{H}_{2}(g)+\mathrm{Cl}_{2}(g) 2 \mathrm{HCl}(g)$

The product of this reaction is a covalent compound, so transfer of electrons in the explicit sense is not involved. To clarify the similarity of this reaction to the previous one and permit an unambiguous definition of redox reactions, a property called oxidation number has been defined.

\section{Chemistry End of Chapter Exercises}

Use the following equations to answer the next four questions:

i. $\mathrm{H}_{2} \mathrm{O}(s) \mathrm{H}_{2} \mathrm{O}(l)$

ii.

$$
\begin{aligned}
& \mathrm{Na}^{+}(a q)+\mathrm{Cl}(\mathrm{aq})+\mathrm{Ag}^{+}(a q)+\mathrm{NO}_{3}(a q) \mathrm{AgCl}(s)+\mathrm{Na}^{+}(a q)+\mathrm{NO}_{3}(a q) \\
& \text { iii. } \mathrm{CH}_{3} \mathrm{OH}(g)+\mathrm{O}_{2}(g) \mathrm{CO}_{2}(g)+\mathrm{H}_{2} \mathrm{O}(g) \\
& \text { iv. } 2 \mathrm{H}_{2} \mathrm{O}(l) \quad 2 \mathrm{H}_{2}(g)+\mathrm{O}_{2}(g) \\
& \text { v. } \mathrm{H}^{+}(a q)+\mathrm{OH}(a q) \quad \mathrm{H}_{2} \mathrm{O}(l)
\end{aligned}
$$

(a) Which equation describes a physical change? 
(b) Which equation identifies the reactants and products of a combustion reaction?

(c) Which equation is not balanced?

(d) Which is a net ionic equation?

Indicate what type, or types, of reaction each of the following represents:

(a) $\mathrm{Ca}(s)+\mathrm{Br}_{2}(l) \mathrm{CaBr}_{2}(s)$

$\mathrm{Ca}(\mathrm{OH})_{2}(a q)+2 \mathrm{HBr}(a q) \mathrm{CaBr}_{2}(a q)+2 \mathrm{H}_{2} \mathrm{O}(l)$

(c) $\mathrm{C}_{6} \mathrm{H}_{12}(l)+9 \mathrm{O}_{2}(g) \quad 6 \mathrm{CO}_{2}(g)+6 \mathrm{H}_{2} \mathrm{O}(g)$

(a) oxidation-reduction (addition); (b) acid-base (neutralization); (c) oxidation-reduction (combustion)

Indicate what type, or types, of reaction each of the following represents:

(a) $\mathrm{H}_{2} \mathrm{O}(g)+\mathrm{C}(s) \mathrm{CO}(g)+\mathrm{H}_{2}(g)$

(b) $2 \mathrm{KClO}_{3}(s) 2 \mathrm{KCl}(s)+3 \mathrm{O}_{2}(g)$

$\mathrm{Al}(\mathrm{OH})_{3}(a q)+3 \mathrm{HCl}(a q) \mathrm{AlCl}_{3}(a q)+3 \mathrm{H}_{2} \mathrm{O}(l)$

$\mathrm{Pb}\left(\mathrm{NO}_{3}\right)_{2}(a q)+\mathrm{H}_{2} \mathrm{SO}_{4}(a q) \quad \mathrm{PbSO}_{4}(s)+2 \mathrm{HNO}_{3}(a q)$

Silver can be separated from gold because silver dissolves in nitric acid while gold does not. Is the dissolution of silver in nitric acid an acid-base reaction or an oxidationreduction reaction? Explain your answer.

It is an oxidation-reduction reaction because the oxidation state of the silver changes during the reaction. 
Classify the following as acid-base reactions or oxidationreduction reactions:

(a) $\mathrm{Na}_{2} \mathrm{~S}(a q)+2 \mathrm{HCl}(a q) \quad 2 \mathrm{NaCl}(a q)+\mathrm{H}_{2} \mathrm{~S}(g)$

(b) $2 \mathrm{Na}(s)+2 \mathrm{HCl}(a q) 2 \mathrm{NaCl}(a q)+\mathrm{H}_{2}(g)$

(c) $\mathrm{Mg}(s)+\mathrm{Cl}_{2}(g) \quad \mathrm{MgCl}_{2}(s)$

(d) $\mathrm{MgO}(s)+2 \mathrm{HCl}(a q) \quad \mathrm{MgCl}_{2}(a q)+\mathrm{H}_{2} \mathrm{O}(l)$

(e) $\mathrm{K}_{3} \mathrm{P}(s)+2 \mathrm{O}_{2}(g) \mathrm{K}_{3} \mathrm{PO}_{4}(s)$

$3 \mathrm{KOH}(a q)+\mathrm{H}_{3} \mathrm{PO}_{4}(a q) \quad \mathrm{K}_{3} \mathrm{PO}_{4}(a q)+3 \mathrm{H}_{2} \mathrm{O}(l)$

(a) acid-base; (b) oxidation-reduction: $\mathrm{Na}$ is oxidized, $\mathrm{H}^{+}$ is reduced; (c) oxidation-reduction: $\mathrm{Mg}$ is oxidized, $\mathrm{Cl}_{2}$ is reduced; (d) acid-base; (e) oxidation-reduction: $\mathrm{P}^{3-}$ is oxidized, $\mathrm{O}_{2}$ is reduced; (f) acid-base

Identify the atoms that are oxidized and reduced, the change in oxidation state for each, and the oxidizing and reducing agents in each of the following equations:

(a) $\mathrm{Mg}(s)+\mathrm{NiCl}_{2}(a q) \mathrm{MgCl}_{2}(a q)+\mathrm{Ni}(s)$

(b) $\mathrm{PCl}_{3}(l)+\mathrm{Cl}_{2}(g) \mathrm{PCl}_{5}(s)$

(c) $\mathrm{C}_{2} \mathrm{H}_{4}(g)+3 \mathrm{O}_{2}(g) 2 \mathrm{CO}_{2}(g)+2 \mathrm{H}_{2} \mathrm{O}(g)$

(d) $\mathrm{Zn}(s)+\mathrm{H}_{2} \mathrm{SO}_{4}(a q) \mathrm{ZnSO}_{4}(a q)+\mathrm{H}_{2}(g)$

(e) $2 \mathrm{~K}_{2} \mathrm{~S}_{2} \mathrm{O}_{3}(s)+\mathrm{I}_{2}(s) \mathrm{K}_{2} \mathrm{~S}_{4} \mathrm{O}_{6}(s)+2 \mathrm{KI}(s)$

$3 \mathrm{Cu}(s)+8 \mathrm{HNO}_{3}(a q) \quad 3 \mathrm{Cu}\left(\mathrm{NO}_{3}\right)_{2}(a q)+2 \mathrm{NO}(g)+4 \mathrm{H}_{2} \mathrm{O}(l)$

Complete and balance the following acid-base equations:

(a) $\mathrm{HCl}$ gas reacts with solid $\mathrm{Ca}(\mathrm{OH})_{2}(s)$.

(b) A solution of $\mathrm{Sr}(\mathrm{OH})_{2}$ is added to a solution of $\mathrm{HNO}_{3}$.

(a)

$2 \mathrm{HCl}(g)+\mathrm{Ca}(\mathrm{OH})_{2}(s) \mathrm{CaCl}_{2}(s)+2 \mathrm{H}_{2} \mathrm{O}(l)$; 
(b)

$\mathrm{Sr}(\mathrm{OH})_{2}(a q)+2 \mathrm{HNO}_{3}(a q) \quad \mathrm{Sr}\left(\mathrm{NO}_{3}\right)_{2}(a q)+2 \mathrm{H}_{2} \mathrm{O}(l)$

Complete and balance the following acid-base equations:

(a) A solution of $\mathrm{HClO}_{4}$ is added to a solution of $\mathrm{LiOH}$.

(b) Aqueous $\mathrm{H}_{2} \mathrm{SO}_{4}$ reacts with $\mathrm{NaOH}$.

(c) $\mathrm{Ba}(\mathrm{OH})_{2}$ reacts with $\mathrm{HF}$ gas.

Complete and balance the equations for the following acid-base neutralization reactions. If water is used as a solvent, write the reactants and products as aqueous ions. In some cases, there may be more than one correct answer, depending on the amounts of reactants used.

(a) $\mathrm{Mg}(\mathrm{OH})_{2}(s)+\mathrm{HClO}_{4}(a q)$

(b)

$\mathrm{SO}_{3}(g)+\mathrm{H}_{2} \mathrm{O}(l)$ (assume an excess of water and that the product dissolves)

(c) $\mathrm{SrO}(s)+\mathrm{H}_{2} \mathrm{SO}_{4}(l)$

(a)

$\mathrm{Mg}(\mathrm{OH})_{2}(s)+2 \mathrm{HClO}_{4}(a q) \mathrm{Mg}^{2+}(a q)+2 \mathrm{ClO}_{4}(a q)+2 \mathrm{H}_{2} \mathrm{O}(l)$;

(b) $\mathrm{SO}_{3}(g)+2 \mathrm{H}_{2} \mathrm{O}(l) \mathrm{H}_{3} \mathrm{O}^{+}(a q)+\mathrm{HSO}_{4}(a q)$,

(a solution of $\left.\mathrm{H}_{2} \mathrm{SO}_{4}\right)$;

$\mathrm{SrO}(s)+\mathrm{H}_{2} \mathrm{SO}_{4}(l) \mathrm{SrSO}_{4}(s)+\mathrm{H}_{2} \mathrm{O}$

When heated to $700-800{ }^{\circ} \mathrm{C}$, diamonds, which are pure carbon, are oxidized by atmospheric oxygen. (They burn!) Write the balanced equation for this reaction.

The military has experimented with lasers that produce very intense light when fluorine combines explosively with hydrogen. What is the balanced equation for this reaction? 


\section{$\mathrm{H}_{2}(g)+\mathrm{F}_{2}(g) 2 \mathrm{HF}(g)$}

Write the molecular, total ionic, and net ionic equations for the following reactions:

(a) $\mathrm{Ca}(\mathrm{OH})_{2}(a q)+\mathrm{HC}_{2} \mathrm{H}_{3} \mathrm{O}_{2}(a q)$

(b) $\mathrm{H}_{3} \mathrm{PO}_{4}(a q)+\mathrm{CaCl}_{2}(a q)$

Great Lakes Chemical Company produces bromine, $\mathrm{Br}_{2}$, from bromide salts such as $\mathrm{NaBr}$, in Arkansas brine by treating the brine with chlorine gas. Write a balanced equation for the reaction of $\mathrm{NaBr}$ with $\mathrm{Cl}_{2}$.

$2 \mathrm{NaBr}(a q)+\mathrm{Cl}_{2}(g) 2 \mathrm{NaCl}(a q)+\mathrm{Br}_{2}(l)$

In a common experiment in the general chemistry laboratory, magnesium metal is heated in air to produce $\mathrm{MgO} . \mathrm{MgO}$ is a white solid, but in these experiments it often looks gray, due to small amounts of $\mathrm{Mg}_{3} \mathrm{~N}_{2}$, a compound formed as some of the magnesium reacts with nitrogen. Write a balanced equation for each reaction.

Lithium hydroxide may be used to absorb carbon dioxide in enclosed environments, such as manned spacecraft and submarines. Write an equation for the reaction that involves $2 \mathrm{~mol}$ of $\mathrm{LiOH}$ per $1 \mathrm{~mol}$ of $\mathrm{CO}_{2}$. (Hint: Water is one of the products.)

$2 \mathrm{LiOH}(a q)+\mathrm{CO}_{2}(g) \mathrm{Li}_{2} \mathrm{CO}_{3}(a q)+\mathrm{H}_{2} \mathrm{O}(l)$

Calcium propionate is sometimes added to bread to retard spoilage. This compound can be prepared by the reaction of calcium carbonate, $\mathrm{CaCO}_{3}$, with propionic acid, $\mathrm{C}_{2} \mathrm{H}_{5} \mathrm{CO}_{2} \mathrm{H}$, which has properties similar to those of acetic 
acid. Write the balanced equation for the formation of calcium propionate.

Complete and balance the equations of the following reactions, each of which could be used to remove hydrogen sulfide from natural gas:

(a) $\mathrm{Ca}(\mathrm{OH})_{2}(s)+\mathrm{H}_{2} \mathrm{~S}(g)$

(b) $\mathrm{Na}_{2} \mathrm{CO}_{3}(a q)+\mathrm{H}_{2} \mathrm{~S}(g)$

(a) $\mathrm{Ca}(\mathrm{OH})_{2}(s)+\mathrm{H}_{2} \mathrm{~S}(g) \mathrm{CaS}(s)+2 \mathrm{H}_{2} \mathrm{O}(l)$; (b) $\mathrm{Na}_{2} \mathrm{CO}_{3}(a q)+\mathrm{H}_{2} \mathrm{~S}(g) \quad \mathrm{Na}_{2} \mathrm{~S}(a q)+\mathrm{CO}_{2}(g)+\mathrm{H}_{2} \mathrm{O}(l)$

Copper(II) sulfide is oxidized by molecular oxygen to produce gaseous sulfur trioxide and solid copper(II) oxide. The gaseous product then reacts with liquid water to produce liquid hydrogen sulfate as the only product. Write the two equations which represent these reactions.

Write balanced chemical equations for the reactions used to prepare each of the following compounds from the given starting material(s). In some cases, additional reactants may be required.

(a) solid ammonium nitrate from gaseous molecular nitrogen via a two-step process (first reduce the nitrogen to ammonia, then neutralize the ammonia with an appropriate acid)

(b) gaseous hydrogen bromide from liquid molecular bromine via a one-step redox reaction

(c) gaseous $\mathrm{H}_{2} \mathrm{~S}$ from solid $\mathrm{Zn}$ and $\mathrm{S}$ via a two-step process (first a redox reaction between the starting materials, then reaction of the product with a strong acid)

(a) step 1: $\mathrm{N}_{2}(g)+3 \mathrm{H}_{2}(g) 2 \mathrm{NH}_{3}(g)$, step 2: 


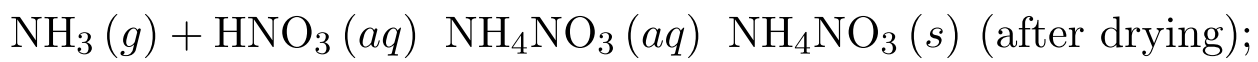

(b)

$\mathrm{H}_{2}(g)+\mathrm{Br}_{2}(l) 2 \mathrm{HBr}(g)$;

$\mathrm{Zn}(s)+\mathrm{S}(s) \mathrm{ZnS}(s)$

and

$\mathrm{ZnS}(s)+2 \mathrm{HCl}(a q) \mathrm{ZnCl}_{2}(a q)+\mathrm{H}_{2} \mathrm{~S}(g)$

Calcium cyclamate $\mathrm{Ca}\left(\mathrm{C}_{6} \mathrm{H}_{11} \mathrm{NHSO}_{3}\right)_{2}$ is an artificial sweetener used in many countries around the world but is banned in the United States. It can be purified industrially by converting it to the barium salt through reaction of the acid $\mathrm{C}_{6} \mathrm{H}_{11} \mathrm{NHSO}_{3} \mathrm{H}$ with barium carbonate, treatment with sulfuric acid (barium sulfate is very insoluble), and then neutralization with calcium hydroxide. Write the balanced equations for these reactions.

Footnotes

- 1The requirement of "charge balance" is just a specific type of "mass balance" in which the species in question are electrons. An equation must represent equal numbers of electrons on the reactant and product sides, and so both atoms and charges must be balanced.

Glossary

acid

substance that produces $\mathrm{H}_{3} \mathrm{O}^{+}$when dissolved in water 


\section{acid-base reaction}

reaction involving the transfer of a hydrogen ion between reactant species base

substance that produces $\mathrm{OH}^{-}$when dissolved in water

\section{combustion reaction}

vigorous redox reaction producing significant amounts of energy in the form of heat and, sometimes, light

\section{half-reaction}

an equation that shows whether each reactant loses or gains electrons in a reaction.

insoluble

of relatively low solubility; dissolving only to a slight extent neutralization reaction

reaction between an acid and a base to produce salt and water

\section{oxidation}

process in which an element's oxidation number is increased by loss of electrons oxidation-reduction reaction

(also, redox reaction) reaction involving a change in oxidation number for one or more reactant elements

\section{oxidation number}

(also, oxidation state) the charge each atom of an element would have in a compound if the compound were ionic 


\section{oxidizing agent}

(also, oxidant) substance that brings about the oxidation of another substance, and in the process becomes reduced

precipitate

insoluble product that forms from reaction of soluble reactants

precipitation reaction

reaction that produces one or more insoluble products; when reactants are ionic compounds, sometimes called doubledisplacement or metathesis

reduction

process in which an element's oxidation number is decreased by gain of electrons reducing agent

(also, reductant) substance that brings about the reduction of another substance, and in the process becomes oxidized

salt

ionic compound that can be formed by the reaction of an acid with a base that contains a cation and an anion other than hydroxide or oxide

\section{single-displacement reaction}

(also, replacement) redox reaction involving the oxidation of an elemental substance by an ionic species

\section{soluble}

of relatively high solubility; dissolving to a relatively large extent 


\section{solubility}

the extent to which a substance may be dissolved in water, or any solvent

\section{strong acid}

acid that reacts completely when dissolved in water to yield hydronium ions

strong base

base that reacts completely when

dissolved in water to yield hydroxide ions

weak acid

acid that reacts only to a slight extent

when dissolved in water to yield hydronium ions

weak base

base that reacts only to a slight extent when dissolved in water to yield hydroxide ions 


\section{2.}

\section{REACTION}

\section{STOICHIOMETRY}

Learning Objectives

By the end of this section, you will be able to:

- Explain the concept of stoichiometry as it pertains to chemical reactions

- Use balanced chemical equations to derive stoichiometric factors relating amounts of reactants and products

- Perform stoichiometric calculations involving mass, moles, and solution molarity

A balanced chemical equation provides a great deal of information in a very succinct format. Chemical formulas provide the identities of the reactants and products involved in the chemical change, allowing classification of the reaction. Coefficients provide the relative numbers 
of these chemical species, allowing a quantitative assessment of the relationships between the amounts of substances consumed and produced by the reaction. These quantitative relationships are known as the reaction's stoichiometry, a term derived from the Greek words stoicheion (meaning "element") and metron (meaning "measure"). In this module, the use of balanced chemical equations for various stoichiometric applications is explored.

The general approach to using stoichiometric relationships is similar in concept to the way people go about many common activities. Food preparation, for example, offers an appropriate comparison. A recipe for making eight pancakes calls for 1 cup pancake mix, $\frac{3}{4}$ cup milk, and one egg. The "equation" representing the preparation of pancakes per this recipe is 1 cup mix $+\frac{3}{4}$ cup milk +1 egg 8 pancakes

If two dozen pancakes are needed for a big family breakfast, the ingredient amounts must be increased proportionally according to the amounts given in the recipe. For example, the number of eggs required to make 24 pancakes is

$24 \overline{\text { pancakes }} \frac{1 \text { egg }}{8 \longdiv { \text { pancakes } }}=3$ eggs

Balanced chemical equations are used in much the same fashion to determine the amount of one reactant required to react with a given amount of another reactant, or to yield a given amount of product, and so forth. The coefficients in the balanced equation are used to derive stoichiometric factors that permit computation of the desired quantity. To illustrate this idea, consider the 
production of ammonia by reaction of hydrogen and nitrogen:

$\mathrm{N}_{2}(g)+3 \mathrm{H}_{2}(g) 2 \mathrm{NH}_{3}(g)$

This equation shows ammonia molecules are produced from hydrogen molecules in a 2:3 ratio, and stoichiometric factors may be derived using any amount (number) unit:

$\frac{2 \mathrm{NH}_{3} \text { molecules }}{3 \mathrm{H}_{2} \text { molecules }}$ or $\frac{2 \mathrm{doz} \mathrm{NH}_{3} \text { molecules }}{3 \mathrm{doz} \mathrm{H}_{2} \text { molecules }}$ or $\frac{2 \mathrm{~mol} \mathrm{NH}_{3} \text { molecules }}{3 \mathrm{~mol} \mathrm{H}_{2} \text { molecules }}$

These stoichiometric factors can be used to compute the number of ammonia molecules produced from a given number of hydrogen molecules, or the number of hydrogen molecules required to produce a given number of ammonia molecules. Similar factors may be derived for any pair of substances in any chemical equation.

Moles of Reactant Required in a Reaction How many moles of $\mathrm{I}_{2}$ are required to react with $0.429 \mathrm{~mol}$ of $\mathrm{Al}$ according to the following equation (see (Figure))?

\section{$2 \mathrm{Al}+3 \mathrm{I}_{2} 2 \mathrm{AlI}_{3}$}

Aluminum and iodine react to produce aluminum iodide. The heat of the reaction vaporizes some of the solid iodine as a purple vapor. (credit: modification of work by Mark Ott)
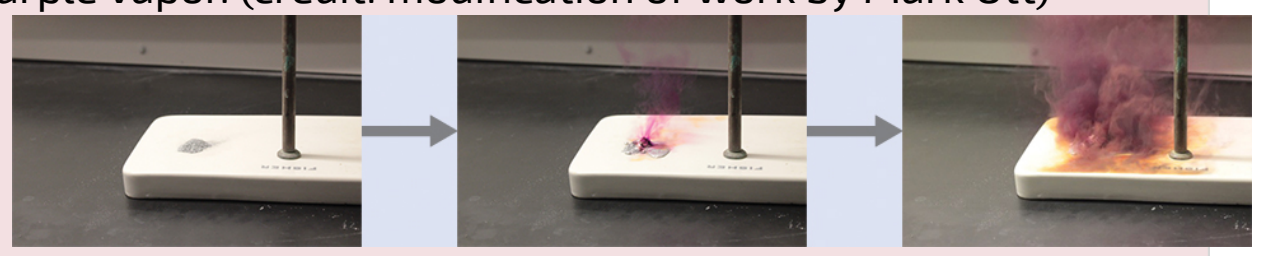

Solution Referring to the balanced chemical equation, the stoichiometric factor relating the two substances of interest is $\frac{3 \mathrm{~mol} \mathrm{I}}{2 \mathrm{~mol} \mathrm{Al}}$. The molar amount of iodine is derived by multiplying the provided molar amount of aluminum by this factor: 


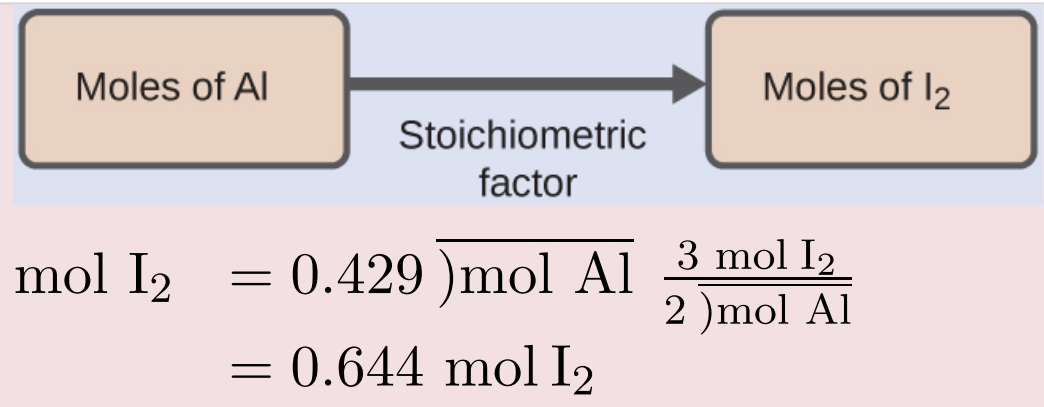

Check Your Learning How many moles of $\mathrm{Ca}(\mathrm{OH})_{2}$ are required to react with $1.36 \mathrm{~mol}$ of $\mathrm{H}_{3} \mathrm{PO}_{4}$ to produce Саз $\left(\mathrm{PO}_{4}\right)_{2}$ according to the equation $3 \mathrm{Ca}(\mathrm{OH})_{2}+2 \mathrm{H}_{3} \mathrm{PO}_{4} \mathrm{Ca}_{3}\left(\mathrm{PO}_{4}\right)_{2}+6 \mathrm{H}_{2} \mathrm{O}$ ?

Answer:

$2.04 \mathrm{~mol}$

Number of Product Molecules Generated by a Reaction How many carbon dioxide molecules are produced when $0.75 \mathrm{~mol}$ of propane is combusted according to this equation?

$$
\mathrm{C}_{3} \mathrm{H}_{8}+5 \mathrm{O}_{2} 3 \mathrm{CO}_{2}+4 \mathrm{H}_{2} \mathrm{O}
$$

Solution The approach here is the same as for (Figure), though the absolute number of molecules is requested, not the number of moles of molecules. This will simply require use of the moles-to-numbers conversion factor, Avogadro's number.

The balanced equation shows that carbon dioxide is produced from propane in a 3:1 ratio:

$\frac{3 \mathrm{~mol} \mathrm{CO}_{2}}{1 \mathrm{~mol} \mathrm{C}_{3} \mathrm{H}_{8}}$

Using this stoichiometric factor, the provided molar amount of propane, and Avogadro's number,

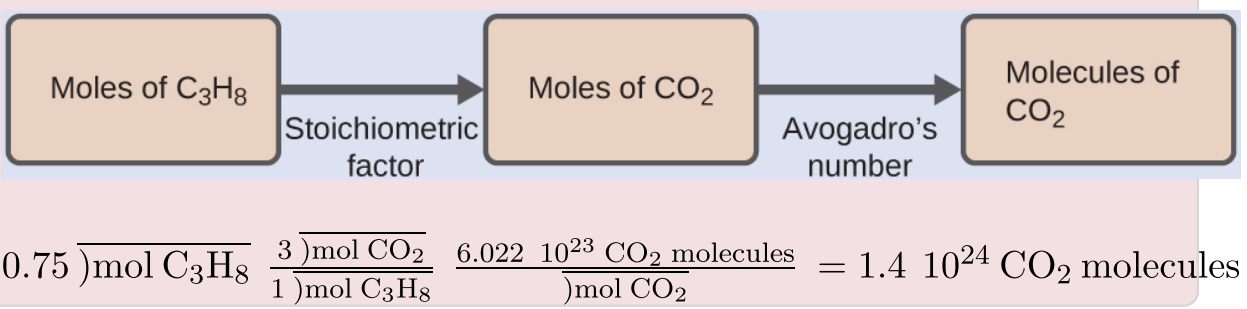


Check Your Learning How many $\mathrm{NH}_{3}$ molecules are produced by the reaction of $4.0 \mathrm{~mol}$ of $\mathrm{Ca}(\mathrm{OH})_{2}$ according to the following equation:

$\left(\mathrm{NH}_{4}\right)_{2} \mathrm{SO}_{4}+\mathrm{Ca}(\mathrm{OH})_{2} 2 \mathrm{NH}_{3}+\mathrm{CaSO}_{4}+2 \mathrm{H}_{2} \mathrm{O}$ Answer:

4.8 $10^{24} \mathrm{NH}_{3}$ molecules

These examples illustrate the ease with which the amounts of substances involved in a chemical reaction of known stoichiometry may be related. Directly measuring numbers of atoms and molecules is, however, not an easy task, and the practical application of stoichiometry requires that we use the more readily measured property of mass.

Relating Masses of Reactants and Products What mass of sodium hydroxide, $\mathrm{NaOH}$, would be required to produce $16 \mathrm{~g}$ of the antacid milk of magnesia [magnesium hydroxide, $\mathrm{Mg}(\mathrm{OH})_{2}$ ] by the following reaction?

$$
\mathrm{MgCl}_{2}(a q)+2 \mathrm{NaOH}(a q) \mathrm{Mg}(\mathrm{OH})_{2}(s)+2 \mathrm{NaCl}(a q)
$$

Solution The approach used previously in (Figure) and (Figure) is likewise used here; that is, we must derive an appropriate stoichiometric factor from the balanced chemical equation and use it to relate the amounts of the two substances of interest. In this case, however, masses (not molar amounts) are provided and requested, so additional steps of the sort learned in the previous chapter are required. The calculations required are outlined in this flowchart: 


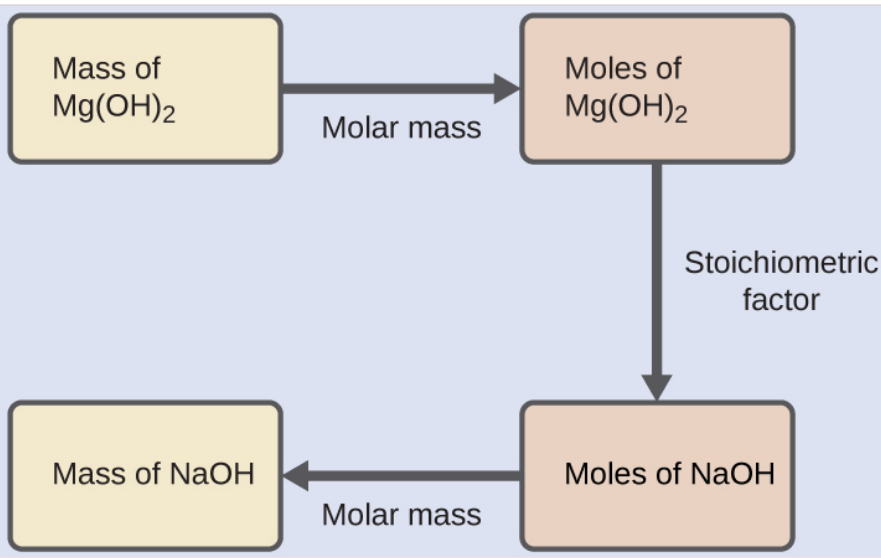

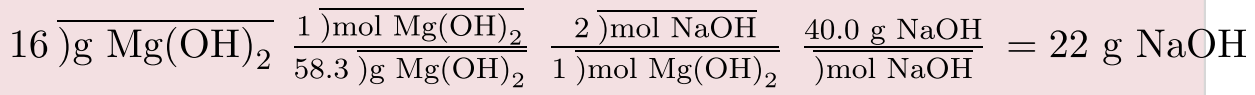

Check Your Learning What mass of gallium oxide, Ga203, can be prepared from $29.0 \mathrm{~g}$ of gallium metal? The equation for the reaction is $4 \mathrm{Ga}+3 \mathrm{O}_{2} 2 \mathrm{Ga}_{2} \mathrm{O}_{3}$.

Answer:

$39.0 \mathrm{~g}$

Relating Masses of Reactants What mass of oxygen gas, $\mathrm{O}_{2}$, from the air is consumed in the combustion of $702 \mathrm{~g}$ of octane, $\mathrm{C}_{8} \mathrm{H}_{18}$, one of the principal components of gasoline?

\section{$2 \mathrm{C}_{8} \mathrm{H}_{18}+25 \mathrm{O}_{2} 16 \mathrm{CO}_{2}+18 \mathrm{H}_{2} \mathrm{O}$}

Solution The approach required here is the same as for the (Figure), differing only in that the provided and requested masses are both for reactant species. 


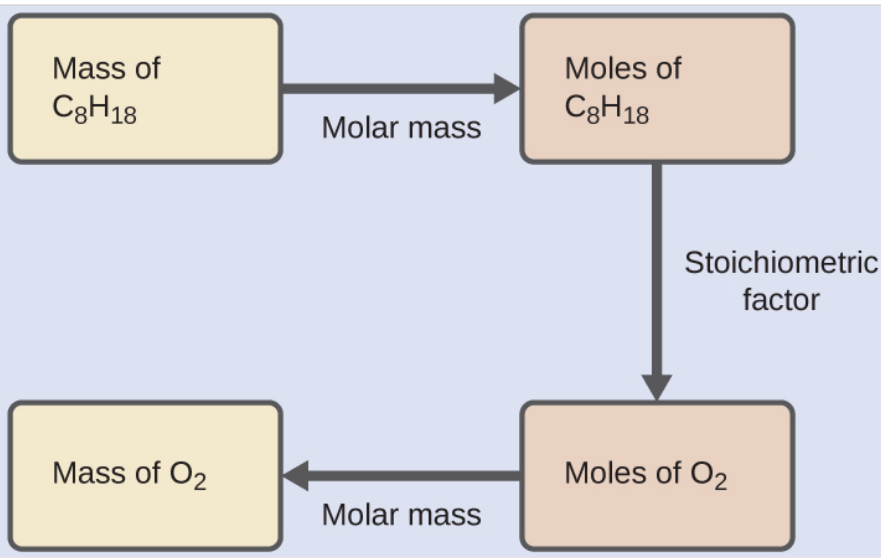

$7 0 2 \longdiv { \mathrm { g } \mathrm { C } _ { 8 } \mathrm { H } _ { 1 8 } } \frac { 1 \overline { \mathrm { mol } \mathrm { C } _ { 8 } \mathrm { H } _ { 1 8 } } } { 1 1 4 . 2 3 \overline { \mathrm { g } \mathrm { C } _ { 8 } \mathrm { H } _ { 1 8 } } } \frac { 2 5 \overline { \mathrm { mol } \mathrm { O } _ { 2 } } } { 2 \overline { \mathrm { mol } \mathrm { C } _ { 8 } \mathrm { H } _ { 1 8 } } } \frac { 3 2 . 0 0 \mathrm { g } \mathrm { O } _ { 2 } } { \overline { \mathrm { mol } \mathrm { O } _ { 2 } } } = 2 . 4 6 1 0 ^ { 3 } \mathrm { g } \mathrm { O } _ { 2 }$

Check Your Learning What mass of CO is required to react

with $25.13 \mathrm{~g}$ of $\mathrm{Fe}_{2} \mathrm{O}_{3}$ according to the equation $\mathrm{Fe}_{2} \mathrm{O}_{3}+3 \mathrm{CO} 2 \mathrm{Fe}+3 \mathrm{CO}_{2}$ ?

Answer:

$13.22 \mathrm{~g}$

These examples illustrate just a few instances of reaction stoichiometry calculations. Numerous variations on the beginning and ending computational steps are possible depending upon what particular quantities are provided and sought (volumes, solution concentrations, and so forth). Regardless of the details, all these calculations share a common essential component: the use of stoichiometric factors derived from balanced chemical equations. (Figure) provides a general outline of the various computational steps associated with many reaction stoichiometry calculations.

The flowchart depicts the various computational steps involved in most reaction stoichiometry calculations. 


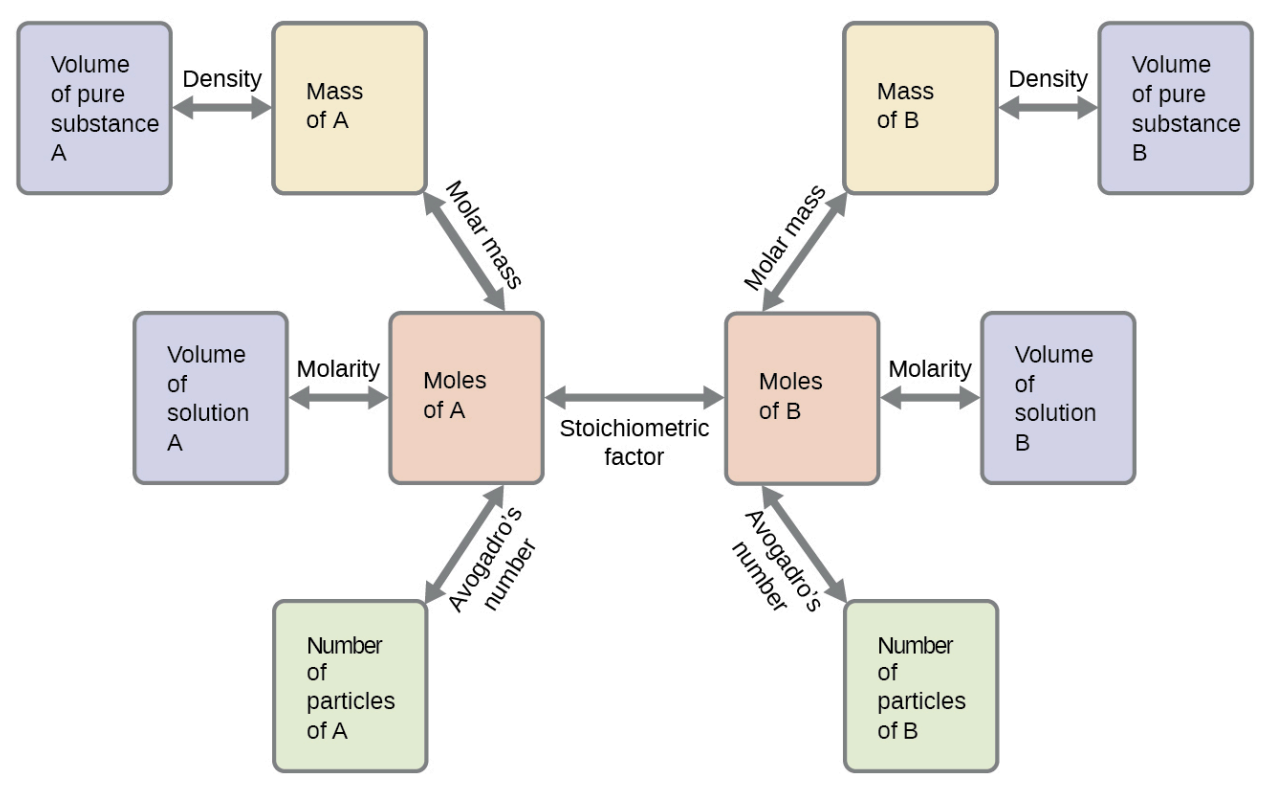

Airbags

Airbags ((Figure)) are a safety feature provided in most automobiles since the 1990s. The effective operation of an airbag requires that it be rapidly inflated with an appropriate amount (volume) of gas when the vehicle is involved in a collision. This requirement is satisfied in many automotive airbag systems through use of explosive chemical reactions, one common choice being the decomposition of sodium azide, $\mathrm{NaN}_{3}$. When sensors in the vehicle detect a collision, an electrical current is passed through a carefully measured amount of $\mathrm{NaN}_{3}$ to initiate its decomposition:

$2 \mathrm{NaN}_{3}(s) 3 \mathrm{~N}_{2}(g)+2 \mathrm{Na}(s)$

This reaction is very rapid, generating gaseous nitrogen that can deploy and fully inflate a typical airbag in a fraction of a second ( 0.03-0.1 s). Among many engineering considerations, the amount of sodium azide used must be appropriate for generating enough nitrogen gas to fully inflate the air bag and ensure its proper 
function. For example, a small mass ( $100 \mathrm{~g}$ ) of $\mathrm{NaN}_{3}$ will generate approximately $50 \mathrm{~L}$ of $\mathrm{N}_{2}$.

Airbags deploy upon impact to minimize serious injuries to passengers. (credit: Jon Seidman)

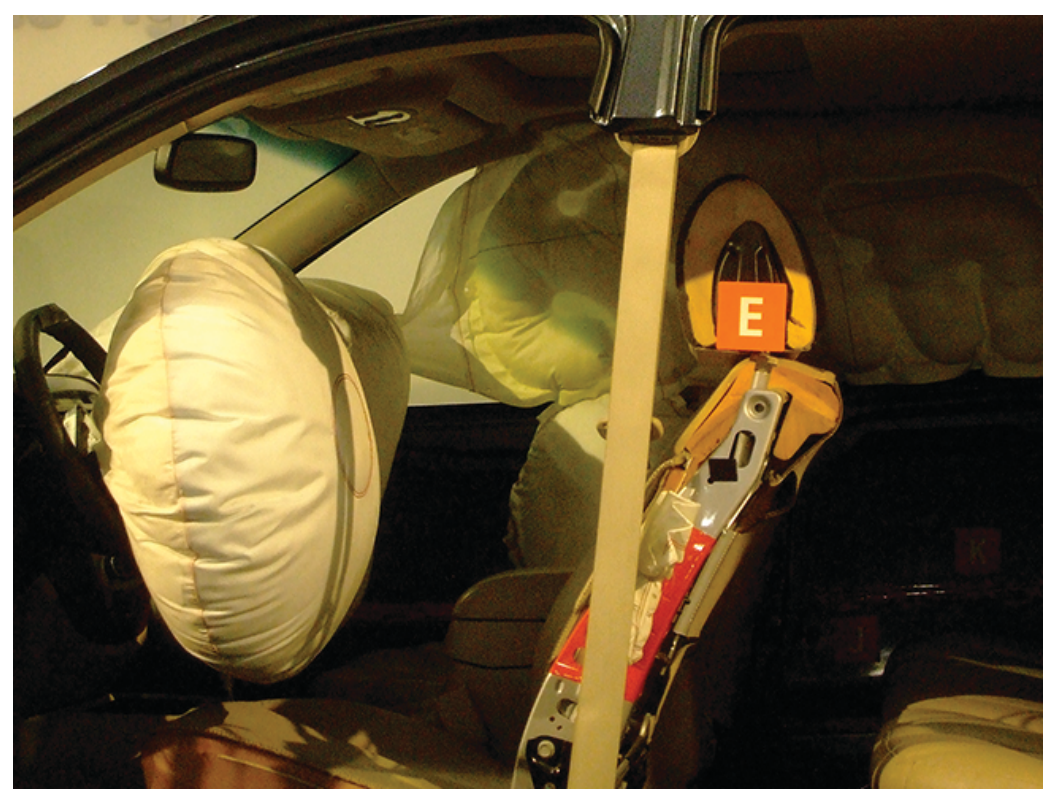

Key Concepts and Summary

A balanced chemical equation may be used to describe a reaction's stoichiometry (the relationships between amounts of reactants and products). Coefficients from the equation are used to derive stoichiometric factors that subsequently may be used for computations relating reactant and product masses, molar amounts, and other quantitative properties.

Chemistry End of Chapter Exercises

Write the balanced equation, then outline the steps necessary to determine the information requested in each of the following: 
(a) The number of moles and the mass of chlorine, $\mathrm{Cl}_{2}$, required to react with $10.0 \mathrm{~g}$ of sodium metal, $\mathrm{Na}$, to produce sodium chloride, $\mathrm{NaCl}$.

(b) The number of moles and the mass of oxygen formed by the decomposition of $1.252 \mathrm{~g}$ of mercury(II) oxide.

(c) The number of moles and the mass of sodium nitrate, $\mathrm{NaNO}_{3}$, required to produce $128 \mathrm{~g}$ of oxygen. ( $\mathrm{NaNO}_{2}$ is the other product.)

(d) The number of moles and the mass of carbon dioxide formed by the combustion of $20.0 \mathrm{~kg}$ of carbon in an excess of oxygen.

(e) The number of moles and the mass of copper(II) carbonate needed to produce $1.500 \mathrm{~kg}$ of copper(II) oxide. $\left(\mathrm{CO}_{2}\right.$ is the other product.)

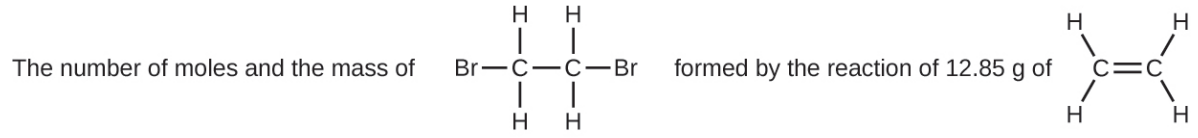
with an excess of $\mathrm{Br}_{2}$.

Determine the number of moles and the mass requested for each reaction in (Figure).

(a) $0.435 \mathrm{~mol} \mathrm{Na}, 0.217 \mathrm{~mol} \mathrm{Cl}_{2}, 15.4 \mathrm{~g} \mathrm{Cl}_{2}$; (b) 0.005780 mol HgO, $2.89010^{-3} \mathrm{~mol} \mathrm{O}_{2}, 9.24810^{-2} \mathrm{~g} \mathrm{O}_{2}$; (c) 8.00 mol $\mathrm{NaNO}_{3}, 6.810^{2} \mathrm{~g} \mathrm{NaNO}_{3}$; (d) $1665 \mathrm{~mol} \mathrm{CO}_{2}, 73.3 \mathrm{~kg}$ $\mathrm{CO}_{2}$; (e) $18.86 \mathrm{~mol} \mathrm{CuO}, 2.330 \mathrm{~kg} \mathrm{CuCO}$; (f) $0.4580 \mathrm{~mol}$ $\mathrm{C}_{2} \mathrm{H}_{4} \mathrm{Br}_{2}, 86.05 \mathrm{~g} \mathrm{C}_{2} \mathrm{H}_{4} \mathrm{Br}_{2}$

Write the balanced equation, then outline the steps necessary to determine the information requested in each of the following:

(a) The number of moles and the mass of $\mathrm{Mg}$ required to react with $5.00 \mathrm{~g}$ of $\mathrm{HCl}$ and produce $\mathrm{MgCl}_{2}$ and $\mathrm{H}_{2}$. 
(b) The number of moles and the mass of oxygen formed by the decomposition of $1.252 \mathrm{~g}$ of silver(I) oxide.

(c) The number of moles and the mass of magnesium carbonate, $\mathrm{MgCO}_{3}$, required to produce $283 \mathrm{~g}$ of carbon dioxide. (MgO is the other product.)

(d) The number of moles and the mass of water formed by the combustion of $20.0 \mathrm{~kg}$ of acetylene, $\mathrm{C}_{2} \mathrm{H}_{2}$, in an excess of oxygen.

(e) The number of moles and the mass of barium peroxide, $\mathrm{BaO}_{2}$, needed to produce $2.500 \mathrm{~kg}$ of barium oxide, $\mathrm{BaO}\left(\mathrm{O}_{2}\right.$ is the other product.)

(f)

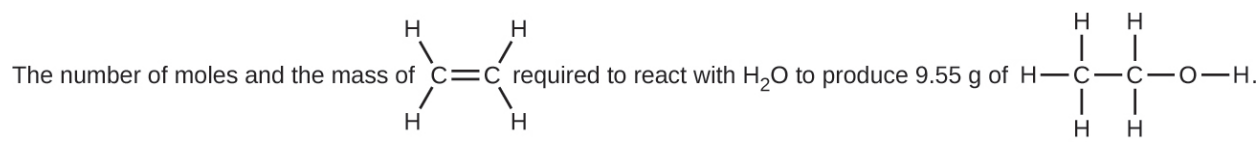

Determine the number of moles and the mass requested for each reaction in (Figure).

(a) $0.0686 \mathrm{~mol} \mathrm{Mg}, 1.67 \mathrm{~g} \mathrm{Mg}$; (b) $2.70110^{-3} \mathrm{~mol} \mathrm{O}_{2}$, $0.08644 \mathrm{~g} \mathrm{O}_{2}$; (c) $6.43 \mathrm{~mol} \mathrm{MgCO}_{3}, 542 \mathrm{~g} \mathrm{MgCO}_{3}$ (d) 713 mol H${ }_{2} \mathrm{O}, 12.8 \mathrm{~kg} \mathrm{H} 2 \mathrm{O}$; (e) $16.31 \mathrm{~mol} \mathrm{BaO}_{2}, 2762 \mathrm{~g} \mathrm{BaO}_{2}$; (f) $0.207 \mathrm{~mol} \mathrm{C}_{2} \mathrm{H}_{4}, 5.81 \mathrm{~g} \mathrm{C}_{2} \mathrm{H}_{4}$

$\mathrm{H}_{2}$ is produced by the reaction of $118.5 \mathrm{~mL}$ of a $0.8775-\mathrm{M}$ solution of $\mathrm{H}_{3} \mathrm{PO}_{4}$ according to the following equation: $2 \mathrm{Cr}+2 \mathrm{H}_{3} \mathrm{PO}_{4} 3 \mathrm{H}_{2}+2 \mathrm{CrPO}_{4}$.

(a) Outline the steps necessary to determine the number of moles and mass of $\mathrm{H}_{2}$.

(b) Perform the calculations outlined.

Gallium chloride is formed by the reaction of $2.6 \mathrm{~L}$ of 
a $1.44 \mathrm{M}$ solution of $\mathrm{HCl}$ according to the following equation: $2 \mathrm{Ga}+6 \mathrm{HCl} 2 \mathrm{GaCl}_{3}+3 \mathrm{H}_{2}$.

(a) Outline the steps necessary to determine the number of moles and mass of gallium chloride.

(b) Perform the calculations outlined.

(a) volume $\mathrm{HCl}$ solution mol $\mathrm{HCl}$ mol $\mathrm{GaCl}_{3}$; (b) $1.25 \mathrm{~mol} \mathrm{GaCl}_{3}, 2.210^{2} \mathrm{~g} \mathrm{GaCl}_{3}$

$\mathrm{I}_{2}$ is produced by the reaction of $0.4235 \mathrm{~mol}$ of $\mathrm{CuCl}_{2}$ according to the following equation: $2 \mathrm{CuCl}_{2}+4 \mathrm{KI} 2 \mathrm{CuI}+4 \mathrm{KCl}+\mathrm{I}_{2}$.

(a) How many molecules of $\mathrm{I}_{2}$ are produced?

(b) What mass of $\mathrm{I}_{2}$ is produced?

Silver is often extracted from ores such as $\mathrm{K}\left[\mathrm{Ag}(\mathrm{CN})_{2}\right]$ and then recovered by the reaction

$2 \mathrm{~K}\left[\mathrm{Ag}(\mathrm{CN})_{2}\right](a q)+\mathrm{Zn}(s) 2 \mathrm{Ag}(s)+\mathrm{Zn}(\mathrm{CN})_{2}(a q)+2 \mathrm{KCN}(a q)$

(a) How many molecules of $\mathrm{Zn}(\mathrm{CN})_{2}$ are produced by the reaction of $35.27 \mathrm{~g}$ of $\mathrm{K}\left[\mathrm{Ag}(\mathrm{CN})_{2}\right]$ ?

(b) What mass of $\mathrm{Zn}(\mathrm{CN})_{2}$ is produced?

(a) $5.33710^{22}$ molecules; (b) $10.41 \mathrm{~g} \mathrm{Zn}(\mathrm{CN})_{2}$

What mass of silver oxide, $\mathrm{Ag}_{2} \mathrm{O}$, is required to produce $25.0 \mathrm{~g}$ of silver sulfadiazine, $\mathrm{AgC}_{10} \mathrm{H}_{9} \mathrm{~N}_{4} \mathrm{SO}_{2}$, from the reaction of silver oxide and sulfadiazine? $2 \mathrm{C}_{10} \mathrm{H}_{10} \mathrm{~N}_{4} \mathrm{SO}_{2}+\mathrm{Ag}_{2} \mathrm{O} 2 \mathrm{AgC}_{10} \mathrm{H}_{9} \mathrm{~N}_{4} \mathrm{SO}_{2}+\mathrm{H}_{2} \mathrm{O}$

Carborundum is silicon carbide, $\mathrm{SiC}$, a very hard material used as an abrasive on sandpaper and in other applications. It is prepared by the reaction of pure sand, $\mathrm{SiO}_{2}$, with carbon at high temperature. Carbon monoxide, 
$\mathrm{CO}$, is the other product of this reaction. Write the balanced equation for the reaction, and calculate how much $\mathrm{SiO}_{2}$ is required to produce $3.00 \mathrm{~kg}$ of $\mathrm{SiC}$.

$\mathrm{SiO}_{2}+3 \mathrm{C} \mathrm{SiC}+2 \mathrm{CO}, 4.50 \mathrm{~kg} \mathrm{SiO} 2$

Automotive air bags inflate when a sample of sodium azide, $\mathrm{NaN}_{3}$, is very rapidly decomposed.

$2 \mathrm{NaN}_{3}(s) \quad 2 \mathrm{Na}(s)+3 \mathrm{~N}_{2}(g)$

What mass of sodium azide is required to produce $2.6 \mathrm{ft}^{3}$ (73.6 $\mathrm{L}$ ) of nitrogen gas with a density of $1.25 \mathrm{~g} / \mathrm{L}$ ?

Urea, $\mathrm{CO}\left(\mathrm{NH}_{2}\right)_{2}$, is manufactured on a large scale for use in producing urea-formaldehyde plastics and as a fertilizer. What is the maximum mass of urea that can be manufactured from the $\mathrm{CO}_{2}$ produced by combustion of $1.0010^{3} \mathrm{~kg}$ of carbon followed by the reaction?

$\mathrm{CO}_{2}(g)+2 \mathrm{NH}_{3}(g) \mathrm{CO}\left(\mathrm{NH}_{2}\right)_{2}(s)+\mathrm{H}_{2} \mathrm{O}(l)$

$5.0010^{3} \mathrm{~kg}$

In an accident, a solution containing $2.5 \mathrm{~kg}$ of nitric acid was spilled. Two kilograms of $\mathrm{Na}_{2} \mathrm{CO}_{3}$ was quickly spread on the area and $\mathrm{CO}_{2}$ was released by the reaction. Was sufficient $\mathrm{Na}_{2} \mathrm{CO}_{3}$ used to neutralize all of the acid?

A compact car gets 37.5 miles per gallon on the highway. If gasoline contains $84.2 \%$ carbon by mass and has a density of $0.8205 \mathrm{~g} / \mathrm{mL}$, determine the mass of carbon dioxide produced during a 500-mile trip (3.785 liters per gallon).

$1.2810^{5} \mathrm{~g} \mathrm{CO}_{2}$ 
What volume of $0.750 \mathrm{M}$ hydrochloric acid solution can be prepared from the $\mathrm{HCl}$ produced by the reaction of $25.0 \mathrm{~g}$ of $\mathrm{NaCl}$ with excess sulfuric acid?

$\mathrm{NaCl}(s)+\mathrm{H}_{2} \mathrm{SO}_{4}(l) \mathrm{HCl}(g)+\mathrm{NaHSO}_{4}(s)$

What volume of a $0.2089 M \mathrm{KI}$ solution contains enough $\mathrm{KI}$ to react exactly with the $\mathrm{Cu}\left(\mathrm{NO}_{3}\right)_{2}$ in $43.88 \mathrm{~mL}$ of a $0.3842 M$ solution of $\mathrm{Cu}\left(\mathrm{NO}_{3}\right)_{2}$ ?

$2 \mathrm{Cu}\left(\mathrm{NO}_{3}\right)_{2}+4 \mathrm{KI} 2 \mathrm{CuI}+\mathrm{I}_{2}+4 \mathrm{KNO}_{3}$

161.40 mL KI solution

A mordant is a substance that combines with a dye to produce a stable fixed color in a dyed fabric. Calcium acetate is used as a mordant. It is prepared by the reaction of acetic acid with calcium hydroxide. $2 \mathrm{CH}_{3} \mathrm{CO}_{2} \mathrm{H}+\mathrm{Ca}(\mathrm{OH})_{2} \mathrm{Ca}\left(\mathrm{CH}_{3} \mathrm{CO}_{2}\right)_{2}+2 \mathrm{H}_{2} \mathrm{O}$

What mass of $\mathrm{Ca}(\mathrm{OH})_{2}$ is required to react with the acetic acid in $25.0 \mathrm{~mL}$ of a solution having a density of $1.065 \mathrm{~g} / \mathrm{mL}$ and containing $58.0 \%$ acetic acid by mass?

The toxic pigment called white lead, $\mathrm{Pb}_{3}(\mathrm{OH})_{2}\left(\mathrm{CO}_{3}\right)_{2}$, has been replaced in white paints by rutile, $\mathrm{TiO}_{2}$. How much rutile ( $\mathrm{g}$ ) can be prepared from $379 \mathrm{~g}$ of an ore that contains $88.3 \%$ ilmenite $\left(\mathrm{FeTiO}_{3}\right)$ by mass?

$2 \mathrm{FeTiO}_{3}+4 \mathrm{HCl}+\mathrm{Cl}_{2} 2 \mathrm{FeCl}_{3}+2 \mathrm{TiO}_{2}+2 \mathrm{H}_{2} \mathrm{O}$

$176 \mathrm{~g} \mathrm{TiO}_{2}$ 
Glossary

stoichiometric factor

ratio of coefficients in a balanced chemical equation, used in computations relating amounts of reactants and products stoichiometry relationships between the amounts of reactants and products of a chemical reaction 


\section{3.}

\section{REACTION YIELDS}

\section{Learning Objectives}

By the end of this section, you will be able to:

- Explain the concepts of theoretical yield and limiting reactants/reagents.

- $\quad$ Derive the theoretical yield for a reaction under specified conditions.

- $\quad$ Calculate the percent yield for a reaction.

The relative amounts of reactants and products represented in a balanced chemical equation are often referred to as stoichiometric amounts. All the exercises of the preceding module involved stoichiometric amounts of reactants. For example, when calculating the amount of product generated from a given amount of reactant, it was assumed that any other reactants required were available in stoichiometric amounts (or greater). In this 
module, more realistic situations are considered, in which reactants are not present in stoichiometric amounts.

Limiting Reactant

Consider another food analogy, making grilled cheese sandwiches ((Figure)):

1 slice of cheese +2 slices of bread 1 sandwich

Stoichiometric amounts of sandwich ingredients for this recipe are bread and cheese slices in a 2:1 ratio. Provided with 28 slices of bread and 11 slices of cheese, one may prepare 11 sandwiches per the provided recipe, using all the provided cheese and having six slices of bread left over. In this scenario, the number of sandwiches prepared has been limited by the number of cheese slices, and the bread slices have been provided in excess.

Sandwich making can illustrate the concepts of limiting and excess reactants.
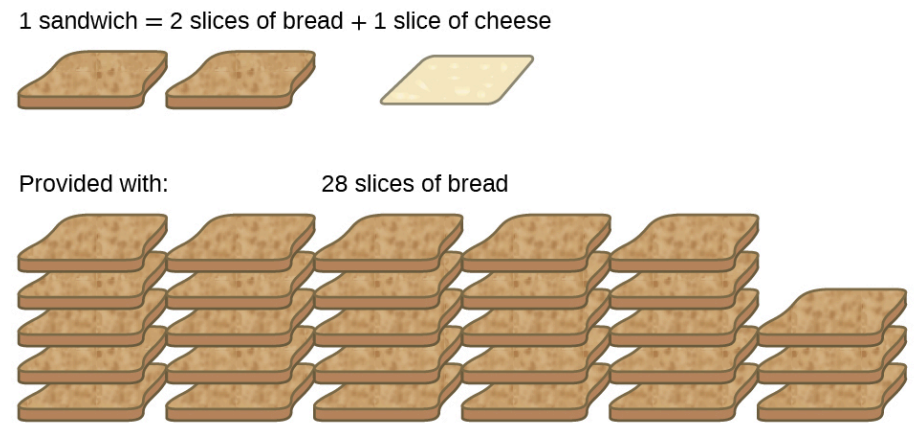

+11 slices of cheese
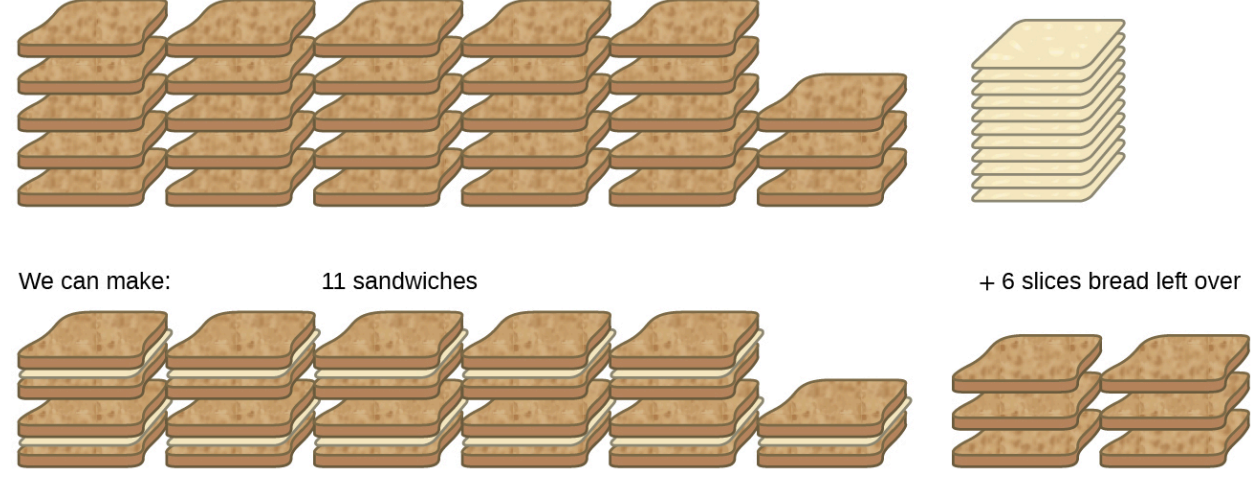

Consider this concept now with regard to a chemical 
process, the reaction of hydrogen with chlorine to yield hydrogen chloride:

$\mathrm{H}_{2}(g)+\mathrm{Cl}_{2}(g) 2 \mathrm{HCl}(g)$

The balanced equation shows the hydrogen and chlorine react in a 1:1 stoichiometric ratio. If these reactants are provided in any other amounts, one of the reactants will nearly always be entirely consumed, thus limiting the amount of product that may be generated. This substance is the limiting reactant, and the other substance is the excess reactant. Identifying the limiting and excess reactants for a given situation requires computing the molar amounts of each reactant provided and comparing them to the stoichiometric amounts represented in the balanced chemical equation. For example, imagine combining 3 moles of $\mathrm{H}_{2}$ and 2 moles of $\mathrm{Cl}_{2}$. This represents a $3: 2$ (or $1.5: 1$ ) ratio of hydrogen to chlorine present for reaction, which is greater than the stoichiometric ratio of $1: 1$. Hydrogen, therefore, is present in excess, and chlorine is the limiting reactant. Reaction of all the provided chlorine ( $2 \mathrm{~mol}$ ) will consume $2 \mathrm{~mol}$ of the $3 \mathrm{~mol}$ of hydrogen provided, leaving $1 \mathrm{~mol}$ of hydrogen unreacted.

An alternative approach to identifying the limiting reactant involves comparing the amount of product expected for the complete reaction of each reactant. Each reactant amount is used to separately calculate the amount of product that would be formed per the reaction's stoichiometry. The reactant yielding the lesser amount of product is the limiting reactant. For the example in the previous paragraph, complete reaction of the hydrogen would yield 
mol $\mathrm{HCl}$ produced $=3 \mathrm{~mol} \mathrm{H}_{2} \frac{2 \mathrm{~mol} \mathrm{HCl}}{1 \mathrm{~mol} \mathrm{H}_{2}}=6 \mathrm{~mol} \mathrm{HCl}$

Complete reaction of the provided chlorine would produce mol $\mathrm{HCl}$ produced $=2 \mathrm{~mol} \mathrm{Cl}_{2} \frac{2 \mathrm{~mol} \mathrm{HCl}}{1 \mathrm{~mol} \mathrm{Cl}_{2}}=4 \mathrm{~mol} \mathrm{HCl}$

The chlorine will be completely consumed once 4 moles of $\mathrm{HCl}$ have been produced. Since enough hydrogen was provided to yield 6 moles of $\mathrm{HCl}$, there will be unreacted hydrogen remaining once this reaction is complete. Chlorine, therefore, is the limiting reactant and hydrogen is the excess reactant ((Figure)).

When $\mathrm{H}_{2}$ and $\mathrm{Cl}_{2}$ are combined in nonstoichiometric amounts, one of these reactants will limit the amount of $\mathrm{HCl}$ that can be produced. This illustration shows a reaction in which hydrogen is present in excess and chlorine is the limiting reactant.
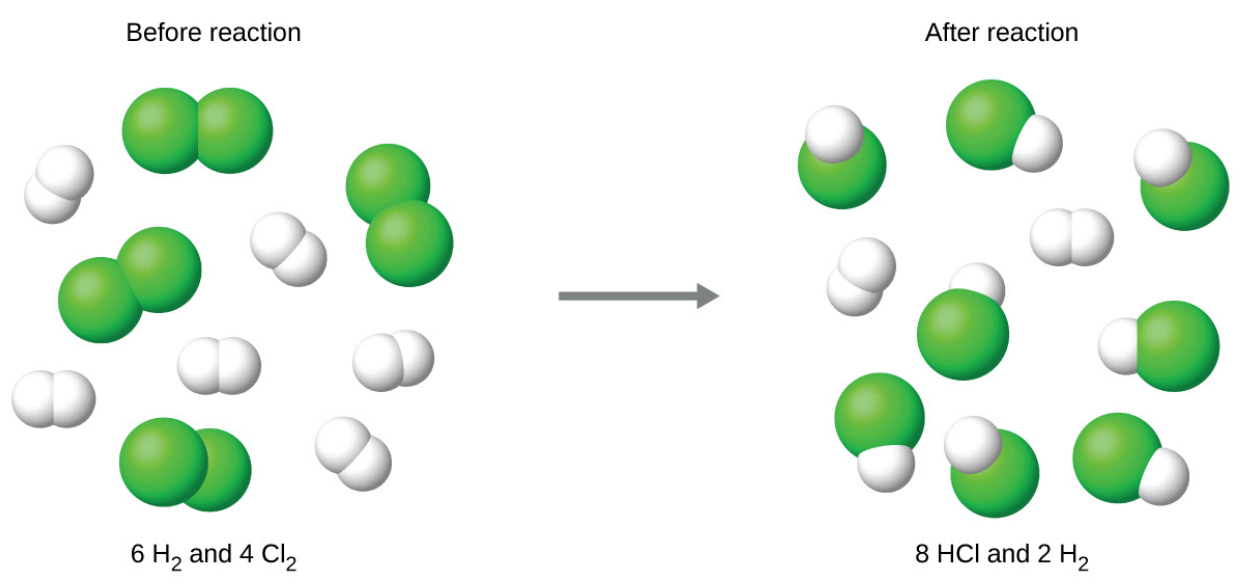

View this interactive simulation illustrating the concepts of limiting and excess reactants.

Identifying the Limiting Reactant Silicon nitride is a very hard, high-temperature-resistant ceramic used as a 
component of turbine blades in jet engines. It is prepared according to the following equation:

$$
3 \mathrm{Si}(s)+2 \mathrm{~N}_{2}(g) \quad \mathrm{Si}_{3} \mathrm{~N}_{4}(s)
$$

Which is the limiting reactant when $2.00 \mathrm{~g}$ of $\mathrm{Si}$ and $1.50 \mathrm{~g}$ of $\mathrm{N}_{2}$ react?

Solution Compute the provided molar amounts of reactants, and then compare these amounts to the balanced equation to identify the limiting reactant.

$$
\begin{aligned}
& \text { mol Si }=2.00 \overline{) g \mathrm{Si}} \frac{1 \mathrm{~mol} \mathrm{Si}}{28.09 \overline{\mathrm{g} \mathrm{Si}}}=0.0712 \mathrm{~mol} \mathrm{Si} \\
& \mathrm{mol} \mathrm{N}_{2}=1.50 \overline{\mathrm{g} \mathrm{N}_{2}} \frac{1 \mathrm{~mol} \mathrm{~N}}{28.02 \overline{\mathrm{g} \mathrm{N}_{2}}}=0.0535 \mathrm{~mol} \mathrm{~N}_{2}
\end{aligned}
$$

The provided Si:N2 molar ratio is:

$$
\frac{0.0712 \mathrm{~mol} \mathrm{Si}}{0.0535 \mathrm{~mol} \mathrm{~N}_{2}}=\frac{1.33 \mathrm{~mol} \mathrm{Si}}{1 \mathrm{~mol} \mathrm{~N}_{2}}
$$

The stoichiometric Si: $\mathrm{N}_{2}$ ratio is:

$$
\frac{3 \mathrm{~mol} \mathrm{Si}}{2 \mathrm{~mol} \mathrm{~N}_{2}}=\frac{1.5 \mathrm{~mol} \mathrm{Si}}{1 \mathrm{~mol} \mathrm{~N}_{2}}
$$

Comparing these ratios shows that $\mathrm{Si}$ is provided in a lessthan-stoichiometric amount, and so is the limiting reactant.

Alternatively, compute the amount of product expected for complete reaction of each of the provided reactants. The 0.0712 moles of silicon would yield

mol Si ${ }_{3} \mathrm{~N}_{4}$ produced $=0.0712 \mathrm{~mol} \mathrm{Si} \frac{1 \mathrm{~mol} \mathrm{Si}_{3} \mathrm{~N}_{4}}{3 \mathrm{~mol} \mathrm{Si}}=0.0237 \mathrm{~mol} \mathrm{Si}_{3} \mathrm{~N}_{4}$ while the 0.0535 moles of nitrogen would produce

mol $\mathrm{Si}_{3} \mathrm{~N}_{4}$ produced $=0.0535 \mathrm{~mol} \mathrm{~N}_{2} \frac{1 \mathrm{~mol} \mathrm{Si}_{3} \mathrm{~N}_{4}}{2 \mathrm{~mol} \mathrm{~N}_{2}}=0.0268 \mathrm{~mol} \mathrm{Si}_{3} \mathrm{~N}_{4}$

Since silicon yields the lesser amount of product, it is the limiting reactant.

Check Your Learning Which is the limiting reactant when $5.00 \mathrm{~g}$ of $\mathrm{H}_{2}$ and $10.0 \mathrm{~g}$ of $\mathrm{O}_{2}$ react and form water?

Answer:

$\mathrm{O}_{2}$ 
Percent Yield

The amount of product that may be produced by a reaction under specified conditions, as calculated per the stoichiometry of an appropriate balanced chemical equation, is called the theoretical yield of the reaction. In practice, the amount of product obtained is called the actual yield, and it is often less than the theoretical yield for a number of reasons. Some reactions are inherently inefficient, being accompanied by side reactions that generate other products. Others are, by nature, incomplete (consider the partial reactions of weak acids and bases discussed earlier in this chapter). Some products are difficult to collect without some loss, and so less than perfect recovery will reduce the actual yield. The extent to which a reaction's theoretical yield is achieved is commonly expressed as its percent yield:

percent yield $=\frac{\text { actual yield }}{\text { theoretical yield }} 100$

Actual and theoretical yields may be expressed as masses or molar amounts (or any other appropriate property; e.g., volume, if the product is a gas). As long as both yields are expressed using the same units, these units will cancel when percent yield is calculated.

\section{Green Chemistry and Atom Economy}

The purposeful design of chemical products and processes that minimize the use of environmentally hazardous substances and the generation of waste is known as green chemistry. Green chemistry is a philosophical approach that is being applied to many areas of science and 
technology, and its practice is summarized by guidelines known as the "Twelve Principles of Green Chemistry" (see details at this website). One of the 12 principles is aimed specifically at maximizing the efficiency of processes for synthesizing chemical products. The atom economy of a process is a measure of this efficiency, defined as the percentage by mass of the final product of a synthesis relative to the masses of all the reactants used:

atom economy $=\frac{\text { mass of product }}{\text { mass of reactants }} 100$

Though the definition of atom economy at first glance appears very similar to that for percent yield, be aware that this property represents a difference in the theoretical efficiencies of different chemical processes. The percent yield of a given chemical process, on the other hand, evaluates the efficiency of a process by comparing the yield of product actually obtained to the maximum yield predicted by stoichiometry.

The synthesis of the common nonprescription pain medication, ibuprofen, nicely illustrates the success of a green chemistry approach ((Figure)). First marketed in the early 1960s, ibuprofen was produced using a six-step synthesis that required $514 \mathrm{~g}$ of reactants to generate each mole (206 g) of ibuprofen, an atom economy of $40 \%$. In the 1990s, an alternative process was developed by the BHC Company (now BASF Corporation) that requires only three steps and has an atom economy of $\sim 80 \%$, nearly twice that of the original process. The BHC process generates significantly less chemical waste; uses lesshazardous and recyclable materials; and provides significant cost-savings to the manufacturer (and, subsequently, the consumer). In recognition of the 
positive environmental impact of the BHC process, the company received the Environmental Protection Agency's Greener Synthetic Pathways Award in 1997.

(a) Ibuprofen is a popular nonprescription pain

medication commonly sold as $200 \mathrm{mg}$ tablets. (b) The BHC process for synthesizing ibuprofen requires only three steps and exhibits an impressive atom economy. (credit a: modification of work by Derrick Coetzee)

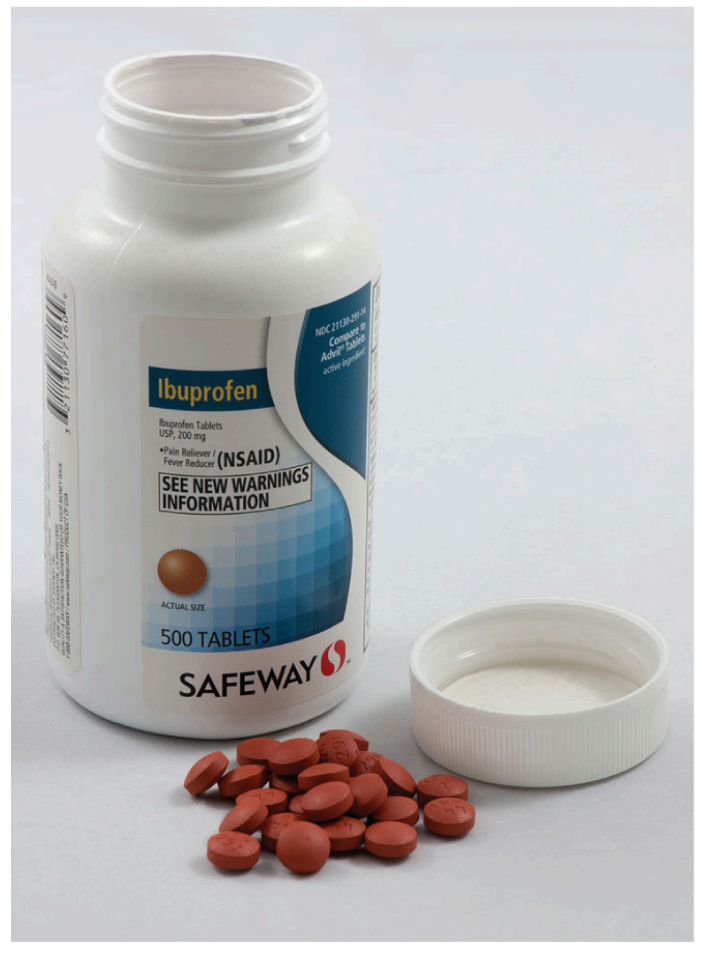

(a)

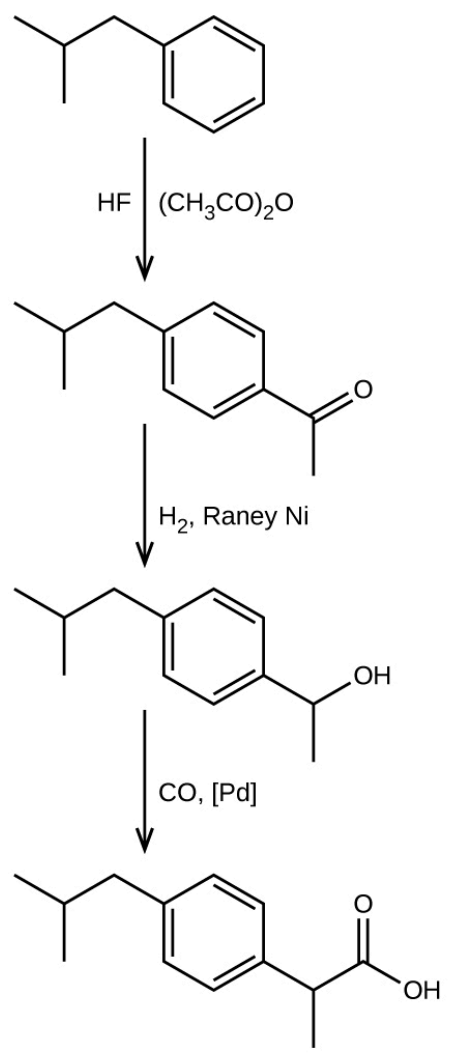

(b)

Key Concepts and Summary

When reactions are carried out using less-thanstoichiometric quantities of reactants, the amount of product generated will be determined by the limiting reactant. The amount of product generated by a chemical 
reaction is its actual yield. This yield is often less than the amount of product predicted by the stoichiometry of the balanced chemical equation representing the reaction (its theoretical yield). The extent to which a reaction generates the theoretical amount of product is expressed as its percent yield.

Key Equations

- percent yield $=\left(\frac{\text { actual yield }}{\text { theoretical yield }}\right) 100$

Chemistry End of Chapter Exercises

The following quantities are placed in a container: 1.5 $10^{24}$ atoms of hydrogen, $1.0 \mathrm{~mol}$ of sulfur, and $88.0 \mathrm{~g}$ of diatomic oxygen.

(a) What is the total mass in grams for the collection of all three elements?

(b) What is the total number of moles of atoms for the three elements?

(c) If the mixture of the three elements formed a compound with molecules that contain two hydrogen atoms, one sulfur atom, and four oxygen atoms, which substance is consumed first?

(d) How many atoms of each remaining element would remain unreacted in the change described in (c)?

What is the limiting reactant in a reaction that produces sodium chloride from $8 \mathrm{~g}$ of sodium and $8 \mathrm{~g}$ of diatomic chlorine?

The limiting reactant is $\mathrm{Cl} 2$. 
Which of the postulates of Dalton's atomic theory explains why we can calculate a theoretical yield for a chemical reaction?

A student isolated $25 \mathrm{~g}$ of a compound following a procedure that would theoretically yield $81 \mathrm{~g}$. What was his percent yield?

A sample of $0.53 \mathrm{~g}$ of carbon dioxide was obtained by heating $1.31 \mathrm{~g}$ of calcium carbonate. What is the percent yield for this reaction?

$$
\mathrm{CaCO}_{3}(s) \mathrm{CaO}(s)+\mathrm{CO}_{2}(s)
$$

Freon-12, $\mathrm{CCl}_{2} \mathrm{~F}_{2}$, is prepared from $\mathrm{CCl}_{4}$ by reaction with $\mathrm{HF}$. The other product of this reaction is $\mathrm{HCl}$. Outline the steps needed to determine the percent yield of a reaction that produces $12.5 \mathrm{~g}$ of $\mathrm{CCl}_{2} \mathrm{~F}_{2}$ from $32.9 \mathrm{~g}$ of $\mathrm{CCl}_{4}$. Freon-12 has been banned and is no longer used as a refrigerant because it catalyzes the decomposition of ozone and has a very long lifetime in the atmosphere. Determine the percent yield.

Citric acid, $\mathrm{C}_{6} \mathrm{H}_{8} \mathrm{O}_{7}$, a component of jams, jellies, and fruity soft drinks, is prepared industrially via fermentation of sucrose by the mold Aspergillus niger. The equation representing this reaction is

$\mathrm{C}_{12} \mathrm{H}_{22} \mathrm{O}_{11}+\mathrm{H}_{2} \mathrm{O}+3 \mathrm{O}_{2} 2 \mathrm{C}_{6} \mathrm{H}_{8} \mathrm{O}_{7}+4 \mathrm{H}_{2} \mathrm{O}$

What mass of citric acid is produced from exactly 1 metric ton $\left(1.00010^{3} \mathrm{~kg}\right)$ of sucrose if the yield is $92.30 \%$ ?

Toluene, $\mathrm{C}_{6} \mathrm{H}_{5} \mathrm{CH}_{3}$, is oxidized by air under carefully controlled conditions to benzoic acid, $\mathrm{C}_{6} \mathrm{H}_{5} \mathrm{CO}_{2} \mathrm{H}$, which is used to prepare the food preservative sodium benzoate, 
$\mathrm{C}_{6} \mathrm{H}_{5} \mathrm{CO}_{2} \mathrm{Na}$. What is the percent yield of a reaction that converts $1.000 \mathrm{~kg}$ of toluene to $1.21 \mathrm{~kg}$ of benzoic acid?

$$
2 \mathrm{C}_{6} \mathrm{H}_{5} \mathrm{CH}_{3}+3 \mathrm{O}_{2} 2 \mathrm{C}_{6} \mathrm{H}_{5} \mathrm{CO}_{2} \mathrm{H}+2 \mathrm{H}_{2} \mathrm{O}
$$

In a laboratory experiment, the reaction of $3.0 \mathrm{~mol}$ of $\mathrm{H}_{2}$ with $2.0 \mathrm{~mol}$ of $\mathrm{I}_{2}$ produced $1.0 \mathrm{~mol}$ of HI. Determine the theoretical yield in grams and the percent yield for this reaction.

Outline the steps needed to solve the following problem, then do the calculations. Ether, $\left(\mathrm{C}_{2} \mathrm{H}_{5}\right)_{2} \mathrm{O}$, which was originally used as an anesthetic but has been replaced by safer and more effective medications, is prepared by the reaction of ethanol with sulfuric acid.

$$
2 \mathrm{C}_{2} \mathrm{H}_{5} \mathrm{OH}+\mathrm{H}_{2} \mathrm{SO}_{4} ?\left(\mathrm{C}_{2} \mathrm{H}_{5}\right)_{2} \mathrm{O}+\mathrm{H}_{2} \mathrm{SO}_{4} \cdot \mathrm{H}_{2} \mathrm{O}
$$

What is the percent yield of ether if $1.17 \mathrm{~L}(\mathrm{~d}=0.7134 \mathrm{~g} /$ $\mathrm{mL}$ ) is isolated from the reaction of $1.500 \mathrm{~L}$ of $\mathrm{C}_{2} \mathrm{H}_{5} \mathrm{OH}$ $(\mathrm{d}=0.7894 \mathrm{~g} / \mathrm{mL})$ ?

Convert mass of ethanol to moles of ethanol; relate the moles of ethanol to the moles of ether produced using the stoichiometry of the balanced equation. Convert moles of ether to grams; divide the actual grams of ether (determined through the density) by the theoretical mass to determine the percent yield; $87.6 \%$

Outline the steps needed to determine the limiting reactant when $30.0 \mathrm{~g}$ of propane, $\mathrm{C}_{3} \mathrm{H}_{8}$, is burned with 75.0 $\mathrm{g}$ of oxygen.

Determine the limiting reactant.

Outline the steps needed to determine the limiting reactant when $0.50 \mathrm{~mol}$ of $\mathrm{Cr}$ and $0.75 \mathrm{~mol}$ of $\mathrm{H}_{3} \mathrm{PO}_{4}$ react 
according to the following chemical equation.

$2 \mathrm{Cr}+2 \mathrm{H}_{3} \mathrm{PO}_{4} 2 \mathrm{CrPO}_{4}+3 \mathrm{H}_{2}$

Determine the limiting reactant.

The conversion needed is mol $\mathrm{Cr}$ mol $\mathrm{H}_{3} \mathrm{PO}_{4}$. Then compare the amount of $\mathrm{Cr}$ to the amount of acid present. $\mathrm{Cr}$ is the limiting reactant.

What is the limiting reactant when $1.50 \mathrm{~g}$ of lithium and $1.50 \mathrm{~g}$ of nitrogen combine to form lithium nitride, a component of advanced batteries, according to the following unbalanced equation?

$\mathrm{Li}+\mathrm{N}_{2} \mathrm{Li}_{3} \mathrm{~N}$

Uranium can be isolated from its ores by dissolving it as $\mathrm{UO}_{2}\left(\mathrm{NO}_{3}\right)_{2}$, then separating it as solid $\mathrm{UO}_{2}\left(\mathrm{C}_{2} \mathrm{O}_{4}\right) \cdot 3 \mathrm{H}_{2} \mathrm{O}$. Addition of $0.4031 \mathrm{~g}$ of sodium oxalate, $\mathrm{Na}_{2} \mathrm{C}_{2} \mathrm{O}_{4}$, to a solution containing $1.481 \mathrm{~g}$ of uranyl nitrate, $\mathrm{UO}_{2}\left(\mathrm{NO}_{3}\right)_{2}$, yields $1.073 \mathrm{~g}$ of solid $\mathrm{UO}_{2}\left(\mathrm{C}_{2} \mathrm{O}_{4}\right) \cdot 3 \mathrm{H}_{2} \mathrm{O}$.

$\mathrm{Na}_{2} \mathrm{C}_{2} \mathrm{O}_{4}+\mathrm{UO}_{2}\left(\mathrm{NO}_{3}\right)_{2}+3 \mathrm{H}_{2} \mathrm{O} ? \mathrm{UO}_{2}\left(\mathrm{C}_{2} \mathrm{O}_{4}\right) \cdot 3 \mathrm{H}_{2} \mathrm{O}+$ $2 \mathrm{NaNO}_{3}$

Determine the limiting reactant and the percent yield of this reaction.

$\mathrm{Na}_{2} \mathrm{C}_{2} \mathrm{O}_{4}$ is the limiting reactant. percent yield $=86.6 \%$

How many molecules of $\mathrm{C}_{2} \mathrm{H}_{4} \mathrm{Cl}_{2}$ can be prepared from 15 $\mathrm{C}_{2} \mathrm{H}_{4}$ molecules and $8 \mathrm{Cl}_{2}$ molecules?

How many molecules of the sweetener saccharin can be prepared from $30 \mathrm{C}$ atoms, $25 \mathrm{H}$ atoms, $12 \mathrm{O}$ atoms, $8 \mathrm{~S}$ atoms, and $14 \mathrm{~N}$ atoms? 


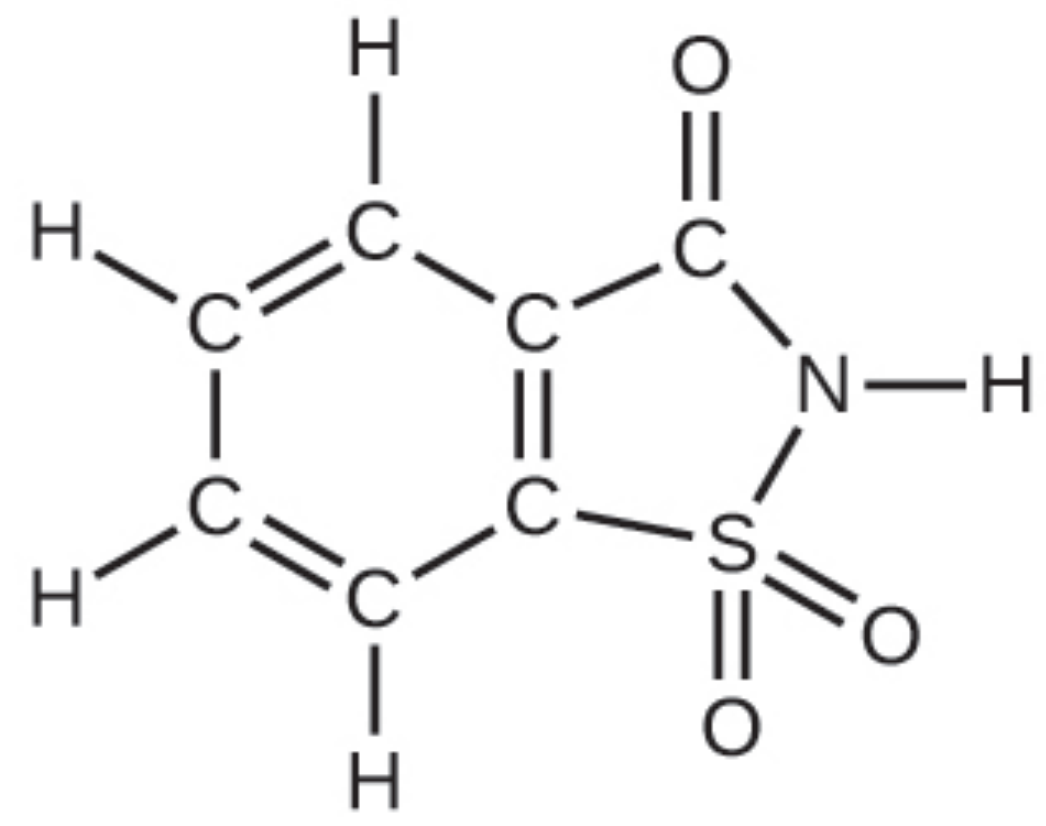

Only four molecules can be made.

The phosphorus pentoxide used to produce phosphoric acid for cola soft drinks is prepared by burning phosphorus in oxygen.

(a) What is the limiting reactant when $0.200 \mathrm{~mol}$ of $\mathrm{P}_{4}$ and 0.200 mol of $\mathrm{O}_{2}$ react according to $\mathrm{P}_{4}+5 \mathrm{O}_{2} \quad \mathrm{P}_{4} \mathrm{O}_{10}$

(b) Calculate the percent yield if $10.0 \mathrm{~g}$ of $\mathrm{P}_{4} \mathrm{O}_{10}$ is isolated from the reaction.

Would you agree to buy 1 trillion $(1,000,000,000,000)$ gold atoms for $\$ 5$ ? Explain why or why not. Find the current price of gold at http:/money.cnn.com/data/commodities/ (1 troy ounce $=31.1 \mathrm{~g}$ )

This amount cannot be weighted by ordinary balances and is worthless. 
Glossary

actual yield

amount of product formed in a reaction

excess reactant

reactant present in an amount greater than

required by the reaction stoichiometry

limiting reactant

reactant present in an amount lower than

required by the reaction stoichiometry, thus

limiting the amount of product generated

percent yield

measure of the efficiency of a reaction, expressed as a percentage of the theoretical yield

\section{theoretical yield}

amount of product that may be produced

from a given amount of reactant(s) according to the reaction stoichiometry 


\section{Focus on Air}

Some simple chemistry is now explored in the context of air and air pollution (ie. what we should be breathing $v s$ what we are breathing). This section deals with gaseous components of the atmosphere, and more specifically, components of the troposphere, the region of the atmosphere where earth's inhabitants live and breathe every day.

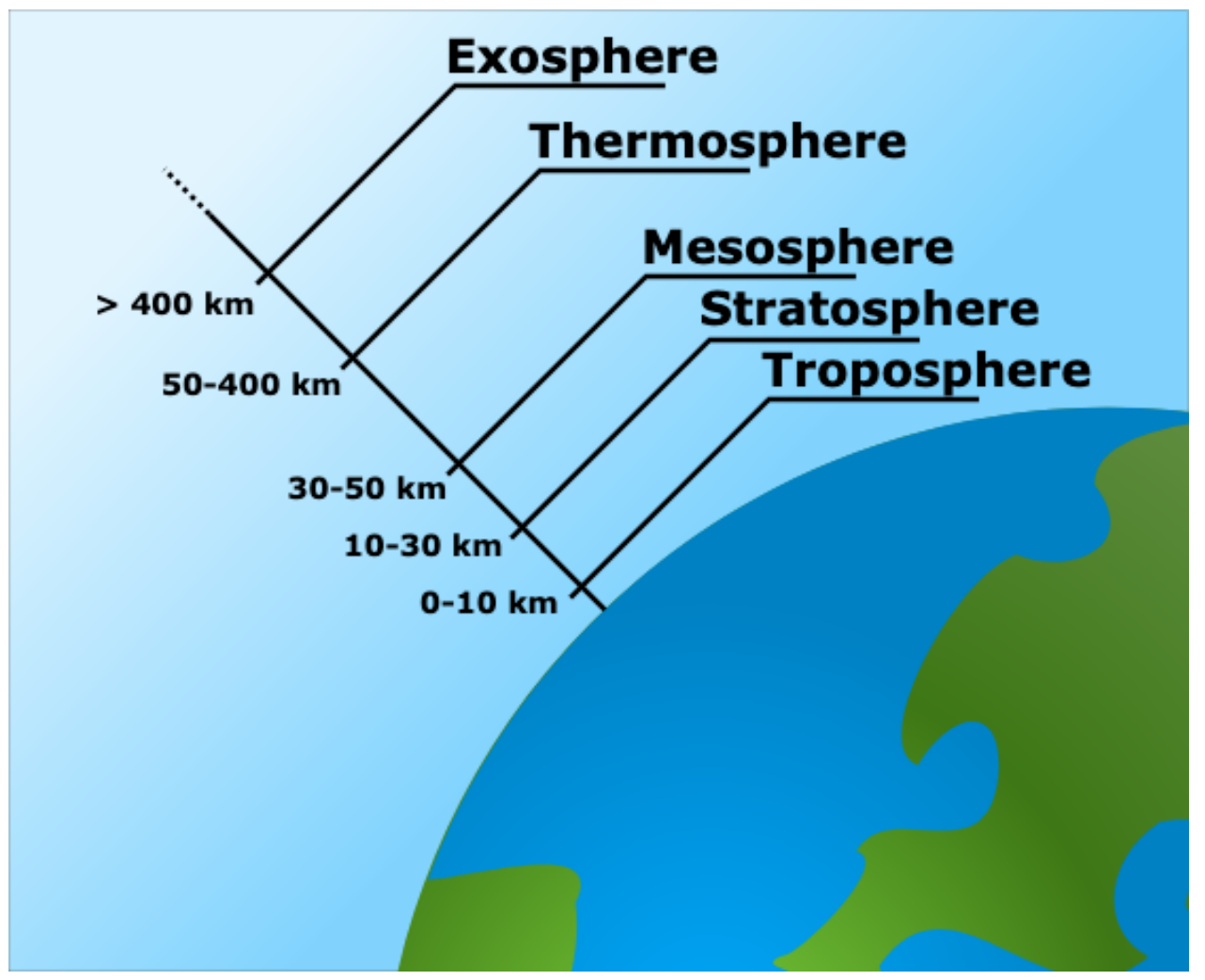

"File:Atmospheric Layers.svg" by Original by en:Bredk, converted to SVG by tiZom, globe borrowed from File:Earth clip art.svg is licensed under CC BY-SA 3.0

As seen in the following figure, air is mostly nitrogen, which is inert to us, and oxygen, which is important for respiration for all aerobic organisms. Argon and carbon dioxide make up $9 / 10$ of the remaining $1 \%$ of air molecules. Thus, 'pollution' is typically found in the remaining $0.1 \%$ of air. 


\section{The composition of dry air}

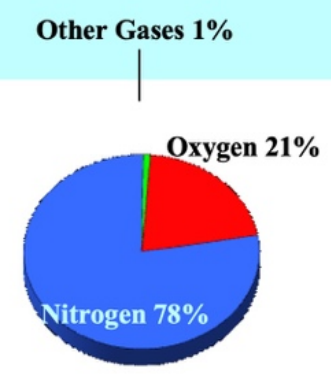

Refer to the information discussed in parts I-IV of this text and elaborate on the following focus boxes, solving the problems that appear. 


\section{The composition of dry air}

Other Gases $1 \%$

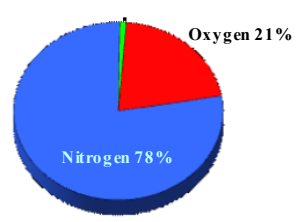

Major common air pollutants (small parts of the 1\%)

- Carbon monoxide (oxygen delivery)

- Ozone (respiratory)

- Sulfur oxides (respiratory)

- Nitrogen oxides (respiratory)

\section{Atoms and Molecules}

- we will normally deal with them in terms of their chemical symbol

- examples:

Carbon $=\mathrm{C}$

Carbon Monoxide $=\mathrm{CO}$

Carbon Dioxide $=\mathrm{CO}_{2}$

Sulfur Oxides $=\mathrm{SO}_{\mathrm{x}}$

Nitrogen Oxide $=\mathrm{NO}_{\mathrm{x}}$ 


\section{chemistry}

- Chemical Reactions

reactants $\longrightarrow$ products

- Law of Conservation of Matter atoms on left $=$ atoms on right

\section{Coal $=$ carbon, trace sulfur}

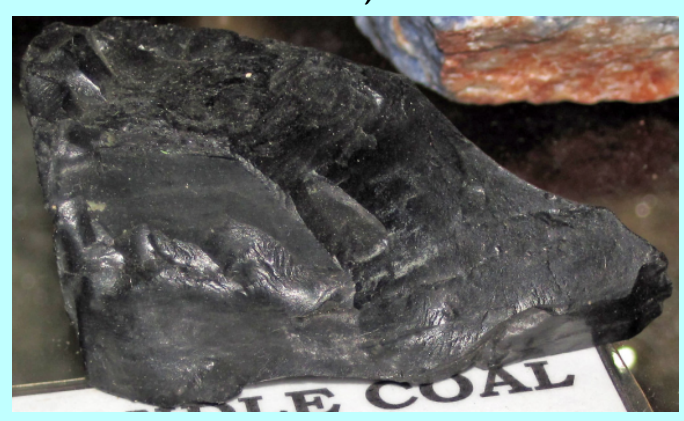

"Cannel coal (Tennessee, USA) 2" by James St. John is licensed under CC BY 2.0
-What is the balanced chemical reation for the combustion of solid carbon $\left(\mathrm{C}_{(\mathrm{s})}\right)$ with oxygen $\left(\mathrm{O}_{2(\mathrm{~g})}\right)$ to form carbon dioxide? 
- Show how these common air pollutants might be produced from the combustion of coal:

- Carbon Monoxide

- Sulfur Dioxide and Sulfur Trioxide

- Nitrogen Monoxide and Nitrogen Dioxide

- Write the balanced chemical reations for the combustion of methane $\left(\mathrm{CH}_{4}\right)$, ethane $\left(\mathrm{C}_{2} \mathrm{H}_{6}\right)$, ethanol $\left(\mathrm{C}_{2} \mathrm{H}_{5} \mathrm{OH}\right)$, and octane $\left(\mathrm{C}_{8} \mathrm{H}_{18}\right)$. Use chemical reactions to demonstrate the difference between the reaction to form carbon dioxide and the formation of carbon monoxide.

\section{Catalytic Converter}

Exhaust emission device that facilitates the conversion of toxic pollutants to less toxic substances.

Catalyst - a substance that increases the rate of a chemical reaction, but does not itself undergo permanent change.

Ex: Show the general reaction for the conversion of carbon monoxide and oxygen gas to carbon dioxide via a platinum catalyst. 



\section{PART V}

\section{THERMOCHEMISTRY}





\section{4.}

\section{INTRODUCTION}

Learning Objectives

- Energy Basics

Sliding a match head along a rough surface initiates a combustion reaction that produces energy in the form of heat and light. (credit: modification of work by Laszlo Ilyes)

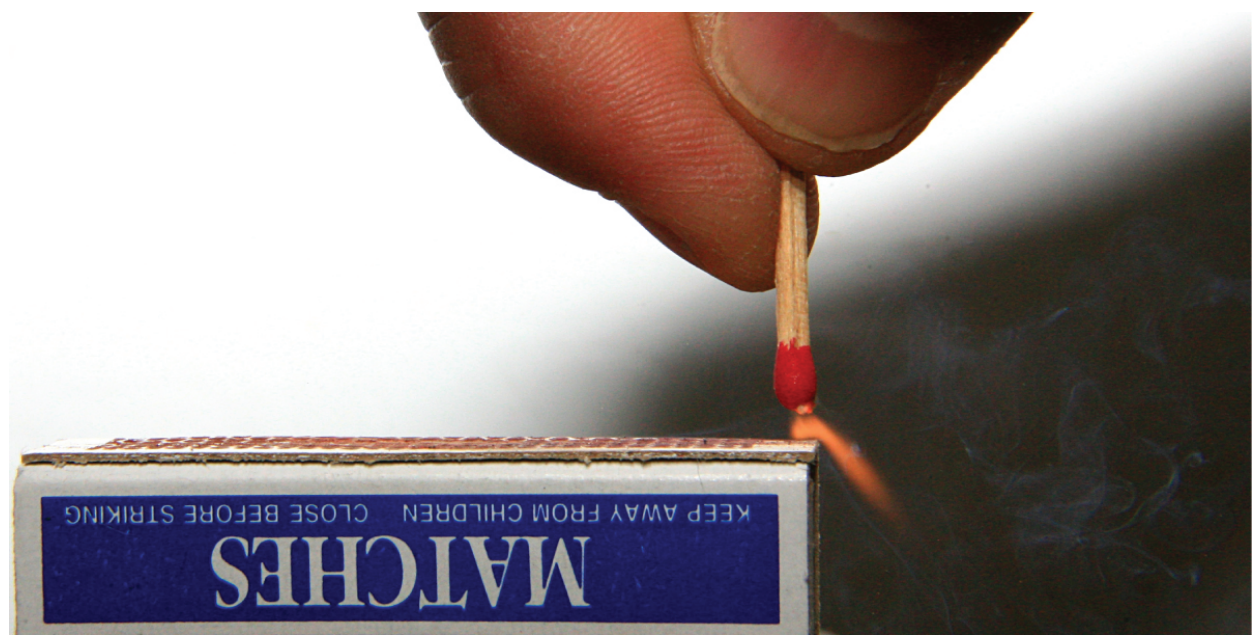


Chemical reactions, such as those that occur when you light a match, involve changes in energy as well as matter. Societies at all levels of development could not function without the energy released by chemical reactions. In 2012 , about $85 \%$ of US energy consumption came from the combustion of petroleum products, coal, wood, and garbage. We use this energy to produce electricity (38\%); to transport food, raw materials, manufactured goods, and people (27\%); for industrial production (21\%); and to heat and power our homes and businesses (10\%). ${ }^{1}$ While these combustion reactions help us meet our essential energy needs, they are also recognized by the majority of the scientific community as a major contributor to global climate change.

Useful forms of energy are also available from a variety of chemical reactions other than combustion. For example, the energy produced by the batteries in a cell phone, car, or flashlight results from chemical reactions. This chapter introduces many of the basic ideas necessary to explore the relationships between chemical changes and energy, with a focus on thermal energy.

Footnotes

- 1 US Energy Information Administration, Primary Energy Consumption by Source and Sector, 2012, http://www.eia.gov/totalenergy/ data/monthly/pdf/flow/css_2012_energy.pdf. Data derived from US Energy Information Administration, Monthly Energy Review (January 2014). 
25.

\section{ENERGY BASICS}

\section{Learning Objectives}

By the end of this section, you will be able to:

- Define energy, distinguish types of energy, and describe the nature of energy changes that accompany chemical and physical changes

- Distinguish the related properties of heat, thermal energy, and temperature

- Define and distinguish specific heat and heat capacity, and describe the physical implications of both

- Perform calculations involving heat, specific heat, and temperature change

Chemical changes and their accompanying changes in energy are important parts of our everyday world ((Figure)). The macronutrients in food (proteins, fats, and carbohydrates) undergo metabolic reactions that provide 
the energy to keep our bodies functioning. We burn a variety of fuels (gasoline, natural gas, coal) to produce energy for transportation, heating, and the generation of electricity. Industrial chemical reactions use enormous amounts of energy to produce raw materials (such as iron and aluminum). Energy is then used to manufacture those raw materials into useful products, such as cars, skyscrapers, and bridges.

The energy involved in chemical changes is important to our daily lives: (a) A cheeseburger for lunch provides the energy you need to get through the rest of the day; (b) the combustion of gasoline provides the energy that moves your car (and you) between home, work, and school; and (c) coke, a processed form of coal, provides the energy needed to convert iron ore into iron, which is essential for making many of the products we use daily. (credit a: modification of work by "Pink Sherbet Photography”/Flickr; credit b: modification of work by Jeffery Turner)

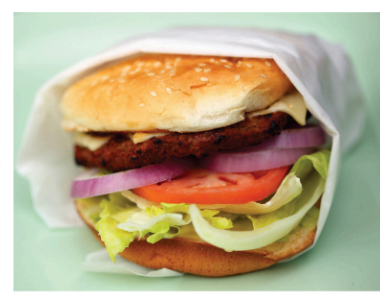

(a)

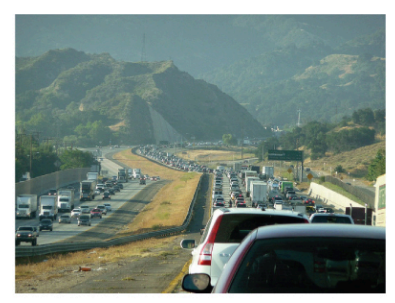

(b)

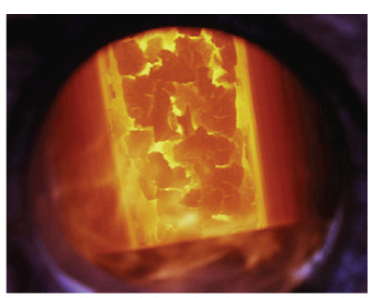

(c)

Over $90 \%$ of the energy we use comes originally from the sun. Every day, the sun provides the earth with almost 10,000 times the amount of energy necessary to meet all of the world's energy needs for that day. Our challenge is to find ways to convert and store incoming solar energy so that it can be used in reactions or chemical processes that 
are both convenient and nonpolluting. Plants and many bacteria capture solar energy through photosynthesis. We release the energy stored in plants when we burn wood or plant products such as ethanol. We also use this energy to fuel our bodies by eating food that comes directly from plants or from animals that got their energy by eating plants. Burning coal and petroleum also releases stored solar energy: These fuels are fossilized plant and animal matter.

This chapter will introduce the basic ideas of an important area of science concerned with the amount of heat absorbed or released during chemical and physical changes - an area called thermochemistry. The concepts introduced in this chapter are widely used in almost all scientific and technical fields. Food scientists use them to determine the energy content of foods. Biologists study the energetics of living organisms, such as the metabolic combustion of sugar into carbon dioxide and water. The oil, gas, and transportation industries, renewable energy providers, and many others endeavor to find better methods to produce energy for our commercial and personal needs. Engineers strive to improve energy efficiency, find better ways to heat and cool our homes, refrigerate our food and drinks, and meet the energy and cooling needs of computers and electronics, among other applications. Understanding thermochemical principles is essential for chemists, physicists, biologists, geologists, every type of engineer, and just about anyone who studies or does any kind of science. 


\section{Energy}

Energy can be defined as the capacity to supply heat or do work. One type of work $(w)$ is the process of causing matter to move against an opposing force. For example, we do work when we inflate a bicycle tire-we move matter (the air in the pump) against the opposing force of the air already in the tire.

Like matter, energy comes in different types. One scheme classifies energy into two types: potential energy, the energy an object has because of its relative position, composition, or condition, and kinetic energy, the energy that an object possesses because of its motion. Water at the top of a waterfall or dam has potential energy because of its position; when it flows downward through generators, it has kinetic energy that can be used to do work and produce electricity in a hydroelectric plant ((Figure)). A battery has potential energy because the chemicals within it can produce electricity that can do work.

(a) Water at a higher elevation, for example, at the top of Victoria Falls, has a higher potential energy than water at a lower elevation. As the water falls, some of its potential energy is converted into kinetic energy. (b) If the water flows through generators at the bottom of a dam, such as the Hoover Dam shown here, its kinetic energy is converted into electrical energy. (credit a: modification of work by Steve Jurvetson; credit b: modification of work by "curimedia"/Wikimedia commons) 


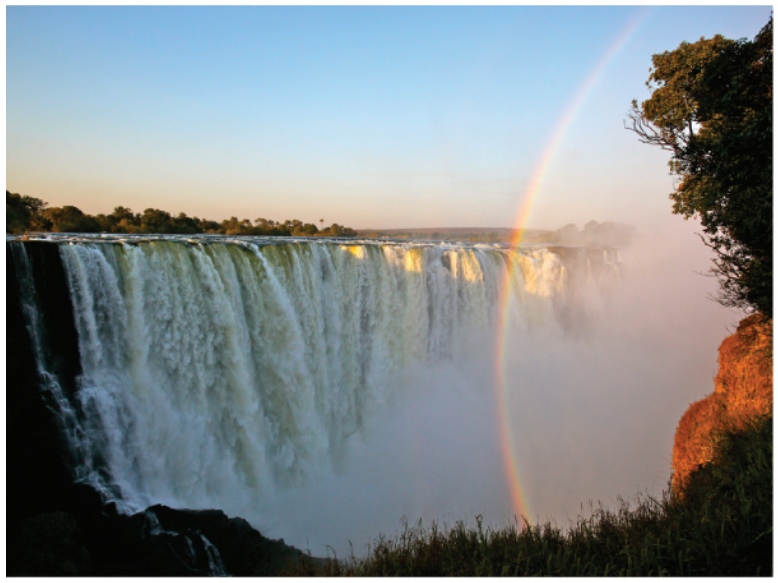

(a)

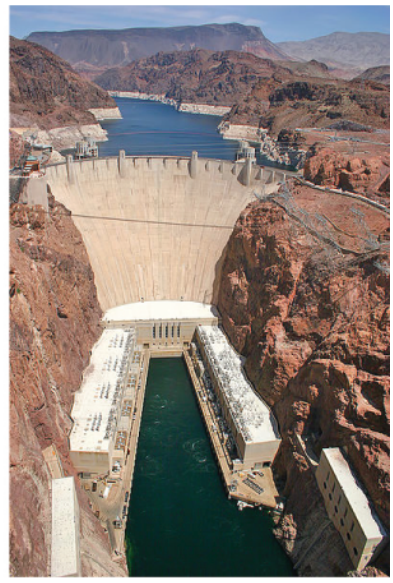

(b)

Energy can be converted from one form into another, but all of the energy present before a change occurs always exists in some form after the change is completed. This observation is expressed in the law of conservation of energy: during a chemical or physical change, energy can be neither created nor destroyed, although it can be changed in form. (This is also one version of the first law of thermodynamics, as you will learn later.)

When one substance is converted into another, there is always an associated conversion of one form of energy into another. Heat is usually released or absorbed, but sometimes the conversion involves light, electrical energy, or some other form of energy. For example, chemical energy (a type of potential energy) is stored in the molecules that compose gasoline. When gasoline is combusted within the cylinders of a car's engine, the rapidly expanding gaseous products of this chemical reaction generate mechanical energy (a type of kinetic energy) when they move the cylinders' pistons.

According to the law of conservation of matter (seen in an earlier chapter), there is no detectable change in the 
total amount of matter during a chemical change. When chemical reactions occur, the energy changes are relatively modest and the mass changes are too small to measure, so the laws of conservation of matter and energy hold well. However, in nuclear reactions, the energy changes are much larger (by factors of a million or so), the mass changes are measurable, and matter-energy conversions are significant. This will be examined in more detail in a later chapter on nuclear chemistry.

Thermal Energy, Temperature, and Heat

Thermal energy is kinetic energy associated with the random motion of atoms and molecules. Temperature is a quantitative measure of "hot" or "cold." When the atoms and molecules in an object are moving or vibrating quickly, they have a higher average kinetic energy (KE), and we say that the object is "hot." When the atoms and molecules are moving slowly, they have lower average KE, and we say that the object is "cold" ((Figure)). Assuming that no chemical reaction or phase change (such as melting or vaporizing) occurs, increasing the amount of thermal energy in a sample of matter will cause its temperature to increase. And, assuming that no chemical reaction or phase change (such as condensation or freezing) occurs, decreasing the amount of thermal energy in a sample of matter will cause its temperature to decrease.

(a) The molecules in a sample of hot water move more rapidly than (b) those in a sample of cold water. 


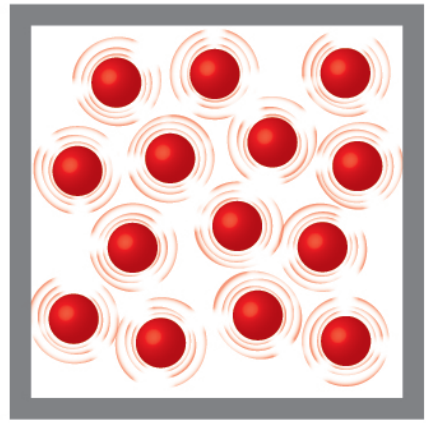

Hot water

(a)

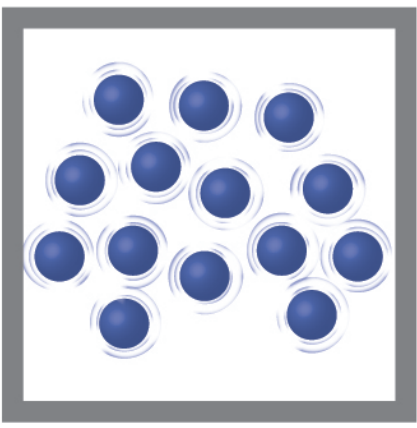

Cold water

(b)

Click on this interactive simulation to view the effects of temperature on molecular motion.

Most substances expand as their temperature increases and contract as their temperature decreases. This property can be used to measure temperature changes, as shown in (Figure). The operation of many thermometers depends on the expansion and contraction of substances in response to temperature changes.

(a) In an alcohol or mercury thermometer, the liquid (dyed red for visibility) expands when heated and contracts when cooled, much more so than the glass tube that contains the liquid. (b) In a bimetallic thermometer, two different metals (such as brass and steel) form a twolayered strip. When heated or cooled, one of the metals (brass) expands or contracts more than the other metal (steel), causing the strip to coil or uncoil. Both types of thermometers have a calibrated scale that indicates the temperature. (credit a: modification of work by "dwstucke"/Flickr) 


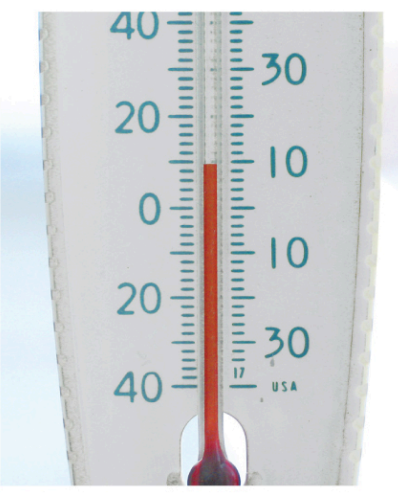

(a)

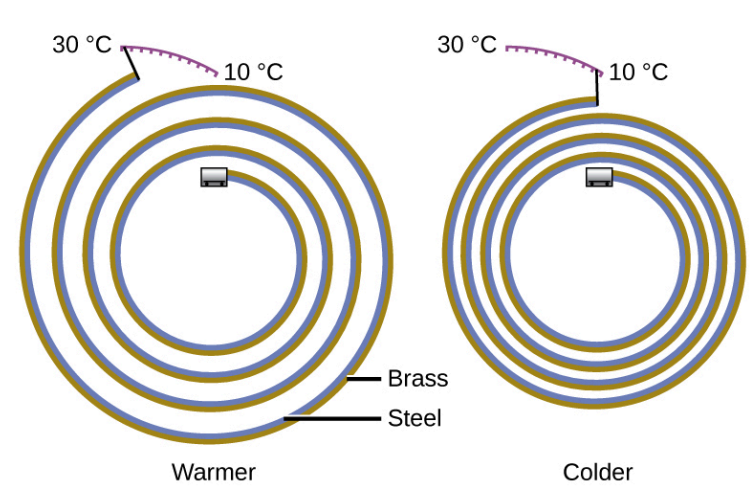

(b)

The following demonstration allows one to view the effects of heating and cooling a coiled bimetallic strip.

Heat $(q)$ is the transfer of thermal energy between two bodies at different temperatures. Heat flow (a redundant term, but one commonly used) increases the thermal energy of one body and decreases the thermal energy of the other. Suppose we initially have a high temperature (and high thermal energy) substance $(\mathrm{H})$ and a low temperature (and low thermal energy) substance (L). The atoms and molecules in $\mathrm{H}$ have a higher average $\mathrm{KE}$ than those in L. If we place substance $\mathrm{H}$ in contact with substance $\mathrm{L}$, the thermal energy will flow spontaneously from substance $\mathrm{H}$ to substance $\mathrm{L}$. The temperature of substance $\mathrm{H}$ will decrease, as will the average $\mathrm{KE}$ of its molecules; the temperature of substance $L$ will increase, along with the average KE of its molecules. Heat flow will continue until the two substances are at the same temperature ((Figure)).

(a) Substances $\mathrm{H}$ and L are initially at different temperatures, and their atoms have different average kinetic energies. (b) When they contact each other, collisions between the molecules result in the transfer of 
kinetic (thermal) energy from the hotter to the cooler matter. (c) The two objects reach "thermal equilibrium" when both substances are at the same temperature and their molecules have the same average kinetic energy.

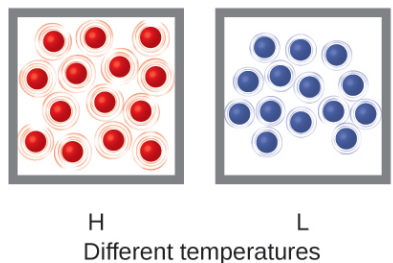

(a)

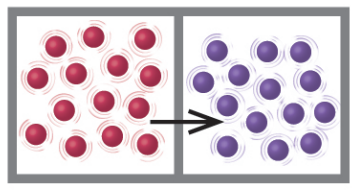

$\mathrm{H}$

(b)

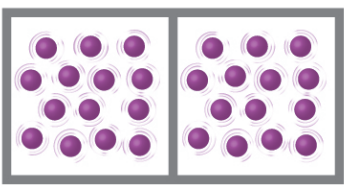

H L

(c)

Click on the PhET simulation to explore energy forms and changes. Visit the Energy Systems tab to create combinations of energy sources, transformation methods, and outputs. Click on Energy Symbols to visualize the transfer of energy.

Matter undergoing chemical reactions and physical changes can release or absorb heat. A change that releases heat is called an exothermic process. For example, the combustion reaction that occurs when using an oxyacetylene torch is an exothermic process-this process also releases energy in the form of light as evidenced by the torch's flame ((Figure)). A reaction or change that absorbs heat is an endothermic process. A cold pack used to treat muscle strains provides an example of an endothermic process. When the substances in the cold pack (water and a salt like ammonium nitrate) are brought together, the resulting process absorbs heat, leading to the sensation of cold.

(a) An oxyacetylene torch produces heat by the combustion of acetylene in oxygen. The energy released by this exothermic reaction heats and then melts the 
metal being cut. The sparks are tiny bits of the molten metal flying away. (b) A cold pack uses an endothermic process to create the sensation of cold. (credit a: modification of work by "Skatebiker"/Wikimedia commons)

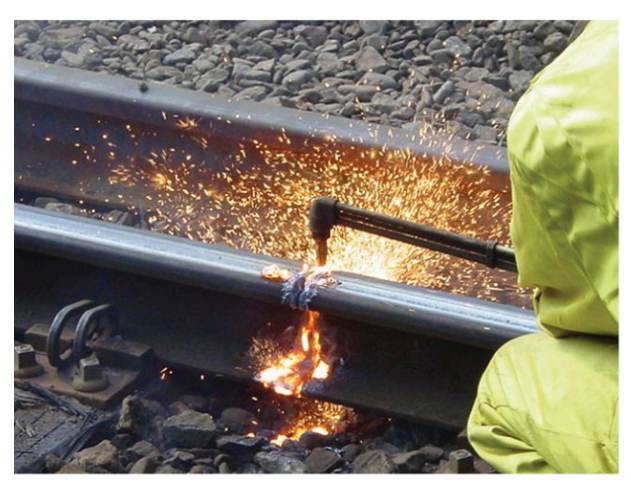

(a)

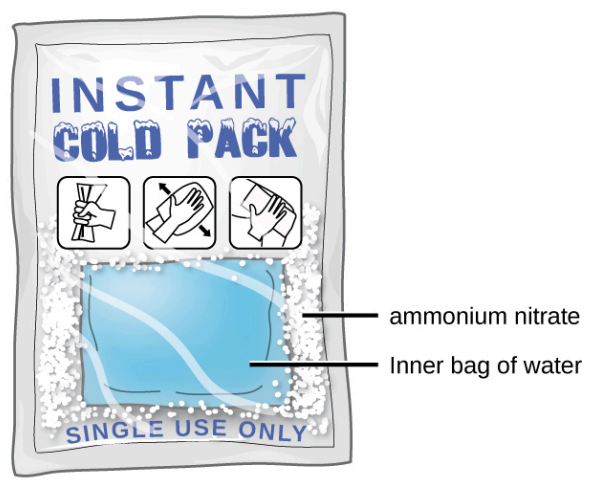

(b)

Historically, energy was measured in units of calories (cal). A calorie is the amount of energy required to raise one gram of water by 1 degree $C$ ( 1 kelvin). However, this quantity depends on the atmospheric pressure and the starting temperature of the water. The ease of measurement of energy changes in calories has meant that the calorie is still frequently used. The Calorie (with a capital C), or large calorie, commonly used in quantifying food energy content, is a kilocalorie. The SI unit of heat, work, and energy is the joule. A joule (J) is defined as the amount of energy used when a force of 1 newton moves an object 1 meter. It is named in honor of the English physicist James Prescott Joule. One joule is equivalent to 1 $\mathrm{kg} \mathrm{m}^{2} / \mathrm{s}^{2}$, which is also called 1 newton-meter. A kilojoule $(\mathrm{kJ})$ is 1000 joules. To standardize its definition, 1 calorie has been set to equal 4.184 joules. 
Solar Thermal Energy Power Plants

The sunlight that reaches the earth contains thousands of times more energy than we presently capture. Solar thermal systems provide one possible solution to the problem of converting energy from the sun into energy we can use. Large-scale solar thermal plants have different design specifics, but all concentrate sunlight to heat some substance; the heat "stored" in that substance is then converted into electricity.

The Solana Generating Station in Arizona's Sonora Desert produces 280 megawatts of electrical power. It uses parabolic mirrors that focus sunlight on pipes filled with a heat transfer fluid (HTF) ((Figure)). The HTF then does two things: It turns water into steam, which spins turbines, which in turn produces electricity, and it melts and heats a mixture of salts, which functions as a thermal energy storage system. After the sun goes down, the molten salt mixture can then release enough of its stored heat to produce steam to run the turbines for 6 hours. Molten salts are used because they possess a number of beneficial properties, including high heat capacities and thermal conductivities.

This solar thermal plant uses parabolic trough mirrors to concentrate sunlight. (credit a: modification of work by Bureau of Land Management) 


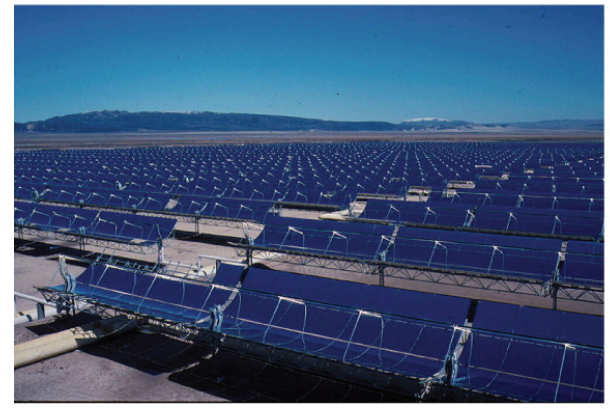

(a)

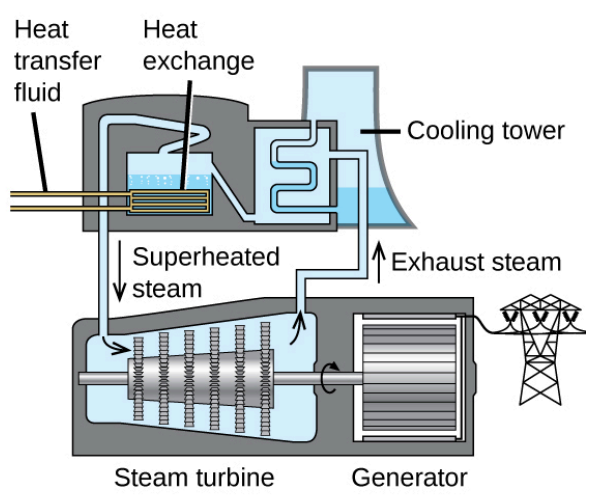

(b)

The 377-megawatt Ivanpah Solar Generating System, located in the Mojave Desert in California, is the largest solar thermal power plant in the world ((Figure)). Its 170,000 mirrors focus huge amounts of sunlight on three water-filled towers, producing steam at over $538{ }^{\circ} \mathrm{C}$ that drives electricity-producing turbines. It produces enough energy to power 140,000 homes. Water is used as the working fluid because of its large heat capacity and heat of vaporization.

(a) The Ivanpah solar thermal plant uses 170,000 mirrors to concentrate sunlight on water-filled towers. (b) It covers 4000 acres of public land near the Mojave Desert and the California-Nevada border. (credit a: modification of work by Craig Dietrich; credit b: modification of work by "USFWS Pacific Southwest Region"/Flickr) 


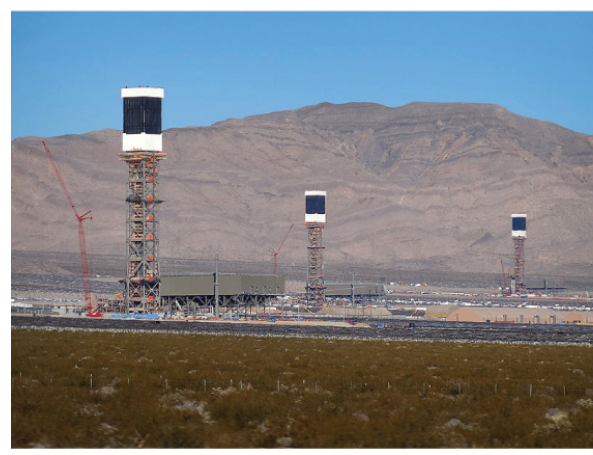

(a)

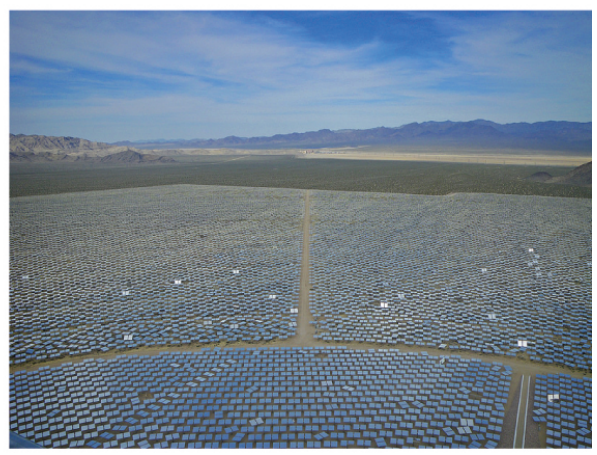

(b)

Key Concepts and Summary

Energy is the capacity to supply heat or do work (applying a force to move matter). Kinetic energy (KE) is the energy of motion; potential energy is energy due to relative position, composition, or condition. When energy is converted from one form into another, energy is neither created nor destroyed (law of conservation of energy or first law of thermodynamics).

The thermal energy of matter is due to the kinetic energies of its constituent atoms or molecules. Temperature is an intensive property of matter reflecting hotness or coldness that increases as the average kinetic energy increases. Heat is the transfer of thermal energy between objects at different temperatures. Chemical and physical processes can absorb heat (endothermic) or release heat (exothermic). The SI unit of energy, heat, and work is the joule (J).

\section{Chemistry End of Chapter Exercises}

A burning match and a bonfire may have the same temperature, yet you would not sit around a burning match on a fall evening to stay warm. Why not? 
The temperature of 1 gram of burning wood is approximately the same for both a match and a bonfire. This is an intensive property and depends on the material (wood). However, the overall amount of produced heat depends on the amount of material; this is an extensive property. The amount of wood in a bonfire is much greater than that in a match; the total amount of produced heat is also much greater, which is why we can sit around a bonfire to stay warm, but a match would not provide enough heat to keep us from getting cold.

Prepare a table identifying several energy transitions that take place during the typical operation of an automobile.

Glossary

calorie (cal)

unit of heat or other energy; the amount of energy required to raise 1 gram of water by 1 degree Celsius; 1 cal is defined as $4.184 \mathrm{~J}$

endothermic process

chemical reaction or physical change that absorbs heat

energy

capacity to supply heat or do work

exothermic process

chemical reaction or physical change that releases heat

heat (q)

transfer of thermal energy between two 
bodies

\section{heat capacity $(C)$}

extensive property of a body of matter that represents the quantity of heat required to increase its temperature by 1 degree Celsius (or 1 kelvin)

joule (J)

SI unit of energy; 1 joule is the kinetic energy of an object with a mass of 2 kilograms moving with a velocity of 1 meter per second, $1 \mathrm{~J}=1 \mathrm{~kg} \mathrm{~m}^{2} / \mathrm{s}$ and $4.184 \mathrm{~J}=1 \mathrm{cal}$

\section{kinetic energy}

energy of a moving body, in joules, equal to $\frac{1}{2} m v^{2}$ (where $m=$ mass and $v=$ velocity) potential energy

energy of a particle or system of particles derived from relative position, composition, or condition

specific heat capacity (c)

intensive property of a substance that represents the quantity of heat required to raise the temperature of $1 \mathrm{gram}$ of the substance by 1 degree Celsius (or 1 kelvin)

\section{temperature}

intensive property of matter that is a quantitative measure of "hotness" and "coldness"

\section{thermal energy}

kinetic energy associated with the random motion of atoms and molecules 


\section{thermochemistry}

study of measuring the amount of heat

absorbed or released during a chemical

reaction or a physical change

work (w)

energy transfer due to changes in external, macroscopic variables such as pressure and volume; or causing matter to move against an opposing force 
PART VI

\section{ELECTRONIC \\ STRUCTURE AND \\ PERIODIC \\ PROPERTIES OF \\ ELEMENTS}



26.

\section{INTRODUCTION}

\section{Learning Objectives}

- Electromagnetic Energy

- The Bohr Model

- Development of Quantum Theory

- Electronic Structure of Atoms (Electron Configurations)

- Periodic Variations in Element Properties

The Crab Nebula consists of remnants of a supernova (the explosion of a star). NASA's Hubble Space Telescope produced this composite image. Measurements of the emitted light wavelengths enabled astronomers to identify the elements in the nebula, determining that it contains specific ions including $\mathrm{S}^{+}$(green filaments) and $\mathrm{O}^{2+}$ (red filaments). (credit: modification of work by NASA and ESA) 


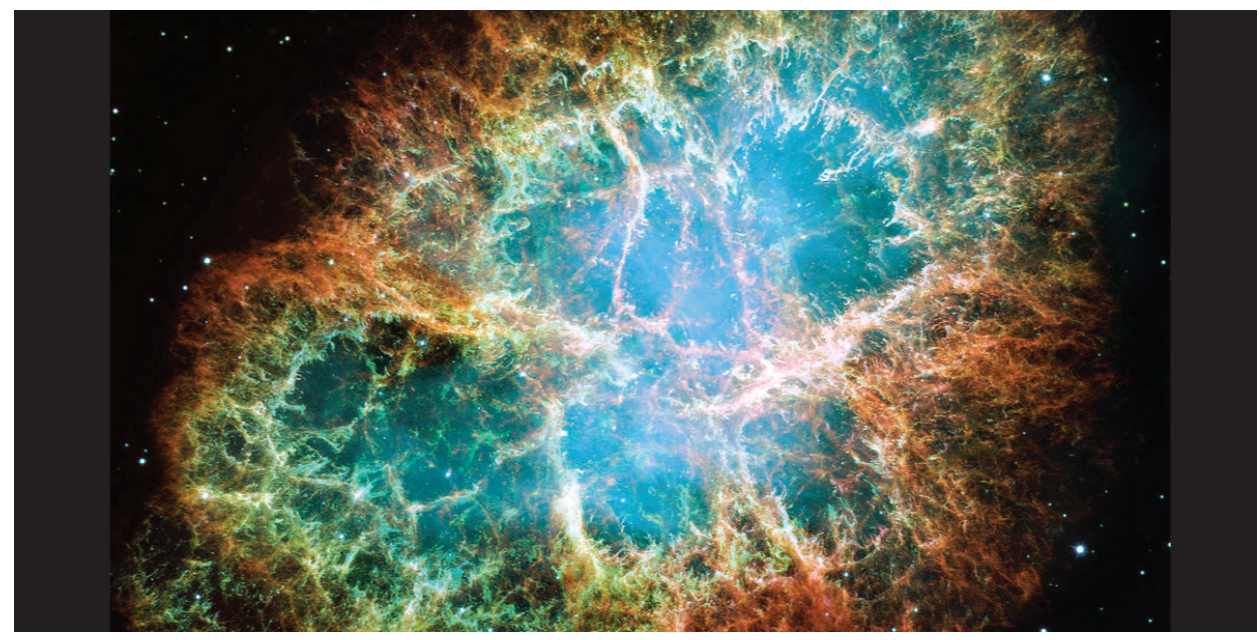

In 1054, Chinese astronomers recorded the appearance of a "guest star" in the sky, visible even during the day, which then disappeared slowly over the next two years. The sudden appearance was due to a supernova explosion, which was much brighter than the original star. Even though this supernova was observed almost a millennium ago, the remaining Crab Nebula ((Figure)) continues to release energy today. It emits not only visible light but also infrared light, X-rays, and other forms of electromagnetic radiation. The nebula emits both continuous spectra (the blue-white glow) and atomic emission spectra (the colored filaments). In this chapter, we will discuss light and other forms of electromagnetic radiation and how they are related to the electronic structure of atoms. We will also see how this radiation can be used to identify elements, even from thousands of light years away. 
27.

\section{ELECTROMAGNETIC ENERGY}

Learning Objectives

By the end of this section, you will be able to:

- Explain the basic behavior of waves, including travelling waves and standing waves

- Describe the wave nature of light

- Use appropriate equations to calculate related light-wave properties such as frequency, wavelength, and energy

- Distinguish between line and continuous emission spectra

- Describe the particle nature of light

The nature of light has been a subject of inquiry since antiquity. In the seventeenth century, Isaac Newton 
performed experiments with lenses and prisms and was able to demonstrate that white light consists of the individual colors of the rainbow combined together. Newton explained his optics findings in terms of a "corpuscular" view of light, in which light was composed of streams of extremely tiny particles travelling at high speeds according to Newton's laws of motion. Others in the seventeenth century, such as Christiaan Huygens, had shown that optical phenomena such as reflection and refraction could be equally well explained in terms of light as waves travelling at high speed through a medium called "luminiferous aether" that was thought to permeate all space. Early in the nineteenth century, Thomas Young demonstrated that light passing through narrow, closely spaced slits produced interference patterns that could not be explained in terms of Newtonian particles but could be easily explained in terms of waves. Later in the nineteenth century, after James Clerk Maxwell developed his theory of electromagnetic radiation and showed that light was the visible part of a vast spectrum of electromagnetic waves, the particle view of light became thoroughly discredited. By the end of the nineteenth century, scientists viewed the physical universe as roughly comprising two separate domains: matter composed of particles moving according to Newton's laws of motion, and electromagnetic radiation consisting of waves governed by Maxwell's equations. Today, these domains are referred to as classical mechanics and classical electrodynamics (or classical electromagnetism). Although there were a few physical phenomena that could not be explained within this framework, scientists at that time were so confident of the overall soundness of this 
framework that they viewed these aberrations as puzzling paradoxes that would ultimately be resolved somehow within this framework. As we shall see, these paradoxes led to a contemporary framework that intimately connects particles and waves at a fundamental level called waveparticle duality, which has superseded the classical view.

Visible light and other forms of electromagnetic radiation play important roles in chemistry, since they can be used to infer the energies of electrons within atoms and molecules. Much of modern technology is based on electromagnetic radiation. For example, radio waves from a mobile phone, X-rays used by dentists, the energy used to cook food in your microwave, the radiant heat from red-hot objects, and the light from your television screen are forms of electromagnetic radiation that all exhibit wavelike behavior.

\section{Waves}

A wave is an oscillation or periodic movement that can transport energy from one point in space to another. Common examples of waves are all around us. Shaking the end of a rope transfers energy from your hand to the other end of the rope, dropping a pebble into a pond causes waves to ripple outward along the water's surface, and the expansion of air that accompanies a lightning strike generates sound waves (thunder) that can travel outward for several miles. In each of these cases, kinetic energy is transferred through matter (the rope, water, or air) while the matter remains essentially in place. An insightful example of a wave occurs in sports stadiums when fans in a narrow region of seats rise simultaneously and stand 
with their arms raised up for a few seconds before sitting down again while the fans in neighboring sections likewise stand up and sit down in sequence. While this wave can quickly encircle a large stadium in a few seconds, none of the fans actually travel with the wave-they all stay in or above their seats.

Waves need not be restricted to travel through matter. As Maxwell showed, electromagnetic waves consist of an electric field oscillating in step with a perpendicular magnetic field, both of which are perpendicular to the direction of travel. These waves can travel through a vacuum at a constant speed of $2.99810^{8} \mathrm{~m} / \mathrm{s}$, the speed of light (denoted by $c$ ).

All waves, including forms of electromagnetic radiation, are characterized by, a wavelength (denoted by $\lambda$, the lowercase Greek letter lambda), a frequency (denoted by $v$, the lowercase Greek letter nu), and an amplitude. As can be seen in (Figure), the wavelength is the distance between two consecutive peaks or troughs in a wave (measured in meters in the SI system). Electromagnetic waves have wavelengths that fall within an enormous range-wavelengths of kilometers $\left(10^{3} \mathrm{~m}\right)$ to picometers $\left(10^{-12} \mathrm{~m}\right)$ have been observed. The frequency is the number of wave cycles that pass a specified point in space in a specified amount of time (in the SI system, this is measured in seconds). A cycle corresponds to one complete wavelength. The unit for frequency, expressed as cycles per second $\left[\mathrm{s}^{-1}\right]$, is the hertz $(\mathrm{Hz})$. Common multiples of this unit are megahertz, $\left(1 \mathrm{MHz}=1 \quad 10^{6}\right.$ $\mathrm{Hz})$ and gigahertz $\left(1 \mathrm{GHz}=1 \quad 10^{9} \mathrm{~Hz}\right)$. The amplitude corresponds to the magnitude of the wave's displacement and so, in (Figure), this corresponds to one-half the height 
between the peaks and troughs. The amplitude is related to the intensity of the wave, which for light is the brightness, and for sound is the loudness.

One-dimensional sinusoidal waves show the relationship among wavelength, frequency, and speed. The wave with the shortest wavelength has the highest frequency. Amplitude is one-half the height of the wave from peak to trough.
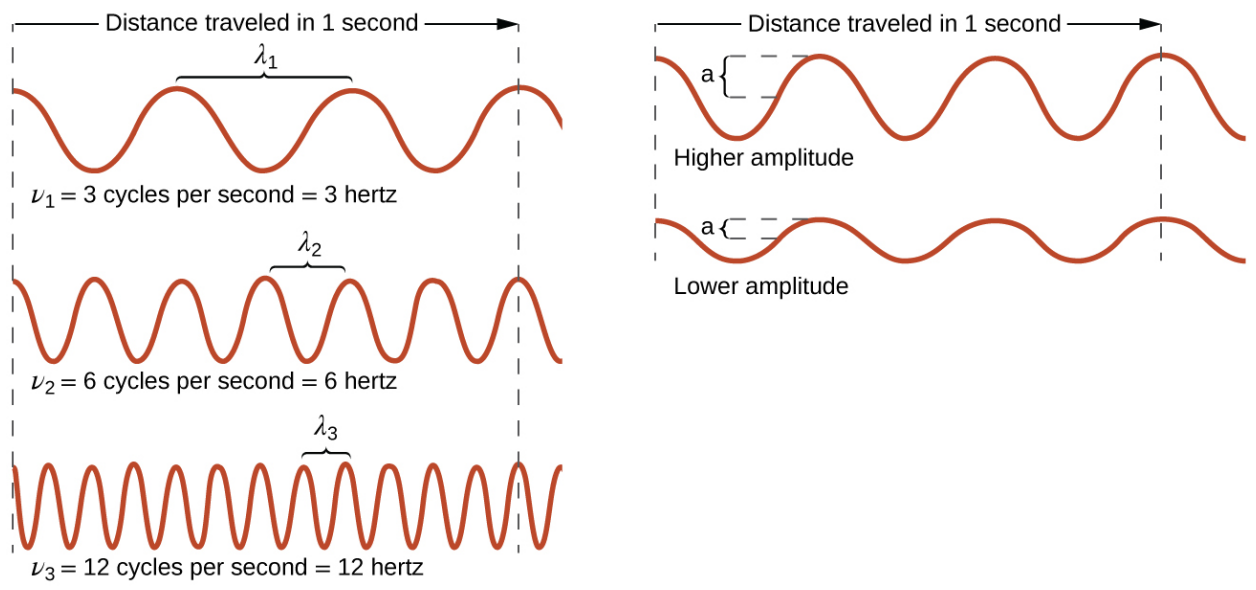

The product of a wave's wavelength $(\lambda)$ and its frequency $(v), \lambda v$, is the speed of the wave. Thus, for electromagnetic radiation in a vacuum, speed is equal to the fundamental constant, $c$ :

$c=2.99810^{8} \mathrm{~ms}^{-1}=\lambda \nu$

Wavelength and frequency are inversely proportional: As the wavelength increases, the frequency decreases. The inverse proportionality is illustrated in (Figure). This figure also shows the electromagnetic spectrum, the range of all types of electromagnetic radiation. Each of the various colors of visible light has specific frequencies and wavelengths associated with them, and you can see that 
visible light makes up only a small portion of the electromagnetic spectrum. Because the technologies developed to work in various parts of the electromagnetic spectrum are different, for reasons of convenience and historical legacies, different units are typically used for different parts of the spectrum. For example, radio waves are usually specified as frequencies (typically in units of $\mathrm{MHz}$ ), while the visible region is usually specified in wavelengths (typically in units of $\mathrm{nm}$ or angstroms).

Portions of the electromagnetic spectrum are shown in order of decreasing frequency and increasing wavelength. (credit "Cosmic ray": modification of work by NASA; credit "PET scan": modification of work by the National Institute of Health; credit "X-ray": modification of work by Dr. Jochen Lengerke; credit “Dental curing”: modification of work by the Department of the Navy; credit "Night vision": modification of work by the Department of the Army; credit "Remote": modification of work by Emilian Robert Vicol; credit "Cell phone": modification of work by Brett Jordan; credit "Microwave oven": modification of work by Billy Mabray; credit "Ultrasound": modification of work by Jane Whitney; credit "AM radio": modification of work by Dave Clausen) 


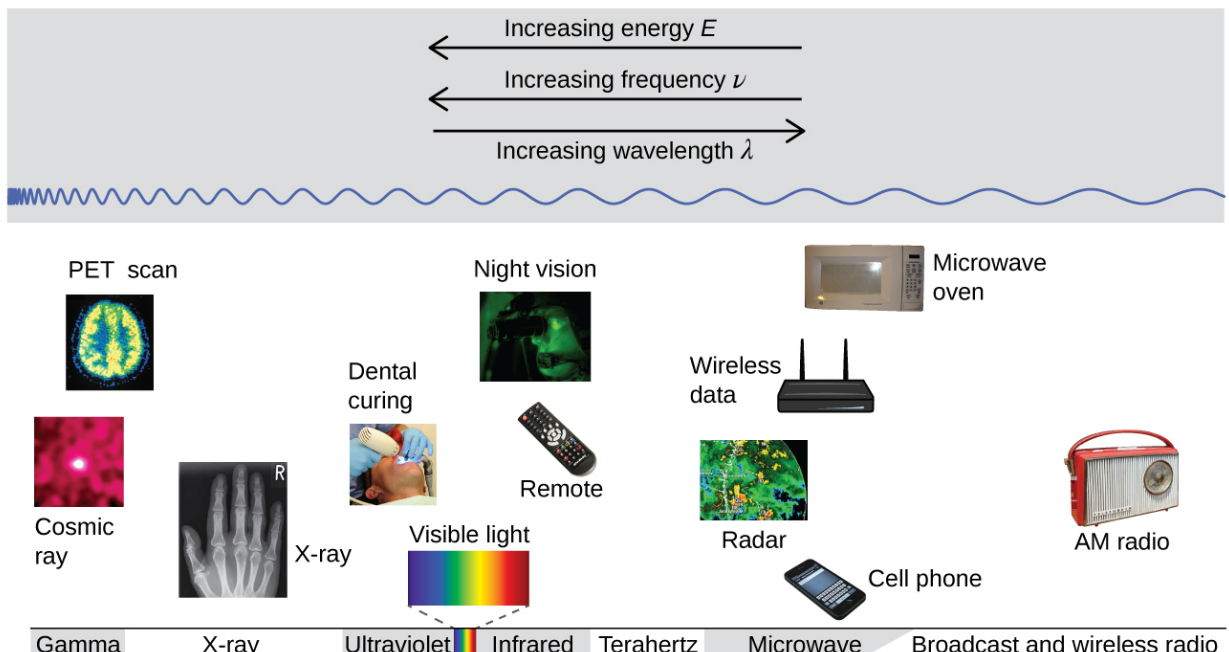

Gamma X-ray Ultraviolet 1 Infrared Terahertz Microwave Broadcast and wireless radio

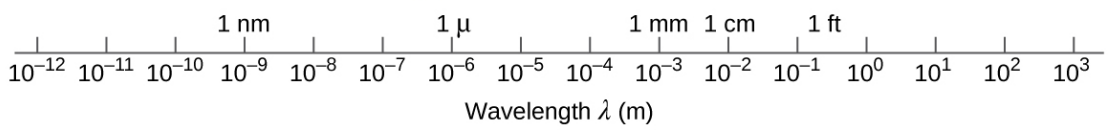

Determining the Frequency and Wavelength of Radiation A sodium streetlight gives off yellow light that has a wavelength of $589 \mathrm{~nm}\left(1 \mathrm{~nm}=110^{-9} \mathrm{~m}\right)$. What is the frequency of this light?

Solution We can rearrange the equation $c=\lambda v$ to solve for the frequency:

$$
\nu=\frac{c}{\lambda}
$$

Since $c$ is expressed in meters per second, we must also convert $589 \mathrm{~nm}$ to meters.

$$
\nu=\left(\frac{2.99810^{8} \overline{\mathrm{ms}^{-1}}}{589 \overline{\mathrm{nm}}}\right)\left(\frac{110^{9} \overline{\mathrm{nm}}}{1) \overline{\mathrm{m}}}\right)=5.0910^{14} \mathrm{~s}^{-1}
$$

Check Your Learning One of the frequencies used to transmit and receive cellular telephone signals in the United States is $850 \mathrm{MHz}$. What is the wavelength in meters of these radio waves?

Answer:

$0.353 \mathrm{~m}=35.3 \mathrm{~cm}$ 
Radio and cell towers are typically used to transmit longwavelength electromagnetic radiation. Increasingly, cell towers are designed to blend in with the landscape, as with the Tucson, Arizona, cell tower (right) disguised as a palm tree. (credit left: modification of work by Sir Mildred Pierce; credit middle: modification of work by M.O.

Stevens)
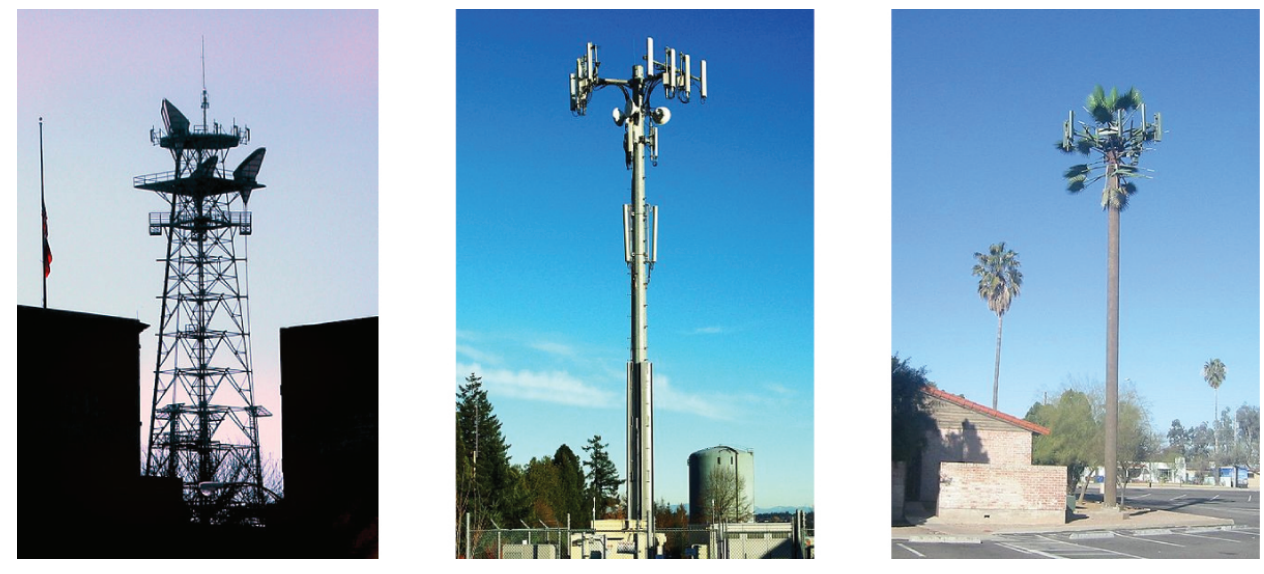

Many valuable technologies operate in the radio (3 $\mathrm{kHz}-300 \mathrm{GHz}$ ) frequency region of the electromagnetic spectrum. At the low frequency (low energy, long wavelength) end of this region are AM (amplitude modulation) radio signals $(540-2830 \mathrm{kHz})$ that can travel long distances. FM (frequency modulation) radio signals are used at higher frequencies (87.5-108.0 MHz). In AM radio, the information is transmitted by varying the amplitude of the wave ((Figure)). In FM radio, by contrast, the amplitude is constant and the instantaneous frequency varies.

This schematic depicts how amplitude modulation (AM) and frequency modulation (FM) can be used to transmit a radio wave. 

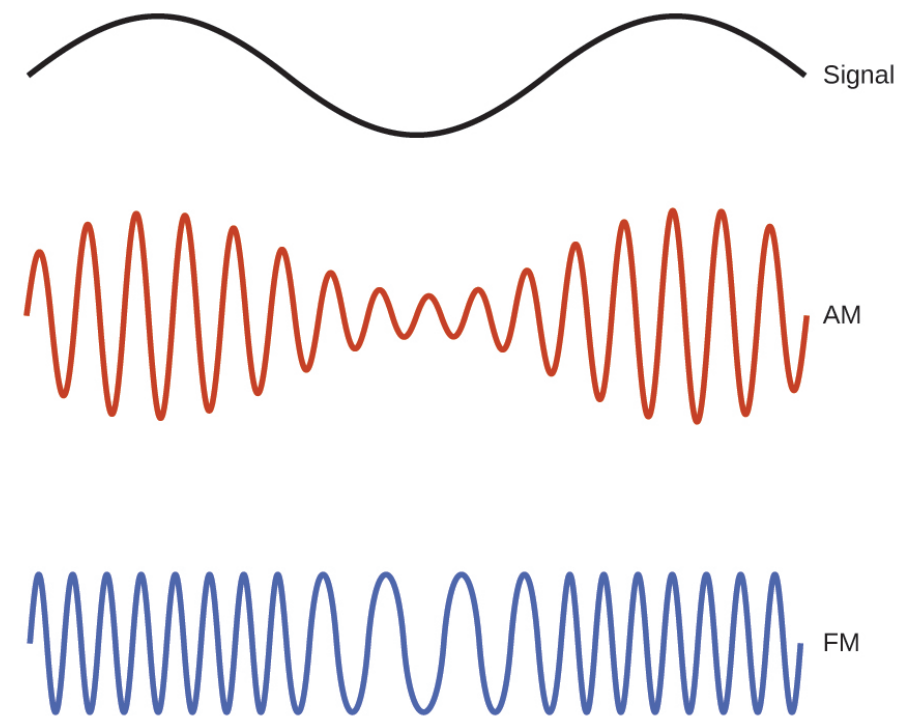

Other technologies also operate in the radio-wave portion of the electromagnetic spectrum. For example, $4 \mathrm{G}$ cellular telephone signals are approximately $880 \mathrm{MHz}$, while Global Positioning System (GPS) signals operate at 1.228 and $1.575 \mathrm{GHz}$, local area wireless technology (Wi-Fi) networks operate at 2.4 to $5 \mathrm{GHz}$, and highway toll sensors operate at $5.8 \mathrm{GHz}$. The frequencies associated with these applications are convenient because such waves tend not to be absorbed much by common building materials.

One particularly characteristic phenomenon of waves results when two or more waves come into contact: They interfere with each other. (Figure) shows the interference patterns that arise when light passes through narrow slits closely spaced about a wavelength apart. The fringe patterns produced depend on the wavelength, with the fringes being more closely spaced for shorter wavelength light passing through a given set of slits. When the light passes through the two slits, each slit effectively acts as a new source, resulting in two closely spaced waves coming 
into contact at the detector (the camera in this case). The dark regions in (Figure) correspond to regions where the peaks for the wave from one slit happen to coincide with the troughs for the wave from the other slit (destructive interference), while the brightest regions correspond to the regions where the peaks for the two waves (or their two troughs) happen to coincide (constructive interference). Likewise, when two stones are tossed close together into a pond, interference patterns are visible in the interactions between the waves produced by the stones. Such interference patterns cannot be explained by particles moving according to the laws of classical mechanics.

Interference fringe patterns are shown for light passing through two closely spaced, narrow slits. The spacing of the fringes depends on the wavelength, with the fringes being more closely spaced for the shorter-wavelength blue light. (credit: PASCO)

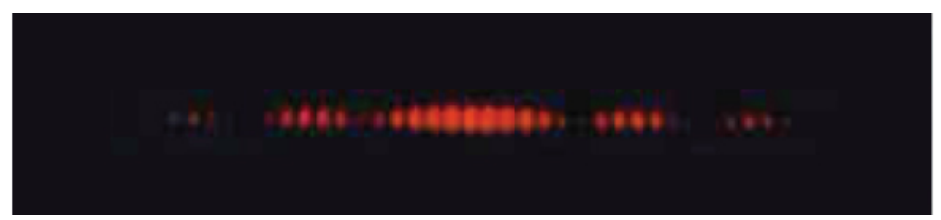

Dorothy Hodgkin

Because the wavelengths of X-rays (10-10,000 picometers $[\mathrm{pm}])$ are comparable to the size of atoms, X-rays can be used to determine the structure of molecules. When a beam of X-rays is passed through molecules packed together in a crystal, the X-rays collide with the electrons and scatter. Constructive and destructive interference of these scattered X-rays creates a specific diffraction pattern. Calculating backward from this pattern, the 
positions of each of the atoms in the molecule can be determined very precisely. One of the pioneers who helped create this technology was Dorothy Crowfoot Hodgkin.

She was born in Cairo, Egypt, in 1910, where her British parents were studying archeology. Even as a young girl, she was fascinated with minerals and crystals. When she was a student at Oxford University, she began researching how X-ray crystallography could be used to determine the structure of biomolecules. She invented new techniques that allowed her and her students to determine the structures of vitamin $\mathrm{B}_{12}$, penicillin, and many other important molecules. Diabetes, a disease that affects 382 million people worldwide, involves the hormone insulin. Hodgkin began studying the structure of insulin in 1934, but it required several decades of advances in the field before she finally reported the structure in 1969. Understanding the structure has led to better understanding of the disease and treatment options.

Not all waves are travelling waves. Standing waves (also known as stationary waves) remain constrained within some region of space. As we shall see, standing waves play an important role in our understanding of the electronic structure of atoms and molecules. The simplest example of a standing wave is a one-dimensional wave associated with a vibrating string that is held fixed at its two end points. (Figure) shows the four lowest-energy standing waves (the fundamental wave and the lowest three harmonics) for a vibrating string at a particular amplitude. Although the string's motion lies mostly within a plane, the wave itself is considered to be one dimensional, since 
it lies along the length of the string. The motion of string segments in a direction perpendicular to the string length generates the waves and so the amplitude of the waves is visible as the maximum displacement of the curves seen in (Figure). The key observation from the figure is that only those waves having an integer number, $n$, of halfwavelengths between the end points can form. A system with fixed end points such as this restricts the number and type of the possible waveforms. This is an example of quantization, in which only discrete values from a more general set of continuous values of some property are observed. Another important observation is that the harmonic waves (those waves displaying more than onehalf wavelength) all have one or more points between the two end points that are not in motion. These special points are nodes. The energies of the standing waves with a given amplitude in a vibrating string increase with the number of half-wavelengths $n$. Since the number of nodes is $n-1$, the energy can also be said to depend on the number of nodes, generally increasing as the number of nodes increases.

A vibrating string shows some one-dimensional standing waves. Since the two end points of the string are held fixed, only waves having an integer number of halfwavelengths can form. The points on the string between the end points that are not moving are called the nodes. 


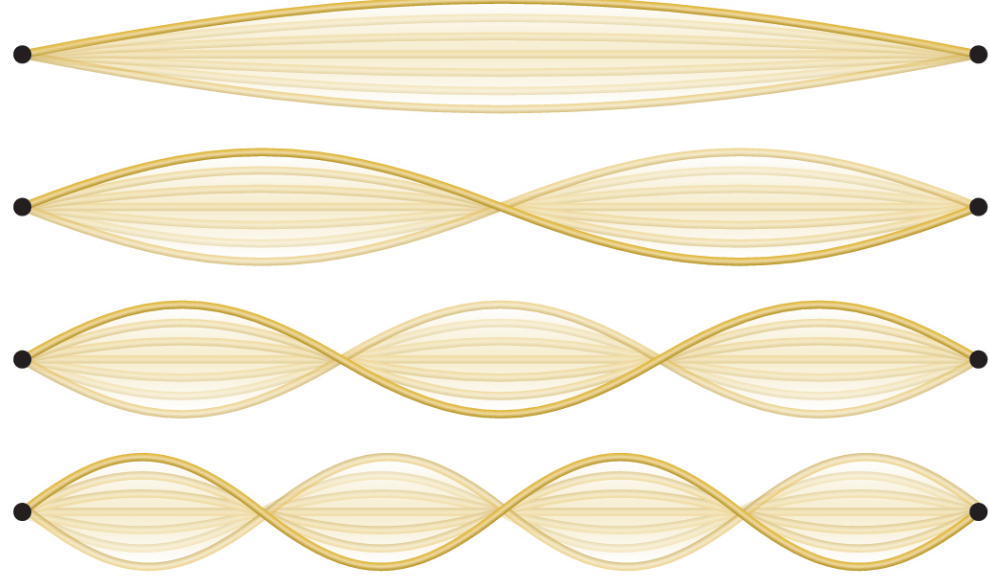

An example of two-dimensional standing waves is shown in (Figure), which shows the vibrational patterns on a flat surface. Although the vibrational amplitudes cannot be seen like they could in the vibrating string, the nodes have been made visible by sprinkling the drum surface with a powder that collects on the areas of the surface that have minimal displacement. For one-dimensional standing waves, the nodes were points on the line, but for twodimensional standing waves, the nodes are lines on the surface (for three-dimensional standing waves, the nodes are two-dimensional surfaces within the threedimensional volume).

Two-dimensional standing waves can be visualized on a vibrating surface. The surface has been sprinkled with a powder that collects near the nodal lines. There are two types of nodes visible: radial nodes (circles) and angular nodes (radii). 

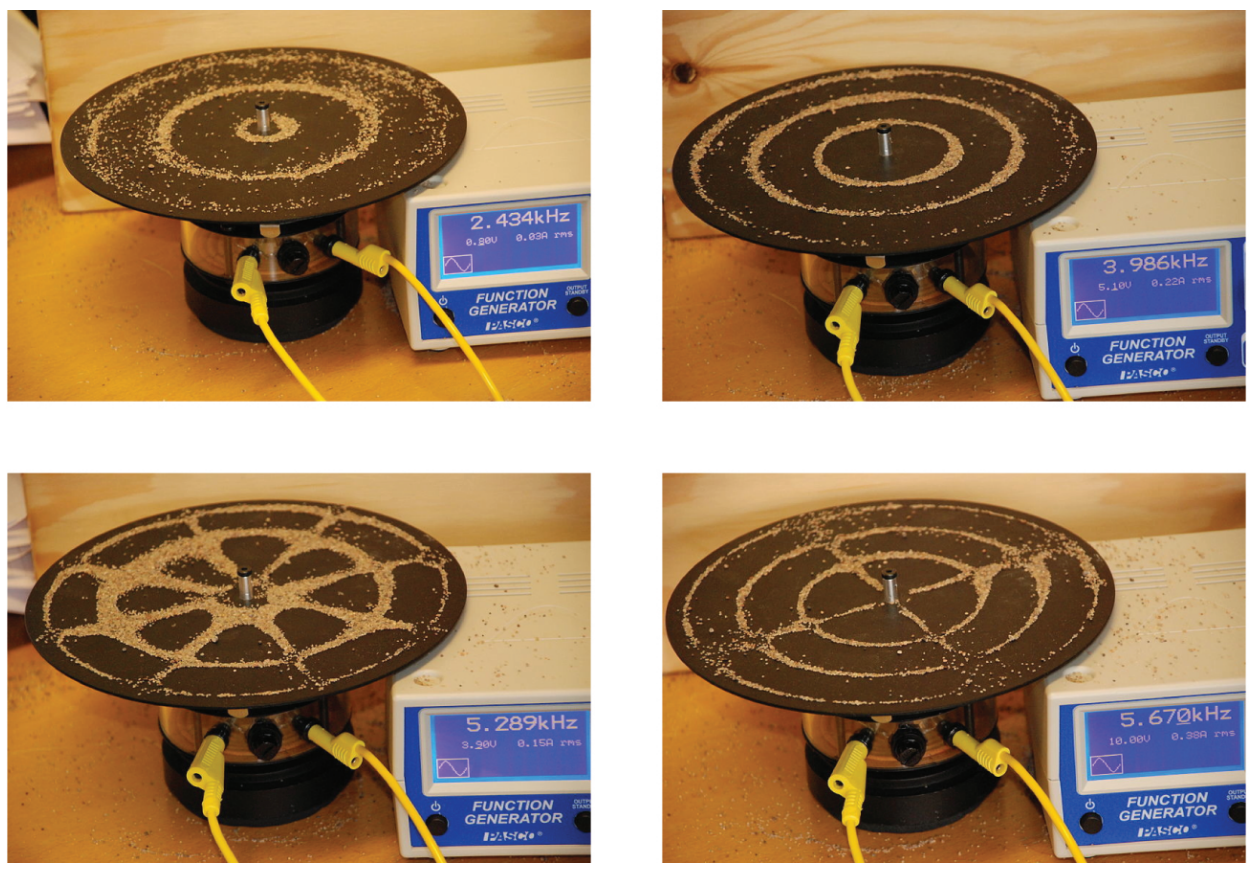

You can watch the formation of various radial nodes here as singer Imogen Heap projects her voice across a kettle drum.

Key Concepts and Summary

Light and other forms of electromagnetic radiation move through a vacuum with a constant speed, $c$, of $2.99810^{8} \mathrm{~m}$ $\mathrm{s}^{-1}$. This radiation shows wavelike behavior, which can be characterized by a frequency, $v$, and a wavelength, $\lambda$, such that $c=\lambda v$. Light is an example of a travelling wave. Other important wave phenomena include standing waves, periodic oscillations, and vibrations. Standing waves exhibit quantization, since their wavelengths are limited to discrete integer multiples of some characteristic lengths. Electromagnetic radiation that passes through two closely spaced narrow slits having dimensions roughly similar to the wavelength will show an interference 
pattern that is a result of constructive and destructive interference of the waves. Electromagnetic radiation also demonstrates properties of particles called photons. The energy of a photon is related to the frequency (or alternatively, the wavelength) of the radiation as $E=h v$ (or $E=\frac{h c}{\lambda}$ ), where $h$ is Planck's constant. That light demonstrates both wavelike and particle-like behavior is known as wave-particle duality. All forms of electromagnetic radiation share these properties, although various forms including X-rays, visible light, microwaves, and radio waves interact differently with matter and have very different practical applications. Electromagnetic radiation can be generated by exciting matter to higher energies, such as by heating it. The emitted light can be either continuous (incandescent sources like the sun) or discrete (from specific types of excited atoms). Continuous spectra often have distributions that can be approximated as blackbody radiation at some appropriate temperature. The line spectrum of hydrogen can be obtained by passing the light from an electrified tube of hydrogen gas through a prism. This line spectrum was simple enough that an empirical formula called the Rydberg formula could be derived from the spectrum. Three historically important paradoxes from the late 19th and early 20th centuries that could not be explained within the existing framework of classical mechanics and classical electromagnetism were the blackbody problem, the photoelectric effect, and the discrete spectra of atoms. The resolution of these paradoxes ultimately led to quantum theories that superseded the classical theories. 
Key Equations

- $c=\lambda v$

- $E=h \nu=\frac{h c}{\lambda}$, where $h=6.62610^{-34} \mathrm{~J} \mathrm{~s}$

- $\frac{1}{\lambda}=R_{\infty}\left(\frac{1}{n_{1}^{2}}-\frac{1}{n_{2}^{2}}\right)$

Chemistry End of Chapter Exercises

The light produced by a red neon sign is due to the emission of light by excited neon atoms. Qualitatively describe the spectrum produced by passing light from a neon lamp through a prism.

The spectrum consists of colored lines, at least one of which (probably the brightest) is red.

An FM radio station found at 103.1 on the FM dial broadcasts at a frequency of $1.03110^{8} \mathrm{~s}^{-1}(103.1 \mathrm{MHz})$. What is the wavelength of these radio waves in meters?

FM-95, an FM radio station, broadcasts at a frequency of $9.5110^{7} \mathrm{~s}^{-1}(95.1 \mathrm{MHz})$. What is the wavelength of these radio waves in meters?

\section{$3.15 \mathrm{~m}$}

A bright violet line occurs at $435.8 \mathrm{~nm}$ in the emission spectrum of mercury vapor. What amount of energy, in joules, must be released by an electron in a mercury atom to produce a photon of this light?

Light with a wavelength of $614.5 \mathrm{~nm}$ looks orange. What 
is the energy, in joules, per photon of this orange light? What is the energy in $\mathrm{eV}\left(1 \mathrm{eV}=1.60210^{-19} \mathrm{~J}\right)$ ?

$3.23310^{-19} \mathrm{~J} ; 2.018 \mathrm{eV}$

Heated lithium atoms emit photons of light with an energy of $2.96110^{-19} \mathrm{~J}$. Calculate the frequency and wavelength of one of these photons. What is the total energy in 1 mole of these photons? What is the color of the emitted light?

A photon of light produced by a surgical laser has an energy of $3.02710^{-19} \mathrm{~J}$. Calculate the frequency and wavelength of the photon. What is the total energy in 1 mole of photons? What is the color of the emitted light?

$v=4.56810^{14} \mathrm{~s} ; \lambda=656.3 \mathrm{~nm} ;$ Energy $\mathrm{mol}^{-1}=1.82310^{5} \mathrm{~J}$
$\mathrm{~mol}^{-1} ;$ red

When rubidium ions are heated to a high temperature, two lines are observed in its line spectrum at wavelengths (a) $7.910^{-7} \mathrm{~m}$ and (b) $4.210^{-7} \mathrm{~m}$. What are the frequencies of the two lines? What color do we see when we heat a rubidium compound?

The emission spectrum of cesium contains two lines whose frequencies are (a) $3.4510^{14} \mathrm{~Hz}$ and (b) $6.5310^{14}$ $\mathrm{Hz}$. What are the wavelengths and energies per photon of the two lines? What color are the lines?

(a) $\lambda=8.6910^{-7} \mathrm{~m} ; E=2.2910^{-19} \mathrm{~J}$; (b) $\lambda=4.5910^{-7} \mathrm{~m} ; E$ $=4.3310^{-19} \mathrm{~J}$; The color of (a) is red; (b) is blue. 
Photons of infrared radiation are responsible for much of the warmth we feel when holding our hands before a fire. These photons will also warm other objects. How many infrared photons with a wavelength of $1.510^{-6} \mathrm{~m}$ must be absorbed by the water to warm a cup of water (175 g) from $25.0{ }^{\circ} \mathrm{C}$ to $40{ }^{\circ} \mathrm{C}$ ?

One of the radiographic devices used in a dentist's office emits an X-ray of wavelength $2.09010^{-11} \mathrm{~m}$. What is the energy, in joules, and frequency of this X-ray?

$E=9.50210^{-15} \mathrm{~J} ; v=1.43410^{19} \mathrm{~s}^{-1}$

The eyes of certain reptiles pass a single visual signal to the brain when the visual receptors are struck by photons of a wavelength of $850 \mathrm{~nm}$. If a total energy of $3.1510^{-14} \mathrm{~J}$ is required to trip the signal, what is the minimum number of photons that must strike the receptor?

RGB color television and computer displays use cathode ray tubes that produce colors by mixing red, green, and blue light. If we look at the screen with a magnifying glass, we can see individual dots turn on and off as the colors change. Using a spectrum of visible light, determine the approximate wavelength of each of these colors. What is the frequency and energy of a photon of each of these colors?

Red: $660 \mathrm{~nm} ; 4.5410^{14} \mathrm{~Hz} ; 3.0110^{-19} \mathrm{~J}$. Green: 520 $\mathrm{nm} ; 5.7710^{14} \mathrm{~Hz} ; 3.8210^{-19} \mathrm{~J}$. Blue: $440 \mathrm{~nm} ; 6.8110^{14}$ $\mathrm{Hz} ; 4.5110^{-19} \mathrm{~J}$. Somewhat different numbers are also possible. 
Glossary

amplitude

extent of the displacement caused by a wave

\section{blackbody}

idealized perfect absorber of all incident electromagnetic radiation; such bodies emit electromagnetic radiation in characteristic continuous spectra called blackbody radiation

\section{continuous spectrum}

electromagnetic radiation given off in an unbroken series of wavelengths (e.g., white light from the sun)

electromagnetic radiation

energy transmitted by waves that have an electric-field component and a magneticfield component electromagnetic spectrum

range of energies that electromagnetic radiation can comprise, including radio, microwaves, infrared, visible, ultraviolet, $X$ rays, and gamma rays

\section{frequency $(v)$}

number of wave cycles (peaks or troughs) that pass a specified point in space per unit time

hertz (Hz)

the unit of frequency, which is the number of cycles per second, $\mathrm{s}^{-1}$ 


\section{intensity}

property of wave-propagated energy

related to the amplitude of the wave, such as

brightness of light or loudness of sound

interference pattern

pattern typically consisting of alternating bright and dark fringes; it results from constructive and destructive interference of waves

\section{line spectrum}

electromagnetic radiation emitted at discrete wavelengths by a specific atom (or atoms) in an excited state

node

any point of a standing wave with zero amplitude

photon

smallest possible packet of electromagnetic radiation, a particle of light quantization

limitation of some property to specific discrete values, not continuous standing wave

(also, stationary wave) localized wave phenomenon characterized by discrete wavelengths determined by the boundary conditions used to generate the waves; standing waves are inherently quantized wave

oscillation of a property over time or space; can transport energy from one point to 
another

wavelength $(\lambda)$

distance between two consecutive peaks or troughs in a wave

wave-particle duality

observation that elementary particles can

exhibit both wave-like and particle-like properties 


\section{8.}

\section{THE BOHR MODEL}

Learning Objectives

By the end of this section, you will be able to:

- Describe the Bohr model of the hydrogen atom

- Use the Rydberg equation to calculate energies of light emitted or absorbed by hydrogen atoms

Following the work of Ernest Rutherford and his colleagues in the early twentieth century, the picture of atoms consisting of tiny dense nuclei surrounded by lighter and even tinier electrons continually moving about the nucleus was well established. This picture was called the planetary model, since it pictured the atom as a miniature "solar system" with the electrons orbiting the nucleus like planets orbiting the sun. The simplest atom is hydrogen, consisting of a single proton as the nucleus about which a single electron moves. The electrostatic force attracting the electron to the proton depends only 
on the distance between the two particles. This classical mechanics description of the atom is incomplete, however, since an electron moving in an elliptical orbit would be accelerating (by changing direction) and, according to classical electromagnetism, it should continuously emit electromagnetic radiation. This loss in orbital energy should result in the electron's orbit getting continually smaller until it spirals into the nucleus, implying that atoms are inherently unstable.

In 1913, Niels Bohr attempted to resolve the atomic paradox by ignoring classical electromagnetism's prediction that the orbiting electron in hydrogen would continuously emit light. Instead, he incorporated into the classical mechanics description of the atom Planck's ideas of quantization and Einstein's finding that light consists of photons whose energy is proportional to their frequency. Bohr assumed that the electron orbiting the nucleus would not normally emit any radiation (the stationary state hypothesis), but it would emit or absorb a photon if it moved to a different orbit. The energy absorbed or emitted would reflect differences in the orbital energies according to this equation:

$|E|=\left|E_{\mathrm{f}}-E_{\mathrm{i}}\right|=h \nu=\frac{h c}{\lambda}$

In this equation, $h$ is Planck's constant and $E_{\mathrm{i}}$ and $E_{\mathrm{f}}$ are the initial and final orbital energies, respectively. The absolute value of the energy difference is used, since frequencies and wavelengths are always positive. Instead of allowing for continuous values of energy, Bohr assumed the energies of these electron orbitals were quantized:

$E_{n}=\frac{k}{n^{2}}, n=1,2,3, \ldots$ 
In this expression, $k$ is a constant comprising fundamental constants such as the electron mass and charge and Planck's constant. Inserting the expression for the orbit energies into the equation for $\Delta E$ gives

$$
E=k\left(\frac{1}{n_{1}^{2}}-\frac{1}{n_{2}^{2}}\right)=\frac{h c}{\lambda}
$$

or

$\frac{1}{\lambda}=\frac{k}{h c}\left(\frac{1}{n_{1}^{2}}-\frac{1}{n_{2}^{2}}\right)$

which is identical to the Rydberg equation in which $R_{\infty}=\frac{k}{h c}$. When Bohr calculated his theoretical value for the Rydberg constant, $R_{\infty}$, and compared it with the experimentally accepted value, he got excellent agreement. Since the Rydberg constant was one of the most precisely measured constants at that time, this level of agreement was astonishing and meant that Bohr's model was taken seriously, despite the many assumptions that Bohr needed to derive it.

The lowest few energy levels are shown in (Figure). One of the fundamental laws of physics is that matter is most stable with the lowest possible energy. Thus, the electron in a hydrogen atom usually moves in the $n=1$ orbit, the orbit in which it has the lowest energy. When the electron is in this lowest energy orbit, the atom is said to be in its ground electronic state (or simply ground state). If the atom receives energy from an outside source, it is possible for the electron to move to an orbit with a higher $n$ value and the atom is now in an excited electronic state (or simply an excited state) with a higher energy. When an electron transitions from an excited state (higher energy orbit) to a less excited state, or ground state, the 
difference in energy is emitted as a photon. Similarly, if a photon is absorbed by an atom, the energy of the photon moves an electron from a lower energy orbit up to a more excited one. We can relate the energy of electrons in atoms to what we learned previously about energy. The law of conservation of energy says that we can neither create nor destroy energy. Thus, if a certain amount of external energy is required to excite an electron from one energy level to another, that same amount of energy will be liberated when the electron returns to its initial state ((Figure)).

Since Bohr's model involved only a single electron, it could also be applied to the single electron ions $\mathrm{He}^{+}, \mathrm{Li}^{2+}$, $\mathrm{Be}^{3+}$, and so forth, which differ from hydrogen only in their nuclear charges, and so one-electron atoms and ions are collectively referred to as hydrogen-like atoms. The energy expression for hydrogen-like atoms is a generalization of the hydrogen atom energy, in which $Z$ is the nuclear charge $(+1$ for hydrogen, +2 for $\mathrm{He},+3$ for $\mathrm{Li}$, and so on) and $k$ has a value of $2.17910^{-18} \mathrm{~J}$.

$E_{n}=\frac{k Z^{2}}{n^{2}}$

The sizes of the circular orbits for hydrogen-like atoms are given in terms of their radii by the following expression, in which 0 is a constant called the Bohr radius, with a value of $5.29210^{-11} \mathrm{~m}$ :

$r=\frac{n^{2}}{Z} a_{0}$

The equation also shows us that as the electron's energy increases (as $n$ increases), the electron is found at greater distances from the nucleus. This is implied by the inverse dependence of electrostatic attraction on distance, since, 
as the electron moves away from the nucleus, the electrostatic attraction between it and the nucleus decreases and it is held less tightly in the atom. Note that as $n$ gets larger and the orbits get larger, their energies get closer to zero, and so the limits $n \infty$ and $r \infty$ imply that $E=0$ corresponds to the ionization limit where the electron is completely removed from the nucleus. Thus, for hydrogen in the ground state $n=1$, the ionization energy would be:

$E=E_{n \infty}-E_{1}=0+k=k$

With three extremely puzzling paradoxes now solved (blackbody radiation, the photoelectric effect, and the hydrogen atom), and all involving Planck's constant in a fundamental manner, it became clear to most physicists at that time that the classical theories that worked so well in the macroscopic world were fundamentally flawed and could not be extended down into the microscopic domain of atoms and molecules. Unfortunately, despite Bohr's remarkable achievement in deriving a theoretical expression for the Rydberg constant, he was unable to extend his theory to the next simplest atom, He, which only has two electrons. Bohr's model was severely flawed, since it was still based on the classical mechanics notion of precise orbits, a concept that was later found to be untenable in the microscopic domain, when a proper model of quantum mechanics was developed to supersede classical mechanics.

Quantum numbers and energy levels in a hydrogen atom. The more negative the calculated value, the lower the energy. 


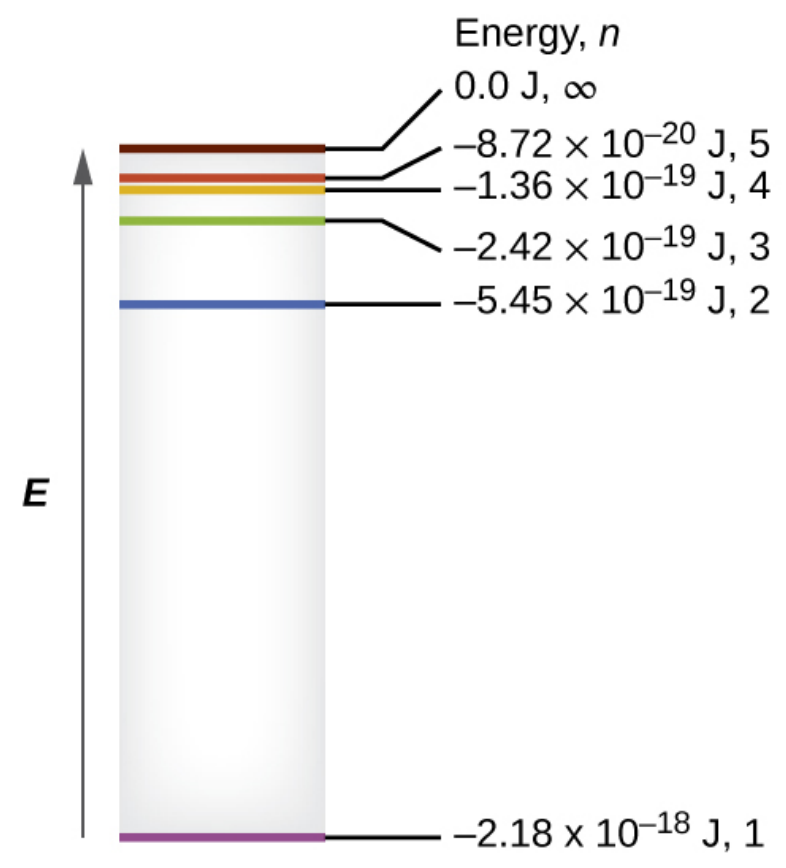

Calculating the Energy of an Electron in a Bohr Orbit Early researchers were very excited when they were able to predict the energy of an electron at a particular distance from the nucleus in a hydrogen atom. If a spark promotes the electron in a hydrogen atom into an orbit with $n=3$, what is the calculated energy, in joules, of the electron?

Solution The energy of the electron is given by this equation:

$$
E=\frac{-k Z^{2}}{n^{2}}
$$

The atomic number, $Z$, of hydrogen is $1 ; k=2.17910^{-18} \mathrm{~J}$; and the electron is characterized by an $n$ value of 3 . Thus,

$$
E=\frac{-\left(2.17910^{-18} \mathrm{~J}\right)(1)^{2}}{(3)^{2}}=-2.42110^{-19} \mathrm{~J}
$$

Check Your Learning The electron in (Figure) is promoted even further to an orbit with $n=6$. What is its new energy?

Answer:

$-6.05310^{-20}$ J 
The horizontal lines show the relative energy of orbits in the Bohr model of the hydrogen atom, and the vertical arrows depict the energy of photons absorbed (left) or emitted (right) as electrons move between these orbits.

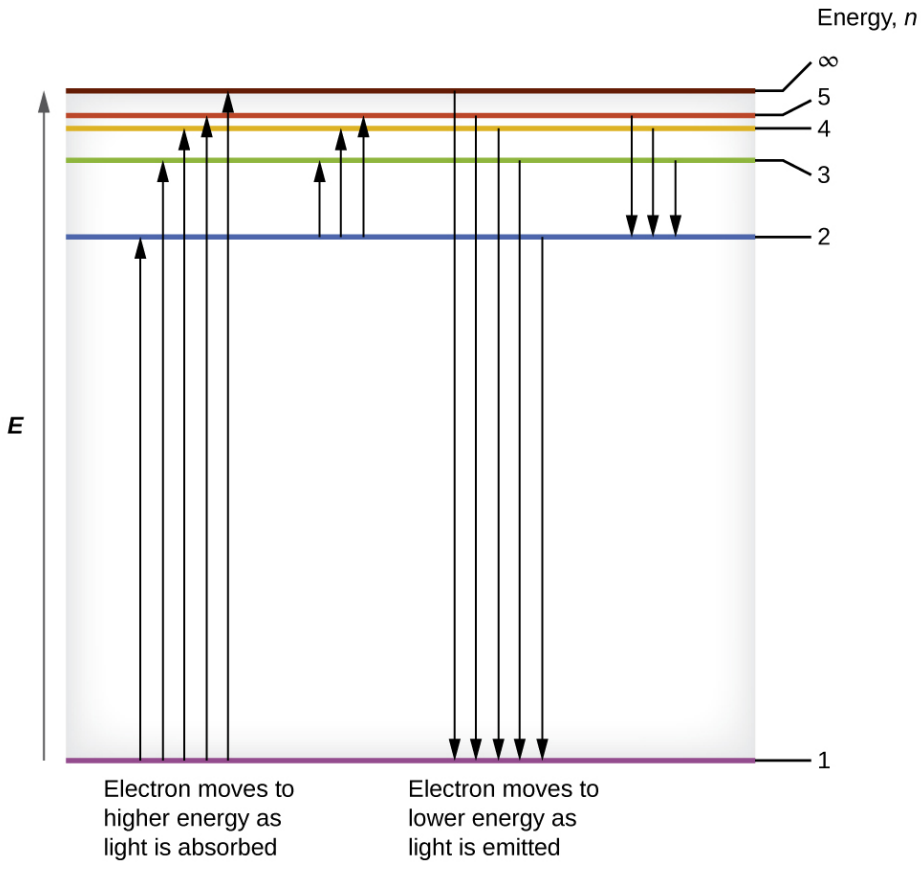

\section{Calculating the Energy and Wavelength of Electron}

Transitions in a One-electron (Bohr) System What is the energy (in joules) and the wavelength (in meters) of the line in the spectrum of hydrogen that represents the movement of an electron from Bohr orbit with $n=4$ to the orbit with $n=6$ ? In what part of the electromagnetic spectrum do we find this radiation?

Solution In this case, the electron starts out with $n=4$, so $n 1$ $=4$. It comes to rest in the $n=6$ orbit, so $n_{2}=6$. The difference in energy between the two states is given by this expression:

$$
\begin{aligned}
& E=E_{1}-E_{2}=2.17910^{-18}\left(\frac{1}{n_{1}^{2}}-\frac{1}{n_{2}^{2}}\right) \\
& E=2.17910^{-18}\left(\frac{1}{4^{2}}-\frac{1}{6^{2}}\right) \mathrm{J}
\end{aligned}
$$




$$
\begin{aligned}
& E=2.17910^{-18}\left(\frac{1}{16}-\frac{1}{36}\right) \mathrm{J} \\
& E=7.56610^{-20} \mathrm{~J}
\end{aligned}
$$

This energy difference is positive, indicating a photon enters the system (is absorbed) to excite the electron from the $n=4$ orbit up to the $n=6$ orbit. The wavelength of a photon with this energy is found by the expression $E=\frac{h c}{\lambda}$.

Rearrangement gives:

$$
\begin{aligned}
& \lambda=\frac{h c}{E} \\
& =\left(6.62610^{-34} \overline{) \mathrm{J}} \overline{) \mathrm{s}}\right) \quad \frac{2.99810^{8} \mathrm{~m} \overline{\mathrm{s}}^{-1}}{7.56610^{-20} \overline{\mathrm{JJ}}} \\
& =2.62610^{-6} \mathrm{~m}
\end{aligned}
$$

From the illustration of the electromagnetic spectrum in Electromagnetic Energy, we can see that this wavelength is found in the infrared portion of the electromagnetic spectrum.

Check Your Learning What is the energy in joules and the wavelength in meters of the photon produced when an electron falls from the $n=5$ to the $n=3$ level in a $\mathrm{He}^{+}$ion $(Z=2$ for $\left.\mathrm{He}^{+}\right)$?

Answer:

$6.19810^{-19} \mathrm{~J} ; 3.20510^{-7} \mathrm{~m}$

Bohr's model of the hydrogen atom provides insight into the behavior of matter at the microscopic level, but it is does not account for electron-electron interactions in atoms with more than one electron. It does introduce several important features of all models used to describe the distribution of electrons in an atom. These features include the following:

- The energies of electrons (energy levels) in an atom are quantized, described by quantum numbers: integer numbers having only specific allowed value and used to characterize the 
arrangement of electrons in an atom.

- An electron's energy increases with increasing distance from the nucleus.

- The discrete energies (lines) in the spectra of the elements result from quantized electronic energies.

Of these features, the most important is the postulate of quantized energy levels for an electron in an atom. As a consequence, the model laid the foundation for the quantum mechanical model of the atom. Bohr won a Nobel Prize in Physics for his contributions to our understanding of the structure of atoms and how that is related to line spectra emissions.

Key Concepts and Summary

Bohr incorporated Planck's and Einstein's quantization ideas into a model of the hydrogen atom that resolved the paradox of atom stability and discrete spectra. The Bohr model of the hydrogen atom explains the connection between the quantization of photons and the quantized emission from atoms. Bohr described the hydrogen atom in terms of an electron moving in a circular orbit about a nucleus. He postulated that the electron was restricted to certain orbits characterized by discrete energies. Transitions between these allowed orbits result in the absorption or emission of photons. When an electron moves from a higher-energy orbit to a more stable one, energy is emitted in the form of a photon. To move an electron from a stable orbit to a more excited one, a photon of energy must be absorbed. Using the Bohr 
model, we can calculate the energy of an electron and the radius of its orbit in any one-electron system.

Key Equations

- $E_{n}=-\frac{k Z^{2}}{n^{2}}, n=1,2,3, \ldots$

- $E=k Z^{2}\left(\frac{1}{n_{1}^{2}}-\frac{1}{n_{2}^{2}}\right)$

- $r=\frac{n^{2}}{Z} a_{0}$

Chemistry End of Chapter Exercises

Why is the electron in a Bohr hydrogen atom bound less tightly when it has a quantum number of 3 than when it has a quantum number of 1 ?

What does it mean to say that the energy of the electrons in an atom is quantized?

Quantized energy means that the electrons can possess only certain discrete energy values; values between those quantized values are not permitted.

Using the Bohr model, determine the energy, in joules, necessary to ionize a ground-state hydrogen atom. Show your calculations.

The electron volt (eV) is a convenient unit of energy for expressing atomic-scale energies. It is the amount of energy that an electron gains when subjected to a potential of 1 volt; $1 \mathrm{eV}=1.60210^{-19} \mathrm{~J}$. Using the Bohr model, determine the energy, in electron volts, of the photon produced when an electron in a hydrogen atom 
moves from the orbit with $n=5$ to the orbit with $n=2$. Show your calculations.

$$
\begin{aligned}
E & =E_{2}-E_{5}=2.17910^{-18}\left(\frac{1}{n_{2}^{2}}-\frac{1}{n_{5}^{2}}\right) \mathrm{J} \\
& =2.17910^{-18}\left(\frac{1}{2^{2}}-\frac{1}{5^{2}}\right)=4.57610^{-19} \mathrm{~J} \\
& =\frac{\left.4.57610^{-19}\right) \mathrm{J}}{1.60210^{-19} \overline{)} \mathrm{eV}^{-1}}=2.856 \mathrm{eV}
\end{aligned}
$$

Using the Bohr model, determine the lowest possible energy, in joules, for the electron in the $\mathrm{Li}^{2+}$ ion.

Using the Bohr model, determine the lowest possible energy for the electron in the $\mathrm{He}^{+}$ion.

$-8.71610^{-18} \mathrm{~J}$

Using the Bohr model, determine the energy of an electron with $n=6$ in a hydrogen atom.

Using the Bohr model, determine the energy of an electron with $n=8$ in a hydrogen atom.

$-3.40510^{-20} \mathrm{~J}$

How far from the nucleus in angstroms ( 1 angstrom $=1$ $10^{-10} \mathrm{~m}$ ) is the electron in a hydrogen atom if it has an energy of $-8.7210^{-20} \mathrm{~J}$ ?

What is the radius, in angstroms, of the orbital of an electron with $n=8$ in a hydrogen atom?

$33.9 \AA$ 
Using the Bohr model, determine the energy in joules of the photon produced when an electron in a $\mathrm{He}^{+}$ion moves from the orbit with $n=5$ to the orbit with $n=2$.

Using the Bohr model, determine the energy in joules of the photon produced when an electron in $\mathrm{Li}^{2+}$ ion moves from the orbit with $n=2$ to the orbit with $n=1$.

\section{$1.47110^{-17} \mathrm{~J}$}

Consider a large number of hydrogen atoms with electrons randomly distributed in the $n=1,2,3$, and 4 orbits.

(a) How many different wavelengths of light are emitted by these atoms as the electrons fall into lower-energy orbitals?

(b) Calculate the lowest and highest energies of light produced by the transitions described in part (a).

(c) Calculate the frequencies and wavelengths of the light produced by the transitions described in part (b).

How are the Bohr model and the Rutherford model of the atom similar? How are they different?

Both involve a relatively heavy nucleus with electrons moving around it, although strictly speaking, the Bohr model works only for one-electron atoms or ions. According to classical mechanics, the Rutherford model predicts a miniature "solar system" with electrons moving about the nucleus in circular or elliptical orbits that are confined to planes. If the requirements of classical electromagnetic theory that electrons in such orbits would emit electromagnetic radiation are ignored, such atoms would be stable, having constant energy and 
angular momentum, but would not emit any visible light (contrary to observation). If classical electromagnetic theory is applied, then the Rutherford atom would emit electromagnetic radiation of continually increasing frequency (contrary to the observed discrete spectra), thereby losing energy until the atom collapsed in an absurdly short time (contrary to the observed long-term stability of atoms). The Bohr model retains the classical mechanics view of circular orbits confined to planes having constant energy and angular momentum, but restricts these to quantized values dependent on a single quantum number, $n$. The orbiting electron in Bohr's model is assumed not to emit any electromagnetic radiation while moving about the nucleus in its stationary orbits, but the atom can emit or absorb electromagnetic radiation when the electron changes from one orbit to another. Because of the quantized orbits, such "quantum jumps" will produce discrete spectra, in agreement with observations.

The spectra of hydrogen and of calcium are shown here. 


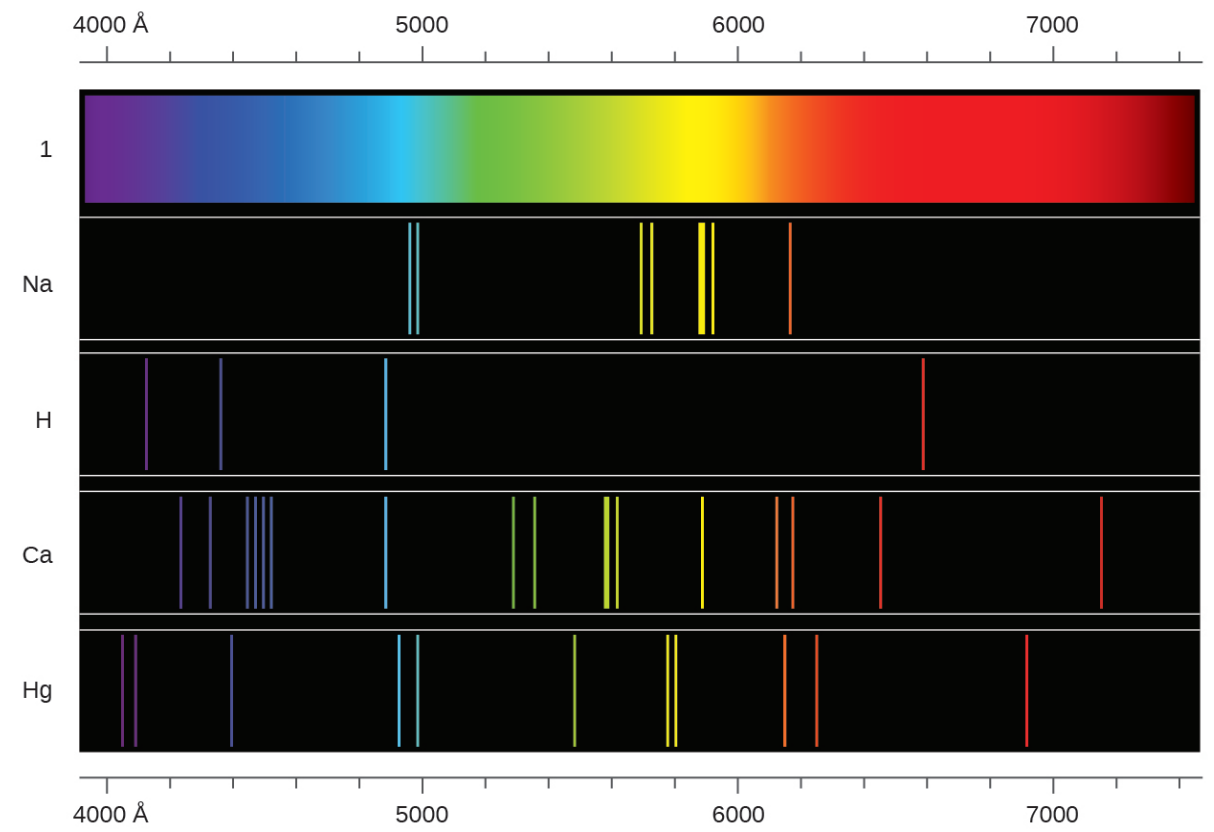

What causes the lines in these spectra? Why are the colors of the lines different? Suggest a reason for the observation that the spectrum of calcium is more complicated than the spectrum of hydrogen.

Glossary

\section{Bohr's model of the hydrogen atom}

structural model in which an electron moves around the nucleus only in circular orbits, each with a specific allowed radius

\section{excited state}

state having an energy greater than the ground-state energy

\section{ground state}

state in which the electrons in an atom, ion, 
or molecule have the lowest energy possible quantum number

number having only specific allowed

values and used to characterize the arrangement of electrons in an atom 
29.

\section{DEVELOPMENT OF QUANTUM THEORY}

\section{Learning Objectives}

By the end of this section, you will be able to:

- Extend the concept of wave-particle duality that was observed in electromagnetic radiation to matter as well

- Understand the general idea of the quantum mechanical description of electrons in an atom, and that it uses the notion of three-dimensional wave functions, or orbitals, that define the distribution of probability to find an electron in a particular part of space

- List and describe traits of the four quantum numbers that form the basis for completely specifying the state of an electron in an atom 
Bohr's model explained the experimental data for the hydrogen atom and was widely accepted, but it also raised many questions. Why did electrons orbit at only fixed distances defined by a single quantum number $n=1,2$, 3 , and so on, but never in between? Why did the model work so well describing hydrogen and one-electron ions, but could not correctly predict the emission spectrum for helium or any larger atoms? To answer these questions, scientists needed to completely revise the way they thought about matter.

Behavior in the Microscopic World

We know how matter behaves in the macroscopic world-objects that are large enough to be seen by the naked eye follow the rules of classical physics. A billiard ball moving on a table will behave like a particle: It will continue in a straight line unless it collides with another ball or the table cushion, or is acted on by some other force (such as friction). The ball has a well-defined position and velocity (or a well-defined momentum, $p=$ $m v$, defined by mass $m$ and velocity $v$ ) at any given moment. In other words, the ball is moving in a classical trajectory. This is the typical behavior of a classical object.

When waves interact with each other, they show interference patterns that are not displayed by macroscopic particles such as the billiard ball. For example, interacting waves on the surface of water can produce interference patters similar to those shown on (Figure). This is a case of wave behavior on the macroscopic scale, and it is clear that particles and waves are very different phenomena in the macroscopic realm. 
An interference pattern on the water surface is formed by interacting waves. The waves are caused by reflection of water from the rocks. (credit: modification of work by Sukanto Debnath)

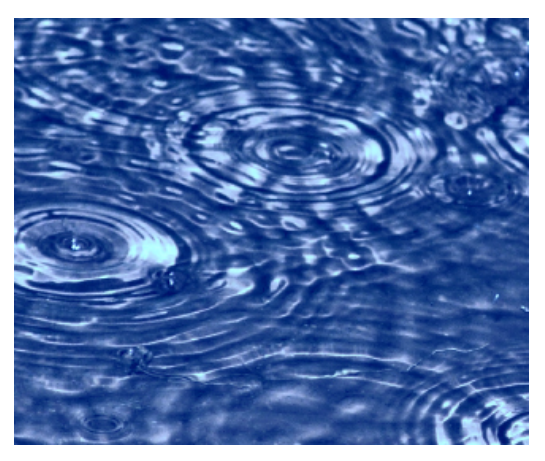

As technological improvements allowed scientists to probe the microscopic world in greater detail, it became increasingly clear by the 1920s that very small pieces of matter follow a different set of rules from those we observe for large objects. The unquestionable separation of waves and particles was no longer the case for the microscopic world.

One of the first people to pay attention to the special behavior of the microscopic world was Louis de Broglie. He asked the question: If electromagnetic radiation can have particle-like character, can electrons and other submicroscopic particles exhibit wavelike character? In his 1925 doctoral dissertation, de Broglie extended the wave-particle duality of light that Einstein used to resolve the photoelectric-effect paradox to material particles. He predicted that a particle with mass $m$ and velocity $v$ (that is, with linear momentum $p$ ) should also exhibit the behavior of a wave with a wavelength value $\lambda$, given by this expression in which $h$ is the familiar Planck's constant:

$\lambda=\frac{h}{m v}=\frac{h}{p}$ 
This is called the de Broglie wavelength. Unlike the other values of $\lambda$ discussed in this chapter, the de Broglie wavelength is a characteristic of particles and other bodies, not electromagnetic radiation (note that this equation involves velocity $[v, \mathrm{~m} / \mathrm{s}]$, not frequency $[v, \mathrm{~Hz}]$. Although these two symbols appear nearly identical, they mean very different things). Where Bohr had postulated the electron as being a particle orbiting the nucleus in quantized orbits, de Broglie argued that Bohr's assumption of quantization can be explained if the electron is considered not as a particle, but rather as a circular standing wave such that only an integer number of wavelengths could fit exactly within the orbit ((Figure)). If an electron is viewed as a wave circling around the nucleus, an integer number of wavelengths must fit into the orbit for this standing wave behavior to be possible.

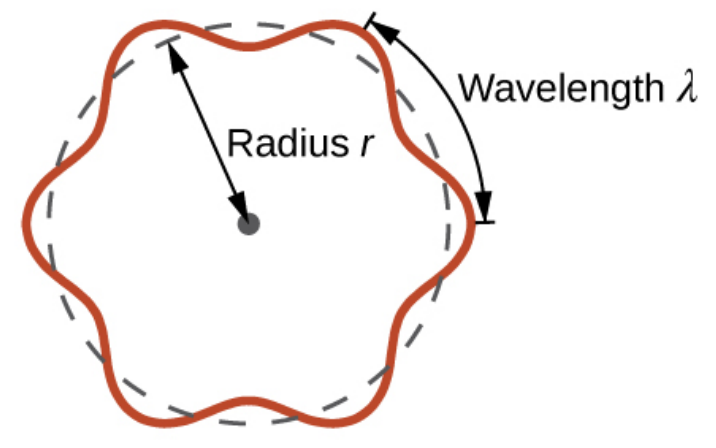

For a circular orbit of radius $r$, the circumference is $2 \pi r$, and so de Broglie's condition is:

$2 \pi r=n \lambda, n=1,2,3, \ldots$

Shortly after de Broglie proposed the wave nature of matter, two scientists at Bell Laboratories, C. J. Davisson and L. H. Germer, demonstrated experimentally that electrons can exhibit wavelike behavior by showing an 
interference pattern for electrons travelling through a regular atomic pattern in a crystal. The regularly spaced atomic layers served as slits, as used in other interference experiments. Since the spacing between the layers serving as slits needs to be similar in size to the wavelength of the tested wave for an interference pattern to form, Davisson and Germer used a crystalline nickel target for their "slits," since the spacing of the atoms within the lattice was approximately the same as the de Broglie wavelengths of the electrons that they used. (Figure) shows an interference pattern. It is strikingly similar to the interference patterns for light shown in Electromagnetic Energy for light passing through two closely spaced, narrow slits. The wave-particle duality of matter can be seen in (Figure) by observing what happens if electron collisions are recorded over a long period of time. Initially, when only a few electrons have been recorded, they show clear particle-like behavior, having arrived in small localized packets that appear to be random. As more and more electrons arrived and were recorded, a clear interference pattern that is the hallmark of wavelike behavior emerged. Thus, it appears that while electrons are small localized particles, their motion does not follow the equations of motion implied by classical mechanics, but instead it is governed by some type of a wave equation. Thus the wave-particle duality first observed with photons is actually a fundamental behavior intrinsic to all quantum particles.

(a) The interference pattern for electrons passing through very closely spaced slits demonstrates that quantum particles such as electrons can exhibit wavelike behavior.

(b) The experimental results illustrated here demonstrate 
the wave-particle duality in electrons.

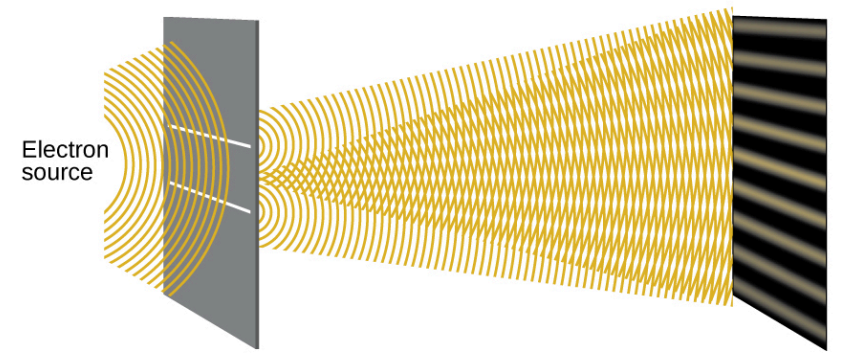

(a)
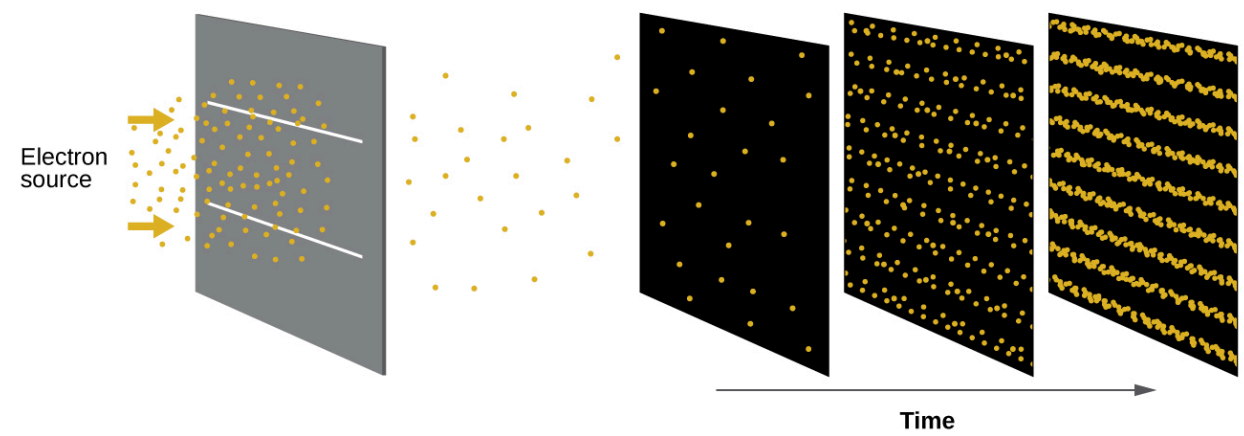

(b)

View the Dr. Quantum - Double Slit Experiment cartoon for an easy-to-understand description of wave-particle duality and the associated experiments.

Werner Heisenberg considered the limits of how accurately we can measure properties of an electron or other microscopic particles. He determined that there is a fundamental limit to how accurately one can measure both a particle's position and its momentum simultaneously. The more accurately we measure the momentum of a particle, the less accurately we can determine its position at that time, and vice versa. This is summed up in what we now call the Heisenberg uncertainty principle: It is fundamentally impossible to determine simultaneously and exactly both the momentum 
and the position of a particle. For a particle of mass $m$ moving with velocity $v_{\mathrm{x}}$ in the $x$ direction (or equivalently with momentum $p_{\mathrm{x}}$ ), the product of the uncertainty in the position, $\Delta x$, and the uncertainty in the momentum, $\Delta p_{\mathrm{X}}$ , must be greater than or equal to $\frac{\hbar}{2}$ (where $\hbar=\frac{h}{2 \pi}$, the value of Planck's constant divided by $2 \pi$ ).

$x p_{x}=(x)(m v) \geq \frac{\hbar}{2}$

This equation allows us to calculate the limit to how precisely we can know both the simultaneous position of an object and its momentum. For example, if we improve our measurement of an electron's position so that the uncertainty in the position $(\Delta x)$ has a value of, say, $1 \mathrm{pm}$ $\left(10^{-12} \mathrm{~m}\right.$, about $1 \%$ of the diameter of a hydrogen atom), then our determination of its momentum must have an uncertainty with a value of at least

$\left[p=m v=\frac{h}{(2 x)}\right]=\frac{\left(\begin{array}{lll}1.05510^{-34} \mathrm{~kg} \mathrm{~m} / \mathrm{s}\end{array}\right)}{\left(210^{-12} \mathrm{~m}\right)}=510^{-23} \mathrm{~kg} \mathrm{~m} / \mathrm{s}$.

The value of $\hbar$ is not large, so the uncertainty in the position or momentum of a macroscopic object like a baseball is too insignificant to observe. However, the mass of a microscopic object such as an electron is small enough that the uncertainty can be large and significant.

It should be noted that Heisenberg's uncertainty principle is not just limited to uncertainties in position and momentum, but it also links other dynamical variables. For example, when an atom absorbs a photon and makes a transition from one energy state to another, the uncertainty in the energy and the uncertainty in the time required for the transition are similarly related, as $\Delta E$ $\Delta t \geqslant \frac{\hbar}{2}$.

Heisenberg's principle imposes ultimate limits on what 
is knowable in science. The uncertainty principle can be shown to be a consequence of wave-particle duality, which lies at the heart of what distinguishes modern quantum theory from classical mechanics.

Read this article that describes a recent macroscopic demonstration of the uncertainty principle applied to microscopic objects.

The Quantum-Mechanical Model of an Atom

Shortly after de Broglie published his ideas that the electron in a hydrogen atom could be better thought of as being a circular standing wave instead of a particle moving in quantized circular orbits, Erwin Schrödinger extended de Broglie's work by deriving what is today known as the Schrödinger equation. When Schrödinger applied his equation to hydrogen-like atoms, he was able to reproduce Bohr's expression for the energy and, thus, the Rydberg formula governing hydrogen spectra. Schrödinger described electrons as three-dimensional stationary waves, or wavefunctions, represented by the Greek letter psi, $\psi$. A few years later, Max Born proposed an interpretation of the wavefunction $\psi$ that is still accepted today: Electrons are still particles, and so the waves represented by $\psi$ are not physical waves but, instead, are complex probability amplitudes. The square of the magnitude of a wavefunction $\psi^{2}$ describes the probability of the quantum particle being present near a certain location in space. This means that wavefunctions can be used to determine the distribution of the electron's density with respect to the nucleus in an atom. In the 
most general form, the Schrödinger equation can be written as:

$H \psi=E \psi$

$H$ is the Hamiltonian operator, a set of mathematical operations representing the total energy of the quantum particle (such as an electron in an atom), $\psi$ is the wavefunction of this particle that can be used to find the special distribution of the probability of finding the particle, and $E$ is the actual value of the total energy of the particle.

Schrödinger's work, as well as that of Heisenberg and many other scientists following in their footsteps, is generally referred to as quantum mechanics.

You may also have heard of Schrödinger because of his famous thought experiment. This story explains the concepts of superposition and entanglement as related to a cat in a box with poison.

Understanding Quantum Theory of Electrons in Atoms

The goal of this section is to understand the electron orbitals (location of electrons in atoms), their different energies, and other properties. The use of quantum theory provides the best understanding to these topics. This knowledge is a precursor to chemical bonding.

As was described previously, electrons in atoms can exist only on discrete energy levels but not between them. It is said that the energy of an electron in an atom is quantized, that is, it can be equal only to certain specific values and can jump from one energy level to another but not transition smoothly or stay between these levels. 
The energy levels are labeled with an $n$ value, where $n$ $=1,2,3, \ldots$. Generally speaking, the energy of an electron in an atom is greater for greater values of $n$. This number, $n$, is referred to as the principal quantum number. The principal quantum number defines the location of the energy level. It is essentially the same concept as the $n$ in the Bohr atom description. Another name for the principal quantum number is the shell number. The shells of an atom can be thought of concentric circles radiating out from the nucleus. The electrons that belong to a specific shell are most likely to be found within the corresponding circular area. The further we proceed from the nucleus, the higher the shell number, and so the higher the energy level ((Figure)). The positively charged protons in the nucleus stabilize the electronic orbitals by electrostatic attraction between the positive charges of the protons and the negative charges of the electrons. So the further away the electron is from the nucleus, the greater the energy it has.

Different shells are numbered by principal quantum numbers.

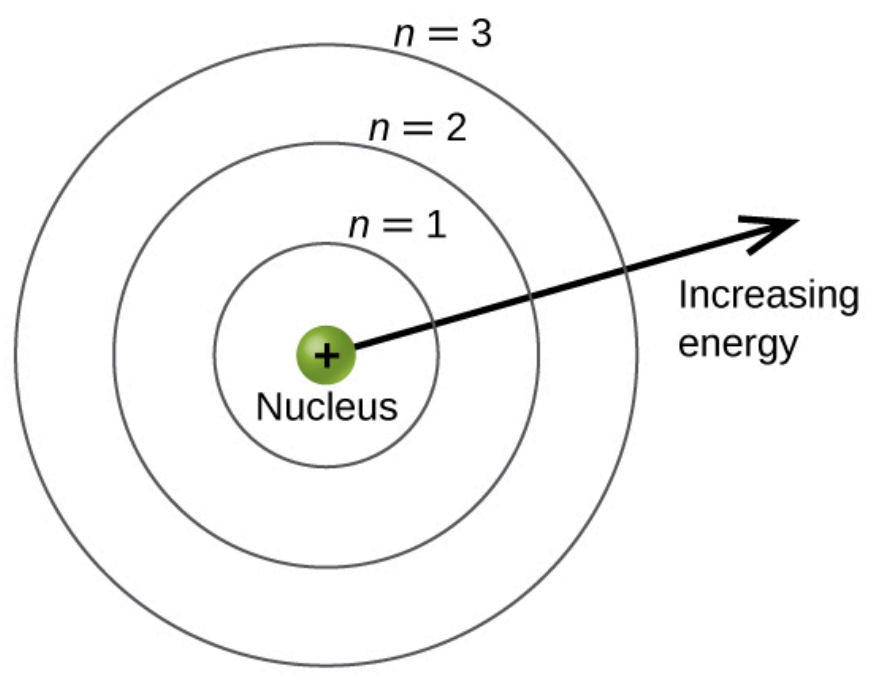


This quantum mechanical model for where electrons reside in an atom can be used to look at electronic transitions, the events when an electron moves from one energy level to another. If the transition is to a higher energy level, energy is absorbed, and the energy change has a positive value. To obtain the amount of energy necessary for the transition to a higher energy level, a photon is absorbed by the atom. A transition to a lower energy level involves a release of energy, and the energy change is negative. This process is accompanied by emission of a photon by the atom. The following equation summarizes these relationships and is based on the hydrogen atom:

$$
E=E_{\text {final }}-E_{\text {initial }}
$$

$=-2.1810^{-18}\left(\frac{1}{n_{\mathrm{f}}^{2}}-\frac{1}{n_{\mathrm{i}}^{2}}\right) \mathrm{J}$

The values $n_{\mathrm{f}}$ and $n_{\mathrm{i}}$ are the final and initial energy states of the electron. (Figure) in the previous section of the chapter demonstrates calculations of such energy changes.

The principal quantum number is one of three quantum numbers used to characterize an orbital. An atomic orbital is a general region in an atom within which an electron is most probable to reside. The quantum mechanical model specifies the probability of finding an electron in the three-dimensional space around the nucleus and is based on solutions of the Schrödinger equation. In addition, the principal quantum number defines the energy of an electron in a hydrogen or hydrogen-like atom or an ion 
(an atom or an ion with only one electron) and the general region in which discrete energy levels of electrons in a multi-electron atoms and ions are located.

Another quantum number is $l$, the secondary (angular momentum) quantum number. It is an integer that may take the values, $l=0,1,2, \ldots, n-1$. This means that an orbital with $n=1$ can have only one value of $l, l=0$, whereas $n=2$ permits $l=0$ and $l=1$, and so on. Whereas the principal quantum number, $n$, defines the general size and energy of the orbital, the secondary quantum number $l$ specifies the shape of the orbital. Orbitals with the same value of $l$ define a subshell.

Orbitals with $l=0$ are called $s$ orbitals and they make up the $s$ subshells. The value $l=1$ corresponds to the $p$ orbitals. For a given $n, p$ orbitals constitute a $p$ subshell (e.g., $3 p$ if $n=3$ ). The orbitals with $l=2$ are called the $d$ orbitals, followed by the $f-, g-$, and $h$-orbitals for $l=3,4$, and 5.

There are certain distances from the nucleus at which the probability density of finding an electron located at a particular orbital is zero. In other words, the value of the wavefunction $\psi$ is zero at this distance for this orbital. Such a value of radius $r$ is called a radial node. The number of radial nodes in an orbital is $n-l-1$.

The graphs show the probability ( $y$ axis) of finding an electron for the $1 s, 2 s, 3 s$ orbitals as a function of distance from the nucleus. 


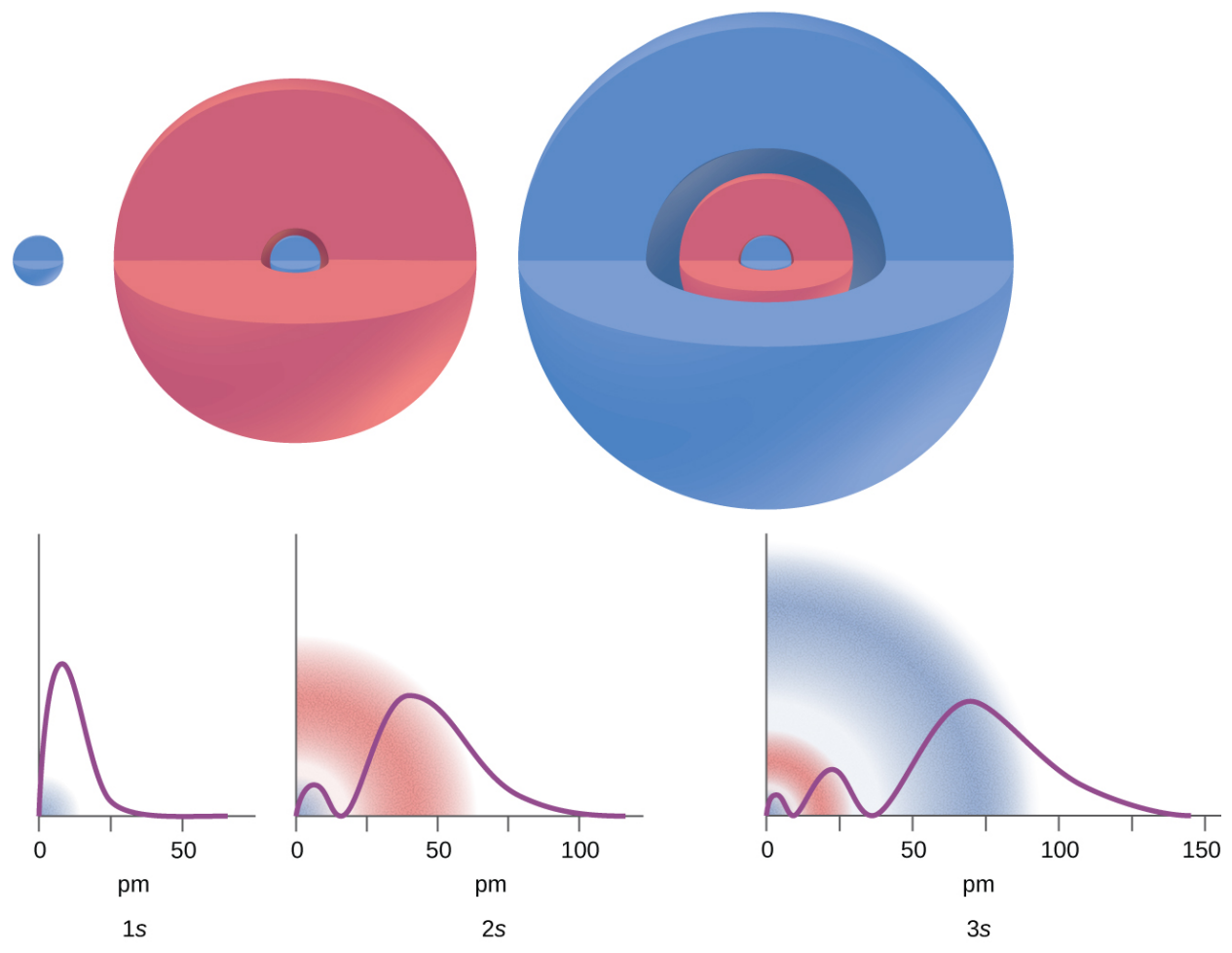

Consider the examples in (Figure). The orbitals depicted are of the $s$ type, thus $l=0$ for all of them. It can be seen from the graphs of the probability densities that there are $1-0-1=0$ places where the density is zero (nodes) for $1 s$ $(n=1), 2-0-1=1$ node for $2 s$, and $3-0-1=2$ nodes for the $3 s$ orbitals.

The $s$ subshell electron density distribution is spherical and the $p$ subshell has a dumbbell shape. The $d$ and $f$ orbitals are more complex. These shapes represent the three-dimensional regions within which the electron is likely to be found.

Shapes of $s, p, d$, and $f$ orbitals. 


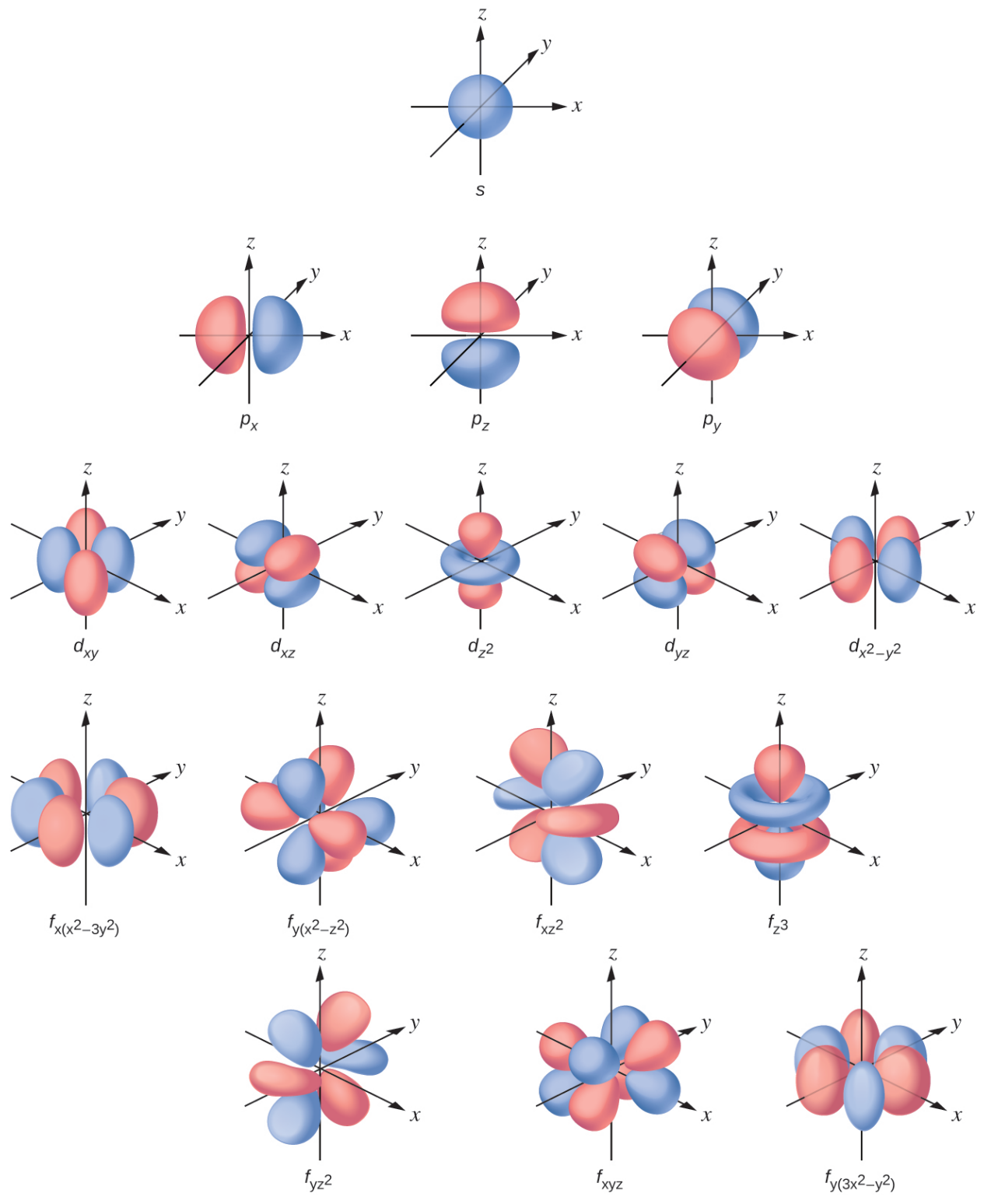

The magnetic quantum number, $m_{1}$, specifies the relative spatial orientation of a particular orbital. Generally speaking, $m_{1}$ can be equal to $-l,-(l-1), \ldots, 0, \ldots,(l-$ $1), l$. The total number of possible orbitals with the same value of $l$ (that is, in the same subshell) is $2 l+1$. Thus, there is one $s$-orbital in an $s$ subshell $(l=0)$, there are three $p$-orbitals in a $p$ subshell $(l=1)$, five $d$-orbitals in a $d$ subshell $(l=2)$, seven $f$-orbitals in an $f$ subshell $(l=$ 
3), and so forth. The principal quantum number defines the general value of the electronic energy. The angular momentum quantum number determines the shape of the orbital. And the magnetic quantum number specifies orientation of the orbital in space, as can be seen in (Figure).

The chart shows the energies of electron orbitals in a multi-electron atom.

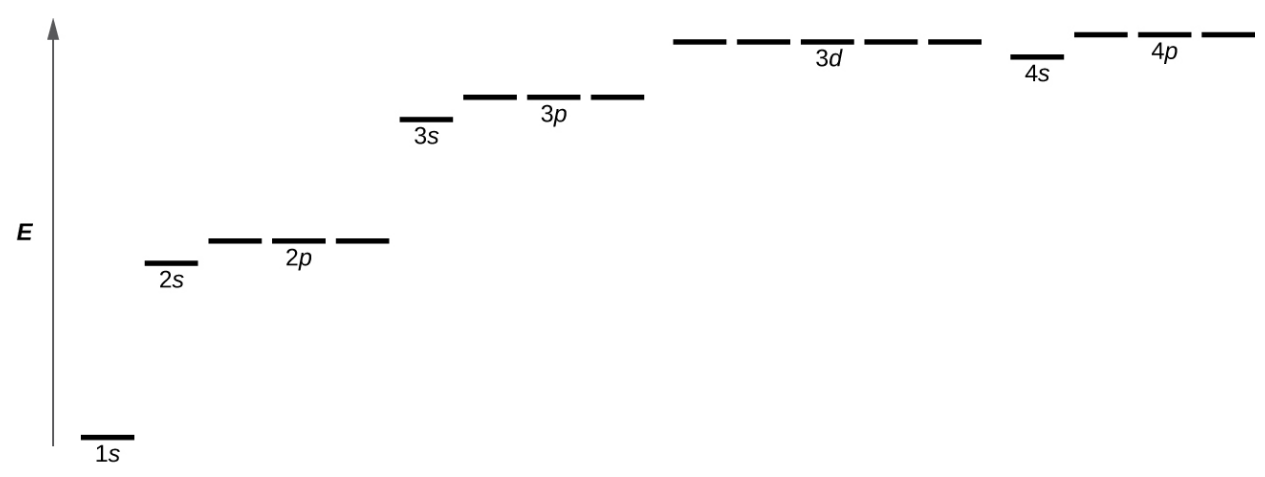

(Figure) illustrates the energy levels for various orbitals. The number before the orbital name (such as $2 s, 3 p$, and so forth) stands for the principal quantum number, $n$. The letter in the orbital name defines the subshell with a specific angular momentum quantum number $l=0$ for $s$ orbitals, 1 for $p$ orbitals, 2 for $d$ orbitals. Finally, there are more than one possible orbitals for $l \geqslant 1$, each corresponding to a specific value of $m_{1}$. In the case of a hydrogen atom or a one-electron ion (such as $\mathrm{He}^{+}, \mathrm{Li}^{2+}$, and so on), energies of all the orbitals with the same $n$ are the same. This is called a degeneracy, and the energy levels for the same principal quantum number, $n$, are called degenerate orbitals. However, in atoms with more than one electron, this degeneracy is eliminated by the electron-electron interactions, and orbitals that belong 
to different subshells have different energies, as shown on (Figure). Orbitals within the same subshell are still degenerate and have the same energy.

While the three quantum numbers discussed in the previous paragraphs work well for describing electron orbitals, some experiments showed that they were not sufficient to explain all observed results. It was demonstrated in the 1920s that when hydrogen-line spectra are examined at extremely high resolution, some lines are actually not single peaks but, rather, pairs of closely spaced lines. This is the so-called fine structure of the spectrum, and it implies that there are additional small differences in energies of electrons even when they are located in the same orbital. These observations led Samuel Goudsmit and George Uhlenbeck to propose that electrons have a fourth quantum number. They called this the spin quantum number, or $m_{\mathrm{s}}$.

The other three quantum numbers, $n, l$, and $m_{1}$, are properties of specific atomic orbitals that also define in what part of the space an electron is most likely to be located. Orbitals are a result of solving the Schrödinger equation for electrons in atoms. The electron spin is a different kind of property. It is a completely quantum phenomenon with no analogues in the classical realm. In addition, it cannot be derived from solving the Schrödinger equation and is not related to the normal spatial coordinates (such as the Cartesian $x, y$, and $z$ ). Electron spin describes an intrinsic electron "rotation" or "spinning." Each electron acts as a tiny magnet or a tiny rotating object with an angular momentum, or as a loop with an electric current, even though this rotation 
or current cannot be observed in terms of spatial coordinates.

The magnitude of the overall electron spin can only have one value, and an electron can only "spin" in one of two quantized states. One is termed the $\alpha$ state, with the $z$ component of the spin being in the positive direction of the $z$ axis. This corresponds to the spin quantum number $m_{s}=\frac{1}{2}$. The other is called the $\beta$ state, with the $z$ component of the spin being negative and $m_{s}=-\frac{1}{2}$. Any electron, regardless of the atomic orbital it is located in, can only have one of those two values of the spin quantum number. The energies of electrons having $m_{s}=-\frac{1}{2}$ and $m_{s}=\frac{1}{2}$ are different if an external magnetic field is applied.

Electrons with spin values $\frac{1}{2}$ in an external magnetic field.

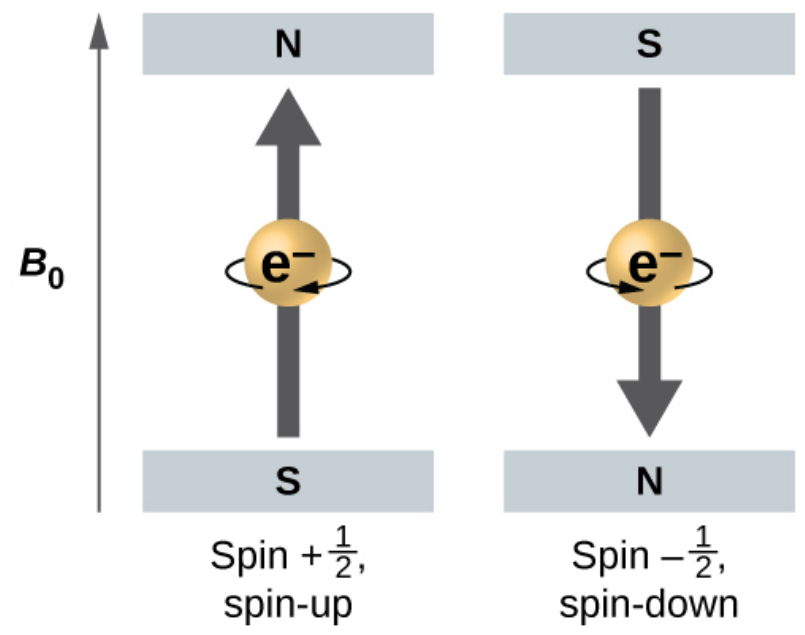

(Figure) illustrates this phenomenon. An electron acts like a tiny magnet. Its moment is directed up (in the positive direction of the $z$ axis) for the $\frac{1}{2}$ spin quantum number and down (in the negative $z$ direction) for the spin quantum number of $-\frac{1}{2}$. A magnet has a lower energy if its 
magnetic moment is aligned with the external magnetic field (the left electron on (Figure)) and a higher energy for the magnetic moment being opposite to the applied field. This is why an electron with $m_{s}=\frac{1}{2}$ has a slightly lower energy in an external field in the positive $z$ direction, and an electron with $m_{s}=-\frac{1}{2}$ has a slightly higher energy in the same field. This is true even for an electron occupying the same orbital in an atom. A spectral line corresponding to a transition for electrons from the same orbital but with different spin quantum numbers has two possible values of energy; thus, the line in the spectrum will show a fine structure splitting.

The Pauli Exclusion Principle

An electron in an atom is completely described by four quantum numbers: $n, l, m_{\mathrm{l}}$, and $m_{\mathrm{s}}$. The first three quantum numbers define the orbital and the fourth quantum number describes the intrinsic electron property called spin. An Austrian physicist Wolfgang Pauli formulated a general principle that gives the last piece of information that we need to understand the general behavior of electrons in atoms. The Pauli exclusion principle can be formulated as follows: No two electrons in the same atom can have exactly the same set of all the four quantum numbers. What this means is that two electrons can share the same orbital (the same set of the quantum numbers $n, l$, and $m_{1}$ ) only if their spin quantum numbers $m_{\mathrm{s}}$ have different values. Since the spin quantum number can only have two values $\left(\frac{1}{2}\right)$, no more than two electrons can occupy the same orbital (and if two electrons are located in the same orbital, they must have 
opposite spins). Therefore, any atomic orbital can be populated by only zero, one, or two electrons.

The properties and meaning of the quantum numbers of electrons in atoms are briefly summarized in (Figure).

\section{Quantum Numbers, Their Properties, and Significance}

$\begin{array}{llll}\text { Name } & \text { Symbol } & \begin{array}{l}\text { Allowed } \\ \text { values }\end{array} & \begin{array}{l}\text { Physical meaning } \\ \text { shell, the general region for }\end{array} \\ \begin{array}{l}\text { principal } \\ \text { quantum number }\end{array} & n & \begin{array}{l}1,2,3,4, \\ \ldots .\end{array} & \begin{array}{l}\text { the value of energy for an } \\ \text { electron on the orbital }\end{array} \\ \begin{array}{l}\text { angular } \\ \text { momentum or } \\ \text { azimuthal } \\ \text { quantum number } \\ \begin{array}{l}\text { magnetic } \\ \text { quantum number }\end{array}\end{array} & l & 0 \leqslant l \leqslant n- & \begin{array}{l}\text { subshell, the shape of the } \\ \text { orbital }\end{array} \\ \begin{array}{l}\text { spin quantum } \\ \text { number }\end{array} & m_{\mathrm{S}} & -1 \leqslant m_{1} \leqslant l & \text { orientation of the orbital } \\ & & \frac{1}{2},-\frac{1}{2} \begin{array}{l}\text { direction of the intrinsic } \\ \text { quantum "spinning" of the } \\ \text { electron }\end{array}\end{array}$

Working with Shells and Subshells Indicate the number of subshells, the number of orbitals in each subshell, and the values of $l$ and $m l$ for the orbitals in the $n=4$ shell of an atom.

Solution For $n=4, l$ can have values of $0,1,2$, and 3 . Thus, $s$, $p, d$, and $f$ subshells are found in the $n=4$ shell of an atom. For $l=0$ (the s subshell), mi can only be 0 . Thus, there is only one 45 orbital. For $l=1$ ( $p$-type orbitals), $m$ can have values of $-1,0$, +1 , so we find three $4 p$ orbitals. For $l=2$ (d-type orbitals), $m l$ can have values of $-2,-1,0,+1,+2$, so we have five $4 d$ orbitals. When $l=3$ ( $f$-type orbitals), $m$ l can have values of $-3,-2,-1,0$, $+1,+2,+3$, and we can have seven $4 f$ orbitals. Thus, we find a total of 16 orbitals in the $n=4$ shell of an atom.

Check Your Learning Identify the subshell in which electrons 
with the following quantum numbers are found: (a) $n=3, l=1$; (b) $n=5, l=3$; (c) $n=2, l=0$.

Answer:

(a) $3 p$ (b) $5 f$ (c) $2<$ !- no-selfclose ->s

Maximum Number of Electrons Calculate the maximum number of electrons that can occupy a shell with (a) $n=2$, (b) $n$ $=5$, and (c) $n$ as a variable. Note you are only looking at the orbitals with the specified $n$ value, not those at lower energies.

Solution (a) When $n=2$, there are four orbitals (a single $2 \mathrm{~s}$ orbital, and three orbitals labeled $2 p$ ). These four orbitals can contain eight electrons.

(b) When $n=5$, there are five subshells of orbitals that we need to sum:

\section{1 orbital labeled $5 \mathrm{~s}$}

3 orbitals labeled $5 \mathrm{p}$

5 orbitals labeled $5 \mathrm{~d}$

7 orbitals labeled $5 \mathrm{f}$

+9 orbitals labeled $5 g$

25 orbitals total

Again, each orbital holds two electrons, so 50 electrons can fit in this shell.

(c) The number of orbitals in any shell $n$ will equal $n^{2}$. There can be up to two electrons in each orbital, so the maximum number of electrons will be $2 n^{2}$.

Check Your Learning If a shell contains a maximum of 32 electrons, what is the principal quantum number, $n$ ?

Answer:

$n=4$ 
Working with Quantum Numbers Complete the following table for atomic orbitals:

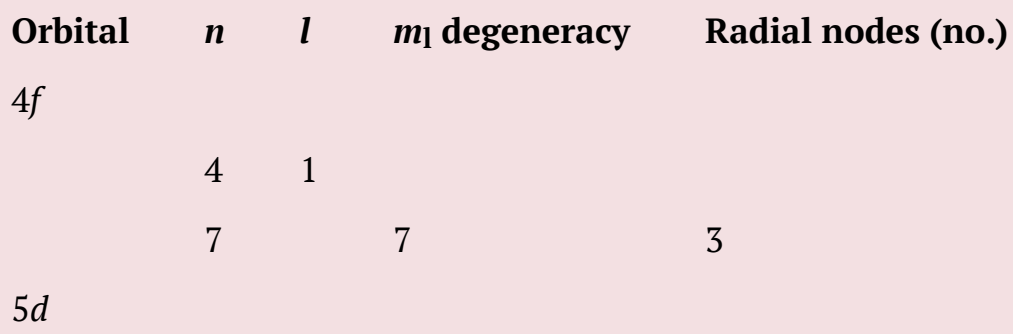

Solution The table can be completed using the following rules:

- The orbital designation is $n l$, where $l=0,1,2,3,4$, $5, \ldots$ is mapped to the letter sequence $s, p, d, f, g, h$, $\cdots$,

- The $m$ l degeneracy is the number of orbitals within an l subshell, and so is $2 l+1$ (there is one $\mathrm{s}$ orbital, three $p$ orbitals, five $d$ orbitals, seven $f$ orbitals, and so forth).

- The number of radial nodes is equal to $n-l-1$.

$\begin{array}{lllll}\text { Orbital } & \boldsymbol{n} & \boldsymbol{l} & \boldsymbol{m}_{\mathbf{l}} \text { degeneracy } & \text { Radial nodes (no.) } \\ 4 f & 4 & 3 & 7 & 0 \\ 4 p & 4 & 1 & 3 & 2 \\ 7 f & 7 & 3 & 7 & 3 \\ 5 d & 5 & 2 & 5 & 2\end{array}$

Check Your Learning How many orbitals have $l=2$ and $n=3$ ? Answer:

The five degenerate $3 d$ orbitals 
Key Concepts and Summary

Macroscopic objects act as particles. Microscopic objects (such as electrons) have properties of both a particle and a wave. Their exact trajectories cannot be determined. The quantum mechanical model of atoms describes the threedimensional position of the electron in a probabilistic manner according to a mathematical function called a wavefunction, often denoted as $\psi$. Atomic wavefunctions are also called orbitals. The squared magnitude of the wavefunction describes the distribution of the probability of finding the electron in a particular region in space. Therefore, atomic orbitals describe the areas in an atom where electrons are most likely to be found.

An atomic orbital is characterized by three quantum numbers. The principal quantum number, $n$, can be any positive integer. The general region for value of energy of the orbital and the average distance of an electron from the nucleus are related to $n$. Orbitals having the same value of $n$ are said to be in the same shell. The secondary (angular momentum) quantum number, $l$, can have any integer value from 0 to $n-1$. This quantum number describes the shape or type of the orbital. Orbitals with the same principal quantum number and the same $l$ value belong to the same subshell. The magnetic quantum number, $m_{1}$, with $2 l+1$ values ranging from $-l$ to $+l$, describes the orientation of the orbital in space. In addition, each electron has a spin quantum number, $m_{\mathrm{s}}$, that can be equal to $\frac{1}{2}$. No two electrons in the same atom can have the same set of values for all the four quantum numbers. 
Chemistry End of Chapter Exercises

How are the Bohr model and the quantum mechanical model of the hydrogen atom similar? How are they different?

Both models have a central positively charged nucleus with electrons moving about the nucleus in accordance with the Coulomb electrostatic potential. The Bohr model assumes that the electrons move in circular orbits that have quantized energies, angular momentum, and radii that are specified by a single quantum number, $n=1,2$, $3, \ldots$, but this quantization is an ad hoc assumption made by Bohr to incorporate quantization into an essentially classical mechanics description of the atom. Bohr also assumed that electrons orbiting the nucleus normally do not emit or absorb electromagnetic radiation, but do so when the electron switches to a different orbit. In the quantum mechanical model, the electrons do not move in precise orbits (such orbits violate the Heisenberg uncertainty principle) and, instead, a probabilistic interpretation of the electron's position at any given instant is used, with a mathematical function $\psi$ called a wavefunction that can be used to determine the electron's spatial probability distribution. These wavefunctions, or orbitals, are three-dimensional stationary waves that can be specified by three quantum numbers that arise naturally from their underlying mathematics (no ad hoc assumptions required): the principal quantum number, $n$ (the same one used by Bohr), which specifies shells such that orbitals having the same $n$ all have the same energy and approximately the same spatial extent; the angular 
momentum quantum number $l$, which is a measure of the orbital's angular momentum and corresponds to the orbitals' general shapes, as well as specifying subshells such that orbitals having the same $l$ (and $n$ ) all have the same energy; and the orientation quantum number $m$, which is a measure of the $z$ component of the angular momentum and corresponds to the orientations of the orbitals. The Bohr model gives the same expression for the energy as the quantum mechanical expression and, hence, both properly account for hydrogen's discrete spectrum (an example of getting the right answers for the wrong reasons, something that many chemistry students can sympathize with), but gives the wrong expression for the angular momentum (Bohr orbits necessarily all have nonzero angular momentum, but some quantum orbitals [ $s$ orbitals] can have zero angular momentum).

What are the allowed values for each of the four quantum numbers: $n, l, m_{\mathrm{l}}$, and $m_{\mathrm{s}}$ ?

Describe the properties of an electron associated with each of the following four quantum numbers: $n, l, m_{1}$, and $m_{\mathrm{s}}$.

$n$ determines the general range for the value of energy and the probable distances that the electron can be from the nucleus. $l$ determines the shape of the orbital. $m_{1}$ determines the orientation of the orbitals of the same $l$ value with respect to one another. $m_{\mathrm{S}}$ determines the spin of an electron.

Answer the following questions:

(a) Without using quantum numbers, describe the 
differences between the shells, subshells, and orbitals of an atom.

(b) How do the quantum numbers of the shells, subshells, and orbitals of an atom differ?

Identify the subshell in which electrons with the following quantum numbers are found:

(a) $n=2, l=1$

(b) $n=4, l=2$

(c) $n=6, l=0$

(a) $2 p$; (b) $4 d$; (c) $6 s$

Which of the subshells described in the previous question contain degenerate orbitals? How many degenerate orbitals are in each?

Identify the subshell in which electrons with the following quantum numbers are found:
(a) $n=3, l=2$
(b) $n=1, l=0$
(c) $n=4, l=3$

(a) $<$ !- no-selfclose -> 3d; (b) 1s; (c) $4 f$

Which of the subshells described in the previous question contain degenerate orbitals? How many degenerate orbitals are in each?

Sketch the boundary surface of a $d_{x^{2} y^{2}}$ and a $p_{\mathrm{y}}$ orbital. Be sure to show and label the axes. 

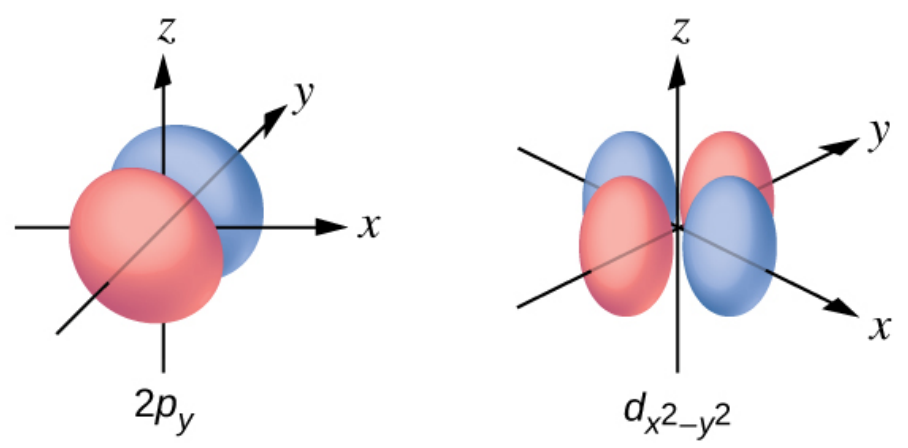

Sketch the $p_{\mathrm{x}}$ and $d_{\mathrm{xz}}$ orbitals. Be sure to show and label the coordinates.

Consider the orbitals shown here in outline.

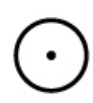

$(\mathrm{x})$

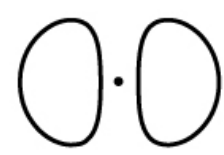

(y)

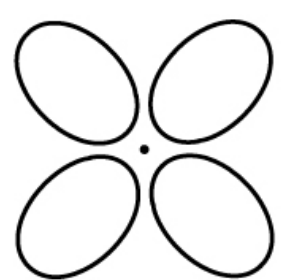

(z)

(a) What is the maximum number of electrons contained in an orbital of type (x)? Of type (y)? Of type (z)?

(b) How many orbitals of type (x) are found in a shell with $n=2$ ? How many of type (y)? How many of type (z)?

(c) Write a set of quantum numbers for an electron in an orbital of type (x) in a shell with $n=4$. Of an orbital of type (y) in a shell with $n=2$. Of an orbital of type (z) in a shell with $n=3$.

(d) What is the smallest possible $n$ value for an orbital of type (x)? Of type (y)? Of type (z)?

(e) What are the possible $l$ and $m_{1}$ values for an orbital of type (x)? Of type (y)? Of type (z)?

(a) x. 2, y. 2, z. 2; (b) x. 1, y. 3, z. 0; (c) x. $400 \frac{1}{2}$, y. $210 \frac{1}{2}$, 
z. $320 \frac{1}{2}$; (d) x. 1 , y. 2 , z. 3 ; (e) x. $l=0, m_{1}=0$, y. $l=1, m_{l}=$ $-1,0$, or +1 , z. $l=2, m_{1}=-2,-1,0,+1,+2$

State the Heisenberg uncertainty principle. Describe briefly what the principle implies.

How many electrons could be held in the second shell of an atom if the spin quantum number $m_{\mathrm{s}}$ could have three values instead of just two? (Hint: Consider the Pauli exclusion principle.)

12

Which of the following equations describe particle-like behavior? Which describe wavelike behavior? Do any involve both types of behavior? Describe the reasons for your choices.

(a) $c=\lambda v$

(b) $E=\frac{m \nu^{2}}{2}$

(c) $r=\frac{n^{2} a_{0}}{Z}$

(d) $E=h v$

(e) $\lambda=\frac{h}{m \nu}$

Write a set of quantum numbers for each of the electrons with an $n$ of 4 in a Se atom. 

$\begin{array}{llll}n & l & m_{1} & s\end{array}$
$\begin{array}{llll}4 & 0 & 0 & +\end{array}$
$\begin{array}{llll}4 & 0 & 0 & -\frac{1}{2}\end{array}$
$\begin{array}{llll}4 & 1 & -1 & +\end{array}$
$\begin{array}{llll}4 & 1 & 0 & +\end{array}$
$41+1+\frac{1}{2}$
$\begin{array}{lllll}4 & 1 & -1 & -\frac{1}{2}\end{array}$

Glossary

secondary (angular momentum) quantum number (l)

quantum number distinguishing the different shapes of orbitals; it is also a measure of the orbital angular momentum atomic orbital

mathematical function that describes the behavior of an electron in an atom (also called the wavefunction)

d orbital

region of space with high electron density that is either four lobed or contains a dumbbell and torus shape; describes orbitals with $l=2$.

degenerate orbitals

orbitals that have the same energy 
electron density

a measure of the probability of locating an electron in a particular region of space, it is equal to the squared absolute value of the wave function $\psi$

$f$ orbital

multilobed region of space with high electron density, describes orbitals with $l=3$

Heisenberg uncertainty principle

rule stating that it is impossible to exactly determine both certain conjugate dynamical properties such as the momentum and the position of a particle at the same time. The uncertainty principle is a consequence of quantum particles exhibiting wave-particle duality

\section{magnetic quantum number $(\mathrm{ml})$}

quantum number signifying the orientation of an atomic orbital around the nucleus

\section{p orbital}

dumbbell-shaped region of space with high electron density, describes orbitals with $l=1$

\section{Pauli exclusion principle}

specifies that no two electrons in an atom can have the same value for all four quantum numbers

principal quantum number ( $n$ )

quantum number specifying the shell an electron occupies in an atom

\section{quantum mechanics}

field of study that includes quantization of 
energy, wave-particle duality, and the Heisenberg uncertainty principle to describe matter

$s$ orbital

spherical region of space with high electron density, describes orbitals with $l=0$ shell

atomic orbitals with the same principal quantum number, $n$

\section{spin quantum number $\left(\boldsymbol{m}_{\mathbf{s}}\right)$}

number specifying the electron spin direction, either $+\frac{1}{2}$ or $-\frac{1}{2}$

\section{subshell}

atomic orbitals with the same values of $n$ and $l$

\section{wavefunction $(\psi)$}

mathematical description of an atomic orbital that describes the shape of the orbital; it can be used to calculate the probability of finding the electron at any given location in the orbital, as well as dynamical variables such as the energy and the angular momentum 
30.

\section{ELECTRONIC STRUCTURE OF ATOMS (ELECTRON CONFIGURATIONS)}

Learning Objectives

By the end of this section, you will be able to:

- Derive the predicted ground-state electron configurations of atoms

- Identify and explain exceptions to predicted electron configurations for atoms and ions

- Relate electron configurations to element classifications in the periodic table

Having introduced the basics of atomic structure and quantum mechanics, we can use our understanding of quantum numbers to determine how atomic orbitals 
relate to one another. This allows us to determine which orbitals are occupied by electrons in each atom. The specific arrangement of electrons in orbitals of an atom determines many of the chemical properties of that atom.

Orbital Energies and Atomic Structure

The energy of atomic orbitals increases as the principal quantum number, $n$, increases. In any atom with two or more electrons, the repulsion between the electrons makes energies of subshells with different values of $l$ differ so that the energy of the orbitals increases within a shell in the order $s<p<d<f$. (Figure) depicts how these two trends in increasing energy relate. The $1 s$ orbital at the bottom of the diagram is the orbital with electrons of lowest energy. The energy increases as we move up to the $2 s$ and then $2 p, 3 s$, and $3 p$ orbitals, showing that the increasing $n$ value has more influence on energy than the increasing $l$ value for small atoms. However, this pattern does not hold for larger atoms. The $3 d$ orbital is higher in energy than the $4 s$ orbital. Such overlaps continue to occur frequently as we move up the chart.

Generalized energy-level diagram for atomic orbitals in an atom with two or more electrons (not to scale). 


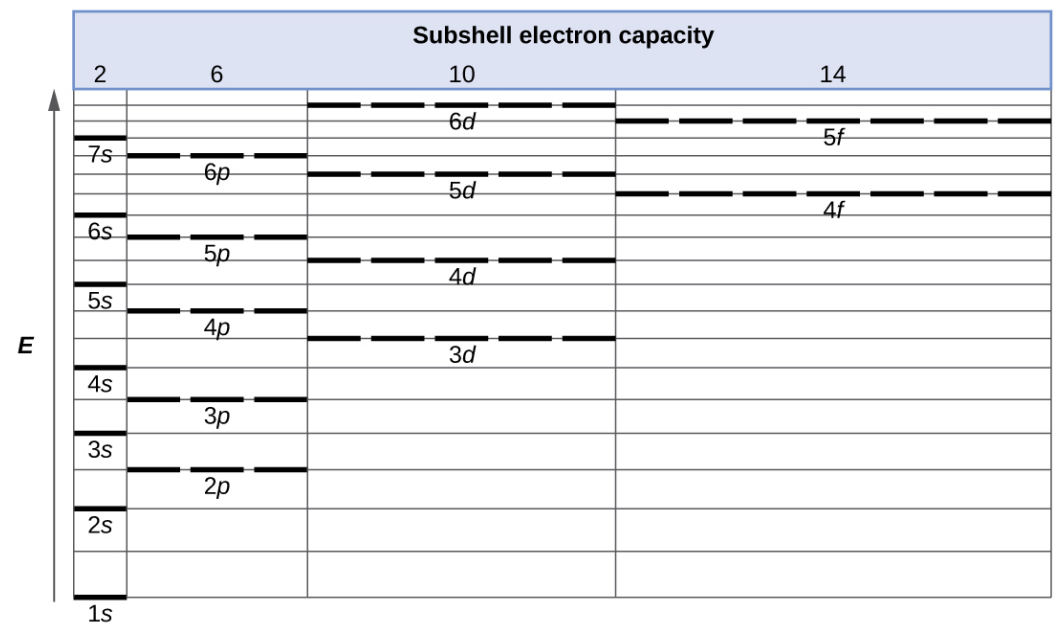

Electrons in successive atoms on the periodic table tend to fill low-energy orbitals first. Thus, many students find it confusing that, for example, the $5 p$ orbitals fill immediately after the $4 d$, and immediately before the $6 s$. The filling order is based on observed experimental results, and has been confirmed by theoretical calculations. As the principal quantum number, $n$, increases, the size of the orbital increases and the electrons spend more time farther from the nucleus. Thus, the attraction to the nucleus is weaker and the energy associated with the orbital is higher (less stabilized). But this is not the only effect we have to take into account. Within each shell, as the value of $l$ increases, the electrons are less penetrating (meaning there is less electron density found close to the nucleus), in the order $s>p$ $>d>f$. Electrons that are closer to the nucleus slightly repel electrons that are farther out, offsetting the more dominant electron-nucleus attractions slightly (recall that all electrons have -1 charges, but nuclei have $+Z$ charges). This phenomenon is called shielding and will be discussed in more detail in the next section. Electrons in 
orbitals that experience more shielding are less stabilized and thus higher in energy. For small orbitals (1s through $3 p$ ), the increase in energy due to $n$ is more significant than the increase due to $l$; however, for larger orbitals the two trends are comparable and cannot be simply predicted. We will discuss methods for remembering the observed order.

The arrangement of electrons in the orbitals of an atom is called the electron configuration of the atom. We describe an electron configuration with a symbol that contains three pieces of information ((Figure)):

1. The number of the principal quantum shell, $n$,

2. The letter that designates the orbital type (the subshell, $l$ ), and

3. A superscript number that designates the number of electrons in that particular subshell.

For example, the notation $2 p^{4}$ (read "two-p-four") indicates four electrons in a $p$ subshell $(l=1)$ with a principal quantum number $(n)$ of 2 . The notation $3 d^{8}$ (read "three-d-eight") indicates eight electrons in the $d$ subshell (i.e., $l=2$ ) of the principal shell for which $n=3$. The diagram of an electron configuration specifies the subshell ( $n$ and $l$ value, with letter symbol) and superscript number of electrons.

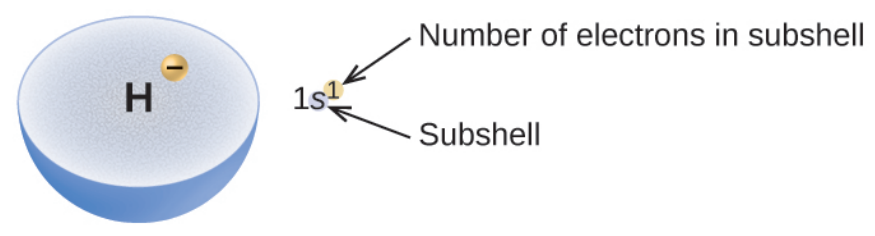


The Aufbau Principle

To determine the electron configuration for any particular atom, we can "build" the structures in the order of atomic numbers. Beginning with hydrogen, and continuing across the periods of the periodic table, we add one proton at a time to the nucleus and one electron to the proper subshell until we have described the electron configurations of all the elements. This procedure is called the Aufbau principle, from the German word Aufbau ("to build up"). Each added electron occupies the subshell of lowest energy available (in the order shown in (Figure)), subject to the limitations imposed by the allowed quantum numbers according to the Pauli exclusion principle. Electrons enter higher-energy subshells only after lower-energy subshells have been filled to capacity. (Figure) illustrates the traditional way to remember the filling order for atomic orbitals. Since the arrangement of the periodic table is based on the electron configurations, (Figure) provides an alternative method for determining the electron configuration. The filling order simply begins at hydrogen and includes each subshell as you proceed in increasing $Z$ order. For example, after filling the $3 p$ block up to Ar, we see the orbital will be $4 \mathrm{~s}(\mathrm{~K}, \mathrm{Ca})$, followed by the $3 d$ orbitals.

This diagram depicts the energy order for atomic orbitals and is useful for deriving ground-state electron configurations. 


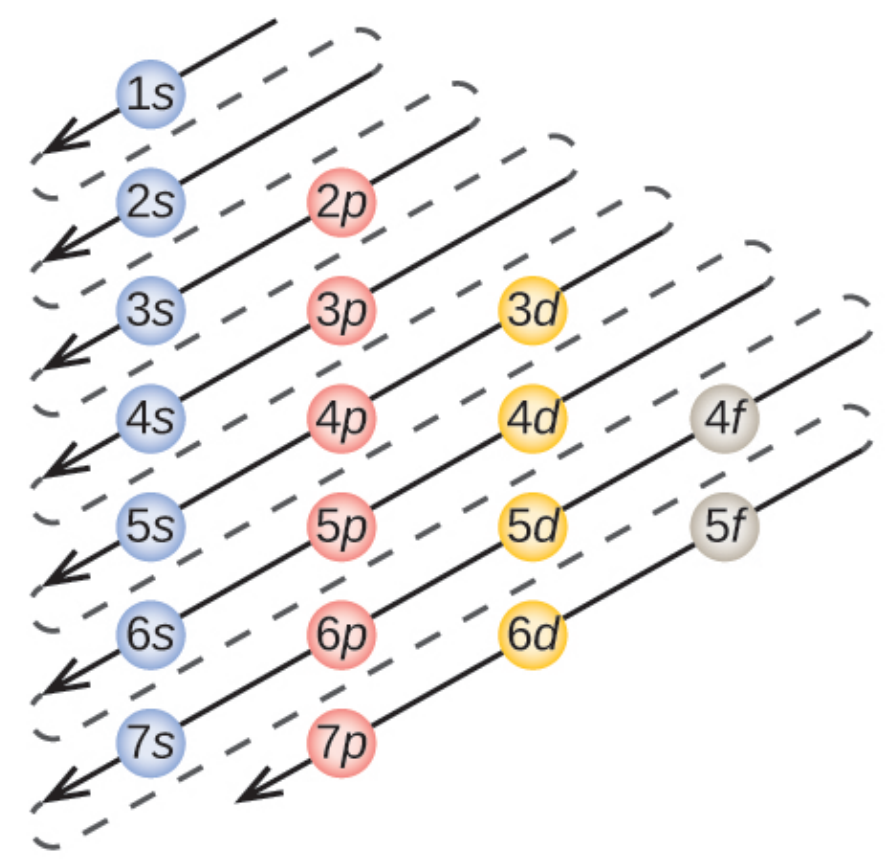

This periodic table shows the electron configuration for each subshell. By "building up" from hydrogen, this table can be used to determine the electron configuration for any atom on the periodic table. 
흠

Electron Configuration Table

$1{ }^{\mathrm{H}^{1}}{ }^{1}$

$2^{\mathrm{Li}}{ }^{1} \mathrm{Be}^{2}$

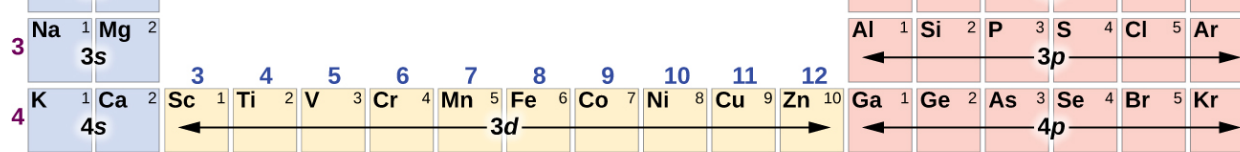

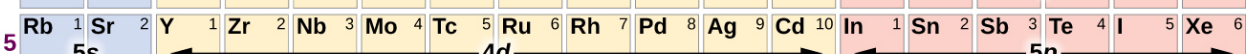

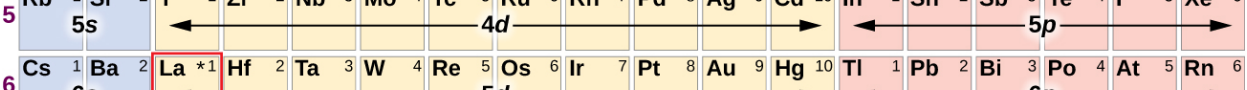

$6{ }_{6 s}{ }_{6 s}{ }_{6 s}$

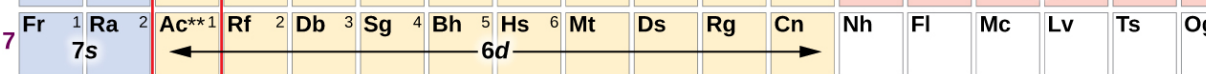

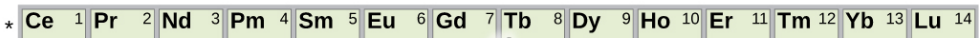

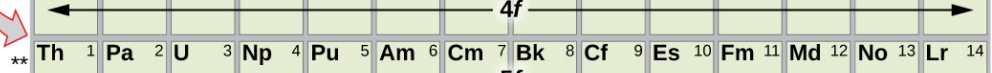

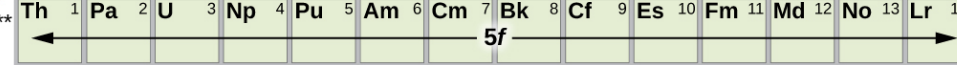

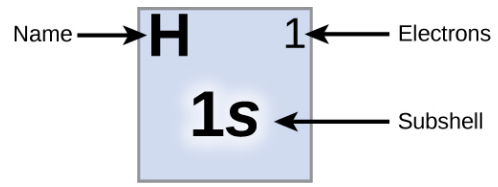

We will now construct the ground-state electron configuration and orbital diagram for a selection of atoms in the first and second periods of the periodic table. Orbital diagrams are pictorial representations of the electron configuration, showing the individual orbitals and the pairing arrangement of electrons. We start with a single hydrogen atom (atomic number 1), which consists of one proton and one electron. Referring to (Figure) or (Figure), we would expect to find the electron in the $1 \mathrm{~s}$ orbital. By convention, the $m_{s}=+\frac{1}{2}$ value is usually filled first. The electron configuration and the orbital diagram are:

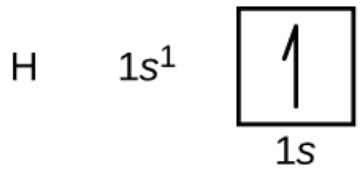

Following hydrogen is the noble gas helium, which has an atomic number of 2 . The helium atom contains two 
protons and two electrons. The first electron has the same four quantum numbers as the hydrogen atom electron ( $n$ $\left.=1, l=0, m_{1}=0, m_{s}=+\frac{1}{2}\right)$. The second electron also goes into the $1 s$ orbital and fills that orbital. The second electron has the same $n, l$, and $m_{1}$ quantum numbers, but must have the opposite spin quantum number, $m_{s}=-\frac{1}{2}$. This is in accord with the Pauli exclusion principle: No two electrons in the same atom can have the same set of four quantum numbers. For orbital diagrams, this means two arrows go in each box (representing two electrons in each orbital) and the arrows must point in opposite directions (representing paired spins). The electron configuration and orbital diagram of helium are:

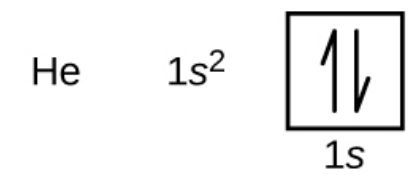

The $n=1$ shell is completely filled in a helium atom.

The next atom is the alkali metal lithium with an atomic number of 3 . The first two electrons in lithium fill the $1 \mathrm{~s}$ orbital and have the same sets of four quantum numbers as the two electrons in helium. The remaining electron must occupy the orbital of next lowest energy, the $2 s$ orbital ((Figure) or (Figure)). Thus, the electron configuration and orbital diagram of lithium are:

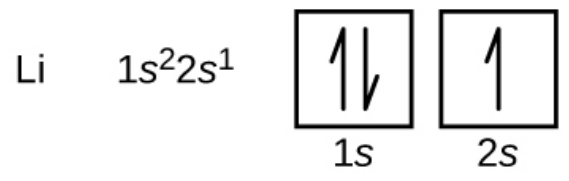

An atom of the alkaline earth metal beryllium, with an atomic number of 4 , contains four protons in the nucleus and four electrons surrounding the nucleus. The fourth electron fills the remaining space in the $2 s$ orbital. 


$$
\text { Be } 1 s^{2} 2 s^{2} \stackrel{1}{\frac{1}{1 s}} \frac{1}{2 s}
$$

An atom of boron (atomic number 5) contains five electrons. The $n=1$ shell is filled with two electrons and three electrons will occupy the $n=2$ shell. Because any $s$ subshell can contain only two electrons, the fifth electron must occupy the next energy level, which will be a $2 p$ orbital. There are three degenerate $2 p$ orbitals $\left(m_{1}=-1\right.$, $0,+1)$ and the electron can occupy any one of these $p$ orbitals. When drawing orbital diagrams, we include empty boxes to depict any empty orbitals in the same subshell that we are filling.

B $\quad 1 s^{2} 2 s^{2} 2 p^{1}$

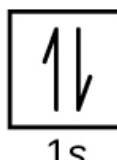

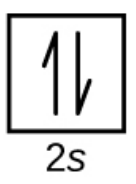

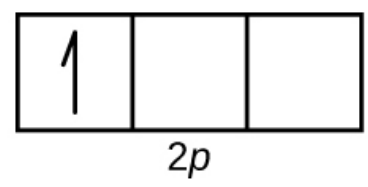

Carbon (atomic number 6) has six electrons. Four of them fill the $1 s$ and $2 s$ orbitals. The remaining two electrons occupy the $2 p$ subshell. We now have a choice of filling one of the $2 p$ orbitals and pairing the electrons or of leaving the electrons unpaired in two different, but degenerate, $p$ orbitals. The orbitals are filled as described by Hund's rule: the lowest-energy configuration for an atom with electrons within a set of degenerate orbitals is that having the maximum number of unpaired electrons. Thus, the two electrons in the carbon $2 p$ orbitals have identical $n, l$, and $m_{\mathrm{s}}$ quantum numbers and differ in their $m_{1}$ quantum number (in accord with the Pauli exclusion principle). The electron configuration and orbital diagram for carbon are: 
C $\quad 1 s^{2} 2 s^{2} 2 p^{2}$
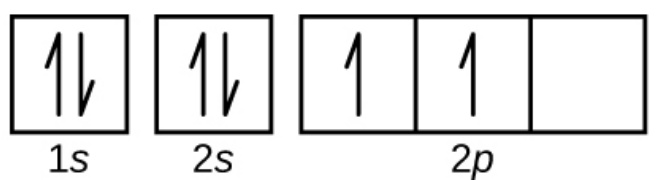

Nitrogen (atomic number 7) fills the $1 s$ and $2 s$ subshells and has one electron in each of the three $2 p$ orbitals, in accordance with Hund's rule. These three electrons have unpaired spins. Oxygen (atomic number 8 ) has a pair of electrons in any one of the $2 p$ orbitals (the electrons have opposite spins) and a single electron in each of the other two. Fluorine (atomic number 9) has only one $2 p$ orbital containing an unpaired electron. All of the electrons in the noble gas neon (atomic number 10) are paired, and all of the orbitals in the $n=1$ and the $n=2$ shells are filled. The electron configurations and orbital diagrams of these four elements are:

$\mathrm{N} \quad 1 s^{2} 2 s^{2} 2 p^{3}$
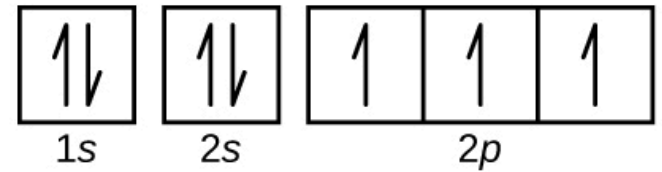

$1 s^{2} 2 s^{2} 2 p^{4}$
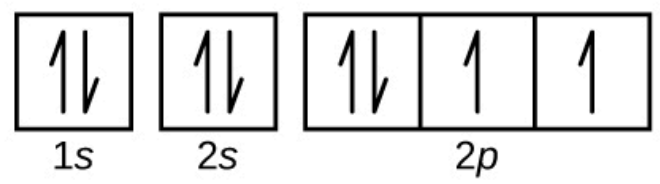

F $\quad 1 s^{2} 2 s^{2} 2 p^{5}$
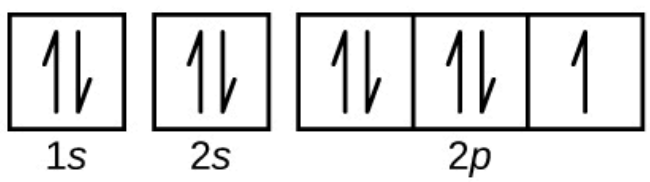

$\mathrm{Ne} \quad 1 s^{2} 2 s^{2} 2 p^{6}$
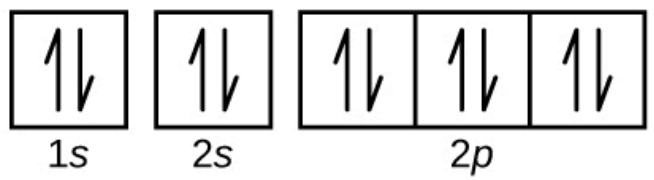

The alkali metal sodium (atomic number 11) has one more electron than the neon atom. This electron must go 
into the lowest-energy subshell available, the $3 s$ orbital, giving a $1 s^{2} 2 s^{2} 2 p^{6} 3 s^{1}$ configuration. The electrons occupying the outermost shell orbital(s) (highest value of $n$ ) are called valence electrons, and those occupying the inner shell orbitals are called core electrons ((Figure)). Since the core electron shells correspond to noble gas electron configurations, we can abbreviate electron configurations by writing the noble gas that matches the core electron configuration, along with the valence electrons in a condensed format. For our sodium example, the symbol $[\mathrm{Ne}]$ represents core electrons, $\left(1 s^{2} 2 s^{2} 2 p^{6}\right)$ and our abbreviated or condensed configuration is $[\mathrm{Ne}] 3 s^{1}$. A core-abbreviated electron configuration (right) replaces the core electrons with the noble gas symbol whose configuration matches the core electron configuration of the other element.

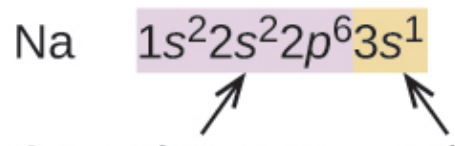

Abbreviation $[\mathrm{Ne}] 3 s^{1}$

Core electrons Valence electron

Similarly, the abbreviated configuration of lithium can be represented as $[\mathrm{He}] 2 s^{1}$, where [He] represents the configuration of the helium atom, which is identical to that of the filled inner shell of lithium. Writing the configurations in this way emphasizes the similarity of the configurations of lithium and sodium. Both atoms, which are in the alkali metal family, have only one electron in a valence $s$ subshell outside a filled set of inner shells.

Li: $[\mathrm{He}] 2 s^{1}$

$\mathrm{Na}$ : [Ne] $3 s^{1}$ 
The alkaline earth metal magnesium (atomic number 12), with its 12 electrons in a $[\mathrm{Ne}] 3 s^{2}$ configuration, is analogous to its family member beryllium, $[\mathrm{He}] 2 s^{2}$. Both atoms have a filled $s$ subshell outside their filled inner shells. Aluminum (atomic number 13), with 13 electrons and the electron configuration [Ne] $3 s^{2} 3 p^{1}$, is analogous to its family member boron, [He] $2 s^{2} 2 p^{1}$.

The electron configurations of silicon (14 electrons), phosphorus (15 electrons), sulfur (16 electrons), chlorine (17 electrons), and argon (18 electrons) are analogous in the electron configurations of their outer shells to their corresponding family members carbon, nitrogen, oxygen, fluorine, and neon, respectively, except that the principal quantum number of the outer shell of the heavier elements has increased by one to $n=3$. (Figure) shows the lowest energy, or ground-state, electron configuration for these elements as well as that for atoms of each of the known elements.

This version of the periodic table shows the outer-shell electron configuration of each element. Note that down each group, the configuration is often similar. 
흠

$\nsubseteq$ Group

$1^{1} \mathrm{H}$

Electron Configuration Table

$1 s^{1}$

$2^{3} \quad \mathrm{Li}^{4} \quad \mathrm{Be}$

$2 s^{1} 2 s^{2}$

$3^{11} \mathrm{Na}^{12} \mathrm{Mg}$

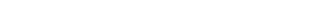

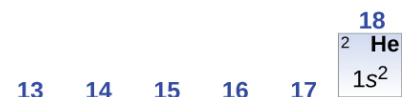

$5^{13}$ B $6^{14}{ }^{14}{ }^{15}{ }_{N} 8{ }^{16}{ }^{17}{ }^{17} F^{10} \mathrm{Ne}$

$2 s^{2} 2 p^{1} 2 s^{2} 2 p^{2} 2 s^{2} 2 p^{3} 2 s^{2} 2 p^{4} 2 s^{2} 2 p^{5} 2 s^{2} 2 p^{6}$

13 Al 14 Si 15 P $16 \quad$ S $17 \quad \mathbf{C l} 18$ Ar

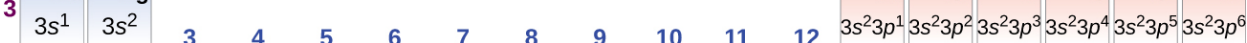

$4 \mathrm{~K}_{20} \mathbf{C a} 21 \mathrm{Sc} 22 \mathrm{Ti} 23 \mathrm{~V} 24 \mathrm{Cr} 25 \mathrm{Mn} 26 \mathrm{Fe} 27 \mathrm{Co} 28 \mathrm{Ni} 29 \mathrm{Cu} 30 \mathrm{Zn} 31 \mathrm{Ga} 32 \mathrm{Ge} 33$ As 34 Se $35 \mathrm{Br} 36 \mathrm{Kr}$

$4 \quad 4 s^{1} \quad 4 s^{2} \quad 4 s^{2} 3 d^{1} 4 s^{2} 3 d^{2} 4 s^{2} 3 d^{3} 4 s^{1} 3 d^{5} 4 s^{2} 3 d^{5} 4 s^{2} 3 d^{10} 4 s^{2} 3 d^{7} 4 s^{2} 3 d^{8} 4 s^{1} 3 d^{10} 4 s^{2} 3 d^{10} 4 s^{2} 3 d^{10} 4 p^{1} 4 s^{2} 3 d^{10} 4 p^{2} 4 s^{2} 3 d^{104} 4 p^{3} 4 s^{2} 3 d^{10} 4 p^{4} 4 s^{2} 3 d^{10} 4 p^{5} 44 s^{3} 3 d^{10} 4 p^{6}$

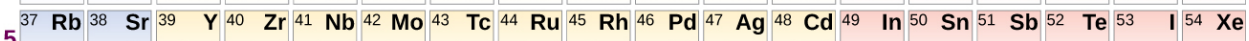

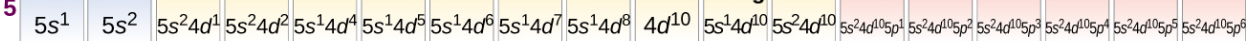

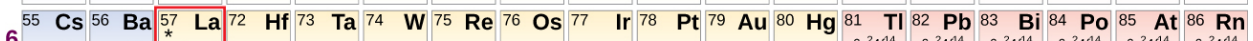

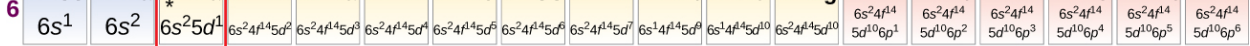

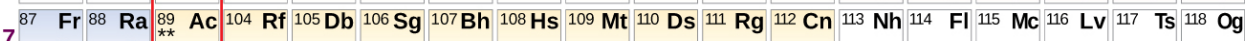

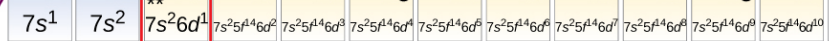

* 58 Ce 59 Pr 60 Nd 61 Pm 62 Sm 63 Eu 64 Gd 65 Tb 66 Dy 67 Ho 68 Er 69 Tm 70 Yb 71 Lu $6 s^{2} 4 f^{2} 6 s^{2} 4 f^{3} 6 s^{2} 4 f^{4} 6 s^{2} 4 f^{5} 6 s^{2} 4 f^{6} 6 s^{2} 4 f^{7} s_{6 s^{2} 47^{7} 5 d^{1}} 6 s^{2} 4 f^{9} 6 s^{2} 4 f^{10} 6 s^{2} 4 f^{11} 6 s^{2} 4 f^{12} 6 s^{2} 4 f^{13} 6 s^{2} 4 f^{14} \cdot 6 s^{2} 4 f^{4} 5 d^{4}$

** 90 Th 91 Pa 92 U 93 Np 94 Pu 95 Am 96 Cm 97 Bk 98 Cf 99 Es $100 \mathrm{Fm} 101 \mathrm{Md} 102 \mathrm{No} 103 \mathrm{Lr}$

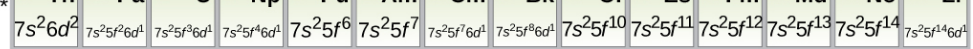

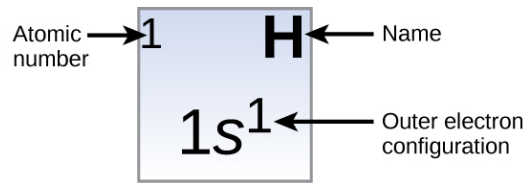

When we come to the next element in the periodic table, the alkali metal potassium (atomic number 19), we might expect that we would begin to add electrons to the $3 d$ subshell. However, all available chemical and physical evidence indicates that potassium is like lithium and sodium, and that the next electron is not added to the $3 d$ level but is, instead, added to the $4 s$ level ((Figure)). As discussed previously, the $3 d$ orbital with no radial nodes is higher in energy because it is less penetrating and more shielded from the nucleus than the $4 s$, which has three radial nodes. Thus, potassium has an electron configuration of $[\mathrm{Ar}] 4 s^{1}$. Hence, potassium corresponds to $\mathrm{Li}$ and $\mathrm{Na}$ in its valence shell configuration. The next electron is added to complete the $4 s$ subshell and calcium has an electron configuration of $[\mathrm{Ar}] 4 s^{2}$. This gives calcium an outer-shell electron configuration 
corresponding to that of beryllium and magnesium. $<$ !no-selfclose $\rightarrow>$

Beginning with the transition metal scandium (atomic number 21), additional electrons are added successively to the $3 d$ subshell. This subshell is filled to its capacity with 10 electrons (remember that for $l=2$ [ $d$ orbitals], there are $2 l+1=5$ values of $m_{1}$, meaning that there are five $d$ orbitals that have a combined capacity of 10 electrons). The $4 p$ subshell fills next. Note that for three series of elements, scandium $(\mathrm{Sc})$ through copper $(\mathrm{Cu})$, yttrium $(\mathrm{Y})$ through silver $(\mathrm{Ag})$, and lutetium $(\mathrm{Lu})$ through gold $(\mathrm{Au})$, a total of $10 d$ electrons are successively added to the $(n-$ 1) shell next to the $n$ shell to bring that $(n-1)$ shell from 8 to 18 electrons. For two series, lanthanum (La) through lutetium (Lu) and actinium (Ac) through lawrencium (Lr), $14 f$ electrons $\left(l=3,2 l+1=7 m_{l}\right.$ values; thus, seven orbitals with a combined capacity of 14 electrons) are successively added to the $(n-2)$ shell to bring that shell from 18 electrons to a total of 32 electrons.

Quantum Numbers and Electron Configurations What is the electron configuration and orbital diagram for a phosphorus atom? What are the four quantum numbers for the last electron added?

Solution The atomic number of phosphorus is 15 . Thus, a phosphorus atom contains 15 electrons. The order of filling of the energy levels is $1 s, 2 s, 2 p, 3 s, 3 p, 4 s, \ldots$ The 15 electrons of the phosphorus atom will fill up to the $3 p$ orbital, which will contain three electrons:

P $\quad 1 s^{2} 2 s^{2} 2 p^{6} 3 s^{2} 3 p^{3}$
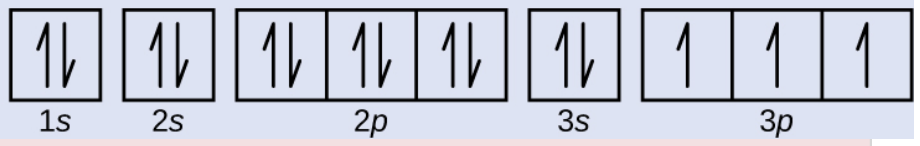
The last electron added is a $3 p$ electron. Therefore, $n=3$ and, for a $p$-type orbital, $l=1$. The $m$ l value could be $-1,0$, or +1 . The three $p$ orbitals are degenerate, so any of these $m$ i values is correct. For unpaired electrons, convention assigns the value of $+\frac{1}{2}$ for the spin quantum number; thus, $m_{s}=+\frac{1}{2}$.

Check Your Learning Identify the atoms from the electron configurations given:

(a) $[\mathrm{Ar}] 4 s^{2} 3 d^{5}$

(b) $[\mathrm{Kr}] 5 s^{2} 4 d^{10} 5 p^{6}$

Answer:

(a) $\mathrm{Mn}$ (b) $\mathrm{Xe}$

The periodic table can be a powerful tool in predicting the electron configuration of an element. However, we do find exceptions to the order of filling of orbitals that are shown in (Figure) or (Figure). For instance, the electron configurations (shown in (Figure)) of the transition metals chromium (Cr; atomic number 24$)$ and copper $(\mathrm{Cu}$; atomic number 29), among others, are not those we would expect. In general, such exceptions involve subshells with very similar energy, and small effects can lead to changes in the order of filling.

In the case of $\mathrm{Cr}$ and $\mathrm{Cu}$, we find that half-filled and completely filled subshells apparently represent conditions of preferred stability. This stability is such that an electron shifts from the $4 s$ into the $3 d$ orbital to gain the extra stability of a half-filled $3 d$ subshell (in $\mathrm{Cr}$ ) or a filled $3 d$ subshell (in $\mathrm{Cu}$ ). Other exceptions also occur. For example, niobium (Nb, atomic number 41$)$ is predicted to have the electron configuration $[\mathrm{Kr}] 5 s^{2} 4 d^{3}$. Experimentally, we observe that its ground-state electron 
configuration is actually $[\mathrm{Kr}] 5 s^{1} 4 d^{4}$. We can rationalize this observation by saying that the electron-electron repulsions experienced by pairing the electrons in the $5 \mathrm{~s}$ orbital are larger than the gap in energy between the $5 \mathrm{~s}$ and $4 d$ orbitals. There is no simple method to predict the exceptions for atoms where the magnitude of the repulsions between electrons is greater than the small differences in energy between subshells.

\section{Electron Configurations and the Periodic Table}

As described earlier, the periodic table arranges atoms based on increasing atomic number so that elements with the same chemical properties recur periodically. When their electron configurations are added to the table ((Figure)), we also see a periodic recurrence of similar electron configurations in the outer shells of these elements. Because they are in the outer shells of an atom, valence electrons play the most important role in chemical reactions. The outer electrons have the highest energy of the electrons in an atom and are more easily lost or shared than the core electrons. Valence electrons are also the determining factor in some physical properties of the elements.

Elements in any one group (or column) have the same number of valence electrons; the alkali metals lithium and sodium each have only one valence electron, the alkaline earth metals beryllium and magnesium each have two, and the halogens fluorine and chlorine each have seven valence electrons. The similarity in chemical properties among elements of the same group occurs because they have the same number of valence electrons. It is the loss, 
gain, or sharing of valence electrons that defines how elements react.

It is important to remember that the periodic table was developed on the basis of the chemical behavior of the elements, well before any idea of their atomic structure was available. Now we can understand why the periodic table has the arrangement it has-the arrangement puts elements whose atoms have the same number of valence electrons in the same group. This arrangement is emphasized in (Figure), which shows in periodic-table form the electron configuration of the last subshell to be filled by the Aufbau principle. The colored sections of (Figure) show the three categories of elements classified by the orbitals being filled: main group, transition, and inner transition elements. These classifications determine which orbitals are counted in the valence shell, or highest energy level orbitals of an atom.

1. Main group elements (sometimes called representative elements) are those in which the last electron added enters an $s$ or a $p$ orbital in the outermost shell, shown in blue and red in (Figure). This category includes all the nonmetallic elements, as well as many metals and the metalloids. The valence electrons for main group elements are those with the highest $n$ level. For example, gallium (Ga, atomic number 31) has the electron configuration $[\mathrm{Ar}] \underline{4 s^{2}} 3 d^{10} \underline{4 p^{1}}$, which contains three valence electrons (underlined). The completely filled $d$ orbitals count as core, not valence, electrons.

2. Transition elements or transition metals. 
These are metallic elements in which the last electron added enters a $d$ orbital. The valence electrons (those added after the last noble gas configuration) in these elements include the $n s$ and $(n-1) d$ electrons. The official IUPAC definition of transition elements specifies those with partially filled $d$ orbitals. Thus, the elements with completely filled orbitals ( $\mathrm{Zn}, \mathrm{Cd}$, $\mathrm{Hg}$, as well as $\mathrm{Cu}, \mathrm{Ag}$, and $\mathrm{Au}$ in (Figure)) are not technically transition elements. However, the term is frequently used to refer to the entire $d$ block (colored yellow in (Figure)), and we will adopt this usage in this textbook.

3. Inner transition elements are metallic elements in which the last electron added occupies an $f$ orbital. They are shown in green in (Figure). The valence shells of the inner transition elements consist of the $(n-2) f$, the $(n$ $-1) d$, and the $n s$ subshells. There are two inner transition series:

a. The lanthanide series: lanthanide (La) through lutetium $(\mathrm{Lu})$

b. The actinide series: actinide (Ac) through lawrencium (Lr)

Lanthanum and actinium, because of their similarities to the other members of the series, are included and used to name the series, even though they are transition metals with no $f$ electrons. 
Electron Configurations of Ions

Ions are formed when atoms gain or lose electrons. A cation (positively charged ion) forms when one or more electrons are removed from a parent atom. For main group elements, the electrons that were added last are the first electrons removed. For transition metals and inner transition metals, however, electrons in the $s$ orbital are easier to remove than the $d$ or $f$ electrons, and so the highest $n s$ electrons are lost, and then the ( $n$ $1) d$ or $(n-2) f$ electrons are removed. An anion (negatively charged ion) forms when one or more electrons are added to a parent atom. The added electrons fill in the order predicted by the Aufbau principle.

Predicting Electron Configurations of lonsWhat is the electron configuration of:

(a) $\mathrm{Na}^{+}$

(b) $\mathrm{P}^{3-}$

(c) $\mathrm{Al}^{2+}$

(d) $\mathrm{Fe}^{2+}$

(e) $\mathrm{Sm}^{3+}$

Solution First, write out the electron configuration for each parent atom. We have chosen to show the full, unabbreviated configurations to provide more practice for students who want it, but listing the core-abbreviated electron configurations is also acceptable.

Next, determine whether an electron is gained or lost. Remember electrons are negatively charged, so ions with a positive charge have lost an electron. For main group elements, the last orbital gains or loses the electron. For 
transition metals, the last $s$ orbital loses an electron before the $d$ orbitals.

(a) Na: $1 s^{2} 2 s^{2} 2 p^{6} 3 s^{1}$. Sodium cation loses one electron, so $\mathrm{Na}^{+}: 1 s^{2} 2 s^{2} 2 p^{6} 3 s^{1}=\mathrm{Na}^{+}: 1 s^{2} 2 s^{2} 2 p^{6}$.

(b) P: $1 s^{2} 2 s^{2} 2 p^{6} 3 s^{2} 3 p^{3}$. Phosphorus trianion gains three electrons, so $\mathrm{P}^{3-}: 1 s^{2} 2 s^{2} 2 p^{6} 3 s^{2} 3 p^{6}$.

(c) Al: $1 s^{2} 2 s^{2} 2 p^{6} 3 s^{2} 3 p^{1}$. Aluminum dication loses two electrons $\mathrm{Al}^{2+}: 1 s^{2} 2 s^{2} 2 p^{6} 3 s^{2} 3 p^{1}=$ $\mathrm{Al}^{2+}: 1 s^{2} 2 s^{2} 2 p^{6} 3 s^{1}$.

(d) Fe: $1 s^{2} 2 s^{2} 2 p^{6} 3 s^{2} 3 p^{6} 4 s^{2} 3 d^{6}$. Iron(II) loses two electrons and, since it is a transition metal, they are removed from the $4 s$ orbital $\mathrm{Fe}^{2+}: 1 s^{2} 2 s^{2} 2 p^{6} 3 s^{2} 3 p^{6} 4 s^{2} 3 d^{6}=1 s^{2} 2 s^{2} 2 p^{6} 3 s^{2} 3 p^{6} 3 d^{6}$.

(e). $5 m: 1 s^{2} 2 s^{2} 2 p^{6} 3 s^{2} 3 p^{6} 4 s^{2} 3 d^{10} 4 p^{6} 5 s^{2} 4 d^{10} 5 p^{6} 6 s^{2} 4 f^{6}$.

Samarium trication loses three electrons. The first two will be lost from the $6 s$ orbital, and the final one is removed from the $4 f$ orbital. $5 m^{3+}: 1 s^{2} 2 s^{2} 2 p^{6} 3 s^{2} 3 p^{6} 4 s^{2} 3 d^{10} 4 p^{6} 5 s^{2} 4 d^{10} 5 p^{6} 6 s^{2} 4 f^{6}$ $=1 s^{2} 2 s^{2} 2 p^{6} 3 s^{2} 3 p^{6} 4 s^{2} 3 d^{10} 4 p^{6} 5 s^{2} 4 d^{10} 5 p^{6} 4 f^{5}$.

Check Your Learning Which ion with $a+2$ charge has the electron configuration $1 s^{2} 2 s^{2} 2 p^{6} 3 s^{2} 3 p^{6} 3 d^{10} 4 s^{2} 4 p^{6} 4 d^{5}$ ? Which ion with $a+3$ charge has this configuration?

Answer:

$\mathrm{Tc}^{2+}, \mathrm{Ru}^{3+}$

Key Concepts and Summary

The relative energy of the subshells determine the order in which atomic orbitals are filled $(1 s, 2 s, 2 p, 3 s, 3 p, 4 s$, $3 d, 4 p$, and so on). Electron configurations and orbital diagrams can be determined by applying the Pauli exclusion principle (no two electrons can have the same set of four quantum numbers) and Hund's rule (whenever 
possible, electrons retain unpaired spins in degenerate orbitals).

Electrons in the outermost orbitals, called valence electrons, are responsible for most of the chemical behavior of elements. In the periodic table, elements with analogous valence electron configurations usually occur within the same group. There are some exceptions to the predicted filling order, particularly when half-filled or completely filled orbitals can be formed. The periodic table can be divided into three categories based on the orbital in which the last electron to be added is placed: main group elements ( $s$ and $p$ orbitals), transition elements ( $d$ orbitals), and inner transition elements $(f$ orbitals).

\section{Chemistry End of Chapter Exercises}

Read the labels of several commercial products and identify monatomic ions of at least four transition elements contained in the products. Write the complete electron configurations of these cations.

Read the labels of several commercial products and identify monatomic ions of at least six main group elements contained in the products. Write the complete electron configurations of these cations and anions.

For example, $\mathrm{Na}^{+}: 1 s^{2} 2 s^{2} 2 p^{6} ; \mathrm{Ca}^{2+}: 1 s^{2} 2 s^{2} 2 p^{6} ; \mathrm{Sn}^{2+}$ : $1 s^{2} 2 s^{2} 2 p^{6} 3 s^{2} 3 p^{6} 3 d^{10} 4 s^{2} 4 p^{6} 4 d^{10} 5 s^{2} ; \mathrm{F}^{-}: 1 s^{2} 2 s^{2} 2 p^{6} ; \mathrm{O}^{2-}$ : $1 s^{2} 2 s^{2} 2 p^{6} ; \mathrm{Cl}^{-}: 1 s^{2} 2 s^{2} 2 p^{6} 3 s^{2} 3 p^{6}$.

Using complete subshell notation (not abbreviations, 
$1 s^{2} 2 s^{2} 2 p^{6}$, and so forth), predict the electron configuration of each of the following atoms:
(a) $\mathrm{C}$
(b) $\mathrm{P}$
(c) $\mathrm{V}$
(d) $\mathrm{Sb}$
(e) $\mathrm{Sm}$

Using complete subshell notation $\left(1 s^{2} 2 s^{2} 2 p^{6}\right.$, and so forth), predict the electron configuration of each of the following atoms:
(a) $\mathrm{N}$
(b) $\mathrm{Si}$
(c) $\mathrm{Fe}$
(d) $\mathrm{Te}$
(e) $\mathrm{Tb}$

(a) $\quad 1 s^{2} 2 s^{2} 2 p^{3}$;

$1 s^{2} 2 s^{2} 2 p^{6} 3 s^{2} 3 p^{6} 4 s^{2} 3 d^{6}$;

(b) $\quad 1 s^{2} 2 s^{2} 2 p^{6} 3 s^{2} 3 p^{2}$; $1 s^{2} 2 s^{2} 2 p^{6} 3 s^{2} 3 p^{6} 4 s^{2} 3 d^{10} 4 p^{6} 5 s^{2} 4 d^{10} 5 p^{4}$; $1 s^{2} 2 s^{2} 2 p^{6} 3 s^{2} 3 p^{6} 4 s^{2} 3 d^{10} 4 p^{6} 5 s^{2} 4 d^{10} 5 p^{6} 6 s^{2} 4 f^{9}$

Is $1 s^{2} 2 s^{2} 2 p^{6}$ the symbol for a macroscopic property or a microscopic property of an element? Explain your answer.

What additional information do we need to answer the question "Which ion has the electron configuration $1 s^{2} 2 s^{2} 2 p^{6} 3 s^{2} 3 p^{6}$ ??

The charge on the ion.

Draw the orbital diagram for the valence shell of each of the following atoms: 
(a) $\mathrm{C}$

(b) $\mathrm{P}$

(c) $\mathrm{V}$

(d) $\mathrm{Sb}$

(e) $\mathrm{Ru}$

Use an orbital diagram to describe the electron configuration of the valence shell of each of the following atoms:
(a) $\mathrm{N}$
(b) $\mathrm{Si}$
(c) $\mathrm{Fe}$
(d) $\mathrm{Te}$
(e) Mo

(a)

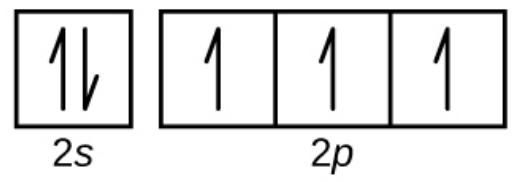

(b)

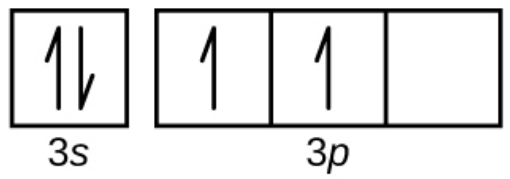

(c)

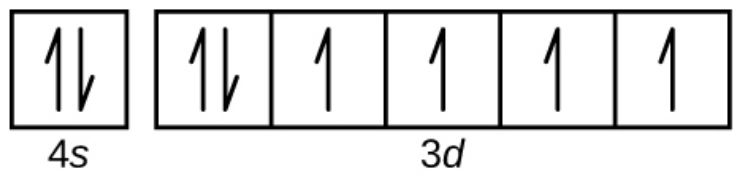

(d) 


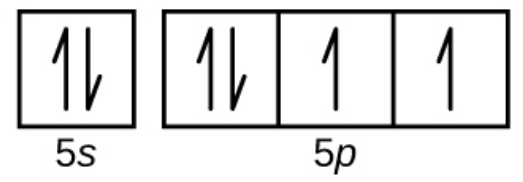

(e)

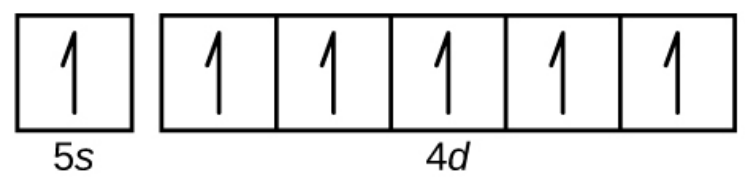

Using complete subshell notation $\left(1 s^{2} 2 s^{2} 2 p^{6}\right.$, and so forth), predict the electron configurations of the following ions.
(a) $\mathrm{N}^{3-}$
(b) $\mathrm{Ca}^{2+}$
(c) $\mathrm{S}^{-}$
(d) $\mathrm{Cs}^{2+}$
(e) $\mathrm{Cr}^{2+}$
(f) $\mathrm{Gd}^{3+}$

Which atom has the electron configuration $1 s^{2} 2 s^{2} 2 p^{6} 3 s^{2} 3 p^{6} 4 s^{2} 3 d^{10} 4 p^{6} 5 s^{2} 4 d^{2}$ ?

$\mathrm{Zr}$

Which atom has the electron configuration $1 s^{2} 2 s^{2} 2 p^{6} 3 s^{2} 3 p^{6} 3 d^{7} 4 s^{2}$ ?

Which ion with a +1 charge has the electron configuration $1 s^{2} 2 s^{2} 2 p^{6} 3 s^{2} 3 p^{6} 3 d^{10} 4 s^{2} 4 p^{6}$ ? Which ion with a -2 charge has this configuration?

$\mathrm{Rb}^{+}, \mathrm{Se}^{2-}$ 
Which of the following atoms contains only three valence electrons: Li, B, N, F, Ne?

Which of the following has two unpaired electrons?
(a) $\mathrm{Mg}$
(b) $\mathrm{Si}$
(c) $\mathrm{S}$
(d) Both Mg and S
(e) Both Si and S.

Although both (b) and (c) are correct, (e) encompasses both and is the best answer.

Which atom would be expected to have a half-filled $6 p$ subshell?

Which atom would be expected to have a half-filled $4 \mathrm{~s}$ subshell?

K

In one area of Australia, the cattle did not thrive despite the presence of suitable forage. An investigation showed the cause to be the absence of sufficient cobalt in the soil. Cobalt forms cations in two oxidation states, $\mathrm{Co}^{2+}$ and $\mathrm{Co}^{3+}$. Write the electron structure of the two cations.

Thallium was used as a poison in the Agatha Christie mystery story "The Pale Horse." Thallium has two possible cationic forms, +1 and +3 . The +1 compounds are the more stable. Write the electron structure of the +1 cation of thallium. 


$$
1 s^{2} 2 s^{2} 2 p^{6} 3 s^{2} 3 p^{6} 3 d^{10} 4 s^{2} 4 p^{6} 4 d^{10} 5 s^{2} 5 p^{6} 6 s^{2} 4 f^{14} 5 d^{10}
$$

Write the electron configurations for the following atoms or ions:
(a) $\mathrm{B}^{3+}$
(b) $\mathrm{O}^{-}$
(c) $\mathrm{Cl}^{3+}$
(d) $\mathrm{Ca}^{2+}$
(e) $\mathrm{Ti}$

Cobalt-60 and iodine-131 are radioactive isotopes commonly used in nuclear medicine. How many protons, neutrons, and electrons are in atoms of these isotopes? Write the complete electron configuration for each isotope.

Co has 27 protons, 27 electrons, and 33 neutrons: $1 s^{2} 2 s^{2} 2 p^{6} 3 s^{2} 3 p^{6} 4 s^{2} 3 d^{7}$. I has 53 protons, 53 electrons, and 78 neutrons: $1 s^{2} 2 s^{2} 2 p^{6} 3 s^{2} 3 p^{6} 3 d^{10} 4 s^{2} 4 p^{6} 4 d^{10} 5 s^{2} 5 p^{5}$.

Write a set of quantum numbers for each of the electrons with an $n$ of 3 in a Sc atom.

Glossary

\section{Aufbau principle}

procedure in which the electron configuration of the elements is determined by "building" them in order of atomic numbers, adding one proton to the nucleus 
and one electron to the proper subshell at a time

core electron

electron in an atom that occupies the orbitals of the inner shells

electron configuration

listing that identifies the electron occupancy of an atom's shells and subshells

Hund's rule

every orbital in a subshell is singly occupied with one electron before any one orbital is doubly occupied, and all electrons in singly occupied orbitals have the same spin orbital diagram pictorial representation of the electron configuration showing each orbital as a box and each electron as an arrow valence electrons electrons in the outermost or valence shell (highest value of $n$ ) of a ground-state atom valence shell

outermost shell of electrons in a groundstate atom 

PART VII

\section{CHEMICAL BONDING AND MOLECULAR GEOMETRY}





\section{1.}

\section{INTRODUCTION}

\section{Learning Objectives}

- $\quad$ lonic Bonding

- Covalent Bonding

- Lewis Symbols and Structures

- Formal Charges and Resonance

- $\quad$ Strengths of Ionic and Covalent Bonds

- Molecular Structure and Polarity

Nicknamed "buckyballs," buckminsterfullerene molecules $\left(\mathrm{C}_{60}\right)$ contain only carbon atoms (left) arranged to form a geometric framework of hexagons and pentagons, similar to the pattern on a soccer ball (center). This molecular structure is named after architect R. Buckminster Fuller, whose innovative designs combined simple geometric shapes to create large, strong structures such as this weather radar dome near Tucson, Arizona (right). (credit 
middle: modification of work by "Petey21"/Wikimedia Commons; credit right: modification of work by Bill Morrow)
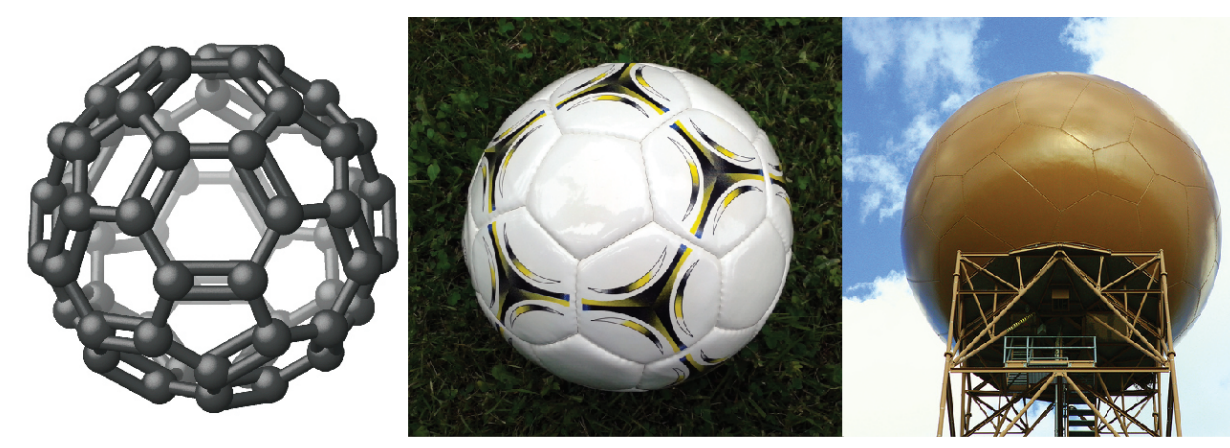

It has long been known that pure carbon occurs in different forms (allotropes) including graphite and diamonds. But it was not until 1985 that a new form of carbon was recognized: buckminsterfullerene. This molecule was named after the architect and inventor R. Buckminster Fuller (1895-1983), whose signature architectural design was the geodesic dome, characterized by a lattice shell structure supporting a spherical surface. Experimental evidence revealed the formula, $\mathrm{C}_{60}$, and then scientists determined how 60 carbon atoms could form one symmetric, stable molecule. They were guided by bonding theory-the topic of this chapter-which explains how individual atoms connect to form more complex structures. 


\section{IONIC BONDING}

Learning Objectives

By the end of this section, you will be able to:

- Explain the formation of cations, anions, and ionic compounds

- $\quad$ Predict the charge of common metallic and nonmetallic elements, and write their electron configurations

As you have learned, ions are atoms or molecules bearing an electrical charge. A cation (a positive ion) forms when a neutral atom loses one or more electrons from its valence shell, and an anion (a negative ion) forms when a neutral atom gains one or more electrons in its valence shell.

Compounds composed of ions are called ionic compounds (or salts), and their constituent ions are held together by ionic bonds: electrostatic forces of attraction between oppositely charged cations and anions. The 
properties of ionic compounds shed some light on the nature of ionic bonds. Ionic solids exhibit a crystalline structure and tend to be rigid and brittle; they also tend to have high melting and boiling points, which suggests that ionic bonds are very strong. Ionic solids are also poor conductors of electricity for the same reason-the strength of ionic bonds prevents ions from moving freely in the solid state. Most ionic solids, however, dissolve readily in water. Once dissolved or melted, ionic compounds are excellent conductors of electricity and heat because the ions can move about freely.

Neutral atoms and their associated ions have very different physical and chemical properties. Sodium atoms form sodium metal, a soft, silvery-white metal that burns vigorously in air and reacts explosively with water. Chlorine atoms form chlorine gas, $\mathrm{Cl}_{2}$, a yellow-green gas that is extremely corrosive to most metals and very poisonous to animals and plants. The vigorous reaction between the elements sodium and chlorine forms the white, crystalline compound sodium chloride, common table salt, which contains sodium cations and chloride anions ((Figure)). The compound composed of these ions exhibits properties entirely different from the properties of the elements sodium and chlorine. Chlorine is poisonous, but sodium chloride is essential to life; sodium atoms react vigorously with water, but sodium chloride simply dissolves in water.

(a) Sodium is a soft metal that must be stored in mineral oil to prevent reaction with air or water. (b) Chlorine is a pale yellow-green gas. (c) When combined, they form white crystals of sodium chloride (table salt). (credit a: modification of work by “Jurii”/Wikimedia Commons) 


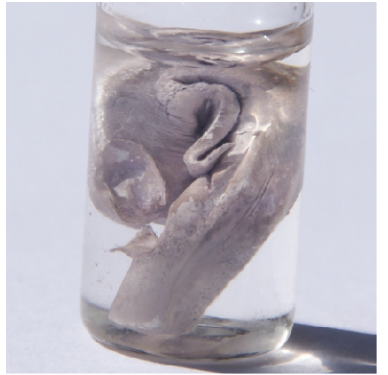

(a)

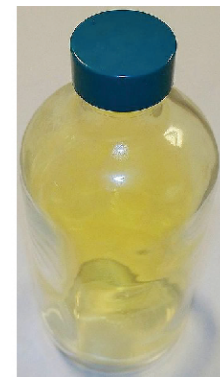

(b)

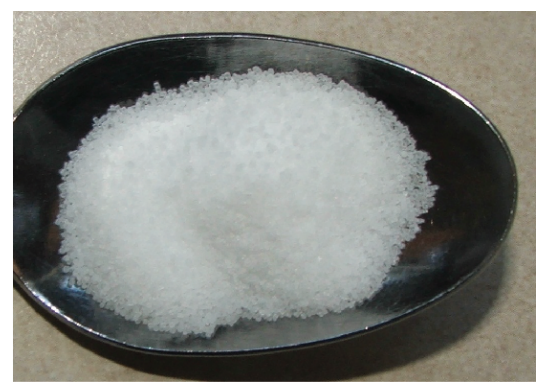

(c)

The Formation of Ionic Compounds

Binary ionic compounds are composed of just two elements: a metal (which forms the cations) and a nonmetal (which forms the anions). For example, $\mathrm{NaCl}$ is a binary ionic compound. We can think about the formation of such compounds in terms of the periodic properties of the elements. Many metallic elements have relatively low ionization potentials and lose electrons easily. These elements lie to the left in a period or near the bottom of a group on the periodic table. Nonmetal atoms have relatively high electron affinities and thus readily gain electrons lost by metal atoms, thereby filling their valence shells. Nonmetallic elements are found in the upper-right corner of the periodic table.

As all substances must be electrically neutral, the total number of positive charges on the cations of an ionic compound must equal the total number of negative charges on its anions. The formula of an ionic compound represents the simplest ratio of the numbers of ions necessary to give identical numbers of positive and negative charges. For example, the formula for aluminum oxide, $\mathrm{Al}_{2} \mathrm{O}_{3}$, indicates that this ionic compound contains two aluminum cations, $\mathrm{Al}^{3+}$, for every three oxide anions, $\mathrm{O}^{2-}$ [thus, $(2+3)+(3-2)=0$ ]. 
It is important to note, however, that the formula for an ionic compound does not represent the physical arrangement of its ions. It is incorrect to refer to a sodium chloride $(\mathrm{NaCl})$ "molecule" because there is not a single ionic bond, per se, between any specific pair of sodium and chloride ions. The attractive forces between ions are isotropic-the same in all directions-meaning that any particular ion is equally attracted to all of the nearby ions of opposite charge. This results in the ions arranging themselves into a tightly bound, three-dimensional lattice structure. Sodium chloride, for example, consists of a regular arrangement of equal numbers of $\mathrm{Na}^{+}$cations and $\mathrm{Cl}^{-}$anions ((Figure)).

The atoms in sodium chloride (common table salt) are arranged to (a) maximize opposite charges interacting. The smaller spheres represent sodium ions, the larger ones represent chloride ions. In the expanded view (b), the geometry can be seen more clearly. Note that each ion is "bonded" to all of the surrounding ions-six in this case.

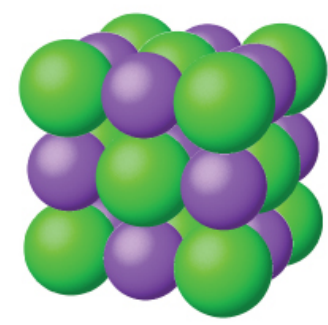

(a)

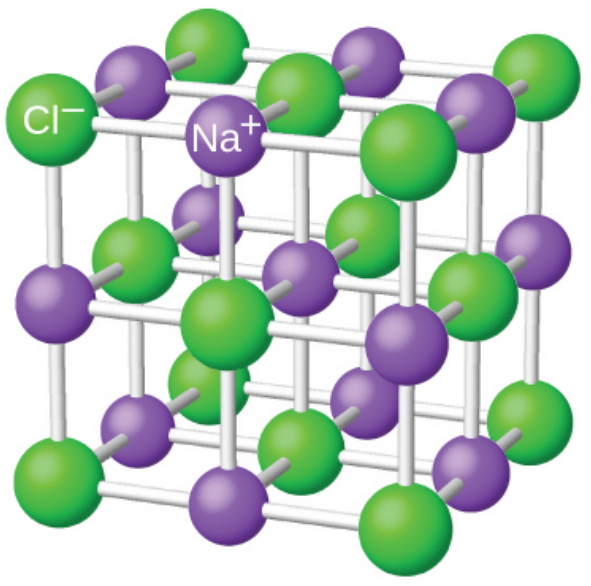

(b) 
The strong electrostatic attraction between $\mathrm{Na}^{+}$and $\mathrm{Cl}^{-}$ ions holds them tightly together in solid $\mathrm{NaCl}$. It requires $769 \mathrm{~kJ}$ of energy to dissociate one mole of solid $\mathrm{NaCl}$ into separate gaseous $\mathrm{Na}^{+}$and $\mathrm{Cl}^{-}$ions:

$\mathrm{NaCl}(s) \mathrm{Na}^{+}(g)+\mathrm{Cl}^{-}(g) \quad H=769 \mathrm{~kJ}$

Electronic Structures of Cations

When forming a cation, an atom of a main group element tends to lose all of its valence electrons, thus assuming the electronic structure of the noble gas that precedes it in the periodic table. For groups 1 (the alkali metals) and 2 (the alkaline earth metals), the group numbers are equal to the numbers of valence shell electrons and, consequently, to the charges of the cations formed from atoms of these elements when all valence shell electrons are removed. For example, calcium is a group 2 element whose neutral atoms have 20 electrons and a ground state electron configuration of $1 s^{2} 2 s^{2} 2 p^{6} 3 s^{2} 3 p^{6} 4 s^{2}$. When a Ca atom loses both of its valence electrons, the result is a cation with 18 electrons, a $2+$ charge, and an electron configuration of $1 s^{2} 2 s^{2} 2 p^{6} 3 s^{2} 3 p^{6}$. The $\mathrm{Ca}^{2+}$ ion is therefore isoelectronic with the noble gas Ar.

For groups 13-17, the group numbers exceed the number of valence electrons by 10 (accounting for the possibility of full $d$ subshells in atoms of elements in the fourth and greater periods). Thus, the charge of a cation formed by the loss of all valence electrons is equal to the group number minus 10 . For example, aluminum (in group 13) forms $3+$ ions $\left(\mathrm{Al}^{3+}\right)$.

Exceptions to the expected behavior involve elements toward the bottom of the groups. In addition to the 
expected ions $\mathrm{Tl}^{3+}, \mathrm{Sn}^{4+}, \mathrm{Pb}^{4+}$, and $\mathrm{Bi}^{5+}$, a partial loss of these atoms' valence shell electrons can also lead to the formation of $\mathrm{Tl}^{+}, \mathrm{Sn}^{2+}, \mathrm{Pb}^{2+}$, and $\mathrm{Bi}^{3+}$ ions. The formation of these $1+, 2+$, and $3+$ cations is ascribed to the inert pair effect, which reflects the relatively low energy of the valence $s$-electron pair for atoms of the heavy elements of groups 13, 14, and 15. Mercury (group 12) also exhibits an unexpected behavior: it forms a diatomic ion, $\mathrm{Hg}_{2}{ }^{2+}$ (an ion formed from two mercury atoms, with an $\mathrm{Hg}-\mathrm{Hg}$ bond), in addition to the expected monatomic ion $\mathrm{Hg}^{2+}$ (formed from only one mercury atom).

Transition and inner transition metal elements behave differently than main group elements. Most transition metal cations have $2+$ or $3+$ charges that result from the loss of their outermost $s$ electron(s) first, sometimes followed by the loss of one or two $d$ electrons from the next-to-outermost shell. For example, iron $\left(1 s^{2} 2 s^{2} 2 p^{6} 3 s^{2} 3 p^{6} 3 d^{6} 4 s^{2}\right)$ forms the ion $\mathrm{Fe}^{2+}$ $\left(1 s^{2} 2 s^{2} 2 p^{6} 3 s^{2} 3 p^{6} 3 d^{6}\right)$ by the loss of the $4 s$ electron and the ion $\mathrm{Fe}^{3+}\left(1 s^{2} 2 s^{2} 2 p^{6} 3 s^{2} 3 p^{6} 3 d^{5}\right)$ by the loss of the $4 s$ electron and one of the $3 d$ electrons. Although the $d$ orbitals of the transition elements are-according to the Aufbau principle-the last to fill when building up electron configurations, the outermost $s$ electrons are the first to be lost when these atoms ionize. When the inner transition metals form ions, they usually have a 3+ charge, resulting from the loss of their outermost $s$ electrons and a $d$ or $f$ electron.

Determining the Electronic Structures of Cations There are at least 14 elements categorized as "essential trace elements" 
for the human body. They are called "essential" because they are required for healthy bodily functions, "trace" because they are required only in small amounts, and "elements" in spite of the fact that they are really ions. Two of these essential trace elements, chromium and zinc, are required as $\mathrm{Cr}^{3+}$ and $\mathrm{Zn}^{2+}$. Write the electron configurations of these cations.

Solution First, write the electron configuration for the neutral atoms:

$\mathrm{Zn:}[\mathrm{Ar}] 3 d^{10} 4 \mathrm{~s}^{2}$

Cr: $[\operatorname{Ar}] 3 d^{5} 4 s^{1}$

Next, remove electrons from the highest energy orbital. For the transition metals, electrons are removed from the s orbital first and then from the $d$ orbital. For the $p$-block elements, electrons are removed from the $p$ orbitals and then from the $s$ orbital. Zinc is a member of group 12, so it should have a charge of $2+$, and thus loses only the two electrons in its 5 orbital. Chromium is a transition element and should lose its 5 electrons and then its $d$ electrons when forming a cation. Thus, we find the following electron configurations of the ions:

$$
\begin{aligned}
& \mathrm{Zn}^{2+}:[\mathrm{Ar}] 3 d^{10} \\
& \mathrm{Cr}^{3+}:[\mathrm{Ar}] 3 d^{3}
\end{aligned}
$$

Check Your Learning Potassium and magnesium are required in our diet. Write the electron configurations of the ions expected from these elements.

Answer:

$\mathrm{K}^{+}:[\mathrm{Ar}], \mathrm{Mg}^{2+}:[\mathrm{Ne}]$

Electronic Structures of Anions

Most monatomic anions form when a neutral nonmetal atom gains enough electrons to completely fill its outer $s$ 
and $p$ orbitals, thereby reaching the electron configuration of the next noble gas. Thus, it is simple to determine the charge on such a negative ion: The charge is equal to the number of electrons that must be gained to fill the $s$ and $p$ orbitals of the parent atom. Oxygen, for example, has the electron configuration $1 s^{2} 2 s^{2} 2 p^{4}$, whereas the oxygen anion has the electron configuration of the noble gas neon (Ne), $1 s^{2} 2 s^{2} 2 p^{6}$. The two additional electrons required to fill the valence orbitals give the oxide ion the charge of 2$\left(\mathrm{O}^{2-}\right)$.

\section{Determining the Electronic Structure of Anions Selenium} and iodine are two essential trace elements that form anions. Write the electron configurations of the anions.

Solution $\mathrm{Se}^{2-}:[\mathrm{Ar}] 3 d^{10} 4 s^{2} 4 p^{6}$

$\mathrm{I}^{-}:[\mathrm{Kr}] 4 d^{10} 5 s^{2} 5 p^{6}$

Check Your Learning Write the electron configurations of a phosphorus atom and its negative ion. Give the charge on the anion.

Answer:

$\mathrm{P}:[\mathrm{Ne}] 3 s^{2} 3 p^{3} ; \mathrm{P}^{3-}:[\mathrm{Ne}] 3 s^{2} 3 p^{6}$

Key Concepts and Summary

Atoms gain or lose electrons to form ions with particularly stable electron configurations. The charges of cations formed by the representative metals may be determined readily because, with few exceptions, the electronic structures of these ions have either a noble gas configuration or a completely filled electron shell. The charges of anions formed by the nonmetals may also be 
readily determined because these ions form when nonmetal atoms gain enough electrons to fill their valence shells.

Chemistry End of Chapter Exercises

Does a cation gain protons to form a positive charge or does it lose electrons?

The protons in the nucleus do not change during normal chemical reactions. Only the outer electrons move. Positive charges form when electrons are lost.

Iron(III) sulfate $\left[\mathrm{Fe}_{2}\left(\mathrm{SO}_{4}\right)_{3}\right]$ is composed of $\mathrm{Fe}^{3+}$ and $\mathrm{SO}_{4}{ }^{2}$ ions. Explain why a sample of iron(III) sulfate is uncharged.

Which of the following atoms would be expected to form negative ions in binary ionic compounds and which would be expected to form positive ions: $\mathrm{P}, \mathrm{I}, \mathrm{Mg}, \mathrm{Cl}, \mathrm{In}, \mathrm{Cs}, \mathrm{O}$, $\mathrm{Pb}, \mathrm{Co}$ ?

$\mathrm{P}, \mathrm{I}, \mathrm{Cl}$, and $\mathrm{O}$ would form anions because they are nonmetals. $\mathrm{Mg}$, In, $\mathrm{Cs}, \mathrm{Pb}$, and $\mathrm{Co}$ would form cations because they are metals.

Which of the following atoms would be expected to form negative ions in binary ionic compounds and which would be expected to form positive ions: $\mathrm{Br}, \mathrm{Ca}, \mathrm{Na}, \mathrm{N}, \mathrm{F}, \mathrm{Al}, \mathrm{Sn}$, $\mathrm{S}, \mathrm{Cd}$ ?

Predict the charge on the monatomic ions formed from the following atoms in binary ionic compounds: 

(a) $\mathrm{P}$
(b) $\mathrm{Mg}$
(c) $\mathrm{Al}$
(d) $\mathrm{O}$
(e) $\mathrm{Cl}$
(f) $\mathrm{Cs}$

(a) $\mathrm{P}^{3-}$; (b) $\mathrm{Mg}^{2+}$; (c) $\mathrm{Al}^{3+}$; (d) $\mathrm{O}^{2-}$; (e) $\mathrm{Cl}^{-}$; (f) $\mathrm{Cs}^{+}$

Predict the charge on the monatomic ions formed from the following atoms in binary ionic compounds:
(a) I
(b) $\mathrm{Sr}$
(c) $\mathrm{K}$
(d) $\mathrm{N}$
(e) $\mathrm{S}$
(f) In

Write the electron configuration for each of the following ions:
(a) $\mathrm{As}^{3-}$
(b) $\mathrm{I}^{-}$
(c) $\mathrm{Be}^{2+}$
(d) $\mathrm{Cd}^{2+}$
(e) $\mathrm{O}^{2-}$
(f) $\mathrm{Ga}^{3+}$
(g) $\mathrm{Li}^{+}$
(h) $\mathrm{N}^{3-}$
(i) $\mathrm{Sn}^{2+}$
(j) $\mathrm{Co}^{2+}$
(k) $\mathrm{Fe}^{2+}$
(l) $\mathrm{As}^{3+}$ 
(a) $[\mathrm{Ar}] 4 s^{2} 3 d^{10} 4 p^{6}$; (b) $[\mathrm{Kr}] 4 d^{10} 5 s^{2} 5 p^{6}$ (c) $1 s^{2}$ (d) $[\mathrm{Kr}] 4 d^{10}$;

(e) $[\mathrm{He}] 2 s^{2} 2 p^{6}$; (f) $[\mathrm{Ar}] 3 d^{10}$; (g) $1 s^{2}$ (h) $[\mathrm{He}] 2 s^{2} 2 p^{6}$ (i) $[\mathrm{Kr}] 4 d^{10} 5 s^{2}$ (j) $[\mathrm{Ar}] 3 d^{7}$ (k) $[\mathrm{Ar}] 3 d^{6}$, (l) $[\mathrm{Ar}] 3 d^{10} 4 s^{2}$

Write the electron configuration for the monatomic ions formed from the following elements (which form the greatest concentration of monatomic ions in seawater):
(a) $\mathrm{Cl}$
(b) $\mathrm{Na}$
(c) $\mathrm{Mg}$
(d) $\mathrm{Ca}$
(e) $\mathrm{K}$
(f) $\mathrm{Br}$
(g) $\mathrm{Sr}$
(h) $\mathrm{F}$

Write out the full electron configuration for each of the following atoms and for the monatomic ion found in binary ionic compounds containing the element:
(a) $\mathrm{Al}$
(b) $\mathrm{Br}$
(c) $\mathrm{Sr}$
(d) $\mathrm{Li}$
(e) As
(f) $\mathrm{S}$

(a) $\quad 1 s^{2} 2 s^{2} 2 p^{6} 3 s^{2} 3 p^{1} ; \quad \mathrm{Al}^{3+}$ : $\quad 1 s^{2} 2 s^{2} 2 p^{6}$; $1 s^{2} 2 s^{2} 2 p^{6} 3 s^{2} 3 p^{6} 3 d^{10} 4 s^{2} 4 p^{5} ; \quad 1 s^{2} 2 s^{2} 2 p^{6} 3 s^{2} 3 p^{6} 3 d^{10} 4 s^{2} 4 p^{6}$; (c) $1 s^{2} 2 s^{2} 2 p^{6} 3 s^{2} 3 p^{6} 3 d^{10} 4 s^{2} 4 p^{6} 5 s^{2} ; \quad \quad \mathrm{Sr}^{2+}$ : $1 s^{2} 2 s^{2} 2 p^{6} 3 s^{2} 3 p^{6} 3 d^{10} 4 s^{2} 4 p^{6}$; (d) $1 s^{2} 2 s^{1} ; \quad \mathrm{Li}^{+}$: $1 s^{2}$; (e) $1 s^{2} 2 s^{2} 2 p^{6} 3 s^{2} 3 p^{6} 3 d^{10} 4 s^{2} 4 p^{3} ; \quad 1 s^{2} 2 s^{2} 2 p^{6} 3 s^{2} 3 p^{6} 3 d^{10} 4 s^{2} 4 p^{6}$; (f) $1 s^{2} 2 s^{2} 2 p^{6} 3 s^{2} 3 p^{4} ; 1 s^{2} 2 s^{2} 2 p^{6} 3 s^{2} 3 p^{6}$ 
From the labels of several commercial products, prepare a list of six ionic compounds in the products. For each compound, write the formula. (You may need to look up some formulas in a suitable reference.)

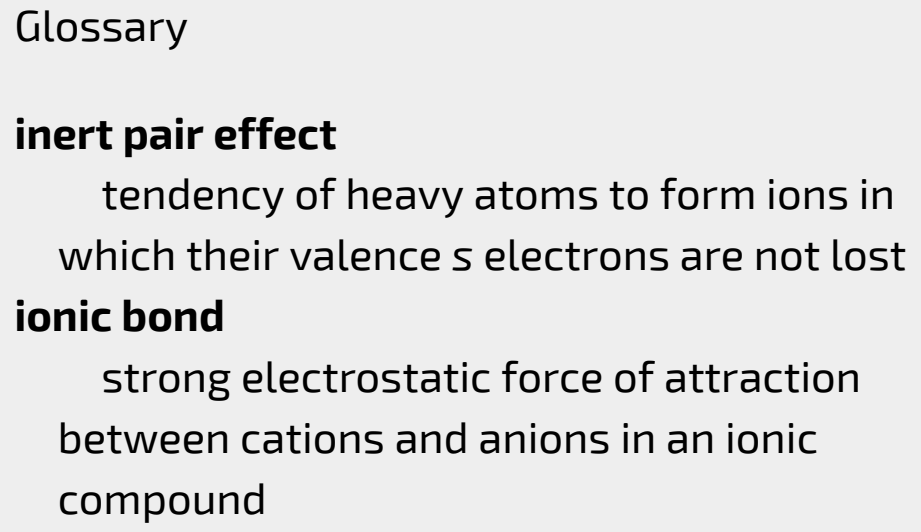


33.

\section{COVALENT BONDING}

\section{Learning Objectives}

By the end of this section, you will be able to:

- Describe the formation of covalent bonds

- Define electronegativity and assess the polarity of covalent bonds

Ionic bonding results from the electrostatic attraction of oppositely charged ions that are typically produced by the transfer of electrons between metallic and nonmetallic atoms. A different type of bonding results from the mutual attraction of atoms for a "shared" pair of electrons. Such bonds are called covalent bonds. Covalent bonds are formed between two atoms when both have similar tendencies to attract electrons to themselves (i.e., when both atoms have identical or fairly similar ionization energies and electron affinities). For example, two hydrogen atoms bond covalently to form an $\mathrm{H}_{2}$ molecule; 
each hydrogen atom in the $\mathrm{H}_{2}$ molecule has two electrons stabilizing it, giving each atom the same number of valence electrons as the noble gas He.

Compounds that contain covalent bonds exhibit different physical properties than ionic compounds. Because the attraction between molecules, which are electrically neutral, is weaker than that between electrically charged ions, covalent compounds generally have much lower melting and boiling points than ionic compounds. In fact, many covalent compounds are liquids or gases at room temperature, and, in their solid states, they are typically much softer than ionic solids. Furthermore, whereas ionic compounds are good conductors of electricity when dissolved in water, most covalent compounds are insoluble in water; since they are electrically neutral, they are poor conductors of electricity in any state.

Formation of Covalent Bonds

Nonmetal atoms frequently form covalent bonds with other nonmetal atoms. For example, the hydrogen molecule, $\mathrm{H}_{2}$, contains a covalent bond between its two hydrogen atoms. (Figure) illustrates why this bond is formed. Starting on the far right, we have two separate hydrogen atoms with a particular potential energy, indicated by the red line. Along the $x$-axis is the distance between the two atoms. As the two atoms approach each other (moving left along the $x$-axis), their valence orbitals (1s) begin to overlap. The single electrons on each hydrogen atom then interact with both atomic nuclei, occupying the space around both atoms. The strong 
attraction of each shared electron to both nuclei stabilizes the system, and the potential energy decreases as the bond distance decreases. If the atoms continue to approach each other, the positive charges in the two nuclei begin to repel each other, and the potential energy increases. The bond length is determined by the distance at which the lowest potential energy is achieved.

The potential energy of two separate hydrogen atoms (right) decreases as they approach each other, and the single electrons on each atom are shared to form a covalent bond. The bond length is the internuclear distance at which the lowest potential energy is achieved.

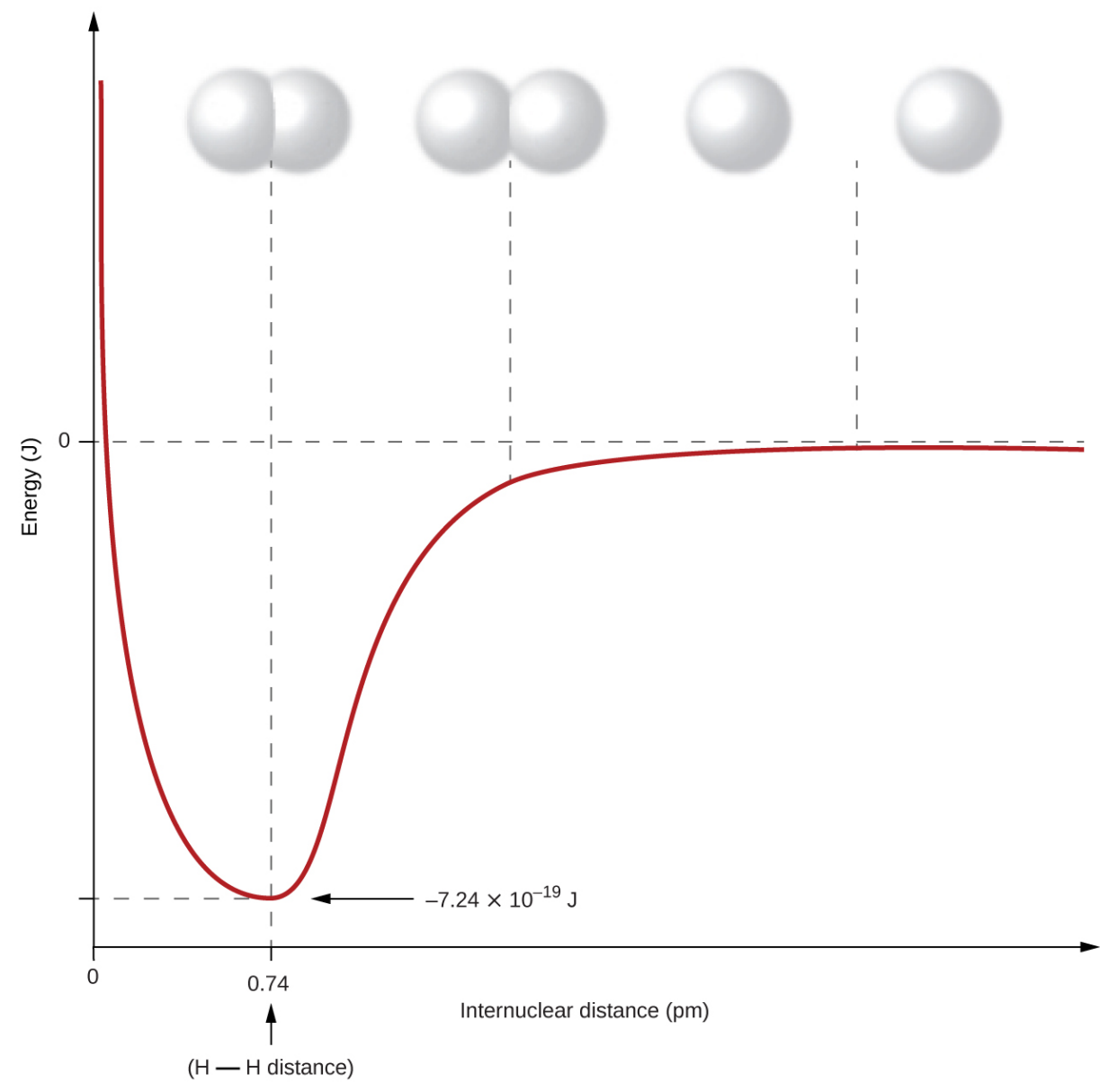


It is essential to remember that energy must be added to break chemical bonds (an endothermic process), whereas forming chemical bonds releases energy (an exothermic process). In the case of $\mathrm{H}_{2}$, the covalent bond is very strong; a large amount of energy, $436 \mathrm{~kJ}$, must be added to break the bonds in one mole of hydrogen molecules and cause the atoms to separate:
$\mathrm{H}_{2}(g) 2 \mathrm{H}(g)$
$H=436 \mathrm{~kJ}$

Conversely, the same amount of energy is released when one mole of $\mathrm{H}_{2}$ molecules forms from two moles of $\mathrm{H}$ atoms:

$2 \mathrm{H}(g) \mathrm{H}_{2}(g) \quad H=-436 \mathrm{~kJ}$

Pure vs. Polar Covalent Bonds

If the atoms that form a covalent bond are identical, as in $\mathrm{H}_{2}, \mathrm{Cl}_{2}$, and other diatomic molecules, then the electrons in the bond must be shared equally. We refer to this as a pure covalent bond. Electrons shared in pure covalent bonds have an equal probability of being near each nucleus.

In the case of $\mathrm{Cl}_{2}$, each atom starts off with seven valence electrons, and each $\mathrm{Cl}$ shares one electron with the other, forming one covalent bond:

$\mathrm{Cl}+\mathrm{Cl} \mathrm{Cl}_{2}$

The total number of electrons around each individual atom consists of six nonbonding electrons and two shared (i.e., bonding) electrons for eight total electrons, matching the number of valence electrons in the noble gas argon. Since the bonding atoms are identical, $\mathrm{Cl}_{2}$ also features a pure covalent bond. 
When the atoms linked by a covalent bond are different, the bonding electrons are shared, but no longer equally. Instead, the bonding electrons are more attracted to one atom than the other, giving rise to a shift of electron density toward that atom. This unequal distribution of electrons is known as a polar covalent bond, characterized by a partial positive charge on one atom and a partial negative charge on the other. The atom that attracts the electrons more strongly acquires the partial negative charge and vice versa. For example, the electrons in the $\mathrm{H}-\mathrm{Cl}$ bond of a hydrogen chloride molecule spend more time near the chlorine atom than near the hydrogen atom. Thus, in an $\mathrm{HCl}$ molecule, the chlorine atom carries a partial negative charge and the hydrogen atom has a partial positive charge. (Figure) shows the distribution of electrons in the $\mathrm{H}-\mathrm{Cl}$ bond. Note that the shaded area around $\mathrm{Cl}$ is much larger than it is around $\mathrm{H}$. Compare this to (Figure), which shows the even distribution of electrons in the $\mathrm{H}_{2}$ nonpolar bond.

We sometimes designate the positive and negative atoms in a polar covalent bond using a lowercase Greek letter "delta," $\delta$, with a plus sign or minus sign to indicate whether the atom has a partial positive charge $\left(\delta^{+}\right)$or a partial negative charge $(\delta-)$. This symbolism is shown for the $\mathrm{H}-\mathrm{Cl}$ molecule in (Figure).

(a) The distribution of electron density in the $\mathrm{HCl}$ molecule is uneven. The electron density is greater around the chlorine nucleus. The small, black dots indicate the location of the hydrogen and chlorine nuclei in the molecule. (b) Symbols $\delta+$ and $\delta$-indicate the polarity of the $\mathrm{H}-\mathrm{Cl}$ bond. 


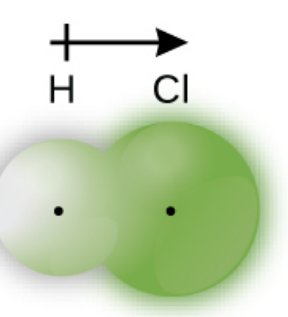

(a)

$$
\begin{aligned}
& \delta+\delta- \\
& \mathrm{H}-\mathrm{Cl}
\end{aligned}
$$

\section{Electronegativity}

Whether a bond is nonpolar or polar covalent is determined by a property of the bonding atoms called electronegativity. Electronegativity is a measure of the tendency of an atom to attract electrons (or electron density) towards itself. It determines how the shared electrons are distributed between the two atoms in a bond. The more strongly an atom attracts the electrons in its bonds, the larger its electronegativity. Electrons in a polar covalent bond are shifted toward the more electronegative atom; thus, the more electronegative atom is the one with the partial negative charge. The greater the difference in electronegativity, the more polarized the electron distribution and the larger the partial charges of the atoms.

(Figure) shows the electronegativity values of the elements as proposed by one of the most famous chemists of the twentieth century: Linus Pauling ((Figure)). In general, electronegativity increases from left to right across a period in the periodic table and decreases down a group. Thus, the nonmetals, which lie in the upper right, tend to have the highest electronegativities, with fluorine the most electronegative element of all $(\mathrm{EN}=4.0)$. Metals tend to be less electronegative elements, and the group 1 metals have the lowest electronegativities. Note that 
noble gases are excluded from this figure because these atoms usually do not share electrons with others atoms since they have a full valence shell. (While noble gas compounds such as $\mathrm{XeO}_{2}$ do exist, they can only be formed under extreme conditions, and thus they do not fit neatly into the general model of electronegativity.)

The electronegativity values derived by Pauling follow predictable periodic trends, with the higher electronegativities toward the upper right of the periodic table.

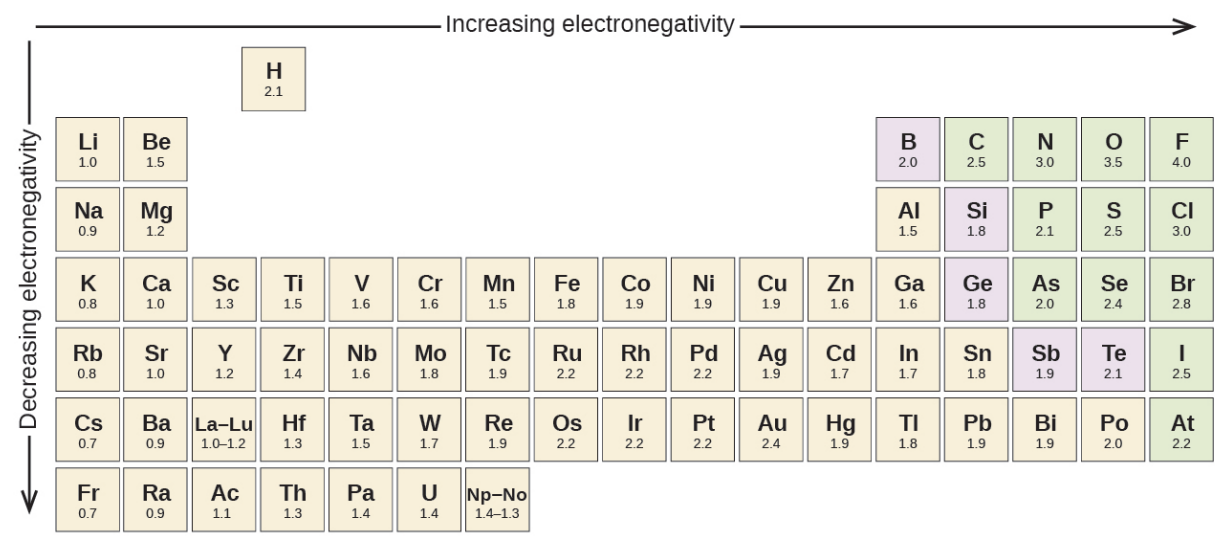

\section{Electronegativity versus Electron Affinity}

We must be careful not to confuse electronegativity and electron affinity. The electron affinity of an element is a measurable physical quantity, namely, the energy released or absorbed when an isolated gas-phase atom acquires an electron, measured in $\mathrm{kJ} / \mathrm{mol}$. Electronegativity, on the other hand, describes how tightly an atom attracts electrons in a bond. It is a dimensionless quantity that is calculated, not measured. Pauling derived the first electronegativity values by comparing the amounts of 
energy required to break different types of bonds. He chose an arbitrary relative scale ranging from 0 to 4 .

Linus Pauling

Linus Pauling, shown in (Figure), is the only person to have received two unshared (individual) Nobel Prizes: one for chemistry in 1954 for his work on the nature of chemical bonds and one for peace in 1962 for his opposition to weapons of mass destruction. He developed many of the theories and concepts that are foundational to our current understanding of chemistry, including electronegativity and resonance structures.

Linus Pauling (1901-1994) made many important contributions to the field of chemistry. He was also a prominent activist, publicizing issues related to health and nuclear weapons. 


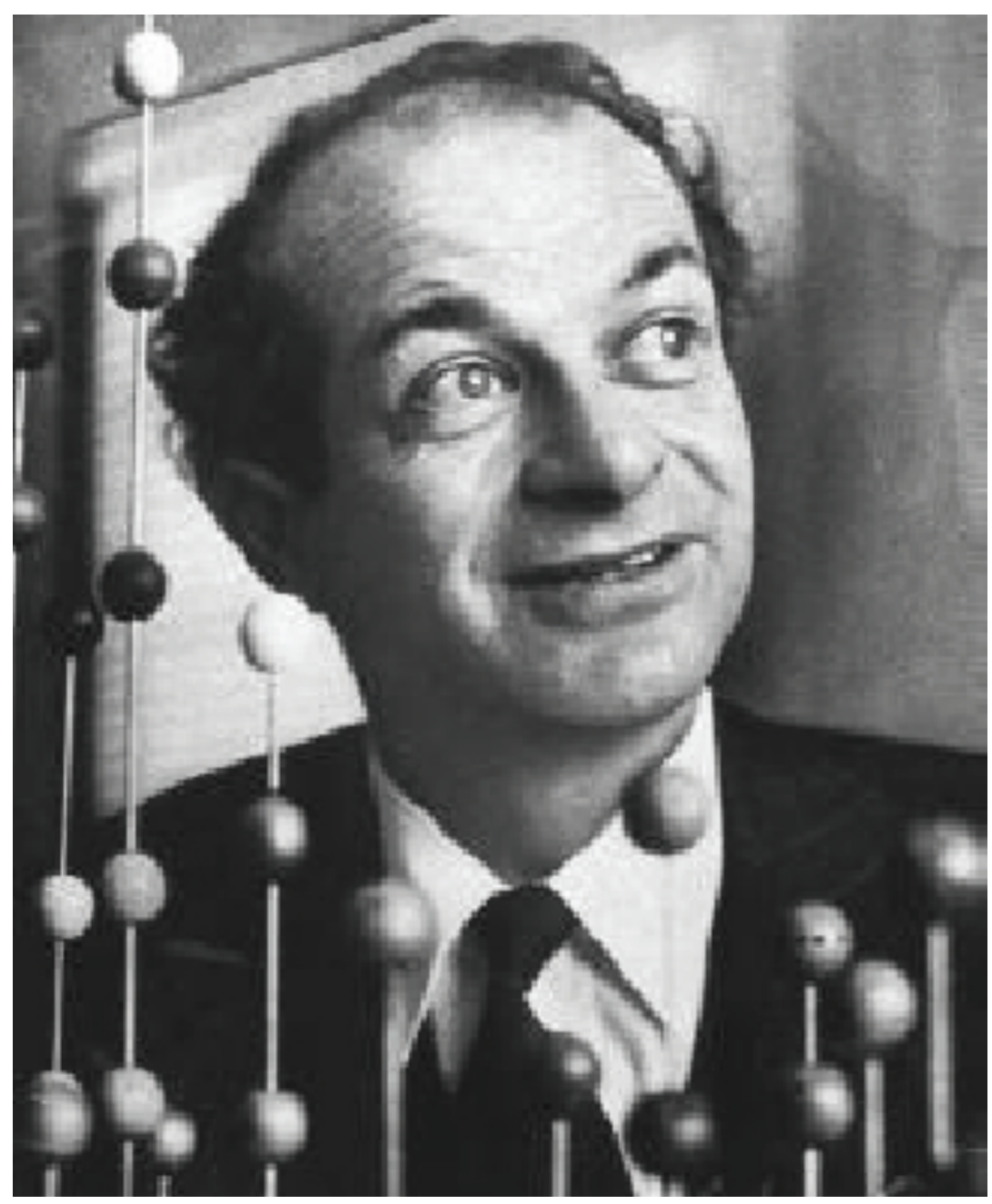

Pauling also contributed to many other fields besides chemistry. His research on sickle cell anemia revealed the cause of the disease-the presence of a genetically inherited abnormal protein in the blood-and paved the way for the field of molecular genetics. His work was also pivotal in curbing the testing of nuclear weapons; he proved that radioactive fallout from nuclear testing posed a public health risk.

Electronegativity and Bond Type

The absolute value of the difference in electronegativity 
$(\triangle \mathrm{EN})$ of two bonded atoms provides a rough measure of the polarity to be expected in the bond and, thus, the bond type. When the difference is very small or zero, the bond is covalent and nonpolar. When it is large, the bond is polar covalent or ionic. The absolute values of the electronegativity differences between the atoms in the bonds $\mathrm{H}-\mathrm{H}, \mathrm{H}-\mathrm{Cl}$, and $\mathrm{Na}-\mathrm{Cl}$ are 0 (nonpolar), 0.9 (polar covalent), and 2.1 (ionic), respectively. The degree to which electrons are shared between atoms varies from completely equal (pure covalent bonding) to not at all (ionic bonding). (Figure) shows the relationship between electronegativity difference and bond type.

As the electronegativity difference increases between two atoms, the bond becomes more ionic.

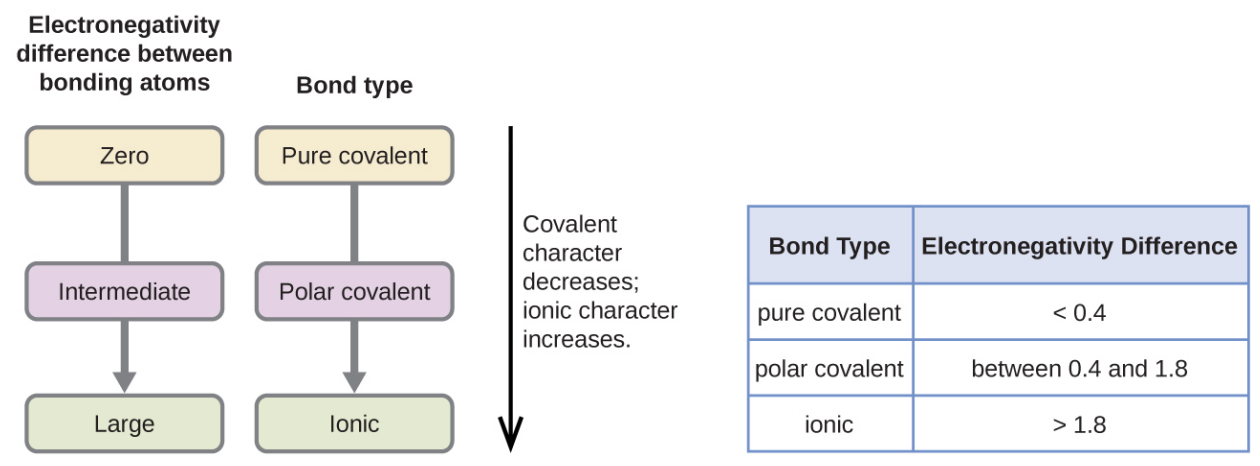

A rough approximation of the electronegativity differences associated with covalent, polar covalent, and ionic bonds is shown in (Figure). This table is just a general guide, however, with many exceptions. For example, the $\mathrm{H}$ and $\mathrm{F}$ atoms in $\mathrm{HF}$ have an electronegativity difference of 1.9 , and the $\mathrm{N}$ and $\mathrm{H}$ atoms in $\mathrm{NH}_{3}$ a difference of 0.9 , yet both of these compounds form bonds that are considered polar covalent. Likewise, the $\mathrm{Na}$ and $\mathrm{Cl}$ atoms in $\mathrm{NaCl}$ have an electronegativity 
difference of 2.1, and the $\mathrm{Mn}$ and I atoms in $\mathrm{MnI}_{2}$ have a difference of 1.0 , yet both of these substances form ionic compounds.

The best guide to the covalent or ionic character of a bond is to consider the types of atoms involved and their relative positions in the periodic table. Bonds between two nonmetals are generally covalent; bonding between a metal and a nonmetal is often ionic.

Some compounds contain both covalent and ionic bonds. The atoms in polyatomic ions, such as $\mathrm{OH}^{-}, \mathrm{NO}_{3}$, and $\mathrm{NH}_{4}{ }^{+}$, are held together by polar covalent bonds. However, these polyatomic ions form ionic compounds by combining with ions of opposite charge. For example, potassium nitrate, $\mathrm{KNO}_{3}$, contains the $\mathrm{K}^{+}$cation and the polyatomic $\mathrm{NO}_{3}$ anion. Thus, bonding in potassium nitrate is ionic, resulting from the electrostatic attraction between the ions $\mathrm{K}^{+}$and $\mathrm{NO}_{3}$, as well as covalent between the nitrogen and oxygen atoms in $\mathrm{NO}_{3}$.

Electronegativity and Bond Polarity Bond polarities play an important role in determining the structure of proteins. Using the electronegativity values in (Figure), arrange the following covalent bonds-all commonly found in amino acids-in order of increasing polarity. Then designate the positive and negative atoms using the symbols $\delta+$ and $\delta-$ :

$$
\mathrm{C}-\mathrm{H}, \mathrm{C}-\mathrm{N}, \mathrm{C}-\mathrm{O}, \mathrm{N}-\mathrm{H}, \mathrm{O}-\mathrm{H}, \mathrm{S}-\mathrm{H}
$$

Solution The polarity of these bonds increases as the absolute value of the electronegativity difference increases. The atom with the $\delta$ - designation is the more electronegative of the two. (Figure) shows these bonds in order of increasing polarity. 


\section{Bond Polarity and Electronegativity Difference}

\begin{tabular}{|c|c|c|}
\hline Bond & $\Delta \mathbf{E N}$ & Polarity \\
\hline $\mathrm{C}-\mathrm{H}$ & 0.4 & $\stackrel{\delta}{\mathrm{C}} \stackrel{\delta+}{\mathrm{H}}$ \\
\hline $\mathrm{S}-\mathrm{H}$ & 0.4 & $\begin{array}{ll}\delta & \delta+ \\
S & H\end{array}$ \\
\hline $\mathrm{C}-\mathrm{N}$ & 0.5 & $\stackrel{\delta+}{\mathrm{C}} \stackrel{\delta}{\mathrm{N}}$ \\
\hline $\mathrm{N}-\mathrm{H}$ & 0.9 & $\begin{array}{cc}\delta & \delta+ \\
\mathrm{N} & \mathrm{H}\end{array}$ \\
\hline $\mathrm{C}-\mathrm{O}$ & 1.0 & $\stackrel{\delta+}{\mathrm{C}} \stackrel{\delta}{\mathrm{O}}$ \\
\hline $\mathrm{O}-\mathrm{H}$ & 1.4 & $\begin{array}{cc}\delta & \delta+ \\
\mathrm{O} & \mathrm{H}\end{array}$ \\
\hline
\end{tabular}

Check Your Learning Silicones are polymeric compounds containing, among others, the following types of covalent bonds: $\mathrm{Si}-\mathrm{O}, \mathrm{Si}-\mathrm{C}, \mathrm{C}-\mathrm{H}$, and $\mathrm{C}-\mathrm{C}$. Using the electronegativity values in (Figure), arrange the bonds in order of increasing polarity and designate the positive and negative atoms using the symbols $\delta+$ and $\delta-$.
Answer:

\begin{tabular}{|c|c|c|}
\hline Bond & Electronegativity Difference & Polarity \\
\hline $\mathrm{C}-\mathrm{C}$ & 0.0 & nonpolar \\
\hline $\mathrm{C}-\mathrm{H}$ & 0.4 & $\stackrel{\delta}{\mathrm{C}} \stackrel{\delta+}{\mathrm{H}}$ \\
\hline $\mathrm{Si}-\mathrm{C}$ & 0.7 & $\stackrel{\delta+}{\mathrm{Si}} \stackrel{\delta}{\mathrm{C}}$ \\
\hline $\mathrm{Si}-\mathrm{O}$ & 1.7 & $\stackrel{\delta+}{\mathrm{Si}} \stackrel{\delta}{\mathrm{O}}$ \\
\hline
\end{tabular}


Key Concepts and Summary

Covalent bonds form when electrons are shared between atoms and are attracted by the nuclei of both atoms. In pure covalent bonds, the electrons are shared equally. In polar covalent bonds, the electrons are shared unequally, as one atom exerts a stronger force of attraction on the electrons than the other. The ability of an atom to attract a pair of electrons in a chemical bond is called its electronegativity. The difference in electronegativity between two atoms determines how polar a bond will be. In a diatomic molecule with two identical atoms, there is no difference in electronegativity, so the bond is nonpolar or pure covalent. When the electronegativity difference is very large, as is the case between metals and nonmetals, the bonding is characterized as ionic.

Chemistry End of Chapter Exercises

Why is it incorrect to speak of a molecule of solid $\mathrm{NaCl}$ ?

$\mathrm{NaCl}$ consists of discrete ions arranged in a crystal lattice, not covalently bonded molecules.

What information can you use to predict whether a bond between two atoms is covalent or ionic?

Predict which of the following compounds are ionic and which are covalent, based on the location of their constituent atoms in the periodic table:

(a) $\mathrm{Cl}_{2} \mathrm{CO}$

(b) $\mathrm{MnO}$

(c) $\mathrm{NCl}_{3}$ 

(d) $\mathrm{CoBr}_{2}$
(e) $\mathrm{K}_{2} \mathrm{~S}$
(f) $\mathrm{CO}$
(g) $\mathrm{CaF}_{2}$
(h) $\mathrm{HI}$
(i) $\mathrm{CaO}$
(j) $\mathrm{IBr}$
(k) $\mathrm{CO}_{2}$

ionic: (b), (d), (e), (g), and (i); covalent: (a), (c), (f), (h), (j), and (k)

Explain the difference between a nonpolar covalent bond, a polar covalent bond, and an ionic bond.

From its position in the periodic table, determine which atom in each pair is more electronegative:

(a) $\mathrm{Br}$ or $\mathrm{Cl}$

(b) $\mathrm{N}$ or $\mathrm{O}$

(c) $\mathrm{S}$ or $\mathrm{O}$

(d) P or S

(e) $\mathrm{Si}$ or N

(f) $\mathrm{Ba}$ or $\mathrm{P}$

(g) $\mathrm{N}$ or $\mathrm{K}$

(a) $\mathrm{Cl}$; (b) $\mathrm{O}$; (c) $\mathrm{O}$; (d) S; (e) $\mathrm{N}$; (f) P; (g) $\mathrm{N}$

From its position in the periodic table, determine which atom in each pair is more electronegative:
(a) $\mathrm{N}$ or $\mathrm{P}$
(b) $\mathrm{N}$ or $\mathrm{Ge}$
(c) $\mathrm{S}$ or $\mathrm{F}$
(d) $\mathrm{Cl}$ or $\mathrm{S}$ 
(e) $\mathrm{H}$ or $\mathrm{C}$

(f) Se or $\mathrm{P}$

(g) $\mathrm{C}$ or $\mathrm{Si}$

From their positions in the periodic table, arrange the atoms in each of the following series in order of increasing electronegativity:

(a) C, F, H, N, O

(b) $\mathrm{Br}, \mathrm{Cl}, \mathrm{F}, \mathrm{H}, \mathrm{I}$

(c) F, H, O, P, S

(d) Al, H, Na, O, P

(e) $\mathrm{Ba}, \mathrm{H}, \mathrm{N}, \mathrm{O}, \mathrm{As}$

(a) H, C, N, O, F; (b) H, I, Br, Cl, F; (c) H, P, S, O, F; (d) Na, $\mathrm{Al}, \mathrm{H}, \mathrm{P}, \mathrm{O}$; (e) Ba, H, As, N, O

From their positions in the periodic table, arrange the atoms in each of the following series in order of increasing electronegativity:

(a) As, H, N, P, Sb

(b) $\mathrm{Cl}, \mathrm{H}, \mathrm{P}, \mathrm{S}, \mathrm{Si}$

(c) $\mathrm{Br}, \mathrm{Cl}, \mathrm{Ge}, \mathrm{H}, \mathrm{Sr}$

(d) $\mathrm{Ca}, \mathrm{H}, \mathrm{K}, \mathrm{N}, \mathrm{Si}$

(e) $\mathrm{Cl}, \mathrm{Cs}, \mathrm{Ge}, \mathrm{H}, \mathrm{Sr}$

Which atoms can bond to sulfur so as to produce a positive partial charge on the sulfur atom?

$\mathrm{N}, \mathrm{O}, \mathrm{F}$, and $\mathrm{Cl}$

Which is the most polar bond?

(a) $\mathrm{C}-\mathrm{C}$

(b) $\mathrm{C}-\mathrm{H}$ 

(c) $\mathrm{N}-\mathrm{H}$
(d) $\mathrm{O}-\mathrm{H}$
(e) $\mathrm{Se}-\mathrm{H}$

Identify the more polar bond in each of the following pairs of bonds:
(a) $\mathrm{HF}$ or $\mathrm{HCl}$
(b) $\mathrm{NO}$ or $\mathrm{CO}$
(c) $\mathrm{SH}$ or $\mathrm{OH}$
(d) $\mathrm{PCl}$ or $\mathrm{SCl}$
(e) $\mathrm{CH}$ or $\mathrm{NH}$
(f) $\mathrm{SO}$ or $\mathrm{PO}$
(g) $\mathrm{CN}$ or $\mathrm{NN}$

(a) HF; (b) $\mathrm{CO}$; (c) $\mathrm{OH}$; (d) $\mathrm{PCl}$; (e) NH; (f) PO; (g) $\mathrm{CN}$

Which of the following molecules or ions contain polar bonds?
(a) $\mathrm{O}_{3}$
(b) $\mathrm{S}_{8}$
(c) $\mathrm{O}_{2}{ }^{2}$
(d) $\mathrm{NO}_{3}$
(e) $\mathrm{CO}_{2}$
(f) $\mathrm{H}_{2} \mathrm{~S}$
(g) $\mathrm{BH}_{4}$ 
Glossary

bond length

distance between the nuclei of two bonded atoms at which the lowest potential energy is achieved

covalent bond

bond formed when electrons are shared between atoms

electronegativity

tendency of an atom to attract electrons in a bond to itself

polar covalent bond

covalent bond between atoms of different electronegativities; a covalent bond with a positive end and a negative end

pure covalent bond

(also, nonpolar covalent bond) covalent bond between atoms of identical electronegativities 


\section{4.}

\section{LEWIS SYMBOLS AND STRUCTURES}

Learning Objectives

By the end of this section, you will be able to:

- Write Lewis symbols for neutral atoms and ions

- Draw Lewis structures depicting the bonding in simple molecules

Thus far in this chapter, we have discussed the various types of bonds that form between atoms and/or ions. In all cases, these bonds involve the sharing or transfer of valence shell electrons between atoms. In this section, we will explore the typical method for depicting valence shell electrons and chemical bonds, namely Lewis symbols and Lewis structures. 
Lewis Symbols

We use Lewis symbols to describe valence electron configurations of atoms and monatomic ions. A Lewis symbol consists of an elemental symbol surrounded by one dot for each of its valence electrons:

$\cdot \mathrm{Ca}$.

(Figure) shows the Lewis symbols for the elements of the third period of the periodic table.

Lewis symbols illustrating the number of valence electrons for each element in the third period of the periodic table.

\begin{tabular}{|l|c|c|}
\hline Atoms & Electronic Configuration & Lewis Symbol \\
\hline sodium & {$[\mathrm{Ne}] 3 s^{1}$} & $\mathrm{Na} \cdot$ \\
\hline magnesium & {$[\mathrm{Ne}] 3 s^{2}$} & $\cdot \mathrm{Mg} \cdot$ \\
\hline aluminum & {$[\mathrm{Ne}] 3 s^{2} 3 p^{1}$} & $\cdot \dot{\mathrm{Al}} \cdot$ \\
\hline silicon & {$[\mathrm{Ne}] 3 s^{2} 3 p^{2}$} & $\cdot \dot{\mathrm{S}} \cdot$ \\
\hline phosphorus & {$[\mathrm{Ne}] 3 s^{2} 3 p^{3}$} & $: \ddot{\mathrm{p}}$ \\
\hline sulfur & {$[\mathrm{Ne}] 3 s^{2} 3 p^{4}$} & $: \ddot{\mathrm{S}} \cdot$ \\
\hline chlorine & {$[\mathrm{Ne}] 3 s^{2} 3 p^{5}$} & $: \ddot{\mathrm{C}} \cdot$ \\
\hline argon & {$[\mathrm{Ne}] 3 s^{2} 3 p^{6}$} & $: \ddot{\mathrm{Ar}}:$ \\
\hline
\end{tabular}

Lewis symbols can also be used to illustrate the formation of cations from atoms, as shown here for sodium and calcium:

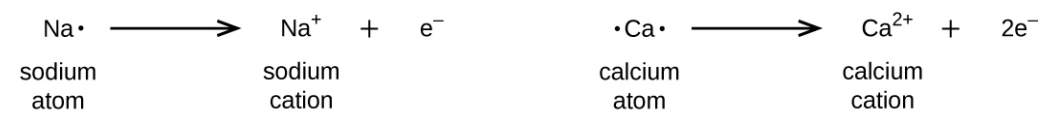

Likewise, they can be used to show the formation of anions from atoms, as shown here for chlorine and sulfur:
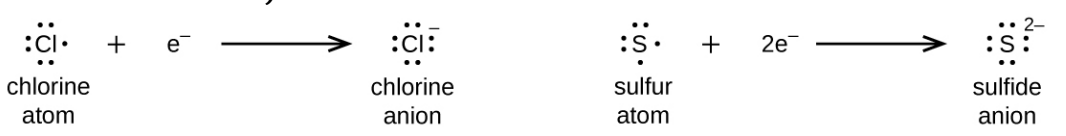
(Figure) demonstrates the use of Lewis symbols to show the transfer of electrons during the formation of ionic compounds.

Cations are formed when atoms lose electrons, represented by fewer Lewis dots, whereas anions are formed by atoms gaining electrons. The total number of electrons does not change.

\begin{tabular}{|c|c|c|c|}
\hline Metal & & Nonmetal & Ionic Compound \\
\hline $\mathrm{Na} \cdot$ & + & $: \ddot{c} \cdot$ & $\rightarrow \quad \mathrm{Na}^{+}[: \ddot{\mathrm{Cl}}:]^{-}$ \\
\hline sodium atom & & chlorine atom & $\begin{array}{c}\text { sodium chloride } \\
\text { (sodium ion and chloride ion) }\end{array}$ \\
\hline$\cdot \mathrm{Mg} \cdot$ & + & $: \ddot{0}$ & $\rightarrow \quad \mathrm{Mg}^{2+}[: \ddot{\mathrm{O}}:]^{2-}$ \\
\hline magnesium atom & & oxygen atom & $\begin{array}{c}\text { magnesium oxide } \\
\text { (magnesium ion and oxide ion) }\end{array}$ \\
\hline - $\mathrm{Ca} \cdot$ & + & $2: \ddot{F}$ & $\rightarrow \quad \mathrm{Ca}^{2+}[: \ddot{\mathrm{F}}:]_{2}^{-}$ \\
\hline calcium atom & & fluorine atoms & $\begin{array}{c}\text { calcium fluoride } \\
\text { (calcium ion and two fluoride ions) }\end{array}$ \\
\hline
\end{tabular}

\section{Lewis Structures}

We also use Lewis symbols to indicate the formation of covalent bonds, which are shown in Lewis structures, drawings that describe the bonding in molecules and polyatomic ions. For example, when two chlorine atoms form a chlorine molecule, they share one pair of electrons:

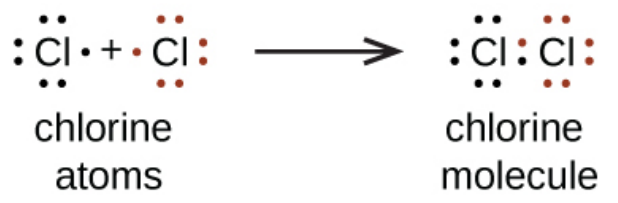

The Lewis structure indicates that each $\mathrm{Cl}$ atom has three pairs of electrons that are not used in bonding (called lone pairs) and one shared pair of electrons 
(written between the atoms). A dash (or line) is sometimes used to indicate a shared pair of electrons:
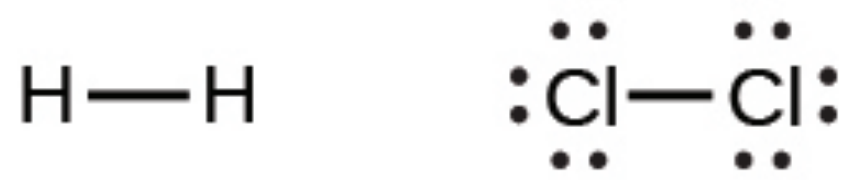

A single shared pair of electrons is called a single bond. Each $\mathrm{Cl}$ atom interacts with eight valence electrons: the six in the lone pairs and the two in the single bond.

The Octet Rule

The other halogen molecules $\left(\mathrm{F}_{2}, \mathrm{Br}_{2}, \mathrm{I}_{2}\right.$, and $\left.\mathrm{At}_{2}\right)$ form bonds like those in the chlorine molecule: one single bond between atoms and three lone pairs of electrons per atom. This allows each halogen atom to have a noble gas electron configuration. The tendency of main group atoms to form enough bonds to obtain eight valence electrons is known as the octet rule.

The number of bonds that an atom can form can often be predicted from the number of electrons needed to reach an octet (eight valence electrons); this is especially true of the nonmetals of the second period of the periodic table $(\mathrm{C}, \mathrm{N}, \mathrm{O}$, and $\mathrm{F})$. For example, each atom of a group 14 element has four electrons in its outermost shell and therefore requires four more electrons to reach an octet. These four electrons can be gained by forming four covalent bonds, as illustrated here for carbon in $\mathrm{CCl}_{4}$ (carbon tetrachloride) and silicon in $\mathrm{SiH}_{4}$ (silane). Because hydrogen only needs two electrons to fill its valence shell, it is an exception to the octet rule. The transition elements and inner transition elements also do not follow the octet rule: 


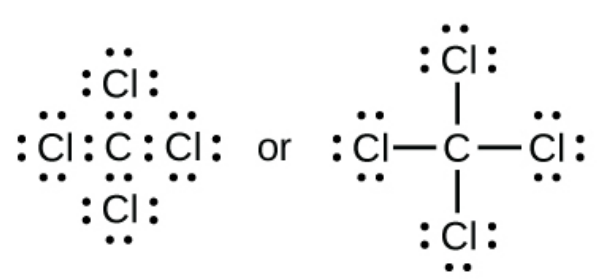

carbon tetrachloride<smiles>C1[Si][Hg]1</smiles>

silane

Group 15 elements such as nitrogen have five valence electrons in the atomic Lewis symbol: one lone pair and three unpaired electrons. To obtain an octet, these atoms form three covalent bonds, as in $\mathrm{NH}_{3}$ (ammonia). Oxygen and other atoms in group 16 obtain an octet by forming two covalent bonds:<smiles>[NH]</smiles>

ammonia<smiles>O</smiles>

Water<smiles>C1#C[C+]1</smiles>

hydrogen fluoride

\section{Double and Triple Bonds}

As previously mentioned, when a pair of atoms shares one pair of electrons, we call this a single bond. However, a pair of atoms may need to share more than one pair of electrons in order to achieve the requisite octet. A double bond forms when two pairs of electrons are shared between a pair of atoms, as between the carbon and oxygen atoms in $\mathrm{CH}_{2} \mathrm{O}$ (formaldehyde) and between the two carbon atoms in $\mathrm{C}_{2} \mathrm{H}_{4}$ (ethylene):<smiles>C[O+]=C=O</smiles>

formaldehyde<smiles>C=CC=C</smiles>

ethylene

A triple bond forms when three electron pairs are 
shared by a pair of atoms, as in carbon monoxide (CO) and the cyanide ion $\left(\mathrm{CN}^{-}\right)$:

$: C::: O:$ or $: C \equiv O: \longrightarrow: C::: \mathrm{N}^{-}$or $: C \equiv \mathrm{N}^{-}$

carbon monoxide

cyanide ion

Writing Lewis Structures with the Octet Rule

For very simple molecules and molecular ions, we can write the Lewis structures by merely pairing up the unpaired electrons on the constituent atoms. See these examples:

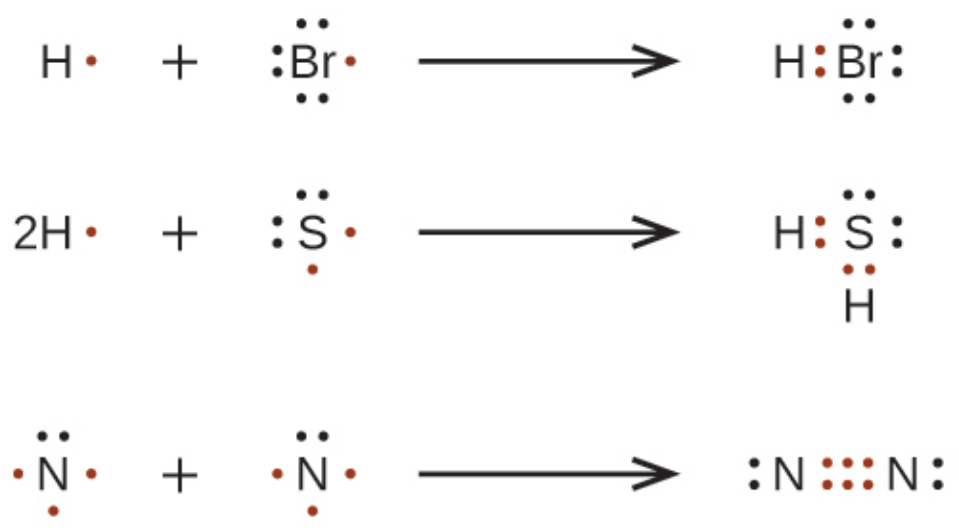

For more complicated molecules and molecular ions, it is helpful to follow the step-by-step procedure outlined here:

1. Determine the total number of valence (outer shell) electrons. For cations, subtract one electron for each positive charge. For anions, add one electron for each negative charge.

2. Draw a skeleton structure of the molecule or ion, arranging the atoms around a central atom. (Generally, the least electronegative element should be placed in the center.) Connect each atom to the central atom with a single bond (one 
electron pair).

3. Distribute the remaining electrons as lone pairs on the terminal atoms (except hydrogen), completing an octet around each atom.

4. Place all remaining electrons on the central atom.

5. Rearrange the electrons of the outer atoms to make multiple bonds with the central atom in order to obtain octets wherever possible.

Let us determine the Lewis structures of $\mathrm{SiH}_{4}, \mathrm{CHO}_{2}$, $\mathrm{NO}^{+}$, and $\mathrm{OF}_{2}$ as examples in following this procedure:

1. Determine the total number of valence (outer shell) electrons in the molecule or ion.

- For a molecule, we add the number of valence electrons on each atom in the molecule:

$\mathrm{SiH}_{4}$

Si: 4 valence electrons/atom 1 atom $=4$

$+\mathrm{H}: 1$ valence electron/atom 4 atoms $=4$

$=8$ valence electrons

- For a negative ion, such as $\mathrm{CHO}_{2}$, we add the number of valence electrons on the atoms to the number of negative charges on the ion (one electron is gained for each single negative charge): 
$\mathrm{CHO}_{2}$

C: 4 valence electrons/atom 1 atom $=4$

$\mathrm{H}$ : 1 valence electron/atom 1 atom $=1$

O: 6 valence electrons/atom 2 atoms $=12$

$+\quad 1$ additional electron $=1$

$=18$ valence electrons

- For a positive ion, such as $\mathrm{NO}^{+}$, we add the number of valence electrons on the atoms in the ion and then subtract the number of positive charges on the ion (one electron is lost for each single positive charge) from the total number of valence electrons:

$\mathrm{NO}^{+}$

$\mathrm{N}$ : 5 valence electrons/atom 1 atom $=5$

O: 6 valence electron/atom 1 atom $=6$

+1 electron (positive charge) $\quad=-1$

$=10$ valence electrons

- Since $\mathrm{OF}_{2}$ is a neutral molecule, we simply add the number of valence electrons:

$\mathrm{OF}_{2}$

O: 6 valence electrons/atom 1 atom $=6$

$+\mathrm{F}: 7$ valence electrons/atom 2 atoms $=14$

$=20$ valence electrons

2. Draw a skeleton structure of the molecule or ion, arranging the atoms around a central atom and connecting each atom to the central atom with a single (one electron pair) bond. (Note that we denote ions with brackets around the structure, indicating the charge outside the brackets:) 
<smiles>[3H]OC(O)C[SiH4]</smiles>

When several arrangements of atoms are possible, as for $\mathrm{CHO}_{2}$, we must use experimental evidence to choose the correct one. In general, the less electronegative elements are more likely to be central atoms. In $\mathrm{CHO}_{2}$, the less electronegative carbon atom occupies the central position with the oxygen and hydrogen atoms surrounding it. Other examples include $\mathrm{P}$ in $\mathrm{POCl}_{3}, \mathrm{~S}$ in $\mathrm{SO}_{2}$, and $\mathrm{Cl}$ in $\mathrm{ClO}_{4}$. An exception is that hydrogen is almost never a central atom. As the most electronegative element, fluorine also cannot be a central atom.

3. Distribute the remaining electrons as lone pairs on the terminal atoms (except hydrogen) to complete their valence shells with an octet of electrons.

- There are no remaining electrons on $\mathrm{SiH}_{4}$, so it is unchanged:

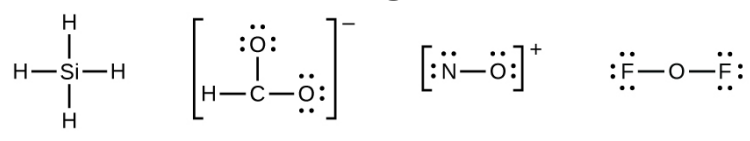

4. Place all remaining electrons on the central atom.

- For $\mathrm{SiH}_{4}, \mathrm{CHO}_{2}$, and $\mathrm{NO}^{+}$, there are no remaining electrons; we already placed all of the electrons determined 
in Step 1.

- For $\mathrm{OF}_{2}$, we had 16 electrons remaining in Step 3, and we placed 12, leaving 4 to be placed on the central atom:

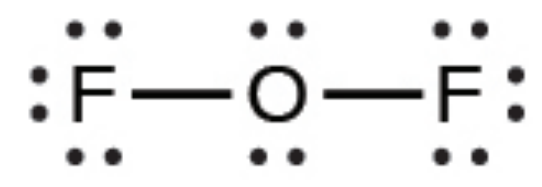

5. Rearrange the electrons of the outer atoms to make multiple bonds with the central atom in order to obtain octets wherever possible.

- $\mathrm{SiH}_{4}$ : Si already has an octet, so nothing needs to be done.

- $\mathrm{CHO}_{2}$ : We have distributed the valence electrons as lone pairs on the oxygen atoms, but the carbon atom lacks an octet:

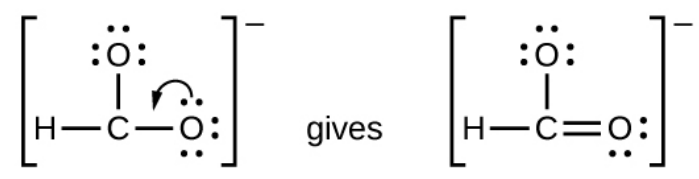

- $\mathrm{NO}^{+}$: For this ion, we added eight valence electrons, but neither atom has an octet. We cannot add any more electrons since we have already used the total that we found in Step 1, so we must move electrons to form a multiple bond:

$$
[\ddot{\mathrm{N}}-\mathscr{\mathrm { O }}:]^{+} \text {gives } \quad[\ddot{\mathrm{N}}=0:]^{+}
$$

This still does not produce an octet, so we must move another pair, forming a 
triple bond:

$$
[: \mathrm{N} \equiv \mathrm{O}:]^{+}
$$

- In $\mathrm{OF}_{2}$, each atom has an octet as drawn, so nothing changes.

Writing Lewis Structures NASA's Cassini-Huygens mission detected a large cloud of toxic hydrogen cyanide (HCN) on Titan, one of Saturn's moons. Titan also contains ethane $\left(\mathrm{H}_{3} \mathrm{CCH}_{3}\right)$, acetylene $(\mathrm{HCCH})$, and ammonia $\left(\mathrm{NH}_{3}\right)$. What are the Lewis structures of these molecules?

\section{Solution}

1. Calculate the number of valence electrons.

HCN: (1 1) + (4 1) + (5 1) $=10$

$\mathrm{H}_{3} \mathrm{CCH}_{3}:\left(\begin{array}{ll}1 & 3\end{array}\right)+\left(\begin{array}{ll}2 & 4\end{array}\right)+\left(\begin{array}{ll}1 & 3\end{array}\right)=14$

$\mathrm{HCCH}:\left(\begin{array}{ll}1 & 1\end{array}\right)+\left(\begin{array}{ll}2 & 4\end{array}\right)+\left(\begin{array}{ll}1 & 1\end{array}\right)=10$

$\mathrm{NH}_{3}:\left(\begin{array}{ll}5 & 1\end{array}\right)+\left(\begin{array}{ll}3 & 1\end{array}\right)=8$

2. Draw a skeleton and connect the atoms with single bonds. Remember that $\mathrm{H}$ is never a central atom:

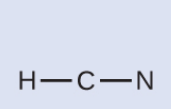<smiles>CCC</smiles><smiles>C=C</smiles><smiles>NN</smiles>

3. Where needed, distribute electrons to the terminal atoms:<smiles>N=N</smiles><smiles>CC</smiles><smiles>C=C</smiles><smiles>NN</smiles>

$\mathrm{HCN}$ : six electrons placed on $\mathrm{N}$ $\mathrm{H}_{3} \mathrm{CCH}_{3}$ : no electrons remain 
HCCH: no terminal atoms capable of accepting electrons

$\mathrm{NH}_{3}$ : no terminal atoms capable of accepting electrons

4. Where needed, place remaining electrons on the central atom:<smiles>N#N</smiles><smiles>CC</smiles><smiles>[CH]C=[CH]</smiles><smiles>NN</smiles>

HCN: no electrons remain $\mathrm{H}_{3} \mathrm{CCH}_{3}$ : no electrons remain $\mathrm{HCCH}$ : four electrons placed on carbon $\mathrm{NH}_{3}$ : two electrons placed on nitrogen

5. Where needed, rearrange electrons to form multiple bonds in order to obtain an octet on each atom:

$\mathrm{HCN}$ : form two more $\mathrm{C}-\mathrm{N}$ bonds $\mathrm{H}_{3} \mathrm{CCH}_{3}$ : all atoms have the correct number of electrons HCCH: form a triple bond between the two carbon atoms

$\mathrm{NH}_{3}$ : all atoms have the correct number of electrons<smiles>N#Cc1cccnc1</smiles><smiles>C</smiles><smiles>CC#CC=CC1CC1</smiles><smiles>[1H]N</smiles>

Check Your Learning Both carbon monoxide, CO, and carbon dioxide, $\mathrm{CO}_{2}$, are products of the combustion of fossil fuels. Both of these gases also cause problems: $\mathrm{CO}$ is toxic and $\mathrm{CO}_{2}$ has been implicated in global climate change. What are the Lewis structures of these two molecules?

Answer:

$$
: c \equiv 0: \quad: \ddot{O}=\mathrm{c}=\ddot{\mathrm{O}} \text { : }
$$




\section{Fullerene Chemistry}

Carbon soot has been known to man since prehistoric times, but it was not until fairly recently that the molecular structure of the main component of soot was discovered. In 1996, the Nobel Prize in Chemistry was awarded to Richard Smalley ((Figure)), Robert Curl, and Harold Kroto for their work in discovering a new form of carbon, the $\mathrm{C}_{60}$ buckminsterfullerene molecule ((Figure)). An entire class of compounds, including spheres and tubes of various shapes, were discovered based on $\mathrm{C}_{60}$. This type of molecule, called a fullerene, shows promise in a variety of applications. Because of their size and shape, fullerenes can encapsulate other molecules, so they have shown potential in various applications from hydrogen storage to targeted drug delivery systems. They also possess unique electronic and optical properties that have been put to good use in solar powered devices and chemical sensors.

Richard Smalley (1943-2005), a professor of physics, chemistry, and astronomy at Rice University, was one of the leading advocates for fullerene chemistry. Upon his death in 2005, the US Senate honored him as the "Father of Nanotechnology." (credit: United States Department of Energy) 


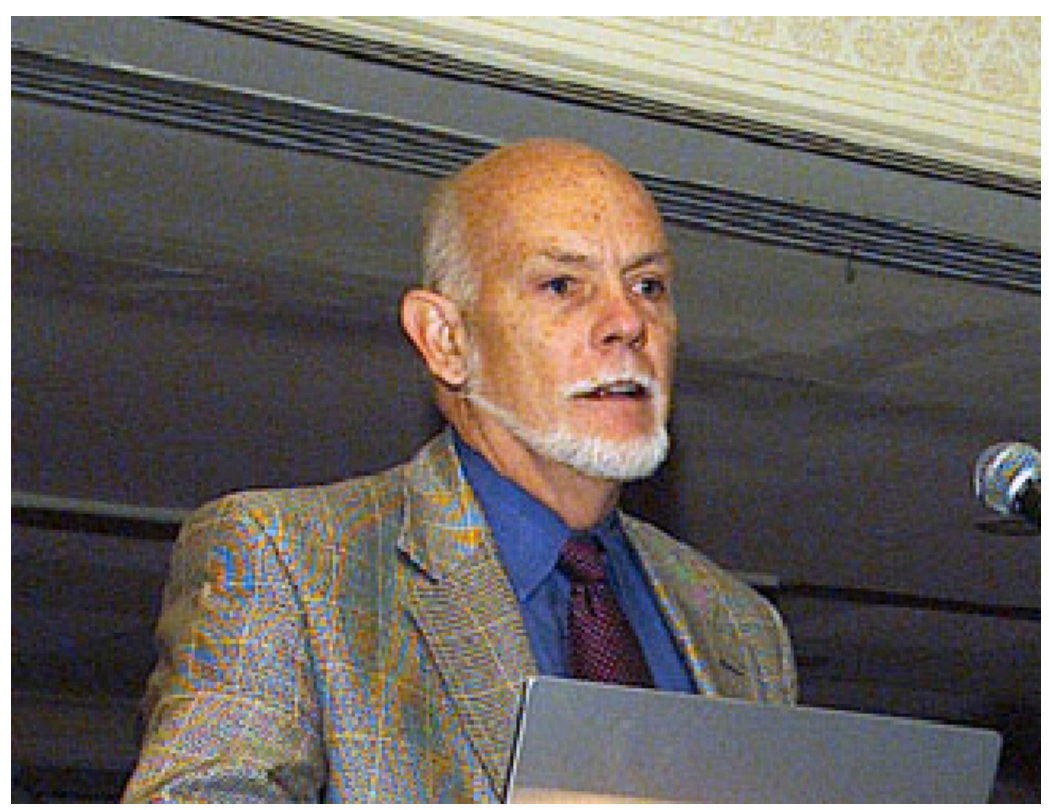

Exceptions to the Octet Rule

Many covalent molecules have central atoms that do not have eight electrons in their Lewis structures. These molecules fall into three categories:

- Odd-electron molecules have an odd number of valence electrons, and therefore have an unpaired electron.

- Electron-deficient molecules have a central atom that has fewer electrons than needed for a noble gas configuration.

- Hypervalent molecules have a central atom that has more electrons than needed for a noble gas configuration.

Odd-electron Molecules

We call molecules that contain an odd number of electrons 
free radicals. Nitric oxide, NO, is an example of an oddelectron molecule; it is produced in internal combustion engines when oxygen and nitrogen react at high temperatures.

To draw the Lewis structure for an odd-electron molecule like NO, we follow the same five steps we would for other molecules, but with a few minor changes:

1. Determine the total number of valence (outer shell) electrons. The sum of the valence electrons is 5 $($ from $\mathrm{N})+6($ from $\mathrm{O})=11$. The odd number immediately tells us that we have a free radical, so we know that not every atom can have eight electrons in its valence shell.

2. Draw a skeleton structure of the molecule. We can easily draw a skeleton with an $\mathrm{N}-\mathrm{O}$ single bond: $\mathrm{N}-\mathrm{O}$

3. Distribute the remaining electrons as lone pairs on the terminal atoms. In this case, there is no central atom, so we distribute the electrons around both atoms. We give eight electrons to the more electronegative atom in these situations; thus oxygen has the filled valence shell:

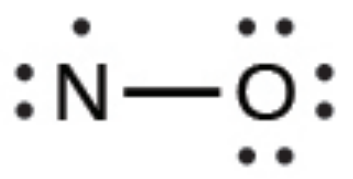

4. Place all remaining electrons on the central atom. Since there are no remaining electrons, this step does not apply.

5. Rearrange the electrons to make multiple bonds 
with the central atom in order to obtain octets wherever possible. We know that an odd-electron molecule cannot have an octet for every atom, but we want to get each atom as close to an octet as possible. In this case, nitrogen has only five electrons around it. To move closer to an octet for nitrogen, we take one of the lone pairs from oxygen and use it to form a NO double bond. (We cannot take another lone pair of electrons on oxygen and form a triple bond because nitrogen would then have nine electrons:)

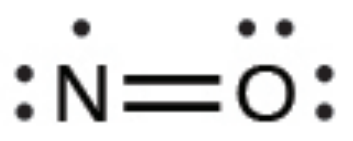

Electron-deficient Molecules

We will also encounter a few molecules that contain central atoms that do not have a filled valence shell. Generally, these are molecules with central atoms from groups 2 and 13, outer atoms that are hydrogen, or other atoms that do not form multiple bonds. For example, in the Lewis structures of beryllium dihydride, $\mathrm{BeH}_{2}$, and boron trifluoride, $\mathrm{BF}_{3}$, the beryllium and boron atoms each have only four and six electrons, respectively. It is possible to draw a structure with a double bond between a boron atom and a fluorine atom in $\mathrm{BF}_{3}$, satisfying the octet rule, but experimental evidence indicates the bond lengths are closer to that expected for B-F single bonds. This suggests the best Lewis structure has three B-F single bonds and an electron deficient boron. The reactivity of the compound is also consistent with an 
electron deficient boron. However, the B-F bonds are slightly shorter than what is actually expected for B-F single bonds, indicating that some double bond character is found in the actual molecule.

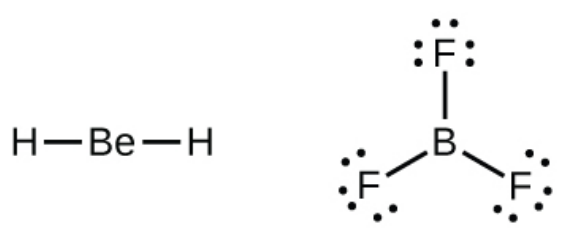

An atom like the boron atom in $\mathrm{BF}_{3}$, which does not have eight electrons, is very reactive. It readily combines with a molecule containing an atom with a lone pair of electrons. For example, $\mathrm{NH}_{3}$ reacts with $\mathrm{BF}_{3}$ because the lone pair on nitrogen can be shared with the boron atom:

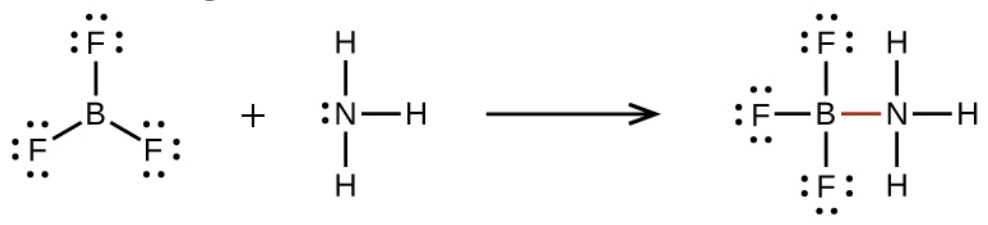

Hypervalent Molecules

Elements in the second period of the periodic table $(n=$ 2) can accommodate only eight electrons in their valence shell orbitals because they have only four valence orbitals (one $2 s$ and three $2 p$ orbitals). Elements in the third and higher periods $(n \geqslant 3)$ have more than four valence orbitals and can share more than four pairs of electrons with other atoms because they have empty $d$ orbitals in the same shell. Molecules formed from these elements are sometimes called hypervalent molecules. (Figure) shows the Lewis structures for two hypervalent molecules, $\mathrm{PCl}_{5}$ and $\mathrm{SF}_{6}$.

In $\mathrm{PCl}_{5}$, the central atom phosphorus shares five pairs of electrons. In $\mathrm{SF}_{6}$, sulfur shares six pairs of electrons. 

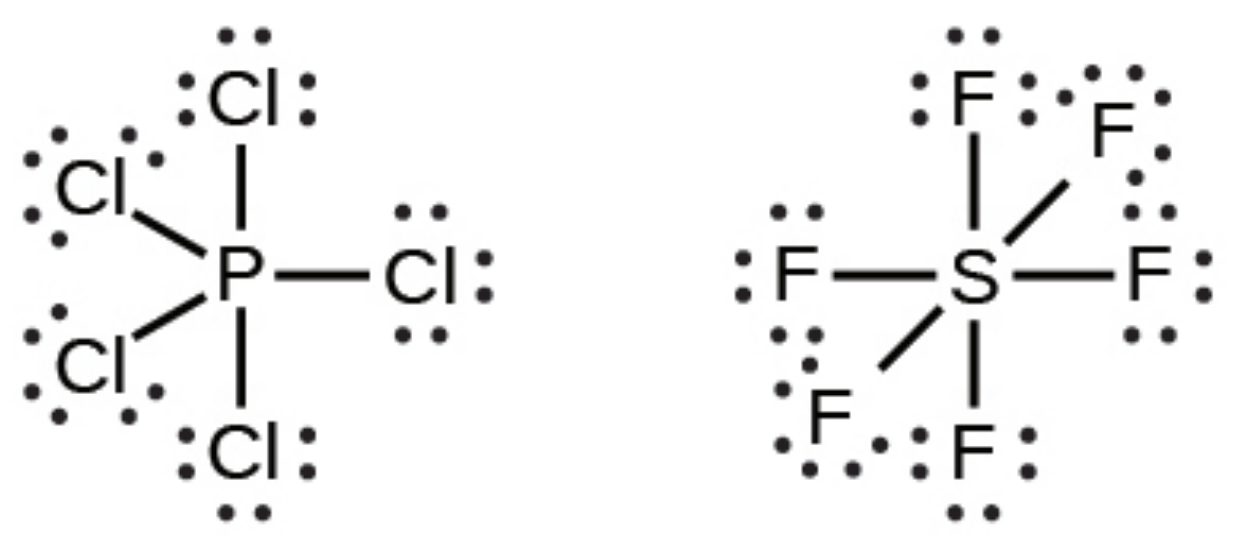

In some hypervalent molecules, such as $\mathrm{IF}_{5}$ and $\mathrm{XeF}_{4}$, some of the electrons in the outer shell of the central atom are lone pairs:
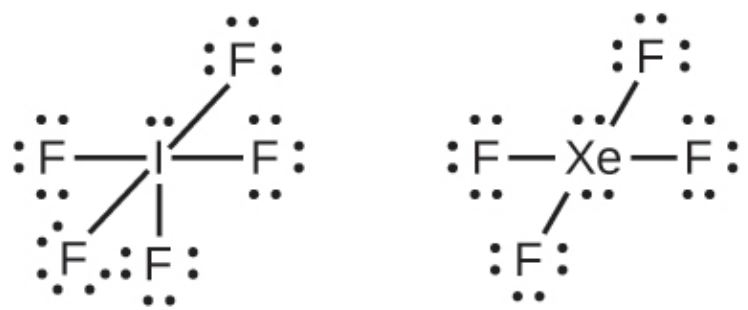

When we write the Lewis structures for these molecules, we find that we have electrons left over after filling the valence shells of the outer atoms with eight electrons. These additional electrons must be assigned to the central atom.

Writing Lewis Structures: Octet Rule Violations Xenon is a noble gas, but it forms a number of stable compounds. We examined $\mathrm{XeF}_{4}$ earlier. What are the Lewis structures of $\mathrm{XeF}_{2}$ and $\mathrm{XeF}_{6}$ ?

Solution We can draw the Lewis structure of any covalent molecule by following the six steps discussed earlier. In this case, we can condense the last few steps, since not all of them apply. 
1. Calculate the number of valence electrons:

$\mathrm{XeF}_{2}: 8+(27)=22$

$\mathrm{XeF}_{6}: 8+(67)=50$

2. Draw a skeleton joining the atoms by single bonds. Xenon will be the central atom because fluorine cannot be a central atom:<smiles>F[X][X](F)(F)(F)(F)F</smiles>

3. Distribute the remaining electrons.

$\mathrm{XeF}_{2}$ : We place three lone pairs of electrons around each $\mathrm{F}$ atom, accounting for 12 electrons and giving each $\mathrm{F}$ atom 8 electrons. Thus, six electrons (three lone pairs) remain. These lone pairs must be placed on the Xe atom. This is acceptable because Xe atoms have empty valence shell $d$ orbitals and can accommodate more than eight electrons. The Lewis structure of XeF 2 shows two bonding pairs and three lone pairs of electrons around the Xe atom:<smiles>[I+]#[Z10]C#[I+]</smiles>

XeF6: We place three lone pairs of electrons around each $\mathrm{F}$ atom, accounting for 36 electrons. Two electrons remain, and this lone pair is placed on the Xe atom:

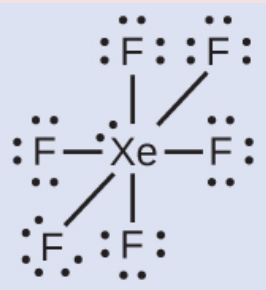

Check Your Learning The halogens form a class of compounds called the interhalogens, in which halogen atoms 
covalently bond to each other. Write the Lewis structures for the interhalogens $\mathrm{BrCl}_{3}$ and $\mathrm{ICl}_{4}$.

Answer:
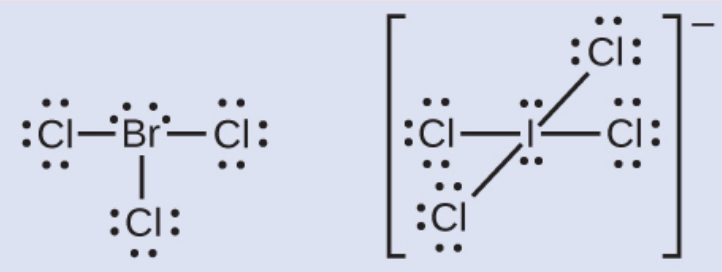

Key Concepts and Summary

Valence electronic structures can be visualized by drawing Lewis symbols (for atoms and monatomic ions) and Lewis structures (for molecules and polyatomic ions). Lone pairs, unpaired electrons, and single, double, or triple bonds are used to indicate where the valence electrons are located around each atom in a Lewis structure. Most structures-especially those containing second row elements-obey the octet rule, in which every atom (except $\mathrm{H}$ ) is surrounded by eight electrons. Exceptions to the octet rule occur for odd-electron molecules (free radicals), electron-deficient molecules, and hypervalent molecules.

\section{Chemistry End of Chapter Exercises}

Write the Lewis symbols for each of the following ions:
(a) $\mathrm{As}^{3-}$
(b) $\mathrm{I}^{-}$
(c) $\mathrm{Be}^{2+}$
(d) $\mathrm{O}^{2-}$
(e) $\mathrm{Ga}^{3+}$
(f) $\mathrm{Li}^{+}$ 
(g) $\mathrm{N}^{3-}$

(a) eight electrons:

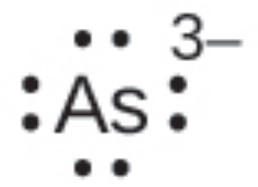

(b) eight electrons:

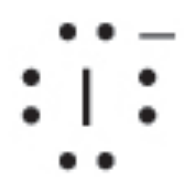

(c) no electrons $\mathrm{Be}^{2+}$

(d) eight electrons:

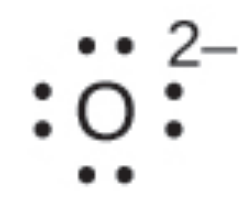

(e) no electrons $\mathrm{Ga}^{3+}$

(f) no electrons $\mathrm{Li}^{+}$

(g) eight electrons:

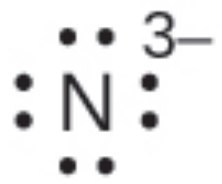

Many monatomic ions are found in seawater, including the ions formed from the following list of elements. Write the Lewis symbols for the monatomic ions formed from the following elements:
(a) $\mathrm{Cl}$
(b) $\mathrm{Na}$ 
(c) $\mathrm{Mg}$

(d) $\mathrm{Ca}$

(e) $\mathrm{K}$

(f) $\mathrm{Br}$

(g) $\mathrm{Sr}$

(h) F

Write the Lewis symbols of the ions in each of the following ionic compounds and the Lewis symbols of the atom from which they are formed:
(a) $\mathrm{MgS}$
(b) $\mathrm{Al}_{2} \mathrm{O}_{3}$
(c) $\mathrm{GaCl}_{3}$
(d) $\mathrm{K}_{2} \mathrm{O}$
(e) $\mathrm{Li}_{3} \mathrm{~N}$
(f) $\mathrm{KF}$

(a)<smiles>[18OH+]</smiles>

(b)

$\mathrm{Al}^{3+} \quad: \ddot{0}^{2-}$

(c)

$\mathrm{Ga}^{3+} \quad: \ddot{\ddot{C l}} \overline{-}$

(d)<smiles>[3H]</smiles>

(e) 


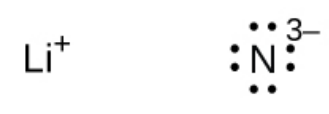

(f)

$$
\mathrm{K}^{+} \quad: \ddot{\mathrm{F}}:
$$

In the Lewis structures listed here, $\mathrm{M}$ and $\mathrm{X}$ represent various elements in the third period of the periodic table. Write the formula of each compound using the chemical symbols of each element:

(a)

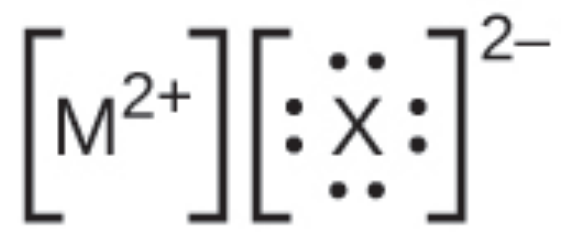

(b)

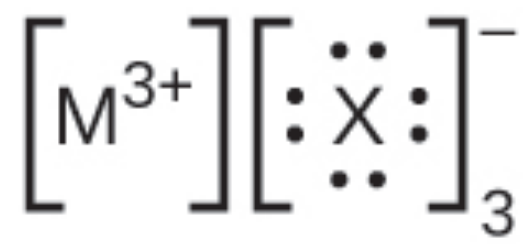

(c)

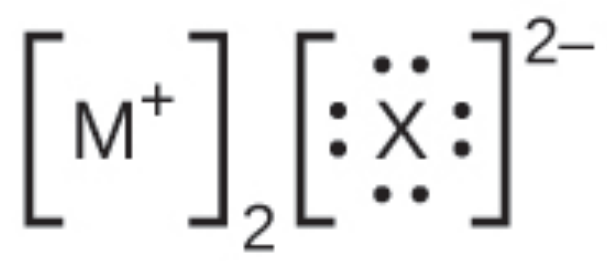

(d)

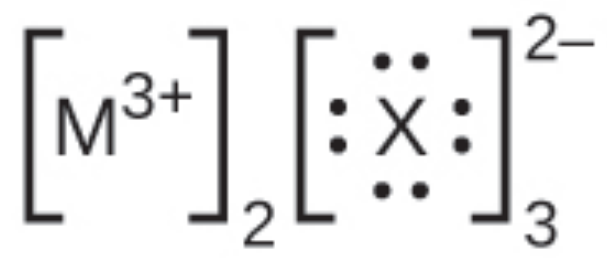

Write the Lewis structure for the diatomic molecule $\mathrm{P}_{2}$, an 
unstable form of phosphorus found in high-temperature phosphorus vapor.

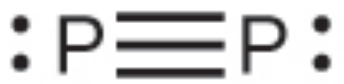

Write Lewis structures for the following:
(a) $\mathrm{H}_{2}$
(b) $\mathrm{HBr}$
(c) $\mathrm{PCl}_{3}$
(d) $\mathrm{SF}_{2}$
(e) $\mathrm{H}_{2} \mathrm{CCH}_{2}$
(f) $\mathrm{HNNH}$
(g) $\mathrm{H}_{2} \mathrm{CNH}$
(h) $\mathrm{NO}^{-}$
(i) $\mathrm{N}_{2}$
(j) $\mathrm{CO}$
(k) $\mathrm{CN}^{-}$

Write Lewis structures for the following:
(a) $\mathrm{O}_{2}$
(b) $\mathrm{H}_{2} \mathrm{CO}$
(c) $\mathrm{AsF}_{3}$
(d) $\mathrm{ClNO}$
(e) $\mathrm{SiCl}_{4}$
(f) $\mathrm{H}_{3} \mathrm{O}^{+}$
(g) $\mathrm{NH}_{4}+$
(h) $\mathrm{BF}_{4}$
(i) $\mathrm{HCCH}$
(j) $\mathrm{ClCN}$
(k) $\mathrm{C}_{2}{ }^{2+}$ 
(a)

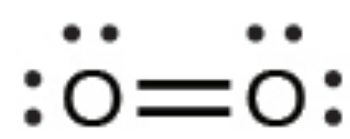

In this case, the Lewis structure is inadequate to depict the fact that experimental studies have shown two unpaired electrons in each oxygen molecule.

(b)

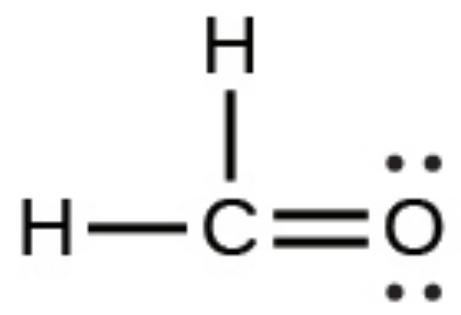

(c)<smiles>FC(F)(F)[As](F)(F)F</smiles>

(d)

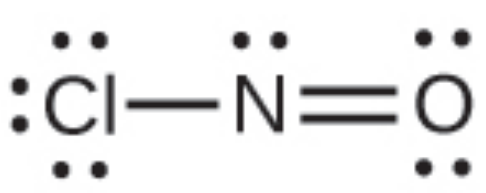

(e) 
Chemistry and the Environment

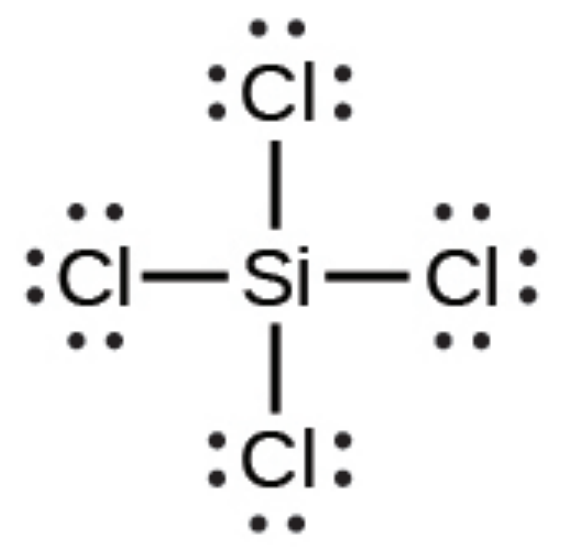

(f)

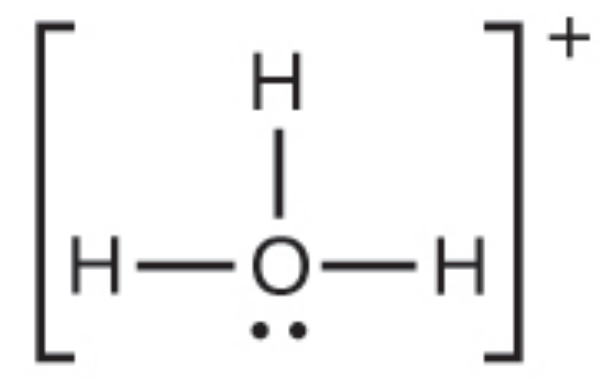

(g)

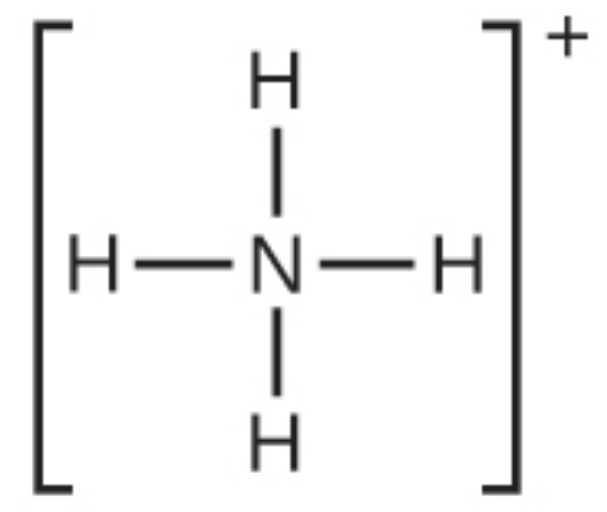

(h) 


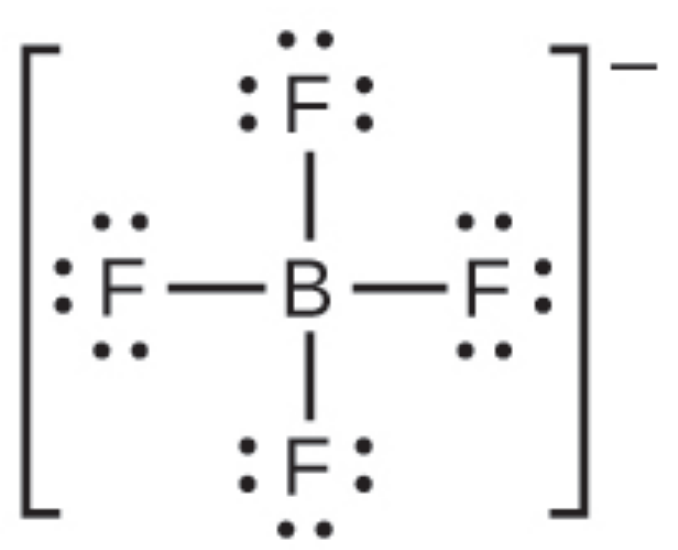

(i)<smiles>C#C</smiles>

(j)

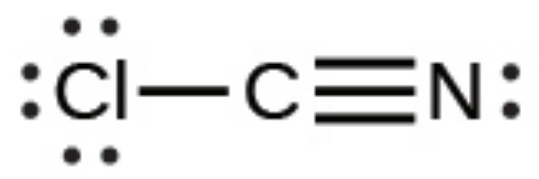

(k)

$$
\mathrm{C} \equiv \mathrm{C}^{2+}
$$

Write Lewis structures for the following:
(a) $\mathrm{ClF}_{3}$
(b) $\mathrm{PCl}_{5}$
(c) $\mathrm{BF}_{3}$
(d) $\mathrm{PF}_{6}$

Write Lewis structures for the following:
(a) $\mathrm{SeF}_{6}$
(b) $\mathrm{XeF}_{4}$ 
(c) $\mathrm{SeCl}_{3}{ }^{+}$

(d) $\mathrm{Cl}_{2} \mathrm{BBCl}_{2}$ (contains a $\mathrm{B}-\mathrm{B}$ bond)

(a) $\mathrm{SeF}_{6}$ :

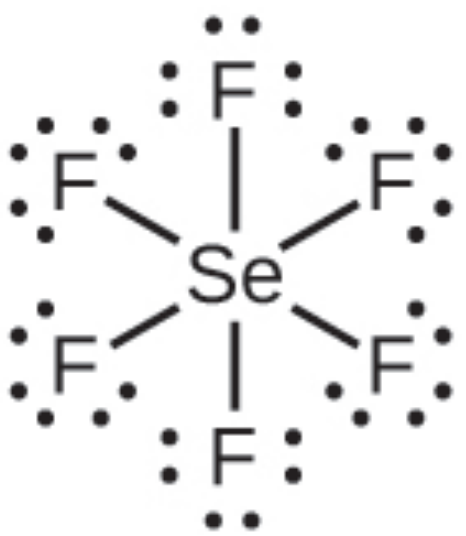

(b) $\mathrm{XeF}_{4}$ :

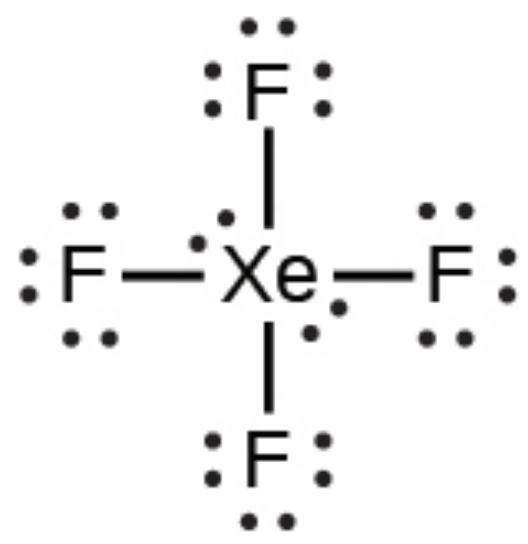

(c) $\mathrm{SeCl}_{3}+$ :

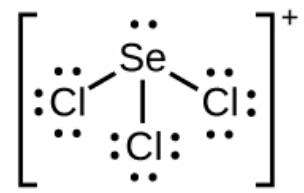

(d) $\mathrm{Cl}_{2} \mathrm{BBCl}_{2}$ : 


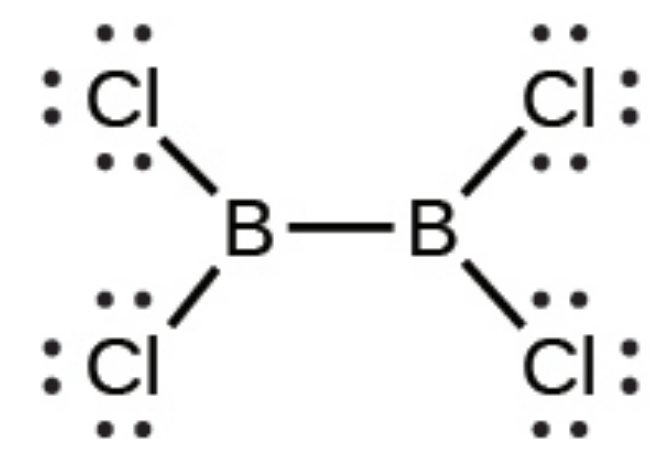

Write Lewis structures for:

(a) $\mathrm{PO}_{4}{ }^{3}$

(b) $\mathrm{ICl}_{4}$

(c) $\mathrm{SO}_{3}^{2}$

(d) HONO

Correct the following statement: "The bonds in solid $\mathrm{PbCl}_{2}$ are ionic; the bond in a $\mathrm{HCl}$ molecule is covalent. Thus, all of the valence electrons in $\mathrm{PbCl}_{2}$ are located on the $\mathrm{Cl}^{-}$ions, and all of the valence electrons in a $\mathrm{HCl}$ molecule are shared between the $\mathrm{H}$ and $\mathrm{Cl}$ atoms."

Two valence electrons per $\mathrm{Pb}$ atom are transferred to $\mathrm{Cl}$ atoms; the resulting $\mathrm{Pb}^{2+}$ ion has a $6 s^{2}$ valence shell configuration. Two of the valence electrons in the $\mathrm{HCl}$ molecule are shared, and the other six are located on the $\mathrm{Cl}$ atom as lone pairs of electrons.

Write Lewis structures for the following molecules or ions:

(a) $\mathrm{SbH}_{3}$

(b) $\mathrm{XeF}_{2}$

(c) $\mathrm{Se}_{8}$ (a cyclic molecule with a ring of eight Se atoms)

Methanol, $\mathrm{H}_{3} \mathrm{COH}$, is used as the fuel in some race cars. 
Ethanol, $\mathrm{C}_{2} \mathrm{H}_{5} \mathrm{OH}$, is used extensively as motor fuel in Brazil. Both methanol and ethanol produce $\mathrm{CO}_{2}$ and $\mathrm{H}_{2} \mathrm{O}$ when they burn. Write the chemical equations for these combustion reactions using Lewis structures instead of chemical formulas.

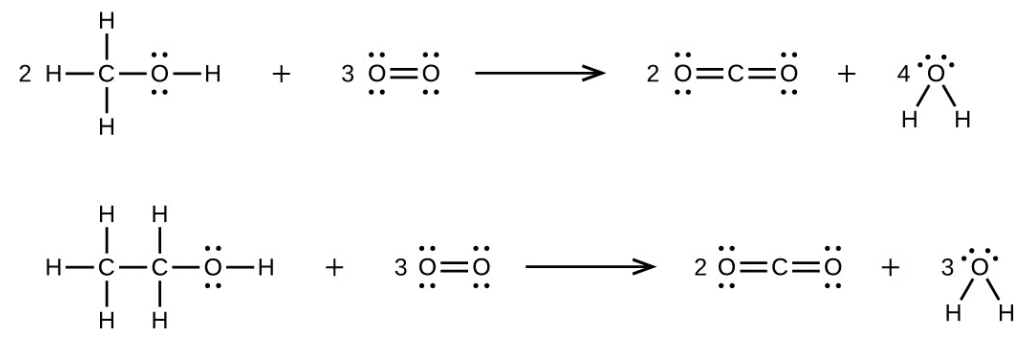

Many planets in our solar system contain organic chemicals including methane $\left(\mathrm{CH}_{4}\right)$ and traces of ethylene $\left(\mathrm{C}_{2} \mathrm{H}_{4}\right)$, ethane $\left(\mathrm{C}_{2} \mathrm{H}_{6}\right)$, propyne $\left(\mathrm{H}_{3} \mathrm{CCCH}\right)$, and diacetylene (HCCCCH). Write the Lewis structures for each of these molecules.

Carbon tetrachloride was formerly used in fire extinguishers for electrical fires. It is no longer used for this purpose because of the formation of the toxic gas phosgene, $\mathrm{Cl}_{2} \mathrm{CO}$. Write the Lewis structures for carbon tetrachloride and phosgene.
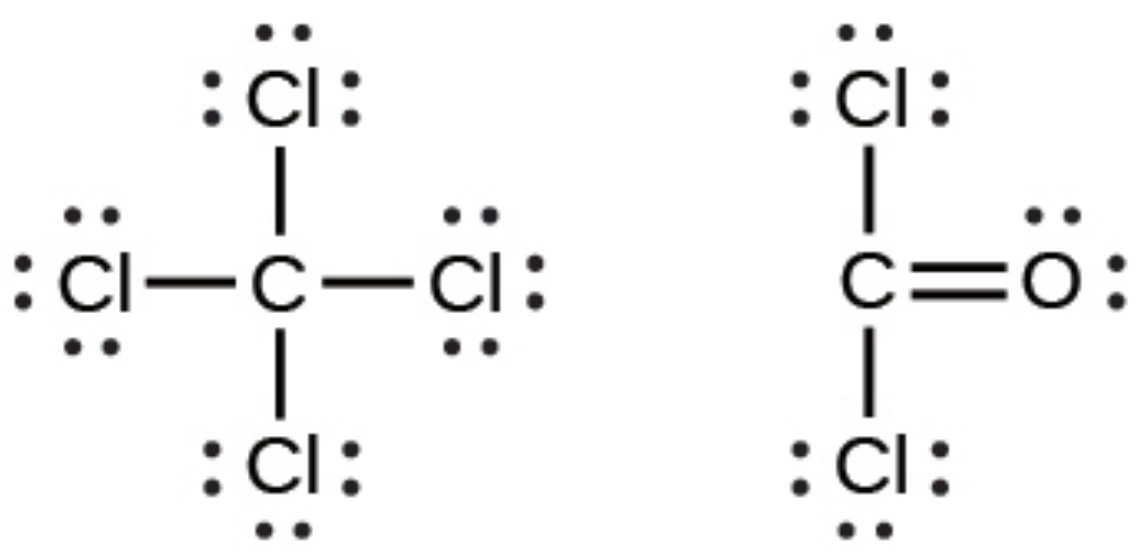

Identify the atoms that correspond to each of the 
following electron configurations. Then, write the Lewis symbol for the common ion formed from each atom:
(a) $1 s^{2} 2 s^{2} 2 p^{5}$
(b) $1 s^{2} 2 s^{2} 2 p^{6} 3 s^{2}$
(c) $1 s^{2} 2 s^{2} 2 p^{6} 3 s^{2} 3 p^{6} 4 s^{2} 3 d^{10}$
(d) $1 s^{2} 2 s^{2} 2 p^{6} 3 s^{2} 3 p^{6} 4 s^{2} 3 d^{10} 4 p^{4}$
(e) $1 s^{2} 2 s^{2} 2 p^{6} 3 s^{2} 3 p^{6} 4 s^{2} 3 d^{10} 4 p^{1}$

The arrangement of atoms in several biologically important molecules is given here. Complete the Lewis structures of these molecules by adding multiple bonds and lone pairs. Do not add any more atoms.

(a) the amino acid serine:

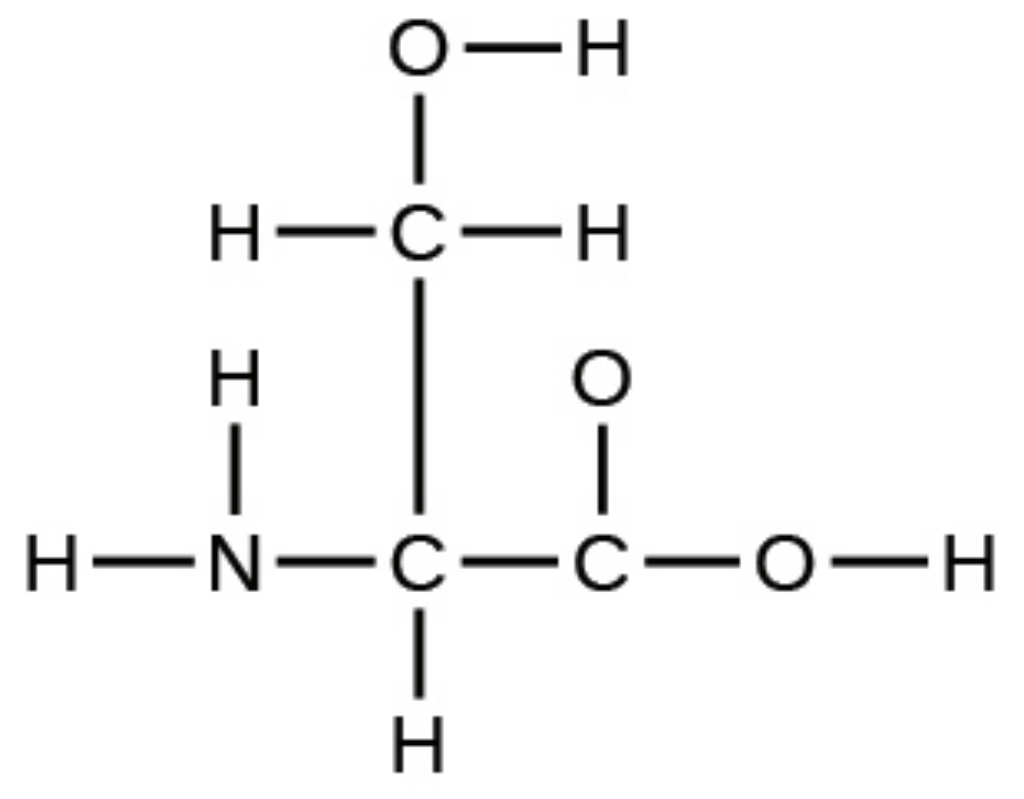

(b) urea:

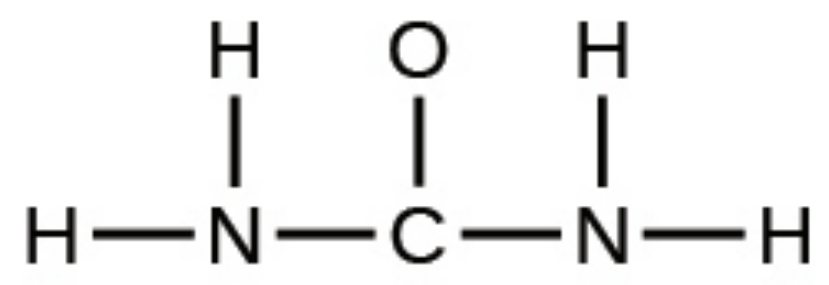

(c) pyruvic acid: 


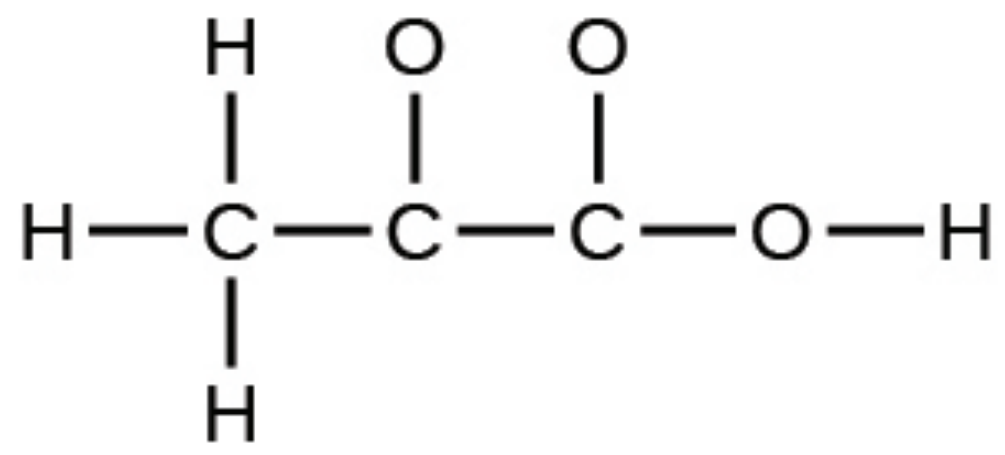

(d) uracil:

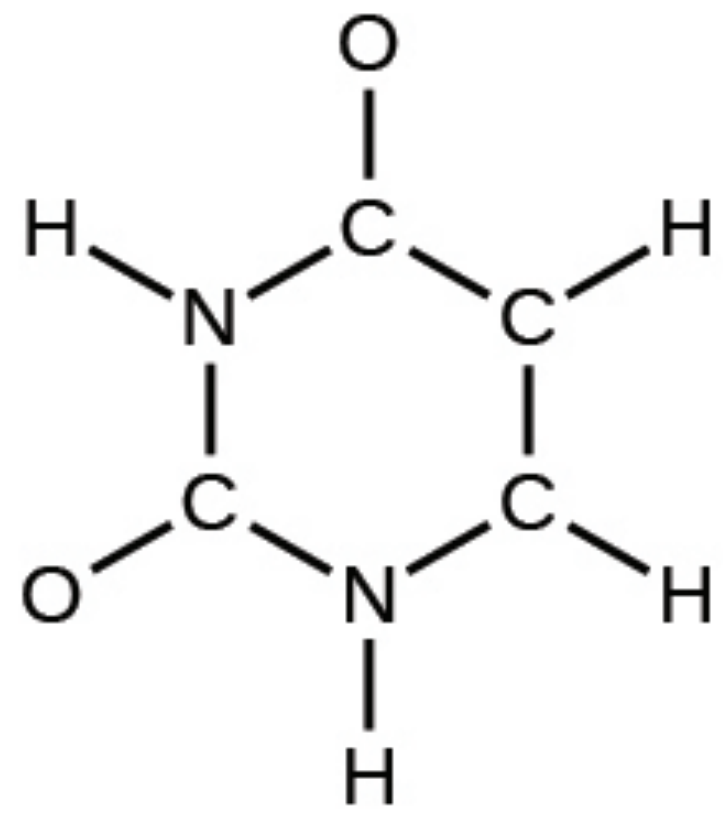

(e) carbonic acid:

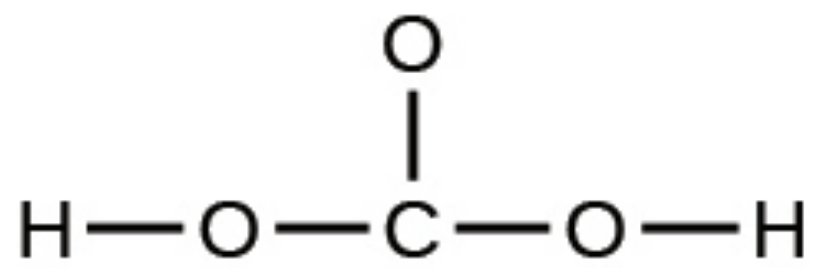

(a) 


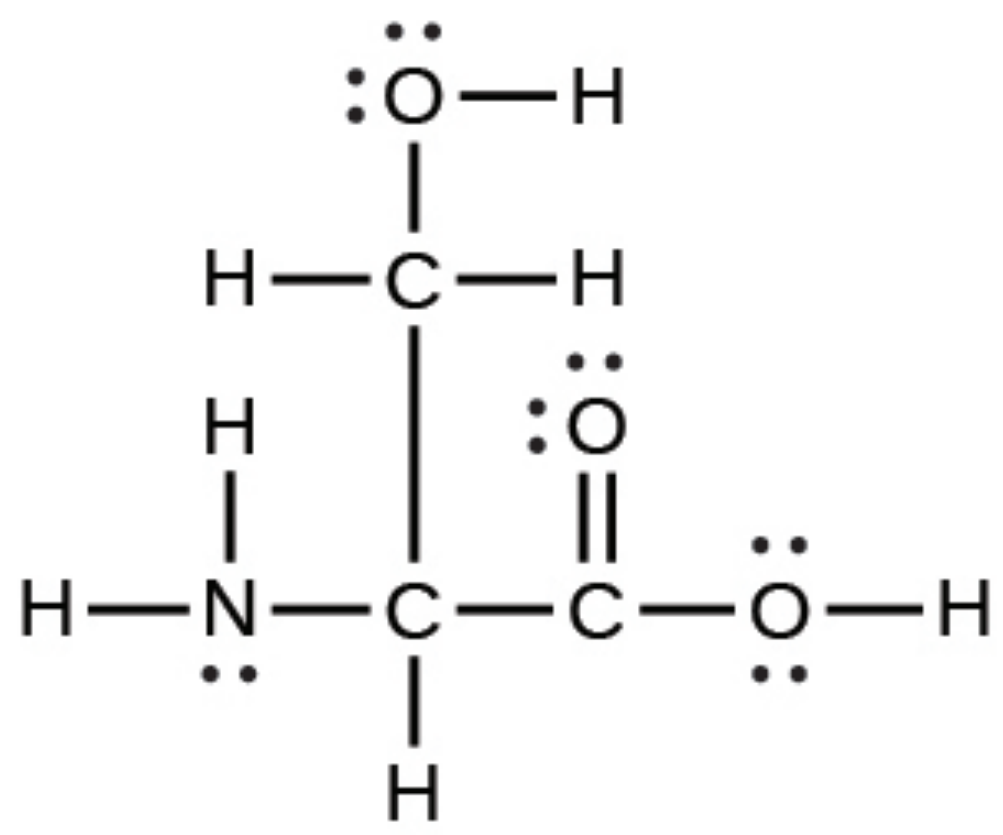

(b)

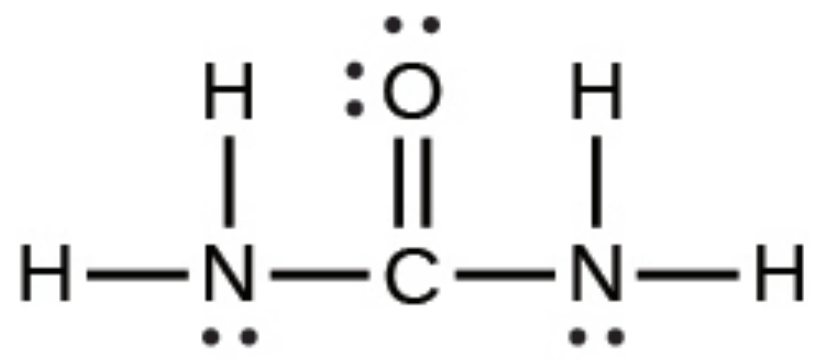

(c)<smiles>CC(=O)C(=O)O</smiles>

(d) 


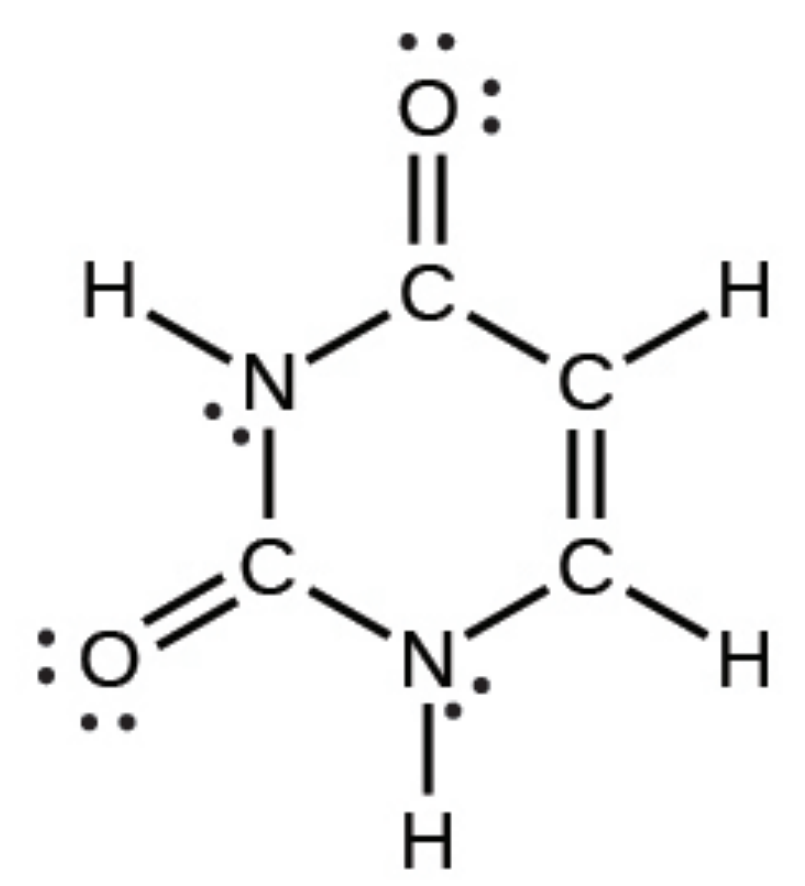

(e)

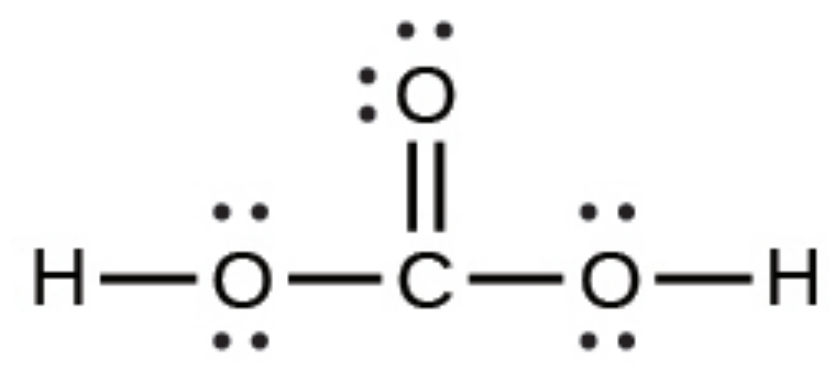

A compound with a molar mass of about $28 \mathrm{~g} / \mathrm{mol}$ contains $85.7 \%$ carbon and $14.3 \%$ hydrogen by mass. Write the Lewis structure for a molecule of the compound.

A compound with a molar mass of about $42 \mathrm{~g} / \mathrm{mol}$ contains $85.7 \%$ carbon and $14.3 \%$ hydrogen by mass. Write the Lewis structure for a molecule of the compound. 
<smiles>C=CC</smiles>

Two arrangements of atoms are possible for a compound with a molar mass of about $45 \mathrm{~g} / \mathrm{mol}$ that contains $52.2 \%$ $\mathrm{C}, 13.1 \% \mathrm{H}$, and $34.7 \% \mathrm{O}$ by mass. Write the Lewis structures for the two molecules.

How are single, double, and triple bonds similar? How do they differ?

Each bond includes a sharing of electrons between atoms. Two electrons are shared in a single bond; four electrons are shared in a double bond; and six electrons are shared in a triple bond.

Glossary

double bond

covalent bond in which two pairs of electrons are shared between two atoms

free radical

molecule that contains an odd number of electrons

hypervalent molecule

molecule containing at least one main group element that has more than eight electrons in its valence shell 


\section{Lewis structure}

diagram showing lone pairs and bonding pairs of electrons in a molecule or an ion

\section{Lewis symbol}

symbol for an element or monatomic ion that uses a dot to represent each valence electron in the element or ion

\section{lone pair}

two (a pair of) valence electrons that are not used to form a covalent bond

\section{octet rule}

guideline that states main group atoms will form structures in which eight valence electrons interact with each nucleus, counting bonding electrons as interacting with both atoms connected by the bond

\section{single bond}

bond in which a single pair of electrons is shared between two atoms

\section{triple bond}

bond in which three pairs of electrons are shared between two atoms 
35.

\section{FORMAL CHARGES AND RESONANCE}

Learning Objectives

By the end of this section, you will be able to:

- Compute formal charges for atoms in any Lewis structure

- Use formal charges to identify the most reasonable Lewis structure for a given molecule

- Explain the concept of resonance and draw Lewis structures representing resonance forms for a given molecule

In the previous section, we discussed how to write Lewis structures for molecules and polyatomic ions. As we have seen, however, in some cases, there is seemingly more than one valid structure for a molecule. We can use the concept of formal charges to help us predict the most 
appropriate Lewis structure when more than one is reasonable.

\section{Calculating Formal Charge}

The formal charge of an atom in a molecule is the hypothetical charge the atom would have if we could redistribute the electrons in the bonds evenly between the atoms. Another way of saying this is that formal charge results when we take the number of valence electrons of a neutral atom, subtract the nonbonding electrons, and then subtract the number of bonds connected to that atom in the Lewis structure.

Thus, we calculate formal charge as follows:

formal charge $=$ valence shell electrons (free atom) - lone pair electrons $\frac{1}{2}$ bonding electrons

We can double-check formal charge calculations by determining the sum of the formal charges for the whole structure. The sum of the formal charges of all atoms in a molecule must be zero; the sum of the formal charges in an ion should equal the charge of the ion.

We must remember that the formal charge calculated for an atom is not the actual charge of the atom in the molecule. Formal charge is only a useful bookkeeping procedure; it does not indicate the presence of actual charges.

Calculating Formal Charge from Lewis Structures Assign formal charges to each atom in the interhalogen ion $\mathrm{ICl}_{4}$.

Solution

1. We divide the bonding electron pairs equally for 
all I-Cl bonds:

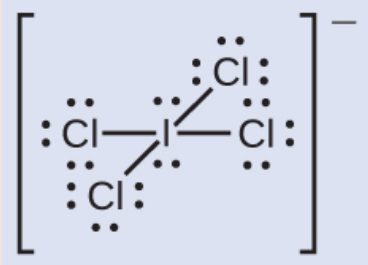

2. We assign lone pairs of electrons to their atoms. Each $\mathrm{Cl}$ atom now has seven electrons assigned to it, and the I atom has eight.

3. Subtract this number from the number of valence electrons for the neutral atom:

I: $7-8=-1$

$\mathrm{Cl}: 7-7=0$

The sum of the formal charges of all the atoms equals -1 , which is identical to the charge of the ion $(-1)$.

Check Your Learning Calculate the formal charge for each atom in the carbon monoxide molecule:

$: C \equiv O:$

Answer:

C $-1,0+1$

Calculating Formal Charge from Lewis Structures Assign formal charges to each atom in the interhalogen molecule $\mathrm{BrCl}_{3}$.

Solution

1. Assign one of the electrons in each $\mathrm{Br}-\mathrm{Cl}$ bond to the $\mathrm{Br}$ atom and one to the $\mathrm{Cl}$ atom in that bond: 
<smiles></smiles>

2. Assign the lone pairs to their atom. Now each $\mathrm{Cl}$ atom has seven electrons and the $\mathrm{Br}$ atom has seven electrons.

3. Subtract this number from the number of valence electrons for the neutral atom. This gives the formal charge:

$\mathrm{Br}: 7-7=0$

$\mathrm{Cl}: 7-7=0$

All atoms in $\mathrm{BrCl}_{3}$ have a formal charge of zero, and the sum of the formal charges totals zero, as it must in a neutral molecule.

Check Your Learning Determine the formal charge for each atom in $\mathrm{NCl}_{3}$.

Answer:

$\mathrm{N}$ : 0; all three $\mathrm{Cl}$ atoms: 0

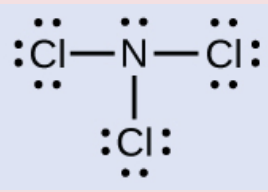

Using Formal Charge to Predict Molecular Structure

The arrangement of atoms in a molecule or ion is called its molecular structure. In many cases, following the steps for writing Lewis structures may lead to more than one possible molecular structure-different multiple bond and lone-pair electron placements or different arrangements of atoms, for instance. A few guidelines involving formal 
charge can be helpful in deciding which of the possible structures is most likely for a particular molecule or ion:

1. A molecular structure in which all formal charges are zero is preferable to one in which some formal charges are not zero.

2. If the Lewis structure must have nonzero formal charges, the arrangement with the smallest nonzero formal charges is preferable.

3. Lewis structures are preferable when adjacent formal charges are zero or of the opposite sign.

4. When we must choose among several Lewis structures with similar distributions of formal charges, the structure with the negative formal charges on the more electronegative atoms is preferable.

To see how these guidelines apply, let us consider some possible structures for carbon dioxide, $\mathrm{CO}_{2}$. We know from our previous discussion that the less electronegative atom typically occupies the central position, but formal charges allow us to understand why this occurs. We can draw three possibilities for the structure: carbon in the center and double bonds, carbon in the center with a single and triple bond, and oxygen in the center with double bonds:

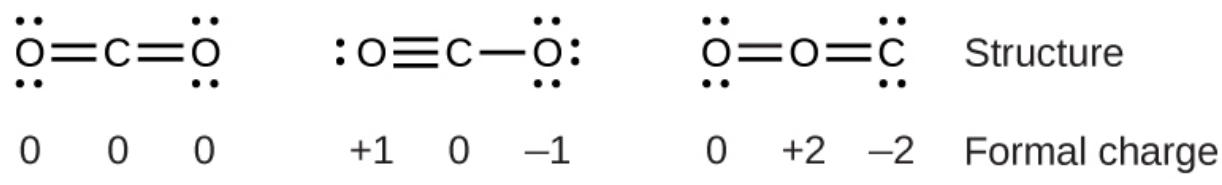

Comparing the three formal charges, we can definitively identify the structure on the left as preferable because it has only formal charges of zero (Guideline 1). 
As another example, the thiocyanate ion, an ion formed from a carbon atom, a nitrogen atom, and a sulfur atom, could have three different molecular structures: $\mathrm{CNS}^{-}$, $\mathrm{NCS}^{-}$, or $\mathrm{CSN}^{-}$. The formal charges present in each of these molecular structures can help us pick the most likely arrangement of atoms. Possible Lewis structures and the formal charges for each of the three possible structures for the thiocyanate ion are shown here:

$$
\begin{array}{lllllll}
\text { Structure } & {[: \ddot{\mathrm{N}}=\mathrm{c}=\ddot{\mathrm{S}}:]^{-}}
\end{array} \quad[\because \ddot{\mathrm{C}}=\mathrm{N}=\ddot{\mathrm{S}}:]^{-} \quad[: \ddot{\mathrm{C}}=\mathrm{s}=\ddot{\mathrm{N}}:]^{-}
$$

Note that the sum of the formal charges in each case is equal to the charge of the ion $(-1)$. However, the first arrangement of atoms is preferred because it has the lowest number of atoms with nonzero formal charges (Guideline 2). Also, it places the least electronegative atom in the center, and the negative charge on the more electronegative element (Guideline 4).

Using Formal Charge to Determine Molecular Structure Nitrous oxide, $\mathrm{N}_{2} \mathrm{O}$, commonly known as laughing gas, is used as an anesthetic in minor surgeries, such as the routine extraction of wisdom teeth. Which is the likely structure for nitrous oxide?

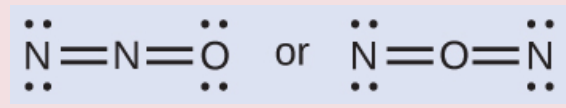

Solution Determining formal charge yields the following:

$$
\begin{aligned}
& : \ddot{N}=N=\ddot{O}: \quad: \ddot{N}=O=\ddot{N}: \\
& \begin{array}{llllll}
-1 & +1 & 0 & -1 & +2 & -1
\end{array}
\end{aligned}
$$

The structure with a terminal oxygen atom best satisfies the criteria for the most stable distribution of formal charge: 


\section{$: \ddot{N}=N=\ddot{O}:$}

The number of atoms with formal charges are minimized (Guideline 2), and there is no formal charge larger than one (Guideline 2). This is again consistent with the preference for having the less electronegative atom in the central position.

Check Your Learning Which is the most likely molecular structure for the nitrite $\left(\mathrm{NO}_{2}\right)$ ion?

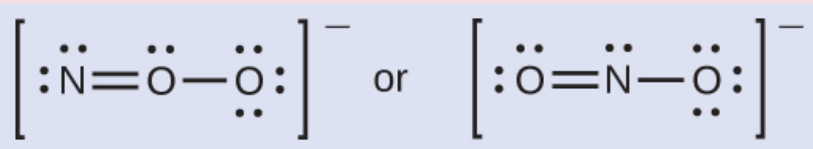

Answer:

$\mathrm{ONO}^{-}$

\section{Resonance}

You may have noticed that the nitrite anion in (Figure) can have two possible structures with the atoms in the same positions. The electrons involved in the $\mathrm{N}-\mathrm{O}$ double bond, however, are in different positions:
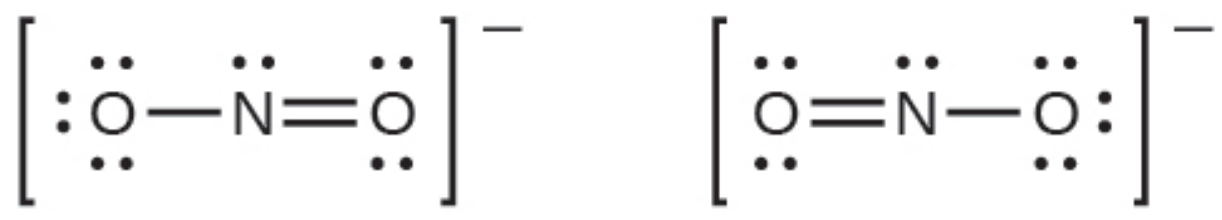

If nitrite ions do indeed contain a single and a double bond, we would expect for the two bond lengths to be different. A double bond between two atoms is shorter (and stronger) than a single bond between the same two atoms. Experiments show, however, that both $\mathrm{N}-\mathrm{O}$ bonds in $\mathrm{NO}_{2}$ have the same strength and length, and are identical in all other properties.

It is not possible to write a single Lewis structure for $\mathrm{NO}_{2}$ in which nitrogen has an octet and both bonds are 
equivalent. Instead, we use the concept of resonance: if two or more Lewis structures with the same arrangement of atoms can be written for a molecule or ion, the actual distribution of electrons is an average of that shown by the various Lewis structures. The actual distribution of electrons in each of the nitrogen-oxygen bonds in $\mathrm{NO}_{2}$ is the average of a double bond and a single bond. We call the individual Lewis structures resonance forms. The actual electronic structure of the molecule (the average of the resonance forms) is called a resonance hybrid of the individual resonance forms. A double-headed arrow between Lewis structures indicates that they are resonance forms. Thus, the electronic structure of the $\mathrm{NO}_{2}$ ion is shown as:

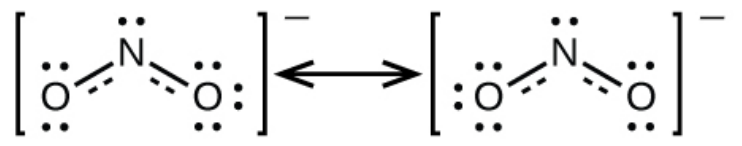

We should remember that a molecule described as a resonance hybrid never possesses an electronic structure described by either resonance form. It does not fluctuate between resonance forms; rather, the actual electronic structure is always the average of that shown by all resonance forms. George Wheland, one of the pioneers of resonance theory, used a historical analogy to describe the relationship between resonance forms and resonance hybrids. A medieval traveler, having never before seen a rhinoceros, described it as a hybrid of a dragon and a unicorn because it had many properties in common with both. Just as a rhinoceros is neither a dragon sometimes nor a unicorn at other times, a resonance hybrid is neither of its resonance forms at any given time. Like a rhinoceros, it is a real entity that experimental evidence 
has shown to exist. It has some characteristics in common with its resonance forms, but the resonance forms themselves are convenient, imaginary images (like the unicorn and the dragon).

The carbonate anion, $\mathrm{CO}_{3}{ }^{2}$, provides a second example of resonance:

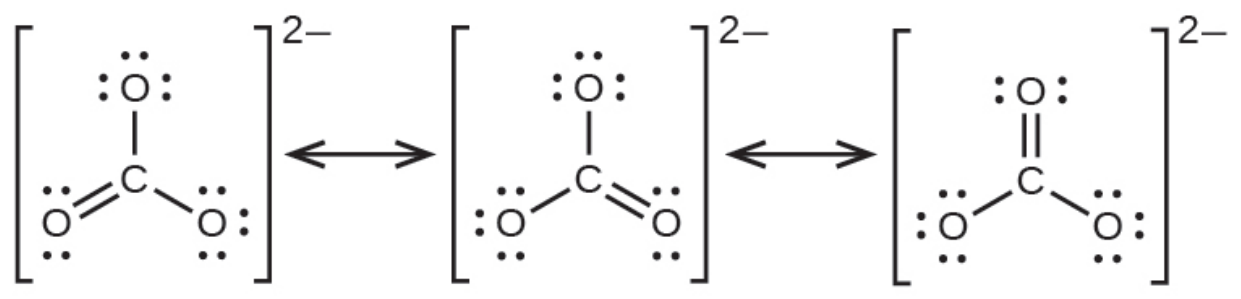

One oxygen atom must have a double bond to carbon to complete the octet on the central atom. All oxygen atoms, however, are equivalent, and the double bond could form from any one of the three atoms. This gives rise to three resonance forms of the carbonate ion. Because we can write three identical resonance structures, we know that the actual arrangement of electrons in the carbonate ion is the average of the three structures. Again, experiments show that all three $\mathrm{C}-\mathrm{O}$ bonds are exactly the same.

The online Lewis Structure Make includes many examples to practice drawing resonance structures.

Key Concepts and Summary

In a Lewis structure, formal charges can be assigned to each atom by treating each bond as if one-half of the electrons are assigned to each atom. These hypothetical formal charges are a guide to determining the most appropriate Lewis structure. A structure in which the formal charges are as close to zero as possible is preferred. Resonance occurs in cases where two or more Lewis 
structures with identical arrangements of atoms but different distributions of electrons can be written. The actual distribution of electrons (the resonance hybrid) is an average of the distribution indicated by the individual Lewis structures (the resonance forms).

Key Equations - formal charge $=$ valence shell electrons (free atom $)-$ one pair electrons -
$\frac{1}{2}$ bonding electrons

Chemistry End of Chapter Exercises

Write resonance forms that describe the distribution of electrons in each of these molecules or ions.

(a) selenium dioxide, $\mathrm{OSeO}$

(b) nitrate ion, $\mathrm{NO}_{3}$

(c) nitric acid, $\mathrm{HNO}_{3}(\mathrm{~N}$ is bonded to an $\mathrm{OH}$ group and two $\mathrm{O}$ atoms)

(d) benzene, $\mathrm{C}_{6} \mathrm{H}_{6}$ :

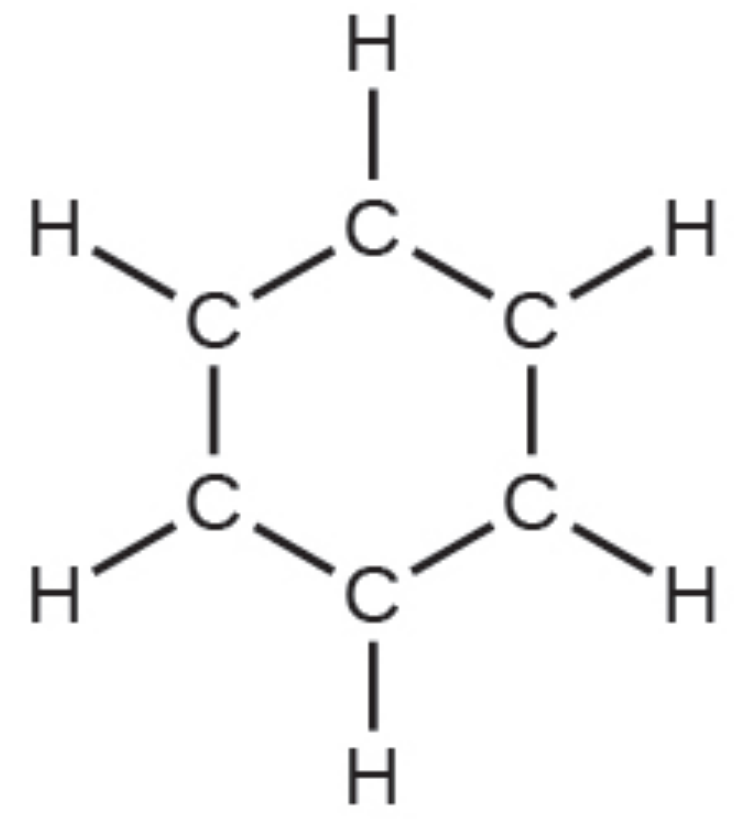

(e) the formate ion: 


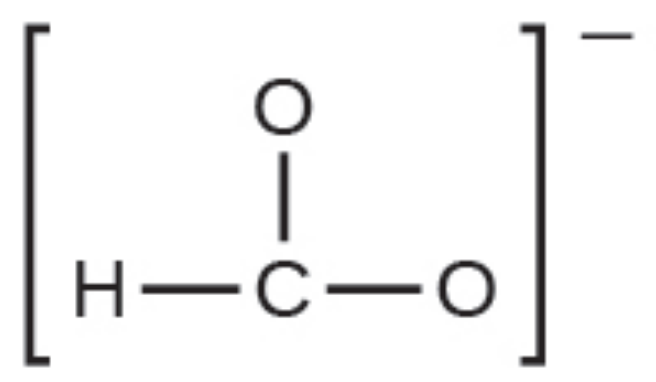

Write resonance forms that describe the distribution of electrons in each of these molecules or ions.

(a) sulfur dioxide, $\mathrm{SO}_{2}$

(b) carbonate ion, $\mathrm{CO}_{3}{ }^{2}$

(c) hydrogen carbonate ion, $\mathrm{HCO}_{3}$ (C is bonded to an $\mathrm{OH}$ group and two $\mathrm{O}$ atoms)

(d) pyridine:

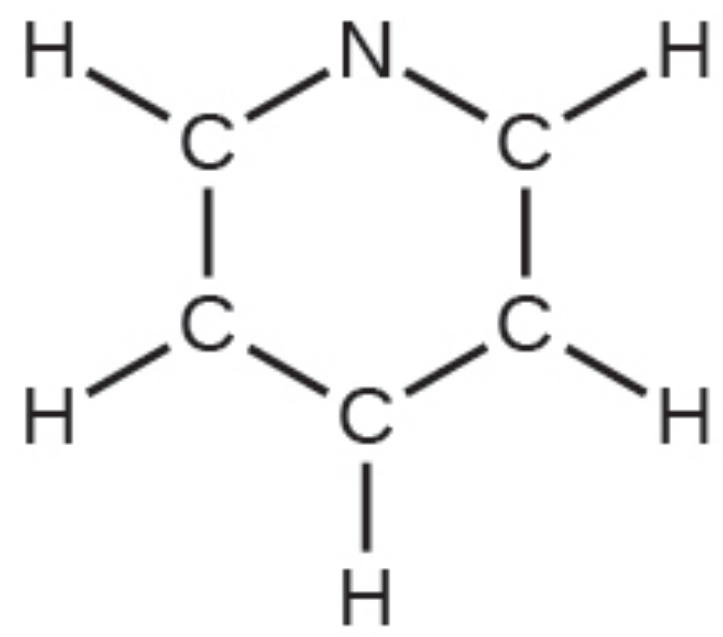

(e) the allyl ion:

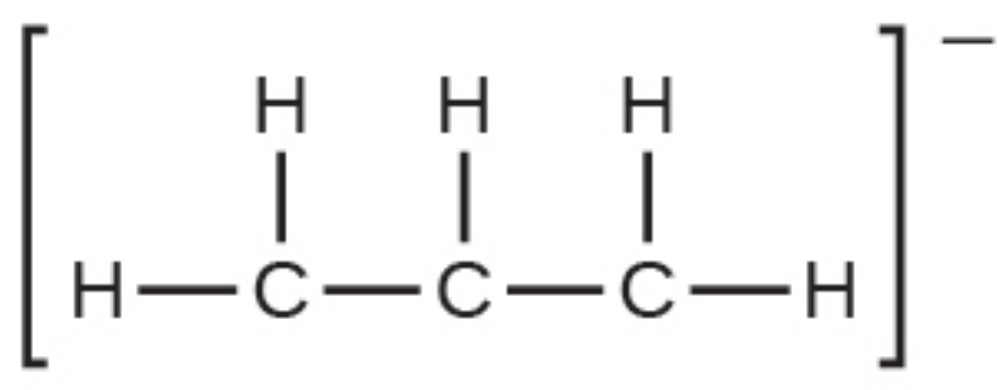


(a)

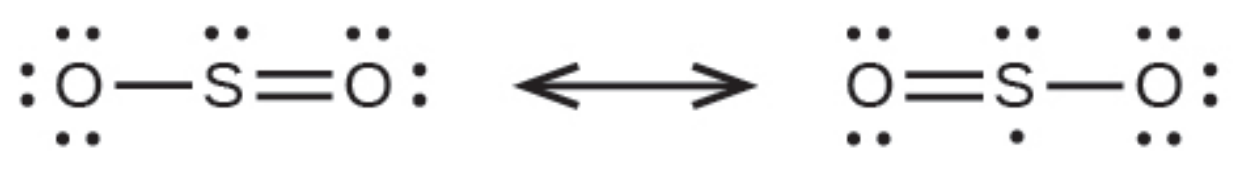

(b)

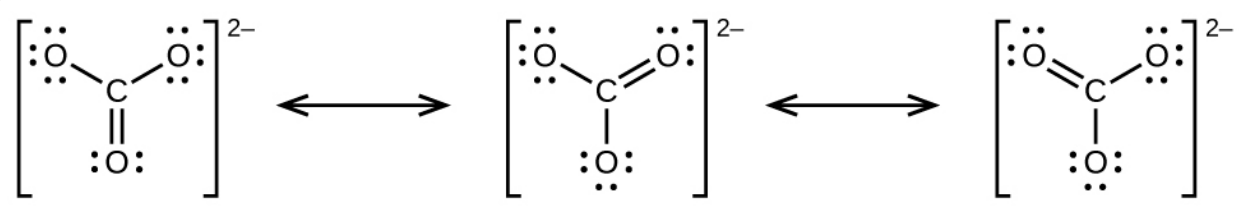

(c)

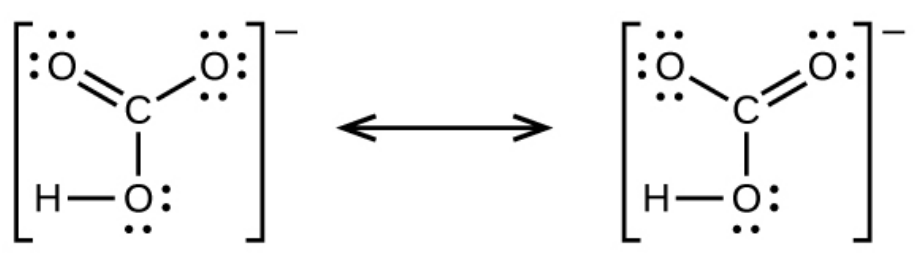

(d)<smiles>c1ccccc1</smiles><smiles>c1ccncc1</smiles>

(e)<smiles>CCCCC</smiles>

Write the resonance forms of ozone, $\mathrm{O}_{3}$, the component of the upper atmosphere that protects the Earth from ultraviolet radiation. 
Sodium nitrite, which has been used to preserve bacon and other meats, is an ionic compound. Write the resonance forms of the nitrite ion, $\mathrm{NO}_{2}{ }^{-}$.

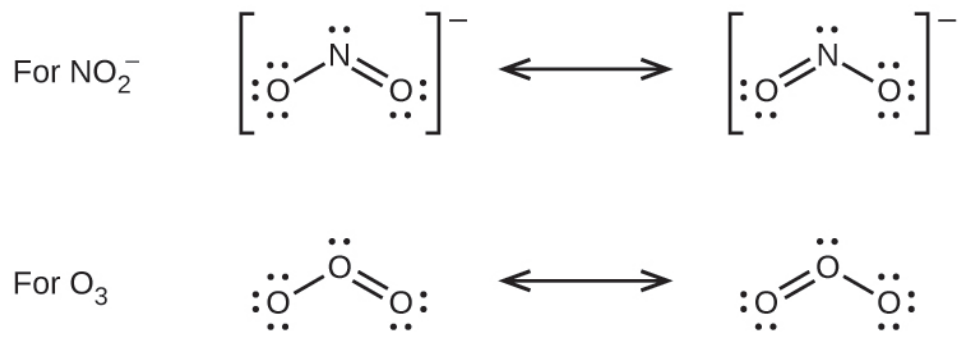

In terms of the bonds present, explain why acetic acid, $\mathrm{CH}_{3} \mathrm{CO}_{2} \mathrm{H}$, contains two distinct types of carbon-oxygen bonds, whereas the acetate ion, formed by loss of a hydrogen ion from acetic acid, only contains one type of carbon-oxygen bond. The skeleton structures of these species are shown:<smiles>CC(C)C(C)O</smiles>

Write the Lewis structures for the following, and include resonance structures where appropriate. Indicate which has the strongest carbon-oxygen bond.

(a) $\mathrm{CO}_{2}$

(b) $\mathrm{CO}$

(a)<smiles>O=C=O</smiles> 
(b)

$$
: c \equiv 0:
$$

CO has the strongest carbon-oxygen bond because there is a triple bond joining $\mathrm{C}$ and $\mathrm{O} . \mathrm{CO}_{2}$ has double bonds.

Toothpastes containing sodium hydrogen carbonate (sodium bicarbonate) and hydrogen peroxide are widely used. Write Lewis structures for the hydrogen carbonate ion and hydrogen peroxide molecule, with resonance forms where appropriate.

Determine the formal charge of each element in the following:
(a) $\mathrm{HCl}$
(b) $\mathrm{CF}_{4}$
(c) $\mathrm{PCl}_{3}$
(d) $\mathrm{PF}_{5}$

(a) H: 0, Cl: 0; (b) C: 0, F: 0; (c) P: 0, Cl 0; (d) P: 0, F: 0

Determine the formal charge of each element in the following:
(a) $\mathrm{H}_{3} \mathrm{O}^{+}$
(b) $\mathrm{SO}_{4}^{2}$
(c) $\mathrm{NH}_{3}$
(d) $\mathrm{O}_{2}{ }^{2}$
(e) $\mathrm{H}_{2} \mathrm{O}_{2}$

Calculate the formal charge of chlorine in the molecules $\mathrm{Cl}_{2}, \mathrm{BeCl}_{2}$, and $\mathrm{ClF}_{5}$. 
$\mathrm{Cl}$ in $\mathrm{Cl}_{2}: 0 ; \mathrm{Cl}$ in $\mathrm{BeCl}_{2}: 0 ; \mathrm{Cl}$ in $\mathrm{ClF}_{5}: 0$

Calculate the formal charge of each element in the following compounds and ions:
(a) $\mathrm{F}_{2} \mathrm{CO}$
(b) $\mathrm{NO}^{-}$
(c) $\mathrm{BF}_{4}$
(d) $\mathrm{SnCl}_{3}$
(e) $\mathrm{H}_{2} \mathrm{CCH}_{2}$
(f) $\mathrm{ClF}_{3}$
(g) $\mathrm{SeF}_{6}$
(h) $\mathrm{PO}_{4}{ }^{3}$

Draw all possible resonance structures for each of these compounds. Determine the formal charge on each atom in each of the resonance structures:
(a) $\mathrm{O}_{3}$
(b) $\mathrm{SO}_{2}$
(c) $\mathrm{NO}_{2}$
(d) $\mathrm{NO}_{3}$

(a)

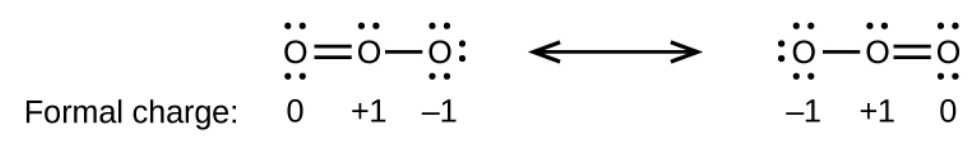

(b)

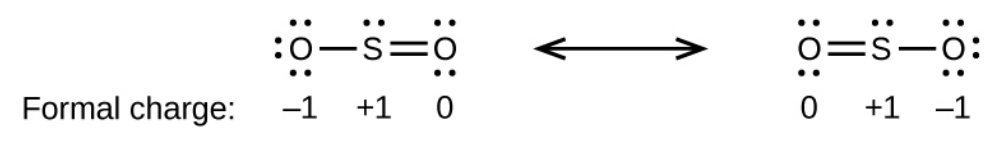

(c) 


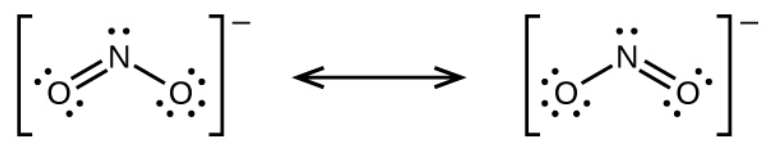

Formal charge: $\quad \begin{array}{lll}0 & 0 & -1\end{array}$

$\begin{array}{lll}-1 & 0 & 0\end{array}$

(d)

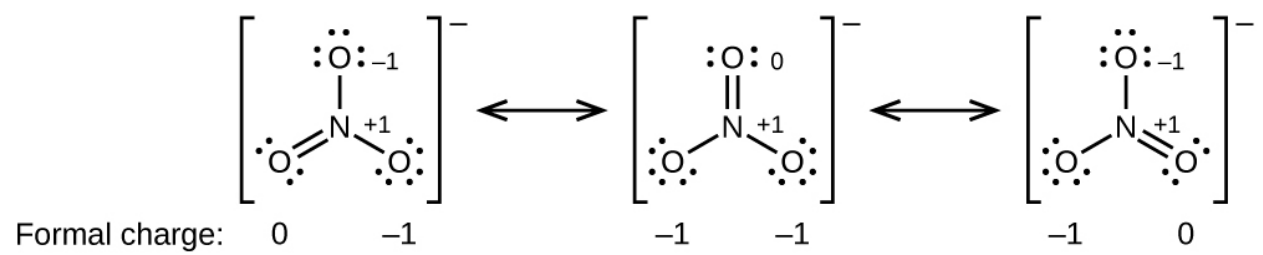

Based on formal charge considerations, which of the following would likely be the correct arrangement of atoms in nitrosyl chloride: CINO or ClON?

Based on formal charge considerations, which of the following would likely be the correct arrangement of atoms in hypochlorous acid: $\mathrm{HOCl}$ or $\mathrm{OClH}$ ?

\section{$\mathrm{HOCl}$}

Based on formal charge considerations, which of the following would likely be the correct arrangement of atoms in sulfur dioxide: OSO or SOO?

Draw the structure of hydroxylamine, $\mathrm{H}_{3} \mathrm{NO}$, and assign formal charges; look up the structure. Is the actual structure consistent with the formal charges?

The structure that gives zero formal charges is consistent with the actual structure: 


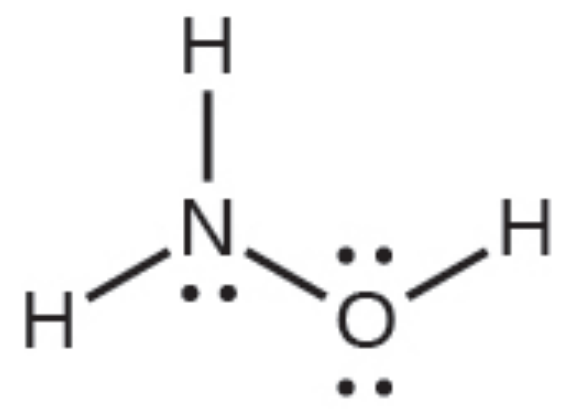

Iodine forms a series of fluorides (listed here). Write Lewis structures for each of the four compounds and determine the formal charge of the iodine atom in each molecule:
(a) IF
(b) $\mathrm{IF}_{3}$
(c) $\mathrm{IF}_{5}$
(d) $\mathrm{IF}_{7}$

Write the Lewis structure and chemical formula of the compound with a molar mass of about $70 \mathrm{~g} / \mathrm{mol}$ that contains $19.7 \%$ nitrogen and $80.3 \%$ fluorine by mass, and determine the formal charge of the atoms in this compound.

$\mathrm{NF}_{3}$;

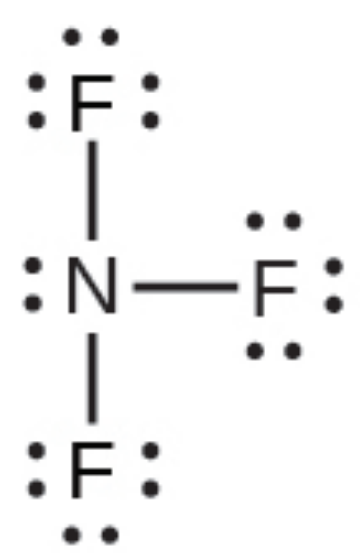


Which of the following structures would we expect for nitrous acid? Determine the formal charges:

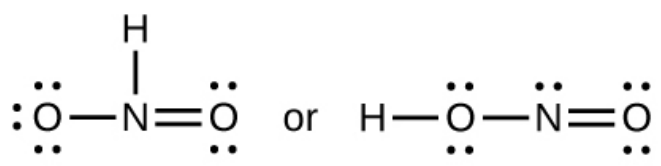

Sulfuric acid is the industrial chemical produced in greatest quantity worldwide. About 90 billion pounds are produced each year in the United States alone. Write the Lewis structure for sulfuric acid, $\mathrm{H}_{2} \mathrm{SO}_{4}$, which has two oxygen atoms and two $\mathrm{OH}$ groups bonded to the sulfur.

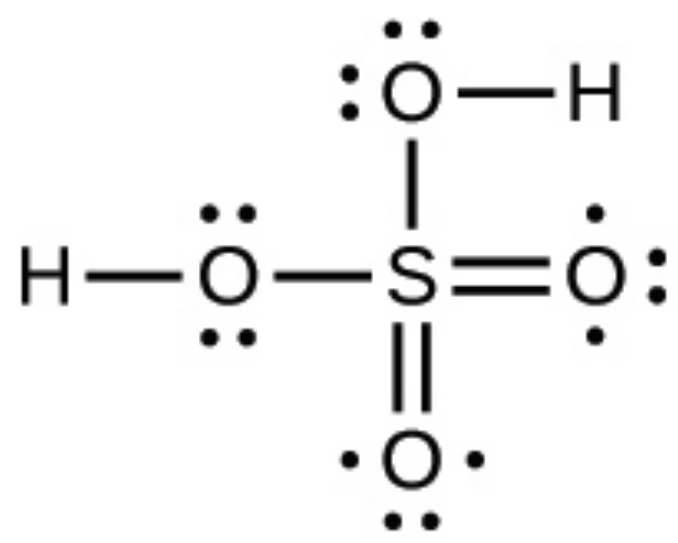

Glossary

formal charge

charge that would result on an atom by taking the number of valence electrons on the neutral atom and subtracting the nonbonding electrons and the number of 
bonds (one-half of the bonding electrons) molecular structure

arrangement of atoms in a molecule or ion resonance

situation in which one Lewis structure is insufficient to describe the bonding in a molecule and the average of multiple structures is observed

\section{resonance forms}

two or more Lewis structures that have the same arrangement of atoms but different arrangements of electrons

\section{resonance hybrid}

average of the resonance forms shown by the individual Lewis structures 
36.

\section{STRENGTHS OF IONIC AND COVALENT BONDS}

Learning Objectives

By the end of this section, you will be able to:

- Describe the energetics of covalent and ionic bond formation and breakage

- Use the Born-Haber cycle to compute lattice energies for ionic compounds

- Use average covalent bond energies to estimate enthalpies of reaction

A bond's strength describes how strongly each atom is joined to another atom, and therefore how much energy is required to break the bond between the two atoms. In this section, you will learn about the bond strength of covalent bonds, and then compare that to the strength 
of ionic bonds, which is related to the lattice energy of a compound.

Bond Strength: Covalent Bonds

Stable molecules exist because covalent bonds hold the atoms together. We measure the strength of a covalent bond by the energy required to break it, that is, the energy necessary to separate the bonded atoms. Separating any pair of bonded atoms requires energy (see (Figure)). The stronger a bond, the greater the energy required to break it.

The energy required to break a specific covalent bond in one mole of gaseous molecules is called the bond energy or the bond dissociation energy. The bond energy for a diatomic molecule, $\mathrm{D}_{\mathrm{X}-\mathrm{Y}}$, is defined as the standard enthalpy change for the endothermic reaction:

$\mathrm{XY}(g) \mathrm{X}(g)+\mathrm{Y}(g) \quad \mathrm{D}_{\mathrm{XY}}=H$

For example, the bond energy of the pure covalent $\mathrm{H}-\mathrm{H}$ bond, $\mathrm{D}_{\mathrm{H}-\mathrm{H}}$, is $436 \mathrm{~kJ}$ per mole of $\mathrm{H}-\mathrm{H}$ bonds broken:

$\mathrm{H}_{2}(g) 2 \mathrm{H}(g) \quad \mathrm{D}_{\mathrm{HH}}=H=436 \mathrm{~kJ}$

Molecules with three or more atoms have two or more bonds. The sum of all bond energies in such a molecule is equal to the standard enthalpy change for the endothermic reaction that breaks all the bonds in the molecule. For example, the sum of the four $\mathrm{C}-\mathrm{H}$ bond energies in $\mathrm{CH}_{4}, 1660 \mathrm{~kJ}$, is equal to the standard enthalpy change of the reaction:

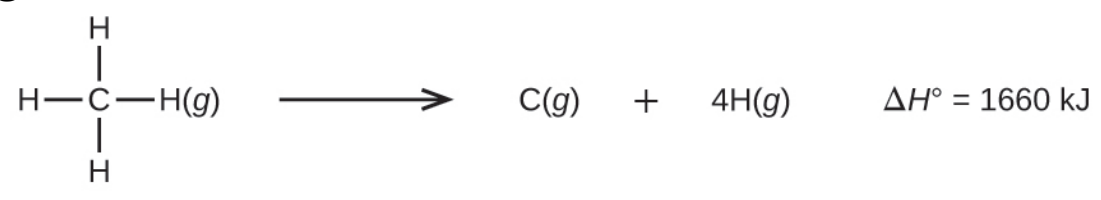


The average $\mathrm{C}-\mathrm{H}$ bond energy, $\mathrm{D}_{\mathrm{C}-\mathrm{H}}$, is $1660 / 4=415 \mathrm{~kJ} /$ mol because there are four moles of $\mathrm{C}-\mathrm{H}$ bonds broken per mole of the reaction. Although the four $\mathrm{C}-\mathrm{H}$ bonds are equivalent in the original molecule, they do not each require the same energy to break; once the first bond is broken (which requires $439 \mathrm{~kJ} / \mathrm{mol}$ ), the remaining bonds are easier to break. The $415 \mathrm{~kJ} / \mathrm{mol}$ value is the average, not the exact value required to break any one bond.

The strength of a bond between two atoms increases as the number of electron pairs in the bond increases. Generally, as the bond strength increases, the bond length decreases. Thus, we find that triple bonds are stronger and shorter than double bonds between the same two atoms; likewise, double bonds are stronger and shorter than single bonds between the same two atoms. Average bond energies for some common bonds appear in (Figure), and a comparison of bond lengths and bond strengths for some common bonds appears in (Figure). When one atom bonds to various atoms in a group, the bond strength typically decreases as we move down the group. For example, $\mathrm{C}-\mathrm{F}$ is $439 \mathrm{~kJ} / \mathrm{mol}, \mathrm{C}-\mathrm{Cl}$ is $330 \mathrm{~kJ} / \mathrm{mol}$, and $\mathrm{C}-\mathrm{Br}$ is $275 \mathrm{~kJ} / \mathrm{mol}$. 
Bond Energies (kJ/mol)

\begin{tabular}{|c|c|c|c|c|c|}
\hline Bond & $\begin{array}{l}\text { Bond } \\
\text { Energy }\end{array}$ & Bond & $\begin{array}{l}\text { Bond } \\
\text { Energy }\end{array}$ & Bond & $\begin{array}{l}\text { Bond } \\
\text { Energy }\end{array}$ \\
\hline $\mathrm{H}-\mathrm{H}$ & 436 & $\mathrm{C}-\mathrm{S}$ & 260 & $\mathrm{~F}-\mathrm{Cl}$ & 255 \\
\hline $\mathrm{H}-\mathrm{C}$ & 415 & $\mathrm{C}-\mathrm{Cl}$ & 330 & $\mathrm{~F}-\mathrm{Br}$ & 235 \\
\hline $\mathrm{H}-\mathrm{N}$ & 390 & $\mathrm{C}-\mathrm{Br}$ & 275 & $\mathrm{Si}-\mathrm{Si}$ & 230 \\
\hline $\mathrm{H}-\mathrm{O}$ & 464 & C-I & 240 & $\mathrm{Si}-\mathrm{P}$ & 215 \\
\hline $\mathrm{H}-\mathrm{F}$ & 569 & $\mathrm{~N}-\mathrm{N}$ & 160 & $\mathrm{Si}-\mathrm{S}$ & 225 \\
\hline $\mathrm{H}-\mathrm{Si}$ & 395 & $\mathrm{~N}=\mathrm{N}$ & 418 & $\mathrm{Si}-\mathrm{Cl}$ & 359 \\
\hline $\mathrm{H}-\mathrm{P}$ & 320 & $\mathrm{~N} \equiv \mathrm{N}$ & 946 & $\mathrm{Si}-\mathrm{Br}$ & 290 \\
\hline $\mathrm{H}-\mathrm{S}$ & 340 & $\mathrm{~N}-\mathrm{O}$ & 200 & $\mathrm{Si}-\mathrm{I}$ & 215 \\
\hline $\mathrm{H}-\mathrm{Cl}$ & 432 & $\mathrm{~N}-\mathrm{F}$ & 270 & $\mathrm{P}-\mathrm{P}$ & 215 \\
\hline $\mathrm{H}-\mathrm{Br}$ & 370 & $\mathrm{~N}-\mathrm{P}$ & 210 & $\mathrm{P}-\mathrm{S}$ & 230 \\
\hline $\mathrm{H}-\mathrm{I}$ & 295 & $\mathrm{~N}-\mathrm{Cl}$ & 200 & $\mathrm{P}-\mathrm{Cl}$ & 330 \\
\hline $\mathrm{C}-\mathrm{C}$ & 345 & $\mathrm{~N}-\mathrm{Br}$ & 245 & $\mathrm{P}-\mathrm{Br}$ & 270 \\
\hline $\mathrm{C}=\mathrm{C}$ & 611 & $\mathrm{O}-\mathrm{O}$ & 140 & $\mathrm{P}-\mathrm{I}$ & 215 \\
\hline $\mathrm{C} \equiv \mathrm{C}$ & 837 & $\mathrm{O}=\mathrm{O}$ & 498 & $S-S$ & 215 \\
\hline $\mathrm{C}-\mathrm{N}$ & 290 & $\mathrm{O}-\mathrm{F}$ & 160 & $\mathrm{~S}-\mathrm{Cl}$ & 250 \\
\hline $\mathrm{C}=\mathrm{N}$ & 615 & $\mathrm{O}-\mathrm{Si}$ & 370 & $\mathrm{~S}-\mathrm{Br}$ & 215 \\
\hline $\mathrm{C} \equiv \mathrm{N}$ & 891 & $\mathrm{O}-\mathrm{P}$ & 350 & $\mathrm{Cl}-\mathrm{Cl}$ & 243 \\
\hline $\mathrm{C}-\mathrm{O}$ & 350 & $\mathrm{O}-\mathrm{Cl}$ & 205 & $\mathrm{Cl}-\mathrm{Br}$ & 220 \\
\hline $\mathrm{C}=\mathrm{O}$ & 741 & $\mathrm{O}-\mathrm{I}$ & 200 & $\mathrm{Cl}-\mathrm{I}$ & 210 \\
\hline $\mathrm{C} \equiv \mathrm{O}$ & 1080 & $\mathrm{~F}-\mathrm{F}$ & 160 & $\mathrm{Br}-\mathrm{Br}$ & 190 \\
\hline $\mathrm{C}-\mathrm{F}$ & 439 & $\mathrm{~F}-\mathrm{Si}$ & 540 & $\mathrm{Br}-\mathrm{I}$ & 180 \\
\hline $\mathrm{C}-\mathrm{Si}$ & 360 & $\mathrm{~F}-\mathrm{P}$ & 489 & I-I & 150 \\
\hline $\mathrm{C}-\mathrm{P}$ & 265 & F-S & 285 & & \\
\hline
\end{tabular}




\begin{tabular}{|c|c|c|}
\hline Bond & Bond Length $(\AA))$ & Bond Energy $(\mathrm{kJ} / \mathrm{mol})$ \\
\hline $\mathrm{C}-\mathrm{C}$ & 1.54 & 345 \\
\hline $\mathrm{C}=\mathrm{C}$ & 1.34 & 611 \\
\hline $\mathrm{C} \equiv \mathrm{C}$ & 1.20 & 837 \\
\hline $\mathrm{C}-\mathrm{N}$ & 1.43 & 290 \\
\hline $\mathrm{C}=\mathrm{N}$ & 1.38 & 615 \\
\hline $\mathrm{C} \equiv \mathrm{N}$ & 1.16 & 891 \\
\hline $\mathrm{C}-\mathrm{O}$ & 1.43 & 350 \\
\hline $\mathrm{C}=\mathrm{O}$ & 1.23 & 741 \\
\hline $\mathrm{C} \equiv \mathrm{O}$ & 1.13 & 1080 \\
\hline
\end{tabular}

We can use bond energies to calculate approximate enthalpy changes for reactions where enthalpies of formation are not available. Calculations of this type will also tell us whether a reaction is exothermic or endothermic. An exothermic reaction ( $\Delta H$ negative, heat produced) results when the bonds in the products are stronger than the bonds in the reactants. An endothermic reaction ( $\Delta H$ positive, heat absorbed) results when the bonds in the products are weaker than those in the reactants.

The enthalpy change, $\Delta H$, for a chemical reaction is approximately equal to the sum of the energy required to break all bonds in the reactants (energy "in", positive sign) plus the energy released when all bonds are formed in the products (energy "out," negative sign). This can be expressed mathematically in the following way:

$H=\mathrm{D}_{\text {bonds broken }}-\mathrm{D}_{\text {bonds formed }}$ 
In this expression, the symbol $\Sigma$ means "the sum of" and D represents the bond energy in kilojoules per mole, which is always a positive number. The bond energy is obtained from a table (like (Figure)) and will depend on whether the particular bond is a single, double, or triple bond. Thus, in calculating enthalpies in this manner, it is important that we consider the bonding in all reactants and products. Because D values are typically averages for one type of bond in many different molecules, this calculation provides a rough estimate, not an exact value, for the enthalpy of reaction.

Consider the following reaction:

$\mathrm{H}_{2}(g)+\mathrm{Cl}_{2}(g) 2 \mathrm{HCl}(g)$

or

$\mathrm{H}-\mathrm{H}(g)+\mathrm{Cl}-\mathrm{Cl}(g) 2 \mathrm{H}-\mathrm{Cl}(g)$

To form two moles of $\mathrm{HCl}$, one mole of $\mathrm{H}-\mathrm{H}$ bonds and one mole of $\mathrm{Cl}-\mathrm{Cl}$ bonds must be broken. The energy required to break these bonds is the sum of the bond energy of the $\mathrm{H}-\mathrm{H}$ bond $(436 \mathrm{~kJ} / \mathrm{mol})$ and the $\mathrm{Cl}-\mathrm{Cl}$ bond $(243 \mathrm{~kJ} / \mathrm{mol})$. During the reaction, two moles of $\mathrm{H}-\mathrm{Cl}$ bonds are formed (bond energy $=432 \mathrm{~kJ} / \mathrm{mol}$ ), releasing $2432 \mathrm{~kJ}$; or $864 \mathrm{~kJ}$. Because the bonds in the products are stronger than those in the reactants, the reaction releases more energy than it consumes:

$$
\begin{aligned}
H & =\mathrm{D}_{\text {bonds broken }}-\mathrm{D}_{\text {bonds formed }} \\
H & =\left[\mathrm{D}_{\mathrm{HH}}+\mathrm{D}_{\mathrm{ClCl}}-2 \mathrm{D}_{\mathrm{HCl}}\right. \\
& =[436+243]-2(432)=-185 \mathrm{~kJ}
\end{aligned}
$$

This excess energy is released as heat, so the reaction is exothermic. Appendix G gives a value for the standard 
molar enthalpy of formation of $\mathrm{HCl}(\mathrm{g}), H_{\mathrm{f}}$, of $-92.307 \mathrm{~kJ} /$ mol. Twice that value is $-184.6 \mathrm{~kJ}$, which agrees well with the answer obtained earlier for the formation of two moles of $\mathrm{HCl}$.

Using Bond Energies to Calculate Approximate Enthalpy Changes Methanol, $\mathrm{CH}_{3} \mathrm{OH}$, may be an excellent alternative fuel. The high-temperature reaction of steam and carbon produces a mixture of the gases carbon monoxide, $\mathrm{CO}$, and hydrogen, $\mathrm{H}_{2}$, from which methanol can be produced. Using the bond energies in (Figure), calculate the approximate enthalpy change, $\Delta H$, for the reaction here:

\section{$\mathrm{CO}(g)+2 \mathrm{H}_{2}(g) \mathrm{CH}_{3} \mathrm{OH}(g)$}

SolutionFirst, we need to write the Lewis structures of the reactants and the products:

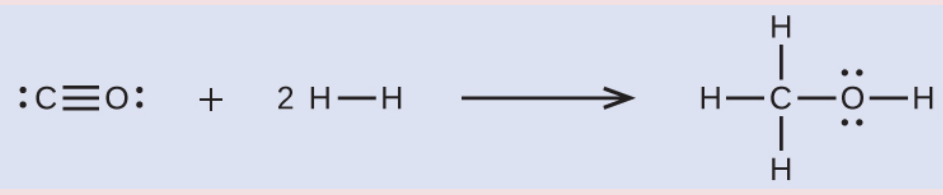

From this, we see that $\Delta H$ for this reaction involves the energy required to break a $\mathrm{C}-\mathrm{O}$ triple bond and two $\mathrm{H}-\mathrm{H}$ single bonds, as well as the energy produced by the formation of three $\mathrm{C}-\mathrm{H}$ single bonds, a $\mathrm{C}-\mathrm{O}$ single bond, and an $\mathrm{O}-\mathrm{H}$ single bond. We can express this as follows:

$$
\begin{aligned}
& H=\mathrm{D}_{\text {bonds broken }}-\mathrm{D}_{\text {bonds formed }} \\
& H=\left[\mathrm{D}_{\mathrm{C} \equiv \mathrm{O}}+2\left(\mathrm{D}_{\mathrm{HH}}\right)\right]-\left[3\left(\mathrm{D}_{\mathrm{CH}}\right)+\mathrm{D}_{\mathrm{CO}}+\mathrm{D}_{\mathrm{OH}}\right]
\end{aligned}
$$

Using the bond energy values in (Figure), we obtain:

$$
\begin{aligned}
H & =[1080+2(436)]-[3(415)+350+464] \\
& =-107 \mathrm{~kJ}
\end{aligned}
$$

We can compare this value to the value calculated based on $H_{\mathrm{f}}$ data from Appendix G: 


$$
\begin{aligned}
H & =\left[H_{\mathrm{f}} \mathrm{CH}_{3} \mathrm{OH}(g)\right]-\left[H_{\mathrm{f}} \mathrm{CO}(g)+2 H_{\mathrm{f}} \mathrm{H}_{2}\right] \\
& =[-201.0]-[-110.52+20] \\
& =-90.5 \mathrm{~kJ}
\end{aligned}
$$

Note that there is a fairly significant gap between the values calculated using the two different methods. This occurs because $D$ values are the average of different bond strengths; therefore, they often give only rough agreement with other data.

Check Your Learning Ethyl alcohol, $\mathrm{CH}_{3} \mathrm{CH}_{2} \mathrm{OH}$, was one of the first organic chemicals deliberately synthesized by humans. It has many uses in industry, and it is the alcohol contained in alcoholic beverages. It can be obtained by the fermentation of sugar or synthesized by the hydration of ethylene in the following reaction:

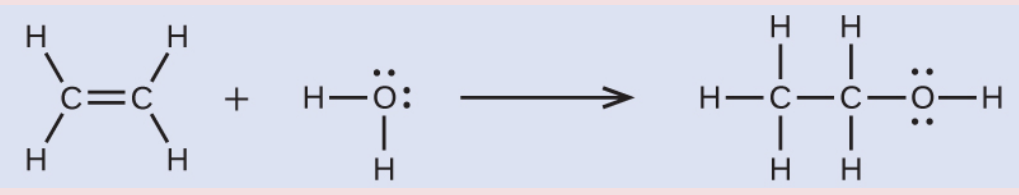

Using the bond energies in (Figure), calculate an approximate enthalpy change, $\Delta H$, for this reaction.

Answer:

$-35 \mathrm{~kJ}$

Ionic Bond Strength and Lattice Energy

An ionic compound is stable because of the electrostatic attraction between its positive and negative ions. The lattice energy of a compound is a measure of the strength of this attraction. The lattice energy ( $\Delta H_{\text {lattice }}$ ) of an ionic compound is defined as the energy required to separate one mole of the solid into its component gaseous ions. For the ionic solid MX, the lattice energy is the enthalpy change of the process: 
$\operatorname{MX}(s) \mathrm{M}^{n+}(g)+\mathrm{X}^{n}(g) \quad H_{\text {lattice }}$

Note that we are using the convention where the ionic solid is separated into ions, so our lattice energies will be endothermic (positive values). Some texts use the equivalent but opposite convention, defining lattice energy as the energy released when separate ions combine to form a lattice and giving negative (exothermic) values. Thus, if you are looking up lattice energies in another reference, be certain to check which definition is being used. In both cases, a larger magnitude for lattice energy indicates a more stable ionic compound. For sodium chloride, $\Delta H_{\text {lattice }}=769 \mathrm{~kJ}$. Thus, it requires $769 \mathrm{~kJ}$ to separate one mole of solid $\mathrm{NaCl}$ into gaseous $\mathrm{Na}^{+}$and $\mathrm{Cl}^{-}$ ions. When one mole each of gaseous $\mathrm{Na}^{+}$and $\mathrm{Cl}^{-}$ions form solid $\mathrm{NaCl}, 769 \mathrm{~kJ}$ of heat is released.

The lattice energy $\Delta H_{\text {lattice }}$ of an ionic crystal can be expressed by the following equation (derived from Coulomb's law, governing the forces between electric charges):

$H_{\text {lattice }}=\frac{\mathrm{C}\left(\mathrm{Z}^{+}\right)(\mathrm{Z})}{\mathrm{R}_{\mathrm{o}}}$

in which $\mathrm{C}$ is a constant that depends on the type of crystal structure; $\mathrm{Z}^{+}$and $\mathrm{Z}^{-}$are the charges on the ions; and $R_{o}$ is the interionic distance (the sum of the radii of the positive and negative ions). Thus, the lattice energy of an ionic crystal increases rapidly as the charges of the ions increase and the sizes of the ions decrease. When all other parameters are kept constant, doubling the charge of both the cation and anion quadruples the lattice energy. For example, the lattice energy of $\operatorname{LiF}\left(\mathrm{Z}^{+}\right.$and $\left.\mathrm{Z}^{-}=1\right)$ is $1023 \mathrm{~kJ} / \mathrm{mol}$, whereas that of $\mathrm{MgO}\left(\mathrm{Z}^{+}\right.$and $\left.\mathrm{Z}^{-}=2\right)$ is 
$3900 \mathrm{~kJ} / \mathrm{mol}\left(\mathrm{R}_{\mathrm{O}}\right.$ is nearly the same-about $200 \mathrm{pm}$ for both compounds).

Different interatomic distances produce different lattice energies. For example, we can compare the lattice energy of $\mathrm{MgF}_{2}(2957 \mathrm{~kJ} / \mathrm{mol})$ to that of $\mathrm{MgI}_{2}(2327 \mathrm{~kJ} / \mathrm{mol})$ to observe the effect on lattice energy of the smaller ionic size of $\mathrm{F}^{-}$as compared to $\mathrm{I}^{-}$.

Lattice Energy Comparisons The precious gem ruby is aluminum oxide, $\mathrm{Al}_{2} \mathrm{O}_{3}$, containing traces of $\mathrm{Cr}^{3+}$. The compound $\mathrm{Al}_{2} \mathrm{Se}_{3}$ is used in the fabrication of some semiconductor devices. Which has the larger lattice energy, $\mathrm{Al}_{2} \mathrm{O}_{3}$ or $\mathrm{Al}_{2} \mathrm{Se}_{3}$ ?

Solution In these two ionic compounds, the charges $\mathrm{Z}^{+}$and $\mathrm{Z}^{-}$are the same, so the difference in lattice energy will depend upon $\mathrm{R}_{0}$. The $\mathrm{O}^{2-}$ ion is smaller than the $\mathrm{Se}^{2-}$ ion. Thus, $\mathrm{Al}_{2} \mathrm{O}_{3}$ would have a shorter interionic distance than $\mathrm{Al}_{2} \mathrm{Se}_{3}$, and $\mathrm{Al}_{2} \mathrm{O}_{3}$ would have the larger lattice energy.

Check Your Learning Zinc oxide, $\mathrm{ZnO}$, is a very effective sunscreen. How would the lattice energy of $\mathrm{ZnO}$ compare to that of $\mathrm{NaCl}$ ?

Answer:

$\mathrm{ZnO}$ would have the larger lattice energy because the $\mathrm{Z}$ values of both the cation and the anion in $\mathrm{ZnO}$ are greater, and the interionic distance of $\mathrm{ZnO}$ is smaller than that of $\mathrm{NaCl}$.

The Born-Haber Cycle

It is not possible to measure lattice energies directly. However, the lattice energy can be calculated using the equation given in the previous section or by using a thermochemical cycle. The Born-Haber cycle is an 
application of Hess's law that breaks down the formation of an ionic solid into a series of individual steps:

- $H_{\mathrm{f}}$, the standard enthalpy of formation of the compound

- $I E$, the ionization energy of the metal

- $E A$, the electron affinity of the nonmetal

- $H_{s}$, the enthalpy of sublimation of the metal

- $D$, the bond dissociation energy of the nonmetal

- $\Delta H_{\text {lattice, }}$ the lattice energy of the compound

(Figure) diagrams the Born-Haber cycle for the formation of solid cesium fluoride.

The Born-Haber cycle shows the relative energies of each step involved in the formation of an ionic solid from the necessary elements in their reference states.

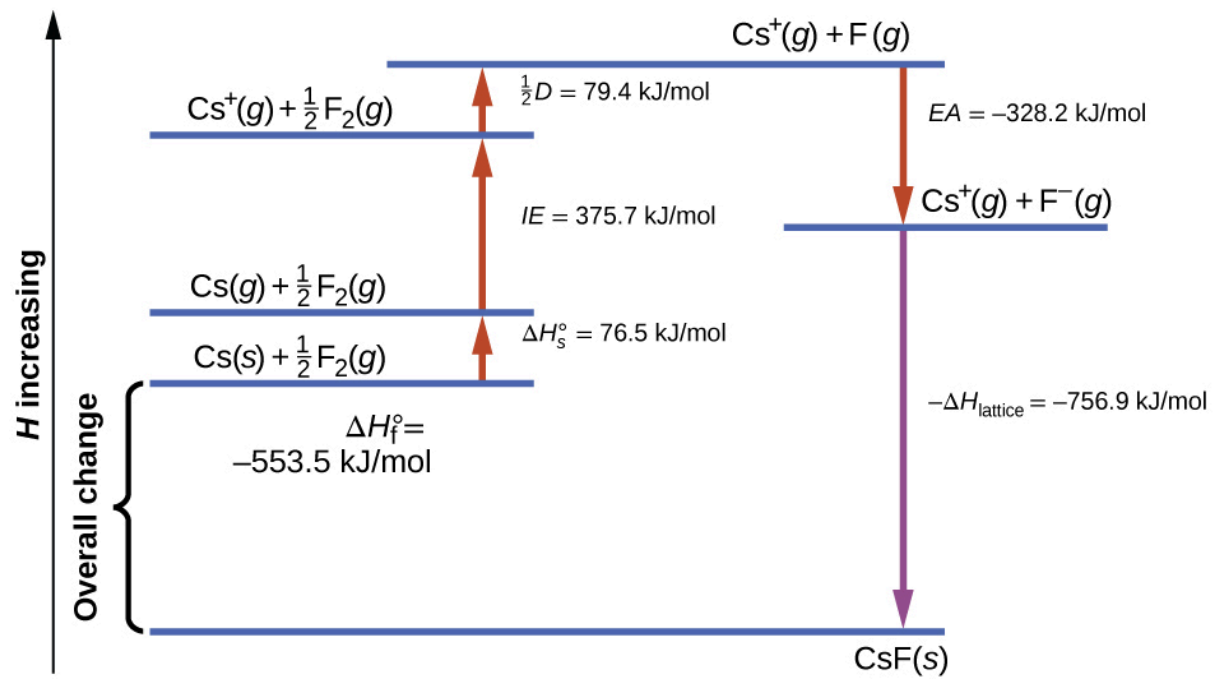

We begin with the elements in their most common states, $\mathrm{Cs}(s)$ and $\mathrm{F}_{2}(g)$. The $H_{s}$ represents the conversion of solid cesium into a gas, and then the ionization energy converts 
the gaseous cesium atoms into cations. In the next step, we account for the energy required to break the F-F bond to produce fluorine atoms. Converting one mole of fluorine atoms into fluoride ions is an exothermic process, so this step gives off energy (the electron affinity) and is shown as decreasing along the $y$-axis. We now have one mole of $\mathrm{Cs}$ cations and one mole of $\mathrm{F}$ anions. These ions combine to produce solid cesium fluoride. The enthalpy change in this step is the negative of the lattice energy, so it is also an exothermic quantity. The total energy involved in this conversion is equal to the experimentally determined enthalpy of formation, $H_{\mathrm{f}}$, of the compound from its elements. In this case, the overall change is exothermic.

Hess's law can also be used to show the relationship between the enthalpies of the individual steps and the enthalpy of formation. (Figure) shows this for fluoride, CsF. 


$$
\begin{aligned}
& \text { Enthalpy of } \\
& \text { sublimation } \mathrm{Cs}(s) \mathrm{Cs}(g) \\
& \text { of } \mathrm{Cs}(s) \\
& \text { One-half of } \\
& \text { the bond } \quad \frac{1}{2} \mathrm{~F}_{2}(g) \mathrm{F}(g) \\
& \mathrm{F}_{2} \\
& \text { Ionization } \\
& \text { energy of } \mathrm{Cs}(g) \mathrm{Cs}^{+}(g)+\mathrm{e} \\
& \mathrm{Cs}(g) \\
& \mathrm{F}(g)+\mathrm{e} \mathrm{F}(g) \\
& \text { affinity of } \mathrm{F} \\
& \mathrm{Cs}^{+}(g)+\mathrm{F}(g) \operatorname{CsF}(s) \\
& H=H_{f}=H_{s}+\frac{1}{2} D+I E+(-E A)+\left(-H_{\text {lattice }}\right) \\
& \mathrm{Cs}(s)+\frac{1}{2} \mathrm{~F}_{2}(g) \mathrm{CsF}(s)
\end{aligned}
$$

Thus, the lattice energy can be calculated from other values. For cesium fluoride, using this data, the lattice energy is:

$H_{\text {lattice }}=(553.5+76.5+79.4+375.7+328.2) \mathrm{kJ} / \mathrm{mol}=1413.3 \mathrm{~kJ} / \mathrm{mol}$

The Born-Haber cycle may also be used to calculate any one of the other quantities in the equation for lattice energy, provided that the remainder is known. For example, if the relevant enthalpy of sublimation $H_{s}$, ionization energy (IE), bond dissociation enthalpy (D), lattice energy $\Delta H_{\text {lattice, and standard enthalpy of }}$ formation $H_{\mathrm{f}}$ are known, the Born-Haber cycle can be used to determine the electron affinity of an atom.

Lattice energies calculated for ionic compounds are 
typically much higher than bond dissociation energies measured for covalent bonds. Whereas lattice energies typically fall in the range of $600-4000 \mathrm{~kJ} / \mathrm{mol}$ (some even higher), covalent bond dissociation energies are typically between 150-400 kJ/mol for single bonds. Keep in mind, however, that these are not directly comparable values. For ionic compounds, lattice energies are associated with many interactions, as cations and anions pack together in an extended lattice. For covalent bonds, the bond dissociation energy is associated with the interaction of just two atoms.

Key Concepts and Summary

The strength of a covalent bond is measured by its bond dissociation energy, that is, the amount of energy required to break that particular bond in a mole of molecules. Multiple bonds are stronger than single bonds between the same atoms. The enthalpy of a reaction can be estimated based on the energy input required to break bonds and the energy released when new bonds are formed. For ionic bonds, the lattice energy is the energy required to separate one mole of a compound into its gas phase ions. Lattice energy increases for ions with higher charges and shorter distances between ions. Lattice energies are often calculated using the Born-Haber cycle, a thermochemical cycle including all of the energetic steps involved in converting elements into an ionic compound.

Key Equations

- Bond energy for a diatomic molecule: 


$$
\mathrm{XY}(g) \mathrm{X}(g)+\mathrm{Y}(g) \quad \mathrm{D}_{\mathrm{X}-\mathrm{Y}}=H
$$

- Enthalpy change: $\Delta H=\Sigma \mathrm{D}_{\text {bonds broken }}-\Sigma \mathrm{D}_{\text {bonds }}$ formed

- Lattice energy for a solid MX:

$$
\operatorname{MX}(s) \mathrm{M}^{n+}(g)+\mathrm{X}^{n}(g) \quad H_{\text {lattice }}
$$

- Lattice energy for an ionic crystal:

$$
H_{\text {lattice }}=\frac{\mathrm{C}\left(\mathrm{Z}^{+}\right)(\mathrm{Z})}{\mathrm{R}_{\mathrm{o}}}
$$

Chemistry End of Chapter Exercises

Which bond in each of the following pairs of bonds is the strongest?

(a) $\mathrm{C}-\mathrm{C}$ or $\mathrm{C}=\mathrm{C}$

(b) $\mathrm{C}-\mathrm{N}$ or $\mathrm{C} \equiv \mathrm{N}$

(c) $\mathrm{C} \equiv \mathrm{O}$ or $\mathrm{C}=\mathrm{O}$

(d) $\mathrm{H}-\mathrm{F}$ or $\mathrm{H}-\mathrm{Cl}$

(e) $\mathrm{C}-\mathrm{H}$ or $\mathrm{O}-\mathrm{H}$

(f) $\mathrm{C}-\mathrm{N}$ or $\mathrm{C}-\mathrm{O}$

Using the bond energies in (Figure), determine the approximate enthalpy change for each of the following reactions:

(a) $\mathrm{H}_{2}(g)+\mathrm{Br}_{2}(g) 2 \mathrm{HBr}(g)$

(b) $\mathrm{CH}_{4}(g)+\mathrm{I}_{2}(g) \mathrm{CH}_{3} \mathrm{I}(g)+\mathrm{HI}(g)$

(c) $\mathrm{C}_{2} \mathrm{H}_{4}(g)+3 \mathrm{O}_{2}(g) 2 \mathrm{CO}_{2}(g)+2 \mathrm{H}_{2} \mathrm{O}(g)$

(a) $-114 \mathrm{~kJ}$; (b) $30 \mathrm{~kJ}$; (c) $-1055 \mathrm{~kJ}$

Using the bond energies in (Figure), determine the approximate enthalpy change for each of the following reactions: 
(a) $\mathrm{Cl}_{2}(g)+3 \mathrm{~F}_{2}(g) 2 \mathrm{ClF}_{3}(g)$

(b) $\mathrm{H}_{2} \mathrm{C}=\mathrm{CH}_{2}(g)+\mathrm{H}_{2}(g) \mathrm{H}_{3} \mathrm{CCH}_{3}(g)$

(c) $2 \mathrm{C}_{2} \mathrm{H}_{6}(g)+7 \mathrm{O}_{2}(g) 4 \mathrm{CO}_{2}(g)+6 \mathrm{H}_{2} \mathrm{O}(g)$

When a molecule can form two different structures, the structure with the stronger bonds is usually the more stable form. Use bond energies to predict the correct structure of the hydroxylamine molecule:<smiles>[O]NC=O</smiles>

The greater bond energy is in the figure on the left. It is the more stable form.

Complete the following Lewis structure by adding bonds (not atoms), and then indicate the longest bond:
$\mathrm{H}$
$\mathrm{H}$
$\mathrm{H} \quad \mathrm{H}$

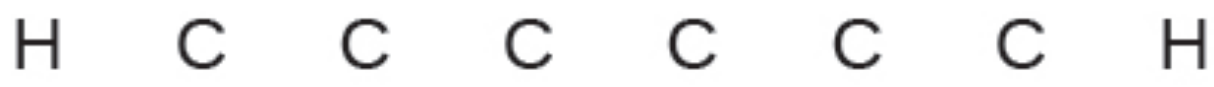

$\mathrm{H} \quad \mathrm{H}$

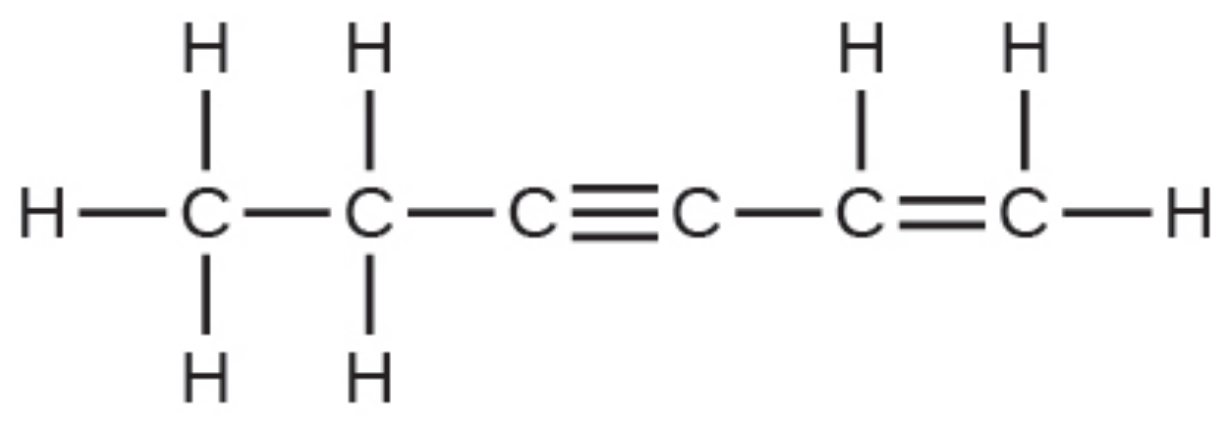


The $\mathrm{C}-\mathrm{C}$ single bonds are longest.

Use the bond energy to calculate an approximate value of $\Delta H$ for the following reaction. Which is the more stable form of $\mathrm{FNO}_{2}$ ?

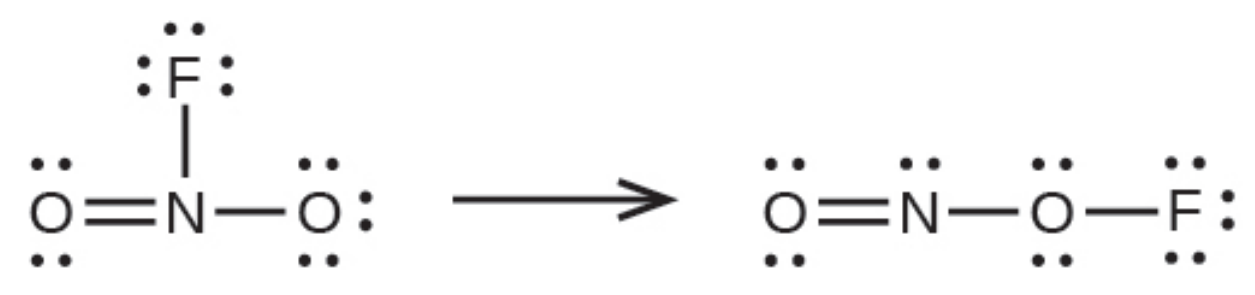

The lattice energy of $\mathrm{LiF}$ is $1023 \mathrm{~kJ} / \mathrm{mol}$, and the $\mathrm{Li}-\mathrm{F}$ distance is $200.8 \mathrm{pm}$. NaF crystallizes in the same structure as LiF but with a Na-F distance of $231 \mathrm{pm}$. Which of the following values most closely approximates the lattice energy of NaF: $510,890,1023,1175$, or $4090 \mathrm{~kJ} /$ mol? Explain your choice.

For which of the following substances is the least energy required to convert one mole of the solid into separate ions?

(a) $\mathrm{MgO}$

(b) $\mathrm{SrO}$

(c) $\mathrm{KF}$

(d) $\mathrm{CsF}$

(e) $\mathrm{MgF}_{2}$

(d)

The reaction of a metal, $\mathrm{M}$, with a halogen, $\mathrm{X}_{2}$, proceeds by an exothermic reaction as indicated by this equation: $\mathrm{M}(s)+\mathrm{X}_{2}(g) \mathrm{MX}_{2}(s)$. For each of the following, 
indicate which option will make the reaction more exothermic. Explain your answers.

(a) a large radius vs. a small radius for $\mathrm{M}^{+2}$

(b) a high ionization energy vs. a low ionization energy for $\mathrm{M}$

(c) an increasing bond energy for the halogen

(d) a decreasing electron affinity for the halogen

(e) an increasing size of the anion formed by the halogen

The lattice energy of LiF is $1023 \mathrm{~kJ} / \mathrm{mol}$, and the $\mathrm{Li}-\mathrm{F}$ distance is $201 \mathrm{pm}$. MgO crystallizes in the same structure as $\mathrm{LiF}$ but with a $\mathrm{Mg}-\mathrm{O}$ distance of $205 \mathrm{pm}$. Which of the following values most closely approximates the lattice energy of MgO: $256 \mathrm{~kJ} / \mathrm{mol}, 512 \mathrm{~kJ} / \mathrm{mol}, 1023 \mathrm{~kJ} / \mathrm{mol}, 2046$ $\mathrm{kJ} / \mathrm{mol}$, or $4008 \mathrm{~kJ} / \mathrm{mol}$ ? Explain your choice.

$4008 \mathrm{~kJ} / \mathrm{mol}$; both ions in $\mathrm{MgO}$ have twice the charge of the ions in LiF; the bond length is very similar and both have the same structure; a quadrupling of the energy is expected based on the equation for lattice energy

Which compound in each of the following pairs has the larger lattice energy? Note: $\mathrm{Mg}^{2+}$ and $\mathrm{Li}^{+}$have similar radii; $\mathrm{O}^{2-}$ and $\mathrm{F}^{-}$have similar radii. Explain your choices.

(a) $\mathrm{MgO}$ or $\mathrm{MgSe}$

(b) $\mathrm{LiF}$ or $\mathrm{MgO}$

(c) $\mathrm{Li}_{2} \mathrm{O}$ or $\mathrm{LiCl}$

(d) $\mathrm{Li}_{2} \mathrm{Se}$ or $\mathrm{MgO}$

Which compound in each of the following pairs has the larger lattice energy? Note: $\mathrm{Ba}^{2+}$ and 
$\mathrm{K}^{+}$have similar radii; $\mathrm{S}^{2-}$ and $\mathrm{Cl}^{-}$have similar radii. Explain your choices.

(a) $\mathrm{K}_{2} \mathrm{O}$ or $\mathrm{Na}_{2} \mathrm{O}$

(b) $\mathrm{K}_{2} \mathrm{~S}$ or $\mathrm{BaS}$

(c) $\mathrm{KCl}$ or $\mathrm{BaS}$

(d) $\mathrm{BaS}$ or $\mathrm{BaCl}_{2}$

(a) $\mathrm{Na}_{2} \mathrm{O} ; \mathrm{Na}^{+}$has a smaller radius than $\mathrm{K}^{+}$; (b) BaS; $\mathrm{Ba}$ has a larger charge than $\mathrm{K}$; (c) $\mathrm{BaS}$; $\mathrm{Ba}$ and $\mathrm{S}$ have larger charges; (d) BaS; S has a larger charge

Which of the following compounds requires the most energy to convert one mole of the solid into separate ions?
(a) $\mathrm{MgO}$
(b) $\mathrm{SrO}$
(c) $\mathrm{KF}$
(d) $\mathrm{CsF}$
(e) $\mathrm{MgF}_{2}$

Which of the following compounds requires the most energy to convert one mole of the solid into separate ions?
(a) $\mathrm{K}_{2} \mathrm{~S}$
(b) $\mathrm{K}_{2} \mathrm{O}$
(c) $\mathrm{CaS}$
(d) $\mathrm{Cs}_{2} \mathrm{~S}$
(e) $\mathrm{CaO}$

(e)

The lattice energy of KF is $794 \mathrm{~kJ} / \mathrm{mol}$, and the interionic distance is $269 \mathrm{pm}$. The $\mathrm{Na}-\mathrm{F}$

distance in $\mathrm{NaF}$, which has the same structure as $\mathrm{KF}$, is $231 \mathrm{pm}$. Which of the following values is the closest 
approximation of the lattice energy of $\mathrm{NaF}: 682 \mathrm{~kJ} / \mathrm{mol}$, $794 \mathrm{~kJ} / \mathrm{mol}, 924 \mathrm{~kJ} / \mathrm{mol}, 1588 \mathrm{~kJ} / \mathrm{mol}$, or $3175 \mathrm{~kJ} / \mathrm{mol}$ ? Explain your answer.

\section{Footnotes}

- 1This question is taken from the Chemistry Advanced Placement Examination and is used with the permission of the Educational Testing Service.

Glossary

\section{bond energy}

(also, bond dissociation energy) energy required to break a covalent bond in a gaseous substance

\section{Born-Haber cycle}

thermochemical cycle relating the various energetic steps involved in the formation of an ionic solid from the relevant elements lattice energy $\left(\Delta H_{\text {lattice})}\right.$ energy required to separate one mole of an ionic solid into its component gaseous ions 


\section{MOLECULAR STRUCTURE AND POLARITY}

\section{Learning Objectives}

By the end of this section, you will be able to:

- $\quad$ Predict the structures of small molecules using valence shell electron pair repulsion (VSEPR) theory

- Explain the concepts of polar covalent bonds and molecular polarity

- Assess the polarity of a molecule based on its bonding and structure

Thus far, we have used two-dimensional Lewis structures to represent molecules. However, molecular structure is actually three-dimensional, and it is important to be able to describe molecular bonds in terms of their distances, angles, and relative arrangements in space ((Figure)). A bond angle is the angle between any two bonds that 
include a common atom, usually measured in degrees. A bond distance (or bond length) is the distance between the nuclei of two bonded atoms along the straight line joining the nuclei. Bond distances are measured in Ångstroms (1 $\left.\AA=10^{-10} \mathrm{~m}\right)$ or picometers $\left(1 \mathrm{pm}=10^{-12} \mathrm{~m}, 100 \mathrm{pm}=1\right.$ $\AA)$.

Bond distances (lengths) and angles are shown for the formaldehyde molecule, $\mathrm{H}_{2} \mathrm{CO}$.
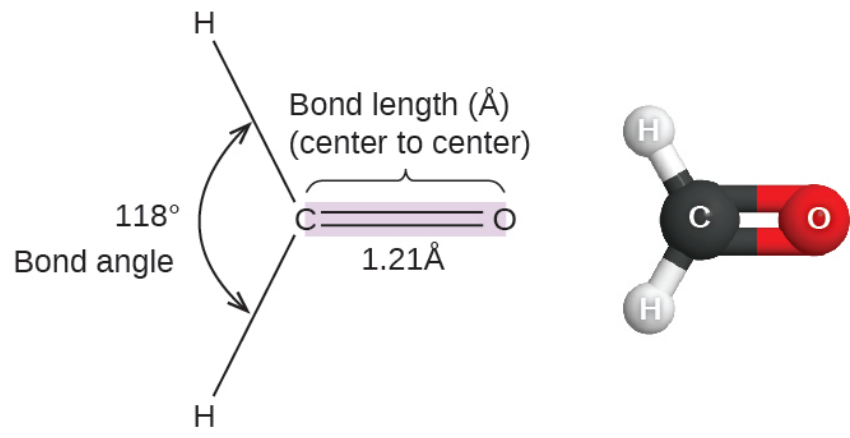

VSEPR Theory

Valence shell electron-pair repulsion theory (VSEPR theory) enables us to predict the molecular structure, including approximate bond angles around a central atom, of a molecule from an examination of the number of bonds and lone electron pairs in its Lewis structure. The VSEPR model assumes that electron pairs in the valence shell of a central atom will adopt an arrangement that minimizes repulsions between these electron pairs by maximizing the distance between them. The electrons in the valence shell of a central atom form either bonding pairs of electrons, located primarily between bonded atoms, or lone pairs. The electrostatic repulsion of these electrons is reduced when the various regions of high 
electron density assume positions as far from each other as possible.

VSEPR theory predicts the arrangement of electron pairs around each central atom and, usually, the correct arrangement of atoms in a molecule. We should understand, however, that the theory only considers electron-pair repulsions. Other interactions, such as nuclear-nuclear repulsions and nuclear-electron attractions, are also involved in the final arrangement that atoms adopt in a particular molecular structure.

As a simple example of VSEPR theory, let us predict the structure of a gaseous $\mathrm{BeF}_{2}$ molecule. The Lewis structure of $\mathrm{BeF}_{2}$ ((Figure)) shows only two electron pairs around the central beryllium atom. With two bonds and no lone pairs of electrons on the central atom, the bonds are as far apart as possible, and the electrostatic repulsion between these regions of high electron density is reduced to a minimum when they are on opposite sides of the central atom. The bond angle is $180^{\circ}$ ((Figure)).

The $\mathrm{BeF}_{2}$ molecule adopts a linear structure in which the two bonds are as far apart as possible, on opposite sides of the Be atom.

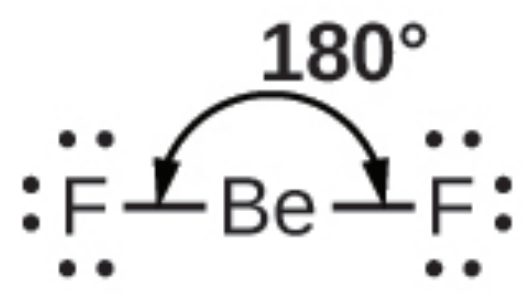

(Figure) illustrates this and other electron-pair geometries that minimize the repulsions among regions of high electron density (bonds and/or lone pairs). Two regions of electron density around a central atom in a 
molecule form a linear geometry; three regions form a trigonal planar geometry; four regions form a tetrahedral geometry; five regions form a trigonal bipyramidal geometry; and six regions form an octahedral geometry. The basic electron-pair geometries predicted by VSEPR theory maximize the space around any region of electron density (bonds or lone pairs).

\begin{tabular}{|c|c|c|c|c|c|}
\hline $\begin{array}{l}\text { Number of } \\
\text { regions }\end{array}$ & $\begin{array}{l}\text { Two regions of } \\
\text { high electron } \\
\text { density (bonds } \\
\text { and/or } \\
\text { unshared } \\
\text { pairs) }\end{array}$ & $\begin{array}{l}\text { Three regions } \\
\text { of high } \\
\text { electron } \\
\text { density (bonds } \\
\text { and/or } \\
\text { unshared } \\
\text { pairs) }\end{array}$ & $\begin{array}{l}\text { Four regions } \\
\text { of high } \\
\text { electron } \\
\text { density (bonds } \\
\text { and/or } \\
\text { unshared } \\
\text { pairs) }\end{array}$ & $\begin{array}{l}\text { Five regions of } \\
\text { high electron } \\
\text { density (bonds } \\
\text { and/or } \\
\text { unshared } \\
\text { pairs) }\end{array}$ & $\begin{array}{l}\text { Six regions of } \\
\text { high electron } \\
\text { density (bonds } \\
\text { and/or } \\
\text { unshared } \\
\text { pairs) }\end{array}$ \\
\hline \multicolumn{6}{|l|}{$\begin{array}{l}\text { Spatial } \\
\text { arrangement }\end{array}$} \\
\hline $\begin{array}{l}\text { Line-dash-wedge } \\
\text { notation }\end{array}$ & $\mathrm{H}-\mathrm{Be}-\mathrm{H}$ & & & & \\
\hline $\begin{array}{l}\text { Electron pair } \\
\text { geometry }\end{array}$ & $\begin{array}{l}\text { Linear; } \\
180^{\circ} \text { angle }\end{array}$ & $\begin{array}{l}\text { Trigonal planar; } \\
\text { all angles } 120^{\circ}\end{array}$ & $\begin{array}{l}\text { Tetrahedral; } \\
\text { all angles } \\
109.5^{\circ}\end{array}$ & $\begin{array}{l}\text { Trigonal } \\
\text { bipyramidal; } \\
\text { angles of } 90^{\circ} \\
\text { or } 120^{\circ} \\
\text { An attached } \\
\text { atom may be } \\
\text { equatorial (in } \\
\text { the plane of } \\
\text { the triangle) or } \\
\text { axial (above or } \\
\text { below the } \\
\text { plane of the } \\
\text { triangle). }\end{array}$ & $\begin{array}{l}\text { Octahedral; } \\
\text { all angles } 90^{\circ} \\
\text { or } 180^{\circ}\end{array}$ \\
\hline
\end{tabular}

\section{Electron-pair Geometry versus Molecular Structure}

It is important to note that electron-pair geometry around a central atom is not the same thing as its molecular structure. The electron-pair geometries shown in (Figure) describe all regions where electrons are located, bonds 
as well as lone pairs. Molecular structure describes the location of the atoms, not the electrons.

We differentiate between these two situations by naming the geometry that includes all electron pairs the electron-pair geometry. The structure that includes only the placement of the atoms in the molecule is called the molecular structure. The electron-pair geometries will be the same as the molecular structures when there are no lone electron pairs around the central atom, but they will be different when there are lone pairs present on the central atom.

For example, the methane molecule, $\mathrm{CH}_{4}$, which is the major component of natural gas, has four bonding pairs of electrons around the central carbon atom; the electronpair geometry is tetrahedral, as is the molecular structure ((Figure)). On the other hand, the ammonia molecule, $\mathrm{NH}_{3}$, also has four electron pairs associated with the nitrogen atom, and thus has a tetrahedral electron-pair geometry. One of these regions, however, is a lone pair, which is not included in the molecular structure, and this lone pair influences the shape of the molecule ((Figure)). The molecular structure of the methane molecule, $\mathrm{CH}_{4}$, is shown with a tetrahedral arrangement of the hydrogen atoms. VSEPR structures like this one are often drawn using the wedge and dash notation, in which solid lines represent bonds in the plane of the page, solid wedges represent bonds coming up out of the plane, and dashed lines represent bonds going down into the plane. 


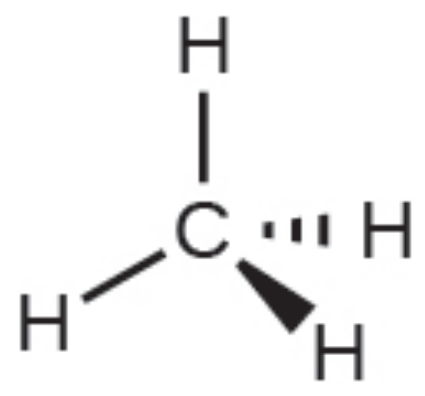

(a) The electron-pair geometry for the ammonia molecule is tetrahedral with one lone pair and three single bonds. (b) The trigonal pyramidal molecular structure is determined from the electron-pair geometry. (c) The actual bond angles deviate slightly from the idealized angles because the lone pair takes up a larger region of space than do the single bonds, causing the $\mathrm{HNH}$ angle to be slightly smaller than $109.5^{\circ}$.

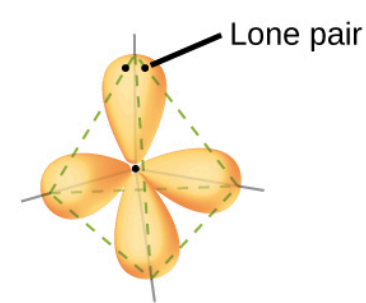

(a)

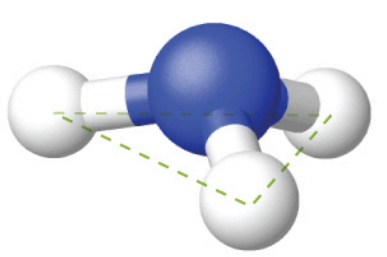

(b)

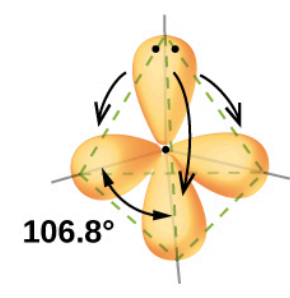

(c)

As seen in (Figure), small distortions from the ideal angles in (Figure) can result from differences in repulsion between various regions of electron density. VSEPR theory predicts these distortions by establishing an order of repulsions and an order of the amount of space occupied by different kinds of electron pairs. The order of electron-pair repulsions from greatest to least repulsion is:

lone pair-lone pair $>$ lone pair-bonding pair $>$ bonding pair-bonding pair $\backslash$ text\{lone pair-bonding pair\}> $\backslash$ text\{bonding pair-bonding 
pair\}" title="Rendered by QuickLaTeX.com" height="16" width="567" style="vertical-align: -4px;">

This order of repulsions determines the amount of space occupied by different regions of electrons. A lone pair of electrons occupies a larger region of space than the electrons in a triple bond; in turn, electrons in a triple bond occupy more space than those in a double bond, and so on. The order of sizes from largest to smallest is: lone pair $>$ triple bond $>$ double bond $>$ single bond $\backslash$ text $\{$ triple bond $\}>\backslash$ text $\{$ double bond $\}>\backslash$ text $\{$ single bond $\} "$ title="Rendered by QuickLaTeX.com" height="16" width="410" style="vertical-align: -4px;">

Consider formaldehyde, $\mathrm{H}_{2} \mathrm{CO}$, which is used as a preservative for biological and anatomical specimens ((Figure)). This molecule has regions of high electron density that consist of two single bonds and one double bond. The basic geometry is trigonal planar with $120^{\circ}$ bond angles, but we see that the double bond causes slightly larger angles $\left(121^{\circ}\right)$, and the angle between the single bonds is slightly smaller $\left(118^{\circ}\right)$.

In the ammonia molecule, the three hydrogen atoms attached to the central nitrogen are not arranged in a flat, trigonal planar molecular structure, but rather in a threedimensional trigonal pyramid ((Figure)) with the nitrogen atom at the apex and the three hydrogen atoms forming the base. The ideal bond angles in a trigonal pyramid are based on the tetrahedral electron pair geometry. Again, there are slight deviations from the ideal because lone pairs occupy larger regions of space than do bonding electrons. The $\mathrm{H}-\mathrm{N}-\mathrm{H}$ bond angles in $\mathrm{NH}_{3}$ are slightly 
smaller than the $109.5^{\circ}$ angle in a regular tetrahedron ((Figure)) because the lone pair-bonding pair repulsion is greater than the bonding pair-bonding pair repulsion ((Figure)). (Figure) illustrates the ideal molecular structures, which are predicted based on the electron-pair geometries for various combinations of lone pairs and bonding pairs.

The molecular structures are identical to the electronpair geometries when there are no lone pairs present (first column). For a particular number of electron pairs (row), the molecular structures for one or more lone pairs are determined based on modifications of the corresponding electron-pair geometry.

\begin{tabular}{|c|c|c|c|c|c|}
\hline $\begin{array}{l}\text { Number of } \\
\text { electron } \\
\text { pairs }\end{array}$ & $\begin{array}{l}\text { Electron pair } \\
\text { geometries: } \\
0 \text { lone pair }\end{array}$ & 1 lone pair & 2 lone pairs & 3 lone pairs & 4 lone pairs \\
\hline 2 & $\overbrace{\mathrm{A}}^{180^{\circ}}$ & & & & \\
\hline 3 & $\begin{array}{c}x^{-A} x_{x} \\
\text { Trigonal planar }\end{array}$ & $\begin{array}{l}x \underbrace{A}_{<120^{\circ}} \lambda_{x} \\
\text { Bent or angular }\end{array}$ & & & \\
\hline 4 & $x_{\text {Tetrahedral }}$ & 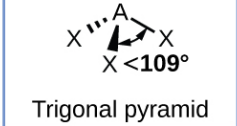 & $\begin{array}{l}x \sum_{<<109^{\circ}}^{A A} x_{x} \\
\text { Bent or angular }\end{array}$ & & \\
\hline 5 & Trigonal bipyramid & $\begin{array}{c}<90^{\circ} X \\
\times \\
\times 120^{\circ} \\
\times \\
\text { Sawhorse or seesaw }\end{array}$ & 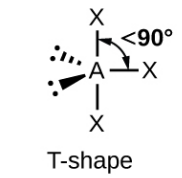 & 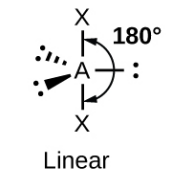 & \\
\hline 6 & Octahedral & Square pyramid & 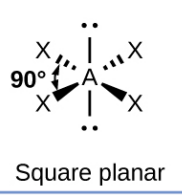 & 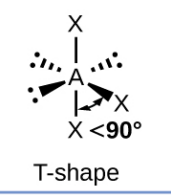 & - \\
\hline
\end{tabular}

According to VSEPR theory, the terminal atom locations (Xs in (Figure)) are equivalent within the linear, trigonal 
planar, and tetrahedral electron-pair geometries (the first three rows of the table). It does not matter which $\mathrm{X}$ is replaced with a lone pair because the molecules can be rotated to convert positions. For trigonal bipyramidal electron-pair geometries, however, there are two distinct $\mathrm{X}$ positions, as shown in (Figure): an axial position (if we hold a model of a trigonal bipyramid by the two axial positions, we have an axis around which we can rotate the model) and an equatorial position (three positions form an equator around the middle of the molecule). As shown in (Figure), the axial position is surrounded by bond angles of $90^{\circ}$, whereas the equatorial position has more space available because of the $120^{\circ}$ bond angles. In a trigonal bipyramidal electron-pair geometry, lone pairs always occupy equatorial positions because these more spacious positions can more easily accommodate the larger lone pairs.

Theoretically, we can come up with three possible arrangements for the three bonds and two lone pairs for the $\mathrm{ClF}_{3}$ molecule ((Figure)). The stable structure is the one that puts the lone pairs in equatorial locations, giving a T-shaped molecular structure.

(a) In a trigonal bipyramid, the two axial positions are located directly across from one another, whereas the three equatorial positions are located in a triangular arrangement. (b-d) The two lone pairs (red lines) in $\mathrm{ClF}_{3}$ have several possible arrangements, but the T-shaped molecular structure (b) is the one actually observed, consistent with the larger lone pairs both occupying equatorial positions. 

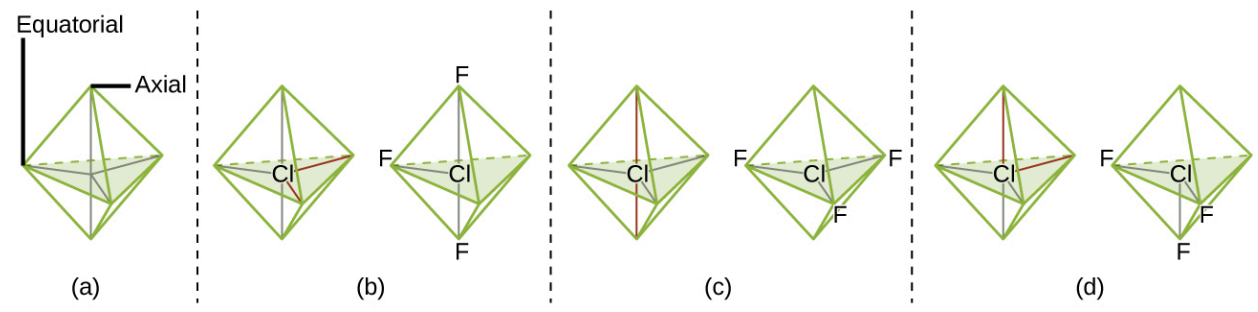

(d)

When a central atom has two lone electron pairs and four bonding regions, we have an octahedral electron-pair geometry. The two lone pairs are on opposite sides of the octahedron (180 ${ }^{\circ}$ apart), giving a square planar molecular structure that minimizes lone pair-lone pair repulsions ((Figure)).

\section{Predicting Electron Pair Geometry and Molecular} Structure

The following procedure uses VSEPR theory to determine the electron pair geometries and the molecular structures:

1. Write the Lewis structure of the molecule or polyatomic ion.

2. Count the number of regions of electron density (lone pairs and bonds) around the central atom. A single, double, or triple bond counts as one region of electron density.

3. Identify the electron-pair geometry based on the number of regions of electron density: linear, trigonal planar, tetrahedral, trigonal bipyramidal, or octahedral ((Figure), first column).

4. Use the number of lone pairs to determine the molecular structure ((Figure)). If more than one 
arrangement of lone pairs and chemical bonds is possible, choose the one that will minimize repulsions, remembering that lone pairs occupy more space than multiple bonds, which occupy more space than single bonds. In trigonal bipyramidal arrangements, repulsion is minimized when every lone pair is in an equatorial position. In an octahedral arrangement with two lone pairs, repulsion is minimized when the lone pairs are on opposite sides of the central atom.

The following examples illustrate the use of VSEPR theory to predict the molecular structure of molecules or ions that have no lone pairs of electrons. In this case, the molecular structure is identical to the electron pair geometry.

Predicting Electron-pair Geometry and Molecular Structure: $\mathrm{CO}_{2}$ and $\mathrm{BCl}_{3}$ Predict the electron-pair geometry and molecular structure for each of the following:

(a) carbon dioxide, $\mathrm{CO}_{2}$, a molecule produced by the combustion of fossil fuels

(b) boron trichloride, $\mathrm{BCl}_{3}$, an important industrial chemical

Solution (a) We write the Lewis structure of $\mathrm{CO}_{2}$ as:<smiles>O=C=O</smiles>

This shows us two regions of high electron density around the carbon atom-each double bond counts as one region, and there are no lone pairs on the carbon atom. Using VSEPR theory, we predict that the two regions of electron density 
arrange themselves on opposite sides of the central atom with a bond angle of $180^{\circ}$. The electron-pair geometry and molecular structure are identical, and $\mathrm{CO}_{2}$ molecules are linear.

(b) We write the Lewis structure of $\mathrm{BCl}_{3}$ as:<smiles>[Si]B([Si])Cl</smiles>

Thus we see that $\mathrm{BCl}_{3}$ contains three bonds, and there are no lone pairs of electrons on boron. The arrangement of three regions of high electron density gives a trigonal planar electron-pair geometry. The $\mathrm{B}-\mathrm{Cl}$ bonds lie in a plane with $120^{\circ}$ angles between them. $\mathrm{BCl}_{3}$ also has a trigonal planar molecular structure ((Figure)).<smiles></smiles>

The electron-pair geometry and molecular structure of $\mathrm{BCl}_{3}$ are both trigonal planar. Note that the VSEPR geometry indicates the correct bond angles $\left(120^{\circ}\right)$, unlike the Lewis structure shown above.

Check Your Learning Carbonate, $\mathrm{CO}_{3}{ }^{2}$, is a common polyatomic ion found in various materials from eggshells to antacids. What are the electron-pair geometry and molecular structure of this polyatomic ion?

\section{Answer:}

The electron-pair geometry is trigonal planar and the molecular structure is trigonal planar. Due to resonance, all three $\mathrm{C}-\mathrm{O}$ bonds are identical. Whether they are single, double, or an average of the two, each bond counts as one region of electron density. 
Predicting Electron-pair Geometry and Molecular Structure: Ammonium Two of the top 50 chemicals produced in the United States, ammonium nitrate and ammonium sulfate, both used as fertilizers, contain the ammonium ion. Predict the electron-pair geometry and molecular structure of the $\mathrm{NH}_{4}{ }^{+}$ cation.

Solution We write the Lewis structure of $\mathrm{NH}_{4}{ }^{+}$as:<smiles></smiles>

We can see that $\mathrm{NH}_{4}{ }^{+}$contains four bonds from the nitrogen atom to hydrogen atoms and no lone pairs. We expect the four regions of high electron density to arrange themselves so that they point to the corners of a tetrahedron with the central nitrogen atom in the middle ((Figure)). Therefore, the electron pair geometry of $\mathrm{NH}_{4}{ }^{+}$is tetrahedral, and the molecular structure is also tetrahedral ((Figure)).

The ammonium ion displays a tetrahedral electron-pair geometry as well as a tetrahedral molecular structure.<smiles>C[NH+](C)C</smiles>

Check Your Learning Identify a molecule with trigonal bipyramidal molecular structure.

Answer:

Any molecule with five electron pairs around the central atoms including no lone pairs will be trigonal bipyramidal. $\mathrm{PF}_{5}$ is a common example. 
The next several examples illustrate the effect of lone pairs of electrons on molecular structure.

Predicting Electron-pair Geometry and Molecular Structure: Lone Pairs on the Central Atom Predict the electron-pair geometry and molecular structure of a water molecule.

Solution The Lewis structure of $\mathrm{H}_{2} \mathrm{O}$ indicates that there are four regions of high electron density around the oxygen atom: two lone pairs and two chemical bonds:<smiles>O</smiles>

We predict that these four regions are arranged in a tetrahedral fashion ((Figure)), as indicated in (Figure). Thus, the electron-pair geometry is tetrahedral and the molecular structure is bent with an angle slightly less than $109.5^{\circ}$. In fact, the bond angle is $104.5^{\circ}$.

(a) $\mathrm{H}_{2} \mathrm{O}$ has four regions of electron density around the central atom, so it has a tetrahedral electron-pair geometry. (b) Two of the electron regions are lone pairs, so the molecular structure is bent.

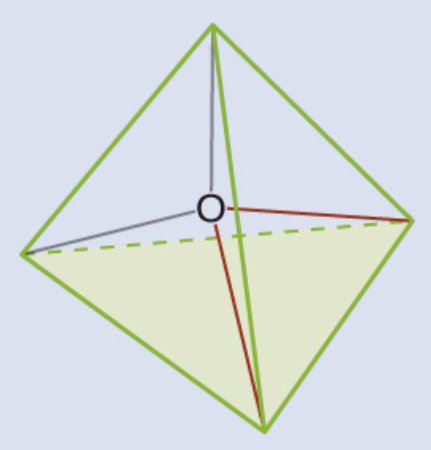

(a)

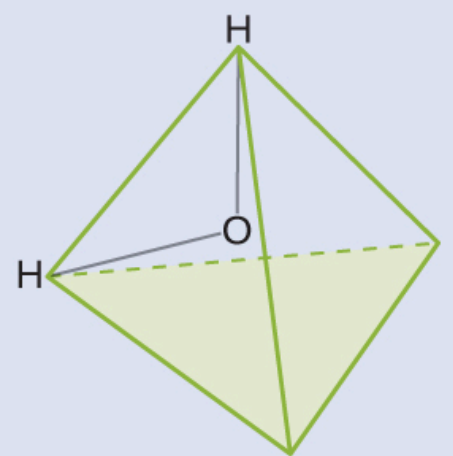

(b)

Check Your Learning The hydronium ion, $\mathrm{H}_{3} \mathrm{O}^{+}$, forms when acids are dissolved in water. Predict the electron-pair geometry and molecular structure of this cation. 
Answer:

electron pair geometry: tetrahedral; molecular structure: trigonal pyramidal

Predicting Electron-pair Geometry and Molecular Structure: $\mathrm{SF}_{4}$ Sulfur tetrafluoride, $\mathrm{SF}_{4}$, is extremely valuable for the preparation of fluorine-containing compounds used as herbicides (i.e., $\mathrm{SF}_{4}$ is used as a fluorinating agent). Predict the electron-pair geometry and molecular structure of a $\mathrm{SF}_{4}$ molecule.

Solution The Lewis structure of $\mathrm{SF}_{4}$ indicates five regions of electron density around the sulfur atom: one lone pair and four bonding pairs:

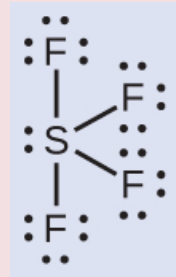

We expect these five regions to adopt a trigonal bipyramidal electron-pair geometry. To minimize lone pair repulsions, the lone pair occupies one of the equatorial positions. The molecular structure ((Figure)) is that of a seesaw ((Figure)).

(a) SF4 has a trigonal bipyramidal arrangement of the five regions of electron density. (b) One of the regions is a lone pair, which results in a seesaw-shaped molecular structure. 


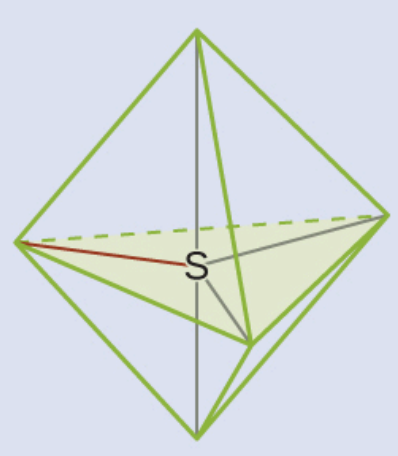

(a)

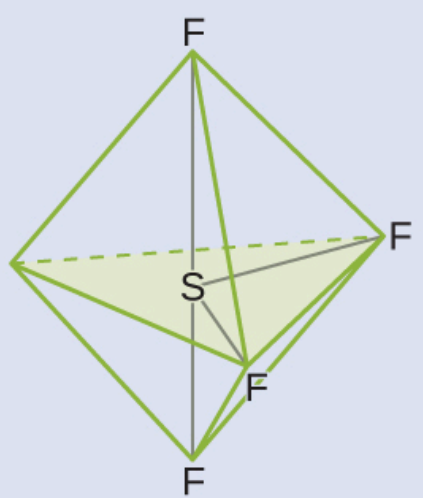

(b)

Check Your Learning Predict the electron pair geometry and molecular structure for molecules of $\mathrm{XeF}_{2}$.

Answer:

The electron-pair geometry is trigonal bipyramidal. The molecular structure is linear.

Predicting Electron-pair Geometry and Molecular Structure: $\mathrm{XeF}_{4} \mathrm{Of}$ all the noble gases, xenon is the most reactive, frequently reacting with elements such as oxygen and fluorine. Predict the electron-pair geometry and molecular structure of the $\mathrm{XeF}_{4}$ molecule.

Solution The Lewis structure of $\mathrm{XeF}_{4}$ indicates six regions of high electron density around the xenon atom: two lone pairs and four bonds:

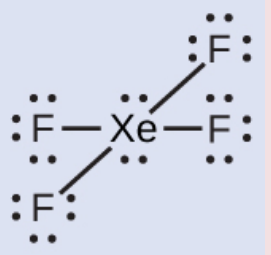

These six regions adopt an octahedral arrangement ((Figure)), which is the electron-pair geometry. To minimize repulsions, the lone pairs should be on opposite sides of the 
central atom ((Figure)). The five atoms are all in the same plane and have a square planar molecular structure.

(a) $\mathrm{XeF}_{4}$ adopts an octahedral arrangement with two lone pairs (red lines) and four bonds in the electron-pair geometry.

(b) The molecular structure is square planar with the lone pairs directly across from one another.

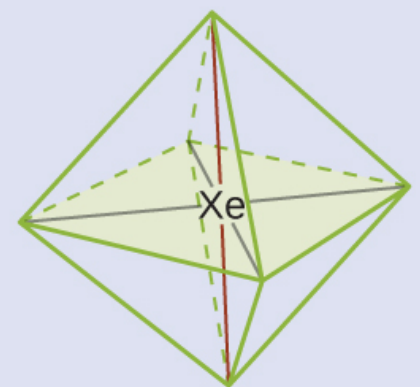

(a)

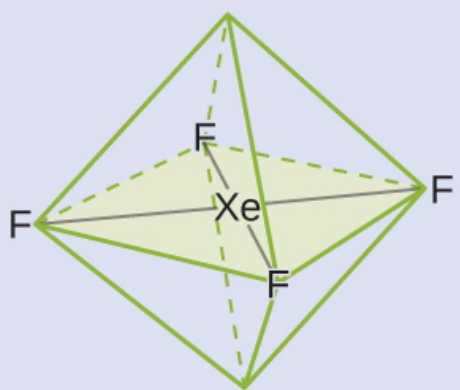

(b)

Check Your Learning In a certain molecule, the central atom has three lone pairs and two bonds. What will the electron pair geometry and molecular structure be?

Answer:

electron pair geometry: trigonal bipyramidal; molecular structure: linear

\section{Molecular Structure for Multicenter Molecules}

When a molecule or polyatomic ion has only one central atom, the molecular structure completely describes the shape of the molecule. Larger molecules do not have a single central atom, but are connected by a chain of interior atoms that each possess a "local" geometry. The way these local structures are oriented with respect to each other also influences the molecular shape, but such considerations are largely beyond the scope of this introductory discussion. For our purposes, we will only focus on determining the local structures. 
Predicting Structure in Multicenter Molecules The Lewis structure for the simplest amino acid, glycine, $\mathrm{H}_{2} \mathrm{NCH}_{2} \mathrm{CO}_{2} \mathrm{H}$, is shown here. Predict the local geometry for the nitrogen atom, the two carbon atoms, and the oxygen atom with a hydrogen atom attached:<smiles>NCC(=O)O</smiles>

Solution<smiles>[1H]N[CH]C(=O)[O-]</smiles>

Consider each central atom independently. The electronpair geometries:

- nitrogen--four regions of electron density; tetrahedral

- carbon $\left(\underline{\mathrm{C}} \mathrm{H}_{2}\right)--$ four regions of electron density; tetrahedral

- carbon $\left(\mathrm{CO}_{2}\right)$-three regions of electron density; trigonal planar

- $\quad$ oxygen $(\underline{\mathrm{O}})$-four regions of electron density; tetrahedral

The local structures:

- nitrogen--three bonds, one lone pair; trigonal pyramidal

- $\quad$ carbon $\left(\underline{\mathrm{CH}_{2}}\right)$-four bonds, no lone pairs; tetrahedral 
- carbon $\left(\mathrm{CO}_{2}\right)$-three bonds (double bond counts as one bond), no lone pairs; trigonal planar

- $\quad$ oxygen $(\underline{\mathrm{OH}})$-two bonds, two lone pairs; bent $\left(109^{\circ}\right)$

Check Your Learning Another amino acid is alanine, which has the Lewis structure shown here. Predict the electron-pair geometry and local structure of the nitrogen atom, the three carbon atoms, and the oxygen atom with hydrogen attached:<smiles>CC(C)C(=O)O</smiles>

\section{Answer:}

electron-pair geometries: nitrogen--tetrahedral; carbon $(\underline{\mathrm{C}} \mathrm{H})$-tetrahedral; carbon $\left(\mathrm{CH}_{3}\right)$-tetrahedral; carbon $\left(\underline{\mathrm{CO}} \mathrm{O}_{2}\right)$-trigonal planar; oxygen $(\underline{\mathrm{O}})$-tetrahedral; local structures: nitrogen-trigonal pyramidal; carbon $(\underline{\mathrm{CH}})$-tetrahedral; carbon $\left(\mathrm{CH}_{3}\right)$-tetrahedral; carbon $\left(\underline{\mathrm{CO}_{2}}\right)$-trigonal planar; oxygen $(\underline{\mathrm{O}} \mathrm{H})$-bent $\left(109^{\circ}\right)$

The molecular shape simulator lets you build various molecules and practice naming their electron-pair geometries and molecular structures.

Molecular Simulation Using molecular shape simulator allows us to control whether bond angles and/or lone pairs are displayed by checking or unchecking the boxes under "Options" on the right. We can also use the "Name" checkboxes at bottom-left to display or hide the electron pair geometry (called "electron geometry" in the simulator) and/or molecular structure (called "molecular shape" in the simulator). 
Build the molecule HCN in the simulator based on the following Lewis structure:

\section{$\mathrm{H}-\mathrm{C} \equiv \mathrm{N}$}

Click on each bond type or lone pair at right to add that group to the central atom. Once you have the complete molecule, rotate it to examine the predicted molecular structure. What molecular structure is this?

Solution The molecular structure is linear.

Check Your Learning Build a more complex molecule in the simulator. Identify the electron-group geometry, molecular structure, and bond angles. Then try to find a chemical formula that would match the structure you have drawn.

Answer:

Answers will vary. For example, an atom with four single bonds, a double bond, and a lone pair has an octahedral electron-group geometry and a square pyramidal molecular structure. $\mathrm{XeOF}_{4}$ is a molecule that adopts this structure.

\section{Molecular Polarity and Dipole Moment}

As discussed previously, polar covalent bonds connect two atoms with differing electronegativities, leaving one atom with a partial positive charge $(\delta+)$ and the other atom with a partial negative charge $(\delta-)$, as the electrons are pulled toward the more electronegative atom. This separation of charge gives rise to a bond dipole moment. The magnitude of a bond dipole moment is represented by the Greek letter mu $(\mu)$ and is given by the formula shown here, where $\mathrm{Q}$ is the magnitude of the partial charges (determined by the electronegativity difference) and $r$ is the distance between the charges:

$\mu=\mathrm{Qr}$ 
This bond moment can be represented as a vector, a quantity having both direction and magnitude ((Figure)). Dipole vectors are shown as arrows pointing along the bond from the less electronegative atom toward the more electronegative atom. A small plus sign is drawn on the less electronegative end to indicate the partially positive end of the bond. The length of the arrow is proportional to the magnitude of the electronegativity difference between the two atoms.

(a) There is a small difference in electronegativity between $\mathrm{C}$ and $\mathrm{H}$, represented as a short vector. (b) The electronegativity difference between $\mathrm{B}$ and $\mathrm{F}$ is much larger, so the vector representing the bond moment is much longer.

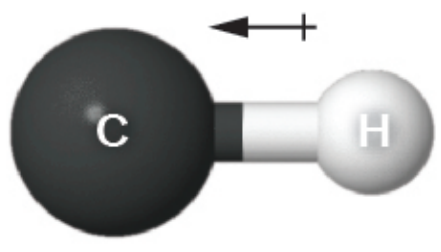

(a)

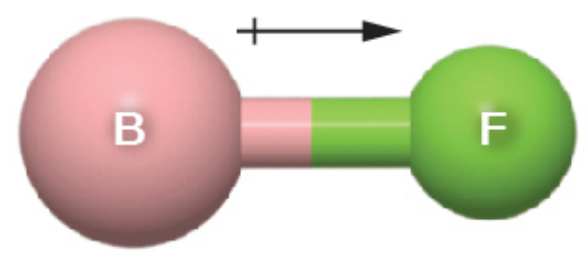

(b)

A whole molecule may also have a separation of charge, depending on its molecular structure and the polarity of each of its bonds. If such a charge separation exists, the molecule is said to be a polar molecule (or dipole); otherwise the molecule is said to be nonpolar. The dipole moment measures the extent of net charge separation in the molecule as a whole. We determine the dipole moment by adding the bond moments in three-dimensional space, taking into account the molecular structure.

For diatomic molecules, there is only one bond, so its bond dipole moment determines the molecular polarity. 
Homonuclear diatomic molecules such as $\mathrm{Br}_{2}$ and $\mathrm{N}_{2}$ have no difference in electronegativity, so their dipole moment is zero. For heteronuclear molecules such as $\mathrm{CO}$, there is a small dipole moment. For HF, there is a larger dipole moment because there is a larger difference in electronegativity.

When a molecule contains more than one bond, the geometry must be taken into account. If the bonds in a molecule are arranged such that their bond moments cancel (vector sum equals zero), then the molecule is nonpolar. This is the situation in $\mathrm{CO}_{2}$ ((Figure)). Each of the bonds is polar, but the molecule as a whole is nonpolar. From the Lewis structure, and using VSEPR theory, we determine that the $\mathrm{CO}_{2}$ molecule is linear with polar $\mathrm{C}=\mathrm{O}$ bonds on opposite sides of the carbon atom. The bond moments cancel because they are pointed in opposite directions. In the case of the water molecule ((Figure)), the Lewis structure again shows that there are two bonds to a central atom, and the electronegativity difference again shows that each of these bonds has a nonzero bond moment. In this case, however, the molecular structure is bent because of the lone pairs on $\mathrm{O}$, and the two bond moments do not cancel. Therefore, water does have a net dipole moment and is a polar molecule (dipole).

The overall dipole moment of a molecule depends on the individual bond dipole moments and how they are arranged. (a) Each CO bond has a bond dipole moment, but they point in opposite directions so that the net $\mathrm{CO}_{2}$ molecule is nonpolar. (b) In contrast, water is polar because the $\mathrm{OH}$ bond moments do not cancel out. 


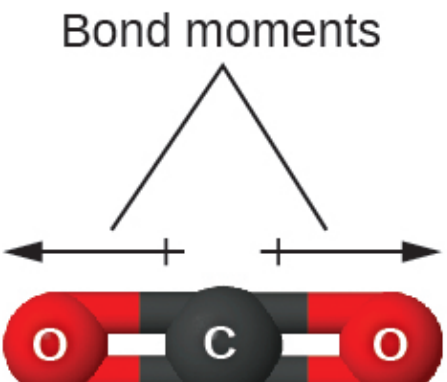

Overall dipole moment $=0$

(a)

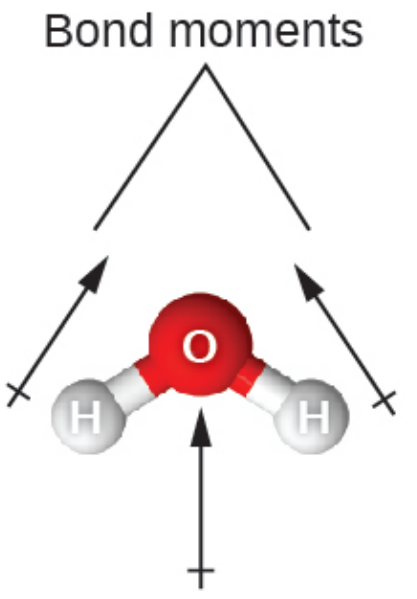

Overall dipole moment

(b)

The OCS molecule has a structure similar to $\mathrm{CO}_{2}$, but a sulfur atom has replaced one of the oxygen atoms. To determine if this molecule is polar, we draw the molecular structure. VSEPR theory predicts a linear molecule:

\section{Bond moments}
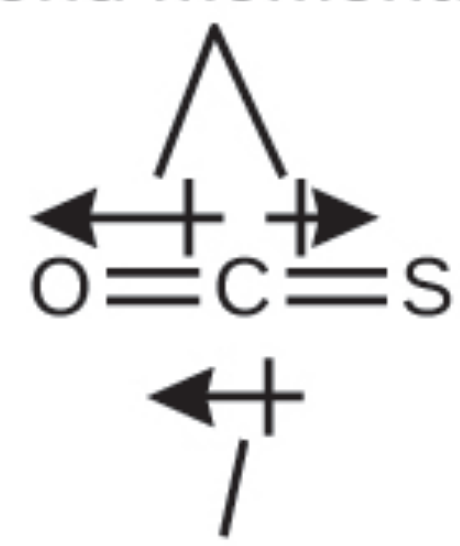

\section{Overall dipole moment}

The $\mathrm{C}-\mathrm{O}$ bond is considerably polar. Although $\mathrm{C}$ and $S$ have very similar electronegativity values, $S$ is slightly more electronegative than $\mathrm{C}$, and so the $\mathrm{C}-\mathrm{S}$ bond is just slightly polar. Because oxygen is more electronegative 
than sulfur, the oxygen end of the molecule is the negative end.

Chloromethane, $\mathrm{CH}_{3} \mathrm{Cl}$, is a tetrahedral molecule with three slightly polar $\mathrm{C}-\mathrm{H}$ bonds and a more polar $\mathrm{C}-\mathrm{Cl}$ bond. The relative electronegativities of the bonded atoms is $\mathrm{H}<\mathrm{C}<\mathrm{Cl}$, and so the bond moments all point toward the $\mathrm{Cl}$ end of the molecule and sum to yield a considerable dipole moment (the molecules are relatively polar).

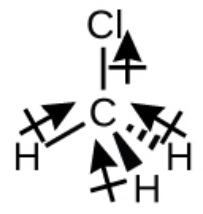

For molecules of high symmetry such as $\mathrm{BF}_{3}$ (trigonal planar), $\mathrm{CH}_{4}$ (tetrahedral), $\mathrm{PF}_{5}$ (trigonal bipymidal), and $\mathrm{SF}_{6}$ (octahedral), all the bonds are of identical polarity (same bond moment) and they are oriented in geometries that yield nonpolar molecules (dipole moment is zero). Molecules of less geometric symmetry, however, may be polar even when all bond moments are identical. For these molecules, the directions of the equal bond moments are such that they sum to give a nonzero dipole moment and a polar molecule. Examples of such molecules include hydrogen sulfide, $\mathrm{H}_{2} \mathrm{~S}$ (nonlinear), and ammonia, $\mathrm{NH}_{3}$ (trigonal pyramidal).
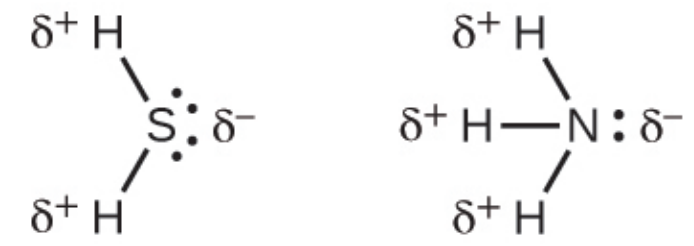

To summarize, to be polar, a molecule must:

1. Contain at least one polar covalent bond.

2. Have a molecular structure such that the sum of the vectors of each bond dipole moment does 
not cancel.

Properties of Polar Molecules

Polar molecules tend to align when placed in an electric field with the positive end of the molecule oriented toward the negative plate and the negative end toward the positive plate ((Figure)). We can use an electrically charged object to attract polar molecules, but nonpolar molecules are not attracted. Also, polar solvents are better at dissolving polar substances, and nonpolar solvents are better at dissolving nonpolar substances.

(a) Molecules are always randomly distributed in the liquid state in the absence of an electric field. (b) When an electric field is applied, polar molecules like HF will align to the dipoles with the field direction.
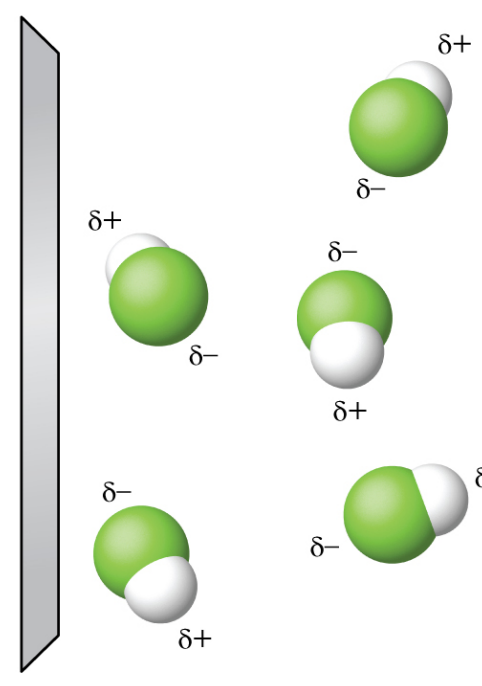

(a)

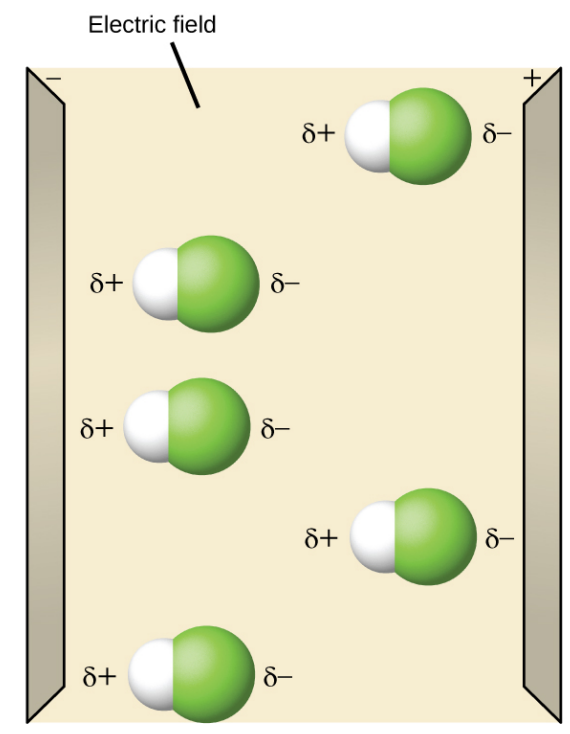

(b)

The molecule polarity simulation provides many ways to explore dipole moments of bonds and molecules. 
Polarity Simulations Open the molecule polarity simulation and select the "Three Atoms" tab at the top. This should display a molecule $A B C$ with three electronegativity adjustors. You can display or hide the bond moments, molecular dipoles, and partial charges at the right. Turning on the Electric Field will show whether the molecule moves when exposed to a field, similar to (Figure).

Use the electronegativity controls to determine how the molecular dipole will look for the starting bent molecule if:

(a) $A$ and $C$ are very electronegative and $B$ is in the middle of the range.

(b) $A$ is very electronegative, and $B$ and $C$ are not.

Solution (a) Molecular dipole moment points immediately between $A$ and $C$.

(b) Molecular dipole moment points along the A-B bond, toward A.

Check Your Learning Determine the partial charges that will give the largest possible bond dipoles.

Answer:

The largest bond moments will occur with the largest partial charges. The two solutions above represent how unevenly the electrons are shared in the bond. The bond moments will be maximized when the electronegativity difference is greatest. The controls for $A$ and $C$ should be set to one extreme, and $B$ should be set to the opposite extreme. Although the magnitude of the bond moment will not change based on whether $B$ is the most electronegative or the least, the direction of the bond moment will.

Key Concepts and Summary

VSEPR theory predicts the three-dimensional 
arrangement of atoms in a molecule. It states that valence electrons will assume an electron-pair geometry that minimizes repulsions between areas of high electron density (bonds and/or lone pairs). Molecular structure, which refers only to the placement of atoms in a molecule and not the electrons, is equivalent to electron-pair geometry only when there are no lone electron pairs around the central atom. A dipole moment measures a separation of charge. For one bond, the bond dipole moment is determined by the difference in electronegativity between the two atoms. For a molecule, the overall dipole moment is determined by both the individual bond moments and how these dipoles are arranged in the molecular structure. Polar molecules (those with an appreciable dipole moment) interact with electric fields, whereas nonpolar molecules do not.

Chemistry End of Chapter Exercises

Explain why the $\mathrm{HOH}$ molecule is bent, whereas the $\mathrm{HBeH}$ molecule is linear.

The placement of the two sets of unpaired electrons in water forces the bonds to assume a tetrahedral arrangement, and the resulting $\mathrm{HOH}$ molecule is bent. The $\mathrm{HBeH}$ molecule (in which Be has only two electrons to bond with the two electrons from the hydrogens) must have the electron pairs as far from one another as possible and is therefore linear.

What feature of a Lewis structure can be used to tell if a molecule's (or ion's) electron-pair geometry and molecular structure will be identical? 
Explain the difference between electron-pair geometry and molecular structure.

Space must be provided for each pair of electrons whether they are in a bond or are present as lone pairs. Electronpair geometry considers the placement of all electrons. Molecular structure considers only the bonding-pair geometry.

Why is the $\mathrm{H}-\mathrm{N}-\mathrm{H}$ angle in $\mathrm{NH}_{3}$ smaller than the $\mathrm{H}-\mathrm{C}-\mathrm{H}$ bond angle in $\mathrm{CH}_{4}$ ? Why is the $\mathrm{H}-\mathrm{N}-\mathrm{H}$ angle in $\mathrm{NH}_{4}{ }^{+}$ identical to the $\mathrm{H}-\mathrm{C}-\mathrm{H}$ bond angle in $\mathrm{CH}_{4}$ ?

Explain how a molecule that contains polar bonds can be nonpolar.

As long as the polar bonds are compensated (for example. two identical atoms are found directly across the central atom from one another), the molecule can be nonpolar.

As a general rule, $\mathrm{MX}_{\mathrm{n}}$ molecules (where $\mathrm{M}$ represents a central atom and $\mathrm{X}$ represents terminal atoms; $\mathrm{n}=2-5$ ) are polar if there is one or more lone pairs of electrons on M. $\mathrm{NH}_{3}(\mathrm{M}=\mathrm{N}, \mathrm{X}=\mathrm{H}, \mathrm{n}=3)$ is an example. There are two molecular structures with lone pairs that are exceptions to this rule. What are they?

Predict the electron pair geometry and the molecular structure of each of the following molecules or ions:
(a) $\mathrm{SF}_{6}$
(b) $\mathrm{PCl}_{5}$
(c) $\mathrm{BeH}_{2}$
(d) $\mathrm{CH}_{3}{ }^{+}$ 
(a) Both the electron geometry and the molecular structure are octahedral. (b) Both the electron geometry and the molecular structure are trigonal bipyramid. (c) Both the electron geometry and the molecular structure are linear. (d) Both the electron geometry and the molecular structure are trigonal planar.

Identify the electron pair geometry and the molecular structure of each of the following molecules or ions:
(a) $\mathrm{IF}_{6}+$
(b) $\mathrm{CF}_{4}$
(c) $\mathrm{BF}_{3}$
(d) $\mathrm{SiF}_{5}$
(e) $\mathrm{BeCl}_{2}$

What are the electron-pair geometry and the molecular structure of each of the following molecules or ions?
(a) $\mathrm{ClF}_{5}$
(b) $\mathrm{ClO}_{2}$
(c) $\mathrm{TeCl}_{4}{ }^{2}$
(d) $\mathrm{PCl}_{3}$
(e) $\mathrm{SeF}_{4}$
(f) $\mathrm{PH}_{2}$

(a) electron-pair geometry: octahedral, molecular structure: square pyramidal; (b) electron-pair geometry: tetrahedral, molecular structure: bent; (c) electron-pair geometry: octahedral, molecular structure: square planar; (d) electron-pair geometry: tetrahedral, molecular structure: trigonal pyramidal; (e) electron-pair geometry: trigonal bypyramidal, molecular structure: seesaw; (f) 
electron-pair geometry: tetrahedral, molecular structure: bent $\left(109^{\circ}\right)$

Predict the electron pair geometry and the molecular structure of each of the following ions:
(a) $\mathrm{H}_{3} \mathrm{O}^{+}$
(b) $\mathrm{PCl}_{4}$
(c) $\mathrm{SnCl}_{3}$
(d) $\mathrm{BrCl}_{4}$
(e) $\mathrm{ICl}_{3}$
(f) $\mathrm{XeF}_{4}$
(g) $\mathrm{SF}_{2}$

Identify the electron pair geometry and the molecular structure of each of the following molecules:

(a) $\mathrm{CINO}$ ( $\mathrm{N}$ is the central atom)

(b) $\mathrm{CS}_{2}$

(c) $\mathrm{Cl}_{2} \mathrm{CO}$ ( $\mathrm{C}$ is the central atom)

(d) $\mathrm{Cl}_{2} \mathrm{SO}$ ( $\mathrm{S}$ is the central atom)

(e) $\mathrm{SO}_{2} \mathrm{~F}_{2}$ ( $\mathrm{S}$ is the central atom)

(f) $\mathrm{XeO}_{2} \mathrm{~F}_{2}$ (Xe is the central atom)

(g) $\mathrm{ClOF}_{2}{ }^{+}(\mathrm{Cl}$ is the central atom)

(a) electron-pair geometry: trigonal planar, molecular structure: bent $\left(120^{\circ}\right)$; (b) electron-pair geometry: linear, molecular structure: linear; (c) electron-pair geometry: trigonal planar, molecular structure: trigonal planar; (d) electron-pair geometry: tetrahedral, molecular structure: trigonal pyramidal; (e) electron-pair geometry: tetrahedral, molecular structure: tetrahedral; (f) electronpair geometry: trigonal bipyramidal, molecular structure: 
seesaw; (g) electron-pair geometry: tetrahedral, molecular structure: trigonal pyramidal

Predict the electron pair geometry and the molecular structure of each of the following:

(a) $\mathrm{IOF}_{5}$ (I is the central atom)

(b) $\mathrm{POCl}_{3}$ ( $\mathrm{P}$ is the central atom)

(c) $\mathrm{Cl}_{2} \mathrm{SeO}$ (Se is the central atom)

(d) $\mathrm{ClSO}^{+}$( $\mathrm{S}$ is the central atom)

(e) $\mathrm{F}_{2} \mathrm{SO}$ ( $\mathrm{S}$ is the central atom)

(f) $\mathrm{NO}_{2}$

(g) $\mathrm{SiO}_{4}^{4}$

Which of the following molecules and ions contain polar bonds? Which of these molecules and ions have dipole moments?

(a) $\mathrm{ClF}_{5}$

(b) $\mathrm{ClO}_{2}$

(c) $\mathrm{TeCl}_{4} 2$

(d) $\mathrm{PCl}_{3}$

(e) $\mathrm{SeF}_{4}$

(f) $\mathrm{PH}_{2}$

(g) $\mathrm{XeF}_{2}$

All of these molecules and ions contain polar bonds. Only $\mathrm{ClF}_{5}, \mathrm{ClO}_{2}, \mathrm{PCl}_{3}, \mathrm{SeF}_{4}$, and $\mathrm{PH}_{2}$ have dipole moments.

Which of these molecules and ions contain polar bonds? Which of these molecules and ions have dipole moments?
(a) $\mathrm{H}_{3} \mathrm{O}^{+}$
(b) $\mathrm{PCl}_{4}$
(c) $\mathrm{SnCl}_{3}$
(d) $\mathrm{BrCl}_{4}$ 

(e) $\mathrm{ICl}_{3}$
(f) $\mathrm{XeF}_{4}$
(g) $\mathrm{SF}_{2}$

Which of the following molecules have dipole moments?
(a) $\mathrm{CS}_{2}$
(b) $\mathrm{SeS}_{2}$
(c) $\mathrm{CCl}_{2} \mathrm{~F}_{2}$
(d) $\mathrm{PCl}_{3}$ (P is the central atom)
(e) ClNO ( $\mathrm{N}$ is the central atom)

$\mathrm{SeS}_{2}, \mathrm{CCl}_{2} \mathrm{~F}_{2}, \mathrm{PCl}_{3}$, and $\mathrm{ClNO}$ all have dipole moments.

Identify the molecules with a dipole moment:
(a) $\mathrm{SF}_{4}$
(b) $\mathrm{CF}_{4}$
(c) $\mathrm{Cl}_{2} \mathrm{CCBr}_{2}$
(d) $\mathrm{CH}_{3} \mathrm{Cl}$
(e) $\mathrm{H}_{2} \mathrm{CO}$

The molecule $\mathrm{XF}_{3}$ has a dipole moment. Is $\mathrm{X}$ boron or phosphorus?

$\mathrm{P}$

The molecule $\mathrm{XCl}_{2}$ has a dipole moment. Is $\mathrm{X}$ beryllium or sulfur?

Is the $\mathrm{Cl}_{2} \mathrm{BBCl}_{2}$ molecule polar or nonpolar?

nonpolar

There are three possible structures for $\mathrm{PCl}_{2} \mathrm{~F}_{3}$ with phosphorus as the central atom. Draw them and discuss 
how measurements of dipole moments could help distinguish among them.

Describe the molecular structure around the indicated atom or atoms:

(a) the sulfur atom in sulfuric acid, $\mathrm{H}_{2} \mathrm{SO}_{4}\left[(\mathrm{HO})_{2} \mathrm{SO}_{2}\right]$

(b) the chlorine atom in chloric acid, $\mathrm{HClO}_{3}\left[\mathrm{HOClO}_{2}\right]$

(c) the oxygen atom in hydrogen peroxide, $\mathrm{HOOH}$

(d) the nitrogen atom in nitric acid, $\mathrm{HNO}_{3}\left[\mathrm{HONO}_{2}\right]$

(e) the oxygen atom in the $\mathrm{OH}$ group in nitric acid, $\mathrm{HNO}_{3}\left[\mathrm{HONO}_{2}\right]$

(f) the central oxygen atom in the ozone molecule, $\mathrm{O}_{3}$

(g) each of the carbon atoms in propyne, $\mathrm{CH}_{3} \mathrm{CCH}$

(h) the carbon atom in Freon, $\mathrm{CCl}_{2} \mathrm{~F}_{2}$

(i) each of the carbon atoms in allene, $\mathrm{H}_{2} \mathrm{CCCH}_{2}$

(a) tetrahedral; (b) trigonal pyramidal; (c) bent $\left(109^{\circ}\right)$; (d) trigonal planar; (e) bent $\left(109^{\circ}\right)$; (f) bent $\left(109^{\circ}\right)$; (g) $\underline{\mathrm{CH}_{3} \mathrm{CCH}}$ tetrahedral, $\mathrm{CH}_{3} \underline{\mathrm{CCH}}$ linear; (h) tetrahedral; (i) $\mathrm{H}_{2} \mathrm{CCCH}_{2}$ linear; $\mathrm{H}_{2} \underline{\mathrm{CC}} \mathrm{CH}_{2}$ trigonal planar

Draw the Lewis structures and predict the shape of each compound or ion:

(a) $\mathrm{CO}_{2}$

(b) $\mathrm{NO}_{2}$

(c) $\mathrm{SO}_{3}$

(d) $\mathrm{SO}_{3}^{2}$

A molecule with the formula $\mathrm{AB}_{2}$, in which $\mathrm{A}$ and $\mathrm{B}$ represent different atoms, could have one of three different shapes. Sketch and name the three different shapes that this molecule might have. Give an example of a molecule or ion for each shape. 


$$
\text { 要 }
$$

A molecule with the formula $A_{3}$, in which $A$ and $B$ represent different atoms, could have one of three different shapes. Sketch and name the three different shapes that this molecule might have. Give an example of a molecule or ion that has each shape.

Draw the Lewis electron dot structures for these molecules, including resonance structures where appropriate:

(a) $\mathrm{CS}_{3}{ }^{2}$

(b) $\mathrm{CS}_{2}$

(c) $\mathrm{CS}$

(d) predict the molecular shapes for $\mathrm{CS}_{3}{ }^{2}$ and $\mathrm{CS}_{2}$ and explain how you arrived at your predictions

(a)

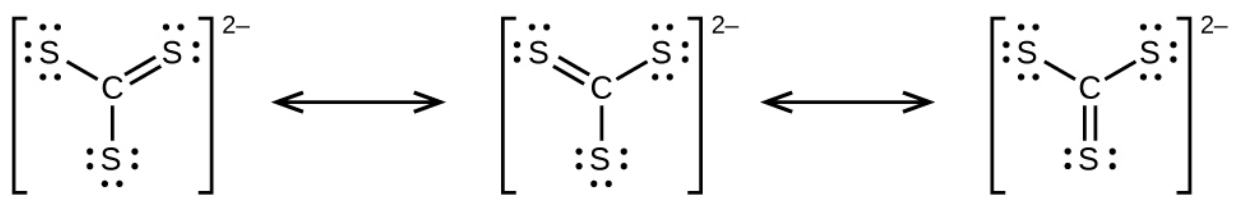

(b)

$$
\ddot{\mathrm{s}}=\mathrm{c}=\ddot{\mathrm{s}}
$$


(c)

$$
: \mathrm{c} \equiv \mathrm{s}:
$$

(d) $\mathrm{CS}_{3}{ }^{2}$ includes three regions of electron density (all are bonds with no lone pairs); the shape is trigonal planar; $\mathrm{CS}_{2}$ has only two regions of electron density (all bonds with no lone pairs); the shape is linear

What is the molecular structure of the stable form of $\mathrm{FNO}_{2}$ ? ( $\mathrm{N}$ is the central atom.)

A compound with a molar mass of about $42 \mathrm{~g} / \mathrm{mol}$ contains $85.7 \%$ carbon and $14.3 \%$ hydrogen. What is its molecular structure?

The Lewis structure is made from three units, but the atoms must be rearranged:

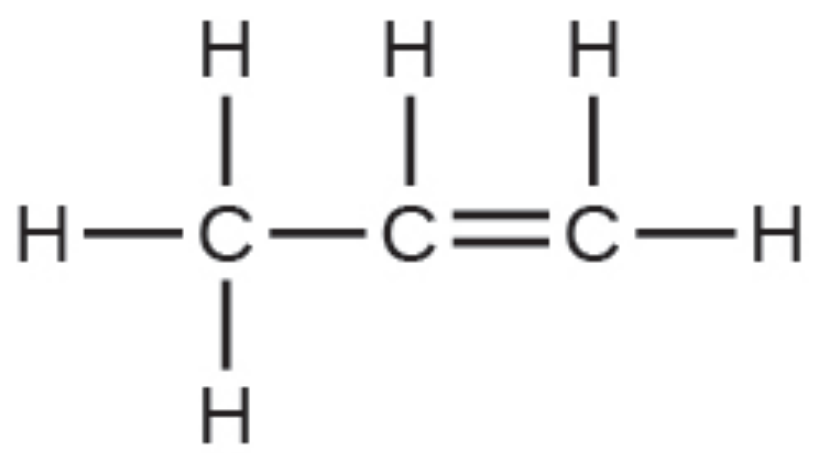

Use the simulation to perform the following exercises for a two-atom molecule:

(a) Adjust the electronegativity value so the bond dipole is pointing toward $B$. Then determine what the electronegativity values must be to switch the dipole so that it points toward A. 
(b) With a partial positive charge on A, turn on the electric field and describe what happens.

(c) With a small partial negative charge on A, turn on the electric field and describe what happens.

(d) Reset all, and then with a large partial negative charge on A, turn on the electric field and describe what happens.

Use the simulation to perform the following exercises for a real molecule. You may need to rotate the molecules in three dimensions to see certain dipoles.

(a) Sketch the bond dipoles and molecular dipole (if any) for $\mathrm{O}_{3}$. Explain your observations.

(b) Look at the bond dipoles for $\mathrm{NH}_{3}$. Use these dipoles to predict whether $\mathrm{N}$ or $\mathrm{H}$ is more electronegative.

(c) Predict whether there should be a molecular dipole for $\mathrm{NH}_{3}$ and, if so, in which direction it will point. Check the molecular dipole box to test your hypothesis.

The molecular dipole points away from the hydrogen atoms.

Use the Molecule Shape simulator to build a molecule. Starting with the central atom, click on the double bond to add one double bond. Then add one single bond and one lone pair. Rotate the molecule to observe the complete geometry. Name the electron group geometry and molecular structure and predict the bond angle. Then click the check boxes at the bottom and right of the simulator to check your answers.

Use the Molecule Shape simulator to explore real molecules. On the Real Molecules tab, select $\mathrm{H}_{2} \mathrm{O}$. Switch 
between the "real" and "model" modes. Explain the difference observed.

The structures are very similar. In the model mode, each electron group occupies the same amount of space, so the bond angle is shown as $109.5^{\circ}$. In the "real" mode, the lone pairs are larger, causing the hydrogens to be compressed. This leads to the smaller angle of $104.5^{\circ}$.

Use the Molecule Shape simulator to explore real molecules. On the Real Molecules tab, select "model" mode and $\mathrm{S}_{2} \mathrm{O}$. What is the model bond angle? Explain whether the "real" bond angle should be larger or smaller than the ideal model angle.

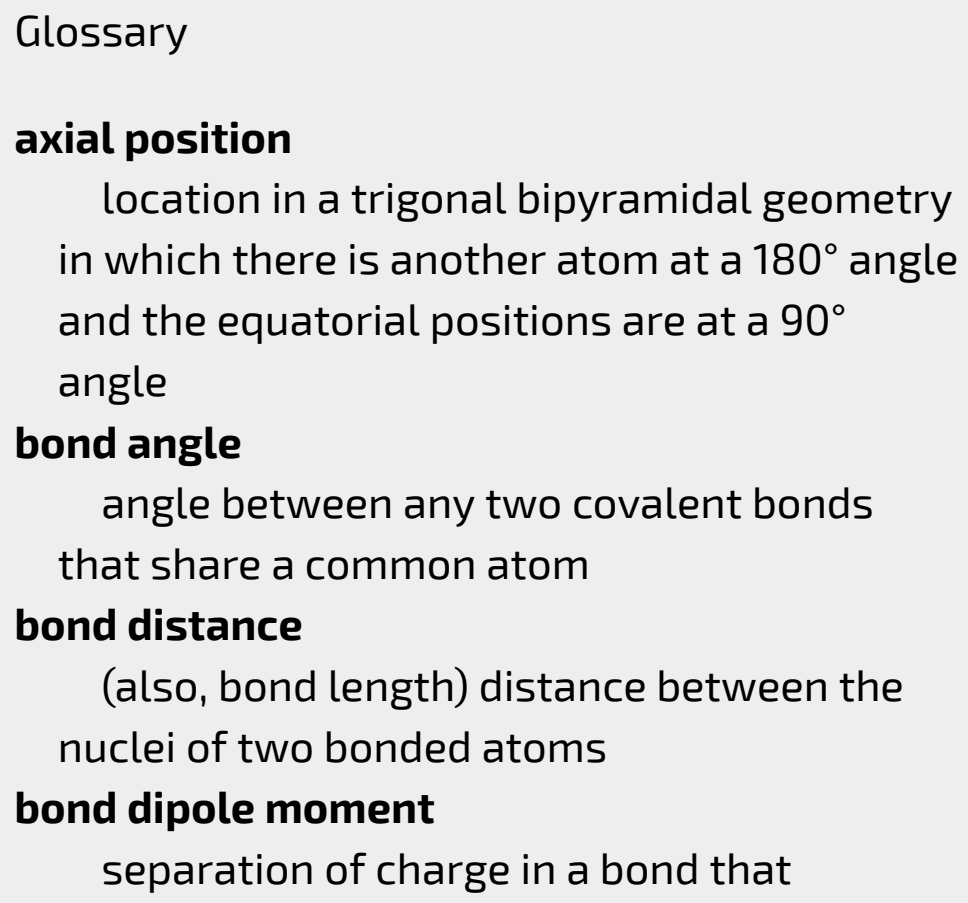


depends on the difference in

electronegativity and the bond distance represented by partial charges or a vector

\section{dipole moment}

property of a molecule that describes the separation of charge determined by the sum of the individual bond moments based on the molecular structure

\section{electron-pair geometry}

arrangement around a central atom of all regions of electron density (bonds, lone pairs, or unpaired electrons)

\section{equatorial position}

one of the three positions in a trigonal bipyramidal geometry with $120^{\circ}$ angles between them; the axial positions are located at a $90^{\circ}$ angle

\section{linear}

shape in which two outside groups are placed on opposite sides of a central atom molecular structure

structure that includes only the placement of the atoms in the molecule

\section{octahedral}

shape in which six outside groups are placed around a central atom such that a three-dimensional shape is generated with four groups forming a square and the other two forming the apex of two pyramids, one above and one below the square plane 


\section{polar molecule}

(also, dipole) molecule with an overall dipole moment

tetrahedral

shape in which four outside groups are placed around a central atom such that a three-dimensional shape is generated with four corners and $109.5^{\circ}$ angles between each pair and the central atom

\section{trigonal bipyramidal}

shape in which five outside groups are placed around a central atom such that three form a flat triangle with $120^{\circ}$ angles between each pair and the central atom, and the other two form the apex of two pyramids, one above and one below the triangular plane

\section{trigonal planar}

shape in which three outside groups are placed in a flat triangle around a central atom with $120^{\circ}$ angles between each pair and the central atom

valence shell electron-pair repulsion theory (VSEPR)

theory used to predict the bond angles in a molecule based on positioning regions of high electron density as far apart as possible to minimize electrostatic repulsion vector

quantity having magnitude and direction 


\section{Focus on The Ozone Layer}

Basic chemical structures, bond energies and the breaking of bonds by electromagnetic radiation is sufficient background to take an elementary look at a very important set of reactions taking place in another part of the atmosphere, the stratosphere.

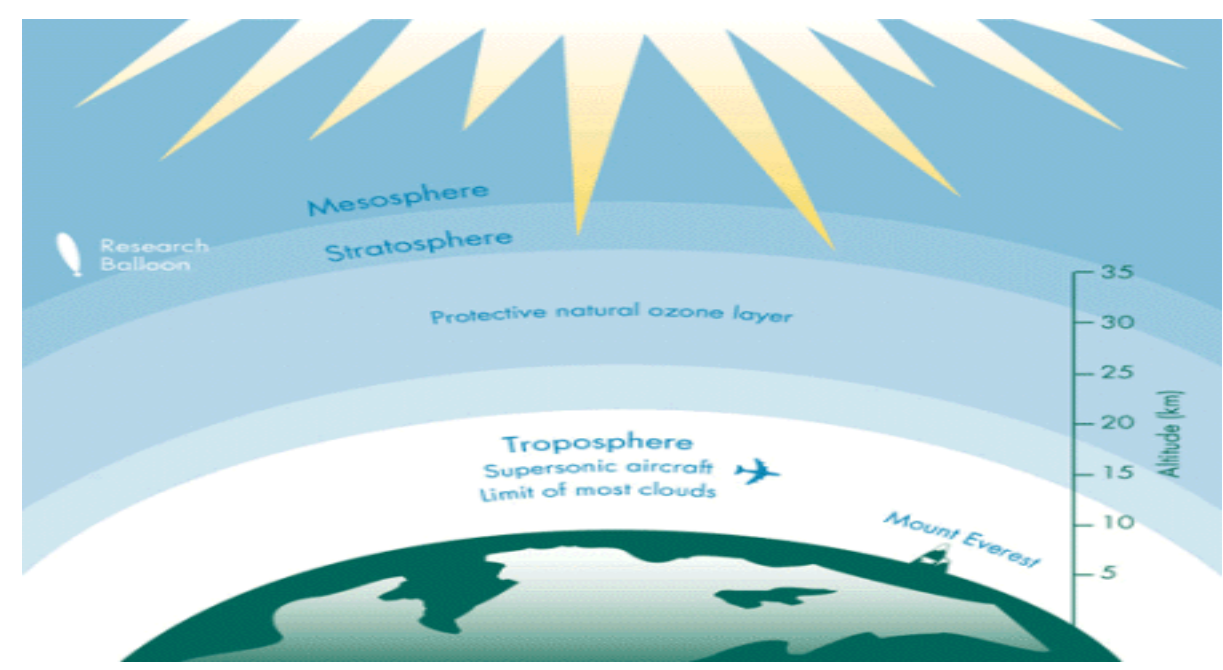

(C) Environment and Climate Change Canada. Layers of the Atmosphere. Government of Canada https://www.canada.ca/en/environment-climatechange/services/air-pollution/issues/ozone-layer/depletionimpacts/about.html

The four reactions of the Chapman cycle are key to protecting life on earth from harmful ultraviolet radiation. It was determined that a set of anthropogenic compounds were putting this protective ozone layer at risk. Refer to the information discussed in the previous Focus section as well as parts VI and VII of this text and elaborate on the following focus boxes, solving the problems that appear. Use the information to frame the story of the thinning ozone layer through the science and events. 

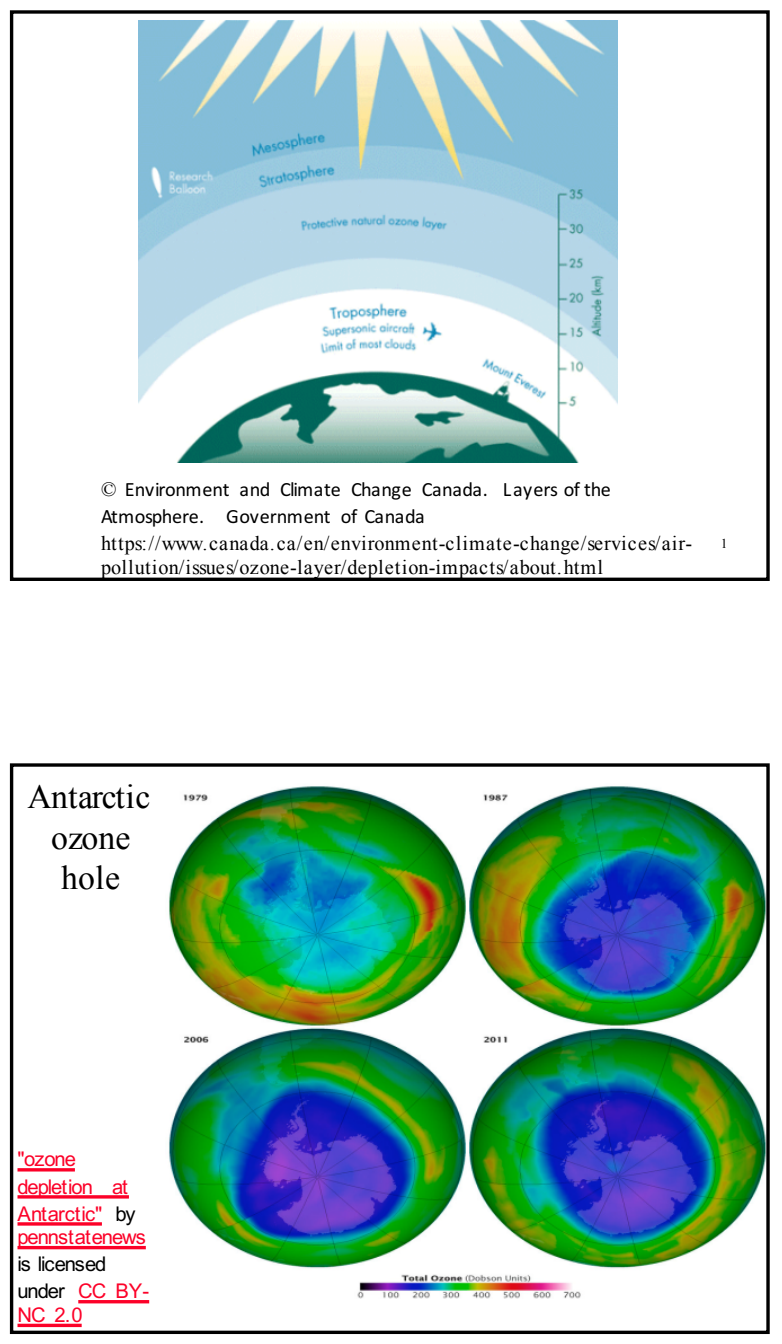

\section{Antarctic Ozone, July - September 2010}

- http://www.flickr.com/photos/gsfc/ 4995772362/

Animation provided by NASA/Goddard Space Flight Center. 


\section{Atomic Theory}

- matter is composed of discrete units called atoms, arranged in unique ways.

- However, atoms are also made up of smaller particles ...
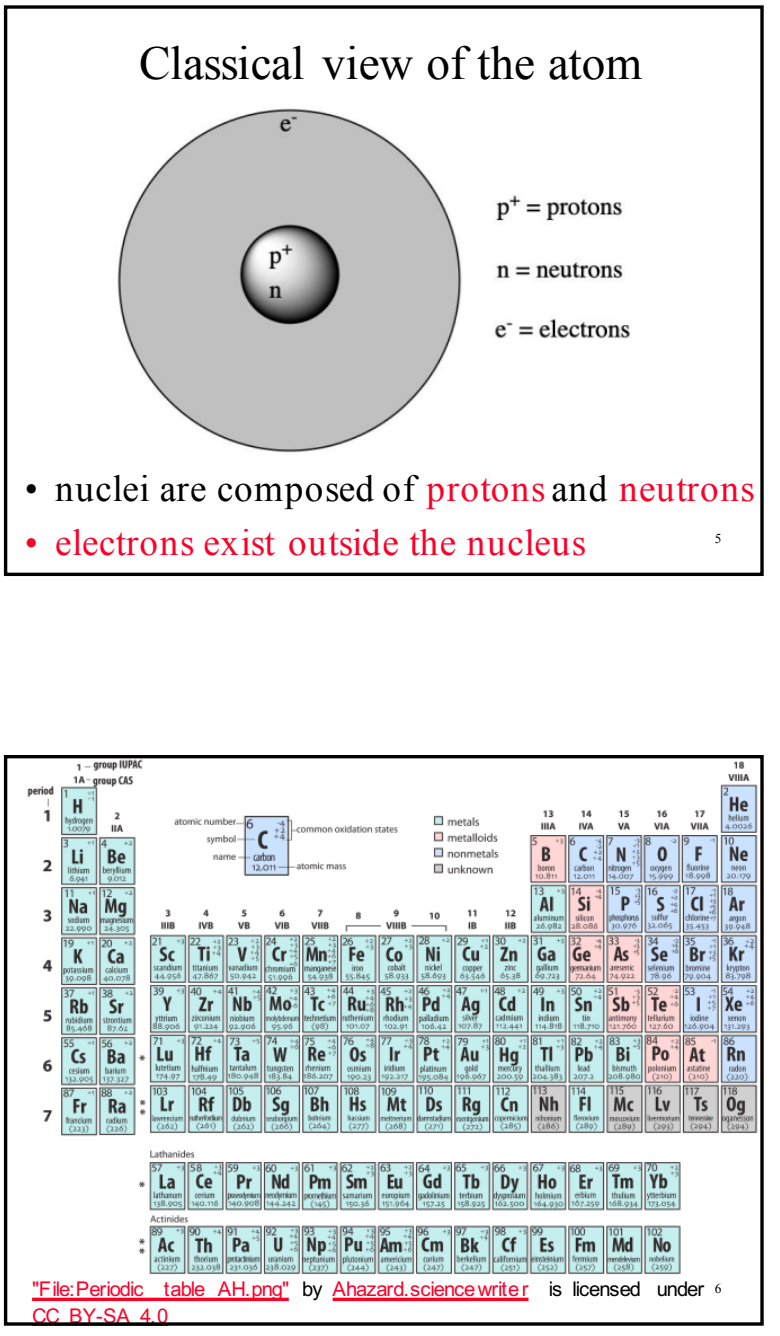


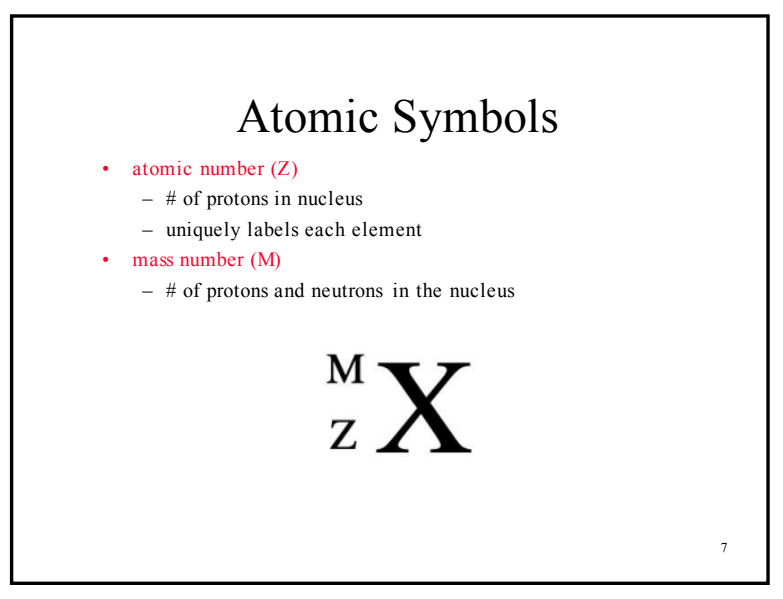

What are the \# of protons and neutrons in ${ }^{238} \mathrm{U}$ ?

- A. 238 protons, 92 neutrons

- B. 92 protons, 156 neutrons

- C. 92 protons, 146 neutrons

- D. 156 protons, 92 neutrons

- E. 146 protons, 92 neutrons

What are the \# of protons and neutrons in ${ }^{1} \mathrm{H}$ ? ${ }^{2} \mathrm{H}$ ? 


\section{Isotopes}

- isotopes: same Z, different M.

- Ex: What is the number of protons and neutrons in the following isotopes?

$$
{ }_{1}^{1} \mathrm{H} \quad{ }_{1}^{2} \mathrm{H} \quad{ }_{1}^{3} \mathrm{H} \quad{ }^{12} \mathrm{C} \quad{ }^{13} \mathrm{C} \quad{ }^{14} \mathrm{C}
$$

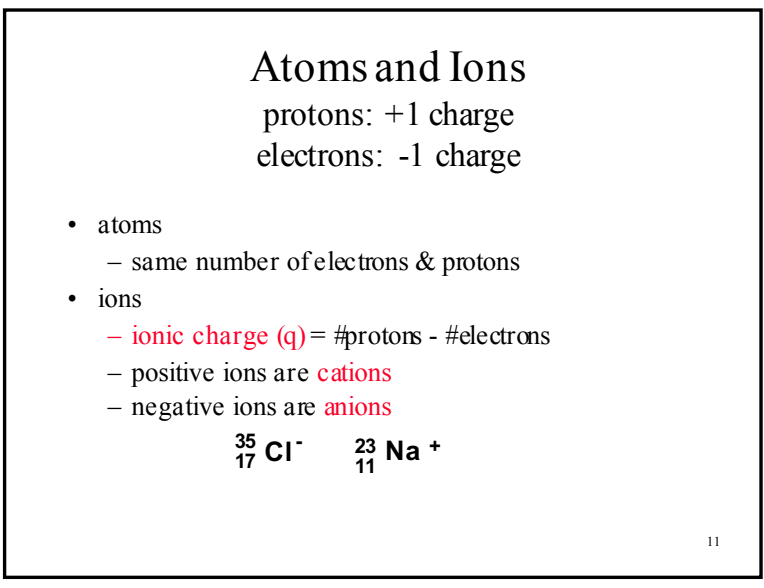

\section{What is the \# of protons, neutrons and electrons in ${ }^{23} \mathrm{Na}^{+}$}

- A. 23 protons, 23 neutrons, 11 electrons

- B. 12 protons, 11 neutrons, 11 electrons

- C. 11 protons, 11 neutrons, 12 electrons

-D. 11 protons, 12 neutrons, 12 electrons

- E. 11 protons, 12 neutrons, 10 electrons 
What is the \# of protons, neutrons and electrons in ${ }^{74} \mathrm{Se}^{2-}$

- A. 74 protons, 34 neutrons, 32 electrons

- B. 34 protons, 40 neutrons, 36 electrons

- C. 40 protons, 74 neutrons, 42 electrons

- D. 34 protons, 40 neutrons, 40 electrons

- E. 32 protons, 34 neutrons, 74 electrons

\section{Quantum Theory}

- both matter and light had to be viewed as having particle properties as well as wave properties.

- light ----> photons

- electrons ---> waves

\section{Classical view of the $\mathrm{H}$ atom}

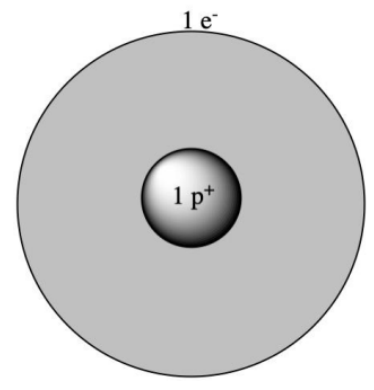




\section{Quantum-mechanical view of the} $\mathrm{H}$ atom

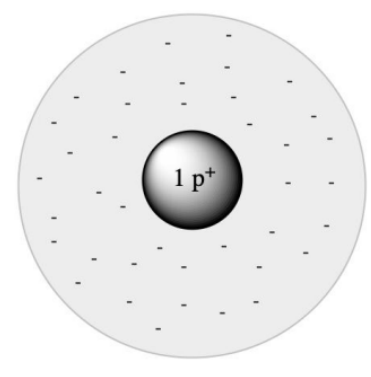

Quantum theory: electrons do not exist in any well-defined area of space and have only certain 'allowed' energy levels. These energy levels have a maximum capacity for electrons.

Exercise: Determine the electron configurations for the first 18 elements $(\mathrm{z}=1$ to $\mathrm{z}=18)$.

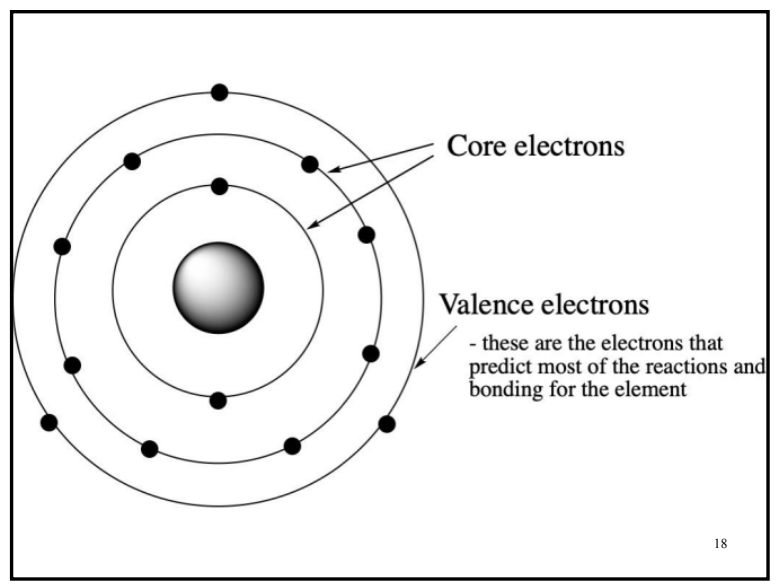


Note: periodic table

- Most periodic tables are labelled such that group A numbers refer to the number of valence electrons.

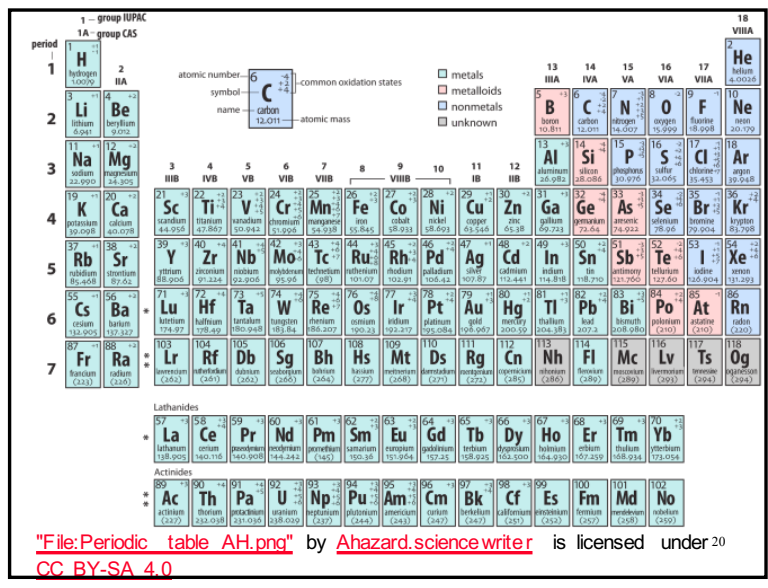

\section{How many valence electrons does} a neutral oxygen atom have?

- (a) 2

- $\underline{\text { (b) }} 6$

- $\underline{\text { (c) } 8}$

- (d) 16

- (e) 32 


\section{How many electrons does a neutral fluorine atom have?}

- (a) 9

- $\underline{\text { (b) }} 8$

- (c) 7

- $\underline{\text { (d) } 6}$

- (e) 5

\section{Lewis Structures}

Draw the atomic symbol

Determine the number of valence electrons for the element

- Place 1 electron at a time in each of 4 spaces around the atomic symbol Each space can hold 2 electrons

- Ex: Draw the Lewis structures for the first 18 elements $(Z=1$ to $Z=18)$.

\section{Noble Gases - Group VIIIA}

-The noble gases, noted for their stability, share a common 'closed shell' electron configuration.

-All of the noble gases have 8 valence electrons (except for helium which has 2). 


\section{Lewis Structures of Molecules}

- many elements will form molecules based on the closed shell rule.

- atoms will

- gain or lose e- (ionic compounds)

- share $\mathrm{e}^{-}$(covalent compounds)

\section{Covalent examples - Draw the}

Lewis structures for the following molecules:

$\begin{array}{llllll}\mathrm{H}_{2} & \mathrm{~F}_{2} & \mathrm{CH}_{4} & \mathrm{CCl}_{4} & \mathrm{NH}_{3} & \mathrm{H}_{2} \mathrm{~S}\end{array}$

$\begin{array}{lll}\mathrm{O}_{2} & \mathrm{CO}_{2} & \mathrm{CO}\end{array}$

\section{Covalent Bonds}

- The first covalent bond formed between two elements is between orbitals that are in the direction of the bond. These are known as $\boldsymbol{\sigma}$ bonds.

- Any further bonding that occurs is between orbitals that are at right angles to the direction of the bond. These are known as $\pi$ bonds. 


\section{Bond Length/Bond Strength}

- The greater the number of bonds (pairs of electrons) between two particular atoms in a molecule, the closer the two atoms will be to each other and the stronger the bond will be.

- for example, it takes more energy to break the $\mathrm{CO}$ bond in carbon monoxide than it does to break the $\mathrm{CO}$ bond in carbon dioxide

Draw the Lewis structures
(including all resonance
structures) of ozone $\left(\mathrm{O}_{3}\right.$, one $\mathrm{O}$ is
central to the other two $)$
- the electron pair forming the $\pi$ bond is
'delocalized' over the three oxygen atoms,
as opposed to being 'localized' between two
atoms like the $\sigma$ bonds are.

\section{Ozone}

- has two equivalent O-O bonds and their properties are intermediate between that normally found for a $\mathrm{O}-\mathrm{O}$ single bond and that normally found for an $\mathrm{O}-\mathrm{O}$ double bond. 


\section{Example}

Draw all of the resonance structures of the Nitrate ion, $\mathrm{NO}_{3}{ }^{-}$

\section{Electromagnetic Radiation}

\section{(Light)}

- A form of energy (photons) that exhibit wave-like behavior as they travel through space. The energy can be characterized by:

- wavelength: the distance between peaks where the level of the field is the same. The longer the wavelength, the lower the energy of the light.

- frequency: how many waves pass a given point in a given amount of time. The higher the frequency, the higher the energy of the light.

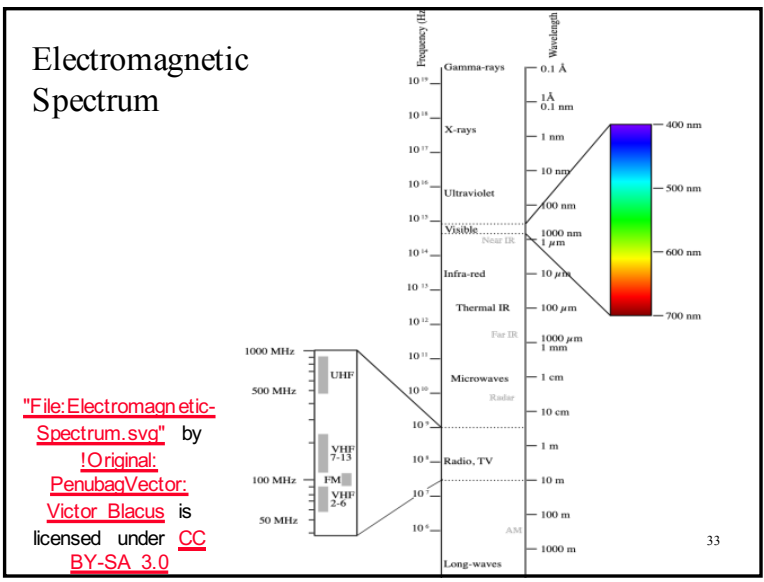




\section{FM radio wave at $3 \mathrm{~m}\left(1 \times 10^{8} \mathrm{~Hz}\right)$}

- The photons have an energy that our bodies are unable to detect unless it is fed through a receiver and converted into sound.

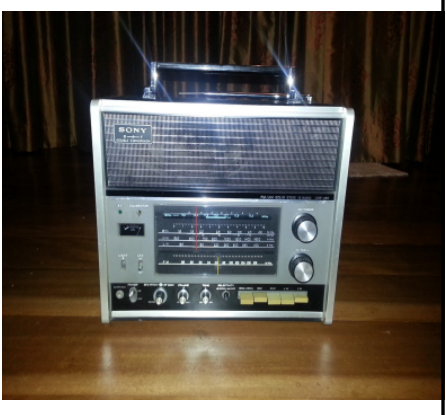

\section{UV light with \\ wavelength $=300 \mathrm{~nm}\left(3 \times 10^{-7} \mathrm{~m}\right)$}

- The photons have an energy which is on the order of covalent bond energies and may break covalent bonds.

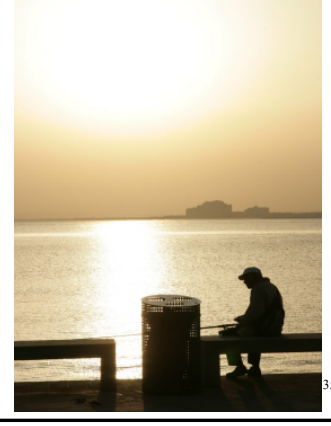

\section{Stratospheric Ozone: Its Formation and Fate}

- each day, $\sim 300,000$ tons of ozone are created and destroyed in the four reactions of the Chapman Cycle. The constant creation and destruction of ozone results in a steady state (constant amount) of the compound. 


\section{Chapman cycle}

- Sketch the reactions of the Chapman cycle and demonstrate how certain frequencies of the sun's UV light are filtered out in the stratosphere.

- Identify any 'free radicals'- an unstable chemical species with an unpaired electron.

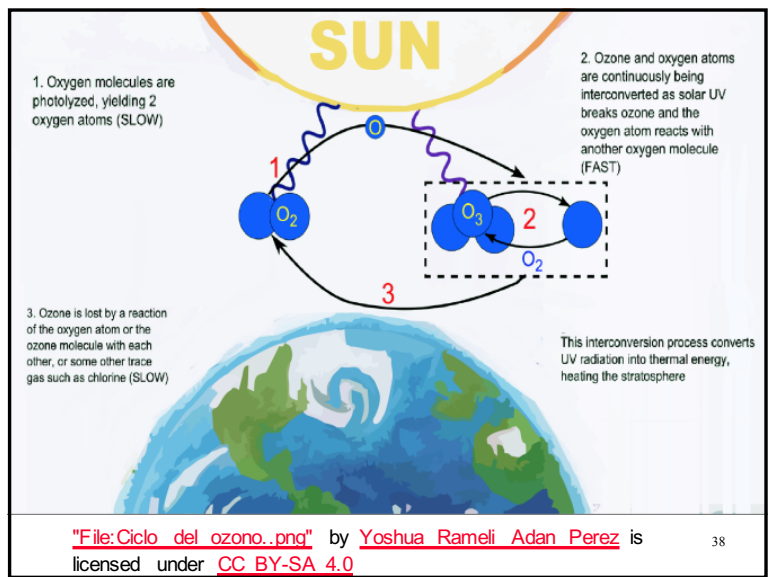

Elaborate on this graph. Why is there less intensity of electromagnetic radiation of lower wavelengths at the surface of the earth?

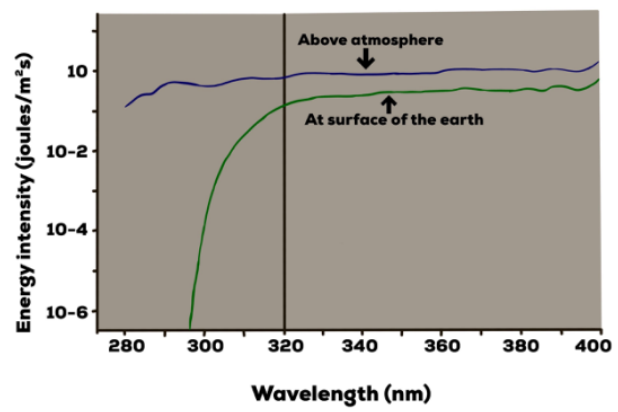


Elaborate on this graph. Why is biological sensitivity higher for electromagnetic radiation of lower wavelengths?

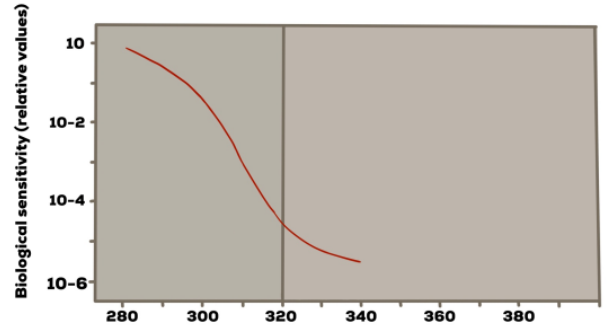

Wavelength $(\mathrm{nm})$

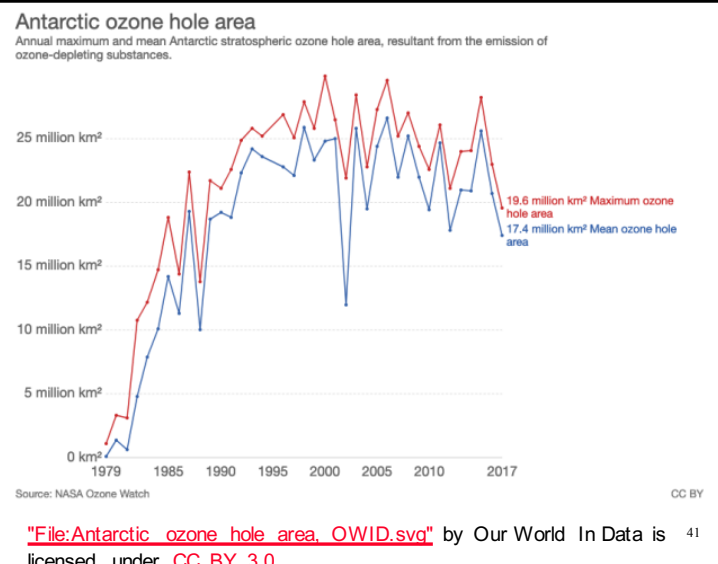

"File:Antarctic ozone hole area, OWID.svg" by Our World In Data is 41 licensed under CC BY 3.0

\section{Chlorofluorocarbons}

- anthropogenic chemicals introduced as a safe alternative to the relatively unsafe substances used in refrigeration.

- Ex: $\mathrm{CF}_{2} \mathrm{Cl}_{2}$

heat $+\mathrm{CF}_{2} \mathrm{Cl}_{2}(\mathrm{i}) \rightarrow \mathrm{CF}_{2} \mathrm{Cl}_{2(\mathrm{~g})}$

- Due in part to their inertness, they soon found many other uses. 


\section{Chlorofluorocarbons}

- Their inertness is due to the great strength of the $\mathrm{C}-\mathrm{F}$ and $\mathrm{C}-\mathrm{Cl}$ bonds. However, rather than remaining harmless in the troposphere, they eventually find their way up to the stratosphere where they react with high energy electromagnetic radiation and $\mathrm{Cl}$ radicals are released.

\section{Show the reaction sequence that} demonstrates catalytic destruction of ozone by chlorine radicals

- one $\mathrm{Cl}$ radical can break down over 100,000 ozone molecules before it is removed from this stratospheric cycle.

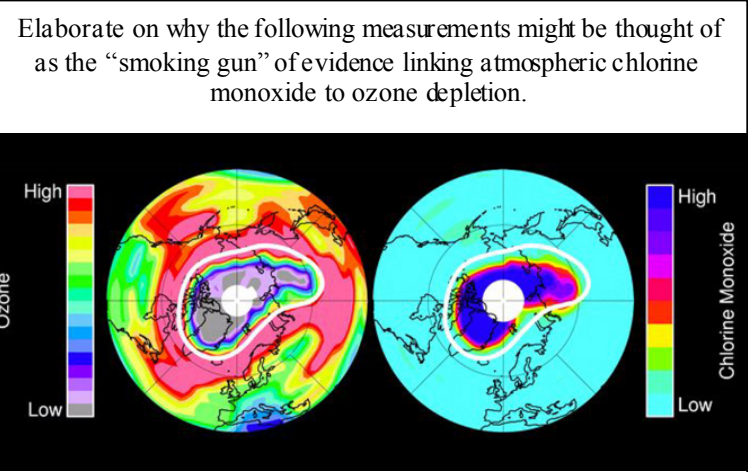

Image courtesy of NASA Jet Propulsion Laboratory at: https://www.jpl.nasa.gov/spaceimages/details.php?id=PIA14824 ${ }^{45}$ 
Antarctic Ozone, July - September 2010

- http://www.flickr.com/photos/gsfc/4995772 $\underline{362 /}$

\section{Antarctic Winter/Spring}

- Extreme cold of winter ( $-90 \mathrm{C})$ freezes atmospheric water into stratospheric clouds of ice crystals. Also condenses other species

- Otherwise safe molecules can be converted to more reactive species. When the sun comes out, the break down of these species may release chlorine radicals.

\section{7 - Montreal Protocol}

- Phaseout of production and use of certain CFC's, development of new compounds.

- Continually being refined, new compounds being added to ban (HCFC's).

- Ex. 2005: $\mathrm{CH}_{3} \mathrm{Br}$ phaseout begins.

- Ex. 2013/2015: HCFC's phaseout begins.

- Many CFC's have a lifetime of $\sim 100$ years, however, some levelling off of effective $[\mathrm{Cl}]$ and $[\mathrm{Br}]$ has been observed. 

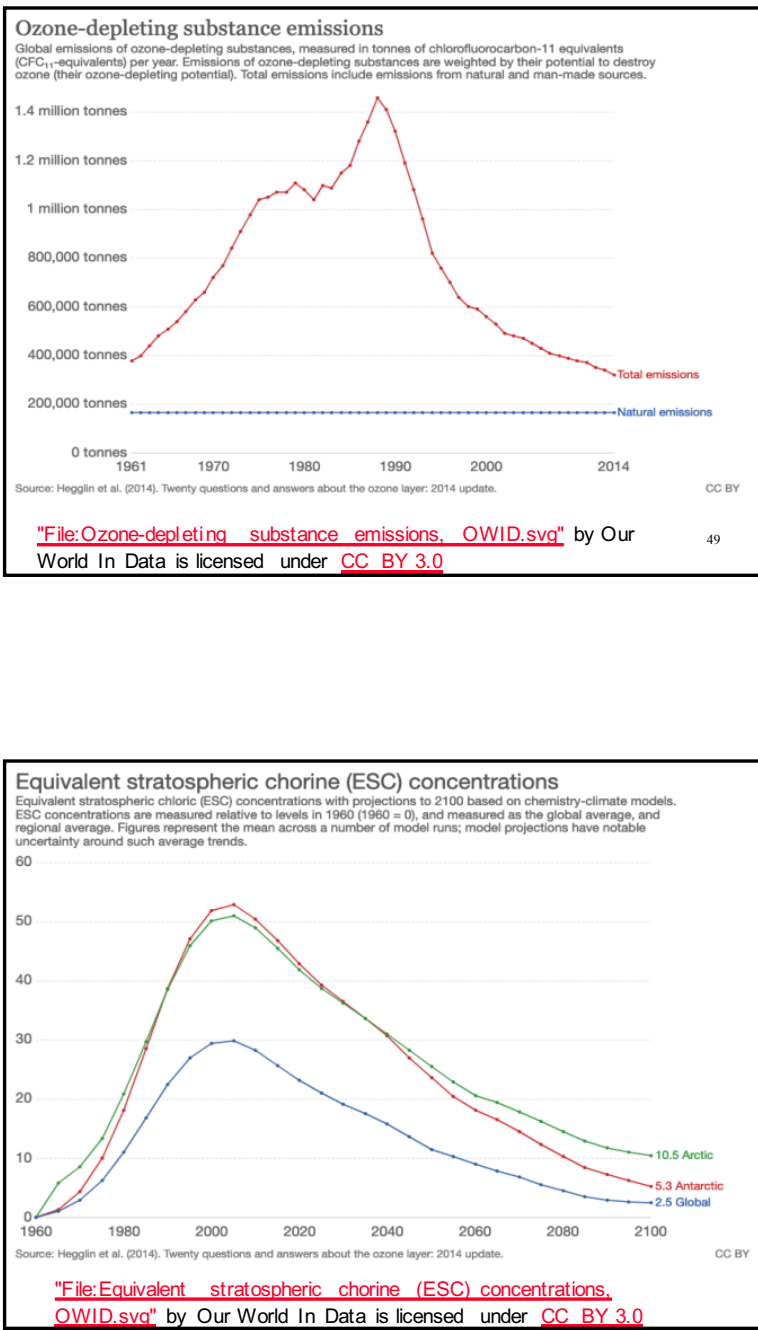

Excess skin cancer cases due to ozone depletion, United States Historic estimates of the number of excess skin cancer cases, measured per million neople, resultant from stratospheric ozone depletion (which increases exposure to UV radiation). Estimated under scenario of $n$ o
restrictions on ozone-depleting substances, the initial Montreal Protocol and Copenhagen Amendment, which

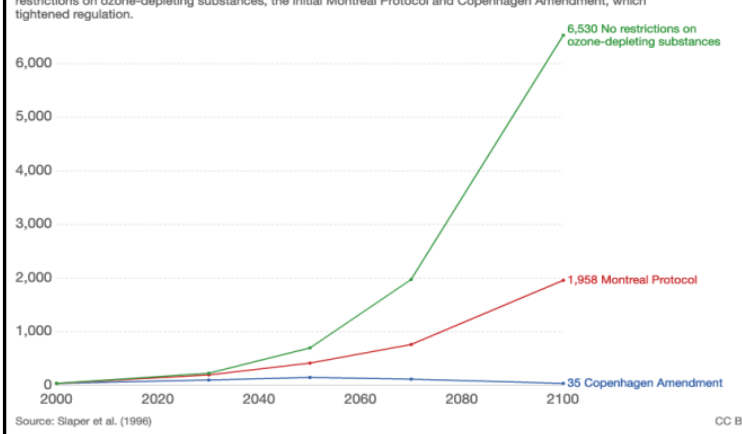

"File:Excess skin cancer cases due to ozone depletion, OWID.svg" 51 by Our World In Data is licensed under CC BY 3.0 


\section{Ozone Watch}

https://earthobservatory.nasa.gov/features/vid eos/the-ozone-hole

Video provided by NASA/Goddard Space Flight Center.

\section{Summary - Ozone/CFC's}

- Chapman cycle

- Potentially catastrophic series of events.

- 'Smoking gun' of evidence.

- Montreal Protocol, and subsequent refinements.

- Substitutes were (are) available.

- Economic setback (but not devastating).

- The ozone 'holes' are shrinking 


\section{Focus on Climate Change}

Molecular shape, polarity and a further look at the interaction of electromagnetic radiation and matter enable one to understand the fundamentals of the greenhouse effect.

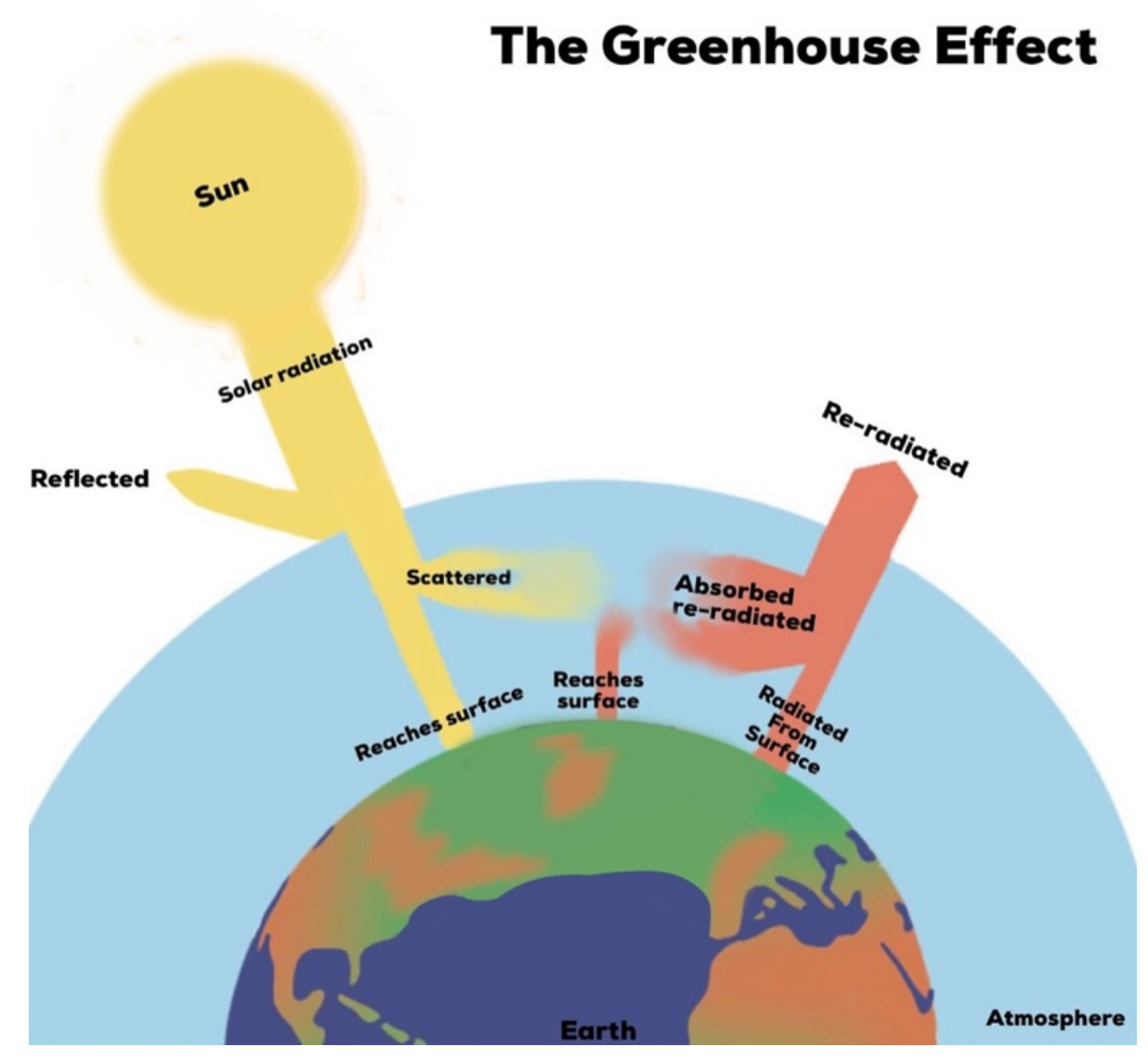

When the suns energy warms the atmosphere and surface of the earth, much of that heat is re-radiated back out into the atmosphere. Molecules in the atmosphere absorb some of that released heat and then re-release it, warming the atmosphere and surface of the earth further. Without this natural greenhouse effect, the earth's average global temperature would be approximately $-18{ }^{\circ} \mathrm{C}$, but instead it is $+15{ }^{\circ} \mathrm{C}$. This $33{ }^{\circ} \mathrm{C}$ temperature difference is crucial to the way that life and society have evolved and a stable average temperature is thought to be an important metric for many climate related issues such as weather and sea levels. 
Anthropogenic release of extra greenhouse gases is being blamed for a measurable increase in recent global temperatures.

Refer to the information discussed in the previous Focus section and elaborate on the following focus boxes, solving the problems that appear. Use the information to frame the story of the climate change debate to where it stands today. It is quite useful to compare the story to that of the ozone layer. 

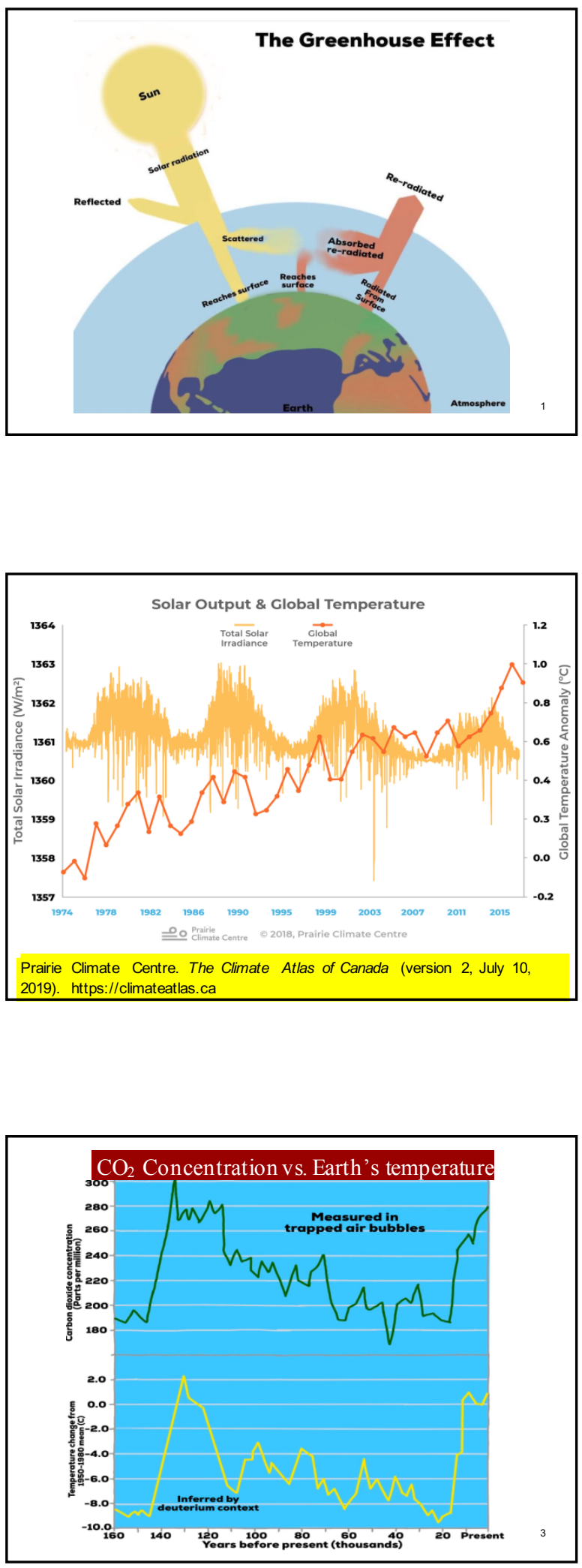

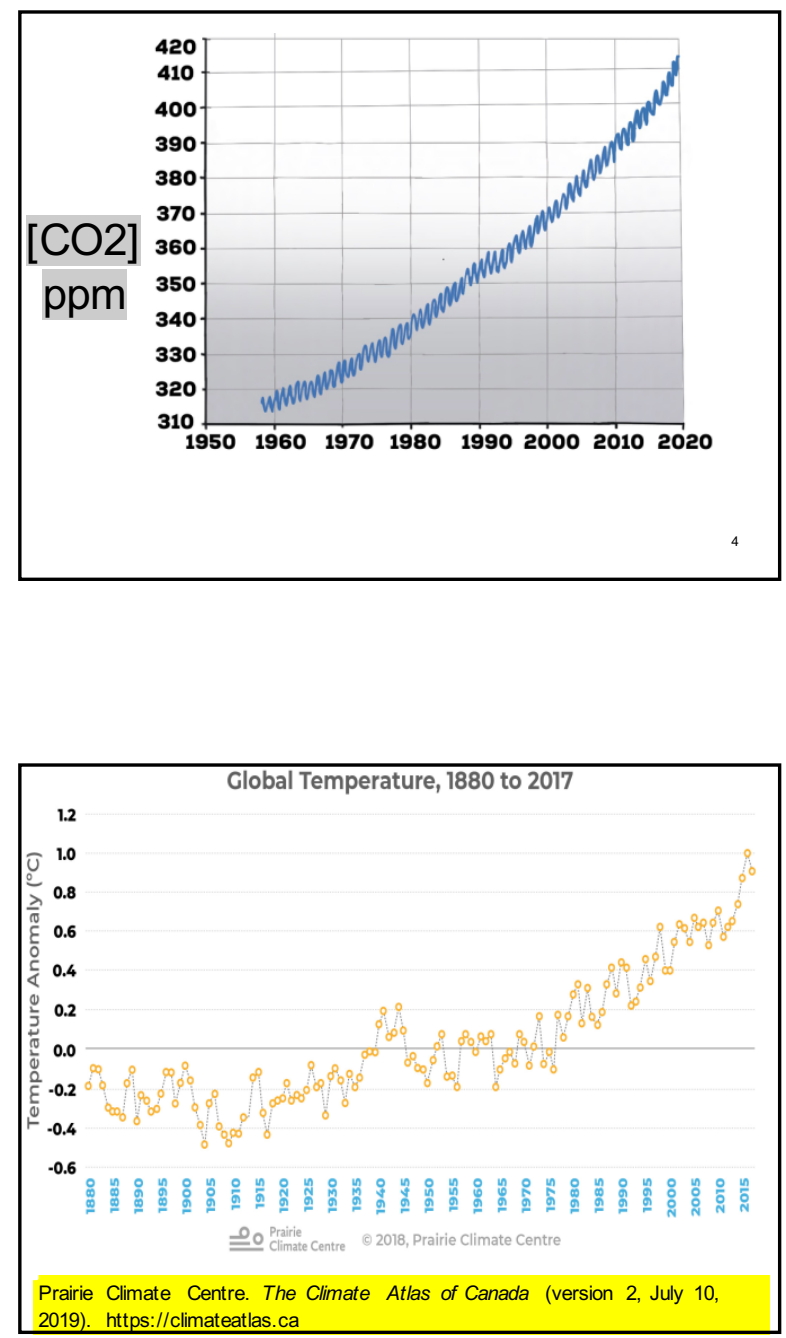

\section{Climate Atlas $-1^{\circ} \mathrm{C}$ and its impacts}

- https://climateatlas.ca/video/one-degreeand-its-impacts

Rebroadcast with permission of the Climate Atlas, Prairie Climate Centre https://climateatlas.ca/ 
Galileo Movement AustraliaAxe the Tax

- https://www.youtube.com/watch?t=7\&v=B C114geSTP8

Rebroadcast with permission of the Galileo Movement Australia

http://www.galileom ov ement.com.au/

\section{Molecular Shape}

- The 3-dimensional positions of bonded atoms in the molecule with respect to a central atom.

- Valence Shell Electron Pair Repulsion (VSEPR)

\section{non-bonding electrons}

- VSEPR predicts the positions of all electrons around a central atom (electron pair geometry).

- Molecular geometry involves the 3dimensional positions of atoms only.

- Further, lone pairs of electrons take up more room than bonding pairs of electrons. 
Ex: Determine the molecular shape of the following molecules.

ie. consider the central atom and determine the number of 'groups of electrons' (symmetry number, SN), then determine the molecular shape.
$\mathrm{Ex}: \mathrm{CO}_{2}$
$\mathrm{Ex}: \mathrm{H}_{2} \mathrm{CO}$
$\mathrm{Ex}: \mathrm{CH}_{4}$

Ex: $\mathbf{C F}_{2} \mathbf{C l}_{2}$

$\mathrm{Ex}: \mathrm{NH}_{3}$

Ex: $\mathrm{H}_{2} \mathrm{~S}$

\begin{tabular}{|c|c|c|}
\hline $\begin{array}{l}\text { Electron } \\
\text { groups }\end{array}$ & Examples & Shape \\
\hline $\begin{array}{l}4 \text { groups of } \\
\text { bonding } \\
\text { electrons }\end{array}$ & $\mathrm{CH}_{4}, \mathrm{CF}_{2} \mathrm{Cl}_{2}$ & Tetrahedral \\
\hline $\begin{array}{l}3 \text { bonding, } \\
1 \text { non-bonding }\end{array}$ & $\mathrm{NH}_{3} \quad \mathrm{PF}_{3}$ & pyramidal \\
\hline $\begin{array}{l}2 \text { bonding, } \\
2 \text { non-bonding }\end{array}$ & $\mathrm{H}_{2} \mathrm{O} \quad \mathrm{SF}_{2}$ & bent \\
\hline
\end{tabular}

\section{What is the shape of the $\mathrm{H}_{2} \mathrm{~S}$ molecule?}

- A. Linear

- B. Triangular planar

- C. Tetrahedral

- D. Triangular pyramid

- E. Bent 
What is the shape of the $\mathrm{CHF}_{3}$ molecule?

- A. Linear

- B. Triangular planar

- C. Tetrahedral

- D. Triangular pyramid

- E. Bent

\section{What is the shape of the $\mathrm{SO}_{3}$} molecule ( $\mathrm{S}$ is central)?

- A. Linear

- B. Triangular planar

- C. Tetrahedral

- D. Triangular pyramid

- E. Bent

\section{What is the shape of the $\mathrm{NF}_{3}$ molecule?}

- A. Linear

- B. Triangular planar

- C. Tetrahedral

- D. Triangular pyramid

- E. Bent 


\section{Bond Polarity}

- when two different atoms form a bond, one attracts the shared pair of electrons more strongly than the other.

- the displacement of the bonding pair causes a partially positive charge on one atom and a partially negative on the other.

- The bond is said to have electric poles and be a polarbond.

\section{Molecular Polarity}

- In a polar molecule, electron density accumulates toward one side of the molecule.

- Ex: Is the $\mathrm{CO}_{2}$ molecule polar? What about $\mathrm{H}_{2} \mathrm{O}$ ?

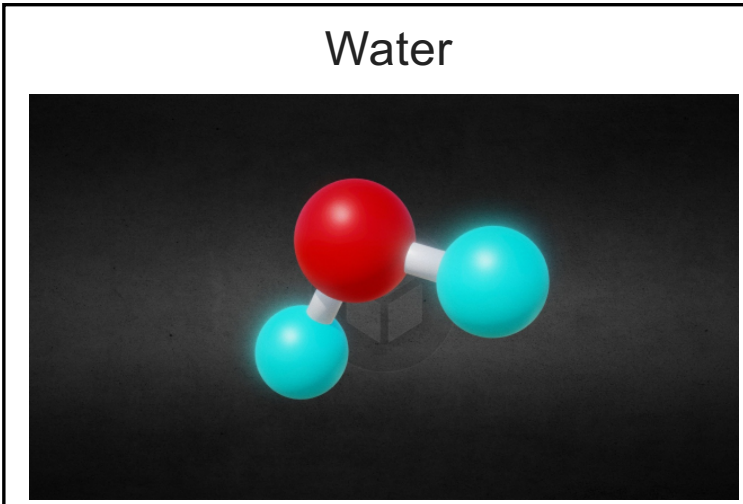

"Water Molecule ball-and-stick model" by borkia is licensed under CC BY-SA 4.0 


\section{Is the $\mathrm{CHF}_{3}$ molecule polar?}

- A. yes

- B. no

- C. Not enough information given

\section{Is the $\mathrm{F}_{2}$ molecule polar?}

- A. yes

- B. no

- C. Not enough information given

Is the $\mathrm{SO}_{3}$ molecule ( $\mathrm{S}$ is central) polar?

- A. yes

- B. no

- C. Not enough information given 


\section{Is the $\mathrm{Cl}_{2} \mathrm{O}$ molecule polar?}

- A. yes

- B. no

- C. Not enough information given

\section{Vibrational States of Molecules}

Based on its molecular geometry, a molecule's bonds are able to undergo certain vibrations. These are known as its 'fundamental modes of vibration'.

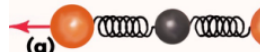

(a)

(c)

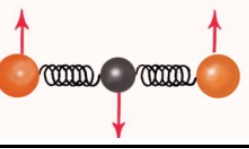

(b)

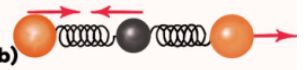

(d)

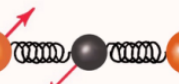

- Like electronic states, there are vibrational states which occur at certain 'fixed' or 'quantized' energy levels.

- Infrared radiation - is not of sufficient energy to break bonds (as in UV/ozone) but can be energetic enough to cause an increase in the amplitude of the 'vibrating'.

- The amount of energy that is absorbed depends on the type of vibration as well as the change in dipole moment as the bonded atoms vibrate. 


\section{Molecular geometry and absorption of IR radiation}

$\underset{\text { (a) }}{\longrightarrow}$

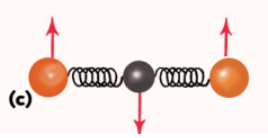

(b)

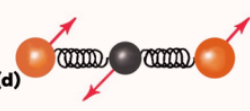

Molecular vibrations in $\mathrm{CO}_{2}$. Each spring represents a $\mathrm{C}=\mathrm{O}$ bond.

(a) = no change in dipole moment - no IR absorption.

$(\mathbf{b}, \mathbf{c}, \mathbf{d})=$ these vibrations have a net change in dipole, so absorption/emission of IR can accompany a vibrational state transition.

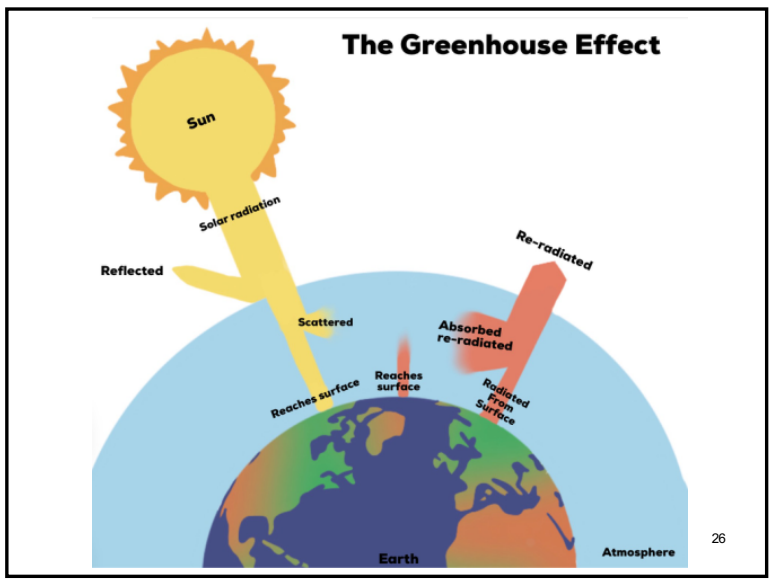

Choose all of the molecules which can absorb infrared radiation?

- A. $\mathrm{N}_{2}$

- B. $\mathrm{O}_{2}$

- C. $\mathrm{Ar}$

- D. $\mathrm{CO}_{2}$

- D. $\mathrm{CH}_{4}$ 


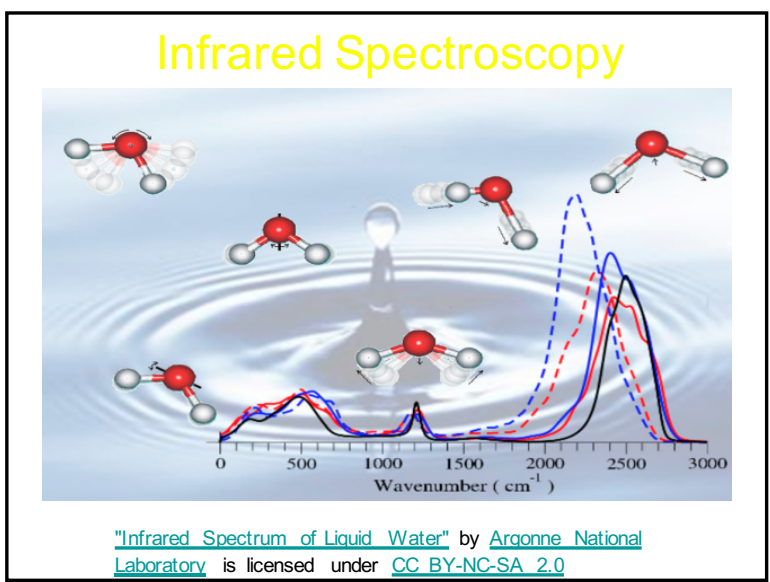

Note: The Composition of Dry Air

In addition to the pie chart below, 0.9 of the " $1 \%$ other gases" is argon. Thus, what percentage of dry air contains greenhouse gases?
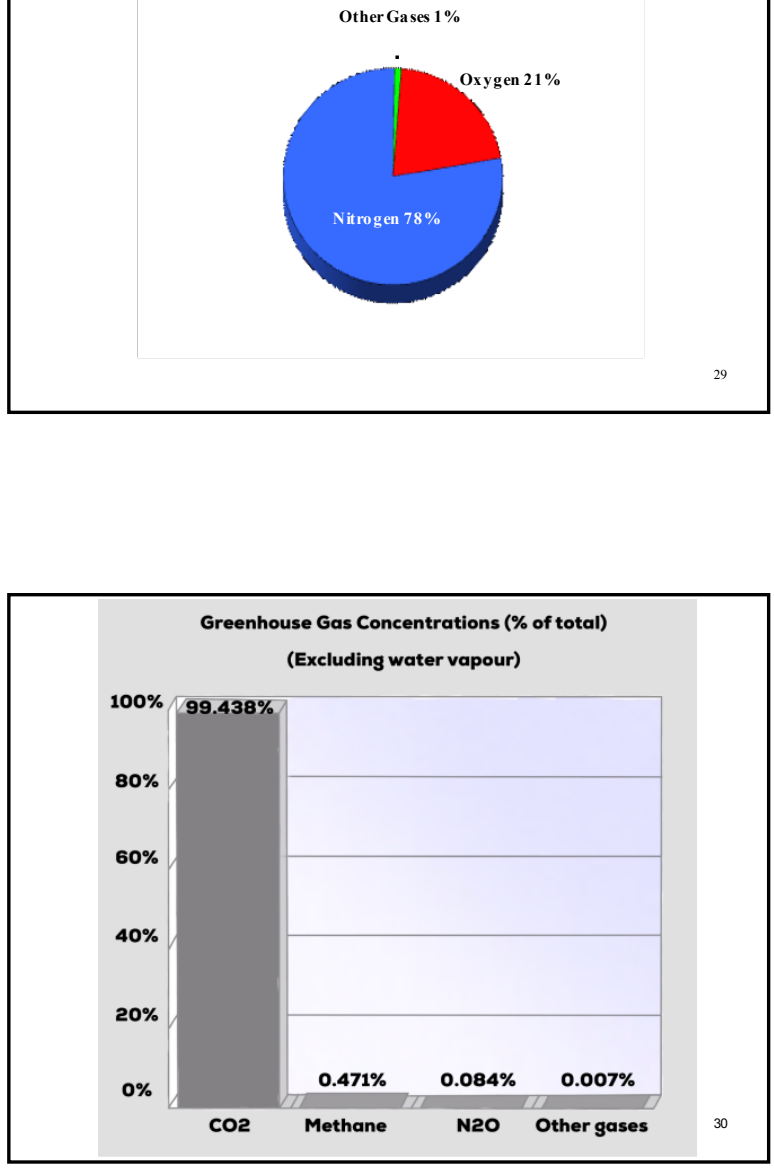
Global Warming Potential (GWP) represents the relative contribution of a molecule of an atmospheric gas to global warming.

$\begin{array}{lllc}\text { Compound } & \text { Name } & \text { anthropogenic sources } & \text { GWP } \\ \mathrm{CO}_{2} & \begin{array}{l}\text { carbon } \\ \text { dioxide }\end{array} & \begin{array}{l}\text { fossil fuel combustion, } \\ \text { deforestation }\end{array} & 1 \\ \mathrm{CH}_{4} & \text { methane } & \text { fossil fuel production, livestock } & 21 \\ \mathrm{~N} 2 \mathrm{O} & \begin{array}{l}\text { nitrous } \\ \text { oxide }\end{array} & \text { combustion, fertilizers } & 310 \\ \mathrm{CCl}_{2} \mathrm{~F}_{2} & \text { CFC-12 } & \text { refrigeration, aerosols } & 8100\end{array}$

Not considering average atmospheric lifetimes, which of the following would you expect to have the greatest global warming

- A. $\mathrm{N}_{2}$ potential?

- B. $\mathrm{CH}_{2} \mathrm{~F}_{2}$

- C. $\mathrm{CO}_{2}$

- D. $\mathrm{CH}_{4}$

- E. $\mathrm{F}_{2}$

Not considering average atmospheric lifetimes, which of the following would you expect to have the greatest global warming potential?

- A. $\mathrm{CO}_{2}$

- B. $\mathrm{CH}_{3} \mathrm{~F}$

-C. $\mathrm{N}_{2}$

- D. $\mathrm{CH}_{4}$

- E. $\mathrm{CCl}_{2} \mathrm{~F}_{2}$ 
Compare the effectiveness of CFC-12 as a ghg to that of $\mathrm{CO}_{2}$ : multiply GWP by concentration.

$\begin{array}{lllcc}\text { Compound } & \text { Name } & \text { anthropogenic sources } & \text { GWP } & \begin{array}{c}2020 \text { atmospheric } \\ \text { concentration }\end{array} \\ \mathrm{CO}_{2} & \begin{array}{l}\text { carbon } \\ \text { dioxide }\end{array} & \begin{array}{l}\text { fossil fuel combustion, } \\ \text { deforestation }\end{array} & 1 & 427 \mathrm{ppm} \\ \mathrm{CH}_{4} & \text { methane } & \begin{array}{l}\text { fossil fuel production, } \\ \text { livestock }\end{array} & 21 & 1875 \mathrm{ppb} \\ \mathrm{N}_{2} \mathrm{O} & \begin{array}{l}\text { nitrous } \\ \text { oxide }\end{array} & \text { combustion, fertilizers } & 310 & 332 \mathrm{ppb} \\ \mathrm{CCl}_{2} \mathrm{~F}_{2} & \text { CFC-12 } & \text { refrigeration, aerosols } & 8100 & 498 \mathrm{ppt} \\ & & & & \end{array}$
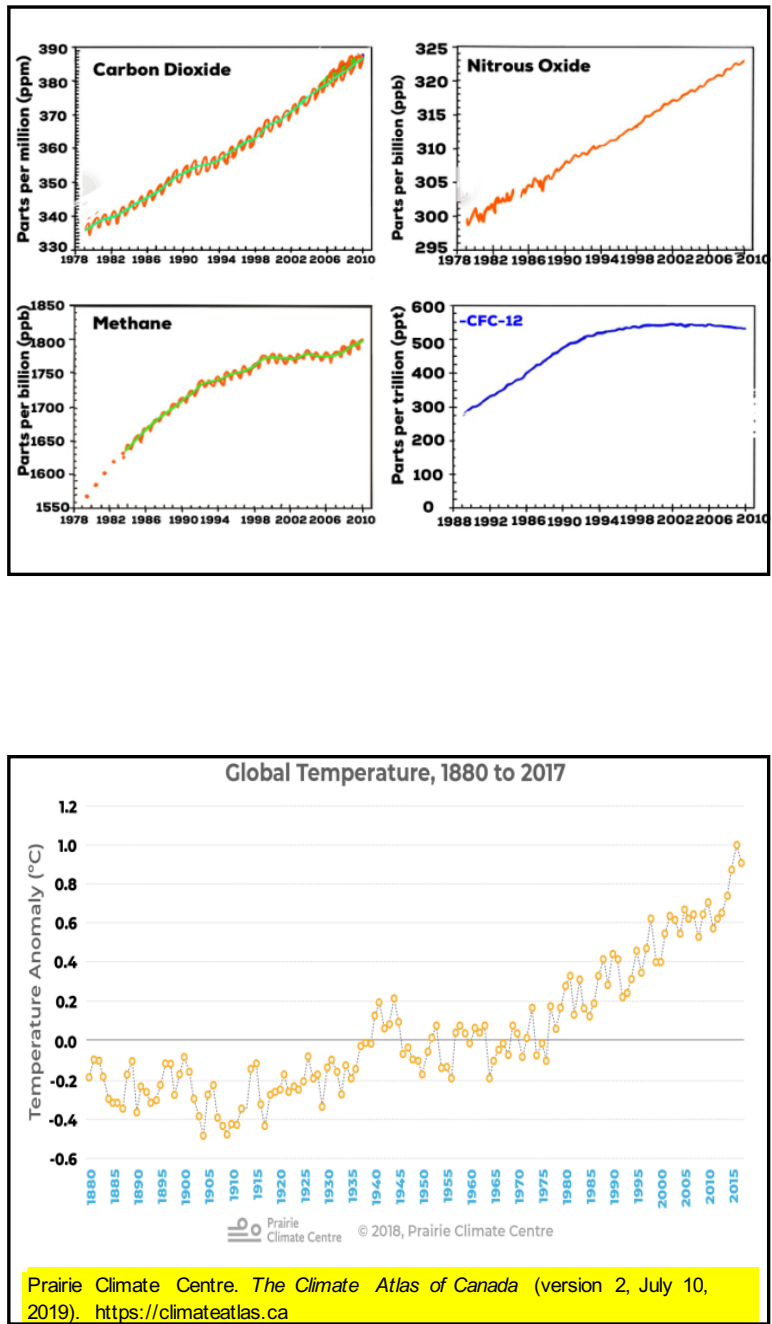

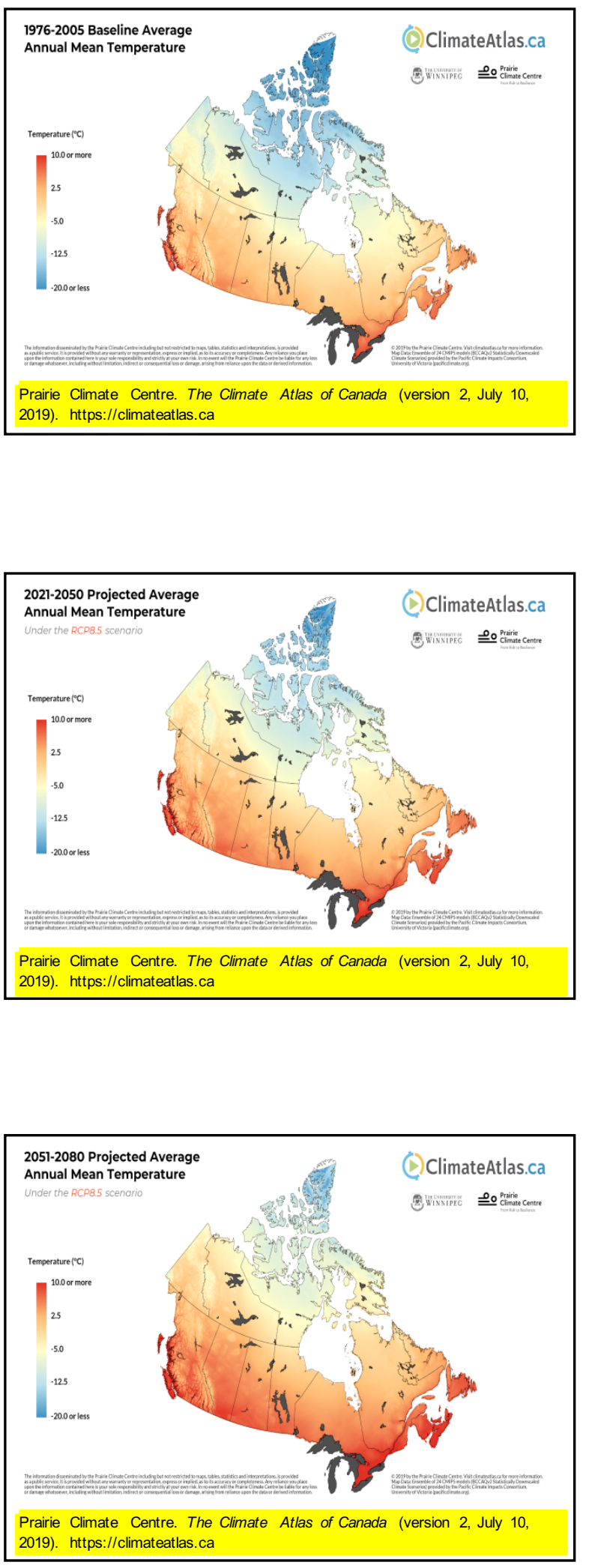

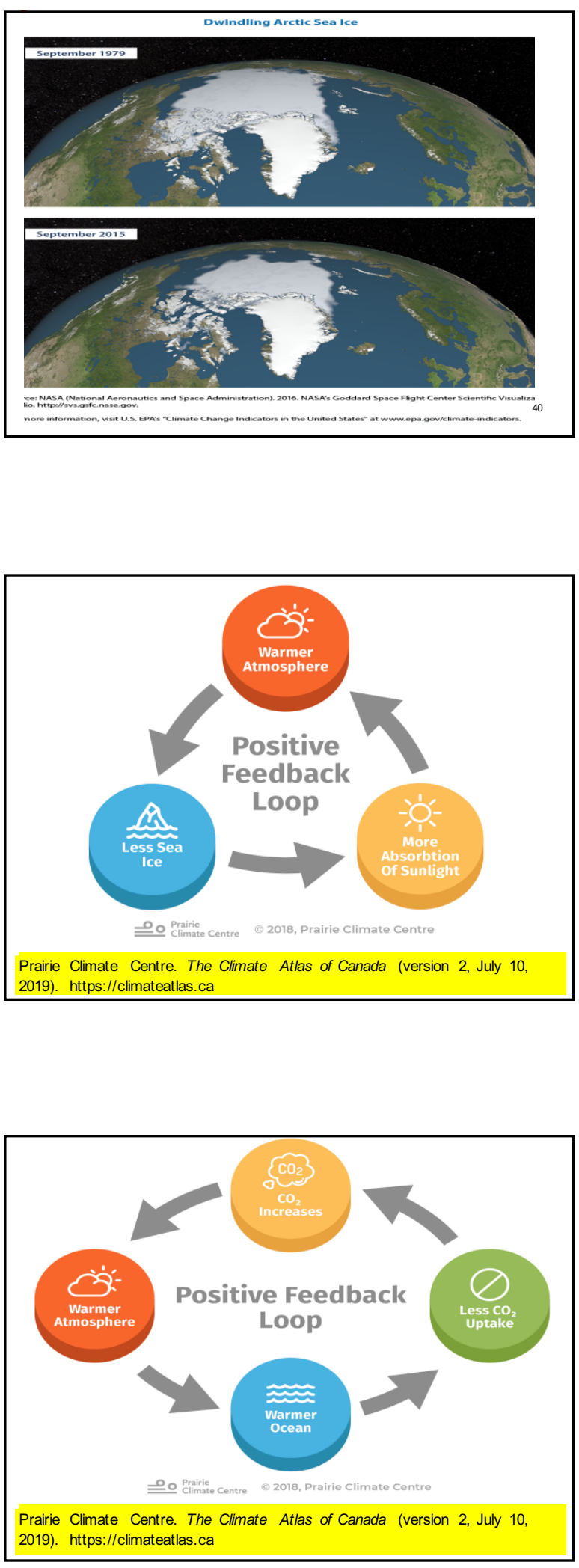


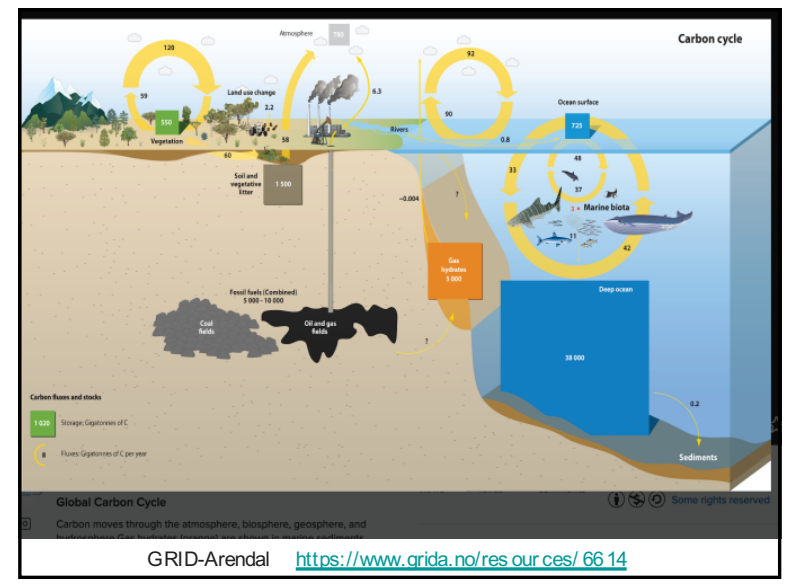

\section{Intergovernmental Panel on Climate Change (IPCC) 1988}

Kyoto Protocol - 1997

- IPCC certified the scientific basis of the greenhouse effect.

-Emission targets set to reduce emissions of six greenhouse gases from 1990 levels.

$\left(\mathrm{CO}_{2}, \mathrm{CH}_{4}, \mathrm{~N}_{2} \mathrm{O}\right.$, HFC's, PFC's, and $\left.\mathrm{SF}_{6}\right)$

-U.S.A. did not ratify

- Many members such as Canada did not meet targets5. Canada has since withdrawn. 


\section{Kyoto successors}

- 'Washington Declaration' - 16 February 2007

- Copenhagen 2009

- Cancun 2010

- South Africa 2011

- Qatar/South Korea 2012 (COP 18)

- Warsaw, 2013

- UN Climate Summit, 2014

- Paris, 2016

\section{Remember: Ozone/CFC's summary}

- Potentially catastrophic series of events.

- Smoking gun.

- Substitutes were (are) available.

- Economic setback (but not devastating).

- Montreal Protocol, and current refinements.

\section{Compare: Climate change} summary

- Scientific events.

- Smoking gun?

- Substitutes available?

- Economic setback?

- Government, industry, personal action? Protocols? 


\section{Focus on Energy}

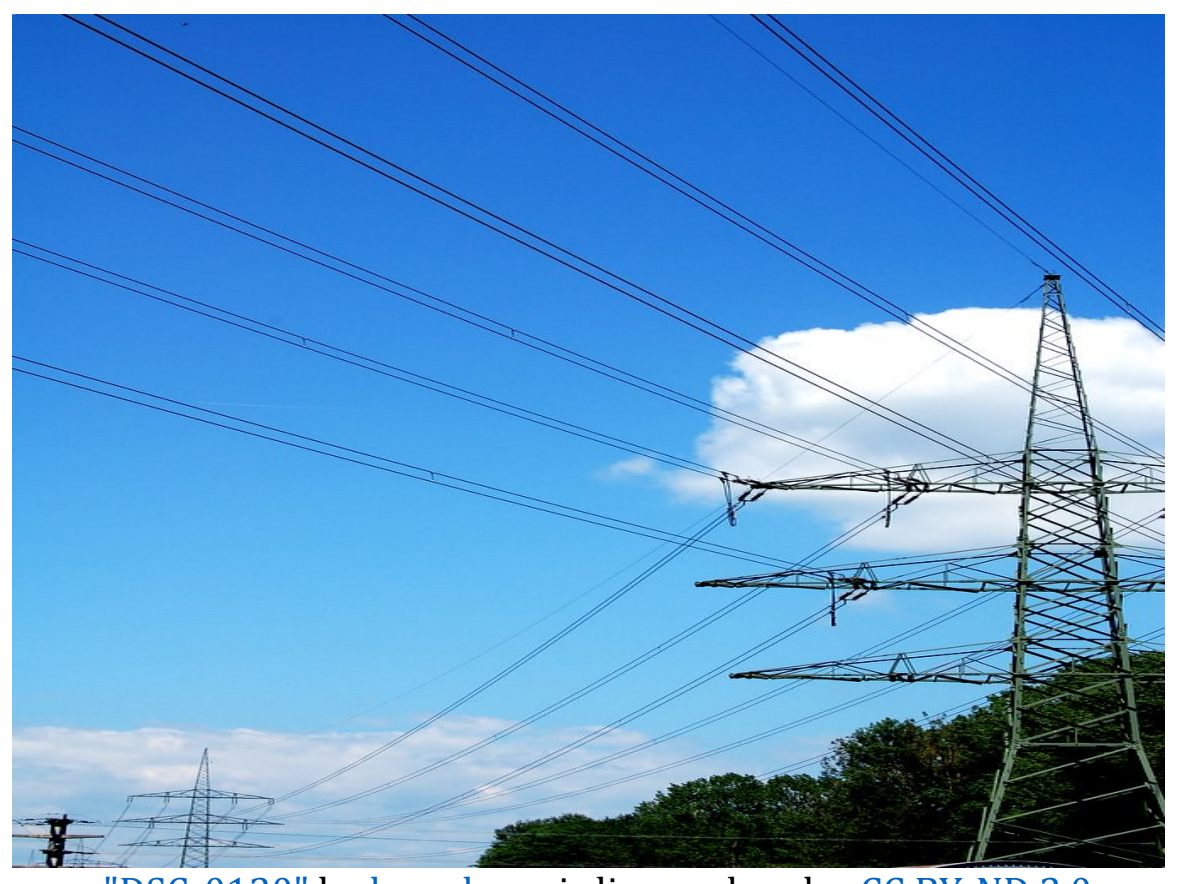

"DSC_0120" by berndnow is licensed under CC BY-ND 2.0

What is energy? Where does the abundance of energy electricity, gasoline, natural gas, etc. - that people have available today come from? Since the industrial revolution began in the $18^{\text {th }}$ century, humankind have discovered a multitude of energy sources to help power our society. The average life expectancy has been extended and the world's population has grown, while our use of external energy sources has continued to grow even faster. With that, there have been rewards, consequences and challenges along the way.

Refer to the information discussed in the previous Focus section as well as Part V of this text and elaborate on the following focus boxes, solving any problems that appear. Make note of the important role that 'fossil' fuels have played and continue to play in meeting our energy demands. In order to determine the best route to meeting our future energy needs, one must understand the benefits and challenges of the processes that are currently in use. 


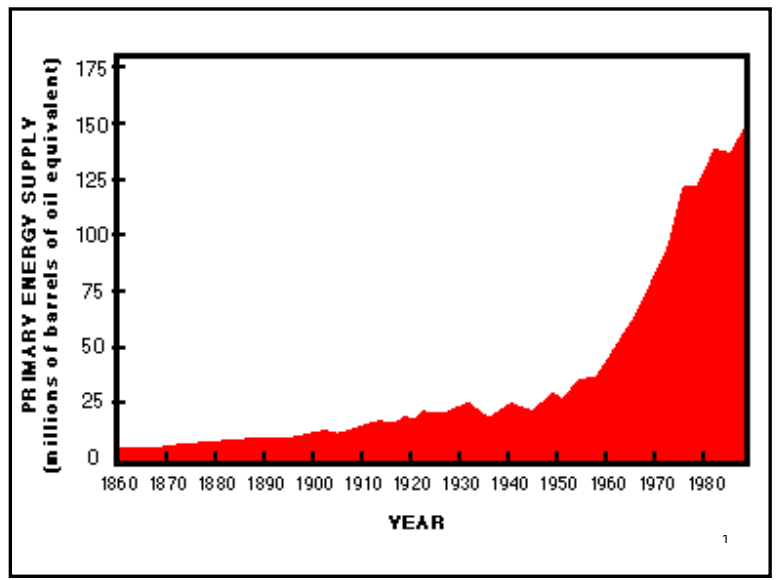

\section{Energy}

Energy can be defined as the capacity to supply heat or do work. One type of work $(w)$ is the process of causing matter to move against an opposing force.

Two types of energy associated with objects can be defined

potential energy - the energy an object has because of its relative position, composition, or condition.

kinetic energy - the energy that an object possesses because of its motion.

Label the following hydroelectric dam with (i) potential energy, (ii) kinetic energy, (iii) work. 


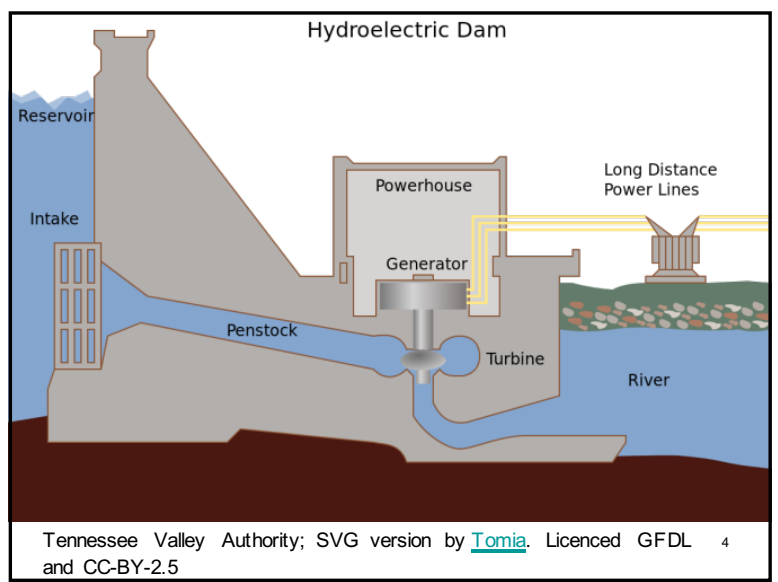

\section{Some sources of Energy}

- Hydro Reservoirs

- Chemical Bonds

- Nuclear Transformations

- Solar Power

Primary energy consumption by source, World

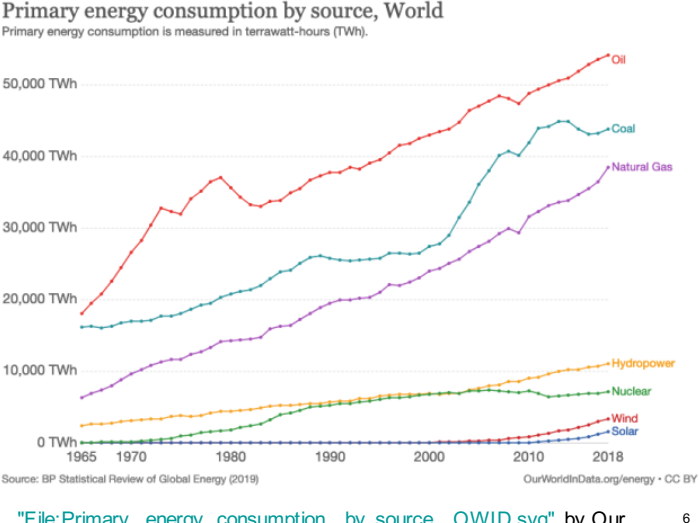

"File:Primary energy consumption by source, OWID.svg" by Our World In Data is licensed under CC BY 3.0 

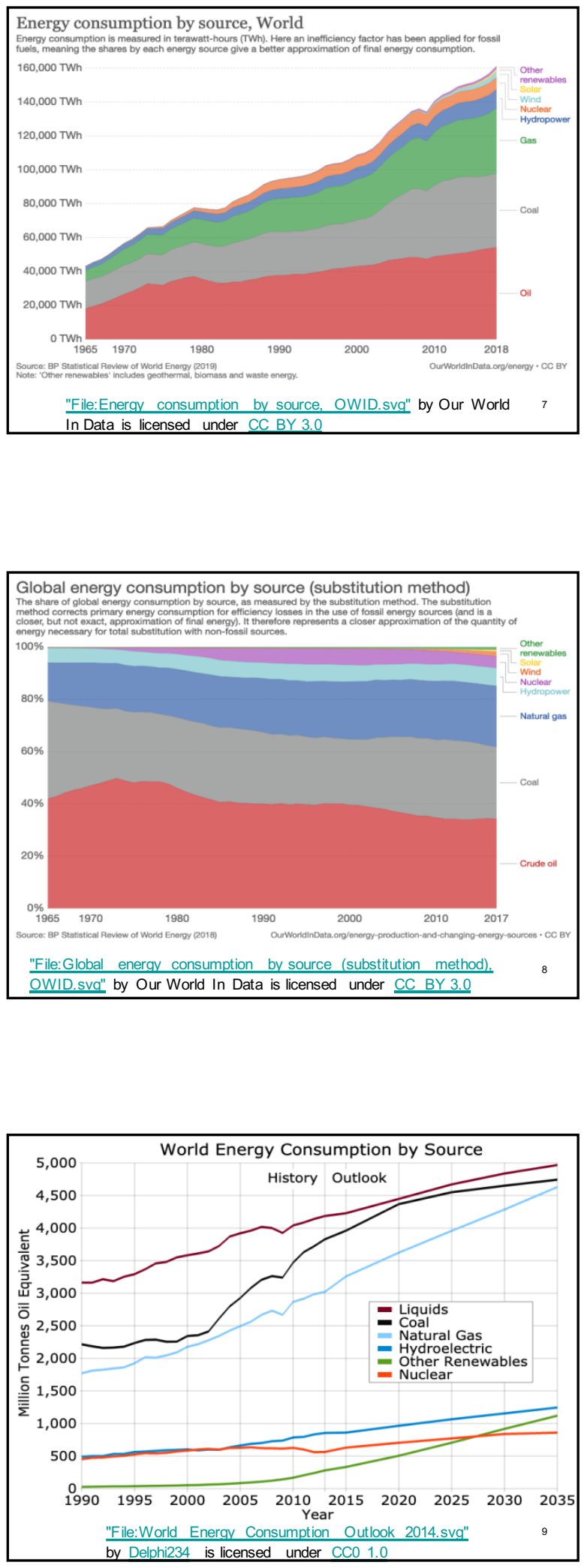


\section{Chemical Bond Energy}

The rearrangement of atoms in chemical reactions... breaking bonds and forming new bonds... may require energy, or it may release energy.

Bond energy - is the amount of energy that is required to break a chemical bond. Thus, it is also the amount of energy that is released when that same chemical bond is formed.

Exothermic - releases energy Endothermic - requires energy

Show the balanced reaction for the combustion of hydrogen and determine the energy change for the reaction (per mole of $\mathrm{H}_{2}$ combusted as

well as per gram of $\mathrm{H}_{2}$ combusted). Is this energy absorbed or released by the reaction? 


\begin{tabular}{|lll|}
\hline \multicolumn{3}{|c|}{ Average Bond Lengths and Bond Energies for Some Common Bonds } \\
Bond & Bond Length $(\AA)$ & Bond Energy $(\mathbf{k J} / \mathbf{m o l})$ \\
$\mathrm{C}-\mathrm{C}$ & 1.54 & 345 \\
$\mathrm{C}=\mathrm{C}$ & 1.34 & 611 \\
$\mathrm{C} \equiv \mathrm{C}$ & 1.20 & 837 \\
$\mathrm{C}-\mathrm{N}$ & 1.43 & 290 \\
$\mathrm{C}=\mathrm{N}$ & 1.38 & 615 \\
$\mathrm{C} \equiv \mathrm{N}$ & 1.16 & 891 \\
$\mathrm{C}-\mathrm{O}$ & 1.43 & 350 \\
$\mathrm{C}=\mathrm{O}$ & 1.23 & 741 \\
$\mathrm{C} \equiv \mathrm{O}$ & 1.13 & 1080 \\
\hline \multicolumn{3}{c}{} \\
Chemistry and the Environment(sec 7.5) by Devin R. Latimer is licensed under a Creative \\
\hline Commons Attribution 4.0 International License \\
\hline
\end{tabular}

\begin{tabular}{|c|c|c|c|c|c|}
\hline \multicolumn{6}{|c|}{ Bond Energies (kl/mol) } \\
\hline Bond & Bond Energy & Bond & Bond Energy & Bond & Bond Energy \\
\hline $\mathrm{H}-\mathrm{H}$ & 436 & C-S & 260 & $\mathrm{~F}-\mathrm{Cl}$ & 255 \\
\hline $\mathrm{H}-\mathrm{C}$ & 415 & $\mathrm{C}-\mathrm{Cl}$ & 330 & $\mathrm{~F}-\mathrm{Br}$ & 235 \\
\hline $\mathrm{H}-\mathrm{N}$ & 390 & $\mathrm{C}-\mathrm{Br}$ & 275 & $\mathrm{Si}-\mathrm{Si}$ & 230 \\
\hline $\mathrm{H}-\mathrm{O}$ & 464 & C-I & 240 & Si-P & 215 \\
\hline H-F & 569 & $\mathrm{~N}-\mathrm{N}$ & 160 & Si-S & 225 \\
\hline $\mathrm{H}-\mathrm{Si}$ & 395 & $\mathrm{~N}=\mathrm{N}$ & 418 & $\mathrm{Si}-\mathrm{Cl}$ & 359 \\
\hline $\mathrm{H}-\mathrm{P}$ & 320 & $\mathrm{~N} \equiv \mathrm{N}$ & 946 & $\mathrm{Si}-\mathrm{Br}$ & 290 \\
\hline $\mathrm{H}-\mathrm{S}$ & 340 & $\mathrm{~N}-\mathrm{O}$ & 200 & $\mathrm{Si}-\mathrm{I}$ & 215 \\
\hline $\mathrm{H}-\mathrm{Cl}$ & 432 & $\mathrm{~N}-\mathrm{F}$ & 270 & P-P & 215 \\
\hline $\mathrm{H}-\mathrm{Br}$ & 370 & $\mathrm{~N}-\mathrm{P}$ & 210 & P-S & 230 \\
\hline $\mathrm{H}-\mathrm{I}$ & 295 & $\mathrm{~N}-\mathrm{Cl}$ & 200 & $\mathrm{P}-\mathrm{Cl}$ & 330 \\
\hline $\mathrm{C}-\mathrm{C}$ & 345 & $\mathrm{~N}-\mathrm{Br}$ & 245 & $\mathrm{P}-\mathrm{Br}$ & 270 \\
\hline
\end{tabular}

\begin{tabular}{|c|c|c|c|}
\hline C-C 345 & $\mathrm{~N}-\mathrm{Br} 245$ & $\mathrm{P}-\mathrm{Br}$ & 270 \\
\hline $\mathrm{C}=\mathrm{C} \quad 611$ & O-O 140 & P-I & 215 \\
\hline $\mathrm{C} \equiv \mathrm{C} \quad 837$ & $\mathrm{O}=\mathrm{O} \quad 498$ & S-S & 215 \\
\hline C-N 290 & O-F 160 & $\mathrm{~S}-\mathrm{Cl}$ & 250 \\
\hline $\mathrm{C}=\mathrm{N} \quad 615$ & O-Si 370 & $\mathrm{~S}-\mathrm{Br}$ & 215 \\
\hline $\mathrm{C} \equiv \mathrm{N} \quad 891$ & O-P 350 & $\mathrm{Cl}-\mathrm{Cl}$ & 1243 \\
\hline C-O 350 & O-Cl 205 & $\mathrm{Cl}-\mathrm{Br}$ & r 220 \\
\hline $\mathrm{C}=\mathrm{O} \quad 741$ & O-I 200 & Cl-I & 210 \\
\hline $\mathrm{C} \equiv \mathrm{O} \quad 1080$ & F-F 160 & $\mathrm{Br}-\mathrm{Br}$ & r190 \\
\hline C-F 439 & F-Si 540 & $\mathrm{Br}-\mathrm{I}$ & 180 \\
\hline C-Si 360 & F-P 489 & I-I & 150 \\
\hline C-P 265 & F-S 285 & & \\
\hline
\end{tabular}




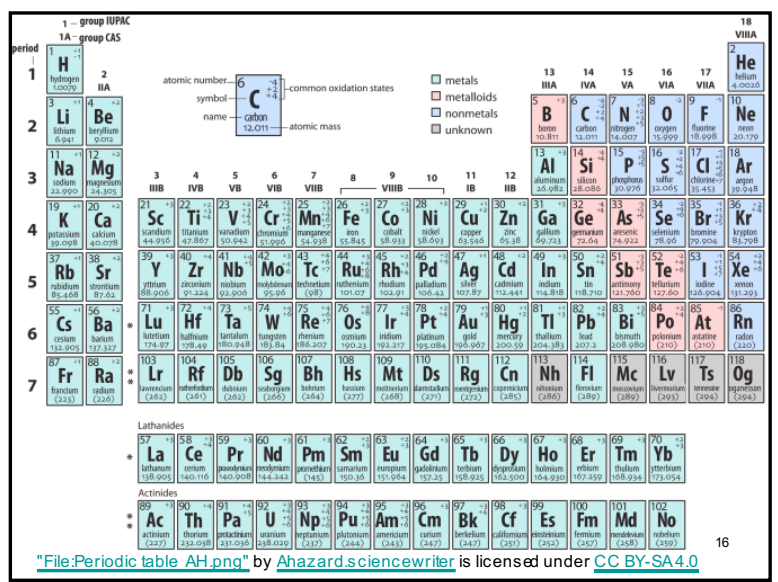

What is the approximate energy change associated with the combustion of methane (per mole of $\mathrm{CH}_{4}$ combusted as well as per gram of $\mathrm{CH}_{4}$ combusted)? Is this energy absorbed or released by the reaction? Do the same determination for ethane $\left(\mathrm{C}_{2} \mathrm{H}_{6}\right)$

\section{Coal}

- $\sim \mathrm{C}_{135} \mathrm{H}_{96} \mathrm{O}_{9} \mathrm{NS}$

- $85 \%$ Carbon

- $30 \mathrm{~kJ}$ per gram

- solid

- various grades

- there's lots of it. ( 400 years) 


\section{http://www.worldometers.info/}

- Energy at:

http://www. worldometers.info/

\section{Petroleum}

- Various Hydrocarbons

- $48 \mathrm{~kJ}$ per gram

- liquid

- must first be refined (distillation)

- value captures/conversions

- there's less known reserves ( $<50$ years) and it's becoming harder to extract ("peak oil")

- 'Where oil is' vs. Where oil is used'

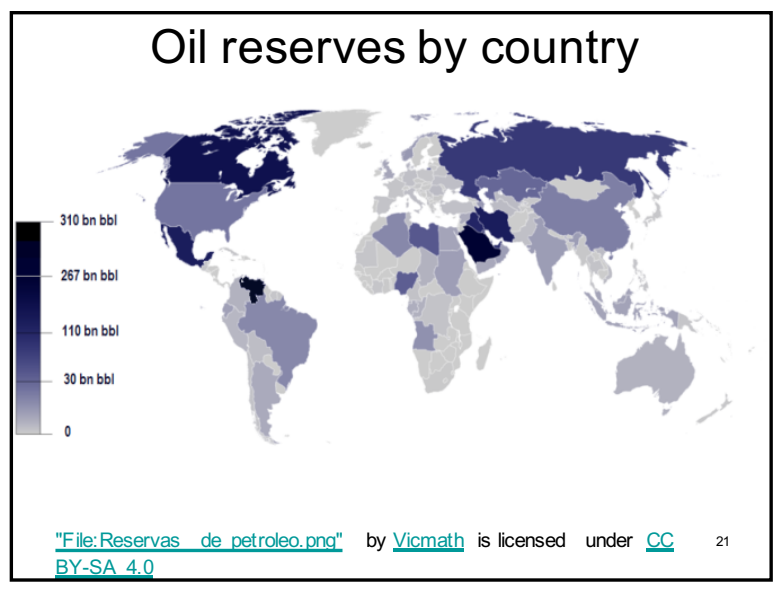




\section{Gasoline additives}

\section{- Tetraethyl lead}

- - used to be added to gasoline to minimize premature firing.

- - Pb would be released to the environment

- $-\mathrm{Pb}$ interferes with the function of certain enzymes

Elimination of octane enhancing tetraethyl lead (TEL) created a need to find substitutes.

MTBE (methyl tertiary-butyl ether) Due to groundwater contamination concerns, MTBE is now banned in the U.S., Canada and Japan.

Ethanol (ethyl alcohol)

\section{Natural Gas}

- $\mathrm{C}_{1}-\mathrm{C}_{4}$ hydrocarbons

- gas

- approximately $55 \mathrm{~kJ}$ per gram

- Approximately 150 years remain of known reserves 


\section{Biomass}

- Wood

- Biodiesel

- Ethanol

- Show the balanced chemical reaction for the combustion of ethanol $\left(\mathrm{CH}_{3} \mathrm{CH}_{2} \mathrm{OH}\right)$. Calculate the energy change per mole and per gram of ethanol combusted.

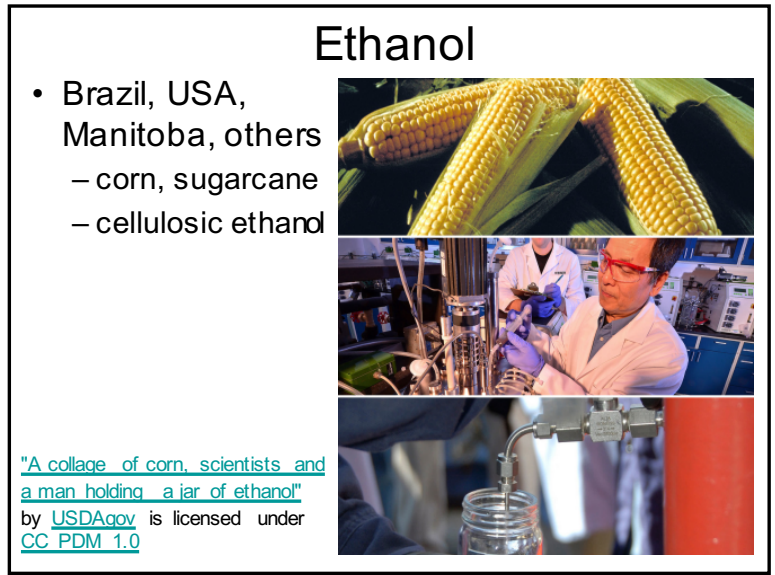

\section{Alternative Combustibles}

- For example:

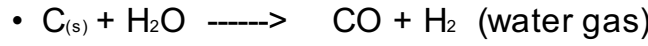

--> Fisher-Tropsch synthesis of gasoline (Fe or Co catalyst) 


\section{Biogas}

- The ferment of animal and vegetable wastes

- $60 \%$ methane

\section{Garbage}

- More than $1 / 3$ is directly combustible, as little as $10 \%$ of the original volume may end up as landfill

- Japan, Germany, US

\section{Fossil Fuels, Biofuels - Photosynthesis}

- Note: the energy in all fossil fuels and biofuels originates from the same place chlorophyll in plants captures solar energy and converts $\mathrm{CO}_{2}$ and $\mathrm{H}_{2} \mathrm{O}$ into carbohydrates, essentially storing the energy in the chemical bonds of the new molecule.

- $6 \mathrm{CO}_{2}+6 \mathrm{H}_{2} \mathrm{O}+$ sunlight $\rightarrow \mathrm{C}_{6} \mathrm{H}_{12} \mathrm{O}_{6}+6 \underset{30}{6 \mathrm{O}_{2}}$ 
Fossil Fuels - the reasons for Conservation and Alternatives

- Pollution

- Climate Change

- Finite Resources - 'Peak oil'

- Political consequences

- Are practically non-renewable. Now is the time to ensure we have this important energy and feedstock source (for chemical synthesis) for the future. 




\section{PART VIII}

\section{LIQUIDS AND SOLIDS}



38.

\section{INTRODUCTION}

\section{Learning Objectives}

- Intermolecular Forces

- $\quad$ Properties of Liquids

- The Solid State of Matter

Solid carbon dioxide (“dry ice", left) sublimes vigorously when placed in a liquid (right), cooling the liquid and generating a dense mist of water above the cylinder. (credit: modification of work by Paul Flowers) 


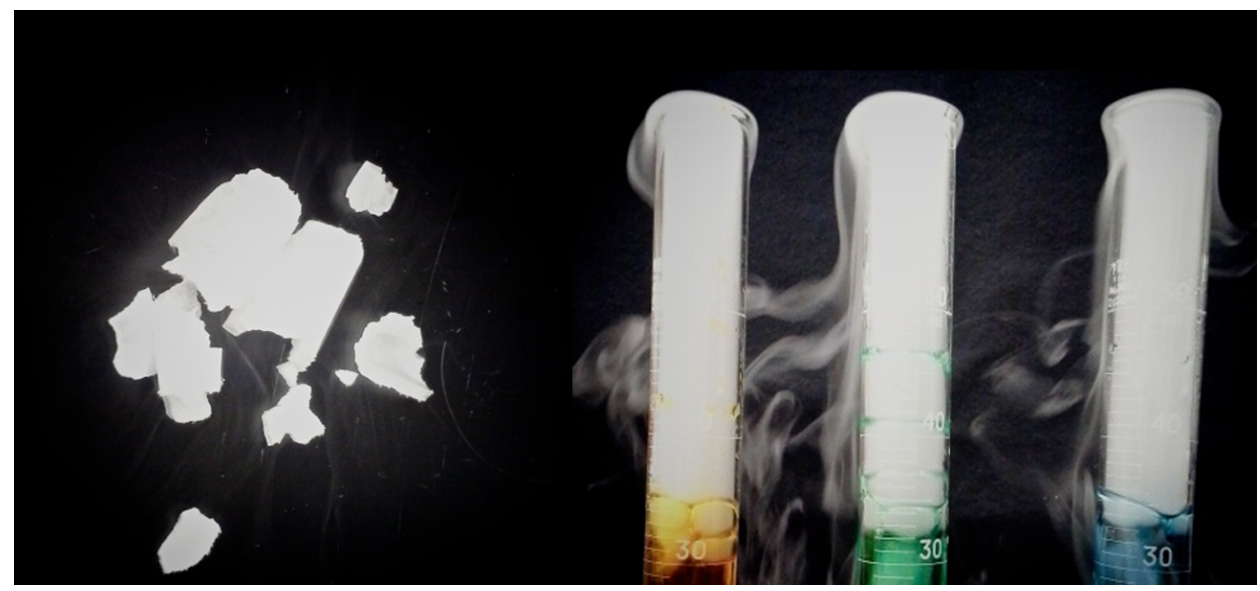

The great distances between atoms and molecules in a gaseous phase, and the corresponding absence of any significant interactions between them, allows for simple descriptions of many physical properties that are the same for all gases, regardless of their chemical identities. As described in the final module of the chapter on gases, this situation changes at high pressures and low temperatures-conditions that permit the atoms and molecules to interact to a much greater extent. In the liquid and solid states, these interactions are of considerable strength and play an important role in determining a number of physical properties that do depend on the chemical identity of the substance. In this chapter, the nature of these interactions and their effects on various physical properties of liquid and solid phases will be examined. 
39.

\section{INTERMOLECULAR FORCES}

Learning Objectives

By the end of this section, you will be able to:

- Describe the types of intermolecular forces possible between atoms or molecules in condensed phases (dispersion forces, dipole-dipole attractions, and hydrogen bonding)

- Identify the types of intermolecular forces experienced by specific molecules based on their structures

- Explain the relation between the intermolecular forces present within a substance and the temperatures associated with changes in its physical state

As was the case for gaseous substances, the kinetic molecular theory may be used to explain the behavior of solids and liquids. In the following description, the term 
particle will be used to refer to an atom, molecule, or ion. Note that we will use the popular phrase "intermolecular attraction" to refer to attractive forces between the particles of a substance, regardless of whether these particles are molecules, atoms, or ions.

Consider these two aspects of the molecular-level environments in solid, liquid, and gaseous matter:

- Particles in a solid are tightly packed together and often arranged in a regular pattern; in a liquid, they are close together with no regular arrangement; in a gas, they are far apart with no regular arrangement.

- Particles in a solid vibrate about fixed positions and do not generally move in relation to one another; in a liquid, they move past each other but remain in essentially constant contact; in a gas, they move independently of one another except when they collide.

The differences in the properties of a solid, liquid, or gas reflect the strengths of the attractive forces between the atoms, molecules, or ions that make up each phase. The phase in which a substance exists depends on the relative extents of its intermolecular forces (IMFs) and the kinetic energies (KE) of its molecules. IMFs are the various forces of attraction that may exist between the atoms and molecules of a substance due to electrostatic phenomena, as will be detailed in this module. These forces serve to hold particles close together, whereas the particles' KE provides the energy required to overcome the attractive forces and thus increase the distance between particles. 
(Figure) illustrates how changes in physical state may be induced by changing the temperature, hence, the average $\mathrm{KE}$, of a given substance.

Transitions between solid, liquid, and gaseous states of a substance occur when conditions of temperature or pressure favor the associated changes in intermolecular forces. (Note: The space between particles in the gas phase is much greater than shown.)

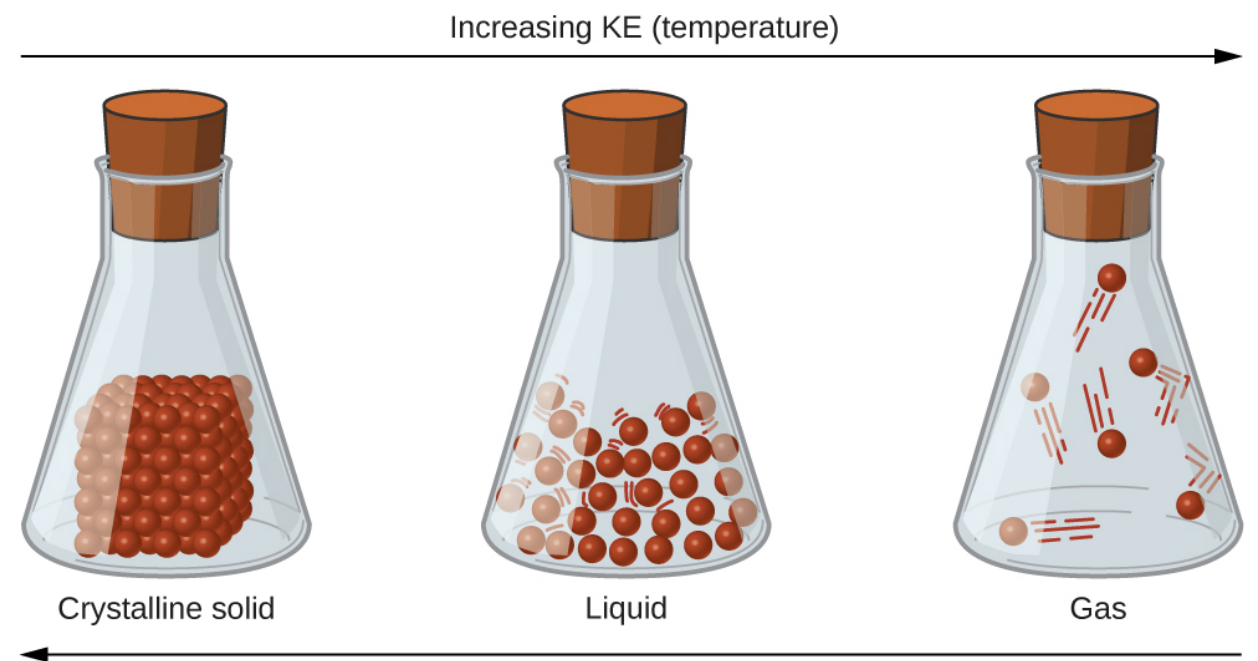

Increasing IMF

As an example of the processes depicted in this figure, consider a sample of water. When gaseous water is cooled sufficiently, the attractions between $\mathrm{H}_{2} \mathrm{O}$ molecules will be capable of holding them together when they come into contact with each other; the gas condenses, forming liquid $\mathrm{H}_{2} \mathrm{O}$. For example, liquid water forms on the outside of a cold glass as the water vapor in the air is cooled by the cold glass, as seen in (Figure).

Condensation forms when water vapor in the air is cooled enough to form liquid water, such as (a) on the outside of a cold beverage glass or (b) in the form of fog. (credit a: modification of work by Jenny Downing; credit $b$ : 
modification of work by Cory Zanker)

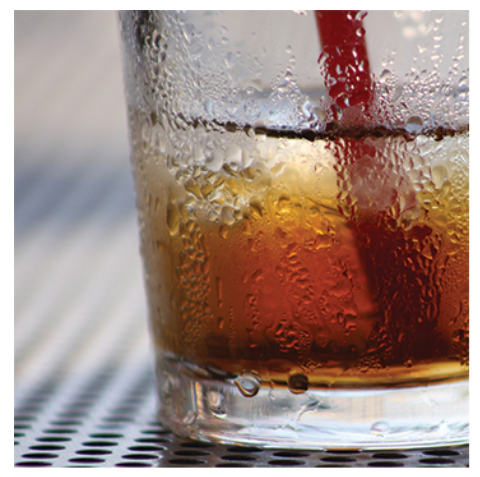

(a)

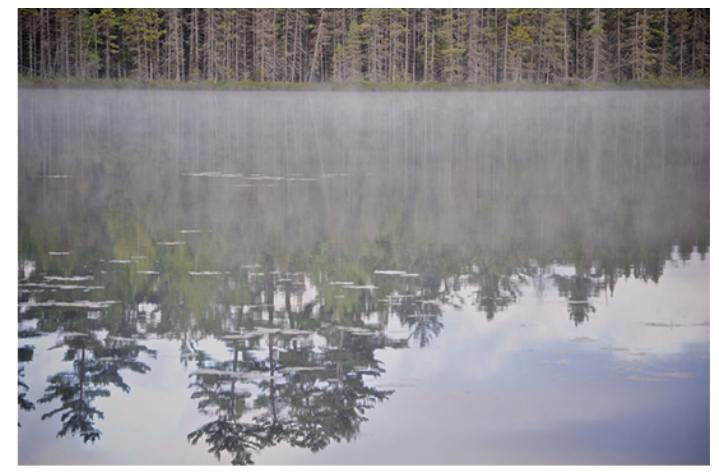

(b)

We can also liquefy many gases by compressing them, if the temperature is not too high. The increased pressure brings the molecules of a gas closer together, such that the attractions between the molecules become strong relative to their KE. Consequently, they form liquids. Butane, $\mathrm{C}_{4} \mathrm{H}_{10}$, is the fuel used in disposable lighters and is a gas at standard temperature and pressure. Inside the lighter's fuel compartment, the butane is compressed to a pressure that results in its condensation to the liquid state, as shown in (Figure).

Gaseous butane is compressed within the storage compartment of a disposable lighter, resulting in its condensation to the liquid state. (credit: modification of work by "Sam-Cat"/Flickr) 


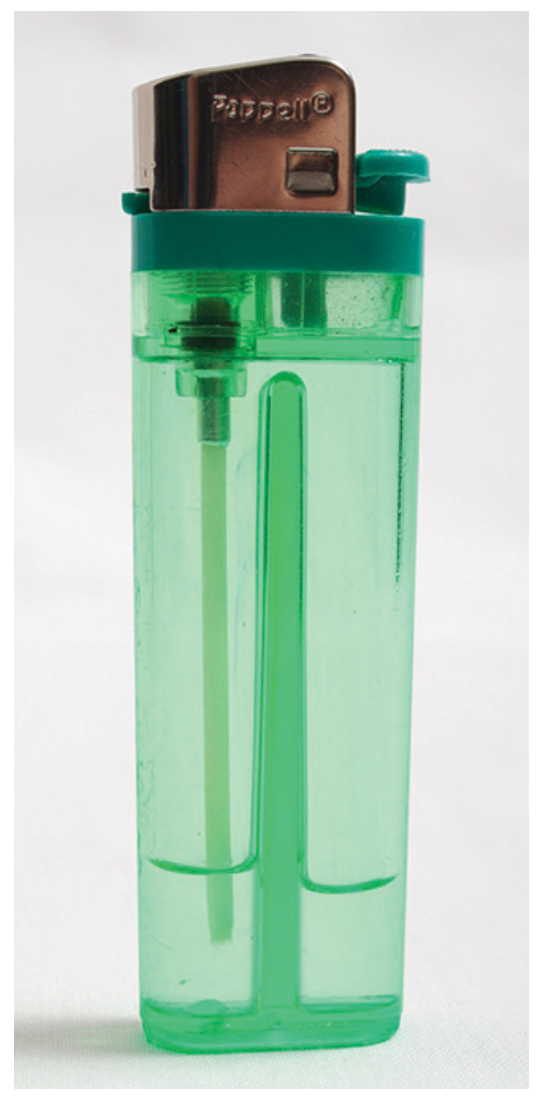

Finally, if the temperature of a liquid becomes sufficiently low, or the pressure on the liquid becomes sufficiently high, the molecules of the liquid no longer have enough KE to overcome the IMF between them, and a solid forms. A more thorough discussion of these and other changes of state, or phase transitions, is provided in a later module of this chapter.

Access this interactive simulation on states of matter, phase transitions, and intermolecular forces. This simulation is useful for visualizing concepts introduced throughout this chapter.

Forces between Molecules

Under appropriate conditions, the attractions between all 
gas molecules will cause them to form liquids or solids. This is due to intermolecular forces, not intramolecular forces. Intramolecular forces are those within the molecule that keep the molecule together, for example, the bonds between the atoms. Intermolecular forces are the attractions between molecules, which determine many of the physical properties of a substance. (Figure) illustrates these different molecular forces. The strengths of these attractive forces vary widely, though usually the IMFs between small molecules are weak compared to the intramolecular forces that bond atoms together within a molecule. For example, to overcome the IMFs in one mole of liquid $\mathrm{HCl}$ and convert it into gaseous $\mathrm{HCl}$ requires only about 17 kilojoules. However, to break the covalent bonds between the hydrogen and chlorine atoms in one mole of $\mathrm{HCl}$ requires about 25 times more energy-430 kilojoules. Intramolecular forces keep a molecule intact.

Intermolecular forces hold multiple molecules together and determine many of a substance's properties.

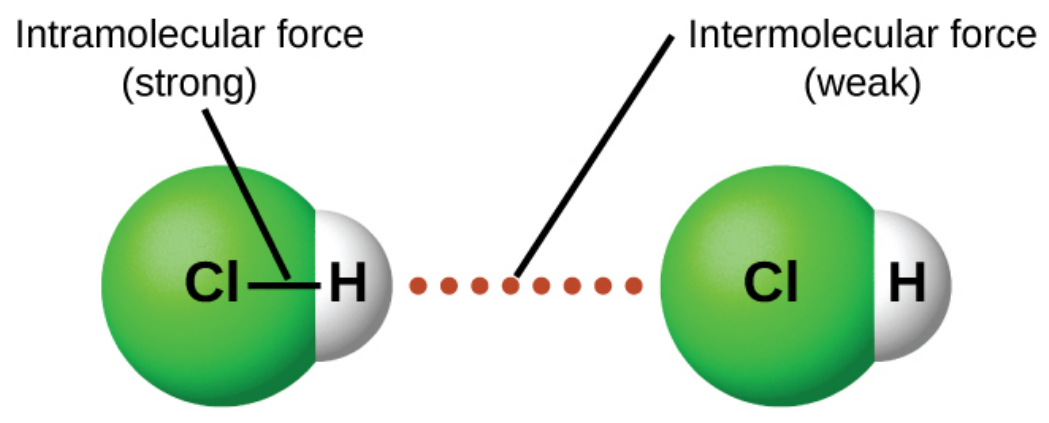

All of the attractive forces between neutral atoms and molecules are known as van der Waals forces, although they are usually referred to more informally as intermolecular attraction. We will consider the various types of IMFs in the next three sections of this module. 
Dispersion Forces

One of the three van der Waals forces is present in all condensed phases, regardless of the nature of the atoms or molecules composing the substance. This attractive force is called the London dispersion force in honor of German-born American physicist Fritz London who, in 1928, first explained it. This force is often referred to as simply the dispersion force. Because the electrons of an atom or molecule are in constant motion (or, alternatively, the electron's location is subject to quantum-mechanical variability), at any moment in time, an atom or molecule can develop a temporary, instantaneous dipole if its electrons are distributed asymmetrically. The presence of this dipole can, in turn, distort the electrons of a neighboring atom or molecule, producing an induced dipole. These two rapidly fluctuating, temporary dipoles thus result in a relatively weak electrostatic attraction between the species-a so-called dispersion force like that illustrated in (Figure).

Dispersion forces result from the formation of temporary dipoles, as illustrated here for two nonpolar diatomic molecules. 


\section{Unequal distribution}

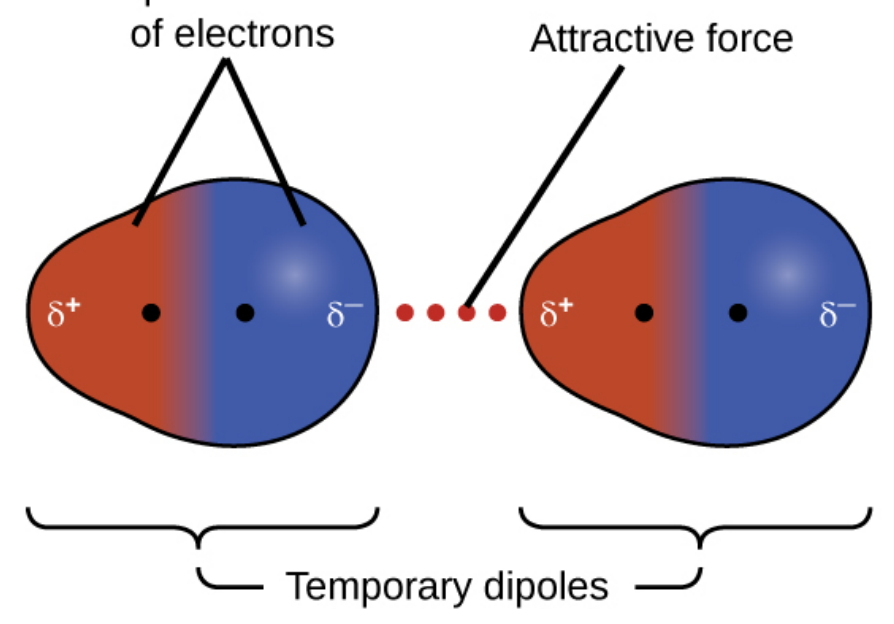

Dispersion forces that develop between atoms in different molecules can attract the two molecules to each other. The forces are relatively weak, however, and become significant only when the molecules are very close. Larger and heavier atoms and molecules exhibit stronger dispersion forces than do smaller and lighter atoms and molecules. $\mathrm{F}_{2}$ and $\mathrm{Cl}_{2}$ are gases at room temperature (reflecting weaker attractive forces); $\mathrm{Br}_{2}$ is a liquid, and $\mathrm{I}_{2}$ is a solid (reflecting stronger attractive forces). Trends in observed melting and boiling points for the halogens clearly demonstrate this effect, as seen in (Figure). 
Melting and Boiling Points of the Halogens

\begin{tabular}{|c|c|c|c|c|}
\hline Halogen & $\begin{array}{l}\text { Molar } \\
\text { Mass }\end{array}$ & $\begin{array}{l}\text { Atomic } \\
\text { Radius }\end{array}$ & $\begin{array}{l}\text { Melting } \\
\text { Point }\end{array}$ & $\begin{array}{l}\text { Boiling } \\
\text { Point }\end{array}$ \\
\hline fluorine, $F_{2}$ & $38 \mathrm{~g} / \mathrm{mol}$ & $72 \mathrm{pm}$ & $53 \mathrm{~K}$ & $85 \mathrm{~K}$ \\
\hline $\begin{array}{l}\text { chlorine, } \\
\mathrm{Cl}_{2}\end{array}$ & $71 \mathrm{~g} / \mathrm{mol}$ & $99 \mathrm{pm}$ & $172 \mathrm{~K}$ & $238 \mathrm{~K}$ \\
\hline $\begin{array}{l}\text { bromine, } \\
\mathrm{Br}_{2}\end{array}$ & $160 \mathrm{~g} / \mathrm{mol}$ & $114 \mathrm{pm}$ & $266 \mathrm{~K}$ & $332 \mathrm{~K}$ \\
\hline iodine, $\mathrm{I}_{2}$ & $254 \mathrm{~g} / \mathrm{mol}$ & $133 \mathrm{pm}$ & $387 \mathrm{~K}$ & $457 \mathrm{~K}$ \\
\hline $\begin{array}{l}\text { astatine, } \\
\mathrm{At}_{2}\end{array}$ & $420 \mathrm{~g} / \mathrm{mol}$ & $150 \mathrm{pm}$ & $575 \mathrm{~K}$ & $610 \mathrm{~K}$ \\
\hline
\end{tabular}

The increase in melting and boiling points with increasing atomic/molecular size may be rationalized by considering how the strength of dispersion forces is affected by the electronic structure of the atoms or molecules in the substance. In a larger atom, the valence electrons are, on average, farther from the nuclei than in a smaller atom. Thus, they are less tightly held and can more easily form the temporary dipoles that produce the attraction. The measure of how easy or difficult it is for another electrostatic charge (for example, a nearby ion or polar molecule) to distort a molecule's charge distribution (its electron cloud) is known as polarizability. A molecule that has a charge cloud that is easily distorted is said to be very polarizable and will have large dispersion forces; one with a charge cloud that is difficult to distort is not very polarizable and will have small dispersion forces.

London Forces and Their Effects Order the following compounds of a group 14 element and hydrogen from lowest 
to highest boiling point: $\mathrm{CH}_{4}, \mathrm{SiH}_{4}, \mathrm{GeH}_{4}$, and $\mathrm{SnH}_{4}$. Explain your reasoning.

Solution Applying the skills acquired in the chapter on chemical bonding and molecular geometry, all of these compounds are predicted to be nonpolar, so they may experience only dispersion forces: the smaller the molecule, the less polarizable and the weaker the dispersion forces; the larger the molecule, the larger the dispersion forces. The molar masses of $\mathrm{CH}_{4}, \mathrm{SiH}_{4}, \mathrm{GeH}_{4}$, and $\mathrm{SnH}_{4}$ are approximately $16 \mathrm{~g} / \mathrm{mol}, 32 \mathrm{~g} / \mathrm{mol}, 77 \mathrm{~g} / \mathrm{mol}$, and $123 \mathrm{~g} / \mathrm{mol}$, respectively. Therefore, $\mathrm{CH}_{4}$ is expected to have the lowest boiling point and $\mathrm{SnH}_{4}$ the highest boiling point. The ordering from lowest to highest boiling point is expected to be $\mathrm{CH}_{4}<\mathrm{SiH}_{4}<\mathrm{GeH}_{4}<$ $\mathrm{SnH}_{4}$.

A graph of the actual boiling points of these compounds versus the period of the group 14 element shows this prediction to be correct:

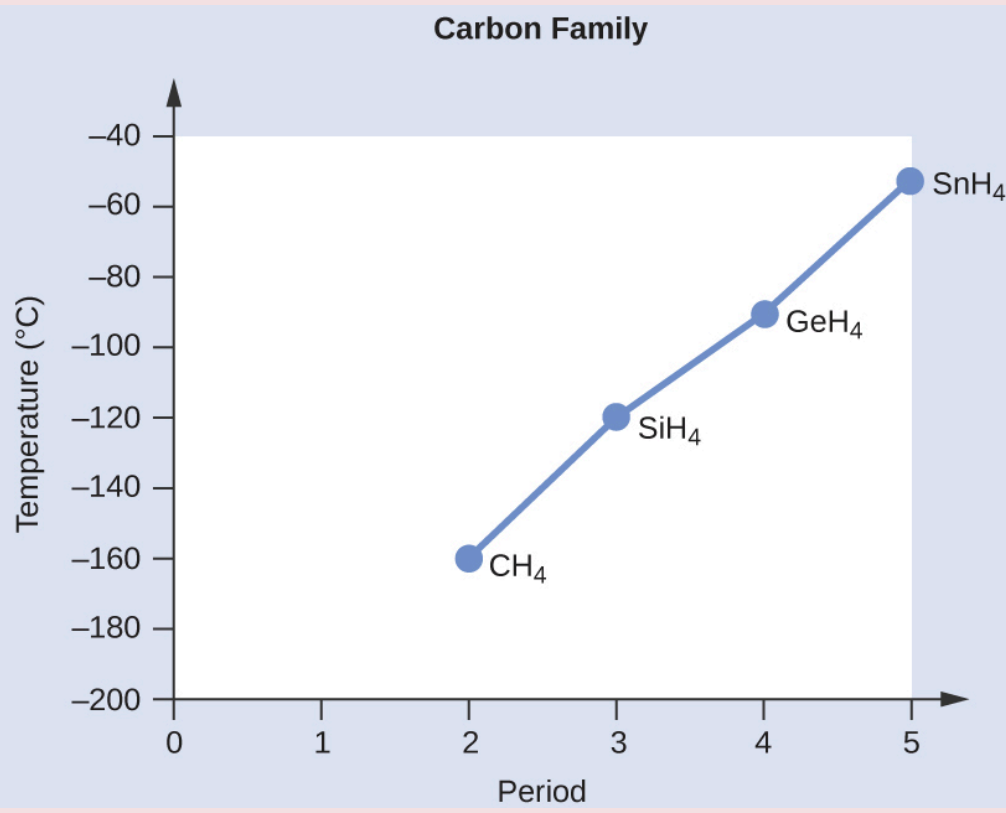

Check Your Learning Order the following hydrocarbons from lowest to highest boiling point: $\mathrm{C}_{2} \mathrm{H}_{6}, \mathrm{C}_{3} \mathrm{H}_{8}$, and $\mathrm{C}_{4} \mathrm{H}_{10}$. 
Answer:

$\mathrm{C}_{2} \mathrm{H}_{6}<\mathrm{C}_{3} \mathrm{H}_{8}<\mathrm{C}_{4} \mathrm{H}_{10}$. All of these compounds are nonpolar and only have London dispersion forces: the larger the molecule, the larger the dispersion forces and the higher the boiling point. The ordering from lowest to highest boiling point is therefore $\mathrm{C}_{2} \mathrm{H}_{6}<\mathrm{C}_{3} \mathrm{H}_{8}<\mathrm{C}_{4} \mathrm{H}_{10}$.

The shapes of molecules also affect the magnitudes of the dispersion forces between them. For example, boiling points for the isomers $n$-pentane, isopentane, and neopentane (shown in (Figure)) are $36{ }^{\circ} \mathrm{C}, 27{ }^{\circ} \mathrm{C}$, and 9.5 ${ }^{\circ} \mathrm{C}$, respectively. Even though these compounds are composed of molecules with the same chemical formula, $\mathrm{C}_{5} \mathrm{H}_{12}$, the difference in boiling points suggests that dispersion forces in the liquid phase are different, being greatest for $n$-pentane and least for neopentane. The elongated shape of $n$-pentane provides a greater surface area available for contact between molecules, resulting in correspondingly stronger dispersion forces. The more compact shape of isopentane offers a smaller surface area available for intermolecular contact and, therefore, weaker dispersion forces. Neopentane molecules are the most compact of the three, offering the least available surface area for intermolecular contact and, hence, the weakest dispersion forces. This behavior is analogous to the connections that may be formed between strips of VELCRO brand fasteners: the greater the area of the strip's contact, the stronger the connection.

The strength of the dispersion forces increases with the contact area between molecules, as demonstrated by the boiling points of these pentane isomers. 


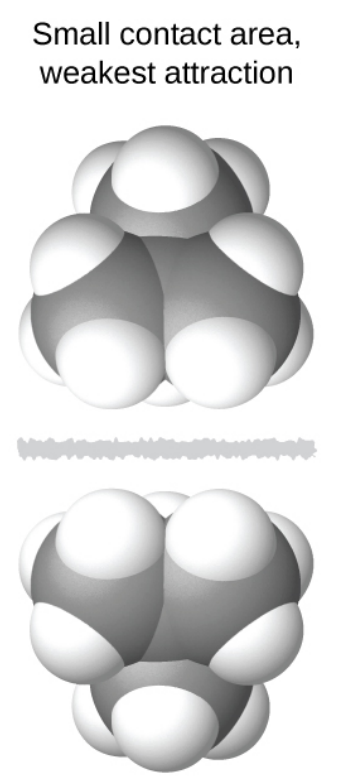

neopentane boiling point: $9.5^{\circ} \mathrm{C}$
Less surface area, less attraction
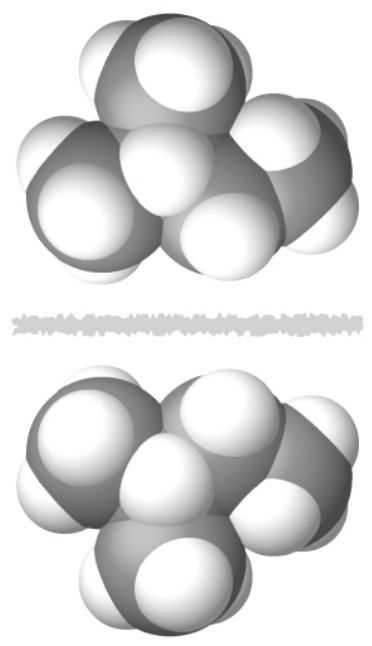

isopentane boiling point: $27^{\circ} \mathrm{C}$
Large contact area, strong attraction
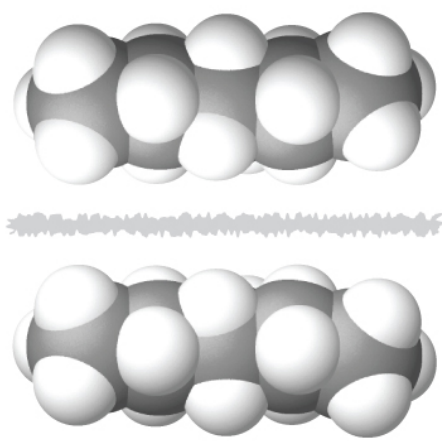

n-pentane boiling point: $36^{\circ} \mathrm{C}$

Geckos and Intermolecular Forces

Geckos have an amazing ability to adhere to most surfaces. They can quickly run up smooth walls and across ceilings that have no toe-holds, and they do this without having suction cups or a sticky substance on their toes. And while a gecko can lift its feet easily as it walks along a surface, if you attempt to pick it up, it sticks to the surface. How are geckos (as well as spiders and some other insects) able to do this? Although this phenomenon has been investigated for hundreds of years, scientists only recently uncovered the details of the process that allows geckos' feet to behave this way.

Geckos' toes are covered with hundreds of thousands of tiny hairs known as setae, with each seta, in turn, branching into hundreds of tiny, flat, triangular tips called spatulae. The huge numbers of spatulae on its setae provide a gecko, shown in (Figure), with a large total surface area for sticking to a surface. In 2000, Kellar 
Autumn, who leads a multi-institutional gecko research team, found that geckos adhered equally well to both polar silicon dioxide and nonpolar gallium arsenide. This proved that geckos stick to surfaces because of dispersion forces-weak intermolecular attractions arising from temporary, synchronized charge distributions between adjacent molecules. Although dispersion forces are very weak, the total attraction over millions of spatulae is large enough to support many times the gecko's weight.

In 2014, two scientists developed a model to explain how geckos can rapidly transition from "sticky" to "nonsticky.” Alex Greaney and Congcong Hu at Oregon State University described how geckos can achieve this by changing the angle between their spatulae and the surface. Geckos' feet, which are normally nonsticky, become sticky when a small shear force is applied. By curling and uncurling their toes, geckos can alternate between sticking and unsticking from a surface, and thus easily move across it. Further investigations may eventually lead to the development of better adhesives and other applications.

Geckos' toes contain large numbers of tiny hairs (setae), which branch into many triangular tips (spatulae). Geckos adhere to surfaces because of van der Waals attractions between the surface and a gecko's millions of spatulae. By changing how the spatulae contact the surface, geckos can turn their stickiness "on" and "off." (credit photo: modification of work by “JC*+A!"/Flickr) 


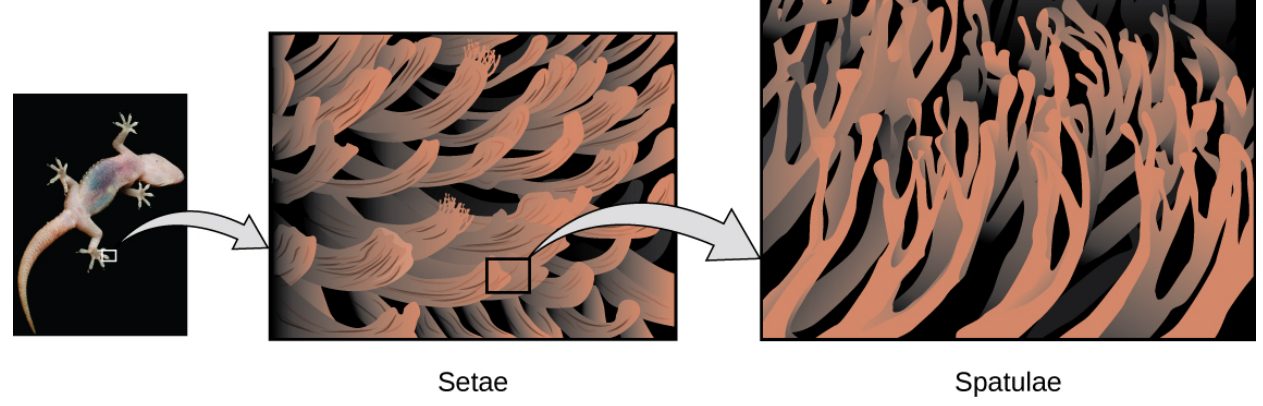

Watch this video to learn more about Kellar Autumn's research that determined that van der Waals forces are responsible for a gecko's ability to cling and climb.

Dipole-Dipole Attractions

Recall from the chapter on chemical bonding and molecular geometry that polar molecules have a partial positive charge on one side and a partial negative charge on the other side of the molecule-a separation of charge called a dipole. Consider a polar molecule such as hydrogen chloride, $\mathrm{HCl}$. In the $\mathrm{HCl}$ molecule, the more electronegative $\mathrm{Cl}$ atom bears the partial negative charge, whereas the less electronegative $\mathrm{H}$ atom bears the partial positive charge. An attractive force between $\mathrm{HCl}$ molecules results from the attraction between the positive end of one $\mathrm{HCl}$ molecule and the negative end of another. This attractive force is called a dipole-dipole attraction-the electrostatic force between the partially positive end of one polar molecule and the partially negative end of another, as illustrated in (Figure). This image shows two arrangements of polar molecules, such as $\mathrm{HCl}$, that allow an attraction between the partial negative end of one molecule and the partial positive end of another. 

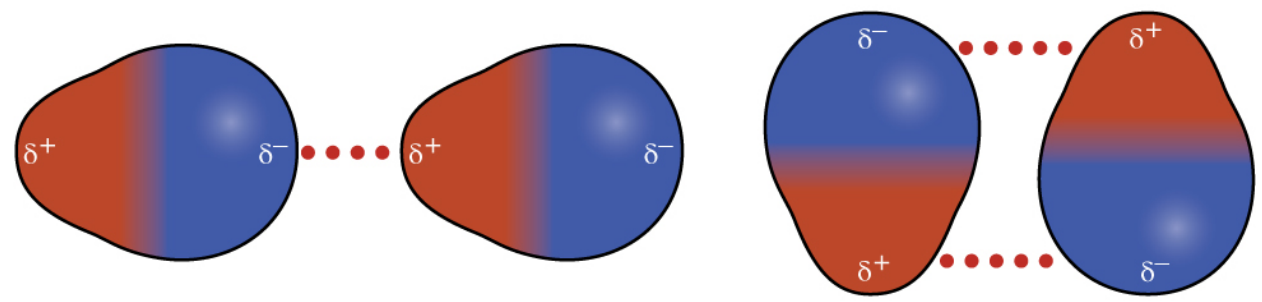

The effect of a dipole-dipole attraction is apparent when we compare the properties of $\mathrm{HCl}$ molecules to nonpolar $\mathrm{F}_{2}$ molecules. Both $\mathrm{HCl}$ and $\mathrm{F}_{2}$ consist of the same number of atoms and have approximately the same molecular mass. At a temperature of $150 \mathrm{~K}$, molecules of both substances would have the same average KE. However, the dipole-dipole attractions between $\mathrm{HCl}$ molecules are sufficient to cause them to "stick together" to form a liquid, whereas the relatively weaker dispersion forces between nonpolar $\mathrm{F}_{2}$ molecules are not, and so this substance is gaseous at this temperature. The higher normal boiling point of $\mathrm{HCl}(188 \mathrm{~K})$ compared to $\mathrm{F}_{2}$ (85 $\mathrm{K})$ is a reflection of the greater strength of dipole-dipole attractions between $\mathrm{HCl}$ molecules, compared to the attractions between nonpolar $\mathrm{F}_{2}$ molecules. We will often use values such as boiling or freezing points, or enthalpies of vaporization or fusion, as indicators of the relative strengths of IMFs of attraction present within different substances.

Dipole-Dipole Forces and Their Effects Predict which will have the higher boiling point: $\mathrm{N}_{2}$ or $\mathrm{CO}$. Explain your reasoning.

Solution $\mathrm{CO}$ and $\mathrm{N}_{2}$ are both diatomic molecules with masses of about $28 \mathrm{amu}$, so they experience similar London dispersion forces. Because $\mathrm{CO}$ is a polar molecule, it experiences dipole-dipole attractions. Because $\mathrm{N}_{2}$ is nonpolar, 
its molecules cannot exhibit dipole-dipole attractions. The dipole-dipole attractions between $\mathrm{CO}$ molecules are comparably stronger than the dispersion forces between nonpolar $\mathrm{N}_{2}$ molecules, so $\mathrm{CO}$ is expected to have the higher boiling point.

Check Your Learning Predict which will have the higher boiling point: $\mathrm{ICl}$ or $\mathrm{Brz}$. Explain your reasoning.

Answer:

$\mathrm{ICl}$. ICl and $\mathrm{Br} 2$ have similar masses ( $160 \mathrm{amu}$ ) and therefore experience similar London dispersion forces. ICl is polar and thus also exhibits dipole-dipole attractions; $\mathrm{Br} 2$ is nonpolar and does not. The relatively stronger dipole-dipole attractions require more energy to overcome, so ICl will have the higher boiling point.

Hydrogen Bonding

Nitrosyl fluoride (ONF, molecular mass $49 \mathrm{amu}$ ) is a gas at room temperature. Water $\left(\mathrm{H}_{2} \mathrm{O}\right.$, molecular mass $\left.18 \mathrm{amu}\right)$ is a liquid, even though it has a lower molecular mass. We clearly cannot attribute this difference between the two compounds to dispersion forces. Both molecules have about the same shape and ONF is the heavier and larger molecule. It is, therefore, expected to experience more significant dispersion forces. Additionally, we cannot attribute this difference in boiling points to differences in the dipole moments of the molecules. Both molecules are polar and exhibit comparable dipole moments. The large difference between the boiling points is due to a particularly strong dipole-dipole attraction that may occur when a molecule contains a hydrogen atom bonded to a fluorine, oxygen, or nitrogen atom (the three most electronegative elements). The very large difference in 
electronegativity between the $\mathrm{H}$ atom (2.1) and the atom to which it is bonded (4.0 for an $\mathrm{F}$ atom, 3.5 for an $\mathrm{O}$ atom, or 3.0 for a $\mathrm{N}$ atom), combined with the very small size of a $\mathrm{H}$ atom and the relatively small sizes of $\mathrm{F}, \mathrm{O}$, or $\mathrm{N}$ atoms, leads to highly concentrated partial charges with these atoms. Molecules with $\mathrm{F}-\mathrm{H}, \mathrm{O}-\mathrm{H}$, or $\mathrm{N}-\mathrm{H}$ moieties are very strongly attracted to similar moieties in nearby molecules, a particularly strong type of dipole-dipole attraction called hydrogen bonding. Examples of hydrogen bonds include $\mathrm{HF} \cdots \mathrm{HF}, \mathrm{H}_{2} \mathrm{O} \cdots \mathrm{HOH}$, and $\mathrm{H}_{3} \mathrm{~N} \cdots \mathrm{HNH}_{2}$, in which the hydrogen bonds are denoted by dots. (Figure) illustrates hydrogen bonding between water molecules.

Water molecules participate in multiple hydrogenbonding interactions with nearby water molecules.

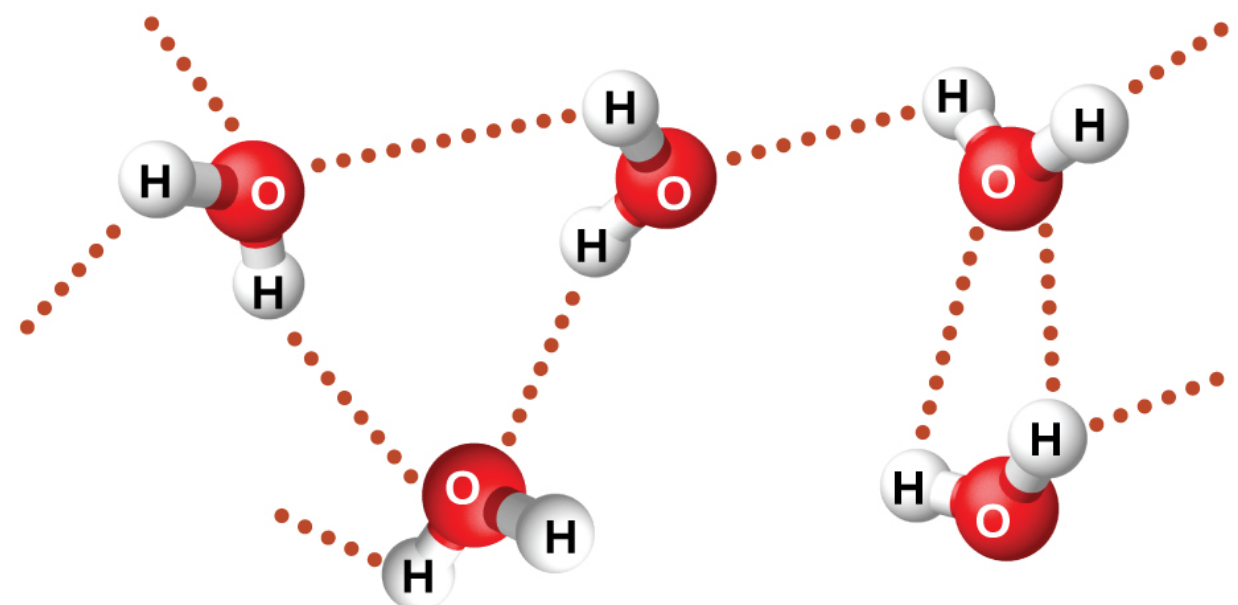

Despite use of the word "bond," keep in mind that hydrogen bonds are intermolecular attractive forces, not intramolecular attractive forces (covalent bonds). Hydrogen bonds are much weaker than covalent bonds, only about 5 to $10 \%$ as strong, but are generally much 
stronger than other dipole-dipole attractions and dispersion forces.

Hydrogen bonds have a pronounced effect on the properties of condensed phases (liquids and solids). For example, consider the trends in boiling points for the binary hydrides of group $15\left(\mathrm{NH}_{3}, \mathrm{PH}_{3}, \mathrm{AsH}_{3}\right.$, and $\left.\mathrm{SbH}_{3}\right)$, group 16 hydrides $\left(\mathrm{H}_{2} \mathrm{O}, \mathrm{H}_{2} \mathrm{~S}, \mathrm{H}_{2} \mathrm{Se}\right.$, and $\left.\mathrm{H}_{2} \mathrm{Te}\right)$, and group 17 hydrides ( $\mathrm{HF}, \mathrm{HCl}, \mathrm{HBr}$, and $\mathrm{HI}$ ). The boiling points of the heaviest three hydrides for each group are plotted in (Figure). As we progress down any of these groups, the polarities of the molecules decrease slightly, whereas the sizes of the molecules increase substantially. The effect of increasingly stronger dispersion forces dominates that of increasingly weaker dipole-dipole attractions, and the boiling points are observed to increase steadily.

For the group 15, 16, and 17 hydrides, the boiling points for each class of compounds increase with increasing molecular mass for elements in periods 3,4 , and 5 . 


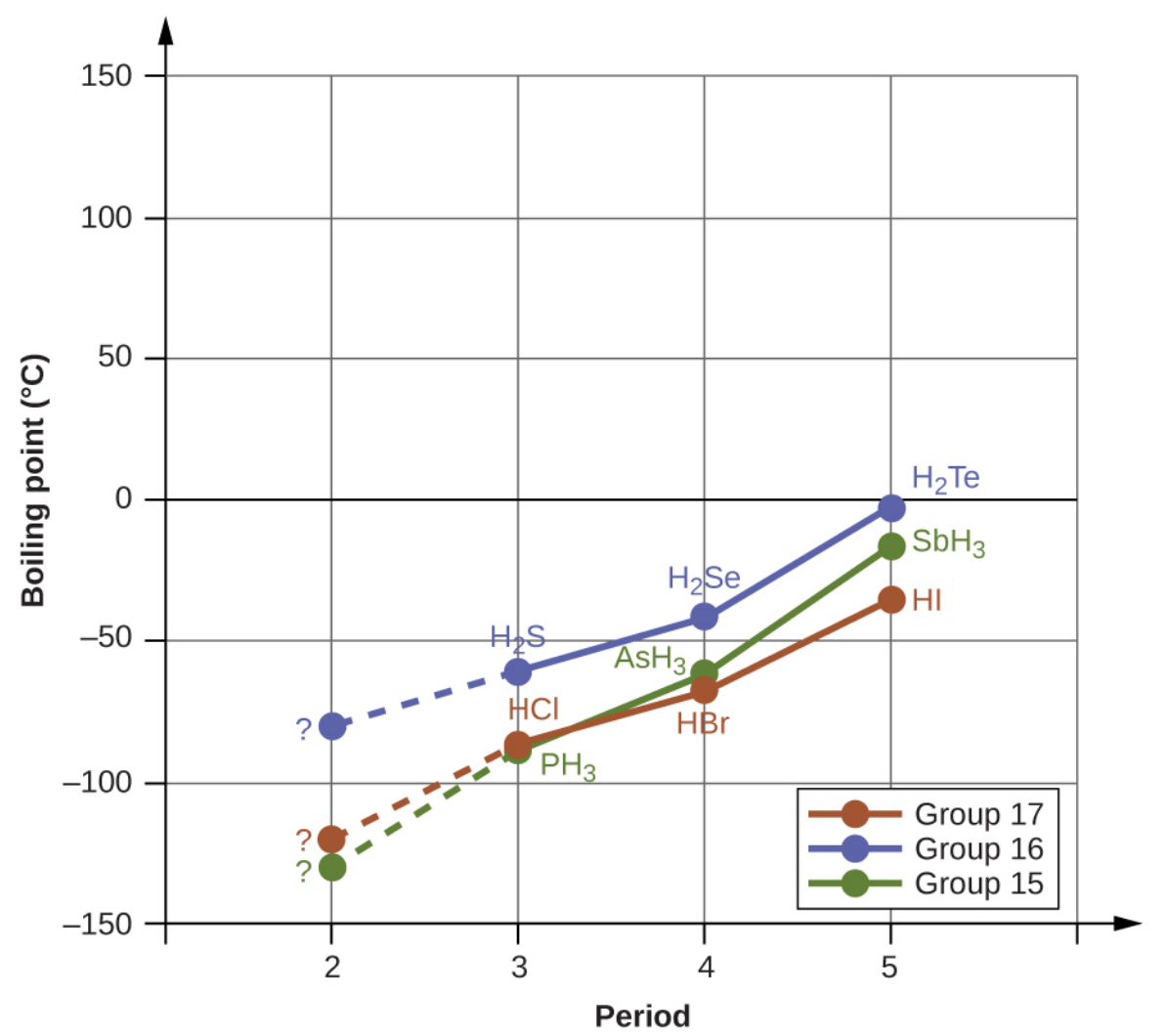

If we use this trend to predict the boiling points for the lightest hydride for each group, we would expect $\mathrm{NH}_{3}$ to boil at about $-120{ }^{\circ} \mathrm{C}, \mathrm{H}_{2} \mathrm{O}$ to boil at about $-80{ }^{\circ} \mathrm{C}$, and $\mathrm{HF}$ to boil at about $-110^{\circ} \mathrm{C}$. However, when we measure the boiling points for these compounds, we find that they are dramatically higher than the trends would predict, as shown in (Figure). The stark contrast between our naïve predictions and reality provides compelling evidence for the strength of hydrogen bonding.

In comparison to periods $3-5$, the binary hydrides of period 2 elements in groups 17, 16 and 15 (F, O and N, respectively) exhibit anomalously high boiling points due to hydrogen bonding. 


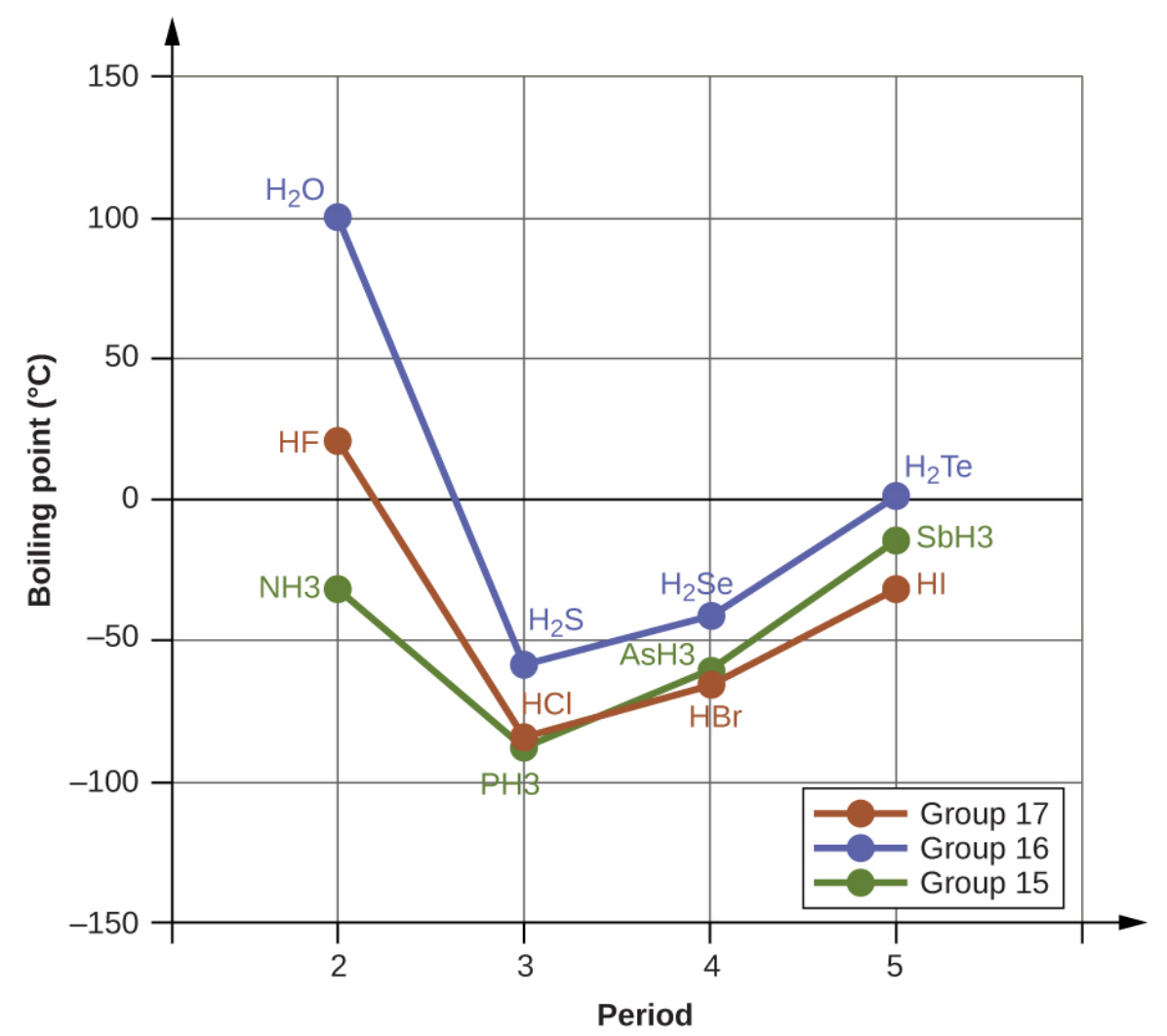

Effect of Hydrogen Bonding on Boiling Points Consider the compounds dimethylether $\left(\mathrm{CH}_{3} \mathrm{OCH}_{3}\right)$, ethanol $\left(\mathrm{CH}_{3} \mathrm{CH}_{2} \mathrm{OH}\right)$, and propane $\left(\mathrm{CH}_{3} \mathrm{CH}_{2} \mathrm{CH}_{3}\right)$. Their boiling points, not necessarily in order, are $-42.1^{\circ} \mathrm{C},-24.8^{\circ} \mathrm{C}$, and $78.4^{\circ} \mathrm{C}$. Match each compound with its boiling point. Explain your reasoning.

Solution The VSEPR-predicted shapes of $\mathrm{CH}_{3} \mathrm{OCH}_{3}$, $\mathrm{CH}_{3} \mathrm{CH}_{2} \mathrm{OH}$, and $\mathrm{CH}_{3} \mathrm{CH}_{2} \mathrm{CH}_{3}$ are similar, as are their molar masses ( $46 \mathrm{~g} / \mathrm{mol}, 46 \mathrm{~g} / \mathrm{mol}$, and $44 \mathrm{~g} / \mathrm{mol}$, respectively), so they will exhibit similar dispersion forces. Since $\mathrm{CH}_{3} \mathrm{CH}_{2} \mathrm{CH}_{3}$ is nonpolar, it may exhibit only dispersion forces. Because $\mathrm{CH}_{3} \mathrm{OCH}_{3}$ is polar, it will also experience dipole-dipole attractions. Finally, $\mathrm{CH}_{3} \mathrm{CH}_{2} \mathrm{OH}$ has an $-\mathrm{OH}$ group, and so it will experience the uniquely strong dipole-dipole attraction known as hydrogen bonding. So the ordering in terms of strength of IMFs, and thus boiling points, is $\mathrm{CH}_{3} \mathrm{CH}_{2} \mathrm{CH}_{3}<\mathrm{CH}_{3} \mathrm{OCH}_{3}<$ 
$\mathrm{CH}_{3} \mathrm{CH}_{2} \mathrm{OH}$. The boiling point of propane is $-42.1^{\circ} \mathrm{C}$, the boiling point of dimethylether is $-24.8^{\circ} \mathrm{C}$, and the boiling point of ethanol is $78.5^{\circ} \mathrm{C}$.

Check Your Learning Ethane $\left(\mathrm{CH}_{3} \mathrm{CH}_{3}\right)$ has a melting point of $-183^{\circ} \mathrm{C}$ and a boiling point of $-89^{\circ} \mathrm{C}$. Predict the melting and boiling points for methylamine $\left(\mathrm{CH}_{3} \mathrm{NH}_{2}\right)$. Explain your reasoning.

Answer:

The melting point and boiling point for methylamine are predicted to be significantly greater than those of ethane. $\mathrm{CH}_{3} \mathrm{CH}_{3}$ and $\mathrm{CH}_{3} \mathrm{NH}_{2}$ are similar in size and mass, but methylamine possesses an -NH group and therefore may exhibit hydrogen bonding. This greatly increases its IMFs, and therefore its melting and boiling points. It is difficult to predict values, but the known values are a melting point of $-93^{\circ} \mathrm{C}$ and a boiling point of $-6^{\circ} \mathrm{C}$.

\section{Hydrogen Bonding and DNA}

Deoxyribonucleic acid (DNA) is found in every living organism and contains the genetic information that determines the organism's characteristics, provides the blueprint for making the proteins necessary for life, and serves as a template to pass this information on to the organism's offspring. A DNA molecule consists of two (anti-)parallel chains of repeating nucleotides, which form its well-known double helical structure, as shown in (Figure).

Two separate DNA molecules form a double-stranded helix in which the molecules are held together via hydrogen bonding. (credit: modification of work by Jerome Walker, Dennis Myts) 
Nitrogenous bases:

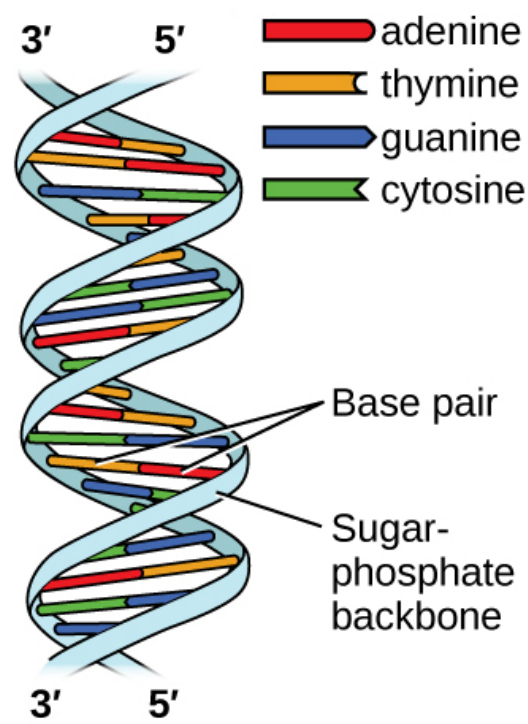

Each nucleotide contains a (deoxyribose) sugar bound to a phosphate group on one side, and one of four nitrogenous bases on the other. Two of the bases, cytosine $(C)$ and thymine $(\mathrm{T})$, are single-ringed structures known as pyrimidines. The other two, adenine (A) and guanine $(G)$, are double-ringed structures called purines. These bases form complementary base pairs consisting of one purine and one pyrimidine, with adenine pairing with thymine, and cytosine with guanine. Each base pair is held together by hydrogen bonding. A and T share two hydrogen bonds, $\mathrm{C}$ and $\mathrm{G}$ share three, and both pairings have a similar shape and structure (Figure).

The geometries of the base molecules result in maximum hydrogen bonding between adenine and thymine (AT) and between guanine and cytosine (GC), so-called “complementary base pairs." 


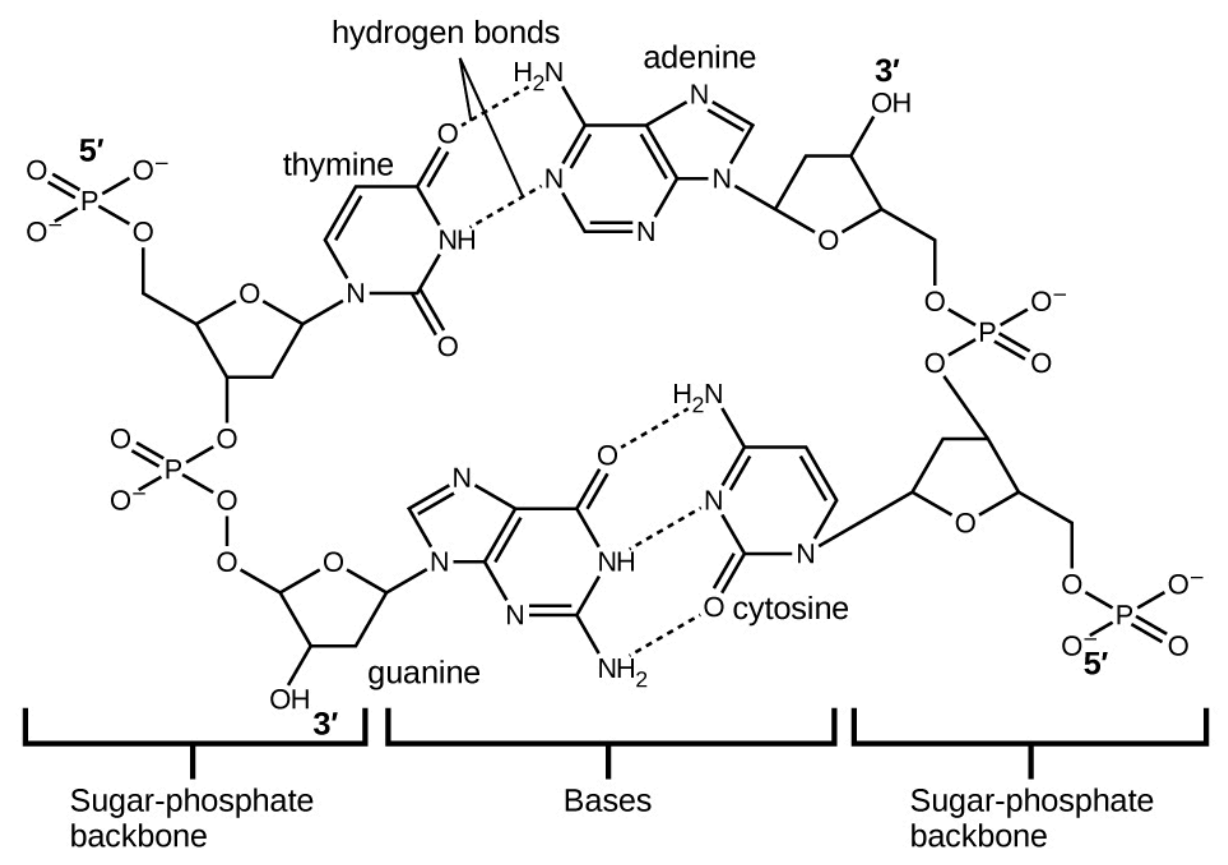

The cumulative effect of millions of hydrogen bonds effectively holds the two strands of DNA together. Importantly, the two strands of DNA can relatively easily "unzip" down the middle since hydrogen bonds are relatively weak compared to the covalent bonds that hold the atoms of the individual DNA molecules together. This allows both strands to function as a template for replication.

Key Concepts and Summary

The physical properties of condensed matter (liquids and solids) can be explained in terms of the kinetic molecular theory. In a liquid, intermolecular attractive forces hold the molecules in contact, although they still have sufficient KE to move past each other.

Intermolecular attractive forces, collectively referred to as van der Waals forces, are responsible for the behavior of liquids and solids and are electrostatic in nature. Dipole- 
dipole attractions result from the electrostatic attraction of the partial negative end of one dipolar molecule for the partial positive end of another. The temporary dipole that results from the motion of the electrons in an atom can induce a dipole in an adjacent atom and give rise to the London dispersion force. London forces increase with increasing molecular size. Hydrogen bonds are a special type of dipole-dipole attraction that results when hydrogen is bonded to one of the three most electronegative elements: F, O, or $\mathrm{N}$.

\section{Chemistry End of Chapter Exercises}

In terms of their bulk properties, how do liquids and solids differ? How are they similar?

Liquids and solids are similar in that they are matter composed of atoms, ions, or molecules. They are incompressible and have similar densities that are both much larger than those of gases. They are different in that liquids have no fixed shape, and solids are rigid.

In terms of the kinetic molecular theory, in what ways are liquids similar to solids? In what ways are liquids different from solids?

In terms of the kinetic molecular theory, in what ways are liquids similar to gases? In what ways are liquids different from gases?

They are similar in that the atoms or molecules are free to move from one position to another. They differ in that the particles of a liquid are confined to the shape of the vessel 
in which they are placed. In contrast, a gas will expand without limit to fill the space into which it is placed.

Explain why liquids assume the shape of any container into which they are poured, whereas solids are rigid and retain their shape.

What is the evidence that all neutral atoms and molecules exert attractive forces on each other?

All atoms and molecules will condense into a liquid or solid in which the attractive forces exceed the kinetic energy of the molecules, at sufficiently low temperature.

Open the PhET States of Matter Simulation to answer the following questions:

(a) Select the Solid, Liquid, Gas tab. Explore by selecting different substances, heating and cooling the systems, and changing the state. What similarities do you notice between the four substances for each phase (solid, liquid, gas)? What differences do you notice?

(b) For each substance, select each of the states and record the given temperatures. How do the given temperatures for each state correlate with the strengths of their intermolecular attractions? Explain.

(c) Select the Interaction Potential tab, and use the default neon atoms. Move the Ne atom on the right and observe how the potential energy changes. Select the Total Force button, and move the Ne atom as before. When is the total force on each atom attractive and large enough to matter? Then select the Component Forces button, and move the Ne atom. When do the attractive (van der Waals) and repulsive (electron overlap) forces 
balance? How does this relate to the potential energy versus the distance between atoms graph? Explain.

Define the following and give an example of each:

(a) dispersion force

(b) dipole-dipole attraction

(c) hydrogen bond

(a) Dispersion forces occur as an atom develops a temporary dipole moment when its electrons are distributed asymmetrically about the nucleus. This structure is more prevalent in large atoms such as argon or radon. A second atom can then be distorted by the appearance of the dipole in the first atom. The electrons of the second atom are attracted toward the positive end of the first atom, which sets up a dipole in the second atom. The net result is rapidly fluctuating, temporary dipoles that attract one another (e.g., Ar). (b) A dipole-dipole attraction is a force that results from an electrostatic attraction of the positive end of one polar molecule for the negative end of another polar molecule (e.g., ICI molecules attract one another by dipole-dipole interaction). (c) Hydrogen bonds form whenever a hydrogen atom is bonded to one of the more electronegative atoms, such as a fluorine, oxygen, or nitrogen atom. The electrostatic attraction between the partially positive hydrogen atom in one molecule and the partially negative atom in another molecule gives rise to a strong dipole-dipole interaction called a hydrogen bond (e.g., HFHF.

The types of intermolecular forces in a substance are 
identical whether it is a solid, a liquid, or a gas. Why then does a substance change phase from a gas to a liquid or to a solid?

Why do the boiling points of the noble gases increase in the order $\mathrm{He}<\mathrm{Ne}<\mathrm{Ar}<\mathrm{Kr}<\mathrm{Xe}$ ?

The London forces typically increase as the number of electrons increase.

Neon and HF have approximately the same molecular masses.

(a) Explain why the boiling points of Neon and HF differ.

(b) Compare the change in the boiling points of $\mathrm{Ne}, \mathrm{Ar}$, $\mathrm{Kr}$, and Xe with the change of the boiling points of $\mathrm{HF}$, $\mathrm{HCl}, \mathrm{HBr}$, and $\mathrm{HI}$, and explain the difference between the changes with increasing atomic or molecular mass.

Arrange each of the following sets of compounds in order of increasing boiling point temperature:

(a) $\mathrm{HCl}, \mathrm{H}_{2} \mathrm{O}, \mathrm{SiH}_{4}$

(b) $\mathrm{F}_{2}, \mathrm{Cl}_{2}, \mathrm{Br}_{2}$

(c) $\mathrm{CH}_{4}, \mathrm{C}_{2} \mathrm{H}_{6}, \mathrm{C}_{3} \mathrm{H}_{8}$

(d) $\mathrm{O}_{2}, \mathrm{NO}, \mathrm{N}_{2}$

(a) $\mathrm{SiH}_{4}<\mathrm{HCl}<\mathrm{H}_{2} \mathrm{O}$; (b) $\mathrm{F}_{2}<\mathrm{Cl}_{2}<\mathrm{Br}_{2}$; (c) $\mathrm{CH}_{4}<\mathrm{C}_{2} \mathrm{H}_{6}<$ $\mathrm{C}_{3} \mathrm{H}_{8} ;$ (d) $\mathrm{N}_{2}<\mathrm{O}_{2}<\mathrm{NO}$

The molecular mass of butanol, $\mathrm{C}_{4} \mathrm{H}_{9} \mathrm{OH}$, is 74.14 ; that of ethylene glycol, $\mathrm{CH}_{2}(\mathrm{OH}) \mathrm{CH}_{2} \mathrm{OH}$, is 62.08, yet their boiling points are $117.2{ }^{\circ} \mathrm{C}$ and $174{ }^{\circ} \mathrm{C}$, respectively. Explain the reason for the difference. 
On the basis of intermolecular attractions, explain the differences in the boiling points of $n$-butane $\left(-1^{\circ} \mathrm{C}\right)$ and chloroethane $\left(12^{\circ} \mathrm{C}\right)$, which have similar molar masses.

Only rather small dipole-dipole interactions from $\mathrm{C}-\mathrm{H}$ bonds are available to hold $n$-butane in the liquid state. Chloroethane, however, has rather large dipole interactions because of the $\mathrm{Cl}-\mathrm{C}$ bond; the interaction, therefore, is stronger, leading to a higher boiling point.

On the basis of dipole moments and/or hydrogen bonding, explain in a qualitative way the differences in the boiling points of acetone $\left(56.2{ }^{\circ} \mathrm{C}\right)$ and 1-propanol $\left(97.4{ }^{\circ} \mathrm{C}\right)$, which have similar molar masses.

The melting point of $\mathrm{H}_{2} \mathrm{O}(s)$ is $0{ }^{\circ} \mathrm{C}$. Would you expect the melting point of $\mathrm{H}_{2} \mathrm{~S}(\mathrm{~s})$ to be $-85^{\circ} \mathrm{C}, 0{ }^{\circ} \mathrm{C}$, or $185^{\circ} \mathrm{C}$ ? Explain your answer.

$-85^{\circ} \mathrm{C}$. Water has stronger hydrogen bonds, so it melts at a higher temperature.

Silane $\left(\mathrm{SiH}_{4}\right)$, phosphine $\left(\mathrm{PH}_{3}\right)$, and hydrogen sulfide $\left(\mathrm{H}_{2} \mathrm{~S}\right)$ melt at $-185{ }^{\circ} \mathrm{C},-133{ }^{\circ} \mathrm{C}$, and $-85^{\circ} \mathrm{C}$, respectively. What does this suggest about the polar character and intermolecular attractions of the three compounds?

Explain why a hydrogen bond between two water molecules is weaker than a hydrogen bond between two hydrogen fluoride molecules.

The hydrogen bond between two hydrogen fluoride molecules is stronger than that between two water 
molecules because the electronegativity of $\mathrm{F}$ is greater than that of $\mathrm{O}$. Consequently, the partial negative charge on $\mathrm{F}$ is greater than that on $\mathrm{O}$. The hydrogen bond between the partially positive $\mathrm{H}$ and the larger partially negative $\mathrm{F}$ will be stronger than that formed between $\mathrm{H}$ and $\mathrm{O}$.

Under certain conditions, molecules of acetic acid, $\mathrm{CH}_{3} \mathrm{COOH}$, form "dimers," pairs of acetic acid molecules held together by strong intermolecular attractions:<smiles>CC(=O)O</smiles>

Draw a dimer of acetic acid, showing how two $\mathrm{CH}_{3} \mathrm{COOH}$ molecules are held together, and stating the type of IMF that is responsible.

Proteins are chains of amino acids that can form in a variety of arrangements, one of which is a helix. What kind of IMF is responsible for holding the protein strand in this shape? On the protein image, show the locations of the IMFs that hold the protein together:

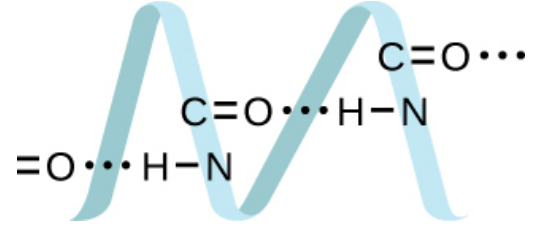

$\mathrm{H}$-bonding is the principle IMF holding the protein strands together. The H-bonding is between the $\mathrm{N}-\mathrm{H}$ and $\mathrm{C}=\mathrm{O}$.

The density of liquid $\mathrm{NH}_{3}$ is $0.64 \mathrm{~g} / \mathrm{mL}$; the density of 
gaseous $\mathrm{NH}_{3}$ at STP is $0.0007 \mathrm{~g} / \mathrm{mL}$. Explain the difference between the densities of these two phases.

Identify the intermolecular forces present in the following solids:
(a) $\mathrm{CH}_{3} \mathrm{CH}_{2} \mathrm{OH}$
(b) $\mathrm{CH}_{3} \mathrm{CH}_{2} \mathrm{CH}_{3}$
(c) $\mathrm{CH}_{3} \mathrm{CH}_{2} \mathrm{Cl}$

(a) hydrogen bonding, dipole-dipole attraction, and dispersion forces; (b) dispersion forces; (c) dipole-dipole attraction and dispersion forces

Glossary

dipole-dipole attraction

intermolecular attraction between two permanent dipoles

dispersion force

(also, London dispersion force) attraction between two rapidly fluctuating, temporary dipoles; significant only when particles are very close together

\section{hydrogen bonding}

occurs when exceptionally strong dipoles attract; bonding that exists when hydrogen is bonded to one of the three most electronegative elements: $\mathrm{F}, \mathrm{O}$, or $\mathrm{N}$ induced dipole

temporary dipole formed when the 
electrons of an atom or molecule are distorted by the instantaneous dipole of a neighboring atom or molecule

instantaneous dipole

temporary dipole that occurs for a brief moment in time when the electrons of an atom or molecule are distributed asymmetrically

intermolecular force

noncovalent attractive force between

atoms, molecules, and/or ions

polarizability

measure of the ability of a charge to distort a molecule's charge distribution (electron cloud)

\section{van der Waals force}

attractive or repulsive force between molecules, including dipole-dipole, dipoleinduced dipole, and London dispersion forces; does not include forces due to covalent or ionic bonding, or the attraction between ions and molecules 
40.

\section{PROPERTIES OF LIQQUIDS}

\section{Learning Objectives}

By the end of this section, you will be able to:

- Distinguish between adhesive and cohesive forces

- Define viscosity, surface tension, and capillary rise

- Describe the roles of intermolecular attractive forces in each of these properties/phenomena

When you pour a glass of water, or fill a car with gasoline, you observe that water and gasoline flow freely. But when you pour syrup on pancakes or add oil to a car engine, you note that syrup and motor oil do not flow as readily. The viscosity of a liquid is a measure of its resistance to flow. Water, gasoline, and other liquids that flow freely have a low viscosity. Honey, syrup, motor oil, and other liquids that do not flow freely, like those shown in (Figure), have 
higher viscosities. We can measure viscosity by measuring the rate at which a metal ball falls through a liquid (the ball falls more slowly through a more viscous liquid) or by measuring the rate at which a liquid flows through a narrow tube (more viscous liquids flow more slowly).

(a) Honey and (b) motor oil are examples of liquids with high viscosities; they flow slowly. (credit a: modification of work by Scott Bauer; credit b: modification of work by David Nagy)

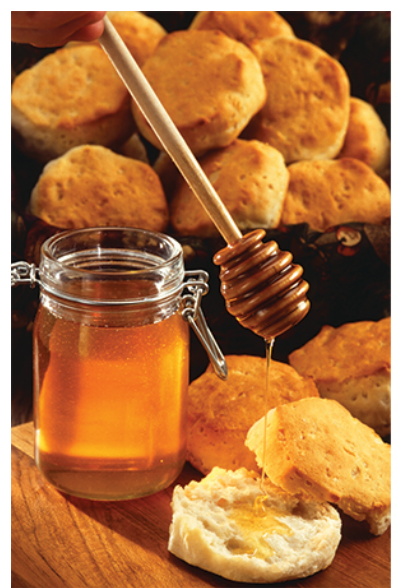

(a)

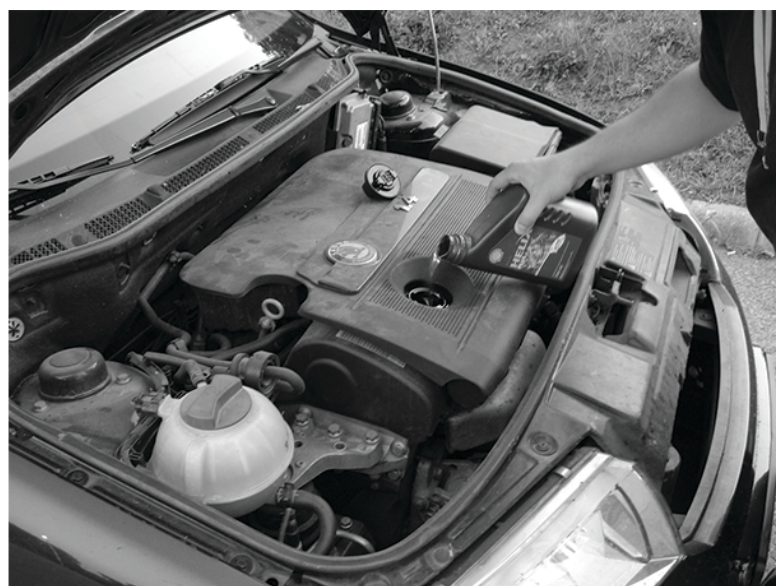

(b)

The IMFs between the molecules of a liquid, the size and shape of the molecules, and the temperature determine how easily a liquid flows. As (Figure) shows, the more structurally complex are the molecules in a liquid and the stronger the IMFs between them, the more difficult it is for them to move past each other and the greater is the viscosity of the liquid. As the temperature increases, the molecules move more rapidly and their kinetic energies are better able to overcome the forces that hold them together; thus, the viscosity of the liquid decreases. 
Viscosities of Common Substances at $25^{\circ} \mathrm{C}$

$\begin{array}{lll}\text { Substance } & \text { Formula } & \text { Viscosity (mPa·s) } \\ \text { water } & \mathrm{H}_{2} \mathrm{O} & 0.890 \\ \text { mercury } & \mathrm{Hg} & 1.526 \\ \text { ethanol } & \mathrm{C}_{2} \mathrm{H}_{5} \mathrm{OH} & 1.074 \\ \text { octane } & \mathrm{C}_{8} \mathrm{H}_{18} & 0.508 \\ \text { ethylene glycol } & \mathrm{CH}_{2}(\mathrm{OH}) \mathrm{CH}_{2}(\mathrm{OH}) & 16.1 \\ \text { honey } & \text { variable } & \sim 2,000-10,000 \\ \text { motor oil } & \text { variable } & \sim 50-500\end{array}$

The various IMFs between identical molecules of a substance are examples of cohesive forces. The molecules within a liquid are surrounded by other molecules and are attracted equally in all directions by the cohesive forces within the liquid. However, the molecules on the surface of a liquid are attracted only by about one-half as many molecules. Because of the unbalanced molecular attractions on the surface molecules, liquids contract to form a shape that minimizes the number of molecules on the surface-that is, the shape with the minimum surface area. A small drop of liquid tends to assume a spherical shape, as shown in (Figure), because in a sphere, the ratio of surface area to volume is at a minimum. Larger drops are more greatly affected by gravity, air resistance, surface interactions, and so on, and as a result, are less spherical. Attractive forces result in a spherical water drop that minimizes surface area; cohesive forces hold the sphere together; adhesive forces keep the drop attached to the web. (credit photo: modification of work by "OliBac"/Flickr) 


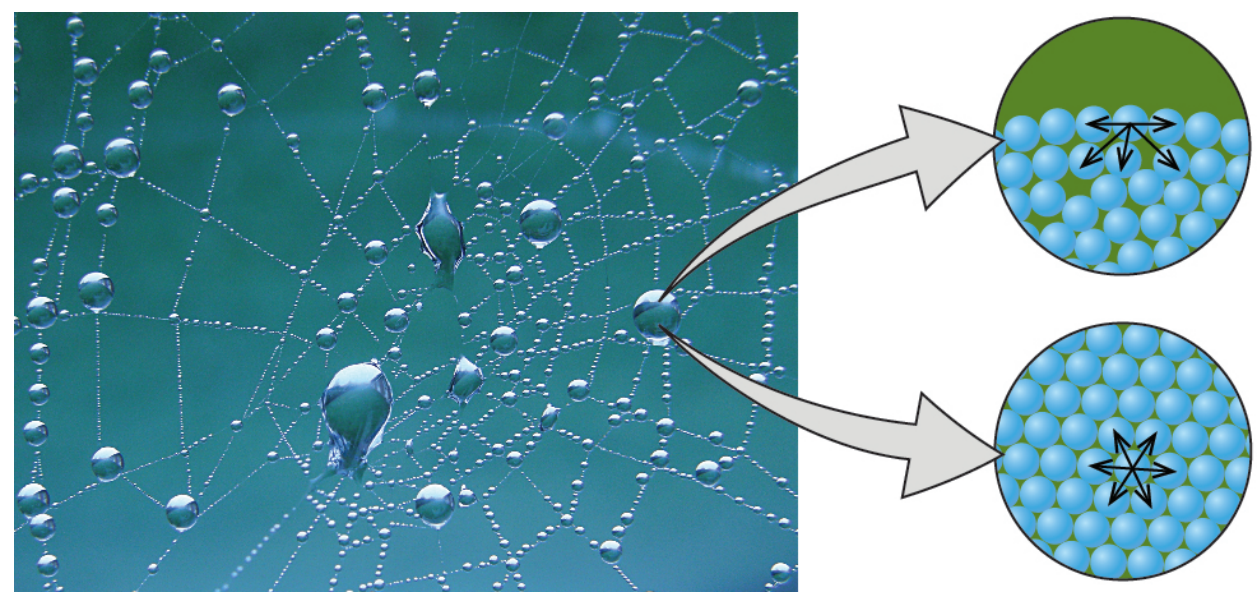

Surface tension is defined as the energy required to increase the surface area of a liquid, or the force required to increase the length of a liquid surface by a given amount. This property results from the cohesive forces between molecules at the surface of a liquid, and it causes the surface of a liquid to behave like a stretched rubber membrane. Surface tensions of several liquids are presented in (Figure). Among common liquids, water exhibits a distinctly high surface tension due to strong hydrogen bonding between its molecules. As a result of this high surface tension, the surface of water represents a relatively "tough skin" that can withstand considerable force without breaking. A steel needle carefully placed on water will float. Some insects, like the one shown in (Figure), even though they are denser than water, move on its surface because they are supported by the surface tension. 
Surface Tensions of Common Substances at $25{ }^{\circ} \mathrm{C}$

$\begin{array}{lll}\text { Substance } & \text { Formula } & \text { Surface Tension }(\mathbf{m N} / \mathbf{m}) \\ \text { water } & \mathrm{H}_{2} \mathrm{O} & 71.99 \\ \text { mercury } & \mathrm{Hg} & 458.48 \\ \text { ethanol } & \mathrm{C}_{2} \mathrm{H}_{5} \mathrm{OH} & 21.97 \\ \text { octane } & \mathrm{C}_{8} \mathrm{H}_{18} & 21.14 \\ \text { ethylene glycol } & \mathrm{CH}_{2}(\mathrm{OH}) \mathrm{CH}_{2}(\mathrm{OH}) & 47.99\end{array}$

Surface tension (right) prevents this insect, a "water strider," from sinking into the water.

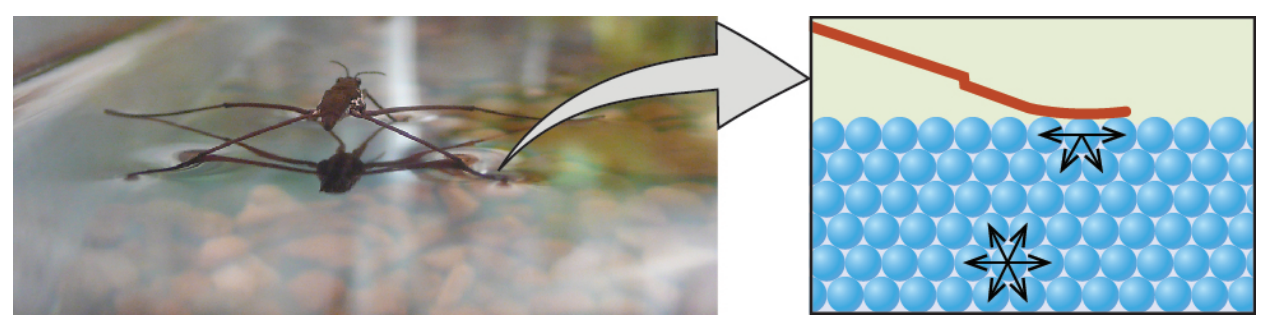

The IMFs of attraction between two different molecules are called adhesive forces. Consider what happens when water comes into contact with some surface. If the adhesive forces between water molecules and the molecules of the surface are weak compared to the cohesive forces between the water molecules, the water does not "wet" the surface. For example, water does not wet waxed surfaces or many plastics such as polyethylene. Water forms drops on these surfaces because the cohesive forces within the drops are greater than the adhesive forces between the water and the plastic. Water spreads out on glass because the adhesive force between water and glass is greater than the cohesive forces within the water. When water is confined in a glass tube, its meniscus (surface) has a concave shape because the water wets the glass and creeps up the side of 
the tube. On the other hand, the cohesive forces between mercury atoms are much greater than the adhesive forces between mercury and glass. Mercury therefore does not wet glass, and it forms a convex meniscus when confined in a tube because the cohesive forces within the mercury tend to draw it into a drop ((Figure)).

Differences in the relative strengths of cohesive and adhesive forces result in different meniscus shapes for mercury (left) and water (right) in glass tubes. (credit: Mark Ott)

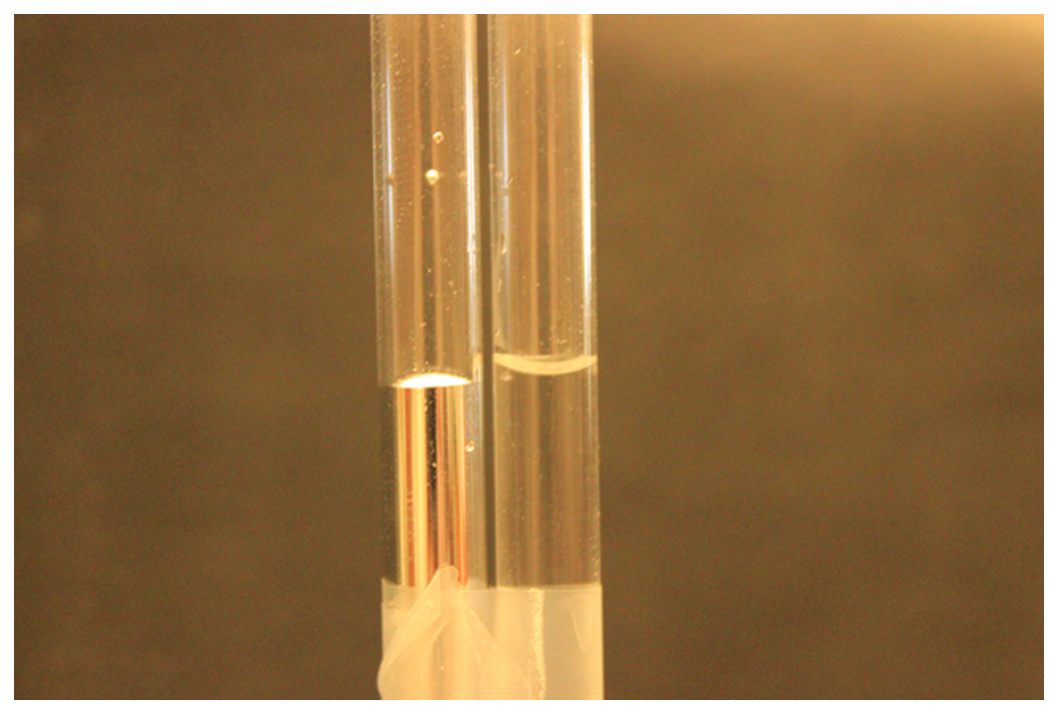

If you place one end of a paper towel in spilled wine, as shown in (Figure), the liquid wicks up the paper towel. A similar process occurs in a cloth towel when you use it to dry off after a shower. These are examples of capillary action-when a liquid flows within a porous material due to the attraction of the liquid molecules to the surface of the material and to other liquid molecules. The adhesive forces between the liquid and the porous material, combined with the cohesive forces within the liquid, may 
be strong enough to move the liquid upward against gravity.

Wine wicks up a paper towel (left) because of the strong attractions of water (and ethanol) molecules to the $-\mathrm{OH}$ groups on the towel's cellulose fibers and the strong attractions of water molecules to other water (and ethanol) molecules (right). (credit photo: modification of work by Mark Blaser)

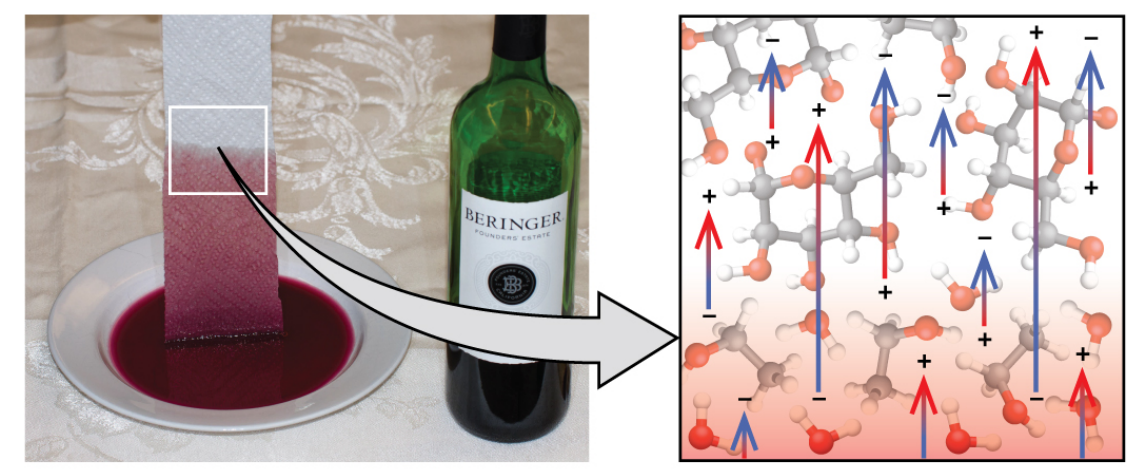

Towels soak up liquids like water because the fibers of a towel are made of molecules that are attracted to water molecules. Most cloth towels are made of cotton, and paper towels are generally made from paper pulp. Both consist of long molecules of cellulose that contain many $-\mathrm{OH}$ groups. Water molecules are attracted to these $-\mathrm{OH}$ groups and form hydrogen bonds with them, which draws the $\mathrm{H}_{2} \mathrm{O}$ molecules up the cellulose molecules. The water molecules are also attracted to each other, so large amounts of water are drawn up the cellulose fibers.

Capillary action can also occur when one end of a small diameter tube is immersed in a liquid, as illustrated in (Figure). If the liquid molecules are strongly attracted to 
the tube molecules, the liquid creeps up the inside of the tube until the weight of the liquid and the adhesive forces are in balance. The smaller the diameter of the tube is, the higher the liquid climbs. It is partly by capillary action occurring in plant cells called xylem that water and dissolved nutrients are brought from the soil up through the roots and into a plant. Capillary action is the basis for thin layer chromatography, a laboratory technique commonly used to separate small quantities of mixtures. You depend on a constant supply of tears to keep your eyes lubricated and on capillary action to pump tear fluid away. Depending upon the relative strengths of adhesive and cohesive forces, a liquid may rise (such as water) or fall (such as mercury) in a glass capillary tube. The extent of the rise (or fall) is directly proportional to the surface tension of the liquid and inversely proportional to the density of the liquid and the radius of the tube.

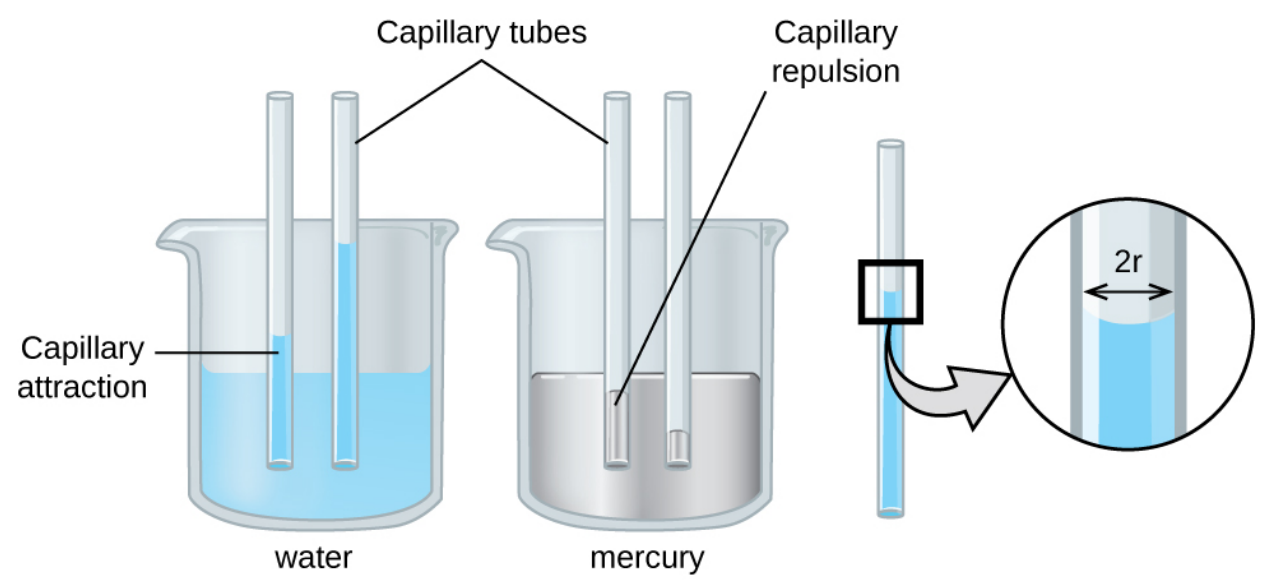

The height to which a liquid will rise in a capillary tube is determined by several factors as shown in the following equation:

$h=\frac{2 T \cos \theta}{r \rho g}$ 
In this equation, $h$ is the height of the liquid inside the capillary tube relative to the surface of the liquid outside the tube, $T$ is the surface tension of the liquid, $\theta$ is the contact angle between the liquid and the tube, $r$ is the radius of the tube, $\rho$ is the density of the liquid, and $g$ is the acceleration due to gravity, $9.8 \mathrm{~m} / \mathrm{s}^{2}$. When the tube is made of a material to which the liquid molecules are strongly attracted, they will spread out completely on the surface, which corresponds to a contact angle of $0^{\circ}$. This is the situation for water rising in a glass tube.

Capillary Rise At $25^{\circ} \mathrm{C}$, how high will water rise in a glass capillary tube with an inner diameter of $0.25 \mathrm{~mm}$ ?

For water, $T=71.99 \mathrm{mN} / \mathrm{m}$ and $\rho=1.0 \mathrm{~g} / \mathrm{cm}^{3}$.

Solution The liquid will rise to a height $h$ given by: $h=\frac{2 T \cos \theta}{r \rho g}$

The Newton is defined as a $\mathrm{kg} \mathrm{m} / \mathrm{s}^{2}$, and so the provided surface tension is equivalent to $0.07199 \mathrm{~kg} / \mathrm{s}^{2}$. The provided density must be converted into units that will cancel appropriately: $\rho=1000 \mathrm{~kg} / \mathrm{m}^{3}$. The diameter of the tube in meters is $0.00025 \mathrm{~m}$, so the radius is $0.000125 \mathrm{~m}$. For a glass tube immersed in water, the contact angle is $\theta=0^{\circ}$, so $\cos \theta=$ 1. Finally, acceleration due to gravity on the earth is $g=9.8 \mathrm{~m} /$ $\mathrm{s}^{2}$. Substituting these values into the equation, and cancelling units, we have:

$$
h=\frac{2\left(0.07199 \mathrm{~kg} / \mathrm{s}^{2}\right)}{(0.000125 \mathrm{~m})\left(1000 \mathrm{~kg} / \mathrm{m}^{3}\right)\left(9.8 \mathrm{~m} / \mathrm{s}^{2}\right)}=0.12 \mathrm{~m}=12 \mathrm{~cm}
$$

Check Your Learning Water rises in a glass capillary tube to a height of $8.4 \mathrm{~cm}$. What is the diameter of the capillary tube?

Answer:

diameter $=0.36 \mathrm{~mm}$ 
Biomedical Applications of Capillary Action

Many medical tests require drawing a small amount of blood, for example to determine the amount of glucose in someone with diabetes or the hematocrit level in an athlete. This procedure can be easily done because of capillary action, the ability of a liquid to flow up a small tube against gravity, as shown in (Figure). When your finger is pricked, a drop of blood forms and holds together due to surface tension-the unbalanced intermolecular attractions at the surface of the drop. Then, when the open end of a narrow-diameter glass tube touches the drop of blood, the adhesive forces between the molecules in the blood and those at the glass surface draw the blood up the tube. How far the blood goes up the tube depends on the diameter of the tube (and the type of fluid). A small tube has a relatively large surface area for a given volume of blood, which results in larger (relative) attractive forces, allowing the blood to be drawn farther up the tube. The liquid itself is held together by its own cohesive forces. When the weight of the liquid in the tube generates a downward force equal to the upward force associated with capillary action, the liquid stops rising.

Blood is collected for medical analysis by capillary action, which draws blood into a small diameter glass tube. (credit: modification of work by Centers for Disease Control and Prevention) 


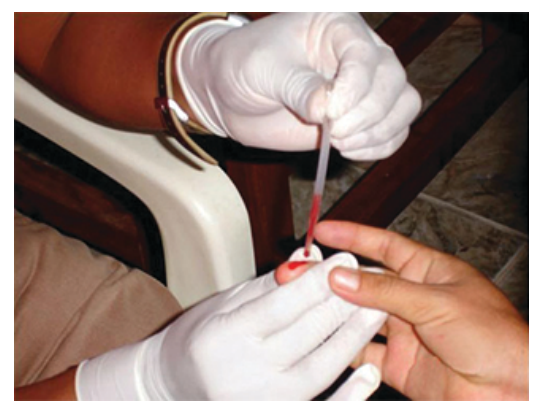

Key Concepts and Summary

The intermolecular forces between molecules in the liquid state vary depending upon their chemical identities and result in corresponding variations in various physical properties. Cohesive forces between like molecules are responsible for a liquid's viscosity (resistance to flow) and surface tension (elasticity of a liquid surface). Adhesive forces between the molecules of a liquid and different molecules composing a surface in contact with the liquid are responsible for phenomena such as surface wetting and capillary rise.

Key Equations

$$
\text { - } h=\frac{2 T \cos \theta}{r \rho g}
$$

\section{Chemistry End of Chapter Exercises}

The test tubes shown here contain equal amounts of the specified motor oils. Identical metal spheres were dropped at the same time into each of the tubes, and a brief moment later, the spheres had fallen to the heights indicated in the illustration.

Rank the motor oils in order of increasing viscosity, and explain your reasoning: 


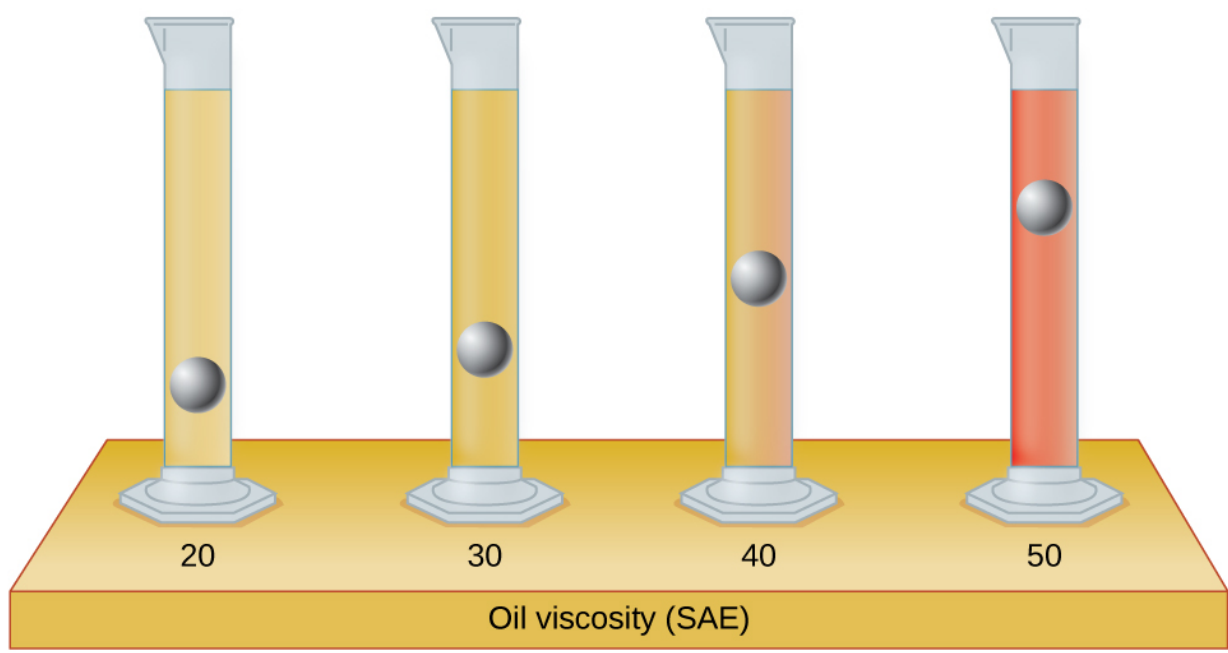

Although steel is denser than water, a steel needle or paper clip placed carefully lengthwise on the surface of still water can be made to float. Explain at a molecular level how this is possible. (credit: Cory Zanker)

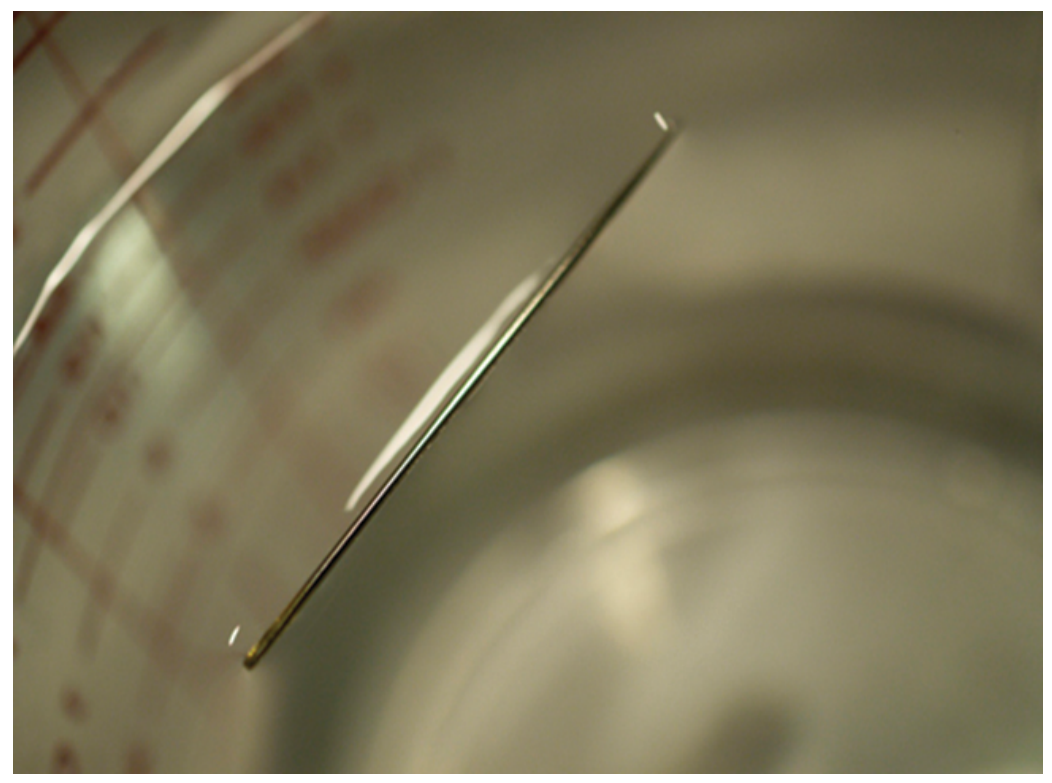

The water molecules have strong intermolecular forces of hydrogen bonding. The water molecules are thus attracted strongly to one another and exhibit a relatively large 
surface tension, forming a type of "skin" at its surface. This skin can support a bug or paper clip if gently placed on the water.

The surface tension and viscosity values for diethyl ether, acetone, ethanol, and ethylene glycol are shown here.

\begin{tabular}{|c|c|c|c|}
\hline Compound & Molecule & $\begin{array}{c}\text { Surface Tension } \\
(\mathrm{mN} / \mathrm{m})\end{array}$ & $\begin{array}{l}\text { Viscosity } \\
\text { (mPa s) }\end{array}$ \\
\hline $\begin{array}{l}\text { diethyl ether } \\
\mathrm{C}_{2} \mathrm{H}_{5} \mathrm{OC}_{2} \mathrm{H}_{5}\end{array}$ & & 17 & 0.22 \\
\hline $\begin{array}{c}\text { acetone } \\
\mathrm{CH}_{3} \mathrm{COCH}_{3}\end{array}$ & & 23 & 0.31 \\
\hline $\begin{array}{l}\text { ethanol } \\
\mathrm{C}_{2} \mathrm{H}_{5} \mathrm{OH}\end{array}$ & & 22 & 1.07 \\
\hline $\begin{array}{l}\text { ethylene glycol } \\
\mathrm{CH}_{2}(\mathrm{OH}) \mathrm{CH}_{2}(\mathrm{OH})\end{array}$ & & 48 & 16.1 \\
\hline
\end{tabular}

(a) Explain their differences in viscosity in terms of the size and shape of their molecules and their IMFs.

(b) Explain their differences in surface tension in terms of the size and shape of their molecules and their IMFs:

You may have heard someone use the figure of speech "slower than molasses in winter" to describe a process that occurs slowly. Explain why this is an apt idiom, using concepts of molecular size and shape, molecular interactions, and the effect of changing temperature.

Temperature has an effect on intermolecular forces: The higher the temperature, the greater the kinetic energies of the molecules and the greater the extent to which their intermolecular forces are overcome, and so the more fluid (less viscous) the liquid. The lower the temperature, the less the intermolecular forces are overcome, and so the less viscous the liquid. 
It is often recommended that you let your car engine run idle to warm up before driving, especially on cold winter days. While the benefit of prolonged idling is dubious, it is certainly true that a warm engine is more fuel efficient than a cold one. Explain the reason for this.

The surface tension and viscosity of water at several different temperatures are given in this table.

$\begin{array}{lll}\text { Water } & \text { Surface Tension }(\mathbf{m N} / \mathbf{m}) & \text { Viscosity (mPa s) } \\ 0{ }^{\circ} \mathrm{C} & 75.6 & 1.79 \\ 20{ }^{\circ} \mathrm{C} & 72.8 & 1.00 \\ 60{ }^{\circ} \mathrm{C} & 66.2 & 0.47 \\ 100{ }^{\circ} \mathrm{C} & 58.9 & 0.28\end{array}$

(a) As temperature increases, what happens to the surface tension of water? Explain why this occurs, in terms of molecular interactions and the effect of changing temperature.

(b) As temperature increases, what happens to the viscosity of water? Explain why this occurs, in terms of molecular interactions and the effect of changing temperature.

(a) As the water reaches higher temperatures, the increased kinetic energies of its molecules are more effective in overcoming hydrogen bonding, and so its surface tension decreases. Surface tension and intermolecular forces are directly related. (b) The same trend in viscosity is seen as in surface tension, and for the same reason. 
At $25^{\circ} \mathrm{C}$, how high will water rise in a glass capillary tube with an inner diameter of $0.63 \mathrm{~mm}$ ? Refer to (Figure) for the required information.

Water rises in a glass capillary tube to a height of $17 \mathrm{~cm}$. What is the diameter of the capillary tube?

\title{
$1.710^{-4} \mathrm{~m}$
}

\author{
Glossary \\ adhesive force \\ force of attraction between molecules of \\ different chemical identities \\ capillary action \\ flow of liquid within a porous material due \\ to the attraction of the liquid molecules to \\ the surface of the material and to other liquid \\ molecules \\ cohesive force \\ force of attraction between identical \\ molecules

\section{surface tension} \\ energy required to increase the area, or \\ length, of a liquid surface by a given amount \\ viscosity \\ measure of a liquid's resistance to flow
}




\section{THE SOLID STATE OF MATTER}

Learning Objectives

By the end of this section, you will be able to:

- Define and describe the bonding and properties of ionic, molecular, metallic, and covalent network crystalline solids

- Describe the main types of crystalline solids: ionic solids, metallic solids, covalent network solids, and molecular solids

- Explain the ways in which crystal defects can occur in a solid

When most liquids are cooled, they eventually freeze and form crystalline solids, solids in which the atoms, ions, or molecules are arranged in a definite repeating pattern. It is also possible for a liquid to freeze before its molecules 
become arranged in an orderly pattern. The resulting materials are called amorphous solids or noncrystalline solids (or, sometimes, glasses). The particles of such solids lack an ordered internal structure and are randomly arranged ((Figure)).

The entities of a solid phase may be arranged in a regular, repeating pattern (crystalline solids) or randomly

(amorphous).

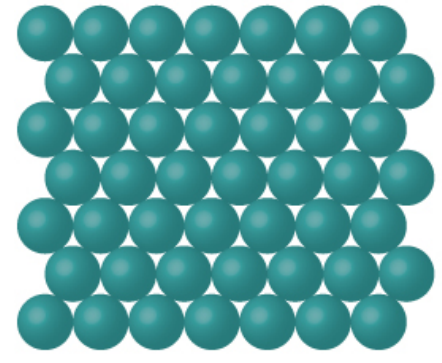

Crystalline

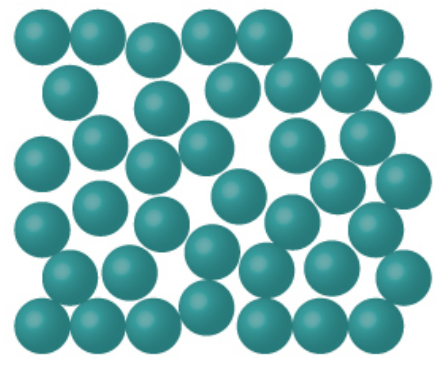

Amorphous

Metals and ionic compounds typically form ordered, crystalline solids. Substances that consist of large molecules, or a mixture of molecules whose movements are more restricted, often form amorphous solids. For examples, candle waxes are amorphous solids composed of large hydrocarbon molecules. Some substances, such as silicon dioxide (shown in (Figure)), can form either crystalline or amorphous solids, depending on the conditions under which it is produced. Also, amorphous solids may undergo a transition to the crystalline state under appropriate conditions.

(a) Silicon dioxide, $\mathrm{SiO}_{2}$, is abundant in nature as one of several crystalline forms of the mineral quartz. (b) Rapid cooling of molten $\mathrm{SiO}_{2}$ yields an amorphous solid known as "fused silica". 
Two sets of molecules are shown. The first set of molecules contains five identical, hexagonal rings composed of alternating red and maroon spheres single bonded together and with a red spheres extending outward from each maroon sphere. The second set of molecules shows four rings with twelve sides each that are joined together. Each ring is composed of alternating red and maroon spheres single bonded together and with a red spheres extending outward from each maroon sphere.

Crystalline solids are generally classified according the nature of the forces that hold its particles together. These forces are primarily responsible for the physical properties exhibited by the bulk solids. The following sections provide descriptions of the major types of crystalline solids: ionic, metallic, covalent network, and molecular.

Ionic Solids

Ionic solids, such as sodium chloride and nickel oxide, are composed of positive and negative ions that are held together by electrostatic attractions, which can be quite strong ((Figure)). Many ionic crystals also have high melting points. This is due to the very strong attractions between the ions-in ionic compounds, the attractions between full charges are (much) larger than those between the partial charges in polar molecular compounds. This will be looked at in more detail in a later discussion of lattice energies. Although they are hard, they also tend to be brittle, and they shatter rather than bend. Ionic solids do not conduct electricity; however, they do conduct when molten or dissolved because their ions are free to move. 
Many simple compounds formed by the reaction of a metallic element with a nonmetallic element are ionic. Sodium chloride is an ionic solid.

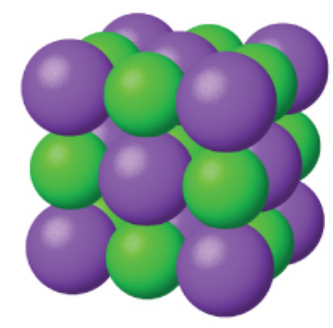

Metallic Solids

Metallic solids such as crystals of copper, aluminum, and iron are formed by metal atoms (Figure). The structure of metallic crystals is often described as a uniform distribution of atomic nuclei within a "sea" of delocalized electrons. The atoms within such a metallic solid are held together by a unique force known as metallic bonding that gives rise to many useful and varied bulk properties. All exhibit high thermal and electrical conductivity, metallic luster, and malleability. Many are very hard and quite strong. Because of their malleability (the ability to deform under pressure or hammering), they do not shatter and, therefore, make useful construction materials. The melting points of the metals vary widely. Mercury is a liquid at room temperature, and the alkali metals melt below $200{ }^{\circ} \mathrm{C}$. Several post-transition metals also have low melting points, whereas the transition metals melt at temperatures above $1000{ }^{\circ} \mathrm{C}$. These differences reflect differences in strengths of metallic bonding among the metals.

Copper is a metallic solid. 


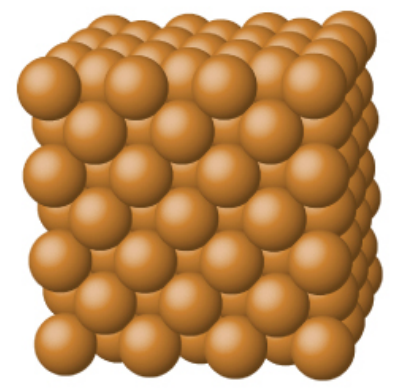

Covalent Network Solid

Covalent network solids include crystals of diamond, silicon, some other nonmetals, and some covalent compounds such as silicon dioxide (sand) and silicon carbide (carborundum, the abrasive on sandpaper). Many minerals have networks of covalent bonds. The atoms in these solids are held together by a network of covalent bonds, as shown in (Figure). To break or to melt a covalent network solid, covalent bonds must be broken. Because covalent bonds are relatively strong, covalent network solids are typically characterized by hardness, strength, and high melting points. For example, diamond is one of the hardest substances known and melts above $3500{ }^{\circ} \mathrm{C}$. A covalent crystal contains a three-dimensional network of covalent bonds, as illustrated by the structures of diamond, silicon dioxide, silicon carbide, and graphite. Graphite is an exceptional example, composed of planar sheets of covalent crystals that are held together in layers by noncovalent forces. Unlike typical covalent solids, graphite is very soft and electrically conductive. 
Four pairs of images are shown. In the first pair, a square box containing a black atom bonded to four other black atoms is shown above a structure composed of many black atoms, each bonded to four other black atoms, where one of the upper atoms is labeled "carbon" and the whole structure is labeled "diamond." In the second pair, a square box containing a white atom bonded to four red atoms is shown above a structure composed of many white atoms, each bonded to four red atoms, where one of the red atoms is labeled "oxygen" and one of the white atoms is labeled "silicon." The whole structure is labeled "silicon dioxide." In the third pair, a square box containing a blue atom bonded to four white atoms is shown above a structure composed of many blue atoms, each bonded to four white atoms, where one of the blue atoms is labeled "carbon" and one of the white atoms is labeled "silicon." The whole structure is labeled "silicon carbide." In the fourth pair, a square box containing six black atoms bonded into a ring is shown above a structure composed of many rings, arranged into sheets layered one atop the other, where one of the black atoms is labeled "carbon." The whole structure is labeled "graphite."

Molecular Solid

Molecular solids, such as ice, sucrose (table sugar), and iodine, as shown in (Figure), are composed of neutral molecules. The strengths of the attractive forces between the units present in different crystals vary widely, as indicated by the melting points of the crystals. Small symmetrical molecules (nonpolar molecules), such as $\mathrm{H}_{2}$, $\mathrm{N}_{2}, \mathrm{O}_{2}$, and $\mathrm{F}_{2}$, have weak attractive forces and form 
molecular solids with very low melting points (below -200 $\left.{ }^{\circ} \mathrm{C}\right)$. Substances consisting of larger, nonpolar molecules have larger attractive forces and melt at higher temperatures. Molecular solids composed of molecules with permanent dipole moments (polar molecules) melt at still higher temperatures. Examples include ice (melting point, $0{ }^{\circ} \mathrm{C}$ ) and table sugar (melting point, $185^{\circ} \mathrm{C}$ ).

Carbon dioxide $\left(\mathrm{CO}_{2}\right)$ consists of small, nonpolar molecules and forms a molecular solid with a melting point of $-78{ }^{\circ} \mathrm{C}$. Iodine $\left(\mathrm{I}_{2}\right)$ consists of larger, nonpolar molecules and forms a molecular solid that melts at 114 ${ }^{\circ} \mathrm{C}$.

Two images are shown and labeled "carbon dioxide" and "iodine." The carbon dioxide structure is composed of molecules, each made up of one gray and two red atoms, stacked together into a cube. The image of iodine shows pairs of purple atoms arranged near one another, but not touching.

\section{Properties of Solids}

A crystalline solid, like those listed in (Figure), has a precise melting temperature because each atom or molecule of the same type is held in place with the same forces or energy. Thus, the attractions between the units that make up the crystal all have the same strength and all require the same amount of energy to be broken. The gradual softening of an amorphous material differs dramatically from the distinct melting of a crystalline solid. This results from the structural nonequivalence of the molecules in the amorphous solid. Some forces are 
weaker than others, and when an amorphous material is heated, the weakest intermolecular attractions break first. As the temperature is increased further, the stronger attractions are broken. Thus amorphous materials soften over a range of temperatures.

\section{Types of Crystalline Solids and Their Properties}

\begin{tabular}{|c|c|c|c|c|}
\hline $\begin{array}{l}\text { Type of } \\
\text { Solid }\end{array}$ & $\begin{array}{l}\text { Type of } \\
\text { Particles }\end{array}$ & $\begin{array}{l}\text { Type of } \\
\text { Attractions }\end{array}$ & Properties & Examples \\
\hline ionic & ions & ionic bonds & $\begin{array}{l}\text { hard, brittle, } \\
\text { conducts } \\
\text { electricity as } \\
\text { a liquid but } \\
\text { not as a } \\
\text { solid, high to } \\
\text { very high } \\
\text { melting } \\
\text { points }\end{array}$ & $\begin{array}{l}\mathrm{NaCl} \\
\mathrm{Al}_{2} \mathrm{O}_{3}\end{array}$ \\
\hline metallic & $\begin{array}{l}\text { atoms of } \\
\text { electropositive } \\
\text { elements }\end{array}$ & $\begin{array}{l}\text { metallic } \\
\text { bonds }\end{array}$ & $\begin{array}{l}\text { shiny, } \\
\text { malleable, } \\
\text { ductile, } \\
\text { conducts } \\
\text { heat and } \\
\text { electricity } \\
\text { well, variable } \\
\text { hardness and } \\
\text { melting } \\
\text { temperature }\end{array}$ & $\begin{array}{l}\mathrm{Cu}, \mathrm{Fe}, \mathrm{Ti}, \\
\mathrm{Pb}, \mathrm{U}\end{array}$ \\
\hline $\begin{array}{l}\text { covalent } \\
\text { network }\end{array}$ & $\begin{array}{l}\text { atoms of } \\
\text { electronegative } \\
\text { elements }\end{array}$ & $\begin{array}{l}\text { covalent } \\
\text { bonds }\end{array}$ & $\begin{array}{l}\text { very hard, } \\
\text { not } \\
\text { conductive, } \\
\text { very high } \\
\text { melting } \\
\text { points }\end{array}$ & $\begin{array}{l}\mathrm{C} \\
\text { (diamond), } \\
\mathrm{SiO}_{2}, \mathrm{SiC}\end{array}$ \\
\hline molecular & $\begin{array}{l}\text { molecules (or } \\
\text { atoms) }\end{array}$ & IMFs & $\begin{array}{l}\text { variable } \\
\text { hardness, } \\
\text { variable } \\
\text { brittleness, } \\
\text { not } \\
\text { conductive, } \\
\text { low melting } \\
\text { points }\end{array}$ & $\begin{array}{l}\mathrm{H}_{2} \mathrm{O}, \mathrm{CO}_{2}, \\
\mathrm{I}_{2}, \\
\mathrm{C}_{12} \mathrm{H}_{22} \mathrm{O}_{11}\end{array}$ \\
\hline
\end{tabular}


Graphene: Material of the Future

Carbon is an essential element in our world. The unique properties of carbon atoms allow the existence of carbonbased life forms such as ourselves. Carbon forms a huge variety of substances that we use on a daily basis, including those shown in (Figure). You may be familiar with diamond and graphite, the two most common allotropes of carbon. (Allotropes are different structural forms of the same element.) Diamond is one of the hardest-known substances, whereas graphite is soft enough to be used as pencil lead. These very different properties stem from the different arrangements of the carbon atoms in the different allotropes.

Diamond is extremely hard because of the strong bonding between carbon atoms in all directions. Graphite (in pencil lead) rubs off onto paper due to the weak attractions between the carbon layers. An image of a graphite surface shows the distance between the centers of adjacent carbon atoms. (credit left photo: modification of work by Steve Jurvetson; credit middle photo: modification of work by United States Geological Survey) 
Three pairs of images are shown, each composed of a photo and a diagram. In the first pair, the photo shows a close-up view of a colorless, multi-faceted crystal and the diagram shows many gray spheres bonded together in a net-like structure. The caption below this pair reads "diamond." In the second pair, the photo shows a rough textured, dark gray solid while the image shows four horizontal sheets, composed of interlocking black spheres, lying atop one another. This pair has a caption that reads "graphite." The third pair shows a photo of twelve black hexagons on a yellow background where two of the hexagons are encircled by a gray border and a caption of "1.4 times 10, superscript negative $10, \mathrm{~m}$, Distance between center of atoms" and an image of many black hexagons evenly arranged on a yellow background. The caption below this pair of images reads "Graphite surface.”

You may be less familiar with a recently discovered form of carbon: graphene. Graphene was first isolated in 2004 by using tape to peel off thinner and thinner layers from graphite. It is essentially a single sheet (one atom thick) of graphite. Graphene, illustrated in (Figure), is not only strong and lightweight, but it is also an excellent conductor of electricity and heat. These properties may prove very useful in a wide range of applications, such as vastly improved computer chips and circuits, better batteries and solar cells, and stronger and lighter structural materials. The 2010 Nobel Prize in Physics was awarded to Andre Geim and Konstantin Novoselov for their pioneering work with graphene. Graphene sheets can be formed into buckyballs, 
nanotubes, and stacked layers.

Four images are shown. In the upper image, labeled "Graphene sheet," a box is drawn around a sheet of interconnected hexagonal rings. In the lower left image, a sphere is composed of hexagonal rings linked together and is labeled "Buckyball." In the lower middle image, a tube is shown that is composed of many hexagonal rings joined together and is labeled "Nanotube." In the lower right image, four horizontal sheets composed of joined, hexagonal rings is shown and labeled "Stacked sheets."

\section{Crystal Defects}

In a crystalline solid, the atoms, ions, or molecules are arranged in a definite repeating pattern, but occasional defects may occur in the pattern. Several types of defects are known, as illustrated in (Figure). Vacancies are defects that occur when positions that should contain atoms or ions are vacant. Less commonly, some atoms or ions in a crystal may occupy positions, called interstitial sites, located between the regular positions for atoms. Other distortions are found in impure crystals, as, for example, when the cations, anions, or molecules of the impurity are too large to fit into the regular positions without distorting the structure. Trace amounts of impurities are sometimes added to a crystal (a process known as doping) in order to create defects in the structure that yield desirable changes in its properties. For example, silicon crystals are doped with varying amounts of different elements to yield suitable electrical properties for their 
use in the manufacture of semiconductors and computer chips.

Types of crystal defects include vacancies, interstitial atoms, and substitutions impurities.

A diagram is shown in which one hundred and forty four spheres are arranged in a twelve by twelve square. A gap in the square is labeled "Vacancy" while one sphere that is a different color from all the rest is labeled "Interstitial impurity." The top right corner of the square is disturbed and has a larger sphere inserted that is labeled "Substitution impurity atom."

Key Concepts and Summary

Some substances form crystalline solids consisting of particles in a very organized structure; others form amorphous (noncrystalline) solids with an internal structure that is not ordered. The main types of crystalline solids are ionic solids, metallic solids, covalent network solids, and molecular solids. The properties of the different kinds of crystalline solids are due to the types of particles of which they consist, the arrangements of the particles, and the strengths of the attractions between them. Because their particles experience identical attractions, crystalline solids have distinct melting temperatures; the particles in amorphous solids experience a range of interactions, so they soften gradually and melt over a range of temperatures. Some crystalline solids have defects in the definite repeating pattern of their particles. These defects (which include vacancies, atoms or ions not in the regular positions, and 
impurities) change physical properties such as electrical conductivity, which is exploited in the silicon crystals used to manufacture computer chips.

Chemistry End of Chapter Exercises

What types of liquids typically form amorphous solids?

At very low temperatures oxygen, $\mathrm{O}_{2}$, freezes and forms a crystalline solid. Which best describes these crystals?

(a) ionic

(b) covalent network

(c) metallic

(d) amorphous

(e) molecular crystals

(e) molecular crystals

As it cools, olive oil slowly solidifies and forms a solid over a range of temperatures. Which best describes the solid?

(a) ionic

(b) covalent network

(c) metallic

(d) amorphous

(e) molecular crystals

Explain why ice, which is a crystalline solid, has a melting temperature of $0{ }^{\circ} \mathrm{C}$, whereas butter, which is an amorphous solid, softens over a range of temperatures.

Ice has a crystalline structure stabilized by hydrogen bonding. These intermolecular forces are of comparable strength and thus require the same amount of energy to 
overcome. As a result, ice melts at a single temperature and not over a range of temperatures. The various, very large molecules that compose butter experience varied van der Waals attractions of various strengths that are overcome at various temperatures, and so the melting process occurs over a wide temperature range.

Identify the type of crystalline solid (metallic, network covalent, ionic, or molecular) formed by each of the following substances:

(a) $\mathrm{SiO}_{2}$

(b) $\mathrm{KCl}$

(c) $\mathrm{Cu}$

(d) $\mathrm{CO}_{2}$

(e) $\mathrm{C}$ (diamond)

(f) $\mathrm{BaSO}_{4}$

(g) $\mathrm{NH}_{3}$

(h) $\mathrm{NH}_{4} \mathrm{~F}$

(i) $\mathrm{C}_{2} \mathrm{H}_{5} \mathrm{OH}$

Identify the type of crystalline solid (metallic, network covalent, ionic, or molecular) formed by each of the following substances:

(a) $\mathrm{CaCl}_{2}$

(b) $\mathrm{SiC}$

(c) $\mathrm{N}_{2}$

(d) $\mathrm{Fe}$

(e) $\mathrm{C}$ (graphite)

(f) $\mathrm{CH}_{3} \mathrm{CH}_{2} \mathrm{CH}_{2} \mathrm{CH}_{3}$

(g) $\mathrm{HCl}$

(h) $\mathrm{NH}_{4} \mathrm{NO}_{3}$

(i) $\mathrm{K}_{3} \mathrm{PO}_{4}$ 
(a) ionic; (b) covalent network; (c) molecular; (d) metallic;

(e) covalent network; (f) molecular; (g) molecular; (h) ionic; (i) ionic

Classify each substance in the table as either a metallic, ionic, molecular, or covalent network solid:

$\begin{array}{lllll}\text { Substance } & \text { Appearance } & \begin{array}{l}\text { Melting } \\ \text { Point }\end{array} & \begin{array}{l}\text { Electrical } \\ \text { Conductivity }\end{array} & \begin{array}{l}\text { Solubility } \\ \text { in Water }\end{array} \\ \mathrm{X} & \begin{array}{l}\text { lustrous, } \\ \text { malleable }\end{array} & 1500^{\circ} \mathrm{C} & \text { high } & \text { insoluble } \\ \mathrm{Y} & \text { soft, yellow } & 113^{\circ} \mathrm{C} & \text { none } & \text { insoluble } \\ \mathrm{Z} & \text { hard, white } & 800^{\circ} \mathrm{C} & \begin{array}{l}\text { only if melted/ } \\ \text { dissolved }\end{array} & \text { soluble }\end{array}$

Classify each substance in the table as either a metallic, ionic, molecular, or covalent network solid:

\begin{tabular}{|c|c|c|c|c|}
\hline Substance & Appearance & $\begin{array}{l}\text { Melting } \\
\text { Point }\end{array}$ & $\begin{array}{l}\text { Electrical } \\
\text { Conductivity }\end{array}$ & $\begin{array}{l}\text { Solubility } \\
\text { in Water }\end{array}$ \\
\hline $\mathrm{X}$ & $\begin{array}{l}\text { brittle, } \\
\text { white }\end{array}$ & $800^{\circ} \mathrm{C}$ & $\begin{array}{l}\text { only if melted/ } \\
\text { dissolved }\end{array}$ & soluble \\
\hline $\mathrm{Y}$ & $\begin{array}{l}\text { shiny, } \\
\text { malleable }\end{array}$ & $1100^{\circ} \mathrm{C}$ & high & insoluble \\
\hline Z & $\begin{array}{l}\text { hard, } \\
\text { colorless }\end{array}$ & $3550^{\circ} \mathrm{C}$ & none & insoluble \\
\hline
\end{tabular}

$\mathrm{X}=$ ionic $; \mathrm{Y}=$ metallic $; \mathrm{Z}=$ covalent network

Identify the following substances as ionic, metallic, covalent network, or molecular solids:

Substance A is malleable, ductile, conducts electricity well, and has a melting point of $1135^{\circ} \mathrm{C}$. Substance B is brittle, does not conduct electricity as a solid but does 
when molten, and has a melting point of $2072{ }^{\circ} \mathrm{C}$. Substance $C$ is very hard, does not conduct electricity, and has a melting point of $3440{ }^{\circ} \mathrm{C}$. Substance D is soft, does not conduct electricity, and has a melting point of $185^{\circ} \mathrm{C}$.

Substance A is shiny, conducts electricity well, and melts at $975^{\circ} \mathrm{C}$. Substance A is likely a(n):

(a) ionic solid

(b) metallic solid

(c) molecular solid

(d) covalent network solid

(b) metallic solid

Substance B is hard, does not conduct electricity, and melts at $1200^{\circ} \mathrm{C}$. Substance B is likely a(n):

(a) ionic solid

(b) metallic solid

(c) molecular solid

(d) covalent network solid

Glossary

amorphous solid

(also, noncrystalline solid) solid in which

the particles lack an ordered internal

structure

covalent network solid

solid whose particles are held together by

covalent bonds 


\section{crystalline solid}

solid in which the particles are arranged in a definite repeating pattern

interstitial sites

spaces between the regular particle positions in any array of atoms or ions ionic solid

solid composed of positive and negative ions held together by strong electrostatic attractions

metallic solid

solid composed of metal atoms molecular solid

solid composed of neutral molecules held together by intermolecular forces of attraction

vacancy

defect that occurs when a position that should contain an atom or ion is vacant 

PART IX

\section{SOLUTIONS AND COLLOIDS}



42.

INTRODUCTION

\section{Learning Objectives}

- The Dissolution Process

- Electrolytes

- Solubility

- Colloids

Coral reefs, such as this one at the Palmyra Atoll National Wildlife Refuge, are vital to the ecosystem of earth's oceans. The health of coral reefs and all marine life depends on the specific chemical composition of the complex mixture known as seawater. (credit: modification of work by "USFWS - Pacific Region"/Wikimedia Commons)

This figure shows an underwater photo of a colorful coral reef. 
Coral reefs are home to about $25 \%$ of all marine species. They are being threatened by climate change, oceanic acidification, and water pollution, all of which change the composition of the solution known as seawater. Dissolved oxygen in seawater is critical for sea creatures, but as the oceans warm, oxygen becomes less soluble. As the concentration of carbon dioxide in the atmosphere increases, the concentration of carbon dioxide in the oceans increases, contributing to oceanic acidification. Coral reefs are particularly sensitive to the acidification of the ocean, since the exoskeletons of the coral polyps are soluble in acidic solutions. Humans contribute to the changing of seawater composition by allowing agricultural runoff and other forms of pollution to affect our oceans.

Solutions are crucial to the processes that sustain life and to many other processes involving chemical reactions. This chapter considers the nature of solutions and examines factors that determine whether a solution will form and what properties it may have. The properties of colloids-mixtures containing dispersed particles larger than the molecules and ions of typical solutions-are also discussed. 


\section{3.}

\section{THE DISSOLUTION PROCESS}

Learning Objectives

By the end of this section, you will be able to:

- Describe the basic properties of solutions and how they form

- $\quad$ Predict whether a given mixture will yield a solution based on molecular properties of its components

- Explain why some solutions either produce or absorb heat when they form

An earlier chapter of this text introduced solutions, defined as homogeneous mixtures of two or more substances. Often, one component of a solution is present at a significantly greater concentration, in which case it is called the solvent. The other components of the solution 
present in relatively lesser concentrations are called solutes. Sugar is a covalent solid composed of sucrose molecules, $\mathrm{C}_{12} \mathrm{H}_{22} \mathrm{O}_{11}$. When this compound dissolves in water, its molecules become uniformly distributed among the molecules of water:

\section{$\mathrm{C}_{12} \mathrm{H}_{22} \mathrm{O}_{11}(s) \quad \mathrm{C}_{12} \mathrm{H}_{22} \mathrm{O}_{11}(a q)$}

The subscript " $a q$ " in the equation signifies that the sucrose molecules are solutes and are therefore individually dispersed throughout the aqueous solution (water is the solvent). Although sucrose molecules are heavier than water molecules, they remain dispersed throughout the solution; gravity does not cause them to "settle out" over time.

Potassium dichromate, $\mathrm{K}_{2} \mathrm{Cr}_{2} \mathrm{O}_{7}$, is an ionic compound composed of colorless potassium ions, $\mathrm{K}^{+}$, and orange dichromate ions, $\mathrm{Cr}_{2} \mathrm{O}_{7}{ }^{2}$. When a small amount of solid potassium dichromate is added to water, the compound dissolves and dissociates to yield potassium ions and dichromate ions uniformly distributed throughout the mixture ((Figure)), as indicated in this equation:

$\mathrm{K}_{2} \mathrm{Cr}_{2} \mathrm{O}_{7}(s) 2 \mathrm{~K}^{+}(a q)+\mathrm{Cr}_{2} \mathrm{O}_{7}^{2}(a q)$

As with the mixture of sugar and water, this mixture is also an aqueous solution. Its solutes, potassium and dichromate ions, remain individually dispersed among the solvent (water) molecules.

When potassium dichromate $\left(\mathrm{K}_{2} \mathrm{Cr}_{2} \mathrm{O}_{7}\right)$ is mixed with water, it forms a homogeneous orange solution. (credit: modification of work by Mark Ott) 
The first photo shows a small mound of an orange crystalline solid. There is a right-facing arrow. The second photo shows a translucent, colorless liquid in a clear container. There is a right-facing arrow. The third photo shows a translucent orange liquid in a clear, covered container.

Visit this virtual lab to view simulations of the dissolution of common covalent and ionic substances (sugar and salt) in water.

Water is used so often as a solvent that the word solution has come to imply an aqueous solution to many people. However, almost any gas, liquid, or solid can act as a solvent. Many alloys are solid solutions of one metal dissolved in another; for example, US five-cent coins contain nickel dissolved in copper. Air is a gaseous solution, a homogeneous mixture of nitrogen, oxygen, and several other gases. Oxygen (a gas), alcohol (a liquid), and sugar (a solid) all dissolve in water (a liquid) to form liquid solutions. (Figure) gives examples of several different solutions and the phases of the solutes and solvents. 


\section{Different Types of Solutions}

$\begin{array}{lll}\begin{array}{ll}\text { Solution } \\ \text { air }\end{array} & \text { Solute } & \text { Solvent } \\ \text { soft drinks }{ }^{1} & \mathrm{O}_{2}(g) & \mathrm{N}_{2}(g) \\ \text { hydrogen in palladium } & \mathrm{CO}_{2}(g) & \mathrm{H}_{2} \mathrm{O}(l) \\ \text { rubbing alcohol } & \mathrm{H}_{2}(g) & \mathrm{Pd}(s) \\ \text { saltwater } & \mathrm{H}_{2} \mathrm{O}(l) & \mathrm{C}_{3} \mathrm{H}_{8} \mathrm{O}(l) \text { (2-propanol) } \\ \text { brass } & \mathrm{NaCl}(s) & \mathrm{H}_{2} \mathrm{O}(l) \\ & \mathrm{Zn}(s) & \mathrm{Cu}(s)\end{array}$

Solutions exhibit these defining traits:

- They are homogeneous; after a solution is mixed, it has the same composition at all points throughout (its composition is uniform).

- The physical state of a solution-solid, liquid, or gas-is typically the same as that of the solvent, as demonstrated by the examples in (Figure).

- The components of a solution are dispersed on a molecular scale; they consist of a mixture of separated solute particles (molecules, atoms, and/or ions) each closely surrounded by solvent species.

- The dissolved solute in a solution will not settle out or separate from the solvent.

- The composition of a solution, or the concentrations of its components, can be varied continuously (within limits determined by the solubility of the components, discussed in detail later in this chapter). 
The Formation of Solutions

The formation of a solution is an example of a spontaneous process, a process that occurs under specified conditions without the requirement of energy from some external source. Sometimes a mixture is stirred to speed up the dissolution process, but this is not necessary; a homogeneous solution will form eventually. The topic of spontaneity is critically important to the study of chemical thermodynamics and is treated more thoroughly in a later chapter of this text. For purposes of this chapter's discussion, it will suffice to consider two criteria that favor, but do not guarantee, the spontaneous formation of a solution:

1. a decrease in the internal energy of the system (an exothermic change, as discussed in the previous chapter on thermochemistry)

2. an increased dispersal of matter in the system (which indicates an increase in the entropy of the system)

In the process of dissolution, an internal energy change often, but not always, occurs as heat is absorbed or evolved. An increase in matter dispersal always results when a solution forms from the uniform distribution of solute molecules throughout a solvent.

When the strengths of the intermolecular forces of attraction between solute and solvent species in a solution are no different than those present in the separated components, the solution is formed with no accompanying energy change. Such a solution is called an 
ideal solution. A mixture of ideal gases (or gases such as helium and argon, which closely approach ideal behavior) is an example of an ideal solution, since the entities comprising these gases experience no significant intermolecular attractions.

When containers of helium and argon are connected, the gases spontaneously mix due to diffusion and form a solution ((Figure)). The formation of this solution clearly involves an increase in matter dispersal, since the helium and argon atoms occupy a volume twice as large as that which each occupied before mixing.

Samples of helium and argon spontaneously mix to give a solution.

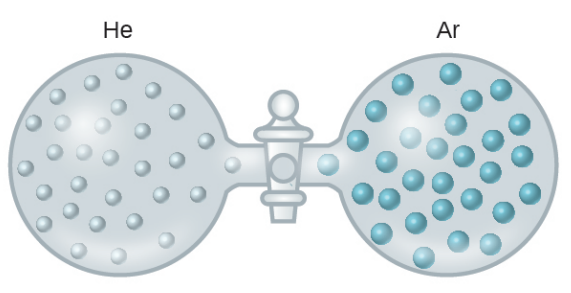

Valve closed

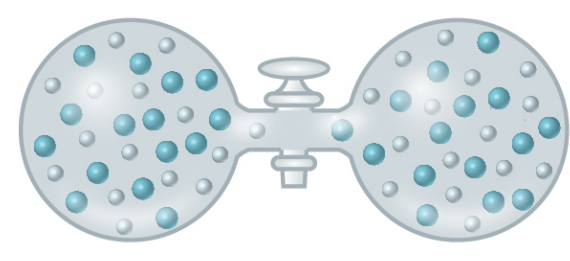

Valve open

Ideal solutions may also form when structurally similar liquids are mixed. For example, mixtures of the alcohols methanol $\left(\mathrm{CH}_{3} \mathrm{OH}\right)$ and ethanol $\left(\mathrm{C}_{2} \mathrm{H}_{5} \mathrm{OH}\right)$ form ideal solutions, as do mixtures of the hydrocarbons pentane, $\mathrm{C}_{5} \mathrm{H}_{12}$, and hexane, $\mathrm{C}_{6} \mathrm{H}_{14}$. Placing methanol and ethanol, or pentane and hexane, in the bulbs shown in (Figure) will result in the same diffusion and subsequent mixing of these liquids as is observed for the He and Ar gases (although at a much slower rate), yielding solutions with no significant change in energy. Unlike a mixture of gases, however, the components of these liquid-liquid solutions do, indeed, experience intermolecular attractive forces. 
But since the molecules of the two substances being mixed are structurally very similar, the intermolecular attractive forces between like and unlike molecules are essentially the same, and the dissolution process, therefore, does not entail any appreciable increase or decrease in energy. These examples illustrate how increased matter dispersal alone can provide the driving force required to cause the spontaneous formation of a solution. In some cases, however, the relative magnitudes of intermolecular forces of attraction between solute and solvent species may prevent dissolution.

Three types of intermolecular attractive forces are relevant to the dissolution process: solute-solute, solventsolvent, and solute-solvent. As illustrated in (Figure), the formation of a solution may be viewed as a stepwise process in which energy is consumed to overcome solutesolute and solvent-solvent attractions (endothermic processes) and released when solute-solvent attractions are established (an exothermic process referred to as solvation). The relative magnitudes of the energy changes associated with these stepwise processes determine whether the dissolution process overall will release or absorb energy. In some cases, solutions do not form because the energy required to separate solute and solvent species is so much greater than the energy released by solvation.

This schematic representation of dissolution shows a stepwise process involving the endothermic separation of solute and solvent species (Steps 1 and 2) and exothermic solvation (Step 3). 


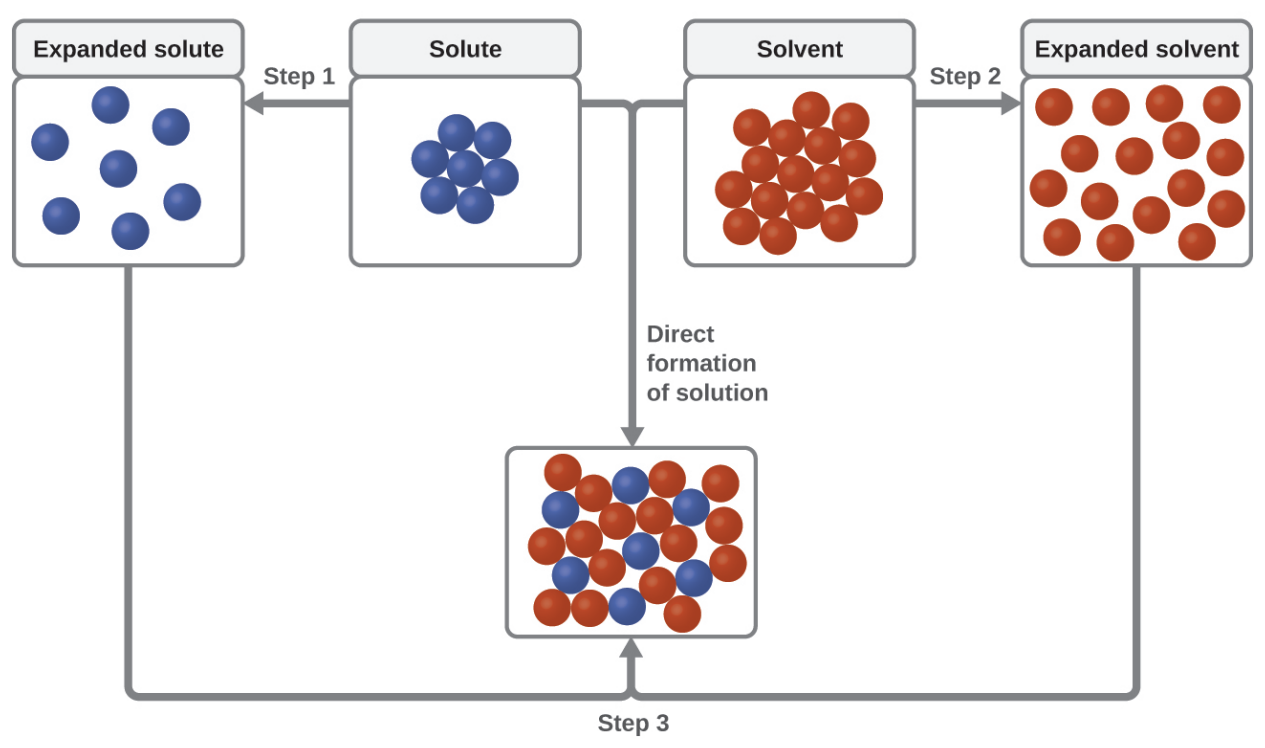

Consider the example of an ionic compound dissolving in water. Formation of the solution requires the electrostatic forces between the cations and anions of the compound (solute-solute) be overcome completely as attractive forces are established between these ions and water molecules (solute-solvent). Hydrogen bonding between a relatively small fraction of the water molecules must also be overcome to accommodate any dissolved solute. If the solute's electrostatic forces are significantly greater than the solvation forces, the dissolution process is significantly endothermic and the compound may not dissolve to an appreciable extent. Calcium carbonate, the major component of coral reefs, is one example of such an "insoluble" ionic compound (see (Figure)). On the other hand, if the solvation forces are much stronger than the compound's electrostatic forces, the dissolution is significantly exothermic and the compound may be highly soluble. A common example of this type of ionic 
compound is sodium chloride, commonly known as table salt.

As noted at the beginning of this module, spontaneous solution formation is favored, but not guaranteed, by exothermic dissolution processes. While many soluble compounds do, indeed, dissolve with the release of heat, some dissolve endothermically. Ammonium nitrate $\left(\mathrm{NH}_{4} \mathrm{NO}_{3}\right)$ is one such example and is used to make instant cold packs for treating injuries like the one pictured in (Figure). A thin-walled plastic bag of water is sealed inside a larger bag with solid $\mathrm{NH}_{4} \mathrm{NO}_{3}$. When the smaller bag is broken, a solution of $\mathrm{NH}_{4} \mathrm{NO}_{3}$ forms, absorbing heat from the surroundings (the injured area to which the pack is applied) and providing a cold compress that decreases swelling. Endothermic dissolutions such as this one require a greater energy input to separate the solute species than is recovered when the solutes are solvated, but they are spontaneous nonetheless due to the increase in disorder that accompanies formation of the solution. An instant cold pack gets cold when certain salts, such as ammonium nitrate, dissolve in water-an endothermic process. 


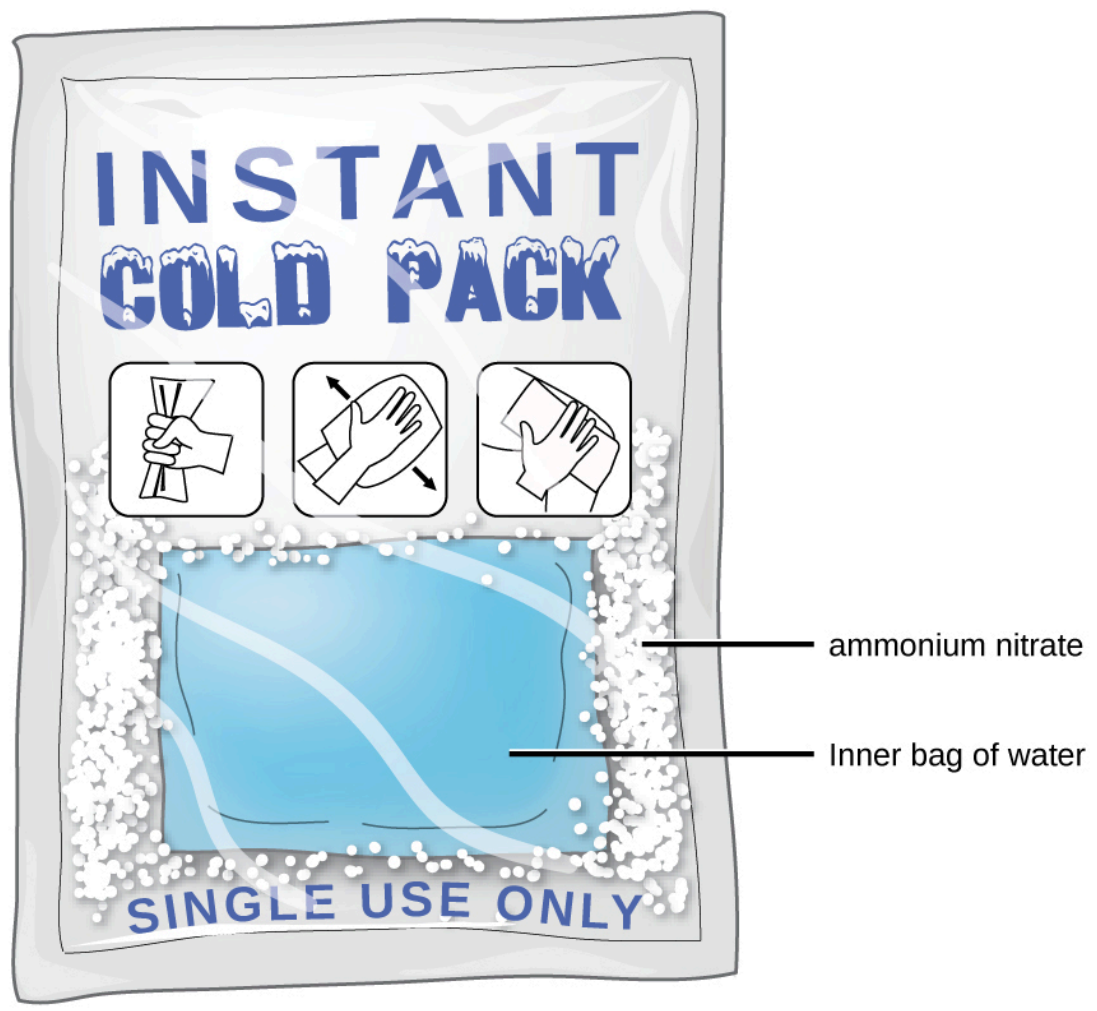

Watch this brief video illustrating endothermic and exothermic dissolution processes.

Key Concepts and Summary

A solution forms when two or more substances combine physically to yield a mixture that is homogeneous at the molecular level. The solvent is the most concentrated component and determines the physical state of the solution. The solutes are the other components typically present at concentrations less than that of the solvent. Solutions may form endothermically or exothermically, depending upon the relative magnitudes of solute and solvent intermolecular attractive forces. Ideal solutions form with no appreciable change in energy. 
Chemistry End of Chapter Exercises

How do solutions differ from compounds? From other mixtures?

A solution can vary in composition, while a compound cannot vary in composition. Solutions are homogeneous at the molecular level, while other mixtures are heterogeneous.

Which of the principal characteristics of solutions are evident in the solutions of $\mathrm{K}_{2} \mathrm{Cr}_{2} \mathrm{O}_{7}$ shown in (Figure)?

When $\mathrm{KNO}_{3}$ is dissolved in water, the resulting solution is significantly colder than the water was originally.

(a) Is the dissolution of $\mathrm{KNO}_{3}$ an endothermic or an exothermic process?

(b) What conclusions can you draw about the intermolecular attractions involved in the process?

(c) Is the resulting solution an ideal solution?

(a) The process is endothermic as the solution is consuming heat. (b) Attraction between the $\mathrm{K}^{+}$and $\mathrm{NO}_{3}$ ions is stronger than between the ions and water molecules (the ion-ion interactions have a lower, more negative energy). Therefore, the dissolution process increases the energy of the molecular interactions, and it consumes the thermal energy of the solution to make up for the difference. (c) No, an ideal solution is formed with no appreciable heat release or consumption.

Give an example of each of the following types of solutions: 
(a) a gas in a liquid

(b) a gas in a gas

(c) a solid in a solid

Indicate the most important types of intermolecular attractions in each of the following solutions:

(a) The solution in (Figure).

(b) $\mathrm{NO}(l)$ in $\mathrm{CO}(l)$

(c) $\mathrm{Cl}_{2}(g)$ in $\mathrm{Br}_{2}(l)$

(d) $\mathrm{HCl}(g)$ in benzene $\mathrm{C}_{6} \mathrm{H}_{6}(l)$

(e) Methanol $\mathrm{CH}_{3} \mathrm{OH}(l)$ in $\mathrm{H}_{2} \mathrm{O}(l)$

(a) ion-dipole forces; (b) dipole-dipole forces; (c) dispersion forces; (d) dispersion forces; (e) hydrogen bonding

Predict whether each of the following substances would be more soluble in water (polar solvent) or in a hydrocarbon such as heptane $\left(\mathrm{C}_{7} \mathrm{H}_{16}\right.$, nonpolar solvent):

(a) vegetable oil (nonpolar)

(b) isopropyl alcohol (polar)

(c) potassium bromide (ionic)

Heat is released when some solutions form; heat is absorbed when other solutions form. Provide a molecular explanation for the difference between these two types of spontaneous processes.

Heat is released when the total intermolecular forces (IMFs) between the solute and solvent molecules are stronger than the total IMFs in the pure solute and in the pure solvent: Breaking weaker IMFs and forming stronger IMFs releases heat. Heat is absorbed when the total IMFs 
in the solution are weaker than the total of those in the pure solute and in the pure solvent: Breaking stronger IMFs and forming weaker IMFs absorbs heat.

Solutions of hydrogen in palladium may be formed by exposing $\mathrm{Pd}$ metal to $\mathrm{H}_{2}$ gas. The concentration of hydrogen in the palladium depends on the pressure of $\mathrm{H}_{2}$ gas applied, but in a more complex fashion than can be described by Henry's law. Under certain conditions, $0.94 \mathrm{~g}$ of hydrogen gas is dissolved in $215 \mathrm{~g}$ of palladium metal (solution density $=10.8 \mathrm{~g} \mathrm{~cm}^{3}$ ).

(a) Determine the molarity of this solution.

(b) Determine the molality of this solution.

(c) Determine the percent by mass of hydrogen atoms in this solution.

Footnotes

- 1If bubbles of gas are observed within the liquid, the mixture is not homogeneous and, thus, not a solution.

Glossary

alloy

solid mixture of a metallic element and one or more additional elements

ideal solution

solution that forms with no accompanying 


\section{energy change \\ solvation}

exothermic process in which

intermolecular attractive forces between the solute and solvent in a solution are established

spontaneous process

physical or chemical change that occurs without the addition of energy from an

external source 


\section{4.}

\section{ELECTROLYTES}

Learning Objectives

By the end of this module, you will be able to:

- Define and give examples of electrolytes

- Distinguish between the physical and chemical changes that accompany dissolution of ionic and covalent electrolytes

- $\quad$ Relate electrolyte strength to solute-solvent attractive forces

When some substances are dissolved in water, they undergo either a physical or a chemical change that yields ions in solution. These substances constitute an important class of compounds called electrolytes. Substances that do not yield ions when dissolved are called nonelectrolytes. If the physical or chemical process that generates the ions is essentially $100 \%$ efficient (all of the dissolved compound yields ions), then the substance 
is known as a strong electrolyte. If only a relatively small fraction of the dissolved substance undergoes the ionproducing process, it is called a weak electrolyte.

Substances may be identified as strong, weak, or nonelectrolytes by measuring the electrical conductance of an aqueous solution containing the substance. To conduct electricity, a substance must contain freely mobile, charged species. Most familiar is the conduction of electricity through metallic wires, in which case the mobile, charged entities are electrons. Solutions may also conduct electricity if they contain dissolved ions, with conductivity increasing as ion concentration increases. Applying a voltage to electrodes immersed in a solution permits assessment of the relative concentration of dissolved ions, either quantitatively, by measuring the electrical current flow, or qualitatively, by observing the brightness of a light bulb included in the circuit ((Figure)). Solutions of nonelectrolytes such as ethanol do not contain dissolved ions and cannot conduct electricity. Solutions of electrolytes contain ions that permit the passage of electricity. The conductivity of an electrolyte solution is related to the strength of the electrolyte.
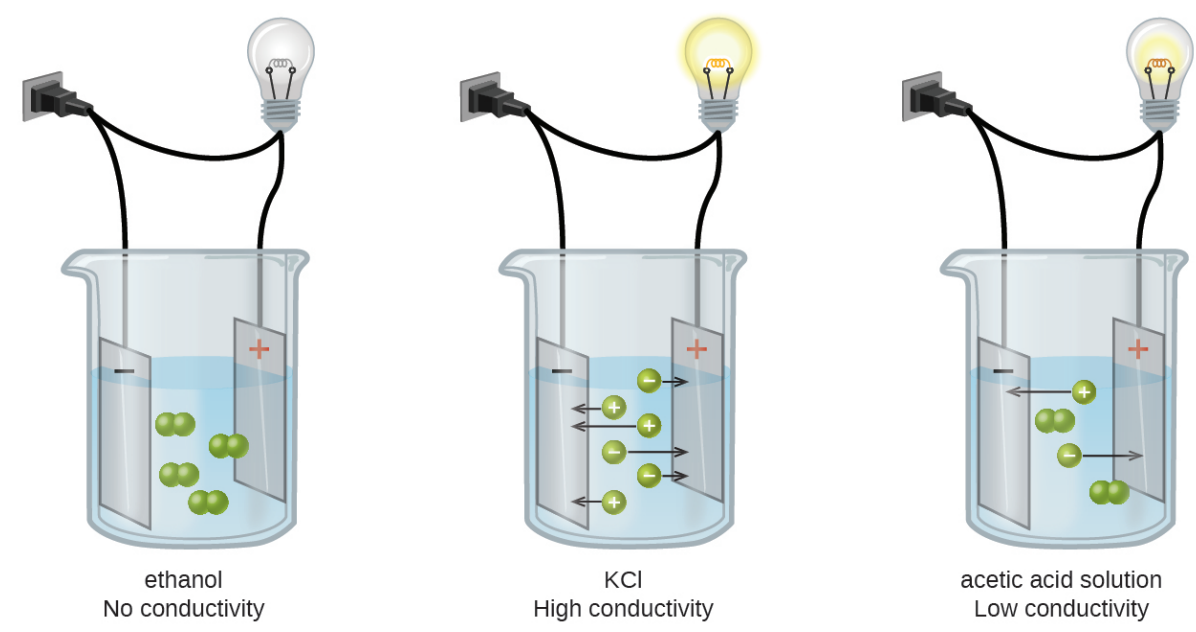
Ionic Electrolytes

Water and other polar molecules are attracted to ions, as shown in (Figure). The electrostatic attraction between an ion and a molecule with a dipole is called an ion-dipole attraction. These attractions play an important role in the dissolution of ionic compounds in water. As potassium chloride $(\mathrm{KCl})$ dissolves in water, the ions are hydrated. The polar water molecules are attracted by the charges on the $\mathrm{K}^{+}$and $\mathrm{Cl}^{-}$ions. Water molecules in front of and behind the ions are not shown.

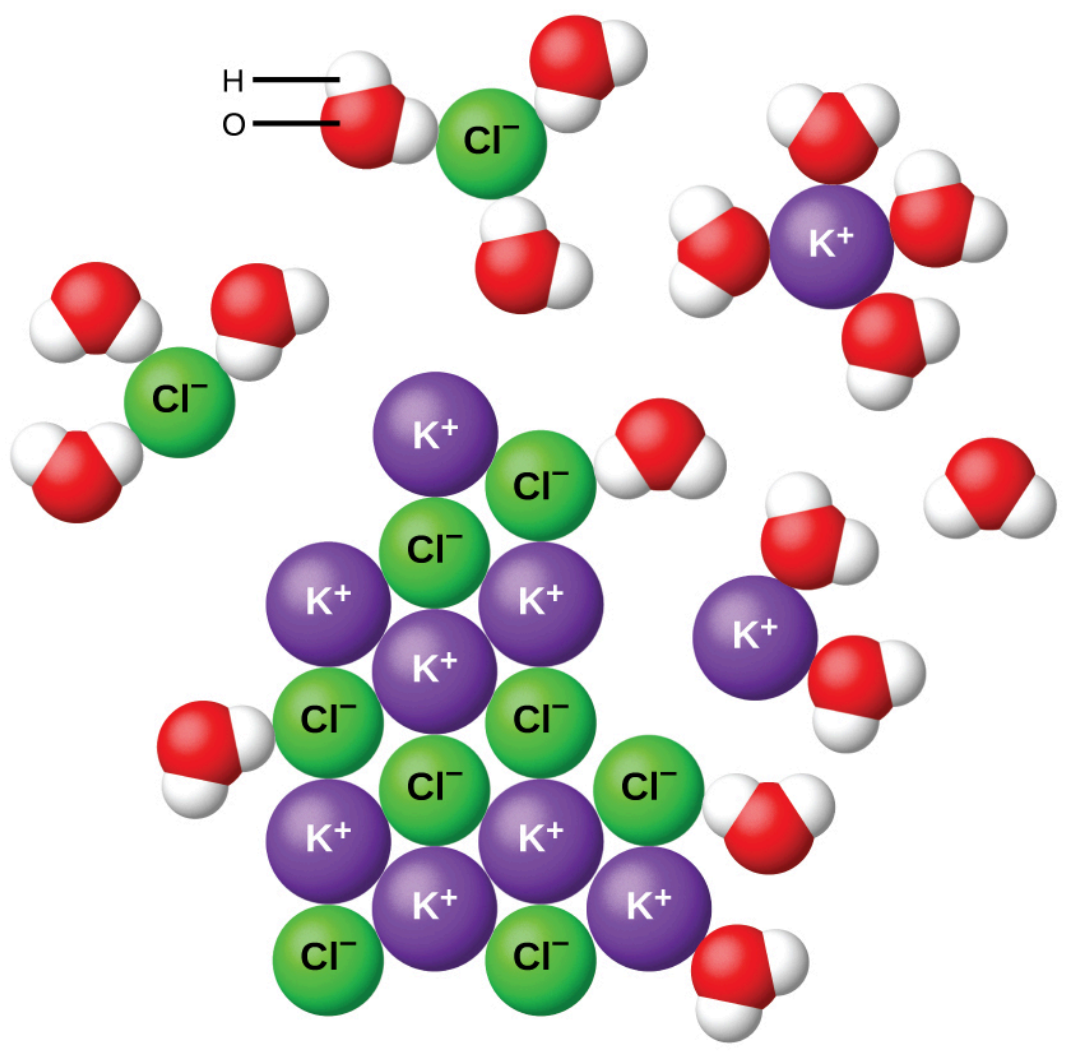

When ionic compounds dissolve in water, the ions in the solid separate and disperse uniformly throughout the solution because water molecules surround and solvate 
the ions, reducing the strong electrostatic forces between them. This process represents a physical change known as dissociation. Under most conditions, ionic compounds will dissociate nearly completely when dissolved, and so they are classified as strong electrolytes. Even sparingly, soluble ionic compounds are strong electrolytes, since the small amount that does dissolve will dissociate completely.

Consider what happens at the microscopic level when solid $\mathrm{KCl}$ is added to water. Ion-dipole forces attract the positive (hydrogen) end of the polar water molecules to the negative chloride ions at the surface of the solid, and they attract the negative (oxygen) ends to the positive potassium ions. The water molecules surround individual $\mathrm{K}^{+}$and $\mathrm{Cl}^{-}$ions, reducing the strong interionic forces that bind the ions together and letting them move off into solution as solvated ions, as (Figure) shows. Overcoming the electrostatic attraction permits the independent motion of each hydrated ion in a dilute solution as the ions transition from fixed positions in the undissolved compound to widely dispersed, solvated ions in solution.

\section{Covalent Electrolytes}

Pure water is an extremely poor conductor of electricity because it is only very slightly ionized-only about two out of every 1 billion molecules ionize at $25{ }^{\circ} \mathrm{C}$. Water ionizes when one molecule of water gives up a proton $\left(\mathrm{H}^{+}\right.$ ion) to another molecule of water, yielding hydronium and hydroxide ions.

$$
\mathrm{H}_{2} \mathrm{O}(l)+\mathrm{H}_{2} \mathrm{O}(l) \mathrm{H}_{3} \mathrm{O}^{+}(a q)+\mathrm{OH}(a q)
$$


In some cases, solutions prepared from covalent compounds conduct electricity because the solute molecules react chemically with the solvent to produce ions. For example, pure hydrogen chloride is a gas consisting of covalent $\mathrm{HCl}$ molecules. This gas contains no ions. However, an aqueous solution of $\mathrm{HCl}$ is a very good conductor, indicating that an appreciable concentration of ions exists within the solution.

Because $\mathrm{HCl}$ is an acid, its molecules react with water, transferring $\mathrm{H}^{+}$ions to form hydronium ions $\left(\mathrm{H}_{3} \mathrm{O}^{+}\right)$and chloride ions $\left(\mathrm{Cl}^{-}\right)$:<smiles>[C-]#[C+]CC(C)OC(C)CC</smiles>

This reaction is essentially $100 \%$ complete for $\mathrm{HCl}$ (i.e., it is a strong acid and, consequently, a strong electrolyte). Likewise, weak acids and bases that only react partially generate relatively low concentrations of ions when dissolved in water and are classified as weak electrolytes. The reader may wish to review the discussion of strong and weak acids provided in the earlier chapter of this text on reaction classes and stoichiometry.

Key Concepts and Summary

Substances that dissolve in water to yield ions are called electrolytes. Electrolytes may be covalent compounds that chemically react with water to produce ions (for example, acids and bases), or they may be ionic compounds that dissociate to yield their constituent cations and anions, when dissolved. Dissolution of an ionic compound is facilitated by ion-dipole attractions between the ions of the compound and the polar water molecules. Soluble 
ionic substances and strong acids ionize completely and are strong electrolytes, while weak acids and bases ionize to only a small extent and are weak electrolytes. Nonelectrolytes are substances that do not produce ions when dissolved in water.

Chemistry End of Chapter Exercises

Explain why the ions $\mathrm{Na}^{+}$and $\mathrm{Cl}^{-}$are strongly solvated in water but not in hexane, a solvent composed of nonpolar molecules.

Crystals of $\mathrm{NaCl}$ dissolve in water, a polar liquid with a very large dipole moment, and the individual ions become strongly solvated. Hexane is a nonpolar liquid with a dipole moment of zero and, therefore, does not significantly interact with the ions of the $\mathrm{NaCl}$ crystals.

Explain why solutions of $\mathrm{HBr}$ in benzene (a nonpolar solvent) are nonconductive, while solutions in water (a polar solvent) are conductive.

Consider the solutions presented:

(a) Which of the following sketches best represents the ions in a solution of $\mathrm{Fe}\left(\mathrm{NO}_{3}\right)_{3}(a q)$ ? 
In this figure, three beakers labeled $\mathrm{x}, \mathrm{y}$, and $\mathrm{z}$ are shown containing various arrangements of blue and red spheres suspended in solution. In beaker $\mathrm{x}$, three small red spheres surround a single central blue sphere in small clusters which in turn are grouped in threes around a single red sphere, forming four larger clusters. In beaker y, the four large clusters are present without the central red spheres. Four individual red spheres are now present. In beaker $\mathrm{z}$, the large clusters are not present. Twelve of the small clusters of three red and one blue sphere are present along with four single red spheres.

(b) Write a balanced chemical equation showing the products of the dissolution of $\mathrm{Fe}\left(\mathrm{NO}_{3}\right)_{3}$.

(a) $\mathrm{Fe}\left(\mathrm{NO}_{3}\right)_{3}$ is a strong electrolyte, thus it should completely dissociate into $\mathrm{Fe}^{3+}$ and $\mathrm{NO}_{3}$ ions. Therefore, (z) best represents the solution. $\mathrm{Fe}\left(\mathrm{NO}_{3}\right)_{3}(s) \mathrm{Fe}^{3+}(a q)+3 \mathrm{NO}_{3}(a q)$

Compare the processes that occur when methanol $\left(\mathrm{CH}_{3} \mathrm{OH}\right)$, hydrogen chloride $(\mathrm{HCl})$, and sodium hydroxide $(\mathrm{NaOH})$ dissolve in water. Write equations and prepare sketches showing the form in which each of these compounds is present in its respective solution.

What is the expected electrical conductivity of the following solutions?
(a) $\mathrm{NaOH}(a q)$
(b) $\mathrm{HCl}(a q)$
(c) $\mathrm{C}_{6} \mathrm{H}_{12} \mathrm{O}_{6}(a q)$ (glucose)
(d) $\mathrm{NH}_{3}(a q)$

(a) high conductivity (solute is an ionic compound that 
will dissociate when dissolved); (b) high conductivity (solute is a strong acid and will ionize completely when dissolved); (c) nonconductive (solute is a covalent compound, neither acid nor base, unreactive towards water); (d) low conductivity (solute is a weak base and will partially ionize when dissolved)

Why are most solid ionic compounds electrically nonconductive, whereas aqueous solutions of ionic compounds are good conductors? Would you expect a liquid (molten) ionic compound to be electrically conductive or nonconductive? Explain.

Indicate the most important type of intermolecular attraction responsible for solvation in each of the following solutions:

(a) the solutions in (Figure)

(b) methanol, $\mathrm{CH}_{3} \mathrm{OH}$, dissolved in ethanol, $\mathrm{C}_{2} \mathrm{H}_{5} \mathrm{OH}$

(c) methane, $\mathrm{CH}_{4}$, dissolved in benzene, $\mathrm{C}_{6} \mathrm{H}_{6}$

(d) the polar halocarbon $\mathrm{CF}_{2} \mathrm{Cl}_{2}$ dissolved in the polar halocarbon $\mathrm{CF}_{2} \mathrm{ClCFCl}_{2}$

(e) $\mathrm{O}_{2}(l)$ in $\mathrm{N}_{2}(l)$

(a) ion-dipole; (b) hydrogen bonds; (c) dispersion forces;

(d) dipole-dipole attractions; (e) dispersion forces 
Glossary

dissociation

physical process accompanying the dissolution of an ionic compound in which the compound's constituent ions are solvated and dispersed throughout the solution electrolyte substance that produces ions when dissolved in water ion-dipole attraction electrostatic attraction between an ion and a polar molecule nonelectrolyte substance that does not produce ions when dissolved in water strong electrolyte

substance that dissociates or ionizes completely when dissolved in water weak electrolyte substance that ionizes only partially when dissolved in water 


\section{5.}

\section{SOLUBILITY}

Learning Objectives

By the end of this module, you will be able to:

- Describe the effects of temperature and pressure on solubility

- State Henry's law and use it in calculations involving the solubility of a gas in a liquid

- Explain the degrees of solubility possible for liquid-liquid solutions

Imagine adding a small amount of sugar to a glass of water, stirring until all the sugar has dissolved, and then adding a bit more. You can repeat this process until the sugar concentration of the solution reaches its natural limit, a limit determined primarily by the relative strengths of the solute-solute, solute-solvent, and solvent-solvent attractive forces discussed in the previous two modules of this chapter. You can be certain that you 
have reached this limit because, no matter how long you stir the solution, undissolved sugar remains. The concentration of sugar in the solution at this point is known as its solubility.

The solubility of a solute in a particular solvent is the maximum concentration that may be achieved under given conditions when the dissolution process is at equilibrium.

When a solute's concentration is equal to its solubility, the solution is said to be saturated with that solute. If the solute's concentration is less than its solubility, the solution is said to be unsaturated. A solution that contains a relatively low concentration of solute is called dilute, and one with a relatively high concentration is called concentrated.

Use this interactive simulation to prepare various saturated solutions.

Solutions may be prepared in which a solute concentration exceeds its solubility. Such solutions are said to be supersaturated, and they are interesting examples of nonequilibrium states (a detailed treatment of this important concept is provided in the text chapters on equilibrium). For example, the carbonated beverage in an open container that has not yet "gone flat" is supersaturated with carbon dioxide gas; given time, the $\mathrm{CO}_{2}$ concentration will decrease until it reaches its solubility.

Watch this impressive video showing the precipitation of sodium acetate from a supersaturated solution. 
Solutions of Gases in Liquids

As for any solution, the solubility of a gas in a liquid is affected by the intermolecular attractive forces between solute and solvent species. Unlike solid and liquid solutes, however, there is no solute-solute intermolecular attraction to overcome when a gaseous solute dissolves in a liquid solvent (see (Figure)) since the atoms or molecules comprising a gas are far separated and experience negligible interactions. Consequently, solutesolvent interactions are the sole energetic factor affecting solubility. For example, the water solubility of oxygen is approximately three times greater than that of helium (there are greater dispersion forces between water and the larger oxygen molecules) but 100 times less than the solubility of chloromethane, $\mathrm{CHCl}_{3}$ (polar chloromethane molecules experience dipole-dipole attraction to polar water molecules). Likewise note the solubility of oxygen in hexane, $\mathrm{C}_{6} \mathrm{H}_{14}$, is approximately 20 times greater than it is in water because greater dispersion forces exist between oxygen and the larger hexane molecules.

Temperature is another factor affecting solubility, with gas solubility typically decreasing as temperature increases ((Figure)). This inverse relation between temperature and dissolved gas concentration is responsible for one of the major impacts of thermal pollution in natural waters.

The solubilities of these gases in water decrease as the temperature increases. All solubilities were measured with a constant pressure of $101.3 \mathrm{kPa}$ (1 atm) of gas above the solutions. 


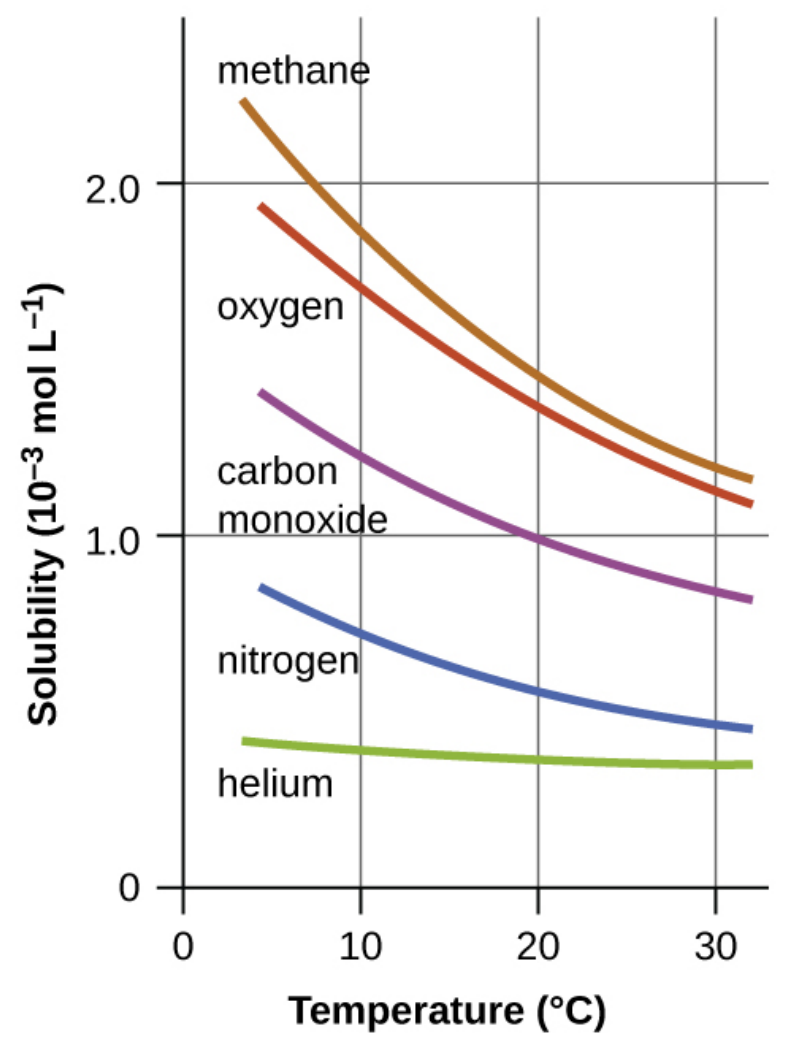

When the temperature of a river, lake, or stream is raised, the solubility of oxygen in the water is decreased. Decreased levels of dissolved oxygen may have serious consequences for the health of the water's ecosystems and, in severe cases, can result in large-scale fish kills ((Figure)).

(a) The small bubbles of air in this glass of chilled water formed when the water warmed to room temperature and the solubility of its dissolved air decreased. (b) The decreased solubility of oxygen in natural waters subjected to thermal pollution can result in large-scale fish kills. (credit a: modification of work by Liz West; credit b: modification of work by U.S. Fish and Wildlife Service) 


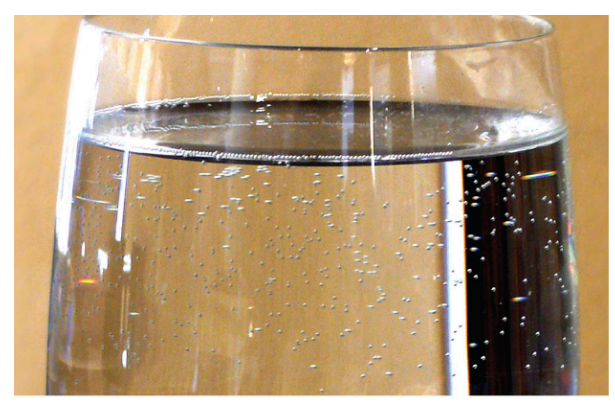

(a)

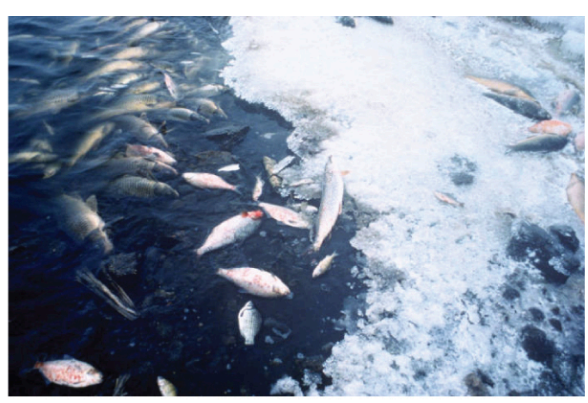

(b)

The solubility of a gaseous solute is also affected by the partial pressure of solute in the gas to which the solution is exposed. Gas solubility increases as the pressure of the gas increases. Carbonated beverages provide a nice illustration of this relationship. The carbonation process involves exposing the beverage to a relatively high pressure of carbon dioxide gas and then sealing the beverage container, thus saturating the beverage with $\mathrm{CO}_{2}$ at this pressure. When the beverage container is opened, a familiar hiss is heard as the carbon dioxide gas pressure is released, and some of the dissolved carbon dioxide is typically seen leaving solution in the form of small bubbles ((Figure)). At this point, the beverage is supersaturated with carbon dioxide and, with time, the dissolved carbon dioxide concentration will decrease to its equilibrium value and the beverage will become "flat." Opening the bottle of carbonated beverage reduces the pressure of the gaseous carbon dioxide above the beverage. The solubility of $\mathrm{CO}_{2}$ is thus lowered, and some dissolved carbon dioxide may be seen leaving the solution as small gas bubbles. (credit: modification of work by Derrick Coetzee) 


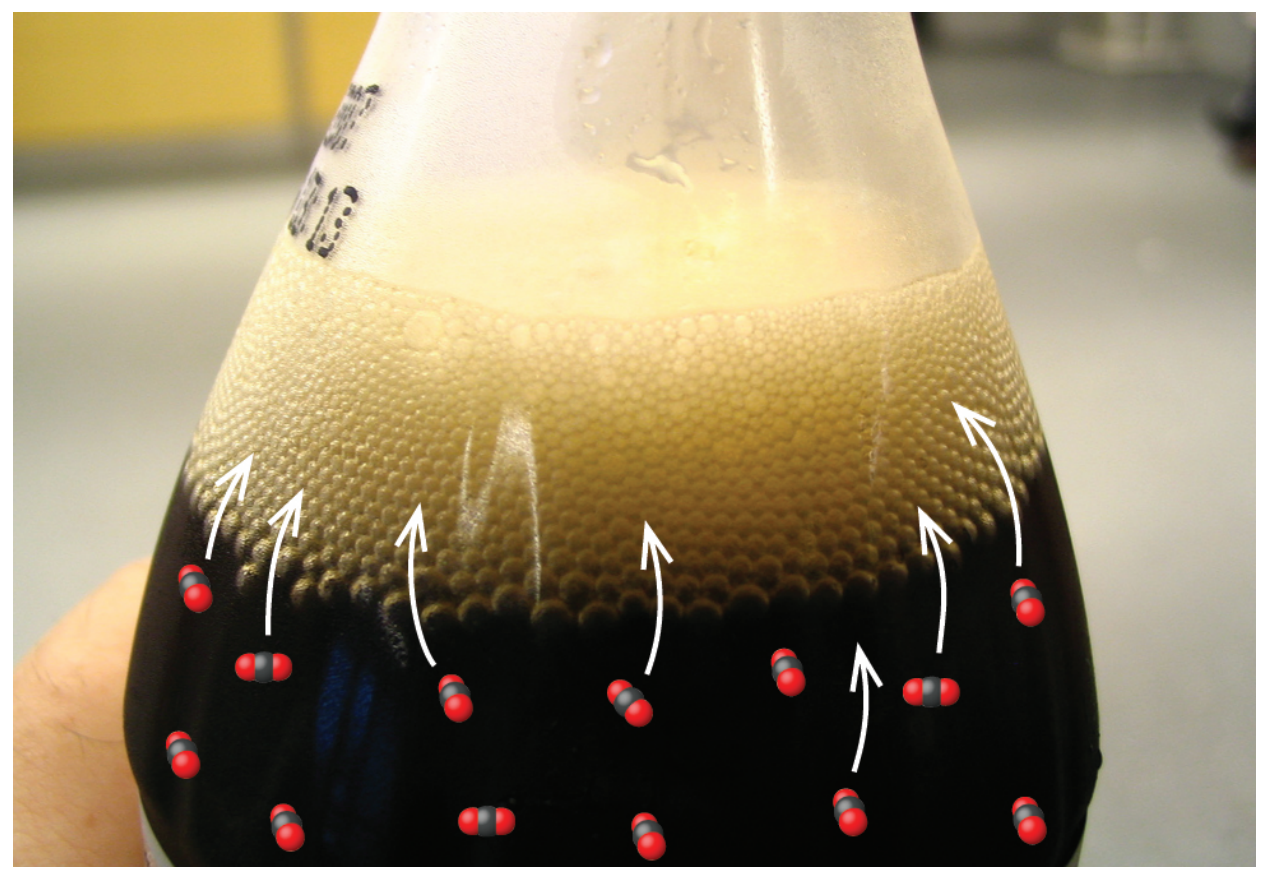

For many gaseous solutes, the relation between solubility, $C_{\mathrm{g}}$, and partial pressure, $P_{\mathrm{g}}$, is a proportional one:

$C_{\mathrm{g}}=k P_{\mathrm{g}}$

where $k$ is a proportionality constant that depends on the identities of the gaseous solute and solvent, and on the solution temperature. This is a mathematical statement of Henry's law: The quantity of an ideal gas that dissolves in a definite volume of liquid is directly proportional to the pressure of the gas.

Application of Henry's Law At $20^{\circ} \mathrm{C}$, the concentration of dissolved oxygen in water exposed to gaseous oxygen at a partial pressure of $101.3 \mathrm{kPa}$ is $1.3810^{-3} \mathrm{~mol} \mathrm{~L}^{-1}$. Use Henry's law to determine the solubility of oxygen when its partial pressure is $20.7 \mathrm{kPa}$, the approximate pressure of oxygen in earth's atmosphere.

Solution According to Henry's law, for an ideal solution the 
solubility, $C_{g}$, of a gas (1.38 $10^{-3} \mathrm{~mol} \mathrm{~L}^{-1}$, in this case) is directly proportional to the pressure, $\mathrm{Pg}_{\mathrm{g}}$ of the undissolved gas above the solution (101.3 kPa in this case). Because both $C_{g}$ and $P_{g}$ are known, this relation can be rearragned and used to solve for $k$.

$$
\begin{aligned}
C_{\mathrm{g}} & =k P_{\mathrm{g}} \\
k & =\frac{C_{\mathrm{g}}}{P_{\mathrm{g}}} \\
& =\frac{1.3810^{-3} \mathrm{~mol} \mathrm{~L}-1}{101.3 \mathrm{kPa}} \\
& =1.3610^{-5} \mathrm{~mol} \mathrm{~L}^{-1} \mathrm{kPa}^{-1}
\end{aligned}
$$

Now, use $k$ to find the solubility at the lower pressure.

$$
\begin{aligned}
C_{\mathrm{g}}= & k P_{\mathrm{g}} \\
& 1.3610^{-5} \mathrm{~mol} \mathrm{~L}{ }^{-1} \mathrm{kPa}^{-1} 20.7 \mathrm{kPa} \\
= & 2.8210^{-4} \mathrm{~mol} \mathrm{~L}^{-1}
\end{aligned}
$$

Note that various units may be used to express the quantities involved in these sorts of computations. Any combination of units that yield to the constraints of dimensional analysis are acceptable.

Check Your Learning Exposing a $100.0 \mathrm{~mL}$ sample of water at $0{ }^{\circ} \mathrm{C}$ to an atmosphere containing a gaseous solute at 152 torr resulted in the dissolution of $1.4510^{-3} \mathrm{~g}$ of the solute. Use Henry's law to determine the solubility of this gaseous solute when its pressure is 760 torr.

Answer:

$7.2510^{-3}$ in $100.0 \mathrm{~mL}$ or $0.0725 \mathrm{~g} / \mathrm{L}$

Thermal Pollution and Oxygen Solubility A certain species of freshwater trout requires a dissolved oxygen concentration of $7.5 \mathrm{mg} / \mathrm{L}$. Could these fish thrive in a thermally polluted mountain stream (water temperature is $30.0^{\circ} \mathrm{C}$, partial pressure of atmospheric oxygen is $0.17 \mathrm{~atm})$ ?

Solution First, compute the Henry's law constant for oxygen in water at the specified temperature of $30.0^{\circ} \mathrm{C}$ (see (Figure)). 
$k=\frac{C_{\mathrm{g}}}{P_{\mathrm{g}}}=1.1510^{-3} \mathrm{~mol} / \mathrm{L} / 1.00 \mathrm{~atm}=1.1510^{-3} \mathrm{~mol} / \mathrm{L} \mathrm{atm}$

Then, use this $k$ value to compute the oxygen solubility at the specified oxygen partial pressure, $0.17 \mathrm{~atm}$.

$$
C_{g}=k P_{g}=\left(1.1510^{-3} \mathrm{~mol} / \mathrm{L} \mathrm{atm}\right)(0.17 \mathrm{~atm})=1.9510^{-4} \mathrm{~mol} / \mathrm{L}
$$

Finally, convert this dissolved oxygen concentration from $\mathrm{mol} / \mathrm{L}$ to $\mathrm{mg} / \mathrm{L}$.

$$
\left(1.9510^{-4} \mathrm{~mol} / \mathrm{L}\right)(32.0 \mathrm{~g} / 1 \mathrm{~mol})(1000 \mathrm{mg} / \mathrm{g})=6.2 \mathrm{mg} / \mathrm{L} .
$$

This concentration is lesser than the required minimum value of $7.5 \mathrm{mg} / \mathrm{L}$, and so these trout would likely not thrive in the polluted stream.

Check Your Learning What dissolved oxygen concentration is expected for the stream above when it returns to a normal summer time temperature of $15^{\circ} \mathrm{C}$ ?

Answer:

$8.2 \mathrm{mg} / \mathrm{L}$

Decompression Sickness or “The Bends”

Decompression sickness (DCS), or "the bends," is an effect of the increased pressure of the air inhaled by scuba divers when swimming underwater at considerable depths. In addition to the pressure exerted by the atmosphere, divers are subjected to additional pressure due to the water above them, experiencing an increase of approximately 1 atm for each $10 \mathrm{~m}$ of depth. Therefore, the air inhaled by a diver while submerged contains gases at the corresponding higher ambient pressure, and the concentrations of the gases dissolved in the diver's blood are proportionally higher per Henry's law.

As the diver ascends to the surface of the water, the ambient pressure decreases and the dissolved gases 
becomes less soluble. If the ascent is too rapid, the gases escaping from the diver's blood may form bubbles that can cause a variety of symptoms ranging from rashes and joint pain to paralysis and death. To avoid DCS, divers must ascend from depths at relatively slow speeds (10 or 20 $\mathrm{m} / \mathrm{min}$ ) or otherwise make several decompression stops, pausing for several minutes at given depths during the ascent. When these preventive measures are unsuccessful, divers with DCS are often provided hyperbaric oxygen therapy in pressurized vessels called decompression (or recompression) chambers ((Figure)).

(a) US Navy divers undergo training in a recompression chamber. (b) Divers receive hyperbaric oxygen therapy.

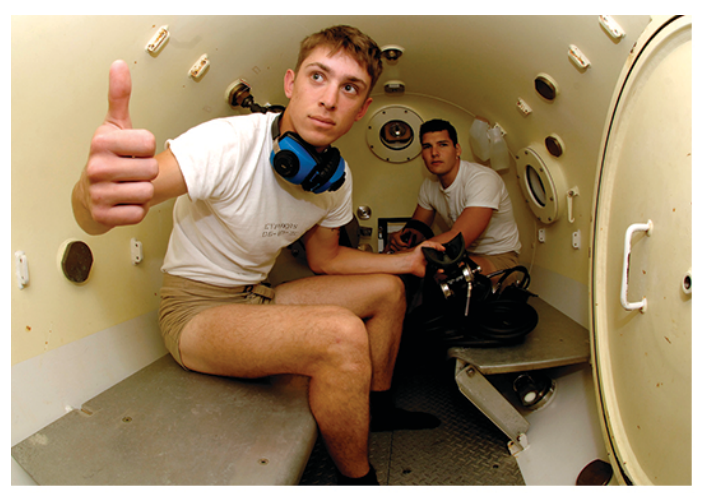

(a)

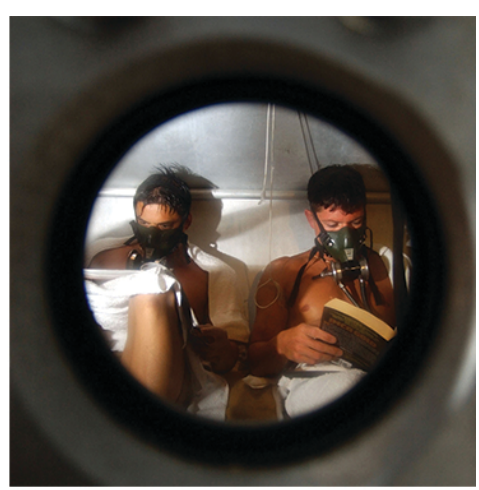

(b)

Deviations from Henry's law are observed when a chemical reaction takes place between the gaseous solute and the solvent. Thus, for example, the solubility of ammonia in water increases more rapidly with increasing pressure than predicted by the law because ammonia, being a base, reacts to some extent with water to form ammonium ions and hydroxide ions. 


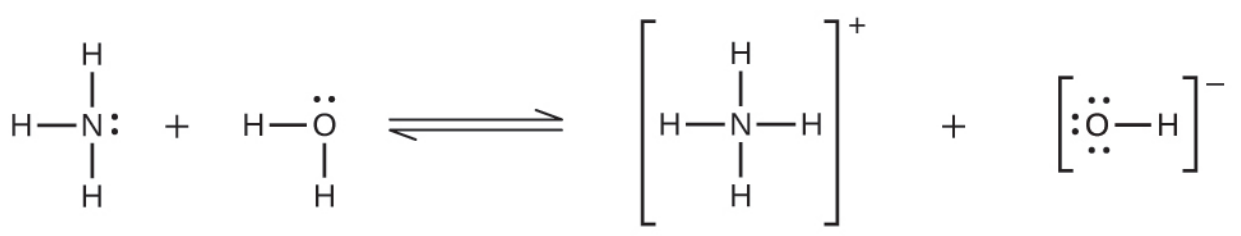

Gases can form supersaturated solutions. If a solution of a gas in a liquid is prepared either at low temperature or under pressure (or both), then as the solution warms or as the gas pressure is reduced, the solution may become supersaturated. In 1986, more than 1700 people in Cameroon were killed when a cloud of gas, almost certainly carbon dioxide, bubbled from Lake Nyos ((Figure)), a deep lake in a volcanic crater. The water at the bottom of Lake Nyos is saturated with carbon dioxide by volcanic activity beneath the lake. It is believed that the lake underwent a turnover due to gradual heating from below the lake, and the warmer, less-dense water saturated with carbon dioxide reached the surface. Consequently, tremendous quantities of dissolved $\mathrm{CO}_{2}$ were released, and the colorless gas, which is denser than air, flowed down the valley below the lake and suffocated humans and animals living in the valley.

(a) It is believed that the 1986 disaster that killed more than 1700 people near Lake Nyos in Cameroon resulted when a large volume of carbon dioxide gas was released from the lake. (b) $\mathrm{A} \mathrm{CO}_{2}$ vent has since been installed to help outgas the lake in a slow, controlled fashion and prevent a similar catastrophe from happening in the future. (credit a: modification of work by Jack Lockwood; credit b: modification of work by Bill Evans) 


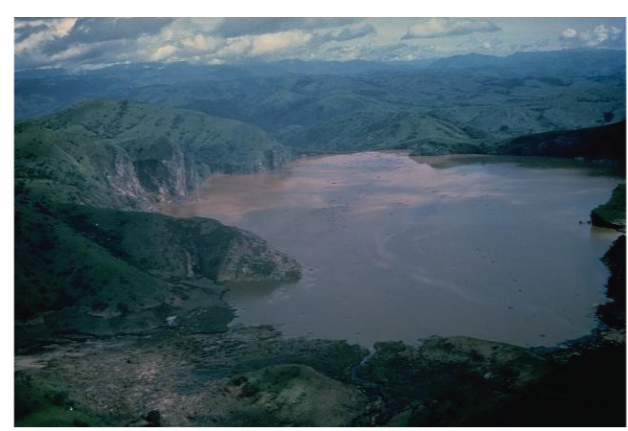

(a)

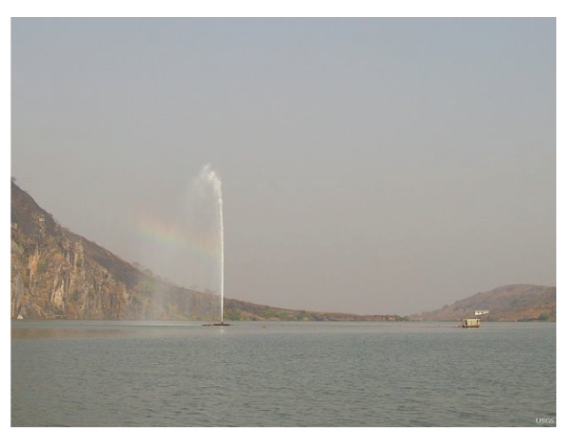

(b)

Solutions of Liquids in Liquids

Some liquids may be mixed in any proportions to yield solutions; in other words, they have infinite mutual solubility and are said to be miscible. Ethanol, sulfuric acid, and ethylene glycol (popular for use as antifreeze, pictured in (Figure)) are examples of liquids that are completely miscible with water. Two-cycle motor oil is miscible with gasoline, mixtures of which are used as lubricating fuels for various types of outdoor power equipment (chainsaws, leaf blowers, and so on).

Water and antifreeze are miscible; mixtures of the two are homogeneous in all proportions. (credit:

“dno1967"/Wikimedia commons) 


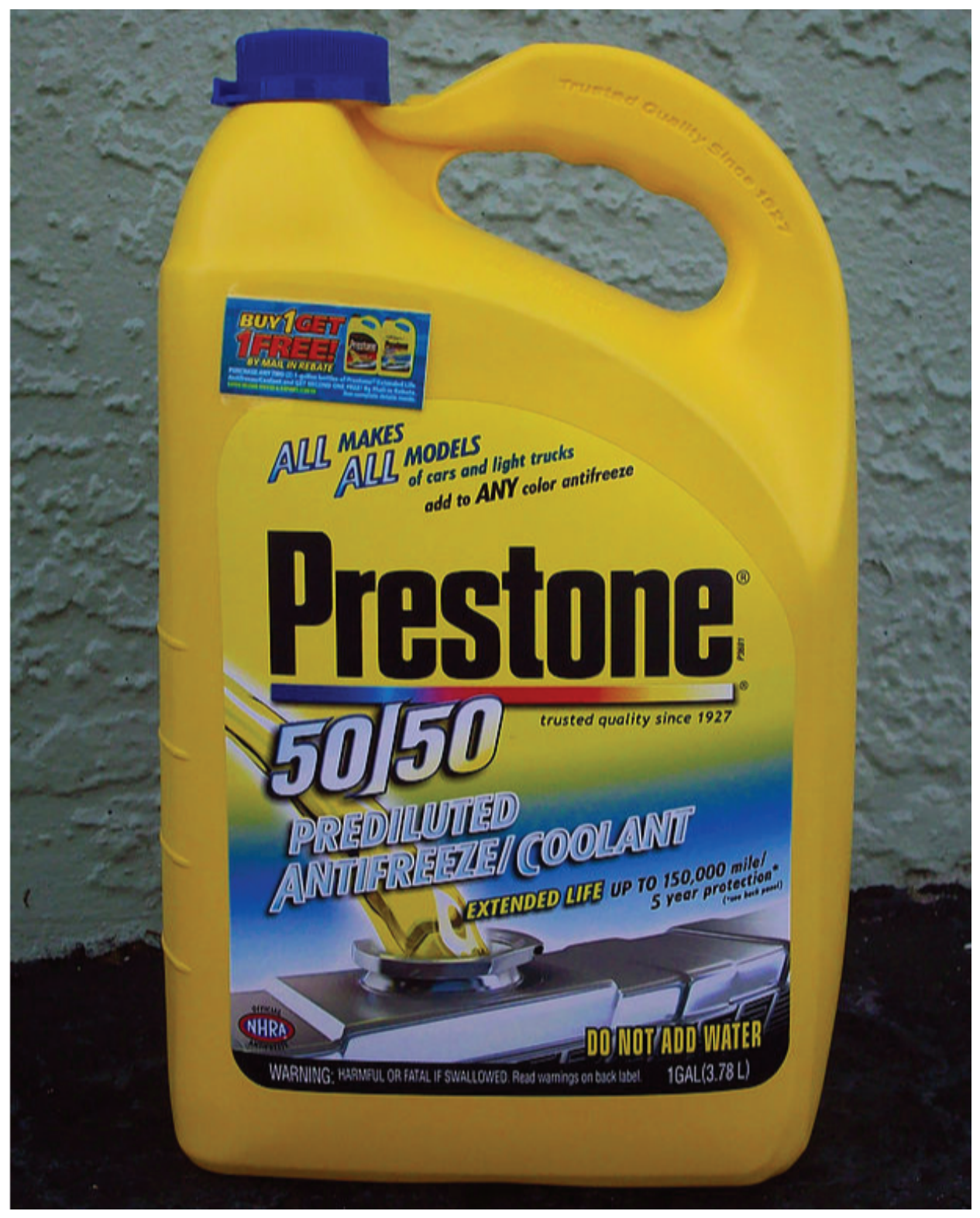

Miscible liquids are typically those with very similar polarities. Consider, for example, liquids that are polar or capable of hydrogen bonding. For such liquids, the dipoledipole attractions (or hydrogen bonding) of the solute molecules with the solvent molecules are at least as strong as those between molecules in the pure solute or in the pure solvent. Hence, the two kinds of molecules mix easily. Likewise, nonpolar liquids are miscible with each 
other because there is no appreciable difference in the strengths of solute-solute, solvent-solvent, and solutesolvent intermolecular attractions. The solubility of polar molecules in polar solvents and of nonpolar molecules in nonpolar solvents is, again, an illustration of the chemical axiom "like dissolves like."

Two liquids that do not mix to an appreciable extent are called immiscible. Separate layers are formed when immiscible liquids are poured into the same container. Gasoline, oil ((Figure)), benzene, carbon tetrachloride, some paints, and many other nonpolar liquids are immiscible with water. Relatively weak attractive forces between the polar water molecules and the nonpolar liquid molecules are not adequate to overcome much stronger hydrogen bonding between water molecules. The distinction between immiscibility and miscibility is really one of extent, so that miscible liquids are of infinite mutual solubility, while liquids said to be immiscible are of very low (though not zero) mutual solubility. Water and oil are immiscible. Mixtures of these two substances will form two separate layers with the less dense oil floating on top of the water. (credit:

"Yortw"/Flickr) 


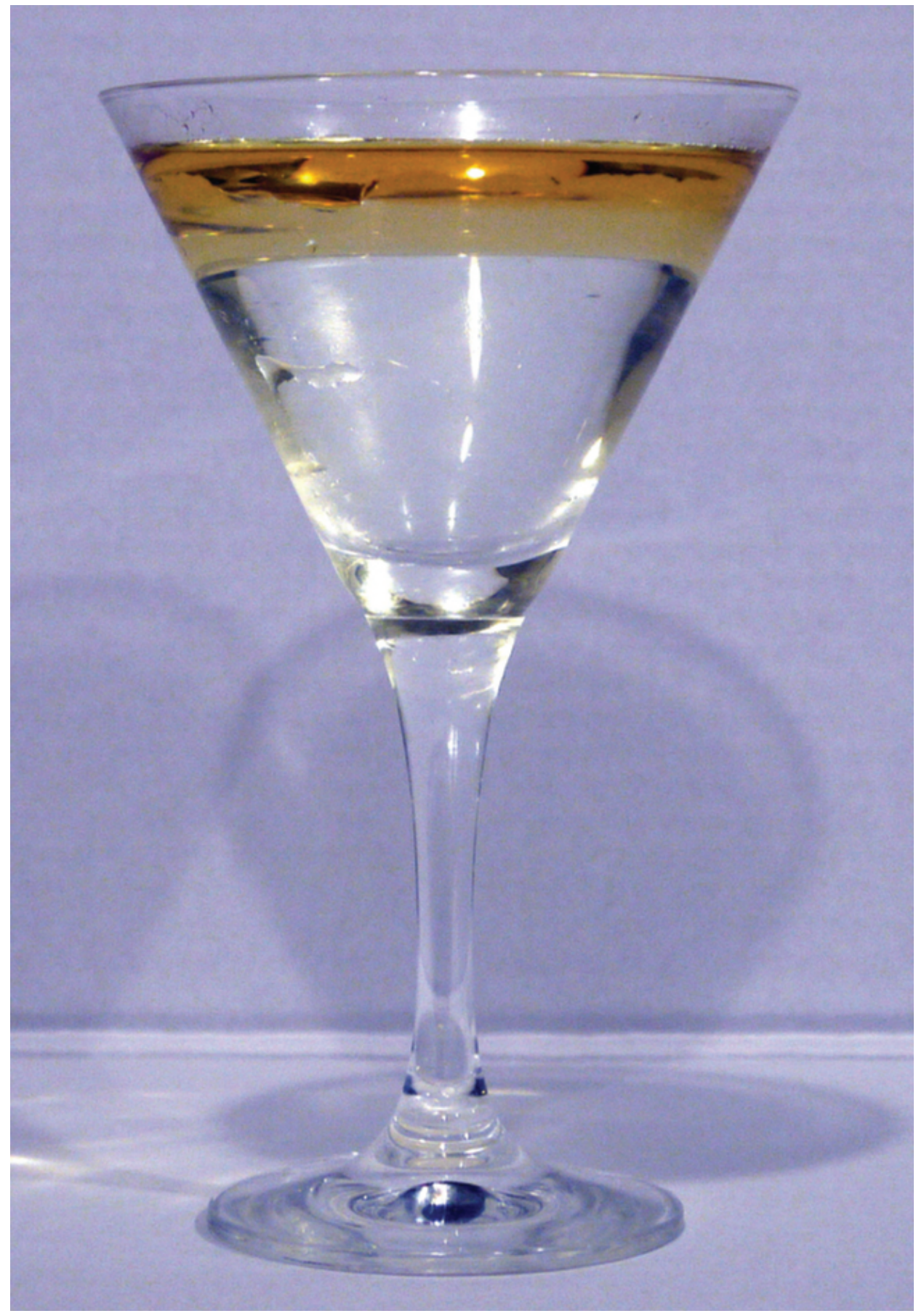

Two liquids, such as bromine and water, that are of moderate mutual solubility are said to be partially miscible. Two partially miscible liquids usually form two layers when mixed. In the case of the bromine and water 
mixture, the upper layer is water, saturated with bromine, and the lower layer is bromine saturated with water. Since bromine is nonpolar, and, thus, not very soluble in water, the water layer is only slightly discolored by the bright orange bromine dissolved in it. Since the solubility of water in bromine is very low, there is no noticeable effect on the dark color of the bromine layer ((Figure)).

Bromine (the deep orange liquid on the left) and water (the clear liquid in the middle) are partially miscible. The top layer in the mixture on the right is a saturated solution of bromine in water; the bottom layer is a saturated solution of water in bromine. (credit: Paul Flowers)

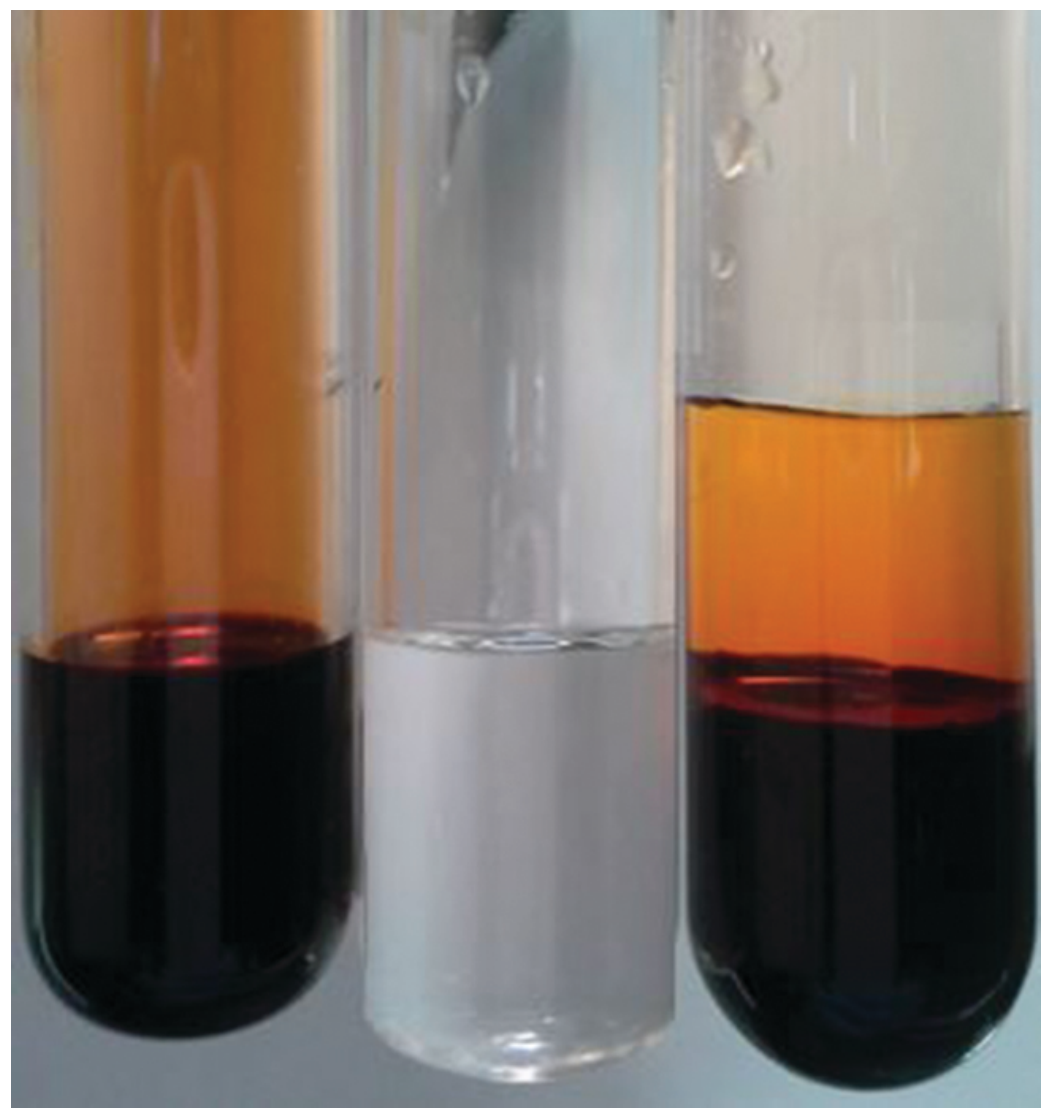


Solutions of Solids in Liquids

The dependence of solubility on temperature for a number of solids in water is shown by the solubility curves in (Figure). Reviewing these data indicates a general trend of increasing solubility with temperature, although there are exceptions, as illustrated by the ionic compound cerium sulfate.

This graph shows how the solubility of several solids changes with temperature.

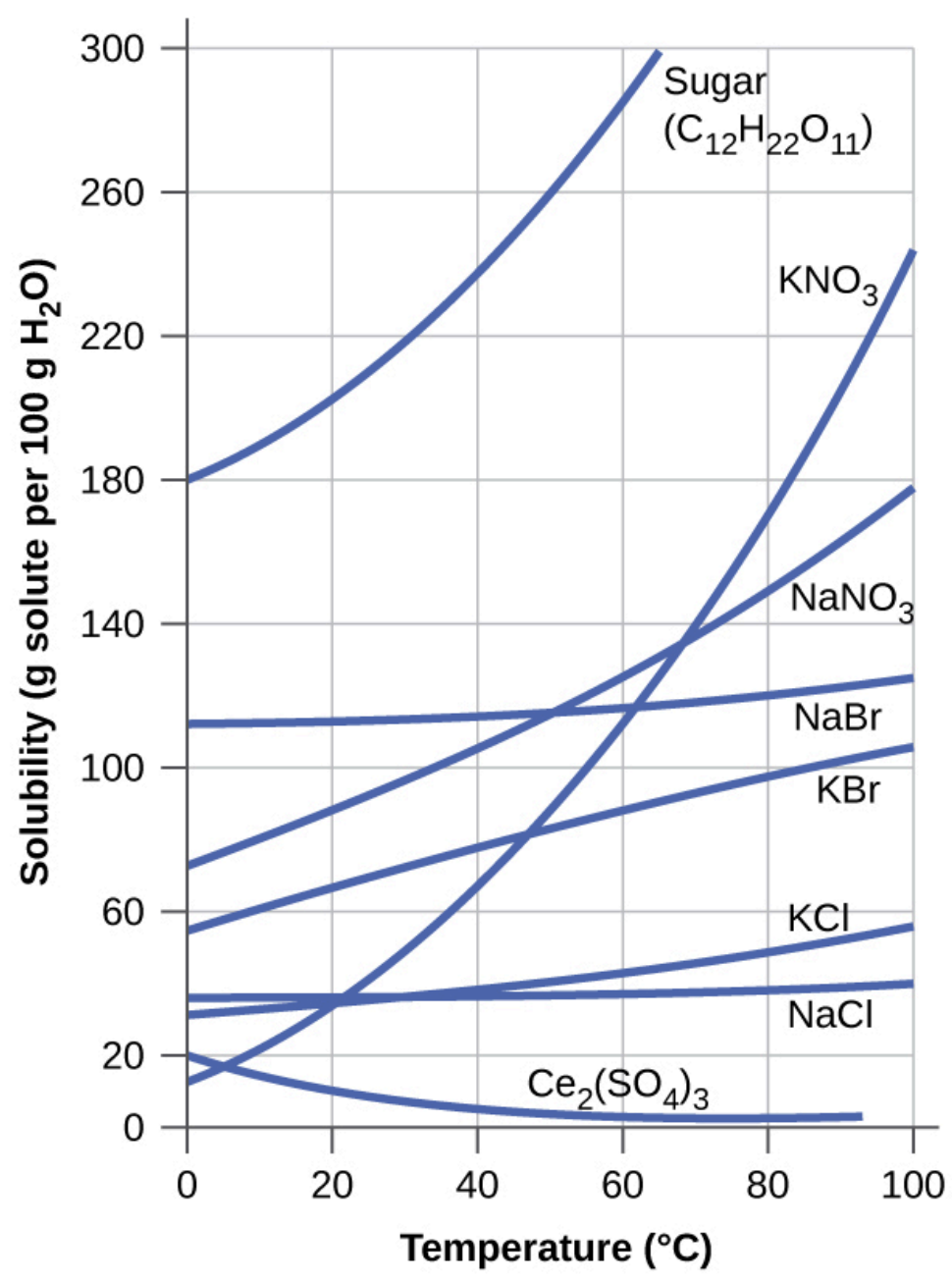

The temperature dependence of solubility can be 
exploited to prepare supersaturated solutions of certain compounds. A solution may be saturated with the compound at an elevated temperature (where the solute is more soluble) and subsequently cooled to a lower temperature without precipitating the solute. The resultant solution contains solute at a concentration greater than its equilibrium solubility at the lower temperature (i.e., it is supersaturated) and is relatively stable. Precipitation of the excess solute can be initiated by adding a seed crystal (see the video in the Link to Learning earlier in this module) or by mechanically agitating the solution. Some hand warmers, such as the one pictured in (Figure), take advantage of this behavior. This hand warmer produces heat when the sodium acetate in a supersaturated solution precipitates. Precipitation of the solute is initiated by a mechanical shockwave generated when the flexible metal disk within the solution is "clicked." (credit: modification of work by "Velela"/Wikimedia Commons)

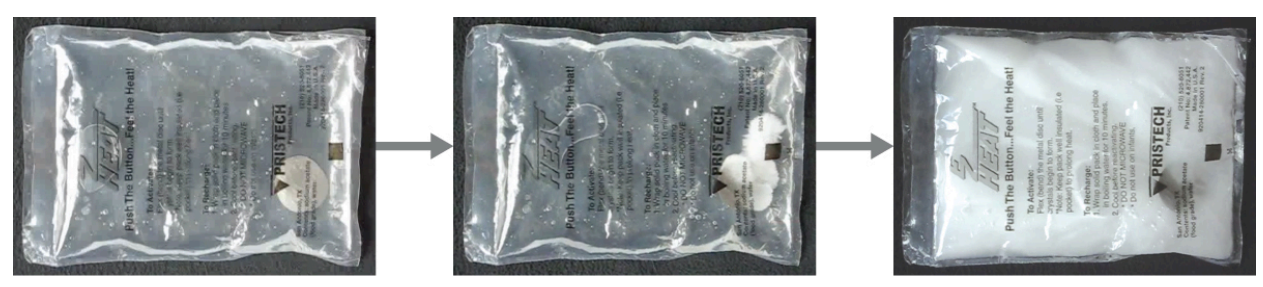

This video shows the crystallization process occurring in a hand warmer.

Key Concepts and Summary

The extent to which one substance will dissolve in another is determined by several factors, including the types and relative strengths of intermolecular attractive forces that 
may exist between the substances' atoms, ions, or molecules. This tendency to dissolve is quantified as a substance's solubility, its maximum concentration in a solution at equilibrium under specified conditions. A saturated solution contains solute at a concentration equal to its solubility. A supersaturated solution is one in which a solute's concentration exceeds its solubility-a nonequilibrium (unstable) condition that will result in solute precipitation when the solution is appropriately perturbed. Miscible liquids are soluble in all proportions, and immiscible liquids exhibit very low mutual solubility. Solubilities for gaseous solutes decrease with increasing temperature, while those for most, but not all, solid solutes increase with temperature. The concentration of a gaseous solute in a solution is proportional to the partial pressure of the gas to which the solution is exposed, a relation known as Henry's law.

Key Equations

$$
\text { - } C_{\mathrm{g}}=k P_{\mathrm{g}}
$$

Chemistry End of Chapter Exercises

Suppose you are presented with a clear solution of sodium thiosulfate, $\mathrm{Na}_{2} \mathrm{~S}_{2} \mathrm{O}_{3}$. How could you determine whether the solution is unsaturated, saturated, or supersaturated?

Supersaturated solutions of most solids in water are prepared by cooling saturated solutions. Supersaturated solutions of most gases in water are prepared by heating saturated solutions. Explain the reasons for the difference in the two procedures. 
The solubility of solids usually decreases upon cooling a solution, while the solubility of gases usually decreases upon heating.

Suggest an explanation for the observations that ethanol, $\mathrm{C}_{2} \mathrm{H}_{5} \mathrm{OH}$, is completely miscible with water and that ethanethiol, $\mathrm{C}_{2} \mathrm{H}_{5} \mathrm{SH}$, is soluble only to the extent of $1.5 \mathrm{~g}$ per $100 \mathrm{~mL}$ of water.

Calculate the percent by mass of $\mathrm{KBr}$ in a saturated solution of $\mathrm{KBr}$ in water at $10{ }^{\circ} \mathrm{C}$. See (Figure) for useful data, and report the computed percentage to one significant digit.

$40 \%$

Which of the following gases is expected to be most soluble in water? Explain your reasoning.

(a) $\mathrm{CH}_{4}$

(b) $\mathrm{CCl}_{4}$

(c) $\mathrm{CHCl}_{3}$

At $0{ }^{\circ} \mathrm{C}$ and $1.00 \mathrm{~atm}$, as much as $0.70 \mathrm{~g}$ of $\mathrm{O}_{2}$ can dissolve in $1 \mathrm{~L}$ of water. At $0{ }^{\circ} \mathrm{C}$ and $4.00 \mathrm{~atm}$, how many grams of $\mathrm{O}_{2}$ dissolve in $1 \mathrm{~L}$ of water?

$2.8 \mathrm{~g}$

Refer to (Figure).

(a) How did the concentration of dissolved $\mathrm{CO}_{2}$ in the beverage change when the bottle was opened?

(b) What caused this change? 
(c) Is the beverage unsaturated, saturated, or supersaturated with $\mathrm{CO}_{2}$ ?

The Henry's law constant for $\mathrm{CO}_{2}$ is $3.410^{-2} \mathrm{M} / \mathrm{atm}$ at $25^{\circ} \mathrm{C}$. Assuming ideal solution behavior, what pressure of carbon dioxide is needed to maintain a $\mathrm{CO}_{2}$ concentration of $0.10 \mathrm{M}$ in a can of lemon-lime soda?

\section{$2.9 \mathrm{~atm}$}

The Henry's law constant for $\mathrm{O}_{2}$ is $1.310^{-3} \mathrm{M} / \mathrm{atm}$ at $25^{\circ} \mathrm{C}$. Assuming ideal solution behavior, what mass of oxygen would be dissolved in a 40 - $\mathrm{L}$ aquarium at $25^{\circ} \mathrm{C}$, assuming an atmospheric pressure of $1.00 \mathrm{~atm}$, and that the partial pressure of $\mathrm{O}_{2}$ is $0.21 \mathrm{~atm}$ ?

Assuming ideal solution behavior, how many liters of $\mathrm{HCl}$ gas, measured at $30.0{ }^{\circ} \mathrm{C}$ and 745 torr, are required to prepare $1.25 \mathrm{~L}$ of a 3.20- $M$ solution of hydrochloric acid?

\section{$102 \mathrm{~L} \mathrm{HCl}$}

Glossary

\section{Henry's law}

the proportional relationship between the concentration of dissolved gas in a solution and the partial pressure of the gas in contact with the solution 


\section{immiscible}

of negligible mutual solubility; typically

refers to liquid substances

miscible

mutually soluble in all proportions;

typically refers to liquid substances

partially miscible

of moderate mutual solubility; typically

refers to liquid substances

saturated

of concentration equal to solubility;

containing the maximum concentration of

solute possible for a given temperature and pressure

solubility

extent to which a solute may be dissolved in water, or any solvent

supersaturated

of concentration that exceeds solubility; a nonequilibrium state

unsaturated

of concentration less than solubility 
46.

\section{COLLOIDS}

Learning Objectives

By the end of this section, you will be able to:

- Describe the composition and properties of colloidal dispersions

- List and explain several technological applications of colloids

As a child, you may have made suspensions such as mixtures of mud and water, flour and water, or a suspension of solid pigments in water, known as tempera paint. These suspensions are heterogeneous mixtures composed of relatively large particles that are visible (or that can be seen with a magnifying glass). They are cloudy, and the suspended particles settle out after mixing. On the other hand, a solution is a homogeneous mixture in which no settling occurs and in which the dissolved species are molecules or ions. Solutions exhibit 
completely different behavior from suspensions. A solution may be colored, but it is transparent, the molecules or ions are invisible, and they do not settle out on standing. Another class of mixtures called colloids (or colloidal dispersions) exhibit properties intermediate between those of suspensions and solutions ((Figure)). The particles in a colloid are larger than most simple molecules; however, colloidal particles are small enough that they do not settle out upon standing.

(a) A solution is a homogeneous mixture that appears clear, such as the saltwater in this aquarium. (b) In a colloid, such as milk, the particles are much larger but remain dispersed and do not settle. (c) A suspension, such as mud, is a heterogeneous mixture of suspended particles that appears cloudy and in which the particles can settle. (credit a photo: modification of work by Adam Wimsatt; credit b photo: modification of work by Melissa Wiese; credit c photo: modification of work by Peter Burgess)
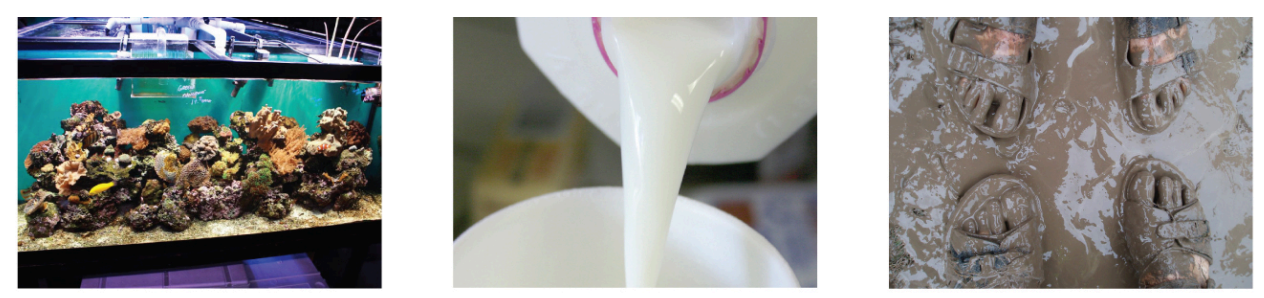

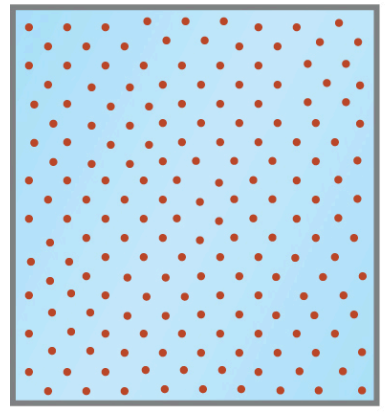

(a)

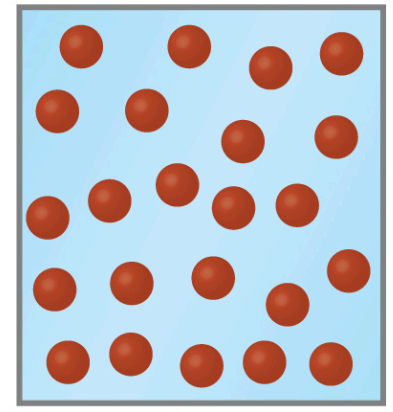

(b)

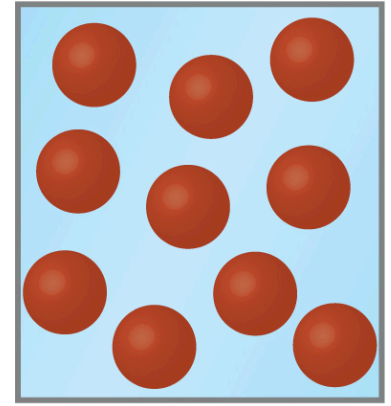

(c) 
The particles in a colloid are large enough to scatter light, a phenomenon called the Tyndall effect. This can make colloidal mixtures appear cloudy or opaque, such as the searchlight beams shown in (Figure). Clouds are colloidal mixtures. They are composed of water droplets that are much larger than molecules, but that are small enough that they do not settle out.

The paths of searchlight beams are made visible when light is scattered by colloidal-size particles in the air (fog, smoke, etc.). (credit: “Bahman"/Wikimedia Commons)

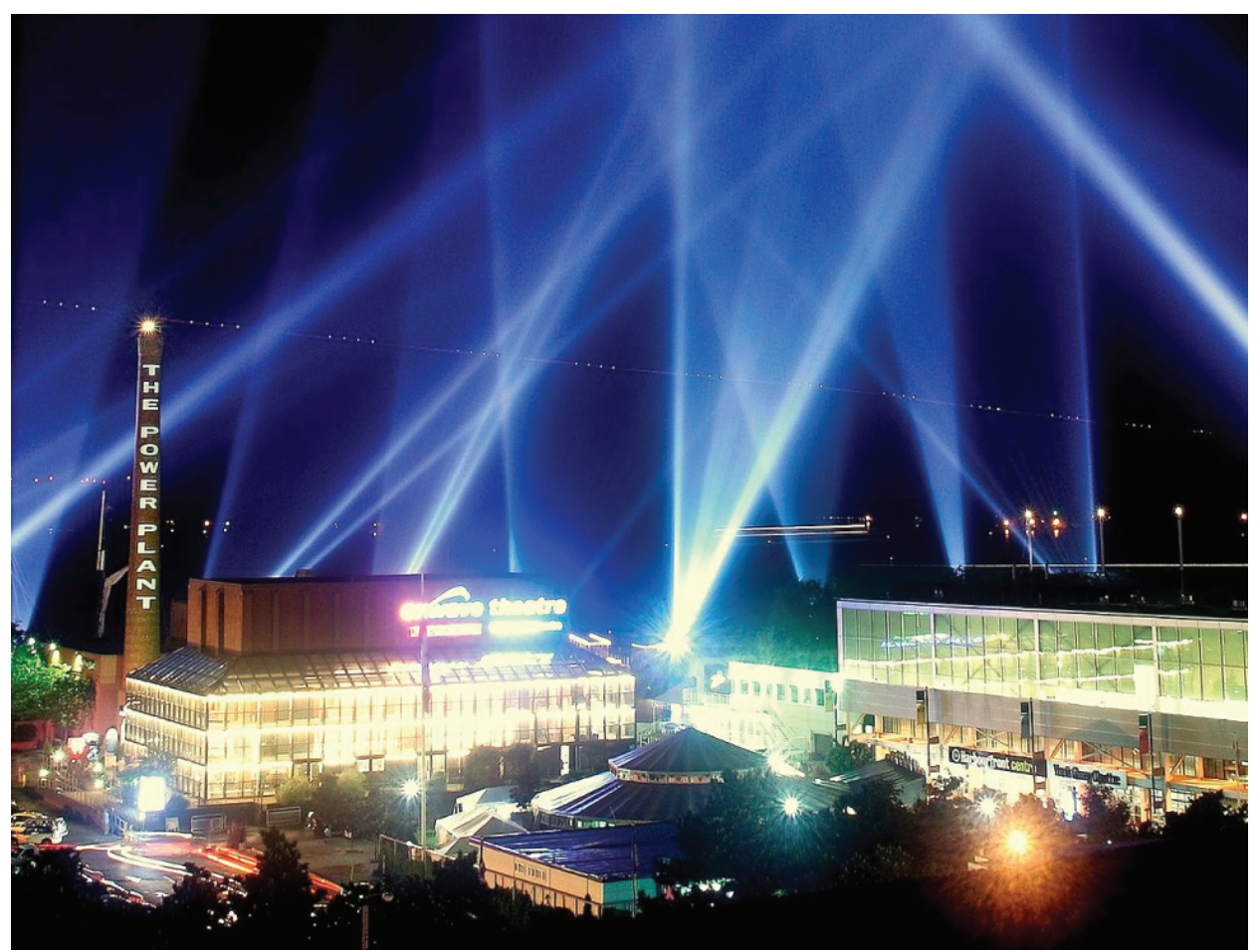

The term "colloid"-from the Greek words kolla, meaning "glue," and eidos, meaning "like"-was first used in 1861 by Thomas Graham to classify mixtures such as starch in water and gelatin. Many colloidal particles are aggregates of hundreds or thousands of molecules, but others (such as proteins and polymer molecules) consist of a single 
extremely large molecule. The protein and synthetic polymer molecules that form colloids may have molecular masses ranging from a few thousand to many million atomic mass units.

Analogous to the identification of solution components as "solute" and "solvent," the components of a colloid are likewise classified according to their relative amounts. The particulate component typically present in a relatively minor amount is called the dispersed phase and the substance or solution throughout which the particulate is dispersed is called the dispersion medium. Colloids may involve virtually any combination of physical states (gas in liquid, liquid in solid, solid in gas, etc.), as illustrated by the examples of colloidal systems given in (Figure).

\section{Examples of Colloidal Systems}

\begin{tabular}{|c|c|c|c|}
\hline $\begin{array}{l}\text { Dispersed } \\
\text { Phase }\end{array}$ & $\begin{array}{l}\text { Dispersion } \\
\text { Medium }\end{array}$ & Common Examples & Name \\
\hline solid & gas & smoke, dust & - \\
\hline solid & liquid & $\begin{array}{l}\text { starch in water, some inks, } \\
\text { paints, milk of magnesia }\end{array}$ & sol \\
\hline solid & solid & $\begin{array}{l}\text { some colored gems, some } \\
\text { alloys }\end{array}$ & - \\
\hline liquid & gas & clouds, fogs, mists, sprays & aerosol \\
\hline liquid & liquid & milk, mayonnaise, butter & emulsion \\
\hline liquid & solid & $\begin{array}{l}\text { jellies, gels, pearl, opal }\left(\mathrm{H}_{2} \mathrm{O} \text { in }\right. \\
\left.\mathrm{SiO}_{2}\right)\end{array}$ & gel \\
\hline gas & liquid & $\begin{array}{l}\text { foams, whipped cream, beaten } \\
\text { egg whites }\end{array}$ & foam \\
\hline gas & solid & pumice, floating soaps & - \\
\hline
\end{tabular}


Preparation of Colloidal Systems

Colloids are prepared by producing particles of colloidal dimensions and distributing these particles throughout a dispersion medium. Particles of colloidal size are formed by two methods:

1. Dispersion methods: breaking down larger particles. For example, paint pigments are produced by dispersing large particles by grinding in special mills.

2. Condensation methods: growth from smaller units, such as molecules or ions. For example, clouds form when water molecules condense and form very small droplets.

A few solid substances, when brought into contact with water, disperse spontaneously and form colloidal systems. Gelatin, glue, starch, and dehydrated milk powder behave in this manner. The particles are already of colloidal size; the water simply disperses them. Powdered milk particles of colloidal size are produced by dehydrating milk spray. Some atomizers produce colloidal dispersions of a liquid in air.

An emulsion may be prepared by shaking together or blending two immiscible liquids. This breaks one liquid into droplets of colloidal size, which then disperse throughout the other liquid. Oil spills in the ocean may be difficult to clean up, partly because wave action can cause the oil and water to form an emulsion. In many emulsions, however, the dispersed phase tends to coalesce, form large drops, and separate. Therefore, emulsions are usually 
stabilized by an emulsifying agent, a substance that inhibits the coalescence of the dispersed liquid. For example, a little soap will stabilize an emulsion of kerosene in water. Milk is an emulsion of butterfat in water, with the protein casein serving as the emulsifying agent. Mayonnaise is an emulsion of oil in vinegar, with egg yolk components as the emulsifying agents.

Condensation methods form colloidal particles by aggregation of molecules or ions. If the particles grow beyond the colloidal size range, drops or precipitates form, and no colloidal system results. Clouds form when water molecules aggregate and form colloid-sized particles. If these water particles coalesce to form adequately large water drops of liquid water or crystals of solid water, they settle from the sky as rain, sleet, or snow. Many condensation methods involve chemical reactions. A red colloidal suspension of iron(III) hydroxide may be prepared by mixing a concentrated solution of iron(III) chloride with hot water:

$\mathrm{Fe}^{3+}(\mathrm{aq})+3 \mathrm{Cl}(\mathrm{aq})+6 \mathrm{H}_{2} \mathrm{O}(l) \mathrm{Fe}(\mathrm{OH})_{3}(s)+\mathrm{H}_{3} \mathrm{O}^{+}(a q)+3 \mathrm{Cl}(a q)$.

A colloidal gold sol results from the reduction of a very dilute solution of gold(III) chloride by a reducing agent such as formaldehyde, tin(II) chloride, or iron(II) sulfate: $\mathrm{Au}^{3+}+3 \mathrm{e} \mathrm{Au}$

Some gold sols prepared in 1857 are still intact (the particles have not coalesced and settled), illustrating the long-term stability of many colloids.

Soaps and Detergents

Pioneers made soap by boiling fats with a strongly basic 
solution made by leaching potassium carbonate, $\mathrm{K}_{2} \mathrm{CO}_{3}$, from wood ashes with hot water. Animal fats contain polyesters of fatty acids (long-chain carboxylic acids). When animal fats are treated with a base like potassium carbonate or sodium hydroxide, glycerol and salts of fatty acids such as palmitic, oleic, and stearic acid are formed. The salts of fatty acids are called soaps. The sodium salt of stearic acid, sodium stearate, has the formula $\mathrm{C}_{17} \mathrm{H}_{35} \mathrm{CO}_{2} \mathrm{Na}$ and contains an uncharged nonpolar hydrocarbon chain, the $\mathrm{C}_{17} \mathrm{H}_{35}-$ unit, and an ionic carboxylate group, the $-\mathrm{CO}_{2}$ unit ((Figure)).

Soaps contain a nonpolar hydrocarbon end (blue) and an ionic end (red). The ionic end is a carboxylate group. The length of the hydrocarbon end can vary from soap to soap.

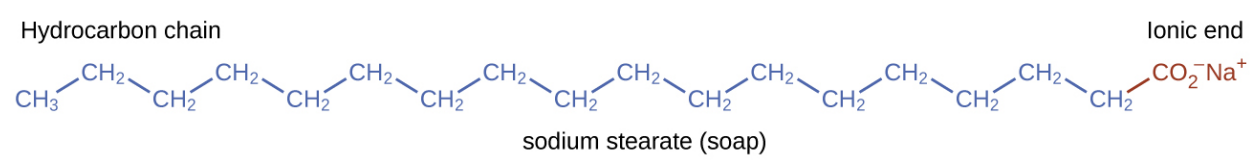

Detergents (soap substitutes) also contain nonpolar hydrocarbon chains, such as $\mathrm{C}_{12} \mathrm{H}_{25}$ - and an ionic group, such as a sulfate- $-\mathrm{OSO}_{3}$, or a sulfonate $-\mathrm{SO}_{3}$ ((Figure)). Soaps form insoluble calcium and magnesium compounds in hard water; detergents form water-soluble products-a definite advantage for detergents.

Detergents contain a nonpolar hydrocarbon end (blue) and an ionic end (red). The ionic end can be either a sulfate or a sulfonate. The length of the hydrocarbon end can vary from detergent to detergent.

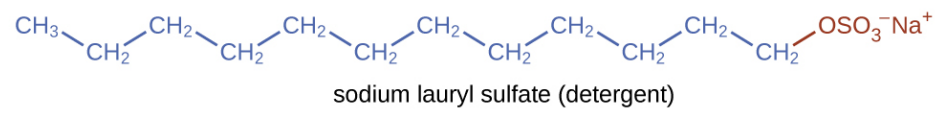


The cleaning action of soaps and detergents can be explained in terms of the structures of the molecules involved. The hydrocarbon (nonpolar) end of a soap or detergent molecule dissolves in, or is attracted to, nonpolar substances such as oil, grease, or dirt particles. The ionic end is attracted by water (polar), illustrated in (Figure). As a result, the soap or detergent molecules become oriented at the interface between the dirt particles and the water so they act as a kind of bridge between two different kinds of matter, nonpolar and polar. Molecules such as this are termed amphiphilic since they have both a hydrophobic ("water-fearing") part and a hydrophilic ("water-loving”) part. As a consequence, dirt particles become suspended as colloidal particles and are readily washed away.

This diagrammatic cross section of an emulsified drop of oil in water shows how soap or detergent acts as an emulsifier. 


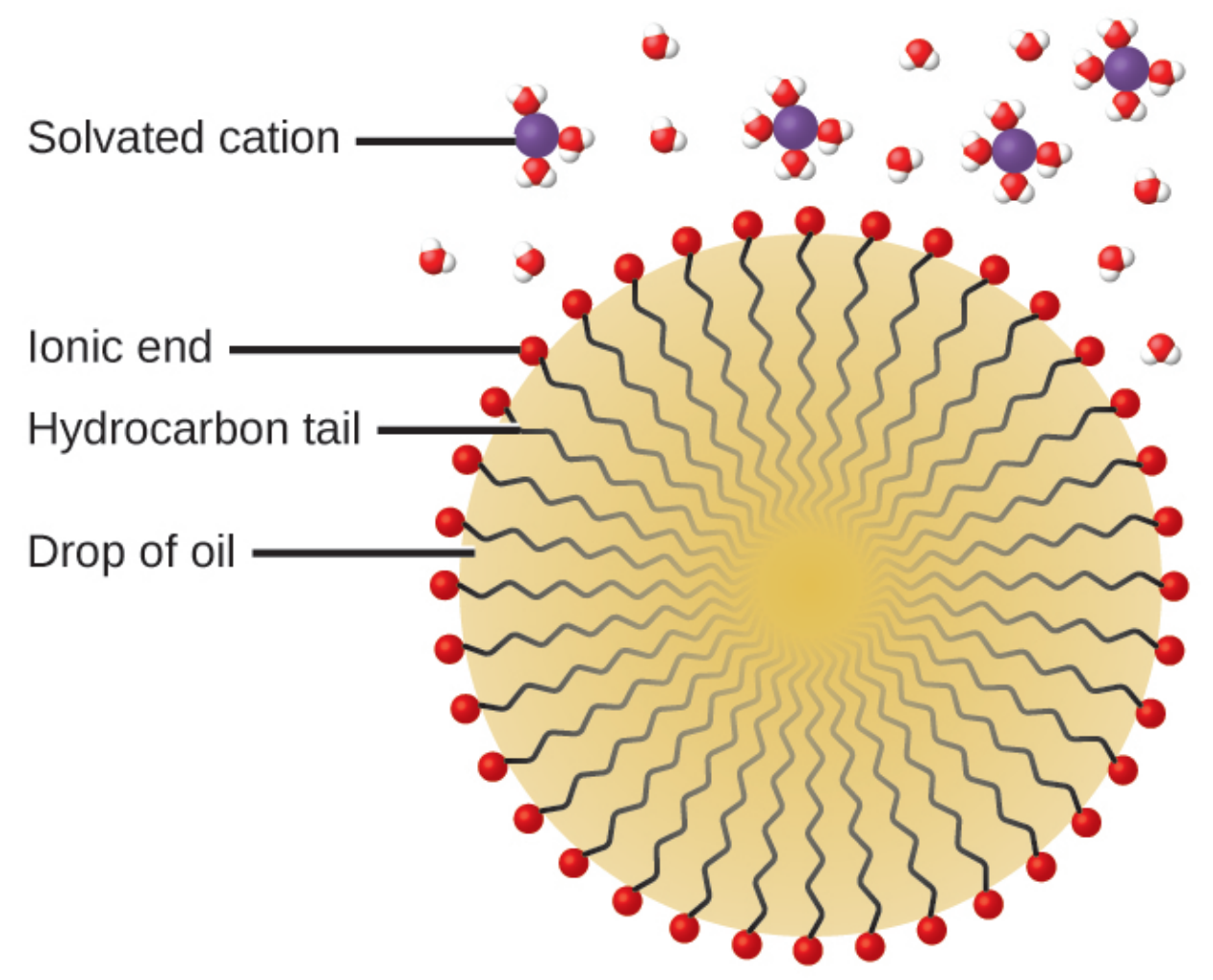

Deepwater Horizon Oil Spill

The blowout of the Deepwater Horizon oil drilling rig on April 20, 2010, in the Gulf of Mexico near Mississippi began the largest marine oil spill in the history of the petroleum industry. In the 87 days following the blowout, an estimated 4.9 million barrels (210 million gallons) of oil flowed from the ruptured well 5000 feet below the water's surface. The well was finally declared sealed on September 19, 2010.

Crude oil is immiscible with and less dense than water, so the spilled oil rose to the surface of the water. Floating booms, skimmer ships, and controlled burns were used to remove oil from the water's surface in an attempt to protect beaches and wetlands along the Gulf coast. In addition to removal of the oil, attempts were also made to 
lessen its environmental impact by rendering it "soluble" (in the loose sense of the term) and thus allowing it to be diluted to hopefully less harmful levels by the vast volume of ocean water. This approach used 1.84 million gallons of the oil dispersant Corexit 9527, most of which was injected underwater at the site of the leak, with small amounts being sprayed on top of the spill. Corexit 9527 contains 2-butoxyethanol $\left(\mathrm{C}_{6} \mathrm{H}_{14} \mathrm{O}_{2}\right)$, an amphiphilic molecule whose polar and nonpolar ends are useful for emulsifying oil into small droplets, increasing the surface area of the oil and making it more available to marine bacteria for digestion ((Figure)). While this approach avoids many of the immediate hazards that bulk oil poses to marine and coastal ecosystems, it introduces the possibility of longterm effects resulting from the introduction of the complex and potential toxic components of petroleum into the ocean's food chain. A number of organizations are involved in monitoring the extended impact of this oil spill, including the National Oceanic and Atmospheric Administration (visit this website for additional details).

(a) This NASA satellite image shows the oil slick from the Deepwater Horizon spill. (b) A US Air Force plane sprays Corexit, a dispersant. (c) The molecular structure of 2-butoxyethanol is shown. (credit a: modification of work by “NASA, FT2, demis.nl”/Wikimedia Commons; credit b: modification of work by "NASA/MODIS Rapid Response Team"/Wikimedia Commons) 


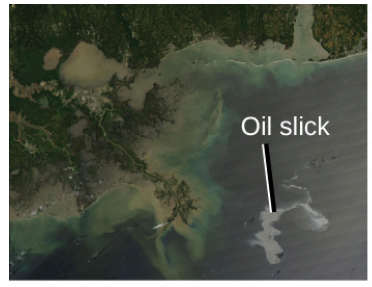

(a)

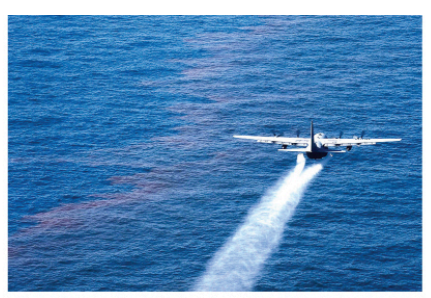

(b)

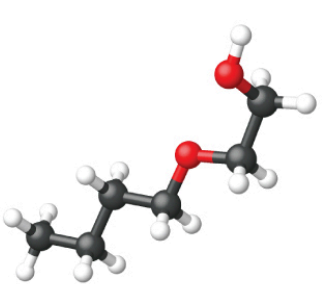

(c)

Electrical Properties of Colloidal Particles

Dispersed colloidal particles are often electrically charged. A colloidal particle of iron(III) hydroxide, for example, does not contain enough hydroxide ions to compensate exactly for the positive charges on the iron(III) ions. Thus, each individual colloidal particle bears a positive charge, and the colloidal dispersion consists of charged colloidal particles and some free hydroxide ions, which keep the dispersion electrically neutral. Most metal hydroxide colloids have positive charges, whereas most metals and metal sulfides form negatively charged dispersions. All colloidal particles in any one system have charges of the same sign. This helps keep them dispersed because particles containing like charges repel each other.

The charged nature of some colloidal particles may be exploited to remove them from a variety of mixtures. For example, the particles comprising smoke are often colloidally dispersed and electrically charged. Frederick Cottrell, an American chemist, developed a process to remove these particles. The charged particles are attracted to highly charged electrodes, where they are neutralized and deposited as dust ((Figure)). This is one of the important methods used to clean up the smoke from a variety of industrial processes. The process is also 
important in the recovery of valuable products from the smoke and flue dust of smelters, furnaces, and kilns. There are also similar electrostatic air filters designed for home use to improve indoor air quality.

Frederick Gardner Cottrell

(a) Frederick Cottrell developed (b) the electrostatic precipitator, a device designed to curb air pollution by removing colloidal particles from air. (credit b: modification of work by "SpLot"/Wikimedia Commons)

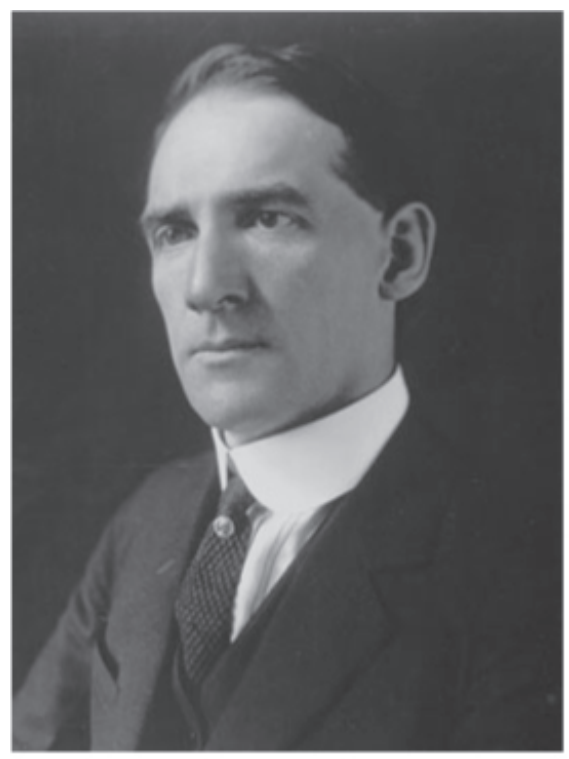

(a)

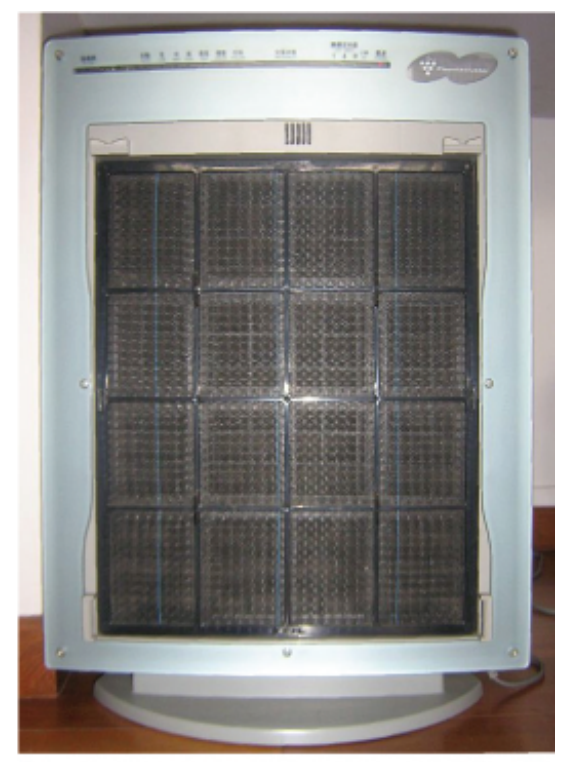

(b)

Born in Oakland, CA, in 1877, Frederick Cottrell devoured textbooks as if they were novels and graduated from high school at the age of 16 . He then entered the University of California (UC), Berkeley, completing a Bachelor's degree in three years. He saved money from his ?1200 annual salary as a chemistry teacher at Oakland High School to fund his studies in chemistry in Berlin with Nobel prize winner Jacobus Henricus van't Hoff, and in Leipzig with 
Wilhelm Ostwald, another Nobel awardee. After earning his $\mathrm{PhD}$ in physical chemistry, he returned to the United States to teach at UC Berkeley. He also consulted for the DuPont Company, where he developed the electrostatic precipitator, a device designed to curb air pollution by removing colloidal particles from air. Cottrell used the proceeds from his invention to fund a nonprofit research corporation to finance scientific research.

In a Cottrell precipitator, positively and negatively charged particles are attracted to highly charged electrodes, where they are neutralized and deposited as dust. 


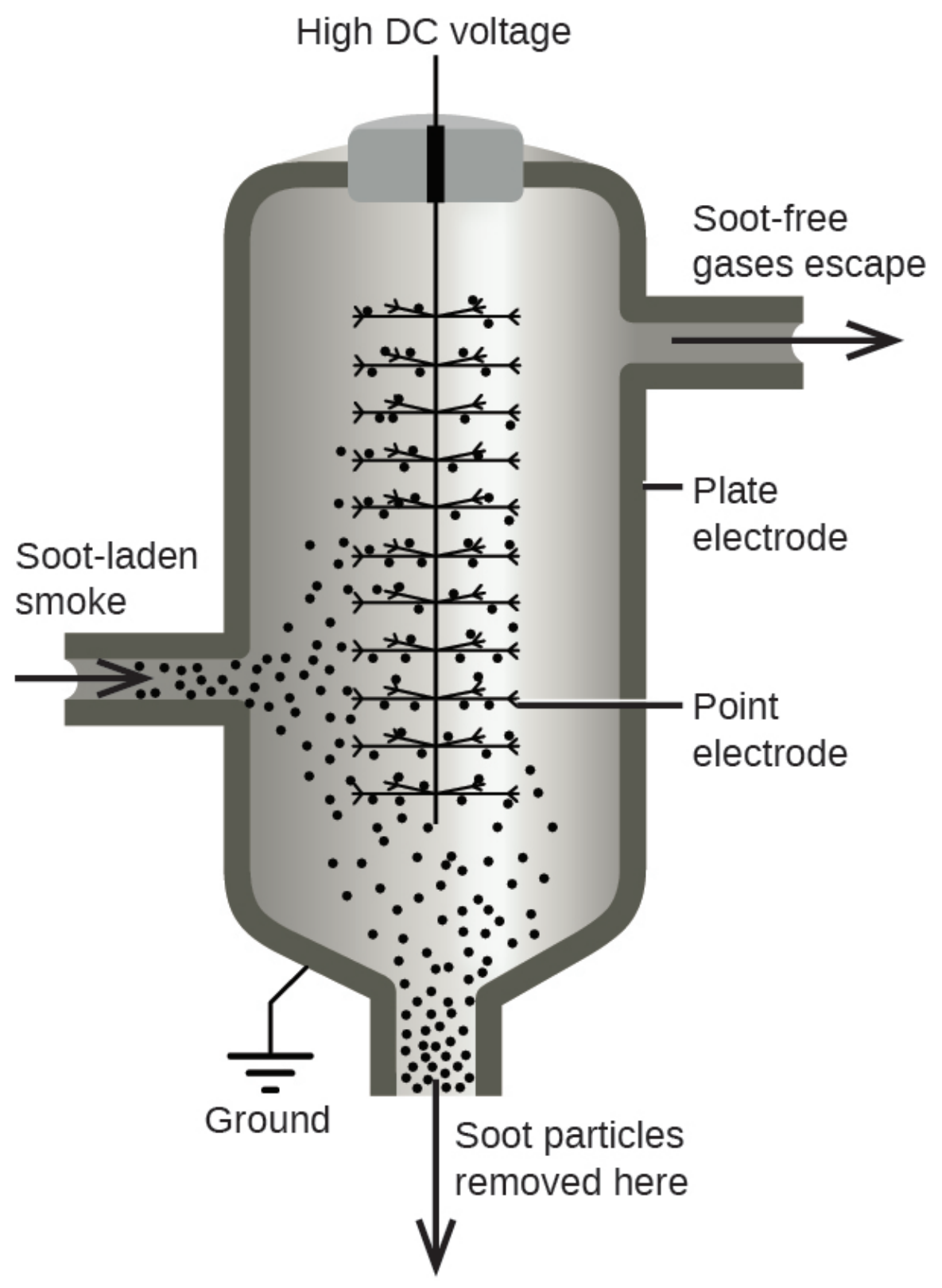

Giels

Gelatin desserts, such as Jell-O, are a type of colloid ((Figure)). Gelatin sets on cooling because the hot aqueous mixture of gelatin coagulates as it cools, yielding an extremely viscous body known as a gel. A gel is a colloidal dispersion of a liquid phase throughout a solid 
phase. It appears that the fibers of the dispersing medium form a complex three-dimensional network, the interstices being filled with the liquid medium or a dilute solution of the dispersing medium.

Gelatin desserts are colloids in which an aqueous solution of sweeteners and flavors is dispersed throughout a medium of solid proteins. (credit photo: modification of work by Steven Depolo)

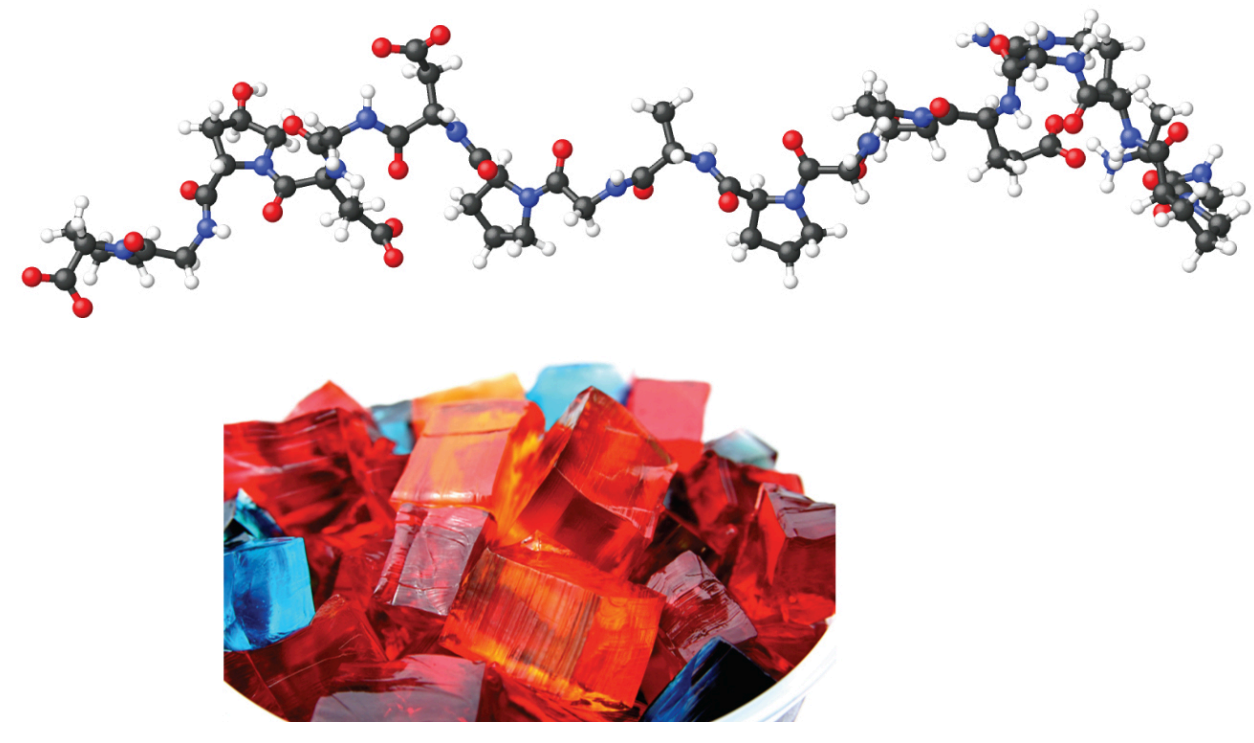

Pectin, a carbohydrate from fruit juices, is a gel-forming substance important in jelly making. Silica gel, a colloidal dispersion of hydrated silicon dioxide, is formed when dilute hydrochloric acid is added to a dilute solution of sodium silicate. Canned Heat is a flammable gel made by mixing alcohol and a saturated aqueous solution of calcium acetate.

Key Concepts and Summary

Colloids are mixtures in which one or more substances are dispersed as relatively large solid particles or liquid 
droplets throughout a solid, liquid, or gaseous medium. The particles of a colloid remain dispersed and do not settle due to gravity, and they are often electrically charged. Colloids are widespread in nature and are involved in many technological applications.

\section{Chemistry End of Chapter Exercises}

Identify the dispersed phase and the dispersion medium in each of the following colloidal systems: starch dispersion, smoke, fog, pearl, whipped cream, floating soap, jelly, milk, and ruby.

$\begin{array}{lll}\begin{array}{l}\text { Colloidal } \\ \text { System }\end{array} & \text { Dispersed Phase } & \text { Dispersion Medium } \\ \begin{array}{l}\text { starch } \\ \text { dispersion }\end{array} & \text { starch } & \text { water } \\ \text { smoke } & \text { solid particles } & \text { air } \\ \text { fog } & \text { water } & \text { air } \\ \text { pearl } & \text { water } & \begin{array}{l}\text { calcium carbonate } \\ \left(\mathrm{CaCO}_{3}\right)\end{array} \\ \text { whipped cream } & \text { air } & \text { cream } \\ \text { floating soap } & \text { air } & \text { soap } \\ \text { jelly } & \text { fruit juice } & \text { pectin gel } \\ \text { milk } & \text { butterfat } & \text { water } \\ \text { ruby } & \begin{array}{l}\text { chromium(III) oxide } \\ \left(\mathrm{Cr}_{2} \mathrm{O}_{3}\right)\end{array} & \text { aluminum oxide }\left(\mathrm{Al}_{2} \mathrm{O}_{3}\right)\end{array}$

Distinguish between dispersion methods and condensation methods for preparing colloidal systems.

How do colloids differ from solutions with regard to dispersed particle size and homogeneity? 
Colloidal dispersions consist of particles that are much bigger than the solutes of typical solutions. Colloidal particles are either very large molecules or aggregates of smaller species that usually are big enough to scatter light. Colloids are homogeneous on a macroscopic (visual) scale, while solutions are homogeneous on a microscopic (molecular) scale.

Explain the cleansing action of soap.

How can it be demonstrated that colloidal particles are electrically charged?

If they are placed in an electrolytic cell, dispersed particles will move toward the electrode that carries a charge opposite to their own charge. At this electrode, the charged particles will be neutralized and will coagulate as a precipitate.

Glossary

amphiphilic

molecules possessing both hydrophobic (nonpolar) and a hydrophilic (polar) parts colloid

(also, colloidal dispersion) mixture in which relatively large solid or liquid particles are dispersed uniformly throughout a gas, liquid, or solid 


\section{dispersion medium}

solid, liquid, or gas in which colloidal particles are dispersed

dispersed phase

substance present as relatively large solid or liquid particles in a colloid emulsifying agent amphiphilic substance used to stabilize the particles of some emulsions

emulsion

colloid formed from immiscible liquids gel

colloidal dispersion of a liquid in a solid suspension

heterogeneous mixture in which relatively large component particles are temporarily dispersed but settle out over time

\section{Tyndall effect}

scattering of visible light by a colloidal dispersion 


\section{Focus on Water Quality}

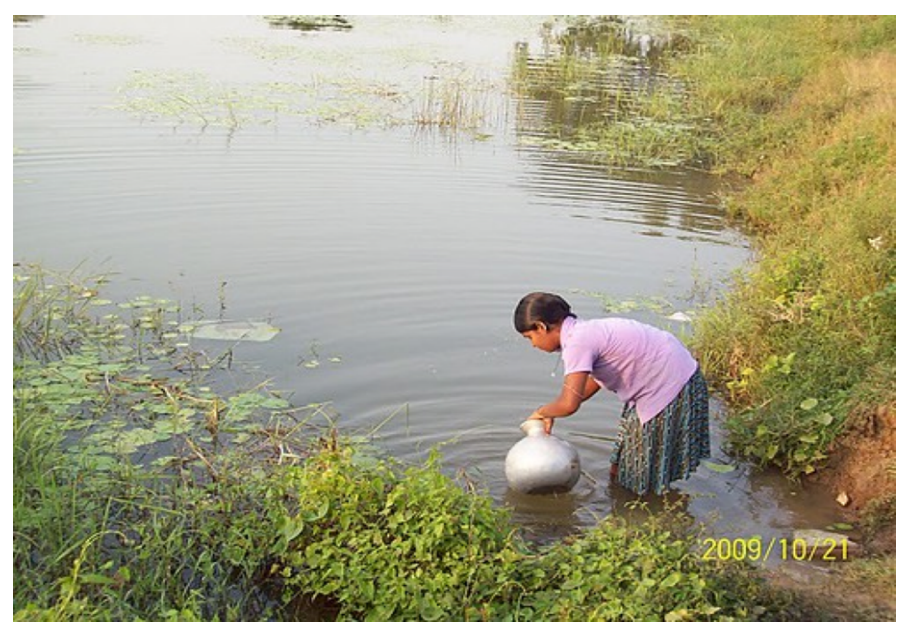

"Safe Drinking Water Supply and Sanitation Project for Puri district" by India Water Portal is licensed under CC BY-NC-SA 2.0

Water - one of the most important and plentiful resources on earth, yet it's availability and quality are lacking to many around the globe. From droughts to pollution, the lack of clean water is a question of health, and ultimately, survival, for millions of people. Water is known to chemists as the 'universal solvent' and in this section the role of solutes and solvents are examined in terms of interactions between molecules to give a glimpse of the field of environmental fate modelling - the prediction of where a chemical might end up in the environment. In order to make a proper assessment of a compound in the environment, there are a number of other factors that must be examined, such as persistence and toxicity, full discussions of which are beyond the scope of this text.

Refer to the information discussed in the previous Focus section as well as Part VIII and IX of this text and elaborate on the following focus boxes, solving any problems that appear. While the field is very complicated, a general ability to approximate the relative solubility of a compound and do rudimentary calculations in concentration terms can provide an important resource for the examination of contaminants in the aqueous environment. 


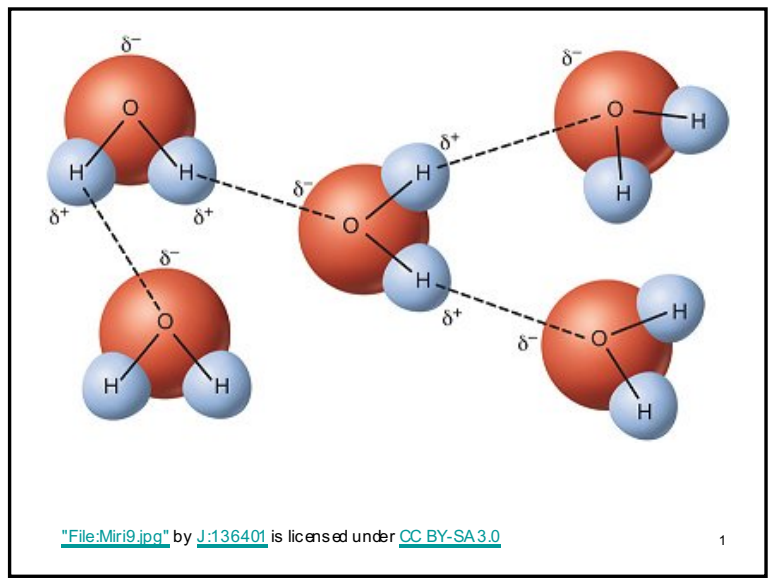

\section{Hydrogen bond}

an especially strong intermolecular force between an electronegative element (such as $\mathrm{N}, \mathrm{O}$ or a halogen) and a hydrogen that is covalently bound to an electronegative element (such as $\mathrm{N}, \mathrm{O}$ or a halogen).

Demonstrate the dominant intermolecular in the following solutions. Does the solution have hydrogen bonding? (i) $\mathrm{H}_{2} \mathrm{O}$, (ii) $\mathrm{NH}_{3}$, (iii) $\mathrm{CH}_{3}-\mathrm{O}-\mathrm{CH}_{3}$, (iv) $\mathrm{NH}_{3}$ in $\mathrm{CH}_{3}-\mathrm{O}-\mathrm{CH}_{3}$.

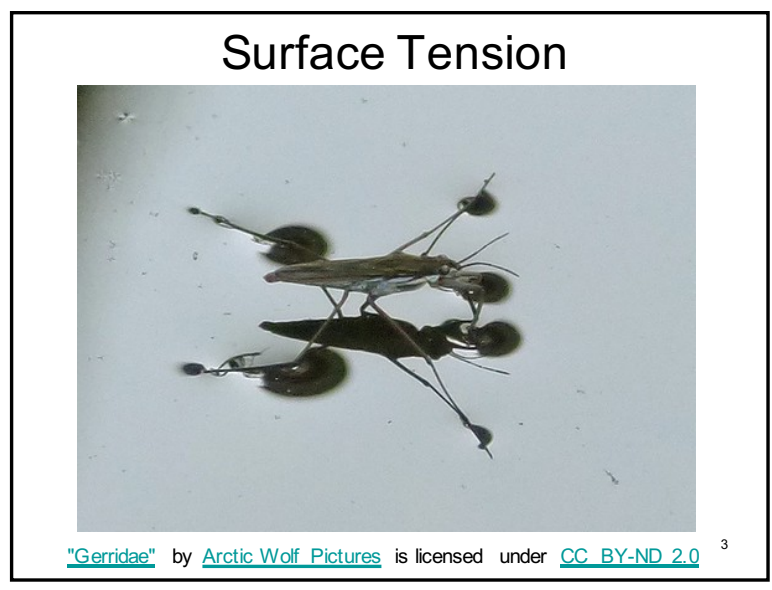




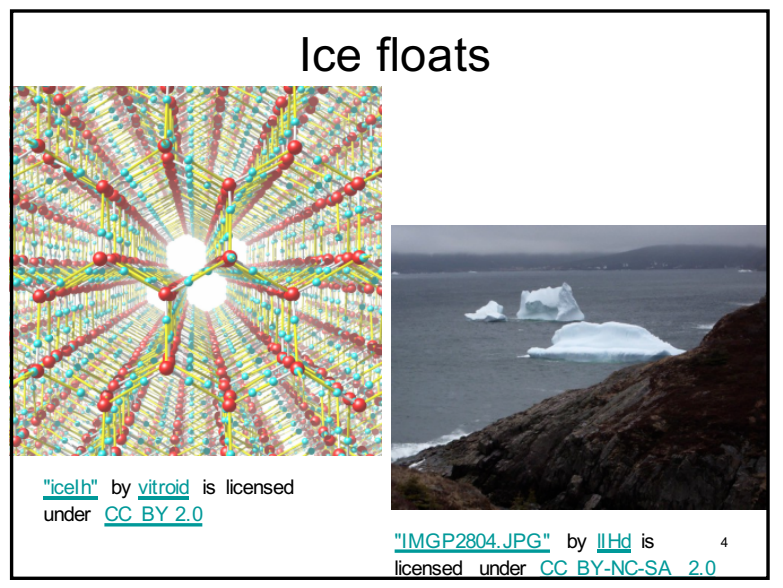

\section{ion}

- an atom or group of atoms that has lost or gained one or more electrons so that it is no longer electrically neutral

- Will form based on the 'stable' electron configuration of the noble gases (Group 8)

- ex: show the reaction of elemental potassium and elemental chlorine to form potassium chloride

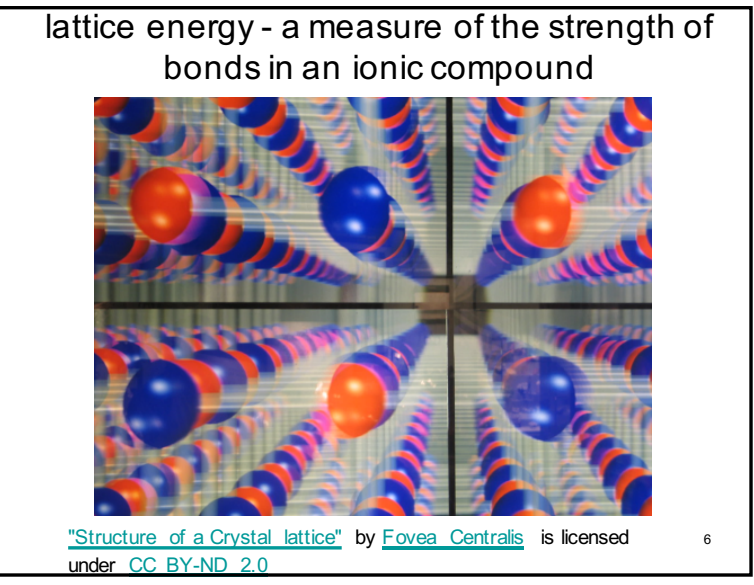




\section{Second Law of Thermodynamics}

Spontaneous processes involve an increase in the entropy (a measure of the disorder of the system) of the universe.

Show the reaction for the dissolution of solid $\mathrm{KCl}$ in water and summarize the interactions that must be overcome and the interactions that result after the dissolution.

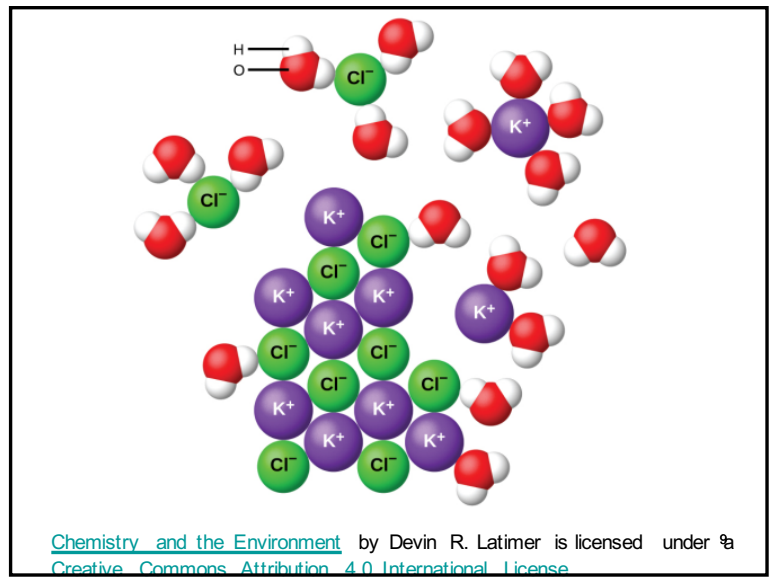


Now consider the following mixtures.

How soluble will they be in one another?

\section{$\mathrm{CH}_{3} \mathrm{CH}_{2} \mathrm{CH}_{2} \mathrm{CH}_{2} \mathrm{CH}_{3}$}

$+$

$\mathrm{H}_{2} \mathrm{O}$

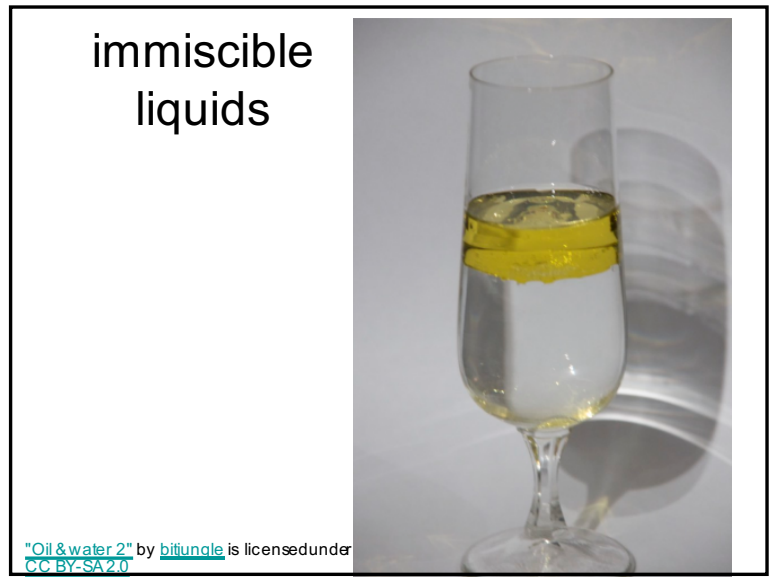

\section{$\mathrm{CH}_{3} \mathrm{CH}_{2} \mathrm{CH}_{2} \mathrm{CH}_{2} \mathrm{CH}_{3}$ \\ $+$}

$\mathrm{CH}_{3} \mathrm{CH}_{2} \mathrm{CH}_{2} \mathrm{CH}_{3}$ 


\section{"Like dissolves like"}

- Polar (hydrophilic) compounds normally dissolve other polar compounds, while non-polar

(hydrophobic) dissolve non-polar.

$\mathrm{CH}_{3} \mathrm{CH}_{2} \mathrm{OH}+\mathrm{H}_{2} \mathrm{O}$
$\mathrm{CH}_{3} \mathrm{CH}_{2} \mathrm{CH}_{2} \mathrm{OH}+\mathrm{H}_{2} \mathrm{O}$
$\mathrm{CH}_{3} \mathrm{CH}_{2} \mathrm{CH}_{2} \mathrm{CH}_{2} \mathrm{CH}_{2} \mathrm{CH}_{2} \mathrm{CH}_{2} \mathrm{CH}_{2} \mathrm{OH}$
$+\mathrm{H}_{2} \mathrm{O}$

\section{$\mathrm{CH}_{3} \mathrm{CH}_{2} \mathrm{CH}_{2} \mathrm{CH}_{2} \mathrm{CH}_{2} \mathrm{CH}_{2} \mathrm{CH}_{2} \mathrm{CH}_{2} \mathrm{OH}$}

$$
+\mathrm{H}_{2} \mathrm{O}
$$

- Now, estimate/measure the differential solubility of a compound between these two solvents.

$\mathrm{K}_{\mathrm{ow}}$ : octanol-water partition coefficient.

Environmental fate modelling 


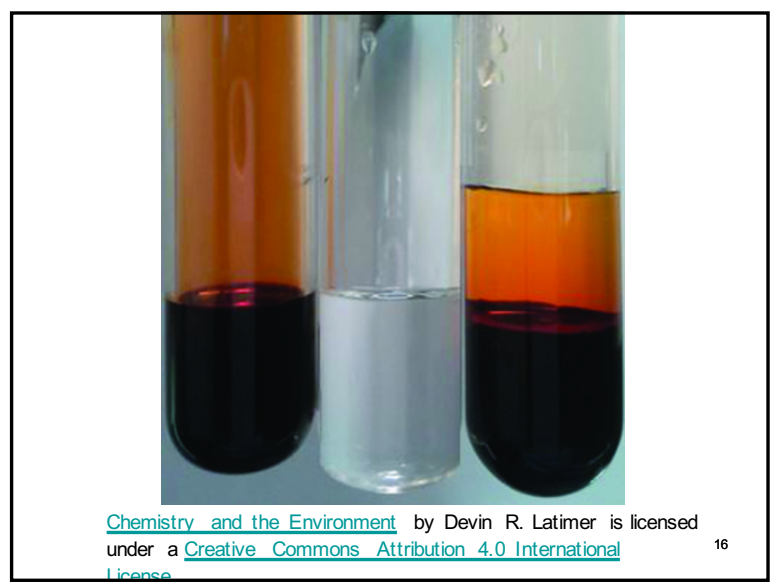

\section{Would you expect $\mathrm{MgCl} 2$ to be more soluble in water or the long chain hydrocarbon, \\ $\mathrm{CH}_{3} \mathrm{CH}_{2} \mathrm{CH}_{2} \mathrm{CH}_{2} \mathrm{CH}_{2} \mathrm{CH}_{2} \mathrm{CH}_{2} \mathrm{CH}_{2} \mathrm{OH}$ ?}

- A. Water

- B. $\mathrm{CH}_{3} \mathrm{CH}_{2} \mathrm{CH}_{2} \mathrm{CH}_{2} \mathrm{CH}_{2} \mathrm{CH}_{2} \mathrm{CH}_{2} \mathrm{CH}_{2} \mathrm{OH}$

- C. Equal solubility.

Would you expect

$\mathrm{CH}_{3} \mathrm{CH}_{2} \mathrm{CH}_{2} \mathrm{CH}_{2} \mathrm{CH}_{2} \mathrm{CH}_{2} \mathrm{CH}_{2} \mathrm{CH}_{2} \mathrm{Cl}$ to be more soluble in water or the long chain hydrocarbon, $\mathrm{CH}_{3} \mathrm{CH}_{2} \mathrm{CH}_{2} \mathrm{CH}_{2} \mathrm{CH}_{2} \mathrm{CH}_{2} \mathrm{CH}_{2} \mathrm{CH}_{2} \mathrm{OH}$ ?

- A. Water

- B. $\mathrm{CH}_{3} \mathrm{CH}_{2} \mathrm{CH}_{2} \mathrm{CH}_{2} \mathrm{CH}_{2} \mathrm{CH}_{2} \mathrm{CH}_{2} \mathrm{CH}_{2} \mathrm{OH}$

- C. Equal solubility. 
Compared to $\mathrm{CH}_{3} \mathrm{CH}_{2} \mathrm{OH}$, what would you expect the solubility properties of $\mathrm{CH}_{3} \mathrm{CH}_{2} \mathrm{CH}_{2} \mathrm{CH}_{2} \mathrm{OH}$ to be?

- A. More soluble in octanol, less soluble in water and organic matter.

- B. Less soluble in octanol, more soluble in water and organic matter.

- C. More soluble in octanol and organic matter, less soluble in water.

- D. Less soluble in octanol and organic matter, less soluble in water.

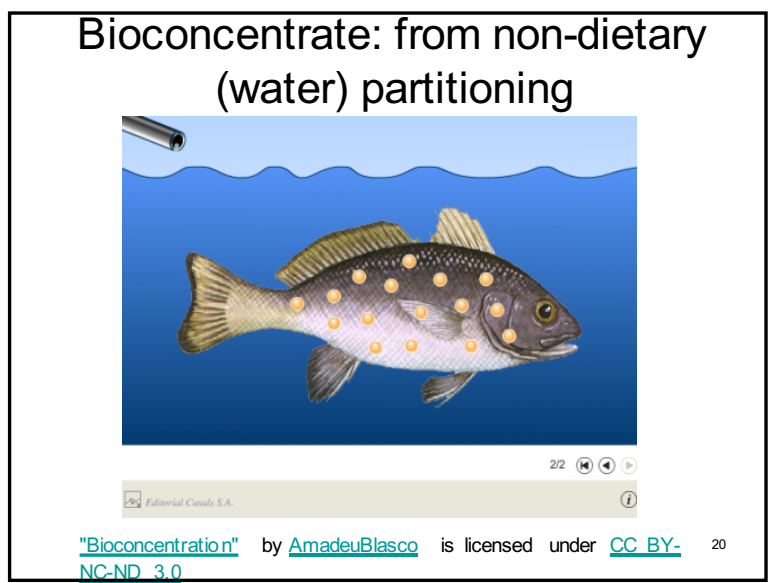

Biomagnify: concentration up the foodchain

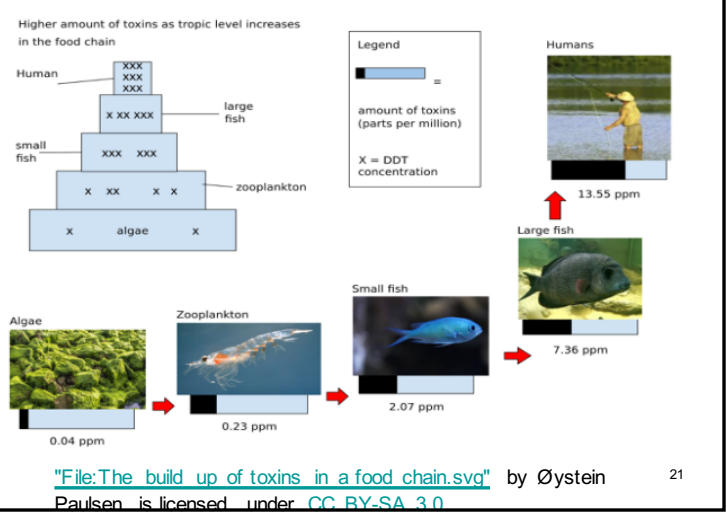



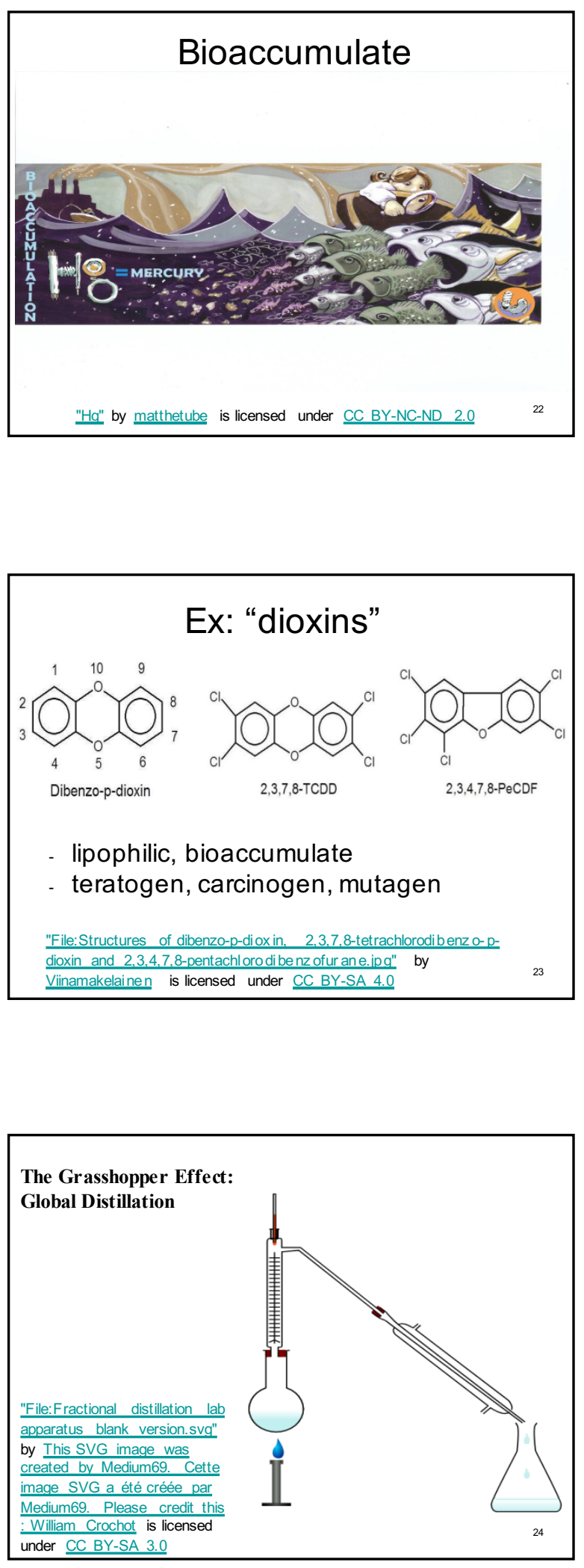


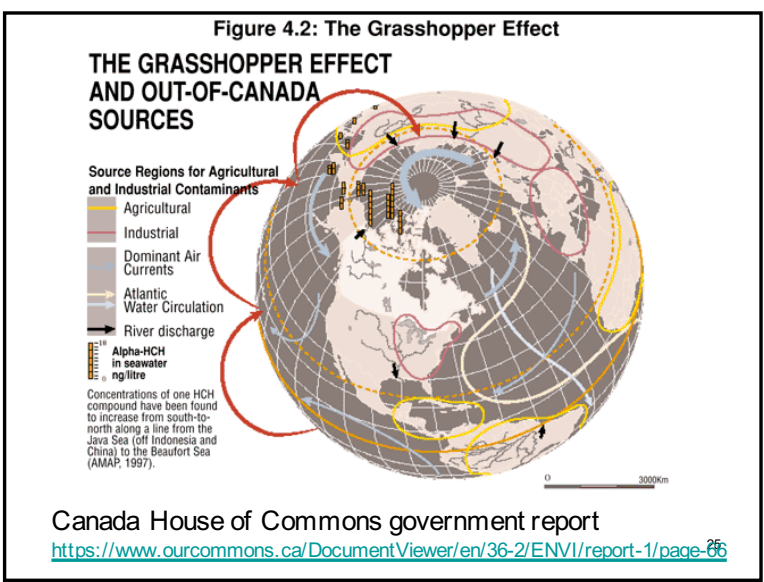

\begin{tabular}{|c|}
\hline Concentration Terms \\
Mass \% $=$ [mass solute/mass solution] x 100 \\
Molarity = moles solute/L of solution \\
Ex: What is the concentration (in mass \% \\
and $M$ ) of the resulting solution when you \\
add 5 grams of NaOH to 95 mL of water? \\
\end{tabular}

\begin{tabular}{|l|} 
Concentration Terms \\
Parts per hundred (percent) \\
$1 \mathrm{~g}$ of $\mathrm{NaCl}$ as part of $100 \mathrm{~g}$ of solution is a $1 \% \mathrm{NaCl}$ solution \\
1 in $100=1$ in $1 \times 10^{2}$ \\
Parts per million (ppm) \\
1 part solute in $1,000,000$ parts solvent $=1$ in $1 \times 10^{6}$ \\
Parts per billion (ppb) \\
1 part solute in $1,000,000,000$ parts solvent $=1$ in $1 \times 10^{9}$ \\
Ex: The maximum contaminant level [MCL] for dioxin in drinking \\
water is set at $3 \times 10^{-8}$ ppm. A sample of drinking water is found to \\
contain $2 \times 10^{-4}$ ppb. Is the sample of water safe to drink? \\
\end{tabular}




\section{The $1 \%$}

- For example, present carbon dioxide concentrations in air 0.040 \%

- $\mathrm{Q}$ - if the indoor $\mathrm{CO}_{2}$ concentration in a building is measured at $4002 \mathrm{ppm}$, would this be considered normal?

-ie. What is the $0.040 \%$ concentration expressed as ppm?

\section{The $1 \%$}

- For example, carbon dioxide $=0.040 \%$ or $400 \mathrm{ppm}$.

So, the measurement of $4002 \mathrm{ppm}$ is 10 times the normal tropospheric $\left[\mathrm{CO}_{2}\right]$. (most likely, more outside air would need to be introduced to the building)

- The permissible upper limit for concern for ground level carbon monoxide is given as $0.009 \mathrm{ppt}$. A researcherfinds the level in Winnipeg one day to be measuring at $6 \mathrm{ppm}$. What is the measured value in ppt and is this permissible?

-A. $0.06 \mathrm{ppt}$, this is not permissible

-B. $0.06 \mathrm{ppt}$, this is permissible

-C. $0.006 \mathrm{ppt}$, this is not permissible

-D. $0.006 \mathrm{ppt}$, this is permissible

-E. $0.0006 \mathrm{ppt}$, this not permissible 
Focus on Water Quality

- The permissible upper limit for ground level ozone is $0.12 \mathrm{ppm}$. A researcher finds the level in Toronto one day to be measuring at $580 \mathrm{ppb}$. What is the measured value in ppm and is this of concern?

-A. $58 \mathrm{ppm}$, this is of concern

-B. $0.00058 \mathrm{ppm}$, this is of no concern

-C. $0.58 \mathrm{ppm}$, this is of concern

-D. $58 \mathrm{ppm}$, this is of no concern

-E. $0.58 \mathrm{ppm}$, this is of no concern. 


\section{PART $X$}

\section{FUNDAMENTAL EQUILIBRIUM CONCEPTS}





\section{INTRODUCTION}

\section{Learning Objectives}

- Introduction to Chemical Equilibria

Transport of carbon dioxide in the body involves several reversible chemical reactions, including hydrolysis and acid ionization (among others).

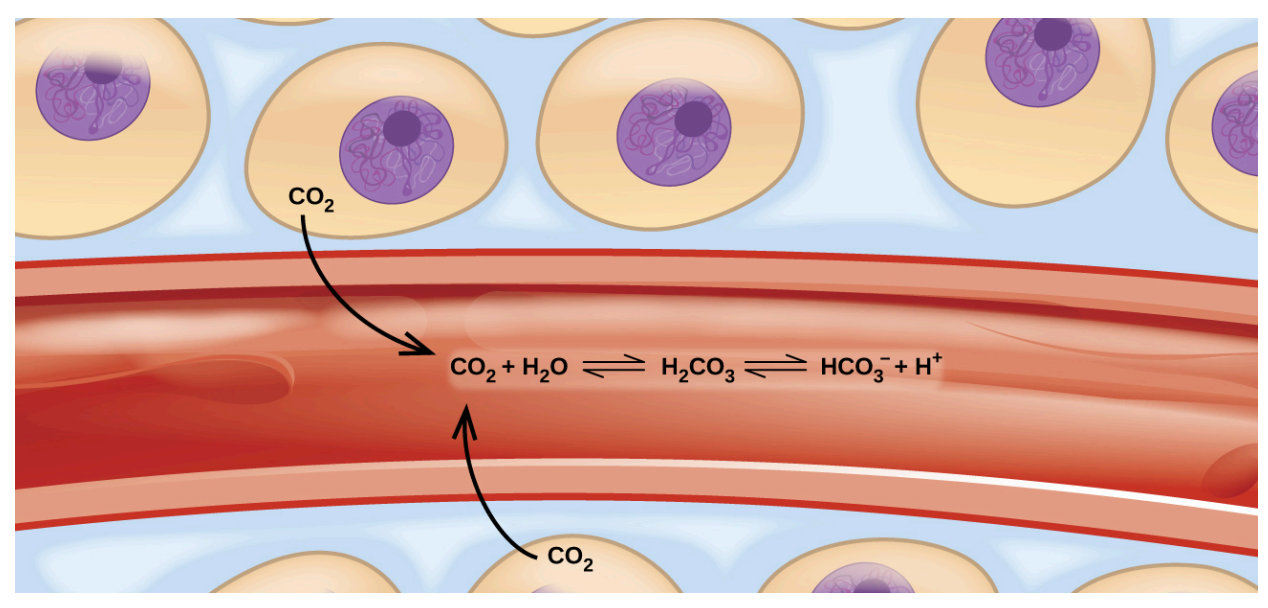

Imagine a beach populated with sunbathers and 
swimmers. As those basking in the sun get too hot, they enter the surf to swim and cool off. As the swimmers tire, they return to the beach to rest. If the rate at which sunbathers enter the surf were to equal the rate at which swimmers return to the sand, then the numbers (though not the identities) of sunbathers and swimmers would remain constant. This scenario illustrates a dynamic phenomenon known as equilibrium, in which opposing processes occur at equal rates. Chemical and physical processes are subject to this phenomenon; these processes are at equilibrium when the forward and reverse reaction rates are equal. Equilibrium systems are pervasive in nature; the various reactions involving carbon dioxide dissolved in blood are examples (see (Figure)). This chapter provides a thorough introduction to the essential aspects of chemical equilibria. 
48.

\section{CHEMICAL EQUILIBRIA}

Learning Objectives

By the end of this section, you will be able to:

- Describe the nature of equilibrium systems

- Explain the dynamic nature of a chemical equilibrium

The convention for writing chemical equations involves placing reactant formulas on the left side of a reaction arrow and product formulas on the right side. By this convention, and the definitions of "reactant" and "product," a chemical equation represents the reaction in question as proceeding from left to right. Reversible reactions, however, may proceed in both forward (left to right) and reverse (right to left) directions. When the rates of the forward and reverse reactions are equal, the concentrations of the reactant and product species remain constant over time and the system is at equilibrium. The 
relative concentrations of reactants and products in equilibrium systems vary greatly; some systems contain mostly products at equilibrium, some contain mostly reactants, and some contain appreciable amounts of both.

(Figure) illustrates fundamental equilibrium concepts using the reversible decomposition of colorless dinitrogen tetroxide to yield brown nitrogen dioxide, an elementary reaction described by the equation:

$\mathrm{N}_{2} \mathrm{O}_{4}(g) 2 \mathrm{NO}_{2}(g)$

Note that a special double arrow is used to emphasize the reversible nature of the reaction.

(a) A sealed tube containing colorless $\mathrm{N}_{2} \mathrm{O}_{4}$ darkens as it decomposes to yield brown $\mathrm{NO}_{2}$. (b) Changes in concentration over time as the decomposition reaction achieves equilibrium. (c) At equilibrium, the forward and reverse reaction rates are equal. 


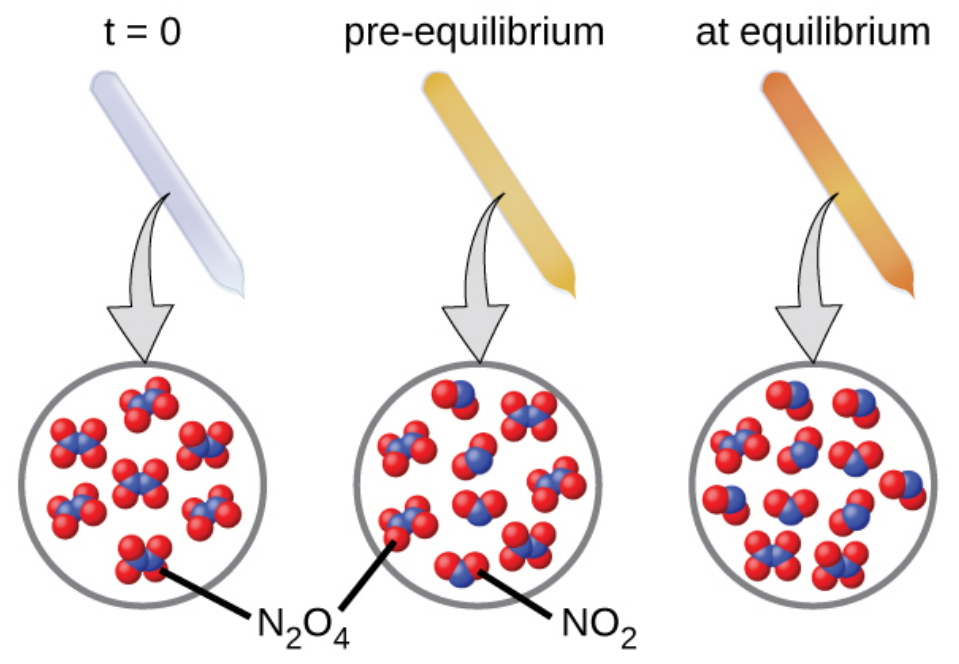

(a)

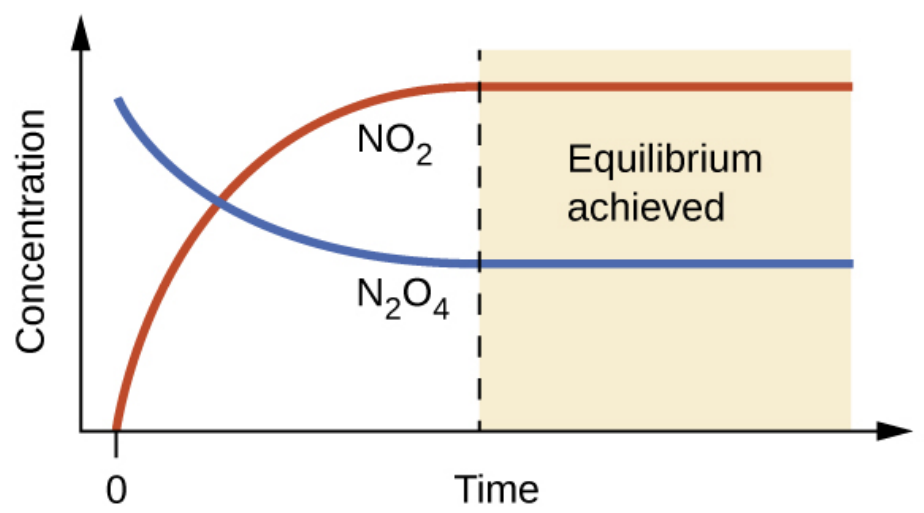

(b)

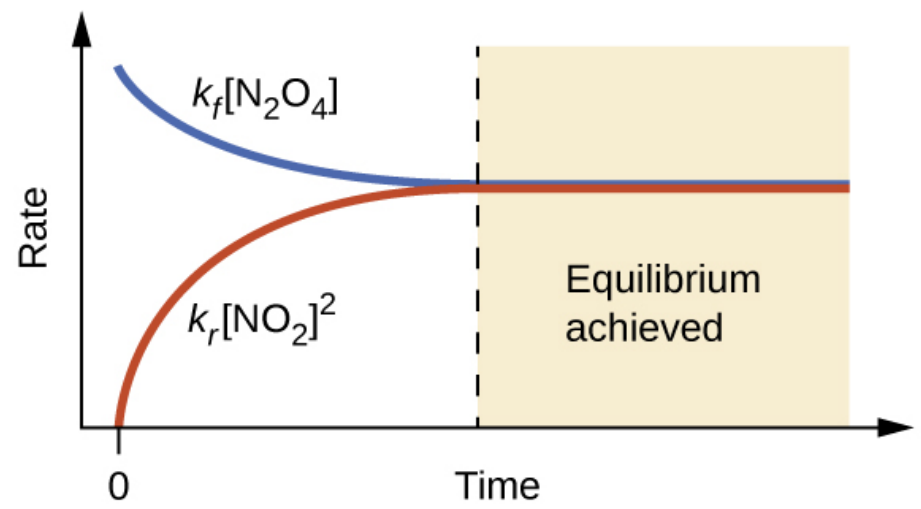

(c)

For this elementary process, rate laws for the forward and 
reverse reactions may be derived directly from the reaction stoichiometry:

rate $_{f}=k_{f}\left[\mathrm{~N}_{-2} \mathrm{O}_{4}\right]$

rate $_{r}=k_{r}\left[\mathrm{NO}_{2}\right]^{2}$

As the reaction begins $(t=0)$, the concentration of the $\mathrm{N}_{2} \mathrm{O}_{4}$ reactant is finite and that of the $\mathrm{NO}_{2}$ product is zero, so the forward reaction proceeds at a finite rate while the reverse reaction rate is zero. As time passes, $\mathrm{N}_{-2} \mathrm{O}_{4}$ is consumed and its concentration falls, while $\mathrm{NO}_{2}$ is produced and its concentration increases ((Figure)b). The decreasing concentration of the reactant slows the forward reaction rate, and the increasing product concentration speeds the reverse reaction rate ((Figure)c). This process continues until the forward and reverse reaction rates become equal, at which time the reaction has reached equilibrium, as characterized by constant concentrations of its reactants and products (shaded areas of (Figure)b and (Figure)c). It's important to emphasize that chemical equilibria are dynamic; a reaction at equilibrium has not "stopped," but is proceeding in the forward and reverse directions at the same rate. This dynamic nature is essential to understanding equilibrium behavior as discussed in this and subsequent chapters of the text.

A two-person juggling act illustrates the dynamic aspect of chemical equilibria. Each person is throwing and catching clubs at the same rate, and each holds a (approximately) constant number of clubs. 


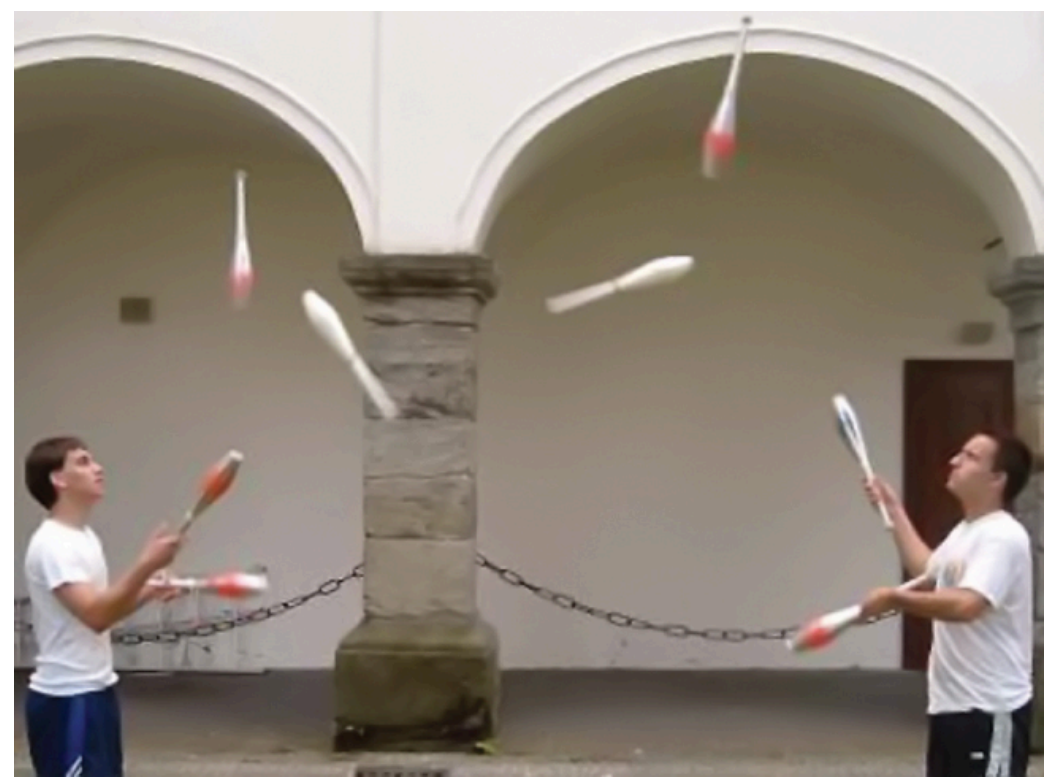

Physical changes, such as phase transitions, are also reversible and may establish equilibria. This concept was introduced in another chapter of this text through discussion of the vapor pressure of a condensed phase (liquid or solid). As one example, consider the vaporization of bromine:

$\mathrm{Br}_{2}(l) \mathrm{Br}_{2}(g)$

When liquid bromine is added to an otherwise empty container and the container is sealed, the forward process depicted above (vaporization) will commence and continue at a roughly constant rate as long as the exposed surface area of the liquid and its temperature remain constant. As increasing amounts of gaseous bromine are produced, the rate of the reverse process (condensation) will increase until it equals the rate of vaporization and equilibrium is established. A photograph showing this phase transition equilibrium is provided in (Figure). A sealed tube containing an equilibrium mixture of liquid and gaseous bromine. (credit: http://images-of- 
elements.com/bromine.php)

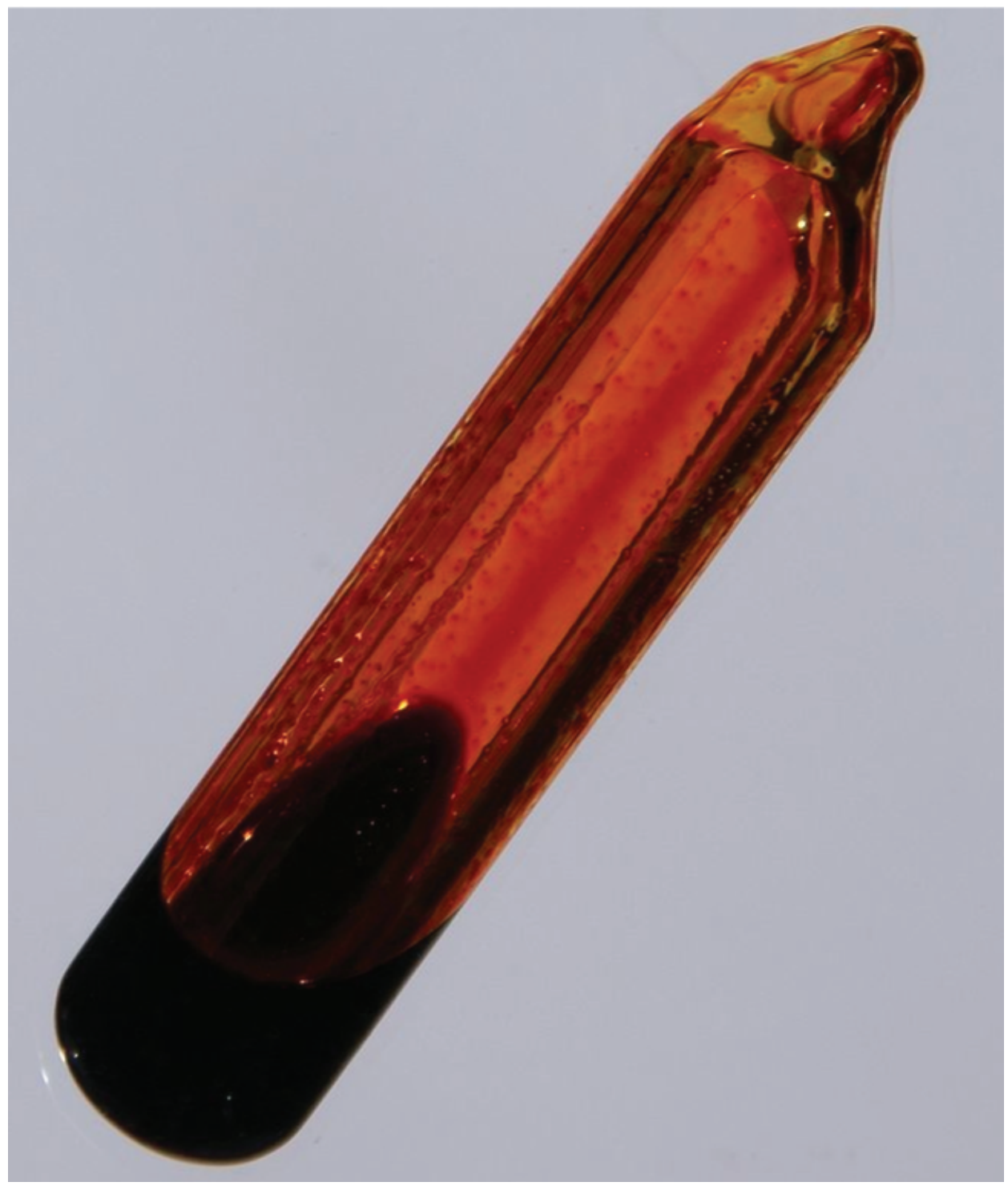

Key Concepts and Summary

A reversible reaction is at equilibrium when the forward and reverse processes occur at equal rates. Chemical equilibria are dynamic processes characterized by constant amounts of reactant and product species. 
Chemistry End of Chapter Exercises

What does it mean to describe a reaction as "reversible"?

The reaction can proceed in both the forward and reverse directions.

When writing an equation, how is a reversible reaction distinguished from a nonreversible reaction?

If a reaction is reversible, when can it be said to have reached equilibrium?

When a system has reached equilibrium, no further changes in the reactant and product concentrations occur; the forward and reverse reactions continue to proceed, but at equal rates.

Is a system at equilibrium if the rate constants of the forward and reverse reactions are equal?

If the concentrations of products and reactants are equal, is the system at equilibrium?

Not necessarily. A system at equilibrium is characterized by constant reactant and product concentrations, but the values of the reactant and product concentrations themselves need not be equal. 
Glossary

equilibrium

state of a reversible reaction in which the forward and reverse processes occur at equal rates

reversible reaction

chemical reaction that can proceed in both the forward and reverse directions under given conditions 


\section{PART XI}

\section{ACID-BASE EQUILIBRIA}



49.

\section{INTRODUCTION}

Learning Objectives

- Brønsted-Lowry Acids and Bases

- $\mathrm{pH}$ and $\mathrm{pOH}$

Sinkholes such as this are the result of reactions between acidic groundwaters and basic rock formations, like limestone. (credit: modification of work by Emil Kehnel)

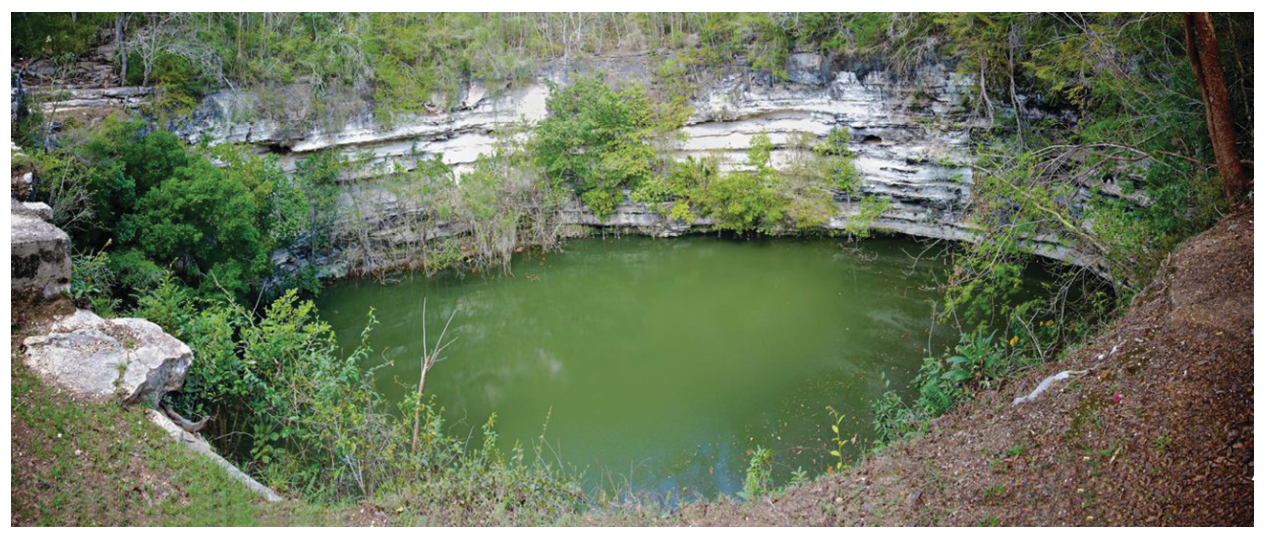

Liquid water is essential to life on our planet, and 
chemistry involving the characteristic ions of water, $\mathrm{H}^{+}$ and $\mathrm{OH}^{\hat{\mathrm{a}}}$, , is widely encountered in nature and society. As introduced in another chapter of this text, acid-base chemistry involves the transfer of hydrogen ions from donors (acids) to acceptors (bases). These $\mathrm{H}+$ transfer reactions are reversible, and the equilibria established by acid-base systems are essential aspects of phenomena ranging from sinkhole formation ((Figure)) to oxygen transport in the human body. This chapter will further explore acid-base chemistry with an emphasis on the equilibrium aspects of this important reaction class. 


\section{0.}

\section{BRDNSTED-LOWRY ACIDS AND BASES}

Learning Objectives

By the end of this section, you will be able to:

- Identify acids, bases, and conjugate acid-base pairs according to the Brønsted-Lowry definition

- Write equations for acid and base ionization reactions

- Use the ion-product constant for water to calculate hydronium and hydroxide ion concentrations

- $\quad$ Describe the acid-base behavior of amphiprotic substances

The acid-base reaction class has been studied for quite some time. In 1680, Robert Boyle reported traits of acid solutions that included their ability to dissolve many 
substances, to change the colors of certain natural dyes, and to lose these traits after coming in contact with alkali (base) solutions. In the eighteenth century, it was recognized that acids have a sour taste, react with limestone to liberate a gaseous substance (now known to be $\mathrm{CO}_{2}$ ), and interact with alkalis to form neutral substances. In 1815, Humphry Davy contributed greatly to the development of the modern acid-base concept by demonstrating that hydrogen is the essential constituent of acids. Around that same time, Joseph Louis Gay-Lussac concluded that acids are substances that can neutralize bases and that these two classes of substances can be defined only in terms of each other. The significance of hydrogen was reemphasized in 1884 when Svante Arrhenius defined an acid as a compound that dissolves in water to yield hydrogen cations (now recognized to be hydronium ions) and a base as a compound that dissolves in water to yield hydroxide anions.

Johannes Brønsted and Thomas Lowry proposed a more general description in 1923 in which acids and bases were defined in terms of the transfer of hydrogen ions, $\mathrm{H}^{+}$. (Note that these hydrogen ions are often referred to simply as protons, since that subatomic particle is the only component of cations derived from the most abundant hydrogen isotope, ${ }^{1} \mathrm{H}$.) A compound that donates a proton to another compound is called a Brønsted-Lowry acid, and a compound that accepts a proton is called a BrønstedLowry base. An acid-base reaction is, thus, the transfer of a proton from a donor (acid) to an acceptor (base).

The concept of conjugate pairs is useful in describing Brønsted-Lowry acid-base reactions (and other reversible reactions, as well). When an acid donates $\mathrm{H}^{+}$, the species 
that remains is called the conjugate base of the acid because it reacts as a proton acceptor in the reverse reaction. Likewise, when a base accepts $\mathrm{H}^{+}$, it is converted to its conjugate acid. The reaction between water and ammonia illustrates this idea. In the forward direction, water acts as an acid by donating a proton to ammonia and subsequently becoming a hydroxide ion, $\mathrm{OH}^{-}$, the conjugate base of water. The ammonia acts as a base in accepting this proton, becoming an ammonium ion, $\mathrm{NH}_{4}{ }^{+}$, the conjugate acid of ammonia. In the reverse direction, a hydroxide ion acts as a base in accepting a proton from ammonium ion, which acts as an acid.

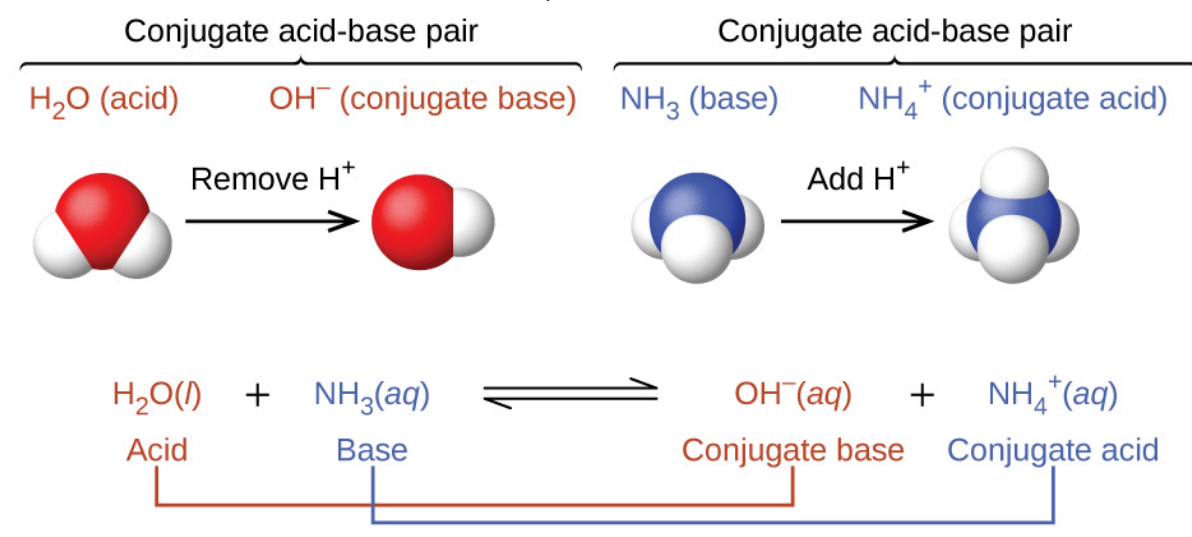

The reaction between a Brønsted-Lowry acid and water is called acid ionization. For example, when hydrogen fluoride dissolves in water and ionizes, protons are transferred from hydrogen fluoride molecules to water molecules, yielding hydronium ions and fluoride ions:

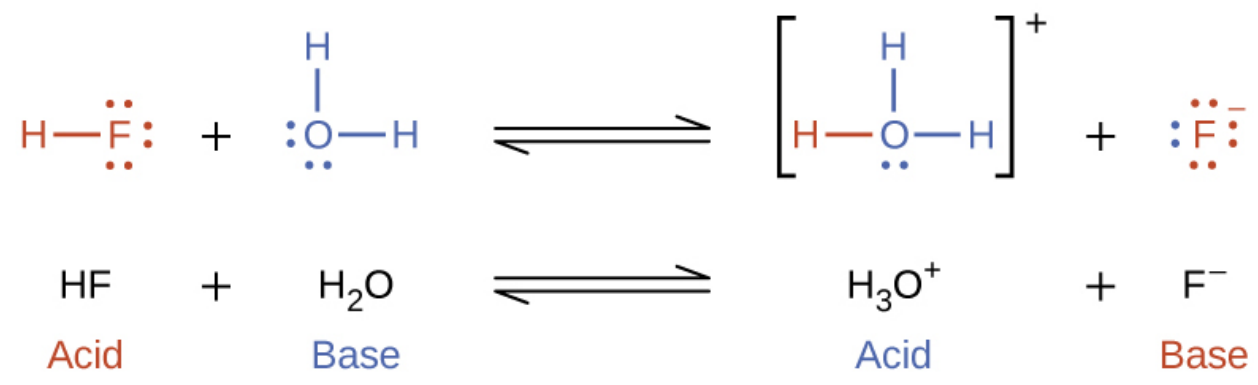

Base ionization of a species occurs when it accepts 
protons from water molecules. In the example below, pyridine molecules, $\mathrm{C}_{5} \mathrm{NH}_{5}$, undergo base ionization when dissolved in water, yielding hydroxide and pyridinium ions:

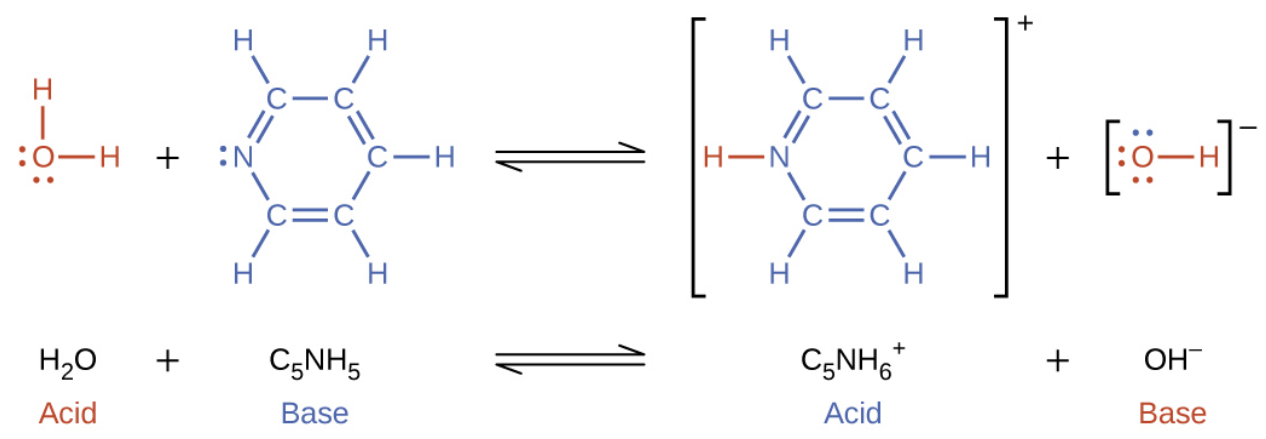

The preceding ionization reactions suggest that water may function as both a base (as in its reaction with hydrogen fluoride) and an acid (as in its reaction with ammonia). Species capable of either donating or accepting protons are called amphiprotric, or more generally, amphoteric, a term that may be used for acids and bases per definitions other than the Brønsted-Lowry one. The equations below show the two possible acid-base reactions for two amphiprotic species, bicarbonate ion and water:

$$
\begin{array}{ll}
\mathrm{HCO}_{3}^{-}(\mathrm{aq})+\mathrm{H}_{2} \mathrm{O}(\mathrm{l}) & \mathrm{CO}_{3}^{2-}(\mathrm{aq})+\mathrm{H}_{3} \mathrm{O}^{+}(\mathrm{aq}) \\
\mathrm{HCO}_{3}{ }^{-}(\mathrm{aq})+\mathrm{H}_{2} \mathrm{O}(\mathrm{l}) & \mathrm{H}_{2} \mathrm{CO}_{3}(\mathrm{aq})+\mathrm{OH}^{-}(\mathrm{aq})
\end{array}
$$

The first equation represents the reaction of bicarbonate as an acid with water as a base, whereas the second represents reaction of bicarbonate as a base with water as an acid. When bicarbonate is added to water, both these equilibria are established simultaneously and the composition of the resulting solution may be determined through appropriate equilibrium calculations, as described later in this chapter.

In the liquid state, molecules of an amphiprotic 
substance can react with one another as illustrated for water in the equations below:

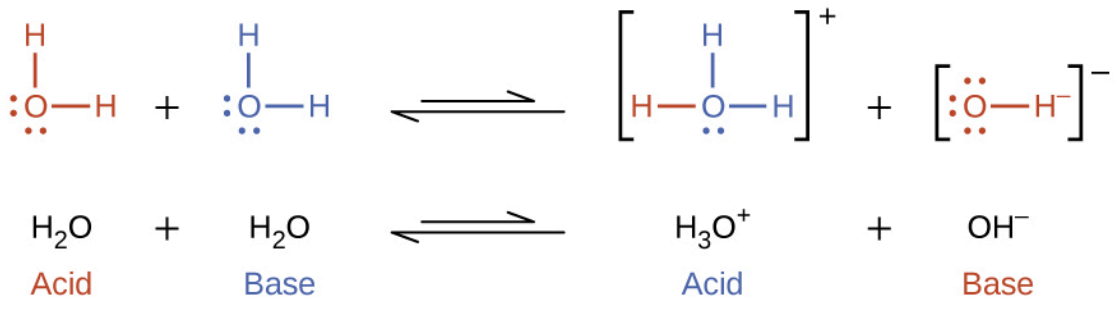

The process in which like molecules react to yield ions is called autoionization. Liquid water undergoes autoionization to a very slight extent; at $25{ }^{\circ} \mathrm{C}$, approximately two out of every billion water molecules are ionized. The extent of the water autoionization process is reflected in the value of its equilibrium constant, the ion-product constant for water, $K_{\mathrm{w}}$ :

$$
\mathrm{H}_{2} \mathrm{O}(l)+\mathrm{H}_{2} \mathrm{O}(l) \mathrm{H}_{3} \mathrm{O}^{+}(a q)+\mathrm{OH}(a q) \quad K_{\mathrm{w}}=\left[\mathrm{H}_{3} \mathrm{O}^{+}\right][\mathrm{OH}]
$$

The slight ionization of pure water is reflected in the small value of the equilibrium constant; at $25^{\circ} \mathrm{C}, K_{\mathrm{W}}$ has a value of $1.010^{-14}$. The process is endothermic, and so the extent of ionization and the resulting concentrations of hydronium ion and hydroxide ion increase with temperature. For example, at $100{ }^{\circ} \mathrm{C}$, the value for $K_{\mathrm{w}}$ is about $5.610^{-13}$, roughly 50 times larger than the value at $25^{\circ} \mathrm{C}$.

Ion Concentrations in Pure Water What are the hydronium ion concentration and the hydroxide ion concentration in pure water at $25^{\circ} \mathrm{C}$ ?

Solution The autoionization of water yields the same number of hydronium and hydroxide ions. Therefore, in pure water, $\left[\mathrm{H}_{3} \mathrm{O}^{+}\right]=\left[\mathrm{OH}^{-}\right]=x$. At $25^{\circ} \mathrm{C}$ : 


$$
K_{\mathrm{w}}=\left[\mathrm{H}_{3} \mathrm{O}^{+}\right][\mathrm{OH}]=x=1.010^{-14}
$$

So:

$$
x=\left[\mathrm{H}_{3} \mathrm{O}^{+}\right]=[\mathrm{OH}]=\sqrt{1.010^{-14}}=1.010^{-7} M
$$

The hydronium ion concentration and the hydroxide ion concentration are the same, $1.010^{-7} \mathrm{M}$.

Check Your Learning The ion product of water at $80{ }^{\circ} \mathrm{C}$ is 2.4 $10^{-13}$. What are the concentrations of hydronium and hydroxide ions in pure water at $80^{\circ} \mathrm{C}$ ?

Answer:

$$
\left[\mathrm{H}_{3} \mathrm{O}^{+}\right]=\left[\mathrm{OH}^{-}\right]=4.910^{-7} \mathrm{M}
$$

The Inverse Relation between $\left[\mathrm{H}_{3} \mathrm{O}^{+}\right]$and $\left[\mathrm{OH}^{-}\right]$A solution of an acid in water has a hydronium ion concentration of 2.0 $10^{-6} \mathrm{M}$. What is the concentration of hydroxide ion at $25^{\circ} \mathrm{C}$ ?

Solution Use the value of the ion-product constant for water at $25^{\circ} \mathrm{C}$

$$
2 \mathrm{H}_{2} \mathrm{O}(l) \mathrm{H}_{3} \mathrm{O}^{+}(a q)+\mathrm{OH}(a q) \quad K_{\mathrm{w}}=\left[\mathrm{H}_{3} \mathrm{O}^{+}\right][\mathrm{OH}]=1.010^{-14}
$$

to calculate the missing equilibrium concentration.

Rearrangement of the $K_{w}$ expression shows that $\left[\mathrm{OH}^{-}\right]$is inversely proportional to $\left[\mathrm{H}_{3} \mathrm{O}^{+}\right]$:

$$
[\mathrm{OH}]=\frac{K_{\mathrm{w}}}{\left[\mathrm{H}_{3} \mathrm{O}^{+}\right]}=\frac{1.010^{-14}}{2.010^{-6}}=5.010^{-9}
$$

Compared with pure water, a solution of acid exhibits a higher concentration of hydronium ions (due to ionization of the acid) and a proportionally lower concentration of hydroxide ions. This may be explained via Le Châtelier's principle as a left shift in the water autoionization equilibrium resulting from the stress of increased hydronium ion concentration.

Substituting the ion concentrations into the $K_{w}$ expression confirms this calculation, resulting in the expected value: 


$$
K_{\mathrm{w}}=\left[\mathrm{H}_{3} \mathrm{O}^{+}\right][\mathrm{OH}]=\left(\begin{array}{lll}
2.0 & 10^{-6}
\end{array}\right)\left(\begin{array}{lll}
5.0 & 10^{-9}
\end{array}\right)=1.010^{-14}
$$

Check Your Learning What is the hydronium ion concentration in an aqueous solution with a hydroxide ion concentration of $0.001 \mathrm{M}$ at $25^{\circ} \mathrm{C}$ ?

Answer:

$\left[\mathrm{H}_{3} \mathrm{O}^{+}\right]=110^{-11} \mathrm{M}$

Representing the Acid-Base Behavior of an Amphoteric Substance Write separate equations representing the reaction of $\mathrm{HSO}_{3}$

(a) as an acid with $\mathrm{OH}^{-}$

(b) as a base with $\mathrm{HI}$

Solution (a)

$\mathrm{HSO}_{3}(a q)+\mathrm{OH}(a q) \mathrm{SO}_{3}^{2}(a q)+\mathrm{H}_{2} \mathrm{O}(l)$

(b) $\mathrm{HSO}_{3}(a q)+\mathrm{HI}(a q) \mathrm{H}_{2} \mathrm{SO}_{3}(a q)+\mathrm{I}(a q)$

Check Your Learning Write separate equations representing the reaction of $\mathrm{H}_{2} \mathrm{PO}_{4}$

(a) as a base with $\mathrm{HBr}$

(b) as an acid with $\mathrm{OH}^{-}$

Answer:

(a)

$\mathrm{H}_{2} \mathrm{PO}_{4}(a q)+\mathrm{HBr}(a q) \mathrm{H}_{3} \mathrm{PO}_{4}(a q)+\mathrm{Br}(a q)$;

(b) $\mathrm{H}_{2} \mathrm{PO}_{4}(a q)+\mathrm{OH}(a q) \mathrm{HPO}_{4}^{2}(a q)+\mathrm{H}_{2} \mathrm{O}(l)$

Key Concepts and Summary

A compound that can donate a proton (a hydrogen ion) to another compound is called a Brønsted-Lowry acid. The compound that accepts the proton is called a BrønstedLowry base. The species remaining after a Brønsted-Lowry 
acid has lost a proton is the conjugate base of the acid. The species formed when a Brønsted-Lowry base gains a proton is the conjugate acid of the base. Thus, an acidbase reaction occurs when a proton is transferred from an acid to a base, with formation of the conjugate base of the reactant acid and formation of the conjugate acid of the reactant base. Amphiprotic species can act as both proton donors and proton acceptors. Water is the most important amphiprotic species. It can form both the hydronium ion, $\mathrm{H}_{3} \mathrm{O}^{+}$, and the hydroxide ion, $\mathrm{OH}^{-}$when it undergoes autoionization:

$2 \mathrm{H}_{2} \mathrm{O}\left(l \mathrm{H}_{3} \mathrm{O}^{+}(a q)+\mathrm{OH}(a q)\right.$

The ion product of water, $K_{\mathrm{W}}$ is the equilibrium constant for the autoionization reaction:

$K_{\mathrm{w}}=\left[\mathrm{H}_{3} \mathrm{O}^{+}\right][\mathrm{OH}]=1.010^{-14}$ at $25 \mathrm{C}$

Key Equations

- $K_{\mathrm{W}}=\left[\mathrm{H}_{3} \mathrm{O}^{+}\right]\left[\mathrm{OH}^{-}\right]=1.010^{-14}\left(\right.$ at $\left.25^{\circ} \mathrm{C}\right)$

Chemistry End of Chapter Exercises

Write equations that show $\mathrm{NH}_{3}$ as both a conjugate acid and a conjugate base.

One example for $\mathrm{NH}_{3}$ as a conjugate acid: $\mathrm{NH}_{2}+\mathrm{H}^{+} \mathrm{NH}_{3}$; as a conjugate base: $\mathrm{NH}_{4}+(a q)+\mathrm{OH}(a q) \mathrm{NH}_{3}(a q)+\mathrm{H}_{2} \mathrm{O}(l)$

Write equations that show $\mathrm{H}_{2} \mathrm{PO}_{4}$ acting both as an acid and as a base. 
Show by suitable net ionic equations that each of the following species can act as a Brønsted-Lowry acid:
(a) $\mathrm{H}_{3} \mathrm{O}^{+}$
(b) $\mathrm{HCl}$
(c) $\mathrm{NH}_{3}$
(d) $\mathrm{CH}_{3} \mathrm{CO}_{2} \mathrm{H}$
(e) $\mathrm{NH}_{4}+$
(f) $\mathrm{HSO}_{4}$

(a)

$$
\mathrm{H}_{3} \mathrm{O}^{+}(a q) \mathrm{H}^{+}(a q)+\mathrm{H}_{2} \mathrm{O}(l) ;
$$

$\mathrm{HCl}(\mathrm{aq}) \mathrm{H}^{+}(a q)+\mathrm{Cl}(a q)$;

$\mathrm{NH}_{3}(a q) \mathrm{H}^{+}(a q)+\mathrm{NH}_{2}(a q)$;

$\mathrm{CH}_{3} \mathrm{CO}_{2} \mathrm{H}(a q) \mathrm{H}^{+}(a q)+\mathrm{CH}_{3} \mathrm{CO}_{2}(a q)$;

$\mathrm{NH}_{4}^{+}(a q) \mathrm{H}^{+}(a q)+\mathrm{NH}_{3}(a q)$;

$\begin{array}{lll}\mathrm{HSO}_{4}(a q) & \mathrm{H}^{+}(a q)+\mathrm{SO}_{4}^{2}(a q)\end{array}$

Show by suitable net ionic equations that each of the following species can act as a Brønsted-Lowry acid:
(a) $\mathrm{HNO}_{3}$
(b) $\mathrm{PH}_{4}{ }^{+}$
(c) $\mathrm{H}_{2} \mathrm{~S}$
(d) $\mathrm{CH}_{3} \mathrm{CH}_{2} \mathrm{COOH}$
(e) $\mathrm{H}_{2} \mathrm{PO}_{4}$
(f) $\mathrm{HS}^{-}$

Show by suitable net ionic equations that each of the following species can act as a Brønsted-Lowry base:
(a) $\mathrm{H}_{2} \mathrm{O}$
(b) $\mathrm{OH}^{-}$
(c) $\mathrm{NH}_{3}$
(d) $\mathrm{CN}^{-}$
(e) $\mathrm{S}^{2-}$ 
(f) $\mathrm{H}_{2} \mathrm{PO}_{4}$

(a)

$$
\mathrm{H}_{2} \mathrm{O}(l)+\mathrm{H}^{+}(a q) \mathrm{H}_{3} \mathrm{O}^{+}(a q) ;
$$

$\mathrm{OH}(a q)+\mathrm{H}^{+}(a q) \mathrm{H}_{2} \mathrm{O}(l)$;

$\mathrm{NH}_{3}(a q)+\mathrm{H}^{+}(a q) \mathrm{NH}_{4}{ }^{+}(a q)$;

$\mathrm{CN}(a q)+\mathrm{H}^{+}(a q) \mathrm{HCN}(a q)$;

$\mathrm{S}^{2-}(a q)+\mathrm{H}^{+}(a q) \mathrm{HS}(a q)$;

$\mathrm{H}_{2} \mathrm{PO}_{4}(a q)+\mathrm{H}^{+}(a q) \quad \mathrm{H}_{3} \mathrm{PO}_{4}(a q)$

Show by suitable net ionic equations that each of the following species can act as a Brønsted-Lowry base:
(a) $\mathrm{HS}^{-}$
(b) $\mathrm{PO}_{4}{ }^{3}$
(c) $\mathrm{NH}_{2}$
(d) $\mathrm{C}_{2} \mathrm{H}_{5} \mathrm{OH}$
(e) $\mathrm{O}^{2-}$
(f) $\mathrm{H}_{2} \mathrm{PO}_{4}$

What is the conjugate acid of each of the following? What is the conjugate base of each?
(a) $\mathrm{OH}^{-}$
(b) $\mathrm{H}_{2} \mathrm{O}$
(c) $\mathrm{HCO}_{3}$
(d) $\mathrm{NH}_{3}$
(e) $\mathrm{HSO}_{4}$
(f) $\mathrm{H}_{2} \mathrm{O}_{2}$
(g) $\mathrm{HS}^{-}$
(h) $\mathrm{H}_{5} \mathrm{~N}_{2}{ }^{+}$

(a) $\mathrm{H}_{2} \mathrm{O}, \mathrm{O}^{2-}$; (b) $\mathrm{H}_{3} \mathrm{O}^{+}, \mathrm{OH}^{-}$; (c) $\mathrm{H}_{2} \mathrm{CO}_{3}, \mathrm{CO}_{3}{ }^{2}$; (d) $\mathrm{NH}_{4}{ }^{+}$, $\mathrm{NH}_{2}$; (e) $\mathrm{H}_{2} \mathrm{SO}_{4}, \mathrm{SO}_{4}^{2}$; (f) $\mathrm{H}_{3} \mathrm{O}_{2}{ }^{+}, \mathrm{HO}_{2}$; (g) $\mathrm{H}_{2} \mathrm{~S} ; \mathrm{S}^{2-}$; (h) $\mathrm{H}_{6} \mathrm{~N}_{2}{ }^{2+}, \mathrm{H}_{4} \mathrm{~N}_{2}$ 
What is the conjugate acid of each of the following? What is the conjugate base of each?
(a) $\mathrm{H}_{2} \mathrm{~S}$
(b) $\mathrm{H}_{2} \mathrm{PO}_{4}$
(c) $\mathrm{PH}_{3}$
(d) $\mathrm{HS}^{-}$
(e) $\mathrm{HSO}_{3}$
(f) $\mathrm{H}_{3} \mathrm{O}_{2}^{+}$
(g) $\mathrm{H}_{4} \mathrm{~N}_{2}$
(h) $\mathrm{CH}_{3} \mathrm{OH}$

Identify and label the Brønsted-Lowry acid, its conjugate base, the Brønsted-Lowry base, and its conjugate acid in each of the following equations:

(a) $\mathrm{HNO}_{3}+\mathrm{H}_{2} \mathrm{O} \mathrm{H} \mathrm{H}_{3} \mathrm{O}^{+}+\mathrm{NO}_{3}$

(b) $\mathrm{CN}+\mathrm{H}_{2} \mathrm{OHCN}+\mathrm{OH}$

(c) $\mathrm{H}_{2} \mathrm{SO}_{4}+\mathrm{Cl} \mathrm{HCl}+\mathrm{HSO}_{4}$

(d) $\mathrm{HSO}_{4}+\mathrm{OH} \mathrm{SO} \mathrm{SO}_{4}^{2}+\mathrm{H}_{2} \mathrm{O}$

(e) $\mathrm{O}^{2-}+\mathrm{H}_{2} \mathrm{O} 2 \mathrm{OH}$

(f)

$\left[\mathrm{Cu}\left(\mathrm{H}_{2} \mathrm{O}\right)_{3}(\mathrm{OH})\right]^{+}+\left[\mathrm{Al}\left(\mathrm{H}_{2} \mathrm{O}\right)_{6}\right]^{3+}\left[\mathrm{Cu}\left(\mathrm{H}_{2} \mathrm{O}\right)_{4}\right]^{2+}+\left[\mathrm{Al}\left(\mathrm{H}_{2} \mathrm{O}\right)_{5}(\mathrm{OH})\right]^{2+}$ (g) $\mathrm{H}_{2} \mathrm{~S}+\mathrm{NH}_{2} \mathrm{HS}+\mathrm{NH}_{3}$

The labels are Brønsted-Lowry acid = BA; its conjugate base $=\mathrm{CB}$; Brønsted-Lowry base $=\mathrm{BB}$; its conjugate acid = CA. (a) $\mathrm{HNO}_{3}(\mathrm{BA}), \mathrm{H}_{2} \mathrm{O}(\mathrm{BB}), \mathrm{H}_{3} \mathrm{O}^{+}(\mathrm{CA}), \mathrm{NO}_{3}(\mathrm{CB})$; (b) $\mathrm{CN}^{-}(\mathrm{BB}), \mathrm{H}_{2} \mathrm{O}(\mathrm{BA}), \mathrm{HCN}(\mathrm{CA}), \mathrm{OH}^{-}(\mathrm{CB})$; (c) $\mathrm{H}_{2} \mathrm{SO}_{4}(\mathrm{BA})$, $\mathrm{Cl}^{-}(\mathrm{BB}), \quad \mathrm{HCl}(\mathrm{CA}), \quad \mathrm{HSO}_{4}(\mathrm{CB}) ; \quad$ (d) $\mathrm{HSO}_{4}(\mathrm{BA})$, $\mathrm{OH}^{-}(\mathrm{BB}), \mathrm{SO}_{4}{ }^{2}(\mathrm{CB}), \mathrm{H}_{2} \mathrm{O}(\mathrm{CA})$; (e) $\mathrm{O}^{2-}(\mathrm{BB}), \mathrm{H}_{2} \mathrm{O}(\mathrm{BA})$ $\mathrm{OH}^{-}(\mathrm{CB}$ and $\mathrm{CA})$; $\left[\mathrm{Al}\left(\mathrm{H}_{2} \mathrm{O}\right)_{6}\right]^{3+}(\mathrm{BA})$,

(f) $\quad\left[\mathrm{Cu}\left(\mathrm{H}_{2} \mathrm{O}\right)_{3}(\mathrm{OH})\right]^{+}(\mathrm{BB})$, $\left[\mathrm{Cu}\left(\mathrm{H}_{2} \mathrm{O}\right)_{4}\right]^{2+}(\mathrm{CA})$, 
$\left[\mathrm{Al}\left(\mathrm{H}_{2} \mathrm{O}\right)_{5}(\mathrm{OH})\right]^{2+}(\mathrm{CB}) ;(\mathrm{g}) \mathrm{H}_{2} \mathrm{~S}(\mathrm{BA}), \mathrm{NH}_{2}(\mathrm{BB}), \mathrm{HS}^{-}(\mathrm{CB})$, $\mathrm{NH}_{3}(\mathrm{CA})$

Identify and label the Brønsted-Lowry acid, its conjugate base, the Brønsted-Lowry base, and its conjugate acid in each of the following equations:

(a) $\mathrm{NO}_{2}+\mathrm{H}_{2} \mathrm{O} \mathrm{HNO}_{2}+\mathrm{OH}$

(b) $\mathrm{HBr}+\mathrm{H}_{2} \mathrm{O} \mathrm{H} \mathrm{H}_{3} \mathrm{O}^{+}+\mathrm{Br}$

(c) $\mathrm{HS}+\mathrm{H}_{2} \mathrm{O} \mathrm{H}_{2} \mathrm{~S}+\mathrm{OH}$

(d) $\mathrm{H}_{2} \mathrm{PO}_{4}+\mathrm{OH} \mathrm{HPO}_{4}^{2}+\mathrm{H}_{2} \mathrm{O}$

(e) $\mathrm{H}_{2} \mathrm{PO}_{4}+\mathrm{HCl} \mathrm{H} \mathrm{PO}_{4}+\mathrm{Cl}$

$\left[\mathrm{Fe}\left(\mathrm{H}_{2} \mathrm{O}\right)_{5}(\mathrm{OH})\right]^{2+}+\left[\mathrm{Al}\left(\mathrm{H}_{2} \mathrm{O}\right)_{6}\right]^{3+}\left[\mathrm{Fe}\left(\mathrm{H}_{2} \mathrm{O}\right)_{6}{ }^{3+}+\left[\mathrm{Al}\left(\mathrm{H}_{2} \mathrm{O}\right)_{5}(\mathrm{OH})\right]^{2+}\right.$ (g) $\mathrm{CH}_{3} \mathrm{OH}+\mathrm{H} \mathrm{CH} \mathrm{CH}_{3} \mathrm{O}+\mathrm{H}_{2}$

What are amphiprotic species? Illustrate with suitable equations.

Amphiprotic species may either gain or lose a proton in a chemical reaction, thus acting as a base or an acid. An example is $\mathrm{H}_{2} \mathrm{O}$. As an acid: $\mathrm{H}_{2} \mathrm{O}(a q)+\mathrm{NH}_{3}(a q) \mathrm{NH}_{4}+(a q)+\mathrm{OH}(a q)$. As a base: $\mathrm{H}_{2} \mathrm{O}(a q)+\mathrm{HCl}(a q) \mathrm{H}_{3} \mathrm{O}^{+}(a q)+\mathrm{Cl}(a q)$

State which of the following species are amphiprotic and write chemical equations illustrating the amphiprotic character of these species:
(a) $\mathrm{H}_{2} \mathrm{O}$
(b) $\mathrm{H}_{2} \mathrm{PO}_{4}$
(c) $\mathrm{S}^{2-}$
(d) $\mathrm{CO}_{3}{ }^{2}$
(e) $\mathrm{HSO}_{4}$ 
State which of the following species are amphiprotic and write chemical equations illustrating the amphiprotic character of these species.

(a) $\mathrm{NH}_{3}$

(b) $\mathrm{HPO}_{4}$

(c) $\mathrm{Br}^{-}$

(d) $\mathrm{NH}_{4}^{+}$

(e) $\mathrm{ASO}_{4}{ }^{3}$

amphiprotic: (a) $\mathrm{NH}_{3}+\mathrm{H}_{3} \mathrm{O}^{+} \mathrm{NH}_{4} \mathrm{OH}+\mathrm{H}_{2} \mathrm{O}$, $\mathrm{NH}_{3}+\mathrm{OCH}_{3} \mathrm{NH}_{2}+\mathrm{CH}_{3} \mathrm{OH}$;

$\mathrm{HPO}_{4}{ }^{2}+\mathrm{OH} \mathrm{PO}_{4}{ }^{3}+\mathrm{H}_{2} \mathrm{O}$

$\mathrm{HPO}_{4}^{2}+\mathrm{HClO}_{4} \mathrm{H}_{2} \mathrm{PO}_{4}+\mathrm{ClO}_{4}$; not amphiprotic:

(c) $\mathrm{Br}^{-} ;$(d) $\mathrm{NH}_{4}^{+}$; (e) $\mathrm{AsO}_{4}^{3}$

Is the self-ionization of water endothermic or exothermic? The ionization constant for water $\left(K_{\mathrm{W}}\right)$ is $2.910^{-14}$ at 40 ${ }^{\circ} \mathrm{C}$ and $9.310^{-14}$ at $60{ }^{\circ} \mathrm{C}$.

Glossary

acid ionization

reaction involving the transfer of a proton from an acid to water, yielding hydronium ions and the conjugate base of the acid amphiprotic

species that may either donate or accept a proton in a Bronsted-Lowry acid-base reaction 


\section{amphoteric}

species that can act as either an acid or a base

\section{autoionization}

reaction between identical species yielding ionic products; for water, this reaction involves transfer of protons to yield hydronium and hydroxide ions

base ionization

reaction involving the transfer of a proton from water to a base, yielding hydroxide ions and the conjugate acid of the base

\section{Bronsted-Lowry acid} proton donor

Bronsted-Lowry base proton acceptor

conjugate acid

substance formed when a base gains a proton

\section{conjugate base}

substance formed when an acid loses a proton ion-product constant for water $\left(K_{\mathrm{w}}\right)$

equilibrium constant for the autoionization of water 


\section{1.}

PH AND POH

\section{Learning Objectives}

By the end of this section, you will be able to:

- Explain the characterization of aqueous solutions as acidic, basic, or neutral

- Express hydronium and hydroxide ion concentrations on the $\mathrm{pH}$ and $\mathrm{pOH}$ scales

- Perform calculations relating $\mathrm{pH}$ and $\mathrm{pOH}$

As discussed earlier, hydronium and hydroxide ions are present both in pure water and in all aqueous solutions, and their concentrations are inversely proportional as determined by the ion product of water $\left(K_{\mathrm{w}}\right)$. The concentrations of these ions in a solution are often critical determinants of the solution's properties and the chemical behaviors of its other solutes, and specific vocabulary has been developed to describe these concentrations in relative terms. A solution is neutral if 
it contains equal concentrations of hydronium and hydroxide ions; acidic if it contains a greater concentration of hydronium ions than hydroxide ions; and basic if it contains a lesser concentration of hydronium ions than hydroxide ions.

A common means of expressing quantities that may span many orders of magnitude is to use a logarithmic scale. One such scale that is very popular for chemical concentrations and equilibrium constants is based on the $p$-function, defined as shown where " $X$ " is the quantity of interest and "log" is the base-10 logarithm:

$\mathrm{pX}=\log \mathrm{X}$

The $\mathrm{pH}$ of a solution is therefore defined as shown here, where $\left[\mathrm{H}_{3} \mathrm{O}^{+}\right]$is the molar concentration of hydronium ion in the solution:

$\mathrm{pH}=\log \left[\mathrm{H}_{3} \mathrm{O}^{+}\right]$

Rearranging this equation to isolate the hydronium ion molarity yields the equivalent expression:

$\left[\mathrm{H}_{3} \mathrm{O}^{+}\right]=10^{\mathrm{pH}}$

Likewise, the hydroxide ion molarity may be expressed as a p-function, or $\mathrm{pOH}$ :

$\mathrm{pOH}=\log [\mathrm{OH}]$

or

$[\mathrm{OH}]=10^{\mathrm{pOH}}$

Finally, the relation between these two ion concentration expressed as p-functions is easily derived from the $K_{\mathrm{W}}$ expression:

$$
K_{\mathrm{w}}=\left[\mathrm{H}_{3} \mathrm{O}^{+}\right][\mathrm{OH}]
$$


$\log K_{\mathrm{w}}=\log \left(\left[\mathrm{H}_{3} \mathrm{O}^{+}\right][\mathrm{OH}]\right)=\log \left[\mathrm{H}_{3} \mathrm{O}^{+}\right]+\log [\mathrm{OH}]$ $\mathrm{p} K_{\mathrm{w}}=\mathrm{pH}+\mathrm{pOH}$

At $25^{\circ} \mathrm{C}$, the value of $K_{\mathrm{W}}$ is $1.010^{-14}$, and so:

$14.00=\mathrm{pH}+\mathrm{pOH}$

As was shown in (Figure), the hydronium ion molarity in pure water (or any neutral solution) is $1.010^{-7} \mathrm{M}$ at $25^{\circ} \mathrm{C}$. The $\mathrm{pH}$ and $\mathrm{pOH}$ of a neutral solution at this temperature are therefore:

$$
\begin{aligned}
& \mathrm{pH}=\log \left[\mathrm{H}_{3} \mathrm{O}^{+}\right]=\log \left(\begin{array}{ll}
1.0 & 10^{-7}
\end{array}\right)=7.00 \\
& \mathrm{pOH}=\log [\mathrm{OH}]=\log \left(\begin{array}{lll}
1.0 & \left.10^{-7}\right)=7.00
\end{array}\right.
\end{aligned}
$$

And so, at this temperature, acidic solutions are those with hydronium ion molarities greater than $1.010^{-7} \mathrm{M}$ and hydroxide ion molarities less than $1.0 \quad 10^{-7} \mathrm{M}$ (corresponding to $\mathrm{pH}$ values less than 7.00 and $\mathrm{pOH}$ values greater than 7.00). Basic solutions are those with hydronium ion molarities less than $1.010^{-7} \mathrm{M}$ and hydroxide ion molarities greater than $1.0 \quad 10^{-7} \mathrm{M}$ (corresponding to $\mathrm{pH}$ values greater than 7.00 and $\mathrm{pOH}$ values less than 7.00).

Since the autoionization constant $K_{\mathrm{W}}$ is temperature dependent, these correlations between $\mathrm{pH}$ values and the acidic/neutral/basic adjectives will be different at temperatures other than $25^{\circ} \mathrm{C}$. For example, the "Check Your Learning” exercise accompanying (Figure) showed the hydronium molarity of pure water at $80{ }^{\circ} \mathrm{C}$ is 4.9 $10^{-7} \mathrm{M}$, which corresponds to $\mathrm{pH}$ and $\mathrm{pOH}$ values of:

$\mathrm{pH}=\log \left[\mathrm{H}_{3} \mathrm{O}^{+}\right]=\log \left(4.910^{-7}\right)=6.31$ $\mathrm{pOH}=\log [\mathrm{OH}]=\log \left(4.910^{-7}\right)=6.31$ 
At this temperature, then, neutral solutions exhibit $\mathrm{pH}=$ $\mathrm{pOH}=6.31$, acidic solutions exhibit $\mathrm{pH}$ less than 6.31 and pOH greater than 6.31, whereas basic solutions exhibit $\mathrm{pH}$ greater than 6.31 and pOH less than 6.31. This distinction can be important when studying certain processes that occur at other temperatures, such as enzyme reactions in warm-blooded organisms at a temperature around 36-40 ${ }^{\circ} \mathrm{C}$. Unless otherwise noted, references to $\mathrm{pH}$ values are presumed to be those at $25^{\circ} \mathrm{C}(($ Figure $))$.

\begin{tabular}{lll}
\multicolumn{3}{l}{ Summary of Relations for Acidic, Basic and Neutral Solutions } \\
Classification & Relative Ion Concentrations & $\mathbf{p H}$ at $25^{\circ} \mathbf{C}$ \\
acidic & {$\left[\mathrm{H}_{3} \mathrm{O}^{+}\right]>\left[\mathrm{OH}^{-}\right]$} & $\mathrm{pH}<7$ \\
neutral & {$\left[\mathrm{H}_{3} \mathrm{O}^{+}\right]=\left[\mathrm{OH}^{-}\right]$} & $\mathrm{pH}=7$ \\
basic & {$\left[\mathrm{H}_{3} \mathrm{O}^{+}\right]<\left[\mathrm{OH}^{-}\right]$} & $\mathrm{pH}>7$
\end{tabular}

(Figure) shows the relationships between $\left[\mathrm{H}_{3} \mathrm{O}^{+}\right],\left[\mathrm{OH}^{-}\right]$, $\mathrm{pH}$, and $\mathrm{pOH}$ for solutions classified as acidic, basic, and neutral.

The $\mathrm{pH}$ and $\mathrm{pOH}$ scales represent concentrations of $\mathrm{H}_{3} \mathrm{O}^{+}$ and $\mathrm{OH}^{-}$, respectively. The $\mathrm{pH}$ and $\mathrm{pOH}$ values of some common substances at $25^{\circ} \mathrm{C}$ are shown in this chart. 
Chemistry and the Environment

\begin{tabular}{|c|c|c|c|c|c|}
\hline$\left[\mathrm{H}_{3} \mathrm{O}^{+}\right](\mathrm{M})$ & {$\left[\mathrm{OH}^{-}\right](\mathrm{M})$} & $\mathrm{pH}$ & pOH & Sample Solution & \\
\hline $10^{1}$ & $10^{-15}$ & -1 & 15 & & \\
\hline $10^{0}$ or 1 & $10^{-14}$ & 0 & 14 & $\longleftarrow 1 \mathrm{M} \mathrm{HCl}$ & acidic \\
\hline $10^{-1}$ & $10^{-13}$ & 1 & 13 & & \\
\hline $10^{-2}$ & $10^{-12}$ & 2 & 12 & $\begin{array}{l}\text { lime juice } \\
1 \mathrm{M} \mathrm{CH}_{3} \mathrm{CO}_{2} \mathrm{H} \text { (vinegar) }\end{array}$ & \\
\hline $10^{-3}$ & $10^{-11}$ & 3 & 11 & F stomach acid & \\
\hline $10^{-4}$ & $10^{-10}$ & 4 & 10 & wine & \\
\hline $10^{-5}$ & $10^{-9}$ & 5 & 9 & $\leftarrow$ coffee & \\
\hline $10^{-6}$ & $10^{-8}$ & 6 & 8 & rain water & \\
\hline $10^{-7}$ & $10^{-7}$ & 7 & 7 & $\longleftarrow$ pure water & neutral \\
\hline $10^{-8}$ & $10^{-6}$ & 8 & 6 & blood & \\
\hline $10^{-9}$ & $10^{-5}$ & 9 & 5 & baking soda & \\
\hline $10^{-10}$ & $10^{-4}$ & 10 & 4 & & \\
\hline $10^{-11}$ & $10^{-3}$ & 11 & 3 & $\longleftarrow$ Milk of Magnesia & \\
\hline $10^{-12}$ & $10^{-2}$ & 12 & 2 & household ammonia, $\mathrm{NH}_{3}$ & \\
\hline $10^{-13}$ & $10^{-1}$ & 13 & 1 & & \\
\hline $10^{-14}$ & $10^{\circ}$ or 1 & 14 & 0 & $\longleftarrow 1 \mathrm{M} \mathrm{NaOH}$ & basic \\
\hline $10^{-15}$ & $10^{1}$ & 15 & -1 & & \\
\hline
\end{tabular}

Calculation of $\mathrm{pH}$ from $\left[\mathrm{H}_{3} \mathrm{O}^{+}\right]$What is the $\mathrm{pH}$ of stomach acid, a solution of $\mathrm{HCl}$ with a hydronium ion concentration of $1.210^{-3} \mathrm{M}$ ?

Solution

$$
\begin{aligned}
& \mathrm{pH}=\log \left[\mathrm{H}_{3} \mathrm{O}^{+}\right] \\
& =\log \left(1.210^{-3}\right. \\
& =(-2.92)=2.92
\end{aligned}
$$

(The use of logarithms is explained in Appendix B. When taking the log of a value, keep as many decimal places in the result as there are significant figures in the value.)

Check Your Learning Water exposed to air contains carbonic acid, $\mathrm{H}_{2} \mathrm{CO}_{3}$, due to the reaction between carbon dioxide and water: 


\section{$\mathrm{CO}_{2}(a q)+\mathrm{H}_{2} \mathrm{O}\left(l \mathrm{H}_{2} \mathrm{CO}_{3}(a q)\right.$}

Air-saturated water has a hydronium ion concentration caused by the dissolved $\mathrm{CO}_{2}$ of $2.010^{-6} \mathrm{M}$, about 20-times larger than that of pure water. Calculate the $\mathrm{pH}$ of the solution at $25^{\circ} \mathrm{C}$.

\section{Answer:}

5.70

Calculation of Hydronium Ion Concentration from $\mathrm{pH}$ Calculate the hydronium ion concentration of blood, the $\mathrm{pH}$ of which is 7.3.

Solution

$$
\begin{aligned}
& \mathrm{pH}=\log \left[\mathrm{H}_{3} \mathrm{O}^{+}\right]=7.3 \\
& \log \left[\mathrm{H}_{3} \mathrm{O}^{+}\right]=-7.3 \\
& {\left[\mathrm{H}_{3} \mathrm{O}^{+}\right]=10^{-7.3} \text { or }\left[\mathrm{H}_{3} \mathrm{O}^{+}\right]=\text {antilog of } 7.3} \\
& {\left[\mathrm{H}_{3} \mathrm{O}^{+}\right]=510^{-8} M}
\end{aligned}
$$

(On a calculator take the antilog, or the "inverse" log, of -7.3 , or calculate $10^{-7.3}$.)

Check Your Learning Calculate the hydronium ion concentration of a solution with a $\mathrm{pH}$ of -1.07 .

Answer:

$12 \mathrm{M}$

\section{Environmental Science}

Normal rainwater has a $\mathrm{pH}$ between 5 and 6 due to the presence of dissolved $\mathrm{CO}_{2}$ which forms carbonic acid:

$\mathrm{H}_{2} \mathrm{O}(l)+\mathrm{CO}_{2}(g) \mathrm{H}_{2} \mathrm{CO}_{3}(a q)$

$\mathrm{H}_{2} \mathrm{CO}_{3}(a q) \mathrm{H}^{+}(a q)+\mathrm{HCO}_{3}(a q)$ 
Acid rain is rainwater that has a $\mathrm{pH}$ of less than 5 , due to a variety of nonmetal oxides, including $\mathrm{CO}_{2}, \mathrm{SO}_{2}, \mathrm{SO}_{3}, \mathrm{NO}$, and $\mathrm{NO}_{2}$ being dissolved in the water and reacting with it to form not only carbonic acid, but sulfuric acid and nitric acid. The formation and subsequent ionization of sulfuric acid are shown here:

$\mathrm{H}_{2} \mathrm{O}(l)+\mathrm{SO}_{3}(g) \mathrm{H}_{2} \mathrm{SO}_{4}(a q)$

$\mathrm{H}_{2} \mathrm{SO}_{4}(a q) \mathrm{H}^{+}(a q)+\mathrm{HSO}_{4}(a q)$

Carbon dioxide is naturally present in the atmosphere because most organisms produce it as a waste product of metabolism. Carbon dioxide is also formed when fires release carbon stored in vegetation or fossil fuels. Sulfur trioxide in the atmosphere is naturally produced by volcanic activity, but it also originates from burning fossil fuels, which have traces of sulfur, and from the process of "roasting" ores of metal sulfides in metal-refining processes. Oxides of nitrogen are formed in internal combustion engines where the high temperatures make it possible for the nitrogen and oxygen in air to chemically combine.

Acid rain is a particular problem in industrial areas where the products of combustion and smelting are released into the air without being stripped of sulfur and nitrogen oxides. In North America and Europe until the 1980s, it was responsible for the destruction of forests and freshwater lakes, when the acidity of the rain actually killed trees, damaged soil, and made lakes uninhabitable for all but the most acid-tolerant species. Acid rain also corrodes statuary and building facades that are made of marble and limestone ((Figure)). Regulations limiting the amount of sulfur and nitrogen oxides that can be released 
into the atmosphere by industry and automobiles have reduced the severity of acid damage to both natural and manmade environments in North America and Europe. It is now a growing problem in industrial areas of China and India.

For further information on acid rain, visit this website hosted by the US Environmental Protection Agency.

(a) Acid rain makes trees more susceptible to drought and insect infestation, and depletes nutrients in the soil. (b) It also is corrodes statues that are carved from marble or limestone. (credit a: modification of work by Chris $\mathrm{M}$ Morris; credit b: modification of work by "Eden, Janine and Jim"/Flickr)

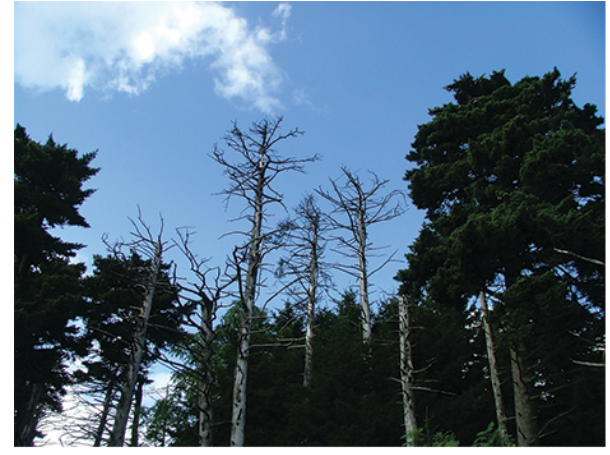

(a)

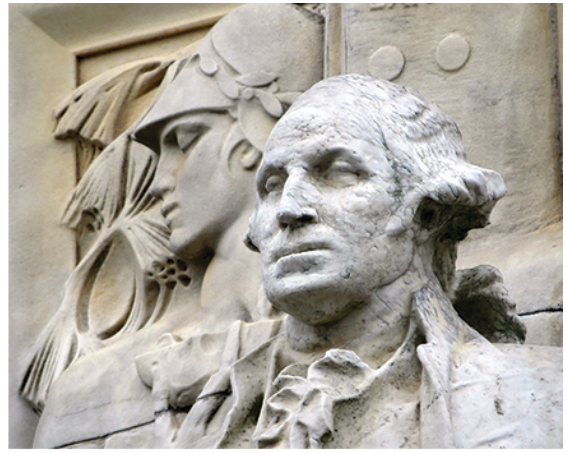

(b)

Calculation of $\mathrm{pOH}$ What are the $\mathrm{pOH}$ and the $\mathrm{pH}$ of a $0.0125-\mathrm{M}$ solution of potassium hydroxide, $\mathrm{KOH}$ ?

Solution Potassium hydroxide is a highly soluble ionic compound and completely dissociates when dissolved in dilute solution, yielding $\left[\mathrm{OH}^{-}\right]=0.0125 \mathrm{M}$ :

$\mathrm{pOH}=\log [\mathrm{OH}]=\log 0.0125$

$=(-1.903)=1.903$

The $\mathrm{pH}$ can be found from the $\mathrm{pOH}$ :

$\mathrm{pH}+\mathrm{pOH}=14.00$ 


$$
\mathrm{pH}=14.00-\mathrm{pOH}=14.00-1.903=12.10
$$

Check Your Learning The hydronium ion concentration of vinegar is approximately $410^{-3} \mathrm{M}$. What are the corresponding values of $\mathrm{pOH}$ and $\mathrm{pH}$ ?

Answer:

$$
\mathrm{pOH}=11.6, \mathrm{pH}=2.4
$$

The acidity of a solution is typically assessed experimentally by measurement of its $\mathrm{pH}$. The $\mathrm{pOH}$ of a solution is not usually measured, as it is easily calculated from an experimentally determined $\mathrm{pH}$ value. The $\mathrm{pH}$ of a solution can be directly measured using a $\mathrm{pH}$ meter ((Figure)).

(a) A research-grade $\mathrm{pH}$ meter used in a laboratory can have a resolution of $0.001 \mathrm{pH}$ units, an accuracy of \pm $0.002 \mathrm{pH}$ units, and may cost in excess of ?1000. (b) A portable $\mathrm{pH}$ meter has lower resolution (0.01 pH units), lower accuracy ( $\pm 0.2 \mathrm{pH}$ units), and a far lower price tag. (credit b: modification of work by Jacopo Werther)

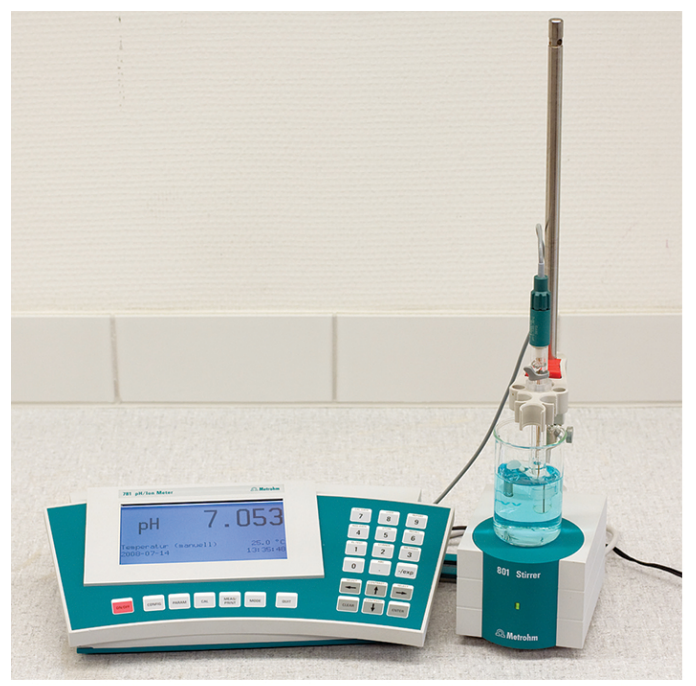

(a)

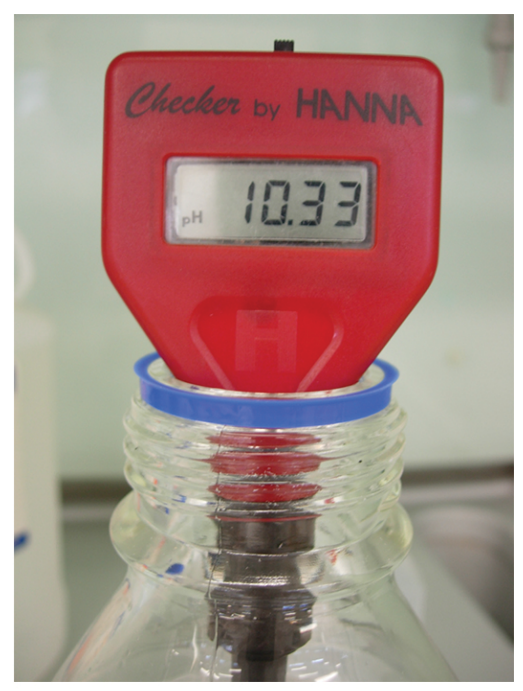

(b)

The $\mathrm{pH}$ of a solution may also be visually estimated using 
colored indicators ((Figure)). The acid-base equilibria that enable use of these indicator dyes for $\mathrm{pH}$ measurements are described in a later section of this chapter.

(a) A solution containing a dye mixture, called universal indicator, takes on different colors depending upon its $\mathrm{pH}$. (b) Convenient test strips, called $\mathrm{pH}$ paper, contain embedded indicator dyes that yield $\mathrm{pH}$-dependent color changes on contact with aqueous solutions.(credit: modification of work by Sahar Atwa)

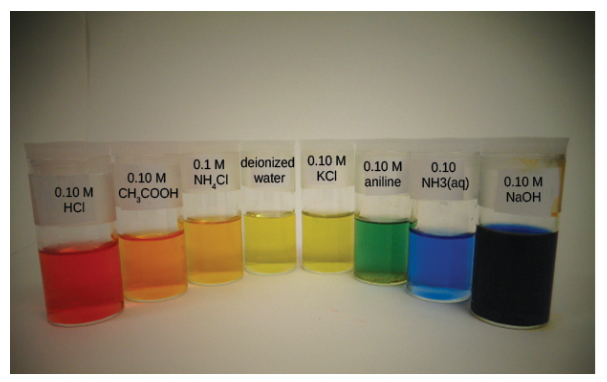

(a)

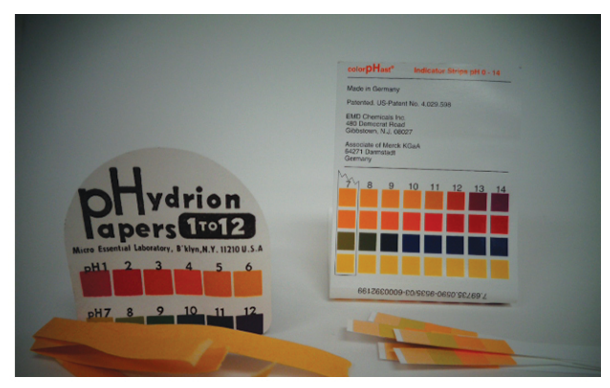

(b)

Key Concepts and Summary

Concentrations of hydronium and hydroxide ions in aqueous media are often represented as logarithmic $\mathrm{pH}$ and $\mathrm{pOH}$ values, respectively. At $25^{\circ} \mathrm{C}$, the autoprotolysis equilibrium for water requires the sum of $\mathrm{pH}$ and $\mathrm{pOH}$ to equal 14 for any aqueous solution. The relative concentrations of hydronium and hydroxide ion in a solution define its status as acidic $\left(\left[\mathrm{H}_{3} \mathrm{O}^{+}\right]>\left[\mathrm{OH}^{-}\right]\right)$, basic $\left(\left[\mathrm{H}_{3} \mathrm{O}^{+}\right]<\left[\mathrm{OH}^{-}\right]\right)$, or neutral $\left(\left[\mathrm{H}_{3} \mathrm{O}^{+}\right]=\left[\mathrm{OH}^{-}\right]\right)$. At $25{ }^{\circ} \mathrm{C}$, a $\mathrm{pH}<7$ indicates an acidic solution, a $\mathrm{pH}>7$ a basic solution, and $\mathrm{a} \mathrm{pH}=7$ a neutral solution.

Key Equations

$$
\text { - } \mathrm{pH}=\log \left[\mathrm{H}_{3} \mathrm{O}^{+}\right]
$$


- $\mathrm{pOH}=-\log \left[\mathrm{OH}^{-}\right]$

- $\left[\mathrm{H}_{3} \mathrm{O}^{+}\right]=10^{-\mathrm{pH}}$

- $\left[\mathrm{OH}^{-}\right]=10^{-\mathrm{pOH}}$

- $\mathrm{pH}+\mathrm{pOH}=\mathrm{p} K_{\mathrm{W}}=14.00$ at $25^{\circ} \mathrm{C}$

Chemistry End of Chapter Exercises

Explain why a sample of pure water at $40{ }^{\circ} \mathrm{C}$ is neutral even though $\left[\mathrm{H}_{3} \mathrm{O}^{+}\right]=1.710^{-7} M . K_{\mathrm{W}}$ is $2.910^{-14}$ at $40^{\circ} \mathrm{C}$.

In a neutral solution $\left[\mathrm{H}_{3} \mathrm{O}^{+}\right]=\left[\mathrm{OH}^{-}\right]$. At $40{ }^{\circ} \mathrm{C},\left[\mathrm{H}_{3} \mathrm{O}^{+}\right]=$ $\left[\mathrm{OH}^{-}\right]=\left(2.910^{-14}\right)^{1 / 2}=1.710^{-7}$.

The ionization constant for water $\left(K_{\mathrm{W}}\right)$ is $2.910^{-14}$ at 40 ${ }^{\circ} \mathrm{C}$. Calculate $\left[\mathrm{H}_{3} \mathrm{O}^{+}\right],\left[\mathrm{OH}^{-}\right], \mathrm{pH}$, and $\mathrm{pOH}$ for pure water at $40{ }^{\circ} \mathrm{C}$.

The ionization constant for water $\left(K_{\mathrm{W}}\right)$ is $9.31110^{-14}$ at 60 ${ }^{\circ} \mathrm{C}$. Calculate $\left[\mathrm{H}_{3} \mathrm{O}^{+}\right],\left[\mathrm{OH}^{-}\right], \mathrm{pH}$, and $\mathrm{pOH}$ for pure water at $60{ }^{\circ} \mathrm{C}$.

$x=3.05110^{-7} M=\left[\mathrm{H}_{3} \mathrm{O}^{+}\right]=\left[\mathrm{OH}^{-}\right] ; \mathrm{pH}=-\log 3.05110^{-7}=$ $-(-6.5156)=6.5156 ; \mathrm{pOH}=\mathrm{pH}=6.5156$

Calculate the $\mathrm{pH}$ and the $\mathrm{pOH}$ of each of the following solutions at $25{ }^{\circ} \mathrm{C}$ for which the substances ionize completely:

(a) $0.200 \mathrm{MHCl}$

(b) $0.0143 \mathrm{M} \mathrm{NaOH}$

(c) $3.0 \mathrm{MHNO}_{3}$

(d) $0.0031 M \mathrm{Ca}(\mathrm{OH})_{2}$ 
Calculate the $\mathrm{pH}$ and the $\mathrm{pOH}$ of each of the following solutions at $25{ }^{\circ} \mathrm{C}$ for which the substances ionize completely:

(a) $0.000259 \mathrm{M} \mathrm{HClO}_{4}$

(b) $0.21 \mathrm{M} \mathrm{NaOH}$

(c) $0.000071 \mathrm{M} \mathrm{Ba}(\mathrm{OH})_{2}$

(d) $2.5 \mathrm{M} \mathrm{KOH}$

(a) $\mathrm{pH}=3.587 ; \mathrm{pOH}=10.413 ;$ (b) $\mathrm{pH}=0.68 ; \mathrm{pOH}=13.32$;

(c) $\mathrm{pOH}=3.85 ; \mathrm{pH}=10.15 ;$ (d) $\mathrm{pH}=-0.40 ; \mathrm{pOH}=14.4$

What are the $\mathrm{pH}$ and $\mathrm{pOH}$ of a solution of $2.0 \mathrm{M} \mathrm{HCl}$, which ionizes completely?

What are the hydronium and hydroxide ion concentrations in a solution whose $\mathrm{pH}$ is 6.52 ?

$\left[\mathrm{H}_{3} \mathrm{O}^{+}\right]=3.010^{-7} M ;\left[\mathrm{OH}^{-}\right]=3.310^{-8} \mathrm{M}$

Calculate the hydrogen ion concentration and the hydroxide ion concentration in wine from its $\mathrm{pH}$. See (Figure) for useful information.

Calculate the hydronium ion concentration and the hydroxide ion concentration in lime juice from its $\mathrm{pH}$. See (Figure) for useful information.

$\left[\mathrm{H}_{3} \mathrm{O}^{+}\right]=1 \quad 10^{-2} M ;\left[\mathrm{OH}^{-}\right]=1 \quad 10^{-12} \mathrm{M}$

The hydronium ion concentration in a sample of rainwater is found to be $1.710^{-6} \mathrm{M}$ at $25^{\circ} \mathrm{C}$. What is the concentration of hydroxide ions in the rainwater?

The hydroxide ion concentration in household ammonia 
is $3.210^{-3} M$ at $25{ }^{\circ} \mathrm{C}$. What is the concentration of hydronium ions in the solution?

$$
\left[\mathrm{OH}^{-}\right]=3.110^{-12} \mathrm{M}
$$

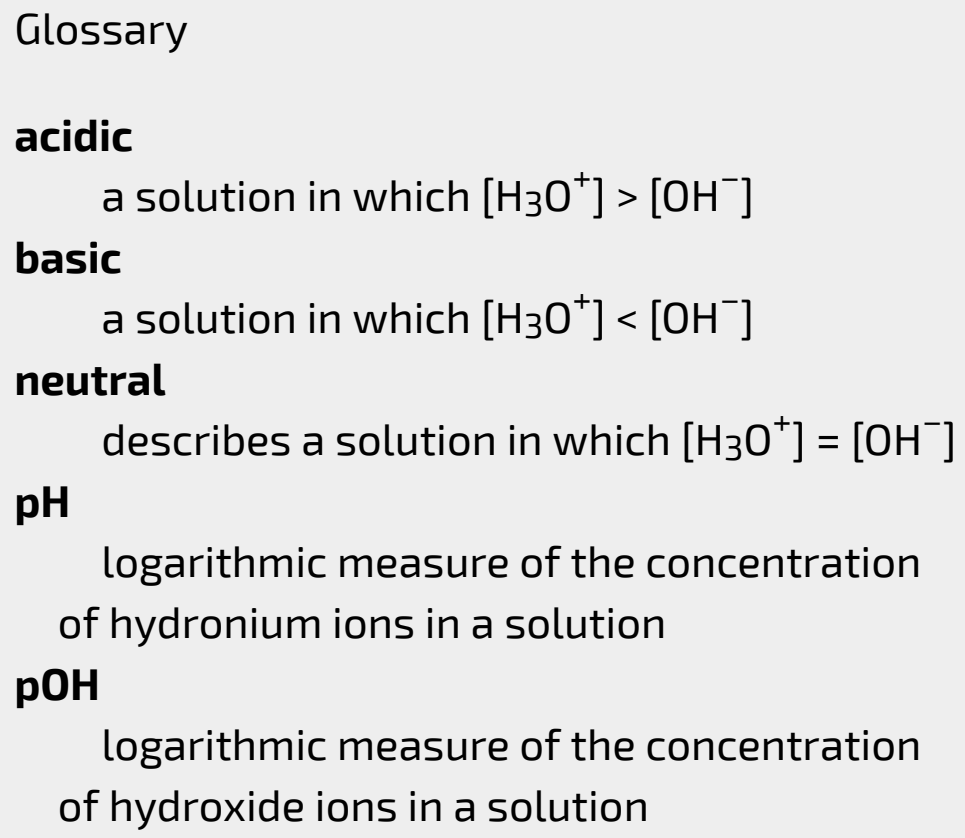




\section{Focus on Acid Precipitation}

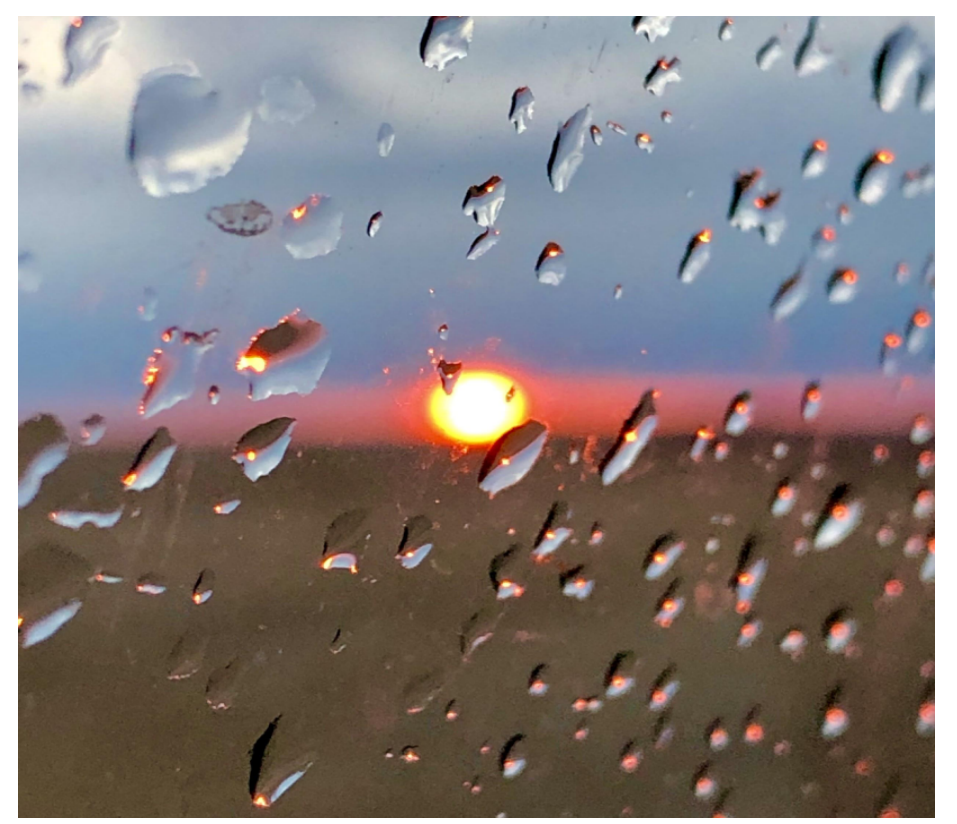

Photo by Steve Schlachter reproduced with permission

At many times, in many places, lakes and forests can experience a surge in acidity causing an unhealthy environment whereby the fish and trees in that ecosystem will die. What is an acid? Where does this extra acidity in the environment come from? Why are some places more prone to acidity than others? In many cases, it seems that the source of the acidity is found in one area, while the environmental effects of the acidity are observed in another. In this section, acids and bases will be introduced in the context of this 'extra acidity' and the source of what is referred to as 'acid precipitation' will be traced. While understanding whole ecosystems is quite complicated, a decent foundation in acidbase chemistry and the calculations involved with a number such as $\mathrm{pH}$ can enable a person to understand the data involved and take part in the discussions required to solve the problem.

Refer to the information discussed in the previous Focus section as well as Part XI of this text and elaborate on the following focus boxes, solving any problems that appear. 
One way to define an acid is as a substance that releases hydrogen ions, $\mathrm{H}^{+}$, in aqueous solution.

Since the hydrogen ion has no electron, and only one proton (hence the positive charge), the hydrogen ion sometimes is referred to as a proton.

Example:

$$
\mathrm{HCl}(g) \stackrel{\mathrm{H}_{2} \mathrm{O}}{\longrightarrow} \underset{\text { (a proton) }}{\mathrm{H}^{+}(a q)}+\mathrm{Cl}^{-}(a q)
$$

However, in aqueous solution, the $\mathrm{H}^{+}$will be associated with a water molecule to form the hydronium ion, $\mathrm{H}_{3} \mathrm{O}^{+}$

One way to define a base is as a substance that releases hydroxide ions, $\mathrm{OH}$, in aqueous solution.

Show how each of these produce hydroxide in water:

$\mathrm{NaOH}$

$\mathrm{NH}_{3}$

When acids and bases react with each other, it is called a neutralization reaction.

$$
\mathrm{HCl}(a q)+\mathrm{NaOH}(a q) \longrightarrow \quad \mathrm{NaCl}(a q)+\mathrm{H}_{2} \mathrm{O}(l)
$$

In neutralization reactions, hydrogen ions from an acid combine with the hydroxide ions from a base to form molecules of water.

The other product is a salt (an ionic compound) and will often be dissolved in the aqueous solution. 


\section{$\mathrm{pH}$ and $\mathrm{pOH}$}

a measure of the concentration of $\mathrm{H}_{3} \mathrm{O}^{+}$ $(\mathrm{pH})$ and $\mathrm{OH}^{-}(\mathrm{pOH})$ in solution.

$$
\begin{aligned}
& \mathrm{pH}=-\log \left[\mathrm{H}_{3} \mathrm{O}^{+}\right] \\
& \mathrm{pOH}=-\log \left[\mathrm{OH}^{-}\right] \\
& \mathrm{pH}+\mathrm{pOH}=14
\end{aligned}
$$

What is the $\mathrm{pH}$ of a solution with $\left[\mathrm{H}_{3} \mathrm{O}^{+}\right]=1.0 \times 10^{-7} \mathrm{M}$ ? What is the $\mathrm{pOH}$

What is the $\mathrm{pH}$ of a solution with $\left[\mathrm{H}_{3} \mathrm{O}^{+}\right]=2.0 \times 10^{-6} \mathrm{M}$ ? What is the $\mathrm{pOH}$

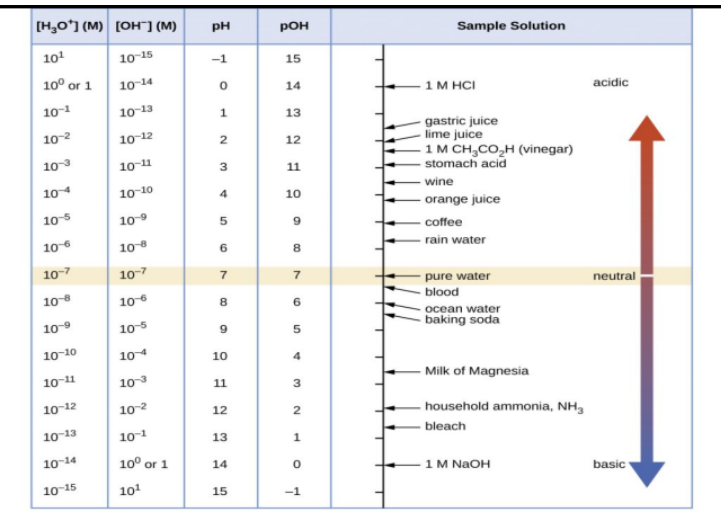

Chemistry and the Environment by Devin R. Latimer is licensed under a Creative Commons Attribution 4.0 International License 
Pure water has a $\mathrm{pH}$ of 7.0, while normal rain has a $\mathrm{pH}$ of around 5.7 because of dissolved $\mathrm{CO}_{2}$. Show how the dissolution of $\mathrm{CO}_{2}$ in water produces carbonic acid $\mathrm{H}_{2} \mathrm{CO}_{3}$ which then reacts with water to produce hydronium ions.

But acid precipitation can have very low $\mathrm{pH}$ values

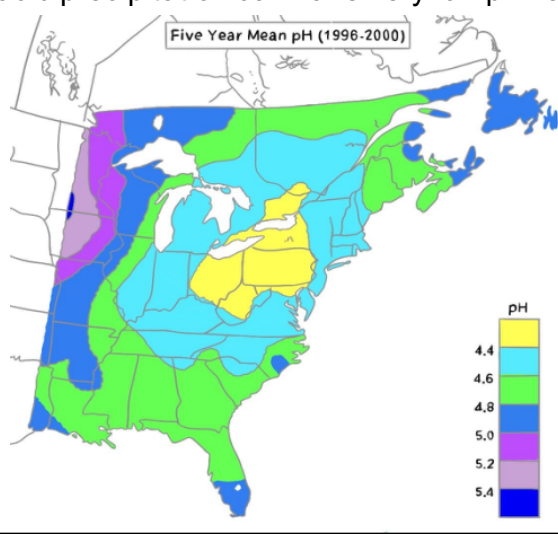

Remember: Because the $\mathrm{pH}$ scale is logarithmic, 1 unit of $\mathrm{pH}$ is a difference of 10 times in the amount of acidity.

Analysis of rain for specific compounds has indicated that the primary components responsible for the extra acidity are the oxides of sulfur and nitrogen. Explain, using chemical reactions, how the combustion of coal can lead to oxides of sulfur and nitrogen, and to acidic aqueous solutions. 

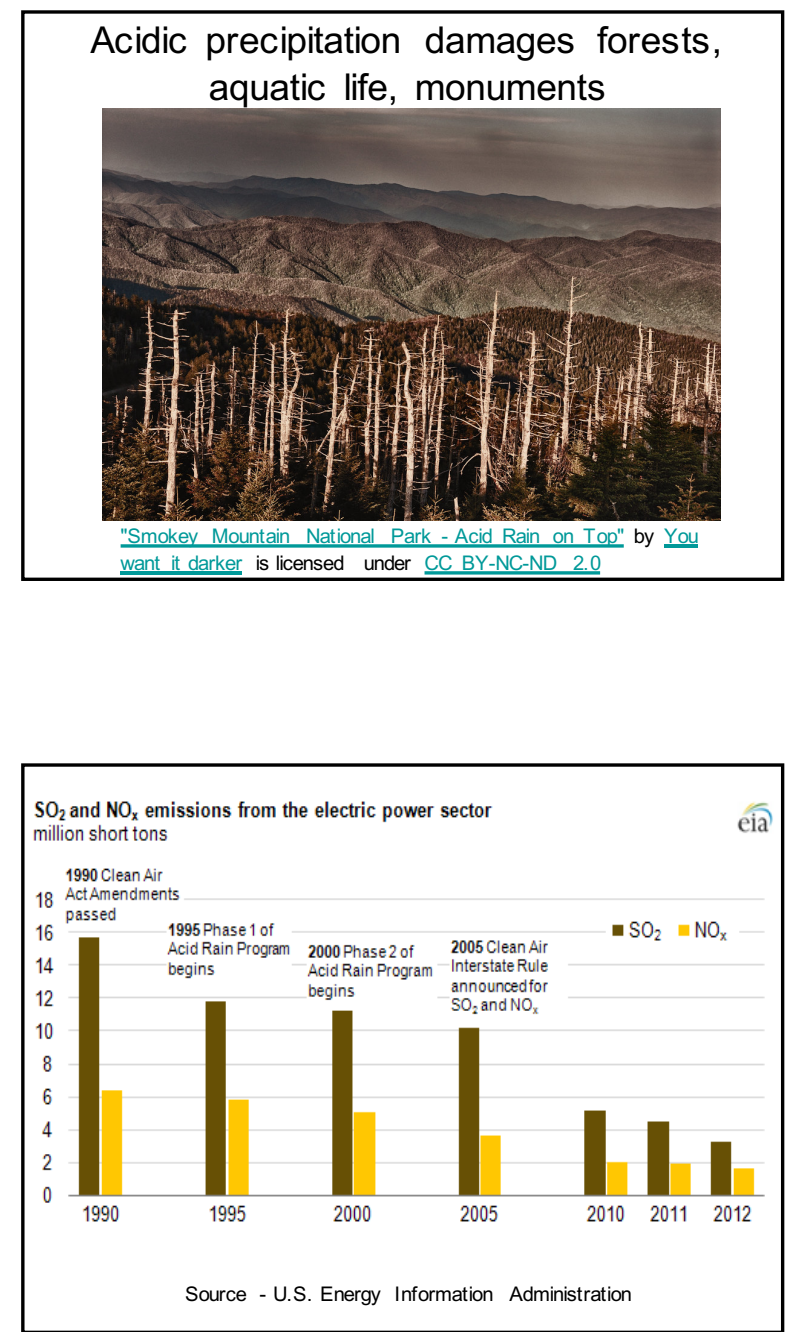

\section{BBC - The bittersweet story of how we stopped acid rain (ELA)}

https://www.bbc.com/future/article/20190823-canlessons-from-acid-rain-help-stop-climate-change 


\section{PART XII}

\section{ELECTROCHEMISTRY}





\section{2.}

\section{INTRODUCTION}

Learning Objectives

- Galvanic Cells

- Batteries and Fuel Cells

Electric vehicles are powered by batteries, devices that harness the energy of spontaneous redox reactions. (credit: modification of work by Robert Couse-Baker)

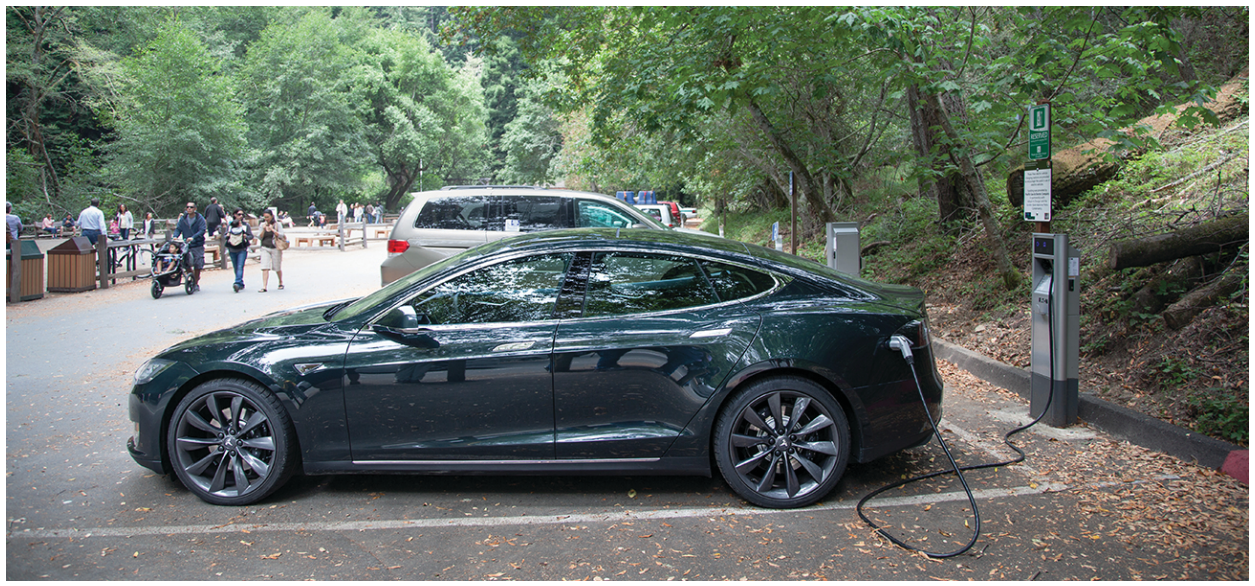


Another chapter in this text introduced the chemistry of reduction-oxidation (redox) reactions. This important reaction class is defined by changes in oxidation states for one or more reactant elements, and it includes a subset of reactions involving the transfer of electrons between reactant species. Around the turn of the nineteenth century, chemists began exploring ways these electrons could be transferred indirectly via an external circuit rather than directly via intimate contact of redox reactants. In the two centuries since, the field of electrochemistry has evolved to yield significant insights on the fundamental aspects of redox chemistry as well as a wealth of technologies ranging from industrial-scale metallurgical processes to robust, rechargeable batteries for electric vehicles ((Figure)). In this chapter, the essential concepts of electrochemistry will be addressed. 


\section{3.}

\section{GALVANIC CELLS}

Learning Objectives

By the end of this section, you will be able to:

- Describe the function of a galvanic cell and its components

- Use cell notation to symbolize the composition and construction of galvanic cells

As demonstration of spontaneous chemical change, (Figure) shows the result of immersing a coiled wire of copper into an aqueous solution of silver nitrate. A gradual but visually impressive change spontaneously occurs as the initially colorless solution becomes increasingly blue, and the initially smooth copper wire becomes covered with a porous gray solid.

A copper wire and an aqueous solution of silver nitrate (left) are brought into contact (center) and a spontaneous transfer of electrons occurs, creating blue $\mathrm{Cu}^{2+}(a q)$ and 
gray $\operatorname{Ag}(s)$ (right).
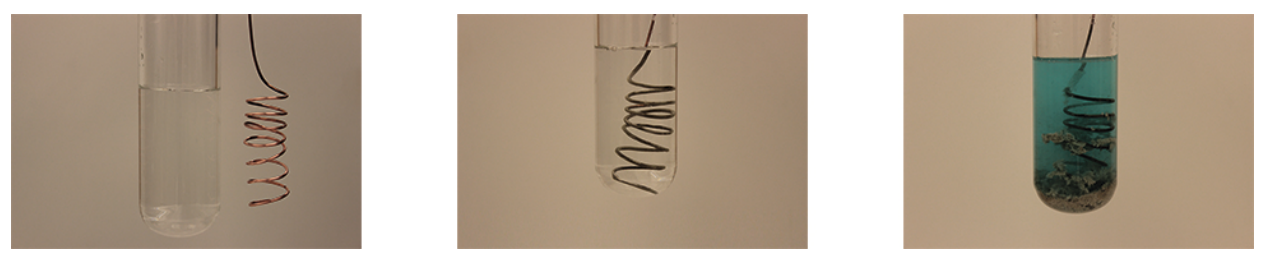

These observations are consistent with (i) the oxidation of elemental copper to yield copper(II) ions, $\mathrm{Cu}^{2+}(a q)$, which impart a blue color to the solution, and (ii) the reduction of silver(I) ions to yield elemental silver, which deposits as a fluffy solid on the copper wire surface. And so, the direct transfer of electrons from the copper wire to the aqueous silver ions is spontaneous under the employed conditions. A summary of this redox system is provided by these equations:

overall reaction:

oxidation half-reaction:

reduction half-reaction:

$\mathrm{Cu}(s)+2 \mathrm{Ag}^{+}(a q) \mathrm{Cu}^{2+}(a q)+2 \mathrm{Ag}(s)$ $\mathrm{Cu}(s) \mathrm{Cu}^{2+}(a q)+2 \mathrm{e}$ $2 \mathrm{Ag}^{+}(a q)+2 \mathrm{e} 2 \mathrm{Ag}(s)$

Consider the construction of a device that contains all the reactants and products of a redox system like the one here, but prevents physical contact between the reactants. Direct transfer of electrons is, therefore, prevented; transfer, instead, takes place indirectly through an external circuit that contacts the separated reactants. Devices of this sort are generally referred to as electrochemical cells, and those in which a spontaneous redox reaction takes place are called galvanic cells (or voltaic cells).

A galvanic cell based on the spontaneous reaction between copper and silver(I) is depicted in (Figure). The cell is comprised of two half-cells, each containing the redox conjugate pair ("couple") of a single reactant. The 
half-cell shown at the left contains the $\mathrm{Cu}(0) / \mathrm{Cu}$ (II) couple in the form of a solid copper foil and an aqueous solution of copper nitrate. The right half-cell contains the $\operatorname{Ag}(\mathrm{I}) / \operatorname{Ag}(0)$ couple as solid silver foil and an aqueous silver nitrate solution. An external circuit is connected to each half-cell at its solid foil, meaning the $\mathrm{Cu}$ and $\mathrm{Ag}$ foil each function as an electrode. By definition, the anode of an electrochemical cell is the electrode at which oxidation occurs (in this case, the $\mathrm{Cu}$ foil) and the cathode is the electrode where reduction occurs (the Ag foil). The redox reactions in a galvanic cell occur only at the interface between each half-cell's reaction mixture and its electrode. To keep the reactants separate while maintaining charge-balance, the two half-cell solutions are connected by a tube filled with inert electrolyte solution called a salt bridge. The spontaneous reaction in this cell produces $\mathrm{Cu}^{2+}$ cations in the anode half-cell and consumes $\mathrm{Ag}^{+}$ions in the cathode half-cell, resulting in a compensatory flow of inert ions from the salt bridge that maintains charge balance. Increasing concentrations of $\mathrm{Cu}^{2+}$ in the anode half-cell are balanced by an influx of $\mathrm{NO}_{3}{ }^{-}$from the salt bridge, while a flow of $\mathrm{Na}^{+}$into the cathode half-cell compensates for the decreasing $\mathrm{Ag}^{+}$ concentration.

A galvanic cell based on the spontaneous reaction between copper and silver(I) ions. 


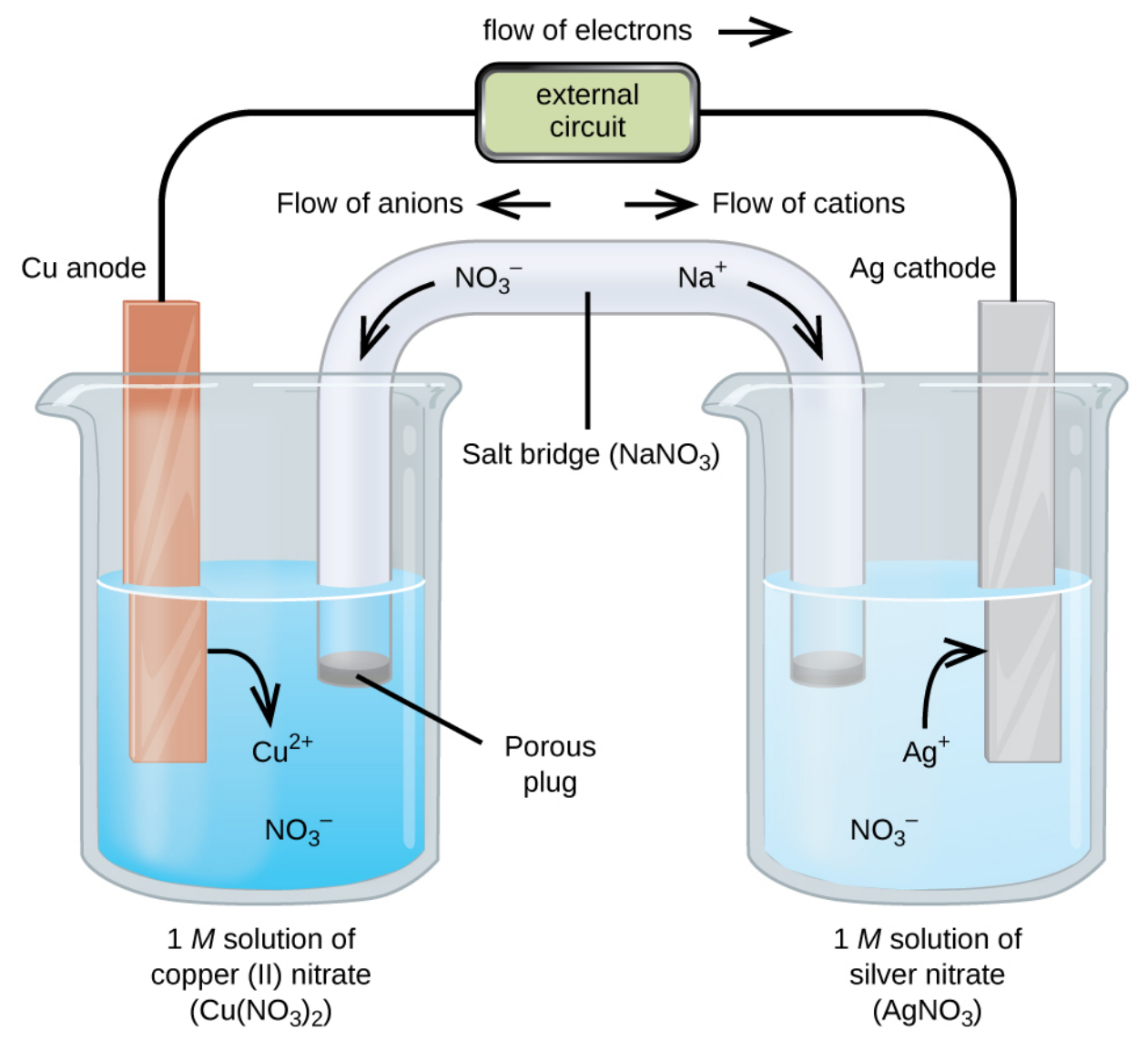

Cell Notation

Abbreviated symbolism is commonly used to represent a galvanic cell by providing essential information on its composition and structure. These symbolic representations are called cell notations or cell schematics, and they are written following a few guidelines:

- The relevant components of each half-cell are represented by their chemical formulas or element symbols

- All interfaces between component phases are represented by vertical parallel lines; if two or 
more components are present in the same phase, their formulas are separated by commas

- By convention, the schematic begins with the anode and proceeds left-to-right identifying phases and interfaces encountered within the cell, ending with the cathode

A verbal description of the cell as viewed from anodeto-cathode is often a useful first-step in writing its schematic. For example, the galvanic cell shown in (Figure) consists of a solid copper anode immersed in an aqueous solution of copper(II) nitrate that is connected via a salt bridge to an aqueous silver(I) nitrate solution, immersed in which is a solid silver cathode. Converting this statement to symbolism following the above guidelines results in the cell schematic:

$\mathrm{Cu}(s) 1 M \mathrm{Cu}\left(\mathrm{NO}_{3}\right)_{2}(a q) 1 M \mathrm{AgNO}_{3}(a q) \mathrm{Ag}(s)$

Consider a different galvanic cell (see (Figure)) based on the spontaneous reaction between solid magnesium and aqueous iron(III) ions:

net cell reaction:

oxidation half-reaction:

reduction half-reaction:

$$
\begin{aligned}
& \mathrm{Mg}(s)+2 \mathrm{Fe}^{3+}(a q) \mathrm{Mg}^{2+}(a q)+2 \mathrm{Fe}^{2+}(a q) \\
& \mathrm{Mg}(s) \mathrm{Mg}^{2+}(a q)+2 \mathrm{e} \\
& 2 \mathrm{Fe}^{3+}(a q)+2 \mathrm{e} 2 \mathrm{Fe}^{2+}(a q)
\end{aligned}
$$

In this cell, a solid magnesium anode is immersed in an aqueous solution of magnesium chloride that is connected via a salt bridge to an aqueous solution containing a mixture of iron(III) chloride and iron(II) chloride, immersed in which is a platinum cathode. The cell schematic is then written as

$\mathrm{Mg}(s) 0.1 M \mathrm{MgCl}_{2}(a q) 0.2 M \mathrm{FeCl}_{3}(a q), 0.3 M \mathrm{FeCl}_{2}(a q) \mathrm{Pt}(s)$ 
Notice the cathode half-cell is different from the others considered thus far in that its electrode is comprised of a substance $(\mathrm{Pt})$ that is neither a reactant nor a product of the cell reaction. This is required when neither member of the half-cell's redox couple can reasonably function as an electrode, which must be electrically conductive and in a phase separate from the half-cell solution. In this case, both members of the redox couple are solute species, and so $\mathrm{Pt}$ is used as an inert electrode that can simply provide or accept electrons to redox species in solution. Electrodes constructed from a member of the redox couple, such as the $\mathrm{Mg}$ anode in this cell, are called active electrodes. A galvanic cell based on the spontaneous reaction between magnesium and iron(III) ions.

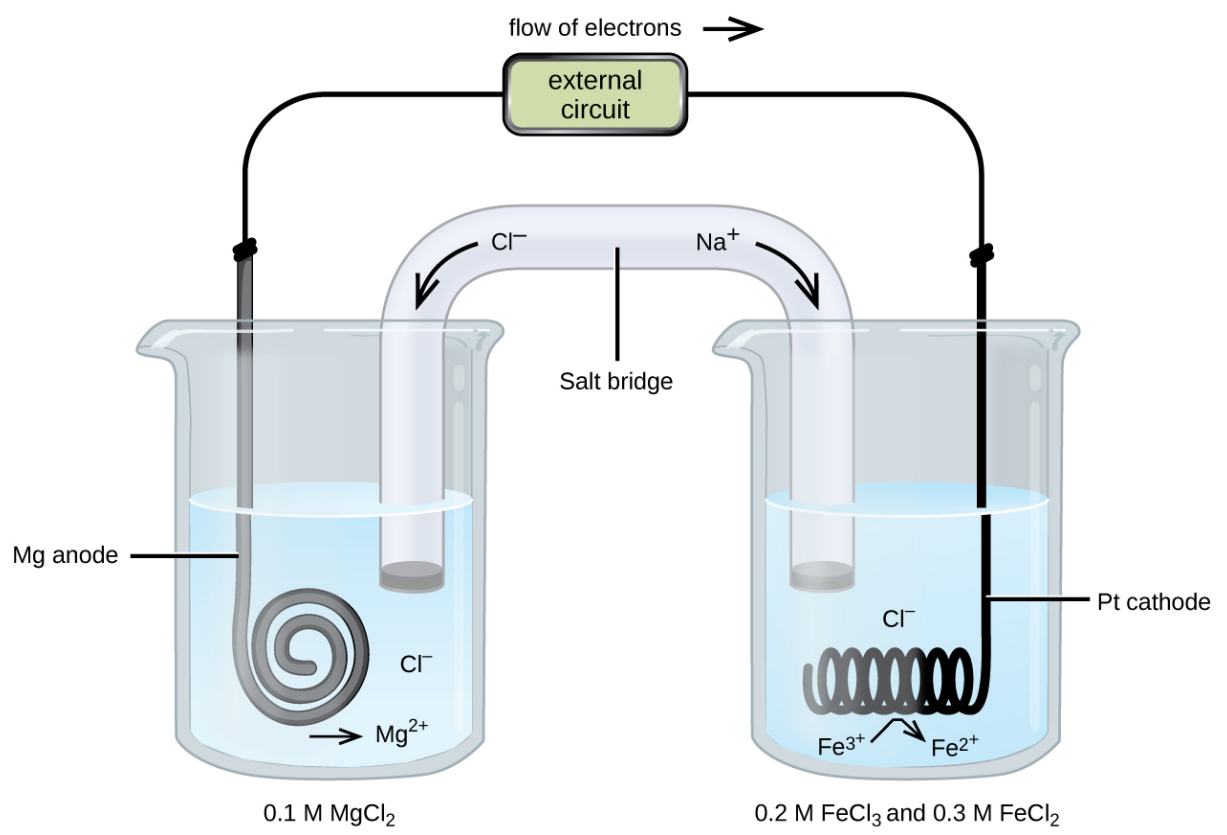

Writing Galvanic Cell Schematics A galvanic cell is fabricated by connecting two half-cells with a salt bridge, one in which a chromium wire is immersed in a $1 \mathrm{M} \mathrm{CrCl}_{3}$ solution and another 
in which a copper wire is immersed in $1 \mathrm{M} \mathrm{CuCl} 2$. Assuming the chromium wire functions as an anode, write the schematic for this cell along with equations for the anode half-reaction, the cathode half-reaction, and the overall cell reaction.

Solution Since the chromium wire is stipulated to be the anode, the schematic begins with it and proceeds left-to-right, symbolizing the other cell components until ending with the copper wire cathode:

\section{$\mathrm{Cr}(s) 1 M \mathrm{CrCl}_{3}(a q) 1 M \mathrm{CuCl}_{2}(a q) \mathrm{Cu}(s)$}

The half-reactions for this cell are

anode (oxidation):

$\mathrm{Cr}(s) \mathrm{Cr}^{3+}(a q)+3 \mathrm{e}$

cathode (oxidation):

$$
\mathrm{Cu}^{2+}(a q)+2 \mathrm{e} \mathrm{Cu}(s)
$$

Multiplying to make the number of electrons lost by $\mathrm{Cr}$ and gained by $\mathrm{Cu}^{2+}$ equal yields

anode (oxidation):

$2 \mathrm{Cr}(s) 2 \mathrm{Cr}^{3+}(a q)+6 \mathrm{e}$

cathode (reducation): $3 \mathrm{Cu}^{2+}(a q)+6 \mathrm{e} 3 \mathrm{Cu}(s)$

Adding the half-reaction equations and simplifying yields an equation for the cell reaction:

$$
2 \mathrm{Cr}(s)+3 \mathrm{Cu}^{2+}(a q) 2 \mathrm{Cr}^{3+}(a q)+3 \mathrm{Cu}(s)
$$

Check Your Learning Omitting solute concentrations and spectator ion identities, write the schematic for a galvanic cell whose net cell reaction is shown below.

$$
\mathrm{Sn}^{4+}(a q)+\mathrm{Zn}(s) \mathrm{Sn}^{2+}(a q)+\mathrm{Zn}^{2+}(a q)
$$

Answer:

$$
\mathrm{Zn}(s) \mathrm{Zn}^{2+}(a q) \mathrm{Sn}^{4+}(a q), \mathrm{Sn}^{2+}(a q) \mathrm{Pt}(s)
$$

\section{Key Concepts and Summary}

Galvanic cells are devices in which a spontaneous redox reaction occurs indirectly, with the oxidant and reductant redox couples contained in separate half-cells. Electrons are transferred from the reductant (in the anode half-cell) 
to the oxidant (in the cathode half-cell) through an external circuit, and inert solution phase ions are transferred between half-cells, through a salt bridge, to maintain charge neutrality. The construction and composition of a galvanic cell may be succinctly represented using chemical formulas and others symbols in the form of a cell schematic (cell notation).

\section{Chemistry End of Chapter Exercises}

Write cell schematics for the following cell reactions, using platinum as an inert electrode as needed.

(a) $\mathrm{Mg}(s)+\mathrm{Ni}^{2+}(a q) \mathrm{Mg}^{2+}(a q)+\mathrm{Ni}(s)$

(b) $2 \mathrm{Ag}^{+}(a q)+\mathrm{Cu}(s) \mathrm{Cu}^{2+}(a q)+2 \mathrm{Ag}(s)$

(c)

$\mathrm{Mn}(s)+\mathrm{Sn}\left(\mathrm{NO}_{32}(a q) \quad \mathrm{Mn}\left(\mathrm{NO}_{32}(a q)+\mathrm{Au}(s)\right.\right.$

(d)

$3 \mathrm{CuNO}_{3}(a q)+\mathrm{Au}\left(\mathrm{NO}_{33}(a q) \quad 3 \mathrm{Cu}\left(\mathrm{NO}_{32}(a q)+\mathrm{Au}(s)\right.\right.$

(a) $\quad \mathrm{Mg}(s) \mathrm{Mg}^{2+}(a q) \mathrm{Ni}^{2+}(a q) \mathrm{Ni}(s)$;

$\mathrm{Cu}(s) \mathrm{Cu}^{2+}(a q) \mathrm{Ag}^{+}(a q) \quad \mathrm{Ag}(s)$;

$\mathrm{Mn}(s) \mathrm{Mn}^{2+}(a q) \mathrm{Sn}^{2+}(a q) \mathrm{Sn}(s)$;

$\mathrm{Pt}(s) \mathrm{Cu}^{+}(a q), \mathrm{Cu}^{2+}(a q) \mathrm{Au}^{3+}(a q) \mathrm{Au}(s)$

Assuming the schematics below represent galvanic cells as written, identify the half-cell reactions occurring in each.
(a) $\mathrm{Mg}(s) \mathrm{Mg}^{2+}(a q) \mathrm{Cu}^{2+}(a q) \mathrm{Cu}(s)$
(b) $\mathrm{Ni}(s) \mathrm{Ni}^{2+}(a q) \mathrm{Ag}^{+}(a q) \mathrm{Ag}(s)$

Write a balanced equation for the cell reaction of each cell in the previous exercise. 
(a) $\mathrm{Mg}(s)+\mathrm{Cu}^{2+}(a q) \mathrm{Mg}^{2+}(a q)+\mathrm{Cu}(s)$; $2 \mathrm{Ag}^{+}(a q)+\mathrm{Ni}(s) \mathrm{Ni}^{2+}(a q)+2 \mathrm{Ag}(s)$

Balance each reaction below, and write a cell schematic representing the reaction as it would occur in a galvanic cell.

(a) $\mathrm{Al}(s)+\mathrm{Zr}^{4+}(a q) \mathrm{Al}^{3+}(a q)+\mathrm{Zr}(s)$

$$
\mathrm{Ag}^{+}(a q)+\mathrm{NO}(g) \mathrm{Ag}(s)+\mathrm{NO}_{3}(a q) \quad \text { (acidic solution) }
$$

(c)

$$
\mathrm{SiO}_{3}{ }^{2-}(a q)+\mathrm{Mg}(s) \mathrm{Si}(s)+\mathrm{Mg}(\mathrm{OH})_{2}(s) \quad \text { (basic solution) }
$$

$$
\mathrm{ClO}_{3}(a q)+\mathrm{MnO}_{2}(s) \mathrm{Cl}(a q)+\mathrm{MnO}_{4}(a q) \quad \text { (basic solution) }
$$

Identify the oxidant and reductant in each reaction of the previous exercise.

Species oxidized = reducing agent: (a) $\mathrm{Al}(s)$; (b) $\mathrm{NO}(g)$; (c) $\mathrm{Mg}(\mathrm{s})$; and (d) $\mathrm{MnO}_{2}(s)$; Species reduced = oxidizing agent: (a) $\mathrm{Zr}^{4+}(a q)$; (b) $\mathrm{Ag}^{+}(a q)$; (c) $\mathrm{SiO}_{3}{ }^{2-}(a q)$; and (d) $\mathrm{ClO}_{3}(a q)$

From the information provided, use cell notation to describe the following systems:

(a) In one half-cell, a solution of $\mathrm{Pt}\left(\mathrm{NO}_{3}\right)_{2}$ forms $\mathrm{Pt}$ metal, while in the other half-cell, $\mathrm{Cu}$ metal goes into a $\mathrm{Cu}\left(\mathrm{NO}_{3}\right)_{2}$ solution with all solute concentrations $1 M$.

(b) The cathode consists of a gold electrode in a 0.55 $M \mathrm{Au}\left(\mathrm{NO}_{3}\right)_{3}$ solution and the anode is a magnesium electrode in $0.75 \mathrm{M} \mathrm{Mg}\left(\mathrm{NO}_{3}\right)_{2}$ solution.

(c) One half-cell consists of a silver electrode in a 1 $M \mathrm{AgNO}_{3}$ solution, and in the other half-cell, a copper electrode in $1 M \mathrm{Cu}\left(\mathrm{NO}_{3}\right)_{2}$ is oxidized. 
Why is a salt bridge necessary in galvanic cells like the one in (Figure)?

Without the salt bridge, the circuit would be open (or broken) and no current could flow. With a salt bridge, each half-cell remains electrically neutral and current can flow through the circuit.

An active (metal) electrode was found to gain mass as the oxidation-reduction reaction was allowed to proceed. Was the electrode an anode or a cathode? Explain.

An active (metal) electrode was found to lose mass as the oxidation-reduction reaction was allowed to proceed. Was the electrode an anode or a cathode? Explain.

Active electrodes participate in the oxidation-reduction reaction. Since metals form cations, the electrode would lose mass if metal atoms in the electrode were to oxidize and go into solution. Oxidation occurs at the anode.

The masses of three electrodes (A, B, and C), each from three different galvanic cells, were measured before and after the cells were allowed to pass current for a while. The mass of electrode A increased, that of electrode B was unchanged, and that of electrode $C$ decreased. Identify each electrode as active or inert, and note (if possible) whether it functioned as anode or cathode. 
Glossary

active electrode

electrode that participates as a reactant or product in the oxidation-reduction reaction of an electrochemical cell; the mass of an active electrode changes during the oxidationreduction reaction

anode

electrode in an electrochemical cell at which oxidation occurs

cathode

electrode in an electrochemical cell at which reduction occurs cell notation (schematic)

symbolic representation of the components and reactions in an electrochemical cell

cell potential ( $\left.E_{\text {cell }}\right)$

difference in potential of the cathode and anode half-cells

galvanic (voltaic) cell

electrochemical cell in which a spontaneous redox reaction takes place; also called a voltaic cell inert electrode

electrode that conducts electrons to and from the reactants in a half-cell but that is not itself oxidized or reduced 


\section{4. BATTERIES AND FUEL CELLS}

Learning Objectives

By the end of this section, you will be able to:

- Describe the electrochemistry associated with several common batteries

- $\quad$ Distinguish the operation of a fuel cell from that of a battery

There are many technological products associated with the past two centuries of electrochemistry research, none more immediately obvious than the battery. A battery is a galvanic cell that has been specially designed and constructed in a way that best suits its intended use a source of electrical power for specific applications. Among the first successful batteries was the Daniell cell, which 
relied on the spontaneous oxidation of zinc by copper(II) ions ((Figure)):

$\mathrm{Zn}(s)+\mathrm{Cu}^{2+}(a q) \mathrm{Zn}^{2+}(a q)+\mathrm{Cu}(s)$

Illustration of a Daniell cell taken from a 1904 journal

publication (left) along with a simplified illustration

depicting the electrochemistry of the cell (right). The 1904 design used a porous clay pot to both contain one of the half-cell's content and to serve as a salt bridge to the other half-cell.
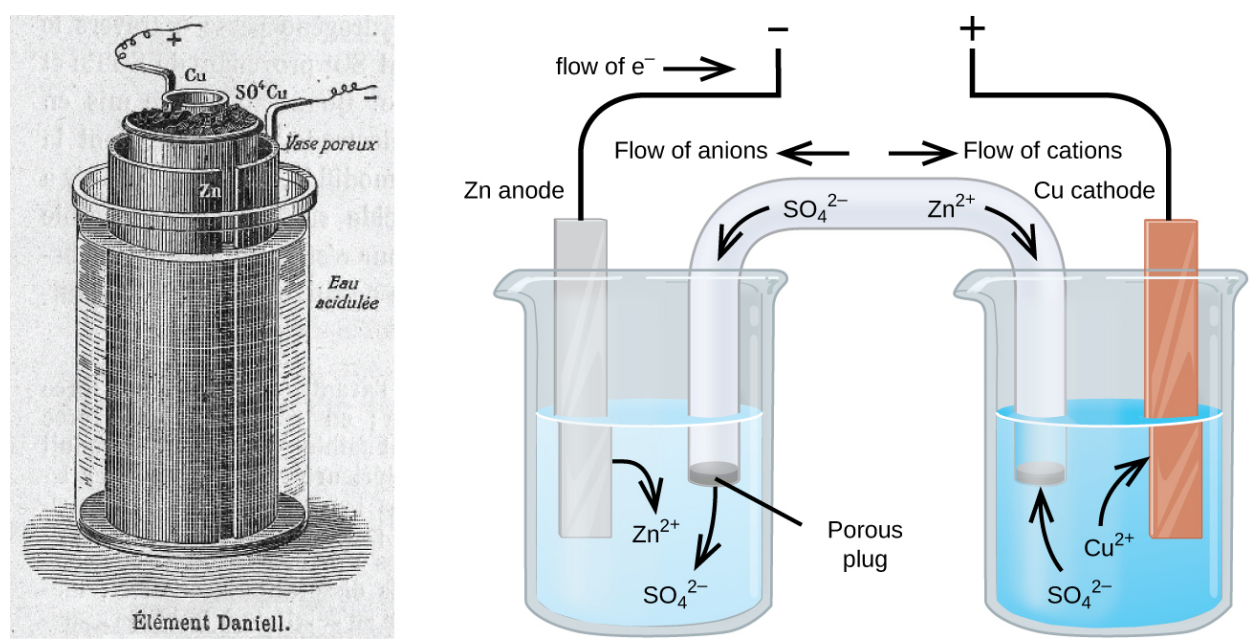

Modern batteries exist in a multitude of forms to accommodate various applications, from tiny button batteries that provide the modest power needs of a wristwatch to the very large batteries used to supply backup energy to municipal power grids. Some batteries are designed for single-use applications and cannot be recharged (primary cells), while others are based on conveniently reversible cell reactions that allow recharging by an external power source (secondary cells). This section will provide a summary of the basic electrochemical aspects of several batteries familiar to most consumers, and will introduce a related 
electrochemical device called a fuel cell that can offer improved performance in certain applications.

Visit this site to learn more about batteries.

Single-Use Batteries

A common primary battery is the dry cell, which uses a zinc can as both container and anode ("-" terminal) and a graphite rod as the cathode ("+" terminal). The $\mathrm{Zn}$ can is filled with an electrolyte paste containing manganese(IV) oxide, zinc(II) chloride, ammonium chloride, and water. A graphite rod is immersed in the electrolyte paste to complete the cell. The spontaneous cell reaction involves the oxidation of zinc:

anode reaction: $\mathrm{Zn}(s) \mathrm{Zn}^{2+}(a q)+2 \mathrm{e}$

and the reduction of manganese(IV)

reduction reaction: $2 \mathrm{MnO}_{2}(s)+2 \mathrm{NH}_{4} \mathrm{Cl}(a q)+2$ e $\mathrm{Mn}_{2} \mathrm{O}_{3}(s)+2 \mathrm{NH}_{3}(a q)+$ $\mathrm{H}_{2} \mathrm{O}(l)+2 \mathrm{Cl}$

which together yield the cell reaction:

cell reaction: $2 \mathrm{MnO}_{2}(s)+2 \mathrm{NH}_{4} \mathrm{Cl}(a q)+\mathrm{Zn}(s) \mathrm{Zn}^{2+}(a q)+\mathrm{Mn}_{2} \mathrm{O}_{3}(s)+$ $2 \mathrm{NH}_{3}(a q)+\mathrm{H}_{2} \mathrm{O}(l)+2 \mathrm{Cl} E_{\text {cell }} 1.5 \mathrm{~V}$

The voltage (cell potential) of a dry cell is approximately 1.5 V. Dry cells are available in various sizes (e.g., D, C, AA, AAA). All sizes of dry cells comprise the same components, and so they exhibit the same voltage, but larger cells contain greater amounts of the redox reactants and therefore are capable of transferring correspondingly greater amounts of charge. Like other galvanic cells, dry cells may be connected in series to yield batteries with greater voltage outputs, if needed.

A schematic diagram shows a typical dry cell. 


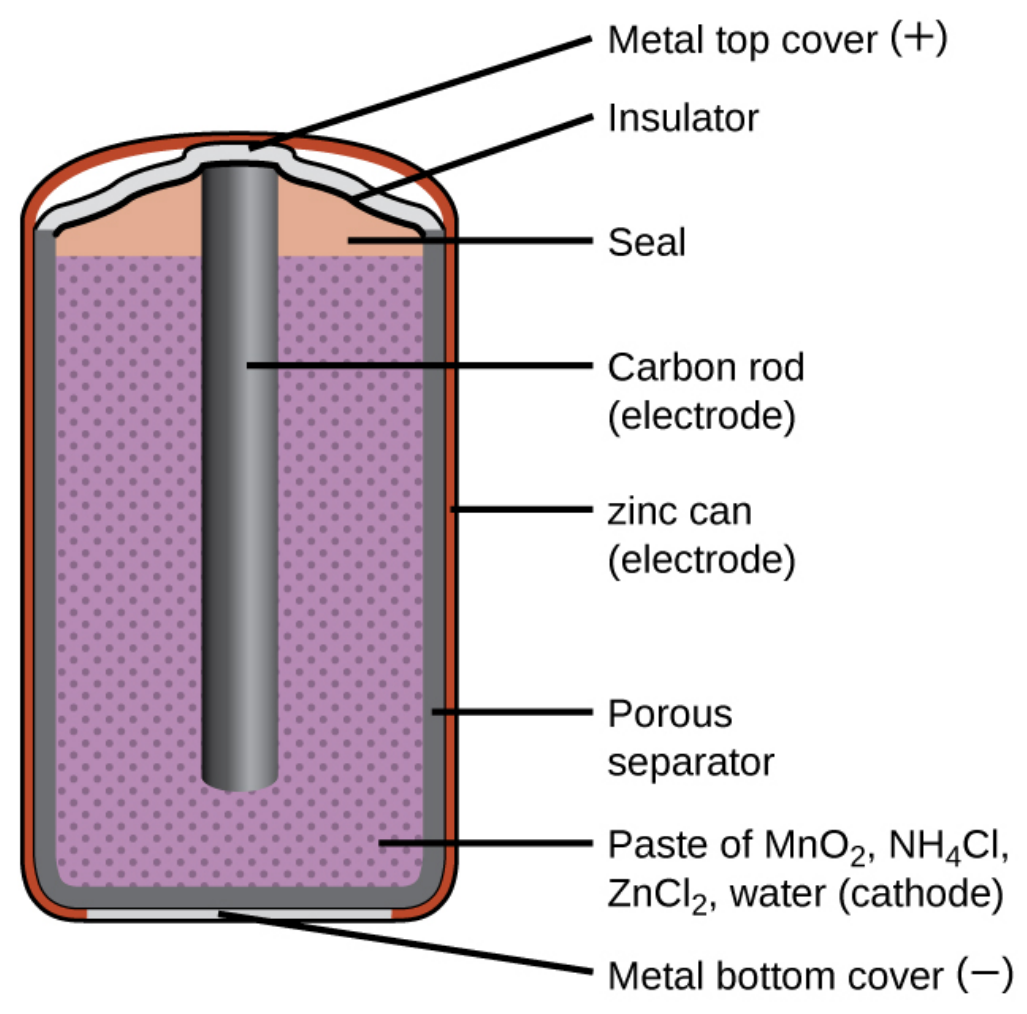

Visit this site to learn more about zinc-carbon batteries.

Alkaline batteries ((Figure)) were developed in the 1950s to improve on the performance of the dry cell, and they were designed around the same redox couples. As their name suggests, these types of batteries use alkaline electrolytes, often potassium hydroxide. The reactions are anode: $\mathrm{Zn}(s)+2 \mathrm{OH}(a q) \quad \mathrm{ZnO}(s)+\mathrm{H}_{2} \mathrm{O}(l)+2 \mathrm{e}$ cathode: $2 \mathrm{MnO}_{2}(s)+\mathrm{H}_{2} \mathrm{O}(l)+2 \mathrm{e} \mathrm{Mn}_{2} \mathrm{O}_{3}(s)+2 \mathrm{OH}(a q)$ cell: $\mathrm{Zn}(s)+2 \mathrm{MnO}_{2}(s) \mathrm{ZnO}(s)+\mathrm{Mn}_{2} \mathrm{O}_{3}(s)$ $E_{\text {cell }}=+1.43 \mathrm{~V}$

An alkaline battery can deliver about three to five times the energy of a zinc-carbon dry cell of similar size. Alkaline batteries are prone to leaking potassium hydroxide, so they should be removed from devices for long-term storage. While some alkaline batteries are rechargeable, most are not. Attempts to recharge an 
alkaline battery that is not rechargeable often leads to rupture of the battery and leakage of the potassium hydroxide electrolyte.

Alkaline batteries were designed as improved replacements for zinc-carbon (dry cell) batteries.

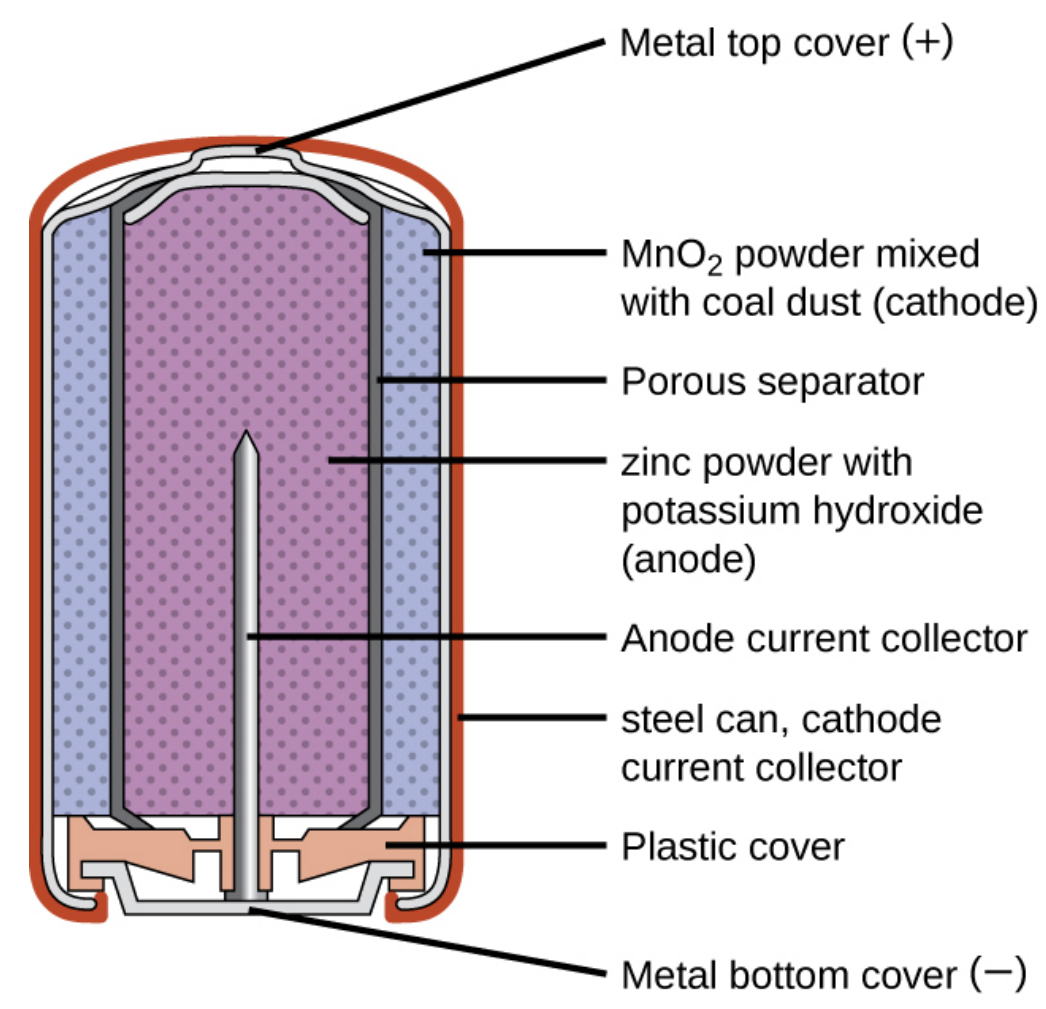

Visit this site to learn more about alkaline batteries.

Rechargeable (Secondary) Batteries

Nickel-cadmium, or NiCd, batteries ((Figure)) consist of a nickel-plated cathode, cadmium-plated anode, and a potassium hydroxide electrode. The positive and negative plates, which are prevented from shorting by the separator, are rolled together and put into the case. This is a "jelly-roll" design and allows the NiCd cell to deliver 
much more current than a similar-sized alkaline battery. The reactions are

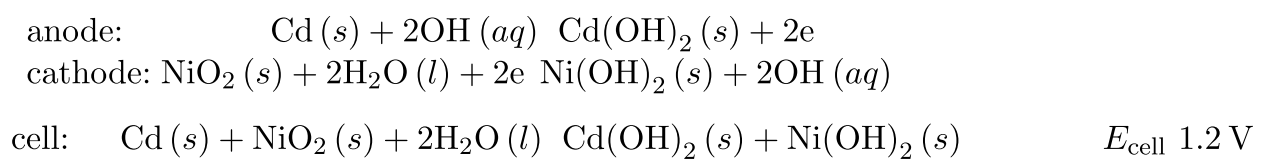

When properly treated, a NiCd battery can be recharged about 1000 times. Cadmium is a toxic heavy metal so NiCd batteries should never be ruptured or incinerated, and they should be disposed of in accordance with relevant toxic waste guidelines.

NiCd batteries use a "jelly-roll" design that significantly increases the amount of current the battery can deliver as compared to a similar-sized alkaline battery.

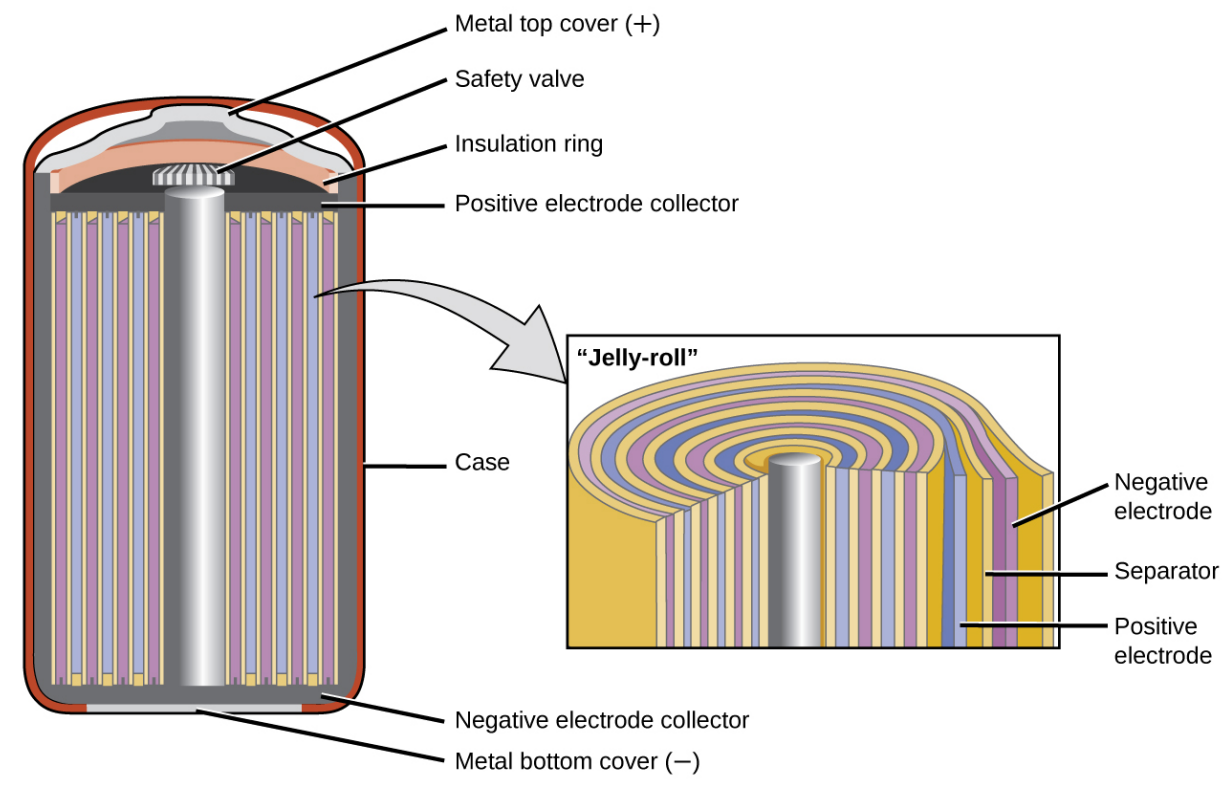

Visit this site for more information about nickel cadmium rechargeable batteries.

Lithium ion batteries ((Figure)) are among the most 
popular rechargeable batteries and are used in many portable electronic devices. The reactions are anode: $\quad \mathrm{LiCoO}_{2} \mathrm{Li}_{1 x} \mathrm{CoO}_{2}+x \mathrm{Li}^{+}+x \mathrm{e}$ cathode: $x \mathrm{Li}^{+}+x \mathrm{e}+x \mathrm{C}_{6} x \mathrm{LiC}_{6}$

cell: $\quad \mathrm{LiCoO}_{2}+x \mathrm{C}_{6} \mathrm{Li}_{1 x} \mathrm{CoO}_{2}+x \mathrm{LiC}_{6} \quad E_{\text {cell }} 3.7 \mathrm{~V}$

The variable stoichiometry of the cell reaction leads to variation in cell voltages, but for typical conditions, $x$ is usually no more than 0.5 and the cell voltage is approximately $3.7 \mathrm{~V}$. Lithium batteries are popular because they can provide a large amount current, are lighter than comparable batteries of other types, produce a nearly constant voltage as they discharge, and only slowly lose their charge when stored.

In a lithium ion battery, charge flows as the lithium ions are transferred between the anode and cathode.

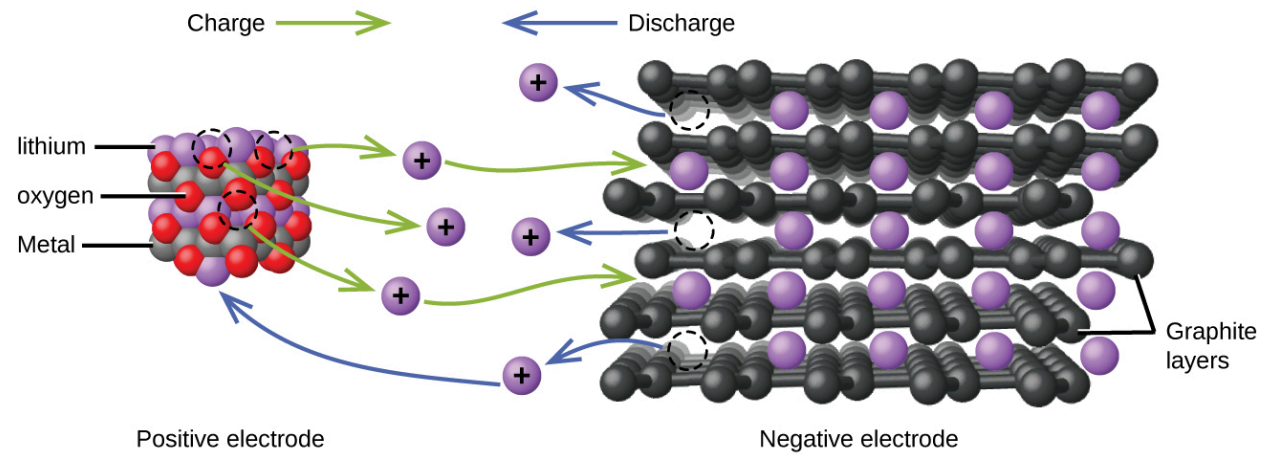

Visit this site for more information about lithium ion batteries.

The lead acid battery ((Figure)) is the type of secondary battery commonly used in automobiles. It is inexpensive and capable of producing the high current required by automobile starter motors. The reactions for a lead acid battery are 
anode: $\quad \mathrm{Pb}(s)+\mathrm{HSO}_{4}(a q) \mathrm{PbSO}_{4}(s)+\mathrm{H}^{+}(a q)+2 \mathrm{e}$

cathode: $\mathrm{PbO}_{2}(s)+\mathrm{HSO}_{4}(a q)+3 \mathrm{H}^{+}(a q)+2 \mathrm{e} \mathrm{PbSO}_{4}(s)+2 \mathrm{H}_{2} \mathrm{O}(l)$

cell:

$\mathrm{Pb}(s)+\mathrm{PbO}_{2}(s)+2 \mathrm{H}_{2} \mathrm{SO}_{4}(a q) 2 \mathrm{PbSO}_{4}(s)+2 \mathrm{H}_{2} \mathrm{O}(l)$

Each cell produces $2 \mathrm{~V}$, so six cells are connected in series to produce a $12-\mathrm{V}$ car battery. Lead acid batteries are heavy and contain a caustic liquid electrolyte, $\mathrm{H}_{2} \mathrm{SO}_{4}(a q)$, but are often still the battery of choice because of their high current density. Since these batteries contain a significant amount of lead, they must always be disposed of properly.

The lead acid battery in your automobile consists of six cells connected in series to give $12 \mathrm{~V}$.

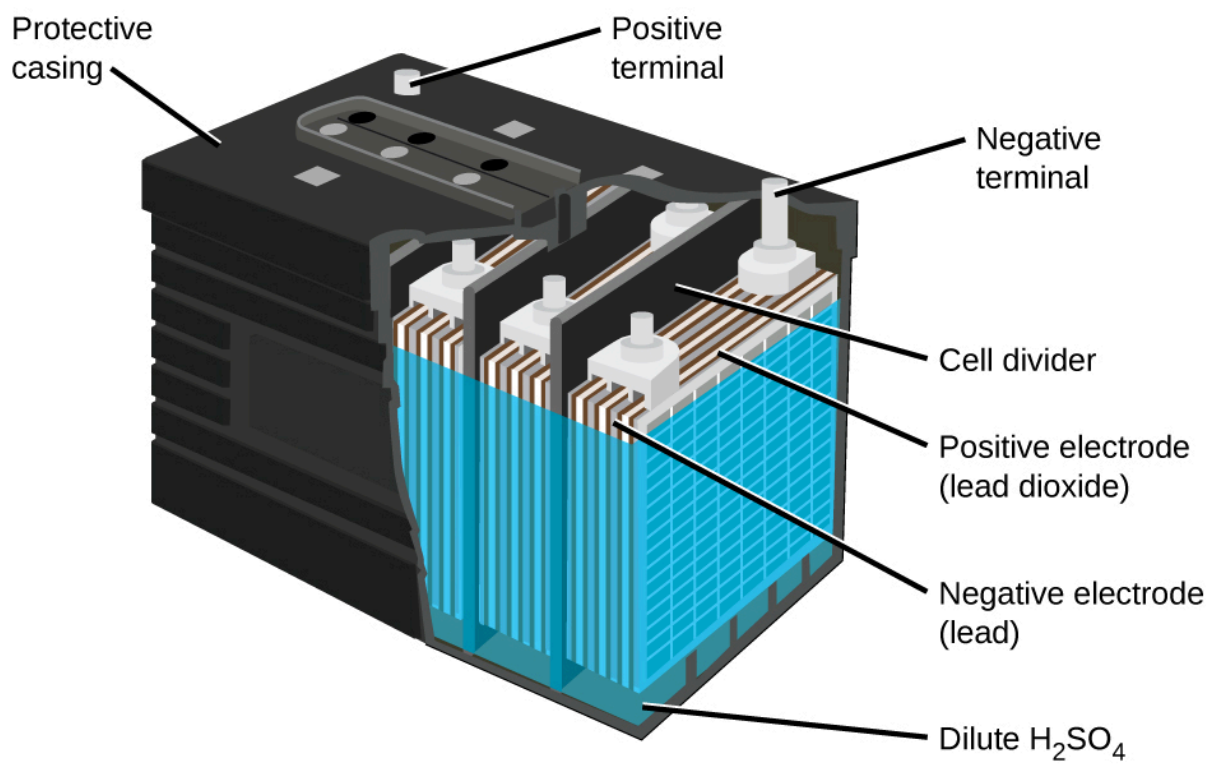

Visit this site for more information about lead acid batteries.

Fuel Cells

A fuel cell is a galvanic cell that uses traditional combustive fuels, most often hydrogen or methane, that 
are continuously fed into the cell along with an oxidant. (An alternative, but not very popular, name for a fuel cell is a flow battery.) Within the cell, fuel and oxidant undergo the same redox chemistry as when they are combusted, but via a catalyzed electrochemical that is significantly more efficient. For example, a typical hydrogen fuel cell uses graphite electrodes embedded with platinum-based catalysts to accelerate the two half-cell reactions:

In this hydrogen fuel cell, oxygen from the air reacts with hydrogen, producing water and electricity.

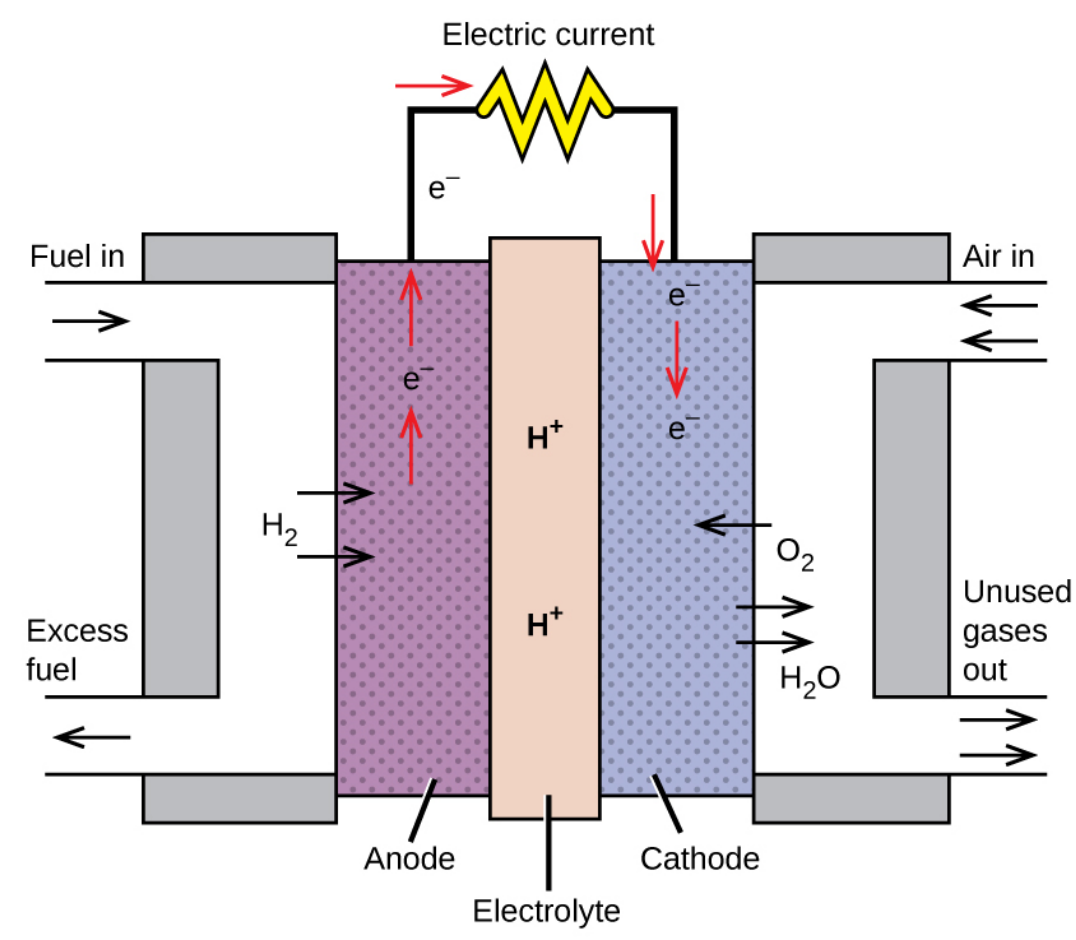

These types of fuel cells generally produce voltages of approximately $1.2 \mathrm{~V}$. Compared to an internal combustion engine, the energy efficiency of a fuel cell using the same redox reaction is typically more than double $(\sim 20 \%-25 \%$ for an engine versus $\sim 50 \%-75 \%$ for a fuel cell). Hydrogen fuel cells are commonly used on extended space missions, 
and prototypes for personal vehicles have been developed, though the technology remains relatively immature.

Check out this link to learn more about fuel cells.

Key Concepts and Summary

Galvanic cells designed specifically to function as electrical power supplies are called batteries. A variety of both single-use batteries (primary cells) and rechargeable batteries (secondary cells) are commercially available to serve a variety of applications, with important specifications including voltage, size, and lifetime. Fuel cells, sometimes called flow batteries, are devices that harness the energy of spontaneous redox reactions normally associated with combustion processes. Like batteries, fuel cells enable the reaction's electron transfer via an external circuit, but they require continuous input of the redox reactants (fuel and oxidant) from an external reservoir. Fuel cells are typically much more efficient in converting the energy released by the reaction to useful work in comparison to internal combustion engines.

\section{Chemistry End of Chapter Exercises}

Consider a battery made from one half-cell that consists of a copper electrode in $1 \mathrm{M} \mathrm{CuSO}_{4}$ solution and another half-cell that consists of a lead electrode in $1 M \mathrm{~Pb}\left(\mathrm{NO}_{3}\right)_{2}$ solution.

(a) What is the standard cell potential for the battery?

(b) What are the reactions at the anode, cathode, and the overall reaction?

(c) Most devices designed to use dry-cell batteries can 
operate between 1.0 and $1.5 \mathrm{~V}$. Could this cell be used to make a battery that could replace a dry-cell battery? Why or why not.

(d) Suppose sulfuric acid is added to the half-cell with the lead electrode and some $\mathrm{PbSO}_{4}(s)$ forms. Would the cell potential increase, decrease, or remain the same?

Consider a battery with the overall reaction: $\mathrm{Cu}(s)+2 \mathrm{Ag}^{+}(a q) 2 \mathrm{Ag}(s)+\mathrm{Cu}^{2+}(a q)$.

(a) What is the reaction at the anode and cathode?

(b) A battery is "dead" when its cell potential is zero. What is the value of $Q$ when this battery is dead?

(c) If a particular dead battery was found to have $\left[\mathrm{Cu}^{2+}\right]$ $=0.11 \mathrm{M}$, what was the concentration of silver ion?

anode: $\mathrm{Cu}(s) \mathrm{Cu}^{2+}(a q)+2 \mathrm{e}$ cathode: $2\left(\mathrm{Ag}^{+}(a q)+\mathrm{e} \mathrm{Ag}(s)\right)$ (b) $3.510^{15}$; (c) $5.610^{-9} \mathrm{M}$

$$
\begin{array}{cc}
E_{\text {anode }}= & 0.34 \mathrm{~V} ; \\
E_{\text {cathode }} & =0.7996 \mathrm{~V}
\end{array}
$$

Why do batteries go dead, but fuel cells do not?

Batteries are self-contained and have a limited supply of reagents to expend before going dead. Alternatively, battery reaction byproducts accumulate and interfere with the reaction. Because a fuel cell is constantly resupplied with reactants and products are expelled, it can continue to function as long as reagents are supplied.

Use the Nernst equation to explain the drop in voltage observed for some batteries as they discharge.

Using the information thus far in this chapter, explain 
why battery-powered electronics perform poorly in low temperatures.

$E_{\text {cell, }}$ as described in the Nernst equation, has a term that is directly proportional to temperature. At low temperatures, this term is decreased, resulting in a lower cell voltage provided by the battery to the device-the same effect as a battery running dead.

Glossary

alkaline battery

primary battery similar to a dry cell that uses an alkaline (often potassium hydroxide) electrolyte; designed to be an improved replacement for the dry cell, but with more energy storage and less electrolyte leakage than typical dry cell

battery

single or series of galvanic cells designed for use as a source of electrical power dry cell

primary battery, also called a zinc-carbon battery, based on the spontaneous oxidation of zinc by manganese(IV)

\section{fuel cell}

devices similar to galvanic cells that require a continuous feed of redox reactants; also called a flow battery 


\section{lead acid battery}

rechargeable battery commonly used in automobiles; it typically comprises six galvanic cells based on $\mathrm{Pb}$ half-reactions in acidic solution

\section{lithium ion battery}

widely used rechargeable battery commonly used in portable electronic devices, based on lithium ion transfer between the anode and cathode nickel-cadmium battery rechargeable battery based on $\mathrm{Ni} / \mathrm{Cd}$ halfcells with applications similar to those of lithium ion batteries

primary cell

nonrechargeable battery, suitable for single use only secondary cell battery designed to allow recharging 


\section{Focus on Alternative Energy}

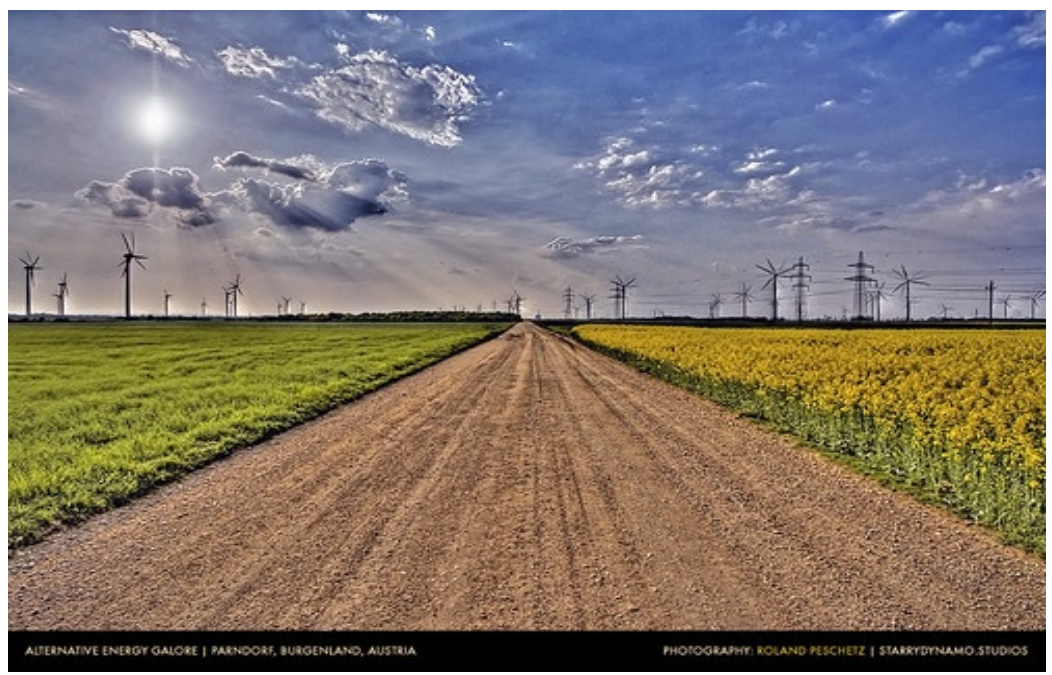

"Alternative Energy Galore" by roliathBrickworx is licensed under CC BY-NC 2.0

There are a multitude of what one might call 'alternative' sources of energy - biofuels, wind, solar, solar-thermal, hydroelectric, fuel cells - simply because they supply a small portion of the world's total energy needs. In this text, we introduced biofuels in the 'Focus on Energy' section as an alternative combustible to the fossil fuels that we spent much of that section on. In this section we will introduce a few other fuels but limit our discussion to hydroelectric power, batteries and hydrogen based power as they all have significant chemistry-based fundamentals at the level of this text.

Refer to the information discussed in the previous Focus section as well as Part XII of this text and elaborate on the following focus boxes, solving any problems that appear. While learning the basics of electrochemistry and using hydrogen as a fuel source, make note of important distinctions between this and other types of fuels in meeting our energy demands. One must understand the benefits and challenges of the processes that are currently in use and those being proposed to meet our future energy demands in order to decide on the best route for moving forward. 


\section{Solar Power}

- Direct Heat

- Wind

- Water - the hydrologic cycle.
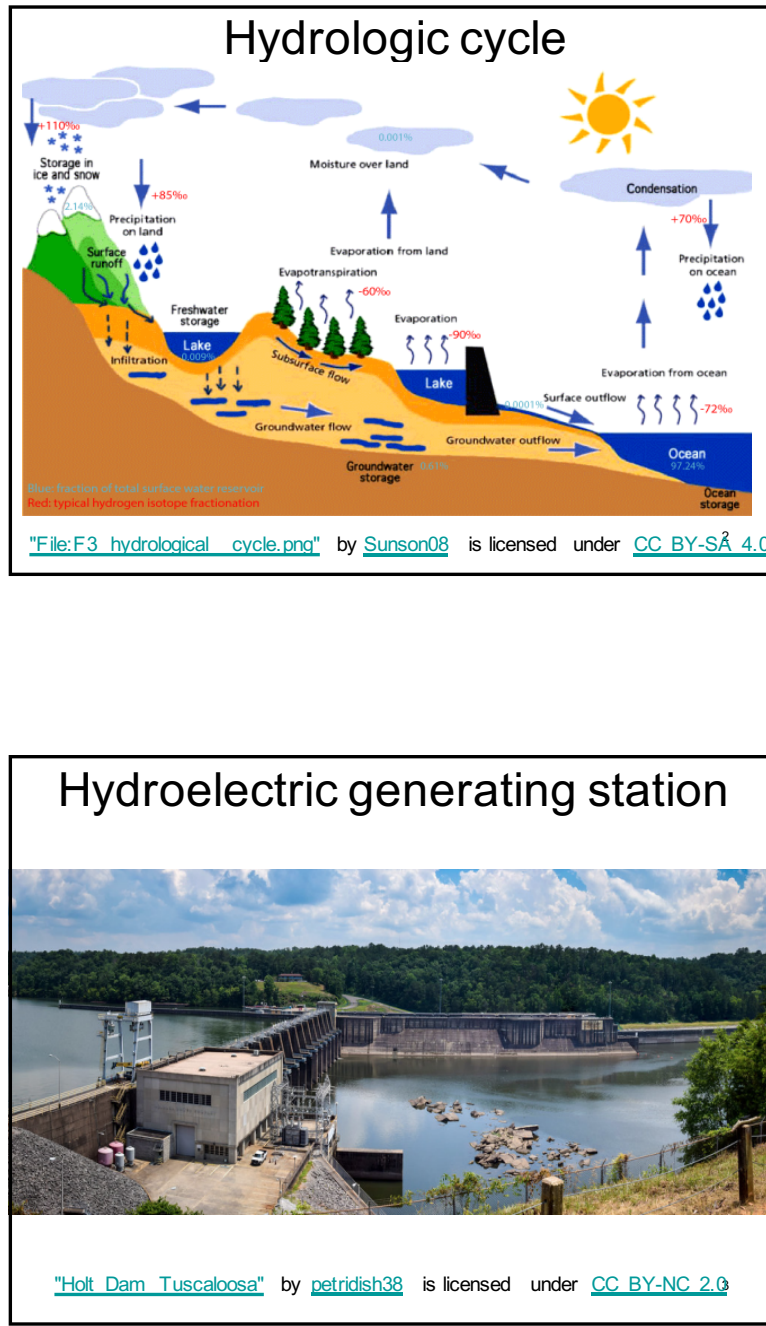


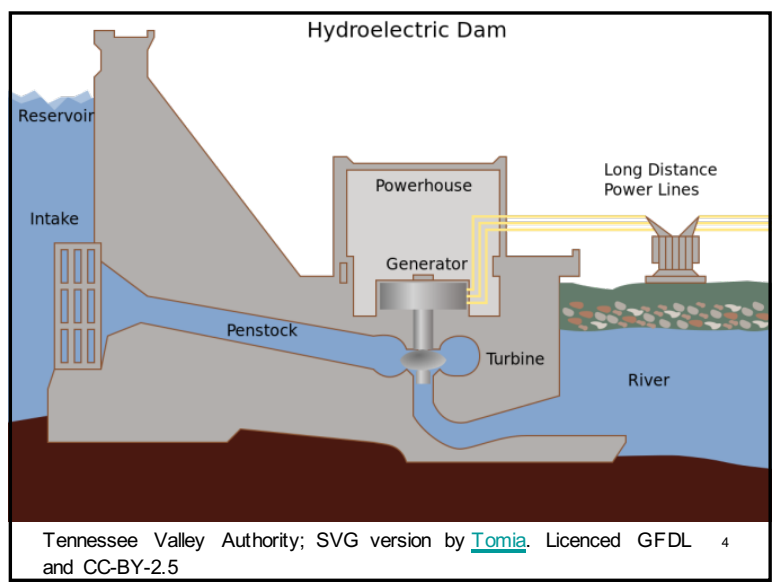

The creation of hydroelectric reservoirs can have a number of environmental consequences. Research and elaborate.

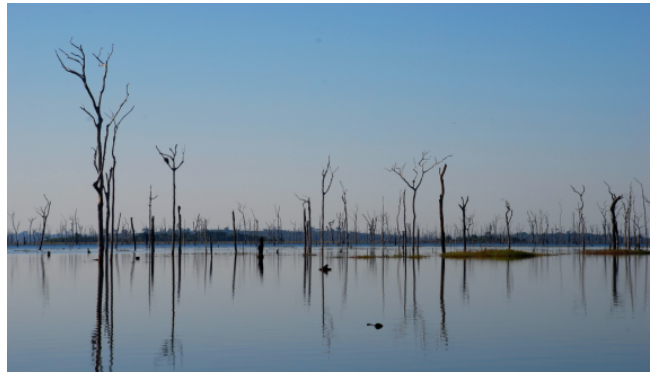

"Dam causes a $100 \mathrm{~km}$ of devastation" by joellehernandez is 5 licensed under CC BY-NC-ND 2.0

\section{A battery (or galvanic cell) is a system for the direct conversion of chemical energy to electrical energy. \\ - convenient, transportable sources of stored energy.}

"AJ Batteries001" by Curious Gregor is

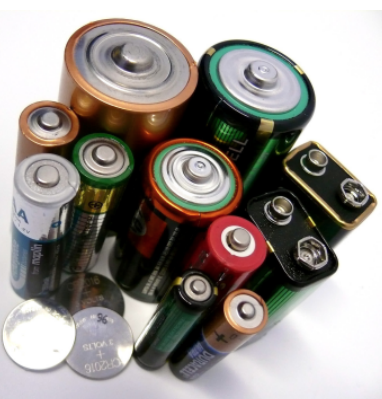

NC-SA 2.0 


\section{Nickel-Cadmium battery}

- a redox reaction between cadmium and nickel. Show the full redox reaction and identify the species that is being oxidized and that which is being reduced.

\section{Fuel Cell}

- chemical reaction corresponding to hydrogen combustion but the energy is released as electricity.

- combustion:

$\mathrm{H}_{2(\mathrm{~g})}+1 / 2 \mathrm{O}_{2(\mathrm{~g})} \Leftrightarrow \mathrm{H}_{2} \mathrm{O}_{(\mathrm{l})}$ yields $143 \mathrm{~kJ} / \mathrm{g}$ and water

- fuel cell redox reaction: has the same overall reaction as combustion

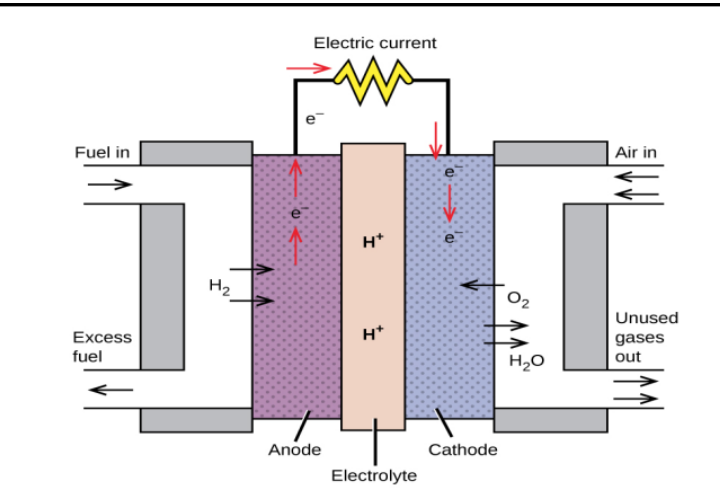

Chemistry and the Environment by Devin R. Latimer is licensed under $a_{9}$ Creative Commons Attribution 4.0 International License 
- Show the half-reactions and overall reaction involved in the release of energy from hydrogen in a fuel cell. Summarize how this compares with the combustion of hydrogen.

- To make the hydrogen economy a reality, where will we get the hydrogen?

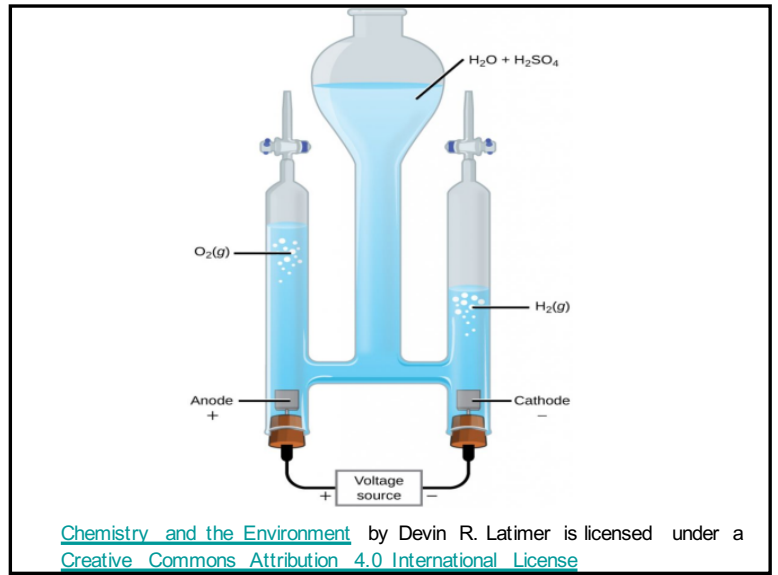

Photovoltaics - involves the flow of electrons directly from the impact of the sun.

- normally using semiconductors

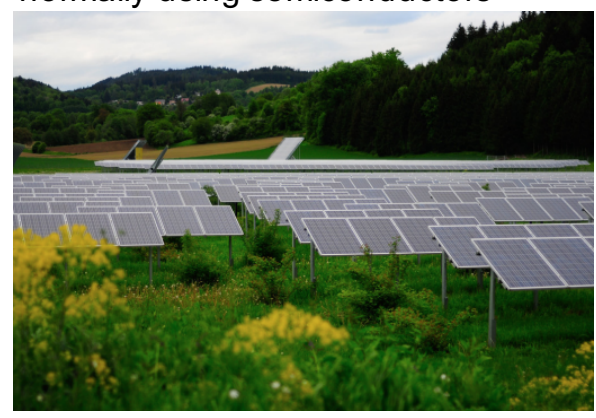


Solar Thermal - concentration of heat energy directly from the sun for use in heating or electricity generation

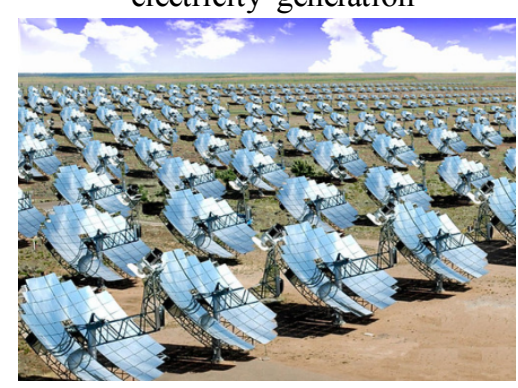

"Solar Thermal" by International Rivers is licensed under $\underline{\text { CC }}$ 13 BY-NC-SA 2.0

Fusion
- of nuclei can produce massive amounts of
energy.
- What is the fusion reaction involved in
the sun? What are the conditions
necessary for this reaction? Do you think
this is a feasible route to power in the
future?

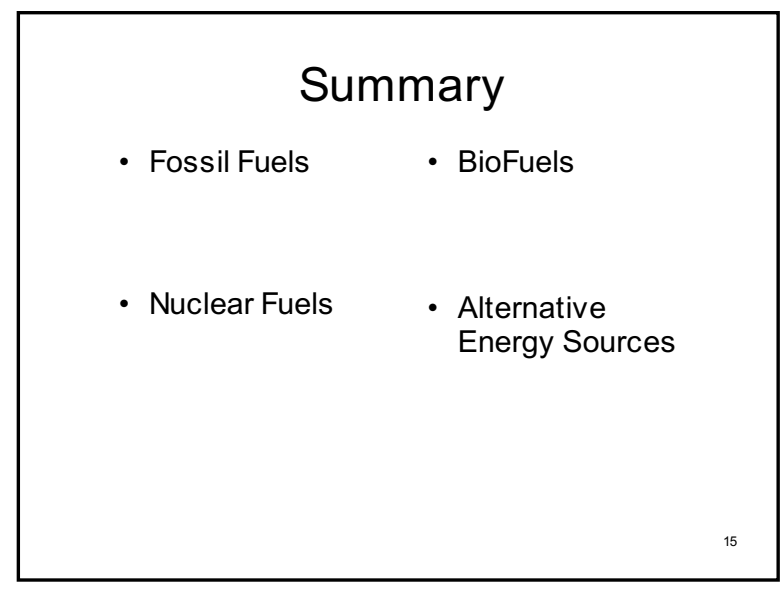






\section{PART XIII}

\section{ORGANIC}

\section{CHEMISTRY}





\section{5.}

\section{INTRODUCTION}

\section{Learning Objectives}

- Hydrocarbons

- Alcohols and Ethers

All organic compounds contain carbon and most are formed by living things, although they are also formed by geological and artificial processes. (credit left:

modification of work by Jon Sullivan; credit left middle: modification of work by Deb Tremper; credit right middle: modification of work by "annszyp"/Wikimedia Commons; credit right: modification of work by George Shuklin) 
This figure includes four photographs. The first is of an orange and black butterfly on a large leaf. The second shows a seam on a worn pair of blue jeans. The third image is of a red plastic drinking cup. The last image shows the blue flames of a lit burner on a gas stove.

All living things on earth are formed mostly of carbon compounds. The prevalence of carbon compounds in living things has led to the epithet "carbon-based" life. The truth is we know of no other kind of life. Early chemists regarded substances isolated from organisms (plants and animals) as a different type of matter that could not be synthesized artificially, and these substances were thus known as organic compounds. The widespread belief called vitalism held that organic compounds were formed by a vital force present only in living organisms. The German chemist Friedrich Wohler was one of the early chemists to refute this aspect of vitalism, when, in 1828, he reported the synthesis of urea, a component of many body fluids, from nonliving materials. Since then, it has been recognized that organic molecules obey the same natural laws as inorganic substances, and the category of organic compounds has evolved to include both natural and synthetic compounds that contain carbon. Some carbon-containing compounds are not classified as organic, for example, carbonates and cyanides, and simple oxides, such as $\mathrm{CO}$ and $\mathrm{CO}_{2}$. Although a single, precise definition has yet to be identified by the chemistry community, most agree that a defining trait of organic molecules is the presence of carbon as the principal element, bonded to hydrogen and other carbon atoms.

Today, organic compounds are key components of 
plastics, soaps, perfumes, sweeteners, fabrics, pharmaceuticals, and many other substances that we use every day. The value to us of organic compounds ensures that organic chemistry is an important discipline within the general field of chemistry. In this chapter, we give a very basic introduction to organic chemistry - why the element carbon gives rise to a vast number and variety of compounds, how some of those compounds are classified, and the role of a few organic compounds in representative biological and industrial settings. 


\section{6.}

\section{HYDROCARBONS}

\section{Learning Objectives}

By the end of this section, you will be able to:

- Explain the importance of hydrocarbons and the reason for their diversity

- Name saturated and unsaturated hydrocarbons, and molecules derived from them

- Describe the reactions characteristic of saturated and unsaturated hydrocarbons

- Identify structural and geometric isomers of hydrocarbons

The largest database ${ }^{1}$ of organic compounds lists about 10 million substances, which include compounds originating from living organisms and those synthesized by chemists. The number of potential organic compounds has been estimated $^{2}$ at $10^{60}$-an astronomically high number. The existence of so many organic molecules is a consequence 
of the ability of carbon atoms to form up to four strong bonds to other carbon atoms, resulting in chains and rings of many different sizes, shapes, and complexities.

The simplest organic compounds contain only the elements carbon and hydrogen, and are called hydrocarbons. Even though they are composed of only two types of atoms, there is a wide variety of hydrocarbons because they may consist of varying lengths of chains, branched chains, and rings of carbon atoms, or combinations of these structures. In addition, hydrocarbons may differ in the types of carbon-carbon bonds present in their molecules. Many hydrocarbons are found in plants, animals, and their fossils; other hydrocarbons have been prepared in the laboratory. We use hydrocarbons every day, mainly as fuels, such as natural gas, acetylene, propane, butane, and the principal components of gasoline, diesel fuel, and heating oil. The familiar plastics polyethylene, polypropylene, and polystyrene are also hydrocarbons. We can distinguish several types of hydrocarbons by differences in the bonding between carbon atoms. This leads to differences in geometries and in the hybridization of the carbon orbitals.

\section{Alkanes}

Alkanes, or saturated hydrocarbons, contain only single covalent bonds between carbon atoms. Each of the carbon atoms in an alkane has $s p^{3}$ hybrid orbitals and is bonded to four other atoms, each of which is either carbon or hydrogen. The Lewis structures and models of methane, ethane, and pentane are illustrated in (Figure). Carbon 
chains are usually drawn as straight lines in Lewis structures, but one has to remember that Lewis structures are not intended to indicate the geometry of molecules. Notice that the carbon atoms in the structural models (the ball-and-stick and space-filling models) of the pentane molecule do not lie in a straight line. Because of the $s p^{3}$ hybridization, the bond angles in carbon chains are close to $109.5^{\circ}$, giving such chains in an alkane a zigzag shape.

The structures of alkanes and other organic molecules may also be represented in a less detailed manner by condensed structural formulas (or simply, condensed formulas). Instead of the usual format for chemical formulas in which each element symbol appears just once, a condensed formula is written to suggest the bonding in the molecule. These formulas have the appearance of a Lewis structure from which most or all of the bond symbols have been removed. Condensed structural formulas for ethane and pentane are shown at the bottom of (Figure), and several additional examples are provided in the exercises at the end of this chapter.

Pictured are the Lewis structures, ball-and-stick models, and space-filling models for molecules of methane, ethane, and pentane. 
$\mathrm{H}-\mathrm{I}_{\mathrm{H}}^{\mathrm{C}}-\mathrm{H}$
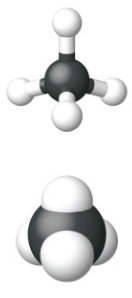

methane $\mathrm{CH}_{4}$
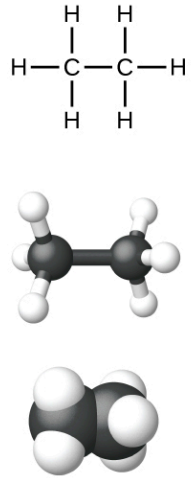

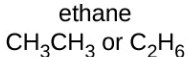
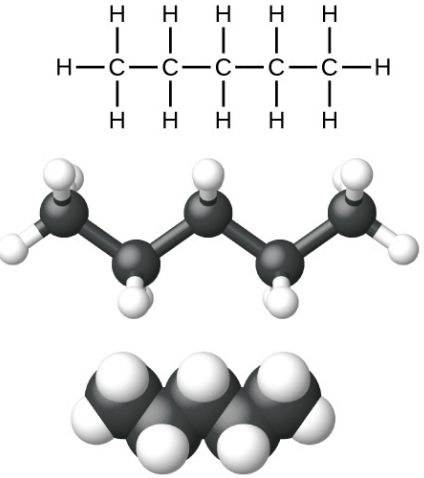

pentane $\mathrm{CH}_{3} \mathrm{CH}_{2} \mathrm{CH}_{2} \mathrm{CH}_{2} \mathrm{CH}_{3}$ or $\mathrm{C}_{5} \mathrm{H}_{12}$

A common method used by organic chemists to simplify the drawings of larger molecules is to use a skeletal structure (also called a line-angle structure). In this type of structure, carbon atoms are not symbolized with a $\mathrm{C}$, but represented by each end of a line or bend in a line. Hydrogen atoms are not drawn if they are attached to a carbon. Other atoms besides carbon and hydrogen are represented by their elemental symbols. (Figure) shows three different ways to draw the same structure. The same structure can be represented three different ways: an expanded formula, a condensed formula, and a skeletal structure.

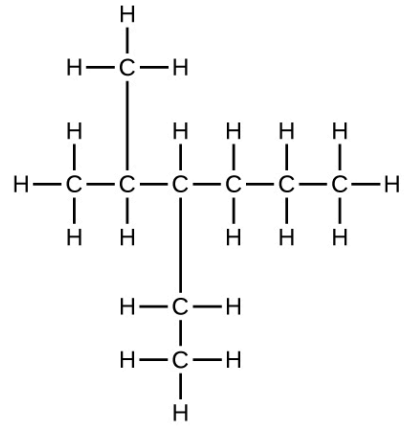

Expanded formula

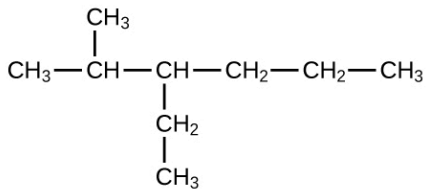

Condensed formula<smiles>CCCC(CC)C(C)C</smiles>

Skeletal structure 
Drawing Skeletal Structures Draw the skeletal structures for these two molecules:<smiles>CC(C)C(C)(C)C</smiles>

(a)

$$
\mathrm{CH}_{3} \mathrm{CH}_{2} \mathrm{CH}_{2} \mathrm{CH}_{2} \mathrm{CH}_{2} \mathrm{CH}_{2} \mathrm{CH}_{3}
$$

(b)

Solution Each carbon atom is converted into the end of a line or the place where lines intersect. All hydrogen atoms attached to the carbon atoms are left out of the structure (although we still need to recognize they are there):

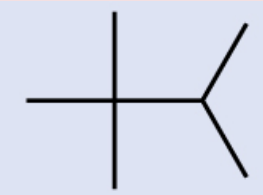

(a)

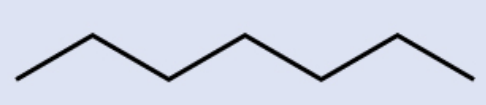

(b)

Check Your Learning Draw the skeletal structures for these two molecules:<smiles>CC1CCCCC1</smiles>

(a)

$$
\mathrm{CH}_{3} \mathrm{CH}_{2} \mathrm{CH}_{2} \mathrm{CH}_{2} \mathrm{CH}_{3}
$$

(b)

Answer:

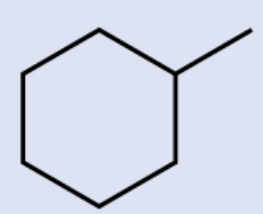

(a)

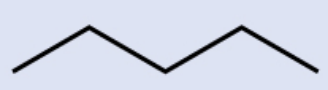

(b) 
Interpreting Skeletal Structures Identify the chemical formula of the molecule represented here:

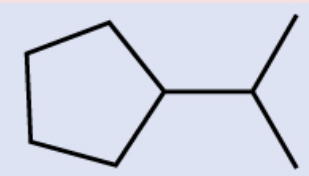

Solution There are eight places where lines intersect or end, meaning that there are eight carbon atoms in the molecule. Since we know that carbon atoms tend to make four bonds, each carbon atom will have the number of hydrogen atoms that are required for four bonds. This compound contains 16 hydrogen atoms for a molecular formula of $\mathrm{C}_{8} \mathrm{H}_{16}$.

Location of the hydrogen atoms:<smiles>CC(C)C1CCCC1</smiles>

Check Your Learning Identify the chemical formula of the molecule represented here:

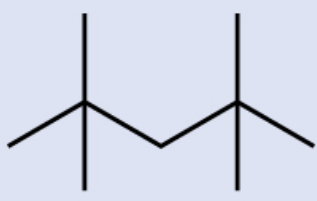

Answer:

$\mathrm{CgH}_{20}$

All alkanes are composed of carbon and hydrogen atoms, and have similar bonds, structures, and formulas; noncyclic alkanes all have a formula of $\mathrm{C}_{n} \mathrm{H}_{2 n+2}$. The number of carbon atoms present in an alkane has no limit. Greater numbers of atoms in the molecules will lead to stronger intermolecular attractions (dispersion forces) 
and correspondingly different physical properties of the molecules. Properties such as melting point and boiling point ((Figure)) usually change smoothly and predictably as the number of carbon and hydrogen atoms in the molecules change.

\section{Properties of Some Alkanes 3}

$\begin{array}{llllll}\text { Alkane } & \begin{array}{l}\text { Molecular } \\ \text { Formula }\end{array} & \begin{array}{l}\text { Melting } \\ \text { Point } \\ \left({ }^{\circ} \mathbf{C}\right)\end{array} & \begin{array}{l}\text { Boiling } \\ \text { Point } \\ \left({ }^{\circ} \mathbf{C}\right)\end{array} & \begin{array}{l}\text { Phase } \\ \text { at } \\ \text { STP }\end{array} & \begin{array}{l}\text { Number of } \\ \text { Structural } \\ \text { Isomers }\end{array} \\ \text { ethane } & \mathrm{CH}_{4} & -182.5 & -161.5 & \text { gas } & 1 \\ \text { propane } & \mathrm{C}_{2} \mathrm{H}_{6} & -183.3 & -88.6 & \text { gas } & 1 \\ \text { butane } & \mathrm{C}_{3} \mathrm{H}_{8} & -187.7 & -42.1 & \text { gas } & 1 \\ \text { pentane } & \mathrm{C}_{4} \mathrm{H}_{10} & -138.3 & -0.5 & \text { gas } & 2 \\ \text { hexane } & \mathrm{C}_{5} \mathrm{H}_{12} & -129.7 & 36.1 & \text { liquid } & 3 \\ \text { heptane } & \mathrm{C}_{6} \mathrm{H}_{14} & -95.3 & 68.7 & \text { liquid } & 5 \\ \text { octane } & \mathrm{C}_{7} \mathrm{H}_{16} & -90.6 & 98.4 & \text { liquid } & 9 \\ \text { nonane } & \mathrm{C}_{8} \mathrm{H}_{18} & -56.8 & 125.7 & \text { liquid } & 18 \\ \text { decane } & \mathrm{C}_{9} \mathrm{H}_{20} & -53.6 & 150.8 & \text { liquid } & 35 \\ \text { tetradecane } & \mathrm{C}_{10} \mathrm{H}_{22} & -29.7 & 174.0 & \text { liquid } & 75 \\ \text { octadecane } & \mathrm{C}_{14} \mathrm{C}_{30} & 5.9 & 253.5 & \text { solid } & 1858 \\ & \mathrm{C}_{18} \mathrm{H}_{38} & 28.2 & 316.1 & \text { solid } & 60,523\end{array}$

Hydrocarbons with the same formula, including alkanes, can have different structures. For example, two alkanes have the formula $\mathrm{C}_{4} \mathrm{H}_{10}$ : They are called $n$-butane and 2-methylpropane (or isobutane), and have the following Lewis structures: 
<smiles>CCCC</smiles><smiles>CC(C)C</smiles>
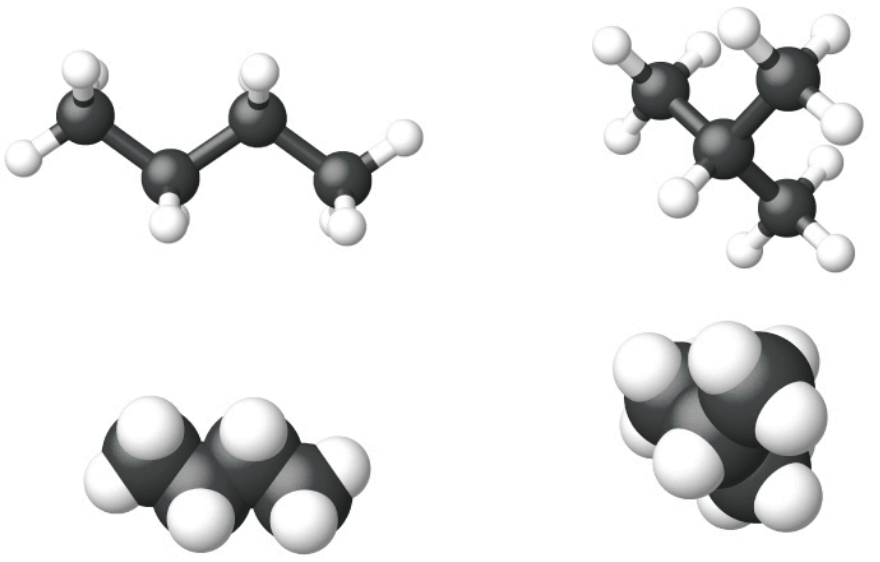

n-butane

2-methylpropane

The compounds $n$-butane and 2-methylpropane are structural isomers (the term constitutional isomers is also commonly used). Constitutional isomers have the same molecular formula but different spatial arrangements of the atoms in their molecules. The $n$-butane molecule contains an unbranched chain, meaning that no carbon atom is bonded to more than two other carbon atoms. We use the term normal, or the prefix $n$, to refer to a chain of carbon atoms without branching. The compound 2-methylpropane has a branched chain (the carbon atom in the center of the Lewis structure is bonded to three other carbon atoms)

Identifying isomers from Lewis structures is not as easy as it looks. Lewis structures that look different may actually represent the same isomers. For example, the three structures in (Figure) all represent the same molecule, $n$-butane, and hence are not different isomers. 
They are identical because each contains an unbranched chain of four carbon atoms.

These three representations of the structure of $n$-butane are not isomers because they all contain the same arrangement of atoms and bonds.<smiles>CCCC</smiles>

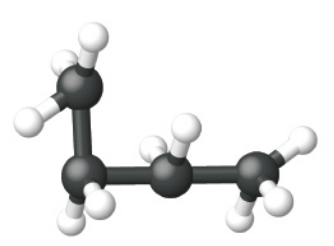<smiles>CCCC</smiles><smiles>CCC</smiles>
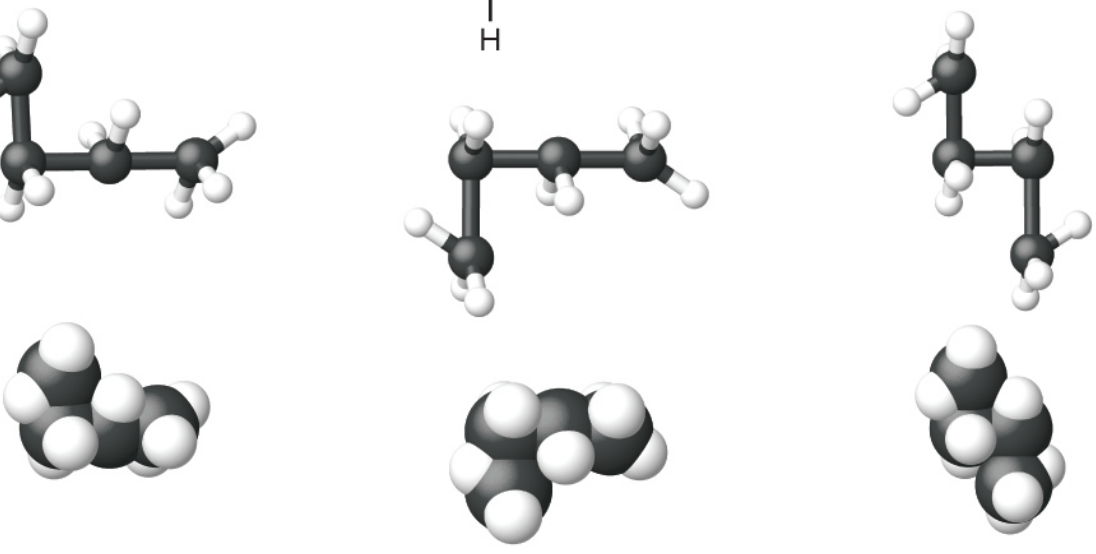

The Basics of Organic Nomenclature: Naming Alkanes

The International Union of Pure and Applied Chemistry (IUPAC) has devised a system of nomenclature that begins with the names of the alkanes and can be adjusted from there to account for more complicated structures. The nomenclature for alkanes is based on two rules:

1. To name an alkane, first identify the longest chain of carbon atoms in its structure. A twocarbon chain is called ethane; a three-carbon chain, propane; and a four-carbon chain, 
butane. Longer chains are named as follows: pentane (five-carbon chain), hexane (6), heptane (7), octane (8), nonane (9), and decane (10). These prefixes can be seen in the names of the alkanes described in (Figure).

2. Add prefixes to the name of the longest chain to indicate the positions and names of substituents. Substituents are branches or functional groups that replace hydrogen atoms on a chain. The position of a substituent or branch is identified by the number of the carbon atom it is bonded to in the chain. We number the carbon atoms in the chain by counting from the end of the chain nearest the substituents. Multiple substituents are named individually and placed in alphabetical order at the front of the name.

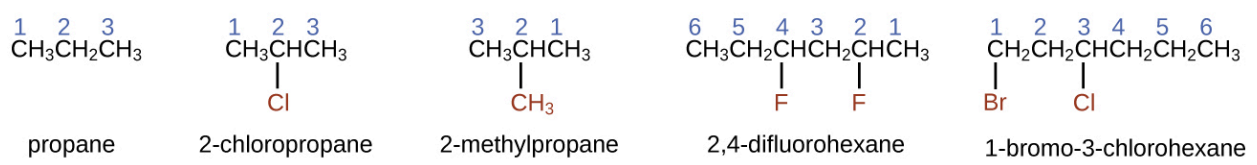

When more than one substituent is present, either on the same carbon atom or on different carbon atoms, the substituents are listed alphabetically. Because the carbon atom numbering begins at the end closest to a substituent, the longest chain of carbon atoms is numbered in such a way as to produce the lowest number for the substituents. The ending - $o$ replaces -ide at the end of the name of an electronegative substituent (in ionic compounds, the negatively charged ion ends with -ide like chloride; in organic compounds, such atoms are treated as substituents and the $-o$ ending is used). The number of 
substituents of the same type is indicated by the prefixes di- (two), tri- (three), tetra- (four), and so on (for example, difluoro- indicates two fluoride substituents).

Naming Halogen-substituted Alkanes Name the molecule whose structure is shown here:<smiles>ClCC(Br)CCl</smiles>

Solution<smiles>CCC(C)Br</smiles>

The four-carbon chain is numbered from the end with the chlorine atom. This puts the substituents on positions 1 and 2 (numbering from the other end would put the substituents on positions 3 and 4). Four carbon atoms means that the base name of this compound will be butane. The bromine at position 2 will be described by adding 2-bromo-; this will come at the beginning of the name, since bromo-comes before chloro- alphabetically. The chlorine at position 1 will be described by adding 1-chloro-, resulting in the name of the molecule being 2-bromo-1-chlorobutane.

Check Your Learning Name the following molecule:<smiles>CCC(Br)(Br)C(C)I</smiles>

Answer:

3,3-dibromo-2-iodopentane 
We call a substituent that contains one less hydrogen than the corresponding alkane an alkyl group. The name of an alkyl group is obtained by dropping the suffix -ane of the alkane name and adding -yl:<smiles>C</smiles>

methane<smiles>CC</smiles>

methyl group<smiles>CC</smiles>

ethane<smiles>CCC</smiles>

ethyl group

The open bonds in the methyl and ethyl groups indicate that these alkyl groups are bonded to another atom.

Naming Substituted Alkanes Name the molecule whose structure is shown here:<smiles>CCCCC(C)C</smiles>

Solution The longest carbon chain runs horizontally across the page and contains six carbon atoms (this makes the base of the name hexane, but we will also need to incorporate the name of the branch). In this case, we want to number from right to left (as shown by the blue numbers) so the branch is connected to carbon 3 (imagine the numbers from left to right-this would put the branch on carbon 4, violating our rules). The branch attached to position 3 of our chain contains two carbon atoms (numbered in red)-so we take our name for two carbons eth- and attach - yl at the end to signify we are 
describing a branch. Putting all the pieces together, this molecule is 3-ethylhexane.

Check Your Learning Name the following molecule:<smiles>CCCCC(C)C(C)CCCC</smiles>

Answer:

4-propyloctane

Some hydrocarbons can form more than one type of alkyl group when the hydrogen atoms that would be removed have different "environments" in the molecule. This diversity of possible alkyl groups can be identified in the following way: The four hydrogen atoms in a methane molecule are equivalent; they all have the same environment. They are equivalent because each is bonded to a carbon atom (the same carbon atom) that is bonded to three hydrogen atoms. (It may be easier to see the equivalency in the ball and stick models in (Figure). Removal of any one of the four hydrogen atoms from methane forms a methyl group. Likewise, the six hydrogen atoms in ethane are equivalent ((Figure)) and removing any one of these hydrogen atoms produces an ethyl group. Each of the six hydrogen atoms is bonded to a carbon atom that is bonded to two other hydrogen atoms and a carbon atom. However, in both propane and 
2-methylpropane, there are hydrogen atoms in two different environments, distinguished by the adjacent atoms or groups of atoms:<smiles>CCC</smiles>

propane<smiles>CC(C)C</smiles>

2-methylpropane

Each of the six equivalent hydrogen atoms of the first type in propane and each of the nine equivalent hydrogen atoms of that type in 2-methylpropane (all shown in black) are bonded to a carbon atom that is bonded to only one other carbon atom. The two purple hydrogen atoms in propane are of a second type. They differ from the six hydrogen atoms of the first type in that they are bonded to a carbon atom bonded to two other carbon atoms. The green hydrogen atom in 2-methylpropane differs from the other nine hydrogen atoms in that molecule and from the purple hydrogen atoms in propane. The green hydrogen atom in 2-methylpropane is bonded to a carbon atom bonded to three other carbon atoms. Two different alkyl groups can be formed from each of these molecules, depending on which hydrogen atom is removed. The names and structures of these and several other alkyl groups are listed in (Figure).

This listing gives the names and formulas for various alkyl groups formed by the removal of hydrogen atoms from different locations. 


\begin{tabular}{|l|l|}
\hline Alkyl Group & \multicolumn{1}{|c|}{ Structure } \\
\hline methyl & $\mathrm{CH}_{3}-$ \\
\hline ethyl & $\mathrm{CH}_{3} \mathrm{CH}_{2}-$ \\
\hline$n$-propyl & $\mathrm{CH}_{3} \mathrm{CH}_{2} \mathrm{CH}_{2}-$ \\
\hline isopropyl & । $\mathrm{CHCH}_{3}$ \\
\hline n-butyl & $\mathrm{CH}_{3} \mathrm{CH}_{2} \mathrm{CH}_{2} \mathrm{CH}_{2}-$ \\
\hline sec-butyl & $\mathrm{CH}_{3} \mathrm{CH}_{2} \mathrm{CHCH}_{3}$ \\
\hline isobutyl & $\mathrm{CH}_{3} \mathrm{CHCH}_{2}-$ \\
\hline & $\stackrel{\mathrm{CH}_{3}}{\mid}$ \\
\hline tert-butyl & $\stackrel{\mathrm{CH}_{3}}{\mathrm{CH}_{3} \mathrm{CH}_{3}}$ \\
\hline
\end{tabular}

Note that alkyl groups do not exist as stable independent entities. They are always a part of some larger molecule. The location of an alkyl group on a hydrocarbon chain is indicated in the same way as any other substituent:

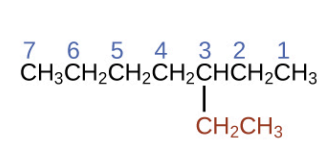

3-ethylheptane

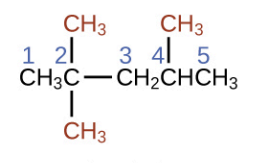

2,2,4-trimethylpentane

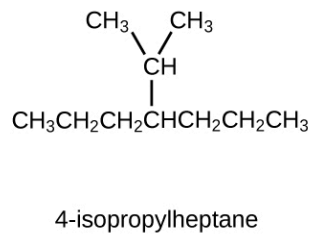

4-isopropylheptane

Alkanes are relatively stable molecules, but heat or light will activate reactions that involve the breaking of $\mathrm{C}-\mathrm{H}$ or $\mathrm{C}-\mathrm{C}$ single bonds. Combustion is one such reaction:

$\mathrm{CH}_{4}(g)+2 \mathrm{O}_{2}(g) \quad \mathrm{CO}_{2}(g)+2 \mathrm{H}_{2} \mathrm{O}(\mathrm{g})$ 
Alkanes burn in the presence of oxygen, a highly exothermic oxidation-reduction reaction that produces carbon dioxide and water. As a consequence, alkanes are excellent fuels. For example, methane, $\mathrm{CH}_{4}$, is the principal component of natural gas. Butane, $\mathrm{C}_{4} \mathrm{H}_{10}$, used in camping stoves and lighters is an alkane. Gasoline is a liquid mixture of continuous- and branched-chain alkanes, each containing from five to nine carbon atoms, plus various additives to improve its performance as a fuel. Kerosene, diesel oil, and fuel oil are primarily mixtures of alkanes with higher molecular masses. The main source of these liquid alkane fuels is crude oil, a complex mixture that is separated by fractional distillation. Fractional distillation takes advantage of differences in the boiling points of the components of the mixture (see (Figure)). You may recall that boiling point is a function of intermolecular interactions, which was discussed in the chapter on solutions and colloids.

In a column for the fractional distillation of crude oil, oil heated to about $425^{\circ} \mathrm{C}$ in the furnace vaporizes when it enters the base of the tower. The vapors rise through bubble caps in a series of trays in the tower. As the vapors gradually cool, fractions of higher, then of lower, boiling points condense to liquids and are drawn off. (credit left: modification of work by Luigi Chiesa) 

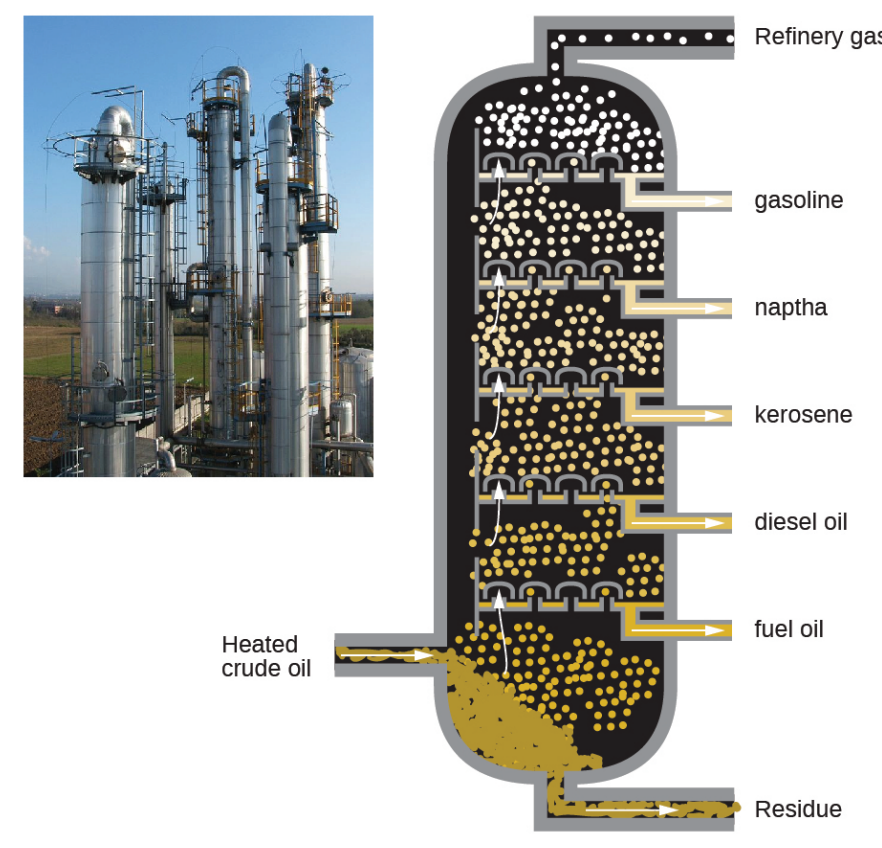

Small molecules:

- Low boiling point

- Very volatile

- Flows easily

- Ignites easily

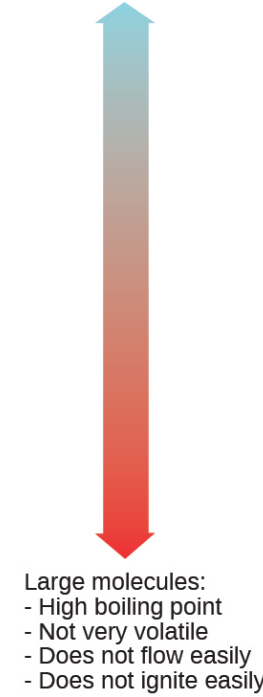

In a substitution reaction, another typical reaction of alkanes, one or more of the alkane's hydrogen atoms is replaced with a different atom or group of atoms. No carbon-carbon bonds are broken in these reactions, and the hybridization of the carbon atoms does not change. For example, the reaction between ethane and molecular chlorine depicted here is a substitution reaction:<smiles>[C]#[C+]CCC=CCCl</smiles>

The $\mathrm{C}-\mathrm{Cl}$ portion of the chloroethane molecule is an example of a functional group, the part or moiety of a molecule that imparts a specific chemical reactivity. The types of functional groups present in an organic molecule are major determinants of its chemical properties and are used as a means of classifying organic compounds as detailed in the remaining sections of this chapter. 
Want more practice naming alkanes? Watch this brief video tutorial to review the nomenclature process.

\section{Alkenes}

Organic compounds that contain one or more double or triple bonds between carbon atoms are described as unsaturated. You have likely heard of unsaturated fats. These are complex organic molecules with long chains of carbon atoms, which contain at least one double bond between carbon atoms. Unsaturated hydrocarbon molecules that contain one or more double bonds are called alkenes. Carbon atoms linked by a double bond are bound together by two bonds, one $\sigma$ bond and one $\pi$ bond. Double and triple bonds give rise to a different geometry around the carbon atom that participates in them, leading to important differences in molecular shape and properties. The differing geometries are responsible for the different properties of unsaturated versus saturated fats.

Ethene, $\mathrm{C}_{2} \mathrm{H}_{4}$, is the simplest alkene. Each carbon atom in ethene, commonly called ethylene, has a trigonal planar structure. The second member of the series is propene (propylene) ((Figure)); the butene isomers follow in the series. Four carbon atoms in the chain of butene allows for the formation of isomers based on the position of the double bond, as well as a new form of isomerism. Expanded structures, ball-and-stick structures, and space-filling models for the alkenes ethene, propene, and 1-butene are shown. 

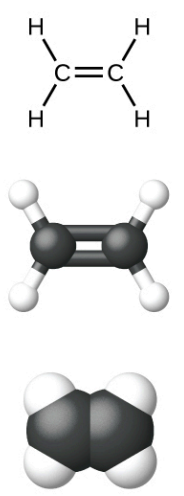

ethene
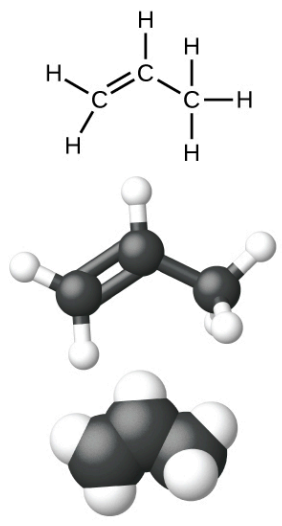

propene
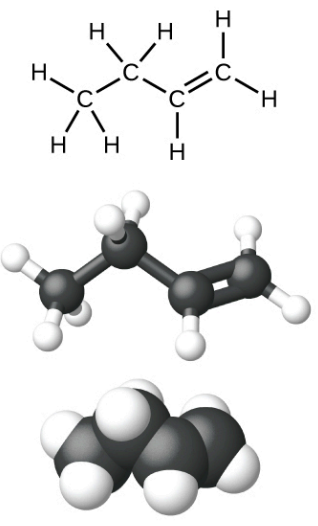

1-butene

Ethylene (the common industrial name for ethene) is a basic raw material in the production of polyethylene and other important compounds. Over 135 million tons of ethylene were produced worldwide in 2010 for use in the polymer, petrochemical, and plastic industries. Ethylene is produced industrially in a process called cracking, in which the long hydrocarbon chains in a petroleum mixture are broken into smaller molecules.

\section{Recycling Plastics}

Polymers (from Greek words poly meaning "many" and mer meaning "parts") are large molecules made up of repeating units, referred to as monomers. Polymers can be natural (starch is a polymer of sugar residues and proteins are polymers of amino acids) or synthetic [like polyethylene, polyvinyl chloride (PVC), and polystyrene]. The variety of structures of polymers translates into a broad range of properties and uses that make them integral parts of our everyday lives. Adding functional groups to the structure of a polymer can result in significantly different properties (see the discussion about Kevlar later in this chapter). 
An example of a polymerization reaction is shown in (Figure). The monomer ethylene $\left(\mathrm{C}_{2} \mathrm{H}_{4}\right)$ is a gas at room temperature, but when polymerized, using a transition metal catalyst, it is transformed into a solid material made up of long chains of $-\mathrm{CH}_{2}-$ units called polyethylene. Polyethylene is a commodity plastic used primarily for packaging (bags and films).

The reaction for the polymerization of ethylene to polyethylene is shown.
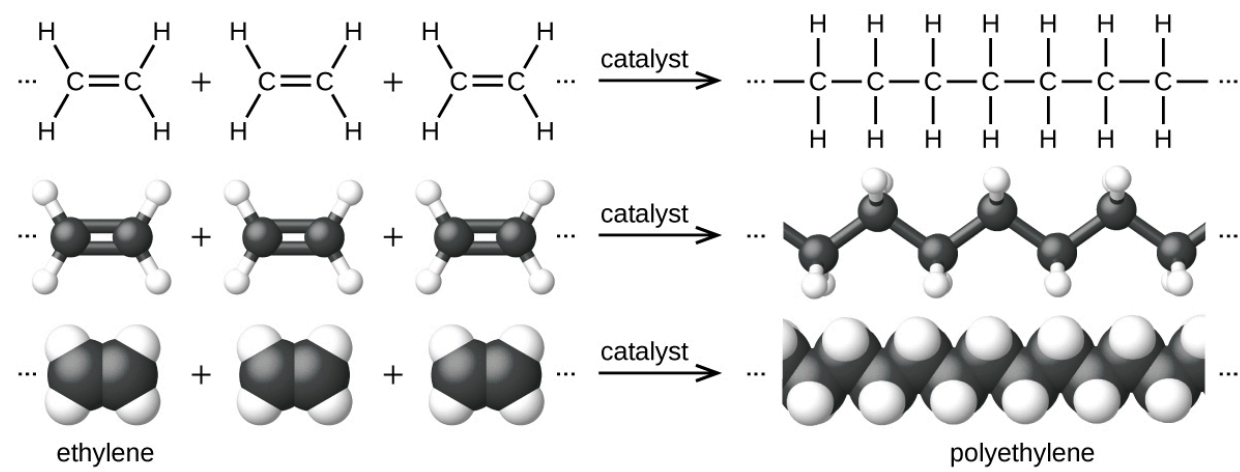

Polyethylene is a member of one subset of synthetic polymers classified as plastics. Plastics are synthetic organic solids that can be molded; they are typically organic polymers with high molecular masses. Most of the monomers that go into common plastics (ethylene, propylene, vinyl chloride, styrene, and ethylene terephthalate) are derived from petrochemicals and are not very biodegradable, making them candidate materials for recycling. Recycling plastics helps minimize the need for using more of the petrochemical supplies and also minimizes the environmental damage caused by throwing away these nonbiodegradable materials.

Plastic recycling is the process of recovering waste, scrap, or used plastics, and reprocessing the material into 
useful products. For example, polyethylene terephthalate (soft drink bottles) can be melted down and used for plastic furniture, in carpets, or for other applications. Other plastics, like polyethylene (bags) and polypropylene (cups, plastic food containers), can be recycled or reprocessed to be used again. Many areas of the country have recycling programs that focus on one or more of the commodity plastics that have been assigned a recycling code (see (Figure)). These operations have been in effect since the 1970s and have made the production of some plastics among the most efficient industrial operations today.

Each type of recyclable plastic is imprinted with a code for easy identification.

\begin{tabular}{|l|l|}
\hline & Soda bottles and oven-ready food trays \\
\hline PETE & Bottles for milk and dishwashing liquids \\
\hline LDPE & Food trays, plastic wrap, bottles for mineral water \\
and shampoo
\end{tabular}

The name of an alkene is derived from the name of the 
alkane with the same number of carbon atoms. The presence of the double bond is signified by replacing the suffix -ane with the suffix -ene.<!- no-selfclose $\rightarrow$ The location of the double bond $<!-$ no-selfclose $->$ is identified by naming the smaller of the numbers of the carbon atoms participating in the double bond:
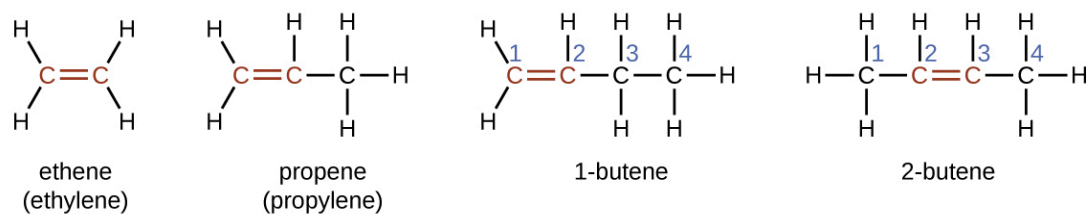

Isomers of Alkenes

Molecules of 1-butene and 2-butene are structural isomers; the arrangement of the atoms in these two molecules differs. As an example of arrangement differences, the first carbon atom in 1-butene is bonded to two hydrogen atoms; the first carbon atom in 2-butene is bonded to three hydrogen atoms.

The compound 2-butene and some other alkenes also form a second type of isomer called a geometric isomer. In a set of geometric isomers, the same types of atoms are attached to each other in the same order, but the geometries of the two molecules differ. Geometric isomers of alkenes differ in the orientation of the groups on either side of a $\mathrm{C}=\mathrm{C}$ bond.

Carbon atoms are free to rotate around a single bond but not around a double bond; a double bond is rigid. This makes it possible to have two isomers of 2-butene, one with both methyl groups on the same side of the double bond and one with the methyl groups on opposite sides. When structures of butene are drawn with $120^{\circ}<$ !no-selfclose $\rightarrow$ bond angles around the $s p^{2}$-hybridized 
carbon atoms participating in the double bond, the isomers are apparent. The 2-butene isomer in which the two methyl groups are on the same side is called a cis-isomer; the one in which the two methyl groups are on opposite sides is called a trans-isomer ((Figure)). The different geometries produce different physical properties, such as boiling point, that may make separation of the isomers possible:

These molecular models show the structural and geometric isomers of butene.
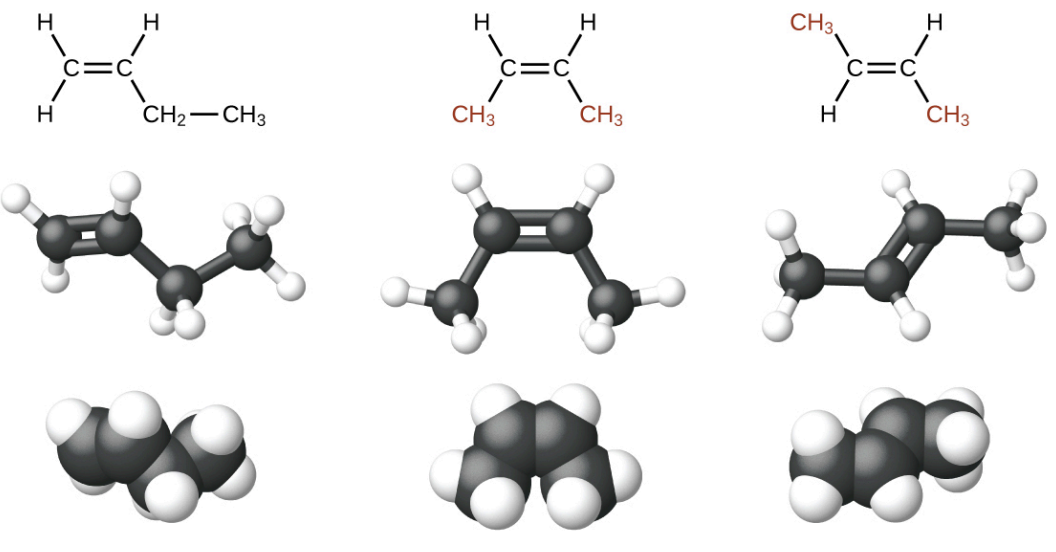

1-butene

cis isomer

trans isomer

Alkenes are much more reactive than alkanes because the $\mathrm{C}=\mathrm{C}$ moiety is a reactive functional group. A $\pi$ bond, being a weaker bond, is disrupted much more easily than a $\sigma$ bond. Thus, alkenes undergo a characteristic reaction in which the $\pi$ bond is broken and replaced by two $\sigma$ bonds. This reaction is called an addition reaction. The hybridization of the carbon atoms in the double bond in an alkene changes from $s p^{2}$ to $s p^{3}$ during an addition reaction. For example, halogens add to the double bond in an alkene instead of replacing hydrogen, as occurs in an alkane: 


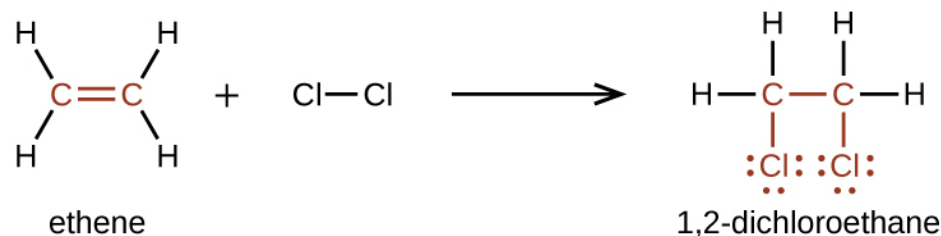

Alkene Reactivity and Naming Provide the IUPAC names for the reactant and product of the halogenation reaction shown here:

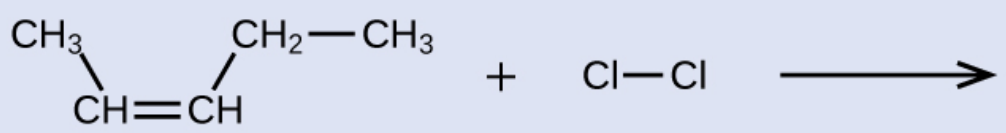

Solution The reactant is a five-carbon chain that contains a carbon-carbon double bond, so the base name will be pentene. We begin counting at the end of the chain closest to the double bond-in this case, from the left-the double bond spans carbons 2 and 3 , so the name becomes 2 -pentene. Since there are two carbon-containing groups attached to the two carbon atoms in the double bond-and they are on the same side of the double bond-this molecule is the cis-isomer, making the name of the starting alkene cis-2-pentene. The product of the halogenation reaction will have two chlorine atoms attached to the carbon atoms that were a part of the carbon-carbon double bond:<smiles>CCC(Cl)C(C)Cl</smiles>

This molecule is now a substituted alkane and will be named as such. The base of the name will be pentane. We will count from the end that numbers the carbon atoms where the chlorine atoms are attached as 2 and 3, making the name of the product 2,3-dichloropentane. 
Check Your Learning Provide names for the reactant and product of the reaction shown:

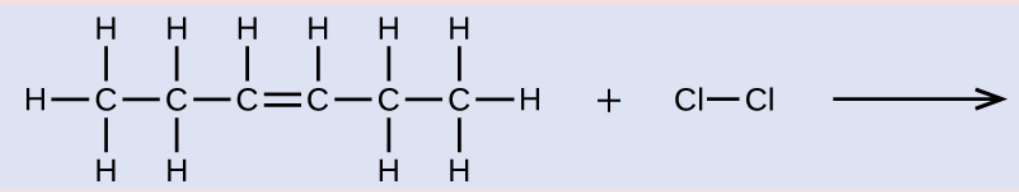

Answer:

reactant: cis-3-hexene product: 3,4-dichlorohexane

\section{Alkynes}

Hydrocarbon molecules with one or more triple bonds are called alkynes; they make up another series of unsaturated hydrocarbons. Two carbon atoms joined by a triple bond are bound together by one $\sigma$ bond and two $\pi$ bonds. The $s p$-hybridized carbons involved in the triple bond have bond angles of $180^{\circ}$, giving these types of bonds a linear, rod-like shape.

The simplest member of the alkyne series is ethyne, $\mathrm{C}_{2} \mathrm{H}_{2}$, commonly called acetylene. The Lewis structure for ethyne, a linear molecule, is:

$$
\begin{aligned}
& \mathrm{H}-\mathrm{C} \equiv \mathrm{C}-\mathrm{H} \\
& \text { ethyne (acetylene) }
\end{aligned}
$$

The IUPAC nomenclature for alkynes is similar to that for alkenes except that the suffix -yne is used to indicate a triple bond in the chain. For example, $\mathrm{CH}_{3} \mathrm{CH}_{2} \mathrm{C} \equiv \mathrm{CH}$ is called 1-butyne.

Structure of Alkynes Describe the geometry and hybridization of the carbon atoms in the following molecule:<smiles>[C]C#CC</smiles> 
Solution Carbon atoms 1 and 4 have four single bonds and are thus tetrahedral with $\mathrm{sp}^{3}$ hybridization. Carbon atoms 2 and 3 are involved in the triple bond, so they have linear geometries and would be classified as sp hybrids.

Check Your Learning Identify the hybridization and bond angles at the carbon atoms in the molecule shown:<smiles>C#CC=CC</smiles>

Answer:

carbon 1: sp, $180^{\circ}$; carbon 2: sp, $180^{\circ}$; carbon 3: $s p^{2}, 120^{\circ}$; carbon $4: s p^{2}, 120^{\circ}$; carbon $5: s p^{3}, 109.5^{\circ}$

Chemically, the alkynes are similar to the alkenes. Since the $\mathrm{C} \equiv \mathrm{C}$ functional group has two $\pi$ bonds, alkynes typically react even more readily, and react with twice as much reagent in addition reactions. The reaction of acetylene with bromine is a typical example:

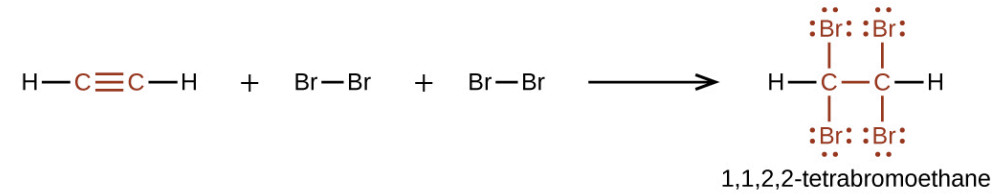

Acetylene and the other alkynes also burn readily. An acetylene torch takes advantage of the high heat of combustion for acetylene.

Aromatic Hydrocarbons

Benzene, $\mathrm{C}_{6} \mathrm{H}_{6}$, is the simplest member of a large family of hydrocarbons, called aromatic hydrocarbons. These compounds contain ring structures and exhibit bonding that must be described using the resonance hybrid 
concept of valence bond theory or the delocalization concept of molecular orbital theory. (To review these concepts, refer to the earlier chapters on chemical bonding). The resonance structures for benzene, $\mathrm{C}_{6} \mathrm{H}_{6}$, are:<smiles></smiles>

Valence bond theory describes the benzene molecule and other planar aromatic hydrocarbon molecules as hexagonal rings of $s p^{2}$-hybridized carbon atoms with the unhybridized $p$ orbital of each carbon atom perpendicular to the plane of the ring. Three valence electrons in the $s p^{2}$ hybrid orbitals of each carbon atom and the valence electron of each hydrogen atom form the framework of $\sigma$ bonds in the benzene molecule. The fourth valence electron of each carbon atom is shared with an adjacent carbon atom in their unhybridized $p$ orbitals to yield the $\pi$ bonds. Benzene does not, however, exhibit the characteristics typical of an alkene. Each of the six bonds between its carbon atoms is equivalent and exhibits properties that are intermediate between those of a $\mathrm{C}-\mathrm{C}$ single bond and a $\mathrm{C}=\mathrm{C}$ double bond. To represent this unique bonding, structural formulas for benzene and its derivatives are typically drawn with single bonds between the carbon atoms and a circle within the ring as shown in (Figure).

This condensed formula shows the unique bonding structure of benzene. 


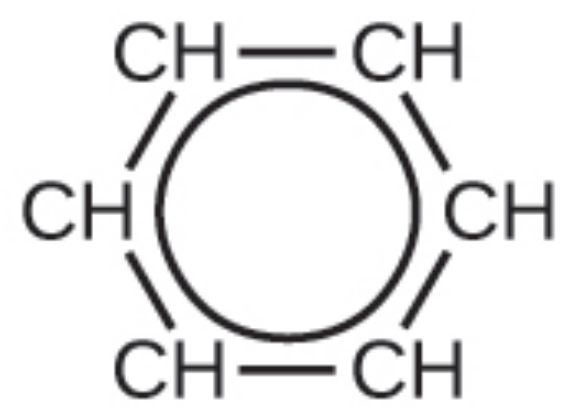

There are many derivatives of benzene. The hydrogen atoms can be replaced by many different substituents. Aromatic compounds more readily undergo substitution reactions than addition reactions; replacement of one of the hydrogen atoms with another substituent will leave the delocalized double bonds intact. The following are typical examples of substituted benzene derivatives:<smiles>Cc1ccccc1</smiles>

toluene<smiles>Cc1ccccc1C</smiles>

xylene<smiles>C=Cc1ccccc1</smiles>

styrene

Toluene and xylene are important solvents and raw materials in the chemical industry. Styrene is used to produce the polymer polystyrene.

Structure of Aromatic Hydrocarbons One possible isomer created by a substitution reaction that replaces a hydrogen atom attached to the aromatic ring of toluene with a chlorine atom is shown here. Draw two other possible isomers in which the chlorine atom replaces a different hydrogen atom attached to the aromatic ring: 

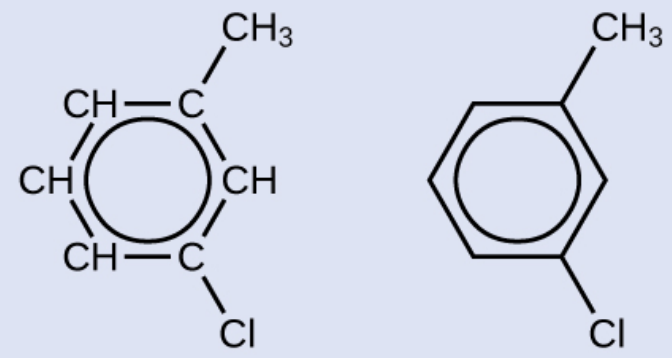

Solution Since the six-carbon ring with alternating double bonds is necessary for the molecule to be classified as aromatic, appropriate isomers can be produced only by changing the positions of the chloro-substituent relative to the methyl-substituent:<smiles>Cc1ccccc1Cl</smiles><smiles>Cc1ccccc1Cl</smiles><smiles>Cc1ccc(Cl)cc1</smiles><smiles>Cc1ccc(Cl)cc1</smiles>

Check Your Learning Draw three isomers of a six-membered aromatic ring compound substituted with two bromines.

Answer:<smiles>Brc1ccccc1Br</smiles><smiles>Brc1cccc(Br)c1</smiles><smiles>Brc1ccc(Br)cc1</smiles>
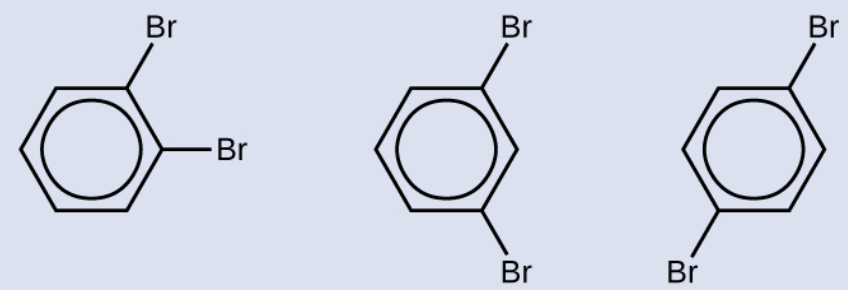

Key Concepts and Summary

Strong, stable bonds between carbon atoms produce complex molecules containing chains, branches, and 
rings. The chemistry of these compounds is called organic chemistry. Hydrocarbons are organic compounds composed of only carbon and hydrogen. The alkanes are saturated hydrocarbons-that is, hydrocarbons that contain only single bonds. Alkenes contain one or more carbon-carbon double bonds. Alkynes contain one or more carbon-carbon triple bonds. Aromatic hydrocarbons contain ring structures with delocalized $\pi$ electron systems.

Chemistry End of Chapter Exercises

Write the chemical formula and Lewis structure of the following, each of which contains five carbon atoms:
(a) an alkane
(b) an alkene
(c) an alkyne

There are several sets of answers; one is:

(a) $\mathrm{C}_{5} \mathrm{H}_{12}$<smiles>CCCC</smiles>

(b) $\mathrm{C}_{5} \mathrm{H}_{10}$<smiles>C/C=C\C</smiles>

(c) $\mathrm{C}_{5} \mathrm{H}_{8}$ 
<smiles>CC#CCC</smiles>

What is the difference between the hybridization of carbon atoms' valence orbitals in saturated and unsaturated hydrocarbons?

On a microscopic level, how does the reaction of bromine with a saturated hydrocarbon differ from its reaction with an unsaturated hydrocarbon? How are they similar?

Both reactions result in bromine being incorporated into the structure of the product. The difference is the way in which that incorporation takes place. In the saturated hydrocarbon, an existing $\mathrm{C}-\mathrm{H}$ bond is broken, and a bond between the $\mathrm{C}$ and the $\mathrm{Br}$ can then be formed. In the unsaturated hydrocarbon, the only bond broken in the hydrocarbon is the $\pi$ bond whose electrons can be used to form a bond to one of the bromine atoms in $\mathrm{Br}_{2}$ (the electrons from the $\mathrm{Br}-\mathrm{Br}$ bond form the other $\mathrm{C}-\mathrm{Br}$ bond on the other carbon that was part of the $\pi$ bond in the starting unsaturated hydrocarbon).

On a microscopic level, how does the reaction of bromine with an alkene differ from its reaction with an alkyne? How are they similar?

Explain why unbranched alkenes can form geometric isomers while unbranched alkanes cannot. Does this 
explanation involve the macroscopic domain or the microscopic domain?

Unbranched alkanes have free rotation about the $\mathrm{C}-\mathrm{C}$ bonds, yielding all orientations of the substituents about these bonds equivalent, interchangeable by rotation. In the unbranched alkenes, the inability to rotate about the $\mathrm{C}=\mathrm{C}$ bond results in fixed (unchanging) substituent orientations, thus permitting different isomers. Since these concepts pertain to phenomena at the molecular level, this explanation involves the microscopic domain.

Explain why these two molecules are not isomers:<smiles>C/C=C\C</smiles><smiles>CCCCC</smiles>

Explain why these two molecules are not isomers:<smiles>CCCCC</smiles><smiles>CCCCC</smiles>

They are the same compound because each is a saturated hydrocarbon containing an unbranched chain of six carbon atoms.

How does the carbon-atom hybridization change when polyethylene is prepared from ethylene? 
Write the Lewis structure and molecular formula for each of the following hydrocarbons:

(a) hexane

(b) 3-methylpentane

(c) cis-3-hexene

(d) 4-methyl-1-pentene

(e) 3-hexyne

(f) 4-methyl-2-pentyne

(a) $\mathrm{C}_{6} \mathrm{H}_{14}$<smiles>CCCC</smiles>

(b) $\mathrm{C}_{6} \mathrm{H}_{14}$<smiles>CCC(C)C</smiles>

(c) $\mathrm{C}_{6} \mathrm{H}_{12}$ 
<smiles>CC=CCC</smiles>

(d) $\mathrm{C}_{6} \mathrm{H}_{12}$<smiles>C=CCCCC</smiles>

(e) $\mathrm{C}_{6} \mathrm{H}_{10}$<smiles>CC#CCC</smiles>

(f) $\mathrm{C}_{6} \mathrm{H}_{10}$<smiles>CC#CCCC</smiles> 
Write the chemical formula, condensed formula, and Lewis structure for each of the following hydrocarbons:
(a) heptane
(b) 3-methylhexane
(c) trans-3-heptene
(d) 4-methyl-1-hexene
(e) 2-heptyne
(f) 3,4-dimethyl-1-pentyne

Give the complete IUPAC name for each of the following compounds:

(a) $\mathrm{CH}_{3} \mathrm{CH}_{2} \mathrm{CBr}_{2} \mathrm{CH}_{3}$

(b) $\left(\mathrm{CH}_{3}\right)_{3} \mathrm{CCl}$

(c)

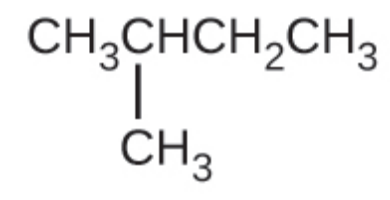

(d) $\mathrm{CH}_{3} \mathrm{CH}_{2} \mathrm{C} \equiv \mathrm{CH} \mathrm{CH} \mathrm{CH}_{2} \mathrm{C} \equiv \mathrm{CH}$

(e)<smiles>C#CCCCCC</smiles>

(f)<smiles>CC=CCl</smiles>

(g) $\left(\mathrm{CH}_{3}\right)_{2} \mathrm{CHCH}_{2} \mathrm{CH}=\mathrm{CH}_{2}$

(a) 2,2-dibromobutane; (b) 2-chloro-2-methylpropane; (c) 2-methylbutane;

(d) 1-butyne; 
4-fluoro-4-methyl-1-octyne; (f) trans-1-chloropropene; (g) 5-methyl-1-pentene

Give the complete IUPAC name for each of the following compounds:

(a) $\left(\mathrm{CH}_{3}\right)_{2} \mathrm{CHF}$

(b) $\mathrm{CH}_{3} \mathrm{CHClCHClCH}$

(c)<smiles>CCC(C)C</smiles>

(d) $\mathrm{CH}_{3} \mathrm{CH}_{2} \mathrm{CH}=\mathrm{CHCH}_{3}$

(e)<smiles>C=CCCC(Br)CC</smiles>

(f) $\left(\mathrm{CH}_{3}\right)_{3} \mathrm{CCH}_{2} \mathrm{C} \equiv \mathrm{CH}$

Butane is used as a fuel in disposable lighters. Write the Lewis structure for each isomer of butane.
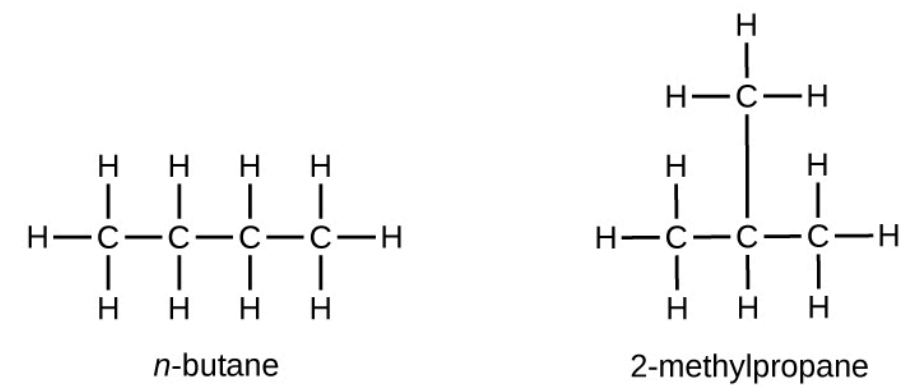

Write Lewis structures and name the five structural isomers of hexane.

Write Lewis structures for the cis-trans isomers of $\mathrm{CH}_{3} \mathrm{CH}=\mathrm{CHCl}$. 
<smiles>CC=CCl</smiles>

cis-<smiles>CC=CCl</smiles>

trans-

Write structures for the three isomers of the aromatic hydrocarbon xylene, $\mathrm{C}_{6} \mathrm{H}_{4}\left(\mathrm{CH}_{3}\right)_{2}$.

Isooctane is the common name of the isomer of $\mathrm{C}_{8} \mathrm{H}_{18}$ used as the standard of 100 for the gasoline octane rating:<smiles>CC(C)C(C)(C)C</smiles>

(a) What is the IUPAC name for the compound?

(b) Name the other isomers that contain a five-carbon chain with three methyl substituents.

(a) 2,2,4-trimethylpentane; (b) 2,2,3-trimethylpentane, 2,3,4-trimethylpentane, and 2,3,3-trimethylpentane:<smiles>CCC(C)C(C)(C)C</smiles><smiles>CC(C)C(C)C(C)C</smiles><smiles>CCC(C)(C)C(C)C</smiles>

Write Lewis structures and IUPAC names for the alkyne isomers of $\mathrm{C}_{4} \mathrm{H}_{6}$.

Write Lewis structures and IUPAC names for all isomers of $\mathrm{C}_{4} \mathrm{H}_{9} \mathrm{Cl}$. 
Four structural formulas are provided. The first has a hydrocarbon chain with a length of four $\mathrm{C}$ atom. All bonds are single. Nine $\mathrm{H}$ atoms are attached and a single $\mathrm{Cl}$ atom is attached at the far right end of the structure which is labeled 1 dash chlorobutane. The second has a hydrocarbon chain with a length four $\mathrm{C}$ atoms. All bonds are single. Nine $\mathrm{H}$ atoms are attached and a single $\mathrm{C} 1$ atom is attached above the second carbon counting left to right. This structure is labeled 2 dash chlorobutane. The third has a hydrocarbon chain with a length of three $\mathrm{C}$ atoms. All bonds are single. A single $\mathrm{C} 1$ atom is bonded beneath the middle $\mathrm{C}$ atom and a $\mathrm{C} H$ subscript 3 group is also bonded above the middle $\mathrm{C}$ atom. Six $\mathrm{H}$ atoms are attached, and the structure is labeled 2 dash chloro dash 2 dash methylpropane. The fourth structure has a hydrocarbon chain with a length of three $\mathrm{C}$ atoms. All bonds are single. A single $\mathrm{C} 1$ atom is bonded beneath the first $\mathrm{C}$ atom (from left to right) and a $\mathrm{C} H$ subscript 3 group is bonded above the middle $\mathrm{C}$ atom. Six $\mathrm{H}$ atoms are attached, and the structure is labeled 1 dash chloro dash 2 dash methylpropane.

Name and write the structures of all isomers of the propyl and butyl alkyl groups.

Write the structures for all the isomers of the $-\mathrm{C}_{5} \mathrm{H}_{11}$ alkyl group.

In the following, the carbon backbone and the appropriate number of hydrogen atoms are shown in condensed form: 
$\mathrm{CH}_{3}-\mathrm{CH}_{2}-\mathrm{CH}_{2}-\mathrm{CH}_{2}-\mathrm{CH}_{2}-$<smiles>CCC(C)CC</smiles><smiles>CCCC(C)C</smiles><smiles>CCCC(C)C</smiles><smiles>CC(C)C(C)C</smiles><smiles>CCC(C)(C)C</smiles><smiles>CCC(C)(C)C</smiles><smiles>CCC(C)CI</smiles>

Write Lewis structures and describe the molecular geometry at each carbon atom in the following compounds:

(a) cis-3-hexene

(b) cis-1-chloro-2-bromoethene

(c) 2-pentyne

(d) trans-6-ethyl-7-methyl-2-octene

Benzene is one of the compounds used as an octane enhancer in unleaded gasoline. It is manufactured by the catalytic conversion of acetylene to benzene:

$3 \mathrm{C}_{2} \mathrm{H}_{2} \quad \mathrm{C}_{6} \mathrm{H}_{6}$

Draw Lewis structures for these compounds, with resonance structures as appropriate, and determine the hybridization of the carbon atoms in each.

Two structural formulas are shown. The first shows two C atoms with a triple bond between them. At each end of the structure, a single $\mathrm{H}$ atom is bonded. The second structure involves a hydrocarbon ring of $6 \mathrm{C}$ atoms with a circle at the center. There are alternating double bonds between $\mathrm{C}$ atoms. Each $\mathrm{C}$ atom is bonded to a single $\mathrm{H}$ atom. 
In acetylene, the bonding uses $s p$ hybrids on carbon atoms and $s$ orbitals on hydrogen atoms. In benzene, the carbon atoms are $s p^{2}$ hybridized.

Teflon is prepared by the polymerization of tetrafluoroethylene. Write the equation that describes the polymerization using Lewis symbols.

Write two complete, balanced equations for each of the following reactions, one using condensed formulas and one using Lewis structures.

(a) 1 mol of 1 -butyne reacts with 2 mol of iodine.

(b) Pentane is burned in air.

(a) $\mathrm{CH} \equiv \mathrm{CCH}_{2} \mathrm{CH}_{3}+2 \mathrm{I}_{2} \mathrm{CHI}_{2} \mathrm{CI}_{2} \mathrm{CH}_{2} \mathrm{CH}_{3}$

A reaction is shown. On the left, a four carbon hydrocarbon chain is shown with a triple bond between $\mathrm{C}$ atoms 1 and 2 moving left to right across the molecule. The first $\mathrm{C}$ is bonded to one $\mathrm{H}$ atom. The second $\mathrm{C}$ atom bonds with the third $\mathrm{C}$ atom. The third $\mathrm{C}$ atom is bonded to two $\mathrm{H}$ atoms and a fourth $\mathrm{C}$ atom. The fourth $\mathrm{C}$ atom is bonded to three $\mathrm{H}$ atoms. A plus sign is shown followed by 2 I dash I, which is followed by a reaction arrow, then a four $\mathrm{C}$ atom hydrocarbon chain with I atoms bonded above and below $\mathrm{C}$ atoms 1 and 2 from left to right. The first $\mathrm{C}$ atom is bonded to one $\mathrm{H}$. The third $\mathrm{C}$ atom is bonded to two $\mathrm{H}$ atoms. The fourth $\mathrm{C}$ atom is bonded to three $\mathrm{H}$ atoms.

(b) $\mathrm{CH}_{3} \mathrm{CH}_{2} \mathrm{CH}_{2} \mathrm{CH}_{2} \mathrm{CH}_{3}+8 \mathrm{O}_{2} \quad 5 \mathrm{CO}_{2}+6 \mathrm{H}_{2} \mathrm{O}$

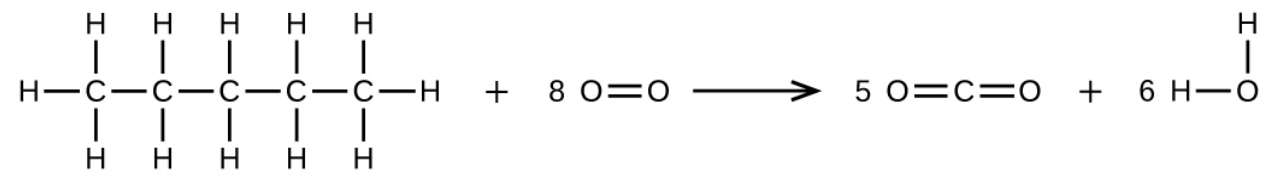


Write two complete, balanced equations for each of the following reactions, one using condensed formulas and one using Lewis structures.

(a) 2-butene reacts with chlorine.

(b) benzene burns in air.

What mass of 2-bromopropane could be prepared from $25.5 \mathrm{~g}$ of propene? Assume a 100\% yield of product.

\section{$65.2 \mathrm{~g}$}

Acetylene is a very weak acid; however, it will react with moist silver(I) oxide and form water and a compound composed of silver and carbon. Addition of a solution of $\mathrm{HCl}$ to a 0.2352 -g sample of the compound of silver and carbon produced acetylene and $0.2822 \mathrm{~g}$ of $\mathrm{AgCl}$.

(a) What is the empirical formula of the compound of silver and carbon?

(b) The production of acetylene on addition of $\mathrm{HCl}$ to the compound of silver and carbon suggests that the carbon is present as the acetylide ion, $\mathrm{C}_{2}{ }^{2-}$. Write the formula of the compound showing the acetylide ion.

Ethylene can be produced by the pyrolysis of ethane: $\mathrm{C}_{2} \mathrm{H}_{6} \mathrm{C}_{2} \mathrm{H}_{4}+\mathrm{H}_{2}$

How many kilograms of ethylene is produced by the pyrolysis of $1.00010^{3} \mathrm{~kg}$ of ethane, assuming a $100.0 \%$ yield?

$9.32810^{2} \mathrm{~kg}$ 
Footnotes

- 1This is the Beilstein database, now available through the Reaxys site (www.elsevier.com/ online-tools/reaxys).

- 2Peplow, Mark. "Organic Synthesis: The RoboChemist," Nature 512 (2014): 20-2.

- 3Physical properties for $\mathrm{C}_{4} \mathrm{H}_{10}$ and heavier molecules are those of the normal isomer, $n$-butane, $n$-pentane, etc.

- 4STP indicates a temperature of $0{ }^{\circ} \mathrm{C}$ and a pressure of $1 \mathrm{~atm}$.

Glossary

addition reaction

reaction in which a double carbon-carbon bond forms a single carbon-carbon bond by the addition of a reactant. Typical reaction for an alkene.

alkane

molecule consisting of only carbon and hydrogen atoms connected by single $(\sigma)$ bonds

alkene

molecule consisting of carbon and hydrogen containing at least one carboncarbon double bond 


\section{alkyl group}

substituent, consisting of an alkane missing one hydrogen atom, attached to a larger structure

alkyne

molecule consisting of carbon and hydrogen containing at least one carboncarbon triple bond

\section{aromatic hydrocarbon}

cyclic molecule consisting of carbon and hydrogen with delocalized alternating carbon-carbon single and double bonds, resulting in enhanced stability

\section{functional group}

part of an organic molecule that imparts a specific chemical reactivity to the molecule

\section{organic compound}

natural or synthetic compound that contains carbon

\section{saturated hydrocarbon}

molecule containing carbon and hydrogen that has only single bonds between carbon atoms

\section{skeletal structure}

shorthand method of drawing organic molecules in which carbon atoms are represented by the ends of lines and bends in between lines, and hydrogen atoms attached to the carbon atoms are not shown (but are understood to be present by the context of the structure) 


\section{substituent}

branch or functional group that replaces

hydrogen atoms in a larger hydrocarbon

chain

substitution reaction

reaction in which one atom replaces

another in a molecule 


\section{7.}

\section{ALCOHOLS AND ETHERS}

\section{Learning Objectives}

By the end of this section, you will be able to:

- Describe the structure and properties of alcohols

- Describe the structure and properties of ethers

- Name and draw structures for alcohols and ethers

In this section, we will learn about alcohols and ethers.

\section{Alcohols}

Incorporation of an oxygen atom into carbon- and hydrogen-containing molecules leads to new functional groups and new families of compounds. When the oxygen atom is attached by single bonds, the molecule is either an alcohol or ether.

Alcohols are derivatives of hydrocarbons in which an 
$-\mathrm{OH}$ group has replaced a hydrogen atom. Although all alcohols have one or more hydroxyl $(-\mathrm{OH})$ functional groups, they do not behave like bases such as $\mathrm{NaOH}$ and $\mathrm{KOH}$. $\mathrm{NaOH}$ and $\mathrm{KOH}$ are ionic compounds that contain $\mathrm{OH}^{-}$ions. Alcohols are covalent molecules; the $-\mathrm{OH}$ group in an alcohol molecule is attached to a carbon atom by a covalent bond.

Ethanol, $\mathrm{CH}_{3} \mathrm{CH}_{2} \mathrm{OH}$, also called ethyl alcohol, is a particularly important alcohol for human use. Ethanol is the alcohol produced by some species of yeast that is found in wine, beer, and distilled drinks. It has long been prepared by humans harnessing the metabolic efforts of yeasts in fermenting various sugars:

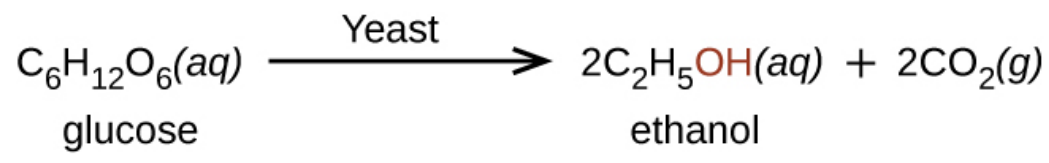

Large quantities of ethanol are synthesized from the addition reaction of water with ethylene using an acid as a catalyst:

This reaction shows two carbons connected by a double bond, each with two bonded $\mathrm{H}$ atoms plus $\mathrm{H} \mathrm{O} \mathrm{H}$ arrow labeled "H subscript $3 \mathrm{O}$ superscript plus" followed by two carbon atoms connected with a single bond with 5 bonded $\mathrm{H}$ atoms and an $\mathrm{O} \mathrm{H}$ group shown in red at the right end of the molecule. The $\mathrm{O}$ of this group is shown with 2 pairs of electron dots.

Alcohols containing two or more hydroxyl groups can be made. Examples include 1,2-ethanediol (ethylene glycol, used in antifreeze) and 1,2,3-propanetriol (glycerine, used as a solvent for cosmetics and medicines): 
Structural formulas for 1 comma 2 dash ethanediol and 1 comma 2 comma 3 dash propanetriol are shown. The first structure has a two $\mathrm{C}$ atom hydrocarbon chain with an $\mathrm{O} \mathrm{H}$ group attached to each carbon. The $\mathrm{O} \mathrm{H}$ groups are shown in red an each $\mathrm{O}$ atom has two sets of electron dots. Each $\mathrm{C}$ atom also has two $\mathrm{H}$ atoms bonded to it. The second structure shows a three $\mathrm{C}$ atom hydrocarbon chain with an $\mathrm{O} \mathrm{H}$ group bonded to each carbon. The $\mathrm{O} \mathrm{H}$ groups are shown in red, and each $\mathrm{O}$ atom has two sets of electron dots. The first $\mathrm{C}$ atom has two $\mathrm{H}$ atoms bonded to it. The second $\mathrm{C}$ atom has one $\mathrm{H}$ atom bonded to it. The third $\mathrm{C}$ atom has two $\mathrm{H}$ atoms bonded to it.

\section{Naming Alcohols}

The name of an alcohol comes from the hydrocarbon from which it was derived. The final $-e$ in the name of the hydrocarbon is replaced by -ol, and the carbon atom to which the $-\mathrm{OH}$ group is bonded is indicated by a number placed before the name. ${ }^{1}$

Naming Alcohols Consider the following example. How should it be named?

A molecular structure of a hydrocarbon chain with a length of five $\mathrm{C}$ atoms is shown. The first $\mathrm{C}$ atom (from left to right) is bonded to three $\mathrm{H}$ atoms. The second $\mathrm{C}$ atom is bonded on one $\mathrm{H}$ atom and an $\mathrm{O}$ atom which is also bonded to an $\mathrm{H}$ atom. The $O$ atom has two sets of electron dots. The third $C$ atom is bonded to two $\mathrm{H}$ atoms. The fourth $\mathrm{C}$ atom is bonded to two $\mathrm{H}$ atoms. The fifth $\mathrm{C}$ atom is bonded to three $\mathrm{H}$ atoms. All bonds shown are single.

Solution The carbon chain contains five carbon atoms. If the 
hydroxyl group was not present, we would have named this molecule pentane. To address the fact that the hydroxyl group is present, we change the ending of the name to -ol. In this case, since the $-\mathrm{OH}$ is attached to carbon 2 in the chain, we would name this molecule 2-pentanol.

Check Your Learning Name the following molecule:

The structure shown has a C H subscript 3 group bonded up and to the right to $\mathrm{a} C$ atom. The $\mathrm{C}$ atom is bonded down and to the right to a $\mathrm{C} H$ subscript 2 group. The $\mathrm{C} H$ subscript 2 group is bonded up and to the right to a $\mathrm{C} H$ subscript 2 group. The $C$ $\mathrm{H}$ subscript 2 group is bonded down and to the right to a $\mathrm{CH}$ subscript 3 group. The second $C$ atom (from left to right) is bonded to a $\mathrm{C} \mathrm{H}$ subscript 3 group and an $\mathrm{O} \mathrm{H}$ group.

Answer:

2-methyl-2-pentanol

\section{Ethers}

Ethers are compounds that contain the functional group -O-. Ethers do not have a designated suffix like the other types of molecules we have named so far. In the IUPAC system, the oxygen atom and the smaller carbon branch are named as an alkoxy substituent and the remainder of the molecule as the base chain, as in alkanes. As shown in the following compound, the red symbols represent the smaller alkyl group and the oxygen atom, which would be named "methoxy." The larger carbon branch would be ethane, making the molecule methoxyethane. Many ethers are referred to with common names instead of the IUPAC system names. For common names, the two branches connected to the oxygen atom are named 
separately and followed by "ether." The common name for the compound shown in (Figure) is ethylmethyl ether:

A molecular structure is shown with a red $\mathrm{C} \mathrm{H}$ subscript 3 group bonded up and to the right to a red $\mathrm{O}$ atom. The $\mathrm{O}$ atom is bonded down and to the right to a $\mathrm{C} \mathrm{H}$ subscript 2 group. The $\mathrm{C} \mathrm{H}$ subscript 2 group is bonded up and to the right to a $\mathrm{C} H$ subscript 3 group.

Naming Ethers Provide the IUPAC and common name for the ether shown here:

A molecular structure shows a $\mathrm{CH}$ subscript 3 group bonded down and to the right to a $\mathrm{C} \mathrm{H}$ subscript 2 group. The $\mathrm{CH}$ subscript 2 group is bonded up and to the right to an $\mathrm{O}$ atom. The $\mathrm{O}$ atom is bonded down and to the right to a $\mathrm{CH}$ subscript 2 group. The $\mathrm{CH}$ subscript 2 group is bonded up and to the right to a C H subscript 3 group.

Solution IUPAC: The molecule is made up of an ethoxy group attached to an ethane chain, so the IUPAC name would be ethoxyethane.

Common: The groups attached to the oxygen atom are both ethyl groups, so the common name would be diethyl ether.

Check Your Learning Provide the IUPAC and common name for the ether shown:

A molecular structure shows a C H subscript 3 group bonded up and to the right to an $\mathrm{O}$ atom. The $\mathrm{O}$ atom is bonded down and to the right to a $\mathrm{CH}$ group. The $\mathrm{CH}$ group is bonded up and to the right to a $\mathrm{CH}$ subscript 3 group. The $\mathrm{C} \mathrm{H}$ group is also bonded down and to the right to another $\mathrm{C} \mathrm{H}$ subscript 3 group. Answer:

IUPAC: 2-methoxypropane; common: isopropylmethyl ether

Ethers can be obtained from alcohols by the elimination of 
a molecule of water from two molecules of the alcohol. For example, when ethanol is treated with a limited amount of sulfuric acid and heated to $140{ }^{\circ} \mathrm{C}$, diethyl ether and water are formed:

This figure shows a reaction. The first molecule, which is labeled, "ethanol," is a two C atom chain. The first C atom is bonded to three $\mathrm{H}$ atoms and a second $\mathrm{C}$ atom. The second $\mathrm{C}$ atom is bonded to a red $\mathrm{O}$ atom with two sets of electron dots. The $\mathrm{O}$ atom has a red bond to a red $\mathrm{H}$ atom. There is a plus sign. The next molecule, which is labeled, "ethanol," is a red $\mathrm{H}$ atom with a red bond to a red $\mathrm{O}$ atom with two pairs of electron dots. The $\mathrm{O}$ atom is bonded to a $\mathrm{C}$ atom which is bonded to two $\mathrm{H}$ atoms and a second $\mathrm{C}$ atom. The second $\mathrm{C}$ atom is bonded to three $\mathrm{H}$ atoms. There is a green dotted box around the red $\mathrm{H}$ atom in the first molecule, the plus sign, and the red $\mathrm{H}$ and $\mathrm{O}$ atoms in the second molecule. To the right o the second molecule there is an arrow labeled $\mathrm{H}$ subscript 2 S O subscript 4 above and Greek capital delta below. The arrow is labeled, "sulfuric acid." The resulting molecules are a $\mathrm{C}$ atom bonded with three $\mathrm{H}$ atoms and a second $\mathrm{C}$ atom. The second $\mathrm{C}$ atom is bonded to two $\mathrm{H}$ atoms and a red $\mathrm{O}$ atom. The red $\mathrm{O}$ atom has two sets of electron dots. The $\mathrm{O}$ atom is bonded to a third $\mathrm{C}$ atom which is bonded to two $\mathrm{H}$ atoms and a fourth $\mathrm{C}$ atom. The fourth $\mathrm{C}$ atom is bonded to three $\mathrm{H}$ atoms. This molecule is labeled, “diethyl ether." There is a plus sign and a red $\mathrm{H} \mathrm{O} \mathrm{H.}$

In the general formula for ethers, $\mathrm{R}-\mathbf{0}-\mathrm{R}$, the hydrocarbon groups (R) may be the same or different. Diethyl ether, the most widely used compound of this class, is a colorless, volatile liquid that is highly flammable. It was first used in 1846 as an anesthetic, but 
better anesthetics have now largely taken its place. Diethyl ether and other ethers are presently used primarily as solvents for gums, fats, waxes, and resins. Tertiary-butyl methyl ether, $\mathrm{C}_{4} \mathrm{H}_{9} \mathrm{OCH}_{3}$ (abbreviated MTBE-italicized portions of names are not counted when ranking the groups alphabetically-so butyl comes before methyl in the common name), is used as an additive for gasoline. MTBE belongs to a group of chemicals known as oxygenates due to their capacity to increase the oxygen content of gasoline.

Want more practice naming ethers? This brief video review summarizes the nomenclature for ethers.

Carbohydrates and Diabetes

Carbohydrates are large biomolecules made up of carbon, hydrogen, and oxygen. The dietary forms of carbohydrates are foods rich in these types of molecules, like pastas, bread, and candy. The name "carbohydrate" comes from the formula of the molecules, which can be described by the general formula $\mathrm{C}_{m}\left(\mathrm{H}_{2} \mathrm{O}\right)_{n}$, which shows that they are in a sense "carbon and water" or "hydrates of carbon." In many cases, $m$ and $n$ have the same value, but they can be different. The smaller carbohydrates are generally referred to as "sugars," the biochemical term for this group of molecules is "saccharide" from the Greek word for sugar ((Figure)). Depending on the number of sugar units joined together, they may be classified as monosaccharides (one sugar unit), disaccharides (two sugar units), oligosaccharides (a few sugars), or polysaccharides (the polymeric version of sugars-polymers were described in the feature box earlier 
in this chapter on recycling plastics). The scientific names of sugars can be recognized by the suffix -ose at the end of the name (for instance, fruit sugar is a monosaccharide called "fructose" and milk sugar is a disaccharide called lactose composed of two monosaccharides, glucose and galactose, connected together). Sugars contain some of the functional groups we have discussed: Note the alcohol groups present in the structures and how monosaccharide units are linked to form a disaccharide by formation of an ether.

The illustrations show the molecular structures of fructose, a five-carbon monosaccharide, and of lactose, a disaccharide composed of two isomeric, six-carbon sugars.

This figure shows structural and ball-and-stick models for the common sugars fructose and lactose. Carbon atoms are illustrated in black, oxygen atoms are red, and hydrogen atoms are white in the ball-and-stick models.

Organisms use carbohydrates for a variety of functions. Carbohydrates can store energy, such as the polysaccharides glycogen in animals or starch in plants. They also provide structural support, such as the polysaccharide cellulose in plants and the modified polysaccharide chitin in fungi and animals. The sugars ribose and deoxyribose are components of the backbones of RNA and DNA, respectively. Other sugars play key roles in the function of the immune system, in cell-cell recognition, and in many other biological roles.

Diabetes is a group of metabolic diseases in which a person has a high sugar concentration in their blood 
((Figure)). Diabetes may be caused by insufficient insulin production by the pancreas or by the body's cells not responding properly to the insulin that is produced. In a healthy person, insulin is produced when it is needed and functions to transport glucose from the blood into the cells where it can be used for energy. The long-term complications of diabetes can include loss of eyesight, heart disease, and kidney failure.

In 2013, it was estimated that approximately 3.3\% of the world's population ( $>380$ million people) suffered from diabetes, resulting in over a million deaths annually. Prevention involves eating a healthy diet, getting plenty of exercise, and maintaining a normal body weight. Treatment involves all of these lifestyle practices and may require injections of insulin.

Diabetes is a disease characterized by high concentrations of glucose in the blood. Treating diabetes involves making lifestyle changes, monitoring bloodsugar levels, and sometimes insulin injections. (credit:

"Blausen Medical Communications"/Wikimedia Commons)

This is a diagram of a hand with a blood droplet on an index finger and a nearby sharp pointed pen-like object. The finger is next shown touching a white and green test strip with arrows pointing to the green region where the bloody finger touches the strip. An arrow points to a small rectangular device in which the green end of the strip is inserted. An L C D display provides a reading. 
Key Concepts and Summary

Many organic compounds that are not hydrocarbons can be thought of as derivatives of hydrocarbons. A hydrocarbon derivative can be formed by replacing one or more hydrogen atoms of a hydrocarbon by a functional group, which contains at least one atom of an element other than carbon or hydrogen. The properties of hydrocarbon derivatives are determined largely by the functional group. The $-\mathrm{OH}$ group is the functional group of an alcohol. The $-\mathrm{R}-\mathrm{O}-\mathrm{R}-$ group is the functional group of an ether.

Chemistry End of Chapter Exercises

Why do the compounds hexane, hexanol, and hexene have such similar names?

Write condensed formulas and provide IUPAC names for the following compounds:

(a) ethyl alcohol (in beverages)

(b) methyl alcohol (used as a solvent, for example, in shellac)

(c) ethylene glycol (antifreeze)

(d) isopropyl alcohol (used in rubbing alcohol)

(e) glycerine

(a) ethyl alcohol, ethanol: $\mathrm{CH}_{3} \mathrm{CH}_{2} \mathrm{OH}$; (b) methyl alcohol, methanol: $\mathrm{CH}_{3} \mathrm{OH}$; (c) ethylene glycol, ethanediol: $\mathrm{HOCH}_{2} \mathrm{CH}_{2} \mathrm{OH}$; (d) isopropyl alcohol, 2-propanol: $\mathrm{CH}_{3} \mathrm{CH}(\mathrm{OH}) \mathrm{CH}_{3}$; (e) glycerine, 1,2,3-trihydroxypropane: $\mathrm{HOCH}_{2} \mathrm{CH}(\mathrm{OH}) \mathrm{CH}_{2} \mathrm{OH}$ 
Give the complete IUPAC name for each of the following compounds:

(a)

This shows a $\mathrm{C} \mathrm{H}$ subscript 3 group bonded to a $\mathrm{C} \mathrm{H}$ group. The $\mathrm{C}$ atom in the $\mathrm{C} \mathrm{H}$ group is bonded above to an $\mathrm{O} \mathrm{H}$ group. The $\mathrm{C}$ in the $\mathrm{C} \mathrm{H}$ group is also bonded below to a $\mathrm{C} \mathrm{H}$ subscript 2 group. The $\mathrm{C} \mathrm{H}$ subscript 2 group is bonded below to a $\mathrm{C} \mathrm{H}$ subscript 3 group.

This shows a $\mathrm{C} H$ subscript 3 group bonded to a $\mathrm{C}$ atom. The $\mathrm{C}$ atom is bonded to an $\mathrm{O} \mathrm{H}$ group and an I atom. It is also bonded to a second $\mathrm{C}$ atom. This second $\mathrm{C}$ atom is bonded above and below to a $\mathrm{C} \mathrm{H}$ subscript 3 group. The second $\mathrm{C}$ atom is bonded to a $\mathrm{C} \mathrm{H}$ subscript 2 group with is bonded to a $\mathrm{C} \mathrm{H}$ subscript 3 group.

This shows a $\mathrm{C} \mathrm{H}$ subscript 3 group bonded to a $\mathrm{C} \mathrm{H}$ group. The $\mathrm{C}$ atom in the $\mathrm{C} \mathrm{H}$ group is bonded to an $\mathrm{O}$ $\mathrm{H}$ group. The $\mathrm{C} \mathrm{H}$ group is bonded to a $\mathrm{C}$ atom. The $\mathrm{C}$ atom is bonded below to a $\mathrm{C} \mathrm{l}$ atom and above to a $\mathrm{C} \mathrm{H}$ subscript 2 group. The $\mathrm{C}$ atom in the $\mathrm{C} \mathrm{H}$ subscript 2 group is also bonded to a $\mathrm{C} \mathrm{H}$ subscript 3 group. The $\mathrm{C}$ atom is also bonded to a $\mathrm{C} \mathrm{H}$ subscript 2 group to the right. This $\mathrm{C} \mathrm{H}$ subscript 2 group is bonded to another $\mathrm{C} \mathrm{H}$ subscript 2 group. Below this second $\mathrm{C} \mathrm{H}$ subscript 2 group a $\mathrm{C} \mathrm{H}$ subscript 3 group is bonded.

Give the complete IUPAC name and the common name for each of the following compounds:

(a) 
This shows a $\mathrm{C} \mathrm{H}$ subscript 3 group bonded to a $\mathrm{C} \mathrm{H}$ subscript 2 group. This $\mathrm{C} \mathrm{H}$ subscript 2 group is bonded to an $\mathrm{O}$ atom which is also bonded to a $\mathrm{C} \mathrm{H}$ subscript 2 group. This $\mathrm{C} \mathrm{H}$ subscript 2 group is bonded to a $\mathrm{C} \mathrm{H}$ subscript 2 group. This $\mathrm{C} \mathrm{H}$ subscript 2 group is bonded to a $\mathrm{C} \mathrm{H}$ subscript 2 group. This $\mathrm{C} \mathrm{H}$ subscript 2 group is bonded to a C H subscritp 3 group. All bonds are in a straight line. (b)

This shows a $\mathrm{C} \mathrm{H}$ subscript 3 group bonded to a $\mathrm{C} \mathrm{H}$ subscript 2 group. This $\mathrm{C} \mathrm{H}$ subscript 2 group is bonded to an $\mathrm{O}$ atom. This $\mathrm{O}$ atom is bonded to a $\mathrm{C} \mathrm{H}$ subscript 2 group which is also bonded to another $\mathrm{C} \mathrm{H}$ subscript 2 group. This $\mathrm{C} \mathrm{H}$ subscript 2 group is bonded to a $\mathrm{C} \mathrm{H}$ subscript 3 group. All bonds are in a straight line.

This figure shows a $\mathrm{C} \mathrm{H}$ subscript 3 group bonded to an $\mathrm{O}$ atom. This $\mathrm{O}$ atom is bonded to a $\mathrm{C} \mathrm{H}$ subscript 2 group which is also bonded to another $\mathrm{C} \mathrm{H}$ subscript 2 group. This C H subscript 2 group is bonded to a $\mathrm{C} \mathrm{H}$ subscript 3 group. All bonds are in a straight line.

(a) 1-ethoxybutane, butyl ethyl ether; 1-ethoxypropane, ethyl propyl ether; 1-methoxypropane, methyl propyl ether

Write the condensed structures of both isomers with the formula $\mathrm{C}_{2} \mathrm{H}_{6} \mathrm{O}$. Label the functional group of each isomer.

Write the condensed structures of all isomers with the formula $\mathrm{C}_{2} \mathrm{H}_{6} \mathrm{O}_{2}$. Label the functional group (or groups) of each isomer. 
$\mathrm{HOCH}_{2} \mathrm{CH}_{2} \mathrm{OH}$, two alcohol groups; $\mathrm{CH}_{3} \mathrm{OCH}_{2} \mathrm{OH}$, ether and alcohol groups

Draw the condensed formulas for each of the following compounds:
(a) dipropyl ether
(b) 2,2-dimethyl-3-hexanol
(c) 2-ethoxybutane

MTBE, Methyl tert-butyl ether, $\mathrm{CH}_{3} \mathrm{OC}\left(\mathrm{CH}_{3}\right)_{3}$, is used as an oxygen source in oxygenated gasolines. MTBE is manufactured by reacting 2-methylpropene with methanol.

(a) Using Lewis structures, write the chemical equation representing the reaction.

(b) What volume of methanol, density $0.7915 \mathrm{~g} / \mathrm{mL}$, is required to produce exactly $1000 \mathrm{~kg}$ of MTBE, assuming a $100 \%$ yield?

(a)

A reaction is shown. The first molecule is a $\mathrm{C}$ atom bonded to another $\mathrm{C}$ atom. The first $\mathrm{C}$ atom (from left to right) is bonded to two $\mathrm{C} \mathrm{H}$ subscript 3 groups. The second $\mathrm{C}$ atom is bonded to two $\mathrm{H}$ atoms. There is a plus sign. The next molecule shows an $\mathrm{H}$ atom bonded to an $\mathrm{O}$ atom bonded to a $\mathrm{C} \mathrm{H}$ subscript 3 group. There is an arrow pointing right. This molecule shows a $\mathrm{C}$ atom bonded to three $\mathrm{C} H$ subscript 3 groups. The $\mathrm{C}$ atom is also bonded to an $\mathrm{O}$ atom which is also bonded to a $\mathrm{C} \mathrm{H}$ subscript 3 group.

(b) $4.59310^{2} \mathrm{~L}$

Write two complete balanced equations for each of the 
following reactions, one using condensed formulas and one using Lewis structures.

(a) propanol is converted to dipropyl ether

(b) propene is treated with water in dilute acid.

Write two complete balanced equations for each of the following reactions, one using condensed formulas and one using Lewis structures.

(a) 2-butene is treated with water in dilute acid

(b) ethanol is dehydrated to yield ethene

(a)

$$
\mathrm{CH}_{3} \mathrm{CH}=\mathrm{CHCH}_{3}+\mathrm{H}_{2} \mathrm{O} \mathrm{CH}_{3} \mathrm{CH}_{2} \mathrm{CH}(\mathrm{OH}) \mathrm{CH}_{3}
$$

$\mathrm{A}$ reaction is shown. The first molecule shows a $\mathrm{C}$ atom bonded with three $\mathrm{H}$ atoms. The first $\mathrm{C}$ atom is bonded to another $\mathrm{C}$ atom. The second $\mathrm{C}$ atom is bonded to an $\mathrm{H}$ atom and also forms a double bond with a third $\mathrm{C}$ atom. The third $\mathrm{C}$ atom is bonded to one $\mathrm{H}$ atom and fourth $\mathrm{C}$ atom. The fourth $\mathrm{C}$ atom is bonded to three $\mathrm{H}$ atoms. There is a plus sign. The second molecule shows an $\mathrm{O}$ atom with two sets of electron dots bonded to two $\mathrm{H}$ atoms. There is an arrow pointing right which is labeled, "acid." The new molecule is a $\mathrm{C}$ atom bonded to three $\mathrm{H}$ atoms and a second $\mathrm{C}$ atom. The second $\mathrm{C}$ atom is bonded to two $\mathrm{H}$ atoms and a third $\mathrm{C}$ atom. The third $\mathrm{C}$ atom is bonded to an $\mathrm{H}$ atom and an $\mathrm{O}$ atom with two sets of electron dots. The $\mathrm{O}$ atom is bonded to an $\mathrm{H}$ atom. The third $\mathrm{C}$ atom is bonded to a fourth $\mathrm{C}$ atom which is bonded to three $\mathrm{H}$ atoms.

(b) $\mathrm{CH}_{3} \mathrm{CH}_{2} \mathrm{OH} \mathrm{CH}_{2}=\mathrm{CH}_{2}+\mathrm{H}_{2} \mathrm{O}$ 
A reaction is shown. The first molecule shows a $\mathrm{C}$ atom which is bonded to three $\mathrm{H}$ atoms and a second $\mathrm{C}$ atom. The second $\mathrm{C}$ atom is bonded to an $\mathrm{O}$ atom as well. The $\mathrm{O}$ atom has two sets of electron dots and is bonded to an $\mathrm{H}$ atom. There is an arrow that points to the right. The next molecule shows two $\mathrm{C}$ atoms forming a double bond between them. Each $\mathrm{C}$ atom is bonded to two $\mathrm{H}$ atoms. There is a plus sign. The next molecule shows an $\mathrm{O}$ atom with two sets of electron dots bonded to two $\mathrm{H}$ atoms.

Footnotes

- 1 The IUPAC adopted new nomenclature guidelines in 2013 that require this number to be placed as an "infix" rather than a prefix. For example, the new name for 2-propanol would be propan-2-ol. Widespread adoption of this new nomenclature will take some time, and students are encouraged to be familiar with both the old and new naming protocols.

Glossary

alcohol

organic compound with a hydroxyl group

$(-\mathrm{OH})$ bonded to a carbon atom

ether

organic compound with an oxygen atom

that is bonded to two carbon atoms 


\section{Focus on Polymers}

Synthetic polymers were developed in the early $20^{\text {th }}$ century, and since that time have become ubiquitous in our world. The clothing, the helmet and the goggles on the people riding these snowmobiles and, indeed, even the material that makes up the housing of the snowmobiles, are all synthetic polymers.

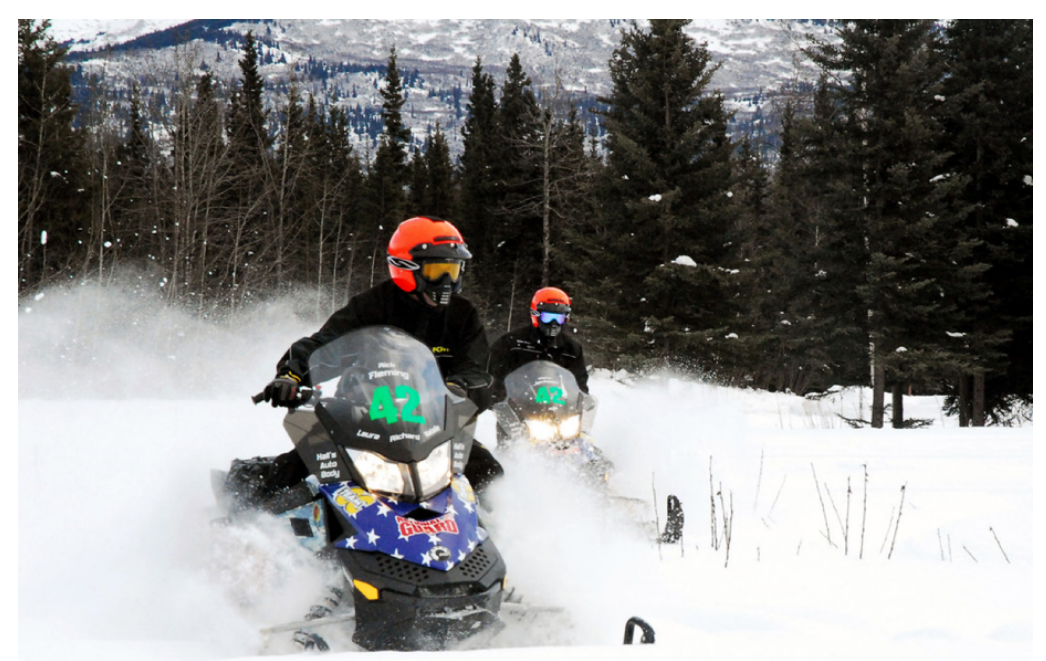

"Alaska National Guard Iron Dog snowmobile race, Camp Denali, Alaska" by The U.S. Army is licensed under CC BY 2.0

The computers we work on, our cell phones and much of the furniture around us all have a large number of different polymers involved in them. The success of these materials, however, is a double-edged sword - their variety continues to provide us with amazing and useful materials, but the fact that they are cheap, vast and persistent is also causing a unique environmental catastrophe. In this section, the basic reactions involved in producing the most common polymers will be examined along with a survey of associated environmental issues and current developments being made to address those issues.

Refer to the information discussed in the previous Focus section as well as Part XIII of this text and elaborate on the following focus boxes, solving any problems that appear. 
A monomer is a molecule that forms the basic unit for polymers. Monomers bind to other monomers to form repeating chain molecules through a process known as polymerization.

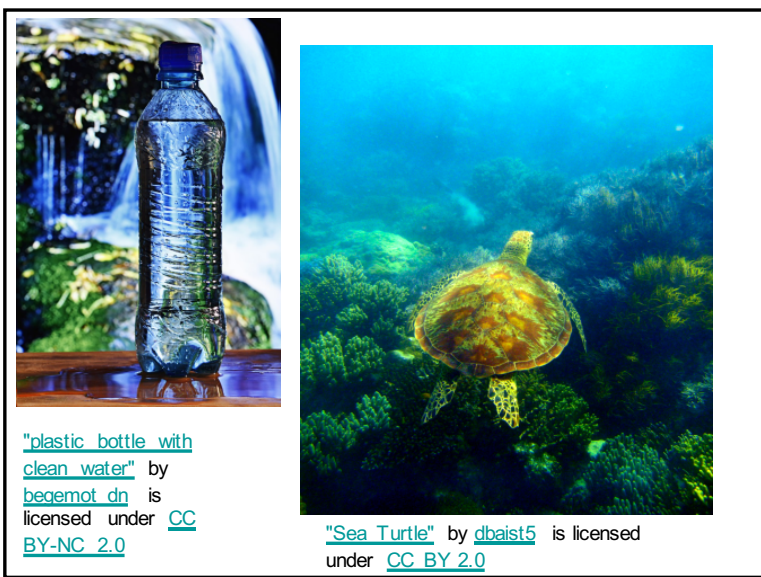

\section{Addition polymers}

Ex: Show the initiation and beginning of chain growth for the formation of polyethylene

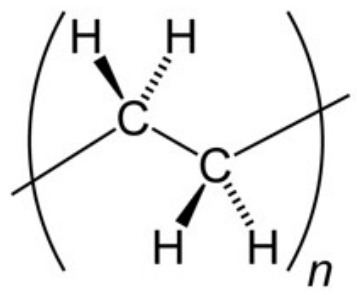




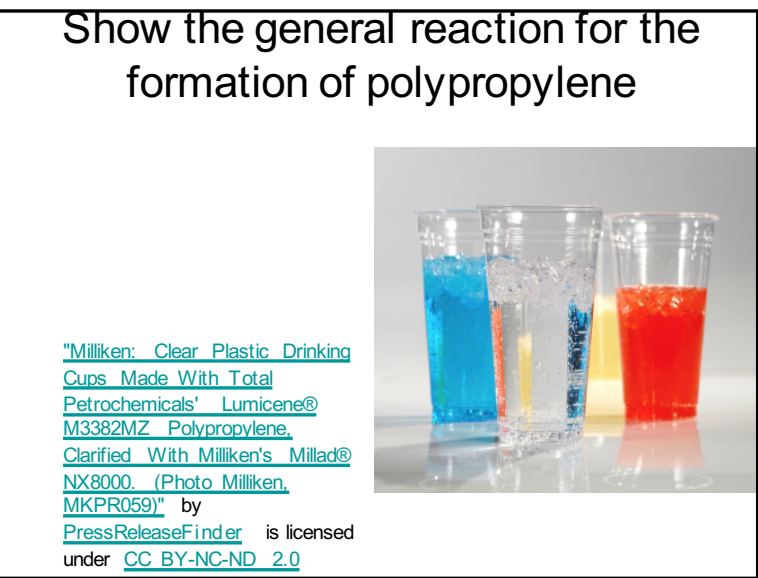

Show the general reaction for the formation of polystyrene

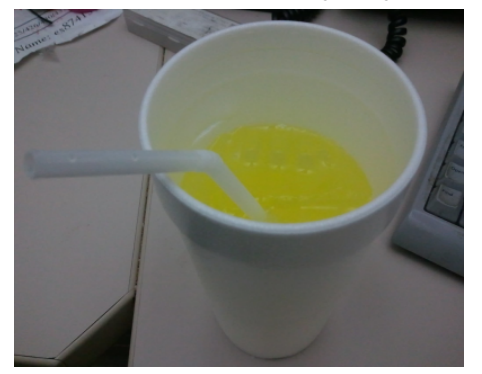

"expanded polystyrene" by Richard Masoner / Cyclelicious is licensed under CC BY-SA 2.0

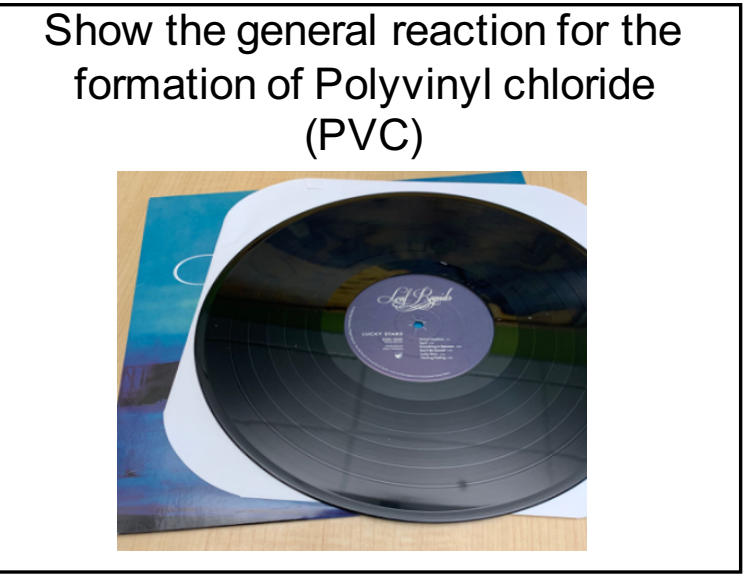


How many chloroethene (vinyl chloride) subunits are in a molecule of polyvinyl chloride with a molar mass of $3122.5 \mathrm{~g} / \mathrm{mole}$ ?

- A. 100

- B. 500

- C. 250

- D. 50

- E. 550

What is the molar mass of one molecule of polypropylene with 300 propylene subunits?

- A. 12,600

- B. 6,300

- C. 25,200

- D. 100,800

- E. 50,400

\section{Condensation Polymers}

- Monomer units join by eliminating (splitting out) a small molecule, often water.

- Ex: show the reaction for the formation of polyethylene terephthalate (PET)

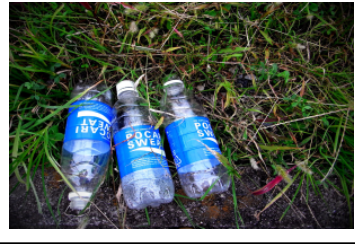




\section{Polycarbonates}

- The main polycarbonate material is produced by the reaction of bisphenol $\mathrm{A}$ and phosgene $\mathrm{COCl} 2$.

管

- strong, transparent

- Have a number of uses and were once used in the production of drinking bottles.

\section{Polycarbonates and bisphenol A}<smiles>CC(C)(c1ccc(O)cc1)c1ccc(O)cc1</smiles>

- Bisphenol A: estrogen-mimicking, hormone-like properties

Various studies have found a range of effects - from 'significant' to 'none'

\section{Polycarbonates and bisphenol A}<smiles>CC(C)(c1ccc(O)cc1)c1ccc(O)cc1</smiles>

- Bisphenol A: estrogen-mimicking, hormone-like properties

- Bisphenol A leachate low-dose effects (Saal and

Hughes; https://ehp.niehs.nih.gov/doi/10.1289/ehp.7713)

- Industry-funded studies tend to find no significant effects

- government-funded studies tend to find significant effects 


\section{Commercial polymers also} normally have a number of other additives and processing agents

- Antioxidants, blowing agents, colorants, coupling agents, flame retardants, heat stabilisers, impact modifiers, lubricants, plasticisers, preservatives, uv stabilisers, etc.
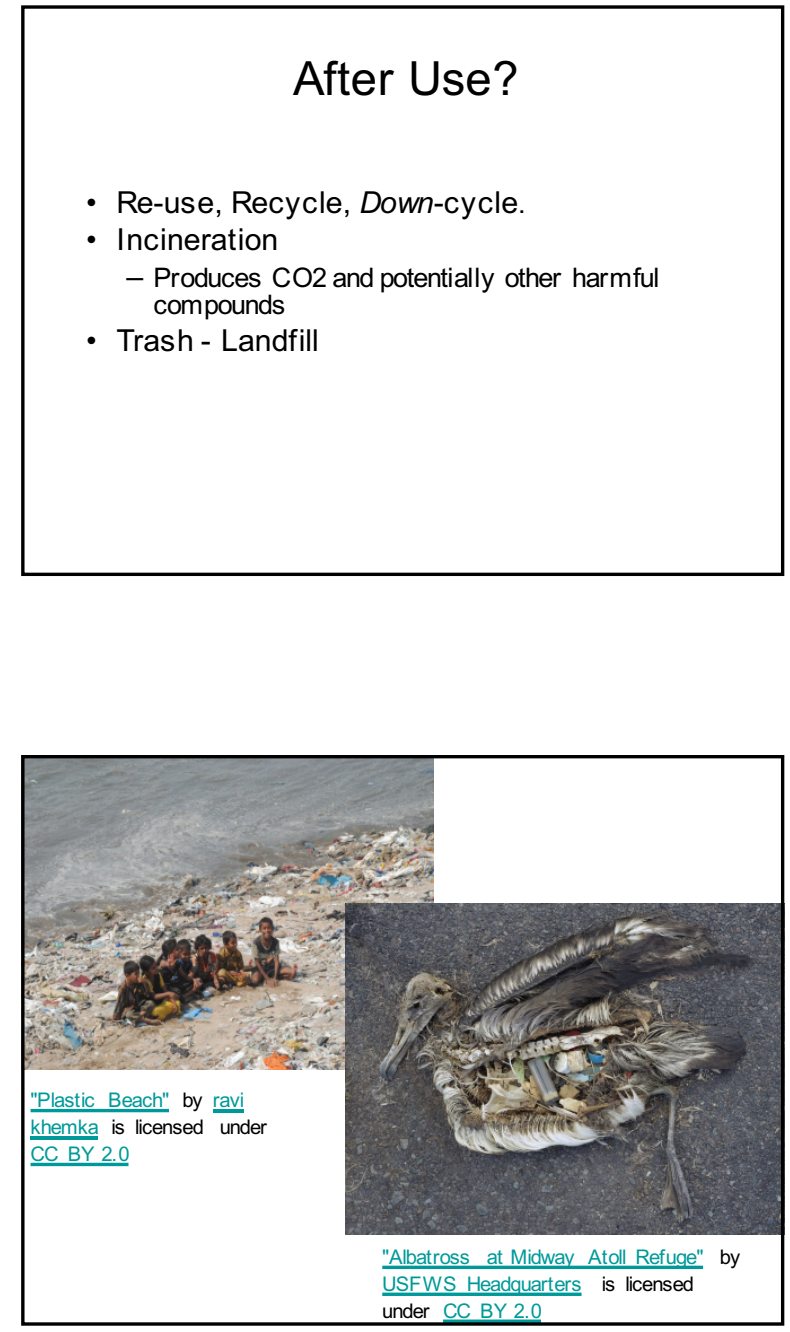
Focus on Polymers

\section{National Geographic - biodegradable polymers}

https://www.nationalgeographic.com/environment 12018/11/are-bioplastics-made-from-plantsbetter-for-environment-ocean-plastic/\#close

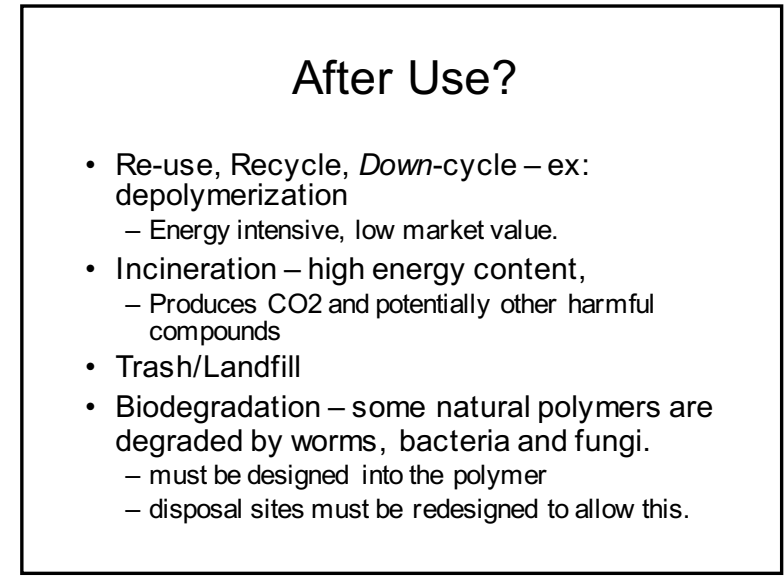





\section{PART XIV}

\section{NUCLEAR \\ CHEMISTRY}

989 



\section{8.}

\section{INTRODUCTION}

\section{Learning Objectives}

- Nuclear Structure and Stability

- Nuclear Equations

- Radioactive Decay

- Transmutation and Nuclear Energy

- Biological Effects of Radiation

Nuclear chemistry provides the basis for many useful diagnostic and therapeutic methods in medicine, such as these positron emission tomography (PET) scans. The PET/computed tomography scan on the left shows muscle activity. The brain scans in the center show chemical differences in dopamine signaling in the brains of addicts and nonaddicts. The images on the right show an oncological application of PET scans to identify lymph node metastasis. 


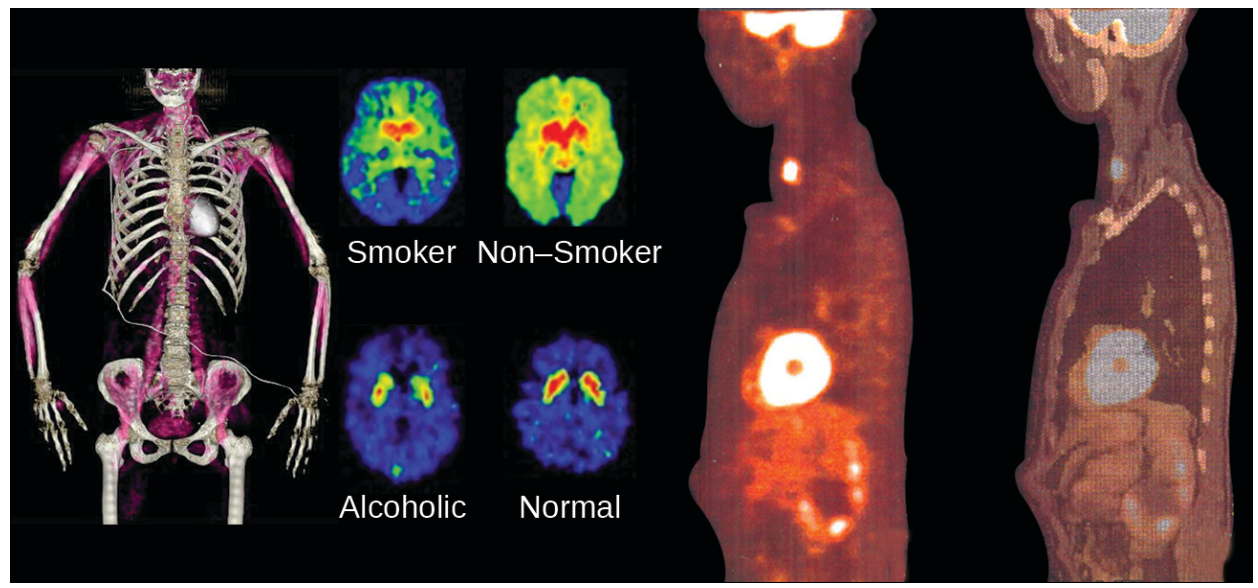

The chemical reactions that we have considered in previous chapters involve changes in the electronic structure of the species involved, that is, the arrangement of the electrons around atoms, ions, or molecules. Nuclear structure, the numbers of protons and neutrons within the nuclei of the atoms involved, remains unchanged during chemical reactions.

This chapter will introduce the topic of nuclear chemistry, which began with the discovery of radioactivity in 1896 by French physicist Antoine Becquerel and has become increasingly important during the twentieth and twenty-first centuries, providing the basis for various technologies related to energy, medicine, geology, and many other areas. 


\section{9.}

\section{NUCLEAR STRUCTURE AND STABILITY}

Learning Objectives

By the end of this section, you will be able to:

- Describe nuclear structure in terms of protons, neutrons, and electrons

- Calculate mass defect and binding energy for nuclei

- Explain trends in the relative stability of nuclei

Nuclear chemistry is the study of reactions that involve changes in nuclear structure. The chapter on atoms, molecules, and ions introduced the basic idea of nuclear structure, that the nucleus of an atom is composed of protons and, with the exception of ${ }_{1}^{1} \mathrm{H}$, neutrons. Recall that the number of protons in the nucleus is called the 
atomic number $(\mathrm{Z})$ of the element, and the sum of the number of protons and the number of neutrons is the mass number (A). Atoms with the same atomic number but different mass numbers are isotopes of the same element. When referring to a single type of nucleus, we often use the term nuclide and identify it by the notation ${ }_{\mathrm{Z}}^{\mathrm{A} X} \mathrm{X}$, where $\mathrm{X}$ is the symbol for the element, $\mathrm{A}$ is the mass number, and $\mathrm{Z}$ is the atomic number (for example, ${ }_{6}^{14} \mathrm{C}$. Often a nuclide is referenced by the name of the element followed by a hyphen and the mass number. For example, ${ }_{6}^{14} \mathrm{C}$ is called "carbon-14."

Protons and neutrons, collectively called nucleons, are packed together tightly in a nucleus. With a radius of about $10^{-15}$ meters, a nucleus is quite small compared to the radius of the entire atom, which is about $10^{-10}$ meters. Nuclei are extremely dense compared to bulk matter, averaging $1.8 \quad 10^{14}$ grams per cubic centimeter. For example, water has a density of 1 gram per cubic centimeter, and iridium, one of the densest elements known, has a density of $22.6 \mathrm{~g} / \mathrm{cm}^{3}$. If the earth's density were equal to the average nuclear density, the earth's radius would be only about 200 meters (earth's actual radius is approximately $6.410^{6}$ meters, 30,000 times larger). (Figure) demonstrates just how great nuclear densities can be in the natural world.

Density of a Neutron Star Neutron stars form when the core of a very massive star undergoes gravitational collapse, causing the star's outer layers to explode in a supernova. Composed almost completely of neutrons, they are the densest-known stars in the universe, with densities comparable to the average density of an atomic nucleus. $A$ 
neutron star in a faraway galaxy has a mass equal to 2.4 solar masses ( 1 solar mass $=\mathrm{M}=$ mass of the sun $=1.9910^{30} \mathrm{~kg}$ ) and a diameter of $26 \mathrm{~km}$.

(a) What is the density of this neutron star?

(b) How does this neutron star's density compare to the density of a uranium nucleus, which has a diameter of about 15 $\mathrm{fm}\left(1 \mathrm{fm}=10^{-15} \mathrm{~m}\right)$ ?

Solution We can treat both the neutron star and the U-235 nucleus as spheres. Then the density for both is given by:
$d=\frac{m}{V}$
with
$V=\frac{4}{3} \pi r^{3}$

(a) The radius of the neutron star is

$\frac{1}{2} 26 \mathrm{~km}=\frac{1}{2} 2.610^{4} \mathrm{~m}=1.310^{4} \mathrm{~m}$, so the density of the neutron star is:

$$
d=\frac{m}{V}=\frac{m}{\frac{4}{3} \pi r^{3}}=\frac{2.4\left(1.9910^{30} \mathrm{~kg}\right)}{\frac{4}{3} \pi\left(1.310^{4} \mathrm{~m}\right)^{3}}=5.210^{17} \mathrm{~kg} / \mathrm{m}^{3}
$$

(b) The radius of the $\mathrm{U}-235$ nucleus is $\frac{1}{2} 1510^{-15} \mathrm{~m}=7.510^{-15} \mathrm{~m}$, so the density of the $\mathrm{U}-235$ nucleus is:

$$
d=\frac{m}{V}=\frac{m}{\frac{4}{3} \pi r^{3}}=\frac{235 \mathrm{amu}\left(\frac{1.6610^{-27} \mathrm{~kg}}{1 \mathrm{amu}}\right)}{\frac{4}{3} \pi\left(7.510^{-15} \mathrm{~m}\right)^{3}}=2.210^{17} \mathrm{~kg} / \mathrm{m}^{3}
$$

These values are fairly similar (same order of magnitude), but the nucleus is more than twice as dense as the neutron star.

Check Your Learning Find the density of a neutron star with a mass of 1.97 solar masses and a diameter of $13 \mathrm{~km}$, and compare it to the density of a hydrogen nucleus, which has a diameter of $1.75 \mathrm{fm}\left(1 \mathrm{fm}=110^{-15} \mathrm{~m}\right)$.

Answer:

The density of the neutron star is $3.410^{18} \mathrm{~kg} / \mathrm{m}^{3}$. The 
density of a hydrogen nucleus is $6.010^{17} \mathrm{~kg} / \mathrm{m}^{3}$. The neutron star is 5.7 times denser than the hydrogen nucleus.

To hold positively charged protons together in the very small volume of a nucleus requires very strong attractive forces because the positively charged protons repel one another strongly at such short distances. The force of attraction that holds the nucleus together is the strong nuclear force. (The strong force is one of the four fundamental forces that are known to exist. The others are the electromagnetic force, the gravitational force, and the nuclear weak force.) This force acts between protons, between neutrons, and between protons and neutrons. It is very different from the electrostatic force that holds negatively charged electrons around a positively charged nucleus (the attraction between opposite charges). Over distances less than $10^{-15}$ meters and within the nucleus, the strong nuclear force is much stronger than electrostatic repulsions between protons; over larger distances and outside the nucleus, it is essentially nonexistent.

Visit this website for more information about the four fundamental forces.

Nuclear Binding Energy

As a simple example of the energy associated with the strong nuclear force, consider the helium atom composed of two protons, two neutrons, and two electrons. The total mass of these six subatomic particles may be calculated as: 
$\underset{\text { protons }}{(21.0073 \mathrm{amu}})+\underset{\text { neutrons }}{(2 \underset{0}{1.0087} \mathrm{amu})}+\underset{\text { electrons }}{(20.00055 \mathrm{amu})}=4.0331 \mathrm{amu}$

However, mass spectrometric measurements reveal that the mass of an ${ }_{2}^{4} \mathrm{He}$ atom is $4.0026 \mathrm{amu}$, less than the combined masses of its six constituent subatomic particles. This difference between the calculated and experimentally measured masses is known as the mass defect of the atom. In the case of helium, the mass defect indicates a "loss" in mass of $4.0331 \mathrm{amu}-4.0026 \mathrm{amu}=$ $0.0305 \mathrm{amu}$. The loss in mass accompanying the formation of an atom from protons, neutrons, and electrons is due to the conversion of that mass into energy that is evolved as the atom forms. The nuclear binding energy is the energy produced when the atoms' nucleons are bound together; this is also the energy needed to break a nucleus into its constituent protons and neutrons. In comparison to chemical bond energies, nuclear binding energies are vastly greater, as we will learn in this section. Consequently, the energy changes associated with nuclear reactions are vastly greater than are those for chemical reactions.

The conversion between mass and energy is most identifiably represented by the mass-energy equivalence equation as stated by Albert Einstein:

$E=m c^{2}$

where $E$ is energy, $m$ is mass of the matter being converted, and $c$ is the speed of light in a vacuum. This equation can be used to find the amount of energy that results when matter is converted into energy. Using this mass-energy equivalence equation, the nuclear binding energy of a nucleus may be calculated from its mass 
defect, as demonstrated in (Figure). A variety of units are commonly used for nuclear binding energies, including electron volts $(\mathrm{eV})$, with $1 \mathrm{eV}$ equaling the amount of energy necessary to the move the charge of an electron across an electric potential difference of 1 volt, making 1 $\mathrm{eV}=1.60210^{-19} \mathrm{~J}$.

Calculation of Nuclear Binding EnergyDetermine the binding energy for the nuclide ${ }_{2}^{4} \mathrm{He}$ in:

(a) joules per mole of nuclei

(b) joules per nucleus

(c) MeV per nucleus

SolutionThe mass defect for a ${ }_{2}^{4} \mathrm{He}$ nucleus is $0.0305 \mathrm{amu}$, as shown previously. Determine the binding energy in joules per nuclide using the mass-energy equivalence equation. To accommodate the requested energy units, the mass defect must be expressed in kilograms (recall that $1 \mathrm{~J}=1 \mathrm{~kg} \mathrm{~m} / \mathrm{s}^{2}$ ).

(a) First, express the mass defect in $\mathrm{g} / \mathrm{mol}$. This is easily done considering the numerical equivalence of atomic mass (amu) and molar mass $(\mathrm{g} / \mathrm{mol})$ that results from the definitions of the amu and mole units (refer to the previous discussion in the chapter on atoms, molecules, and ions if needed). The mass defect is therefore $0.0305 \mathrm{~g} / \mathrm{mol}$. To accommodate the units of the other terms in the mass-energy equation, the mass must be expressed in $\mathrm{kg}$, since $1 \mathrm{~J}=1 \mathrm{~kg}$ $\mathrm{m}^{2} / \mathrm{s}^{2}$. Converting grams into kilograms yields a mass defect of $3.0510^{-5} \mathrm{~kg} / \mathrm{mol}$. Substituting this quantity into the massenergy equivalence equation yields:

$$
\begin{aligned}
& \mathrm{E}=\mathrm{mc}^{2}=\frac{3.0510^{-5} \mathrm{~kg}}{\mathrm{~mol}}\left(\frac{2.99810^{8} \mathrm{~m}}{\mathrm{~s}}\right)^{2}=2.7410^{12} \mathrm{~kg} \mathrm{~m}^{2} \mathrm{~s}^{-2} \mathrm{~mol}^{-1} \\
& =2.7410^{12} \mathrm{~J} \mathrm{~mol}^{-1}
\end{aligned}
$$

Note that this tremendous amount of energy is associated 
with the conversion of a very small amount of matter (about $30 \mathrm{mg}$, roughly the mass of typical drop of water).

(b) The binding energy for a single nucleus is computed from the molar binding energy using Avogadro's number:

$E=2.7410^{12} \mathrm{~J} \mathrm{~mol}^{-1} \frac{1 \mathrm{~mol}}{6.02210^{23} \text { nuclei }}=4.5510^{-12} \mathrm{~J}=4.55 \mathrm{pJ}$

(c) Recall that $1 \mathrm{eV}=1.60210^{-19} \mathrm{~J}$. Using the binding energy computed in part (b):

$E=4.5510^{-12} \mathrm{~J} \frac{1 \mathrm{eV}}{1.60210^{-19} \mathrm{~J}}=2.8410^{7} \mathrm{eV}=28.4 \mathrm{MeV}$

Check Your LearningWhat is the binding energy for the nuclide ${ }_{9}^{19} \mathrm{~F}$ (atomic mass: $18.9984 \mathrm{amu}$ ) in MeV per nucleus?

Answer:

$148.4 \mathrm{MeV}$

Because the energy changes for breaking and forming bonds are so small compared to the energy changes for breaking or forming nuclei, the changes in mass during all ordinary chemical reactions are virtually undetectable. As described in the chapter on thermochemistry, the most energetic chemical reactions exhibit enthalpies on the order of thousands of $\mathrm{kJ} / \mathrm{mol}$, which is equivalent to mass differences in the nanogram range $\left(10^{-9} \mathrm{~g}\right)$. On the other hand, nuclear binding energies are typically on the order of billions of $\mathrm{kJ} / \mathrm{mol}$, corresponding to mass differences in the milligram range $\left(10^{-3} \mathrm{~g}\right)$.

Nuclear Stability

A nucleus is stable if it cannot be transformed into another configuration without adding energy from the outside. Of the thousands of nuclides that exist, about 250 are stable. A plot of the number of neutrons versus the number of protons for stable nuclei reveals that the stable 
isotopes fall into a narrow band. This region is known as the band of stability (also called the belt, zone, or valley of stability). The straight line in (Figure) represents nuclei that have a 1:1 ratio of protons to neutrons (n:p ratio). Note that the lighter stable nuclei, in general, have equal numbers of protons and neutrons. For example, nitrogen-14 has seven protons and seven neutrons. Heavier stable nuclei, however, have increasingly more neutrons than protons. For example: iron-56 has 30 neutrons and 26 protons, an n:p ratio of 1.15 , whereas the stable nuclide lead-207 has 125 neutrons and 82 protons, an n:p ratio equal to 1.52 . This is because larger nuclei have more proton-proton repulsions, and require larger numbers of neutrons to provide compensating strong forces to overcome these electrostatic repulsions and hold the nucleus together.

This plot shows the nuclides that are known to exist and those that are stable. The stable nuclides are indicated in blue, and the unstable nuclides are indicated in green. Note that all isotopes of elements with atomic numbers greater than 83 are unstable. The solid line is the line where $\mathrm{n}=\mathrm{Z}$. 


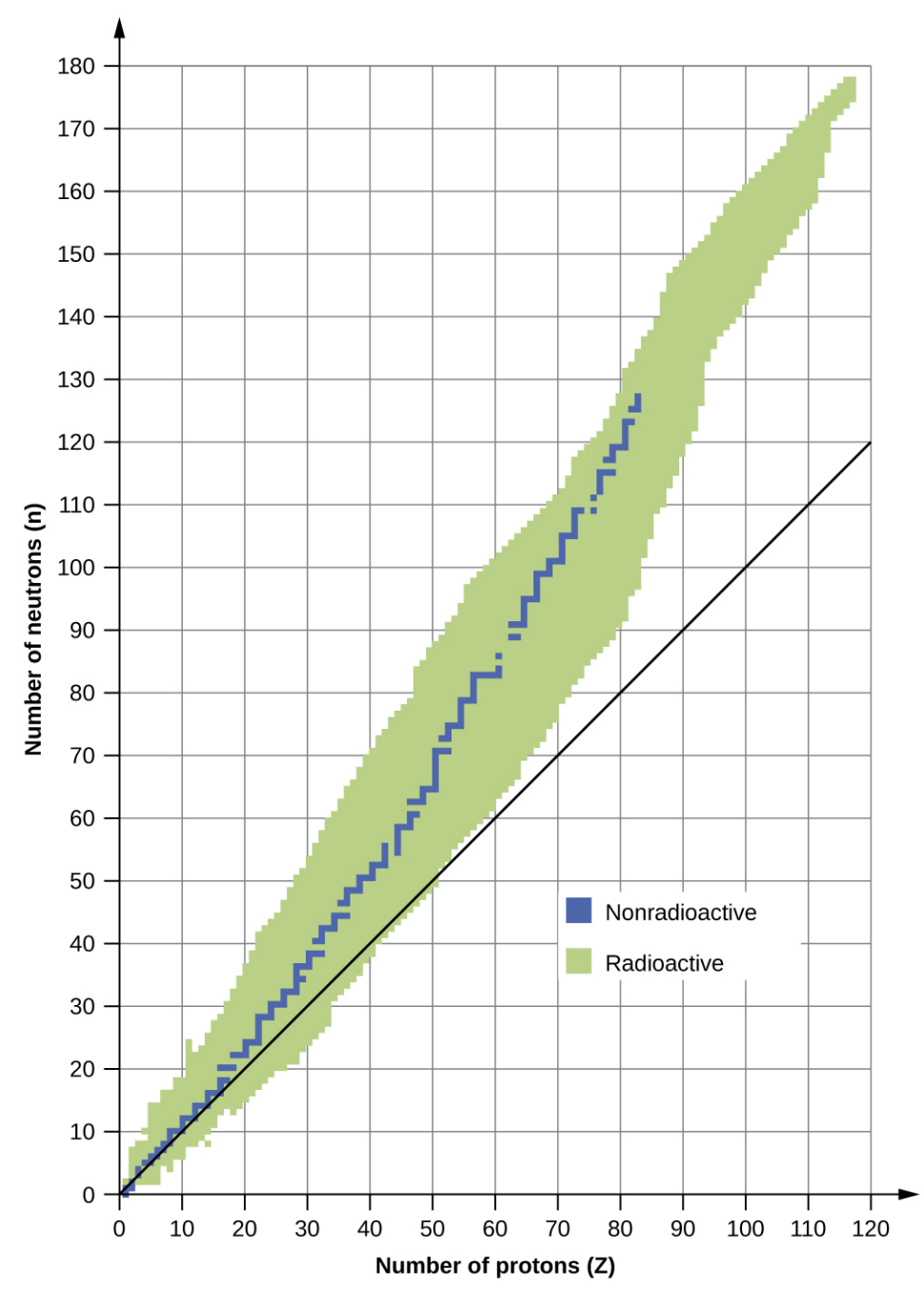

The nuclei that are to the left or to the right of the band of stability are unstable and exhibit radioactivity. They change spontaneously (decay) into other nuclei that are either in, or closer to, the band of stability. These nuclear decay reactions convert one unstable isotope (or radioisotope) into another, more stable, isotope. We will discuss the nature and products of this radioactive decay in subsequent sections of this chapter.

Several observations may be made regarding the relationship between the stability of a nucleus and its structure. Nuclei with even numbers of protons, neutrons, 
or both are more likely to be stable (see (Figure)). Nuclei with certain numbers of nucleons, known as magic numbers, are stable against nuclear decay. These numbers of protons or neutrons $(2,8,20,28,50,82$, and 126) make complete shells in the nucleus. These are similar in concept to the stable electron shells observed for the noble gases. Nuclei that have magic numbers of both protons and neutrons, such as ${ }_{2}^{4} \mathrm{He},{ }_{8}^{16} \mathrm{O},{ }_{20}^{40} \mathrm{Ca}$, and ${ }_{82}^{208} \mathrm{~Pb}$, are called "double magic" and are particularly stable. These trends in nuclear stability may be rationalized by considering a quantum mechanical model of nuclear energy states analogous to that used to describe electronic states earlier in this textbook. The details of this model are beyond the scope of this chapter.

\section{Stable Nuclear Isotopes}

$\begin{array}{lll}\text { Number of Stable Isotopes } & \text { Proton Number } & \text { Neutron Number } \\ 157 & \text { even } & \text { even } \\ 53 & \text { even } & \text { odd } \\ 50 & \text { odd } & \text { even } \\ 5 & \text { odd } & \text { odd }\end{array}$

The relative stability of a nucleus is correlated with its binding energy per nucleon, the total binding energy for the nucleus divided by the number or nucleons in the nucleus. For instance, we saw in (Figure) that the binding energy for a ${ }_{2}^{4} \mathrm{He}$ nucleus is $28.4 \mathrm{MeV}$. The binding energy per nucleon for a ${ }_{2}^{4} \mathrm{He}$ nucleus is therefore: $\frac{28.4 \mathrm{MeV}}{4 \text { nucleons }}=7.10 \mathrm{MeV} /$ nucleon 
In (Figure), we learn how to calculate the binding energy per nucleon of a nuclide on the curve shown in (Figure). The binding energy per nucleon is largest for nuclides with mass number of approximately 56.

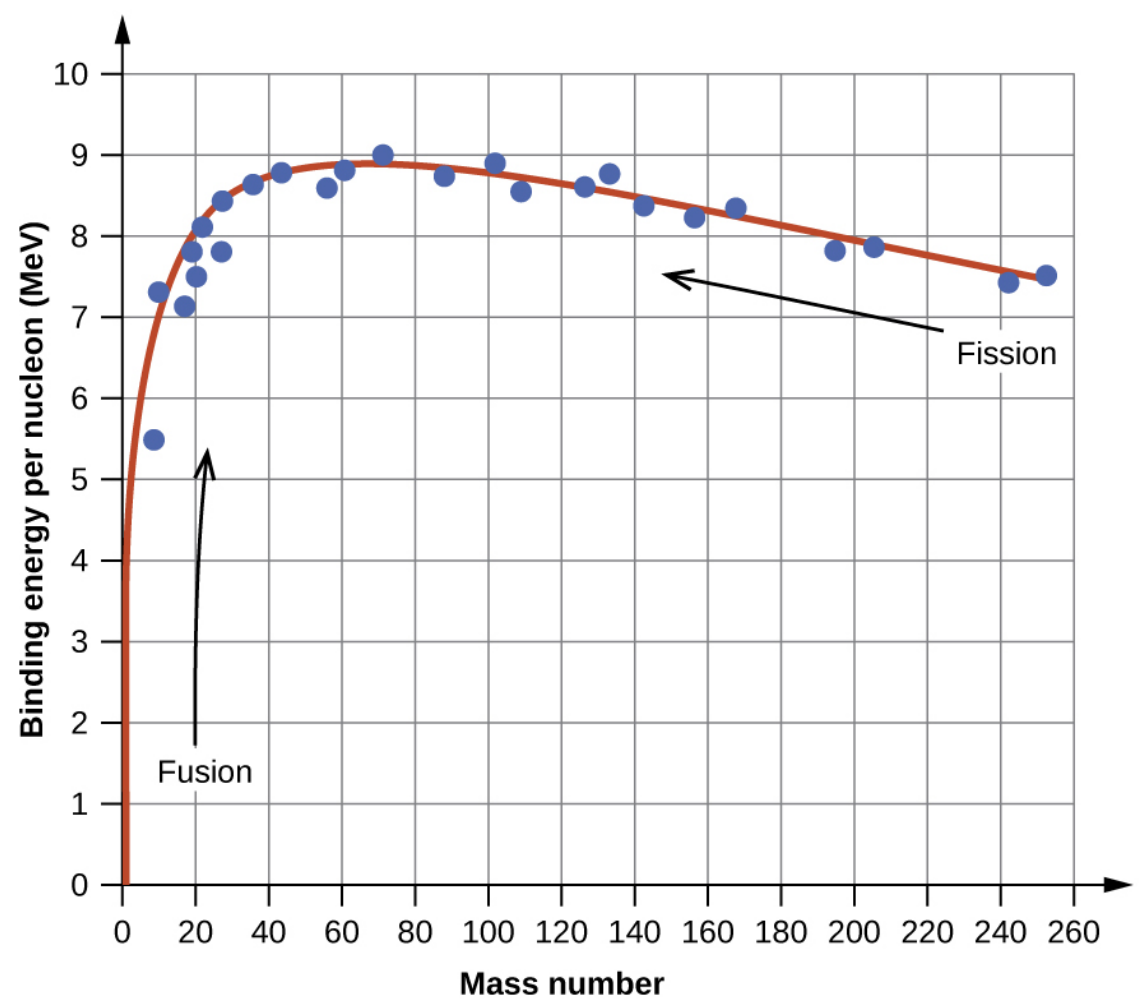

Calculation of Binding Energy per NucleonThe iron nuclide ${ }_{26}^{56}$ Fe lies near the top of the binding energy curve ((Figure)) and is one of the most stable nuclides. What is the binding energy per nucleon (in MeV) for the nuclide ${ }_{26}^{56} \mathrm{Fe}$ (atomic mass of $55.9349 \mathrm{amu})$ ?

SolutionAs in (Figure), we first determine the mass defect of the nuclide, which is the difference between the mass of 26 protons, 30 neutrons, and 26 electrons, and the observed mass of an ${ }_{26}^{56} \mathrm{Fe}$ atom:

Mass defect $=\left[\left(\begin{array}{ll}26 & 1.0073 \mathrm{amu}\end{array}\right)+\left(\begin{array}{ll}30 & 1.0087 \mathrm{amu}\end{array}\right)+\left(\begin{array}{ll}26 & 0.00055 \mathrm{amu}\end{array}\right)\right]-55.9349 \mathrm{amu}$

$=56.4651 \mathrm{amu}-55.9349 \mathrm{amu}$

$=0.5302 \mathrm{amu}$ 
We next calculate the binding energy for one nucleus from the mass defect using the mass-energy equivalence equation:

$$
\begin{aligned}
& \mathrm{E}=\mathrm{mc}^{2}=0.5302 \mathrm{amu} \frac{1.660510^{-27} \mathrm{~kg}}{1 \mathrm{amu}}\left(2.99810^{8} \mathrm{~m} / \mathrm{s}\right)^{2} \\
& =7.91310^{-11} \mathrm{kgm} / \mathrm{s}^{2} \\
& =7.91310^{-11} \mathrm{~J}
\end{aligned}
$$

We then convert the binding energy in joules per nucleus into units of $\mathrm{MeV}$ per nuclide:

$$
7.91310^{-11} \mathrm{~J} \frac{1 \mathrm{MeV}}{1.60210^{-13} \mathrm{~J}}=493.9 \mathrm{MeV}
$$

Finally, we determine the binding energy per nucleon by dividing the total nuclear binding energy by the number of nucleons in the atom:

Binding energy per nucleon $=\frac{493.9 \mathrm{MeV}}{56}=8.820 \mathrm{MeV} /$ nucleon

Note that this is almost 25\% larger than the binding energy per nucleon for ${ }_{2}^{4} \mathrm{He}$.

(Note also that this is the same process as in (Figure), but with the additional step of dividing the total nuclear binding energy by the number of nucleons.)

Check Your LearningWhat is the binding energy per nucleon in ${ }_{9}^{19} \mathrm{~F}$ (atomic mass, $18.9984 \mathrm{amu}$ )?

Answer:

7.810 MeV/nucleon

Key Concepts and Summary

An atomic nucleus consists of protons and neutrons, collectively called nucleons. Although protons repel each other, the nucleus is held tightly together by a shortrange, but very strong, force called the strong nuclear force. A nucleus has less mass than the total mass of its constituent nucleons. This "missing" mass is the mass defect, which has been converted into the binding energy 
that holds the nucleus together according to Einstein's mass-energy equivalence equation, $E=m c^{2}$. Of the many nuclides that exist, only a small number are stable. Nuclides with even numbers of protons or neutrons, or those with magic numbers of nucleons, are especially likely to be stable. These stable nuclides occupy a narrow band of stability on a graph of number of protons versus number of neutrons. The binding energy per nucleon is largest for the elements with mass numbers near 56; these are the most stable nuclei.

Key Equations

$$
\text { - } E=m c^{2}
$$

Chemistry End of Chapter Exercises

Write the following isotopes in hyphenated form (e.g., "carbon-14")
(a) ${ }_{11}^{24} \mathrm{Na}$
(b) ${ }_{13}^{29} \mathrm{Al}$
(c) ${ }_{36}^{73} \mathrm{Kr}$
(d) ${ }_{77}^{194} \mathrm{Ir}$

(a) sodium-24; (b) aluminum-29; (c) krypton-73; (d) iridium-194

Write the following isotopes in nuclide notation (e.g., $"{ }_{6}^{14} \mathrm{C} "$
(a) oxygen-14
(b) copper-70
(c) tantalum-175
(d) francium-217 
For the following isotopes that have missing information, fill in the missing information to complete the notation
(a) ${ }_{14}^{34} \mathrm{X}$
(b) ${ }^{36} \mathrm{P}$
(c) ${ }_{\mathrm{X}}^{57} \mathrm{Mn}$
(d) ${ }_{56}^{121} \mathrm{X}$

(a) ${ }_{14}^{34} \mathrm{Si}$; (b) ${ }_{15}^{36} \mathrm{P}$; (c) ${ }_{25}^{57} \mathrm{Mn}$; (d) ${ }_{56}^{121} \mathrm{Ba}$

For each of the isotopes in (Figure), determine the numbers of protons, neutrons, and electrons in a neutral atom of the isotope.

Write the nuclide notation, including charge if applicable, for atoms with the following characteristics:

(a) 25 protons, 20 neutrons, 24 electrons

(b) 45 protons, 24 neutrons, 43 electrons

(c) 53 protons, 89 neutrons, 54 electrons

(d) 97 protons, 146 neutrons, 97 electrons

(a) ${ }_{25}^{45} \mathrm{Mn}^{+1}$; (b) ${ }_{45}^{69} \mathrm{Rh}^{+2}$; (c) ${ }_{53}^{142} \mathrm{I}^{-1}$; (d) ${ }_{97}^{243} \mathrm{Bk}$

Calculate the density of the ${ }_{12}^{24} \mathrm{Mg}$ nucleus in $\mathrm{g} / \mathrm{mL}$, assuming that it has the typical nuclear diameter of 1 $10^{-13} \mathrm{~cm}$ and is spherical in shape.

What are the two principal differences between nuclear reactions and ordinary chemical changes?

Nuclear reactions usually change one type of nucleus into another; chemical changes rearrange atoms. Nuclear reactions involve much larger energies than chemical reactions and have measureable mass changes. 
The mass of the atom ${ }_{11}^{23} \mathrm{Na}$ is $22.9898 \mathrm{amu}$.

(a) Calculate its binding energy per atom in millions of electron volts.

(b) Calculate its binding energy per nucleon.

Which of the following nuclei lie within the band of stability shown in (Figure)?
(a) chlorine-37
(b) calcium- 40
(c) ${ }^{204} \mathrm{Bi}$
(d) ${ }^{56} \mathrm{Fe}$
(e) ${ }^{206} \mathrm{~Pb}$
(f) ${ }^{211} \mathrm{~Pb}$
(g) ${ }^{222} \mathrm{Rn}$
(h) carbon-14

(a), (b), (c), (d), and (e)

Which of the following nuclei lie within the band of stability shown in (Figure)?
(a) argon-40
(b) oxygen-16
(c) ${ }^{122} \mathrm{Ba}$
(d) ${ }^{58} \mathrm{Ni}$
(e) ${ }^{205} \mathrm{Tl}$
(f) ${ }^{210} \mathrm{Tl}$
(g) ${ }^{226} \mathrm{Ra}$
(h) magnesium-24 
Glossary

band of stability

(also, belt of stability, zone of stability, or valley of stability) region of graph of number of protons versus number of neutrons containing stable (nonradioactive) nuclides

\section{binding energy per nucleon}

total binding energy for the nucleus divided by the number of nucleons in the nucleus electron volt (eV)

measurement unit of nuclear binding energies, with $1 \mathrm{eV}$ equaling the amount energy due to the moving an electron across an electric potential difference of 1 volt magic number

nuclei with specific numbers of nucleons that are within the band of stability mass defect

difference between the mass of an atom and the summed mass of its constituent subatomic particles (or the mass "lost" when nucleons are brought together to form a nucleus)

\section{mass-energy equivalence equation}

Albert Einstein's relationship showing that mass and energy are equivalent

\section{nuclear binding energy}

energy lost when an atom's nucleons are bound together (or the energy needed to break a nucleus into its constituent protons 
and neutrons)

nuclear chemistry

study of the structure of atomic nuclei and

processes that change nuclear structure

nucleon

collective term for protons and neutrons in a nucleus

nuclide

nucleus of a particular isotope

radioactivity

phenomenon exhibited by an unstable

nucleon that spontaneously undergoes

change into a nucleon that is more stable; an

unstable nucleon is said to be radioactive

radioisotope

isotope that is unstable and undergoes

conversion into a different, more stable isotope

strong nuclear force

force of attraction between nucleons that holds a nucleus together 
60.

NUCLEAR EQUUATIONS

Learning Objectives

By the end of this section, you will be able to:

- Identify common particles and energies involved in nuclear reactions

- Write and balance nuclear equations

Changes of nuclei that result in changes in their atomic numbers, mass numbers, or energy states are nuclear reactions. To describe a nuclear reaction, we use an equation that identifies the nuclides involved in the reaction, their mass numbers and atomic numbers, and the other particles involved in the reaction.

Types of Particles in Nuclear Reactions

Many entities can be involved in nuclear reactions. The most common are protons, neutrons, alpha particles, beta 
particles, positrons, and gamma rays, as shown in (Figure). Protons $\left({ }_{1}^{1} \mathrm{p}\right.$, also represented by the symbol ${ }_{1}^{1} \mathrm{H}$ and neutrons $\left({ }_{0}^{1} \mathrm{n}\right)$ are the constituents of atomic nuclei, and have been described previously. Alpha particles $\left({ }_{2}^{4} \mathrm{He}\right.$, also represented by the symbol ${ }_{2}^{4}$ are high-energy helium nuclei. Beta particles $\left({ }_{-1}^{0}\right.$, also represented by the symbol ${ }_{-1}^{0} \mathrm{e}$ are high-energy electrons, and gamma rays are photons of very high-energy electromagnetic radiation. Positrons $\left({ }_{+1}^{0} \mathrm{e}\right.$, also represented by the symbol ${ }_{+1}^{0}$ are positively charged electrons ("anti-electrons"). The subscripts and superscripts are necessary for balancing nuclear equations, but are usually optional in other circumstances. For example, an alpha particle is a helium nucleus (He) with a charge of +2 and a mass number of 4 , so it is symbolized ${ }_{2}^{4} \mathrm{He}$. This works because, in general, the ion charge is not important in the balancing of nuclear equations.

Although many species are encountered in nuclear reactions, this table summarizes the names, symbols, representations, and descriptions of the most common of these. 


\begin{tabular}{|c|c|c|c|}
\hline Name & Symbol(s) & Representation & Description \\
\hline Alpha particle & ${ }_{2}^{4} \mathrm{He}$ or ${ }_{2}^{4} \alpha$ & & $\begin{array}{l}\text { (High-energy) helium nuclei consisting of two protons } \\
\text { and two neutrons }\end{array}$ \\
\hline Beta particle & ${ }_{-1}^{0} \mathrm{e}$ or ${ }_{-1}^{0} \beta$ & $\Theta$ & (High-energy) electrons \\
\hline Positron & ${ }_{+1}^{0} \mathrm{e}$ or ${ }_{+1}^{0} \beta$ & $\oplus$ & $\begin{array}{l}\text { Particles with the same mass as an electron but with } \\
1 \text { unit of positive charge }\end{array}$ \\
\hline Proton & ${ }_{1}^{1} \mathrm{H}$ or ${ }_{1}^{1} \mathrm{p}$ & & Nuclei of hydrogen atoms \\
\hline Neutron & ${ }_{0}^{1} n$ & & $\begin{array}{l}\text { Particles with a mass approximately equal to } \\
\text { that of a proton but with no charge }\end{array}$ \\
\hline Gamma ray & $\gamma$ & $\sim \sim \gamma$ & Very high-energy electromagnetic radiation \\
\hline
\end{tabular}

Note that positrons are exactly like electrons, except they have the opposite charge. They are the most common example of antimatter, particles with the same mass but the opposite state of another property (for example, charge) than ordinary matter. When antimatter encounters ordinary matter, both are annihilated and their mass is converted into energy in the form of gamma rays $(\gamma)$-and other much smaller subnuclear particles, which are beyond the scope of this chapter-according to the mass-energy equivalence equation $E=m c^{2}$, seen in the preceding section. For example, when a positron and an electron collide, both are annihilated and two gamma ray photons are created:

${ }_{-1}^{0} \mathrm{e}+{ }_{+1}^{0} \mathrm{e}+$

As seen in the chapter discussing light and electromagnetic radiation, gamma rays compose short wavelength, high-energy electromagnetic radiation and are (much) more energetic than better-known X-rays that can behave as particles in the wave-particle duality sense. Gamma rays are a type of high energy electromagnetic 
radiation produced when a nucleus undergoes a transition from a higher to a lower energy state, similar to how a photon is produced by an electronic transition from a higher to a lower energy level. Due to the much larger energy differences between nuclear energy shells, gamma rays emanating from a nucleus have energies that are typically millions of times larger than electromagnetic radiation emanating from electronic transitions.

\section{Balancing Nuclear Reactions}

A balanced chemical reaction equation reflects the fact that during a chemical reaction, bonds break and form, and atoms are rearranged, but the total numbers of atoms of each element are conserved and do not change. A balanced nuclear reaction equation indicates that there is a rearrangement during a nuclear reaction, but of nucleons (subatomic particles within the atoms' nuclei) rather than atoms. Nuclear reactions also follow conservation laws, and they are balanced in two ways:

1. The sum of the mass numbers of the reactants equals the sum of the mass numbers of the products.

2. The sum of the charges of the reactants equals the sum of the charges of the products.

If the atomic number and the mass number of all but one of the particles in a nuclear reaction are known, we can identify the particle by balancing the reaction. For instance, we could determine that ${ }_{8}^{17} \mathrm{O}$ is a product of the nuclear reaction of ${ }_{7}^{14} \mathrm{~N}$ and ${ }_{2}^{4} \mathrm{He}$ if we knew that a proton, 
${ }_{1}^{1} \mathrm{H}$, was one of the two products. (Figure) shows how we can identify a nuclide by balancing the nuclear reaction.

Balancing Equations for Nuclear ReactionsThe reaction of an a particle with magnesium- $25\left({ }_{12}^{25} \mathrm{Mg}\right)$ produces a proton and a nuclide of another element. Identify the new nuclide produced.

Solution The nuclear reaction can be written as:

$$
{ }_{12}^{25} \mathrm{Mg}+{ }_{2}^{4} \mathrm{He}{ }_{1}^{1} \mathrm{H}+{ }_{\mathrm{Z}}^{\mathrm{A}} \mathrm{X}
$$

where $A$ is the mass number and $Z$ is the atomic number of the new nuclide, $X$. Because the sum of the mass numbers of the reactants must equal the sum of the mass numbers of the products:

$25+4=\mathrm{A}+1$, or $\mathrm{A}=28$

Similarly, the charges must balance, so:

$$
12+2=\mathrm{Z}+1 \text {, and } \mathrm{Z}=13
$$

Check the periodic table: The element with nuclear charge $=$ +13 is aluminum. Thus, the product is ${ }_{13}^{28} \mathrm{Al}$.

Check Your LearningThe nuclide ${ }_{53}^{125} \mathrm{I}$ combines with an electron and produces a new nucleus and no other massive particles. What is the equation for this reaction?

\section{Answer:}

$$
{ }_{53}^{125} \mathrm{I}+{ }_{-1}^{0} \mathrm{e}^{1252} \mathrm{Te}
$$

Following are the equations of several nuclear reactions that have important roles in the history of nuclear chemistry:

- The first naturally occurring unstable element that was isolated, polonium, was discovered by the Polish scientist Marie Curie and her husband 
Pierre in 1898. It decays, emitting $\alpha$ particles: ${ }_{84}^{212} \mathrm{Po}{ }_{82}^{208} \mathrm{~Pb}+{ }_{2}^{4} \mathrm{He}$

- The first nuclide to be prepared by artificial means was an isotope of oxygen, ${ }^{17} \mathrm{O}$. It was made by Ernest Rutherford in 1919 by bombarding nitrogen atoms with $\alpha$ particles: ${ }_{7}^{14} \mathrm{~N}+{ }_{2}^{4} \mathrm{He}{ }_{8}^{17} \mathrm{O}+{ }_{1}^{1} \mathrm{H}$

- James Chadwick discovered the neutron in 1932, as a previously unknown neutral particle produced along with ${ }^{12} \mathrm{C}$ by the nuclear reaction between ${ }^{9} \mathrm{Be}$ and ${ }^{4} \mathrm{He}$ : ${ }_{4}^{9} \mathrm{Be}+{ }_{2}^{4} \mathrm{He}{ }_{6}^{12} \mathrm{C}+{ }_{0}^{1} \mathrm{n}$

- The first element to be prepared that does not occur naturally on the earth, technetium, was created by bombardment of molybdenum by deuterons (heavy hydrogen, ${ }_{1}^{2} \mathrm{H}$, by Emilio Segre and Carlo Perrier in 1937: ${ }_{1}^{2} \mathrm{H}+{ }_{42}^{97}$ Mo $2{ }_{0}^{1} \mathrm{n}+{ }_{43}^{97} \mathrm{Tc}$

- The first controlled nuclear chain reaction was carried out in a reactor at the University of Chicago in 1942. One of the many reactions involved was:

$$
{ }_{92}^{235} \mathrm{U}+{ }_{0}^{1} \mathrm{n}{ }_{35}^{87} \mathrm{Br}+{ }_{57}^{146} \mathrm{La}+3{ }_{0}^{1} \mathrm{n}
$$

Key Concepts and Summary

Nuclei can undergo reactions that change their number of protons, number of neutrons, or energy state. Many different particles can be involved in nuclear reactions. The most common are protons, neutrons, positrons (which are positively charged electrons), alpha $(\alpha)$ 
particles (which are high-energy helium nuclei), beta $(\beta)$ particles (which are high-energy electrons), and gamma $(\gamma)$ rays (which compose high-energy electromagnetic radiation). As with chemical reactions, nuclear reactions are always balanced. When a nuclear reaction occurs, the total mass (number) and the total charge remain unchanged.

Chemistry End of Chapter Exercises

Write a brief description or definition of each of the following:

(a) nucleon

(b) $\alpha$ particle

(c) $\beta$ particle

(d) positron

(e) $\gamma$ ray

(f) nuclide

(g) mass number

(h) atomic number

(a) A nucleon is any particle contained in the nucleus of the atom, so it can refer to protons and neutrons. (b) An $\alpha$ particle is one product of natural radioactivity and is the nucleus of a helium atom. (c) A $\beta$ particle is a product of natural radioactivity and is a high-speed electron. (d) A positron is a particle with the same mass as an electron but with a positive charge. (e) Gamma rays compose electromagnetic radiation of high energy and short wavelength. (f) Nuclide is a term used when referring to a single type of nucleus. (g) The mass number is the sum of the number of protons and the number of neutrons in an 
element. (h) The atomic number is the number of protons in the nucleus of an element.

Which of the various particles ( $\alpha$ particles, $\beta$ particles, and so on) that may be produced in a nuclear reaction are actually nuclei?

Complete each of the following equations by adding the missing species:
(a) ${ }_{13}^{27} \mathrm{Al}+{ }_{2}^{4} \mathrm{He} ?+{ }_{0}^{1} \mathrm{n}$
(b) ${ }_{94}^{239} \mathrm{Pu}+?{ }_{96}^{242} \mathrm{Cm}+{ }_{0}^{1} \mathrm{n}$
(c) ${ }_{7}^{14} \mathrm{~N}+{ }_{2}^{4} \mathrm{He} ?+{ }_{1}^{1} \mathrm{H}$
(d) ${ }_{92}^{235} \mathrm{U} ?+{ }_{55}^{135} \mathrm{Cs}+4{ }_{0}^{1} \mathrm{n}$

(a)

${ }_{94}^{239} \mathrm{Pu}+{ }_{2}^{4} \mathrm{He}{ }_{96}^{242} \mathrm{Cm}+{ }_{0}^{1} \mathrm{n}$;

${ }_{7}^{14} \mathrm{~N}+{ }_{2}^{4} \mathrm{He}{ }^{17} \mathrm{O}+{ }_{1}^{1} \mathrm{H}$;

${ }_{92}^{235} \mathrm{U}{ }_{37}^{96} \mathrm{Rb}+{ }_{55}^{135} \mathrm{Cs}+4{ }_{0}^{1} \mathrm{n}$

Complete each of the following equations:
(a) ${ }_{3}^{7} \mathrm{Li}+? 22_{2}^{4} \mathrm{He}$
(b) ${ }_{6}^{14} \mathrm{C}{ }_{7}^{14} \mathrm{~N}+$ ?
(c) ${ }_{13}^{27} \mathrm{Al}+{ }_{2}^{4} \mathrm{He} ?+{ }_{0}^{1} \mathrm{n}$
(d) ${ }_{96}^{250} \mathrm{Cm} ?+{ }_{38}^{98} \mathrm{Sr}+4{ }_{0}^{1} \mathrm{n}$

Write a balanced equation for each of the following nuclear reactions:

(a) the production of ${ }^{17} \mathrm{O}$ from ${ }^{14} \mathrm{~N}$ by $\alpha$ particle bombardment

(b) the production of ${ }^{14} \mathrm{C}$ from ${ }^{14} \mathrm{~N}$ by neutron bombardment

(c) the production of ${ }^{233} \mathrm{Th}$ from ${ }^{232} \mathrm{Th}$ by neutron bombardment 
(d) the production of ${ }^{239} \mathrm{U}$ from ${ }^{238} \mathrm{U}$ by ${ }_{1}^{2} \mathrm{H}$ bombardment

(a) ${ }_{7}^{14} \mathrm{~N}+{ }_{2}^{4} \mathrm{He}{ }_{8}^{17} \mathrm{O}+{ }_{1}^{1} \mathrm{H}$; (b) ${ }_{7}^{14} \mathrm{C}+{ }_{0}^{1} \mathrm{n}{ }_{6}^{14} \mathrm{C}+{ }_{1}^{1} \mathrm{H}$;

(c) ${ }_{90}^{232} \mathrm{Th}+{ }_{0}^{1} \mathrm{n}{ }_{90}^{233} \mathrm{Th}$; (d) ${ }_{92}^{238} \mathrm{U}+{ }_{1}^{2} \mathrm{H}{ }_{92}^{239} \mathrm{U}+{ }_{1}^{1} \mathrm{H}$

Technetium-99 is prepared from ${ }^{98}$ Mo. Molybdenum-98 combines with a neutron to give molybdenum-99, an unstable isotope that emits a $\beta$ particle to yield an excited form of technetium-99, represented as ${ }^{99}{ }^{\mathrm{Tc}}{ }^{*}$. This excited nucleus relaxes to the ground state, represented as ${ }^{99} \mathrm{Tc}$, by emitting a $\gamma$ ray. The ground state of ${ }^{99} \mathrm{Tc}$ then emits a $\beta$ particle. Write the equations for each of these nuclear reactions.

The mass of the atom ${ }_{9}^{19} \mathrm{~F}$ is $18.99840 \mathrm{amu}$.

(a) Calculate its binding energy per atom in millions of electron volts.

(b) Calculate its binding energy per nucleon.

(a) $148.8 \mathrm{MeV}$ per atom; (b) $7.808 \mathrm{MeV} /$ nucleon

For the reaction ${ }_{6}^{14} \mathrm{C}{ }_{7}^{14} \mathrm{~N}+$ ?, if $100.0 \mathrm{~g}$ of carbon reacts, what volume of nitrogen gas $\left(\mathrm{N}_{2}\right)$ is produced at $273 \mathrm{~K}$ and $1 \mathrm{~atm}$ ? 
Glossary

alpha particle

(a or ${ }_{2}^{4} \mathrm{He}$ or ${ }_{\mathbf{2}}^{\mathbf{4}}$ high-energy helium

nucleus; a helium atom that has lost two

electrons and contains two protons and two

neutrons

antimatter

particles with the same mass but opposite properties (such as charge) of ordinary particles

beta particle

( or ${ }_{-1}^{0}$ e or ${ }_{-1}^{0}$ ) high-energy electron

gamma ray

( $\gamma$ or $\left.\begin{array}{l}\mathbf{0} \\ \mathbf{0}\end{array}\right)$ short wavelength, high-energy

electromagnetic radiation that exhibits wave-particle duality

nuclear reaction

change to a nucleus resulting in changes in the atomic number, mass number, or energy state

positron $\left({ }_{+1}^{0}\right.$ or $\left.{ }_{+1}^{0} \mathrm{e}\right)$

antiparticle to the electron; it has identical properties to an electron, except for having the opposite (positive) charge 
61.

\section{RADIOACTIVE DECAY}

\section{Learning Objectives}

By the end of this section, you will be able to:

- Recognize common modes of radioactive decay

- Identify common particles and energies involved in nuclear decay reactions

- Write and balance nuclear decay equations

- Calculate kinetic parameters for decay processes, including half-life

- Describe common radiometric dating techniques

Following the somewhat serendipitous discovery of radioactivity by Becquerel, many prominent scientists began to investigate this new, intriguing phenomenon. Among them were Marie Curie (the first woman to win a Nobel Prize, and the only person to win two Nobel Prizes in different sciences-chemistry and physics), who was 
the first to coin the term "radioactivity," and Ernest Rutherford (of gold foil experiment fame), who investigated and named three of the most common types of radiation. During the beginning of the twentieth century, many radioactive substances were discovered, the properties of radiation were investigated and quantified, and a solid understanding of radiation and nuclear decay was developed.

The spontaneous change of an unstable nuclide into another is radioactive decay. The unstable nuclide is called the parent nuclide; the nuclide that results from the decay is known as the daughter nuclide. The daughter nuclide may be stable, or it may decay itself. The radiation produced during radioactive decay is such that the daughter nuclide lies closer to the band of stability than the parent nuclide, so the location of a nuclide relative to the band of stability can serve as a guide to the kind of decay it will undergo ((Figure)).

A nucleus of uranium-238 (the parent nuclide) undergoes $\alpha$ decay to form thorium-234 (the daughter nuclide). The alpha particle removes two protons (green) and two neutrons (gray) from the uranium-238 nucleus.

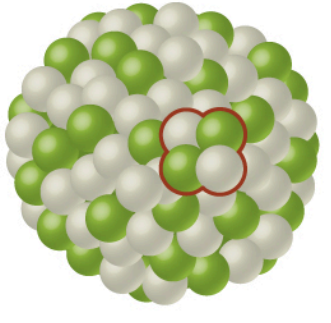

Parent nucleus uranium-238

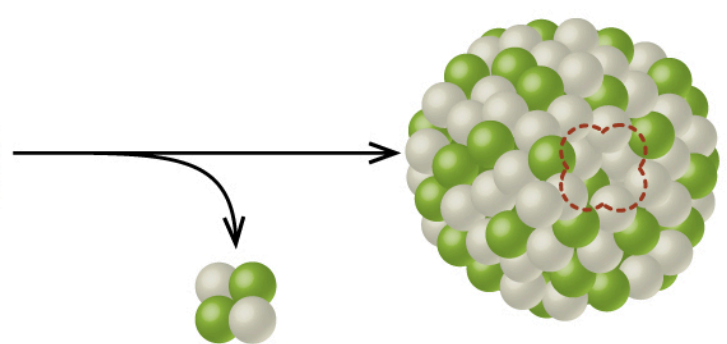

$\alpha$ particle
Daughter nucleus thorium-234

Although the radioactive decay of a nucleus is too small to see with the naked eye, we can indirectly view radioactive 
decay in an environment called a cloud chamber. Click here to learn about cloud chambers and to view an interesting Cloud Chamber Demonstration from the Jefferson Lab.

\section{Types of Radioactive Decay}

Ernest Rutherford's experiments involving the interaction of radiation with a magnetic or electric field ((Figure)) helped him determine that one type of radiation consisted of positively charged and relatively massive $\alpha$ particles; a second type was made up of negatively charged and much less massive $\beta$ particles; and a third was uncharged electromagnetic waves, $\gamma$ rays. We now know that $\alpha$ particles are high-energy helium nuclei, $\beta$ particles are high-energy electrons, and $\gamma$ radiation compose highenergy electromagnetic radiation. We classify different types of radioactive decay by the radiation produced.

Alpha particles, which are attracted to the negative plate and deflected by a relatively small amount, must be positively charged and relatively massive. Beta particles, which are attracted to the positive plate and deflected a relatively large amount, must be negatively charged and relatively light. Gamma rays, which are unaffected by the electric field, must be uncharged. 


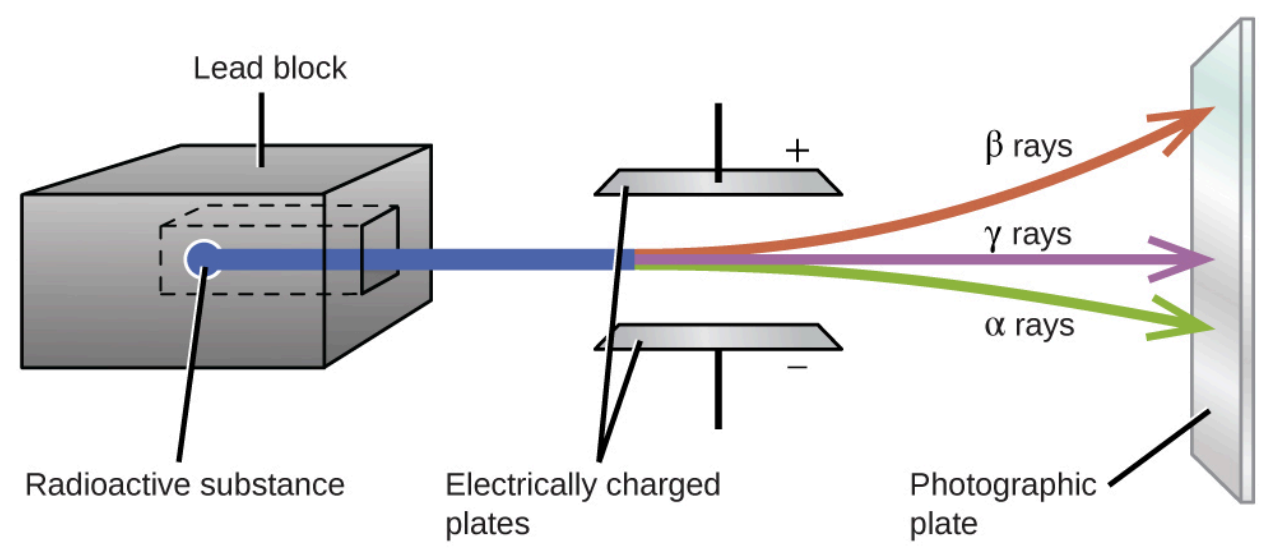

Alpha ( $\alpha$ ) decay is the emission of an $\alpha$ particle from the nucleus. For example, polonium-210 undergoes $\alpha$ decay: ${ }_{84}^{210} \mathrm{Po}{ }_{2}^{4} \mathrm{He}+{ }_{82}^{206} \mathrm{~Pb} \quad$ or $\quad{ }_{84}^{210} \mathrm{Po}{ }_{2}^{4}+{ }_{82}^{206} \mathrm{~Pb}$

Alpha decay occurs primarily in heavy nuclei (A > 200, Z $>$ 83). Because the loss of an $\alpha$ particle gives a daughter nuclide with a mass number four units smaller and an atomic number two units smaller than those of the parent nuclide, the daughter nuclide has a larger n:p ratio than the parent nuclide. If the parent nuclide undergoing $\alpha$ decay lies below the band of stability (refer to (Figure)), the daughter nuclide will lie closer to the band.

Beta ( $\beta$ ) decay is the emission of an electron from a nucleus. Iodine-131 is an example of a nuclide that undergoes $\beta$ decay:
${ }_{53}^{131} \mathrm{I}_{-1}^{0} \mathrm{e}+{ }_{54}^{131} \mathrm{Xe}$
or
${ }_{53}^{131} \mathrm{I}-{ }_{-1}^{0}+{ }_{54}^{131} \mathrm{Xe}$

Beta decay, which can be thought of as the conversion of a neutron into a proton and a $\beta$ particle, is observed in nuclides with a large $\mathrm{n}: \mathrm{p}$ ratio. The beta particle (electron) emitted is from the atomic nucleus and is not one of the electrons surrounding the nucleus. Such nuclei lie above the band of stability. Emission of an electron does not 
change the mass number of the nuclide but does increase the number of its protons and decrease the number of its neutrons. Consequently, the n:p ratio is decreased, and the daughter nuclide lies closer to the band of stability than did the parent nuclide.

Gamma emission ( $\gamma$ emission) is observed when a nuclide is formed in an excited state and then decays to its ground state with the emission of a $\gamma$ ray, a quantum of high-energy electromagnetic radiation. The presence of a nucleus in an excited state is often indicated by an asterisk $\left(^{*}\right)$. Cobalt-60 emits $\gamma$ radiation and is used in many applications including cancer treatment:

${ }_{27}^{60} \mathrm{Co} *{ }_{0}^{0}+{ }_{27}^{60} \mathrm{Co}$

There is no change in mass number or atomic number during the emission of a $\gamma$ ray unless the $\gamma$ emission accompanies one of the other modes of decay.

Positron emission $\left(\beta^{+}\right.$decay) is the emission of a positron from the nucleus. Oxygen-15 is an example of a nuclide that undergoes positron emission:
${ }_{8}^{15} \mathrm{O}+{ }_{+1}^{0} \mathrm{e}+{ }_{7}^{15} \mathrm{~N}$
or
${ }_{8}^{15} \mathrm{O}+1+{ }_{7}^{0} \mathrm{~N}$

Positron emission is observed for nuclides in which the n:p ratio is low. These nuclides lie below the band of stability. Positron decay is the conversion of a proton into a neutron with the emission of a positron. The n:p ratio increases, and the daughter nuclide lies closer to the band of stability than did the parent nuclide.

Electron capture occurs when one of the inner electrons in an atom is captured by the atom's nucleus. For example, potassium-40 undergoes electron capture:

${ }_{19}^{40} \mathrm{~K}+{ }_{-1}^{0} \mathrm{e}{ }_{18}^{40} \mathrm{Ar}$ 
Electron capture occurs when an inner shell electron combines with a proton and is converted into a neutron. The loss of an inner shell electron leaves a vacancy that will be filled by one of the outer electrons. As the outer electron drops into the vacancy, it will emit energy. In most cases, the energy emitted will be in the form of an $\mathrm{X}$-ray. Like positron emission, electron capture occurs for "proton-rich" nuclei that lie below the band of stability. Electron capture has the same effect on the nucleus as does positron emission: The atomic number is decreased by one and the mass number does not change. This increases the $n: p$ ratio, and the daughter nuclide lies closer to the band of stability than did the parent nuclide. Whether electron capture or positron emission occurs is difficult to predict. The choice is primarily due to kinetic factors, with the one requiring the smaller activation energy being the one more likely to occur.

(Figure) summarizes these types of decay, along with their equations and changes in atomic and mass numbers. This table summarizes the type, nuclear equation, representation, and any changes in the mass or atomic numbers for various types of decay. 


\begin{tabular}{|c|c|c|c|}
\hline Type & Nuclear equation & Representation & $\begin{array}{c}\text { Change in } \\
\text { mass/atomic numbers }\end{array}$ \\
\hline Alpha decay & ${ }_{Z}^{A} X \quad{ }_{2}^{4} \mathrm{He}+{ }_{\mathrm{Z}-2}^{\mathrm{A}-4} \mathrm{Y}$ & 890 & $\begin{array}{l}\text { A: decrease by } 4 \\
\text { Z: decrease by } 2\end{array}$ \\
\hline Beta decay & ${ }_{Z}^{A} X \quad{ }_{-1}^{0} e+\underset{Z+1}{A} Y$ & $\nabla$ & $\begin{array}{l}\text { A: unchanged } \\
\text { Z: increase by } 1\end{array}$ \\
\hline $\begin{array}{l}\text { Gamma } \\
\text { decay }\end{array}$ & ${ }_{0}^{0} \gamma+{ }_{Z}^{A} Y$ & $\nabla$ & $\begin{array}{l}\text { A: unchanged } \\
\text { Z: unchanged }\end{array}$ \\
\hline $\begin{array}{l}\text { Positron } \\
\text { emission }\end{array}$ & ${ }_{Z}^{A} X \quad{ }_{+1}^{0} e+Y-1$ & $\begin{array}{l}\nabla \\
\oplus\end{array}$ & $\begin{array}{l}\text { A: unchanged } \\
\text { Z: decrease by } 1\end{array}$ \\
\hline $\begin{array}{l}\text { Electron } \\
\text { capture }\end{array}$ & ${ }_{Z}^{A} X \quad{ }_{-1}^{0} e+Y-{ }_{1}^{A} Y$ & ) & $\begin{array}{l}\text { A: unchanged } \\
\text { Z: decrease by } 1\end{array}$ \\
\hline
\end{tabular}

\section{PET Scan}

Positron emission tomography (PET) scans use radiation to diagnose and track health conditions and monitor medical treatments by revealing how parts of a patient's body function ((Figure)). To perform a PET scan, a positron-emitting radioisotope is produced in a cyclotron and then attached to a substance that is used by the part of the body being investigated. This "tagged" compound, or radiotracer, is then put into the patient (injected via IV or breathed in as a gas), and how it is used by the tissue reveals how that organ or other area of the body functions. A PET scanner (a) uses radiation to provide an image of how part of a patient's body functions. The scans it produces can be used to image a healthy brain (b) or can be used for diagnosing medical conditions such as Alzheimer's disease (c). (credit a: modification of work by Jens Maus) 


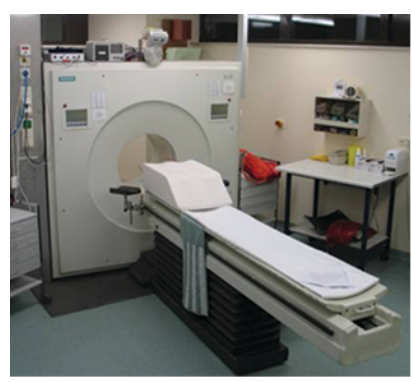

(a)

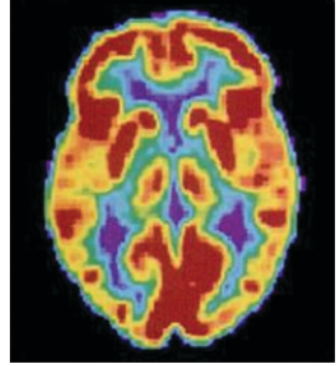

(b)

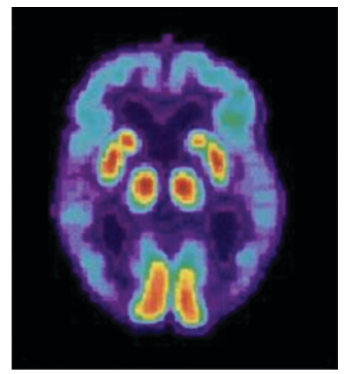

(c)

For example, $\mathrm{F}-18$ is produced by proton bombardment of ${ }^{18} \mathrm{O}\left({ }_{8}^{18} \mathrm{O}+{ }_{1}^{1} \mathrm{p}{ }_{9}^{18} \mathrm{~F}+{ }_{0}^{1} \mathrm{n}\right)$ and incorporated into a glucose analog called fludeoxyglucose (FDG). How FDG is used by the body provides critical diagnostic information; for example, since cancers use glucose differently than normal tissues, FDG can reveal cancers. The ${ }^{18} \mathrm{~F}$ emits positrons that interact with nearby electrons, producing a burst of gamma radiation. This energy is detected by the scanner and converted into a detailed, three-dimensional, color image that shows how that part of the patient's body functions. Different levels of gamma radiation produce different amounts of brightness and colors in the image, which can then be interpreted by a radiologist to reveal what is going on. PET scans can detect heart damage and heart disease, help diagnose Alzheimer's disease, indicate the part of a brain that is affected by epilepsy, reveal cancer, show what stage it is, and how much it has spread, and whether treatments are effective. Unlike magnetic resonance imaging and X-rays, which only show how something looks, the big advantage of PET scans is that they show how something functions. PET scans are now usually performed in conjunction with a computed tomography scan. 
Radioactive Decay Series

The naturally occurring radioactive isotopes of the heaviest elements fall into chains of successive disintegrations, or decays, and all the species in one chain constitute a radioactive family, or radioactive decay series. Three of these series include most of the naturally radioactive elements of the periodic table. They are the uranium series, the actinide series, and the thorium series. The neptunium series is a fourth series, which is no longer significant on the earth because of the short halflives of the species involved. Each series is characterized by a parent (first member) that has a long half-life and a series of daughter nuclides that ultimately lead to a stable end-product-that is, a nuclide on the band of stability ((Figure)). In all three series, the end-product is a stable isotope of lead. The neptunium series, previously thought to terminate with bismuth-209, terminates with thallium-205.

Uranium-238 undergoes a radioactive decay series consisting of 14 separate steps before producing stable lead-206. This series consists of eight $\alpha$ decays and $\operatorname{six} \beta$ decays. 


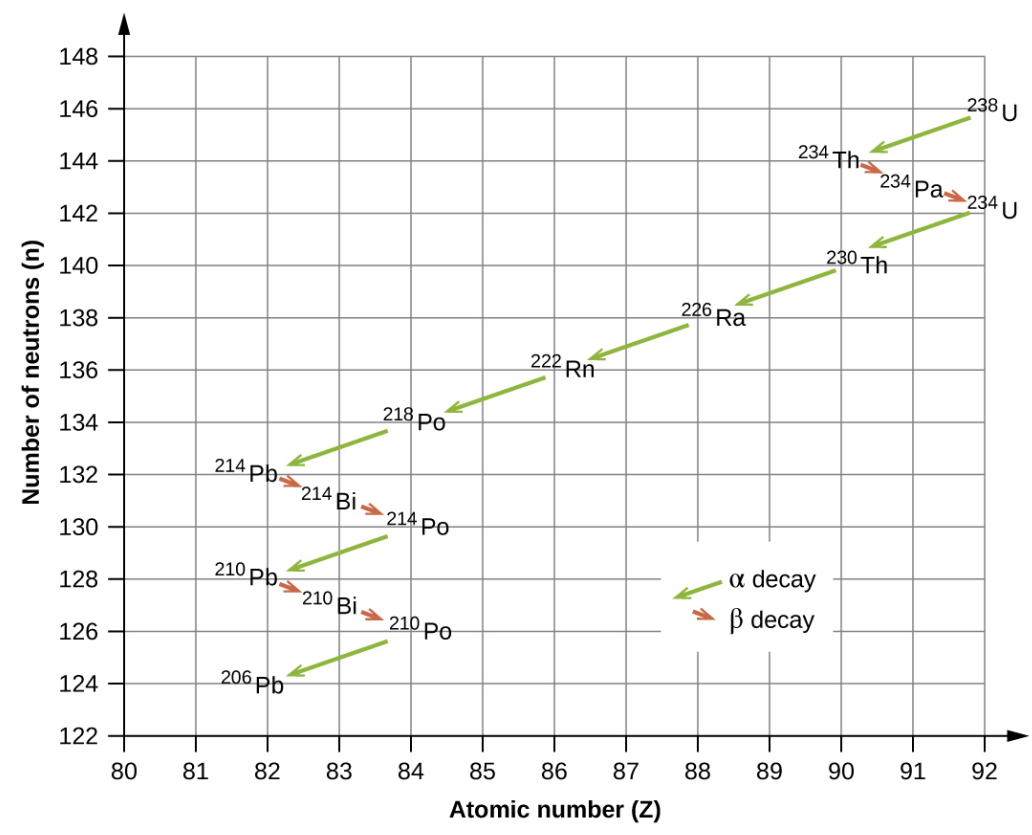

Radioactive Half-Lives

Radioactive decay follows first-order kinetics. Since firstorder reactions have already been covered in detail in the kinetics chapter, we will now apply those concepts to nuclear decay reactions. Each radioactive nuclide has a characteristic, constant half-life $\left(t_{1 / 2}\right)$, the time required for half of the atoms in a sample to decay. An isotope's half-life allows us to determine how long a sample of a useful isotope will be available, and how long a sample of an undesirable or dangerous isotope must be stored before it decays to a low-enough radiation level that is no longer a problem.

For example, cobalt-60, an isotope that emits gamma rays used to treat cancer, has a half-life of 5.27 years ((Figure)). In a given cobalt-60 source, since half of the ${ }_{27}^{60} \mathrm{Co}$ nuclei decay every 5.27 years, both the amount of material and the intensity of the radiation emitted is cut in half every 5.27 years. (Note that for a given substance, 
the intensity of radiation that it produces is directly proportional to the rate of decay of the substance and the amount of the substance.) This is as expected for a process following first-order kinetics. Thus, a cobalt-60 source that is used for cancer treatment must be replaced regularly to continue to be effective.

For cobalt-60, which has a half-life of 5.27 years, 50\%

remains after 5.27 years (one half-life), 25\% remains after 10.54 years (two half-lives), $12.5 \%$ remains after 15.81 years (three half-lives), and so on.

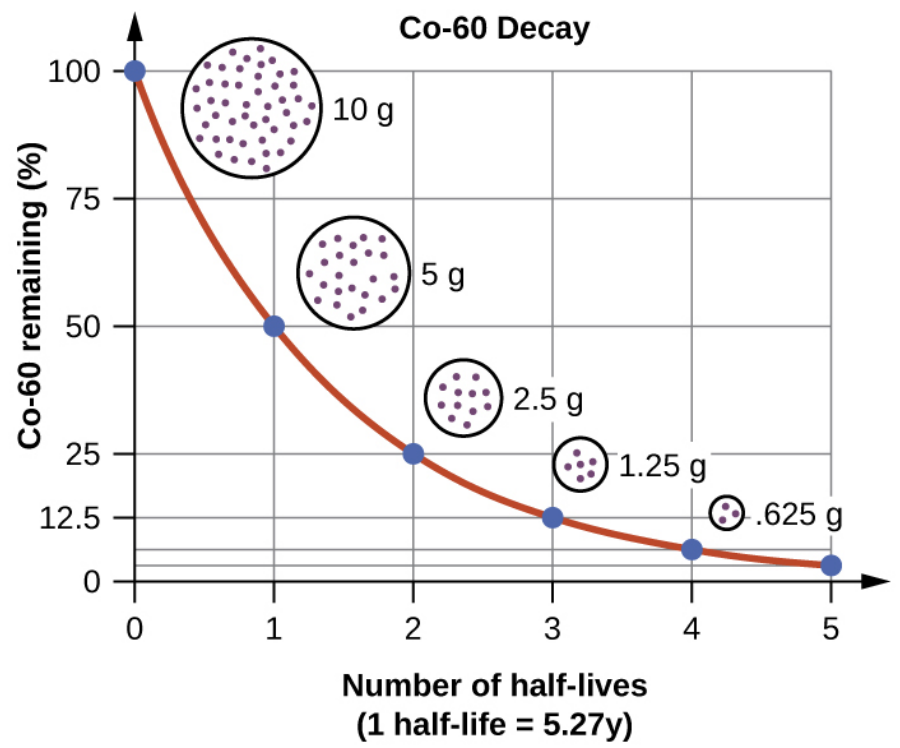

Since nuclear decay follows first-order kinetics, we can adapt the mathematical relationships used for first-order chemical reactions. We generally substitute the number of nuclei, $N$, for the concentration. If the rate is stated in nuclear decays per second, we refer to it as the activity of the radioactive sample. The rate for radioactive decay is:

decay rate $=\lambda N$ with $\lambda=$ the decay constant for the particular radioisotope

The decay constant, $\lambda$, which is the same as a rate 
constant discussed in the kinetics chapter. It is possible to express the decay constant in terms of the half-life, $t_{1 / 2}$ :
$\lambda=\frac{\ln 2}{t_{1 / 2}}=\frac{0.693}{t_{1 / 2}}$
or
$t_{1 / 2}=\frac{\ln 2}{\lambda}=\frac{0.693}{\lambda}$

The first-order equations relating amount, $N$, and time are:

$$
N_{t}=N_{0} e^{-k t} \quad \text { or } \quad t=-\frac{1}{\lambda} \ln \left(\frac{N_{t}}{N_{0}}\right)
$$

where $N_{0}$ is the initial number of nuclei or moles of the isotope, and $N_{\mathrm{t}}$ is the number of nuclei/moles remaining at time $t$. (Figure) applies these calculations to find the rates of radioactive decay for specific nuclides.

Rates of Radioactive Decay ${ }_{27}^{60} \mathrm{Co}$ decays with a half-life of 5.27 years to produce ${ }_{28}^{60} \mathrm{Ni}$.

(a) What is the decay constant for the radioactive disintegration of cobalt-60?

(b) Calculate the fraction of a sample of the ${ }_{27}^{60} \mathrm{Co}$ isotope that will remain after 15 years.

(c) How long does it take for a sample of ${ }_{27}^{60} \mathrm{Co}$ to disintegrate to the extent that only $2.0 \%$ of the original amount remains?

Solution (a) The value of the rate constant is given by:

$$
\lambda=\frac{\ln 2}{t_{1 / 2}}=\frac{0.693}{5.27 \mathrm{y}}=0.132 \mathrm{y}^{-1}
$$

(b) The fraction of ${ }_{27}^{60} \mathrm{Co}$ that is left after time $t$ is given by $\frac{N_{t}}{N_{0}}$. Rearranging the first-order relationship $N_{\mathrm{t}}=N_{0} e^{-\lambda t}$ to solve for this ratio yields:

$$
\frac{N_{t}}{N_{0}}=e^{-\lambda t}=e^{-(0.132 / \mathrm{y})(15.0 / \mathrm{y})}=0.138
$$

The fraction of ${ }_{27}^{60} \mathrm{Co}$ that will remain after 15.0 years is 
0.138 . Or put another way, $13.8 \%$ of the ${ }_{27}^{60}$ Co originally present will remain after 15 years.

(c) $2.00 \%$ of the original amount of ${ }_{27}^{60} \mathrm{Co}$ is equal to 0.0200 $N_{0}$. Substituting this into the equation for time for first-order kinetics, we have:

$$
t=-\frac{1}{\lambda} \ln \left(\frac{N_{t}}{N_{0}}\right)=-\frac{1}{0.132 \mathrm{y}^{-1}} \ln \left(\frac{0.0200 N_{0}}{N_{0}}\right)=29.6 \mathrm{y}
$$

Check Your LearningRadon-222, ${ }_{86}^{222} \mathrm{Rn}$, has a half-life of

3.823 days. How long will it take a sample of radon-222 with a mass of $0.750 \mathrm{~g}$ to decay into other elements, leaving only 0.100 g of radon-222?

Answer:

11.1 days

Because each nuclide has a specific number of nucleons, a particular balance of repulsion and attraction, and its own degree of stability, the half-lives of radioactive nuclides vary widely. For example: the half-life of ${ }_{83}^{209} \mathrm{Bi}$ is $1.910^{19}$ years; ${ }_{94}^{239} \mathrm{Ra}$ is 24,000 years; ${ }_{86}^{222} \mathrm{Rn}$ is 3.82 days; and element-111 ( $\mathrm{Rg}$ for roentgenium) is $1.510^{-3}$ seconds. The half-lives of a number of radioactive isotopes important to medicine are shown in (Figure), and others are listed in Appendix M. 
Half-lives of Radioactive Isotopes Important to Medicine

\begin{tabular}{|c|c|c|c|}
\hline Type $^{1}$ & $\begin{array}{l}\text { Decay } \\
\text { Mode }\end{array}$ & Half-Life & Uses \\
\hline $\mathrm{F}-18$ & $\beta^{+}$decay & $\begin{array}{l}110 . \\
\text { minutes }\end{array}$ & PET scans \\
\hline Co-60 & $\begin{array}{l}\beta \text { decay, } \gamma \\
\text { decay }\end{array}$ & $\begin{array}{l}5.27 \\
\text { years }\end{array}$ & cancer treatment \\
\hline Tc-99m & $\gamma$ decay & $\begin{array}{l}8.01 \\
\text { hours }\end{array}$ & scans of brain, lung, heart, bone \\
\hline $\mathrm{I}-131$ & $\beta$ decay & 8.02 days & thyroid scans and treatment \\
\hline Tl-201 & $\begin{array}{l}\text { electron } \\
\text { capture }\end{array}$ & 73 hours & $\begin{array}{l}\text { heart and arteries scans; cardiac } \\
\text { stress tests }\end{array}$ \\
\hline
\end{tabular}

\section{Radiometric Dating}

Several radioisotopes have half-lives and other properties that make them useful for purposes of "dating" the origin of objects such as archaeological artifacts, formerly living organisms, or geological formations. This process is radiometric dating and has been responsible for many breakthrough scientific discoveries about the geological history of the earth, the evolution of life, and the history of human civilization. We will explore some of the most common types of radioactive dating and how the particular isotopes work for each type.

Radioactive Dating Using Carbon-14

The radioactivity of carbon-14 provides a method for dating objects that were a part of a living organism. This method of radiometric dating, which is also called radiocarbon dating or carbon-14 dating, is accurate for dating carbon-containing substances that are up to about 
30,000 years old, and can provide reasonably accurate dates up to a maximum of about 50,000 years old.

Naturally occurring carbon consists of three isotopes: ${ }_{6}^{12} \mathrm{C}$, which constitutes about $99 \%$ of the carbon on earth; ${ }_{6}^{13} \mathrm{C}$, about $1 \%$ of the total; and trace amounts of ${ }_{6}^{14} \mathrm{C}$. Carbon-14 forms in the upper atmosphere by the reaction of nitrogen atoms with neutrons from cosmic rays in space:

${ }_{7}^{14} \mathrm{~N}+{ }_{0}^{1} \mathrm{n}{ }_{6}^{14} \mathrm{C}+{ }_{1}^{1} \mathrm{H}$

All isotopes of carbon react with oxygen to produce $\mathrm{CO}_{2}$ molecules. The ratio of ${ }_{6}^{14} \mathrm{CO}_{2}$ to ${ }_{6}^{12} \mathrm{CO}_{2}$ depends on the ratio of ${ }_{6}^{14} \mathrm{CO}$ to ${ }_{6}^{12} \mathrm{CO}$ in the atmosphere. The natural abundance of ${ }_{6}^{14} \mathrm{CO}$ in the atmosphere is approximately 1 part per trillion; until recently, this has generally been constant over time, as seen is gas samples found trapped in ice. The incorporation of ${ }_{6}^{14} \mathrm{C}_{6}^{14} \mathrm{CO}_{2}$ and ${ }_{6}^{12} \mathrm{CO}_{2}$ into plants is a regular part of the photosynthesis process, which means that the ${ }_{6}^{14} \mathrm{C}:{ }_{6}^{12} \mathrm{C}$ ratio found in a living plant is the same as the ${ }_{6}^{14} \mathrm{C}:{ }_{6}^{12} \mathrm{C}$ ratio in the atmosphere. But when the plant dies, it no longer traps carbon through photosynthesis. Because ${ }_{6}^{12} \mathrm{C}$ is a stable isotope and does not undergo radioactive decay, its concentration in the plant does not change. However, carbon-14 decays by $\beta$ emission with a half-life of 5730 years:

${ }_{6}^{14} \mathrm{C}{ }_{7}^{12} \mathrm{~N}+{ }_{-1}^{0} \mathrm{e}$

Thus, the ${ }_{6}^{14} \mathrm{C}:{ }_{6}^{12} \mathrm{C}$ ratio gradually decreases after the plant dies. The decrease in the ratio with time provides a measure of the time that has elapsed since the death of 
the plant (or other organism that ate the plant). (Figure) visually depicts this process.

Along with stable carbon-12, radioactive carbon-14 is taken in by plants and animals, and remains at a constant level within them while they are alive. After death, the $\mathrm{C}-14$ decays and the $\mathrm{C}-14: \mathrm{C}-12$ ratio in the remains decreases. Comparing this ratio to the $\mathrm{C}-14: \mathrm{C}-12$ ratio in living organisms allows us to determine how long ago the organism lived (and died).

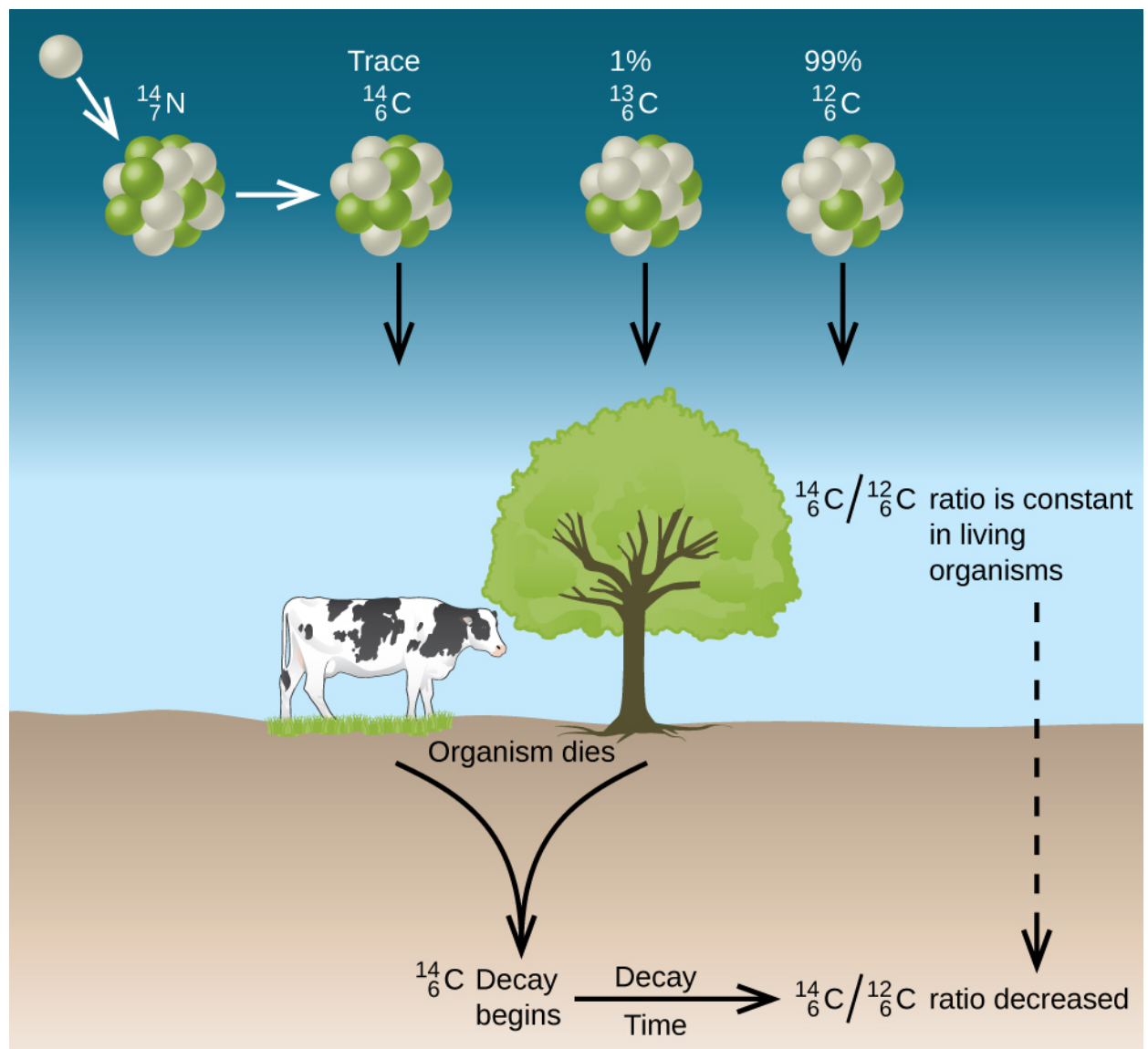

For example, with the half-life of ${ }_{6}^{14} \mathrm{C}$ being 5730 years, if the ${ }_{6}^{14} \mathrm{C}:{ }_{6}^{12} \mathrm{C}$ ratio in a wooden object found in an archaeological dig is half what it is in a living tree, this indicates that the wooden object is 5730 years old. Highly 
accurate determinations of ${ }_{6}^{14} \mathrm{C}:{ }_{6}^{12} \mathrm{C}$ ratios can be obtained from very small samples (as little as a milligram) by the use of a mass spectrometer.

Visit this website to perform simulations of radiometric dating.

Radiocarbon Dating A tiny piece of paper (produced from formerly living plant matter) taken from the Dead Sea Scrolls has an activity of 10.8 disintegrations per minute per gram of carbon. If the initial C-14 activity was 13.6 disintegrations/ $\mathrm{min} / \mathrm{g}$ of C, estimate the age of the Dead Sea Scrolls.

Solution The rate of decay (number of disintegrations/ minute/gram of carbon) is proportional to the amount of radioactive $\mathrm{C}-14$ left in the paper, so we can substitute the rates for the amounts, $N$, in the relationship:

$$
t=-\frac{1}{\lambda} \ln \left(\frac{N_{t}}{N_{0}}\right) t=-\frac{1}{\lambda} \ln \left(\frac{\text { Rate }_{t}}{\text { Rate }_{0}}\right)
$$

where the subscript 0 represents the time when the plants were cut to make the paper, and the subscript $t$ represents the current time.

The decay constant can be determined from the half-life of C-14, 5730 years:

$$
\lambda=\frac{\ln 2}{t_{1 / 2}}=\frac{0.693}{5730 \mathrm{y}}=1.2110^{-4} \mathrm{y}^{-1}
$$

Substituting and solving, we have:

$$
t=-\frac{1}{\lambda} \ln \left(\frac{\text { Rate }_{t}}{\text { Rate }_{0}}\right)=-\frac{1}{1.2110^{-4} \mathrm{y}^{-1}} \ln \left(\frac{10.8 \mathrm{dis} / \mathrm{min} / \mathrm{g} \mathrm{C}}{13.6 \mathrm{dis} / \mathrm{min} / \mathrm{g} \mathrm{C}}\right)=1910 \mathrm{y}
$$

Therefore, the Dead Sea Scrolls are approximately 1900 years old ((Figure)).

Carbon-14 dating has shown that these pages from the Dead Sea Scrolls were written or copied on paper made from plants that died between $100 \mathrm{BC}$ and $\mathrm{AD} 50$. 


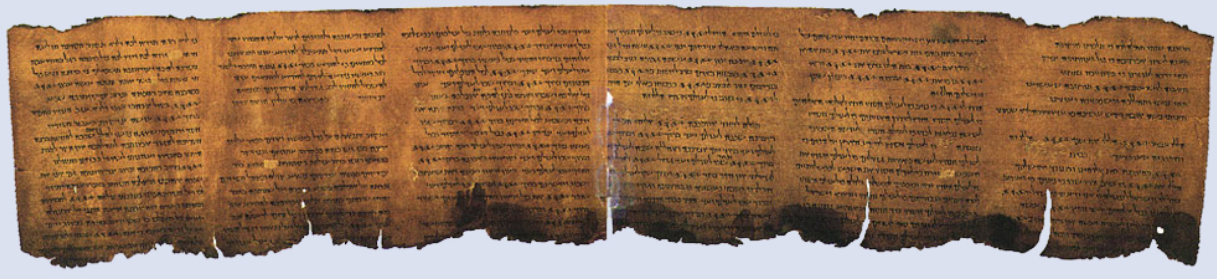

Check Your Learning More accurate dates of the reigns of ancient Egyptian pharaohs have been determined recently using plants that were preserved in their tombs. Samples of seeds and plant matter from King Tutankhamun's tomb have a C-14 decay rate of 9.07 disintegrations $/ \mathrm{min} / \mathrm{g}$ of C. How long ago did King Tut's reign come to an end?

Answer:

about 3350 years ago, or approximately 1340 BC

There have been some significant, well-documented changes to the ${ }_{6}^{14} \mathrm{C}:{ }_{6}^{12} \mathrm{C}$ ratio. The accuracy of a straightforward application of this technique depends on the ${ }_{6}^{14} \mathrm{C}:{ }_{6}^{12} \mathrm{C}$ ratio in a living plant being the same now as it was in an earlier era, but this is not always valid. Due to the increasing accumulation of $\mathrm{CO}_{2}$ molecules (largely ${ }_{6}^{12} \mathrm{CO}_{2}$ in the atmosphere caused by combustion of fossil fuels (in which essentially all of the ${ }_{6}^{14} \mathrm{C}$ has decayed), the ratio of ${ }_{6}^{14} \mathrm{C}:{ }_{6}^{12} \mathrm{C}$ in the atmosphere may be changing. This manmade increase in ${ }_{6}^{12} \mathrm{CO}_{2}$ in the atmosphere causes the ${ }_{6}^{14} \mathrm{C}:{ }_{6}^{12} \mathrm{C}$ ratio to decrease, and this in turn affects the ratio in currently living organisms on the earth. Fortunately, however, we can use other data, such as tree dating via examination of annual growth rings, to calculate correction factors. With these correction factors, accurate dates can be determined. In general, radioactive dating only works for about 10 half-lives; therefore, the limit for carbon-14 dating is about 57,000 years. 
Radioactive Dating Using Nuclides Other than Carbon-14

Radioactive dating can also use other radioactive nuclides with longer half-lives to date older events. For example, uranium-238 (which decays in a series of steps into lead-206) can be used for establishing the age of rocks (and the approximate age of the oldest rocks on earth). Since U-238 has a half-life of 4.5 billion years, it takes that amount of time for half of the original U-238 to decay into $\mathrm{Pb}$-206. In a sample of rock that does not contain appreciable amounts of $\mathrm{Pb}-208$, the most abundant isotope of lead, we can assume that lead was not present when the rock was formed. Therefore, by measuring and analyzing the ratio of $\mathrm{U}-238: \mathrm{Pb}-206$, we can determine the age of the rock. This assumes that all of the lead-206 present came from the decay of uranium-238. If there is additional lead-206 present, which is indicated by the presence of other lead isotopes in the sample, it is necessary to make an adjustment. Potassium-argon dating uses a similar method. K-40 decays by positron emission and electron capture to form Ar-40 with a halflife of 1.25 billion years. If a rock sample is crushed and the amount of Ar-40 gas that escapes is measured, determination of the Ar- $40: \mathrm{K}-40$ ratio yields the age of the rock. Other methods, such as rubidium-strontium dating ( $\mathrm{Rb}-87$ decays into $\mathrm{Sr}-87$ with a half-life of 48.8 billion years), operate on the same principle. To estimate the lower limit for the earth's age, scientists determine the age of various rocks and minerals, making the assumption that the earth is older than the oldest rocks and minerals in its crust. As of 2014, the oldest known rocks on earth 
are the Jack Hills zircons from Australia, found by uranium-lead dating to be almost 4.4 billion years old.

Radioactive Dating of Rocks An igneous rock contains 9.58 $10^{-5} \mathrm{~g}$ of $\mathrm{U}-238$ and $2.5110^{-5} \mathrm{~g}$ of $\mathrm{Pb}-206$, and much, much smaller amounts of $\mathrm{Pb}-208$. Determine the approximate time at which the rock formed.

Solution The sample of rock contains very little $\mathrm{Pb}-208$, the most common isotope of lead, so we can safely assume that all the $\mathrm{Pb}-206$ in the rock was produced by the radioactive decay of U-238. When the rock formed, it contained all of the $\mathrm{U}-238$ currently in it, plus some $\mathrm{U}-238$ that has since undergone radioactive decay.

The amount of U-238 currently in the rock is:

$$
9.5810^{-5} \overline{) \mathrm{g} \mathrm{U}}\left(\frac{1 \mathrm{~mol} \mathrm{U}}{238 \overline{\operatorname{gg~}}}\right)=4.0310^{-7} \mathrm{~mol} \mathrm{U}
$$

Because when one mole of $\mathrm{U}-238$ decays, it produces one mole of $\mathrm{Pb}-206$, the amount of $\mathrm{U}-238$ that has undergone radioactive decay since the rock was formed is:

$$
2.5110^{-5} \overline{\mathrm{g} \mathrm{Pb}}\left(\frac{1 \overline{\mathrm{mol} \mathrm{Pb}}}{206 \overline{\overline{\mathrm{g} \mathrm{Pb}}}}\right)\left(\frac{1 \mathrm{~mol} \mathrm{U}}{1 \overline{\operatorname{mol~Pb}}}\right)=1.2210^{-7} \mathrm{~mol} \mathrm{U}
$$

The total amount of $\mathrm{U}-238$ originally present in the rock is therefore:

$$
4.0310^{-7} \mathrm{~mol}+1.2210^{-7} \mathrm{~mol}=5.2510^{-7} \mathrm{~mol} \mathrm{U}
$$

The amount of time that has passed since the formation of the rock is given by:

$$
t=-\frac{1}{\lambda} \ln \left(\frac{N_{t}}{N_{0}}\right)
$$

with $N_{0}$ representing the original amount of U-238 and $N_{t}$ representing the present amount of $\mathrm{U}-238$.

$\mathrm{U}-238$ decays into $\mathrm{Pb}-206$ with a half-life of $4.510^{9} \mathrm{y}$, so the decay constant $\lambda$ is: 


$$
\lambda=\frac{\ln 2}{t_{1 / 2}}=\frac{0.693}{4.510^{9} \mathrm{y}}=1.5410^{-10} \mathrm{y}^{-1}
$$

Substituting and solving, we have:

$$
t=-\frac{1}{1.5410^{-10} \mathrm{y}^{-1}} \ln \left(\frac{4.0310^{-7}}{5.2510^{-7} \overline{\mathrm{mol} \mathrm{U}}}\right)=1.710^{9} \mathrm{y}
$$

Therefore, the rock is approximately 1.7 billion years old.

Check Your Learning A sample of rock contains $6.1410^{-4} \mathrm{~g}$ of $\mathrm{Rb}-87$ and $3.5110^{-5} \mathrm{~g}$ of Sr-87. Calculate the age of the rock. (The half-life of the $\beta$ decay of $\mathrm{Rb}-87$ is $4.710^{10} \mathrm{y}$.)

Answer:

$3.710^{9} \mathrm{y}$

Key Concepts and Summary

Nuclei that have unstable n:p ratios undergo spontaneous radioactive decay. The most common types of radioactivity are $\alpha$ decay, $\beta$ decay, $\gamma$ emission, positron emission, and electron capture. Nuclear reactions also often involve $\gamma$ rays, and some nuclei decay by electron capture. Each of these modes of decay leads to the formation of a new nucleus with a more stable n:p ratio. Some substances undergo radioactive decay series, proceeding through multiple decays before ending in a stable isotope. All nuclear decay processes follow firstorder kinetics, and each radioisotope has its own characteristic half-life, the time that is required for half of its atoms to decay. Because of the large differences in stability among nuclides, there is a very wide range of half-lives of radioactive substances. Many of these substances have found useful applications in medical diagnosis and treatment, determining the age of archaeological and geological objects, and more. 
Key Equations

- decay rate $=\lambda N$

- $t_{1 / 2}=\frac{\ln 2}{\lambda}=\frac{0.693}{\lambda}$

Chemistry End of Chapter Exercises

What are the types of radiation emitted by the nuclei of radioactive elements?

$\alpha$ (helium nuclei), $\beta$ (electrons), $\beta^{+}$(positrons), and $\eta$ (neutrons) may be emitted from a radioactive element, all of which are particles; $\gamma$ rays also may be emitted.

What changes occur to the atomic number and mass of a nucleus during each of the following decay scenarios?

(a) an $\alpha$ particle is emitted

(b) a $\beta$ particle is emitted

(c) $\gamma$ radiation is emitted

(d) a positron is emitted

(e) an electron is captured

What is the change in the nucleus that results from the following decay scenarios?

(a) emission of a $\beta$ particle

(b) emission of a $\beta^{+}$particle

(c) capture of an electron

(a) conversion of a neutron to a proton: ${ }_{0}^{1} \mathrm{n}{ }_{1}^{1} \mathrm{p}+{ }_{+1}^{0} \mathrm{e}$;

(b) conversion of a proton to a neutron; the positron has the same mass as an electron and the same magnitude of positive charge as the electron has negative charge; when the n:p ratio of a nucleus is too low, a proton is 
converted into a neutron with the emission of a positron: ${ }_{1}^{1} \mathrm{p}{ }_{0}^{1} \mathrm{n}+{ }_{+1}^{0} \mathrm{e}$; (c) In a proton-rich nucleus, an inner atomic electron can be absorbed. In simplest form, this changes a proton into a neutron: ${ }_{1}^{1} \mathrm{p}+{ }_{-1}^{0} \mathrm{e}{ }_{0}^{1} \mathrm{p}$

Many nuclides with atomic numbers greater than 83 decay by processes such as electron emission. Explain the observation that the emissions from these unstable nuclides also normally include $\alpha$ particles.

Why is electron capture accompanied by the emission of an X-ray?

The electron pulled into the nucleus was most likely found in the $1 s$ orbital. As an electron falls from a higher energy level to replace it, the difference in the energy of the replacement electron in its two energy levels is given off as an X-ray.

Explain, in terms of (Figure), how unstable heavy nuclides (atomic number > 83) may decompose to form nuclides of greater stability (a) if they are below the band of stability and (b) if they are above the band of stability.

Which of the following nuclei is most likely to decay by positron emission? Explain your choice.

(a) chromium-53

(b) manganese-51

(c) iron-59

Manganese- 51 is most likely to decay by positron emission. The n:p ratio for $\mathrm{Cr}-53$ is $\frac{29}{24}=1.21$; for $\mathrm{Mn}-51$, it is $\frac{26}{25}=1.04$; for Fe-59, it is $\frac{33}{26}=1.27$. Positron decay occurs 
when the $n: p$ ratio is low. $\mathrm{Mn}-51$ has the lowest $\mathrm{n}: \mathrm{p}$ ratio and therefore is most likely to decay by positron emission. Besides, ${ }_{24}^{53} \mathrm{Cr}$ is a stable isotope, and ${ }_{26}^{59} \mathrm{Fe}$ decays by beta emission.

The following nuclei do not lie in the band of stability. How would they be expected to decay? Explain your answer.
(a) ${ }_{15}^{34} \mathrm{P}$
(b) ${ }_{92}^{239} \mathrm{U}$
(c) ${ }_{20}^{38} \mathrm{Ca}$
(d) ${ }_{1}^{3} \mathrm{H}$
(e) ${ }_{94}^{245} \mathrm{Pu}$

The following nuclei do not lie in the band of stability. How would they be expected to decay?
(a) ${ }_{15}^{28} \mathrm{P}$
(b) ${ }_{92}^{235} \mathrm{U}$
(c) ${ }_{20}^{37} \mathrm{Ca}$
(d) ${ }_{3}^{9} \mathrm{Li}$
(e) ${ }_{96}^{245} \mathrm{Cm}$

(a) $\beta$ decay; (b) $\alpha$ decay; (c) positron emission; (d) $\beta$ decay;

(e) $\alpha$ decay

Predict by what mode(s) of spontaneous radioactive decay each of the following unstable isotopes might proceed:
(a) ${ }_{2}^{6} \mathrm{He}$
(b) ${ }_{30}^{60} \mathrm{Zn}$
(c) ${ }_{91}^{235} \mathrm{~Pa}$
(d) ${ }_{94}^{241} \mathrm{~Np}$
(e) ${ }^{18} \mathrm{~F}$ 
(f) ${ }^{129} \mathrm{Ba}$

(g) ${ }^{237} \mathrm{Pu}$

Write a nuclear reaction for each step in the formation of ${ }_{84}^{218} \mathrm{Po}$ from ${ }_{98}^{238} \mathrm{U}$, which proceeds by a series of decay reactions involving the step-wise emission of $\alpha, \beta, \beta, \alpha, \alpha$, $\alpha$ particles, in that order.

$$
\begin{aligned}
& { }_{92}^{238} \mathrm{U}{ }_{90}^{234} \mathrm{Th}+{ }_{2}^{4} \mathrm{He} ;{ }_{90}^{234} \mathrm{Th}{ }_{91}^{234} \mathrm{~Pa}+{ }_{-1}^{0} \mathrm{e} ; \\
& { }_{91}^{234} \mathrm{~Pa}{ }_{92}^{234} \mathrm{U}+{ }_{-1} \mathrm{e} ;{ }_{92}^{234} \mathrm{U}{ }_{90}^{230} \mathrm{Th}+{ }_{2}^{4} \mathrm{He} \\
& { }_{930} \mathrm{Th}{ }_{90}^{226} \mathrm{Ra}+{ }_{2}^{4} \mathrm{He}_{82}^{226} \mathrm{Ra}{ }_{88}^{222} \mathrm{Rn}+{ }_{2}^{4} \mathrm{He} ; \\
& { }_{86}^{22} \mathrm{Rn}{ }_{84}^{218} \mathrm{Po}+{ }_{2}^{4} \mathrm{He}
\end{aligned}
$$

Write a nuclear reaction for each step in the formation of ${ }_{82}^{208} \mathrm{~Pb}$ from ${ }_{90}^{228} \mathrm{Th}$, which proceeds by a series of decay reactions involving the step-wise emission of $\alpha, \alpha, \alpha, \alpha, \beta$, $\beta$, $\alpha$ particles, in that order.

Define the term half-life and illustrate it with an example.

Half-life is the time required for half the atoms in a sample to decay. Example (answers may vary): For C-14, the halflife is 5770 years. A 10 -g sample of $\mathrm{C}-14$ would contain 5 $\mathrm{g}$ of $\mathrm{C}-14$ after 5770 years; a 0.20 -g sample of $\mathrm{C}-14$ would contain $0.10 \mathrm{~g}$ after 5770 years.

A $1.0010^{-6}$-g sample of nobelium, ${ }_{102}^{254} \mathrm{No}$, has a half-life of 55 seconds after it is formed. What is the percentage of ${ }_{102}^{254}$ No remaining at the following times?

(a) $5.0 \mathrm{~min}$ after it forms

(b) $1.0 \mathrm{~h}$ after it forms

${ }^{239} \mathrm{Pu}$ is a nuclear waste byproduct with a half-life of 
$24,000 \mathrm{y}$. What fraction of the ${ }^{239} \mathrm{Pu}$ present today will be present in $1000 \mathrm{y}$ ?

$$
\left(\frac{1}{2}\right)^{0.04}=0.973 \text { or } 97.3 \%
$$

The isotope ${ }^{208} \mathrm{Tl}$ undergoes $\beta$ decay with a half-life of 3.1 $\min$.

(a) What isotope is produced by the decay?

(b) How long will it take for $99.0 \%$ of a sample of pure ${ }^{208}$ Tl to decay?

(c) What percentage of a sample of pure ${ }^{208} \mathrm{Tl}$ remains un-decayed after $1.0 \mathrm{~h}$ ?

If $1.000 \mathrm{~g}$ of ${ }_{88}^{226} \mathrm{Ra}$ produces $0.0001 \mathrm{~mL}$ of the gas ${ }_{86}^{222} \mathrm{Rn}$ at STP (standard temperature and pressure) in $24 \mathrm{~h}$, what is the half-life of ${ }^{226} \mathrm{Ra}$ in years?

\section{$210^{3} \mathrm{y}$}

The isotope ${ }_{38}^{90} \mathrm{Sr}$ is one of the extremely hazardous species in the residues from nuclear power generation. The strontium in a $0.500 \mathrm{~g}$ sample diminishes to $0.393 \mathrm{~g}$ in 10.0 y. Calculate the half-life.

Technetium-99 is often used for assessing heart, liver, and lung damage because certain technetium compounds are absorbed by damaged tissues. It has a half-life of $6.0 \mathrm{~h}$. Calculate the rate constant for the decay of ${ }_{43}^{99} \mathrm{Tc}$.

$0.12 \mathrm{~h}^{-1}$

What is the age of mummified primate skin that contains $8.25 \%$ of the original quantity of ${ }^{14} \mathrm{C}$ ? 
A sample of rock was found to contain $8.23 \mathrm{mg}$ of rubidium-87 and $0.47 \mathrm{mg}$ of strontium-87.

(a) Calculate the age of the rock if the half-life of the decay of rubidium by $\beta$ emission is $4.710^{10} \mathrm{y}$.

(b) If some ${ }_{38}^{87} \mathrm{Sr}$ was initially present in the rock, would the rock be younger, older, or the same age as the age calculated in (a)? Explain your answer.

(a) 3.8 billion years; (b) The rock would be younger than the age calculated in part (a). If Sr was originally in the rock, the amount produced by radioactive decay would equal the present amount minus the initial amount. As this amount would be smaller than the amount used to calculate the age of the rock and the age is proportional to the amount of Sr, the rock would be younger.

A laboratory investigation shows that a sample of uranium ore contains $5.37 \mathrm{mg}$ of ${ }_{92}^{238} \mathrm{U}$ and $2.52 \mathrm{mg}$ of ${ }_{82}^{206} \mathrm{~Pb}$. Calculate the age of the ore. The half-life of ${ }_{92}^{238} \mathrm{U}$ is 4.5 $10^{9} \mathrm{yr}$.

Plutonium was detected in trace amounts in natural uranium deposits by Glenn Seaborg and his associates in 1941. They proposed that the source of this ${ }^{239} \mathrm{Pu}$ was the capture of neutrons by ${ }^{238} \mathrm{U}$ nuclei. Why is this plutonium not likely to have been trapped at the time the solar system formed $4.710^{9}$ years ago?

$c=0$; This shows that no $\mathrm{Pu}-239$ could remain since the formation of the earth. Consequently, the plutonium now present could not have been formed with the uranium.

A ${ }_{4}^{7}$ Be atom (mass $=7.0169 \mathrm{amu}$ ) decays into a ${ }_{3}^{7} \mathrm{Li}$ atom 
(mass $=7.0160 \mathrm{amu}$ ) by electron capture. How much energy (in millions of electron volts, $\mathrm{MeV}$ ) is produced by this reaction?

A ${ }_{5}^{8} \mathrm{~B}$ atom (mass $=8.0246 \mathrm{amu}$ ) decays into a ${ }_{4}^{8} \mathrm{~B}$ atom (mass $=8.0053 \mathrm{amu}$ ) by loss of a $\beta^{+}$particle (mass $=$ $0.00055 \mathrm{amu}$ ) or by electron capture. How much energy (in millions of electron volts) is produced by this reaction?

\section{$17.5 \mathrm{MeV}$}

Isotopes such as ${ }^{26} \mathrm{Al}$ (half-life: $7.210^{5}$ years) are believed to have been present in our solar system as it formed, but have since decayed and are now called extinct nuclides.

(a) ${ }^{26} \mathrm{Al}$ decays by $\beta^{+}$emission or electron capture. Write the equations for these two nuclear transformations.

(b) The earth was formed about $4.710^{9}$ (4.7 billion) years ago. How old was the earth when $99.999999 \%$ of the ${ }^{26} \mathrm{Al}$ originally present had decayed?

Write a balanced equation for each of the following nuclear reactions:

(a) bismuth-212 decays into polonium-212

(b) beryllium- 8 and a positron are produced by the decay of an unstable nucleus

(c) neptunium-239 forms from the reaction of uranium-238 with a neutron and then spontaneously converts into plutonium-239

(d) strontium-90 decays into yttrium-90

(a) ${ }_{83}^{212} \mathrm{Bi}{ }_{84}^{212} \mathrm{Po}+{ }_{-1}^{0} \mathrm{e} ; \quad$ (b) ${ }_{5}^{8} \mathrm{~B}{ }_{4}^{8} \mathrm{Be}+{ }_{-1}^{0} \mathrm{e} ; \quad$ (c)
${ }_{238} \mathrm{U}+{ }_{0} \mathrm{n}{ }_{939}^{239} \mathrm{~Np}+{ }_{0}^{0} \mathrm{~Np},{ }_{93}^{239} \mathrm{~Np}{ }_{94}^{239} \mathrm{Pu}+{ }_{-1}^{0} \mathrm{e} ;($ (d)
${ }_{90} \mathrm{Sr}{ }_{39}^{90} \mathrm{Y}+{ }_{-1}^{0} \mathrm{e}$ 
Write a balanced equation for each of the following nuclear reactions:

(a) mercury-180 decays into platinum-176

(b) zirconium-90 and an electron are produced by the decay of an unstable nucleus

(c) thorium-232 decays and produces an alpha particle and a radium-228 nucleus, which decays into actinium- 228 by beta decay

(d) neon-19 decays into fluorine-19

Footnotes

- 1The " $\mathrm{m}$ " in Tc-99m stands for "metastable," indicating that this is an unstable, high-energy state of Tc-99. Metastable isotopes emit $\gamma$ radiation to rid themselves of excess energy and become (more) stable.

Glossary

alpha (a) decay

loss of an alpha particle during radioactive decay

beta $(\beta)$ decay

breakdown of a neutron into a proton, which remains in the nucleus, and an electron, which is emitted as a beta particle daughter nuclide

nuclide produced by the radioactive decay of another nuclide; may be stable or may 


\section{decay further \\ electron capture}

combination of a core electron with a proton to yield a neutron within the nucleus gamma $(\gamma)$ emission

decay of an excited-state nuclide accompanied by emission of a gamma ray half-life ( $\left.\boldsymbol{t}_{\mathbf{1} / \mathbf{2}}\right)$

time required for half of the atoms in a radioactive sample to decay

\section{parent nuclide}

unstable nuclide that changes spontaneously into another (daughter) nuclide

\section{positron emission}

(also, $\beta^{+}$decay) conversion of a proton into a neutron, which remains in the nucleus, and a positron, which is emitted

\section{radioactive decay}

spontaneous decay of an unstable nuclide into another nuclide

\section{radioactive decay series}

chains of successive disintegrations (radioactive decays) that ultimately lead to a stable end-product

\section{radiocarbon dating}

highly accurate means of dating objects 30,000-50,000 years old that were derived from once-living matter; achieved by calculating the ratio of ${ }_{6}^{14} \mathrm{C}:{ }_{6}^{12} \mathrm{C}$ in the object vs. the ratio of ${ }_{6}^{14} \mathrm{C}:{ }_{6}^{12} \mathrm{C}$ in the 


\section{present-day atmosphere radiometric dating}

use of radioisotopes and their properties to date the formation of objects such as archeological artifacts, formerly living organisms, or geological formations 
62.

\section{TRANSMUTATION AND \\ NUCLEAR ENERGY}

Learning Objectives

By the end of this section, you will be able to:

- Describe the synthesis of transuranium nuclides

- Explain nuclear fission and fusion processes

- Relate the concepts of critical mass and nuclear chain reactions

- Summarize basic requirements for nuclear fission and fusion reactors

After the discovery of radioactivity, the field of nuclear chemistry was created and developed rapidly during the early twentieth century. A slew of new discoveries in the 1930s and 1940s, along with World War II, combined to usher in the Nuclear Age in the mid-twentieth century. 
Scientists learned how to create new substances, and certain isotopes of certain elements were found to possess the capacity to produce unprecedented amounts of energy, with the potential to cause tremendous damage during war, as well as produce enormous amounts of power for society's needs during peace.

Synthesis of Nuclides

Nuclear transmutation is the conversion of one nuclide into another. It can occur by the radioactive decay of a nucleus, or the reaction of a nucleus with another particle. The first manmade nucleus was produced in Ernest Rutherford's laboratory in 1919 by a transmutation reaction, the bombardment of one type of nuclei with other nuclei or with neutrons. Rutherford bombarded nitrogen atoms with high-speed $\alpha$ particles from a natural radioactive isotope of radium and observed protons resulting from the reaction:

${ }_{7}^{14} \mathrm{~N}+{ }_{2}^{4} \mathrm{He}{ }_{8}^{17} \mathrm{O}+{ }_{1}^{1} \mathrm{H}$

The ${ }_{8}^{17} \mathrm{O}$ and ${ }_{1}^{1} \mathrm{H}$ nuclei that are produced are stable, so no further (nuclear) changes occur.

To reach the kinetic energies necessary to produce transmutation reactions, devices called particle accelerators are used. These devices use magnetic and electric fields to increase the speeds of nuclear particles. In all accelerators, the particles move in a vacuum to avoid collisions with gas molecules. When neutrons are required for transmutation reactions, they are usually obtained from radioactive decay reactions or from various nuclear reactions occurring in nuclear reactors. The Chemistry in 
Everyday Life feature that follows discusses a famous particle accelerator that made worldwide news.

CERN Particle Accelerator

Located near Geneva, the CERN ("Conseil Européen pour la Recherche Nucléaire,” or European Council for Nuclear Research) Laboratory is the world's premier center for the investigations of the fundamental particles that make up matter. It contains the 27-kilometer (17 mile) long, circular Large Hadron Collider (LHC), the largest particle accelerator in the world ((Figure)). In the LHC, particles are boosted to high energies and are then made to collide with each other or with stationary targets at nearly the speed of light. Superconducting electromagnets are used to produce a strong magnetic field that guides the particles around the ring. Specialized, purpose-built detectors observe and record the results of these collisions, which are then analyzed by CERN scientists using powerful computers.

A small section of the LHC is shown with workers

traveling along it. (credit: Christophe Delaere) 


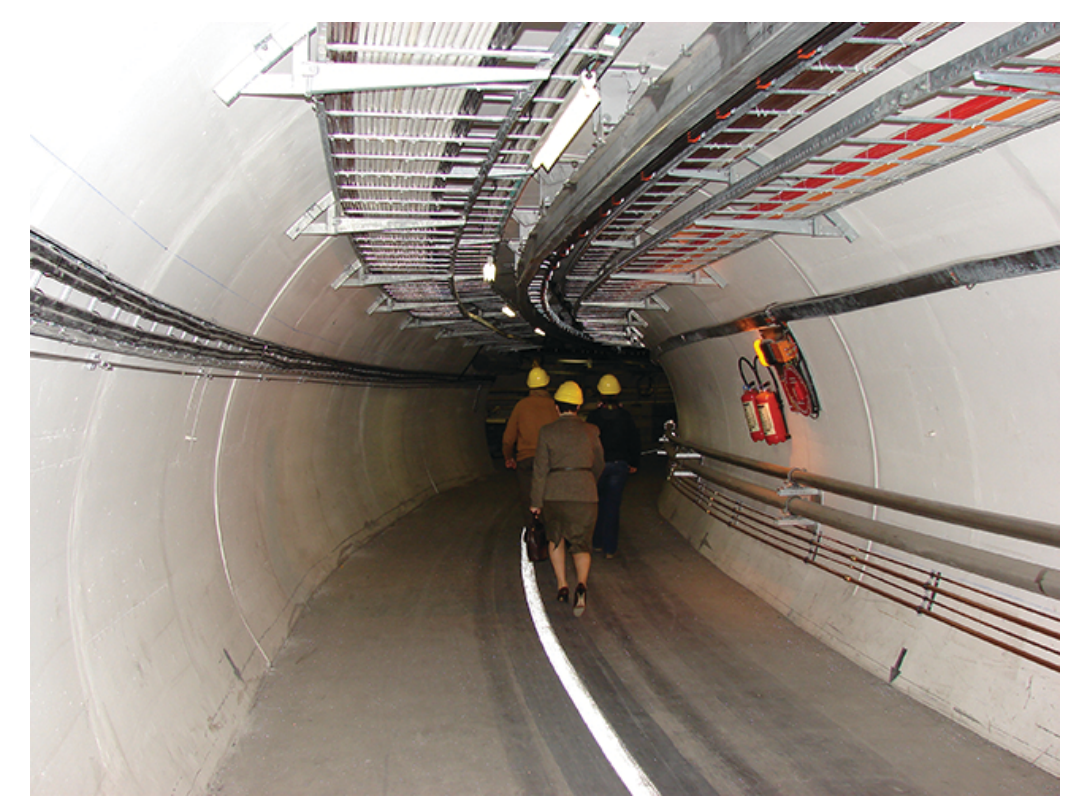

In 2012, CERN announced that experiments at the LHC showed the first observations of the Higgs boson, an elementary particle that helps explain the origin of mass in fundamental particles. This long-anticipated discovery made worldwide news and resulted in the awarding of the 2013 Nobel Prize in Physics to François Englert and Peter Higgs, who had predicted the existence of this particle almost 50 years previously.

Famous physicist Brian Cox talks about his work on the Large Hadron Collider at CERN, providing an entertaining and engaging tour of this massive project and the physics behind it.

View a short video from CERN, describing the basics of how its particle accelerators work.

Prior to 1940, the heaviest-known element was uranium, whose atomic number is 92. Now, many artificial elements have been synthesized and isolated, including several on such a large scale that they have had a profound effect on 
society. One of these-element 93, neptunium (Np)-was first made in 1940 by McMillan and Abelson by bombarding uranium-238 with neutrons. The reaction creates unstable uranium-239, with a half-life of 23.5 minutes, which then decays into neptunium-239. Neptunium-239 is also radioactive, with a half-life of 2.36 days, and it decays into plutonium-239. The nuclear reactions are:

${ }_{92}^{238} \mathrm{U}+{ }_{0}^{1} \mathrm{n}{ }_{92}^{239} \mathrm{U}$

$$
\begin{aligned}
{ }_{92}^{239} \mathrm{U}{ }_{93}^{239} \mathrm{~Np}+{ }_{-1}^{0} \mathrm{e} & \text { half-life }=23.5 \mathrm{~min} \\
{ }_{93}^{239} \mathrm{~Np}{ }_{94}^{239} \mathrm{Pu}+{ }_{-1}^{0} \mathrm{e} & \text { half-life }=2.36 \text { days }
\end{aligned}
$$

Plutonium is now mostly formed in nuclear reactors as a byproduct during the decay of uranium. Some of the neutrons that are released during U-235 decay combine with U-238 nuclei to form uranium-239; this undergoes $\beta$ decay to form neptunium-239, which in turn undergoes $\beta$ decay to form plutonium-239 as illustrated in the preceding three equations. It is possible to summarize these equations as:

${ }_{92}^{238} \mathrm{U}+{ }_{0}^{1} \mathrm{n}{ }_{92}^{239} \mathrm{U} \rightarrow{ }_{93}^{239} \mathrm{~Np} \rightarrow{ }_{94}^{239} \mathrm{Pu}$

Heavier isotopes of plutonium-Pu-240, $\mathrm{Pu}-241$, and $\mathrm{Pu}-242$-are also produced when lighter plutonium nuclei capture neutrons. Some of this highly radioactive plutonium is used to produce military weapons, and the rest presents a serious storage problem because they have half-lives from thousands to hundreds of thousands of years.

Although they have not been prepared in the same 
quantity as plutonium, many other synthetic nuclei have been produced. Nuclear medicine has developed from the ability to convert atoms of one type into other types of atoms. Radioactive isotopes of several dozen elements are currently used for medical applications. The radiation produced by their decay is used to image or treat various organs or portions of the body, among other uses.

The elements beyond element 92 (uranium) are called transuranium elements. As of this writing, 22 transuranium elements have been produced and officially recognized by IUPAC; several other elements have formation claims that are waiting for approval. Some of these elements are shown in (Figure).

\section{Preparation of Some of the Transuranium Elements}

\begin{tabular}{|c|c|c|c|}
\hline Name & Symbol & $\begin{array}{l}\text { Atomic } \\
\text { Number }\end{array}$ & Reaction \\
\hline americium & $\mathrm{Am}$ & 95 & ${ }_{94}^{239} \mathrm{Pu}+{ }_{0}^{1} \mathrm{n}{ }_{95}^{240} \mathrm{Am}+{ }_{-1}^{0} \mathrm{e}$ \\
\hline curium & $\mathrm{Cm}$ & 96 & ${ }_{94}^{239} \mathrm{Pu}+{ }_{2}^{4} \mathrm{He}{ }_{96}^{242} \mathrm{Cm}+{ }_{0}^{1} \mathrm{n}$ \\
\hline californium & $\mathrm{Cf}$ & 98 & ${ }_{96}^{242} \mathrm{Cm}+{ }_{2}^{4} \mathrm{He}{ }_{98}^{245} \mathrm{Cf}+{ }_{0}^{1} \mathrm{n}$ \\
\hline einsteinium & Es & 99 & ${ }_{92}^{238} \mathrm{U}+15_{0}^{1} \mathrm{n} \quad{ }_{99}^{253} \mathrm{Es}+7_{-1}^{0} \mathrm{e}$ \\
\hline mendelevium & Md & 101 & ${ }_{99}^{253} \mathrm{Es}+{ }_{2}^{4} \mathrm{He}{ }_{101}^{256} \mathrm{Md}+{ }_{0}^{1} \mathrm{n}$ \\
\hline nobelium & No & 102 & ${ }_{96}^{246} \mathrm{Cm}+{ }_{6}^{12} \mathrm{C}{ }_{102}^{254} \mathrm{No}+4{ }_{0}^{1} \mathrm{n}$ \\
\hline rutherfordium & $\mathrm{Rf}$ & 104 & ${ }_{98}^{249} \mathrm{Cf}+{ }_{6}^{12} \mathrm{C}{ }_{104}^{257} \mathrm{Rf}+4{ }_{0}^{1} \mathrm{n}$ \\
\hline seaborgium & $\mathrm{Sg}$ & 106 & $\begin{array}{l}{ }_{82}^{206} \mathrm{~Pb}+{ }_{24}^{54} \mathrm{Cr}{ }_{106}^{257} \mathrm{Sg}+3{ }_{0}^{1} \mathrm{n} \\
249 \\
{ }_{98}^{4} \mathrm{Cf}+{ }_{8}^{18} \mathrm{O}{ }_{106}^{263} \mathrm{Sg}+4{ }_{0}^{1} \mathrm{n}\end{array}$ \\
\hline meitnerium & Mt & 107 & ${ }_{83}^{209} \mathrm{Bi}+{ }_{26}^{58} \mathrm{Fe}{ }_{109}^{266} \mathrm{Mt}+{ }_{0}^{1} \mathrm{n}$ \\
\hline
\end{tabular}


Nuclear Fission

Many heavier elements with smaller binding energies per nucleon can decompose into more stable elements that have intermediate mass numbers and larger binding energies per nucleon-that is, mass numbers and binding energies per nucleon that are closer to the "peak" of the binding energy graph near 56 (see (Figure)). Sometimes neutrons are also produced. This decomposition is called fission, the breaking of a large nucleus into smaller pieces. The breaking is rather random with the formation of a large number of different products. Fission usually does not occur naturally, but is induced by bombardment with neutrons. The first reported nuclear fission occurred in 1939 when three German scientists, Lise Meitner, Otto Hahn, and Fritz Strassman, bombarded uranium-235 atoms with slow-moving neutrons that split the U-238 nuclei into smaller fragments that consisted of several neutrons and elements near the middle of the periodic table. Since then, fission has been observed in many other isotopes, including most actinide isotopes that have an odd number of neutrons. A typical nuclear fission reaction is shown in (Figure).

When a slow neutron hits a fissionable U-235 nucleus, it is absorbed and forms an unstable U-236 nucleus. The U-236 nucleus then rapidly breaks apart into two smaller nuclei (in this case, Ba-141 and Kr-92) along with several neutrons (usually two or three), and releases a very large amount of energy. 


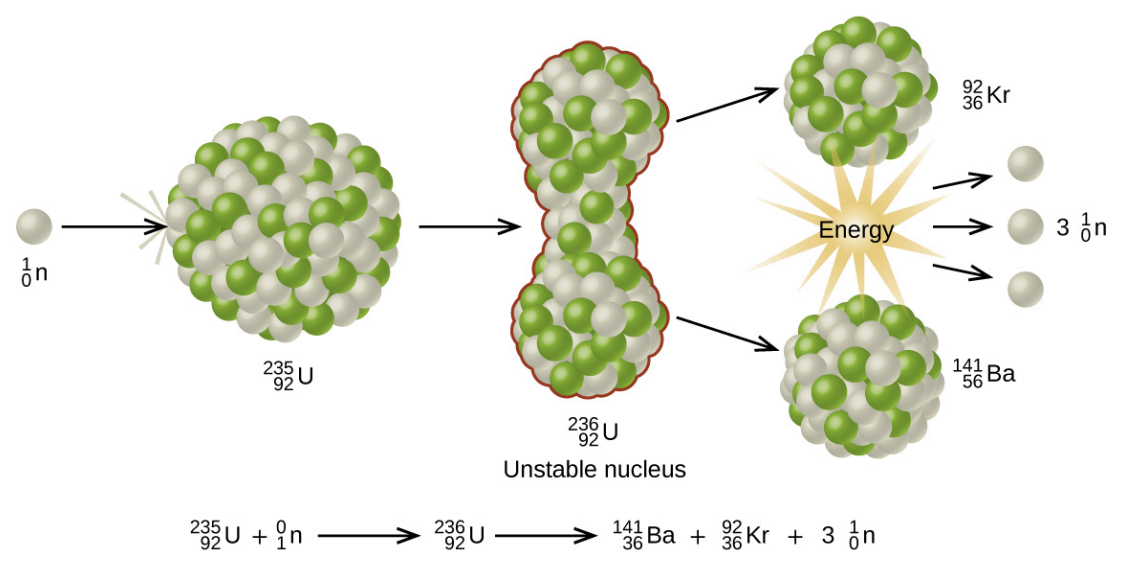

Among the products of Meitner, Hahn, and Strassman's fission reaction were barium, krypton, lanthanum, and cerium, all of which have nuclei that are more stable than uranium-235. Since then, hundreds of different isotopes have been observed among the products of fissionable substances. A few of the many reactions that occur for $\mathrm{U}-235$, and a graph showing the distribution of its fission products and their yields, are shown in (Figure). Similar fission reactions have been observed with other uranium isotopes, as well as with a variety of other isotopes such as those of plutonium.

(a) Nuclear fission of U-235 produces a range of fission products. (b) The larger fission products of U-235 are typically one isotope with a mass number around 85-105, and another isotope with a mass number that is about 50\% larger, that is, about 130-150. 


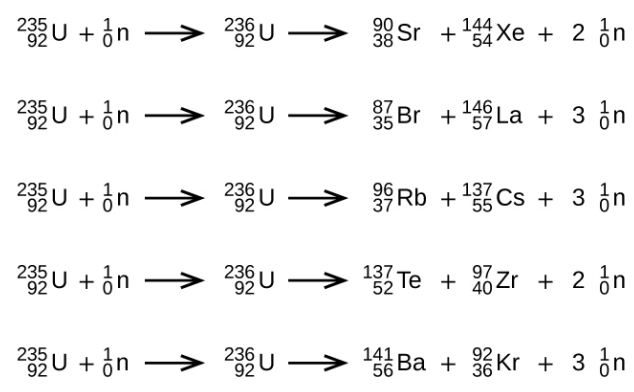

(a)

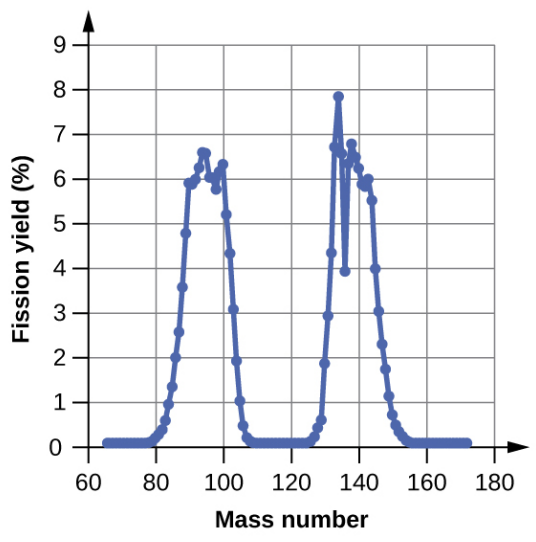

(b)

View this link to see a simulation of nuclear fission.

A tremendous amount of energy is produced by the fission of heavy elements. For instance, when one mole of U-235 undergoes fission, the products weigh about 0.2 grams less than the reactants; this "lost" mass is converted into a very large amount of energy, about $1.810^{10} \mathrm{~kJ}$ per mole of U-235. Nuclear fission reactions produce incredibly large amounts of energy compared to chemical reactions. The fission of 1 kilogram of uranium-235, for example, produces about 2.5 million times as much energy as is produced by burning 1 kilogram of coal.

As described earlier, when undergoing fission U-235 produces two "medium-sized" nuclei, and two or three neutrons. These neutrons may then cause the fission of other uranium-235 atoms, which in turn provide more neutrons that can cause fission of even more nuclei, and so on. If this occurs, we have a nuclear chain reaction (see (Figure)). On the other hand, if too many neutrons escape the bulk material without interacting with a nucleus, then no chain reaction will occur.

The fission of a large nucleus, such as U-235, produces 
two or three neutrons, each of which is capable of causing fission of another nucleus by the reactions shown. If this process continues, a nuclear chain reaction occurs.

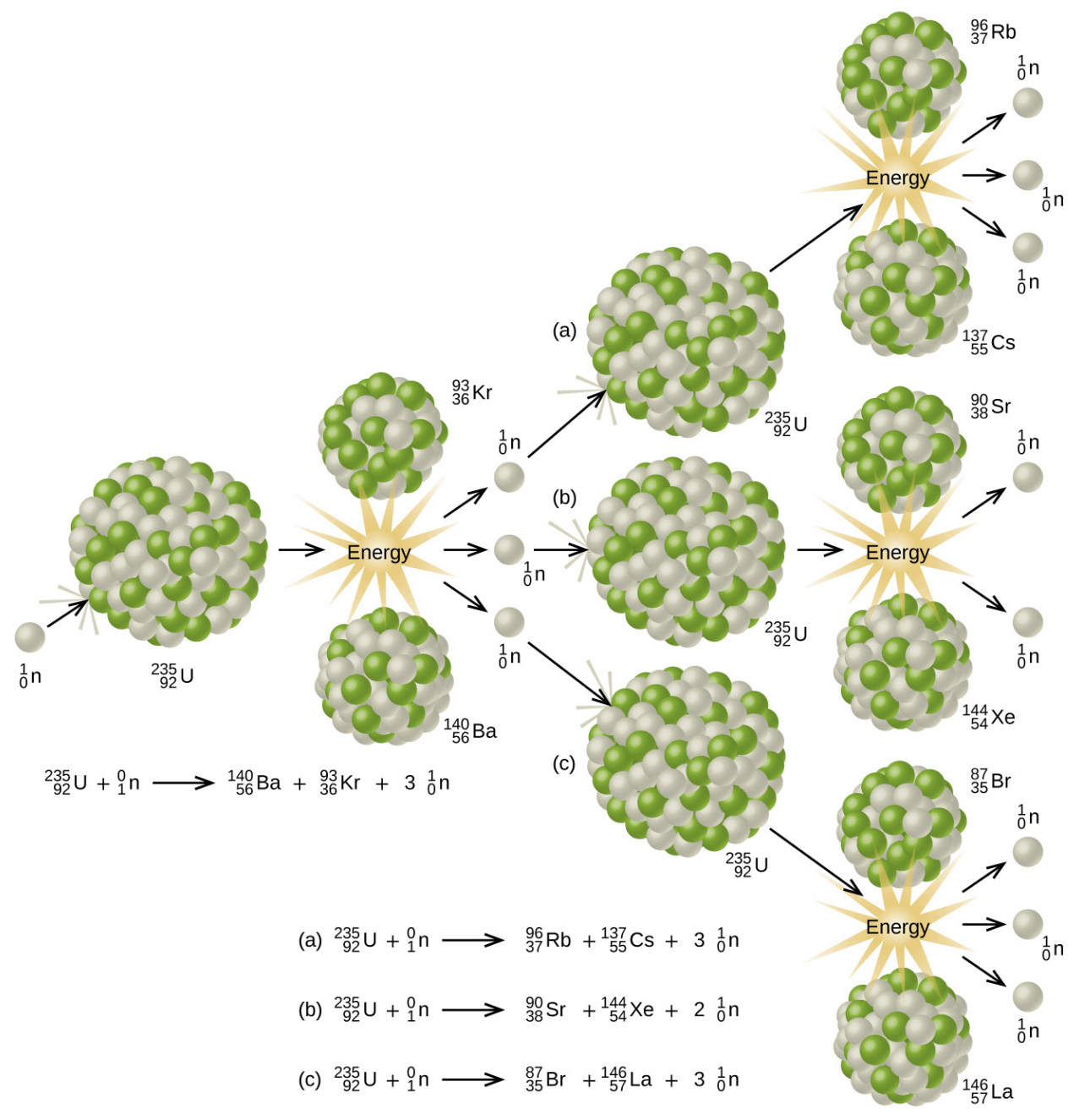

Material that can sustain a nuclear fission chain reaction is said to be fissile or fissionable. (Technically, fissile material can undergo fission with neutrons of any energy, whereas fissionable material requires high-energy neutrons.) Nuclear fission becomes self-sustaining when the number of neutrons produced by fission equals or exceeds the number of neutrons absorbed by splitting nuclei plus the number that escape into the surroundings. 
The amount of a fissionable material that will support a self-sustaining chain reaction is a critical mass. An amount of fissionable material that cannot sustain a chain reaction is a subcritical mass. An amount of material in which there is an increasing rate of fission is known as a supercritical mass. The critical mass depends on the type of material: its purity, the temperature, the shape of the sample, and how the neutron reactions are controlled ((Figure)).

(a) In a subcritical mass, the fissile material is too small and allows too many neutrons to escape the material, so a chain reaction does not occur. (b) In a critical mass, a large enough number of neutrons in the fissile material induce fission to create a chain reaction.

Sub-critical mass

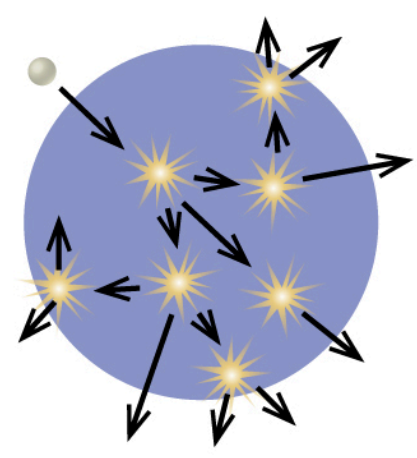

(a)

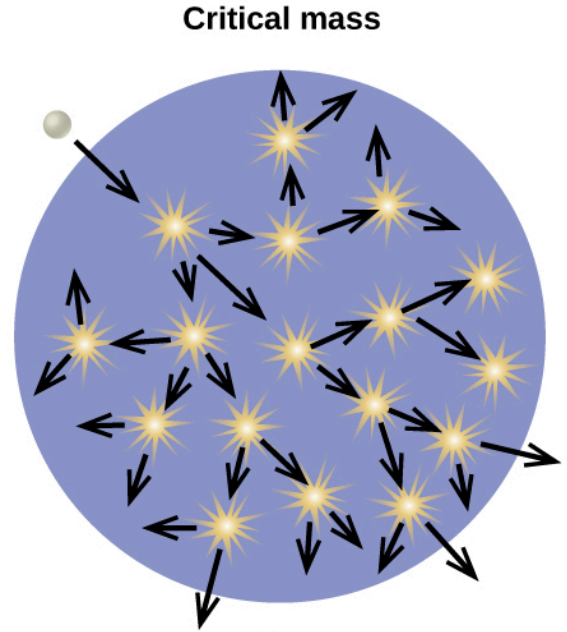

(b)

An atomic bomb ((Figure)) contains several pounds of fissionable material, ${ }_{92}^{235} \mathrm{U}$ or ${ }_{94}^{239} \mathrm{Pu}$, a source of neutrons, and an explosive device for compressing it quickly into a small volume. When fissionable material is in small pieces, the proportion of neutrons that escape through the relatively large surface area is great, and a 
chain reaction does not take place. When the small pieces of fissionable material are brought together quickly to form a body with a mass larger than the critical mass, the relative number of escaping neutrons decreases, and a chain reaction and explosion result.

(a) The nuclear fission bomb that destroyed Hiroshima on August 6,1945, consisted of two subcritical masses of U-235, where conventional explosives were used to fire one of the subcritical masses into the other, creating the critical mass for the nuclear explosion. (b) The plutonium bomb that destroyed Nagasaki on August 9, 1945, consisted of a hollow sphere of plutonium that was rapidly compressed by conventional explosives. This led to a concentration of plutonium in the center that was greater than the critical mass necessary for the nuclear explosion.

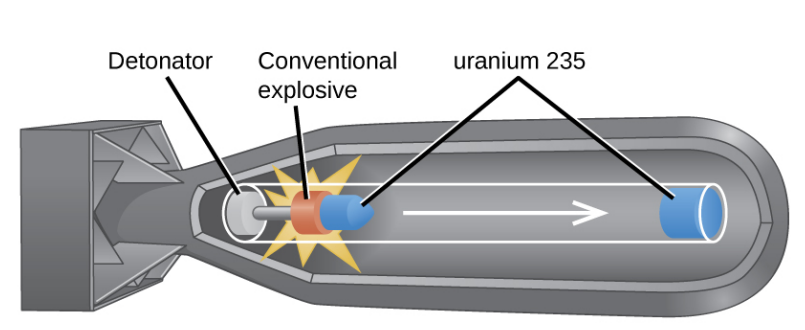

(a)

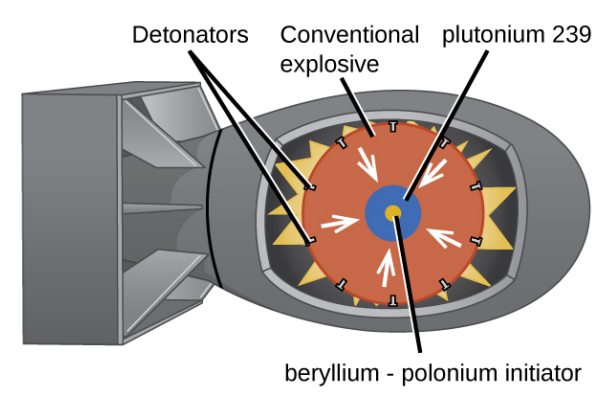

(b)
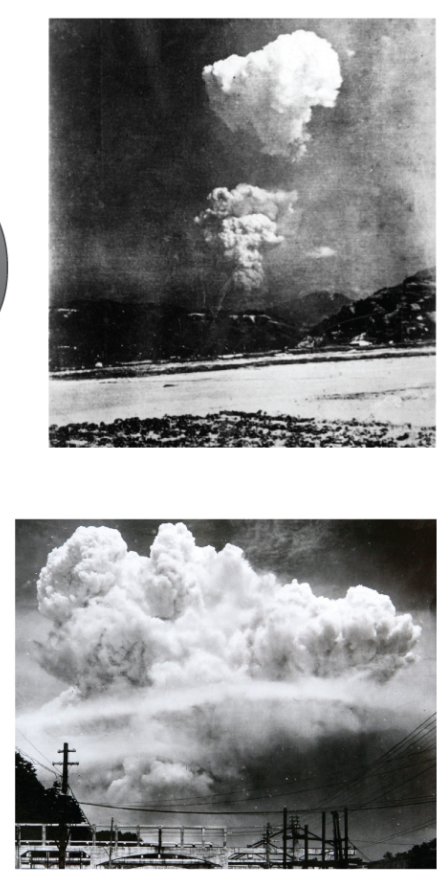

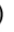


Fission Reactors

Chain reactions of fissionable materials can be controlled and sustained without an explosion in a nuclear reactor ((Figure)). Any nuclear reactor that produces power via the fission of uranium or plutonium by bombardment with neutrons must have at least five components: nuclear fuel consisting of fissionable material, a nuclear moderator, reactor coolant, control rods, and a shield and containment system. We will discuss these components in greater detail later in the section. The reactor works by separating the fissionable nuclear material such that a critical mass cannot be formed, controlling both the flux and absorption of neutrons to allow shutting down the fission reactions. In a nuclear reactor used for the production of electricity, the energy released by fission reactions is trapped as thermal energy and used to boil water and produce steam. The steam is used to turn a turbine, which powers a generator for the production of electricity.

(a) The Diablo Canyon Nuclear Power Plant near San Luis Obispo is the only nuclear power plant currently in operation in California. The domes are the containment structures for the nuclear reactors, and the brown building houses the turbine where electricity is generated. Ocean water is used for cooling. (b) The Diablo Canyon uses a pressurized water reactor, one of a few different fission reactor designs in use around the world, to produce electricity. Energy from the nuclear fission reactions in the core heats water in a closed, pressurized system. Heat from this system produces steam that drives a turbine, which in turn produces electricity. (credit a: 
modification of work by “Mike” Michael L. Baird; credit b: modification of work by the Nuclear Regulatory Commission)

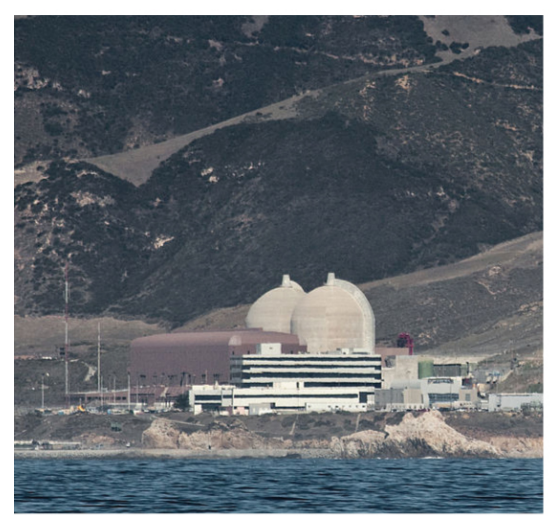

(a)

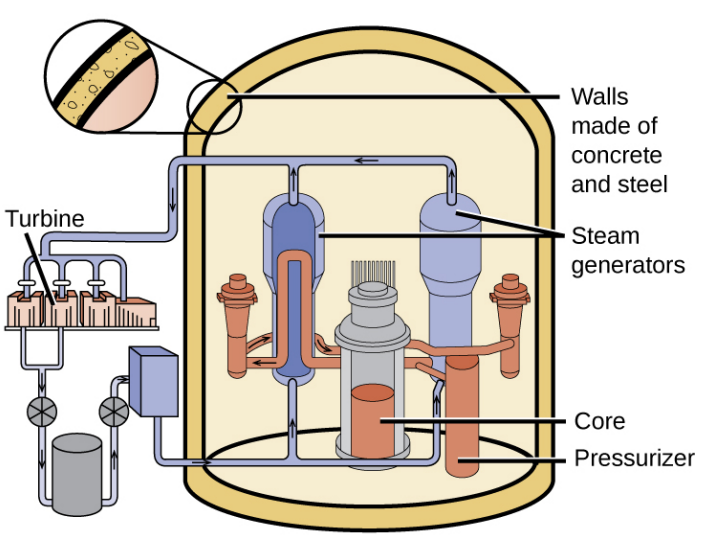

(b)

Nuclear Fuels

Nuclear fuel consists of a fissionable isotope, such as uranium-235, which must be present in sufficient quantity to provide a self-sustaining chain reaction. In the United States, uranium ores contain from $0.05-0.3 \%$ of the uranium oxide $\mathrm{U}_{3} \mathrm{O}_{8}$; the uranium in the ore is about 99.3\% nonfissionable U-238 with only $0.7 \%$ fissionable U-235. Nuclear reactors require a fuel with a higher concentration of U-235 than is found in nature; it is normally enriched to have about $5 \%$ of uranium mass as $\mathrm{U}-235$. At this concentration, it is not possible to achieve the supercritical mass necessary for a nuclear explosion. Uranium can be enriched by gaseous diffusion (the only method currently used in the US), using a gas centrifuge, or by laser separation.

In the gaseous diffusion enrichment plant where U-235 fuel is prepared, $\mathrm{UF}_{6}$ (uranium hexafluoride) gas at low pressure moves through barriers that have holes just 
barely large enough for $\mathrm{UF}_{6}$ to pass through. The slightly lighter ${ }^{235} \mathrm{UF}_{6}$ molecules diffuse through the barrier slightly faster than the heavier ${ }^{238} \mathrm{UF}_{6}$ molecules. This process is repeated through hundreds of barriers, gradually increasing the concentration of ${ }^{235} \mathrm{UF}_{6}$ to the level needed by the nuclear reactor. The basis for this process, Graham's law, is described in the chapter on gases. The enriched $\mathrm{UF}_{6}$ gas is collected, cooled until it solidifies, and then taken to a fabrication facility where it is made into fuel assemblies. Each fuel assembly consists of fuel rods that contain many thimble-sized, ceramicencased, enriched uranium (usually $\mathrm{UO}_{2}$ ) fuel pellets. Modern nuclear reactors may contain as many as 10 million fuel pellets. The amount of energy in each of these pellets is equal to that in almost a ton of coal or 150 gallons of oil.

Nuclear Moderators

Neutrons produced by nuclear reactions move too fast to cause fission (refer back to (Figure)). They must first be slowed to be absorbed by the fuel and produce additional nuclear reactions. A nuclear moderator is a substance that slows the neutrons to a speed that is low enough to cause fission. Early reactors used high-purity graphite as a moderator. Modern reactors in the US exclusively use heavy water $\left({ }_{1}^{2} \mathrm{H}_{2} \mathrm{O}\right)$ or light water (ordinary $\mathrm{H}_{2} \mathrm{O}$ ), whereas some reactors in other countries use other materials, such as carbon dioxide, beryllium, or graphite.

Reactor Coolants

A nuclear reactor coolant is used to carry the heat 
produced by the fission reaction to an external boiler and turbine, where it is transformed into electricity. Two overlapping coolant loops are often used; this counteracts the transfer of radioactivity from the reactor to the primary coolant loop. All nuclear power plants in the US use water as a coolant. Other coolants include molten sodium, lead, a lead-bismuth mixture, or molten salts.

\section{Control Rods}

Nuclear reactors use control rods ((Figure)) to control the fission rate of the nuclear fuel by adjusting the number of slow neutrons present to keep the rate of the chain reaction at a safe level. Control rods are made of boron, cadmium, hafnium, or other elements that are able to absorb neutrons. Boron-10, for example, absorbs neutrons by a reaction that produces lithium-7 and alpha particles: ${ }_{5}^{10} \mathrm{~B}+{ }_{0}^{1} \mathrm{n}{ }_{3}^{7} \mathrm{Li}+{ }_{2}^{4} \mathrm{He}$

When control rod assemblies are inserted into the fuel element in the reactor core, they absorb a larger fraction of the slow neutrons, thereby slowing the rate of the fission reaction and decreasing the power produced. Conversely, if the control rods are removed, fewer neutrons are absorbed, and the fission rate and energy production increase. In an emergency, the chain reaction can be shut down by fully inserting all of the control rods into the nuclear core between the fuel rods.

The nuclear reactor core shown in (a) contains the fuel and control rod assembly shown in (b). (credit: modification of work by E. Generalic, http://glossary.periodni.com/ 
glossary.php?en=control+rod)

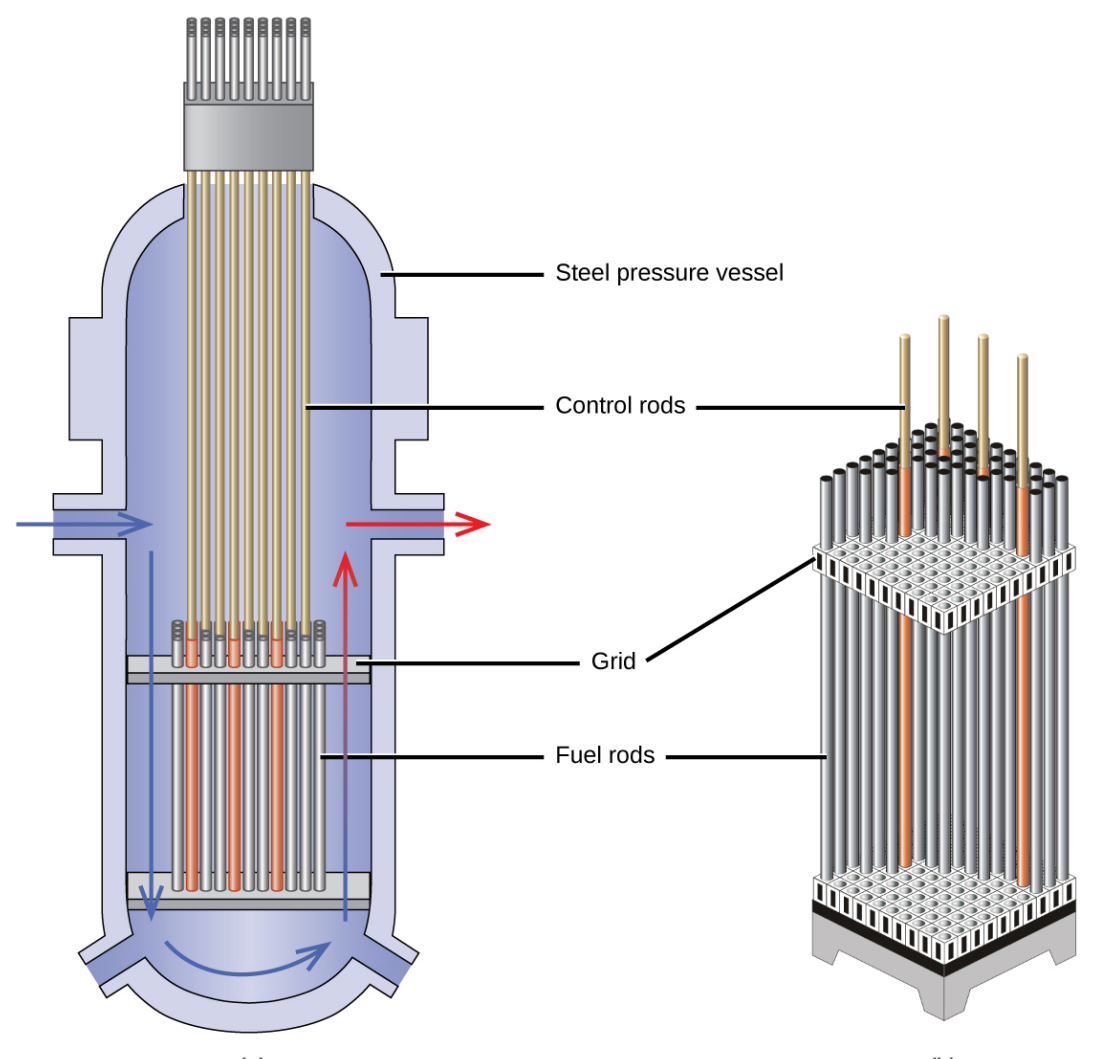

(a)

(b)

Shield and Containment System

During its operation, a nuclear reactor produces neutrons and other radiation. Even when shut down, the decay products are radioactive. In addition, an operating reactor is thermally very hot, and high pressures result from the circulation of water or another coolant through it. Thus, a reactor must withstand high temperatures and pressures, and must protect operating personnel from the radiation. Reactors are equipped with a containment system (or shield) that consists of three parts:

1. The reactor vessel, a steel shell that is 3-20-centimeters thick and, with the moderator, 
absorbs much of the radiation produced by the reactor

2. A main shield of 1-3 meters of high-density concrete

3. A personnel shield of lighter materials that protects operators from $\gamma$ rays and X-rays

In addition, reactors are often covered with a steel or concrete dome that is designed to contain any radioactive materials might be released by a reactor accident.

Click here to watch a 3-minute video from the Nuclear Energy Institute on how nuclear reactors work.

Nuclear power plants are designed in such a way that they cannot form a supercritical mass of fissionable material and therefore cannot create a nuclear explosion. But as history has shown, failures of systems and safeguards can cause catastrophic accidents, including chemical explosions and nuclear meltdowns (damage to the reactor core from overheating). The following Chemistry in Everyday Life feature explores three infamous meltdown incidents.

Nuclear Accidents

The importance of cooling and containment are amply illustrated by three major accidents that occurred with the nuclear reactors at nuclear power generating stations in the United States (Three Mile Island), the former Soviet Union (Chernobyl), and Japan (Fukushima). 
In March 1979, the cooling system of the Unit 2 reactor at Three Mile Island Nuclear Generating Station in Pennsylvania failed, and the cooling water spilled from the reactor onto the floor of the containment building. After the pumps stopped, the reactors overheated due to the high radioactive decay heat produced in the first few days after the nuclear reactor shut down. The temperature of the core climbed to at least $2200{ }^{\circ} \mathrm{C}$, and the upper portion of the core began to melt. In addition, the zirconium alloy cladding of the fuel rods began to react with steam and produced hydrogen:

$\mathrm{Zr}(s)+2 \mathrm{H}_{2} \mathrm{O}(g) \mathrm{ZrO}_{2}(s)+2 \mathrm{H}_{2}(g)$

The hydrogen accumulated in the confinement building, and it was feared that there was danger of an explosion of the mixture of hydrogen and air in the building. Consequently, hydrogen gas and radioactive gases (primarily krypton and xenon) were vented from the building. Within a week, cooling water circulation was restored and the core began to cool. The plant was closed for nearly 10 years during the cleanup process.

Although zero discharge of radioactive material is desirable, the discharge of radioactive krypton and xenon, such as occurred at the Three Mile Island plant, is among the most tolerable. These gases readily disperse in the atmosphere and thus do not produce highly radioactive areas. Moreover, they are noble gases and are not incorporated into plant and animal matter in the food chain. Effectively none of the heavy elements of the core of the reactor were released into the environment, and no cleanup of the area outside of the containment building was necessary ((Figure)). 
(a) In this 2010 photo of Three Mile Island, the remaining structures from the damaged Unit 2 reactor are seen on the left, whereas the separate Unit 1 reactor, unaffected by the accident, continues generating power to this day (right). (b) President Jimmy Carter visited the Unit 2 control room a few days after the accident in 1979.

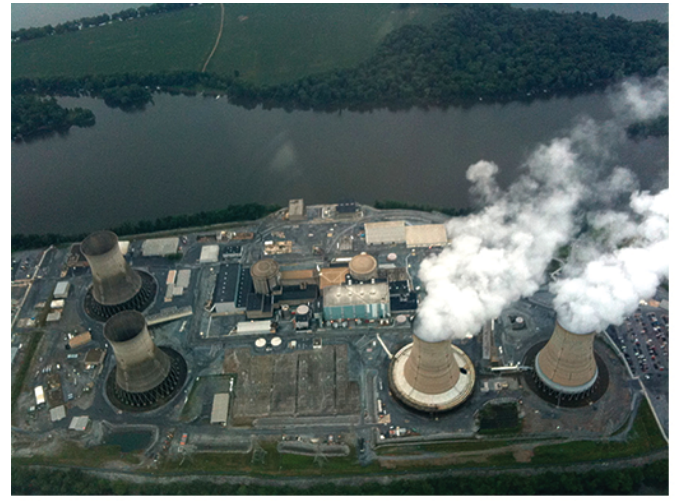

(a)

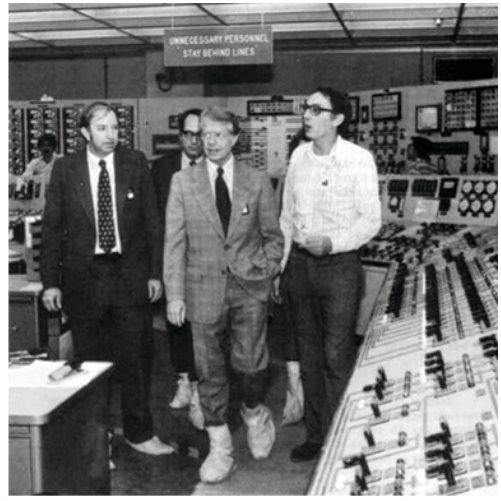

(b)

Another major nuclear accident involving a reactor occurred in April 1986, at the Chernobyl Nuclear Power Plant in Ukraine, which was still a part of the former Soviet Union. While operating at low power during an unauthorized experiment with some of its safety devices shut off, one of the reactors at the plant became unstable. Its chain reaction became uncontrollable and increased to a level far beyond what the reactor was designed for. The steam pressure in the reactor rose to between 100 and 500 times the full power pressure and ruptured the reactor. Because the reactor was not enclosed in a containment building, a large amount of radioactive material spewed out, and additional fission products were released, as the graphite (carbon) moderator of the core ignited and burned. The fire was controlled, but over 200 plant workers and firefighters developed acute radiation 
sickness and at least 32 soon died from the effects of the radiation. It is predicted that about 4000 more deaths will occur among emergency workers and former Chernobyl residents from radiation-induced cancer and leukemia. The reactor has since been encapsulated in steel and concrete, a now-decaying structure known as the sarcophagus. Almost 30 years later, significant radiation problems still persist in the area, and Chernobyl largely remains a wasteland.

In 2011, the Fukushima Daiichi Nuclear Power Plant in Japan was badly damaged by a 9.0-magnitude earthquake and resulting tsunami. Three reactors up and running at the time were shut down automatically, and emergency generators came online to power electronics and coolant systems. However, the tsunami quickly flooded the emergency generators and cut power to the pumps that circulated coolant water through the reactors. Hightemperature steam in the reactors reacted with zirconium alloy to produce hydrogen gas. The gas escaped into the containment building, and the mixture of hydrogen and air exploded. Radioactive material was released from the containment vessels as the result of deliberate venting to reduce the hydrogen pressure, deliberate discharge of coolant water into the sea, and accidental or uncontrolled events.

An evacuation zone around the damaged plant extended over 12.4 miles away, and an estimated 200,000 people were evacuated from the area. All 48 of Japan's nuclear power plants were subsequently shut down, remaining shuttered as of December 2014. Since the disaster, public opinion has shifted from largely favoring to largely opposing increasing the use of nuclear power 
plants, and a restart of Japan's atomic energy program is still stalled ((Figure)).

(a) After the accident, contaminated waste had to be removed, and (b) an evacuation zone was set up around the plant in areas that received heavy doses of radioactive fallout. (credit a: modification of work by "Live Action Hero"/Flickr)

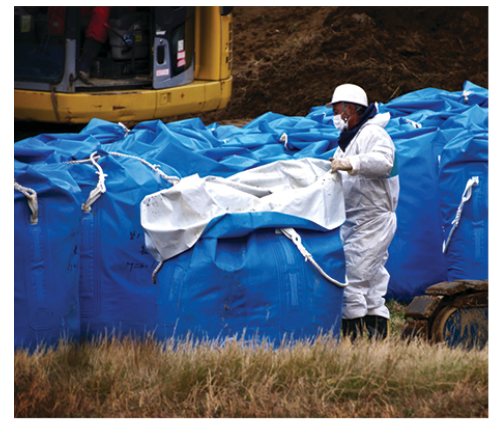

(a)

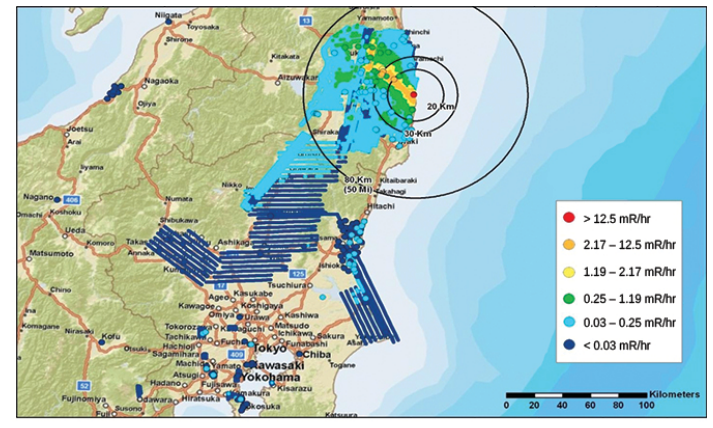

(b)

The energy produced by a reactor fueled with enriched uranium results from the fission of uranium as well as from the fission of plutonium produced as the reactor operates. As discussed previously, the plutonium forms from the combination of neutrons and the uranium in the fuel. In any nuclear reactor, only about $0.1 \%$ of the mass of the fuel is converted into energy. The other $99.9 \%$ remains in the fuel rods as fission products and unused fuel. All of the fission products absorb neutrons, and after a period of several months to a few years, depending on the reactor, the fission products must be removed by changing the fuel rods. Otherwise, the concentration of these fission products would increase and absorb more neutrons until the reactor could no longer operate.

Spent fuel rods contain a variety of products, consisting of unstable nuclei ranging in atomic number from 25 to 
60, some transuranium elements, including plutonium and americium, and unreacted uranium isotopes. The unstable nuclei and the transuranium isotopes give the spent fuel a dangerously high level of radioactivity. The long-lived isotopes require thousands of years to decay to a safe level. The ultimate fate of the nuclear reactor as a significant source of energy in the United States probably rests on whether or not a politically and scientifically satisfactory technique for processing and storing the components of spent fuel rods can be developed.

Explore the information in this link to learn about the approaches to nuclear waste management.

Nuclear Fusion and Fusion Reactors

The process of converting very light nuclei into heavier nuclei is also accompanied by the conversion of mass into large amounts of energy, a process called fusion. The principal source of energy in the sun is a net fusion reaction in which four hydrogen nuclei fuse and produce one helium nucleus and two positrons. This is a net reaction of a more complicated series of events: $4_{1}^{1} \mathrm{H}{ }_{2}^{4} \mathrm{He}+2{ }_{+1}^{0}$

A helium nucleus has a mass that is $0.7 \%$ less than that of four hydrogen nuclei; this lost mass is converted into energy during the fusion. This reaction produces about $3.610^{11} \mathrm{~kJ}$ of energy per mole of ${ }_{2}^{4} \mathrm{He}$ produced. This is somewhat larger than the energy produced by the nuclear fission of one mole of U-235 (1.8 $\left.10^{10} \mathrm{~kJ}\right)$, and over 3 million times larger than the energy produced by the (chemical) combustion of one mole of octane (5471 kJ). 
It has been determined that the nuclei of the heavy isotopes of hydrogen, a deuteron, ${ }_{1}^{2}$ and a triton, ${ }_{1}^{3}$, undergo fusion at extremely high temperatures (thermonuclear fusion). They form a helium nucleus and a neutron:

${ }_{1}^{2} \mathrm{H}+{ }_{1}^{3} \mathrm{H}{ }_{2}^{4} \mathrm{He}+2{ }_{0}^{1} \mathrm{n}$

This change proceeds with a mass loss of $0.0188 \mathrm{amu}$, corresponding to the release of $1.6910^{9}$ kilojoules per mole of ${ }_{2}^{4} \mathrm{He}$ formed. The very high temperature is necessary to give the nuclei enough kinetic energy to overcome the very strong repulsive forces resulting from the positive charges on their nuclei so they can collide.

Useful fusion reactions require very high temperatures for their initiation-about $15,000,000 \mathrm{~K}$ or more. At these temperatures, all molecules dissociate into atoms, and the atoms ionize, forming plasma. These conditions occur in an extremely large number of locations throughout the universe-stars are powered by fusion. Humans have already figured out how to create temperatures high enough to achieve fusion on a large scale in thermonuclear weapons. A thermonuclear weapon such as a hydrogen bomb contains a nuclear fission bomb that, when exploded, gives off enough energy to produce the extremely high temperatures necessary for fusion to occur.

Another much more beneficial way to create fusion reactions is in a fusion reactor, a nuclear reactor in which fusion reactions of light nuclei are controlled. Because no solid materials are stable at such high temperatures, mechanical devices cannot contain the plasma in which fusion reactions occur. Two techniques to contain plasma 
at the density and temperature necessary for a fusion reaction are currently the focus of intensive research efforts: containment by a magnetic field and by the use of focused laser beams ((Figure)). A number of large projects are working to attain one of the biggest goals in science: getting hydrogen fuel to ignite and produce more energy than the amount supplied to achieve the extremely high temperatures and pressures that are required for fusion. At the time of this writing, there are no self-sustaining fusion reactors operating in the world, although smallscale controlled fusion reactions have been run for very brief periods.

(a) This model is of the International Thermonuclear Experimental Reactor (ITER) reactor. Currently under construction in the south of France with an expected completion date of 2027, the ITER will be the world's largest experimental Tokamak nuclear fusion reactor with a goal of achieving large-scale sustained energy production. (b) In 2012, the National Ignition Facility at Lawrence Livermore National Laboratory briefly produced over 500,000,000,000 watts (500 terawatts, or 500 TW) of peak power and delivered 1,850,000 joules $(1.85 \mathrm{MJ})$ of energy, the largest laser energy ever produced and 1000 times the power usage of the entire United States in any given moment. Although lasting only a few billionths of a second, the 192 lasers attained the conditions needed for nuclear fusion ignition. This image shows the target prior to the laser shot. (credit a: modification of work by Stephan Mosel) 


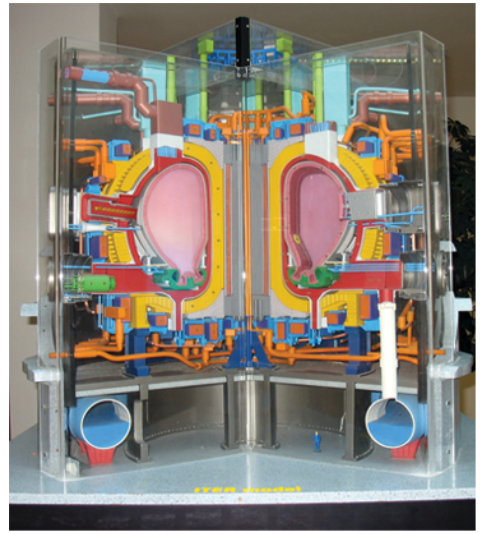

(a)

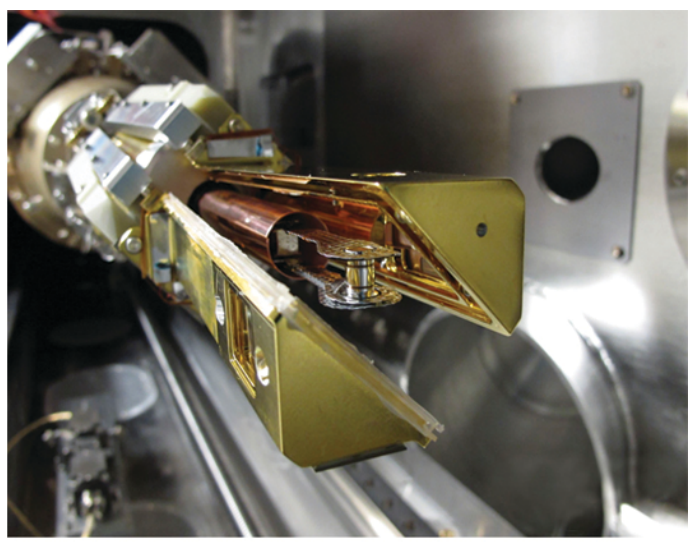

(b)

Key Concepts and Summary

It is possible to produce new atoms by bombarding other atoms with nuclei or high-speed particles. The products of these transmutation reactions can be stable or radioactive. A number of artificial elements, including technetium, astatine, and the transuranium elements, have been produced in this way.

Nuclear power as well as nuclear weapon detonations can be generated through fission (reactions in which a heavy nucleus is split into two or more lighter nuclei and several neutrons). Because the neutrons may induce additional fission reactions when they combine with other heavy nuclei, a chain reaction can result. Useful power is obtained if the fission process is carried out in a nuclear reactor. The conversion of light nuclei into heavier nuclei (fusion) also produces energy. At present, this energy has not been contained adequately and is too expensive to be feasible for commercial energy production. 
Chemistry End of Chapter Exercises

Write the balanced nuclear equation for the production of the following transuranium elements:

(a) berkelium-244, made by the reaction of Am-241 and He-4

(b) fermium-254, made by the reaction of $\mathrm{Pu}-239$ with a large number of neutrons

(c) lawrencium-257, made by the reaction of Cf-250 and B-11

(d) dubnium-260, made by the reaction of Cf-249 and $\mathrm{N}-15$

${ }_{94}^{239} \mathrm{Pu}+15_{0}^{1} \mathrm{n}{ }_{100}^{954} \mathrm{Fm}+6{ }_{-1}^{0} \mathrm{e}$;

${ }_{98}^{250} \mathrm{Cf}+{ }_{5}^{11} \mathrm{~B}{ }_{103}^{257} \mathrm{Lr}+4{ }_{0}^{1} \mathrm{n}$;

${ }_{98}^{249} \mathrm{Cf}+{ }_{7}^{15} \mathrm{~N}{ }_{105}^{260} \mathrm{Db}+4{ }_{0}^{1} \mathrm{n}$

How does nuclear fission differ from nuclear fusion? Why are both of these processes exothermic?

Both fusion and fission are nuclear reactions. Why is a very high temperature required for fusion, but not for fission?

Two nuclei must collide for fusion to occur. High temperatures are required to give the nuclei enough kinetic energy to overcome the very strong repulsion resulting from their positive charges.

Cite the conditions necessary for a nuclear chain reaction to take place. Explain how it can be controlled to produce energy, but not produce an explosion. 
1078

Describe the components of a nuclear reactor.

A nuclear reactor consists of the following:

1. A nuclear fuel. A fissionable isotope must be present in large enough quantities to sustain a controlled chain reaction. The radioactive isotope is contained in tubes called fuel rods.

2. A moderator. A moderator slows neutrons produced by nuclear reactions so that they can be absorbed by the fuel and cause additional nuclear reactions.

3. A coolant. The coolant carries heat from the fission reaction to an external boiler and turbine where it is transformed into electricity.

4. A control system. The control system consists of control rods placed between fuel rods to absorb neutrons and is used to adjust the number of neutrons and keep the rate of the chain reaction at a safe level.

5. A shield and containment system. The function of this component is to protect workers from radiation produced by the nuclear reactions and to withstand the high pressures resulting from high-temperature reactions.

In usual practice, both a moderator and control rods are necessary to operate a nuclear chain reaction safely for the purpose of energy production. Cite the function of each and explain why both are necessary. 
Describe how the potential energy of uranium is converted into electrical energy in a nuclear power plant.

The fission of uranium generates heat, which is carried to an external steam generator (boiler). The resulting steam turns a turbine that powers an electrical generator.

The mass of a hydrogen atom $\left({ }_{1}^{1} \mathrm{H}\right)$ is $1.007825 \mathrm{amu}$; that of a tritium atom $\left({ }_{1}^{3} \mathrm{H}\right)$ is $3.01605 \mathrm{amu}$; and that of an $\alpha$ particle is $4.00150 \mathrm{amu}$. How much energy in kilojoules per mole of ${ }_{2}^{4} \mathrm{He}$ produced is released by the following fusion reaction: ${ }_{1}^{1} \mathrm{H}+{ }_{1}^{3} \mathrm{H}{ }_{2}^{4} \mathrm{He}$.

Glossary

chain reaction

repeated fission caused when the neutrons released in fission bombard other atoms containment system

(also, shield) a three-part structure of materials that protects the exterior of a nuclear fission reactor and operating personnel from the high temperatures, pressures, and radiation levels inside the reactor

\section{control rod}

material inserted into the fuel assembly that absorbs neutrons and can be raised or lowered to adjust the rate of a fission reaction 


\section{critical mass}

amount of fissionable material that will support a self-sustaining (nuclear fission) chain reaction

\section{fissile (or fissionable)}

when a material is capable of sustaining a nuclear fission reaction

\section{fission}

splitting of a heavier nucleus into two or more lighter nuclei, usually accompanied by the conversion of mass into large amounts of energy

\section{fusion}

combination of very light nuclei into heavier nuclei, accompanied by the conversion of mass into large amounts of energy

\section{fusion reactor}

nuclear reactor in which fusion reactions of light nuclei are controlled

\section{nuclear fuel}

fissionable isotope present in sufficient quantities to provide a self-sustaining chain reaction in a nuclear reactor

\section{nuclear moderator}

substance that slows neutrons to a speed low enough to cause fission

\section{nuclear reactor}

environment that produces energy via nuclear fission in which the chain reaction is controlled and sustained without explosion 


\section{nuclear transmutation}

conversion of one nuclide into another nuclide

\section{particle accelerator}

device that uses electric and magnetic

fields to increase the kinetic energy of nuclei used in transmutation reactions

\section{reactor coolant}

assembly used to carry the heat produced

by fission in a reactor to an external boiler and turbine where it is transformed into electricity

\section{subcritical mass}

amount of fissionable material that cannot sustain a chain reaction; less than a critical mass

\section{supercritical mass}

amount of material in which there is an increasing rate of fission

\section{transmutation reaction}

bombardment of one type of nuclei with other nuclei or neutrons

\section{transuranium element}

element with an atomic number greater than 92; these elements do not occur in nature 


\section{3.}

\section{BIOLOGICAL EFFECTS OF RADIATION}

Learning Objectives

By the end of this section, you will be able to:

- Describe the biological impact of ionizing radiation

- Define units for measuring radiation exposure

- Explain the operation of common tools for detecting radioactivity

- List common sources of radiation exposure in the US

The increased use of radioisotopes has led to increased concerns over the effects of these materials on biological systems (such as humans). All radioactive nuclides emit high-energy particles or electromagnetic waves. When 
this radiation encounters living cells, it can cause heating, break chemical bonds, or ionize molecules. The most serious biological damage results when these radioactive emissions fragment or ionize molecules. For example, alpha and beta particles emitted from nuclear decay reactions possess much higher energies than ordinary chemical bond energies. When these particles strike and penetrate matter, they produce ions and molecular fragments that are extremely reactive. The damage this does to biomolecules in living organisms can cause serious malfunctions in normal cell processes, taxing the organism's repair mechanisms and possibly causing illness or even death ((Figure)).

Radiation can harm biological systems by damaging the DNA of cells. If this damage is not properly repaired, the cells may divide in an uncontrolled manner and cause cancer.

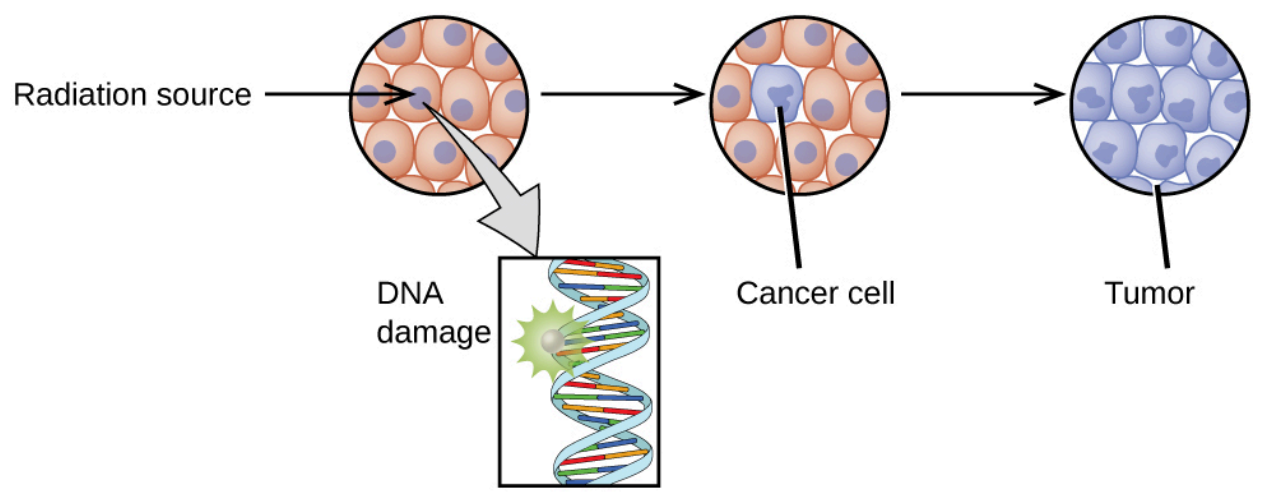

lonizing and Nonionizing Radiation

There is a large difference in the magnitude of the biological effects of nonionizing radiation (for example, light and microwaves) and ionizing radiation, emissions energetic enough to knock electrons out of molecules (for 
example, $\alpha$ and $\beta$ particles, $\gamma$ rays, $\mathrm{X}$-rays, and high-energy ultraviolet radiation) ((Figure)).

Lower frequency, lower-energy electromagnetic radiation is nonionizing, and higher frequency, higher-energy electromagnetic radiation is ionizing.

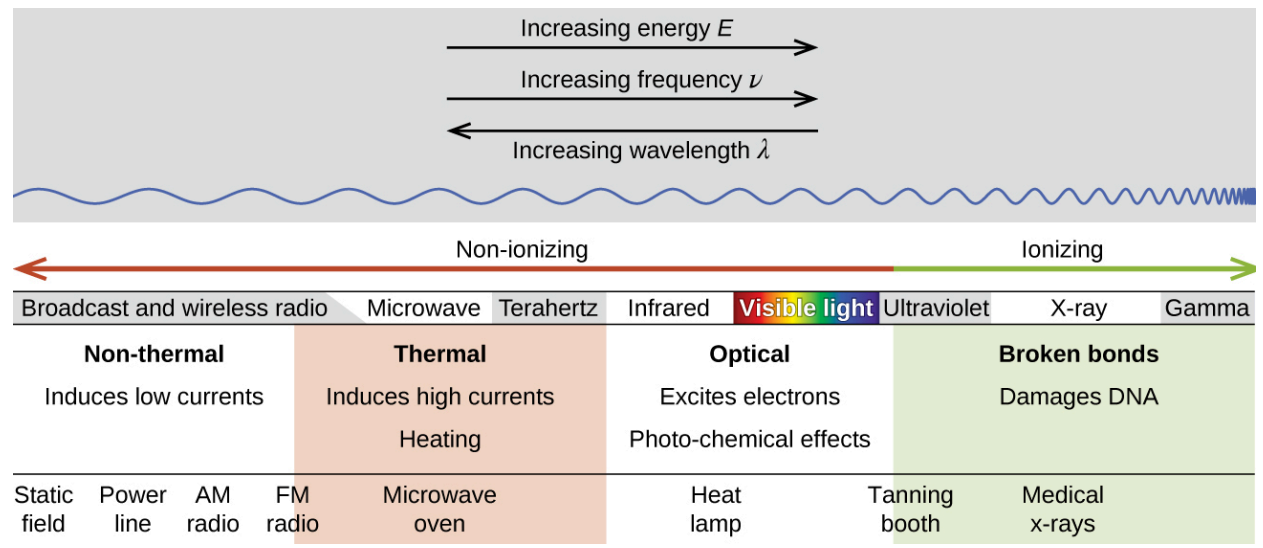

Energy absorbed from nonionizing radiation speeds up the movement of atoms and molecules, which is equivalent to heating the sample. Although biological systems are sensitive to heat (as we might know from touching a hot stove or spending a day at the beach in the sun), a large amount of nonionizing radiation is necessary before dangerous levels are reached. Ionizing radiation, however, may cause much more severe damage by breaking bonds or removing electrons in biological molecules, disrupting their structure and function. The damage can also be done indirectly, by first ionizing $\mathrm{H}_{2} \mathrm{O}$ (the most abundant molecule in living organisms), which forms a $\mathrm{H}_{2} \mathrm{O}^{+}$ion that reacts with water, forming a hydronium ion and a hydroxyl radical:

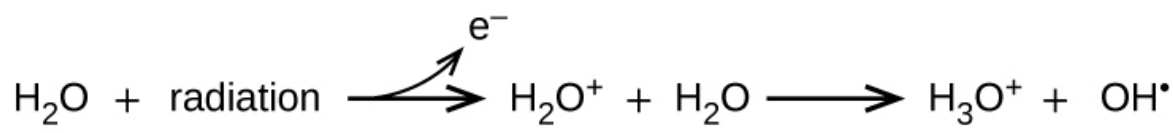

Because the hydroxyl radical has an unpaired electron, 
it is highly reactive. (This is true of any substance with unpaired electrons, known as a free radical.) This hydroxyl radical can react with all kinds of biological molecules (DNA, proteins, enzymes, and so on), causing damage to the molecules and disrupting physiological processes. Examples of direct and indirect damage are shown in (Figure).

Ionizing radiation can (a) directly damage a biomolecule by ionizing it or breaking its bonds, or (b) create an $\mathrm{H}_{2} \mathrm{O}^{+}$ ion, which reacts with $\mathrm{H}_{2} \mathrm{O}$ to form a hydroxyl radical, which in turn reacts with the biomolecule, causing damage indirectly.

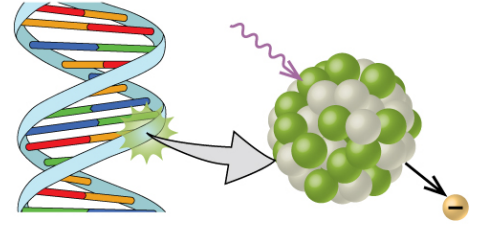

Direct effect

(a)

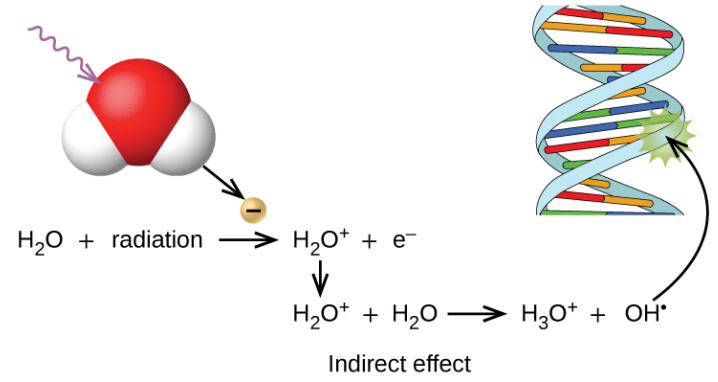

(b)

Biological Effects of Exposure to Radiation

Radiation can harm either the whole body (somatic damage) or eggs and sperm (genetic damage). Its effects are more pronounced in cells that reproduce rapidly, such as the stomach lining, hair follicles, bone marrow, and embryos. This is why patients undergoing radiation therapy often feel nauseous or sick to their stomach, lose hair, have bone aches, and so on, and why particular care must be taken when undergoing radiation therapy during pregnancy.

Different types of radiation have differing abilities to 
pass through material ((Figure)). A very thin barrier, such as a sheet or two of paper, or the top layer of skin cells, usually stops alpha particles. Because of this, alpha particle sources are usually not dangerous if outside the body, but are quite hazardous if ingested or inhaled (see the Chemistry in Everyday Life feature on Radon Exposure). Beta particles will pass through a hand, or a thin layer of material like paper or wood, but are stopped by a thin layer of metal. Gamma radiation is very penetrating and can pass through a thick layer of most materials. Some high-energy gamma radiation is able to pass through a few feet of concrete. Certain dense, high atomic number elements (such as lead) can effectively attenuate gamma radiation with thinner material and are used for shielding. The ability of various kinds of emissions to cause ionization varies greatly, and some particles have almost no tendency to produce ionization. Alpha particles have about twice the ionizing power of fast-moving neutrons, about 10 times that of $\beta$ particles, and about 20 times that of $\gamma$ rays and X-rays.

The ability of different types of radiation to pass through material is shown. From least to most penetrating, they are alpha $<$ beta $<$ neutron $<$ gamma.

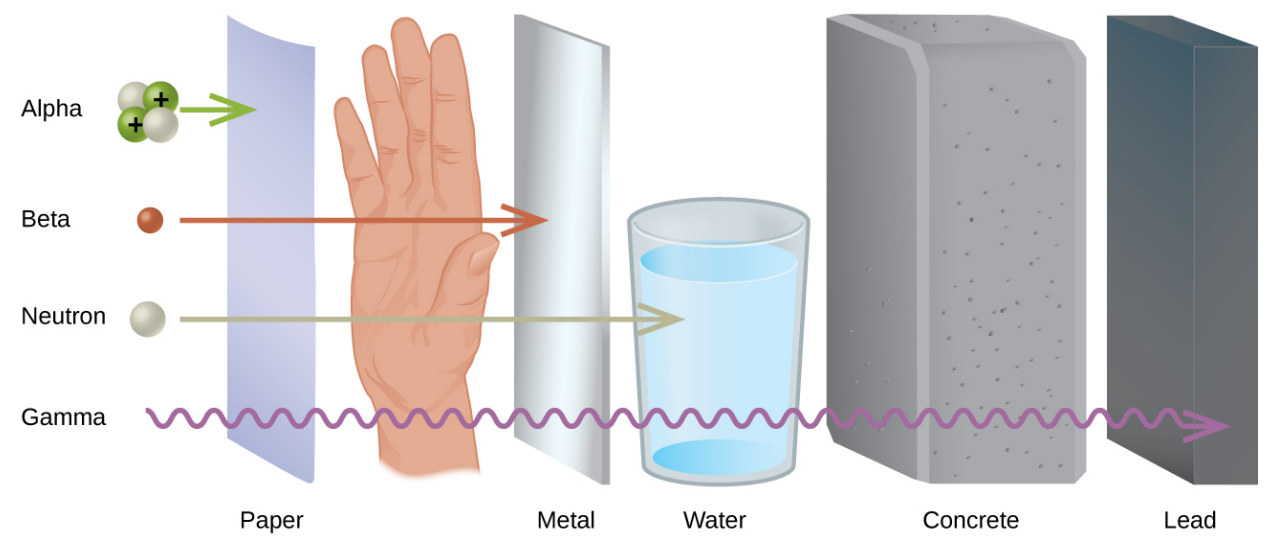




\section{Radon Exposure}

For many people, one of the largest sources of exposure to radiation is from radon gas (Rn-222). Radon-222 is an $\alpha$ emitter with a half-life of 3.82 days. It is one of the products of the radioactive decay series of U-238 ((Figure)), which is found in trace amounts in soil and rocks. The radon gas that is produced slowly escapes from the ground and gradually seeps into homes and other structures above. Since it is about eight times more dense than air, radon gas accumulates in basements and lower floors, and slowly diffuses throughout buildings ((Figure)). Radon-222 seeps into houses and other buildings from rocks that contain uranium-238, a radon emitter. The radon enters through cracks in concrete foundations and basement floors, stone or porous cinderblock foundations, and openings for water and gas pipes. 


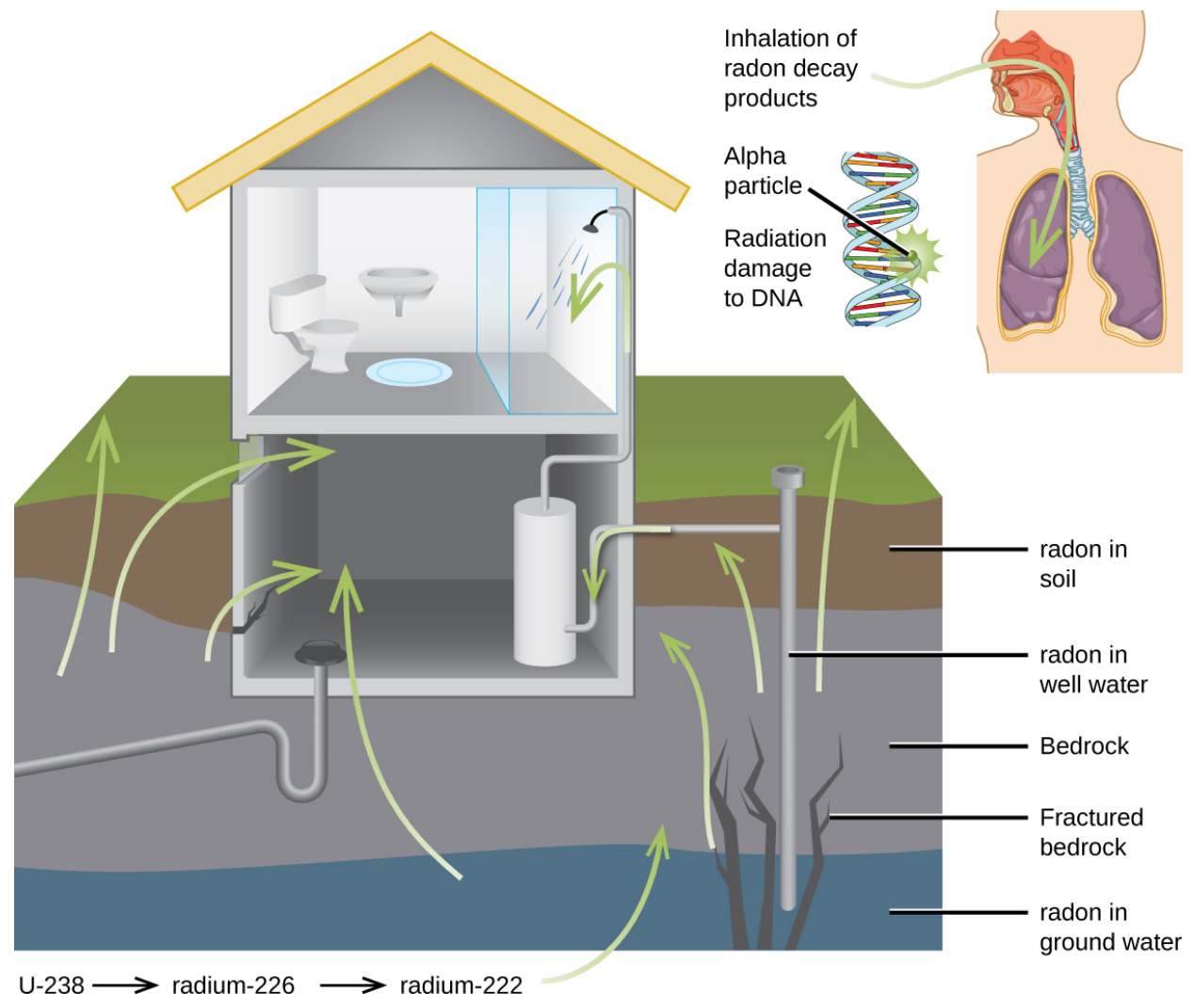

Radon is found in buildings across the country, with amounts depending on where you live. The average concentration of radon inside houses in the US $(1.25 \mathrm{pCi}$ / $\mathrm{L}$ ) is about three times the levels found in outside air, and about one in six houses have radon levels high enough that remediation efforts to reduce the radon concentration are recommended. Exposure to radon increases one's risk of getting cancer (especially lung cancer), and high radon levels can be as bad for health as smoking a carton of cigarettes a day. Radon is the number one cause of lung cancer in nonsmokers and the second leading cause of lung cancer overall. Radon exposure is believed to cause over 20,000 deaths in the US per year. 
Measuring Radiation Exposure

Several different devices are used to detect and measure radiation, including Geiger counters, scintillation counters (scintillators), and radiation dosimeters ((Figure)). Probably the best-known radiation instrument, the Geiger counter (also called the Geiger-Müller counter) detects and measures radiation. Radiation causes the ionization of the gas in a Geiger-Müller tube. The rate of ionization is proportional to the amount of radiation. A scintillation counter contains a scintillator-a material that emits light (luminesces) when excited by ionizing radiation-and a sensor that converts the light into an electric signal. Radiation dosimeters also measure ionizing radiation and are often used to determine personal radiation exposure. Commonly used types are electronic, film badge, thermoluminescent, and quartz fiber dosimeters.

Devices such as (a) Geiger counters, (b) scintillators, and (c) dosimeters can be used to measure radiation. (credit c: modification of work by "osaMu”/Wikimedia commons)

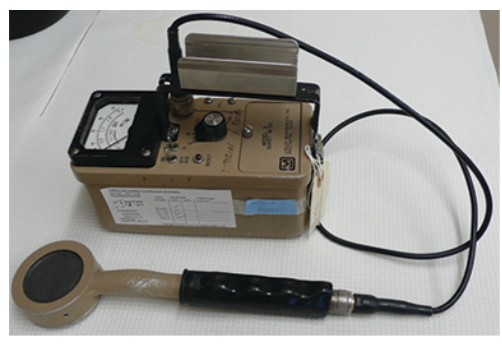

(a)

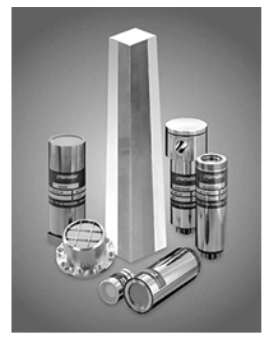

(b)

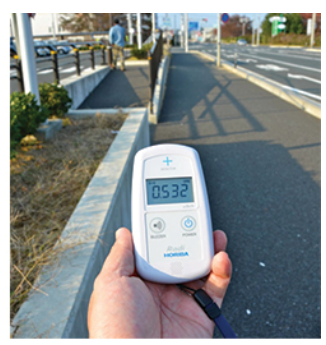

(c)

A variety of units are used to measure various aspects of radiation ((Figure)). The SI unit for rate of radioactive decay is the becquerel $(\mathrm{Bq})$, with $1 \mathrm{~Bq}=1$ disintegration 
per second. The curie (Ci) and millicurie (mCi) are much larger units and are frequently used in medicine (1 curie $=1 \mathrm{Ci}=3.710^{10}$ disintegrations per second). The SI unit for measuring radiation dose is the gray $(\mathrm{Gy})$, with $1 \mathrm{~Gy}=$ $1 \mathrm{~J}$ of energy absorbed per kilogram of tissue. In medical applications, the radiation absorbed dose (rad) is more often used ( $1 \mathrm{rad}=0.01 \mathrm{~Gy} ; 1$ rad results in the absorption of $0.01 \mathrm{~J} / \mathrm{kg}$ of tissue). The SI unit measuring tissue damage caused by radiation is the sievert (Sv). This takes into account both the energy and the biological effects of the type of radiation involved in the radiation dose. The roentgen equivalent for man (rem) is the unit for radiation damage that is used most frequently in medicine (100 rem $=1 \mathrm{~Sv}$ ). Note that the tissue damage units (rem or Sv) includes the energy of the radiation dose (rad or Gy) along with a biological factor referred to as the RBE (for relative biological effectiveness) that is an approximate measure of the relative damage done by the radiation. These are related by:

number of rems $=$ RBE number of rads

with RBE approximately 10 for $\alpha$ radiation, 2(+) for protons and neutrons, and 1 for $\beta$ and $\gamma$ radiation.

Different units are used to measure the rate of emission from a radioactive source, the energy that is absorbed from the source, and the amount of damage the absorbed radiation does. 


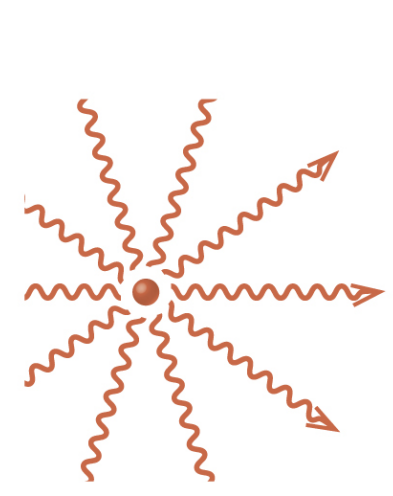

Rate of radioactive decay measured in bequerels or curies

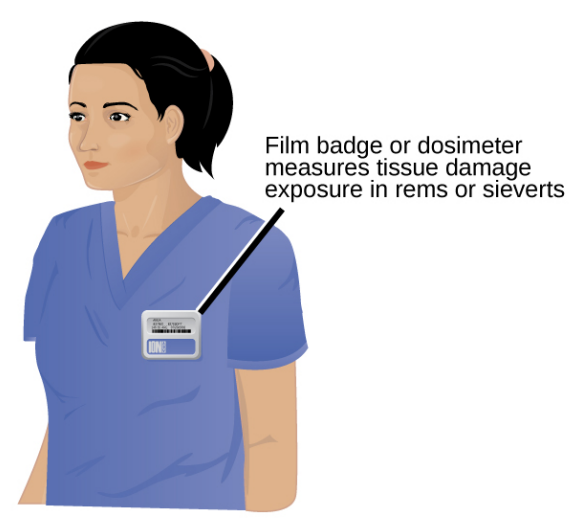

Absorbed dose measured in grays or rads

\section{Units of Radiation Measurement}

(Figure) summarizes the units used for measuring radiation.

\section{Units Used for Measuring Radiation}

\begin{tabular}{|c|c|c|c|}
\hline $\begin{array}{l}\text { Measurement } \\
\text { Purpose }\end{array}$ & Unit & $\begin{array}{l}\text { Quantity } \\
\text { Measured }\end{array}$ & Description \\
\hline \multirow{2}{*}{$\begin{array}{l}\text { activity of } \\
\text { source }\end{array}$} & $\begin{array}{l}\text { becquerel } \\
\text { (Bq) }\end{array}$ & \multirow{2}{*}{$\begin{array}{l}\text { radioactive } \\
\text { decays or } \\
\text { emissions }\end{array}$} & $\begin{array}{l}\text { amount of sample that } \\
\text { undergoes } 1 \text { decay/ } \\
\text { second }\end{array}$ \\
\hline & curie (Ci) & & $\begin{array}{l}\text { amount of sample that } \\
\text { undergoes } 3.710^{10} \\
\text { decays/second }\end{array}$ \\
\hline \multirow[b]{2}{*}{ absorbed dose } & gray (Gy) & \multirow{3}{*}{$\begin{array}{l}\text { energy } \\
\text { absorbed } \\
\text { per kg of } \\
\text { tissue }\end{array}$} & $1 \mathrm{~Gy}=1 \mathrm{~J} / \mathrm{kg}$ tissue \\
\hline & $\begin{array}{l}\text { radiation } \\
\text { absorbed } \\
\text { dose (rad) }\end{array}$ & & $1 \mathrm{rad}=0.01 \mathrm{~J} / \mathrm{kg}$ tissue \\
\hline & sievert (Sv) & & $\mathrm{Sv}=\mathrm{RBE} \quad \mathrm{Gy}$ \\
\hline $\begin{array}{l}\text { biologically } \\
\text { effective dose }\end{array}$ & $\begin{array}{l}\text { roentgen } \\
\text { equivalent } \\
\text { for man } \\
\text { (rem) }\end{array}$ & $\begin{array}{l}\text { tissue } \\
\text { damage }\end{array}$ & $\mathrm{Rem}=\mathrm{RBE} \mathrm{rad}$ \\
\hline
\end{tabular}


Body

The effects of radiation depend on the type, energy, and location of the radiation source, and the length of exposure. As shown in (Figure), the average person is exposed to background radiation, including cosmic rays from the sun and radon from uranium in the ground (see the Chemistry in Everyday Life feature on Radon Exposure); radiation from medical exposure, including CAT scans, radioisotope tests, X-rays, and so on; and small amounts of radiation from other human activities, such as airplane flights (which are bombarded by increased numbers of cosmic rays in the upper atmosphere), radioactivity from consumer products, and a variety of radionuclides that enter our bodies when we breathe (for example, carbon-14) or through the food chain (for example, potassium-40, strontium-90, and iodine-131). The total annual radiation exposure for a person in the US is about 620 mrem. The various sources and their relative amounts are shown in this bar graph. (source: U.S. Nuclear Regulatory Commission) 
Radiation Doses and Regulatory Limits (in Millirems)

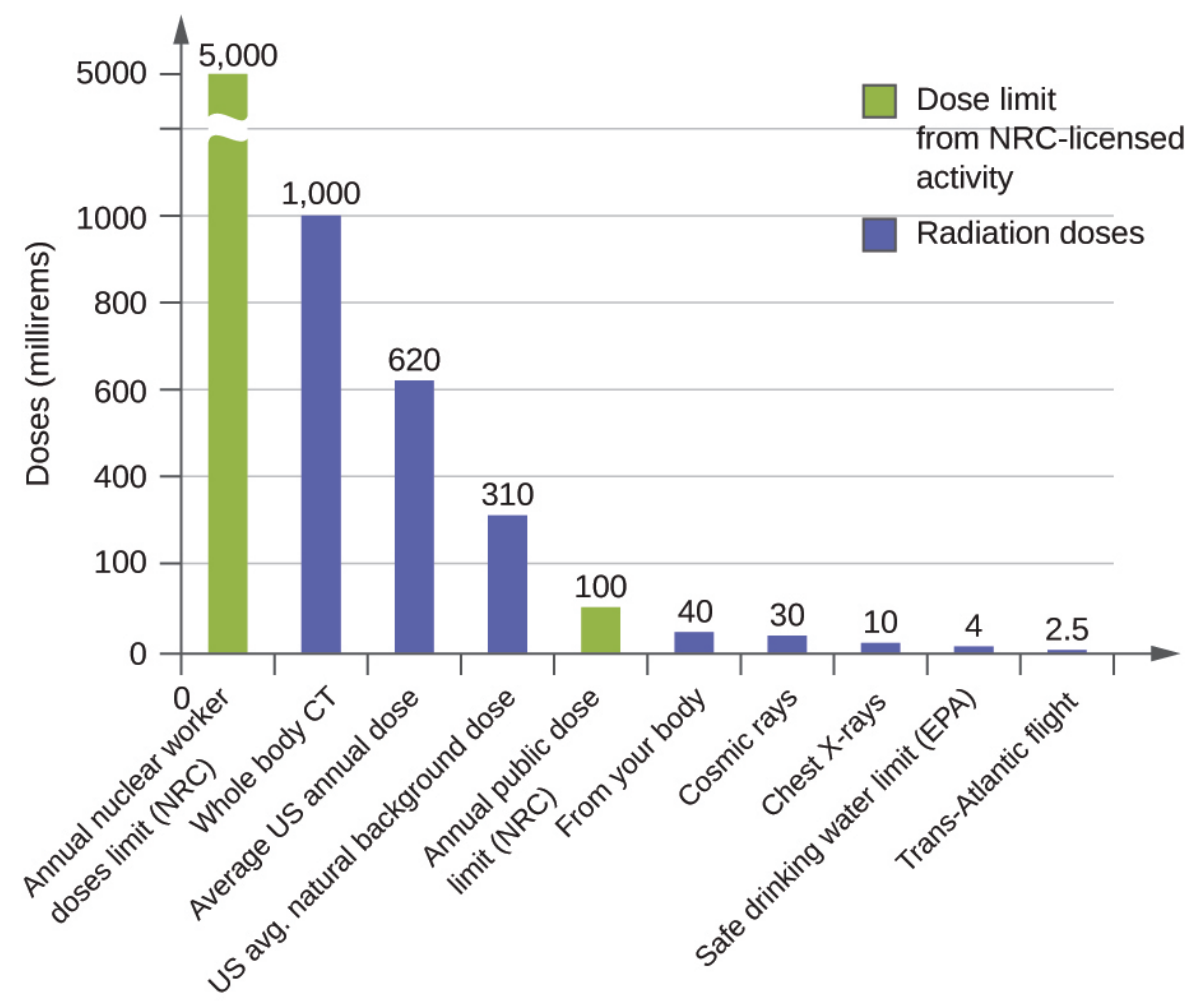

A short-term, sudden dose of a large amount of radiation can cause a wide range of health effects, from changes in blood chemistry to death. Short-term exposure to tens of rems of radiation will likely cause very noticeable symptoms or illness; a dose of about 500 rems is estimated to have a $50 \%$ probability of causing the death of the victim within 30 days of exposure. Exposure to radioactive emissions has a cumulative effect on the body during a person's lifetime, which is another reason why it is important to avoid any unnecessary exposure to radiation. Health effects of short-term exposure to radiation are shown in (Figure). 


$\begin{array}{lll}\begin{array}{l}\text { Health Effects of Radiation } \\ \text { Exposure } \\ \text { (rem) }\end{array} & \text { Health Effect } & \begin{array}{l}\text { Time to Onset (without } \\ \text { treatment) }\end{array} \\ 5-10 & \begin{array}{l}\text { changes in blood } \\ \text { chemistry }\end{array} & - \\ 50 & \text { nausea } \\ 55 & \text { fatigue } & \text { hours } \\ 70 & \text { vomiting } & - \\ 75 & \text { hair loss } & - \\ 90 & \text { diarrhea } & 2-3 \text { weeks } \\ 100 & \text { hemorrhage } & - \\ 400 & \text { possible death } & - \\ 1000 & \begin{array}{l}\text { destruction of intestinal } \\ \text { lining }\end{array} & - \\ & \text { internal bleeding } & - \\ & \text { death } & 1-2 \text { weeks } \\ & \begin{array}{l}\text { damage to central } \\ \text { nervous system } \\ \text { loss of consciousness; }\end{array} & - \\ & \text { death } & \text { minutes } \\ & & \text { hours to days }\end{array}$

It is impossible to avoid some exposure to ionizing radiation. We are constantly exposed to background radiation from a variety of natural sources, including cosmic radiation, rocks, medical procedures, consumer products, and even our own atoms. We can minimize our exposure by blocking or shielding the radiation, moving farther from the source, and limiting the time of exposure.

Key Concepts and Summary

We are constantly exposed to radiation from a variety of 
naturally occurring and human-produced sources. This radiation can affect living organisms. Ionizing radiation is the most harmful because it can ionize molecules or break chemical bonds, which damages the molecule and causes malfunctions in cell processes. It can also create reactive hydroxyl radicals that damage biological molecules and disrupt physiological processes. Radiation can cause somatic or genetic damage, and is most harmful to rapidly reproducing cells. Types of radiation differ in their ability to penetrate material and damage tissue, with alpha particles the least penetrating but potentially most damaging and gamma rays the most penetrating.

Various devices, including Geiger counters, scintillators, and dosimeters, are used to detect and measure radiation, and monitor radiation exposure. We use several units to measure radiation: becquerels or curies for rates of radioactive decay; gray or rads for energy absorbed; and rems or sieverts for biological effects of radiation. Exposure to radiation can cause a wide range of health effects, from minor to severe, and including death. We can minimize the effects of radiation by shielding with dense materials such as lead, moving away from the source, and limiting time of exposure.

Key Equations

- $\operatorname{rem}=\mathrm{RBE} \operatorname{rad}$

- $\mathrm{Sv}=\mathrm{RBE} \mathrm{Gy}$ 
Chemistry End of Chapter Exercises

If a hospital were storing radioisotopes, what is the minimum containment needed to protect against:

(a) cobalt-60 (a strong $\gamma$ emitter used for irradiation)

(b) molybdenum-99 (a beta emitter used to produce technetium-99 for imaging)

Based on what is known about Radon-222's primary decay method, why is inhalation so dangerous?

Alpha particles can be stopped by very thin shielding but have much stronger ionizing potential than beta particles, $\mathrm{X}$-rays, and $\gamma$-rays. When inhaled, there is no protective skin covering the cells of the lungs, making it possible to damage the DNA in those cells and cause cancer.

Given specimens uranium-232 $\left(t_{1 / 2}=68.9 \mathrm{y}\right)$ and uranium-233 $\left(t_{1 / 2}=159,200 \mathrm{y}\right)$ of equal mass, which one would have greater activity and why?

A scientist is studying a $2.234 \mathrm{~g}$ sample of thorium-229 $\left(t_{1 /}\right.$ $2=7340 \mathrm{y}$ ) in a laboratory.

(a) What is its activity in Bq?

(b) What is its activity in $\mathrm{Ci}$ ?

(a) $7.6410^{9} \mathrm{~Bq}$; (b) $2.0610^{-2} \mathrm{Ci}$

Given specimens neon-24 $\left(t_{1 / 2}=3.38 \mathrm{~min}\right)$ and bismuth-211 $\left(t_{1 / 2}=2.14 \mathrm{~min}\right)$ of equal mass, which one would have greater activity and why? 
Footnotes

- 1Source: US Environmental Protection Agency

Glossary

becquerel (Bq)

SI unit for rate of radioactive decay; $1 \mathrm{~Bq}=1$ disintegration/s

\section{curie (Ci)}

larger unit for rate of radioactive decay frequently used in medicine; $1 \mathrm{Ci}=3.710^{10}$ disintegrations/s

\section{Geiger counter}

instrument that detects and measures radiation via the ionization produced in a Geiger-Müller tube

\section{gray (Gy)}

SI unit for measuring radiation dose; 1 Gy =

$1 \mathrm{~J}$ absorbed/kg tissue

ionizing radiation

radiation that can cause a molecule to lose an electron and form an ion

\section{millicurie (mCi)}

larger unit for rate of radioactive decay

frequently used in medicine; $1 \mathrm{Ci}=3.710^{10}$

disintegrations/s

\section{nonionizing radiation}

radiation that speeds up the movement of atoms and molecules; it is equivalent to heating a sample, but is not energetic enough 
to cause the ionization of molecules

radiation absorbed dose (rad)

SI unit for measuring radiation dose,

frequently used in medical applications; 1 rad

$=0.01$ Gy

radiation dosimeter

device that measures ionizing radiation

and is used to determine personal radiation

exposure

relative biological effectiveness (RBE)

measure of the relative damage done by

radiation

roentgen equivalent man (rem)

unit for radiation damage, frequently used

in medicine; $100 \mathrm{rem}=1 \mathrm{~Sv}$

scintillation counter

instrument that uses a scintillator-a

material that emits light when excited by

ionizing radiation-to detect and measure

radiation

sievert (Sv)

SI unit measuring tissue damage caused by radiation; takes into account energy and biological effects of radiation 


\section{Focus on Nuclear Energy}

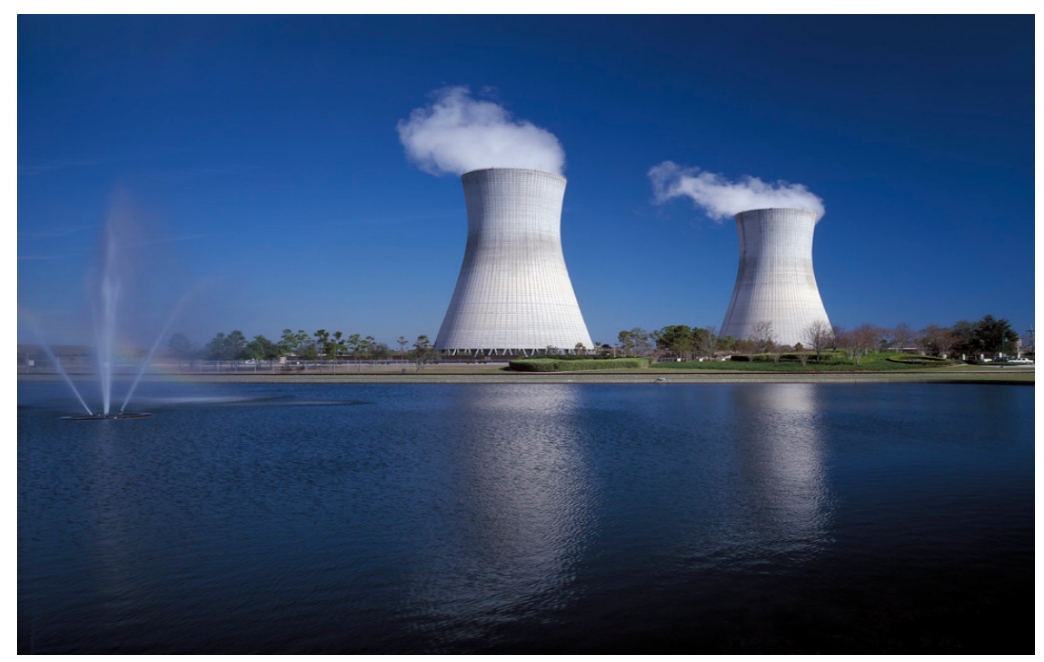

"Cooling towers" by US Department of State is licensed under CC BY-NC 2.0

When nuclear energy came into the mainstream in the 1950's, it was envisioned, by then chairman of the U.S. Atomic Energy Commission Lewis L Strauss, to be such a cheap and powerful source of energy that "our children would enjoy in their homes electrical energy too cheap to meter." While some countries like France have since adopted and developed 'nuclear' as the primary source of energy for electricity generation, in most countries the development of nuclear power has slowed considerably. While the energy potential might remain, accidents and other complications have led to considerable costs and political opposition to the development of nuclear power programs.

Refer to the information discussed in the previous Focus section as well as Part XIV of this text and elaborate on the following focus boxes, solving any problems that appear. While learning the basics of balanced nuclear reactions and radioactivity, make note of important distinctions between nuclear and other types of fuels in meeting our energy demands. In order to determine the best route to meeting our future energy needs, one must understand the benefits and challenges of the processes that are currently in use. 


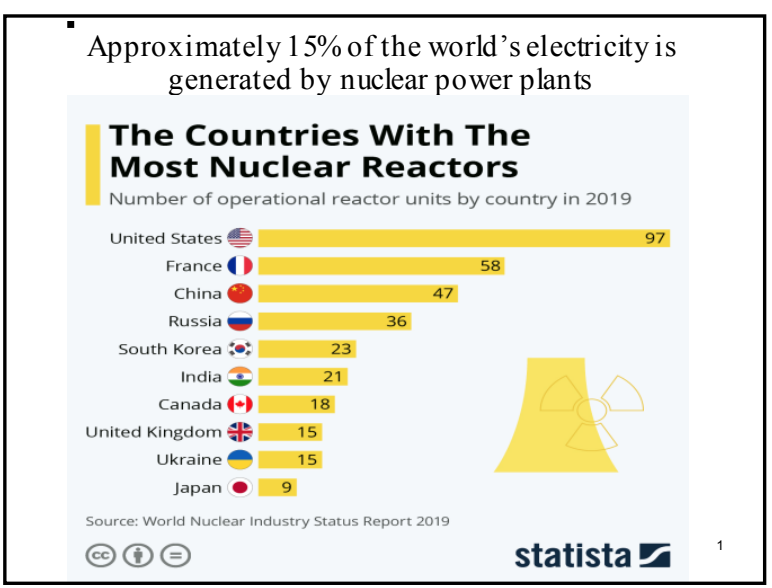

Nuclear fission - the splitting of a large nucleus into smaller ones with the release of energy.

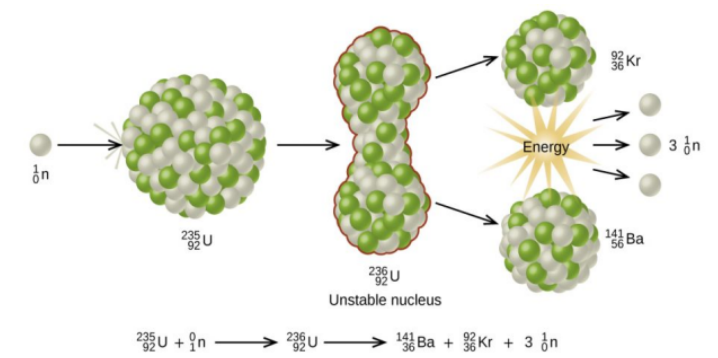

- Show the balanced nuclear reaction for U235 absorbing a neutron and undergoing fission to produce Ba-141, Kr-92 and neutrons. 
Energy is released because the sum of the masses of these fragments is less than the original mass, and this missing mass (about 0.1 percent of the original mass of U-235) has been converted into energy according to Einstein's equation:

\section{$\mathrm{E}=\mathrm{mc}^{2}$}

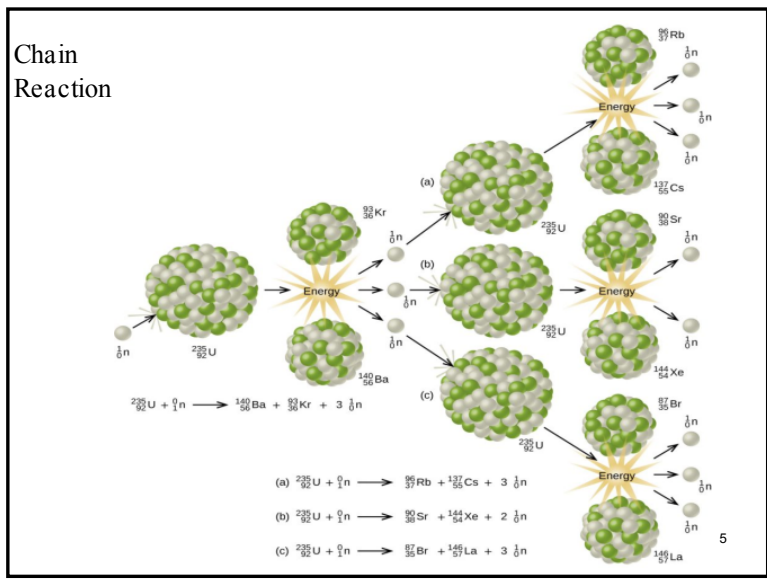

\section{Using $\mathrm{E}=\mathrm{mc}^{2}$}

Ex: Calculate the amount of energy that is released when $1.0 \mathrm{~kg}$ of U-235 undergoes nuclear fission.

$0.1 \%$ of the mass is converted to energy 


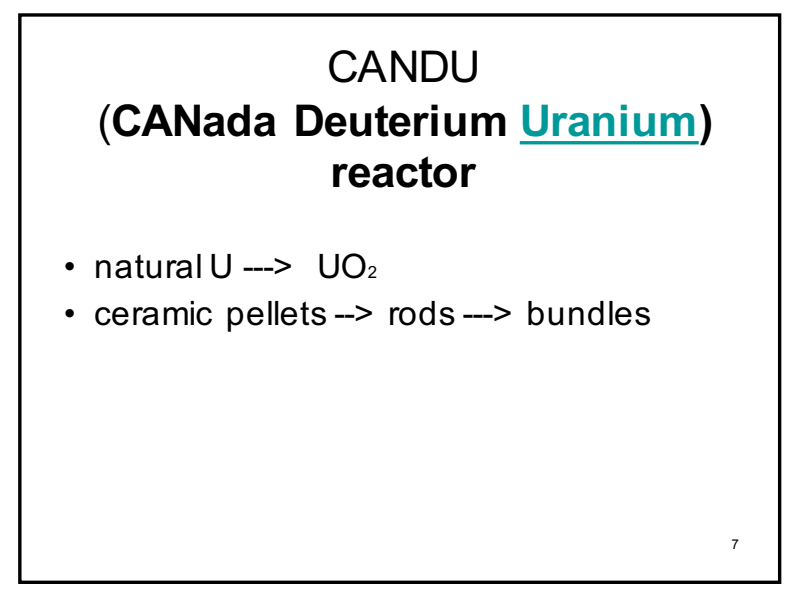

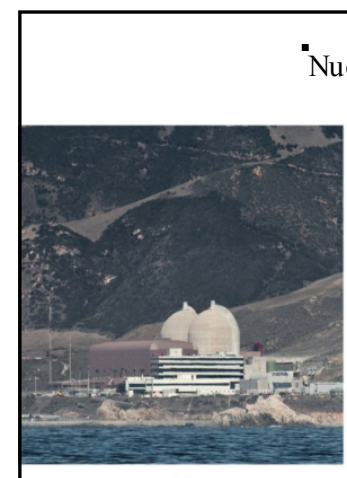

(a)

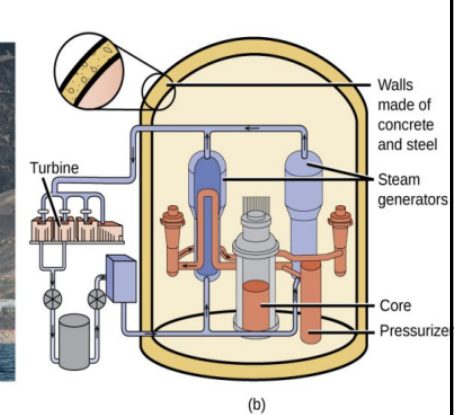

(b)

Chemistry and the Environment by Devin R. Latimer is licensed under a Creative Commons Attribution 4.0 International License

\section{For equivalent energy production}

- 3-400 tons coal - one CANDU

- emitting: bundle

-1400 tons $\mathrm{CO} 2 \cdot$ no C, S or $\mathrm{N}$

-12 tons SOx

-4 tons NOx

- Expensive and becoming more so

oxides emitted into air

- Postulated to potentially be a very cheap source of energy 
Could a nuclear power plant undergo a nuclear explosion?

Power plant (controlled fission)

$=0.7-5 \%$ U-235

Nuclear explosion (critical mass) $=90 \%$ U-235
Sub-critical mass

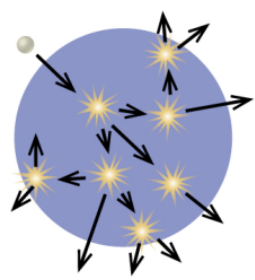

(a)

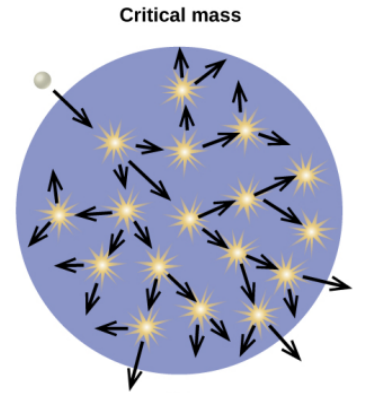

(b)

Chemistry and the Environment by Devin R. Latimer is licensed under 11 a Creative Commons Attribution 4.0 International License

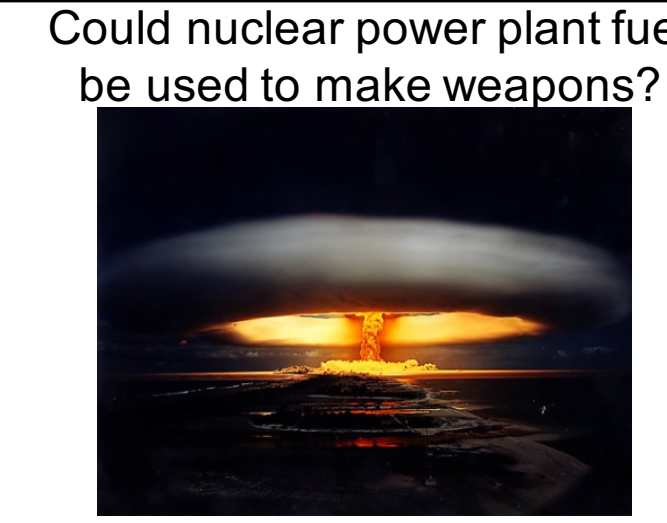

"Nuclear explosion" by Fotos CGQ is licensed under CC BY-NC 2.0 ${ }^{12}$ 
The isotopes U-235 and U-238 behave essentially the same in all chemical reactions, so the separation/purification of either of these isotopes is extremely difficult.

However, plutonium is a much more likely candidate. Show the balanced nuclear reaction of how U-238 absorbing neutrons can create Pu-239 via the release of beta particles.

\section{Accidents/Waste}

Large amounts of radioactivity can be very dangerous.

Radioactivity - the spontaneous emission of radiation by certain elements

\begin{tabular}{|c|c|c|c|}
\hline Type & Nuclear equation & Representation & $\begin{array}{l}\text { Change in } \\
\text { mass/atomic numbers }\end{array}$ \\
\hline Alpha decay & ${ }_{2}^{A} X \quad{ }_{2}^{4} \mathrm{He}+\frac{\mathrm{A}-4}{2-2} \mathrm{Y}$ & 890 & $\begin{array}{l}\text { A: decrease by } 4 \\
\text { Z: decrease by } 2\end{array}$ \\
\hline Beta decay & ${ }_{Z}^{A} X \quad{ }_{-1}^{0} \mathrm{e}+{ }_{Z+1}^{A} Y$ & 3 & $\begin{array}{l}\text { A: unchanged } \\
\text { Z: increase by } 1\end{array}$ \\
\hline $\begin{array}{l}\text { Gamma } \\
\text { decay }\end{array}$ & $\frac{A}{2} x \quad \delta \gamma+\frac{A}{2} y$ & $(823) \stackrel{v}{\text { Excited nuclear state }^{2}}$ & $\begin{array}{l}\text { A: unchanged } \\
Z \text { : unchanged }\end{array}$ \\
\hline $\begin{array}{l}\text { Positron } \\
\text { emission }\end{array}$ & ${ }_{2}^{A} X \quad{ }_{+1}^{0} \mathrm{e}+Y-{ }_{-1}^{A} Y$ & 800 & $\begin{array}{l}\text { A: unchanged } \\
\text { Z: decrease by } 1\end{array}$ \\
\hline $\begin{array}{l}\text { Electron } \\
\text { capture }\end{array}$ & ${ }_{z}^{A} X \quad-00+Y-1 Y$ & $8909 \longrightarrow \underset{x \text {-ray } m \rightarrow}{\longrightarrow} 890$ & $\begin{array}{l}\text { A: unchanged } \\
\text { Z: decrease by } 1\end{array}$ \\
\hline
\end{tabular}



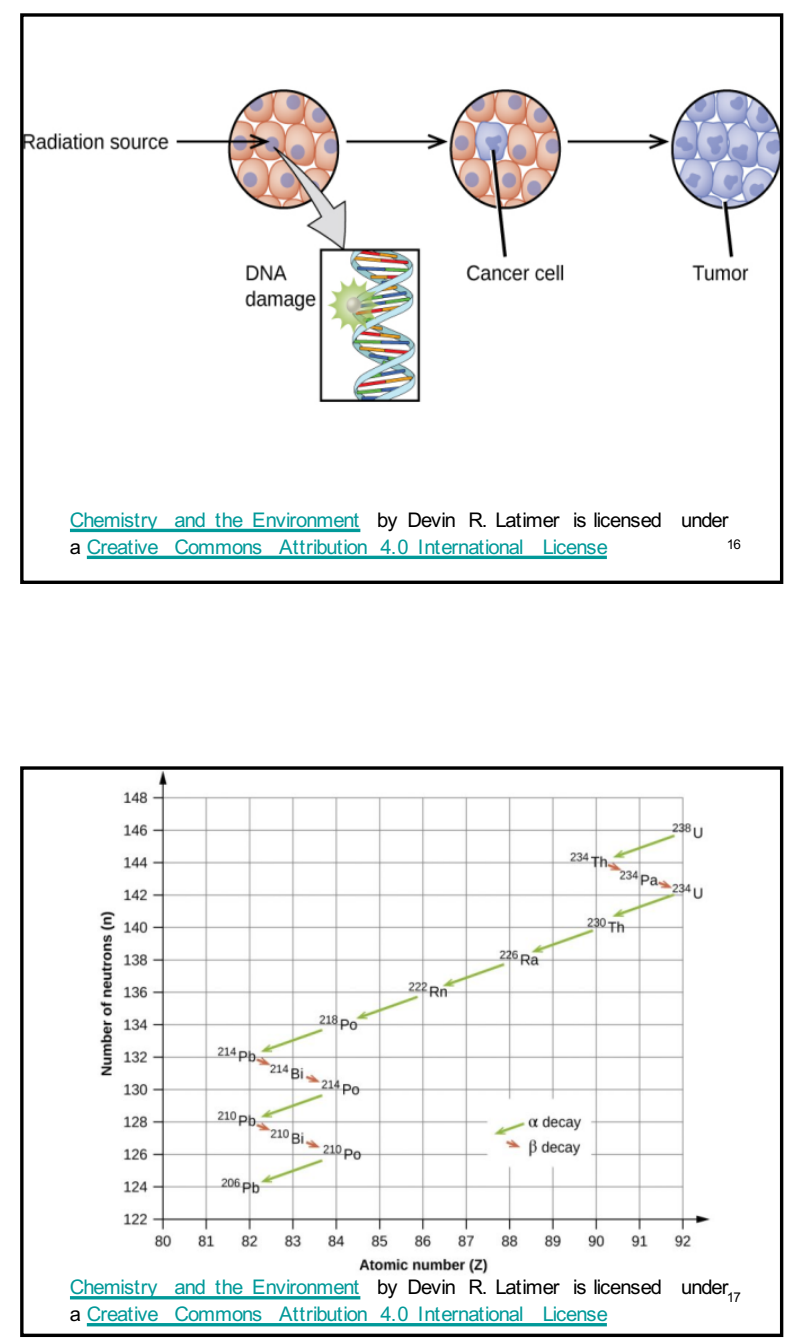

Half-life $\left(t_{1 / 2}\right)$ : the time required for a quantity to reduce to half of its initial value.

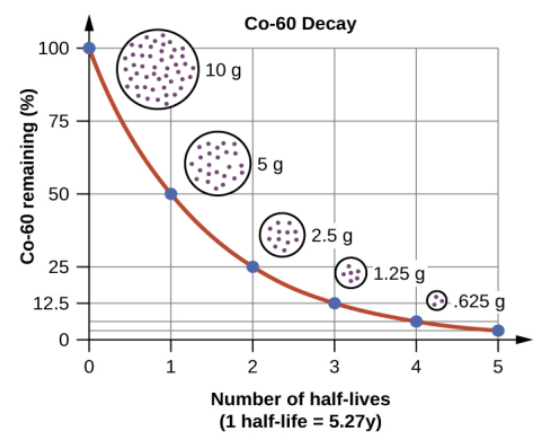

Chemistry and the Environment by Devin R. Latimer is licensed under18 a Creative Commons Attribution 4.0 International License 

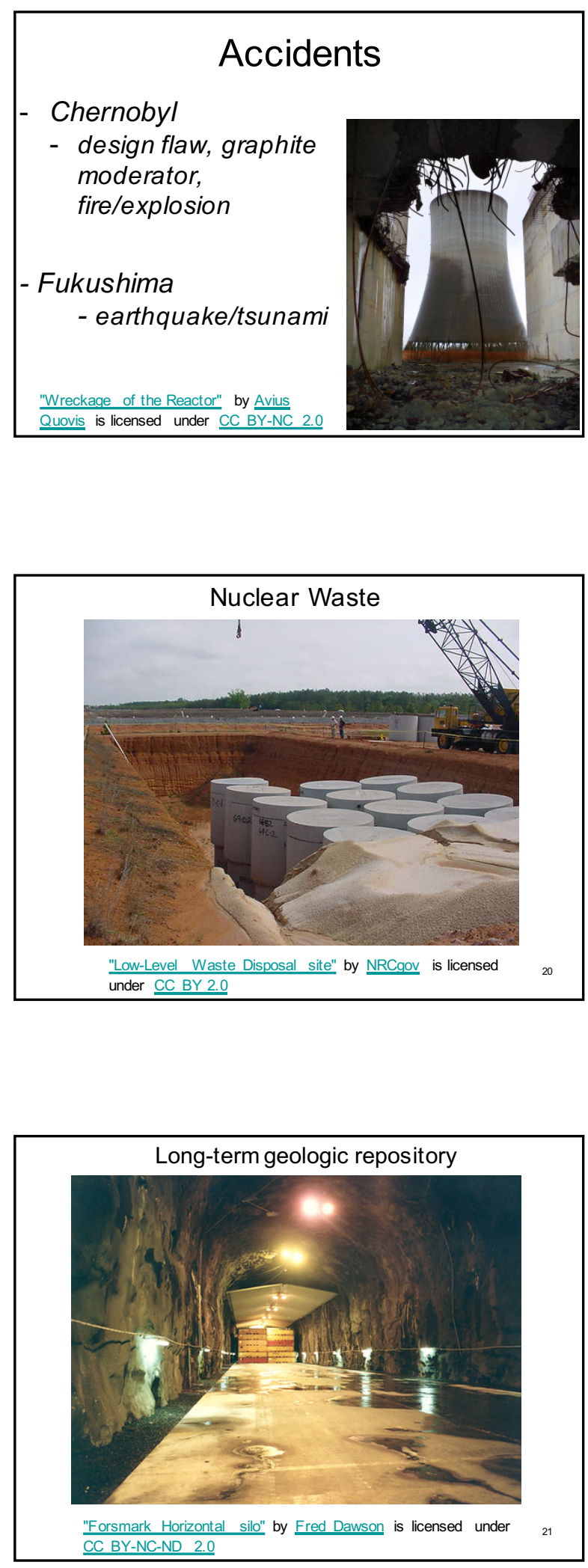


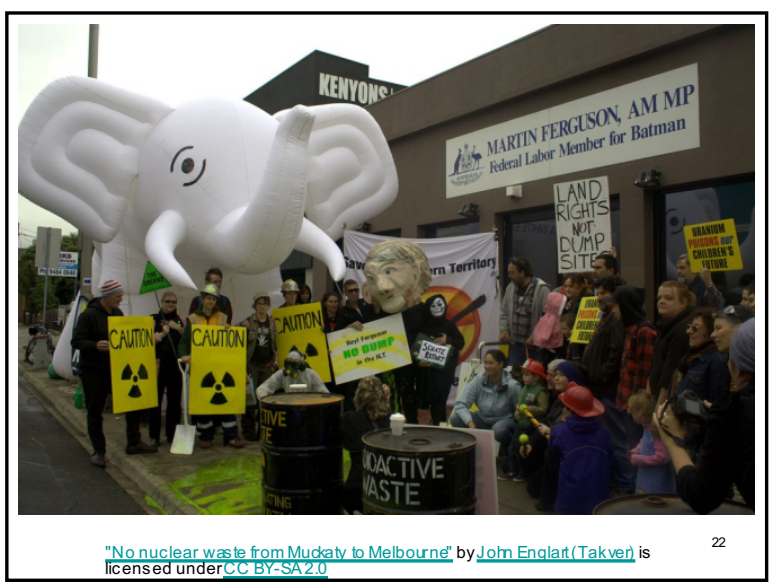

\section{Spent Nuclear Fuel: A Trash} Heap Deadly for 250,000 Years or a Renewable Energy Source?

- http://www.scientificamerican.com/article.c fm? id=nuclear-waste-lethal-trash-orrenewable-energy-source

A sample of $10 \mathrm{Kg}$ of Thorium-234 is allowed to radioactively decay for

96.4 days. Approximately, how much Th-234 remains? [Th-234 $\mathrm{t}_{1 / 2}=24.1$ days]

- A. $7.5 \mathrm{Kg}$

- B. $5 \mathrm{Kg}$

- C. $2.5 \mathrm{Kg}$

-D. $1.25 \mathrm{Kg}$

- E. $0.625 \mathrm{Kg}$ 
Focus on Nuclear Energy

\section{Carbon Dating}

- A sample of fossilized remains is found to contain $6 \%$ of the original (living organism)

${ }^{14} \mathrm{C}$. Approximately, how old are the

remains? [C-14 $\mathrm{t}_{1 / 2}=5715$ years]

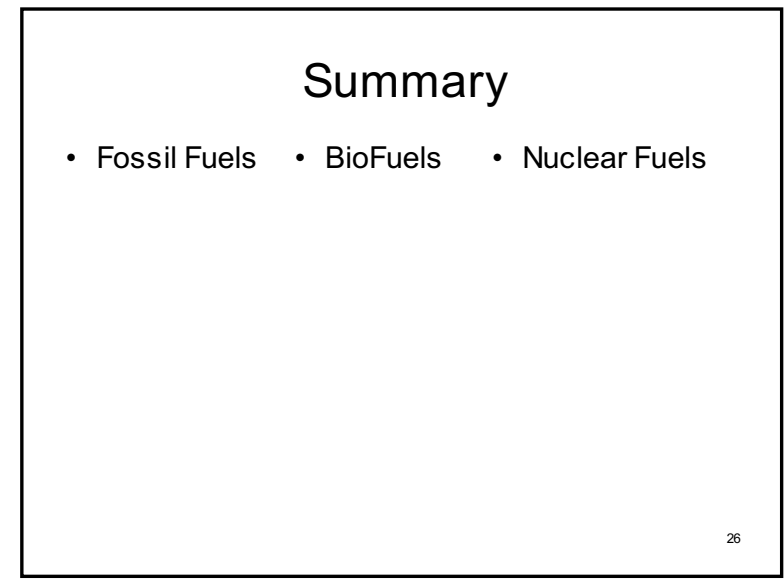


Appendix 


\section{THE PERIODIC TABLE}

음

Group Periodic Table of the Elements

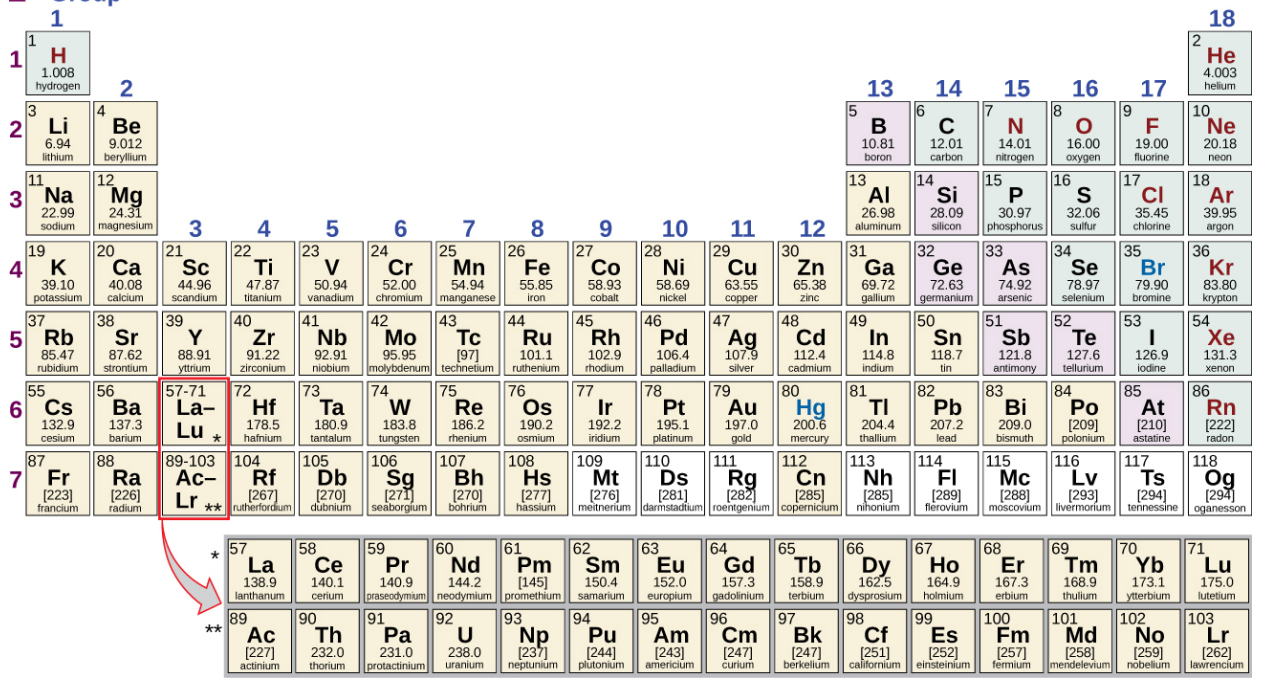

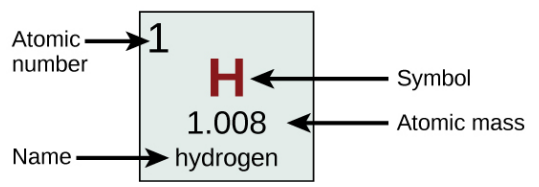

\begin{tabular}{|ll}
$\square$ Metal & Solid \\
$\square$ Metalloid & Liquid \\
$\square$ Nonmetal & Gas
\end{tabular}




\section{ESSENTIAL MATHEMATICS}

Exponential Arithmetic

Exponential notation is used to express very large and very small numbers as a product of two numbers. The first number of the product, the digit term, is usually a number not less than 1 and not equal to or greater than 10. The second number of the product, the exponential term, is written as 10 with an exponent. Some examples of exponential notation are:

$$
\begin{aligned}
1000 & =110^{3} \\
100 & =110^{2} \\
10 & =110^{1} \\
1 & =110^{0} \\
0.1 & =110^{-1} \\
0.001 & =110^{-3} \\
2386 & =2.3861000=2.38610^{3} \\
0.123 & =1.230 .1=1.2310^{-1}
\end{aligned}
$$

The power (exponent) of 10 is equal to the number of places the decimal is shifted to give the digit number. The exponential method is particularly useful notation 
for every large and very small numbers. For example, $1,230,000,000=1.2310^{9}$, and $0.00000000036=3.610^{-10}$.

\section{Addition of Exponentials}

Convert all numbers to the same power of 10 , add the digit terms of the numbers, and if appropriate, convert the digit term back to a number between 1 and 10 by adjusting the exponential term.

Adding Exponentials Add $5.0010^{-5}$ and $3.0010^{-3}$.

Solution

$$
\begin{aligned}
3.0010^{-3} & =30010^{-5} \\
\left(5.0010^{-5}\right)+\left(30010^{-5}\right) & =30510^{-5}=3.0510^{-3}
\end{aligned}
$$

Subtraction of Exponentials

Convert all numbers to the same power of 10 , take the difference of the digit terms, and if appropriate, convert the digit term back to a number between 1 and 10 by adjusting the exponential term.

Subtracting Exponentials Subtract 4.0 $10^{-7}$ from $5.010^{-6}$.

Solution

$$
\begin{aligned}
& 4.010^{-7}=\quad 0.4010^{-6} \\
& \left(5.010^{-6}\right)-\left(0.4010^{-6}\right)=4.610^{-6}
\end{aligned}
$$

Multiplication of Exponentials

Multiply the digit terms in the usual way and add the exponents of the exponential terms. 
Multiplying Exponentials Multiply $4.210^{-8}$ by $2.010^{3}$.

Solution

$$
\left(\begin{array}{ll}
4.210^{-8}
\end{array}\right)\left(2.010^{3}\right)=\left(\begin{array}{ll}
4.2 & 2.0
\end{array}\right) 10^{(-8)+(+3)}=8.410^{-5}
$$

Division of Exponentials

Divide the digit term of the numerator by the digit term of the denominator and subtract the exponents of the exponential terms.

Dividing Exponentials Divide $3.610^{5}$ by $6.010^{-4}$.

Solution

$$
\frac{3.610^{-5}}{6.010^{-4}}=\left(\frac{3.6}{6.0}\right) \quad 10^{(-5)-(-4)}=0.6010^{-1}=6.010^{-2}
$$

Squaring of Exponentials

Square the digit term in the usual way and multiply the exponent of the exponential term by 2 .

Squaring Exponentials Square the number $4.010^{-6}$.

Solution

$\left(4.010^{-6}\right)^{2}=4410^{2}(-6)=1610^{-12}=1.610^{-11}$

Cubing of Exponentials

Cube the digit term in the usual way and multiply the exponent of the exponential term by 3 .

Cubing Exponentials Cube the number $210^{4}$. 


\section{Solution}

$$
\left(210^{4}\right)^{3}=22210^{34}=810^{12}
$$

\section{Taking Square Roots of Exponentials}

If necessary, decrease or increase the exponential term so that the power of 10 is evenly divisible by 2 . Extract the square root of the digit term and divide the exponential term by 2 .

Finding the Square Root of Exponentials Find the square root of $1.610^{-7}$.

Solution

$$
\begin{aligned}
1.610^{-7} & =1610^{-8} \\
\sqrt{1610^{-8}}=\sqrt{16} \sqrt{10^{-8}} & =\sqrt{16} 10^{-\frac{8}{2}}=4.010^{-4}
\end{aligned}
$$

\section{Significant Figures}

A beekeeper reports that he has 525,341 bees. The last three figures of the number are obviously inaccurate, for during the time the keeper was counting the bees, some of them died and others hatched; this makes it quite difficult to determine the exact number of bees. It would have been more reasonable if the beekeeper had reported the number 525,000. In other words, the last three figures are not significant, except to set the position of the decimal point. Their exact values have no useful meaning in this situation. When reporting quantities, use only as many significant figures as the accuracy of the measurement warrants.

The importance of significant figures lies in their 
application to fundamental computation. In addition and subtraction, the sum or difference should contain as many digits to the right of the decimal as that in the least certain of the numbers used in the computation (indicated by underscoring in the following example).

Addition and Subtraction with Significant Figures Add 4.383 $\mathrm{g}$ and $0.0023 \mathrm{~g}$.

Solution

$4.383 \mathrm{~g}$

$0.0023 \mathrm{~g}$

$4.385 \mathrm{~g}$

In multiplication and division, the product or quotient should contain no more digits than that in the factor containing the least number of significant figures.

Multiplication and Division with Significant Figures Multiply 0.6238 by 6.6 .

Solution

$0.6238 \quad 6.6=4.1$

When rounding numbers, increase the retained digit by 1 if it is followed by a number larger than 5 ("round up"). Do not change the retained digit if the digits that follow are less than 5 ("round down"). If the retained digit is followed by 5 , round up if the retained digit is odd, or round down if it is even (after rounding, the retained digit will thus always be even). 
The Use of Logarithms and Exponential Numbers

The common logarithm of a number (log) is the power to which 10 must be raised to equal that number. For example, the common logarithm of 100 is 2, because 10 must be raised to the second power to equal 100 . Additional examples follow.

\section{Logarithms and Exponential Numbers}

Number Number Expressed Exponentially Common Logarithm

$1000 \quad 10^{3}$

$10 \quad 10^{1}-1$

$1 \quad 10^{0} \quad 0$

$\begin{array}{lll}0.1 & 10^{-1} & -1\end{array}$

$\begin{array}{lll}0.001 & 10^{-3} & -3\end{array}$

What is the common logarithm of 60 ? Because 60 lies between 10 and 100, which have logarithms of 1 and 2, respectively, the logarithm of 60 is 1.7782 ; that is, $60=10^{1.7782}$

The common logarithm of a number less than 1 has a negative value. The logarithm of 0.03918 is -1.4069 , or $0.03918=10^{-1.4069}=\frac{1}{10^{1.4069}}$

To obtain the common logarithm of a number, use the $\log$ button on your calculator. To calculate a number from its logarithm, take the inverse log of the logarithm, or calculate $10^{x}$ (where $x$ is the logarithm of the number).

The natural logarithm of a number (ln) is the power to which $e$ must be raised to equal the number; $e$ is the 
constant 2.7182818. For example, the natural logarithm of 10 is 2.303 ; that is,

$$
10=e^{2.303}=2.7182818^{2.303}
$$

To obtain the natural logarithm of a number, use the ln button on your calculator. To calculate a number from its natural logarithm, enter the natural logarithm and take the inverse $\ln$ of the natural logarithm, or calculate $e^{\mathrm{x}}$ (where $x$ is the natural logarithm of the number).

Logarithms are exponents; thus, operations involving logarithms follow the same rules as operations involving exponents.

1. The logarithm of a product of two numbers is the sum of the logarithms of the two numbers. $\log x y=\log x+\log y$, and $\ln x y=\ln x+\ln y$

2. The logarithm of the number resulting from the division of two numbers is the difference between the logarithms of the two numbers. $\log \frac{x}{y}=\log x-\log y$, and $\ln \frac{x}{y}=\ln x-\ln y$

3. The logarithm of a number raised to an exponent is the product of the exponent and the logarithm of the number. $\log x^{n}=n \log x$ and $\ln x^{n}=n \ln x$

The Solution of Quadratic Equations

Mathematical functions of this form are known as secondorder polynomials or, more commonly, quadratic functions.

$a x^{2}+b x+c=0$ 
The solution or roots for any quadratic equation can be calculated using the following formula:

$x=\frac{-b \sqrt{b^{2}-4 a c}}{2 a}$

Solving Quadratic Equations Solve the quadratic equation $3 x^{2}+13 x-10=0$

Solution Substituting the values $a=3, b=13, c=-10$ in the formula, we obtain

$$
\begin{aligned}
& x=\frac{-13 \sqrt{(13)^{2}-43(-10)}}{23} \\
& x=\frac{-13 \sqrt{169+120}}{6}=\frac{-13 \sqrt{289}}{6}=\frac{-1317}{6}
\end{aligned}
$$

The two roots are therefore

$$
x=\frac{-13+17}{6}=\frac{2}{3} \text { and } x=\frac{-13-17}{6}=-5
$$

Quadratic equations constructed on physical data always have real roots, and of these real roots, often only those having positive values are of any significance.

Two-Dimensional $(x-y)$ Graphing

The relationship between any two properties of a system can be represented graphically by a two-dimensional data plot. Such a graph has two axes: a horizontal one corresponding to the independent variable, or the variable whose value is being controlled $(x)$, and a vertical axis corresponding to the dependent variable, or the variable whose value is being observed or measured $(y)$.

When the value of $y$ is changing as a function of $x$ (that is, different values of $x$ correspond to different values of $y$ ), a graph of this change can be plotted or sketched. The graph can be produced by using specific values for $(x, y)$ data pairs. 
Graphing the Dependence of $y$ on $x$

$\begin{array}{ll}\boldsymbol{x} & \boldsymbol{y} \\ 1 & 5 \\ 2 & 10 \\ 3 & 7 \\ 4 & 14\end{array}$

This table contains the following points: $(1,5),(2,10),(3,7)$, and $(4,14)$. Each of these points can be plotted on a graph and connected to produce a graphical representation of the dependence of $y$ on $x$.

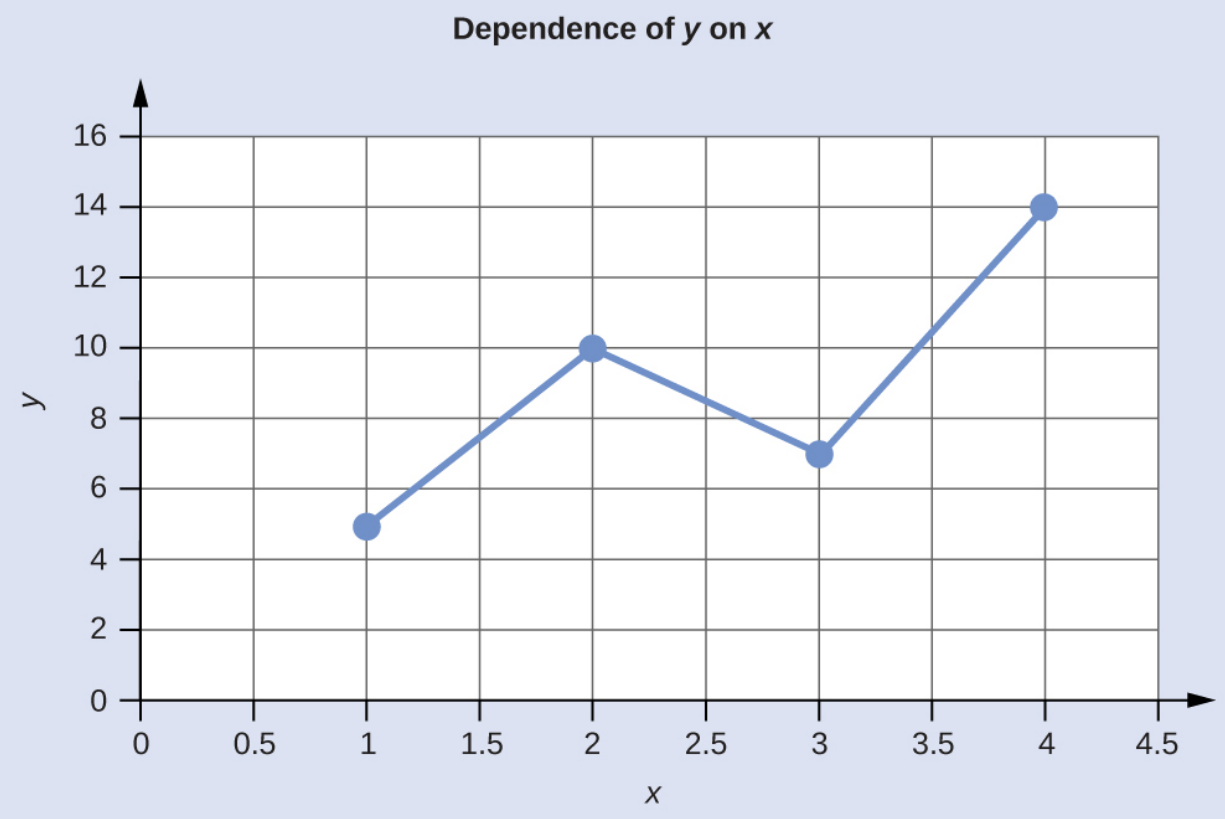

If the function that describes the dependence of $y$ on $x$ is known, it may be used to compute $\mathrm{x}, \mathrm{y}$ data pairs that may subsequently be plotted.

Plotting Data Pairs If we know that $y=x^{2}+2$, we can 
produce a table of a few $(x, y)$ values and then plot the line based on the data shown here.

$\begin{array}{ll}\boldsymbol{x} & \boldsymbol{y}=\boldsymbol{x}^{2}+2 \\ 1 & 3 \\ 2 & 6 \\ 3 & 11 \\ 4 & 18\end{array}$

A graph is titled " $Y$ equals $x$ superscript 2 plus 2. " The $x$-axis ranges from 0 to 4.5. The $y$-axis ranges from 0 to 20 . Four points are plotted as a line graph; the points are 1 and 3,2 and 6,3 and 11 , and 4 and 18. 


\title{
UNITS AND CONVERSION FACTORS
}

\author{
Units of Length \\ meter $(\mathrm{m}) \quad=39.37$ inches (in.) \\ $=1.094$ yards $(\mathrm{yd})$ \\ centimeter $(\mathrm{cm}) \quad=0.01 \mathrm{~m}$ (exact, definition) \\ millimeter $(\mathrm{mm})=0.001 \mathrm{~m}$ (exact, definition) \\ kilometer $(\mathrm{km}) \quad=1000 \mathrm{~m}$ (exact, definition) \\ $\begin{aligned} \operatorname{angstrom}(\AA) & =10^{-8} \mathrm{~cm}(\text { exact, definition) } \\ & =10^{-10} \mathrm{~m}(\text { exact }, \text { definition) }\end{aligned}$ \\ yard $(\mathrm{yd}) \quad=0.9144 \mathrm{~m}$ \\ inch (in.) $\quad=2.54 \mathrm{~cm}$ (exact, definition) \\ mile (US) $\quad=1.60934 \mathrm{~km}$
}




\section{Units of Volume}

$\begin{array}{ll}\text { liter (L) } & =0.001 \mathrm{~m}^{3} \text { (exact, definition) } \\ & =1000 \mathrm{~cm}^{3} \text { (exact, definition) } \\ & =1.057(\mathrm{US}) \text { quarts } \\ \text { milliliter (mL) } & =0.001 \mathrm{~L} \text { (exact, definition) } \\ & =1 \mathrm{~cm}^{3} \text { (exact, definition) } \\ \text { microliter (L) } & =10^{-6} \mathrm{~L} \text { (exact, definition) } \\ & =10^{-3} \mathrm{~cm}^{3} \text { (exact, definition) } \\ \text { liquid quart (US) } & =32(\mathrm{US}) \text { liquid ounces (exact, definition) } \\ & =0.25 \text { (US) gallon (exact, definition) } \\ \text { dry quart } & =1.1012 \mathrm{~L} \\ \text { cubic foot (US) } & =28.316 \mathrm{~L}\end{array}$

\section{Units of Mass}

$$
\begin{array}{ll}
\text { gram }(\mathrm{g}) & =0.001 \mathrm{~kg}(\text { exact, definition) } \\
\operatorname{milligram}(\mathrm{mg}) & =0.001 \mathrm{~g}(\text { exact, definition) } \\
\text { kilogram }(\mathrm{kg}) & =1000 \mathrm{~g}(\text { exact, definition) } \\
& =2.205 \mathrm{lb} \\
\text { ton (metric) } & =1000 \mathrm{~kg}(\text { exact, definition) } \\
\text { ounce }(\mathrm{oz}) & =2204.62 \mathrm{lb} \\
\text { pound }(\mathrm{lb}) & =0.4535924 \mathrm{~kg} \\
\text { ton (short) } & =2000 \mathrm{lb}(\text { exact, definition) } \\
& =907.185 \mathrm{~kg} \\
\text { ton (long) } & =2240 \mathrm{lb}(\text { exact }, \text { definition) } \\
& =1.016 \text { metric ton }
\end{array}
$$




\section{Units of Energy}

\begin{tabular}{|c|c|}
\hline 4.184 joule $(\mathrm{J})$ & $=1$ thermochemical calorie $(\mathrm{cal})$ \\
\hline $\begin{array}{l}1 \text { thermochemical calorie } \\
\text { (cal) }\end{array}$ & $=4.18410^{7} \mathrm{erg}$ \\
\hline erg & $=10^{-7} \mathrm{~J}$ (exact, definition) \\
\hline electron-volt (eV) & $=1.6021810^{-19} \mathrm{~J}=23.061 \mathrm{kcal} \mathrm{mol}^{-1}$ \\
\hline liter-atmosphere & $\begin{array}{l}=24.217 \mathrm{cal}=101.325 \mathrm{~J} \text { (exact, } \\
\text { definition) }\end{array}$ \\
\hline nutritional calorie (Cal) & $=1000 \mathrm{cal}$ (exact, definition) $=4184 \mathrm{~J}$ \\
\hline British thermal unit (BTU) & $=1054.804 \mathrm{~J}^{1}$ \\
\hline
\end{tabular}

\section{Units of Pressure}

torr

$=1 \mathrm{~mm} \mathrm{Hg}$ (exact, definition)

pascal $(\mathrm{Pa})$

$=\mathrm{N} \mathrm{m}^{-2}$ (exact, definition)

$=\mathrm{kg} \mathrm{m}^{-1} \mathrm{~s}^{-2}$ (exact, definition)

$=760 \mathrm{~mm} \mathrm{Hg}$ (exact, definition)

$\begin{aligned} \text { atmosphere }(\mathrm{atm}) & =760 \text { torr }(\text { exact }, \text { definition) } \\ & =101,325 \mathrm{~N} \mathrm{~m}^{-2} \text { (exact, definition) }\end{aligned}$

$=101,325 \mathrm{~Pa}$ (exact, definition)

bar

$=10^{5} \mathrm{~Pa}$ (exact, definition)

$=10^{5} \mathrm{~kg} \mathrm{~m}^{-1} \mathrm{~s}^{-2}$ (exact, definition)

\section{Footnotes}

- 1BTU is the amount of energy needed to heat one pound of water by one degree Fahrenheit. Therefore, the exact relationship of BTU to joules and other energy units depends on the temperature at which BTU is measured. $59^{\circ} \mathrm{F}$ $\left(15^{\circ} \mathrm{C}\right)$ is the most widely used reference temperature for BTU definition in the United States. At this temperature, the conversion factor is the one provided in this table. 


\section{FUNDAMENTAL PHYSICAL CONSTANTS}




\section{Fundamental Physical Constants}

Name and Symbol

atomic mass unit (amu)

Avogadro's number

Boltzmann's constant $(k)$

charge-to-mass ratio for electron $\left(e / m_{\mathrm{e}}\right)$

fundamental unit of charge (e)

electron rest mass $\left(m_{\mathrm{e}}\right)$

Faraday's constant $(F)$

gas constant $(R)$
Value

$1.660540210^{-27} \mathrm{~kg}$

$6.0221407610^{23} \mathrm{~mol}^{-1}$

$1.38064910^{-23} \mathrm{~J} \mathrm{~K}^{-1}$

$1.7588196210^{11} \mathrm{C} \mathrm{kg}^{-1}$

$1.60217663410^{-19} \mathrm{C}$

$9.109389710^{-31} \mathrm{~kg}$

$9.648530910^{4} \mathrm{C} \mathrm{mol}^{-1}$

$8.20578410^{-2} \mathrm{~L}_{\text {atm mol }}^{-1} \mathrm{~K}^{-1}=$ $8.314510 \mathrm{~J} \mathrm{~mol}^{-1} \mathrm{~K}^{-1}$

22.41409 $\mathrm{L} \mathrm{mol}^{-1}$

$1 \mathrm{~atm}, 0{ }^{\circ} \mathrm{C}$

molar volume of an ideal gas, 1 bar, $0{ }^{\circ} \mathrm{C}$

22.71108 $\mathrm{L} \mathrm{mol}^{-1}$

neutron rest mass $\left(m_{\mathrm{n}}\right)$

$1.674927410^{-27} \mathrm{~kg}$

Planck's constant $(h)$

$6.6260701510^{-34} \mathrm{~J} \mathrm{~s}$

proton rest mass $\left(m_{\mathrm{p}}\right)$

$1.672623110^{-27} \mathrm{~kg}$

Rydberg constant (R)

$1.097373153410^{7} \mathrm{~m}^{-1}=2.1798736$ $10^{-18} \mathrm{~J}$

speed of light (in vacuum) (c) $2.9979245810^{8} \mathrm{~m} \mathrm{~s}^{-1}$ 


\section{HALF-LIVES FOR SEVERAL RADIOACTIVE ISOTOPES}


Half-Lives for Several Radioactive Isotopes

\begin{tabular}{|c|c|c|c|c|c|}
\hline Isotope & Half-Life $^{1}$ & $\begin{array}{l}\text { Type of } \\
\text { Emission }^{2}\end{array}$ & Isotope & Half-Life $^{3}$ & $\begin{array}{l}\text { Type of } \\
\text { Emission }\end{array}$ \\
\hline${ }_{6}^{14} \mathrm{C}$ & $5730 \mathrm{y}$ & $(\beta)$ & ${ }_{83}^{210} \mathrm{Bi}$ & $5.01 \mathrm{~d}$ & $(\beta)$ \\
\hline${ }_{7}^{13} \mathrm{~N}$ & $9.97 \mathrm{~m}$ & $\left(\beta^{+}\right)$ & ${ }_{83}^{212} \mathrm{Bi}$ & $60.55 \mathrm{~m}$ & $(\alpha$ or $\beta)$ \\
\hline${ }_{9}^{15} \mathrm{~F}$ & $\begin{array}{l}4.110^{-22} \\
\mathrm{~s}\end{array}$ & $(p)$ & ${ }_{84}^{210} \mathrm{Po}$ & $138.4 \mathrm{~d}$ & $(\alpha)$ \\
\hline${ }_{11}^{24} \mathrm{Na}$ & $15.00 \mathrm{~h}$ & $(\beta)$ & ${ }_{84}^{212} \mathrm{Po}$ & $310^{-7} \mathrm{~s}$ & $(\alpha)$ \\
\hline${ }_{15}^{32} \mathrm{P}$ & $14.29 \mathrm{~d}$ & $(\beta)$ & ${ }_{84}^{216} \mathrm{Po}$ & $0.15 \mathrm{~s}$ & $(\alpha)$ \\
\hline${ }_{19}^{40} \mathrm{~K}$ & $1.2710^{9} \mathrm{y}$ & $(\beta$ or E.C. $)$ & ${ }_{84}^{218} \mathrm{Po}$ & $3.05 \mathrm{~m}$ & $(\alpha)$ \\
\hline${ }_{26}^{49} \mathrm{Fe}$ & $0.08 \mathrm{~s}$ & $\left(\beta^{+}\right)$ & ${ }_{85}^{215} \mathrm{At}$ & $1.010^{-4} \mathrm{~s}$ & $(\alpha)$ \\
\hline${ }_{26}^{60} \mathrm{Fe}$ & $2.610^{6} y$ & $(\beta)$ & ${ }_{85}^{218} \mathrm{At}$ & $1.6 \mathrm{~s}$ & $(\alpha)$ \\
\hline${ }_{27}^{60} \mathrm{Co}$ & $5.27 \mathrm{y}$ & $(\beta)$ & ${ }_{86}^{220} \mathrm{Rn}$ & $55.6 \mathrm{~s}$ & $(\alpha)$ \\
\hline${ }_{37}^{87} \mathrm{Rb}$ & $4.710^{10} \mathrm{y}$ & $(\beta)$ & ${ }_{86}^{222} \mathrm{Rn}$ & $3.82 \mathrm{~d}$ & $(\alpha)$ \\
\hline${ }_{38}^{90} \mathrm{Sr}$ & $29 y$ & $(\beta)$ & ${ }_{88}^{224} \mathrm{Ra}$ & $3.66 \mathrm{~d}$ & $(\alpha)$ \\
\hline${ }_{49}^{115} \operatorname{In}$ & $5.110^{15} \mathrm{y}$ & $(\beta)$ & ${ }_{88}^{226} \mathrm{Ra}$ & $1600 \mathrm{y}$ & $(\alpha)$ \\
\hline${ }_{53}^{131} \mathrm{I}$ & $8.040 \mathrm{~d}$ & $(\beta)$ & ${ }_{88}^{228} \mathrm{Ra}$ & $5.75 \mathrm{y}$ & $(\beta)$ \\
\hline${ }_{58}^{142} \mathrm{Ce}$ & $510^{15} \mathrm{y}$ & $(\alpha)$ & ${ }_{89}^{228} \mathrm{Ac}$ & $6.13 \mathrm{~h}$ & $(\beta)$ \\
\hline${ }_{81}^{208} \mathrm{Tl}$ & $3.07 \mathrm{~m}$ & $(\beta)$ & ${ }_{90}^{228} \mathrm{Th}$ & $1.913 \mathrm{y}$ & $(\alpha)$ \\
\hline${ }_{82}^{210} \mathrm{~Pb}$ & $22.3 \mathrm{y}$ & $(\beta)$ & ${ }_{90}^{232} \mathrm{Th}$ & $1.410^{10} \mathrm{y}$ & $(\alpha)$ \\
\hline${ }_{82}^{212} \mathrm{~Pb}$ & $10.6 \mathrm{~h}$ & $(\beta)$ & ${ }_{90}^{233} \mathrm{Th}$ & $22 \mathrm{~m}$ & $(\beta)$ \\
\hline${ }_{82}^{214} \mathrm{~Pb}$ & $26.8 \mathrm{~m}$ & $(\beta)$ & ${ }_{90}^{234} \mathrm{Th}$ & $24.10 \mathrm{~d}$ & $(\beta)$ \\
\hline${ }_{83}^{206} \mathrm{Bi}$ & $6.243 \mathrm{~d}$ & $(E . C)$. & ${ }_{91}^{233} \mathrm{~Pa}$ & $27 \mathrm{~d}$ & $(\beta)$ \\
\hline
\end{tabular}


Half-Lives for Several Radioactive Isotopes

\begin{tabular}{|c|c|c|c|c|c|}
\hline Isotope & Half-Life $^{1}$ & $\begin{array}{l}\text { Type of } \\
\text { Emission }^{2}\end{array}$ & Isotope & Half-Life ${ }^{3}$ & $\begin{array}{l}\text { Type of } \\
\text { Emission }\end{array}$ \\
\hline${ }_{92}^{233} \mathrm{U}$ & $1.5910^{5} \mathrm{y}$ & $(\alpha)$ & ${ }_{96}^{242} \mathrm{Cm}$ & $162.8 \mathrm{~d}$ & $(\alpha)$ \\
\hline${ }_{92}^{234} \mathrm{U}$ & $2.4510^{5} \mathrm{y}$ & $(\alpha)$ & ${ }_{97}^{243} \mathrm{Bk}$ & $4.5 \mathrm{~h}$ & $(\alpha$ or E.C.) \\
\hline${ }_{92}^{235} \mathrm{U}$ & $7.0310^{8} \mathrm{y}$ & $(\alpha)$ & ${ }_{99}^{253} \mathrm{Es}$ & $20.47 \mathrm{~d}$ & $(\alpha)$ \\
\hline${ }_{92}^{238} \mathrm{U}$ & $4.4710^{9} \mathrm{y}$ & $(\alpha)$ & ${ }_{100}^{254} \mathrm{Fm}$ & $3.24 \mathrm{~h}$ & $(\alpha$ or $S . F)$. \\
\hline${ }_{92}^{239} \mathrm{U}$ & $23.54 \mathrm{~m}$ & $(\beta)$ & ${ }_{100}^{255} \mathrm{Fm}$ & $20.1 \mathrm{~h}$ & $(\alpha)$ \\
\hline${ }_{93}^{239} \mathrm{~Np}$ & $2.3 \mathrm{~d}$ & $(\beta)$ & ${ }_{101}^{256} \mathrm{Md}$ & $76 \mathrm{~m}$ & $(\alpha$ or E.C.) \\
\hline${ }_{94}^{239} \mathrm{Pu}$ & $\begin{array}{ll}2.407 & 10^{4} \\
y\end{array}$ & $(\alpha)$ & ${ }_{102}^{254} \mathrm{No}$ & $55 \mathrm{~s}$ & $(\alpha)$ \\
\hline${ }_{94}^{239} \mathrm{Pu}$ & $6.5410^{3} \mathrm{y}$ & $(\alpha)$ & $\begin{array}{l}257 \\
103\end{array}$ & $0.65 \mathrm{~s}$ & $(\alpha)$ \\
\hline${ }_{94}^{241} \mathrm{Pu}$ & $14.4 \mathrm{y}$ & $(\alpha$ or $\beta)$ & $\begin{array}{l}260 \\
105\end{array}$ & $1.5 \mathrm{~s}$ & $(\alpha$ or $S . F)$. \\
\hline${ }_{95}^{241} \mathrm{Am}$ & $432.2 \mathrm{y}$ & $(\alpha)$ & ${ }_{106}^{263} \mathrm{Sg}$ & $0.8 \mathrm{~s}$ & $(\alpha$ or S.F. $)$ \\
\hline
\end{tabular}

Footnotes

- $1 \mathrm{y}=$ years, $\mathrm{d}=$ days, $\mathrm{h}=$ hours, $\mathrm{m}=$ minutes, $\mathrm{s}=$ seconds

- 2E.C. = electron capture, S.F. = Spontaneous fission

- $3 y=$ years, $d=$ days, $\mathrm{h}=$ hours, $\mathrm{m}=$ minutes, $\mathrm{s}=$ seconds

- 4E.C. = electron capture, S.F. = Spontaneous fission 